Wirtz, Katharina

\title{
Qualitätsbausteine schulischer Inklusion. Organisations-, Personal- und Unterrichtsentwicklung an inklusiven Schulen aus der Sicht unterschiedlicher Beteiligter
}

Bad Heilbrunn : Verlag Julius Klinkhardt 2020, 511 S. - (Perspektiven sonderpädagogischer Forschung) (Dissertation, Universität Koblenz-Landau, 2019)

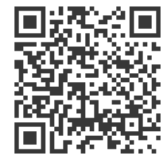

\section{Quellenangabe/ Reference:}

Wirtz, Katharina: Qualitätsbausteine schulischer Inklusion. Organisations-, Personal- und Unterrichtsentwicklung an inklusiven Schulen aus der Sicht unterschiedlicher Beteiligter. Bad Heilbrunn : Verlag Julius Klinkhardt 2020, 511 S. - (Perspektiven sonderpädagogischer Forschung) - (Dissertation, Universität Koblenz-Landau, 2019) - URN: urn:nbn:de:0111-pedocs-210754 - DOI: 10.25656/01:21075

https://nbn-resolving.org/urn:nbn:de:0111-pedocs-210754

https://doi.org/10.25656/01:21075

in Kooperation mit / in cooperation with:

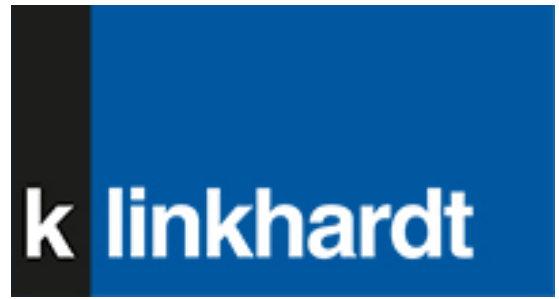

http://www.klinkhardt.de

\section{Nutzungsbedingungen}

Dieses Dokument steht unter folgender Creative Commons-Lizenz: http://creativecommons.org/licenses/by-nc-sa/4.0/deed.de - Sie dürfen das Werk bzw. den Inhalt unter folgenden Bedingungen vervielfältigen, verbreiten und öffentlich zugänglich machen sowie Abwandlungen und Bearbeitungen des Werkes bzw. Inhaltes anfertigen: Sie müssen den Namen des Autors/Rechteinhabers in der von ihm festgelegten Weise nennen. Dies Werk bzw. der Inhalt darf nicht für kommerzielle Zwecke verwendet werden. Die neu entstandenen Werke bzw. Inhalte dürfen nur unter Verwendung von
Lizenzbedingungen weitergegeben werden, die mit denen dieses Lizenzbedingungen weitergegeben werden,
Lizenzvertrages identisch oder vergleichbar sind.

Mit der Verwendung dieses Dokuments erkennen Sie die Nutzungsbedingungen an.

\section{Terms of use}

This document is published under following Creative Commons-License: http://creativecommons.org/licenses/by-nc-sa/4.0/deed.en - You may copy, distribute and transmit, adapt or exhibit the work in the public and alter, transform or change this work as long as you attribute the work in the manner specified by the author or licensor. You are not allowed to make commercial use of the work. If you alter, transform, or change this work in any way, you may distribute the resulting work only under this or a comparable license.

By using this particular document, you accept the above-stated conditions of use.

\section{Kontakt / Contact:}

\section{peDOCS}

DIPF | Leibniz-Institut für Bildungsforschung und Bildungsinformation Informationszentrum (IZ) Bildung

E-Mail: pedocs@dipf.de

Internet: www.pedocs.de

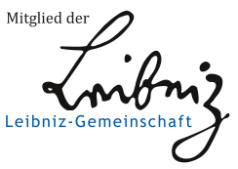




\section{Perspektiven sonderpädagogischer Forschung}

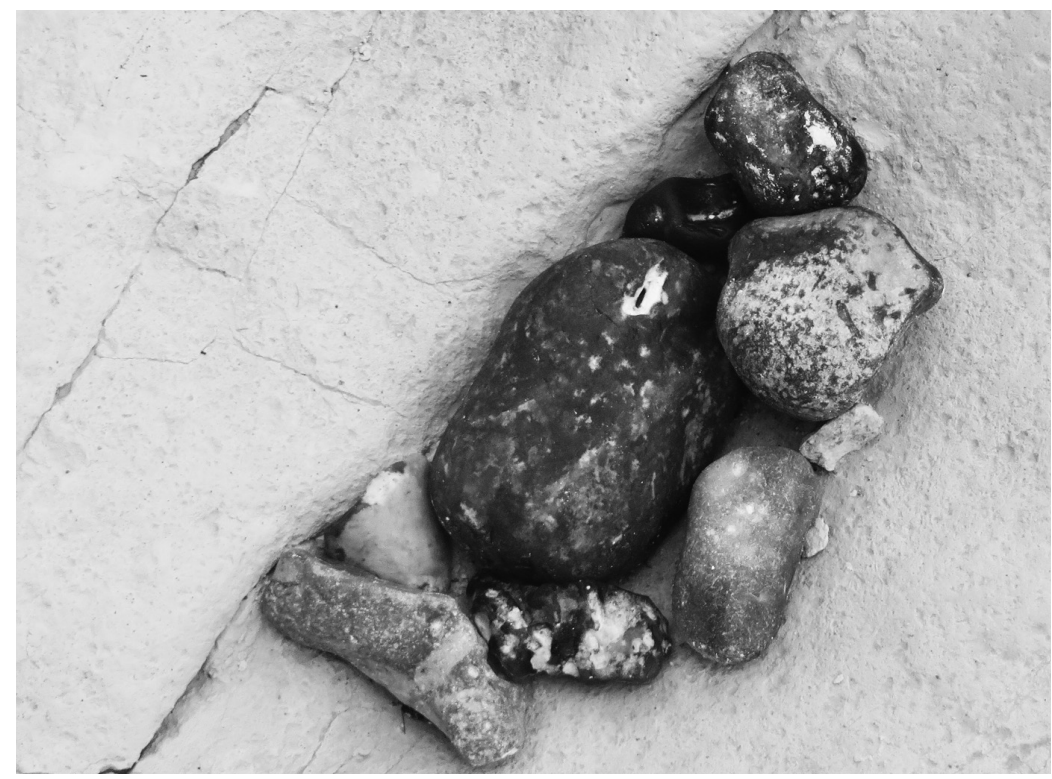

Katharina Wirtz

\section{Qualitätsbausteine schulischer Inklusion}

Organisations-, Personal- und Unterrichtsentwicklung an inklusiven Schulen aus der Sicht unterschiedlicher Beteiligter 
Wirtz

Qualitätsbausteine schulischer Inklusion 


\section{Perspektiven sonderpädagogischer Forschung}

im Namen der Sektion Sonderpädagogik

der Deutschen Gesellschaft für Erziehungswissenschaft (DGfE)

herausgegeben von

Christian Lindmeier, Birgit Lütje-Klose und Anja Hackbarth 


\section{Qualitätsbausteine schulischer Inklusion}

Organisations-, Personal- und Unterrichtsentwicklung an inklusiven Schulen aus der Sicht unterschiedlicher Beteiligter 
Die vorliegende Arbeit wurde vom Fachbereich Erziehungswissenschaften der Universität Koblenz-Landau, Campus Landau, unter dem Titel „Die Qualität schulischer Integration unter dem Blickwinkel der Entwicklung inklusiver Bildungsangebote" als Dissertation angenommen.

Gutachter: Prof. Dr. Sven Jennessen, Prof. Dr. Anja Wildemann.

Tag der Disputation: 13.12.2019.

Dieser Titel wurde in das Programm des Verlages mittels eines Peer-Review-Verfahrens aufgenommen. Für weitere Informationen siehe www.klinkhardt.de.

Bibliografische Information der Deutschen Nationalbibliothek Die Deutsche Nationalbibliothek verzeichnet diese Publikation in der Deutschen Nationalbibliografie; detaillierte bibliografische Daten sind im Internet abrufbar über http://dnb.d-nb.de.

2020.kg (C) by Julius Klinkhardt.

Foto Umschlagseite 1: (C) Autorin, Bearbeitung Isabell Wirtz.

Satz: Kay Fretwurst, Spreeau.

Druck und Bindung: AZ Druck und Datentechnik, Kempten.

Printed in Germany 2020.

Gedruckt auf chlorfrei gebleichtem alterungsbeständigem Papier.

(c) () Die Publikation (mit Ausnahme aller Fotos, Grafiken und Abbildungen) ist veröffentlicht unter der cc. ${ }_{\mathrm{BY}} \mathrm{NC}$ SA Creative Commons-Lizenz: CC BY-NC-SA 4.0 International

https://creativecommons.org/licenses/by-nc-sa/4.0/ 


\section{Zusammenfassung}

Im Zusammenhang mit der UN-Behindertenrechtskonvention stehen Schulen vor der Herausforderung Schülerinnen und Schüler mit und ohne sonderpädagogischen Förderbedarf gemeinsam zu beschulen und zu unterrichten. Die vorliegende Arbeit analysiert, welche qualitativen Bausteine bei der damit verbundenen Umgestaltung des Bildungssystems eine Rolle spielen und welche Bedeutung die einzelnen Bausteine für die (Weiter-) Entwicklung inklusiver Bildungsangebote haben können.

Damit die Qualitätsbausteine in den Bereichen Organisations-, Personal- und Unterrichtsentwicklung von Schulen in einen theoretischen Gesamtrahmen eingebettet werden können, werden im zweiten Kapitel zunächst geschichtliche Eckpunkte der Umsetzung schulischer Integration in den letzten Jahrzehnten erfasst. Es zeigt sich, dass Reformprozesse Wellenbewegungen vergleichbar sind, die nach einem ersten Reformschwung häufig in restaurativen Bemühungen enden. Darüber hinaus stehen Forderungen zur Gestaltung integrativen Unterrichts aus den 70er-Jahren erstaunlich unverändert auf der Agenda des aktuellen Inklusionsdiskurses. Dieser Diskurs wird in unterschiedlichen Fachwissenschaften (Soziologie, Bildungssoziologie, Allgemeine Pädagogik, Sonderpädagogik) wiederum durch eine Vielfalt von Begriffen und Sichtweisen geprägt, deren Analyse die Schlussfolgerung nahelegt, dass eine ,inklusive Schule“ nicht gleichzusetzen ist mit der umfassenden gesellschaftlichen Inklusion von Kindern und Jugendlichen.

Im dritten Kapitel werden bisherige Forschungsergebnisse zur Qualität schulischer Integration aus der sonderpädagogischen Integrations- bzw. Inklusionsforschung sowie der allgemeinpädagogischen Schul(entwicklungs)- und Unterrichtsforschung zusammengeführt. Qualitätskategorien, -subkategorien und -indikatoren aus den Bereichen Organisations-, Personal- und Unterrichtsentwicklung von (integrativ arbeitenden) Schulen werden mit Hilfe einer qualitativen und quantitativen Inhaltsanalyse der bislang vorliegenden Forschungsliteratur in einem Kategoriensystem gesammelt, systematisierend strukturiert und gewichtet, wodurch sowohl ein Gesamtüberblick zu dieser komplexen Thematik entsteht als auch eine wissenschaftliche Grundlage für die Forschungsvorhaben geschaffen wird.

Im empirischen Teil werden die Ergebnisse von zwei Erhebungen dargestellt und interpretiert, die im Saarland durchgeführt worden sind - einem Bundesland, das über jahrzehntelange Erfahrung mit der schulischen Integration von Kindern und Jugendlichen mit sonderpädagogischem Förderbedarf verfügt. Die qualitative Teilstudie umfasst 25 Interviews, die quantitative Teilstudie bezieht 32 Grund- und Gemeinschaftsschulen sowie vier Förder- und Beratungszentren ein. Beide Erhebungen sind mehrperspektivisch angelegt und analysieren die Wahrnehmung und Einschätzung der Qualität schulischer Integration aus der Sicht von Schulleitungen von Förder- und Beratungszentren sowie Grund- und weiterführenden Schulen, Regel- und Förderschullehrkräften, Eltern von Kindern und Jugendlichen mit sonderpädagogischem Förderbedarf sowie den Schülerinnen und Schülern selbst. Die Verknüpfung dieser unterschiedlichen Perspektiven ermöglicht einen umfassenden Einblick in das Innenleben inklusiver Schulen. Aus den Ergebnissen der Studien werden sowohl Schlussfolgerungen für die Entwicklung von Einzelschulen als auch für das Bildungssystem insgesamt abgeleitet. Im Fazit werden diese Schlussfolgerungen vor dem Hintergrund des im zweiten Kapitel erarbeiteten theoretischen Rahmens zudem kritisch eingeordnet. 


\section{Abstract}

As a result of the UN-Convention on the Rights of Persons with Disabilities schools are faced with the challenge to develop inclusive education for pupils with and without special educational needs. This doctoral thesis puts its focus on the question, which elements of quality play an important role concerning the reorganization of the educational system in Germany into an inclusive one.

To be able to embed these elements including organizational change, personnel development and the development of teaching in a theoretical framework the second chapter first of all describes historical aspects of inclusive schooling in Germany during the last decades as well as different viewpoints of scientific theories. It seems obvious that reform processes can be compared to wave movements, which end up in restorative efforts after an initial swing of reforms. In addition, demands for inclusive education from the 70 s are surprisingly unchanged in the current discourse. Different scientific theories (sociology, educational sociology, theories of education and special education) shape this discourse by a various understanding and use of terms like exclusion and inclusion. The analysis of this scientific discussion leads to the conclusion that attending a so-called ,inclusive school“ is not to be equated with the social inclusion of children and young people in general.

In the third chapter empirical research concerning the quality of inclusive education from two scientific viewpoints is brought together: the view of special pedagogy and the view of research on schools and teaching in general. Categories, sub-categories and indicators of this quality in the fields of organization, personnel and teaching of (inclusive) schools are collected, systematically structured and weighted by a qualitative and quantitative content analysis of the previous research literature. This approach results in a system of categories that gives an overview about this complex research field and provides a basis for further research.

The results of two surveys, which have been carried out in the federal state of Saarland, are described and interpreted in the fourth chapter. In this state inclusive education has been practiced for more than thirty years. The qualitative sub-study includes 25 interviews, the quantitative sub-study involves 32 primary and secondary schools as well as four support- and advice centres for inclusive education. Both surveys analyse the perception und evaluation of the quality of inclusion from multiple perspectives: the views of headmasters, mainstream and special school teachers, parents of children with special educational needs and the viewpoints of the pupils themselves. Describing the connections between those different perspectives gives a deep insight into the inner life of inclusive schools.

Conclusions for the development of individual schools as well as for the education system as a whole are derived from the results of the studies. Moreover those conclusions are critically classified against the background of the theoretical framework that has been described in the second chapter. 


\section{Danksagung}

Diese Arbeit verdanke ich vor allem meinen Eltern Eva-Maria und Gerd: ihr habt mir das notwendige Rüstzeug, die Kraft und das Durchhaltevermögen mit auf den Weg gegeben. Peter hat diese Arbeit initiiert („ich solle doch endlich mal was Gescheites machen“) und mich immer wieder motiviert diese fertigzustellen, sprich: ohne dich gäbe es diese Arbeit nicht.

Ich verdanke sie auch allen Menschen, denen ich während meiner Tätigkeit in Schulen begegnet bin, darunter insbesondere dem Kollegium der Förderschule und des Förder- und Beratungszentrums Blieskastel sowie dem Kollegium der Schloss-Schule in Ludwigshafen-Oggersheim, den Kolleginnen und Kollegen aus den jeweils umliegenden Regel- bzw. Schwerpunktschulen und allen Schulleitungen, mit denen ich kooperieren durfte: von euch habe ich unglaublich viel gelernt und ohne euch wäre diese Arbeit nicht entstanden.

Besonders herzlich bedanken möchte ich mich bei allen Schülerinnen und Schülern, mit denen ich in den vergangenen über zwanzig Jahren Zeit verbringen durfte: ihr habt mir viel Geduld geschenkt. Eure Fröhlichkeit und euer Vertrauen waren und sind die beste Motivation zum Arbeiten.

Ein großes Dankeschön geht an die Schulleitungen, Lehrkräfte, Eltern und Schülerinnen und Schüler der Grundschulen, Gemeinschaftsschulen sowie Förder- und Beratungszentren, die sich bereitgefunden haben an den Interviews sowie an den schriftlichen Befragungen teilzunehmen: ohne Ihre und eure Antworten hätte diese Arbeit nicht entstehen können.

Besonders bedanken möchte ich mich bei Hans-Jürgen Latz, meinem „Ziehvater“ am Förderund Beratungszentrum Blieskastel: von deinem Erfahrungsschatz und unermüdlichem pädagogischen Engagement durfte ich jahrelang profitieren!

Ein Dank geht auch an die Mitarbeiterinnen und Mitarbeiter der Referate Grund-, Gemeinschafts- und Förderschulen des Ministeriums für Bildung des Saarlandes: Sie waren und ihr wart immer zu konstruktiv-kritischen Diskussionen zum Thema inklusive Bildung bereit.

Im universitären Rahmen danke ich insbesondere Prof. Dr. Sven Jennessen und Prof. Dr. Anja Wildemann: das Wohlwollen und Vertrauen deiner und Ihrerseits, die Beratung und Unterstützung dieser Arbeit waren der wichtigste „Begleitschutz“. Herzlich bedanken möchte ich mich auch bei den wissenschaftlichen Hilfskräften: Jan Dörendahl, Sabine Brunnengräber und Heiko Sahm, die die Dateneingabe und Auswertung in SPSS unterstützt haben. Danken möchte ich außerdem den Kolleginnen und Kollegen des Instituts für Sonderpädagogik der Universität Landau sowie den Mitarbeiterinnen des Sekretariats: Ihr und euer Zuspruch, konstruktive Fragen und Diskussionsbereitschaft haben - auch nach der Zeit des Lehrauftrags - immer wieder gut getan.

Ein letzter Dank geht an meine Schwestern und alle Freundinnen und Freunde, die Verständnis für und Interesse an meinem Projekt gezeigt haben. 



\section{Inhalt}

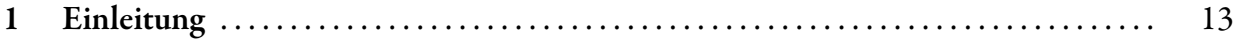

2 Theoretische Einbettung des Themas ........................... 19

2.1 Geschichtliche Eckdaten $\ldots \ldots \ldots \ldots \ldots \ldots \ldots \ldots \ldots \ldots \ldots \ldots \ldots \ldots$

2.1.1 Sondereinrichtungen versus integrative Bildungsangebote:

zwei Entwicklungslinien der frühen (Sonder-) Pädagogik ........... 20

2.1.2 Die Gesamtschule - (k)eine Schule für alle Kinder?.............. 23

2.1.3 Bildungspolitische Entwicklungen der 70er Jahre .............. 27

2.1.4 Integration (nur) unter bestimmten Bedingungen? Modellversuche als Möglichkeit der Umsetzung integrativer Beschulung $\ldots \ldots \ldots \ldots \ldots . \quad 31$

2.1.5 Bildungspolitische Dokumente der 90er Jahre ................. 33

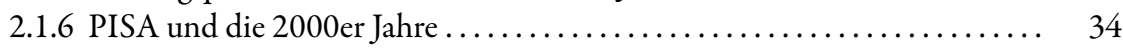

2.1.7 Der Stand der inklusiven Förderung in der Bundesrepublik Deutschland

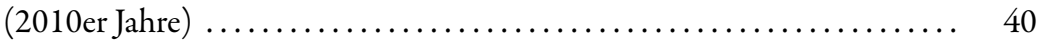

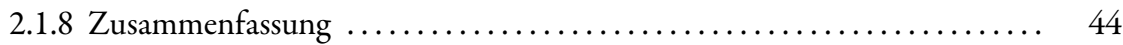

2.2 Unterschiedliche Perspektiven auf Begriffe und Diskurs .............. 48

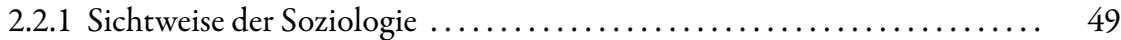

2.2.2 Sichtweise der Bildungssoziologie $\ldots \ldots \ldots \ldots \ldots \ldots \ldots \ldots \ldots \ldots \ldots \quad 57$

2.2.3 Sichtweise der Allgemeinen Pädagogik ................... 61

2.2.4 Sichtweise der Sonderpädagogik $\ldots \ldots \ldots \ldots \ldots \ldots \ldots \ldots \ldots \ldots .68$

2.2.5 Zusammenfassung der unterschiedlichen Perspektiven auf Begriffe

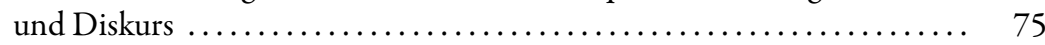

3 Systematischer Forschungsüberblick zu Qualitätsbereichen schulischer Integration/Inklusion $\ldots \ldots \ldots \ldots \ldots \ldots \ldots \ldots \ldots \ldots \ldots \ldots \ldots \ldots \ldots \ldots \ldots \ldots$

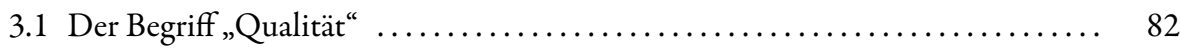

3.2 Auswahl eines Rahmenmodells zur Systematisierung der

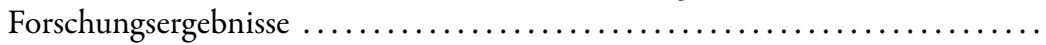

3.2.1 Modelle zur Systematisierung schulischer Integration aus der

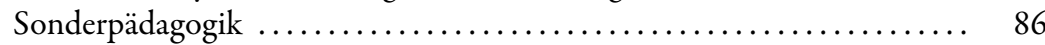

3.2.2 Modelle zur Systematisierung schulischer Entwicklungsprozesse aus der Allgemeinen Pädagogik $\ldots \ldots \ldots \ldots \ldots \ldots \ldots \ldots \ldots \ldots . \ldots \ldots$

3.2.3 Zusammenhang zwischen sonderpädagogischen und allgemeinpädagogischen Ansätzen zur Systematisierung ........... 90

3.2.4 Begründung der Auswahl des Rahmenmodells für die Kategorienbildung ... 92

3.3 Forschung zu Integration und Inklusion in den vergangenen vier Jahrzehnten ..... 95

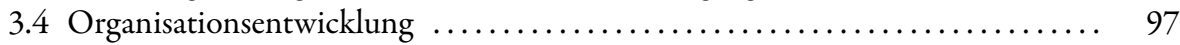

3.4.1 Ressourcen im Bereich der Organisationsentwicklung ............. 98

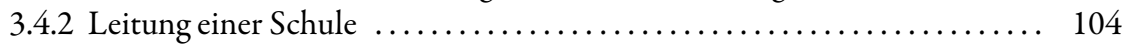

3.4 .3 Schule als sozialer Raum ................................. 107

3.4.4 Organisationsstrukturen einer Schule ..................... 113

3.4.5 Ergebnisse im Bereich der Organisationsentwicklung ............ 117

3.4.6 Zusammenfassung zum Bereich der Organisationsentwicklung ........ 120 


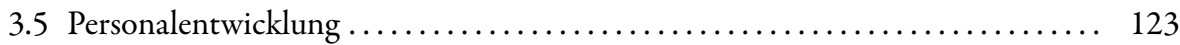

3.5.1 Ressourcen im Bereich der Personalentwicklung ............... 125

3.5.2 Kooperation ........................................ 129

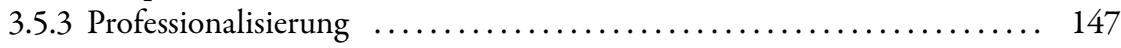

3.5.4 Ergebnisse im Bereich der Personalentwicklung ................ 151

3.5.5 Zusammenfassung zum Bereich der Personalentwicklung ............ 154

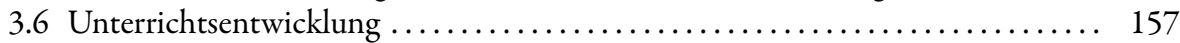

3.6.1 Ressourcen im Bereich der Unterrichtsentwicklung .............. 161

3.6.2 Schulklasse als sozialer Raum $\ldots \ldots \ldots \ldots \ldots \ldots \ldots \ldots \ldots \ldots \ldots \ldots$

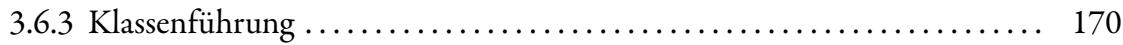

3.6.4 Didaktik ....................................... 174

3.6.5 Methodik ......................................... 180

3.6.6 Differenzierung und Individualisierung $\ldots \ldots \ldots \ldots \ldots \ldots \ldots \ldots \ldots \ldots \ldots \ldots$

3.6.7 Ergebnisse im Bereich der Unterrichtsentwicklung ............... 199

3.6.8 Zusammenfassung zum Bereich der Unterrichtsentwicklung ......... 211

3.7 Zusammenfassung mit Blick auf die forschungsleitenden Fragestellungen ...... 216

4 Empirischer Teil: Studie zur Qualität schulischer Integration im Saarland ...... 223

4.1 Schulische Integration bzw. Inklusion im Saarland $\ldots \ldots \ldots \ldots \ldots \ldots \ldots . .224$

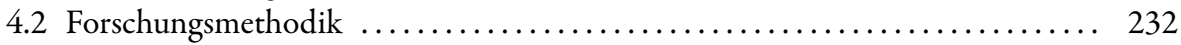

4.3 Qualitative Studie .................................... 235

4.3.1 Forschungsmethodik der qualitativen Studie .................. 235

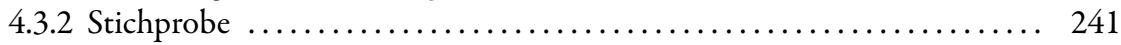

4.3.3 Darstellung der Ergebnisse zur Organisationsentwicklung .......... 242

4.3.4 Darstellung der Ergebnisse zur Personalentwicklung .............. 250

4.3.5 Darstellung der Ergebnisse zur Unterrichtsentwicklung ............ 273

4.3.6 Interpretation der Ergebnisse zur Organisationsentwicklung ........... 292

4.3.7 Interpretation der Ergebnisse zur Personalentwicklung ............. 295

4.3.8 Interpretation der Ergebnisse zur Unterrichtsentwicklung ............ 304

4.3.9 Zusammenfassung und Weiterentwicklung des Kategoriensystems mit Hilfe der Ergebnisse sowie Konsequenzen für die inhaltliche

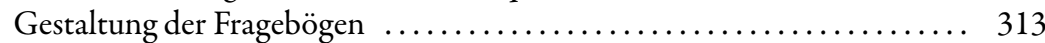

4.4 Quantitative Studie ..................................... 315

4.4.1 Forschungsmethodik der quantitativen Studie ................ 315

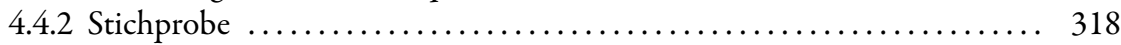

4.4.3 Darstellung der Ergebnisse zur Organisationsentwicklung .......... 323

4.4.4 Darstellung der Ergebnisse zur Personalentwicklung ............... 341

4.4.5 Darstellung der Ergebnisse zur Unterrichtsentwicklung ............ 367

4.4.6 Interpretation der Ergebnisse zur Organisationsentwicklung ........... 390

4.4.7 Interpretation der Ergebnisse zur Personalentwicklung .............. 396

4.4.8 Interpretation der Ergebnisse zur Unterrichtsentwicklung .......... 403

4.5 Zusammenführende Darstellung, kritische Einordnung und Perspektiven der zentralen Ergebnisse der qualitativen und der quantitativen Studie zur

Qualität der schulischen Integration im Saarland . . . . . . . . . . . . . . . 408

4.5 .1 Organisationsentwicklung $\ldots \ldots \ldots \ldots \ldots \ldots \ldots \ldots \ldots \ldots \ldots \ldots \ldots \ldots \ldots \ldots \ldots$

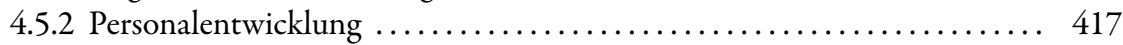

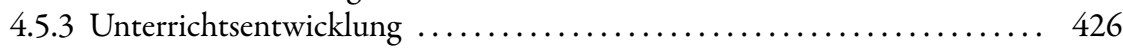


5 Fazit und Perspektiven für das Bildungssystem

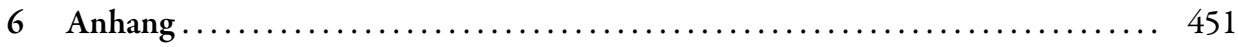

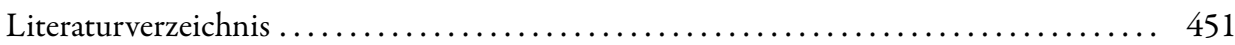

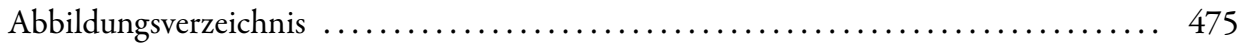

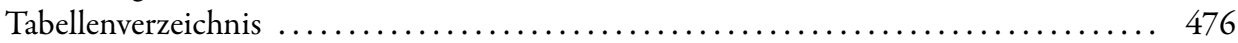

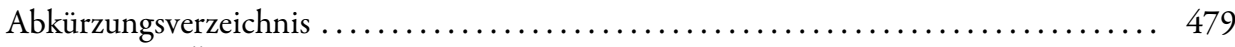

Tabellarische Übersicht des Kategoriensystems für schulische Integration $\ldots \ldots \ldots \ldots .481$

Beispiel für einen Interviewleitfaden (Regelschullehrkraft Grundschule) . ........... 500

Beispiel für einen Fragebogen (Regelschullehrkraft Grundschule) ................ 501 



\section{Einleitung}

Schulen unterliegen Veränderungserwartungen. Als Institutionen sollen sie über Transformationskompetenz verfügen, also die Fähigkeit zu aktiver Veränderung mit Blick auf zeitlichen und gesellschaftlichen Wandel. So stehen Schulen immer wieder vor der Aufgabe pädagogische Antworten auf Fragen zu finden, die sich bspw. mit der Sorge um Fachkräftemangel, nach der Integration von Kindern und Jugendlichen mit Migrationshintergrund, nach dem Kompetenzerwerb von Schülerinnen und Schülern im internationalen Vergleich, nach Digitalisierung oder nach der integrativen bzw. inklusiven Beschulung ${ }^{1}$ von Kindern und Jugendlichen mit sonderpädagogischem Förderbedarf ${ }^{2}$ befassen.

Im Zusammenhang mit der UN-Behindertenrechtskonvention wird erwartet, dass sich eine Transformation der sogenannten „Regelschulen“ in sogenannte „inklusive Schulen“ vollzieht, ein Prozess, der vom UN-Ausschuss als sehr umfassend beschriebend wird:

Der UN-Ausschuss betont, dass die Umsetzung des Rechts auf inklusive Bildung als transformativer Prozess im Rahmen einer systemischen Reform zu begreifen sei, die einen tiefgreifenden Wandel der Bildungssysteme nach sich ziehe. Dafür müssten Gesetze, Politikkonzepte sowie die Finanzierung, Verwaltung, Ausgestaltung (Inhalt, Lehrmethoden, Ansätze, Strukturen und Strategien), Erbringung und Überwachung von Bildung angepasst werden [...]. (Dt. Institut für Menschenrechte, 2017, S. 1)

Die vorliegende Arbeit wirft einen Blick darauf, welche Qualitätsbausteine bei dieser Umgestaltung des Bildungssystems eine Rolle spielen und welche Bedeutung die einzelnen Bausteine für die (Weiter-)Entwicklung inklusiver Bildungsangebote haben können.

Aus dieser Blickrichtung heraus ergibt sich eine Fülle von Forschungsfragen:

Welche Qualität - im Sinne von „Beschaffenheit“ - hat das, was als schulische Integration oder schulische Inklusion bezeichnet wird? Wie stellen sich die gemeinsame Beschulung und der gemeinsame Unterricht von Schülern und Schülerinnen mit und ohne Behinderungen in so genannten „Regelschulen“ dar? Wie sind solche Schulen organisiert? Wie arbeiten die Menschen in diesen Schulen zusammen? Wie wird der Unterricht gestaltet? Welche Qualität haben Organisations-, Personal- und Unterrichtsentwicklung in diesen Schulen? Wie wird die Qualität dieser Bereiche von den Prozessbeteiligten - von Schülern, Eltern, Regel- und Förderschullehrkräften sowie Schulleitungen - wahrgenommen? Welche dieser Qualitätsbereiche sind aus allgemeinpädagogischer und sonderpädagogischer Perspektive relevant für die schulische Integration von Kindern und Jugendlichen mit Behinderungen? Das sind die zentralen Fragen dieser Forschungsarbeit.

Schulen sind komplexe Systeme, in denen eine Vielzahl von Menschen miteinander agiert, Kommunikation und Kooperation auf einer Fülle unterschiedlicher Ebenen stattfinden und unterschiedliche Zielsetzungen im Rahmen des Bildungs- und Erziehungsauftrags gleichzeitig verfolgt werden. Die unterschiedlichen „Bausteine“ von Schulen und deren Entwicklung - Or-

1 Die Begriffe ,integrativ“ sowie „inklusiv“ werden in dieser Arbeit mit Blick auf eine zeitliche Abfolge genutzt. Während der Begriff der schulischen Integration den Zeitraum bis um die 2010er Jahre kennzeichnet (bis zur Verabschiedung der UN-BRK), findet der Begriff der Inklusion in der deutschen Diskussion seit etwa diesem Zeitpunkt Verwendung.

2 Der Begriff ,sonderpädagogischer Förderbedarf“ wird in dieser Arbeit genutzt, da er auf die Kinder und Jugendlichen bezogen werden kann, die - aus schulischer Sicht - integrativ bzw. inklusiv unterrichtet werden können (zur Problematisierung s. 2.1.7). 
ganisation, Personal und Unterricht (Bohl et al., 2010) - sowie die dynamischen Beziehungen zwischen diesen in Schulen wirksamen Teilbereichen sind nur punktuell und begrenzt zu erfassen. Ein Austausch mit der Umwelt findet in Schulen in vielfältige Richtungen statt: über die Lehrkräfte, Schulleitungen, Eltern und Schüler mit einer Vielzahl an Personen, mit den Kommunen, die als Träger der Schulen fungieren, mit der Schulverwaltung und Schulaufsicht in einem bestimmten Bezirk, innerhalb der Bundesländer, die durch ihre Bildungspolitik das Geschehen in den Schulen beeinflussen. Geprägt werden Schulen auch durch die Haltungen, Einstellungen und Hintergründe der Personen, die in ihnen arbeiten. Diese wiederum resultieren aus persönlichen Kenntnissen wie Erfahrungen und sind gesellschaftlich geprägt.

Aufgrund der Komplexität der zu untersuchenden Systeme stellt in diesem Zusammenhang zunächst die Entscheidung für einen „brauchbaren Komplexitätsgrad“ (Vester, 1999, S. 20) eine zentrale Aufgabe für die Wahl eines Analyse- und Forschungszugangs dar. Dass diese Wahl trotz aller Bemühungen problematisch bleibt, lässt sich anhand der Überlegungen von Vester verdeutlichen:

Daher sei hier kurz erklärt, was Systeme grundsätzlich von Einzeldingen unterscheidet: Wie jeder Organismus besteht ein komplexes System aus mehreren verschiedenen Teilen (Organen), die in einer bestimmten dynamischen Ordnung zueinander stehen, zu einem Wirkungsgefüge vernetzt sind. In dieses kann man nicht eingreifen, ohne dass sich die Beziehung aller Teile zueinander und damit der Gesamtcharakter des Systems ändern würde. Reale Systeme sind darüber hinaus auch immer offen und erhalten sich durch ständigen Austausch mit der Umwelt. (Vester, 1999, S. 25)

Erkenntnistheoretisch ist die Aufgabe der Analyse der Qualität schulischer Integration auch deshalb mit Schwierigkeiten konfrontiert, da sie eine spezifische Auswahl von Perspektiven auf einen Wirklichkeitsbereich erfordert:

Nur wo Dinge, ohne ihre Identität zu verlieren, von Vielen in einer Vielfalt von Perspektiven erblickt werden, so daß die um sie Versammelten wissen, daß ein Selbes sich ihnen in äußerster Verschiedenheit darbietet, kann weltliche Wirklichkeit eigentlich und zuverlässig in Erscheinung treten. (Arendt, 1967, S. 72)

So wird die Qualität der schulischen Integration von der Allgemeinen Pädagogik und der Sonderpädagogik unter unterschiedlichen Blickwinkeln analysiert.

Die Struktur der vorliegenden Arbeit versucht diese komplexe Grundsituation zu berücksichtigen, indem drei inhaltliche Schwerpunkte gesetzt werden: eine fundierte theoretische Einbettung des Themas, eine umfassende Analyse bisheriger Forschungsergebnisse sowie die konkrete Untersuchung der Wahrnehmung und Einschätzung der Umsetzung schulischer Integration durch beteiligte Personen mit Hilfe einer qualitativen und einer quantitativen Studie. „Erfahrungen der pädagogischen Praxis, die in dieser wirksamen Theorien und die verzweigten Anstrengungen und Resultate erziehungswissenschaftlicher Forschung" (Benner, 2015, S. 5) sollen also ein Gesamtbild ergeben, das Antworten auf die eingangs gestellten Fragen ermöglicht. Während im zweiten Kapitel zunächst geschichtliche Eckpunkte der schulischen Integration von Kindern und Jugendlichen mit Behinderungen in Regelschulen sowie unterschiedliche Sichtweisen auf Inklusion bzw. Exklusion beschrieben werden (Soziologie, Bildungssoziologie, Allgemeine Pädagogik, Sonderpädagogik), umfasst das dritte Kapitel eine umfassende Analyse sonder- und allgemeinpädagogischer Forschungsergebnisse und strukturiert und systematisiert diese zu einem Kategoriensystem der Qualität schulischer Integration. Auf der Basis dieses Kategoriensystems werden eine qualitative Untersuchung sowie eine quantitative Studie durch- 
geführt, deren Ergebnisse im vierten Kapitel vorgestellt und interpretiert werden, bevor im 5. Kapitel Fazit und Perspektiven folgen. Die Komplexität des Forschungsgegenstands wird auf der einen Seite also durch das auf die Schulen und deren Entwicklungsbereiche bezogene Kategoriensystem reduziert, auf der anderen Seite wird versucht verschiedene Perspektiven auf die Qualität schulischer Integration zu berücksichtigen: Perspektiven unterschiedlicher Fachwissenschaften, geschichtliche und aktuelle Sichtweisen sowie Perspektiven unterschiedlicher Beteiligter wie Schüler, Eltern, Regel- und Förderschullehrkräfte sowie Schulleitungen von Grund- und weiterführenden Schulen bzw. Förder- und Beratungszentren.

Das zweite Kapitel soll zunächst die Frage danach beantworten, wie schulische Integration in den vergangenen Jahrzehnten umgesetzt wurde. Die Realität, die derzeit in Schulen vorzufinden ist, steht in engem Zusammenhang mit zurückliegenden geschichtlichen Entwicklungen wie bspw. den bildungspolitischen Entscheidungen der vergangenen 70 Jahre. Diese werden im ersten Teil dieses Kapitels unter der Blickrichtung der integrativen Beschulung kritisch zusammengefasst. Anschließend wird beschrieben, welche Bedeutung die Begriffe „Integration“ und „Separation“ bzw. „Inklusion“ und „Exklusion“ im soziologischen, bildungssoziologischen, pädagogischen und sonderpädagogischen Kontext haben. Die begriffliche Annäherung an das Thema aus mehreren wissenschaftlichen Perspektiven heraus wird vollzogen, damit ein grundlegendes Verständnis zentraler Begriffe der Thematik für diese Arbeit gegeben ist. Theoretisch konstruierte Begriffe versuchen praktische Erfahrungen zu erfassen bzw. zu vermitteln. Sie spiegeln damit Realitätswahrnehmung aus unterschiedlichen Blickrichtungen und ermöglichen den Diskurs zur Fragestellung der schulischen Integration. Dieses Kapitel dient also insgesamt der theoretischen Einbettung der Thematik schulischer Integration bzw. Inklusion in einen geschichtlichen und begrifflichen Rahmen. Als pädagogische Institutionen agieren Schulen innerhalb dieser und weiterer Rahmungen, so dass die Auseinandersetzung mit der Qualität der Arbeit in diesen Schulen voraussetzt, dass der Rahmen auch dann erfasst wird, wenn er nicht selbst Inhalt des Forschungsvorhabens ist, damit empirische Ergebnisse entsprechend eingeordnet werden können. Der theoretischen Fundierung des Themenfelds wird - mit Blick darauf, dass der Forschungsschwerpunkt die Qualität schulischer Integration umfasst - viel Raum gegeben. Eine sorgfältige Darstellung, die diesen Schwerpunkt theoretisch fundiert einbettet, ist allerdings aus systemischer Sicht notwendig, damit Schulen als Systeme, die sich im komplexen Austausch mit ihrer Umwelt befinden, analysierbar werden und sich diese Analyse nicht in einem „luftleeren“ Raum bewegt.

Im dritten Kapitel geht es darum bisherige Forschungsergebnisse zur Qualität schulischer Integration bzw. Inklusion darzustellen. Während zurückliegende Forschungsarbeiten sich vor allem mit Fragen nach den möglichen Vor- und Nachteilen integrativer versus separativer Beschulung von Kindern und Jugendlichen mit Behinderungen befasst haben, möchte diese Arbeit einen Beitrag dazu leisten, mit Hilfe von Forschungsergebnissen aus der Sonderpädagogik wie der Allgemeinen Pädagogik ein umfassendes Kategoriensystem zur Qualität schulischer Integration bzw. Inklusion zu entwickeln. Im Hinblick auf das Forschungsprojekt spielt die vorhergehende systematische Erschließung von Qualitätskategorien, -subkategorien und -indikatoren aus den Bereichen Organisations-, Personal- und Unterrichtsentwicklung von (integrativ arbeitenden) Schulen mit Hilfe einer qualitativen und quantitativen Inhaltsanalyse der bislang vorliegenden Forschungsliteratur eine zentrale Rolle, da diese die Grundlage für das eigene Forschungsvorhaben darstellen. Die Kategorien, Subkategorien und Indikatoren werden mit Hilfe dieser Analyse gesammelt, strukturiert und gewichtet. Ziel des dritten Kapitels ist es in diesem Zusammenhang auch Ergebnisse aus der der sonderpädagogischen Integrations- bzw. Inklusi- 
onsforschung mit Ergebnissen der allgemeinpädagogischen Schul(entwicklungs)- und Unterrichtsforschung zusammenzuführen. Bei der Erfassung von Qualitätskategorien für den Bereich der inklusiven Beschulung spielt eine vernetzte Analyse von Forschungen aus der Allgemeinen Pädagogik sowie der Sonderpädagogik eine wesentliche Rolle, da diese sich in Regel- und nicht in Förderschulen vollzieht. Die Qualität schulischer Integration lässt sich nicht unabhängig von der qualitativen Arbeit dieser Schulen allgemein erfassen.

Um die Qualität schulischer Integration in der praktischen Umsetzung greifbar zu machen, ist es notwendig in die Schulen „hineinzuschauen“ und deren Innenleben empirisch - das heißt von Erfahrungen ausgehend - zu erschließen. Dieses Ziel verfolgen die beiden im vierten Kapitel vorgestellten Studien, die im Saarland durchgeführt werden. Dieses Bundesland bietet sich für die Untersuchung der Qualität schulischer Integration an, da dort zwischen 1987 und 2015 die so genannte Integrationsverordnung galt (Ministerium für Bildung des Saarlandes, 1986), die den Eltern behinderter Kinder weitgehende Rechte hinsichtlich der Entscheidung zwischen dem Besuch einer Förderschule oder der Einrichtung einer integrativen Maßnahme in einer Regelschule einräumte. Im Jahr 2015 trat die Inklusionsverordnung in Kraft, die diese Rechte noch einmal erweiterte. Der Anteil integrativ unterrichteter Kinder lag in diesem Bundesland bereits 2006 bei etwa 26 Prozent (Speck, 2010, S. 36), im Schuljahr 2008/2009 bei 31,22\% (Klemm, 2018, S. 18) und 2017 bei 56,6\% (Lange, 2017, S. 13). Die saarländischen Schulen verfügen also über jahrzehntelange Erfahrung mit der schulischen Integration von Kindern und Jugendlichen mit sonderpädagogischem Förderbedarf. Nach den wissenschaftlichen Berichten von Sander u.a. in den 90er-Jahren (Bsp.: Sander et al., 1994; Sander et al., 1995) und abgesehen von der wissenschaftlichen Begleitung des Modellversuchs inklusive Schule (Schön \& Stark, 2012, 2014) wurde allerdings noch nicht umfassend wissenschaftlich untersucht, welche Qualität die schulische Integration in diesem Bundesland aufweist. Das Saarland bietet sich daher als räumlicher Schwerpunkt für eine wissenschaftliche Arbeit an. Im Rahmen des Forschungsvorhabens erfolgen zwei Erhebungen mit Schulleitungen von Förder- und Beratungszentren, Grund- und weiterführenden Schulen, mit Regel- und Förderschullehrkräften, Eltern von Kindern und Jugendlichen mit sonderpädagogischem Förderbedarf ( $\mathrm{spF}$ ) sowie den Schülerinnen und Schülern mit spF selbst. In einem ersten Schritt werden 25 Experteninterviews durchgeführt, die mit Hilfe der qualitativ strukturierenden Inhaltsanalyse nach Kuckartz kategorienorientiert ausgewertet und interpretiert werden. Mit Hilfe dieser qualitativen Analyse wird das in Kapitel 3 erstellte Kategoriensystem überarbeitet und erweitert. In einem zweiten Schritt werden eine quantitative Erhebung mit Fragebögen an 32 Grund- und Gemeinschaftsschulen sowie vier Förder- und Beratungszentren, die Auswertung der Daten sowie ihre Interpretation vollzogen. Eine zusammenführende Interpretation beider Teilstudien, die auch Perspektiven für die Weiterentwicklung inklusiver Bildungsangebote aufgreift, rundet dieses Kapitel ab.

Im fünften Kapitel - Fazit und Perspektiven - werden die wichtigsten Forschungsergebnisse kritisch eingeordnet. Aufgrund der inhaltlichen Vielfalt der Ergebnisse werden dafür zentral erscheinende Bereiche ausgewählt und mit Blick auf die eingangs aufgeführten Fragestellungen dieser Arbeit abschließend analysiert. Im Anschluss werden Perspektiven für den wissenschaftlichen Bereich wie für die Weiterentwicklung inklusiver Bildungsangebote in der Praxis thematisiert. Ein wichtiges Ziel der Dissertation ist es in diesem Zusammenhang einen wissenschaftlichen Beitrag zur Analyse der Qualität schulischer Integration in Regelschulen zu leisten und damit das Augenmerk auf die weitere Qualitätsentwicklung inklusiver Bildungsangebote zu lenken. 
Folgende Grafik veranschaulicht den Aufbau dieser Arbeit:

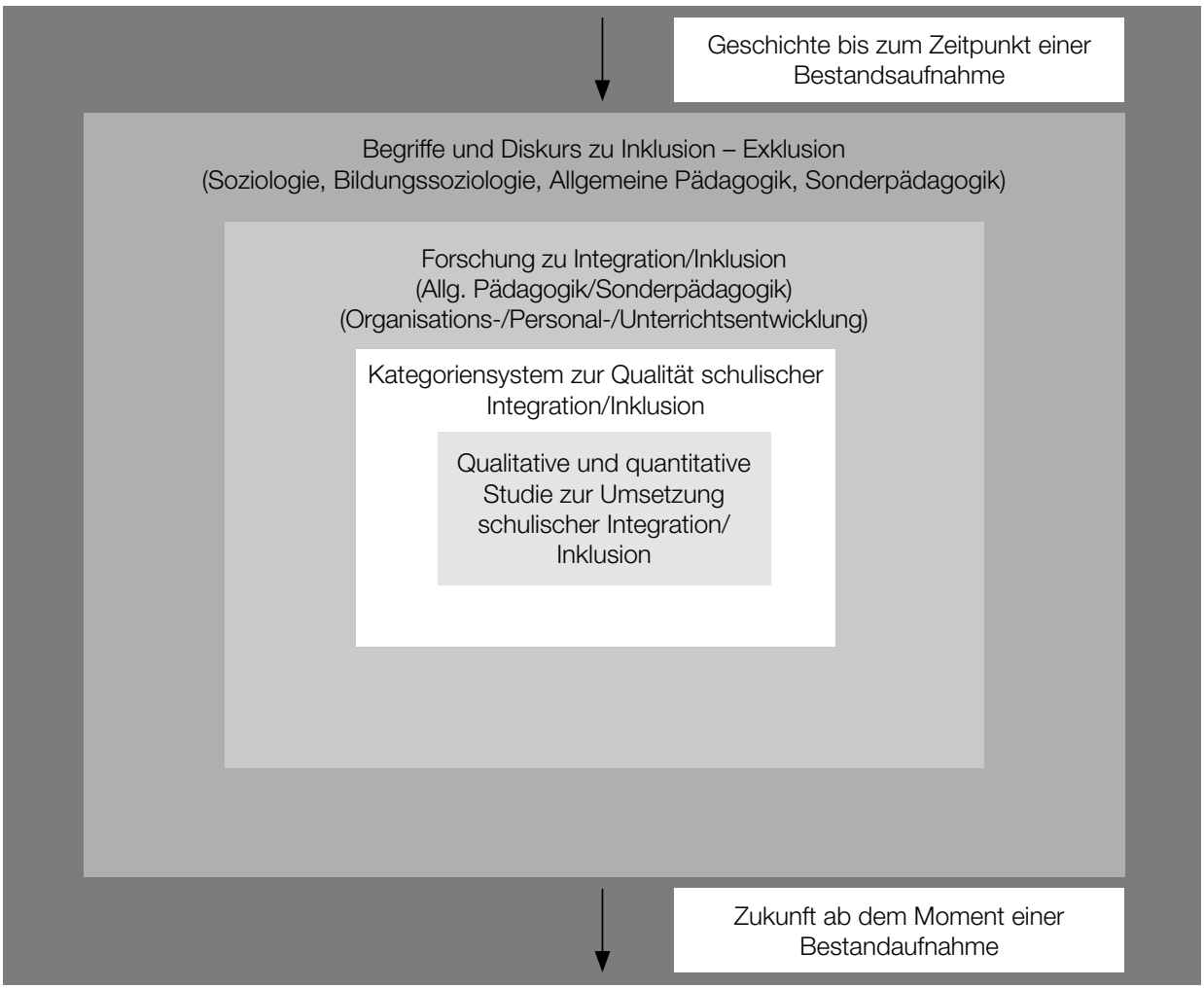

Abb. 1: Grafische Darstellung des Aufbaus der Dissertation 



\section{Theoretische Einbettung des Themas}

Dieses Kapitel befasst sich mit der theoretischen Rahmengebung für das Themenfeld der Qualität schulischer Integration und analysiert geschichtliche Eckdaten, Begriffe und Diskurs. Der Versuch der theoretischen Einbettung des Themas ist aus unterschiedlichen Gründen sehr komplex: sonderpädagogisches Arbeiten in Regelschulen spielt sich einem Kontext ab, der von rechtlichen, bildungspolitischen sowie schulaufsichtlichen Entwicklungen und Entscheidungen geprägt ist, die pädagogische bzw. sonderpädagogische Theoriebildung zu den Themenfeldern Integration bzw. Inklusion ist teilweise nur in Ansätzen erfolgt, Perspektiven auf diese Themenfelder sind so vielgestaltig wie kontrovers und entstammen unterschiedlichen Fachwissenschaften (u.a. Soziologie, Allgemeine Pädagogik, Sonderpädagogik). Daher konzentriert sich die theoretische Aufarbeitung auf folgende zentrale Fragestellungen:

- Welche Entwicklungen haben sich mit Blick auf die schulische Integration von Kindern und Jugendlichen mit Einschränkungen oder Behinderungen in den vergangenen etwa 70 Jahren in der Bundesrepublik Deutschland ergeben?

- Welche Antworten können die Soziologie, die Bildungssoziologie, die Allgemeine Pädagogik und die Sonderpädagogik auf Fragen nach dem Verständnis der Begrifflichkeiten Integration und Separation bzw. Inklusion und Exklusion geben?

Die weiterführende Fragestellung, welche Schlussfolgerungen aus der Darstellung dieser Entwicklungen, Begriffsklärungen und diskursiven Impulse für die Fragestellung nach der Qualität schulischer Integration unter dem Blickwinkel der Entwicklung inklusiver Bildungsangebote gezogen werden können, wird im letzten Kapitel dieser Arbeit unter Fazit und Perspektiven aufgegriffen.

\subsection{Geschichtliche Eckdaten}

Geschichte als Wissenschaft versucht Entwicklungsprozesse zu beschreiben. Im Zusammenhang mit der schulischen Integration von Schülerinnen und Schülern mit Behinderungen geht es darum aufzuzeigen, wie sich dieser Teilbereich in den vergangenen Jahrzehnten beeinflusst durch gesellschaftliche, bildungspolitische, schulaufsichtliche, pädagogische und sonderpädagogische Auffassungen und Aktivitäten entwickelt hat. Dieses Unterkapitel befasst sich daher mit einer Fülle von Fragen, die vor allem Verknüpfungen zwischen bildungspolitischen Entwicklungen bzw. Entscheidungen sowie der Wahrnehmung und Beschreibung derselben durch allgemein- wie sonderpädagogische Autoren herstellen sollen: Wie haben Pädagogik und Sonderpädagogik sich im bildungspolitischen Kontext in den vergangenen Jahrzehnten mit der Frage nach integrativer bzw. separativer Beschulung auseinandergesetzt? Welche Wege hat die Bildungspolitik in diesem Zusammenhang geebnet bzw. verhindert? Ist die Frage nach Inklusion versus Exklusion, die u.a. aufgrund der UN-Behindertenrechtskonvention diskutiert wird, tatsächlich eine „neue“ Fragestellung oder gründet sie auf einer jahrzehntelangen Entwicklung? Und inwiefern spielt die Darstellung geschichtlicher Eckdaten mit Blick auf die dieser Arbeit zugrundeliegende Forschungsfragestellung, welche Qualität integrative Schulen und integrativer Unterricht haben, eine Rolle? Die Ausführungen zur Geschichte der integrativen Unterrichtung sollen u.a. erläutern, welche Faktoren dazu beigetragen haben, dass die Entwicklung schulischer Integration in der BRD über einen langen Zeitraum hinweg in kleinen Schritten 
und - mit Blick auf die Anzahl der integrativ unterrichteten Schüler - in geringem Umfang verlaufen ist um darauf zu schließen, welche Bedingungen schulische Integration und ihre Qualität möglicherweise auch heute noch behindern bzw. fördern. Das Kapitel fragt daher besonders intensiv nach Merkmalen von Veränderungsprozessen, die sich in diesem Bereich in den vergangenen etwa 70 Jahren vollzogen oder nicht vollzogen haben.

Ziel dieses Unterkapitels ist es darüber hinaus aufzuzeigen, dass pädagogische und sonderpädagogische Fragestellungen in den vergangenen Jahrzehnten in einem engen Zusammenhang standen. Immer wieder bewegten sich Theorie und Praxis von Pädagogik und Sonderpädagogik wie auf nebeneinander verlaufenden Schienen, die sich zwischenzeitlich annähern oder auch wieder voneinander entfernen. So genannter „integrativer“ oder "gemeinsamer“ Unterricht fand und findet in Regelschulen, nicht in Förderschulen statt. Irritierend wäre in diesem Zusammenhang eine geschichtliche Aufarbeitung der Pädagogik für Kinder mit Behinderungen unabhängig von der Geschichte der allgemeinbildenden Schulen. Eine solche "getrennte“ Darstellung ist mit Blick auf die Fragestellung der Qualität integrativer sonderpädagogischer Förderung nicht Ziel führend. Stattdessen sollen in den folgenden Abschnitten zentrale Entwicklungslinien herausgearbeitet werden, die die enge Verknüpfung der Gegenstandsbereiche verdeutlichen ${ }^{3}$. Eine dieser Entwicklung zugrunde liegende These lautet, dass das Thema der integrativen Beschulung von Kindern und Jugendlichen mit Behinderungen nicht in ausreichendem Maß zu einem Thema der Allgemeinen Pädagogik geworden ist (Bleidick \& Ellger-Rüttgardt, 2008, S. 250). Dies könnte wiederum damit zusammenhängen, dass bspw. innerhalb der schulischen Modellversuche zwar gute Erfahrungen mit gemeinsamer Unterrichtung gesammelt und diese positiven Erfahrungen zum Teil auch wissenschaftlich dokumentiert wurden, offensichtlich jedoch u.a. ein engerer produktiver Austausch zwischen Schulen, Hochschulen und Bildungspolitik ausblieb. So behauptet Bleidick, dass es „Strukturen der Beharrung in unserem Bildungswesen, politische Widerstände und Ressourcenknappheit“ (Bleidick \& Ellger-Rüttgardt, 2008, S. 49) waren, die dazu geführt haben, dass eine flächendeckende Verwirklichung der Integration nicht erreicht werden konnte. In Anlehnung an die Grundgedanken der Empfehlungen des Deutschen Bildungsrates (1973) waren es die Rahmenbedingungen, vor allem in den allgemeinen Schulen, die den Anforderungen integrativen Unterrichts nicht gerecht wurden, während Preuss-Lausitz ebenfalls schon in den 70er Jahren - die Annahme formuliert, „daß die wichtigsten Probleme der Sonderschule die Probleme des gegenwärtigen übrigen vertikalen Schulsystems sind“ (Preuss-Lausitz, 1977, S. 45).

\subsubsection{Sondereinrichtungen versus integrative Bildungsangebote: zwei Entwicklungslinien der frühen (Sonder-) Pädagogik}

Seit Beginn der „Anerkennung der Bildbarkeit Behinderter“ (Bleidick \& Ellger-Rüttgardt, 2008, S. 17) im Verlauf des 18. Jahrhunderts (Unterrichtung taubstummer Schüler durch den Abbé de l'Epée in Paris 1763, Eröffnung der ersten Blindenschule durch Valentin Haüy 1785 oder Erziehungsversuche bei einem geistig behinderten Jungen durch Jean Itard (1800) (Bleidick \& Ellger-Rüttgardt, 2008, S. 17)) lassen sich mit Blick auf besondere pädagogische Förderung zwei konträr erscheinende Entwicklungsstränge erkennen: zum einen der Ansatz das Schulsystem von Schülern mit Behinderungen zu „entlasten“, indem Differenz in allgemeinen Schulen durch

3 Aufgrund der spezifischen Fragestellung wird der Schwerpunkt der Ausführungen die wesentlichen Entwicklungslinien der integrativen sonderpädagogischen Förderung ab dem Ende des zweiten Weltkriegs betreffen, nicht aber die sonderpädagogische Theorieentwicklung in diesem Zeitraum. Dabei erfolgt eine Beschränkung auf die Ereignisse in der Bundesrepublik Deutschland. 
Sondereinrichtungen in ihrer Komplexität reduziert werden sollte (Muth, 1976, S. 15; Romey, 1977, S. 115; Bleidick, 1990, S. 10; Bleidick \& Ellger-Rüttgardt, 2008, S. 17), zum anderen, Bildungsangebote für diese Schüler zu „verallgemeinern“, also bspw. sinnesgeschädigte Kinder in allgemeinen Schulen zu unterrichten oder die gesellschaftliche Teilhabe schwerbehinderter und psychisch kranker Menschen in einer Gemeinde zu ermöglichen (Bleidick, 1990, S. 12; Speck, 2010, S. 11). Das heißt, dass von Anbeginn der Entwicklung von allgemeiner bzw. gesonderter Bildung für Kinder und Jugendliche mit Behinderungen bereits beide Entwicklungsansätze der Aufbau eines spezifisch ausgerichteten sonderpädagogischen Schulwesens und Ideen zur integrativen Unterrichtung in allgemeinen Schulen - vorhanden waren.

Nach dem Ende des Zweiten Weltkriegs kam es in Deutschland zunächst zu einer „Rennaissance der Heilpädagogik“ (Preuß, 1985, S. 11), das heißt, dass versucht wurde, die Entwicklung des Sonderschulwesens an der Stelle anzusetzen, an der sie mit der Weimarer Republik geendet war. Ähnlich wie beim Wiederaufbau der deutschen Städte und Gemeinden insgesamt ging es für die Hilfsschullehrer ab 1945 in beiden Teilen Deutschlands zunächst einmal darum, die Schulen, die vor bzw. während der Zeit der Diktatur bestanden hatten, wieder herzurichten und weitere Schulen aufzubauen (Bleidick, 1990, S. 13). Ab 1947/1948 begann die akademische Ausbildung von Sonderpädagogen an der Humboldt-Universität zu Berlin (Ellger-Rüttgardt, 2008, S. 372). Es erfolgte die Neukonstitution des Verbands Deutscher Hilfsschulen (VDH), der einige Jahre später eine „Denkschrift zu dem Ausbau des Heilpädagogischen Sonderschulwesens" verfasste (Verband deutscher Hilfsschulen, 1955, S. 3-9), die zur Beschlussfassung dem Deutschen Städtetag übergeben wurde, da die Gemeinden für die Finanzierung und Organisation der Schulen zuständig sind (Preuß, 1985, S. 11; Bleidick \& Ellger-Rüttgardt, 2008, S. 19). Diese Schrift umfasste ein Programm zur Ausgestaltung des Sonderschulwesens im westlichen Teil Deutschlands. Konzeptionell war die Einrichtung von fünf Typen von Sonderschulen vorgesehen. Lediglich die Hilfsschule wurde hinsichtlich möglicher didaktischer Prinzipien in der Denkschrift näher beschrieben. Insgesamt wurden vor allem strukturelle Bedingungen, die die Schulgebäude und Räumlichkeiten betreffen, dargestellt (Bleidick \& Ellger-Rüttgardt, 2008, S. 19).

Im „Gutachten zur Ordnung des Sonderschulwesens“, das 1960 von der Kultusministerkonferenz (KMK) veröffentlicht wurde, erfolgte eine Ausdifferenzierung in zwölf Sonderschulen: Sehbehindertenschule, Blindenschule, Gehörlosenschule, Schwerhörigenschule, Sprachheilschule, Körperbehindertenschule, Krankenschule und Hausunterricht, Hilfsschule, Beobachtungsschule, Erziehungsschwierigenschule, Gefängnisschule (Schule im Jugendstrafvollzug) und Sonderberufsschule (als eigene Schule oder in Verbindung mit anderen Sonderschulen) (Bleidick \& Ellger-Rüttgardt, 2008, S. 27; KMK, 1960). ${ }^{4}$ An der Redaktion maßgeblich beteiligt war der Arbeitskreis Sonderschulen im Schulausschuss der KMK. Verfolgt wurde eine vor allem pragmatische Zielsetzung mit dem „Zweck, die in den einzelnen Ländern auseinanderdriftenden, unterschiedlichen Entwicklungen zu koordinieren." (Bleidick \& Ellger-Rüttgardt, 2008, S. 24-35). Das Gutachten war damit eine „administrative Standorterklärung mit dem Ziel, schulorganisatorische Vereinheitlichungen und Ausbau des Sonderschulwesens zu ermöglichen und kein bildungspolitisch perspektivisches Grundsatzpapier." (Preuß, 1985, S. 25). Die im Gutachten formulierten Ziele wurden bundesweit verfolgt, die Ausweitung der Anzahl

\footnotetext{
4 Bis auf die Schule für geistige Entwicklung waren damit alle heutigen Formen gegeben. Es folgte 1958 die Gründung der Lebenshilfe für das geistig behinderte Kind (heute Lebenshilfe e.V.) in Marburg. Eine Denkschrift von 1960 forderte die Verankerung der Schulpflicht. Ab 1959: Gründung einer ersten Schule für Kinder mit geistigen Behinderungen (Mühl, 2018).
} 
der Sonderschulen führte zu einer Verdreifachung der Anzahl der Sonderschüler von 133.000 Schülern im Jahr 1960 auf 387.000 im Jahr 1973 (Preuss-Lausitz, 1981, S. 45).

$\mathrm{Zu}$ unterscheiden ist mit Blick auf die bildungspolitischen Auseinandersetzungen im Anschluss an die Gründung der beiden deutschen Staaten $1949 \mathrm{im}$ bundesrepublikanischen Teil insgesamt zwischen „verwaltungsorientierten Gremien, wie der Ständigen Konferenz der Kultusminister (KMK), oder den Kommunalvertretungen des Deutschen Städtetages zum einen, und den bildungspolitischen Gremien, wie dem Deutschen Ausschuß, dem Deutschen Bildungsrat und, besonders den zentralen Aspekt einer einheitlichen Bildungspolitik akzentuierend, der BundLänder-Kommission [zum anderen]“ (Preuß, 1985, S. 11f). Maßgeblich für die Entwicklung des Sonderschulwesens im Westen Deutschlands waren also zunächst die an Verwaltung orientierten Gremien. Bildungspolitische Diskussionen zu einer möglichen integrativen Beschulung wurden dagegen - anders als in anderen europäischen Ländern - in der Bundesrepublik nicht geführt (Speck, 2010, S. 10). Speck bewertet die Ausrichtung des Gutachtens auf den Ausbau der Sonderschulen in der Rückschau aus gesellschaftlicher Perspektive folgendermaßen:

Angesichts der damals [1960] verbreiteten gesellschaftlichen Distanz gegenüber „den Behinderten“ und damit auch dieser Aufgabe wurde Wert auf die „Eigenständigkeit“ der Sonderschulen gelegt. Mit der gleichen fachlichen Intention erfolgte der Wiederaufbau des Sonderschulwesens in der damaligen DDR. Eine Neuorientierung in integrativer Richtung, wie sie sich z.B. in den USA und in den skandinavischen Ländern schon Jahrzehnte vorher angebahnt hatte, war kein Thema, auch nicht für die allgemeine Schule, etwa für die damals entstehenden „Gesamtschulen“ in den 1960er Jahren. (Speck, 2010, S. 10)

Die isolierte Entwicklung der Sonderschulpädagogik in beiden deutschen Staaten erläutert Ellger-Rüttgardt (2008) ebenfalls rückblickend:

Dabei hatte es in Deutschland [...] zur Zeit der Weimarer Republik im Zusammenhang mit den vielfältigen Diskussionen um eine Reform der Pädagogik durchaus Bestrebungen gegeben, eine stärkere Verbindung zwischen allgemeiner und spezieller Pädagogik herzustellen. Aber das Verdrängen der Zeit des Nationalsozialismus und damit von Geschichte überhaupt sowie der Mangel an Internationalität bleiben wirksam und prägend bis zu Beginn der 70er Jahre. (Ellger-Rüttgardt, 2008, S. 298)

Obwohl Muth als Vorsitzender des Deutschen Bildungsrats in seiner Rückschau (1976) das Gutachten von 1960 als wesentlichen Meilenstein für den Ausbau des Sonderschulwesens in Deutschland einschätzt, sieht er es kritisch, dass diese Schulen eine Entlastungsfunktion übernahmen und erläutert die Wechselwirkung zwischen den beiden schulischen Systemen:

Im Zusammenhang mit der Entlastung der allgemeinen Schulen von behinderten Kindern setzten negative schulische Selektionsprozesse ein, in deren Gefolge sich ein unpädagogisches Leistungsverständnis schon in den Grundschulen durchsetzte, das sich neben den Überweisungen in Sonderschulen in relativ hohen Rückstellungsquoten schulpflichtiger Kinder vom Schulbesuch und in hohen Sitzenbleiberquoten konkretisierte. Diese Praxis führte dazu, daß in der allgemeinen Schule die sonderpädagogische Verantwortung in Verlust geriet, was zu einer pädagogischen Verarmung der allgemeinen Schule insgesamt führte; denn gerade in außergewöhnlichen Situationen, also etwa in der Förderung behinderter Kinder, zeigt sich, was pädagogisches Engagement und pädagogische Verantwortung sein kann. (Muth, 1976, S. 15)

Ein fruchtbarer Diskurs zwischen den beiden Disziplinen Pädagogik und Sonderpädagogik blieb nach Auffassung vieler Autoren also aus. Interessant ist auch, dass die Sonderschulpädagogik im Rahmen der vom Deutschen Ausschuss (nach Preuß (1985) ideeller Vorläufer des Deutschen Bildungsrats) verfassten bildungspolitischen Rahmenvorgaben oder Gutachten zur 
Lehrerausbildung in diesem Zeitraum (Mitte der 50er- bis Mitte der 60er-Jahre) schlicht nicht berücksichtigt wurde (Preuß, 1985, S. 13-16). Preuß erläutert bezüglich der „Vergessenen Sonderpädagogik“ (Preuß, 1985, S. 16), dass eine Erklärungsmöglichkeit für diese Nichtberücksichtigung darin liegen könnte, dass der Ausschuss vor allem aus Gymnasiallehrern zusammengesetzt war. Sie ergänzt allerdings:

Eine andere Ebene der Interpretation scheint aber auch zulässig: die Sonderpädagogik der 50er und 60er Jahre befindet sich ihrerseits außerhalb der Diskussion um eine bildungspolitische Neuorientierung. Dies wird z.B. offenkundig in der fachspezifischen Enthaltsamkeit hinsichtlich der Diskussion bildungspolitischer Dokumente in der Zeitschrift für Heilpädagogik während der 50er und 60er Jahre. Auch das Fehlen sonderpädagogischer Äußerungen in der von Schorb 1966 zusammengestellten Dokumentation von Stellungnahmen zum „Rahmenplan zur Umgestaltung.... [5] vom Februar 1959 kann als Indiz der bildungspolitischen Enthaltsamkeit der Sonderpädagogik interpretiert werden. Insgesamt also ein Problem von gegenseitigem Nichtwahrnehmen, verhängnisvoll dann, wenn - wie es zu prüfen gilt - auch in anderen Phasen der bildungspolitischen Neuorientierung dieses Phänomen nachweisbar wird. (Preuß, 1985, S. 17f)

Schon 1985 sieht Preuß als eine kritische Autorin der Berliner Sonderpädagogik demnach Anteile für eine fehlende Wahrnehmung und öffentliche Darstellung der Sonderpädagogik und des Sonderschulwesens auf beiden Seiten: der Seite der Vertreter der allgemeinen Pädagogik wie auch der Seite der Vertreter der Sonderschulpädagogik (Bsp.: fehlende bildungspolitische Einflussnahme der sonderpädagogischen Verbände). Ein möglicher Begründungsansatz für fehlende Innovationen im Bereich der Sonderpädagogik ist also nach Preuß (1985) u.a. die fehlende Innovationsbereitschaft seitens der Sonderpädagogen selbst.

\subsubsection{Die Gesamtschule - (k)eine Schule für alle Kinder?}

\section{Die Grundidee der Gesamtschulreform}

Besonders auffällig zeigt sich die „bildungspolitische Enthaltsamkeit der Sonderpädagogen“ nach Bleidick und Ellger-Rüttgardt (2008) im Zusammenhang mit der wichtigsten Diskussion der 60er- und 70er-Jahre: der Frage nach der Möglichkeit einer Beschulung von Kindern und Jugendlichen mit unterschiedlichen Voraussetzungen (Herkunft, Sozialisation, Leistungsvermögen) in einer gemeinsamen Schule, der sogenannten Gesamtschule.

Der durch die 68er-Studentenrevolte ausgelöste Ruf nach Demokratisierung der westdeutschen Gesellschaft führte in der Pädagogik zu der Forderung nach Überwindung des dreigliedrigen Schulwesens und zur Einführung von Gesamtschulen, deren Hauptziele die Herstellung von gleichen Bildungschancen vor allem für sozial unterprivilegierte Kinder, eine weitgehende Vermeidung früher Ausleseprozesse und Laufbahnentscheidungen, eine Ausschöpfung von Bildungsreserven sowie eine Reformierung von Lerninhalten und -methoden waren. [...] Die politische Intervention hieß Kompensatorische Erziehung, der Ausgleich sozial bedingter Versäumnisse. Schichtenspezifische Sozialisation wurde zum soziologischen Schlüsselbegriff. (Bleidick \& Ellger-Rüttgardt, 2008, S. 38f)

Ausgangspunkt der Gesamtschuldiskussion waren dabei nicht in erster Linie pädagogische Beweggründe, sondern die u.a. von Picht (1964) aufgrund statistischer Daten der OECD (1963) vermutete und prognostizierte „Bildungskatastrophe“, von der angenommen wurde, dass sie

5 Gemeint sind der „Rahmenplan zur Umgestaltung und Vereinheitlichung des allgemeinbildenden öffentlichen Schulwesens" (Deutscher Ausschuss, 4. Febrar 1959) und die Veröffentlichung von Schorb, A.-O. (Hrsg.) (1966): Für und Wider den Rahmenplan, Stuttgart: Klett-Verlag. 
zu einem erheblichen Fachkräftemangel führen könnte und damit zusammenhängen, dass Bildungsreserven bei Kindern und Jugendlichen aufgrund der frühen Zuweisung in unterschiedliche Leistungsgruppen nicht ausgenutzt werden würden:

Durch das Schulsystem werden schon zehnjährige Kinder - und in der Regel definitiv - in Leistungsgruppen eingewiesen, die durch das Berechtigungswesen einer entsprechenden Gruppierung den sozialen Positionen zugeordnet sind. [...] Die Schule ist deshalb ein sozialpolitischer Direktionsmechanismus, der die soziale Struktur stärker bestimmt als die gesamte Sozialgesetzgebung der letzten 15 Jahre. (Picht, 1964, S. 6)

Benner (2015) erläutert in diesem Zusammenhang, dass sich „alle politischen Parteien für eine ausgleichende, kompensatorische Erziehung aus[sprachen], um das Schreckgespenst einer auf das Beschäftigungssystem übergreifenden Bildungskatastrophe abzuwenden, Wirtschaft und Verwaltung höherqualifizierte Absolventen des Schulsystems in ausreichendem Maße zuzuführen und Bildung als Produktivkraft eher auf Wachstumssteigerung ausgerichteten gesellschaftlichen Gesamtkonzeption zu nutzen“ (Benner, 2015, S. 142). Als „Kernstück der Bildungsreform der Siebzigerjahre" (Bleidick \& Ellger-Rüttgardt, 2008, S. 40) erfuhr die Gesamtschule zur Zeit der sozial-liberalen Koalition politische Unterstützung, was allerdings nicht dazu führte, dass Gesamtschulen flächendeckend eingerichtet werden konnten und zwar weder in SPD-regierten Ländern, noch in CDU-bzw. CSU-regierten Ländern, in denen die Einrichtung von Gesamtschulen eine Ausnahme darstellte (Bleidick \& Ellger-Rüttgardt, 2008, S. 40).

Wie das Gutachten zur „Einrichtung von Schulversuchen mit Gesamtschulen“ vom Januar 1969 belegt, war die Integration von behinderten Kindern und Jugendlichen in der Gesamtschule nicht geplant: „In der Mittelstufe der Gesamtschule wird die Trennung von Schulzweigen mit unterschiedlichen Bildungszielen aufgehoben. Alle Schüler - ausgenommen die Sonderschüler - besuchen in der Mittelstufe eine gemeinsame Schule“ (Gutachten-Gesamtschule S. 16). (zitiert n. Preuß, 1985, S. 19), weshalb Romey 1977 forderte, dass „unter bildungsökonomischen, gesellschaftspolitisch-ideologischen und dynamischen Gesichtspunkten die faktische Ausklammerung des Sonderschulwesens von Gesamtschulversuchen (DEUTSCHER BILDUNGSRAT, 1969, S. 16) zu untersuchen [sei].“ (Romey, 1977, S. 111). Im Strukturplan für das Bildungswesen, veröffentlicht 1970 von der Bildungskommission des Deutschen Bildungsrats, zeigte sich darüber hinaus, dass auch die Sonderschulen an sich „vergessen“ wurden (Bleidick \& Ellger-Rüttgardt, 2008, S. 35-38), obwohl dieser Plan die wichtigste Planungsgrundlage für Erziehung und Bildung im Westdeutschland der Nachkriegszeit darstellte. Zwar formulierte die sozial-liberale Bundesregierung im Bildungsbericht von 1970 die politische Zielvorstellung möglichst alle schulpflichtigen Kinder in die allgemeine Schule aufzunehmen sowie eine Rückführung von Sonderschülern in die Regelschule zu ermöglichen (BMBW, 1970, S. 45) und verknüpfte diese politische Zielvorstellung mit Kritik am extensiven Ausbau der Sonderschulen (Preuss-Lausitz, 1981, S. 89f); ein Ansatz, der sich in ähnlicher Form auch in dem Konzept der Bund-Länder-Kommission von 1971 findet (Bleidick \& Ellger-Rüttgardt, 2008, S. 35). Aufgrund der Nichtidentifikation der Kultusminister mit den Zielvorstellungen der BrandtRegierung haben diese sich nach Preuss-Lausitz allerdings nicht auf tatsächliche Planungen der Länder ausgewirkt (Preuss-Lausitz, 1981, S. 90). Wichtige Lehrerverbände (GEW und VDS) wiederum brachten sich erst Ende der 60er Jahre in die Gesamtschuldiskussion ein und konnten daher u.a. nicht verhindern, dass die Grundstufe (Klasse 1-4) in die Gesamtschulplanung nicht einbezogen wurde. Diese Tatsache wiederum wertet Bleidick als erhebliche Hürde mit Blick auf die Beschulung von Schülerinnen und Schülern mit Behinderung in der Gesamtschule, da 
diese häufig schon vor dem Wechsel in die Sekundarstufe in Sonderschulen überwiesen wurden (Bleidick, 1978, S. 234).

\section{Inhaltliche Bausteine der Gesamtschulreform}

Erst in den 70er Jahren äußerten einzelne Allgemein- und Sonderpädagogen den Wunsch, die Rahmenbedingungen in Regelschulen bzw. Gesamtschulen vor allem für Kinder mit Lernbehinderungen oder Verhaltensauffälligkeiten so zu gestalten, dass eine Ausgliederung von vorneherein auszuschließen wäre (Bleidick, 1974, S. 225f; Muth, 1977; S. 90ff; Weigt, 1977, S. 11-24; PreussLausitz, 1981, S. 79). So forderten sie für die mögliche Integration von Schülern mit Behinderungen in den Gesamtschulen die Früherkennung von Behinderungen und (kompensatorische) vorschulische Erziehung bzw. Frühförderung im Elementarbereich (Muth, 1977, S. 95; PreussLausitz, 1977, S. 50; Weigt, 1977, S. 13), eine Individualisierung des Bildungsangebotes und den Ausbau des Primarbereichs (Preuss-Lausitz, 1977, S. 50; Weigt, 1977, S. 13), den Abbau des Leistungsdrucks in den Schulen (Muth, 1977, S. 96), Flexibilität bei der Schüler-Lehrer-Relation für individualisierte Lernprozesse (Muth, 1977, S. 96), die Senkung und Flexibilisierung von Klassenfrequenzen (Muth, 1977, S. 97f), die Ausstattung der Schulen mit technischen Medien und behinderungsspezifischen Fördermitteln (Weigt, 1977, S. 14; Muth, 1977, S. 96) sowie die Einbeziehung sonderpädagogischer Fachinhalte in die allgemeine Lehrerausbildung (Weigt, 1977, S. 14; Muth, 1977, S. 95). Muth betont in diesem Zusammenhang:

Die entscheidende Aufgabe des Integrationsbemühens ist deshalb in einer Veränderung der sozialen Bedingungen des Lernens zu sehen; das impliziert, daß die Eigenart des Einzelnen in den Lern- und Sozialisationsprozessen respektiert wird. Vorrangig ist in diesem Verständnis darum die dem Einzelnen gemäße individuelle Förderung, nicht die von vermeintlichen allgemeinen Verbindlichkeiten her vorzunehmende Selektion. (Muth, 1977, S. 91)

Zu den schulorganisatorischen Möglichkeiten für die Umsetzung dieses Anspruchs zählten Allgemeinpädagogen wie Muth (1977) oder Heese (1977) innere Differenzierung, Förderunterricht, behinderungsspezifische Förderung wie Bewegungstherapie oder Sprachtherapie, die additive Angliederung von Gruppen behinderter Kinder mit Teilintegration oder eine additive Angliederung ohne Teilintegration, aber mit Einbeziehung in das Schulleben, und forderten somit bereits Ende der 70er-Jahre ein flexibles integratives System (Muth, 1977, S. 97ff). Heese weist in diesem Zusammenhang auch auf die Notwendigkeit einer entsprechenden Lehrerbildung für die Umsetzung dieser Aspekte hin (Heese, 1977, S. 103) und fordert, dass schulische Integration „durch Abklärung ihrer Bedingungsfaktoren zu einem differenzierten lehrbaren System von praktikabler Schulorganisation und Methodik gemacht werden [müsse]“ (Heese, 1977, S. 104). Etwa gegen Ende der 70er Jahre verlagerte sich die Fragestellung der Integration von Schülern und Schülerinnen mit Behinderungen vom Sekundarstufen- in den Primarbereich, in dem vor allem präventive Maßnahmen dazu dienen sollten eine so genannte "Schulschwäche“ bereits vor ihrer Entstehung zu vermeiden:

Schulschwäche wird [...] als Sammelbezeichnung für Lernstörungen, Teilleistungsstörungen, Verhaltensstörungen, Sprachschwierigkeiten, drohende und leichtere manifeste Behinderungen aller Art sowie für ähnliche Beeinträchtigungen verwendet, unabhängig davon, ob die Beeinträchtigung überwiegend psychosozial, soziokulturell oder organisch bedingt ist. Die Bezeichnung Schulschwäche soll auch die Schwäche der Schule bei der Förderung der betroffenen Kinder zum Ausdruck bringen. In besonderer Weise von Schulschwäche bedroht sind Kinder aus Familien ausländischer Arbeitnehmer. [...] In Anbetracht der bisher vorliegenden Erfahrungen ist unter den gegenwärtigen Bedingungen jedoch mit einem Anteil von 25-30\% schulschwacher Kinder je Schülerjahrgang zu rechnen. (Reinartz \& Sander, 1982, S. 13) 
Im Thesenpapier „Maßnahmenkatalog zur Verminderung von Schulschwäche in der Grundschule" (Arbeitskreis Grundschule, 1977) machten die Autoren vielfältige Vorschläge zur Lernatmosphäre, $\mathrm{zu}$ individualisierenden und differenzierenden Lernangeboten sowie zum Einsatz von Medien und speziellem Förderunterricht (Bleidick \& Ellger-Rüttgardt, 2008, S. 42). Die Thesen und Empfehlungen sind „aus der Arbeit einer bundesweit zusammengesetzten Fachgruppe hervorgegangen, die aus Grundschul- und Sonderpädagogen, Hochschullehrern dieser Fächer, Schulpsychologen, Soziologen und Schulverwaltungsbeamten bestand“ (Reinartz \& Sander, 1982, S. 7). Interessant daran ist, dass diese Veröffentlichung sich explizit in erster Linie an Grundschullehrkräfte richtete. Die Zielsetzung des Maßnahmenkatalogs war, dass „die Quote der Kinder, die in den gegenwärtigen Grund- und Hauptschulen versagen, und [...] die Quote der Kinder, die in die Sonderschule überwiesen werden“ (Bleidick \& Ellger-Rüttgardt, 2008, S. 42) gesenkt werden sollte. Ähnliche Ziele formulierte auch die GEW, die sich bis dahin nicht vehement in die Integrationsdiskussion eingeschaltet hatte, auf ihrem Gewerkschaftstag 1977. Auch sie forderte als Verband eine mögliche Integration von Sonderschülern in die Gesamtschulen, Fördermaßnahmen in den Grundschulen sowie einen Ausbau der Frühförderung (Preuss-Lausitz, 1981, S. 101). Die für die Umsetzung pädagogischer Maßnahmen notwendigen Veränderungen beziehen sich dabei ausschließlich auf die Regelschulen, so dass PreussLausitz (1981) zu dieser Zeit kommentiert: „So wurde insbesondere von Sonderpädagogen der 'Schwarze Peter' an die Gesamtschulen und, in den Jahren nach der Bildungsratsempfehlung von 1973, zunehmend auch an die Grundschulen zurückgegeben." (Preuss-Lausitz, 1981, S. 79).

\section{Aspekte der Umsetzung der Gesamtschulreform - Grenzen integrativer Möglichkeiten, Problematisierung der Umsetzung der Gesamtschulidee}

Die inhaltliche Diskussion um Möglichkeiten und Grenzen einer integrativen Unterrichtung von Schülern mit Behinderungen in der Gesamtschule wurde in den 70er-Jahren sowohl von Allgemein- wie von Sonderpädagogen geführt und war Thema in Stellungnahmen oder bei Tagungen der Verbände (Muth, 1977; Heese, 1977; VDS, 1970; GEW, 1977; GGG, 1971). Am entschiedensten äußerte sich nach Preuss-Lausitz (1981) die Gemeinnützige Gesellschaft Gesamtschule (GGG) für integrative Unterrichtung. Sie forderte bei ihrer Jahresversammlung 1971 ein Schulwesen, „das die Vorschulerziehung als Elementarbereich einbezieht, den Primarbereich neu gestaltet und im Sekundarbereich Möglichkeiten schafft, behinderte Schüler bei sich zu behalten" (GGG, 1971, S. 11). Gleichzeitig gab es eine Reihe von Sonderpädagogen (Bachmann, 1974; Berg, 1975), die die Integration vehement ablehnten (Preuss-Lausitz, 1981, S. 79ff). Grenzen im schulorganisatorischen Bereich wurden dahingehend gesehen, dass die Integration von Schülern mit Behinderungen Einzelfallentscheidungen bleiben sollten (Muth, 1977, S: 94; Heese, 1977, S. 105):

Das Integrationsbemühen kann nicht so verstanden werden, daß es gilt, alle behinderten Kinder in die allgemeinen Unterrichtsprozesse zu integrieren. Das Spektrum dessen, was der Begriff Behinderung deckt, ist vielfältig. Es reicht, wenn man Extreme aufweist, von der einfachen Lernbehinderung [...] bis zur schweren geistigen Behinderung. Deshalb läßt sich in der Regel immer nur für den individuellen Fall auf dem Hintergrund einer flexiblen Schulorganisation die Integrationsmöglichkeit und Separationsnotwendigkeit für ein behindertes Kind ausmachen; denn das sollte die Devise der Schulorganisation der Zukunft sein: Integration des behinderten Kindes sollte gewährleistet werden, soweit sie möglich ist; Separation des behinderten Kindes sollte beschränkt werden auf das, was unabdingbar notwendig ist. (Muth, 1977, S. 94)

Weitere Grenzen wurden auch im Prinzip der Leistungsorientierung gesehen, das die Gesamtschulen genau wie andere Schulformen zu berücksichtigen habe: 
Man kann fragen, ob die Scheu vor der Diskussion um Integration darin begründet ist, daß man die Gesamtschule nicht aus dem ungebrochenen Leistungsanspruch entlassen möchte, einem Anspruch, der sich vor allem in einer auf die wichtigsten Fächer erstreckenden äußeren Differenzierung (Fachleistungskurssystem) und in einer Vielzahl informeller Tests konkretisiert. (Preuss-Lausitz, 1977, S. 45)

Dieses Problem bestehe nach Preuss-Lausitz auch deshalb, da die Gesamtschule sich als Angebotsschule neben den anderen vorhandenen Regelschulen des Sekundarstufenbereichs profilieren müsse. Eine äußere Strukturreform reiche zu einer integrativen Unterrichtung von Kindern mit Behinderungen nicht aus, dazu bedürfe es einer inneren Veränderung des Systems Gesamtschule (Preuss-Lausitz, 1977, S. 45). Auch die Notwendigkeit der Bereitstellung von Ressourcen wurde im Rahmen der Diskussion der Voraussetzungen einer möglichen Integration von Kindern und Jugendlichen in die Gesamtschulen thematisiert (Heese, 1977, S. 105, Weigt, 1977, S. 22):

Die integrative Förderung behinderter Kinder wird nicht nur keine Budgeteinschränkungen möglich machen; eher dürfte sie höhere Mittel erfordern. Denn die bestehenden Sonderschulen werden nicht geschlossen werden; sie werden nur voraussichtlich weniger Schüler haben. Aber die Integration bedarf vieler Sonderpädagogen, die an Regelschulen zugleich behinderte und nichtbehinderte Kinder unterrichten, vermutlich mehr Sonderpädagogen als bisher. Ferner braucht die integrative Förderung solche Arbeitsbedingungen, die eine Individualisierung des Unterrichts begünstigen (z.B. Kleinklassen, Gruppenarbeit bei team-teaching usw.); dergleichen kostet vermehrte Lehrerstunden, Einsatz von mehr therapeutischem Personal in den Schulen und mehr Räume für unterschiedliche Förderungszwecke. (Heese, 1977, S. 105)

Dass aufgrund dieser Notwendigkeit Probleme mit Blick auf die Umsetzung einer integrativen Unterrichtung entstehen könnten, wurde zum damaligen Zeitpunkt ebenfalls bereits klar erfasst:

In der Tat dürfte in dem letztgenannten eines der Hauptprobleme der Realisierung einer schulischen Integration Behinderter liegen: Solange die Bildungshaushalte durch finanzpolitische Entscheidungen zu drastischen Einsparungen von zusätzlichen Ausgaben angehalten sind, lassen sich pädagogische Utopien für behinderte Schüler nicht verwirklichen. (Weigt, 1977, S. 22)

Abgesehen von der aus unterschiedlichen Gründen nur unzureichend umsetzbar erscheinenden Forderung Kinder und Jugendliche mit Behinderungen in den Gesamtschulen zu unterrichten, wird in diesem Zusammenhang ebenfalls Ende der 70er-Jahre erkannt, dass die Gesamtschulen ihren eigenen Ansprüchen an eine kompensatorische Erziehung u.a. aufgrund mangelhafter materieller Ausstattung nicht gerecht werden konnten:

Durch ein differenziertes Lernangebot, Schaffung kleiner Lerngruppen sowie eigens entwickelter Curricula war beabsichtigt, ungleiche Bildungschancen, die in einem mehrgliedrigen Schulsystem typisch sind, durch ein integriertes Gesamtschulsystem aufzuheben. Allerdings sind zehn Jahre nach der Einleitung derartiger Reformvorhaben die meisten Gesamtschulen noch nicht aus dem Versuchsstadium herausgewachsen und es häufen sich unter anderem etwa aufgrund halbherziger Unterstützung seitens der Bildungsbürokratien planerisch-organisatorische Schwierigkeiten sowie materielle Ausstattungsmängel dieser auf zu große Schülerzahlen angelegten Bildungseinrichtung. Arbeitsüberlastung und Resignation von Schülern und Lehrern sind trotz positiver Grundhaltung gegenüber der Gesamtschule die Folge. (Weigt, 1977, S. 11f)

\subsubsection{Bildungspolitische Entwicklungen der 70er Jahre}

Zeitgleich zur Gesamtschuldiskussion erfolgten politische Grundsatzüberlegungen zur „Ordnung des Sonderschulwesens“ durch die KMK (1972) unter redaktioneller Führung durch den Verband Deutscher Sonderschulen (Bleidick \& Ellger-Rüttgardt, 2008, S. 25) sowie - durch 
den Deutschen Bildungsrat (1973) - die Veröffentlichung der Schrift „Zur pädagogischen Förderung behinderter und von Behinderung bedrohter Kinder und Jugendlicher“. Während die Empfehlungen der KMK den Ausbau des Sonderschulwesens für die auch heute noch vorhandenen Sonderschulformen festlegten (Bleidick \& Ellger-Rüttgardt, 2008, S. 29), und damit nach Preuß (1985) eine konsequente Fortführung der Empfehlungen von 1960 darstellen, wies die vom Deutschen Bildungsrat 1973 herausgegebene Schrift eine überwiegend integrativ orientierte Zielsetzung aus und hat nach Bleidick (1990) „das Stichwort „Integration“ schulpolitisch verbreitet“ (S. 36) oder kennzeichnete - wie Speck (2010) es formuliert - eine „Wendemarke in Richtung einer integrativen Neuordnung des Schulwesens in der Bundesrepublik Deutschland“ (S. 17). Bei der Erarbeitung dieser Schrift waren sowohl Allgemeinpädagogen wie Muth als auch Fachleute der Behindertenpädagogik sowie Regierungsvertreter beteiligt (Preuss-Lausitz, 1981, S. 91). Die Empfehlung des Deutschen Bildungsrats beinhaltete drei konzeptionelle Akzente: eine horizontale Gliederung des Schulwesens, die Individualisierung der Lernanforderungen sowie die Bedeutung früher Lernprozesse (Dt. Bildungsrat, 1973, S. 16-19). Die Grundgedanken der „Empfehlungen des Bildungsrates“ waren, dass gemeinsame Erziehung möglich ist, dafür aber bestimmte Rahmenbedingungen notwendig sind, dass sie prinzipiellen Vorrang vor spezialisiertem Unterricht haben sollte und dass sie eine strukturelle Umgestaltung der allgemeinen Schule erfordert (Speck, 2010, S. 17).

Als bedeutsam wurden in diesem Zusammenhang die Früherkennung von Behinderungen sowie eine den Anforderungen schulischer Integration angepasste Aus- bzw. Fortbildung der Lehrkräfte bzw. des pädagogischen Personals eingeschätzt (Dt. Bildungsrat, 1973, S. 12). Tragendes Modell der Empfehlung war das Fördersystem des Kooperativen Schulzentrums, das als Organisationsform ein flexibles System der Förderung behinderter Kinder innerhalb und außerhalb allgemeiner Schulen gewähren sollte (Dt. Bildungsrat, 1973, S. 77-19), wie folgende Grafik veranschaulicht:

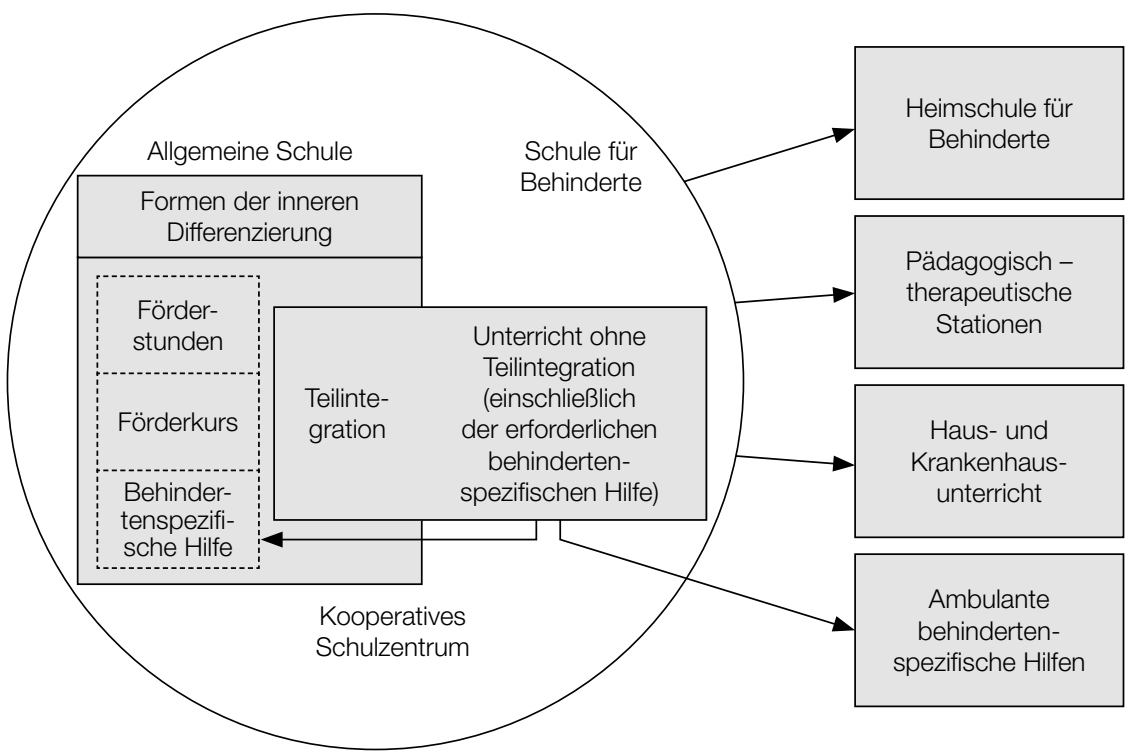

Abb. 2: Fördersystem eines kooperativen Schulzentrums (in Anlehnung an Deutscher Bildungsrat, 1973, S. 79) 
Es ging also um ein „flexibles System von Fördermaßnahmen, das einer Aussonderungstendenz der allgemeinen Schule begegnet, gemeinsame soziale Lernprozesse Behinderter und Nichtbehinderter ermöglicht und den individuellen Möglichkeiten und Bedürfnissen behinderter Kinder und Jugendlicher entgegenkommt" (Bleidick, 1990, S. 37). Rahmenbedingungen für die gemeinsame Unterrichtung (Bsp.: Vorbereitung der Lehrer, Schüler-Lehrer-Relation, Tätigkeit von Sonderpädagogen in allgemeinen Schulen, Ausstattung der Schulen, bauliche Voraussetzungen) (Dt. Bildungsrat, 1973, S. 72-76) werden in den Empfehlungen genauso präzise beschrieben wie mögliche entstehende Investitionskosten (S. 139-164) sowie Veränderungen auf unterrichtlicher Ebene wie Formen der inneren Differenzierung oder allgemeine Fördermaßnahmen. Besonders wichtig ist den Autoren die Durchlässigkeit des schulischen Systems. Die Herausgeber der Empfehlungen sahen damals eine

[...] reelle Chance für die Verwirklichung der neuen Konzeption [...] unter anderem darin, daß sich das bestehende allgemeine Schulwesen in einer umfassenden Reform befindet und daß das bestehende Sonderschulwesen eines erheblichen weiteren Ausbaus bedarf, der nicht in traditionellen Formen vorgenommen werden muß, sondern im Sinne der integrativen Konzeption eingeleitet werden kann. (Dt. Bildungsrat, 1973, S. 16)

Tatsächlich hätte diese Idee allerdings einer kompletten und vor allem bundesweiten Veränderung des Schulwesens bedurft. Bleidick geht diesbezüglich bereits in den 70er Jahren

[...] von der deutlich artikulierten Voraussetzung aus, daß den veränderten gesellschaftlichen Verhältnissen gemäß die Schule der Zukunft eine Gesamtschule in der Überwindung des drei- bis viergliedrigen vertikalen Schulsystems sein wird, die die Erziehung von Behinderten und den Gegenstand der Behindertenpädagogik in ein neues gesellschaftliches Bezugsfeld und an einen anderen wissenschaftlichen Ort der Pädagogik bringen könnte. (Bleidick, 1974, S. 213)

Problematisch hinsichtlich der Wirkkraft der Empfehlungen und damit auch mit Blick auf Bleidicks Einschätzung ist zu werten, dass diese zu einem Zeitpunkt erscheinen, zu dem die Gesamtschuldiskussion ihren Höhepunkt bereits hinter sich gelassen hat und die Veröffentlichung des Strukturplans für das Bildungswesen schon drei Jahre zurückliegt. Preuß führt dazu aus: „Es erscheint allein vom Zeitpunkt der Veröffentlichung als Reformnachzügler“ (Preuß, 1985, S. 21) und benennt diese Empfehlungen daher als „bildungspolitischen Appendix“ (Preuß, 1985, S. 22). Dass die Empfehlungen zumindest kurzfristig nicht zu nachhaltigen (bildungspolitischen) Veränderungen geführt haben, belegt nach Preuß u.a. der 1975 vom Deutschen Bildungsrat veröffentlichte Bericht „Entwicklungen im Bildungswesen“:

Denn wären diese Empfehlungen von 1973 nicht nur fachwissenschaftlich bedeutsam, sondern auch bildungspolitisch relevant für die Neustrukturierung des Bildungswesens in der Bundesrepublik Deutschland geworden, dann müßten bereits im Bericht ' 75 [...] veränderte Grundstrukturen, z.B. im Primarbereich der Grundschule erkennbar sein: Behinderte in die Planung des Bildungswesens explizit - auch in ausgewiesenen Formen einer integrativen Erziehung - einzubeziehen, das wäre ein Indiz für ein neues bildungspolitisches Grundverständnis gewesen. Diese Erwartungen aus der sonderpädagogischen Sicht werden jedoch im Bericht '75 nicht erfüllt. (Preuß, 1985, S. 22)

Muth sah das größte Hemmnis bei der Umsetzung der Bildungsratsempfehlung in fehlenden Bewusstseinsprozessen gesellschaftlicher Entscheidungsträger wie bspw. den öffentlichen Verwaltungen: 
In einer Verwaltung, die ein grundschulfähiges körperbehindertes Kind auf einen weiten Schulweg schickt, weil in der Schule, die der Wohnung des Kindes benachbart liegt, keine für das Kind geeignete Toilette vorhanden ist, hat sich diese Bewußtseinsänderung ebensowenig angebahnt wie bei der Errichtung einer Sonderschule zum Beispiel für hörgeschädigte Kinder auf einer grünen Wiese weitab vom Leben der Gesellschaft. (Muth, 1976, S. 17)

Darüber hinaus forderte Muth 1976, dass sich Bewusstseinsveränderungen auch bei den Regelund Sonderschullehrern vollziehen müssen. Auf der einen Seite dahingehend, dass die Lehrer der allgemeinen Schulen bereits in ihrer Ausbildung mit sonderpädagogischen Inhalten konfrontiert werden sollten, damit sie sich später in ihren Schulen für Kinder mit Einschränkungen verantwortlich fühlen; auf der anderen Seite in der Richtung, dass Sonderschullehrer die Bereitschaft entwickeln, Kinder mit Behinderungen in den Regelschulen zu fördern. Darüber hinaus seien Bewusstseinsänderungen in der Gesellschaft generell notwendig (Muth, 1976, S. 17).

Innerhalb der Sonderpädagogik lassen sich nach Preuss-Lausitz (1981) und Preuß (1985) für die 70er-Jahre verschiedene „Krisen“ ausmachen. Während Preuss-Lausitz in diesem Zusammenhang Anfang der 80er-Jahre die Krise der Sonderschule beschreibt (Preuss-Lausitz, 1981, S. 17ff), spricht Preuß für die 70er-Jahre von einer „Identitätskrise in der Sonderpädagogik“ (Preuß, 1985, S. 44ff) insgesamt und spielt dabei insbesondere auf das problematisch gewordene berufliche Selbstverständnis von Sonderschullehrern bzw. Sonderpädagogen an. Da es innerhalb (sonder-) pädagogischer Diskussionen verstärkt um die Thematik der Sonderpädagogik in allgemeinen Schulen ging (u.a. Sander, 1976; Kleber, 1977; Reinartz \& Sander, 1982) mussten sich die Sonderschullehrer in dieser Zeit intensiv mit der Frage auseinandersetzen, wodurch sich ihr spezifisches sonderpädagogisches Handeln auszeichnet. Gleichzeitig musste wieder gesehen werden, worin die gemeinsamen pädagogischen Aufgabenstellungen der allgemeinen und der besonderen Pädagogik bestanden. Für eine ganze Reihe von Sonderschullehrern - so Preuß - verursachte dieses Infragestellen der eigenen - institutionsgebundenen - Tätigkeit Unsicherheiten, die durch das damalige Ausbildungssystem an den Hochschulen, das eigene Studiengänge für das Sonderschullehramt vorsah, nicht verringert werden konnten (Preuß, 1985, S. 47f).

Preuss-Lausitz schätzte zusammenfassend die Zeit zwischen dem Ende der 60er- und den ausgehenden 70er-Jahren folgendermaßen ein:

Der Zeitraum [...] in der Bundesrepublik und in West-Berlin ließe sich für den Bildungsbereich als Wandel von einer reformorientierten zu einer restaurativen Phase beschreiben. [...] Innerhalb der Integrationsdiskussion lassen sich diese Krisen und Verschiebungen durch eine Verlagerung der Diskussion über Gesamtschulen (als Ort der Integration potentieller Hilfsschüler) bin zur Grundschule feststellen und zugleich durch Ersetzung des Themas von Chancengleichheit und kompensatorischer Erziehung durch das über Didaktik, soziales Lernen und 'Mut zur Erziehung'. (Preuss-Lausitz, 1981, S. 76, Herv. im Orig.).

Preuß formuliert:

Insgesamt für die Sonderpädagogik der 60er und 70er Jahre eher eine deprimierende Grundposition, sich „ausgenommen“ zu sehen - „ausgenommen“ von der Neukonzeption eines offenen Schulsystems! (Preuß, 1985, S. 19) Die bildungspolitisch anfänglich ,Vergessene Sonderpädagogik‘ wird zu Beginn der siebziger Jahre durch die Empfehlungen des Deutschen Bildungsrates (1973) korrigiert. Die späte bildungspolitische Korrektur wird zwar schulorganisatorisch nicht mehr wirksam, produziert aber auf Bundes- und Landesebene einen pädagogischen Widerspruch zwischen bildungspolitischen Zielen und schulorganisatorischen Umsetzungen von Sonderpädagogik in der allgemeinen Schule. Der Ausbau und die Differenzierung des Sonderschulwesens erfolgen entsprechend den KMK-Aussagen und erscheinen in sich lange Zeit widerspruchsfrei, weil die bildungspolitischen Zielformulierungen der Sonderpädagogik für die allgemeine Schule weitgehend negiert werden. (Preuß, 1985, S. 40f) 


\subsubsection{Integration (nur) unter bestimmten Bedingungen? Modellversuche als Möglichkeit der Umsetzung integrativer Beschulung}

Obwohl es also in den 70er Jahren Überlegungen zur Integration von Schülern mit Behinderungen in das allgemeine Schulsystem gab, konnten sich diese nur wenig durchsetzen. Anstelle einer umfassenden Umgestaltung des Schulsystems, wie die Gesamtschulbefürworter sie gefordert hatten, blieb diese Schulform eine Erweiterung des Angebots. Für die Umsetzung integrativer Unterrichtung wurde eine Fülle von Modellversuchen gestartet, die teilweise bis heute fortbestehen. Muth (1976) beschreibt in diesem Zusammenhang den Modellversuch an der Fläming-Grundschule als Weg, Integration bei fehlenden umfassenden bildungspolitischen Entscheidungen, die Veränderungen im Schulwesen insgesamt erfordert hätten, zumindest im Kleinen umzusetzen:

Die Empfehlung des Deutschen Bildungsrates war 1973 von den Kultusministern der Länder positiv aufgenommen worden. Aber kein Minister oder Senator dachte daran, ihre Vorschläge in seinem Wirkungsbereich auf den Weg der Verwirklichung zu bringen. [...] Das Vakuum, das nach 1973 hinsichtlich der Ausbreitung des Integrationsgedankens und der Einrichtung integrativer Schulen entstanden war, wurde 1975 durch den integrativen Zug der Fläming-Grundschule beendet. (Muth, 1988, S. 19f)

Dies wiederum wertete er schon zu einem früheren Zeitpunkt wie folgt:

In der Schulpolitik in der Bundesrepublik hat sich in kontroversen Problemen und in offenen Fragestellungen in den letzten Jahren die Praxis herausgebildet, auf eine Klärung und Antwort in Schulversuchen oder Versuchsprogrammen zu verweisen. Häufig ist das aber nichts weiter als ein Ausweichen vor politischen Entscheidungen oder das Kaschieren konservativer Grundhaltungen. (Muth, 1976, S. 15)

Tatsächlich wurden zahlreiche Modellversuche in verschiedenen Schulformen (Grundschulen, Orientierungsstufen, Gesamtschulen, Gymnasien) und teilweise gezielt für Schüler mit spezifischen Behinderungen (Bsp.: blinde oder gehörlose Schüler) eingerichtet. Eine der ersten Modellschulen für integrativen Unterricht war die private Schule Aktion Sonnenschein in München (1970), gefolgt von der staatlichen Fläming-Grundschule in Berlin (eingerichtet 1975) und der Uckermarck-Grundschule (1982). Ähnlich wie Ende der 70er Jahre wurden auch in den 80er Jahren konkrete qualitative Kriterien für die Umsetzung integrativer Unterrichtung benannt, diesmal allerdings auf der Grundlage der vorangegangenen Schulversuche (PreussLausitz, 1981; Reiser et al., 1984; Mühl, 1987; Wocken, 1987; Dumke et al., 1989; Bächthold et al., 1990; s. dazu auch 3.2-3.5). Beispielhaft dafür sind die - in diesem Fall mit Blick auf das gemeinsame Lernen geistig behinderter und nichtbehinderter Kinder und Jugendlicher in der Grundschule fokussierten - Qualitätsmerkmale nach Müller (1987), die u.a. Rahmenbedingungen wie die Klassengröße (nicht mehr als 20 Schüler mit 2-4 Schülern mit Behinderung) und ein Zwei-Pädagogen System (bedarfsorientiert, situationsbezogen, flexibel, Sonderschullehrer mit vollem Deputat) umfassen sowie neue binnendifferenzierende und handlungsorientierte Lernformen in den Grundschulen und eine gemeinsame Erstellung von integrativen Konzepten durch Sonder- und Grundschullehrer fordern (Müller, 1987, S. 158-164).

Die Schlussfolgerungen, die Autoren der Sonderpädagogik aus der Phase der Schulversuche ziehen, sind unterschiedlich. Reiser u.a. (1984) forderten beispielsweise, dass die Schulen sich verändern müssen um die entstehende größere Heterogenität nutzen zu können:

Damit müßte entfallen: Der Zeitdruck, der herkömmliche Frontalunterricht, das Notensystem, die Jahrgangsklassen, die Einengung des Leistungsbegriffs, die Vorstellung eines von einer Lerngruppe im Gleichschritt durchexerzierten Curriculums. (Reiser et al., 1984, S. 316) 
Die Autoren gehen davon aus, dass die Umsetzung schulischer Integration ein langer Weg mit mehreren Etappen sein werde, bei dem auf die Individualhilfe von Sonderschullehrern in Regelschulen eine umfassende Unterstützung von Grundschulen durch dieselben erfolge, und damit eine „Trendwende in der Grundschulpädagogik“ eingeleitet werden könne (Reiser et al., 1984, S. 316f). Feuser und Meyer (1987) formulieren Vermutungen dafür, warum diese Reform nicht stattfinde. So biete das vertikal gegliederte Schulsystem die Möglichkeit, dass Unterricht ohne bzw. mit wenig aufwendiger Binnendifferenzierung erteilt werden könne. Der mangelnde Reformwille an sich hänge damit zusammen, dass die Politik keine echten Veränderungen im Bildungssystem wünsche (Feuser \& Meyer, 1987, S. 32). Darüber hinaus habe die Grundschule „keine Didaktik, Methodik und Unterrichtsorganisation entwickelt, die sie besonders für integrativen Unterricht prädestinieren würde." (Feuser \& Meyer, 1987, S. 33). Kanter resümiert 1991, „dass die Frage nach der richtigen Institution und der Ruf nach Reformen [...] mehr denn je nur denkbar und machbar innerhalb einer Reform des gesamten Bildungs- und Erziehungswesens zu beantworten ist“ (Kanter, 1991, S. 103; Herv. im Orig.). Für Bleidick erscheint 1990 das „in integrativen Schulversuchen auszählbare Ergebnis enttäuschend“ (Bleidick, 1990, S. 17). Speck verweist aus Sicht der 2000er Jahre in positiver Hinsicht auf die Ergebnisebene, da die Modellversuche belegt hätten, dass gemeinsamer Unterricht unter passenden Rahmenbedingungen möglich sei (Speck, 2010, S. 31). Insgesamt gehen die sonderpädagogischen Autoren also davon aus, dass weder ein umfassendes politisches noch pädagogisches Interesse an der integrativen Unterrichtung von Schülern mit Behinderungen in Regelschulen bestanden habe.

Kritisch zu den Schulversuchen äußern sich auch die Sonderschulreferenten der Länder in ihrem 1983 für die Kultusministerkonferenz erstellten „Bericht über Bedingungen und Grenzen des gemeinsamen Unterrichts von lernbehinderten und nichtbehinderten Schülern in allgemeinbildenden Schulen“. Sie verweisen darauf, dass „nur ein sehr geringer Teil der insgesamt ca. 90 Modellversuche den Fragen des gemeinsamen Unterrichts von Behinderten und Nichtbehinderten gewidmet ist" (KMK, 1983, S. 9). Stattdessen hätte sich der überwiegende Teil der Modellversuche mit der Früherkennung und Frühförderung befasst und ergeben, dass einige Schüler nicht aber alle - mit Hilfe von Fördermaßnahmen durch Sonderschullehrer in den Regelschulen verbleiben können. Für diese präventiven Maßnahmen sei ein hoher personeller Aufwand von einem Sonderschullehrer je vier Klassen (Grundschule) notwendig (KMK, 1983, S. 9f). Für die Modellversuche, die eine gemeinsame Unterrichtung im engeren Sinn erprobt hätten (Bsp.: Aktion Sonnenschein, Fläming-Grundschule), seien Schüler ausgewählt worden. Offene Fragen wie die zum Leistungsanspruch und zur Leistungsbeurteilung, zur Lehrplangestaltung oder zu integrationsfördernden Methoden seien ungeklärt (KMK, 1983, S. 11). Als Rahmenbedingungen formulieren die Referenten folgende Aspekte: eine Klassengröße von nicht mehr als 20 Schülern, darunter höchstens ein Viertel lernbehinderter Schüler, sechs Sonderschullehrerstunden je Klasse, sonderpädagogisch geeignete Lehr- und Lernmittel, Sicherung der fachlichen Kompetenzen durch Einsatz von Sonderschullehrern, positive Haltung von Lehrern und Eltern gegenüber Schülern mit Lernbehinderungen, gemeinsamer Unterricht, Binnendifferenzierung, Einzel- und Gruppenförderung (KMK, 1983, S. 13f). Die Sonderschulreferenten gehen davon aus, dass die entstehenden Kosten höher liegen werden als die, die zum damaligen Zeitpunkt in den Sonderschulen anfallen (KMK, 1983, S. 16).

In der Rückschau fasst Bleidick zusammen, dass sich über mehrere Jahrzehnte hinweg eine „unübersehbare Vielfalt nebeneinander bestehender Organisationsstrukturen“ (Bleidick \& EllgerRüttgardt, 2008, S. 47) entwickelt habe. Einen Grund dafür sieht er - mit Bezug auf Füssel und Kretschmann (1993) - darin, dass keine klaren schulrechtlichen Bedingungen für schuli- 
sche Integration geschaffen wurden (Füssel \& Kretschmann, 1993, S. 14-22). Nach Füssel \& Kretschmann erfülle lediglich die Grundschule „die ihr zugedachte Integrationsleistung, indem es ihr gelingt, eine gemeinsame Lernsituation für Kinder unterschiedlicher sozialer Herkunft und auch mit einer durchaus beträchtlichen Streuung der intellektuellen Fertigkeiten herzustellen“ (Füssel \& Kretschmann, 1993, S. 52). Sie vertreten damit eine andere Haltung als die o.g. von Feuser und Meyer (1987).

\subsubsection{Bildungspolitische Dokumente der 90er Jahre}

Zeitgleich wurden 1994 zwei Dokumente veröffentlicht, die sich nachhaltig auf die sonderpädagogische Förderung auswirkten: die Salamanca-Erklärung zur „Pädagogik für besondere Bedürfnisse“ (UNESCO, 1994), die eine Verankerung des Diskriminierungsverbots für Menschen mit Behinderungen im Grundgesetz der BRD zur Folge hatte (Ergänzung des Artikels 3 des GG um Punkt 4: „Niemand darf wegen seiner Behinderung benachteiligt werden." (Bundesministerium der Justiz, 1994, GG Artikel 3)) sowie die „Empfehlungen zur sonderpädagogischen Förderung in den Ländern der BRD“ (KMK, 1994), die mit 22 Jahren Abstand zur vorangegangenen „Empfehlung zur Ordnung des Sonderschulwesens“ veröffentlicht wurden. Die Salamanca-Erklärung war Ergebnis einer Tagung, bei der auf Einladung der spanischen Regierung hin „höhere Erziehungs- und Verwaltungsbeamte, Politiker und Spezialisten zusammen mit Vertretern der Vereinten Nationen und Speziellen Behörden, anderen internationalen Regierungsorganisationen, sowie nichtstaatlichen und Spenderorganisationen"(UNESCO, 1994, S. 1) gemeinsam über integrative Pädagogik und „Bildung für Alle“ diskutierten. Sie stellt einen „Aktionsrahmen“ dar und fordert als Leitprinzip:

[...] dass Schulen alle Kinder, unabhängig von ihren physischen, intellektuellen, sozialen, emotionalen, sprachlichen oder anderen Fähigkeiten aufnehmen sollen. Das soll behinderte und begabte Kinder einschließen, Straßen- ebenso wie arbeitende Kinder, Kinder von entlegenen oder nomadischen Völkern, von sprachlichen, kulturellen oder ethnischen Minoritäten sowie Kinder von anders benachteiligten Randgruppen oder -gebieten. Diese Bestimmungen schaffen eine Reihe von Herausforderungen an Schulsysteme. Im Zusammenhang mit diesem Aktionsrahmen bezieht sich der Begriff „besondere pädagogische Bedürfnisse" auf all jene Kinder und Jugendliche, deren Bedürfnisse von Behinderungen oder Lernschwierigkeiten herrühren. (UNESCO, 1994, S. 4)

Sie umfasst Ausführungen zu Politik und Organisation, zu Schulfaktoren, zur Auswahl und Ausbildung pädagogischen Personals, zu externen unterstützenden Systemen, Prioritäten, Perspektiven in der Gemeinde sowie erforderlichen Mitteln (UNESCO, 1994, S. 7-16) und formuliert in diesem Zusammenhang klare Qualitätskriterien für integrative Bildungspolitik, integrative Schulen und integrative Gemeinden (UNESCO, 1994).

Die „Empfehlungen zur sonderpädagogischen Förderung“ schärften eine eher personenbezogene und individuelle Sichtweise anstelle des Blicks auf Institutionen und definierten statt der Sonderschulbedürftigkeit den so genannten sonderpädagogischen Förderbedarf (KMK, 1994, S. 2), so dass die schon in den 70er Jahren geforderte Lernortunabhängigkeit der sonderpädagogischen Förderung erneut in den Vordergrund rückte: „Die Erfüllung Sonderpädagogischen Förderbedarfs ist nicht an Sonderschulen gebunden; ihm kann auch in allgemeinen Schulen, zu denen auch berufliche Schulen zählen, vermehrt entsprochen werden." (KMK, 1994, S. 2). Dieser Auffassung gemäß erfolgte die Bezeichnung von Förderschwerpunkten (Bsp.: Hören, Sehen, körperliche und motorische Entwicklung, kranke Schüler, Sprache, geistige Entwicklung, Lernen, emotionale und soziale Entwicklung, autistisches Verhalten). Bleidick spricht in 
diesem Zusammenhang von einer „Kopernikanischen Wende“ der Bildungspolitik (Bleidick \& Ellger-Rüttgardt, 2008, S. 34), obwohl die Ausführungen mit Blick auf integrativen Unterricht im Vergleich zur Salamanca-Erklärung sehr vorsichtig erscheinen, wenn als Ziel formuliert wird, die „Weiterentwicklung der schulischen Förderung aller behinderten und von Behinderung bedrohten Kinder und Jugendlichen abzusichern und die Bemühungen um gemeinsame Erziehung und gemeinsamen Unterricht für Behinderte und Nichtbehinderte zu unterstützen. (Bleidick \& Ellger-Rüttgardt, 2008, S. 33). Vorgesehen war ein flexibles und qualitativ hochwertiges gestuftes System von Förderangeboten (Bleidick \& Ellger-Rüttgardt, 2008, S. 34). Auch die bereits in den 70er Jahren entstandene Idee zu Sonderpädagogischen Förderzentren wurde in den 90er Jahren vom Verband Sonderpädagogik wieder aufgegriffen, um damit „eine Organisationseinheit zur Überbrückung der Fronten zu schaffen“ (Bleidick \& Ellger-Rüttgardt, 2008, S. 66). Der Fachverband forderte 1997 „eine weitgehende Umsetzung des Gemeinsamen Unterrichts für alle Kinder und Jugendlichen auf der Grundlage ihres individuellen Förderbedarfs“ (vds, 1997, S. 2) und ein „bedarfsangemessenes und schulbezogenes Kontingent an sonderpädagogischen Ressourcen in der allgemeinen Schule“ (vds, 1997, S. 3). Nach Auffassung des Verbands sollten Sonderpädagogische Förderzentren diese Aufgaben koordinieren (vds, 1997, S. 3).

In den Jahren zwischen 1995 und 2003 stieg sowohl die Anzahl der Schülerinnen und Schüler in Förderschulen um etwa 10\% (1995: 390.500; 2002: 429.300) und die Förderschulbesuchsquote damit von 4,3\% auf 4,8\% als auch die Anzahl integrativ unterrichteter Schüler mit spF in den Regelschulen (1999: 54.350; 2003: 63.113) und damit der Inklusionsanteil auf 14,77\% (KMK, 2005, S. XII u.S. 53). In den Folgejahren bis 2012 blieb zwar die Förderschulbesuchsquote relativ unverändert (2012: 4,8\%), es stiegen allerdings sowohl der Inklusionsanteil (auf $24,8 \%$ in 2012) als auch die Förderquote (von 5,4\% 2002 auf 6,4\% 2012) (Dietze, 2013, S. 36).

\subsubsection{PISA und die 2000er Jahre}

Nach der zu Beginn der 60er Jahre befürchteten Bildungskatastrophe stellten die Ergebnisse der PISA (Programme for International Student Assessment)-Untersuchung im Jahr 2000 in den Augen vieler deutscher Pädagogen und Politiker tatsächlich eine bundesrepublikanische Bildungskatastrophe dar. Und wie Weiner (2009) erläutert, ging davon - ähnlich wie in den 60er Jahren - erneut ein (bildungs-)politischer Reformwille aus:

Es wurde die überragende Bedeutung der Bildung für die Konkurrenzfähigkeit Deutschlands in der globalisierten Wissensgesellschaft und die Teilhabe des Einzelnen am kulturellen und gesellschaftlichen Leben betont. Plötzlich schien jeder genau zu wissen, was getan werden muss, um die von der OECD aufgelisteten Defizite unseres Bildungssystems zu beseitigen. In den Medien übertrumpfte man sich gegenseitig mit Reformforderungen, die man vor PISA noch aus wahltaktischen oder ideologischen Gründen bekämpft hatte. (Weiner, 2009, S. 3)

Die PISA-Studie - eine Vergleichsuntersuchung zwischen 32 Staaten, durchgeführt von der OECD - untersuchte Schülerleistungen in unterschiedlichen Kompetenzbereichen (Lesekompetenz, mathematische Kompetenz, naturwissenschaftliche Kompetenz sowie fachübergreifende Kompetenzen). Einbezogen wurden in der BRD etwa 1500 Schulen und über 50.000 Schüler (Deutsches PISA-Konsortium, 2001, S. 13). Die Ergebnisse der PISA-Studie schockierten die deutsche Bildungspolitik vor allem mit Blick auf zwei wesentliche Ergebnisse: 1. Die Schülerleistungen in den Bereichen Lesen, Mathematik und Naturwissenschaften waren im weltweiten Vergleich unterdurchschnittlich. 2. Es gab einen sehr engen Zusammenhang zwischen sozialer Herkunft und den Leistungen der Schüler und Schülerinnen: 
In keinem anderen der untersuchten Länder besteht eine so enge Koppelung von niedriger Schulleistung und sozioökonomisch prekärem Herkunftsmilieu (Armut, Arbeitslosigkeit, niedrige Sozialschicht, Migrantenfamilien). (Bleidick \& Ellger-Rüttgardt, 2008, S. 57)

Noch etwas hat PISA an den Tag gebracht: Die soziale Herkunft entscheidet in Deutschland so stark wie in kaum einem anderen Land darüber, ob ein Kind gute oder schlechte Leistungen erbringt. Erhält ein Kind von seinen Eltern zu wenig Unterstützung für die Entwicklung seiner Kompetenzen, dann können die Lehrerinnen und Lehrer in der Schule ein solches Defizit kaum noch ausgleichen. Das Elternhaus gehört in diesem Sinn mit zum „Bildungssystem“ eines Landes. (Hurrelmann \& Timm, 2011, S. 7)

Aufgrund des durch die PISA-Ergebnisse entstandenen Veränderungsdrucks wurde versucht „verschiedene Arrangements evidenzbasierter oder outputorientierter Steuerung in den deutschsprachigen Schulsystemen“" (Altrichter \& Feyerer, 2017, S. 31, Herv. im Orig.) zu implementieren, und zwar vor allem in Form von Bildungsstandards und Qualitätsrahmen (Klieme, 2007). Während die 90er Jahre durch eine Fokussierung auf die Entwicklung von Einzelschulen (Rolff, 1998) gekennzeichnet waren, spielte nun die Veränderung des gesamten Schulsystems wieder eine Rolle. Im Anschluss an PISA erfolgte u.a. ein Ausbau des Ganztagsangebots in Deutschland und damit eine Strukturveränderung, die in allen Bundesländern vollzogen wurde (Lange, 2015, S. 7). Fend u.a. plädieren aufgrund PISA-ähnlicher Ergebnisse aus einer Studie zu Absolventen des Bildungssystems für eine pädagogische Umgestaltung von Schulen mit dem Fokus auf einer umfassenderen Förderung der Schüler ergänzt durch behutsame organisatorische Veränderungen hinsichtlich der Erhöhung der Durchlässigkeit von Bildungsgängen (Fend et al., 2009) - Forderungen, die in ähnlicher Form bereits im Rahmen der Gesamtschuldiskussion in den 60er- und 70er-Jahren formuliert wurden (s. 2.1.2). Da Schüler mit sonderpädagogischem Förderbedarf in der PISA-Studie nicht berücksichtigt wurden, war die Sonderpädagogik nach Bleidick und Ellger-Rüttgardt (2008) von den Ergebnissen nicht unmittelbar betroffen, mittelbar allerdings schon:

Gleichwohl ergibt sich aus dem Abschneiden der schlechten Schüler ein offenkundiger sonderpädagogischer Zusammenhang. Die ernüchternden Zahlen korrelieren nach Meinung der PISA-Interpreten mit einem hoch selektiven Schulsystem der äußeren Differenzierung und dem international höchsten Anteil von 4,8\% an Sonderschülern. (Bleidick \& Ellger-Rüttgardt, 2008, S. 57)

Mit Blick auf umfassende Veränderungen im Schulsystem, wie diese nach der PISA-Studie gefordert wurden, geht Ellger-Rüttgardt bspw. auch mit Blick auf die Einführung inklusiver Schulen davon aus, dass diese „nur durch eine grundlegende Strukturveränderung des Schulwesens sowie einen Mentalitätswandel des pädagogischen Personals zu erreichen ist (EllgerRüttgardt, 2007)“ (Bleidick \& Ellger-Rüttgardt, 2008, S. 242). Dass in diesem Zusammenhang nur politische Entscheidungen zu einer flächendeckenden Umsetzung integrativer Angebote in der Bundesrepublik führen können, postulieren Demmer-Dieckmann und Textor in ihrer Veröffentlichung zu Integrationsforschung und Bildungspolitik und beziehen sich dabei auf den Aufruf von Feuser und Eberwein ein „Bündnis Integrationspolitik“6 zu bilden. Sie fordern in diesem Zusammenhang, dass Integrationspädagogen sich an den politischen Diskussionen zur Gemeinschaftsschule beteiligen sollten, damit nicht - wie bei der Einführung der Gesamtschulen - die Einrichtung derselben ohne Berücksichtigung von Schülerinnen und Schülern mit sonderpädagogischem Förderbedarf erfolge (Demmer-Dieckmann \& Textor, 2007, S. 12f).

6 Diskussion und Verabschiedung eines Gründungsmanifests „Integrationspolitik“ und Etablierung einer integrationspolitischen Institution, Moderation: Prof. Dr. Hans Eberwein, FU Berlin; Prof. Dr. Georg Feuser, Universität Zürich, bei der 21. Jahrestagung der Integrationsforschung, 2007 
Hinsichtlich der rechtlichen Grundlegung sowie der möglichen Umsetzung der inklusiven Beschulung in der BRD stellte die Ratifizierung der UN-Behindertenrechtskonvention (BMAS, 2010) einen wesentlichen Einschnitt in den 2000er-Jahren dar. In Deutschland wurde sie im Dezember 2008 vom Bundestag beschlossen, am 26.03.2009 für die Bundesrepublik völkerrechtlich und mit einem entsprechenden Beschluss der Kultusministerkonferenz vom 18.11.2010 als einfaches Bundesrecht verbindlich. Im Gegensatz zur Salamanca-Erklärung bildet sie also nicht nur einen Aktionsrahmen ab, sondern verändert die nationale Rechtslage. Kern der Konvention ist mit Bezug auf integrative Beschulung und integrativen Unterricht Art. 24 der Konvention, in dem es in der Originalfassung mit Blick auf die Realisierung des Bildungsrechts heißt:

(a) Persons with disabilities are not excluded from the general education system on the basis of disability, and that children with disabilities are not excluded from free and compulsory primary education, or from secondary education, on the basis of disability;(b) Persons with disabilities can access an inclusive, quality and free primary education and secondary education on an equal basis with others in the communities in which they live; (c) Reasonable accommodation of the individual's requirements is provided;(d) Persons with disabilities receive the support required, within the general education system, to facilitate their effective education;(e) Effective individualized support measures are provided in environments that maximize academic and social development, consistent with the goal of full inclusion. (United Nations, 2006, S. 17)

Die UN-Behindertenrechtskonvention „verpflichtet den Staat dazu, die gleichberechtigte, barrierefreie Rechtsausübung jedes Menschen trotz individueller Beeinträchtigung zu ermöglichen." (Hirschberg, 2011, S. 1) und ist damit eng verknüpft mit einem Verständnis von Behinderung als Bündel verschiedener Faktoren, die als Barrieren aufgefasst werden können, was wiederum einen Paradigmenwechsel von der Fürsorge hin zur Verwirklichung von Menschenrechten einschließt (Hirschberg, 2011, S. 4), oder - wie Rothfritz (2010) formuliert - einen "Paradigmenwechsel vom medizinischen zum menschenrechtlichen Modell der Behinderung“ (Rothfritz, 2010, S. 119) ${ }^{7}$. Die Zielvorstellung der UN-BRK liegt bei einem 80-90 prozentigen Anteil inklusiv unterrichteter Schüler:

Experience has shown that as many as 80 to 90 per cent of children with specific education needs, including children with intellectual disabilities, can easily be integrated into regular schools and classrooms, as long as there is basic support for their inclusion. (UN-Handbook for Parlamentarians, 2007, S. 85; zitiert n. Poscher et al., 2008, S. 28)

Aufgrund dieser Zielvorgabe sei zu schlussfolgern, dass die UN-BRK das Bestehen von Förderschulen nicht grundsätzlich ausschließe (Poscher et al., 2008, S. 28). Im Vordergrund der UN-BRK stehe vorrangig das Wohl des Kindes, d.h. das Recht auf inklusive Bildung werde durch die Konvention grundsätzlich, allerdings nicht für jeden Einzelfall vorbehaltlos gewährleistet (Dt. Städtetag, 2011, S. 6). Mögliche Grenzen des Rechts auf inklusive Bildung können nach Poscher et al. (2008) neben dem Kindeswohl auch die Rechte von Mitschülern (bspw. auf Durchführung ordnungsgemäßen Unterrichts) oder entstehende Kosten (s. dazu 3.3.1) sein (Poscher et al., 2008, S. 35f). Nach Rothfritz (2011) ziele die BRK darauf ab

\footnotetext{
7 s. dazu Deutsches Institut für Medizinische Dokumentation und Information (DIMDI) (2005): ICF (Internationale Klassifikation der Funktionsfähigkeit, Behinderung und Gesundheit. Unter: https://www.dimdi.de/dynamic/de/ klassifikationen/icf/; Stand: 18.05.2013; und Bundesteilhabegesetz vom 23.12.2016; Unter: https://www.bmas.de/ SharedDocs/Downloads/DE/PDF-Meldungen/2016/ bundesteilhabegesetz.pdf?_blob=publicationFile\&v=7; Stand: 21.04.2019
} 
[...] Menschen mit Behinderungen das Recht zu vermitteln frei zu entscheiden, ob sie integrativ beschult werden möchten oder nicht. Es soll keine Verpflichtung begründet werden, das allgemeine Schulsystem zu nutzen, wenn dort ausnahmsweise die besonderen Bedürfnisse nach individuellen Anpassungen von Menschen mit Behinderungen nicht entsprechend befriedigt werden können. ${ }^{8}$ (Rothfritz, 2010, S. 385)

Unabhängig von der Konvention kommt daher der Entscheidung der Eltern für den schulischen Bereich eine zentrale Bedeutung zu. Zwar habe „der Staat einen eigenen Erziehungsanspruch, der vom Grundsatz der staatlichen Schulaufsicht gemäß Art 7 Abs. 1 GG vorausgesetzt wird und der mit dem Erziehungsrecht der Eltern in Ausgleich gebracht werden muss" (Poscher et al., 2008, S, 66), gleichzeitig belege Art. 6 Abs. 2 S. 1 GG die Vorstellung, „dass die Eltern grundsätzlich dazu in der Lage sind, die Interessen ihrer Kinder wahrzunehmen" (Poscher et al., 2008, S. 66). Dagegen vertritt das Deutsche Institut für Menschenrecht im Zusammenhang mit der UN-BRK die Position, dass das Wahlrecht der Eltern zwischen inklusiver Beschulung und Beschulung in einer Förderschulform nur übergangsweise vertretbar sei und formuliert:

Sollte die Existenz eines Elternwahlrechts nachweislich den Aufbau eines inklusiven Bildungssystems verzögern oder untergraben, beispielsweise weil es die erforderliche Reorganisation von Kompetenzen und Ressourcen für das Regelschulsystem erschwert und in diesem Zuge das Sonderschulwesen stärkt, ist das Elternwahlrecht mit dem Gebot der progressiven Verwirklichung des Rechts auf inklusive Bildung nicht in Einklang zu bringen. Das Recht auf Inklusion ist ein Recht der Person mit Behinderung. Die Eltern haben bei der Ausübung der elterlichen Sorge den Leitgedanken der Inklusion zu beachten und ggf. zu erklären, warum sie keine inklusiven Bildungsangebote wahrnehmen. (Dt. Institut für Menschenrecht, 2011, S. 14)

Textor verweist darauf, dass die UN-BRK zwar eine „zwangsweise Zuweisung an eine Sondereinrichtung" (Dt. Institut für Menschrechte, 2012, S. 275) ausschließe, nicht aber ein freiwilliges Beschulungsangebot an Förderschulen. Mit Verweis auf Avenarius (2012, S. 68) sei bei der Wahl dieses Beschulungsangebots die Entscheidung der Eltern gegen ein inklusives Bildungsangebot zu rechtfertigen (Textor, 2015, S. 50f).

Trotz der klaren Formulierungen zum Anspruch auf inklusive Bildung' wurde und wird die UN-BRK von deutschen Gremien, Verbänden, Juristen wie auch von den einzelnen Bundesländern sehr unterschiedlich ausgelegt. Während die KMK laut Spaenle die Auffassung vertritt, dass die „gleichberechtigte Teilnahme [...] nicht als Individualrecht angesehen [wird], sondern als zu verfolgendes allgemeines Rechtsgut." (Dt. Philologenverband, 2010, S. 1) fordern GEW und VBE deutlich, dass inklusive schulische Bildung als Rechtsanspruch verankert werden muss (VBE, 2010, o.S.). Auch das Institut für Menschenrechte geht davon aus, dass das „Recht auf inklusive Bildung im Sinne der Konvention [...] als individuelles Recht ausgestaltet [ist]“ (Dt. Institut für Menschenrechte, 2011, S. 1) und daher „für den schrittweisen Aufbau eines inklusiven Bildungssystems als auch für den Zugang zu diesem Bildungssystem im Einzelfall verbindliche Maßstäbe [setzt]" (Dt. Institut für Menschenrechte, 2011, S. 1). Ebenso argumentiert Riedel, der als Völkerrechtler von einer „subjektivrechtlichen Anspruchsnorm“ spricht (Riedel, 2010, S. 4), die sich aus der UN-BRK ergebe. Seine Auffassung begründet er damit, dass „Deutschland

8 Unter Verweis auf Fußnote 60 des Abschlussberichts zur dritten Sitzung des Ad-hoc-Ausschusses vom 09. Juni 2004, UN-Doc. A/AC.265/2004/5

9 Hinweis auf ungünstige Übersetzung durch Gutachten von Krajewski et al. (2008), das belegt, dass neben „dem Wortlaut auch historischer Kontext, Systematik und Ziel der UN-BRK“ belegen, dass „ein inklusives und kein integratives System gewollt ist“" (Dt. Städtetag, 2011, S. 4) 
neben der Konvention auch das Zusatzprotokoll ratifiziert hat, welches dem Staatenberichtsverfahren ein Individualverfahren an die Seite stellt" (Riedel, 2010, S. 3).

Die Ratifizierung der Konvention durch die Bundesrepublik Deutschland verpflichtet die Bundesländer dazu Transformationsgesetze zu verabschieden, die ein inklusiven Bildungssystems auf allen Ebenen gewährleisten (Dt. Städtetag, 2011, S. 3f). Unabhängig von der Gesetzgebung geht der Deutsche Städtetag davon aus, dass die UN-BRK mit Blick auf schulrechtliche Regelungen im Einzelfall bereits kurz nach der Beschlussfassung durch die KMK Wirkungen zeigen könne, da

[...] innerstaatliche Behörden und Gerichte im Falle von Ermessensentscheidungen und bei der Konkretisierung von Generalklauseln bei mehreren Auslegungsmöglichkeiten des nationalen Rechts die völkerrechtskonforme zu wählen [haben]. (Dt. Städtetag, 2011, S. 5)

Rothfritz (2010) nimmt aus juristischer Sicht an, dass man bei der Auslegung des Rechts auf Bildung zwar aus dem Wortlaut der BRK schlussfolgern könne, „dass aus Artikel 24 Abs. 1 Satz 1 ein echtes, originäres Leistungsrecht folgt" (Rothfritz, 2010, S. 381), da diese Auslegung die Gewährleistung allerdings zu stark strapazieren könnte (Bsp.: Entwicklungsländer), sei dieses Recht „lediglich als derivates Teilhaberecht, das ein Recht auf Zugang zu bestehenden staatlichen Bildungseinrichtungen ohne diskriminierende Einschränkungen gewährt" (Rothfritz, 2010, S. 381) zu verstehen.

Das Monitoring der Gewährleistung der Rechte, die sich aus der UN-BRK ergeben, übernimmt in der BRD das „Institut für Menschenrechte“. Das Institut schätzt das Innovationspotenzial der Behindertenrechtskonvention als hoch ein und begründet dies mit drei Argumenten. Zum einen entspreche die Konvention dem „diversity-Ansatz“, indem sie ein Verständnis von Behinderung zugrundelege, das diese als normalen „Bestandteil menschlichen Leben und menschlicher Gesellschaft ausdrücklich bejaht und darüber hinaus als Quelle möglicher kultureller Bereicherung" (Bielefeldt, 2009, S. 6f) wertschätzt. Zum anderen problematisiere die Konvention Behinderung als ,ausgrenzenden und diskriminierenden gesellschaftlichen Umgang“ (Bielefeldt, 2009, S. 8) und damit als Konstruktion, die Behinderungen als Resultat „der Beziehung zwischen Personen mit Beeinträchtigungen und den in Grundhaltungen und Umweltfaktoren bestehenden Barrieren“" (Bielefeldt, 2009, S. 8f) verstehe. Darüber hinaus postuliere die UNBRK, dass individuelle Autonomie und soziale Inklusion untrennbar zusammengehören.

Anders als gelegentlich unterstellt wird, stehen Autonomie und Inklusion nicht nur keineswegs in Widerspruch zueinander. Vielmehr bedingen sie einander wechselseitig: Ohne soziale Inklusion kann Autonomie praktisch nicht gelebt werden, und ohne Autonomie nimmt soziale Inklusion fast zwangsläufig Züge von Bevormundung an. Mit anderen Worten: Erst in der wechselseitigen Verwiesenheit wird klar, dass Autonomie gerade nicht die Selbstmächtigkeit des ganz auf sich gestellten Einzelnen (frei nach Wilhelm Tells Ausspruch: „Der Starke ist am mächtigsten allein“) meint, sondern auf selbstbestimmtes Leben in sozialen Bezügen zielt; und im Gegenzug wird deutlich, dass soziale Inklusion ihre Qualität gerade dadurch gewinnt, dass sie Raum und Rückhalt für persönliche Lebensgestaltung bietet. (Bielefeldt, 2009, S. 10f)

Das Innovationspotenzial der Konvention bestehe darin, dass die Menschenrechtsthematik mit Blick auf „die besonderen Gefährdungslagen von Menschen mit Behinderungen hin konkretisiert und präzisiert [werde]“ (Bielefeldt, 2009, S. 16), wichtige Impulse für das Spannungsfeld zwischen sozialer Inklusion und individueller Autonomie liefere und eine „Bedeutung für die Humanisierung der Gesellschaft im Ganzen“" (Bielefeldt, 2009, S. 16) habe. 
In ihrem Beschluss vom 18.11.2010 verweist die KMK allerdings darauf, dass es sich bei inklusiver Bildung um einen ständigen Prozess handle und diese daher nicht kurzfristig zu realisieren sei, zumal die Verantwortung für die Umsetzung bei den einzelnen Bundesländern liege:

Soweit die schulische Bildung betroffen ist, liegt die Umsetzung nach der Kompetenzordnung des Grundgesetzes vor allem in Händen der Länder und der Kommunen. Artikel 24 des Übereinkommens begründet für die schulische Bildung eine staatliche Verpflichtung, die dem Vorbehalt der progressiven Realisierung unterliegt. Das heißt, dass die Verwirklichung nicht innerhalb eines kurzen Zeitraums erreicht werden kann und dass eine Konkurrenz zu anderen gleichrangigen staatlichen Aufgaben besteht. Die Umsetzung des Übereinkommens ist damit als gesamtgesellschaftliches komplexes Vorhaben längerfristig und schrittweise angelegt. (KMK, 2010, S. 2)

Sie beruft sich auch darauf, dass die BRK keine Vorgaben zur Gliederung des Schulwesens mache (KMK, 2010, S. 4). Das Institut für Menschenrechte stimmt zwar dieser Auffassung insofern zu, dass die Konvention „keine Antwort auf die Zwei- beziehungsweise Dreigliedrigkeit der allgemeinen Schule [gibt]“ (Dt. Institut für Menschenrechte, 2011, S. 6), hält den Ansatz an dem Parallelsystem von Förderschulen weiter festzuhalten allerdings für unvereinbar mit der UN-BRK (Dt. Institut für Menschenrechte, 2011, S. 8).

Auch vom Deutschen Städtetag wurde das innovative Potenzial der UN-BRK grundsätzlich als hoch eingeschätzt:

Der UN-BRK wird ein Paradigmenwechsel weg von einer am Fürsorgeprinzip und an der medizinischen Versorgung hin zu einer an Menschenrechten und dem Prinzip der Teilhabe orientierten Behindertenpolitik zugeschrieben. Das Fakultativprotokoll ist ein eigenständiger völkerrechtlicher Vertrag. Es erweitert die Kompetenzen des Ausschusses für Menschen mit Behinderungen nach Art. 34 der UN-BRK um das Verfahren der Individualbeschwerde und das Untersuchungsverfahren. Beide Verfahren zielen darauf, die Umsetzung und Überwachung des Übereinkommens zu stärken. Eine Besonderheit gegenüber anderen völkerrechtlichen Konventionen besteht also darin, dass Verbände und einzelne Bürger den Ausschuss anrufen und Vertragsverletzungen durch die Staaten anzeigen können. (Dt. Städtetag, 2011, S. 3)

Hinsichtlich der möglichen schulbezogenen Auswirkungen der UN-BRK bezieht der Deutsche Städtetag Stellung und differenziert dabei zwischen Integration und Inklusion:

Zwischen Integration und Inklusion besteht ein Unterschied: Während Integration im Bereich Schule verkürzt dargestellt in erster Linie auf eine Anpassungsleistung von Schülern mit Behinderungen an die bestehenden Schulstrukturen setzt, fordert Inklusion im Wesentlichen, dass sich das Schulsystem an den Fähigkeiten und Bedürfnissen der einzelnen Schüler orientiert. Die Erreichung dieses Ziels setzt im Unterschied zum Konzept der Integration eine systemische Veränderung im Schulwesen voraus. (Dt. Städtetag, 2011, S. 4).

Die Kommunen verstehen sich in diesem Zusammenhang als Ausgangspunkt für Bildungsprozesse und geben als Leitbild eine „kommunale Bildungslandschaft im Sinne eines vernetzten Systems von Erziehung, Bildung und Betreuung “ an, der mit der Prämisse verknüpft wird: „Kein Kind, kein Jugendlicher darf verloren gehen.“ (Dt. Städtetag, 2007, S. 2).

Neben der UN-BRK spielen auch das "Gesetz zur Gleichstellung behinderter Menschen“ (BGG, vom 01.05.2002), das SGB IX sowie das Bundesteilhabegesetz (23.12.2016) im Zusammenhang mit der Umsetzung der Inklusion auf kommunaler Ebene eine wichtige Rolle, da diese u.a. die monetären Zuweisungswege (Bsp.: Persönliches Budget) verändert haben (Wansing, 2005, S. 176): 
Eine weitere wichtige Impulswirkung ging von der Einführung des SGB IX aus. Selbstbestimmung und Teilhabe an der Gesellschaft werden als zentrale rehabilitationspolitische Zielperspektiven hervorgehoben und zum neuen Schlüsselbegriff einer zielorientierten sozialen Rehabilitation (vgl. III.2.2). (Wansing, 2005, S. 138)

In Folge der Konvention wurden alle „Empfehlungen zur sonderpädagogischen Förderung“ unter Einbeziehung der Verbände durch die Kultusminister überarbeitet und zielen jetzt u.a. darauf „die gemeinsame Bildung und Erziehung für Kinder und Jugendliche zu verwirklichen und die erreichten Standards sonderpädagogischer Bildungs-, Beratungs- und Unterstützungsangebote [...] weiterzuentwickeln" (KMK, 2011, S. 3). Zunächst passen vor allem die Bundesländer, die bereits vor der UN-BRK eine integrative Beschulung durch eine entsprechende Gesetzgebung unterstützt haben (Bsp.: Saarland, Hessen), ihre Schulgesetzgebung weiter an und ermöglichen so eine zumindest in quantitativer Hinsicht relevante Veränderung hin zu einer höheren Anzahl „,inklusiv“ unterrichteter Schüler. In den Folgejahren wurden auch in den anderen Bundesländern gesetzliche Rahmenbedingungen für inklusiven Unterricht geschaffen. Nach Lange ermöglicht in diesem Zusammenhang nur Hamburg durch sein Schulgesetz einen unbedingten Rechtsanspruch der Schüler aufZugang zu einer Regelschule (HmbSG \$12 (1), n. Lange, 2017, S. 23), für Thüringen gelte dies eingeschränkt, während im Saarland und in Rheinland-Pfalz die Entscheidung zwischen Regel- und Förderschule ausschließlich bei den Eltern liege (SchulG Rheinland-Pfalz \$59 (4); SchoG \$4 (3), n. Lange, 2017, S. 23). Die Bundesländer Berlin, Brandenburg, Bremen, Hessen, Mecklenburg-Vorpommern, Niedersachsen, NordrheinWestfalen und Schleswig-Holstein hätten der inklusiven Beschulung durch die Änderung ihrer Schulgesetze Vorrang vor der Beschulung an Förderschulen eingeräumt, allerdings ohne einen expliziten Rechtsanspruch auf inklusive Beschulung vorzusehen (SchulG Berlin $\$ 36$ (2); BbgSchulG \$3 (4); BremSchulG $\$ 4$ (5); HSchulG $\$ 51$ (1); FöSoVO \$6 (2); NSchG \$4 (1); SchulG NRW $\$ 20$ (2); SchulG Schleswig-Holstein $\$ 5$ (2), n. Lange, 2017, S. 23). Die Schulgesetze von Baden-Württemberg, Bayern, Sachsen und Sachsen-Anhalt wiederum

[...] sehen den gemeinsamen Unterricht von Schüler_innen mit und ohne sonderpädagogischem Förderbedarf als gemeinsame Aufgabe von allgemeinen Schulen und Förderschulen, gewähren diesem aber keinen Vorrang vor dem Förderschulbesuch (vgl. SchG $\$ 15$ (2); BayEUG $\$ 30 b(2)$; SchulG Sachsen $\$ 30$ (1); SchulG LSA $\$ 1$ (3a)) (Lange, 2017, S. 23)

Nur Hamburg, Rheinland-Pfalz und das Saarland sowie mit Einschränkungen Niedersachsen und Bremen verzichten in ihren Schulgesetzen auf Formulierungen zu einem Ressourcenvorbehalt, während dieser in allen anderen Bundesländern enthalten ist (Lange, 2017, S. 24).

\subsubsection{Der Stand der inklusiven Förderung in der Bundesrepublik Deutschland (2010er Jahre)}

Insgesamt steht in Folge der UN-BRK nicht in erster Linie die qualitative Auseinandersetzung mit der Thematik inklusiver Beschulung bzw. inklusiven Unterrichts im Fokus. Es ist zunächst wenig sichtbar, dass Allgemein- oder Sonderpädagogen sich mit den bildungspolitischen Erfahrungen der vergangenen Jahrzehnte oder den bereits vorliegenden Qualitätsbausteinen bspw. aus den zurückliegenden Modellversuchen auseinandersetzen. Stattdessen entstehen zum Teil einseitig orientierte Diskussionen zum Pro und Kontra inklusiver Beschulung (s. dazu 2.2). Quantitativ führte die Änderung der Schulgesetzgebung in den einzelnen Bundesländern als Folge der UN-Konvention tatsächlich zu einer beachtlich höheren Anzahl inklusiv unterrichteter Schüler. Versucht man statistische Daten zur inklusiven schulischen Förderung in der Bundesrepublik Deutschland zu analysieren, ist allerdings zunächst festzuhalten, dass diese Analyse 
mit mehreren Schwierigkeiten konfrontiert ist. Zum einen sind die Diagnostik und Feststellung des sonderpädagogischen Förderbedarfs bundesweit nicht vereinheitlicht, was u.a. zu unterschiedlich hohen Förderquoten in den einzelnen Bundesländern führt (Speck, 2010. S. 44f), zum anderen müssen mehrere Datensätze bzw. Anteile miteinander in Beziehung gesetzt werden um aufschlussreiche Aussagen über inklusiv bzw. in Förderschulen unterrichtete Schülerinnen und Schüler treffen zu können (Klemm, 2018, S. 7ff).

Klemm benennt mit Blick auf die Analyse der Daten der KMK (2018) die vier unterscheidenden Fachbegriffe „Förderquote“ (= Anteil der SuS mit spF an allen SuS mit Vollzeitschulpflicht in den Jahrgangsstufen 1-9), „Exklusionsquote“ (= Anteil der SuS mit spF, die in Förderschulen unterrichtet werden, an allen SuS), „Inklusionsquote“ (= Anteil der SuS mit spF, die inklusiv in allgemeinen Schulen unterrichtet werden) sowie „Inklusionsanteile“ (= Anteil der SuS mit spF, die inklusiv unterrichtet werden, an allen SuS mit spF) (Klemm, 2018, S. 7). In diesem Zusammenhang erläutert er, dass der Indikator „Inklusionsquote“ seiner Auffassung nach nicht geeignet sei um den statistischen Stand der Inklusion in der BRD zu beschreiben. Die Tatsache, dass einige Bundesländer SuS mit den Förderschwerpunkten Lernen, Sprache und soziale und emotionale Entwicklung in den Regelschulen nicht mehr erfassen, führe dazu, dass sowohl die Förderquote als auch der Inklusionsanteil statistisch unterschätzt werden. Der „Inklusionsanteil“, der im Zeitraum zwischen 2008 und 2016 von 18,4\% auf 39,3\% gestiegen sei, könne für die Beschreibung der Entwicklung bzw. des IST-Stands nicht verwendet werden, da dieser u.a. durch den Anstieg der Förderquote von 6,0 \% auf 7,1 \% in diesem Zeitraum bedingt sei. Lediglich die „Exklusionsquote“ könne diesbezüglich verlässlich Auskunft geben.

Die Entwicklung der absoluten Anzahl der Schülerinnen und Schüler in allgemeinen ${ }^{10}$ Schulen insgesamt und differenziert nach verschiedenen Förderbedarfen stellt sich zwischen 2008 und 2016 folgendermaßen dar:

Tab. 1: Schüler mit sonderpäd. Förderung in allgemeinen Schulen (absolut) (in Anlehnung an KMK, 2018a, S. 34)

\begin{tabular}{llllllllll}
\hline SuS & 2008 & 2009 & 2010 & 2011 & 2012 & 2013 & 2014 & 2015 & 2016 \\
\hline insg. & 88.664 & 95.475 & 108.642 & 121.999 & 139.605 & 157.201 & 173.392 & 194.866 & 205.811 \\
L & 39.839 & 42.563 & 47.259 & 53.334 & 61.124 & 68.351 & 75.675 & 85.634 & 92.973 \\
SE & 19.912 & 22.167 & 25.478 & 28.266 & 33.193 & 38.256 & 42.990 & 47.638 & 48.961 \\
S & 13.574 & 14.553 & 16.550 & 18.725 & 20.306 & 22.320 & 23.306 & 25.538 & 26.228 \\
K & 6.208 & 6.671 & 7.341 & 7.951 & 8.864 & 9.812 & 10.899 & 12.497 & 12.683 \\
G & 2.554 & 2.713 & 3.189 & 4.229 & 5.312 & 6.327 & 7.192 & 9.065 & 10.373 \\
Hören & 3.914 & 4.084 & 5.210 & 5.583 & 6.331 & 7.179 & 7.813 & 8.632 & 8.779 \\
Sehen & 1.899 & 1.995 & 2.232 & 2.393 & 2.543 & 2.806 & 3.132 & 3.400 & 3.549 \\
Kranke & 140 & 294 & 174 & 566 & 770 & 836 & 911 & 954 & 275 \\
übergr. & 624 & 435 & 1.209 & 952 & 1.162 & 1.314 & 1.474 & 1.498 & 1.990 \\
\hline
\end{tabular}

Die Daten aus Tabelle 1 zeigen, dass diese Anzahl seit 2008 um 117.157 Schülerinnen und Schüler mit spF gestiegen ist, sich also mehr als verdoppelt hat. Der prozentual höchste Anstieg ist dabei mit über $300 \%$ in der Gruppe der SuS mit spF geistige Entwicklung (G) zu verzeichnen. Tabelle 2 zeigt, dass auch der prozentuale Anteil von SuS an der Gesamtzahl der SuS mit 
spF in allgemeinen Schulen bei den Förderbedarfen geistige und sozial-emotionale Entwicklung (SE) in diesem Zeitraum gestiegen ist.

Tab 2: Schüler mit sonderpäd. Förderung in allg. Schulen, Verteilung nach Förderschwerpunkten in \% (in Anlehnung an KMK, 2018a, S. 34)

\begin{tabular}{llllllllll}
\hline SuS & 2008 & 2009 & 2010 & 2011 & 2012 & 2013 & 2014 & 2015 & 2016 \\
\hline L & 44,9 & 44,6 & 43,5 & 43,7 & 43,8 & 43,5 & 43,6 & 43,9 & 45,17 \\
SE & 22,5 & 23,2 & 23,5 & 23,2 & 23,8 & 24,3 & 24,8 & 24,4 & 23,79 \\
S & 15,3 & 15,2 & 15,2 & 15,3 & 14,5 & 14,2 & 13,4 & 13,1 & 12,74 \\
K & 7,0 & 7,0 & 6,8 & 6,5 & 6,3 & 6,2 & 6,3 & 6,4 & 6,16 \\
G & 2,9 & 2,8 & 2,9 & 3,5 & 3,8 & 4,0 & 4,1 & 4,7 & 5,04 \\
Hören & 4,4 & 4,3 & 4,8 & 4,6 & 4,5 & 4,6 & 4,5 & 4,4 & 4,27 \\
Sehen & 2,1 & 2,1 & 2,1 & 2,0 & 1,8 & 1,8 & 1,8 & 1,7 & 1,72 \\
Kranke & 0,2 & 0,3 & 0,2 & 0,5 & 0,6 & 0,5 & 0,5 & 0,5 & 0,13 \\
übergr. & 0,7 & 0,5 & 1,1 & 0,8 & 0,8 & 0,8 & 0,9 & 0,8 & 0,97 \\
\hline
\end{tabular}

Im Zeitraum zwischen 2007 und 2016 haben sich Verschiebungen bei den Anteilen von SuS mit bestimmten Förderschwerpunkten an der Gesamtheit der SuS mit spF, die in Förder- und in allgemeinen Schulen unterrichtet werden, ergeben. Während sich der Anteil der SuS mit dem spF Lernen verringert hat (von 43,8\% auf 36,5\%), hat sich der Anteil der SuS mit dem spF emotionale und soziale Entwicklung erhöht, und zwar von 11,5\% auf 16,6\%, was einem prozentualen Anstieg von nahezu $65 \%$ entspricht (KMK, 2018a, S. 16). Bei den anderen Förderschwerpunkten haben sich diese Anteile - wenn überhaupt - nur sehr geringfügig verändert (Klemm, 2018, S. 14). Die Exklusionsquote hat sich in den Jahren zwischen 2008 und 2016 wiederum nur bei den Förderschwerpunkten „Lernen“ (von 2,1 \% auf 1,3\%) und „Sprache“ (von 0,5\% auf 0,4\%) verringert, während sie bei allen anderen Förderschwerpunkten gleich geblieben ist (Klemm, 2018, S. 14f).

Die Förderquote ist nach Klemm im angegebenen Zeitraum von 6,0 \% auf 7,1 \% gestiegen. Der Inklusionsanteil hat sich von 18,4\% auf 39,3\% erhöht (Klemm, 2018, S. 7).

Tab. 3: Vergleich Schüler mit sonderpäd. Förderbedarf in allg. Schulen und in Förderschulen, Quoten und Anteile (in Anlehnung an Klemm, 2018, S. 7; Berechnungen durch Klemm auf der Grundlage von KMK. 2016 und KMK, 2018a11)

\begin{tabular}{lllllllll}
\hline Jahr & $\begin{array}{l}\text { Jg. 1-9/10 } \\
\text { insg. }\end{array}$ & $\begin{array}{l}\text { mit spF } \\
\text { insg. }\end{array}$ & $\begin{array}{l}\text { in allg. } \\
\text { Schulen }\end{array}$ & $\begin{array}{l}\text { in Fö- } \\
\text { Schulen }\end{array}$ & $\begin{array}{l}\text { Förder- } \\
\text { quote }\end{array}$ & $\begin{array}{l}\text { Exklusions- } \\
\text { quote }\end{array}$ & $\begin{array}{l}\text { Inklusions- } \\
\text { quote }\end{array}$ & $\begin{array}{l}\text { Inklusion- } \\
\text { anteil }\end{array}$ \\
\hline 2008 & 7.992 .000 & 482.000 & 89.000 & 393.000 & $6,0 \%$ & $4,9 \%$ & $1,1 \%$ & $18,4 \%$ \\
2016 & 7.334 .000 & 524.000 & 206.000 & 318.000 & $7,1 \%$ & $4,3 \%$ & $2,8 \%$ & $39,3 \%$ \\
\hline
\end{tabular}

Die Exklusionsquote habe sich in den acht Jahren allerdings nur um $0,6 \%$ von 4,9\% auf 4,3\% verringert (Klemm, 2018, S. 8f). Dieser Rückgang bedeutet nach Klemm,

11 Unterschiede in Quoten ergeben sich dadurch, dass kranke SuS bei Klemm weiter einbezogen werden, während diese in den KMK-Daten nicht mehr erfasst werden (Klemm, 2018, S. 7) 
[...] dass die Verkleinerung der Exklusionsquote, die von 2008/09 bis 2016/17 erreicht wurde, bundesweit bei Berücksichtigung der insgesamt sinkenden Schülerzahlen zu einer Verringerung der exklusiv in Förderschulen unterrichteten Kinder und Jugendlichen im Umfang von 42.847 geführt hat [...]. All die Anstrengungen der acht Jahre nach Deutschlands Beitritt zur UN-Konvention haben dazu geführt, dass knapp 43.000 Kinder und Jugendliche, die ohne den Prozess der Inklusion in Förderschulen zur Schule gegangen wären, jetzt in allgemeinen Schulen unterrichtet werden. (Klemm, 2018, S. 9)

Im Ländervergleich zeigt sich, dass die Exklusionsquoten der einzelnen Bundesländer sich 2016 zum Teil erheblich voneinander unterscheiden und zwischen der niedrigsten Quote von $1,2 \%$ in Bremen sowie der höchsten Quote von 6,0\% in Mecklenburg-Vorpommern variieren (Klemm, 2018, S. 11):

Entgegen dem Bundestrend sind die Exklusionsquoten in Südwestdeutschland zwischen 2008 und 2017 sogar gestiegen: In Bayern, Baden-Württemberg und Rheinland-Pfalz gehen wieder mehr Kinder auf eine Förderschule. In Ostdeutschland hingegen geht die Exklusionsquote prozentual erheblich zurück. In Nordrhein-Westfalen und Hessen gab es moderate Rückgänge, im Saarland dagegen nur kleine. Besonders niedrig sind die Anteile der separat in Förderschulen beschulten Schüler*innen in Niedersachsen, Schleswig-Holstein und in den Stadtstaaten: Hier ist die Exklusionsquote stark gesunken, insbesondere in Bremen. (Klemm, 2018, S. 4f)

Tab. 4: Förder-/Exklusions-/Inklusionsquoten und Inklusionsanteile in den verschiedenen Bundesländern (Schuljahr 2016/17) (in Anlehnung an Klemm, 2018, S. 18, auf der Basis von KMK, 2016 und KMK 2018a12

\begin{tabular}{lllll}
\hline Bundesland & Förderquote & Exklusionsquote & Inklusionsquote & Inklusionsanteil \\
\hline Baden-Württ. & $7,6 \%$ & $4,9 \%$ & $2,6 \%$ & $34,9 \%$ \\
Bayern & $6,5 \%$ & $4,8 \%$ & $1,7 \%$ & $26,3 \%$ \\
Berlin & $7,8 \%$ & $2,8 \%$ & $4,8 \%$ & $63,5 \%$ \\
Brandenburg & $7,8 \%$ & $4,0 \%$ & $3,8 \%$ & $48,5 \%$ \\
Bremen & $7,1 \%$ & $1,2 \%$ & $5,9 \%$ & $82,3 \%$ \\
Hamburg & $8,7 \%$ & $3,1 \%$ & $5,7 \%$ & $64,7 \%$ \\
Hessen & $5,6 \%$ & $4,1 \%$ & $1,5 \%$ & $27,0 \%$ \\
Meck.-Vorp. & $9,8 \%$ & $6,0 \%$ & $3,8 \%$ & $38,5 \%$ \\
Niedersachsen & $6,7 \%$ & $3,4 \%$ & $3,3 \%$ & $49,5 \%$ \\
Nord.-Westf. & $7,7 \%$ & $4,6 \%$ & $3,0 \%$ & $39,7 \%$ \\
Rheinl.-Pfalz & $5,8 \%$ & $4,0 \%$ & $1,8 \%$ & $31,2 \%$ \\
Saarland & $4,2 \%$ & $4,2 \%$ & - & - \\
Sachsen & $8,5 \%$ & $5,7 \%$ & $2,8 \%$ & $32,8 \%$ \\
Sachsen-Anhalt & $8,9 \%$ & $5,9 \%$ & $3,0 \%$ & $33,7 \%$ \\
Schlesw.-Holst. & $6,4 \%$ & $2,1 \%$ & $4,3 \%$ & $67,3 \%$ \\
Thüringen & $6,6 \%$ & $4,0 \%$ & $2,6 \%$ & $39,9 \%$ \\
\hline BRD & $7,1 \%$ & $4,3 \%$ & $2,8 \%$ & $39,3 \%$ \\
\hline
\end{tabular}

12 Für das Saarland können Inklusionsquote und Inklusionsanteil nicht mehr angegben werden, da SuS mit spF Lernen, Sprache und soziale und emotionale Entwicklung in den allgemeinen Schulen nicht mehr erfasst werden. 
Nicht nur mit Blick auf die quantitative Entwicklung schulischer Inklusion schlussfolgert das Deutsche Institut für Menschenrechte zehn Jahre nach Inkrafttreten der UN-BRK, dass

[...] der Umsetzungsstand weit hinter den Erwartungen zurückbleibt. Einzelne Länder haben zwar entscheidende gesetzliche Schritte hin zu einem inklusiven System geschafft und praktische Erfolge zu verzeichnen, andere wiederum haben trotz Novellierung der Schulgesetze keine richtungsweisenden Schritte unternommen. In keinem Bundesland ist der notwendige gesetzliche Rahmen für die Schaffung und Gewährleistung einer inklusiven Schule abschließend entwickelt worden. Und auch in der Praxis ist es nur einrichtungsbezogen, maximal regional gelungen, qualitativ hochwertige inklusive Bildung anzubieten. Hier ist also nach wie vor politischer Wille gefordert, inklusive Bildung nicht nur rhetorisch zu unterstützen, sondern die nötigen finanziellen und personellen Ressourcen für einen erfolgreichen gemeinsamen Unterricht im allgemeinen Schulsystem zur Verfügung zu stellen. (Dt. Institut für Menschenrechte, 2019, S. 35)

\subsubsection{Zusammenfassung}

Während zu Beginn der Unterrichtung von Menschen mit Behinderungen beide Entwicklungsstränge - Sonderbeschulung und allgemeine Beschulung - mit den Zielrichtungen Reduktion von Komplexität versus Verallgemeinerung von bzw. Teilhabe an allgemeinen Bildungsangeboten vorhanden waren, sah die Realität im Westen Deutschlands ab 1945 so aus, dass ein konsequenter Ausbau des Sonderschulwesens erfolgte, der durch verwaltungsorientierte Gremien forciert wurde. Auch die bildungspolitischen Auseinandersetzungen wurden von diesen Gremien dominiert. Stärker bildungsorientierte Gremien wie der Deutsche Ausschuss, der Deutsche Bildungsrat oder die Bund-Länder-Kommission beschäftigten sich - anders als in anderen Ländern - nicht umfassend mit Fragen einer möglichen integrativen Beschulung. Im Vordergrund der Veränderungen standen strukturelle und nicht inhaltliche Bedingungen (Bsp.: Schulgebäude, Räumlichkeiten), während qualitativ-inhaltliche pädagogische Konzepte in Ansätzen lediglich für die „Hilfsschule“ ausformuliert wurden. Es ging also um pragmatische Entscheidungen, während eine bildungspolitische Diskussion zum Thema schulische Integration genauso ausblieb wie ein fruchtbarer inhaltlicher Diskurs zwischen der Allgemeinen Pädagogik und der Sonderpädagogik. Diskussionsansätze zur stärkeren Verbindung von allgemeiner und spezieller Pädagogik aus der Zeit der Weimarer Republik wurden nicht aufgegriffen. Der Querschnittsbereich der Wechselwirkung zwischen beiden Systemen wurde nicht untersucht. Die Frage danach, wie sich die Sonderpädagogik im bildungspolitischen Kontext mit der Frage nach integrativer bzw. separativer Beschulung auseinandergesetzt hat, lässt sich also für diesen Zeitraum dahingehend beantworten, dass diese sich selbst nicht an Diskussionen zu einer bildungspolitischen Neuorientierung beteiligt hat. Preuß (1985) spricht in diesem Zusammenhang von der „bildungspolitischen Enthaltsamkeit“ und fehlenden Innovationsbereitschaft der Sonderpädagogen. Gleichzeitig wurden die Sonderschulen und damit die Sonderpädagogik bildungspolitisch mehrfach „vergessen“, bspw. in den bildungspolitischen Rahmenvorgaben des Deutschen Ausschusses (1959).

In den 60er Jahren erfolgte zunächst keine Einmischung von Sonderpädagogen in die Gesamtschuldiskussion. Grundidee der Einrichtung von Gesamtschulen war die Schaffung einer gemeinsamen Schule für Kinder aller sozialen Schichten mit dem Ziel des Ausschöpfens von Bildungsreserven zur Vermeidung einer möglichen Bildungskatastrophe. Nicht geplant war die Integration von Schülern mit Behinderungen in die Gesamtschule. Ideen der sozial-liberalen Koalition, die in diese Richtung gingen, wurden von der KMK (aus politischen Gründen) nicht berücksichtigt. Stellungnahmen von Verbänden (GEW, VDS) zum Thema der integrativen 
Beschulung wurden erst Ende der 60er Jahre getätigt, einzelne Diskussionsbeiträge von Allgemeinpädagogen und Sonderpädagogen folgten in den 70er Jahren. Diese erfassten Rahmenbedingungen für integrative Angebote in Regelschulen wie eine behindertengerechte Ausstattung von Schulen, die Flexibilität der Klassengrößen und eine Anpassung der Lehrerausbildung, aber auch inhaltliche Qualitätskriterien wie Früherkennung, Frühförderung, Differenzierung und Individualisierung, behinderungsspezifische Förderung, Abbau des Leistungsdrucks und eine enge Zusammenarbeit des Lehrerkollegiums bzw. zwischen Klassen- und Förderlehrern. Aus sonderpädagogischer Sicht war also klar, dass neben äußeren strukturellen Veränderungen auch Veränderungen auf innerer schulischer Ebene (Unterricht und Kooperation) für eine Umsetzung schulischer Integration notwendig waren. Angedacht war in diesem Zusammenhang ein flexibles integratives System. Auch Grenzen integrativer Beschulung (Integration als Einzelfallentscheidung, Leistungsorientierung der Regelschulen, mangelnde Bereitstellung von Ressourcen) wurden analysiert. Ende der 70er Jahre verlagerte sich der Diskussionsschwerpunkt auf mögliche präventive und integrative Maßnahmen in den Grundschulen.

Die „Enthaltsamkeit“ der Sonderpädagogik zeigte sich also auch mit Bezug auf das größte bildungspolitische Projekt der 60er- und 70er-Jahre. Unabhängig von der Sonderpädagogik mussten die Gesamtschulbefürworter die Erfahrung machen, dass schulstrukturelle und -organisatorische Veränderungen sich nicht gleichzeitig in der Tiefe eines komplexen Systems auf das pädagogisch-didaktische Arbeiten auswirken. So richtet sich die Reformkritik Mitte der 80er-Jahre darauf, dass der Ansatz strukturelle Reformen als entscheidend für ein höheres $\mathrm{Ma}$ an Bildungsgleichheit anzusehen gescheitert sei, was sich unter anderem daran zeige, dass sich die Zahl der Arbeiterkinder, die auf ein Gymnasium wechselten, zwischen 1972 und 1982 lediglich von 6\% auf $10 \%$ erhöht habe (Klemm et al., 1985, S. 26).

Während die KMK unter redaktioneller Führung des VDS mit der 1972 veröffentlichten „Empfehlung zur Ordnung des Sonderschulwesens“ weiterhin den Ausbau des Sonderschulwesens forderte, nahm die vom Deutschen Bildungsrat 1973 veröffentlichte Schrift „Zur pädagogischen Förderung behinderter und von Behinderung bedrohter Kinder und Jugendlicher" das gesamte Schulwesen und dessen mögliche Neuorientierung in Richtung einer Überwindung der Drei- bzw. Viergliedrigkeit in den Blick. Die gemischte Zusammensetzung des Deutschen Bildungsrats (Allgemeinpädagogen, Fachleute der Behindertenpädagogik, Regierungsvertreter) führte zu einer deutlich integrativen Ausrichtung dieser Empfehlungen, die sowohl strukturelle Rahmenbedingungen wie inhaltlich-qualitative Veränderungen in den Regelschulen thematisierte. Die Schrift umfasst pädagogisch-didaktische Bausteine für schulische Integration sowie die Idee eines „Kooperativen Schulzentrums“, das als flexibles Fördersystem für integrative und teilintegrative Beschulungsformen für Kinder und Jugendliche mit Behinderungen gedacht war. Obwohl die Herausgeber von realistischen Umsetzungsmöglichkeiten ihrer Ideen ausgingen, konnte die Schrift keine (bildungs-) politische Wirkkraft entwickeln, da sie mit Blick auf die Gesamtschuldiskussion zu spät erschien, keine umfassende politische Unterstützung erfuhr und gesellschaftliche Entscheidungsträger sowie die Bildungsverwaltung nicht über ein entsprechendes Bewusstsein für die Anforderungen integrativer Beschulung verfügten. Auch von der Allgemeinen Pädagogik bzw. in den Regelschulen wurden die Ideen nicht aufgegriffen oder umgesetzt. Bildungspolitische Relevanz hatte lediglich die Veröffentlichung der KMK zur Ordnung des Sonderschulwesens, die zur Einrichtung der auch heute noch vorhandenen Sonderschulformen führte.

Einen positiven Anstoß zu einem pädagogisch fundierten Änderungsprozess stellte der Ansatz des Arbeitskreises Grundschule (1977) zur Verminderung von Schulschwäche dar. Dabei ging es in diesem Fall nicht darum - wie Preuss-Lausitz es Anfang der 80er-Jahre formuliert - von der 
Sonderpädagogik aus den ,Schwarzen Peter ' an die Grund- bzw. Gesamtschulen zurückzugeben, sondern darum gemeinsam und fachübergreifend pädagogische Fragen, die innerhalb der Regelschulen bearbeitet werden müssen, zu erörtern und mögliche Lösungsansätze zu diskutieren.

Eine Vielzahl von Modellversuchen zur gemeinsamen Unterrichtung von Schülern mit und ohne Behinderungen wurde vor allem in den 80er Jahren eingerichtet um die Umsetzung von Integration zumindest „im Kleinen“ zu ermöglichen. In Folge der Versuche war es möglich eine Fülle von Qualitätskriterien für integrative Beschulung zu benennen (s. dazu Kap. 3). Auch die Modellversuche haben allerdings - trotz positiver Erfahrungen - nicht zu einer flächendeckenden Einführung integrativer Schulen geführt, da eine entsprechende politische Unterstützung fehlte. Dieser Mangel an Unterstützung hing u.a. damit zusammen, dass sich die Sonderschulreferenten der Länder in einem Bericht für die KMK kritisch-einschränkend zu den Ergebnissen der Modellversuche äußerten und auf höhere Kosten eines integrativen Systems hinwiesen. Auch der schulischen Integration offen gegenüberstehende Autoren der Sonderpädagogik gehen zum damaligen Zeitpunkt davon aus, dass schulische Integration als langer Weg gedacht werden müsse, der u.a. abhängig von finanziellen Rahmenbedingungen sei. Probleme werden in der Beharrlichkeit des gegliederten Schulsystems gesehen sowie im fehlenden Austausch zwischen Sonderpädagogik und Allgemeiner Pädagogik. Trotz überwiegend positiver Ergebnisse konnten die vielfältigen Modellversuche also weder eine qualitative Wirkkraft mit Blick auf methodische oder didaktische Veränderungen im Regelschulsystem, noch eine quantitative Wirkkraft hinsichtlich des Anstiegs der Anzahl integrativ unterrichteter Schüler entwickeln. Das Thema der integrativen Beschulung von Kindern und Jugendlichen mit Behinderungen ist tatsächlich nicht in ausreichendem Maß zu einem Thema der Allgemeinen Pädagogik geworden (Bleidick \& Ellger-Rüttgardt, 2008). Dies hängt wiederum damit zusammen, dass innerhalb der schulischen Modellversuche zwar gute Erfahrungen mit gemeinsamer Unterrichtung gesammelt und diese positiven Erfahrungen zum Teil auch wissenschaftlich dokumentiert wurden, offensichtlich jedoch u.a. ein engerer produktiver Austausch zwischen Schulen, Hochschulen und Bildungspolitik ausblieb.

Bildungspolitische Dokumente der 90er Jahre thematisieren eine „Pädagogik für besondere Bedürfnisse“ bzw. „Empfehlungen zur sonderpädagogischen Förderung“ und fördern eine personen- statt institutionsbezogene Sichtweise auf Menschen mit Behinderungen. Damit rücken die schon in den 70er Jahren geforderte Lernortunabhängigkeit sonderpädagogischer Förderung sowie die ebenfalls bereits in dieser Zeit entstandene Idee der Einrichtung flexibler integrativer Systeme wie sonderpädagogischer Förderzentren erneut in den Fokus. Da die Salamanca-Erklärung wie die Empfehlungen zwar Leitprinzipien und Rahmenbedingungen formulieren, diese aber nicht schulpolitisch bzw. -gesetzlich bindend waren, wirkten sie sich allerdings statistisch betrachtet nur geringfügig auf die Anzahl integriert unterrichteter Schüler aus.

Entscheidende Reformansätze für das Bildungssystem insgesamt gingen dagegen von der PISA-Studie bzw. ihren Ergebnissen aus. Aufgrund der wesentlichen Ergebnisse der PISAStudie (2001), die u.a. belegten, dass die Kompetenzen deutscher Schüler im internationalen Vergleich unterdurchschnittlich waren und die soziale Herkunft in Deutschland maßgeblich über schulische Leistungen und Abschlüsse entscheidet, wurden Reformen eingeleitet, die nicht nur schulorganisatorische bzw. -strukturelle Veränderungen umfassten, sondern - im Rahmen der Auseinandersetzung mit Problemen beim Erwerb schulischer Kompetenzen - auch methodisch-didaktische Fragestellungen, die direkt den Unterricht betreffen (Bsp.: problembezogene Aufgabenstellungen im Mathematikunterricht). Es ergab sich also die Notwendigkeit über Unterricht zu sprechen und unterrichtsbezogene Veränderungen zu initiieren (Bsp.: Rahmen- 
lehrpläne, Bildungsstandards, Kompetenzorientierung als Kernmerkmal der Unterrichtsgestaltung). Als Ergebnis wurden in den Folgestudien (PISA 2009, 2015) tatsächlich bessere, d.h. im Vergleich zu anderen teilnehmenden Ländern überdurchschnittliche, Kompetenzen gemessen (PISA, 2015, S. 9). Gleichzeitig blieben Zusammenhänge zwischen Geschlecht, sozialer Herkunft sowie Migrationshintergrund und schulischen Leistungen im Sinne von Ungleichheiten erhalten (PISA, 2015, S. 10).

Auffällig ist mit Blick auf die Ergebnisse dabei, dass sich Schülerleistungen in den Gymnasien bei leistungsstarken Schülerinnen und Schülern auf der einen Seite gesteigert haben, während auf der anderen Seite eine Zunahme besonders leistungsschwacher Jugendlicher in den Bereichen Lesen und Mathematik festgestellt werden kann (PISA, 2018, S. 6f). So besteht der Anteil der Schüler, die außerhalb der Gymnasien nur über sehr grundlegende mathematische Kenntnisse verfügen, laut PISA 2018 bei rund $30 \%$ (PISA, 2018, S. 14). Die Autorinnen und Autoren der Studie schlussfolgern daher:

So ist die Förderung schwächerer Schülerinnen und Schüler beziehungsweise die breite Förderung von allen Jugendlichen immer noch eine zentrale Aufgabe, die gezielte Aufmerksamkeit erfodert. In Bezug auf das Lesen genauso wie in Bezug auf Mathematik und Naturwissenschaften ist der Anteil leistungsschwacher Jugendlicher fraglos zu hoch. [...] Ähnlich gilt es auch, die Förderung leistungsstarker Schülerinnen und Schüler zu intensivieren. (PISA, 2018, S. 17).

Es besteht also weiterhin ein hoher Grad der Streuung innerhalb der Ergebnisse und damit ein Hinweis darauf, dass zwar sozusagen die „Durchschnittsförderung“ mit Hilfe der ergriffenen Maßnahmen gut funktioniert, eine Differenzierung von Leistungsanforderungen mit dem Ziel differenzierter Förderung für besonders leistungsstarke und besonders leistungsschwache Schülerinnen und Schüler allerdings anhand dieser Ergebnisse nicht ablesbar ist.

Wie diese Zusammenfassung zeigt, sind Entwicklungen, die im Zusammenhang mit der schulischen Integration von Kindern und Jugendlichen mit Einschränkungen oder Behinderungen stehen, in den vergangenen sieben Jahrzehnten wellenförmig erfolgt. Während im Anschluss an den Zweiten Weltkrieg in einer ersten Welle der Ausbau des Sonderschulwesens im Vordergrund stand und bildungspolitische Diskussionen sich in den 60er Jahren mit der Idee der Gesamtschulbewegung auseinandersetzten, befassten sich Allgemein- wie Sonderpädagogen erst im Lauf der 70er Jahre mit Fragestellungen der schulischen Integration und ihrer Rahmenbedingungen in den weiterführenden Schulen sowie im Primarbereich. Bildungspolitische Empfehlungen zu einer stärker integrativ ausgerichteten Beschulung hatten in dieser Zeit allerdings keine konkreten Auswirkungen auf quantitative und qualitative Entwicklungen in diesem Bereich. Als Konsequenz aus dieser mangelnden Berücksichtigung startete in den 80er Jahren als „neue Welle“ eine ganze Reihe von Modellversuchen, die wiederum - obwohl Erfahrungsberichte positive Umsetzungsbeispiele dokumentierten - von den Sonderschulreferenten der Bundesländer selbst u.a. mit Blick auf höhere Kosten für das Bildungssystem kritisch eingeordnet wurden und keine flächendeckende Wirkung erzeugten. Eine kleinere Wellenbewegung forderte Anfang der 90er Jahre eine stärkere Lernortunabhängigkeit sonderpädagogischer Förderung und formulierte Leitlinien für integrative Unterrichtung, führte aber ebenfalls - auch aufgrund des rein empfehlenden Charakters - nicht zu einer umfassenden Weiterentwicklung schulischer Integration. 
Die Salamanca-Erklärung postulierte in diesem Zusammenhang sehr allgemeine Forderungen, die vor allem die Schulorganisation betrafen. Gleichzeitig blieben die Empfehlungen zur sonderpädagogischen Förderung hinsichtlich schulischer Integration sehr vorsichtig, forderten aber immerhin ein flexibles diesbezügliches Modell. Dagegen schaffte die UN-BRK eine rechtliche Grundlage für inklusive schulische Bildung. Ob aus dieser Grundlage individuelle Rechtsansprüche abgeleitet werden können oder ob diese - aufgrund des Progressionsvorbehalts - das Recht auf inklusive Bildung lediglich grundsätzlich festlegen, ist juristisch bislang nicht eindeutig geklärt. In Folge der UN-BRK werden Vor- und Nachteile inklusiver Beschulung - ähnlich wie in den 80er Jahren - erneut diskutiert (vor allem in der Sonderpädagogik, s. dazu 2.2), ohne dass die Frage nach der Qualität in den Vordergrund gerückt wurde. Quantitativ wird inzwischen ein höherer Anteil an Schülerinnen und Schülern mit sonderpädagogischem Förderbedarf in den Regelschulen inklusiv unterrichtet, gleichzeitig ist allerdings auch die Förderquote in der Bundesrepublik gestiegen, so dass die Exklusionsquote sich bundesweit nur um wenige Prozentpunkte verringert hat.

Während die Bildungsstudie PISA in der BRD eine Fülle konkreter Reformen auf den Weg brachte und dadurch positive Veränderungen bei den durchschnittlichen Schülerleistungen erreicht werden konnten, sind die Auswirkungen der UN-Konvention auf Veränderungen im Schulsystem noch weitgehend offen. Das hängt u.a. mit der sehr unterschiedlichen Auslegung dieser Rechtskonvention durch die einzelnen Bundesländer, KMK, Städtetag, Verbände und Kommunen zusammen. Obwohl eine klare rechtliche Grundlage für ein „inklusives Schulsystem" vorhanden ist und es sich damit um eine rechtlich bindende Innovation im Vergleich zu vorangegangenen bildungspolitischen Dokumenten handelt, wird das Potenzial nicht umfassend gesehen bzw. genutzt. Eine grundlegende „systemische Veränderung im Schulwesen“, wie sie der Deutsche Städtetag 2011 gefordert hat, findet bislang nicht statt. Gesetzlich festgelegte Veränderungen wie bspw. die Inklusionsverordnung im Saarland bilden quasi ein „Plus“ zu vorliegenden Schulgesetzen und Verwaltungsvorschriften, werden aber nicht Bestandteil schulstruktureller oder -inhaltlicher Veränderungen wie bspw. der Schaffung der Gemeinschaftsschule. Tief greifende schulstrukturelle Veränderungen wie die Schließung von Förderschulen werden wiederum in einzelnen Bundesländern wie NRW oder Bremen umgesetzt, ohne dass das Regelschulsystem sich qualitativ auf diese Veränderungen vorbereiten konnte.

Es ist also bislang nicht abzusehen, inwiefern eine den Reformen als Folge der PISA-Studien vergleichbare Welle im Zusammenhang mit der UN-Behindertenrechtskonvention nachhaltige Auswirkungen auf ein tatsächlich als „inklusiv“ zu bezeichnendes Schulsystem hat. Als mögliche Schlussfolgerung aus der mehrere Jahrzehnte umfassenden Analyse ergibt sich die Annahme, dass auf eine erste Welle von durch diese Rechtskonvention ausgelösten strukturellen Veränderungen im Bildungssystem eine „Ruhephase“ in Form eher restaurativer Bemühungen folgen könnte, in der vor allem qualitativ-tiefgreifende Reformen mit Blick auf schulische Inklusion im Regelschulsystem keine wesentliche Rolle spielen. Diese These wird unter „Fazit und Perspektiven“ für das Bildungssystem näher ausgeführt.

\subsection{Unterschiedliche Perspektiven auf Begriffe und Diskurs}

Wie können die Begriffe „Inklusion“ und „Exklusion“ bzw. „Integration“ und „Separation“ erfasst werden? Worin unterscheiden sie sich? Welche theoretischen Beiträge leisten Soziologie, Bildungssoziologie, Pädagogik und Sonderpädagogik mit Blick auf Begriffe und Diskurs? Mit diesen Fragen befasst sich das folgende Unterkapitel. 
Die Darstellung unterschiedlicher Sichtweisen auf das Themenfeld (schulische) Integration bzw. Inklusion dient dazu die Einbettung des Themas dieser Arbeit in einen theoretisch-begrifflichen Gesamtzusammenhang vorzunehmen. Eine Annäherung an die Begriffe wird dabei orientiert an der Grundbedeutung des Verbs „definieren“ vorgenommen, das ursprünglich „definire, eigentlich ,abgrenzen“" (Kluge, 1999, S. 166, Herv. im Orig.) als Bedeutungsinhalt umfasste. In den folgenden Abschnitten geht es also nicht darum Definitionen für Begriffe zu bestimmen und damit feste Bedeutungszuweisungen vorzunehmen. Vielmehr besteht der Schwerpunkt dieses Kapitels darin, in der vorliegenden Arbeit verwendete Begriffe hinsichtlich möglicher Bedeutungen zu beschreiben und damit zu anderen Begriffen abzugrenzen. Verfolgt wird diese Zielsetzung in der Form, dass Diskursansätze aus soziologischer, bildungssoziologischer, pädagogischer und sonderpädagogischer Sicht zumindest in Ansätzen umrissen werden, da eine umfassende Darstellung aufgrund der Komplexität der Thematik und des damit zusammenhängenden Diskurses nicht möglich erscheint. Da die Begriffe Integration und Inklusion ursprünglich aus der Soziologie stammen, wird diese Sichtweise zu Beginn des Unterkapitels vorgestellt und damit zunächst eine Perspektive gewählt, die Integration und Separation bzw. Inklusion und Exklusion in der Gesellschaft fokussiert. Eng mit dieser Sichtweise zusammen hängt die der Bildungssoziologie, die sich mit dem Schulsystem und unterschiedlichen Heterogenitätsdimensionen befasst. Im Anschluss werden das Begriffsverständnis sowie die Diskurse der Allgemeinen Pädagogik und der Sonderpädagogik vorgestellt.

\subsubsection{Sichtweise der Soziologie}

Das soziologische Interesse an Bildung und Erziehung richtet sich [...] auf die gesellschaftlichen Voraussetzungen von Erziehung und Bildung, den Einfluss von gesellschaftlichen Strukturen und Dynamiken auf pädagogische Institutionen, Theorien und Praktiken sowie auf die sozialen Folgen dessen, was im Bildungs- und Erziehungssystem der Gesellschaft geschieht. (Bauer et al., 2012, S. 13)

Während die Bildungssoziologie (s. 2.2.2) in diesem Zusammenhang bildungs- oder institutionsbezogen argumentiert, nehmen soziologische Sichtweisen im weiteren Sinn die Thematik Inklusion bzw. Exklusion zunächst ohne direkten Bezug zum Bildungssystem auf der Ebene gesellschaftlicher Strukturen in den Blick. Beide Blickrichtungen bilden für das Verständnis von Inklusion und Exklusion einen Rahmen, der die dynamische Wechselwirkung zwischen Gesellschaft und Schule als Bildungseinrichtung bzw. Schülern und Schülerinnen als beteiligten Individuen in den Fokus nimmt.

\section{Theoretische Ansätze zur Beschreibung von Inklusion bzw. Exklusion}

Veränderungen auf gesellschaftlicher Ebene sind gekennzeichnet durch Modernisierung und Individualisierung (Beck, 1986; Nollmann \& Strasser, 2004; Goetze, 2004), durch Überforderung und Verunsicherung, bspw. bedingt durch technologische, soziokulturelle und ökonomische Veränderungen (Beck, 1986; SINUS, 2018), oder durch Entgrenzung und Segregation (SINUS, 2017, S. 17), die wiederum durch das von „Globalisierung und Digitalisierung getriebene Auseinanderdriften der Lebens- und Wertewelten“ (SINUS, 2017, S. 17) bedingt sind. Ein tiefgreifender Wandel in der Gesellschaft hängt u.a. mit der demografischen Entwicklung zusammen, durch die eine Verschiebung von Wertevorstellungen und eine Ausdifferenzierung in unterschiedlichen Bereichen der Gesellschaft erfolgt (Bsp.: Prekarisierung von Teilen der Unterschicht, Modernisierung der Funktionselite (Bsp.: digital divide) (SINUS, 2017, S. 20)), was von Soziologen teilweise sehr kritisch bewertet wird. So spricht Bauman vom „Ende der Eindeutigkeit" und der „flüchtigen Moderne“ (Bauman, 2003), in der soziale Orientierung schwieriger werde und Modernisierungsprozesse zu Exklusion führen (Bauman, 2005). 
Exklusion könne dabei auf der einen Seite als Prozess verstanden werden, der Gesellschaftsmitglieder aus bestimmten Lebensformen ausschließt, auf der anderen Seite allerdings von bestimmten Mitgliedern der Gesellschaft als „inszenierte Nichtzugehörigkeit“ (Precht, 2011) durchaus gewollt sein könne. Aufgrund der gesellschaftlichen Entwicklung gehen soziologische Ansätze zwar weiterhin grundsätzlich von einem Schichtenmodell aus, differenzieren aber sowohl innerhalb der Schichten als auch schichtübergreifend darüber hinaus zwischen verschiedenen Grundorientierungen. Plastisch veranschaulicht wird diese stärkere Differenzierung durch die (vom Marktforschungsinstitut SINUS entwickelte) Übersicht zu unterschiedlichen Milieus, bei der innerhalb der unteren Mittelschicht bspw. zwischen prekärem und hedonistischem Milieu unterschieden wird, die wiederum Überlappungsbereiche zu Milieus der mittleren Mittelschicht aufweisen. Das traditionelle und bürgerliche sowie das sogenannte adaptiv-pragmatische Milieu erstrecken sich ebenfalls überlappend zwischen unterer und mittlerer Mittelschicht, während konservativ-etablierte, liberal-intellektuelle, sozialökologische oder expeditive Milieus sich als Bereiche ergeben, die sich von der mittleren hin zu oberen Mittelschicht bzw. Oberschicht bewegen (SINUS Institut, 2018, o.S.).

Mit Hilfe unterschiedlicher theoretischer Ansätze versucht die Soziologie die genannten gesellschaftlichen Entwicklungen zu erfassen und verwendet im Zusammenhang mit dem Themenfeld Inklusion bzw. Exklusion unterschiedliche begriffliche Gegensatzpaare, die diesen Ansätzen zuzuordnen sind: Inklusion/Exklusion (Luhmann, 1995; Stichweh, 1997; Kronauer, 2007a, 2007b), soziale Gleichheit/soziale Ungleichheit (Nassehi, 2004; Kronauer, 2010) oder soziale Integration/soziale Ausgrenzung (Böhnke, 2006). Der Begriff der Exklusion lässt sich in diesem Zusammenhang nach Stichweh (1997) auf die Französische Revolution zurückführen, in denen „les exclus" diejenigen bezeichnete, die vom französischen Nationalismus und der französischen Kultur ausgeschlossen waren (Stichweh, 1997, S. 2). Exklusion werde in Frankreich als „Bruch der nationalen oder der gesamtgesellschaftlichen Solidarität" (Stichweh, 1997, S. 2) gedacht und damit als Bestandteil gesamtgesellschaftlicher Differenzierung (in unterschiedlichen Kategorien wie Armut, Behinderung, etc.) (Stichweh, 1997, S. 2). Das Verständnis hängt aus soziologischer Sicht damit zusammen, dass die Gesellschaft als Kollektiv aufgefasst werde, „das für seine Mitglieder die erforderlichen Lebensbedingungen ermöglicht“ (Engels, 2006, S. 10) und dem Anspruch „sozialer Kohäsion und organischer Solidarität, erwachsen aus der gesellschaftlich (und nicht allein marktbestimmt-ökonomisch) verfassten Arbeitsteilung und informeller Reziprozität“ (Kronauer, 2010, S. 32) gerecht werden müsse (Vertreter z. Bsp.: Emile Durkheim, Robert Castel). Gemäß Stichweh hat sich der Begriff der Exklusion ,in wenigen Jahren in den Sozialwissenschaften und im öffentlich-politischen Diskurs etabliert" (Stichweh, 1997, S. 1). Er stellt fest, dass der Exklusionsbegriff an Stelle der Begriffe der sozialen Ungleichheit sowie Armut getreten sei (Stichweh, 1997, S. 2). Engels weist darauf hin, dass der Begriff der „sozialen Exklusion“ in Deutschland in den 90er Jahren den Armutsbegriff abgelöst habe, da im Rahmen der Armutsprogramme der europäischen Sozialpolitik der mehrdimensionale Charakter von Armut in den Vordergrund gerückt sei (Engels, 2006, S. 110). Der Begriff der (sozialen) Exklusion wird nach Bude (2004) in Deutschland seit den 90er Jahren verwendet und diente im politischen Bereich dazu soziale Probleme zu beschreiben, „die den sozialen Zusammenhalt unserer Gesellschaften gefährden." (Bude, 2004, S. 4). Ein anderer Theoriestrang (n. Kronauer, 2010, S. 32) sei auf das in England und Amerika verbreitete Verständnis der so genannten „underclass“"(Myrdal, Gunnar, n. Stichweh, 1997, S. 3) zurückzuführen und damit schichtbezogen. In England stehe dabei das „citizenship“ mit Blick auf die Dimensionen rechtlicher, politischer 
und sozialer Teilhabe (Bürgerstatus) im Vordergrund, in den USA das meritokratische Marktmodell (Bude, 2004, S. 7; Kronauer, 2010, S. 32) (Bsp.: Marshall, Thomas, Wilson (1991)), das davon ausgeht, dass persönliche Leistungsanstrengungen zur Positionierung von Individuen auf dem „Markt“ führen.

Der Begriff der Exklusion (und damit auch der der Inklusion) ist demnach ursprünglich kein pädagogisch, sondern ein soziologisch geprägter Begriff, der geschichtlich auf zwei unterschiedlichen Verständnisweisen beruht (Frankreich: mangelnde gesamtgesellschaftliche Kohäsion; England und USA: Ausgrenzung der „underclass“). Eine schichtbezogene Sichtweise scheint mit Blick auf die gesellschaftliche Ausdifferenzierung nicht ausreichend zu sein, da es - wie insbesondere die SINUS-Milieus zeigen - auch innerhalb verschiedener nach Grundorientierungen differenzierter Schichten ein Innen bzw. Außen von Gesellschaftsmitgliedern geben kann. Die Soziologie sieht Prozesse der Individualisierung und Verunsicherung u.a. als Folge von Globalisierung und Digitalisierung.

\section{Theoriestränge der Soziologie}

Der Schwerpunkt der folgenden Darstellung liegt auf der genaueren Differenzierung der Begriffe Inklusion und Exklusion und in diesem Zusammenhang auf der Gegenüberstellung der zwei wesentlichen soziologischen Theoriestränge der Systemtheorie (Luhmann, 1995; Nassehi, 2004; Engels, 2006; Emmerich \& Hormel, 2013) und der Sozialstrukturanalyse (Bude, 2004; Böhnke, 2006; Bude \& Willisch, 2006; Kronauer, 2007a, 2007b, 2010):

Die Soziologie sozialer Ungleichheit und die Theorie funktionaler Differenzierung können als Theorieangebote verstanden werden, die sich im Kern dem Problem gesellschaftlicher Differenzierung widmen. Allerdings unterscheiden sie sich hinsichtlich ihrer erkenntnistheoretischen Prämissen und methodologischen Grundannahmen, weshalb auch von den „zwei Soziologien“ (Schimank 1996; Schwinn 2004) die Rede ist. Eine einfache und sehr grobe Unterscheidung zwischen beiden Differenzierungstheorien kann darin gesehen werden, dass in der Ungleichheitssoziologie von der ,vertikalen Struktur gesellschaftlicher Verteilungsordnungen und von Verteilungskonflikten ausgegangen wird, um von dort aus die Struktur lebensweltlich-symbolischer Orientierungen zu erschließen. Demgegenüber geht die Theorie funktionaler Differenzierung von einem Strukturprimat der , horizontalen Ausdifferenzierung sozialer Handlungssysteme (vgl. Parsons 1986) oder Kommunikationssysteme (vgl. Luhmannn 1994) aus und behandelt ,vertikale‘ Strukturbildungen als ein sekundäres Phänomen, dem keine eigentliche ,Funktion' mehr für die moderne Gesellschaft zukommt. (Emmerich \& Hormel, 2013, S. 20, Herv. im Orig.)

\section{Theorie funktionaler Differenzierung}

Luhmann bezieht sich in seinen Ausführungen zu Inklusion und Exklusion zunächst auf den Begriff der sozialen Differenzierung, in dessen "Schatten“ der Begriff der „Integration“ mitgeführt worden sei (Luhmann, 1995, S. 237). In Rückbezug auf Parsons (1986) geht er von der Ausdifferenzierung der Gesellschaft in Teilsysteme aus und versteht Integration nicht als „Einheitsgarant“, sondern als „wechselseitige Einschränkung von Freiheitsgraden von strukturell gekoppelten Systemen“ (Luhmann, 1995, S. 238). Das Konzept der Differenzierung wiederum hat nach Nassehi (2004) in der Soziologie eine lange Tradition und fasst „gesellschaftliche Modernisierung als Differenzierungsprozess auf." (Nassehi, 2004, S. 98). Beispiele für gesellschaftliche Teilsysteme sind Wirtschaft, Recht, Wissenschaft, Politik, Religion, Erziehung, Kunst (Nassehi, 2004, S. 107). Um „Inklusion“ als Begriff zu fassen, führt Luhmann (1995) zunächst den Begriff der „Form“ ein: 
Der Begriff der Form bezeichnet [...] das Postulat, daß Operationen, soweit sie Beobachtungen sind, immer die eine Seite einer Unterscheidung bezeichnen, aktualisieren, als Ausgangspunkt für weitere Operationen markieren - und nicht die andere Seite, die im Moment gleichsam leer mitgeführt wird. [...] Operationen sind [...], wenn sie Beobachtungen sind, unterscheidende Bezeichnungen. Sie bezeichnen die eine Seite einer Unterscheidung unter der Voraussetzung, daß es eine andere gibt. (Luhmann, 1995, S. 240)

Von diesem Begriff ausgehend postuliert er, dass „man auch einen Formbegriff der Inklusion bilden [könne]“ (Luhmann, 1995, S. 241):

„Inklusion“ bezeichnet dann die innere Seite der Form, deren äußere Seite „Exklusion“ ist, Von Inklusion kann man also sinnvoll nur sprechen, wenn es Exklusion gibt. Die Theorieaufgabe besteht demnach darin, die Differenz von Inklusion und Exklusion in Beziehung zu setzen zu den Erfordernissen der Systembildung und insbesondere zu den Konsequenzen bestimmter Formen der Differenzierung, die sich im Laufe der gesellschaftlichen Evolution herausgebildet haben. Für eine bei systembildenden Operationen ansetzende Theorie versteht es sich von selbst, daß mit Inklusion nicht gemeint sein kann der Einschluß aller Leben fortsetzenden (organischen) oder Bewußtsein fortsetzenden (psychischen) Operationen in das soziale System. Inklusion (und entsprechend Exklusion) kann sich nur auf die Art und Weise beziehen, in der im Kommunikationszusammenhang Menschen bezeichnet, also für relevant gehalten werden. Man kann, an eine traditionelle Bedeutung des Terminus anschließend, auch sagen: die Art und Weise, in der sie als „Personen“ behandelt werden. (Luhmann, 1995, S. 241, Herv. im Orig.)

Nach Luhmann resultieren individuelle Teilhabechancen nicht mehr automatisch aus Herkunft und Gruppenzugehörigkeit, stattdessen werden Personen in unserer Gesellschaft unterschiedlich „adressiert“ und erhalten damit unterschiedliche „Kommunikationschancen“ (Emmerich \& Hormel, 2013, S. 43f), so dass sie gleichzeitig in einigen Teilsystemen zugehörig und in anderen nicht-zugehörig sein können (Engels, 2006, S. 11) bzw. Exklusion sich nicht auf Personen bezieht, „sondern [...] bestimmte Aspekte [betrifft], die vom jeweiligen Teilsystem als Kriterien der Zugehörigkeit bestimmt worden sind““ (Engels, 2006, S. 12). Exklusion sei nach diesem Verständnis ,unter anderem daran zu erkennen, daß Reziprozität unterbrochen wird." (Luhmann, 1995, S. 244). Nassehi (2004) führt dazu erläuternd aus:

Unter Inklusion ist der kommunikative Mechanismus zu verstehen, wie Menschen im Kommunikationszusammenhang für relevant gehalten werden, wie sie ansprechbar, zurechnungsfähig, positionierbar oder auch nur thematisierbar sind. Der Mechanismus der Inklusion vermittelt also die sachliche und soziale Sinndimension, indem er auf den Begriff bringt, wie Ereignisse der sachlich formierten Funktionssysteme sozial zugerechnet werden. (Nassehi, 2004, S. 110f, Herv. im Orig.)

Da die Gesellschaft in eine Vielzahl von Untersystemen ausdifferenziert sei, könne „Inklusion keineswegs als Integration gedacht werden, also als Passungsprozeß zwischen Gesellschaft und Individuum. Inklusion in der Moderne sei vielmehr Multiinklusion, d.h. gleichzeitige und unvermittelte Teilhabe an unterschiedlichen Funktionszusammenhängen." (Nassehi, 1997, S. 620).

Die Unterscheidung Inklusion/Exklusion ist eine systeminterne Unterscheidung. Sie kann nur zur Ordnung der Kommunikation verwendet werden. Hier macht es aber einen Unterschied, ob Personen als mitwirkungsrelevant oder nichtmitwirkungsrelevant bezeichnet werden. In einem Fall hängt etwas davon ab, wie sie agieren und reagieren; im anderen Falle nicht [...] Mit den Modi der Inklusion beschreibt die Gesellschaft das, was sie als Teilnahmebedingung setzt bzw. als Teilnahmechance in Aussicht stellt. Exklusion ist demgegenüber das, was unmarkiert bleibt, wenn diese Bedingungen bzw. Chancen formuliert werden. Sie ergibt sich gleichsam aus der Operation der Selbstbeschreibung als Nebeneffekt - so wie jede Fixierung einer Identität etwas außer Acht lässt, was nicht dazugehört. (Luhmann, 1995, S. 261f) 
Anders als in segmentaren oder stratifizierten Gesellschaften liegt in funktional differenzierten Gesellschaften nach Luhmann keine gesellschaftseinheitliche Regelung von Inklusion vor, sondern die Frage nach Inklusion bzw. Exklusion wird ihren Funktionssystemen überlassen (Luhmann, 1995, S. 242-246):

Die Teilnahme am Wirtschaftssystem regelt sich über Eigentum und Einkommen, die Teilnahme am Erziehungssystem über Schule, Schulzeugnisse und Schulabschlüsse [...] An wissenschaftlicher Forschung kann man nur teilnehmen, wenn man über den Forschungsstand informiert ist. (Luhmann, 1995, S. 246)

Von besonderer Bedeutung seien in diesem Zusammenhang soziale Kontakte und Netzwerke, wobei ein Netzwerk wiederum selbst „über einen eigenen Mechanismus der Inklusion bzw. Exklusion“ (Luhmann, 1995, S. 253) verfüge: „Mitmachen oder Herausfallen - das ist eine Entscheidung, die laufend getroffen und erneuert werden muß, und dies, ohne dass Außenfaktoren (etwa durch Inflationierung der Möglichkeiten oder durch Ressourcenentzug), entscheidend eingreifen könnten.“ (Luhmann, 1995, S. 254).

\section{Sozialstrukturanalyse}

Die Sozialstrukturanalyse „schärft ihren Blick an der Sozialdimension, indem sie Gesellschaft(en) als Kollektive konzipiert, deren interne Differenziertheit über die Zugehörigkeit zu Einkommensklassen, Schichten oder Lebensformen organisiert wird“ (Nassehi, 2004, S. 110, Herv. im Orig.). Sie geht nicht davon aus, dass die funktionale Differenzierung einer Gesellschaft als Erklärungsansatz für Inklusion bzw. Exklusion ausreicht, sondern nimmt vielmehr an, dass Dimensionen sozialer Gleichheit bzw. Ungleichheit, sozialer Gerechtigkeit bzw. Ungerechtigkeit, sozialer Teilhabe bzw. sozialer Ausgrenzung relevant sind (Stichweh, 1997; Kronauer, 2010). Stichweh (1997) vertritt die Auffassung, dass die Unterscheidung der Begriffe Inklusion und Exklusion innerhalb der Weltgesellschaft keine „binäre Alternative verkörpere, demzufolge man in einem System entweder inkludiert oder exkludiert ist und eine dritte Möglichkeit nicht gegeben ist" (Stichweh, 1997, S. 12). Er sieht die beiden Begriff daher in einer hierarchischen Opposition: Inklusion als Oberbegriff für Exklusion:

Die Frage ist genau die, ob nicht vielleicht kompakte Sachverhalte des Ausschlusses aus der Gesellschaft beobachtbar sind, die nicht mehr als soziale Ungleichheit und Schichtung - im Sinne eines mehr oder weniger der Teilhabe an sozialen Gütern - beschrieben werden können, die vielmehr eine innergesellschaftliche Trennlinie schaffen, die ein „innen“ und ein „außen“ deutlich voneinander unterscheidet. (Stichweh, 1997, S. 4)

Kronauer nimmt in diesem Zusammenhang eine Abgrenzung des Begriffs der Inklusion zum Begriff der Integration vor:

Der deutlichste Unterschied zwischen dem Begriff der „Integration“ und dem der „Inklusion“ [...] besteht darin, dass Integration von einer vorgegebenen Gesellschaft ausgeht, in die integriert werden kann und soll, Inklusion aber erfordert, dass gesellschaftliche Verhältnisse, die exkludieren, überwunden werden müssen. (Kronauer, 2010, S. 56)

Zwar sieht beispielsweise Stichweh (1997) eine Stärke des Exklusionsbegriffs nach Luhmann in seiner Mehrdimensionalität, durch die „die Aufmerksamkeit auf die Frage gelenkt [werde], wie und durch wen Exklusion vollzogen wird und wie sich soziale Beziehungen dadurch ändern." (Stichweh, 1997, S. 5) (Bsp.: Familie/Verwandtschaft als eine mögliche Quelle bzw. bestim- 
mende Variable von Exklusionsrisiken (Stichweh, 1997, S. 6) oder Schulerziehung, über die ein gesellschaftlicher Status zugewiesen wird (Stichweh, 1997, S. 7)). Grenzen dieser Mehrdimensionalität erkennt Stichweh (1997) allerdings mit Blick auf „Exklusionsursachen, die nicht auf funktionale Differenzierung zugerechnet werden können“" (Stichweh, 1997, S. 9) wie ethnische Segregation oder Migration. Exklusion könne in diesem Zusammenhang räumliche Trennung bedeuten, muss es aber nicht, da auch „exclusion sur place“ (Foucault, 1974, S. 590) möglich sei (n. Stichweh, 1997, S. 9). Kronauer (2010) spricht im Zusammenhang mit dem Begriffspaar Inklusion/Exklusion von der Entwicklung einer neuen sozialen Frage („vergleichbar der Arbeiterfrage im 19. und frühen 20. Jahrhundert" (Kronauer, 2010, S. 24)) und stellt damit einen Bezug zur Sozialpolitik her: „Wie damals stehen auch heute wieder die Institutionen infrage, die das gesellschaftliche Zusammenleben regeln." (Kronauer, 2010, S. 24). Nach Kronauer (2010) wird der der Begriff, „Exklusion“ soziologisch häufig mit „Ausschließung“" gleichgesetzt, was auf die Kategorie der „sozialen Schließung“ nach Max Weber zurückgehe (Kronauer, 2007a, S. 25). Soziale Exklusion (als Ergebnis von Ausgrenzung sowie als Prozess der Ausgrenzung) könne auf „alle Aspekte des menschlichen Lebens übergreifen und auf Dauer gestellt werden.“ (Kronauer, 2010, S. 26). Er grenzt seine eigene Auffassung von der soziologischen Systemtheorie ab, da die Grundkategorie der sozialen Schließung nicht mit dem Postulat der „Vollinklusion“, „der prinzipiellen Offenheit der Funktionssysteme“ (Kronauer, 2010, S. 27) vereinbar sei:

[...] im Anschluss an Luhmann gehört die Unterscheidung zwischen Inklusion und Exklusion zu den Konstitutionsbedingungen moderner Gesellschaften. Dieser Lesart entsprechend folgen solche Gesellschaften einer Logik der Differenzierung, in der sich Funktionssysteme mit jeweils eigenen Formen interner Kommunikation herausbilden, indem sie sich gegeneinander und gegen ihre sonstigen Umwelten abgrenzen. (Kronauer, 2010, S. 26)

Gemäß Kronauer (2010) könne die Systemtheorie weder die „Mehrdimensionalität von Ausgrenzungsprozessen“ (Kronauer, 2010, S. 27) noch die „sozialstrukturelle Ungleichverteilung von Ausgrenzungsrisiken“ (Kronauer, 2010, S. 27) erklären. Kronauer (2010, S. 47) wie Böhnke (2006, S. 4193) gehen davon aus, dass Exklusionsprozesse zum einen über verschiedene Dimensionen hinweg erfolgen können und zum anderen, dass diese kumulieren und sich gegenseitig verstärken können (Kronauer, 2010, S. 47) oder wie Bude (2004) es formuliert: „Institutionelle Unerreichbarkeit [...], soziale Abgeschlossenheit [...] und kulturelle Selbstausschließung [...] ergänzen sich zu einem unaufhaltsamen Prozeß der Entkopplung vom Ganzen." (Bude, 2004, S. 5). Exklusion könne demnach als Zuspitzung sozialer Ungleichheit aufgefasst werden (Kronauer, 2010, S. 26). Sie kann sich nach Kronauer (2010) auf drei Ebenen beziehen: den Ausschluss am Arbeitsmarkt, die Aufösung sozialer Bindungen und den Verlust von sozialen Teilhabemöglichkeiten. Dabei habe die Exklusion „als Ausschluss aus Institutionen“ (Kronauer, 2010, S. 43) eine wesentliche Bedeutung:

Inklusion bemisst sich [...] nicht allein an der formalen Einbeziehung in Institutionen, sondern auch und vor allem an der sozial-materiellen Qualität möglicher Teilhabe, die durch die Institutionen vermittelt wird. Das gleiche gilt für Exklusion. (Kronauer, 2010, S. 44)

In der Zone der Ausgrenzung befinden sich gemäß Kronauer Menschen, die von Erwerbsarbeit dauerhaft ausgeschlossen sind (oder allenfalls einen sporadischen Zugang haben) und die eine Verengung sozialer Beziehungen bzw. im äußersten Fall „Vereinzelung“ erleben (Kronauer, 2007b, S. 370): „An die Stelle der Einbindung in wechselseitige Sozialbezüge, der aktiven Teilhabe am gesellschaftlichen Leben, tritt immer stärker die einseitige Abhängigkeit von fremder, 
mit sozialer Kontrolle und Sanktionen verbundener institutioneller Hilfe." (Kronauer, 2007b, S. 370). Demgegenüber gebe es in der Zone der Exklusivität (globale Elite) hohe außertarifliche Einkommen und gute Netzwerke (Kronauer, 2007b, S. 370). Während Böhnke in diesem Zusammenhang den Begriff der sozialen Ausgrenzung (Böhnke, 2006) verwendet, sprechen Bude und Willisch (2006) von sozialer Exklusion:

Man spricht nicht mehr von relativer Unterprivilegierung nach Maßgabe allgemein geschätzter Güter wie Einkommen, Bildung oder Prestige, sondern von sozialer Exklusion aus den dominanten Anerkennungszusammenhängen und Zugehörigkeitskontexten unserer Gesellschaft (Byrne 1999; Büchel et al. 2000; Kronauer 2002; Hills/Le Grand/Piachaud 2002). Nach der für die Sozialberichterstattung der EU gültigen Definition handelt es sich um einen „Prozess, durch den bestimmte Personen an den Rand der Gesellschaft gedrängt und durch ihre Armut bzw. wegen unzureichender Grundfertigkeiten oder fehlender Angebote für lebenslanges Lernen oder aber infolge von Diskriminierung an der vollwertigen Teilhabe gehindert werden" (Europäische Kommission 2004, S. 12). (Bude \& Willisch, 2006, S. 8)

\section{Zusammenführung der beiden Gesellschaftstheorien}

Die Kritik an der Theorie funktionaler Differenzierung führt Nassehi u.a. darauf zurück, dass Sozialstruktur innerhalb dieser Theorie „nicht als Struktur sozialer Stratifikation geführt [wird], sondern als Struktur sachlicher Differenzierung in Funktionen“" (Nassehi, 2004, S. 99f), was die Frage nach der Deutung sozialer Ungleichheit offen lasse. Er versucht beide Theoriestränge zusammenzuführen:

Die Attraktivität des Exklusionsbegriffs scheint [...] unter anderem darin zu liegen, dass er mit der anderen Seite seiner Unterscheidung die Lösung des Problems gleich mitliefert, Inklusion nämlich. [...] Inklusion blieb weitgehend die dunkle Seite dieser Unterscheidung, aber gerade auf dieser Seite wäre anzusetzen, um die beiden Gesellschaftstheorien zusammenzuführen. (Nassehi, 2004, S. 111; Herv. im Orig.)

Um die Thematik der sozialen Ungleichheit in der Theorie der funktionalen Differenzierung aufzugreifen, hält Nassehi (2004) es für wesentlich, nach der Funktion sozialer Ungleichheiten innerhalb der Gesellschaft zu fragen: „Welches Bezugsproblem wird durch Ungleichheit gelöst, und warum gelingt es solchen Ungleichheiten, sich auf Dauer zu stellen?" (Nassehi, 2004, S. 112). Und als eine mögliche Antwort formuliert er: „Wenn sich soziale Ungleichheit nicht kausal aus der funktionalen Differenzierungsform ableiten lässt, müssten die Gründe eher in der stabilisierenden Funktion des Unterscheidungsgebrauchs gesucht werden." (Nassehi, 2004, S. 112, Herv. im Orig.) und führt aus, dass Asymmetrien kennzeichnend für unterscheidende Operationen allgemein sind (Nassehi, 2004, S. 112f). Beispielsweise habe „das Bildungssystem [...] geradezu die sachliche gesellschaftliche Funktion, asymmetrische Positionen zuzuweisen und für soziale Ungleichheit zu sorgen [...]“ (Nassehi, 2004, S. 113; Herv. im Orig.) Die Etablierung solcher Asymmetrien wiederum habe strukturbildende Effekte für die Funktionssysteme (Nassehi, 2004, S. 113). Soziale Ungleichheit könne demnach innerhalb der Theorie funktionaler Differenzierung „als eine Struktur angesehen werden, die quer zur sachlichen Ungleichheit der Funktionssysteme liegt." (Nassehi, 2004, S. 114; Herv. im Orig.).

Engels (2006) schlägt in diesem Zusammenhang beispielsweise vor, das „Lebenslagen-Konzept“, das „auf die Mehrdimensionalität unterschiedlicher Lebensbereiche“ (S. 1) fokussiert, mit Luhmanns Systemtheorie dahingehend zu verbinden, dass die Dichotomie zwischen In- und Exklusion in ein "graduelles Kontinuum“ (S. 1) transformiert wird. In diesem Zusammenhang würden über das Lebenslagen-Konzept „Zugangsvoraussetzungen zu einzelnen Teilsystemen“ (S. 1) identifiziert, deren Relevanz als individuell variabel eingestuft werden könne. Er definiert 
entsprechend: „Der Begriff, Lebenslagen“ meint die Gesamtheit der sozialen Zusammenhänge, in denen Personen ibre materiellen und immateriellen Möglichkeiten nutzen. " (Engels, 2006, S. 5; Herv. im Orig.).

\section{Exkurs: Behinderung als Kategorie im Sinne von möglicher Ausgrenzung}

Wie stuft die Soziologie (genauer: die Theorie der Sozialstrukturanalyse) in diesem Zusammenhang Behinderung als mögliches Exklusionsrisiko ein? Ausgehend vom Modell der Theorie der sozialen Desintegration (Anhut \& Heitmeyer, 2000) stellt nicht eine Behinderung an sich ein mögliches Ausgrenzungsrisiko dar, sondern die Kumulation möglicher benachteiligender bzw. desintegrierender Faktoren, die mit einer Behinderung einhergehen können. Dabei unterscheiden Anhut und Heitmeyer zwischen der sozial-strukturellen Dimension (Bsp.: Zugang zu materiellen Gütern, zum Bildungs- oder Arbeitsmarkt), der institutionellen Dimension (Bsp.: Einhaltung von Grundprinzipien wie Gerechtigkeit oder Solidarität) und der personalen Dimension (Bsp.: Anerkennung personaler Identität) (Anhut \& Heitmeyer, 2000, S. 48).

Problematisch ist laut Armuts- und Reichtumsbericht der Bundesregierung zu werten, dass die Armutsgefährdung von Menschen mit Behinderungen im Vergleich zur Gesamtbevölkerung hoch ist:

Menschen mit Beeinträchtigungen sind im Vergleich zur Gesamtbevölkerung überdurchschnittlich häufig armutsgefährdet. Ihre Erwerbslosenquote ist nahezu doppelt so hoch wie der Durchschnitt. Beeinträchtigungen oder Behinderungen stellen weiterhin Risiken für die soziale Mobilität dar. Bei chronisch Kranken ist das Armutsrisiko besonders hoch. (BMAS, 2017, S. 36)

Während die Armutsgefährdung nichtbehinderter Menschen zwischen 2005 und 2013 etwa gleich geblieben ist, stieg dieses Risiko für Menschen mit Beeinträchtigungen in diesem Zeitraum von $13 \%$ auf $20 \%$ und für Menschen mit Schwerbehinderungen von $12 \%$ auf $19 \%$. Am höchsten ist dieses Risiko für Menschen mit chronischen Erkrankungen, das von 19\% auf 26\% gestiegen ist. (Bundesregierung, 2017, S. 473). Die gesellschaftliche Abwertung von Menschen mit Behinderung ist nach Zick und Klein (2014) im Vergleich zur Abwertung anderer Personengruppen vergleichsweise gering. Während bspw. langzeitarbeitslose Menschen von insgesamt 47,8\% der Befragten abgewertet werden und dies für 44,3\% aller Asylsuchenden gilt, werden behinderte Menschen von 4,1\% der 1915 Befragten abgelehnt. Allerdings verweist Heitmeyer darauf, dass vor allem die Menschen abgewertet werden, die keine Arbeitsleistung erbringen und damit als „wenig nützlich“ erscheinen ${ }^{13}$. Zu dieser Gruppe zählt er auch Menschen mit Behinderungen:

Verbreitet ist diese Einstellung u.a. bei Menschen aus niedrigen Einkommensgruppen (vgl. Zick et al. 2015, 70), aber auch in den oberen Einkommensgruppen: Insbesondere in bezug auf Langzeitarbeitslose und Hartz-IV-Empfänger plädiert man für Leistungskürzungen. Im Sinne des Kapitals und im Einklang mit dem ökonomistischen Denken werden diese Menschen als nutzlos etikettiert. Sie müßten nun endlich selbst Verantwortung übernehmen, die Solidargemeinschaft könne nicht alle auffangen. Tatsächlich gibt es statistisch eindeutige Zusammenhänge zwischen der Forderung, die sozial Schwachen sollten ihr Leben endlich selbst in die Hand nehmen und der Abwertung von Langzeitarbeitslosen, niedrigqualifizierten Zuwanderern und Behinderten: Angehörige höherer Einkommensgruppen betrachten diese Gruppen immer seltener als gleichwertig. (Heitmeyer, 2015b, S. 27)

13 Dazu können weitere Interdependenzen von Kategorien wie Geschlecht, Migrationshintergrund oder sozialem Milieu kommen, eine Sichtweise, die als Intersektionalität bezeichnet wird (Wansing, G. \& Westphal, M. (2014): Behinderung und Migration. Inklusion, Diversität, Intersektionalität. Wiesbaden: Springer). 
7,7\% der Befragten stimmen in diesem Zusammenhang der Auffassung zu, dass für Behinderte in Deutschland zu viel Aufwand betrieben werde und 11,3\% unterstützen die Aussage, dass viele Forderungen von Menschen mit Behinderungen überzogen seien (Heitmeyer, 2015b, S. 39). Aus soziologischer Sicht formuliert Bude (2004) dazu:

Eine zweite Art der gesellschaftlichen Reaktion auf festgestellte Exklusion ist Kompensation. Das kennzeichnet den Umgang mit allen Formen definierter Behinderung in unserer Gesellschaft. Insbesondere fordert ein „natürlich“ gegebener Ausschluß aus allgemein als relevant erachteten Tätigkeitsbereichen und Beziehungsweisen einen „gesellschaftlichen“ Ausgleich. So erklärt sich, daß die Anerkennung einer prinzipiellen Exklusion die beste Voraussetzung für eine sekundär vermittelte Inklusion darstellt. Die Anormalen werden gerade nicht aus-, sondern eingeschlossen. Allerdings beschränkt sich die Kompensation auf den nationalstaatlichen Rahmen der Sozialpflege. (Bude, 2004, S. 13f)

Mögliche Ursachen von Abgrenzungen bzw. Abwertungen sind nach Zick \& Klein wiederum die Zugehörigkeit zu einer Gruppe und die Gefährdung der Identität der eigenen Gruppe, das Motiv positiver Bewertung und sozialer Anerkennung (Vergleich mit anderen Menschen), Sicherheitsbedürfnis und Autoritarismus, das Bedürfnis nach Verständnis sozialer Umstände und Verstehensverluste sowie Kontrollmotive (Zick \& Klein, 2015, S. 71). Als bedeutsam im Kontext der Frage nach Exklusion oder Inklusion gesellschaftlicher Gruppen bzw. Individuen wird auch die Frage nach der Wahrnehmung von Zusammenhalt innerhalb der Gesellschaft sowie der Frage nach einer möglichen Hinwendung zu Homogenität oder Heterogenität als Idealvorstellung bewertet:

Die Antwort auf die Frage nach Zusammenhalt und Vielfalt ist letztlich davon bestimmt, wieviel Gleichwertigkeit verschiedenen sozialen Gruppen zugebilligt wird und inwieweit ihre soziale, politische und ökonomische Integration gewollt ist. Stets geht es hierbei um die Frage, welche Gruppen von der dominanten Mehrheit als legitim, gleichwertig und normal oder aber als störend, ungleich, abweichend oder sogar bedrohlich wahrgenommen werden. (Zick \& Klein, 2015, S. 153)

Diesbezüglich erweisen sich die Ergebnisse in der BRD als ambivalent:

In 2011 sind rund 37 Prozent der Deutschen der Ansicht, daß „zu viele kulturelle Unterschiede [...] dem Zusammenhalt der Deutschen“ schaden, und fünfzig Prozent meinen: „Deutschland wird in einem gefährlichen Maß überfremdet. [...] Zugleich wird kulturelle Vielfalt von einer überwältigenden Mehrheit der Befragten als etwas Positives bewertet. 85 Prozent sind beispielsweise der Ansicht, daß „Verschiedene kulturelle Gruppen [...] eine Gesellschaft bereichern, [...]. In den Meinungen wird eine Ambivalenz deutlich zwischen einer positiven Haltung zur Vielfalt auf der einen Seite und der Überzeugung, Vielfalt schade dem Zusammenhalt in Deutschland, auf der anderen. (Zick \& Klein, 2015, S. 167)

Positive Vorstellungen mit Blick auf Heterogenität sind also - zumindest hinsichtlich kultureller Vielfalt - in der BRD sehr verbreitet, gleichzeitig sind homogenitätsorientierte Einstellungen dafür verantwortlich, dass auch Ängste vor Überfremdung benannt werden. Tendenzen zur Exklusion bestimmter Gruppen beziehen sich - wie weiter oben dargestellt - allerdings eher auf sozial schwache Personen sowie Zuwanderer als auf Menschen mit Behinderungen bzw. beinhalten die Kumulation mehrerer Faktoren.

\subsubsection{Sichtweise der Bildungssoziologie}

Bildungssoziologisch orientierte Analysen des deutschen Schulsystems legen den Fokus auf die gesellschaftlichen Funktionen von Schule, wozu die Qualifikationsfunktion, die Selektionsbzw. Allokationsfunktion sowie die Legitimations-/Integrationsfunktion gehören. Sie lassen sich nicht immer streng von allgemeinpädagogischen bzw. schulpädagogischen Analysen tren- 
nen, da diese sich wiederum auf (bildungs-)soziologische Theorieansätze beziehen (u.a. Solga \& Dombrowski, 2009; Trautmann \& Wischer, 2011; Emmerich \& Hormel, 2013; Wenning, 2013). Die Qualifikationsfunktion der Schule hängt eng mit Entwicklungen auf dem Arbeitsmarkt zusammen, für den Nachwuchs ausgebildet werden muss, während die Integrationsfunktion u.a. „die Reproduktion von Normen, Werten und Deutungen“ (Trautmann und Wischer, 2011, S. 90) beinhaltet. Starke Auswirkungen auf alle Fragen, die mit dem Thema Heterogenität zu tun haben, haben dabei die Allokations- und Selektionsfunktion, indem am Ende der Bildungslaufbahn unterschiedliche Zugangsberechtigungen bzw. Abschlüsse vergeben und dafür zuvor zwischen „schlechteren“ und „besseren“ Schülern unterschieden werden muss (Trautmann und Wischer, 2011, S. 91f), was Picht schon in den 60er Jahren beschrieb:

In der modernen „Leistungsgesellschaft“ heißt soziale Gerechtigkeit nichts anderes als gerechte Verteilung der Bildungschancen; denn von Bildungschancen hängen der soziale Aufstieg und die Verteilung des Einkommens ab. (...) Der gesamte soziale Status, vor allem aber der Spielraum an persönlicher Freiheit, ist wesentlich durch die Bildungsqualifikationen definiert, die von dem Schulwesen vermittelt werden. (Picht, 1964, S. 6)

Nach Vester (2006) grenzt die Bildungssoziologie sich trotz unterschiedlicher Strömungen insgesamt von dem in der Öffentlichkeit dominanten „meritokratischen“ Marktmodell ab, das davon ausgeht, dass Ungleichheit dadurch entstehe, dass Menschen „sich im freien Leistungswettbewerb einen angemessenen Platz in der Bildungs- und Berufshierarchie erarbeiten“ (Vester, 2006, S. 14). Sie gehe stattdessen davon aus, dass „Kinder mit ungleichen sozialen und kulturellen „Startkapitalien" in das Bildungssystem eintreten und dort dann nach ihrer sozialen Herkunft schrittweise so „sortiert“ werden, dass sie überwiegend in das ebenfalls sozial gestufte Berufssystem gelenkt werden“" (Vester, 2006, S. 14). Die Analyse der Prozesse, die zu ungleichen Bildungschancen führen würden, kommt allerdings innerhalb der Sozialwissenschaften zu unterschiedlichen Schlüssen, wobei zwischen dem konflikttheoretischen Ansatz nach Bourdieu \& Passeron (1971) sowie der Rational-Choice-Theorie (Boudon, 1974; Becker, 2000; Becker \& Lauterbach, 2004) unterschieden wird (Vester, 2006, S. 15ff). Bourdieu geht davon aus, dass es vor allem sozio-kulturelle Segregationsmechanismen wie das kulturelle Kapital im Elternhaus (Hinz \& Groß, 2006, S. 201) sind, die sich auf die Bildungschancen von Kindern auswirken. Gemäß einem makrosoziologischen Ansatz seien es die ständische Organisation von Klasseninteressen und die soziale Klasse (ökonomisch und kulturell definiert), die diese bestimmen. Ziel sei die Sicherung von Rangstellungen innerhalb von Klassen und mit Blick auf Klassen untereinander, was auch bedeute, dass Lehrer eher Zugang zu Kindern mit einer hohen Ausstattung an kulturellem Kapital fänden (Hinz \& Groß, 2006, S. 201). Ähnlich wie bei beim Terminus „Exklusion“ allgemein spricht Bourdieu auch mit Blick auf das Bildungssystem von „interner Ausgrenzung“ (Bourdieu u.a. 1997, S. 527) und Kronauer fasst mit Blick auf das System zusammen:

Dieses wird für die Zuteilung gesellschaftlicher Positionen und Laufbahnen immer wichtiger, verspricht, Chancengleichheit zu fördern, und erhält dennoch - gerade in Deutschland - in vielfältiger Weise Ungleichheiten auf der Basis von sozialer Herkunft aufrecht, wenn es sie nicht gar verstärkt. Wer heute im Bildungswesen scheitert, ohne Schulabschluss oder Ausbildung bleibt, hat kaum Aussicht, im Erwerbsleben Fuß zu fassen. (Kronauer, 2010, S. 46)

Boudon u.a. unterscheiden zwischen vertikalen und institutionellen Dimensionen der Bildungssegregation (Vester, 2006, S. 21). Maßgeblich für Bildungschancen sei die Ressourcenausstattung der Eltern (Hinz \& Groß, 2006, S. 201). Gemäß einem mesosoziologischen Ansatz gingen Herkunftseffekte auf Familienmilieus zurück. Ziel seien der Erhalt oder die Verbesserung 
des sozialen Status unter der Abwägung von Bildungskosten, erzielbaren Bildungsrenditen und der Erfolgswahrscheinlichkeit (Hinz \& Groß, 2006, S. 199ff). Als primäre Herkunftseffekte werden die Auswirkungen von an die soziale Herkunft gekoppelten Lernvoraussetzungen und schulischen Leistungen bezeichnet. Sekundäre Herkunftseffekte bezeichnen den Zusammenhang von sozialer Herkunft und Bildungsentscheidungen der Eltern (Becker \& Lauterbach, 2004). Trotz der Expansion des Bildungssystems hinsichtlich der Bildungsbeteiligung (von 1952-1999 stieg der Besuch der Gymnasien von 13\% auf 31\%, der der Realschulen von 6\% auf 24\%, der Besuch von Fachhochschulen und Hochschulen von 110.000 auf 1,8 Millionen Personen), bestehen weiterhin Ungleichheiten: „Arbeiterkinder können [...] tatsächlich eher studieren, aber die Chance, dadurch in die obere Dienstklasse aufzusteigen, ist bei Kindern der Mittelklassen doppelt so hoch und bei Kindern aus der Dienstklasse viermal so hoch." (Vester, 2006, S. 36). Müller (2004) formuliert aufgrund der Forschungsergebnisse zum Ansatz von Boudon sogar die These, dass das deutsche Schulsystem eine „optimale Konstruktion [sei], wenn man möglichst große Ungleichheiten produzieren will.“ (Müller, 2004, S. 8; zitiert n. Vester, 2006, S. 19).

Beide Theorien können nach Vester allerdings nicht erfassen, welche „sozialen Mechanismen“ tatsächlich zu Bildungsungleichheiten führen (Vester, 2006, S. 22f). Er selbst geht davon aus, dass ,institutionelle und sozialkulturelle Segregation sich zueinander verhalten wie Form und Inhalt, dass sie sich gegenseitig stützen und verstärken und ohne einander weit weniger wirken könnten." (Vester, 2006, S. 25; Herv. im Orig.). Solga und Dombrowski wiederum unterscheiden in ihrem Modell der Strukturebenen sozialer (Bildungs-)Ungleichheiten zwischen Determinanten wie sozialer Schicht, Migrationshintergrund oder Geschlecht als Input, Dimensionen wie Kompetenzen oder Schulabschlüssen als Output und Mechanismen, „durch die aus Determinanten soziale Ungleichheiten entstehen und weiter bestehen" (Solga \& Dombrowski, 2009, S. 12). Sie beschreiben die Habitusproblematik, von der Boudon ausgeht, sehr plastisch:

Wenn im familiären Rahmen nur sehr eingeschränkte Spielräume für den Erwerb von Erfahrungen vorhanden sind, dann werden Kinder in ihren Lernmöglichkeiten und infolgedessen in ihrer kognitiven Entwicklung beeinträchtigt. Des Weiteren geht die Familiensoziologie davon aus, dass Entscheidungs-, Denk- und Sprechgewohnheiten, Verhaltensmuster etc. über die Zugehörigkeit zu einer bestimmten sozialen Gruppe geprägt werden und wiederum Einfluss auf die Bildungschancen haben. [...] Die sich im Erziehungsstil der Eltern widerspiegelnden Werte und Normen sowie deren Persönlichkeit und Kompetenzen in den Bereichen Bildung, Leistungsorientierung, Motivation, Alltagskompetenzen, sprachliche Fähigkeiten, Allgemeinwissen etc. haben in hohem Maße Einfluss auf die kindliche Entwicklung. Ungleichheiten im familiären kulturellen Kapital einer Familie werden insbesondere im deutschen Schulsystem in Bildungsungleichheiten transferiert. (Solga \& Dombrowki, 2009, S. 22)

Diese Annahmen wären noch nicht umfassend durch Forschungsergebnisse belegt, die IGLUStudien ${ }^{14}$ (2000 und 2003) hätten allerdings „deutliche Kompetenzunterschiede am Ende der Grundschule nach sozialer Herkunft" (Solga \& Dombrowski, 2009, S. 24) festgestellt. Hinz \& Groß wiederum verweisen in diesem Zusammenhang darauf, dass ein

[...] hoher sozioökonomischer Status des Haushalts (ISEI) [...] ebenso wie eine hohe Bildung beider Elternteile mit einer höheren Wahrscheinlichkeit einer Schulempfehlung für das Gymnasium einher [gehen], während mit sinkendem sozioökonomischem Status des Haushalts und niedrigerer Bildung der Eltern die Wahrscheinlichkeit einer Schulempfehlung für die Hauptschule steigt. (Hinz \& Groß, 2006, S. 210) 
Aussagekräftig sei diesbezüglich vor allem das PISA-Ergebnis, das belegt, dass $71 \%$ der Schüler im empfohlenen Bildungsgang verbleiben, $26 \%$ in einen niedrigeren Bildungsgang ab- und lediglich $3 \%$ in einen höheren Bildungsgang aufsteigen (Hinz \& Groß, 2006, S. 213). Gleichzeitig belege PISA, dass die von Schülern und Schülerinnen in niedrigeren Bildungsgängen erreichten Kompetenzstufen für den erfolgreichen Besuch höherer Bildungsgänge ausreichen würden, so dass Hinz und Groß die These der Reproduktion von Bildungsungleichheit durch das deutsche Schulsystem durch frühe Selektion am ersten schulischen Übergang in die Sekundarstufe zustimmen (2006, S. 201 u. 213). Insgesamt gehen die Autoren davon aus, dass „sich bei der Schulempfehlung die gezeigte Leistung der Kinder in der Grundschule (Noten im Übertrittszeugnis) mit einer Art „Sozialprognose“ durch die Lehrkräfte“ (Hinz \& Groß, 2006, S. 220) vermische. Emmerich und Hormel beschreiben das Erziehungssystem mit Bezug auf Luhmann als solches hinsichtlich inkludierender und exkludierender Operationen:

Das Erziehungssystem adressiert Individuen einer definierten Alterskohorte, es inkludiert diese, indem es ihnen ein spezifisches Rollenmuster zuschreibt (SchülerIn) und in einem spezifischen Modus der Kommunikation (pädagogische Kommunikation) in Anspruch nimmt. Erst mit der Inklusion [...] einer gesamten Alterskohorte entfaltet sich innerhalb der organisatorischen und interaktionellen Strukturen eine Dynamik des In- und Exkludierens von SchülerInnen: Schulsysteme schaffen durch ihre organisatorisch differenzierte Struktur die Möglichkeitsbedingungen für ein ständiges Re-Gruppieren von AdressatInnen, sei es unter Ausnutzung der Differenzierung von Sekundarschulformen, der Differenzierung von Klassen und Niveaukursen oder auf der Ebene von Unterrichtsinteraktion durch zeitliche begrenzte Binnendifferenzierungen von Lerngruppen. Auf der Basis dieser internen Operationen prädisponiert das Erziehungssystem Optionen für die Inklusions-/ Exklusionsoperationen anderer Teilsysteme, insbesondere des Wirtschaftssystems. [...] (Emmerich \& Hormel, 2013, S. 74f, Herv. im Orig.)

Sie verstehen Bildungsungleichheit als „Inklusionseffekt [...], den das Erziehungssystem nach internen Kriterien produziert." (Emmerich \& Hormel, 2013, S. 74), so dass diese nur im und durch das System als solches erzeugt werden könne. Inklusion und Exklusion würden „in Form von Homogenisierung/Heterogenisierung in Erscheinung " (Emmerich \& Hormel, 2013, S. 74, Herv. im Orig.) treten. Sie bezeichnen auch zeitlich begrenzte Formen wie binnendifferenzierten Unterricht als Exklusion:

Exklusion findet auch dann statt, wenn sie zeitlich begrenzt, d.h. temporalisiert vollzogen wird bspw. in Form binnendifferenzierenden Unterrichts: Auch hier ist die Teilhabe an dieser und nicht einer anderen Gruppe immer exklusiv, jedoch im Unterschied zur äußeren Differenzierung nach Schulformen und Niveaukursen prinzipiell reversibel. (Emmerich \& Hormel, 2013, S. 75; Herv. im Orig.)

Während Schulleistungen ein grundlegendes Kriterium für unterschiedliche Zuweisungen von Schülern seien, würden weitere Kriterien wie Geschlecht, Ethnie, Religion, Kultur, Schicht, Milieu ebenfalls zur unterschiedlichen Adressierung von Schülern genutzt (Emmerich \& Hormel, 2013, S. 75; Zinnecker \& Stecher, 2006, S. 296).

Das Interaktionssystem Unterricht wiederum sei hochkomplex, so dass die Beobachtung und Bewertung von Leistungsunterschieden schwierig und mehrdeutig erscheine (Emmerich \& Hormel, 2013, S. 78). Gleichzeitig sei die Bedeutung von Leistungsdifferenzen für die Schule zentral:

Die zentrale Stellung von Leistung ist dabei in doppelter Hinsicht augenfällig. Denn erstens gelten Leistungsdifferenzen - im Gegensatz zu vielen anderen Differenzen - als natürlich und legitim zugleich. Während eine Ungleichbewertung aufgrund von Milieu, Geschlecht oder Ethnizität zumeist illegi- 
tim erscheint, sind Leistungsdifferenzen nicht nur akzeptiert. Im Gegenteil, die Hervorbringung von Leistungsunterschieden und deren differentielle Bewertung ist geradezu eine wichtige Funktion von Schule, unabhängig davon, ob sie sich im Einzelfalle der individuellen Förderung oder der größtmöglichen Gleichheit verschrieben hat (vgl. Sacher 2009; Sacher/Grunder 2011; Schrader/Helmke 2001). (Budde, 2013, S. 9)

Im Erziehungssystem sind also Organisation und Interaktion miteinander gekoppelt: durch die Organisation ist festgelegt, dass selektive Entscheidungen getroffen werden müssen; die Interaktion muss dazu beitragen, dass diese Entscheidungen getroffen werden können: „Offenbar bleibt es dabei der Profession überlassen, wie beide Codes miteinander gekoppelt werden und in welchem Umfang bspw. pädagogisches Beobachten selektions- und/oder vermittlungscodiert abläuft." (Emmerich \& Hormel, 2013, S. 78f).

Die sozialen Unterschiede der AdressatInnen pädagogischer Kommunikation liegen ebensowenig ,als solche'vor, wie vermeintliche Begabungsunterschiede. Auch soziale Differenz stellt vielmehr eine genuin systemeigene Beobachtungsleistung dar, die mit systemeigenen Klassifikationen und Askriptionen arbeitet, die allerdings - und das ist der neuralgische Punkt - weder durch Kommunikationscodes noch durch pädagogische Programme kontrolliert werden können, sondern in Form ,wilden` Klassifizierens und Askribierens ablaufen. (Emmerich \& Hormel, 2013, S. 88)

\subsubsection{Sichtweise der Allgemeinen Pädagogik}

Problematisch bei der Darstellung der Sichtweise der Allgemeinen Pädagogik auf die Konstrukte Exklusion und Inklusion ist zunächst, dass eine Systematisierung aufgrund der Fülle unterschiedlicher Zugänge schwerfällt. Aus diesem Grund werden beispielhaft Grundauffassungen mehrerer Allgemeinpädagogen zusammengestellt, ohne die Gesamtdiskussion der Allgemeinen Pädagogik zu Heterogenität, Vielfalt etc. umfassend abbilden zu können.

Benner (2015) differenziert mit Blick auf eine Systematisierung der Allgemeinen Pädagogik zunächst drei Grundpositionen: „eine pädagogisch-naive, eine politisch-pragmatische und eine den Prinzipien pädagogischen Denkens und Handelns verpflichtete Position“ (Benner, 2015, S. 139). Die pädagogisch-naive Position gehe davon aus, dass eine Überführung sozialisatorischer Benachteiligung von Bildungskarrieren in Chancengleichheit möglich sei (Benner, 2015, S. 139). Dieser Position liegen wiederum zwei unterschiedliche Hypothesen zugrunde: die Defizithypothese und die Differenzhypothese. „Die Anhänger der Defizithypothese erklärten die Sozialisationsprozesse benachteiligter Heranwachsender für defizitär und erhoben die Sozialisationsprozesse der nicht-benachteiligten Heranwachsenden zum Ideal einer erstrebenswerten Normalität." (Benner, 2015, S. 140). Daher sei kompensatorische Erziehung Ziel führend. „Die Befürworter der Differenzhypothese kritisierten diese Strategie und warnten davor, die Sozialisationsprozesse nicht-benachteiligter Gruppen zum Ideal für alle zu erheben." (Benner, 2105, S. 140). Einen interessanten Aspekt dieser Problematik im Kontext Schule benannte schon 1970 Basil Bernstein:

Wenn Kinder „kulturell depriviert“ genannt werden, folgt daraus, daß die Eltern unzulänglich sind, den kulturellen Anforderungen nicht entsprechen. Die spontanen Verwirklichungen ihrer Kultur, deren Bilder und symbolische Repräsentationen sind von verringertem Wert und minderer Bedeutung. Die Lehrer haben dann geringere Erwartungen von den Kindern, die diese zweifellos erfüllen. Alles, was das Kind außerhalb der Schule beeinflußt, für das Kind Bedeutung und Zweck hat, hört auf, wertvoll zu sein; weder wird ihm Bedeutung zuerkannt, noch bietet es Chancen für das Vorwärtskommen in der Schule. (Bernstein, 1970, n. Bauer et al., 2012, S. 152) 
Die politisch-pragmatische Position wiederum gehe davon aus, dass kompensatorische Erziehung der passenden Zuführung von Arbeitskräften (Wirtschaft, Verwaltung) diene (Benner, 2015, S. 141f). Die dritte Position pädagogischen Denken und Handelns wehre sich gegen die Verkürzungen der „Kausalität pädagogischer Einwirkungen auf intentionale und funktionale Modelle" und stelle stattdessen pädagogische Prinzipien wie Selbsttätigkeit in den Mittelpunkt (Benner, 2015, S. 144).

Insgesamt befasst sich die allgemeine Pädagogik im Zusammenhang mit den Begriffen Inklusion und Exklusion - ähnlich wie die Bildungssoziologie - vor allem mit den Funktionen von Schule (Qualifikation, Selektion, Allokation) (Fend, 2012) und denen des Schulsystems, die Fend als Reproduktion kultureller Systeme, der Sozialstruktur und gesellschaftlicher Herrschaftsverhältnisse (politische Funktion) aufschlüsselt (Fend, 2012, S. 161ff). Im Gegensatz zur Auseinandersetzung mit Integration oder Inklusion (mit Blick auf Menschen mit Behinderungen) in der Sonderpädagogik bewegt sich der Diskurs in der Pädagogik u.a. um die Begriffe „Einheit und Differenz“ (Tenorth, 1994), „Pädagogik der Vielfalt (Prengel, 1993) sowie „Heterogenität“ (Trautmann \& Wischer, 2011; Wenning, 2013; Wischer, 2013). Als einer der ersten Allgemeinpädagogen beschäftigte sich Jakob Muth mit Fragen der integrativen Unterrichtung von Schülern mit Behinderungen und analysiert, dass es dabei um gesellschaftspolitische Prozesse und die Verminderung sozialer Diskriminierung geht:

Der identische Grundzug dieses Integrationsbemühens in allen Bereichen dürfte darin zu sehen sein, $\mathrm{da}$ es sich um ein primär gesellschaftspolitisches Phänomen handelt, das in der fortschreitenden Profilierung demokratischen Bewußtseins seinen Grund hat. Er richtet sich konkret auf die Überwindung hierarchischer Strukturen im Bildungswesen und mithin auf den Abbau sozialer Diskriminierung. Denn die getrennten Einrichtungen im Hochschulbereich und die jeweils separat nebeneinander bestehenden Schulformen sind keine generell gleichrangigen Institutionen, sondern sie stellen eine Rangordnung dar. In ihnen gibt es ein Oben und ein Unten, ein Hoch und Nieder, ein Zugelassensein, aber auch ein Ausgesperrtsein. Darum ist heute noch der Weg junger Menschen durch diese Einrichtungen von Selektionsprozessen gesteuert, die einerseits zu einer sozialen Favorisierung, andererseits aber auch zu einer sozialen Diskriminierung führen, die der gesellschaftlichen Situation des Menschen in einem demokratisch verfaßten Gemeinwesen widersprechen. (Muth, 1977, S. 91)

Tenorth (1994) thematisiert in seiner Veröffentlichung „Alle alles zu lehren“ intensiv die Frage nach dem Konstrukt der „allgemeinen Bildung“. Die Diskussion dessen, was Allgemeinbildung sei, müsse sich mit vier Fragestellungen auseinandersetzen, die sich auf Inhalt, Adressaten, Ort sowie Ziel und Funktion beziehen (Tenorth, 1994, S. 7). Mit Blick auf soziologische und bildungssoziologische Überlegungen geht er davon aus, dass die Schule in der Moderne an sozialer Bedeutung gewonnen habe und sie Differenzen erzeuge, die sich folgenreich auf Lebensläufe von Schülerinnen und Schülern auswirken (Tenorth, 1994, S. 58). Da sich die primäre Sozialisation der Schüler allerdings in der Herkunftsfamilie ereigne und dadurch Differenzen vorbereitet werden würden, könne die pädagogische Arbeit in der Institution Schule immer nur „Überformung von Sozialisation, spezifische Bemühung, substantielle Erziehung, die ein Allgemeines modifizieren will, das schon existiert“ (Tenorth, 1994, S. 65) sein. Die Kernfrage allgemeiner Bildung betreffe daher die „Einheit und Differenz von Bildungsprozessen“ und die damit verbundene komplexe Zielsetzung gleichzeitig die Bildung von Individualität, die Herstellung von Chancengleichheit und die Steigerung von Lernleistungen zu erreichen. Dass diese Zielsetzung zu „Zielkonflikten und Realisierungsproblemen“ führe, sei naheliegend, da es „unrealistisch [sei] zu erwarten, daß sich alle Zielkomplexe zugleich und gleichzeitig realisieren oder gar steigern ließen“ (Tenorth, 1994, S. 143). Nach Tenorth ist 
[...] das Ergebnis zunehmender Homogenisierung der Bevölkerung in den öffentlichen Einrichtungen des Lernens [...] die Erzeugung von Differenz, von Unterschieden in der Verteilung von Zertifikaten, Interessen und Handlungsmustern, von Lebensperspektiven und Merkmalen der Persönlichkeit. Allgemeine Bildung ist ein Prozeß, der Differenzen verstärkt, indem er egalisiert - das scheint das zunächst paradox klingende Ergebnis, und wird entsprechend der Anstoß für Kontroversen. (Tenorth, 1994, S. 156)

Mit Blick auf die Frage der Gliederung des Schulsystems versus der Gesamtschulidee (Dreigliedrigkeit als Versuch möglichst homogene Gruppen zu bilden, Gesamtschulen als Versuch heterogene Gruppen zu gestalten) sei die Bildungsforschung nicht zu einheitlichen Ergebnissen für oder gegen einen dieser Wege gelangt (Tenorth, 1994, S. 143-146). Vorteile biete die Gesamtschule allerdings dahingehend, dass eine höhere Durchlässigkeit zwischen den verschiedenen Bildungsgängen gewährleistet sei (Tenorth, 1994, S. 144f). Allerdings reicht es seiner Meinung nach auch nicht aus, lediglich Effekte unterschiedlicher Schulstrukturen zu messen, notwendig sei es vielmehr unterrichtliche Prozesse in den Blick zu nehmen (z.B. didaktische Planung, Motivierung von Schülern, Lehrerstrategien). Dabei könne sich die innere Differenzierung „als das Medium [erweisen], zwischen Qualifizierung und Egalisierung zu vermitteln“. (Tenorth, 1994, S. 146). Die Untersuchung der Systemfrage habe

[...] zu der Einsicht geführt, daß die Unterschiede innerhalb der Systeme größer sind als zwischen ihnen und daß die entscheidenden Ursachen für die Unterschiede nicht Bedingungen des Schulsystems, sondern Faktoren der Schulkultur zuzurechnen sind. Damit rücken Faktoren ins Zentrum des Interesses, die nicht die strukturellen, systemischen Prämissen der Organisation, sondern die konkrete pädagogische Arbeit berühren. Vereinfacht gesagt, Kontroversen über Differenzierungsformen führen zurück zu den Möglichkeiten und Alternativen pädagogischer Arbeit selbst und zu den selbstgesetzten Zielen und Kompetenzen der Lehrenden. (Tenorth, 1994, S. 146f, Herv. im Orig.)

Als Schlussfolgerung zu seinen Überlegungen gelangt Tenorth zu folgenden möglichen konstruktiven Antworten auf die eingangs gestellten Fragen nach dem Wer, Was, Wo und Wie allgemeiner Bildung: Allgemeine Bildung richte sich an alle Heranwachsenden. Kritisch sei in diesem Zusammenhang allerdings die Frage nach der Vereinbarungsmöglichkeit der Freiheit individuellen Lernens mit der Forderung gleicher Bildungsprozesse. Dies zeige sich am Beispiel der Diskussion zu Hochbegabten, für die spezielle Einrichtungen vorgeschlagen werden würden, während für „Sonderschüler“ integrative Angebote diskutiert werden würden. Dabei werde nicht das Recht auf allgemeine Bildung in Frage gestellt, sondern dessen Realisierungsmöglichkeit innerhalb eines Angebots bspw. mit Blick auf die Finanzierung (Tenorth, 1994, S. 169-173). Allgemeine Bildung habe die Aufgabe „ein Bildungsminimum für alle zu sichern und zugleich die Kultivierung von Lernfähigkeiten zu eröffnen“" (Tenorth, 1994, S. 166; Herv. im Orig.). Als Organisationsmodell für den Ort allgemeiner Bildung empfiehlt Tenorth ein einheitliches schulisches Angebot für die Sekundarstufe I, gefolgt von einem ausdifferenzierten Angebot für die Sekundarstufe II (Tenorth, 1994, S. 183f). Ziel und Funktion allgemeiner Bildung entsprechen seiner Meinung nach der o.g. Aufgabe allgemeiner Bildung. Auch wenn sich Tenorth in seinen Ausführungen eingangs auf Luhmanns Inklusionsverständnis bezieht, differenziert er selbst zwischen den Begriffen Einheit, Gleichheit und Differenz. Er geht nicht davon aus, dass Differenzen sich durch das System Schule hin zu einem größeren $\mathrm{Maß}$ an Gleichheit verändern lassen, sondern im Gegenteil davon, dass schulische Bildung Differenzen verstärke. 
Auch Prengel befasst sich intensiv mit den Begriffen Gleichheit und Verschiedenheit (Differenz). Sie nimmt an, dass die „,in der alltäglichen Schulpädagogik realisierte Vielschichtigkeit [...] kaum eine Entsprechung in der pädagogischen Theoriebildung und den damit einhergehenden erziehungswissenschaftlichen Diskursen [findet]“ (Prengel, 2006, S. 11). Um diese Theoriebildung zu gewährleisten, greift Prengel u.a. auf den philosophischen Diskurs zurück. Orientiert an den Ausführungen von Dann (1980) beschreibt sie Gleichheit zunächst als „eine Form der Übereinstimmung von Verschiedenen“" (Prengel, 2006, S. 30), während sie Verschiedenheit als qualitative Differenz versteht, die klar und präzise mit Blick auf bestimmte Merkmale bestimmbar sei (Prengel, 2006, S. 31). Gleichheit und Verschiedenheit seien also Kategorien, die in einem spezifischen Verhältnis zueinander stehen. Daraus schlussfolgert sie:

Die für die Pädagogik der Vielfalt wesentliche Einsicht aus dieser Begriffsklärung ist, daß sowohl Gleichheit als auch Verschiedenheit ausschließlich in der Lage sind, Aussagen über ein partielles Verhältnis, nie die generelle Beziehung mit allen Aspekten zu machen. Darum ist es erforderlich, einen Maßstab des Vergleichens, die Hinsicht in welcher etwas gleich oder verschieden sei, zu bestimmen. Es muss ein ,tertium comparationis' klar definiert werden. (Prengel, 2006, S. 33)

Prengel definiert „Gleichberechtigung“ als eine Variante der Gleichheit (S. 35) und postuliert daher, dass Emanzipationsbewegungen nicht universelle Gleichheit (Gefahr der Assimilation), sondern Gleichberechtigung fordern sollten. Ihre zentralen Fragestellungen richten sich demnach darauf, inwiefern pädagogisches Handeln der Verschiedenheit der Menschen gerecht werden und Pädagogik das demokratische Prinzip der Gleichberechtigung verwirklichen kann. In diesem Zusammenhang vertritt sie die Auffassung:

Pädagogik der Vielfalt versteht sich als Pädagogik der intersubjektiven Anerkennung zwischen gleichberechtigten Verschiedenen. Indem sie Mißachtung im Bildungswesen zu vermeiden sucht, fördert sie persönliche Bildungsprozesse, sowie Qualifikations- und Sozialisationsprozesse und wirkt den schädlichen Folgen des im Bildungssystem vorherrschenden Selektionsprinzips entgegen. (Prengel, 2006, S. 62)

Unter Integration im schulischen Bereich wiederum versteht Prengel „das gemeinsame Lernen aller“ (Prengel, 2006, S. 139), das weder als "gelegentliches Zusammensein“ (Prengel, 2006, S. 139) noch als „kompensatorische Förderung“ (Prengel, 2006, S. 139) misszuverstehen sei. Prengel erkennt in diesem Zusammenhang als Leistung der Sonderpädagogik an, dass diese spezifische Formen der Unterstützung und Förderung für Kinder und Jugendliche mit Behinderungen entwickelt habe, kritisiert aber, dass diese auch zur „Inferiorisierung“ dieser Gruppe von Menschen beigetragen habe (Prengel, 2006, S. 145-155). Im Gegensatz zur ausführlichen, differenzierten und weitgehend „normfreien“ Beschreibung des Begriffspaars „Gleichheit und Differenz“ arbeitet Prengel mit Blick auf die Frage nach der schulischen Integration mit starken normativen Setzungen und vertritt die Auffassung, dass gemeinsamer Unterricht in extrem heterogenen Gruppen möglich sei (Prengel, 2006, S. 158f). Mit Hilfe der integrativen Didaktik, bei der Differenzierung und Individualisierung sowie offene Unterrichtsformen (aus der Reformpädagogik) im Mittelpunkt stehen, könne dieser Unterricht umgesetzt werden. Äußere Differenzierung solle so gering wie möglich gehalten werden (Prengel, 2006, S. 158-163). Neben dem Bezug zu - diese Auffassung einheitlich stützenden - Forschungsergebnissen argumentiert sie dabei auch mit eigenen Forschungsergebnissen aus einer qualitativen Interviewstudie mit 30 Personen (Deppe-Wolfinger et al., 1990). 
Die einzigen Einschränkungen zu dieser Auffassung, die Prengel selbst formuliert, beziehen sich zum einen auf das Dilemma, dass die Gemeinsamkeiten von Kindern und Jugendlichen mit ähnlichen Lebenserfahrungen bei der sogenannten wohnortnahen Integration vernachlässigt werden müssen, da diese unter Umständen nicht in einer Gruppe zusammenfinden. Als Beispiel nennt sie die Gruppe der gehörlosen Schüler, die ihre „Kollektivität“ durch Verständigung mit Hilfe der Gebärdensprache in einem integrativen Setting nicht umsetzen können (Prengel, 2006, S. 170). Zum anderen sieht sie eine mögliche Grenze des gemeinsamen Lernens darin, dass Gewalt durch Schülerinnen und Schüler mit massiven Verhaltensauffälligkeiten und damit zusammenhängenden „regressiven und aggressiven Verarbeitungsformen“ (Prengel, 2006, S. 194) ggf. verhindert werden müsse. Als Schlussfolgerungen zu ihrem Ansatz fasst Prengel u.a. zusammen, dass die Legitimation von Hierarchien durch „egalitäre Differenz" beendet werden müsse (Prengel, 2006, S. 181), dass Selbstachtung und Anerkennung anderer zentrale Werte seien (Prengel, 2006, S. 185-189), dass möglicherweise auch Schulen für homogene Gruppen (Bsp.: Mädchen, blinde oder gehörlose Schüler) und damit Schulpluralismus (Prengel, 2006, S. 188) möglich seien, wenn diese Schulen sich „demokratischen Prinzipien verpflichten “ (Prengel, 2006. S. 196) und dass eine Didaktik des offenen Unterrichts ohne Ziffernnoten umzusetzen sei (Prengel, 2006, S. 193).

Als Lösungsansatz zur pädagogischen Bearbeitung von Qualifikation und Sozialisation formuliert Prengel für den schulischen Bereich:

Pädagogische Institutionen haben beschränkte gesellschaftliche Einflußmöglichkeiten, so können sie ökonomisches Elend nicht beseitigen. Die Möglichkeit eine eigene ,Sphäre der Gerechtigkeit' [...] zu schaffen aber liegt in ihrer Verantwortung. Das gesellschaftlich wertvolle Gut, das Schulen und andere pädagogische Einrichtungen aus eigener Machtbefugnis und eigenen Ressourcen zu verteilen haben, heißt intersubjektive Anerkennung jeder einzelnen Person in ihrer je einmaligen Lebenslage. (Prengel, 2006, S. 61)

Aus diesem Grund solle die Selektionsfunktion von Schule stärker nach außen verlagert werden (Prengel, 2006, S. 61).

Eine kritischere Sicht auf Möglichkeiten zum Umgang mit Heterogenität in der Schule stellen Trautmann und Wischer (2011) bzw. Wischer (2013) dar. Schulen befinden sich im Umgang mit Heterogenität nach Wischer in einem Grunddilemma, da zwar durch schulische Institutionalisierung ein flächendeckendes Bildungsangebot für alle ermöglicht werden kann, gleichzeitig dadurch aber immer auch Einschränkungen für Individuen in Kauf genommen werden (Wischer, 2013, S. 100f). Festzustellen bleibt mit Trautmann und Wischer zunächst, dass neben der aus pädagogischer Sicht zu unterstützenden Perspektive der individuellen Förderung auch zu berücksichtigen ist, dass es in der Institution Schule

[...] in aller Regel zunächst einmal darum [geht], Massenlernprozesse zu organisieren: Es müssen große Schülerströme kanalisiert, Laufbahnen strukturiert und Übergänge wie auch Gruppenzugehörigkeiten verbindlich geregelt werden, was Strategien von Vereinheitlichung voraussetzt und Maßnahmen zur Reduzierung von Heterogenität durch Klassifizierung und Sortierung durchaus nahe legt. (Trautmann \& Wischer, 2011, S. 10)

Sachlich und mehrperspektivisch betrachtet ergebe sich aufgrund der Struktur der Institution Schule also ein Widerspruch zwischen Institutionalisierung und Individualisierung, der durch pädagogische Maßnahmen allein nicht aufzuheben sei. Institutionalisierung und Individualisierung stehen dabei grundsätzlich in einem Spannungsverhältnis, wie folgende Tabelle veranschaulicht: 
Tab. 5: Spannungsverhältnis zwischen Institutionalisierung und Individualisierung

\begin{tabular}{l|l}
\hline Institutionalisierung & Individualisierung \\
\hline $\begin{array}{l}\text { organisatorisch-strukturelle Rahmenbedingungen } \\
\text { (Bsp.: mehrgliedriges Schulsystem, Klassenstufen) }\end{array}$ & $\begin{array}{l}\text { individuelle Entwicklung von Schülerinnen und } \\
\text { Schülern (Bsp.: individuelle Förderung) }\end{array}$ \\
\hline $\begin{array}{l}\text { formalisierte Regelungen (Bsp.: Klassenarbeiten, } \\
\text { Zeugnisse) }\end{array}$ & $\begin{array}{l}\text { Berücksichtigung individueller Bedürfnisse } \\
\text { (individuelle Leistungsbewertung) }\end{array}$ \\
\hline $\begin{array}{l}\text { Bildungs- und Erziehungsaufgaben für alle Schüler } \\
\text { und Schülerinnen (Bsp.: Lehrpläne) }\end{array}$ & $\begin{array}{l}\text { auf Einzelschüler zugeschnittene Bildung und } \\
\text { Erziehung (Bsp.: individuelle Förderpläne) }\end{array}$ \\
\hline $\begin{array}{l}\text { gesellschaftliche Funktion von Schule (Allokation: } \\
\text { Zuweisung von Bildungsabschlüssen) }\end{array}$ & $\begin{array}{l}\text { Offenhaltung von Bildungsabschlüssen über } \\
\text { einen langen Zeitraum (Bsp.: Gesamtschulidee) }\end{array}$ \\
\hline $\begin{array}{l}\text { Umgang mit Heterogenität = schulorganisatori- } \\
\text { sche Aufgabe }\end{array}$ & $\begin{array}{l}\text { Umgang mit Heterogenität = unterrichtliche } \\
\text { Aufgabe für Lehrer }\end{array}$ \\
\hline $\begin{array}{l}\text { Schülerinnen und Schüler werden gruppiert und } \\
\text { auf unterschiedliche Lernangebote verteilt }\end{array}$ & $\begin{array}{l}\text { Schülerinnen und Schüler bleiben in ihrer Grup- } \\
\text { pe und erhalten unterschiedliche Lernangebote } \\
\text { (quantitativ und qualitativ differenziert) }\end{array}$ \\
\hline $\begin{array}{l}\text { Interaktionsbeziehungen in der Schule sind nicht } \\
\text { auf Zweierbeziehung zwischen Lehrer und Einzel- }\end{array}$ & $\begin{array}{l}\text { Unterricht wird vom einzelnen Kind aus gedacht } \\
\text { und so organisiert, dass individuelle Förderung }\end{array}$ \\
$\begin{array}{l}\text { schüler angelegt, sondern auf Interaktion zwischen } \\
\text { Lehrer und Gruppe (bspw. etwa 30 Schüler) }\end{array}$ & und gemeinsames Lernen sich abwechseln \\
\hline
\end{tabular}

(Quelle: eigene Darstellung; in Anlehnung an Wischer, 2013, S. 101-115)

Einem Verzicht „auf jegliche Formen der Kategorisierung, Klassifizierung und Schülerzuweisung" (Wischer, 2013, S. 109) könne demnach nur durch innere Differenzierung und die damit verknüpfte Individualisierung von Lernprozessen begegnet werden. Diese Form der Differenzierung wiederum könne auf der einen Seite zwar ungünstige Lernvoraussetzungen kompensieren, auf der anderen Seite könne sie allerdings auch zu einer Verstärkung von (sozialer) Ungleichheit führen, da differenzierte Lernangebote ein unterschiedlicheres Leistungsniveau zur Folge hätten (Wischer, 2013, S. 110, 112). Schulische Selektion würde dadurch „nach innen“ verschoben, also beispielsweise in eine gemeinsame Schule hinein und selbst wenn erst am Ende der Schulzeit leistungsbezogene Unterschiede durch Noten und Zeugnisse dokumentiert werden würden, blieben gesellschaftliche Platzierunggsprozesse im Anschluss an die Schulzeit bestehehen, so dass durch „Bildungsgerechtigkeit keine soziale Gleichheit hergestellt" (Wischer, 2013, S. 121) werden könne. Auch Demmer (2007) problematisiert diesen Aspekt, allerdings dahingehend, dass Lehrkräfte sich im dem Paradox bewegen, auf der einen Seite eine Orientierung an den Schülern und deren Bildungsprozessen vorzunehmen, auf der anderen Seite einer Institution verpflichtet zu sein, die diesen Bildungsprozessen zum Teil aufgrund der Allokations- und Selektionsfunktion im Weg steht (Demmer, 2007, S. 163). Fend (2008) beschreibt dieses Dilemma als Abarbeiten von Antinomien. Dabei gehe es auf der einen Seite um eine Orientierung an Leistungsnormen, auf der anderen Seite um die Gewährleistung von Individualansprüchen (Fend, 2008, S. 28).

Heterogenität an sich wiederum wird von Wenning definiert als „Feststellung einer Ungleichheit bzw. Uneinheitlichkeit in einer sozialen Situation. Sie ist eine momentane Zustandsbeschreibung, die von einer Person oder einer Gruppe einer sozialen Situation zugemessen wird.“ (Wenning, 2013, S. 133), eine Aussage, die mit bildungssoziologischen Vorstellungen wie 
denen von Emmerich \& Hormel (2013) verknüpft ist. Nach Trautmann und Wischer (2011) hängt der Diskurs zum Thema Heterogenität eng mit einer Kind- und Individualitätszentrierung zusammen (Trautmann \& Wischer, 2011, S. 22). Aus diesem Begründungsansatz heraus würde der Unterschiedlichkeit von Schülern besondere Bedeutung zugemessen und der Anspruch formuliert, ein an die individuellen Ansprüche von Kindern angepasster Unterricht müsse entsprechend individualisierend gestaltet sein - eine Orientierung, die ihre Ursprünge in der Reformpädagogik habe (Trautmann \& Wischer, 2011, S. 23). Das „,Feiern“ von Vielfalt in der Literatur" (Trautmann \& Wischer, 2011, S. 34) kritisieren sie, da Heterogenität als moralisch gut einstuft werde, ohne Homogenisierung unter Umständen ebenfalls als berechtigte strategische Herangehensweise in den Blick zu nehmen, ohne zu prüfen, welche systembestimmten Grenzen von Schule als Institution diesem Anspruch entgegenstehen und ob dadurch u.a. eine „Pädagogisierung gesellschaftlicher Probleme“" (Trautmann \& Wischer, 2011, S. 24) erfolge. Ähnlich äußert sich Wenning:

Konsequenz daraus ist die Zuschreibung von Verantwortung an die pädagogisch Handelnden - und führt so unmittelbar zur Forderung der Verbesserung des didaktischen Umgangs mit Heterogenität. Umgekehrte Folge dieses Vorgehens ist die Entlastung von anderen möglichen Verantwortlichen, etwa politisch Handelnden oder der sozialen, finanziellen usw. Rahmenbedingungen, unter denen das pädagogische Handeln stattfindet. (Wenning, 2013, S. 144)

Das Autorenteam Trautmann und Wischer schlussfolgert aus uneindeutigen empirischen Ergebnissen zur tatsächlichen Heterogenität der Schülerschaft, dass hinsichtlich der Klärung des Begriffs Heterogenität eine Systematisierung, Konkretisierung und Priorisierung von Merkmalen erfolgen müsste, damit er sich nicht nur als ein Schlagwort auffassen lässt, „das sich zwar für viele Erfahrungen mit schulischer Förderung als irgendwie anschlussfähig erweist, die dahinter stehenden Probleme und Herausforderungen aber eher verdeckt als aufklärt" (Trautmann \& Wischer, 2011, S. 65). Sie gehen in diesem Zusammenhang nicht davon aus, dass ein integratives System einem differenzierten Schulsystem überlegen sei. Mit Bezug auf Ergebnisse von Schümer und Weiß (2008), Fend (1986) sowie Helmke und Weinert (1997) weisen sie darauf hin, dass Unterschiede zwischen einzelnen Schulen derselben Schulform größer sein können als bspw. zwischen einer Gesamtschule und einem Gymnasium und die Anzahl der Bedingungsfaktoren für schulische und unterrichtliche Prozesse zu hoch sei um lineare Schlussfolgerungen aus den Gegebenheiten eines gegliederten Schulsystems ziehen zu können (Trautmann \& Wischer, 2011, S. 84f).

Trautmann und Wischer (2011) sprechen in diesem Zusammenhang von ,starren Fronten' zwischen den Befürworten eines inklusiven und den Befürwortern eines nach Schulformen differenzierten Schulsystems in Deutschland:

Der schulische Umgang mit Heterogenität ist nicht allein ein technisches Problem (Wie lassen sich Massenlernprozesse am effektivsten organisieren?). Und es spielen keineswegs nur pädagogische Argumente (Durch welche Variante werden alle SchülerInnen am besten gefördert?) eine Rolle, sondern auch ökonomische, politische oder rechtliche Argumente. Anhand der Schulformdifferenzierung zeigt sich im historischen Rückblick (z.B. Diederich/Tenorth 1997: 13ff.; Oelkers 2006), aber auch bei aktuellen Debatten, dass mit dieser Kontroverse grundsätzliche gesellschaftspolitische Präferenzen und gruppenspezifische Interessen verknüpft sind, was den gesellschaftlichen Umgang mit Vielfalt betrifft. Es ging früher, und es geht durchaus heute noch auch darum, gesellschaftliche Unterschiede (und Ungleichheiten!) fortzuführen, zu legitimieren oder aufzuheben bzw. aufzubauen. (Trautmann \& Wischer, 2011, S. 80) 


\subsubsection{Sichtweise der Sonderpädagogik}

Ähnlich wie die Pädagogik stellt auch die Sonderpädagogik hinsichtlich der Definition der Begriffe Integration, Separation, Inklusion und Exklusion teilweise Bezüge zum soziologischen bzw. bildungssoziologischen Begriffsverständnis her (Romey, 1977; Bleidick, 1990; Wansing, 2005; Ziemen u.a., 2011). So analysiert Romey bereits 1977 Bezug nehmend auf die Funktion des Schulsystems:

Das allgemeine Schulsystem der BRD ist nicht auf Einbeziehung aller Schüler in einen gemeinsamen Lernprozeß, sondern auf Aussonderung in verschiedene Schulformen mit divergierenden Lernzielen ausgerichtet. Entsprechend den jeweiligen Abschlußebenen werden Berechtigungen erteilt, die Berufschancen und dadurch bedingt Statuspositionen, z.B. Aufstiegs- und Verdienstmöglichkeiten, in der Gesellschaft festlegen. (Romey, 1977, S. 107)

Seiner Auffassung nach ist die soziale Schichtzugehörigkeit entscheidend für schulische Werdegänge (Romey, 1977, S. 107). Das „drei- bzw. viergliedrige Schulsystem hat eine stabilisierende Funktion auf das System sozialer Schichtung, indem es Schulerfolgschancen - beispielsweise über Curricula, Vermittlungsformen, Lehrerverhalten - schichtenspezifisch unverhältnismäßig verteilt und schichtenspezifische Erwartungshaltungen aufbaut bzw. verstärkt [...].“ (Romey, 1977, S. 108), woraus er schlussfolgert, dass soziale Integration erst in einem einheitlichen Schulsystem möglich sei (Romey, 1977, S. 109). Bleidick wiederum geht davon aus, dass schulorganisatorische Veränderungen die pädagogische Förderung von Schülern mit Behinderungen nicht automatisch verbessern würden, und führt die Gesamtschulen als Beispiel an:

Gesamtschulen haben dort, wo sie flächendeckend und über einen Zeitraum hinweg zum Regelangebot wurden, keineswegs die Schichtenmobilität verbessert und soziale Benachteiligungen abgebaut, vielmehr wurde eher die Leistungskonkurrenz zum Gymnasium verstärkt. (Bleidick, 1990, S. 27)

Insbesondere die Fragen nach der Problematik von Schulleistungen und damit nach zieldifferentem Lernen seien in der Sekundarstufe unbeantwortet (Bleidick, 1990, S. 22). Werning dagegen kritisiert die starke Gewichtung von Bildungsstandards und Vergleichsarbeiten, die die Entwicklung einer inklusiven Schulkultur erschwere (Werning, 2013, S. 20). Dyson belegt wiederum am Beispiel Englands, dass Lehrkräfte die Orientierung an Bildungsstandards durchaus auch als förderlich mit Blick auf die Unterrichtung von Schülern mit sonderpädagogischem Förderbedarf einschätzen:

Specifically, the focus on standards directed their attention to students who were doing badly in the schools' current arrangements, and whose difficulties stimulated teachers to develop new practices. At the same time, by studying these children's difficulties closely, teachers typically became convinced that the practices prescribed by the standards agenda were inadequate, and that more creative and ambitious approaches needed to be developed. In this way, working in the context of the standards agenda also enabled schools to escape the limitations of that agenda. (Dyson, 2010, S. 9)

Kronig (2007) hat als Heil- und Sonderpädagoge einen umfassenden empirischen Beitrag zur „systematische[n] Zufälligkeit des Bildungserfolgs“ geliefert und stellt damit ein inhaltliches Bindeglied zu den obigen (bildungs-) soziologischen Ausführungen dar. Er beschreibt ausführlich, dass das Leistungsprinzip als maßgebliches und grundlegendes Prinzip des (vertikal gegliederten) Schulsystems von einer Vergleichbarkeit schulischer Leistungen ausgeht, die tatsächlich allerdings nicht gegeben sei. Stattdessen seien Bildungserfolge (bspw. von Schülern mit 
Lernbehinderungen) maßgeblich durch standortabhängige Faktoren sowie durch bildungspolitische Entscheidungsprozesse beeinflusst, so dass das meritokratische Prinzip keine Gültigkeit beanspruchen könne (Kronig, 2007, S. 17f). Er referiert in diesem Zusammenhang eine Fülle empirischer Ergebnisse, die bspw. belegen, dass Leistungsüberschneidungen sowohl bei Schülern in Sonderschulen und Schülern in Regelklassen bestehen (mit Bezug auf Kronig, 2003; Orthmann, 1993, S. 59ff; Willand, 1983, S. 24ff; Klauer, 1964; Schmalohr, 1962) als auch zwischen anderen Schülern unterschiedlicher Regelschulformen (mit Bezug auf Brühwiler \& Biedermann, 2002; Moser \& Rhyn, 2000; Haefeli \& Schräder-Naef, 1979) (Kronig, 2007, S. 26f). In anspruchsarmen Schultypen liege allerdings ein komplexes Benachteiligungsmuster vor: eine Entwertung des Bildungsabschlusses, soziale Entmischung, anregungsärmeres Umfeld. Diese Form der Benachteiligung beziehe sich nicht speziell auf Schüler mit Lernbehinderungen, sondern auf alle Schüler, die bspw. Hauptschulen besuchen, darunter vor allem auf Kinder und Jugendliche mit Migrationshintergrund (Kronig, 2007, S. 53f).

Bezüge zur Soziologie werden in der Sonderpädagogik ansonsten vor allem von Autorinnen und Autoren hergestellt, die sich mit Inklusion im Sinne von gesellschaftlicher Teilhabe auseinandersetzen und sich in diesem Zusammenhang nicht nur auf schulische Integration bzw. Inklusion beziehen (Wansing, 2005; Stein et al., 2010). Wansing weist bspw. darauf hin, dass mit Behinderung ein hohes Exklusionsrisiko einhergehe (Wansing, 2005, S. 78). Dieses Risiko hänge mit einem möglicherweise erschwerten Zugang zu ökonomischen, sozialen und kulturellen Ressourcen zusammen (Wansing, 2005, S. 76). Teilhabe bedeute personale Inklusion durch die verschiedenen Gesellschaftssysteme sowie Herstellung und Aufrechterhaltung einer individuellen Lebensführung (Wansing, 2005, S. 191). Damit Teilhabe gewährleistet werden könne, müsse dieses Thema „aus den besonderen, exklusiven Kommunikationszusammenhängen des Wohlfahrts- und Rehabilitationssystems deutlich herausgelöst und in den allgemeinen demokratischen Diskurs einer Bürgergesellschaft verankert werden“ (Wansing, 2005, S. 187, Herv. im Orig.), wofür auch eine Analyse ökonomischer Zusammenhänge notwendig sei (Stein et al., 2010, S. 9). Auch Bürli et al. betrachten die Fragestellung nach Integration bzw. Inklusion als Frage nach dem Verhältnis von Individuum und Gesellschaft und halten in diesem Zusammenhang eine wissenschaftliche Fundierung für wesentlich (Bürli et al., 2009). Moser (2017) bezieht sich auf die von Fend (2008) beschriebenen Funktionen von Schule und denkt - ähnlich wie Prengel (2006) - darüber nach, inwiefern die Allokationsfunktion aus der allgemeinbildenden Schule heraus in den berufsbildenden Bereich verlagert werden könne (Moser, 2017, S. 17). Sie sieht außerdem enge Verknüpfungen zwischen den Begriffen Heterogenität und Inklusion, da beide Diskurse auf der einen Seite ein höheres Maß an Bildungsgerechtigkeit fordern und auf der anderen Seite Zuschreibungen aufgrund von Kategorien wie Behinderung oder Geschlecht ablehnen (Moser, 2017, S. 18f).

In vielfacher Hinsicht ist die Sonderpädagogik allerdings um eigene Begriffsbildung bemüht, ohne sich im engeren Sinn auf(bildungs-) soziologische Grundlagen zu berufen oder einer pädagogischen Systematik zu folgen, wie Preuss-Lausitz schon 1981 feststellte:

[...] die pädagogisch-wissenschaftliche Diskussion ist jedoch wenig systematisch: Selten werden Pro oder Contra oder die überwiegenden sowohl-als-auch-Positionen systematisch gesellschaftspolitisch, schultheoretisch, sozialisationstheoretisch oder im engeren Sinne lerntheoretisch entfaltet. [...] Aufgrund dieser theoretisch unbefriedigenden Situation der Integrationsdiskussion sind folgerichtig auch vielfältige, oft unverbunden aneinandergefügte Forderungen oder Bedingungen für die Veränderung der Regelschule formuliert. (Preuss-Lausitz, 1981, S. 75) 
Kobi differenziert beispielsweise zwischen „bedingter“ und „unbedingter“ Integration, wobei er mit „bedingt“ meint, dass Integration nur unter bestimmten Bedingungen umgesetzt werden kann, während die unbedingte Integration davon ausgeht, dass Individuen ein bedingungsloses Recht auf Integration haben (Kobi, 1997, S. 77). Lee weist darauf hin, dass einige Sonderpädagogen das Verhältnis von Inklusion und Exklusion nicht - wie aus soziologischer Sicht üblich - synchronisch, sondern stattdessen diachronisch fassen (Lee, 2010, S. 25). Ein bekanntes entsprechendes Stufenmodell zu den Begriffen „Exklusion, Separation, Integration und Inklusion" hat Sander (2003) in Anlehnung an Bürli (1997) entwickelt. Er ordnet diese Begriffe „historischen Phasen“ zu, die einen Wandel von pädagogischen Leitkonzepten beinhalten (Sander, 2003, S. 122f). Wocken fasst die von Sander beschriebenen Stufen als „Qualitätsstufen der Behindertenpolitik und Pädagogik“ (Wocken, 2012, S. 75) auf, während Moser von vier Paradigmen spricht, die bestimmten Phasen zugeordnet werden können, auch wenn diese nicht strikt chronologisierbar seien. Sie differenziert zwischen „Perfektibilität, Verbesonderung, Förderung und Teilhabe/Inklusion“ (Moser, 2009). Nach Lindmeier (2012) geht dieses Modell wiederum auf den Allgemeinpädagogen Tenorth (2006) zurück. Perfektibilität meint in diesem Zusammenhang „die Entdeckung der Bildsamkeit der Behinderten und ihre Einbeziehung in Bildung und Schule durch Sonderpädagogen (Hänsel \& Miller, 2014, S. 95).

Auch Speck (2010) ordnet Begrifflichkeiten unterschiedlichen zeitlichen Phasen zu, indem er davon ausgeht, dass lange Zeit die individuelle Förderung im Sinne personaler Integration von Menschen mit Behinderung im Vordergrund stand, die in spezifischen Einrichtungen erfolgte, während die Zielsetzung sozialer Integration und damit auch der Abbau der Separation in Institutionen erst ab den 70er Jahren erfolgte (Speck, 2010, S. 9). Mühl (1987) und Speck (2010) beschreiben darüber hinaus mehrere Dimensionen des Integrationsbegriffs wie bspw. die physische, funktionale, personale und gesellschaftliche Integration (Mühl, 1987, S. 73), die normative Dimension (Ziel und Weg für die Erziehung und Bildung von Kindern mit Behinderung; Ableitung aus allgemeinen Menschenrechten) oder die pädagogische Dimension (Integration als konkreter Prozess gemeinsamen Lebens und Lernens) (Speck, 2010, S. 19f).

Was die Abgrenzung der Begriffe „Integration“ und „Inklusion“ betrifft, gehen die Auffassungen innerhalb der Sonderpädagogik weit auseinander (Hinz, 2002, 2004 u. 2009; Reiser, 2003; Sander, 2003; Holzinger \& Wohlhart, 2009; Stein et al., 2010; Speck-Hamdan, 2015; Moser, 2017). So vertritt Stein (2008) die Auffassung, dass es keine „theoretisch begründete Notwendigkeit der Ablösung des Integrationsbegriffs durch den der Inklusion [gibt] " (Stein, 2008, S. 81), da der Begriff der Integration im deutschen Verständnis nicht auf Menschen mit Behinderung begrenzt war, sondern weitere Dimensionen von Heterogenität berücksichtigt habe. Auf diese „Weite“ des Inklusionsbegriffs im Vergleich zum Integrationsbegriff beziehen sich aber u.a. Hinz, der in diesem Zusammenhang neben der Kategorie Behinderung auch die Kategorien Lebenslage, Geschlecht oder Migrationshintergrund benennt (Hinz, 2009, S. 172), oder Moser, die ebenfalls auch die „nicht alleinige Inblicknahme ausschließlich behinderter Menschen“ (Moser, 2017, S. 15) verweist. Nach Hinz werde die sogenannte „Zwei-GruppenTheorie“ durch Inklusion abgelöst, da diese ,jegliche gruppenbezogene Kategorisierung [...] als diskriminierend ablehnt [und] [...] anders als bei soziologischer Betrachtung - die Gruppe der „Menschen mit Behinderung “ keine eigene Kategorie mehr bilden [kann].“ (Hinz, 2010, S. 5) Die begriffliche Diskussion an sich wird in diesem Zusammenhang zum Teil als wenig Ziel führend eingeschätzt:

In der Begriffsdiskussion um Integration und Inklusion werden beide Begriffe inzwischen häufig als Gegensatzpaar formuliert bzw. wird der Integrationsbegriff als der zu überwindende, der Begriff der 
Inklusion als der weiterführende bezeichnet. Ohne hier näher darauf eingehen zu können ist es unsere Auffassung, dass diese Debatte in dieser Gegenüberstellung wenig dazu beiträgt, die eigentliche Dimension des Problems der uneingeschränkten Teilhabe von Menschen mit unterschiedlichsten Beeinträchtigungen und Benachteiligungen neu zu fassen: die Dimension der Notwendigkeit, diese Forderung als politische Forderung zu begreifen. Entsprechende pädagogisch-didaktische Konzepte, sowie die Gemeinwesenorientierung sind notwendige Transformationsprozesse, im Kern geht es jedoch um gesellschaftspolitische Fragen, die entsprechendes politisches Handeln verlangen. (Stein et al., 2010, S. 10)

Dabei gehen Stein et al. bei ihrer Begriffsbeschreibung über den schulischen Rahmen hinaus und beschreiben sozialraumbezogene Strategien (Stein et al., 2010). In diesem Kontext nutzen sie vor allem den Begriff der „Teilhabe“ als Leitprinzip. Auch Moser und Egger (2017) vertreten die These,

[...] dass alle Merkmale einer inklusiven Schule bereits in den Anfängen der Theoriebildung der Integrationspädagogik vertreten waren (vgl. Prengel, 1993; Hinz, 1993; Feuser, 1993). Eher aus historischen Gründen verliert der Begriff, Integration' an definitorischer Kraft, weil er zunehmend in den ausgehenden 1990er-Jahren für die Thematik ,Migration' Anwendung findet - der in der Folge einsetzende wissenschaftliche und bildungspolitische Disput um Integration vs. Inklusion scheint hingegen eher strategisch motiviert. (Moser \& Egger, 2017, S. 12)

Als Fachbegriff spielt „Inklusion“ in der deutschen Diskussion erst seit der Salamanca-Erklärung - also ab etwa Mitte der 90er Jahre - eine Rolle (Sander, 2003, S. 122; Speck-Hamdan, 2015, S. 14). Speck-Hamdan weist diesbezüglich auf den programmatischen bzw. normativen Gehalt des Begriffs in der sonderpädagogischen Diskussion hin:

Inklusion gehe von der Tatsache der Verschiedenheit in einem Ganzen aus, während Integration auf der Unterscheidung einer Gruppe hinsichtlich eines Merkmals beruhe, einer Minderheitengruppe, die in die Mehrheitsgruppe zu integrieren sei. Integration kategorisiere, während Inklusion auf Kategorien verzichten könne, weil eben jedes Kind anders sei. Damit verbunden wird stets die Vorstellung, es gäbe sozusagen ein „Richtig“ und ein „Falsch“. Integration sei falsch, Inklusion sei richtig. Hier nun wird der Begriff Inklusion zu einem programmatischen. Er verbindet sich mit einer festen Zielvorstellung, die eine äußerst starke normative Kraft in der Diskussion entwickelt hat. (Speck-Hamdan, 2015, S. 14)

Dieser programmatische Gehalt findet sich bspw. bei Holzinger und Wohlhart (2009): „Integration löst sich in Inklusion auf, wenn alle Kinder integriert sind.“ (S. 16) oder Feyerer (2009):

Im Konzept der Inklusion ist nicht mehr die Integration der Minorität in die Majorität das Ziel, sondern eine Schule für alle. [...] Dabei geht es nicht darum, wo das betroffene Kind am besten platziert werden kann, sondern darum, wie das jeweilige Lernsystem an die Erfordernisse aller angepasst werden kann. [...] Grundlage für den Unterricht ist ein gemeinsames Curriculum für alle. Differenzen werden als produktiv wahrgenommen und im Unterricht fruchtbar gemacht. (Feyerer, 2009, S. 241f)

Auch Frühauf (2008) vertritt eine ähnliche Ansicht:

Aus dem Blick eines inklusiven Ansatzes ist das heute vorherrschende schulische Integrationsmodell immer noch eine spezielle Maßnahme, da zumeist eine wohnortsübergreifende Bündelung von Kindern mit sonderpädagogischem Förderbedarf sowie eine Zentrierung sonderpädagogischer, insbesondere personeller Ressourcen die wesentliche Grundlage dieser Praxis ist. In einem inklusiven Ansatz würde dieses Modell an Bedeutung verlieren, da die Klassifikation bestimmter Schülergruppen und ihre Zusammenführung an zentralen Orten grundsätzlich unterbleiben würden. (Frühauf, 2008, S. 21) 
Kullmann et al. definieren Inklusion als ,gleichrangige gesellschaftliche Partizipation aller Menschen einschließlich derjenigen mit Behinderungen unter Gewährung dafür notwendiger Hilfen" (Kullmann et al., 2014, S. 90) und nehmen dabei Bezug auf Inklusion als Menschenrecht. Inklusive Bildung unter dem Aspekt des rechtlichen Anspruchs wird auch bei Münch (2010, S. 90f) oder Wocken (2010, S. 219) aufgegriffen (n. Textor, 2015, S. 27).

Feuser vertritt zwar eine programmatische Deutung, benutzt die Begriffe Integration und Inklusion dabei allerdings synonym:

Integration kennzeichnet heute eine breite internationale Bewegung der Durchsetzung des Menschenrechts auf gleichwertige und gleichberechtigte Teilhabe und Teilnahme an einer gemeinsamen, nicht ausgrenzenden und nicht separierenden Kultur. Inklusion kennzeichnet eine entsprechende, am Gemeinsinn orientierte Lebenskultur. (Feuser, 2010, S. 17)

Speck-Hamdan weist darauf hin, dass die Begriffe Integration und Inklusion in den Veröffentlichungen der UN und der UNESCO bedeutungsgleich verwendet werden würden und die ideologischen Bedeutungszumessungen in der internationalen Diskussion weniger bedeutsam seien als in Deutschland. Der Begriff der Inklusion habe sich allerdings ihrer Auffassung nach inzwischen durchgesetzt (Speck-Hamdan, 2015, S. 13). Textor (2015) unterscheidet die Begriffe Integration und Inklusion dahingehend, dass der Integrationsbegriff auf die zu integrierenden Schüler orientiert sei, während der Inklusionsbegriff die Perspektive auf das System richte (Zwei-vs.-Ein-Gruppen-Theorie). Moser beschreibt vier Dimensionen von Inklusion auf der Ebene pädagogischer Prozesse sowie der Systemebene. Die Dimension der Anerkennung sei als sozialphilosophische Kategorie von Prengel (1993) als pädagogische Herausforderung benannt und durch eine entsprechende Gesetzgebung um die sozialrechtliche Dimension der Teilhabe ergänzt worden. Antidiskriminierung als weitere Dimension von Inklusion sei als Gebot aus der UN-BRK juristisch relevant, werde in der Umsetzung in der BRD allerdings durch die Aufrechterhaltung eines Parallelsystems von inklusiver Bildung und Förderschulen nicht ausreichend berücksichtigt. Die Dimension der Bildungsgerechtigkeit spiele mit Blick auf mögliche Systemveränderungen eine entscheidende Rolle. Durch die Berücksichtigung dieser vier Dimensionen ergebe sich ein umfassendes Inklusionsverständnis (Moser, 2017, S. 20-26). Bei diesem Verständnis zeigen sich Verknüpfungen zum Lebenslagen-Ansatz von Engels (2006), wenn Moser formuliert:

Bei Inklusion, die auf Teilhabe und Partizipation zielt, handelt es sich [...], zudem um einen opportunity basierten Zugang, der outcome orientierte Perspektiven, wie sie die bildungspolitische Diskussion hierzulande derzeit dominieren, ergänzt. Opportunity basiert bedeutet hier, Möglichkeiten bereitzustellen, die die Wählbarkeit von Lebenschancen in Aussicht stellen. Eine Gesellschaft, so argumentiert der Träger des Nobelpreises für Ökonomie, Amartya Sen, ist nicht daran zu bemessen, welche outcomes sie produziert, sondern an der Anzahl real wählbarerer Möglichkeiten, die den einzelnen Individuen zur Verfügung stehen, um ihr Leben zu gestalten (Sen 2009). Und Bildung ist hierzu, wie beschrieben, eine Schlüsselstelle. (Moser, 2012, S. 16)

Auch in der internationalen sonderpädagogischen Literatur wird ein relativ breites Begriffsverständnis von Inklusion vertreten (Dyson, 2010; Ainscow et al., 2006), das dadurch allerdings zum Teil auch wenig greifbar erscheint:

However, it is also the case that inclusion is a notoriously ,slippery" concept, with its roots in many different streams of thought (Clough, 2000; Dyson, 1999), and deeply embedded in the structures, histories and cultures of different education systems (Artiles \& Dyson, 2005). (Dyson, 2010, S. 2) 
Daraus schlussfolgert Dyson, dass es entsprechend keine einzelne und übereinstimmungsfähige Definition von Inklusion oder inklusiven Schulen gebe (Dyson, 2010, S. 2). Ainscow u.a. sehen die Unbestimmtheit des Konzepts auch darin begründet, dass es werte- und praxisbezogen orientiert sei und daher immer wieder neu diskutiert werden müsse (Ainscow et al., 2006, S. 3). Es stelle einen Prozess dar, der sich auf eine Verbesserung der Partizipation von Schülern und eine Verringerung der Exklusion von Curricula, Kulturen und Gemeinschaften in Schulen beziehe, auf die Veränderung der Schulkultur und -politik sowie auf alle Schülerinnen und Schüler, die von Exklusion bedroht seien (Ainscow et al., 2006, S. 25). Sie beschreiben einen engen Zusammenhang von Inklusion und Exklusion:

[...] inclusion and exclusion are linked together such that inclusion involves the active combating of exclusion; and inclusion is seen as a never-ending process. Thus an inclusive school is one that is on the move, rather than one that has reached a perfect state. (Ainscow et al., 2006, S. 25)

Mit Blick auf den deutschen Diskurs hinsichtlich der Umsetzung der „einen Schule für alle“ werden die o.g. normativen Setzungen wiederum sehr kritisch bewertet. So bezeichnet Anken den Diskurs als „quasi-axiomatische Setzung“ (Anken, 2012, S. 34) oder "teilweise normativ überhöht bis moralisierend“ (Anken, 2012, S. 35), Kobi (2008) spricht von „ekklesialen Alleinseligmachungs-Ansprüchen“ und Ahrbeck (2011) vom „moralischen Impetus“. Auch Klemenz und Paschen (2012) ordnen den Inklusionsbegriff unter die „pädagogischen Dogmen“ ein (n. Speck-Hamdan, 2015, S. 15), die u.a. Alleinstelligkeit, Alternativlosigkeit und kontingente Voraussetzungen postulieren, ohne diesen Ansprüchen gerecht werden zu können (Klemenz \& Paschen, 2012, S. 482). Nach Speck-Hamdan erschwere diese Form der ideologischen Zuspitzung die fachliche Diskussion (Speck-Hamdan, 2015, S. 15) und Schäper befürchtet, dass die Ideologie die „Praxis des Nicht-Gelingens von Inklusion“ (Schäper, 2010, S. 33) verschleiern könne. Auch Speck sieht mögliche Gefahren einer ideologisierten Inklusionsdiskussion, bspw. die Gefahr des „Wegkürzens“ von Einschränkungen oder Behinderungen, die Gefahr einer erweiterten Stigmatisierung von Kindern und Jugendlichen in sonderpädagogischen Schulen oder Einrichtungen sowie die Gefahr einer fehlenden Praktikabilität der Umsetzung:

Das fachliche Interesse an einer Klärung bezieht sich im Besonderen darauf, dass bei einer Radikallösung unverzichtbare heilpädagogische Erfordernisse vernachlässigt werden und sich für einen Teil der Kinder mit Behinderungen nicht verantwortbare Nachteile ergeben könnten, was freilich auf der anderen Seite bestritten wird. Da damit reale Erfahrungen gegen Zukunftsvisionen stehen, liegt der Verdacht auf ideologische Verzerrungen nahe. Sie lassen sich in doppelter und gegenläufiger Hinsicht ausmachen: In dem einen Fall wird durch die Absolutsetzung eines Ideals Realität verkürzt und im anderen wird durch eine Überbetonung heilpädagogischer Erfordernisse eine progressive Entwicklung konterkariert. [...] Wenn akzeptable Lösungen gefunden werden sollen, kann eine solche ideologische Divergenz und Frontstellung der Sache nicht dienlich sein. Es muss eine rationale Klärung gewagt werden, ohne das Ideal aufzugeben. (Speck, 2011, S. 84)

Den Diskurs insgesamt beschreibt Speck folgendermaßen:

Es ist ein ausgesprochen heißes Thema, d.h., es ist hoch aufgeladen mit z.T. gegensätzlichen Emotionen. Die einen verbinden mit ihm Hoffnung und die Erfüllung eines Traumes, nämlich das Ende der schulischen Separation und der vielfältigen Barrieren in der Lebenswelt von Menschen mit Behinderungen; die anderen trauen den Verheißungen nicht recht und sehen unberechenbare Risiken für die Zukunft. Als Wissenschaftler, der um Objektivität und Realitätsanalyse bemüht sein muss, bewegt man sich bei diesem Thema quasi auf einem ideologischen Minenfeld. (Speck, 2010, S. 7, Herv. im Orig.) 
Im Rahmen dieser ideologisierenden Diskussion werden auch wenig wissenschaftlich orientierte und aus sprachlicher Hinsicht normativ aufgeladene Beiträge veröffentlicht, die bspw. „Inklusive Missverständnisse“ (Speck, 2014) auf der einen bzw. einen „Einspruch gegen Falschmeldungen über Inklusion“ oder das „Scheitern der Pseudo-Inklusion“ auf der anderen Seite thematisieren (Wocken, 2014 u. 2018) und die sonderpädagogische Diskussion dadurch in die Öffentlichkeit transportieren.

Jennessen \& Wagner (2012) wählen in ihren Ausführungen dagegen einen sachlichen Zugang zur Thematik, indem sie zunächst begriffliche und inhaltliche Unschärfen benennen. Die Debatte um Inklusion sei u.a. deshalb problematisch, da diese „über keine einheitliche und konsensuale Sprache verfügt" (Jennessen \& Wagner, 2012, S. 336). Aus pragmatischer Sicht bezeichne Inklusion „soziale Zugehörigkeit und das Recht auf Teilhabe in allen Lebensbereichen“ (Jennessen \& Wagner, 2012, S. 336) sowie eine Fülle unterschiedlicher Heterogenitätsdimensionen. Anstelle von „Absolutheitsfor-derungen“ vertreten sie u.a. die These, dass „Inklusion gelingt, wenn die Autonomie der situativen Entscheidung für oder gegen Teilhabe als Bestandteil von Inklusion begriffen wird.“ (Jennessen \& Wagner, 2012, S. 338). Darüber hinaus sei eine Einbeziehung aller Akteure in schulische Entwicklungsprozesse Richtung weisend sowie die Berücksichtigung außerschulischer Ebenen wie Freizeit oder anderer Lebensbereiche (Jennessen \& Wagner, 2012, S. 338f).

Im Rahmen der Inklusionsdiskussion nehmen zum Teil auch Schulen selbst eine Positionierung vor. In diesem Zusammenhang „verteidigt“ die private Montessori-Schule Aktion Sonnenschein ihre Haltung zur Unterrichtung von schwer bzw. mehrfach behinderten Schülern in so genannten Spezialklassen:

Seit Verabschiedung der UN-Konvention zur INKLUSION diskutieren auch wir immer wieder diesen Begriff und sind natürlich der Meinung, dass inklusive Schule - also eine Schule für ALLE Kinder das Natürlichste sein sollte. Durch unsere langjährige Erfahrung ist uns aber bewusst, dass man nicht einfach jedes noch so schwer behinderte Kind ohne zusätzliche Hilfe und Unterstützung in eine Schule aufnehmen kann. Wir plädieren bei unseren Schulaufnahmeverfahren in die Grund- und Hauptschulstufe sehr dafür, dass die Vielfalt der Schülerschaft optimal ist, gleichzeitig aber jeden Tag auch pädagogisch verantwortungsvoll leistbar sein muss. Das heißt, dass wir sehr wohl Grenzen in dieser Vielfalt setzen müssen. Aufgrund unserer Ressourcen können wir derzeit keine blinden, gehörlosen oder körperlich schwerstbehinderten Kinder aufnehmen. Vielleicht können wir dies aber in einigen Jahren, wenn sich Schul- und Sozialpolitik den „neuen“ gesellschaftlichen Forderungen bezüglich der Inklusion geöffnet haben und/oder wir in einen entsprechenden neuen Schulversuch eingebunden wären. (Montessori-Schule Aktion Sonnenschein, o.J., o.S.)

Gleichzeitig wird die Problematik der möglichen Verstärkung der „Besonderung“ der Menschen mit Behinderung durch Inklusionsbemühungen thematisiert:

Der Versuch, die bis dahin Exkludierten nun auch zu inkludieren, trifft auf das Problem, dass Behinderung in der Umwelt von Sozialsystemen in diesen Systemen nichtignorable Belastungen hervorruft, die zur Ausdifferenzierung einer Expertenkultur zwingt, die die gleichsam naturläufige Exklusionsdrift stoppen soll. (Fuchs, 2002, zitiert n. Lee, 2010, S. 27)

Menschen mit Behinderungen nehmen zum Teil sehr kritisch Stellung zur Inklusionsdiskussion. Exner geht bspw. davon aus, dass die Integrationsdiskussion Behinderung als soziale Kategorie festige (Exner, 2007, S. 86). Er äußert sich dahingehend, dass der Integrationsbegriff seit Jahrzehnten durch Menschen ohne Behinderung geprägt werde und bezieht sich in diesem Zusammenhang auf Daoud-Harms, die ausführt, dass 
[...] bisher kein von ,Nichtbehinderten' gestaltetes Projekt integrativer Erziehung in der Bundesrepublik bekannt geworden ist, an dem ,Behinderte' in der Planung, Ausarbeitung, Durchführung und Auswertung in irgendeiner Form als Mitarbeiter und nicht als Projektgegenstand beteiligt worden wären. (Daoud-Harms, 1997, S. 431).

Ebenso werde die Diskussion zur Beschulung behinderter Kinder und Jugendlicher nach Deppe-Wolfinger (1990, S. 323) aus der Blickrichtung nichtbehinderter Menschen geführt. Die Perspektive behinderter Menschen werde nicht genutzt um bspw. deren Erfahrungen mit integrativer oder nicht-integrativer Beschulung zu untersuchen (Exner, 2007, S. 92f). Es sei in Frage zu stellen, inwiefern die Prämisse „mit ,Nichtbehinderten' ist grundsätzlich besser als ohne“ (Exner, 2007, S. 92) Gültigkeit beanspruchen könne, da diese die „Integrationskompetenz“ (Exner, 2007, S. 94) von Menschen mit Behinderungen indirekt in Frage stelle.

Darüber hinaus wird behinderten Menschen im Rahmen der vielschichtigen Integrationsauseinandersetzung in Theorie und Praxis - wenn auch ungewollt - vermittelt, daß sie als Person das eigentliche Problem sind. So sind sie z.B. in der theoretischen Auseinandersetzung zwischen Sonder- und Integrationspädagogik das zentrale Streitobjekt, um dessen schulische Versorgung es geht. (Exner, 2007, S. 95)

Auch Ainscow problematisiert diesen Aspekt und weist darauf hin, dass neben den Werten Gleichheit, Partizipation, Respekt vor Diversität etc. der Wert der Freiheit und der echter Partizipation auch darin bestehen könne zu entscheiden nicht zu partizipieren (Ainscow et al., 2006, S. 24).

\subsubsection{Zusammenfassung der unterschiedlichen Perspektiven auf Begriffe und Diskurs}

Die Theorie funktionaler Differenzierung nach Luhmann geht davon aus, dass die Gesellschaft sich immer stärker in Teilsysteme ausdifferenziert. Inklusion und Exklusion werden in diesem Zusammenhang als kommunikative Prozesse verstanden, innerhalb derer Personen unterschiedlich „adressiert“ werden. Da diese Prozesse in verschiedenen Teilsystemen ablaufen, ist es möglich, dass Individuen in bestimmten Teilsystemen zugehörig und in anderen nicht-zugehörig sein können. Inklusion sei daher kein Passungsprozess zwischen Individuum und Gesellschaft allgemein, sondern umfasse die Teilhabe bzw. Nicht-Teilhabe von Individuen in differenzierten Funktionsbereichen und könne deshalb als „Multiinklusion bzw. -exklusion“ aufgefasst werden. Die Sozialstrukturanalyse als weiterer soziologischer Theoriestrang hält den Ansatz rein funktionaler Differenzierung nicht für ausreichend, um Inklusions- bzw. Exklusionsprozesse zu erklären. Sie versteht die Teilsysteme der Gesellschaft nicht als prinzipiell offen, sondern nimmt an, dass es Exklusionsursachen und -risiken wie Migration oder ethnische Segregation gibt, die als Dimension sozialer Ungleichheit bspw. im Sinne „der sozial-materiellen Qualität möglicher Teilhabe“ (Kronauer, 2010, S. 44) gesellschaftlich relevant sind. Versuche zur Zusammenführung dieser zwei soziologischen Theoriestränge fragen nach der Funktion sozialer Ungleichheit oder verbinden Systemtheorie und Sozialstrukturanalyse bspw. in Form des „Lebenslagen-Konzepts“.

Die bildungssoziologische Perspektive befasst sich u.a. mit den gesellschaftlichen Funktionen von Schule (Qualifikations-/ Selektions-/ Allokations-/ Legitimations-/ Integrationsfunktion). Eine besondere Rolle mit Blick auf Exklusion bzw. Inklusion spielen dabei Unterscheidungsmechanismen wie die Ressourcenausstattung von Haushalten und das kulturelle Kapital im Elternhaus (primäre und sekundäre Herkunftseffekte), die sich sowohl auf die Lernvoraussetzungen und die schulischen Leistungen von Kindern auswirken als auch die Bildungsentscheidungen von Eltern beeinflussen. Die Bildungssoziologie geht davon aus, dass (Bildungs-) Ungleichhei- 
ten u.a. durch Faktoren wie soziale Schicht, Migrationshintergrund oder Geschlecht determiniert werden. Für die Schule bedeutet dies, dass ein ungleiches soziales und kulturelles Startkapital von Kindern fortwirkt. Insbesondere die Ergebnisse der Bildungsstudie PISA sowie die Armuts- und Reichtumsberichte der Bundesregierung belegen entsprechend, dass in Deutschland ein ausgeprägter Zusammenhang zwischen sozialer Herkunft und erreichten Bildungszielen besteht. Dabei entscheidet nach Emmerich und Hormel nicht die Schulform über inklusive oder exklusive Prozesse. Das sei darin begründet, dass Leistungsdifferenzen nicht nur akzeptiert werden würden, sondern aufgrund der o.g. Funktionen von Schule funktional-institutionell notwendig seien. Soziale Differenz wiederum werde in Schulen u.a. durch Zuschreibungsprozesse von Lehrkräften hergestellt und sei demnach eine systemeigene Beobachtungsleistung. Interne (leistungsorientierte) Selektionsentscheidungen umfassen inkludierende und exkludierende Operationen, die internen Kriterien unterliegen.

Problematisch hinsichtlich der Vergleichbarkeit der Sichtweise der Pädagogik auf die soziologischen Kategorien Inklusion und Exklusion ist zunächst, dass diese Wissenschaft mit anderen Fachbegriffen bzw. Kategorien wie bspw. Heterogenität, Differenz, Einheit oder Gleichheit arbeitet, so dass eine direkte Gegenüberstellung mit den soziologischen Ausführungen schwierig ist. Benner systematisiert die Pädagogik insgesamt dahingehend, dass sie von verschiedenen Grundpositionen ausgeht. Während seiner Auffassung nach intentionale Modelle (Bsp.: Überwindung sozialisatorischer Benachteiligung) oder funktionale Modelle (Bsp.: kompensatorische Erziehung zur Bildung von Arbeitskräften) eine Verkürzung der Pädagogik bedeuten, solle pädagogisches Handeln und Denken die Überführung gesellschaftlicher Festlegung in pädagogische Festlegung prüfen. Muth thematisiert in den 70er Jahren die Funktion des Schulwesens dahingehend, dass dieses Selektionsprozesse steuere, innerhalb derer es ein ,Oben' und ein ,Unten' gebe und Fend spricht davon, dass das Schulsystem eine Reproduktionsfunktion für Kultur, Sozialstruktur und gesellschaftliche Herrschaftssystem übernehme.

Tenorth thematisiert die Frage nach der Einheit bzw. Differenz von Bildungsprozessen. Einerseits versuche Bildung Gleichheit herzustellen, andererseits erzeuge sie Verschiedenheit, da sie bereits bestehende Differenzen zwischen Schülerinnen und Schülern, die diese aufgrund ihrer Sozialisation mitbringen, lediglich überformen und durch Qualifikation verstärken könne. Kritisch hinterfragt er daraufhin schulsystemische Bedingungen und empfiehlt eine gemeinsame Beschulung bis zum Ende der Sekundarstufe I, auch wenn die Realisierung von Lernangeboten bspw. für hochbegabte oder kognitiv beeinträchtigte Schüler in diesem Rahmen schwierig sein könne. Wie Prengel geht er davon aus, dass das Problem durch Differenzierung pädagogisch bearbeitet werden könne. Prengel schlüsselt Gleichheit und Differenz zunächst philosophisch auf, indem sie die Begriffe "Gleichheit“ und „Verschiedenheit“ als in einem spezifischen Verhältnis zueinander stehende Kategorien analysiert. Im Sinne der Verwirklichung des demokratischen Prinzips der Gleichberechtigung bedeutet Integration im schulischen Bereich für sie "gemeinsamer Unterricht" oder „eine Schule für alle“. Eine möglicherweise spezifische Beschulung hält sie in Ausnahmefällen für gerechtfertigt (Bsp.: gehörlose oder verhaltensauffällige Schüler). Trotz der offen-philosophischen Herleitung überwiegen bei Prengel mit Blick auf die Vorschläge der pädagogischen Bearbeitung von Gleichheit und Verschiedenheit also normative Setzungen. Während Tenorth mögliche gesellschaftliche Probleme im Anschluss an die von ihm empfohlene gemeinsame Sekundarstufe I beschreibt, wird Schule bei Prengel idealistisch aus einer gesellschaftlichen Struktur herausgegriffen und soll als „Idealort“ fungieren, ohne dass Spannungen im Anschluss (Bsp.: fehlende Gerechtigkeit auf beruflicher Ebene, Notwendigkeit von Abschlüssen für nachschulische Bildungsangebote etc.) von ihr einbezogen bzw. aufgegriffen werden. 
Kritisch auf Individualisierung als Ansatz zum Umgang mit Heterogenität schauen Trautmann und Wischer, die einen grundlegenden Widerspruch zwischen der institutionellen Struktur von Schule und der pädagogischen Idee individueller Förderung sehen. Eine stärkere Differenzierung von Lernangeboten in der Schule habe ein noch höheres Maß an Unterschiedlichkeit im Leistungsniveau zur Folge, so dass Ungleichheit durch diesen Ansatz verstärkt werde. Wenning erläutert, dass Heterogenität etwas sei, dass Lehrkräfte situations- und zustandsbezogen zumessen. Er kritisiert, dass gesellschaftliche und politische Verantwortung an Pädagogen delegiert werde, indem erwartet werde, dass diese das Problem innerschulisch durch didaktische Veränderungen aufzuschlüsseln hätten.

Die sonderpädagogische Begriffsdiskussion lässt sich nur schwer mit der soziologischen bzw. bildungssoziologischen Begriffswelt verknüpfen. Häufig werden Auffassungen vertreten, die sich inhaltlich widersprechen. So geht bspw. Romey (1977) davon aus, dass soziale Integration nur in einem einheitlichen Schulsystem möglich sei, während Bleidick (1990) darauf hinweist, dass die Gesamtschule soziale Benachteiligungen nicht abgebaut habe. Problematisiert wird in diesem Zusammenhang die Leistungsorientierung, -differenzierung und -bewertung in Schulen, die nicht geklärt sei, wobei Kronig (2007) durch seine Untersuchungen belegt, dass eine Vergleichbarkeit von Leistungen im Schulsystem insgesamt nicht gegeben sei. Die Benachteiligung von Schülern und Schülerinnen erfolge vielmehr aufgrund von Schulstandorten sowie Bildungspolitik.

Innerhalb der Sonderpädagogik werden vor allem Versuche unternommen die Begriffe ,Integration' und ,Inklusion' inhaltlich und konzeptionell voneinander abzugrenzen. Dazu wird nach Lee (2010) häufig eine diachrone anstelle der in der Soziologie üblichen synchronen Sichtweise auf Inklusion eingenommen (Sander, 2003; Moser, 2009). Darüber hinaus richtet sich der Diskurs vorwiegend auf mögliche Vor- bzw. Nachteile inklusiver vs. besonderer Beschulung von Kindern und Jugendlichen. Es findet also eine starke Verengung, Verkürzung und normative Vereinfachung der begrifflichen Dimensionen von Inklusion bspw. auf die sogenannte ,Schule für alle' statt. Exklusion wird von der Sonderpädagogik als Kategorie selten definiert oder genutzt. Anders verhält sich dies bei Autoren, die sich nicht nur mit dem schulischen Bereich, sondern bspw. mit dem Themenfeld gesellschaftlicher Teilhabe insgesamt befassen (Wansing, 2005; Ziemen et al., 2011). Diese stellen auch Bezüge zwischen Inklusion und Gesellschaft her (Wansing, 2005; Bürli, 2009; Stein, 2010). Dyson (2010) und Ainscow et al. (2006) weisen darauf hin, dass das Begriffsverständnis der „Inklusion“ nicht scharf, sondern eher „slippery“ sei. Auch sie verstehen unter Inklusion einen Prozess (diachron), der international unter anderem abhängig von sehr unterschiedlichen Schulsystemen sei.

Kronauer (2013) versucht in diesem Zusammenhang die soziologische Diskussion zu Exklusion und Inklusion sowie die (sonder-) pädagogische Diskussion zu Inklusion und Diskriminierung aufeinander zu beziehen. Während Inklusion im behindertenpädagogischen Verständnis vor allem die „Öffnung gesellschaftlicher Organisationen und Infrastruktur zur gleichberechtigten Teilhabe aller an deren Leistungen" (Kronauer, 2013, S. 18, Herv. im Orig.) meine und als Gegenbegriff der Inklusion den der Diskriminierung verwende, gehe es bei der soziologisch orientierten gesellschaftspolitischen Debatte um Inklusion in erster Linie um die "Qualität der durch sie ermöglichten gesellschaftlichen Teilhabe“ (Kronauer, 2013, S. 18, Herv. im Orig.). Die soziologische Auseinandersetzung gehe über die (behinderten-) pädagogische hinaus, da sie sich auch mit den „Regeln der zentralen Institutionen“ (Kronauer, 2013, S. 24) befasse, die zum Teil soziale Ausgrenzung beinhalten (Bsp.: Schulsystem), so dass es nicht ausreiche eine „Inklusion in sozial ausgrenzende Verhältnisse“ (Kronauer, 2013, S. 24) anzustreben, sondern es um die Überwindung dieser Verhältnisse in einem breiten gesellschaftspolitischen Rahmen gehe. 
Die soziologische Sichtweise auf Inklusion und Exklusion fokussiert Inklusion und Exklusion also als relationale, mehrdimensionale und prozesshaft angelegte Konstrukte und vertritt demnach eine synchrone Auffassung dieses Gegensatzpaars, während die Sonderpädagogik ein vorwiegend diachrones Verständnis (Bsp.: Separation und Integration etc. als geschichtlich fassbare Phasen) vermittelt. Bildungssoziologische Ansätze beschreiben u.a. die mit den unterschiedlichen Funktionen von Schule zusammenhängenden Widersprüche, in denen Schulen sich befinden, und gehen davon aus, dass exklusive und inklusive Prozesse sich auch innerhalb von Schulen ereignen. Pädagogische Ansätze arbeiten mit anderen Begriffen und fordern zum Teil, dass Schulen als pädagogische Institutionen exklusiven Prozessen durch Anerkennung und allgemeine Bildung entgegenwirken sollten.

Die sonderpädagogische Sichtweise auf die Kategorien Inklusion und Exklusion erscheint insgesamt teilweise wenig verknüpft mit anderen geisteswissenschaftlichen Ansätzen. Sie setzt sich nur marginal mit soziologischen Theorien oder pädagogisch-kritischen Ansätzen auseinander, es überwiegen teilweise stark vereinfachende bzw. ideologisierende Darstellungen, die weder für die theoretische Verortung noch für die Analyse der Umsetzung schulischer Integration bzw. Inklusion hilfreich sind. Besonders problematisch zu werten ist die - in der Sonderpädagogik vorherrschende - erhebliche Vereinfachung des Diskurses zu Inklusion und Exklusion auf das Gegensatzpaar Inklusion $=$ Besuch einer gemeinsamen Schule vs. Exklusion $=$ Besuch einer För derschule.

Diese Vereinfachung ist - mit Blick auf die theoretische Fundierung des Begriffsverständnisses in der Soziologie - in mehrfacher Hinsicht unzulässig. Sie fokussiert eine eindimensionale Wahrnehmung und konstruiert ein klar fassbares „Teilsystem Schule“, während die Soziologie von einem nur mehrdimensional greifbaren Verständnis und hochgradig ausdifferenzierten Teilsystemen ausgeht. Sonderpädagogische Diskussionsbeiträge beschreiben „Inklusion in der einen Schule für alle“ zum Teil als Idealzustand, der Unterschiede zwischen Kindern und Jugendlichen schulstrukturell aufhebt, und damit als statisches Modell aufgefasst werden kann, während ein soziologischer Zugang „Inklusion“ als Prozess begreift, der durch eine Vielfalt gradueller Unterschiede gekennzeichnet sei und damit nur als dynamisches Modell verständlich erscheint. Während die sonderpädagogische Diskussion ein vorwiegend diachrones Begriffsverständnis vertritt, bei dem sich eine „Zunahme von Inklusion“ in geschichtlich greifbaren Phasen nachvollziehen lässt, ist das soziologische Begriffsverständnis durch eine synchrone Wahrnehmung geprägt, in der sich gleichzeitig ablaufende Differenzierungsprozesse in unterschiedlichen Teilsystemen immer wieder überlagern bzw. ablösen können.

So stellen bspw. die in der Sonderpädagogik entwickelten Stufenmodelle, die historische Entwicklungsphasen von „der Exklusion“ bis zur „Inklusion“ annehmen, zwar Teilaspekte der Entwicklung integrativer Beschulung dar (vgl. dazu 2.1), bilden die mehrdimensionale, prozesshafte und graduell mögliche Inklusion und Exklusion von Menschen in sehr unterschiedliche und hochgradig funktional ausdifferenzierte Teilsysteme aus soziologischer Sicht allerdings nicht - und im soziologischen Verständnis nicht einmal unzureichend - ab. Sie beziehen sich ausschließlich auf das schulische System und nutzen das o.g. Gegensatzpaar dazu, davon auszugehen, dass Inklusion sich quasi automatisch in einer Regelschule „abspiele“, so dass diese normative Setzung einen offenen, kritischen und gesellschaftspolitischen Diskurs eher behindert als fördert. Die in der Sonderpädagogik häufig favorisierte „Eine Schule für alle“ scheint Inklusion beobachterunabhängig zu garantieren, während (bildungs-) soziologische Ausführungen immer wieder auf die Beobachterabhängigkeit mit Blick auf die Analyse inklusiver bzw. exklusiver 
Prozesse verweisen. Die sonderpädagogische Form der „Inklusionsdiskussion“ setzt damit zum Teil wertende Prämissen, die einem soziologischen Verständnis zuwider laufen:

- Der Aufenthalt unter Menschen ohne Behinderung ist „inklusiver“ und damit „besser“ als der Aufenthalt unter Menschen mit Behinderung.

- Nur bestimmte mit diesem Auftrag bildungspolitisch bzw. von der Schulverwaltung aus versehene Schulen können „inklusiv“ arbeiten.

- Schüler mit Behinderungen und Schüler ohne Behinderungen, die eine Regelschule besuchen, sind automatisch ,inkludiert“, unabhängig davon in welchen anderen Teilsystemen sie zugehörig oder nicht zugehörig sind und unabhängig davon, welche ,inklusiven“ und ,exklusiven“ Prozesse innerhalb des Teilsystems Schule ablaufen.

In Anlehnung an Feuser (2010) ist dagegen davon auszugehen,

[...] dass es in einer sich funktional immer weiter differenzierenden Gesellschaft keine für alle Menschen gleichzeitig besetzbaren Sozialräume gibt, an denen sie partizipieren könnten. Entsprechend bleibt nicht nur die Notwendigkeit bestehen, die verschiedenen Sozialräume eines Gemeinwesens auf ihre Exklusions-Inklusionsverhältnisse zu untersuchen, sondern für die Beschreibung der Analyseergebnisse entsprechend differenzierte Begrifflichkeiten zu verwenden und diese hinsichtlich ihrer strukturbildenden Funktionen und prozesssteuernden Eigenschaften im jeweiligen Sozialraum, in dem sie wirksam werden, zu präzisieren. (Feuser, 2010, S. 17)

Insgesamt ergibt sich aus der begrifflichen Analyse der beschriebenen Fachwissenschaften also der Eindruck, dass Soziologie und Bildungssoziologie hilfreiche Ansätze für ein differenziertes begriffliches Verständnis und damit für eine differenzierte Beschreibung inklusiver und exklusiver Prozesse anbieten, die dazu führen können, dass Schülerinnen und Schüler als Teile einer hochausdifferenzierten Gesellschaft wahrgenommen werden, in der diese Prozesse auch unabhängig vom Teilsystem Schule unablässig und veränderlich ablaufen. Dieser Gedankengang wird unter Fazit und Perspektiven erneut aufgegriffen und weiter ausgeführt. 



\section{Systematischer Forschungsüberblick zu Qualitätsbereichen schulischer Integration/Inklusion}

In der Welt zusammenleben heißt wesentlich, daß eine Welt von Dingen zwischen denen liegt, deren gemeinsamer Wohnort sie ist, und zwar in dem gleichen Sinne, in dem etwa ein Tisch zwischen denen steht, die um ihn herum sitzen; wie jedes Zwischen verbindet und trennt die Welt diejenigen, denen sie jeweils gemeinsam ist. (Arendt, 1967, S. 66)

Um dieses Bild zu verwenden: der „Tisch“, den Allgemein- wie Sonderpädagogen betrachten und zu erfassen versuchen, ist derselbe. Es handelt sich um „eine“ Schule und „einen“ Unterricht, die untersucht werden. Die Trennung besteht lediglich darin, dass unterschiedliche Perspektiven auf den „Tisch“ eingenommen werden. Allgemein- wie Sonderpädagogen betrachten ihn von unterschiedlichen Seiten, während der „Tisch“ derselbe bleibt. Während die Schul(entwicklungs)- und Unterrichtsforschung u.a. unterschiedliche schulische Entwicklungsbereiche und ihre Auswirkungen auf Schüler allgemein untersuchen (Bsp.: organisatorische Strukturen, Kooperation von Lehrkräften, Klassenführung usw.), nimmt die sonderpädagogische Forschung diese Bereiche vor allem unter der Perspektive in den Blick, inwiefern schulische Prozesse sich auf die Unterrichtung von Schülern mit sonderpädagogischem Förderbedarf auswirken (Bsp.: Leistungsentwicklung, Selbstbild). Die Prozesse an sich unterscheiden sich nicht voneinander, beruhen auf einem dynamischen Wirkungsgefüge und haben Auswirkungen auf alle Beteiligten.

Ziel dieses Kapitels ist es Ergebnisse aus der der Integrations- bzw. Inklusionsforschung (vorwiegend sonderpädagogisch fundiert) mit Ergebnissen der Schul- und Unterrichtsforschung (vorwiegend allgemeinpädagogisch fundiert) in einer kategorial geordneten Struktur zusammenzuführen. Dieser Ansatz beruht auf der Grundannahme, dass Schulen und Unterricht in den vergangenen Jahrzehnten aus den zwei genannten Blickrichtungen heraus untersucht worden sind, ohne dass die daraus gewonnenen Ergebnisse jeweils (eng) miteinander verknüpft wurden. Im vorliegenden Kapitel wird aus diesem Grund inhaltlich systematisierend dargestellt, welche Kategorien, Subkategorien und Indikatoren die Qualität schulischer Integration hinsichtlich des derzeitigen Forschungsstands aus Blickrichtung der Sonderpädagogik kennzeichnen und welche Forschungsergebnisse der Allgemeinen Pädagogik sie inhaltlich stützen und fundieren können. Diese Darstellungsform der bisherigen Forschungsergebnisse soll erfolgen um einerseits einen möglichst umfassenden Überblick über wesentliche Kategorien, Subkategorien und Indikatoren für die Qualität schulischer Integration zu erhalten und diese andererseits auch quantitativ gewichten zu können, so dass ein umfassendes Kategoriensystem für das Forschungsprojekt entstehen kann. Trotz der Unübersichtlichkeit und des umfassenden zeitlichen Rahmens der Veröffentlichungen lassen sich aus vielfältigen Forschungsarbeiten Qualitätskriterien herauskristallisieren, die Hinweise darauf geben, welche Bereiche und Variablen für die Qualität integrativer bzw. inklusiver Beschulung und Unterrichtung entscheidend sind. Alle Forschungsarbeiten wurden also unter der Fragestellung analysiert, welche Faktoren die Qualität schulischer Integration beeinflussen können. Die Auswahl der Kategorien, Subkategorien und Indikatoren erfolgte fast ausschließlich auf der Grundlage forschungsbasierter Veröffentlichungen (Heese, 1977; Reiser et al., 1984; Reiser, 1987; Wocken, 1987; Dumke et al., 1989; Bächthold et al., 1990; Innerhofer \& Klicpera, 1991; Hug, 1994b; Husinsky, 1994; Köbberling, 1994; Berges, 1996; Heyer et al., 1997; Bless, 2003; Haeberlin et al., 2003; Bürli et al., 2009). 
Einige Ergänzungen wurden Qualitätsübersichten entnommen (Boban \& Hinz, 2003; Brugger-Paggi, 2003; Specht et al., 2006; Wember \& Prändl, 2009), die gezielt versuchen die Qualität schulischer Integration bzw. Inklusion kategorienorientiert zu erfassen. Erst nachdem diese Übersicht auf der Grundlage sonderpädagogischer Veröffentlichungen erstellt wurde, wurde eine Zuordnung zu den drei Bereichen Organisations-, Personal- und Unterrichtsentwicklung vorgenommen. Im Anschluss wurden allgemeinpädagogische Forschungsveröffentlichungen analysiert, die sich auf inhaltlich deckungsgleiche Kategorien (Bsp.: Klassenführung, Binnendifferenzierung) beziehen. Diese wurden nicht in die Kategorienübersicht aufgenommen (s. Tabellen im Anhang), ergänzen allerdings den Forschungsüberblick zu den einzelnen Kategorien.

\subsection{Der Begriff „Qualität“}

Die vorliegende Arbeit geht davon aus, dass eine systematische Analyse schulischer Qualität möglich ist. Qualität wird in diesem Zusammenhang als inhaltlich offener (Wember, 2009, S. 24) und dadurch komplexer sowie vielschichtiger Begriff aufgefasst, unter dem ursprünglich - wie in der Einleitung bereits erwähnt - die „Beschaffenheit“ einer Sache verstanden wird. Beeinträchtigt wird dieses eigentlich neutrale Verständnis allerdings dadurch, dass im alltäglichen Sprachgebrauch mit Blick auf diesen Begriff „fast immer eine implizite Wertung mit[schwingt]“ (Wember, 2009, S. 24). Diese wertende Konnotation hängt mit den zwei unterschiedlichen begrifflichen Bedeutungsebenen von „Qualität“ zusammen. Auf der einen Seite wird diese im neutralen Sinn als die „Gesamtheit der charakteristischen Eigenschaften (einer Sache, Person) “ (DUDEN) verstanden, auf der anderen Seite aber auch wertend mit Blick auf die besondere „Güte“ (DUDEN) des- oder derselben. Grundlage einer vorwiegend neutralen Begriffsverwendung ist mit Bezug auf die erstgenannte Ebene das Qualitätsverständnis, das den DIN EN ISO Normen 9000, 9001 und 9004 vergleichbar ist, die Qualität als „Gesamtheit inhärenter Merkmale eines Produkts, Systems oder Prozesses“ (Kamiske \& Brauer, 2002, S. 58) beschreiben und eine prozessorientierte Denkweise beinhalten, die annimmt, dass „miteinander verknüpfte Einzelprozesse [...] die Kernprozesse einer Organisation [formen] “ (Kamiske \& Brauer, 2002, S. 58). Bezogen auf Schule und Unterricht geht es in diesem Zusammenhang also sowohl um die systematische Erfassung qualitativer Merkmale als auch um die Beschreibung der mit diesen Merkmalen zusammenhängenden Prozesse sowie möglicher „Beziehungen zwischen einzelnen Qualitätsfaktoren“ (Dubs, 2005, S. 205). Nach Sauerwein und Klieme muss mit Blick auf die zwei unterschiedlichen Bedeutungsebenen bei der Nutzung des Begriffs Qualität diesbezüglich zwischen beschreibenden Aussagen, die wissenschaftlich überpüfbar sind, und bewertenden Aussagen, die das Ergebnis der Beurteilung dieser Beschreibungen sind, unterschieden werden (Sauerwein \& Klieme, 2016, S. 462). Wember geht davon aus, dass der Qualitätsbegriff für ein konkretes Produkt seine ursprüngliche Neutralität verliere, sobald dieser „konkretisiert und verbindlich definiert wird“ (Wember, 2009, S. 25).

Inwiefern und in welchem Maß bestimmte Merkmale oder Prozesse in Schulen gegeben sind, kann wiederum nicht per se als "qualitativ gut“ oder „qualitativ schlecht“ bewertet werden. Dagegen sprechen mehrere Gründe, die mit dem Bezugsrahmen, möglicherweise unterschiedlichen Auswirkungen auf bestimmte Personengruppen, der Beobachterabhängigkeit, der Abhängigkeit von Beziehungen zwischen Merkmalen sowie dem Problem einer ggf. zu stark leistungsorientierten Ergebnisorientierung zusammenhängen.

Im Rahmen der Bildungsforschung der vergangenen Jahrzehnte wurden für die Untersuchung schulischer Qualität nach Sauerwein und Klieme „Modelle entwickelt, die zwischen verschieden 
strukturellen Bedingungen (Inputs), Aktivitäten und Maßnahmen (Prozesse) und den (Lern-) Ergebnissen der Schüler/-Innen (Outcomes) unterscheiden“ (Sauerwein \& Klieme, 2016, S. 260). Der Bezugsrahmen (Werte, Normen, etc.), innerhalb dessen diese Ressourcen, Prozesse und Ergebnisse analysiert werden, ist veränderlich (durch die letzten Jahrzehnte hindurch, abhängig von politischen Rahmenbedingungen, etc.). Erst wenn Anforderungen und damit auch Zielsetzungen wie Lernergebnisse oder Kompetenzen für bestimmte Merkmale und Prozesse definiert sind, kann im übertragenen Sinn geprüft werden, in welchem Ausmaß „unterschiedliche Produkte die erwünschten Kriterien erfüllen“" (Wember, 2009, S. 25; Huber \& Schneider, 2011, S. 355). Die Frage nach einer qualitativ guten Schule erfordert also zunächst eine Klärung dahingehend, „was Schule eigentlich ist und tut (Fend 2008)“"(Schmidt, 2016, S. 143). Sauerwein und Klieme gehen davon aus, „dass Qualität sich nur über eine normative Setzung erschließt, weil die Bestimmung der Ziele bzw. Zwecke oder der moralischen bzw. ethischen Standards des zu betrachten Objekts normativ ist." (Sauerwein \& Klieme, 2016, S. 468). Bildungsforschung sei daher

[...] werturteilsbehaftet, weil entweder (implizite) normative Zuschreibungen der Ziele des Objekts die Qualität bedingen - die Förderung der Leistung der Schülerinnen und Schüler als Zweck des Unterrichts, die Herstellung von Chancengerechtigkeit als Zweck des Schulsystems - oder professionelle, moralische oder ethische Vorstellungen gesetzt werden. (Sauerwein \& Klieme, 2016, S. 469)

Die Anforderungen an Schule in einem staatlich verantworteten Schulsystem sind (anders als ggf. bei Industrieunternehmen) also nicht ohne Weiteres aus den Wünschen der Kunden (Eltern, Schüler, Arbeitsmarkt) mit unterschiedlichen, möglicherweise widersprüchlichen Interessen abzuleiten, sondern vorrangig aus dem Bildungs- und Erziehungsauftrag in der Verfassung und den Schulgesetzen. Aufgrund unterschiedlicher Ausgestaltung, verschiedener Ausgangsbedingungen und unterschiedlicher Schulkontexte kommt es entsprechend auch zu unterschiedlichen „Qualitäten“ (Huber \& Schneider, 2011, S. 355). Dubs geht daher davon aus, dass der Begriff Qualität im Bildungswesen

[...] die bewertete Beschaffenheit eines Bildungssystems, einer Schule oder einer Klasse gemessen an den in einem politischen Aushandlungsprozess gefundenen Zielvorstellungen und Ansprüchen aller am Bildungswesen interessierten Anspruchsgruppen und Personen [meint]. (Dubs, 2005, S. 205)

Da Ansprüche an das Bildungswesen sehr unterschiedliche Merkmale betreffen (Bsp.: strukturelle oder personenbezogene Aspekte), die je nach normativem oder gesellschaftlichem Einordungsrahmen eine unterschiedliche Gewichtung erfahren, könne keine Festlegung verbindlicher Qualitätskriterien für alle Bildungseinrichtungen über längere Zeiträume hinweg erfolgen (Dubs, 2005, S. 205). Mit Blick auf die Beschreibung von Unterrichtsqualität formuliert Eikenbusch dieses Grundproblem folgendermaßen:

$\mathrm{Zu}$ bestimmen, was guter Unterricht ist, erfordert also normative Entscheidungen. Er lässt sich nur in Bezug auf Vorgaben und Ziele definieren und ist nicht „objektiv“ und eindeutig feststellbar. Die Entscheidung hängt ab von Qualitätskriterien, die gemäß ihrer pädagogischen Intentionalität unterschiedlich gesetzt und gewichtet werden können, die aber auch mit bestimmten fachlichen Qualitätsansprüchen sowie mit gesellschaftlichen und institutionellen Rahmenbedingungen und Vorgaben (z.B. Lehrplänen) verträglich sein müssen. (Eikenbusch, 2011, S. 9)

Rihm wiederum problematisiert in diesem Zusammenhang den strukturellen Zielkonflikt von Schulen im Rahmen der Qualitätsdiskussion, der sowohl darin bestehe Unterschiede auszugleichen als auch Unterschiede zwischen Schülern herzustellen (Rihm, 2006, S. 399) (s. dazu auch 2.2.2). 
Bestimmte Qualitätsmerkmale können sich darüber hinaus auf verschiedene Personengruppen unterschiedlich auswirken, so dass sie in ihrer Wirksamkeit, ihrer Wirkrichtung oder -ausdehnung auch in sich nicht einheitlich beschrieben werden können. Während sich die Größe einer Klasse aus schulorganisatorischer Sicht günstig auf eine Stundenplanung auswirken kann (Bsp.: wenige und dafür große Klassen - weniger komplexe Stundenpläne), kann ihre Wirkung auf Lernprozesse der Schülerinnen und Schüler unter Umständen ungünstige Auswirkungen haben (Bsp.: große Klasse - weniger individualisierte Lernangebote).

Sowohl die Beschreibung als auch die Bewertung qualitativer Merkmale ist außerdem abhängig von der Wahrnehmung der Beobachtenden. Sie kann sich daher auch unterscheiden, wenn verschiedene Beobachter ein Qualitätsmerkmal aus ihrer Perspektive wahrnehmen bzw. bewerten (Bsp.: Wahrnehmung der Klassenführung durch eine Lehrkraft bzw. die Schülerinnen und Schüler). Nach Rihm bewerten Beteiligte die Qualität von Unterricht und Schule je nach Handlungsinteressen unterschiedlich (Bsp.: Schüler als unmittelbar Betroffene, Lehrkräfte oder Schulleitungen als mittelbar Betroffene von Unterricht) (Rihm, 2006, S. 399). Deshalb ist seiner Einschätzung nach die Einbeziehung der unterschiedlichen Beteiligten ein zentraler Aspekt, wenn es um die Erfassung (und Messung) von Qualitätskriterien geht. Aus diesem Grund werden laut Holzäpfel in Erhebungen zu schulischer Qualität mehrere Personengruppen befragt:

[...] daher stellt nun die Einbeziehung von Eltern und Schülern sowie Schulträgern und Gemeinden in den Prozess der Schulgestaltung eine entscheidende Neuerung dar. Dies wird mitunter an Erhebungsinstrumenten wie z.B. SEIS deutlich, welche derzeit für Überblicksevaluationen eingesetzt werden und verschiedene Personengruppen in die Befragung einbeziehen. (Holzäpfel, 2008, S. 34f)

Neben der beobachterabhängigen Wahrnehmung und Bewertung schulischer Qualität spielt für den schulbezogenen Qualitätsbegriff auch die Abhängigkeit von Beziehungen zwischen Merkmalen untereinander eine Rolle. So kann beispielsweise die Klassengröße ein Qualitätsmerkmal darstellen, für das erst im Zusammenhang mit anderen Merkmalen erfasst werden kann, ob oder inwiefern dieses sich auf das Lernen in einer Klasse auswirkt. Ähnlich verhält es sich mit Qualitätsmerkmalen wie der Kooperation zwischen Lehrkräften oder der Nutzung unterschiedlicher Sozialformen im Unterricht.

Hinzu kommt, dass es im Zusammenhang mit der Analyse und Beschreibung schulischer oder pädagogischer Qualität grundsätzlich schwierig erscheint die Qualität von Input-, Prozess- und Outputkriterien zu messen. Wann beispielsweise verfügt eine Schule über „ausreichende“ Ressourcen? Wann kann davon gesprochen werden, dass Unterricht tatsächlich weiterentwickelt wird? Werden bei Prozessbeschreibungen Kommunikation und Interaktion beteiligter Personen untersucht (Wember, 2009, S. 35) oder stehen Fragen der Inhaltsvermittlung im Fokus? Welcher Output soll gemessen werden?

$\mathrm{Zu}$ Recht darf hierbei kritisch betrachtet werden, dass oftmals nur Leistung als Bewertungskriterium fungiert (Scharenberg, 2015) oder andere Bewertungskriterien in den Hintergrund rücken. Wie eingangs erwähnt, werden immerhin in PISA und StEG vielfältige Bildungsziele operationalisiert, in der öffentlichen Wahrnehmung scheint jedoch Leistung zu überwiegen. [...] Die Qualität von Schule ausschließlich auf Schülerleistung zu reduzieren, also auf messbare Ergebnisse durch reine Outputkontrolle im Sinne der reinen Qualitätssicherung, zeigt allerdings ein einseitig begrenztes Bild von Schulqualität. Schülerleistungen sind nur ein im Kontext von Schule zu betrachtendes Merkmal von Schulqualität im Sinne von Leistungen einer Schule. (Sauerwein \& Klieme, 2016, S. 469)

Nach Sauerwein und Klieme definiert Mortimore (1991) beispielsweise eine gute bzw. wirksame Schule als eine, „in welcher der Lernfortschritt der Schüler größer ist bzw. deren in Noten 
gemessenen Leistungen besser sind, als von ihren Eingangsvoraussetzungen her zu erwarten gewesen wäre." (Sauerwein \& Klieme, 2016, S. 469). Für die Messung von Schülerleistungen im sonderpädagogischen Bereich weist Wember zusätzlich darauf hin, dass die Messung von Lernfortschritten hinsichtlich der Effektivität und Effizienz sonderpädagogischer Förderung „immer individuell und relativ zu den Eingangsvoraussetzungen beurteilt werden [müsse]“ (Wember, 2009, S. 35). Insbesondere im sonderpädagogischen Bereich schätzt er daher eine Outputerfassung als schwierig ein. Noch problematischer und weniger prüfbar wird diese, wenn zusätzlich Auswirkungen auf den beruflichen Erfolg ehemaliger Schülerinnen und Schülern erfasst werden sollen (Dubs, 2005, S. 206). Auch ist es laut Dubs nicht zulässig von linearen Beziehungen zwischen verschiedenen Qualitätsfaktoren auszugehen (Bsp.: finanzielle Rahmenbedingungen - Lernerfolge von Schülerinnen und Schülern): „Solche linearen Beziehungen werden der Komplexität der Schule nicht gerecht." (Dubs, 2005, S. 206).

Sind es also Kenntnisse bzw. Kompetenzen in verschiedenen Unterrichtsfächern oder ist es die soziale bzw. emotionale Entwicklung von Schülerinnen und Schülern, die als besonders relevant eingestuft werden? Wird bei dieser Messung die Persönlichkeitsentwicklung von Kindern und Jugendlichen als ganzheitlicher Prozess in den Blick genommen? Wie kann diese gemessen werden? Wird der Output mit Effizienz gleichgesetzt (erreichte Kompetenzen) oder geht es um die Effektivität des Outcomes? (beruflicher Erfolg) (Dubs, 2005, S. 205). Sollte diese „Orientierung auf nachweisbare Wirkungen“ (Helmke, 2009, S. 16) bei der Qualitätsmessung pädagogischer Prozesse im Vordergrund stehen?

Viele dieser Fragen können zwar immer wieder diskursiv erschlossen, nicht aber letztgültig beantwortet werden und stehen wiederum im Zusammenhang mit der Frage der Festlegung eines normativen Bezugsrahmens für die Qualitätsmessung im Zusammenhang mit Schulen.

Zielsetzung dieser Arbeit ist es zunächst den Begriff Qualität vorwiegend in seiner neutralen Bedeutungsebene zu verwenden und so wenig wertend wie möglich zu erschließen, wie integrative Schulen organisiert sind, wie das Personal in diesen Schulen arbeitet und wie der Unterricht gestaltet wird. Nach Sauerwein und Klieme geht es bei diesem Ansatz um eine „systematisch deskriptive Qualitätsbestimmung“ (Sauerwein \& Klieme, 2016, S. 467). Dazu erfolgt im vorliegenden Kapitel eine präzise Beschreibung der Qualitätskategorien, -subkategorien und -indikatoren, die aus Sicht der empirischen Bildungsforschung im Zusammenhang mit schulischer Integration stehen. Diesbezüglich werden mögliche Einflussgrößen, Prozesse, die sich im Rahmen von Schulen bzw. auch gezielter schulischer Entwicklung ereignen, sowie Ergebnisse beschrieben und analysiert. Auch diese möglichst neutrale Beschreibung und Analyse kann allerdings nicht frei von normativen Setzungen erfolgen, sondern wird im Bewusstsein dahingehend vorgenommen, dass beispielsweise bei der Beschreibung der Merkmale „guten“ Unterrichts die zugrundliegende Forschung bereits von Prämissen ausgeht, die im Zusammenhang mit bestimmten normativen Rahmungen stehen und daher implizite Wertungen enthalten (Bsp.: Bewertung der Effekte auf Leistungen, nicht auf die Persönlichkeitsentwicklung von Schülern).

Durch qualitative Interviews und eine schriftliche Befragung wird im Anschluss versucht festzustellen, ob bzw. auch in welcher Form und Ausprägung diese Qualitätsmerkmale in den untersuchten Schulen gegeben sind. Es geht dabei in Anlehnung an Sauerwein und Klieme vor allem darum

[...] das Vorhandensein bestimmter zuvor festgelegter Qualitätsmerkmale festzustellen [...]. Die Bewertungskriterien für Qualität werden in diesem Modell nicht über bestimmte Outcomes repräsentiert, deren Erreichen über Zusammenhangsanalysen geprüft wird. (Sauerwein \& Klieme, 2016, S. 466) 
Gleichzeitig wird durchaus versucht Beziehungen zwischen einzelnen Merkmalen inhaltlichqualitativ darzustellen. Damit die Wahrnehmung und Bewertung verschiedener Qualitätsmerkmale durch unterschiedliche Personengruppen erfasst werden kann, werden sowohl Schulleitungen, also auch Regel- wie Förderschullehrkräfte, Eltern und Schülerinnen und Schüler zu diesen Merkmalen befragt. Um keine rein leistungsorientierte Ergebnisdarstellung vorzunehmen, werden im Zusammenhang mit schulischer Integration neben der kognitven Lernentwicklung auch die emotionale und soziale Entwicklung von Kindern und Jugendlichen in den Blick genommen. Wertende Aussagen zu Qualitätskategorien, -subkategorien bzw. -indikatoren im Sinne der oben beschriebenen zweiten Bedeutungsebene des Qualitätsbegriffs werden dann aufgegriffen, wenn diese im Rahmen der Interviews oder schriftlichen Befragung durch die befragten Personengruppen getätigt werden oder wenn es - wie in Fazit und Perspektiven um eine kritische Einordnung von Qualitätsmerkmalen hinsichtlich ihrer Umsetzung geht.

\subsection{Auswahl eines Rahmenmodells zur Systematisierung der Forschungsergebnisse}

Um eine Systematisierung der bisherigen Forschungsergebnisse und Qualitätskriterien vorzunehmen, ist es zunächst hilfreich sich bisherige Systematisierungsversuche für schulische Entwicklungsprozesse aus der Sonderpädagogik und der Allgemeinen Pädagogik vergleichend anzuschauen.

\subsubsection{Modelle zur Systematisierung schulischer Integration aus der Sonderpädagogik}

Reiser (1984) beispielsweise definiert mit Blick auf Integrationsbemühungen in Grundschulen ein Bedingungsgefüge aus Lehrern (Lehrerrolle und -persönlichkeit), Schülern (Schülerrolle und -persönlichkeit) sowie der Grundschule selbst (Institution und lokalspezifische Ausprägung) (Reiser et al., 1984, S. 286f). Dieses Bedingungsgefüge wiederum sei in ein Umfeld von Eltern, Verwaltung, direkten politischen Einflüssen und indirekten gesellschaftlichen Einflüssen eingebettet. Wissenschaftliche Forschung zu Integration müsse daher komplexe Interaktionen analysieren:

Wir fanden bestätigt, daß Lern- und Verhaltensstörungen sich in der Grundschule im Rahmen eines hochkomplexen Bedingungsgefüges entwickeln und stellten fest, daß verschiedene wissenschaftliche Ansätze stets nur Ausschnitte aus diesem Bedingungsgefüge von einer Perspektive her beleuchten. Eine Strukturierung der Komplexität der Interaktionsprozesse führte uns zu einer situationsbezogenen Definition des Begriffes Integration. [...] Die Analyse der Daten bestätigte die aus der Praxis gewonnene Vermutung, daß Interventionen, die in diese Interaktionsprozesse eingreifen, mehrdeutige Auswirkungen haben, und daß hochkomplexe Interaktionen häufig differentielle Effekte aufweisen. (Reiser et al., 1984, S. 285)

Bach (1987) schlussfolgert, dass einfache und wenig komplexe integrative Maßnahmen (Bsp.: Nachhilfeunterricht) daher auch keine so hohe integrative Wirksamkeit entfalten können wie komplexe Maßnahmen, die das komplette „Kommunikationssystem Schule und Unterricht“ (Bach, 1987, S. 144) erfassen (Bsp.: fallbezogene Beratung, persönlichkeitsbezogene Fortbildung, Ko-Unterricht, Klima im Lehrerkollegium etc.).

Preuß (1985) wiederum nimmt eine Systematisierung von Kriterien integrativer Arbeit nach Strukturmerkmalen sonderpädagogischer Intervention vor und definiert dabei unterschiedliche Merkmale für die drei Bereiche individuelle Lernprozessorientierung (Bsp.: Lernprozessdokumentation), kooperative Handlungsstruktur (Bsp.: Indikatoren für erfolgreiche Kooperationsprozesse) und Lernortunabhängigkeit (Bsp.: Beratungszentrum). Daraus ergeben sich wiederum praxis- bzw. handlungsorientierte Aspekte, kollegiale Aspekte und institutionelle Aspekte integrativer Tätigkeit (Preuß, 1985, S. 99). Auch Feuser und Meyer (1987) systematisieren drei übergeordnete Aspekte integrativer Pädagogik, wobei sie vor allem unterrichtliche Prozesse 
berücksichtigen: curriculare, didaktisch-mediale und methodisch-therapeutische Zusammenhänge (Bsp.: offener Unterricht), die Qualität von Kooperation und integrierter Therapie sowie die Entwicklung von Tätigkeitsniveau, Handlungskompetenzen und sozialer Beziehungen von Schülern durch Individualisierung (Lernzielebene) und innere Differenzierung (Handlungsebene der Schüler) (Feuser \& Meyer, 1987, S. 24).

Ein bekanntes Instrument für die Analyse und Förderung inklusiver Prozesse in Schulen stellt der „Index für Inklusion“ dar, der in Großbritannien erarbeitet (Booth \& Ainscow, 2002) und von Boban und Hinz (2003) für den deutschen Sprachraum adaptiert wurde. Dieser Index unterscheidet zwischen drei Dimensionen (Inklusive Kulturen schaffen, Inklusive Strukturen etablieren, Inklusive Praktiken entwickeln), sechs Bereichen (jeweils zwei je Dimension) und insgesamt 44 diesen Dimensionen und Bereichen zugeordneten Indikatoren. Je Indikator sind wiederum zwischen sechs und 17 Leitfragen formuliert, die während des durch den Index gesteuerten Schulentwicklungsprozesses reflektiert werden können. Diese Systematisierung stellt einen ersten in mehrjähriger Zusammenarbeit von Lehrern, Eltern, Schulvorständen, Forschern sowie einem Vertreter von Behindertenorganisationen entstandenen Versuch dar, Schulen ein Hilfsmittel für qualitätsorientierte inklusive Schulentwicklung anzubieten. Insbesondere die Leitfragen zu den einzelnen Indikatoren sind sehr stark ausdifferenziert.

Maykus et al. (2014) erläutern unabhängig vom schulischen Kontext drei übergeordnete Teilbereiche im „Praxisentwicklungskontext“ Inklusion:

1. Inklusive Bildung als Entwicklungsprozess auf den Ebenen Person/Akteur/Konzept und Struktur (Interdependenz von Organisationsgestaltungsfaktoren)

2. Inklusive Bildung als Implementationsgefüge (Dynamisches Gefüge von Einflussfaktoren)

3. Inklusive Bildung als Anpassungsimpuls (Rationalitätsmythos einer Fachmaxime) (Maykus et al., 2014, S. 13ff)

Sie weisen darauf hin, dass das „Programm Inklusion“ nicht unbedingt in einem engen Zusammenhang mit der Umsetzungspraxis stehe:

Programmvorgaben stehen nur in einem lockeren Zusammenhang mit den Organisationen und ihren Akteurinnen und Akteuren und schließlich sind es genau diese, die in ihrer Vielzahl und auf der Grundlage unterschiedlicher Aufträge, Erfahrungen und Selbstverständnisse die Zielvorstellungen von Inklusion modifizieren, (um-) deuten, dadurch mithin ein offenes Ergebnis des Umsetzungsprozesses bedingen. (Maykus et al., 2014, S. 13)

Querschnittsthemen für inklusive Bildungskontexte in Kindertageseinrichtungen, Schulen, schulbezogener Kinder- und Jugendhilfe sowie Institutionen der beruflichen Bildung seien: Organisationsgestaltung und Personalentwicklung, (sozial-) rechtliche Grundlagen und Gestaltungsspielräume sowie die Kommune als Raum der System- und Lebensweltvermittlung (Maykus et al., 2014, S. 19). Maykus et al. machen also deutlich, dass inklusive Prozesse auf unterschiedlichen Ebenen und auf diesen Ebenen in mehreren Bereichen ablaufen, so dass sein Systematisierungsansatz als Mehrebenenmodell aufgefasst werden kann.

Einen ähnlichen Ansatz stellt Dlugosch (2013) vor, die ebenfalls davon ausgeht, dass Inklusion als Mehrebenenkonstellation eine Fülle von Akteuren, Faktoren und Handlungsdimensionen umfasst. Forschung müsse analysieren, welche Einflussfaktoren vorhanden sind, wie diese aufeinander wirken, welche Faktoren als träge oder kritisch einzustufen seien und welche Handlungsansätze sich daraus ergeben. Dieses Modell weist eine hohe Komplexität auf und setzt sich mit Makro-, Meso- und Mikrostrukturen des Schulsystems und der Einzelschule auseinander (Dlugosch, 2013, S. 21-33). 


\subsubsection{Modelle zur Systematisierung schulischer Entwicklungsprozesse aus der Allgemeinen Pädagogik}

Einen übergreifenden theoriebasierten Ansatz für die Analyse des Schulsystems und einzelner Schulen hat Fend mit seiner "Gestaltungslehre des Bildungswesens“ (2008) ausgearbeitet. Im Rahmen dieser Gestaltungslehre geht der Autor von Fragen danach aus, wie das Bildungswesen funktioniert, wie Möglichkeiten und Instrumente der Gestaltung schulischer Lernprozesse aussehen und wie ,man gute Bildungssysteme, gute Schulen und guten Unterricht [macht]" (Fend, 2008, S. 12ff). Damit gelangt er zu einem ganzheitlichen Modell, das erfasst, wie Bildungssysteme gestaltet sind und wie die Qualität des Bildungswesens gesichert werden kann. Im Rahmen des Modells unterscheidet Fend Makroebene (Bsp.: Verfassung, Schulgesetze, Bildungspolitik), Mesoebene (Bsp.: Schulleitung, Einbettung der Schule in eine Kommune), Mikroebene Lehrer (Bsp.: Methodik, Didaktik) sowie Mikroebene Schüler (Bsp.: Verhalten, Leistung) (Fend, 2008, S. 17) und postuliert in diesem Zusammenhang:

Wir können nicht davon ausgehen, dass alles, was vom Gemeinwesen auf bildungspolitischer Ebene gewollt ist, auf unverfälschte Weise bei Lehrern und Schülern ankommt. Viele Menschen sind an der Umsetzung beteiligt und sie alle interpretieren die Vorgaben wieder auf ihre Weise. (Fend, 2008, S. 27)

Diese Interpretation bildungspolitischer Maßnahmen durch unterschiedliche Akteure beschreibt Fend als „Rekontextualisierung“ (Fend, 2008, S. 26), einen Prozess, bei dem Vorgaben aus einem vorgesehenen Zusammenhang gelöst und für spezifische Handlungsbedingungen angepasst werden (Fend, 2008, S. 27). Eine besondere Bedeutung kommt in diesem Modell dem „aktive[n] Gestaltungsanteil von Akteuren auf der jeweiligen Ebene“ (Fend, 2008, S. 26) zu. Aufgrund dieser Annahme, die den Kerngedanken der Gestaltungslehre des Bildungswesens kennzeichnet, geht Fend davon aus, dass das eigentliche Veränderungspotenzial auf der Mesoebene der Einzelschule liegt. Fends Modell stellt mit Blick auf Schulentwicklungsprozesse eine systemische Analysehilfe dar. Ähnlich wie das Mehrebenenmodell von Dlugosch (2013) kann das Modell als umfängliches Rahmenmodell aufgefasst werden, innerhalb dessen sich ein engeres Modell von Schulentwicklung im eigentlichen Sinn verorten lässt.

Ein forschungsbasiertes einzelschulbezogenes Modell haben Bryk et al. (2010) als Ergebnis einer auf mehr als zehn Jahre angelegten Langzeitstudie generiert, die die Schulentwicklung von 22 Grundschulen (aus einer Stichprobe von insgesamt mehr als 200 Primarschulen mit über 150.000 Schülern) im Großraum Chicago untersucht hat. Diesen Schulen - sowie den Stadtteilen bzw. Gemeinden, in denen sich die Schulen befinden - wurden im Rahmen des Chicago School Reform Acts von 1988 weitgehende Autonomie und Selbständigkeit mit Blick auf Entwicklungsentscheidungen gewährt:

The Chicago School Reform Act of 1988 had changed all the rules. It devolved significant resources and authority to newly formed Local School Councils and mandated these leaders to reform their schools. Contrary to what has since become the accepted wisdom about how best to advance large system change, the Reform Act deliberately contained the power of the central bureaucracy to interfere with local initiative. Rather than centrally developed plans for the improvement of its schools, Chicago chose a very different course: democratic localism as a lever for change.At base here was a simple but powerful belief. If local school professionals reconnected with the parents and communities they were supposed to serve, and if everyone was empowered to reform their schools, together they could be much more effective in solving local problems than some impersonal bureaucracy. (Bryk et al., 2010, S. 12) 
Bryk et al. haben vor allem substantielle Veränderungen hinsichtlich des Schulbesuchs sowie der Lernergebnisse von Schülern und Schülerinnen mit Blick auf Lesekompetenzen und Mathematik an diesen Schulen untersucht und festgestellt, dass sich nach mehreren Jahren (1990-1996) in diesen Bereichen große Unterschiede in den Einzelschulen ergaben. Beispielsweise verbesserten sich die Leseleistungen von Schülern (orientiert an nationalen Normen in den verschiedenen Klassenstufen) in Schulen mit positiver Entwicklung um 15\% (von 22\% auf 37\%) ${ }^{15}$, während sich in Schulen mit wenig Entwicklung kaum Veränderungen ergaben. Mathematische Leistungen stiegen in positiv entwickelten Schulen um ebenfalls $15 \%$ (von $27 \%$ auf $42 \%$ ), während sich diese in Schulen ohne systematische Weiterentwicklung zum Teil um 4\% (von 29\% auf $25 \%$ ) verschlechterten (Bryk et al., 2010, S. 18f). Ungefähr ein Drittel der Grundschulen zeigte positive Entwicklungstendenzen, entwickelte eigene Initiativen und setzte aktive Umstrukturierungen um, ein anderes Drittel startete ähnliche Prozesse, ohne erfolgreich zu sein, und ein weiteres Drittel zeigte keine Veränderungsanzeichen (Bryk et al., 2010, S. 16). Im Mittelpunkt der Forschung stand daher die Fragestellung: ,what did the improving schools actually do to realize these gains in student learning?" (Bryk et al., 2010, S. 20). Sehr knapp zusammengefasst kamen sie zu dem Ergebnis, dass fünf essentielle Unterstützungsbereiche den Rahmen für Schulentwicklung bilden:

School leadership sits in the first position. It acts as driver for improvements in four organizational subsystems: parent and community ties, professional capacity of the faculty and staff, a student-centered learning climate, and an instructional guidance system. [...] the evidence presented here attests that these systems stand in strong interaction with one another. As a consequence of this interactivity, meaningful improvement typically entails orchestrated initiatives across multiple domains. (Bryk et al., 2010, S. 197; Herv. im Orig.)

Dieses Modell geht aufgrund der Untersuchungsergebnisse von konkreten Querverbindungen zwischen den genannten fünf Unterstützungsbereichen aus und lässt sich damit als dynamisches Modell kennzeichnen (Bryk, 2010, S. 24-27).

Einen ebenfalls systematisierenden Ansatz stellt das von Hans-Günter Rolff entwickelte DreiWege-Modell dar, das bezüglich schulischer Entwicklungsprozesse zwischen Organisations-, Personal- und Unterrichtsentwicklung unterscheidet (Rolff, 2010, S. 30). Sein Ansatz bezieht sich - anders als die Mehrebenenmodelle - ausschließlich auf einzelne Schulen und begreift Schulentwicklung als „bewusste und systematische Weiterentwicklung von Einzelschulen“ (Rolff, 2010, S. 36). Rolff geht von den Prämissen aus, „dass die Entwicklung von Einzelschulen primär ist gegenüber der Systementwicklung." (Rolff, 2010, S. 30) und die zentrale Zielsetzung darin besteht, Schülerinnen und Schülern Lernfortschritte zu ermöglichen:

Gelingende Schulentwicklung hat zum ultimativen Ziel, die Lerngelegenheiten der Schülerinnen und Schüler zu verbessern. Daran gemessen sind Organisations-, Personal- und selbst Unterrichtsentwicklung nur Mittel. (Rolff, 2010, S. 35)

Eine Gewichtung der drei Bausteine bzw. einzelner Kategorien innerhalb dieser Bausteine nimmt Rolff nicht vor. Querverbindungen zwischen allen Bereichen werden in diesem Modell angenommen, anders als bspw. bei Bryk et al. (2010) allerdings nicht präzise definiert und beschrieben. Im Mittelpunkt des Modells steht die Vorstellung der einzelnen Schule als Haus des

15 Dieser Wert bezieht sich auf eine Steigerung im Verlauf von vier Jahren und bedeutet, dass 1996 im Vergleich zu $199315 \%$ mehr Schüler in den sich positiv entwickelnden Schulen Leistungen erzielten, die nationalen Normen entsprachen oder darüber lagen. 
Lernens (Bildungskommission NRW, 1995, S. 86-100), in dem die Entwicklung einer nachhaltigen Lernkultur wesentliches Leitziel ist. Grundlegend ist außerdem die Annahme, dass sich Qualitätsentwicklung als Prozess in einem ständigen Kreislauf von Zielsetzung, Prozessen, Ergebnissen und Bewertungen befindet. Das Modell geht von der Organisationsentwicklung als Ausgangspunkt schulischer Entwicklung aus. Der Bereich der Personalentwicklung umfasst nach Rolff ein Gesamtkonzept, das „Personalfortbildung, Personalführung und Personalförderung umfasst“ (Rolff, 2010, S. 33), während die Unterrichtsentwicklung Bausteine wie Methodik, Fachdidaktik, soziales Lernen etc. betrifft (Rolff, 2010, S. 34). Steuerungsprozesse auf übergeordneter Ebene (Bsp.: Bildungspolitik) spielen in dem Modell insofern eine Rolle, als dass sie die Rahmenbedingungen für die Entwicklung einzelner Schulen festlegen (Rolff, 2010, S. 36). Eine umfassende bzw. geschlossene Theorie der Schulentwicklung liegt nicht vor. Forschung erfolgt in den meisten Fällen zu einzelnen Bausteinen der Schulentwicklung, selten aber zu Schulentwicklungsprozessen an sich (Rolff, 2010, S. 35f; Moser \& Egger, 2017, S. 9f).

\subsubsection{Zusammenhang zwischen sonderpädagogischen und allgemeinpädagogischen Ansätzen zur Systematisierung}

Der wesentliche Zusammenhang zwischen sonder- und allgemeinpädagogischen Ansätzen zur Systematisierung von schulischer Integration bzw. schulischen Entwicklungsprozessen besteht darin, dass „Integration“ oder „Inklusion“ in den meisten Veröffentlichungen jeweils als Teilbereich von Schulentwicklung aufgefasst werden. In Veröffentlichungen zur Schulentwicklung aus allgemeinpädagogischer Sicht steht schulische Integration als Handlungsfeld der Schulentwicklung neben weiteren Feldern wie "Schulentwicklung und Differenz: Gender" (Horstkemper, 2010,S. 37-42) oder „SchulentwicklungundDifferenz: Migration“(Hornberg, 2010,S. 42-45). Preuss-Lausitz (2010) wiederum sieht Integration als Teil der Unterrichts- und Schulentwicklung, indem er die von Meijer 2003 zusammengefassten best-practice-Erfahrungen (z.B. differenzierte Leistungsbewertung, unterschiedliche Sozialformen, individuelle Förderpläne) als Elemente guten Unterrichts allgemein einstuft (Preuss-Lausitz, 2010, S. 47).

Specht (1997) konnte im Rahmen einer großen Untersuchung zur Evaluation der Schulautonomie in Österreich auch der Frage nachgehen, was die Führung von Integrationsklassen für die Schule als Ganzes bedeutet. Dabei stellte er fest,

[...] daß sich an Schulen mit Integration häufiger als an anderen Veränderungen vollzogen haben, die auf eine aktive, innovationsorientierte Unterrichts- und Schulentwicklungsarbeit hinweisen. Veränderungen der Lehr- und Lernformen in Richtung Ganzheitlichkeit und Schülerorientierung, Aktivierung von Lehrern und Schülern im Entwicklungsprozeß der Schule und ausgeprägtere Zusammenarbeit sind die wichtigsten beobachtbaren Effekte an solchen Schulen. (Specht, 1997a, S. 183)

Einerseits hoben die befragten Personen hervor, „dass der gemeinsame Unterricht dort, wo er praktiziert wird, insgesamt zu einer Verbesserung der Schul- und Unterrichtsqualität an Volksund Hauptschulen führe, weil mehr Gewicht auf Individualisierung und Differenzierung im Unterricht gelegt wird und die Teamorientierung der Lehrer/innen gefördert wird“ (Specht, 2006, S. 34). Andererseits wurden auch häufig Qualitätsprobleme hinsichtlich mangelnder Bereitschaft seitens der Lehrer, ungenügender Rahmenbedingungen, problematischem Ressourcenmanagement und ungünstiger struktureller Bedingungen vor allem in der Sekundarstufe I genannt.

Einen verbindenden Ansatz zwischen Inklusion und Schulentwicklung vertritt Werning (2013): 
Inklusive Schulentwicklung umfasst wie jeder schulische Entwicklungsprozess Unterrichtsentwicklung, Personalentwicklung, Organisationsentwicklung und die Vernetzung der Schule mit der familiären und außerschulischen Lebenswelt der Schülerinnen und Schüler. Dabei ist es das Ziel, die Möglichkeiten von Schule zu vergrößern für alle Schülerinnen und Schüler ein lernförderlicher Entwicklungsraum zu werden. Bei der Entwicklung inklusiver Schulen muss nicht auf optimale Bedingungen gewartet werden. Vielmehr kann auf jeder Ebene begonnen werden. (Werning, 2013, S. 60)

Diese Annahme wird auch durch eine Studie von Dyson und anderen gestützt, die zwischen 1999 und 2003 an mehr als 20 englischen Schulen durchgeführt wurde (Unterrichtsbeobachtungen, Erhebung von Schülerleistungen und Interviews mit Schülern). Bei der Begleitung dieser Schulen kristallisierte sich u.a. folgender Entwicklungsaspekt heraus:

[...] the engine driving the development of these schools was not the advent of a charismatic head teacher, or a sudden conversion to inclusive values. Rather, it was being asked to engage with evidence about the actual nature of the school's practices and the impacts of those practices on actual students. Teachers certainly brought values to bear on this engagement, but they were the values of 'doing the best for all students' that we also encountered in the highly-inclusive schools [...]. However, what changed thinking and practice was not a commitment to new sets of values, but a collaborative engagement with evidence about real children in real classrooms, in a way which disturbed the assumptions teachers held about themselves and their students. (Dyson, 2010, S. 9)

Lehrkräfte in den begleiteten Schulen tauschten sich bspw. über die Wirksamkeit oder Unwirksamkeit bestimmter Unterrichtsmethoden oder didaktischer Ansätze (z.B. im Rahmen des Lese- und Schreibunterrichts) aus und veränderten diese in Folge. So kommt Dyson, was die Schulentwicklung inklusiver Schulen betrifft, insgesamt zu folgendem Ergebnis:

The process of development is not one that depends on the presence of exceptional individuals, but one in which ordinary teachers engage with the realities of their own students and classrooms and seek to extend the responsiveness of their work. Moreover, that process is not one which conflicts hopelessly with the other aims of national policy, but which interacts with, and may even be supported by, those aims. Whatever the limitations of this model may be in developing showpiece inclusive schools, it seems like a more promising way of bringing some form of inclusion to the school system as a whole. (Dyson, 2010, S. 11)

Dyson geht aufgrund der vorliegenden Forschungsergebnisse davon aus, dass Schulen, in denen proportional viele Schüler mit Schwierigkeiten unterrichtet werden, ihre Organisation und ihren Unterricht flexibel und gedankenvoll gestalten, sich dadurch allerdings nicht grundlegend von anderen gut organisierten Schulen unterscheiden. Die Unterstützung, die diese Schulen bspw. Schülern mit Förderbedarf zukommen lassen würden, sei vorwiegend pragmatisch und weniger ideologisch ausgerichtet (Dyson, 2010, S. 6). Anstelle eines klaren Einzelmodells für inklusive Schulen zeige sich, dass eine hohe Bandbreite und Flexibilität pädagogischer Zugänge wichtiger scheine als ein bestimmter Maßnahmenkatalog (Dyson, 2010, S. 3f). Zu einem ähnlichen Schluss kommt Deppe-Wolfinger auch für deutsche Schulen:

Die Schulen, die für alle Kinder ihres Einzugsgebietes Verantwortung übernehmen, sie individuell und gemeinsam bilden und außerdem kontinuierlich Schulentwicklung betreiben, sind nicht nur gute Schulen, sondern beste Schulen im Lande. So jedenfalls sehen es die Stiftungen (Robert-Boschstiftung, Heidehofstiftung) und die Medien (ZDF, Stern), die den Deutschen Schulpreis ausgelobt haben. (DeppeWolfinger, 2009, S. 54, Herv. im Orig.) 
Das Verhältnis zwischen „Inklusion“ und Schulentwicklung ist also nicht umfassend geklärt. Es bleibt fraglich, inwiefern schulische Integration einen Teilbereich schulischer Qualitätsentwicklung darstellt oder aber inwiefern qualitative Entwicklungsprozesse in Schulen sich auf Aspekte schulischer Integration auswirken bzw. diese quasi „automatisch mitbearbeiten“. Im Kapitel Fazit und Perspektiven wird die Frage nach diesem Verhältnis wieder aufgegriffen.

\subsubsection{Begründung der Auswahl des Rahmenmodells für die Kategorienbildung}

Welche Systematisierung erscheint sowohl für die Analyse von Forschungsergebnissen als auch als Kategoriensystem für das Forschungsprojekt innerhalb dieser Arbeit geeignet? Die Auseinandersetzung mit dieser Fragestellung hat einerseits im Blick, dass es im Rahmen der Forschungsarbeit darum gehen wird sich mit der qualitativen Umsetzung von Integration in einzelnen Schulen zu befassen ohne weitere Ebenen (Bsp.: Kommune, politische Rahmenbedingungen) detailliert einbeziehen zu können. Andererseits wird es - anders als bei der Längsschnittstudie von Bryk et al. - nicht möglich sein, die dynamischen Wechselbeziehungen zwischen den einzelnen Bereichen der Qualitäts- bzw. Schulentwicklung zu untersuchen. Daher kommen die o.g. Mehrebenenmodelle (Fend, Dlugosch, Maykus), aber auch das von Bryk et al. entwickelte dynamische Modell für die vorliegende Arbeit nicht in Frage.

Grundlage der Entscheidung für ein Modell ist vielmehr zunächst die unter 3.1.2 bereits erwähnte Auffassung, dass „die Einzelschule als Organisation einen empirisch nachweisbaren Einfluss auf den Lernzuwachs von Schülerinnen und Schülern hat.“ (Bos et al., 2010, S. 62) und damit als „Motor der Schulentwicklung“ (Dalin \& Rolff, 1990, S. 54) angesehen werden kann:

Baumert et al. (2003) beziffern die Varianzaufklärung der Einzelschule nach Analyse der BIJU-Datenbasis mit circa 16 Prozent, womit diese an Erklärungskraft für das Zustandekommen von Leistungsunterschieden sowohl die Klassenebene (5 Prozent) als auch die Schulformzugehörigkeit (10 Prozent) übertrifft. Die Autoren resümieren: „Der Schluss, den man aus den Ergebnissen ziehen muss, ist offenkundig. Sowohl Schulformen als auch Einzelschulen innerhalb derselben Schulform stellen institutionell vorgeformte differenzielle Entwicklungsmilieus dar. Schüler und Schülerinnen mit gleicher Begabung, gleicher Fachleistung und gleicher Sozialschichtzugehörigkeit erhalten je nach Schulformzugehörigkeit und je nach besuchter Einzelschule unterschiedliche Entwicklungschancen. (Baumert et al., 2003, S. 288) $)^{16}$

Auch die anglo-amerikanische Schuleffektivitätsforschung (Silins \& Mulford, 2002; zitiert nach Keller-Schneider \& Albisser, 2013, S. 36f) kommt zu dem Ergebnis, dass etwa $20 \%$ der Leistungsunterschiede durch Einzelschulen bedingt sind. Hattie weist in diesem Zusammenhang darauf hin, dass schulstrukturelle Effekte wie Klassengröße, Sitzenbleiben, äußere Differenzierung usw. seiner Metastudie nach nur sehr geringe Auswirkungen auf die Lernerfolge von Schülern haben, im Gegensatz zu schulinternen Maßgaben wie einem lernförderlichen Klima (Hattie, 2009, S. 33).

Die oben dargestellten einzelschulbezogenen Modelle von Reiser, Preuß sowie Feuser \& Meyer greifen zwar einzelne qualitative Bausteine schulischer Integration auf, bieten allerdings jeweils keinen übergeordneten Ansatz, der alle in Schulen möglichen relevanten Aspekte einschließt. Der Index für Inklusion wiederum ist so stark ausdifferenziert, dass eine gezielte Zuordnung

$16 \mathrm{BIJU}=$ Bildungsverläufe und psychosoziale Entwicklung im Jugend- und jungen Erwachsenenalter (Baumert, J., Gruehn, S., Heyn, S., Köller, O. \& Schnabel, K.-U. (1997). Bildungsverläufe und psychosoziale Entwicklung im Jugendalter (BIJU). Dokumentation - Band 1. Skalen Längsschnitt I, Welle 1-4. Berlin: Max-Planck-Institut für Bildungsforschung 
von Forschungsergebnissen schwer fallen kann. Dieses Modell bietet dementsprechend nicht die - vor allem für die qualitative Teilstudie - entscheidende Offenheit für mögliche erweiternde Kategorien.

Aus diesen Gründen wird als Kategorienrahmen für die vorliegende Arbeit das vergleichsweise offene Modell der Schulentwicklung nach Rolff gewählt. Bei der Analyse der Forschungsveröffentlichungen aus den Bereichen der Allgemeinen Pädagogik und der Sonderpädagogik stellt sich heraus, dass sich alle Kategorien, Subkategorien und Indikatoren den Bereichen Organisations-, Personal- und Unterrichtsentwicklung zuordnen lassen und damit innerhalb dieses Modells zu verorten sind. Dabei werden für diese drei Teilbereiche nicht die von Rolff gewählten Subkategorien (Rolff, 2010, S. 37) übernommen. Stattdessen werden lediglich die Oberbegriffe verwendet, für die eine eigene Kategorien-, Subkategorien- und Indikatorenbildung möglich erscheint. In Anlehnung an Wember \& Prändl (2009) werden dabei die drei Ebenen Ressourcen, Prozesse und Ergebnisse unterschieden. Die Forschungsergebnisse werden daher im Folgenden (ab 3.3) entsprechend dieser Systematisierung geordnet.

Die Auswahl der Kategorien und Subkategorien zur Organisations-, Personal- und Unterrichtsentwicklung orientiert sich an der Anzahl der Nennungen dieser Subkategorien in den aufgeführten Veröffentlichungen und ist somit keine subjektive Auswahl. Mit der Zusammenfassung des Forschungsstands in dieser Form werden zwei Zielsetzungen verfolgt: zum einen eine perspektivisch verschränkte Sicht auf die Qualität schulischer Integration, die in Regelschulen erfolgt, die sich hinsichtlich ihrer Organisations-, Personal- und Unterrichtsentwicklung teilweise erheblich unterscheiden, zum anderen die Entwicklung eines Kategoriensystems als Grundlage für die Entwicklung und Bearbeitung eigener Forschungsfragestellungen.

Begrenzungen ergeben sich bei diesem Ansatz dahingehend, dass zwar eine klare Systematisierung vorgenommen wird, diese aber ein Konstrukt bleibt, da sich inhaltlich abgegrenzte Kategorien in der tatsächlichen Schul- und Unterrichtspraxis sowie auch auf theoretischer Ebene überlappen können. Dadurch können sich innerhalb des Kategorienmodells Zuordnungsschwierigkeiten ergeben. Aus sonderpädagogischer Sicht könnte kritisiert werden, dass bspw. spezifische Förderbedürfnisse von Schülern der Kategorie „Individualisierung “ zugeordnet werden, so dass „sonderpädagogische“ Kategorien in „allgemeinpädagogische“ Kategorien eingehen. Darüber hinaus kann das Modell zwar Qualitätskategorien erfassen, ermöglicht allerdings nicht eine Messung der Effektivität dieser Kriterien. Trotz quantitativer Auszählung von Kategorien, Subkategorien und Indikatoren bleibt die Auswahl der Kategorien wegen der zugrundeliegenden Auswahl von Forschungsarbeiten und Qualitätsübersichten letztendlich begrenzt, da nicht alle relevanten Forschungsarbeiten berücksichtigt werden können. Darüber hinaus ist die Qualität der Forschungsveröffentlichungen sehr unterschiedlich. Zum Teil handelt es sich dabei um Erfahrungsberichte zu kleinen Stichproben. Dennoch wurden diese einbezogen, da es im deutschsprachigen Raum Anfang der 2010er Jahre nur wenige fundierte und umfassende sonderpädagogische Studien gab, die versucht haben die Qualität schulischer Integration gezielt zu erfassen. Kategorien und Subkategorien des Modells stellen keine kohärenten Theorien oder homogene Konstrukte dar (Helmke, 2015, S. 169), sondern sind letztlich eine pragmatische Auswahl (Begrenzung der Anzahl der Bereiche). Sie können daher als "Schubladen“ verstanden werden, „die inhaltlich verwandte, aber durchaus heterogene Facetten und Forschungstraditionen enthalten" (Helmke, 2015, S. 169). Insgesamt gehen die Kategorien hauptsächlich aus Veröffentlichungen hervor, die die Perspektiven von Lehrkräften erschließen, Eltern bzw. Schülerinnen und Schüler sind bislang bislang nur zum Teil in Forschungsansätze einbezogen. 
Um die Orientierung in den Kapiteln 3.5 bis 3.7 zu erleichtern, zeigt die folgende Grafik alle Kategorien, die sich bei der Analyse ergeben haben.

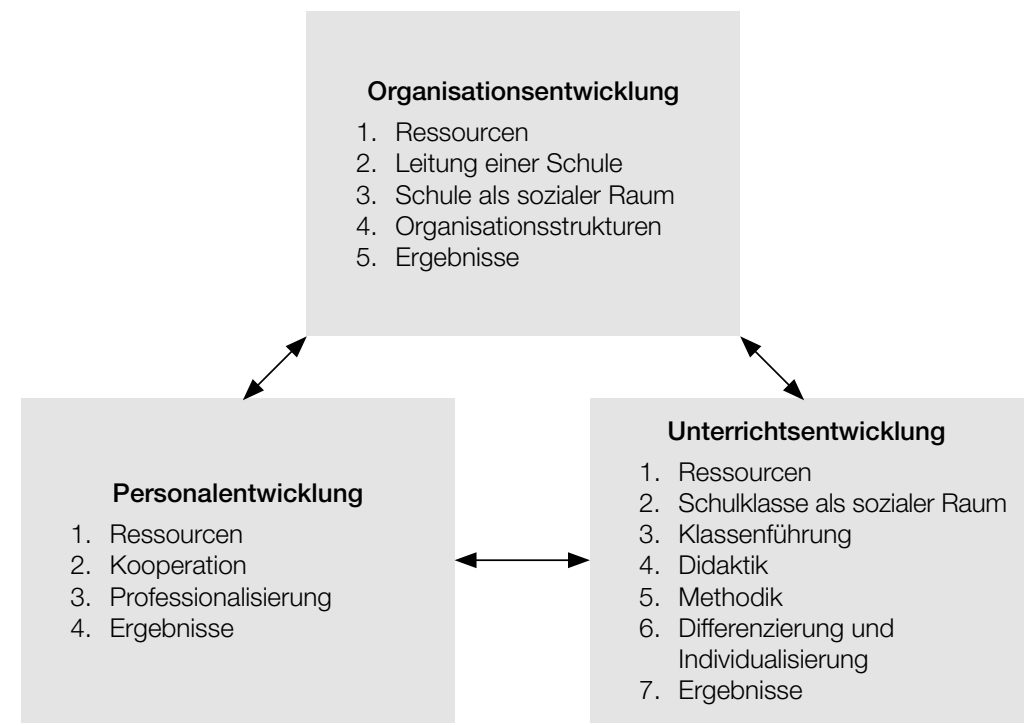

Abb. 3: Übersicht über das Kategoriensystem für schulische Integration

Das Besondere des Forschungsfeldes „Schulentwicklung“ liegt darin begründet, dass es dabei um die Untersuchung der (Weiter-)Entwicklung der Qualität von Schulen geht und somit um die Analyse vielschichtiger Prozesse, die (zeitlich) parallel ablaufen, sich in Kreisläufen bewegen und über die Einzelschulen hinaus als zentrale Wirkfaktoren von Entwicklung aufgefasst werden können. Die Schulentwicklungsforschung im engeren Sinne prüft, „[...] wie sich Autonomie von Schulen auswirken kann und welchen Beitrag eine erweiterte Selbständigkeit zur Verbesserung von Qualität von Schule und Unterricht leisten kann.“ (Pfeiffer, 2010, S. 21) Ein weiteres Verständnis dieses Forschungsansatzes kennzeichnet Holtappels wie folgt:

Schulentwicklungsforschung umfasst ein Spezialgebiet, das zwischen Bildungs-, Schul- und Innovationsforschung anzusiedeln ist und mit diesen Forschungsgebieten korrespondiert, aber eine eigene Forschungsrichtung ausmacht. Schulentwicklungsforschung untersucht mit besonderem Fokus auf Wandel und Reform die Voraussetzungen und Bedingungen, Formen und Prozesse sowie Ergebnisse und Wirkungen im Schulbereich, und zwar auf mehreren Ebenen: Systemebene, Ebene der einzelnen Schule sowie innerhalb der Schule auf der Ebene der Lerngruppen und des Lehrerhandelns. (Holtappels, 2010d, S. 26)

Problematisch erweist sich in diesem Zusammenhang vor allem die Tatsache, dass die Wirkfaktoren von Schulentwicklungsprozessen zumeist isoliert betrachtet werden, obwohl diese sich in einem hochkomplexen Wirkungsgefüge befinden:

Schools are complex organizations consisting of multiple interacting subsystems. Each subsystem involves a mix of human and social factors that shape the actual activities that occur and the meaning that individuals attribute to these events. These social interactions are bounded by various rules, roles, and prevailing practices that, in combination with technical resources, constitute schools as formal organi- 
zations. In a simple sense, almost everything interacts with everything else. A key in theory formation for both analytic and clinical purposes is to figure out how "to carve this complexity“ at the joints. (Bryk et al., 2010, S. 45)

Auch wenn die vorliegende Arbeit weder dieses Wirkungsgefüge an sich noch die Schulen umgebenden Makroebenen untersucht, bleibt für die Einordnung von Forschungsergebnissen wesentlich, dass sowohl das komplexe Wirkungsgefüge als auch der Makrorahmen (Bsp.: Bildungspolitik, gesellschaftliche Prozesse) gesehen werden und bei der Einordnung der Ergebnisse in Fazit und Perspektiven eine Rolle spielen. Sie soll dazu dienen Qualitätsbausteine guter (inklusiver) Schulen zu erfassen, die im Anschluss in dynamische Modelle oder Mehrebenenmodelle überführt werden können:

Künftig wird es vermehrt darum gehen müssen, mit Hilfe von theoretisch stark fundierten Mehrebenenmodellen die jeweiligen Effekte, die die Einzelschule auf Unterricht und darüber vermittelt auf Schülerleistungen hat, zu identifizieren. [...] Des Weiteren gilt es, die bekannten Qualitätskriterien von Einzelschulen weiter auszudifferenzieren. (Bos, W.; Bonsen, M.; Berkemeyer, N., 2010, S. 65; in Bohl et al. 2010, 62-65)

Bevor die Forschungsergebnisse im Einzelnen beschrieben werden, erfolgt zunächst ein grober Überblick über Forschungsveröffentlichungen der Integrations- bzw. Inklusionsforschung sowie eine kurze Übersicht zu übergreifenden Forschungsergebnissen zu (inklusiver) Schulentwicklung.

\subsection{Forschung zu Integration und Inklusion in den vergangenen vier Jahrzehnten}

Bei der Auseinandersetzung mit der wissenschaftlichen Aufarbeitung der Qualität integrativer Arbeit in Regelschulen wird schnell deutlich, dass diese im Verlauf der vergangenen Jahrzehnte mit sehr unterschiedlichen Schwerpunktsetzungen verknüpft war. In den Anfängen stand dabei die Begleitforschung zu Schulversuchen im Vordergrund (z.B. Preuss-Lausitz, 1981; Reiser et al., 1984; Wocken \& Antor, 1987; Hug, 1994b). Diese Begleitforschung wurde oft von den Bildungsministerien in Auftrag gegeben. Neben der empirischen Zielsetzung erfolgte häufig eine Prozessbegleitung, zum Teil verknüpft mit einer Beratung der Akteure (Preuss-Lausitz, 2014, S. 1). Das Forschungsdesideratum dieser Veröffentlichungen hing u.a. damit zusammen, dass „Integrationsklassen in der Grundschule [...] gleichsam theorielos [starteten]“ (Bleidick \& Ellger-Rüttgardt, 2008, S. 42). Muth äußerte zur damaligen Zeit dazu:

Einschränkend muß allerdings darauf hingewiesen werden, daß bis heute noch weithin empirische Untersuchungen fehlen, deren Befunde eine optimale Gestaltung der gemeinsamen Erfahrung und des gemeinsamen Umgangs von Behinderten und Nichtbehinderten ermöglichen. Hier hat die Forschung noch ein weites Feld. (Muth, 1977, S. 93)

Die Begleitforschung zu schulischen Modellversuchen im Zusammenhang mit der Integration von Schülern mit Behinderungen untersuchte mehrere Forschungsschwerpunkte: den gemeinsamen Unterricht im engeren Sinn, die Darstellung unterschiedlicher Modelle der Umsetzung schulischer Integration, Untersuchungen mit Fokus auf verschiedene Behinderungen, die Kooperation von Regel- und Förderschullehrkräften, Maßnahmen der Regelschulen sowie Einstellungen von Lehrkräften und Eltern. Die Berichte zu den Modellversuchen fragen also nicht übergreifend nach der Qualität dieser Maßnahmen, sondern nach spezifischen Schwerpunkten (Bsp.: Vergleich der Beschulung von Schülern mit spF in Regel- und Förderschulen, Integration von Schülern mit spezifischen Behinderungen, Kooperation von Förder- und Regelschullehrern). 
Gemeinsamer Unterricht im engeren Sinn fand in Anbetracht der Fülle der Modellversuche in den 70er- und 80er- Jahren nur an wenigen Einzelschulen statt. Als exemplarische Vorreiter integrativer Unterrichtung galten damals u.a. die Integrationsklassen an der Fläming-Grundschule in Berlin sowie die Grundschule Aktion Sonnenschein in München (Hellbrügge, 1977; Prell \& Link, 1977; Projektgruppe Integrationsversuch, 1988; Stoellger, 1988). Die Darstellung unterschiedlicher integrativer Modelle bezog sich bspw. auf kooperative Modelle zwischen mehreren Schulen (Muth et al., 1976) oder die parallele Förderung innerhalb von Regelschulen (Preuss-Lausitz, 1981; Wocken \& Antor, 1987). Im Rahmen der wissenschaftlichen Begleitung dieser Modellversuche standen bspw. Aspekte wie die Aufnahmebedingungen für Schüler, die Sozialentwicklung (Mühl, 1987; Maikowski \& Podlesch, 1988; Dumke et al. 1989), die Leistungsentwicklung von Schülern mit und ohne Behinderungen (Maikowski \& Podlesch, 1988; Dumke et al., 1989) sowie mögliche Grenzen der gemeinsamen Unterrichtung in integrativen Klassen (Mühl, 1987; Bleidick, 1990, S. 9-32) im Blickpunkt. Einige Forschungsarbeiten bezogen die Elternperspektive ein (Dumke et al., 1989; Hetzner, 1988, S. 281-292).

Eine ganze Reihe von Autoren untersuchte die Möglichkeiten integrativer Unterrichtung für bestimmte Behinderungen bzw. Förderbereiche (Muth et al., 1976; Feuser \& Meyer, 1987; Mühl, 1987; Projektgruppe Integrationsversuch, 1988; Dumke et al., 1989). Einige Regelschulen spezialisierten sich auf die Integration von Schülern mit unterschiedlichen Förderschwerpunkten bzw. auf unterschiedliche integrative Ansätze. So konzentrierten sich die Max-Tau Grundschule in Kiel sowie die Grundschule Pürschweg in Bremen auf Sprachförderung oder Grundschulen in Frankfurt a.M. und Braunschweig auf die Arbeit mit verhaltensauffälligen Schülern (Muth et al., 1976). Untersuchungen zu Veränderungen des Unterrichts bezogen sich bspw. auf „open education“ (Grundschule Hannover-Glocksee, Grundschule „Tempelhof “ Berlin) oder die Umsetzung des Team-Kleingruppen-Modells an Gesamtschulen (IGS Hannover-Linden; Grundschule Berlin-Tempelhof) (Preuss-Lausitz, 1981, S. 111, S. 227ff). Ein weiteres Forschungsfeld stellte die Untersuchung der Kooperation von Regel- und Förderschullehrkräften dar (Reiser et al., 1984). Unterschiede zwischen diesen Forschungsarbeiten bestehen dabei in verschiedenen Dimensionen: zum einen mit Blick auf den jeweiligen wissenschaftlichen Forschungsansatz (eine Fülle qualitativer wie quantitativer Studien unterschiedlicher Qualität), zum anderen hinsichtlich der Wahl der befragten bzw. untersuchten Personengruppe (Schüler, Eltern, Lehrkräfte). Deutlich wird die große Bandbreite, wenn man sich die geradezu unüberschaubare Vielfalt der Modellversuche aus den 70er- und 80er-Jahren vor Augen hält, die selbstverständlich nicht nach einheitlichen Kriterien bewertet wurden. Im Gegenteil - in den meisten Fällen überwiegen bei der Darstellung der unterschiedlichen integrativen Ansätze in den Schulen eher persönlich gehaltene Erfahrungsberichte, die sich teilweise auf einzelne Schüler, also Fallbeispiele, beziehen. So interessant diese Berichte auch für pädagogisch interessierte Leser sind, für die sonderpädagogische Forschung und Theorieentwicklung im Hinblick auf integrative Unterrichtung reichten diese Darstellungen offensichtlich nicht aus.

Im Anschluss wurden spezifische Fragestellungen herausgegriffen, die sich bspw. auf Prozesse im Zusammenhang mit schulischer Integration beziehen (Innerhofer \& Klicpera, 1991; Feyerer, 1998), die Leistungsentwicklung behinderter und nichtbehinderter Kinder in Integrationsklassen untersuchen (Aigner, 1989; Unterleitner, 1990; Dumke, 1991; Bless \& Klaghofer 1991; Dumke \& Schäfer, 1993; Hildeschmidt \& Sander, 1995; Haeberlin et al., 2003; Bless, 2003; Wocken, 2005) oder sich mit Emotionalität und Selbstkonzept befassen (Huber, 2009; Venetz \& Tarnutzer, 2012). In den 2010er Jahren setzte - u.a. als Folge der UN-BRK - eine neue Welle von Forschungsprojekten ein, die inklusive Entwicklungen untersuchen (Arndt \& Werning, 2013; Textor, 
2015; Lütje-Klose et al., 2018) und erneut Pilotprojekte begleiten (Schön \& Stark, 2014; Landesinstitut für Schule und Medien des Landes Brandenburg (LISUM), 2015) oder die Umsetzung von Inklusion in bestimmten Bundesländern in den Blick nehmen (Laubenstein et al., 2015) (s. dazu Übersicht von Preuss-Lausitz, 2014, S. 5ff). Weitere Veröffentlichungen, die zumindest zum Teil auf Forschungsergebnissen beruhen, formulieren qualitative Kriterien für schulische Integration bzw. Inklusion (Boban \& Hinz, 2003; Brugger-Paggi, 2003; Deutsches Schulamt, 2004; Specht et al., 2006; BMBF Österreich, 2008; Wember \& Prändl, 2009).

Umfassendere Forschungsarbeiten mit Blick auf die Qualität integrativer bzw. inklusiver Schulen gibt es bislang nur wenige:

However, widespread as this view was in the literature we reviewed, the evidence supporting it was decidedly flimsy. For the most part, studies took the form of single school or small- $n$ case studies where the schools were identified as inclusive on the basis of nomination or self-identification, rather than through some independent assessment of their inclusivity. Fieldwork tended to be limited and to draw heavily on interviews with teachers and school leaders rather than on observations of practice or impacts on children. There were very few comparative studies between more and less inclusive schools to identify the distinguishing features of either. All of this leaves a nagging doubt about much of the research literature on inclusive schools. (Dyson, 2010, S. 4)

Zu diesen Arbeiten zählen u.a. die von Innerhofer \& Klicpera (1991), Specht et al. (2006) oder neuere Begleitforschung zu inklusiven Schulen aus den 2010er Jahren (Laubenstein et al., 2015; LISUM, 2015). Parallel zu den Aufarbeitungen aus sonderpädagogischer Perspektive gab und gibt es jeweils auch Forschungsbemühungen der allgemeinen Schul- und Unterrichtsforschung (Brophy, 2000; Bryk et al., 2010; Helmke, 2010; Hattie, 2013), die Qualitätsmerkmale von Schulen bzw. Unterricht fokussieren. Erst seit den 2000er Jahren häufen sich Veröffentlichungen, die Forschungsergebnisse zu Integration bzw. Inklusion als relevanten Bestandteil von Schul- und Unterrichtsforschung einordnen (Moser \& Egger, 2017; Lütje-Klose et al., 2018). Insbesondere aus Forschungsberichten zu Modellversuchen sowie aus Studien, die bestimmte Aspekte schulischer Integration untersuchen, lassen sich inhaltsanalytisch zentrale Qualitätskategorien, -subkategorien und -indikatoren ableiten. Wesentliche Ergebnisse werden daher in den folgenden Unterkapiteln jeweils thematisch geordnet dargestellt.

\subsection{Organisationsentwicklung}

Was versteht man unter der Organisationsentwicklung von Schulen? Welche Qualitätsbausteine spielen im Zusammenhang mit Organisationsentwicklung eine Rolle? Welche davon sind für schulische Integration von besonderer Relevanz? Welche Rolle spielt die Schulleitung in integrativ arbeitenden Schulen? Wie werden Eltern und Schüler in den Schulen einbezogen? Mit welchen Institutionen und Vernetzungspartnern arbeiten sie zusammen und wie wird diese $\mathrm{Zu}$ sammenarbeit gestaltet? Welche Organisationsstrukturen werden in diesen Schulen umgesetzt (Bsp.: Formen äußerer Differenzierung, jahrgangsübergreifender Unterricht)? Diese Fragen versucht das folgende Kapitel zu beantworten. Am Beginn steht dabei eine kurze theoriebezogene Beschreibung dieses Bereichs, dessen Grundlagen vor allem aus der Organisationspsychologie hervorgegangen sind. Im Anschluss werden die Kategorien der Organisationsentwicklung vorgestellt, die sich bei der Analyse von sonderpädagogischen Forschungsarbeiten als bedeutsam für schulische Integration herausgestellt haben. Dazu gehören neben den Ressourcen und Ergebnissen von Organisationsentwicklung die Prozesskategorien Leitung einer Schule, Funktion der Schule als sozialer Raum sowie Organisationsstrukturen. 
Nach Holtappels (2010c) ist das organisationale Lernen als Konzept von Argyris und Schön (1978) entwickelt worden. Es geht davon aus, dass Organisationen Lernsysteme sind, in denen Individuen kollektiv bspw. Normen und Handlungsstrategien entwickeln:

Organizational learning is defined [here] as the process within the organization by which knowledge about action-outcome, relationships and the effect of the environment on these relationships is developed. (Duncan \& Weiss, 1979, S. 84; zitiert n. Holtappels, 2010c, S. 100)

Senge (1990) und Argyris (1996) haben die Idee der Lernenden Organisation weiter ausgearbeitet und differenzieren hinsichtlich des Ablaufs zwischen verschiedenen Phasen (Planung, Aktion, Reflexion, Transfer (Schley, 1998, S. 22; n. Holtappels, 2010c, S. 101)). Rolff selbst definiert Organisationsentwicklung (OE) wie folgt und unterscheidet dabei drei aufeinander folgende Phasen (Initiation, Implementation und Inkorporation):

Organisationsentwicklung bedeutet, eine Organisation von innen heraus weiterzuentwickeln und zwar im Wesentlichen durch deren Mitglieder selbst, wobei der Leitung eine zentrale Bedeutung zukommt und nicht selten Prozessberater von außen hinzugezogen werden (vgl. dazu French \& Bell 1990). OE wird als Lernprozess von Menschen und Organisationen verstanden. (Rolff, 2010, S. 30)

Eine Weiterentwicklung haben Holtappels \& Feldhoff mit dem Konzept des „Change-Managements" gestaltet, das die Rolle der Führung einer Organisation stärker in den Blick nimmt:

Change Management bezieht sich auf das Management von Veränderungsprozessen und kann als bewusster Steuerungsprozess verstanden werden, der die Veränderungen in einer Organisation auf formaler Ebene, vor allem durch Änderung der Aufbauorganisation und auf der Prozessebene für Organisation und Personal initiiert und steuert. (Holtappels \& Feldhoff, 2010a, S. 160)

Holtappels und Rolff ergänzen, dass Organisationsentwicklung ein geplantes Verfahren „zur Veränderung des Sozialverhaltens von Organisationsmitgliedern und zugleich der Organisationsstrukturen zum Zweck verbesserter Aufgabenerfüllung [sei]“ (Holtappels \& Rolff, 2010b, S. 76). Im Mittelpunkt der Organisationsentwicklung an Schulen stehen eine förderliche Schulkultur und die Entwicklung eines Schulprogramms (Holtappels \& Rolff, 2010b, S. 76). Für Bryk et al. ist wesentlich, dass Organisationsentwicklung eng mit Personal- und Unterrichtsentwicklung verknüpft ist und nur dann als effektiv bewertet werden kann, wenn sie sich auf die Arbeit von Lehrkräften sowie die Leistungen von Schülern positiv auswirkt: „To the point, if restructuring school organization matters, its effects must largely accrue through influencing the conditions under which teachers work and engage with students around subject matter in the classroom." (Bryk et al., 2010, S. 47). Forschungsergebnisse zum organisationalen Lernen an sich liegen nach Holtappels \& Feldhoff (2010) bislang nicht in umfassender Form vor. Die Nutzung eines Indexes von Marks und Louis (1999) im Rahmen der Begleitforschung zum Modellvorhaben Selbständige Schule in Nordrhein-Westfalen ermittelte positive Effekte auf Arbeitsklima sowie Arbeitszufriedenheit, Unterrichtsqualität und Schülerlernen (Feldhoff, 2008; n. Holtappels, 2010c, S. 104).

\subsubsection{Ressourcen im Bereich der Organisationsentwicklung}

Kinder und Schule - ob Regel- oder Förderschule - taugen nicht für politische Experimente. Deshalb muss eines ganz klar sein: Ein qualitativ hochwertiges, inklusives Bildungssystem gibt es nicht über Nacht und nicht umsonst. Wer schulische Inklusion will, der muss investieren: in Infrastruktur, in Personal, in individuelle Unterstützungsangebote. (Evers-Meyer, 2010, S. 30) 
Schulen verursachen Kosten. Während die Träger (Kommunen, Städte, freie Träger) als Sachaufwandsträger für die Kosten aufkommen, die mit den Gebäuden, der Gebäudeunterhaltung und den Materialien zusammenhängen, die in Schulen benötigt werden, zahlen die Länder die Personalkosten. Daher betrifft die Bereitstellung von finanziellen Mitteln sowohl die Schulträger im Hinblick auf bauliche Maßnahmen sowie die Ausstattung mit Lehr- und Lernmitteln an den Schulen, als auch die Landesministerien für Bildung bzw. die Sozialministerien der Länder bezüglich der Einstellung des Personals und des Angebots von umfassenden Fortbildungsmaßnahmen. In diesem Zusammenhang sind auch finanzielle Vorgaben, die das Regelschulsystem an sich im Fokus haben, zu berücksichtigen (Bsp.: Lehrerwochenstunden pro Schüler, Klassenteiler etc.).

An Schulen, die qualitativ hochwertige Arbeit leisten wollen, spielen sowohl die räumlichen Bedingungen und die Materialausstattung als auch die personelle Ausstattung eine wesentliche Rolle. Die Schulen, an denen Kinder mit Einschränkungen oder Behinderungen unterrichtet werden, sind zum Teil auf eine barrierefreie Gestaltung angewiesen. Der Personalschlüssel an diesen Schulen ist höher. Neben Regel- und Förderschullehrern werden dort unter Umständen auch Pädagogische Fachkräfte, Integrationshelfer, medizinische Fachkräfte, Bundesfreiwilligendienstler etc. beschäftigt. Während die Ressourcen, die das Personal betreffen, im Kapitel 3.4.1 dargestellt werden, und diejenigen, die den Unterricht direkt betreffen, in Kapitel 3.5.1, beschreibt die Kategorie „Ressourcen“ im Bereich der Organisationsentwicklung zunächst diejenigen, die Gebäude und Material umfassen.

Die Problematik, dass begrenzte finanzielle Ressourcen die Entwicklung schulischer Integration einschränken könnten, wurde - wie unter 2.1 aufgegriffen - schon Ende der 70er Jahre erkannt: „Auch kann und darf die in finanzpolitischen Gründen liegende Beschränkung der Realisierungsmöglichkeiten denkbarer Konzeptionen nicht übersehen werden.“ (Vds, 1975, S. 662). Aufgrund der Modellversuche gingen die Sonderschulreferenten der Länder bereits 1983 davon aus, dass „bei einer verstärkten gemeinsamen Unterrichtung behinderter Schüler mit nichtbehinderten Schülern höhere Aufwendungen notwendig werden, als sie derzeit in Sonderschulen anfallen“ (KMK, 1983, S. 16). Teilweise wurde daher der so genannte „Finanzierungsvorbehalt“ in Landesgesetzen zur schulischen Integration konkret festgehalten (Sander, 2003, S. 11; Speck, 2010, S. 48f), wie im folgenden Abschnitt der Integrationsverordnung des Saarlandes:

\section{$\S 2$ Formen integrativer Unterrichtung}

(2) Bei der Entscheidung über die integrative Unterrichtung eines Schülers/einer Schülerin ist die Organisationsform zu wählen, die auf der Grundlage der an der betreffenden Schule der Regelform jeweils vorhandenen baulichen, räumlichen, sächlichen, personellen und organisatorischen Gegebenheiten den individuellen Bedürfnissen des betreffenden Schülers/der betreffenden Schülerin, die sich aus der Art und Schwere seiner/ihrer Behinderung sowie aus dem Umfeld seines/ihres schulischen Lernens ergeben, am besten gerecht wird. (Ministerium für Bildung des Saarlandes, 2003, S. 2)

Daher geht Sander (2003) in Anlehnung an Lersch (2001) davon aus, dass schulische Integration sich nur entsprechend begrenzt entwickeln konnte:

Der überall geltende Haushaltsvorbehalt bremst die weitere Entwicklung schulischer Integration von Kindern und Jugendlichen mit Behinderungen auch dort, wo sie politisch gewollt ist, bzw. lässt sie nur unter verschlechterten Bedingungen zu - hier ist Wachsamkeit geboten, wenn das Recht auf Nichtaussonderung droht, um den Preis einer nicht hinreichenden besonderen Förderung erkauft zu werden! (Lersch, 2001, S. 58; zitiert n. Sander, 2003, S. 119) 
Und Ende der 2000er Jahre betont die Europäische Kommission (2009), dass

[...] Finanzierungsstrategien und -strukturen [...] nach wie vor zu den wichtigsten inklusionsbestimmenden Faktoren [zählen]. Eingeschränkter oder fehlender Zugang zu bestimmten Einrichtungen und Angeboten kann die Inklusion und Chancengleichheit von Lernenden mit sonderpädagogischem Förderbedarf behindern. (Europäische Agentur für Entwicklungen in der sonderpädagogischen Förderung, 2009, S. 23)

Textor äußert in diesem Zusammenhang die Befürchtung, dass von der mangelhaften Ressourcenausstattung der Regelschulen politisch die „Gefahr indirekter Steuerung“ (Textor, 2015, S. 52) ausgehe, da Eltern sich für den Besuch einer Förderschule entscheiden könnten, wenn die Rahmenbedingungen für individuelle Förderung in den Regelschulen nicht ausreichend seien. Auch wenn die Bundesregierung 2001 betont, dass sie sich für inklusives Lernen einsetzt (BMAS, 2011, S. 45), enthält der Nationale Aktionsplan - anders als in den Ausführungen zu beschäftigungspolitischen Maßnahmen, bei denen eine Summe von 100 Millionen Euro zur Finanzierung der „Initiative Inklusion“ benannt wird - keine Hinweise darauf, dass Ressourcen für das schulische Bildungswesen von den Ländern bereitzustellen sind. Die dazu passende Formulierung lautet stattdessen:

Im Rahmen ihrer Zuständigkeiten und Möglichkeiten wird die Bundesregierung Länder und Schulträger zum Ausbau der Angebote des gemeinsamen schulischen Lernens aktiv auffordern und in diesem Prozess weiterhin unterstützen. (BMAS, 2011, S. 46)

Die Länder wiederum „[... s sollen eine Bestandsaufnahme vornehmen, Schritte der Weiterentwicklung festlegen, entsprechende Maßnahmen veranlassen und die gegebenenfalls erforderlichen rechtlichen Maßnahmen zur Steigerung der inklusiven Bildung an allgemeinbildenden Schulen entwickeln." (BMAS, 2011, S. 46). Die Kultusministerkonferenz fordert diesbezüglich:

Insbesondere sind die Träger von Eingliederungshilfen nach dem Sozialgesetzbuch entsprechend ihren jeweiligen Zuständigkeiten, Kompetenzen und Aufgaben frühzeitig einzubeziehen. Dies gilt insbesondere für die Sicherstellung der Barrierefreiheit, Sicherstellung der Schülerbeförderung, Ausstattung mit vielfältigen Lehr- und Lernmitteln, Gewährleisten von Nachteilsausgleich, Assistenz und angemessener Kommunikationsmöglichkeiten sowie das Einbeziehen anderer Fachdienste. (KMK, 2010, S.9f)

Eine genaue Festlegung der Ressourcen, die zur Verfügung stehen müssen um inklusive Unterrichtung erfolgreich und qualitativ hochwertig zu gestalten, erfolgt seitens der Bildungspolitik also nicht. Im Zusammenhang mit den möglichen Kosten, die für inklusive Bildung entstehen könnten, sind zwei Gutachten erstellt worden (Klemm, 2009; Dohmen \& Fuchs, 2009). Während Klemm in seinem Gutachten zum Ausbau eines inklusiven Bildungssystems in Deutschland - je nach Berechnungsvariante - zu Ergebnissen jährlicher Mehrausgaben zwischen 0,24 bis 0,66 Milliarden Euro kommt (Klemm, 2009, S. 29), beziffern Dohmen und Fuchs in ihrem von den GRÜNEN in Auftrag gegebenem Gutachten diese Summe mit etwa 1,8 bis 4,3 Milliarden Euro pro Jahr (Dohmen \& Fuchs, 2009, S. 29). So weist Klemm in seinem Gutachten darauf hin, dass eine inklusive Unterrichtung zwar höhere Personalkosten erfordere, dafür aber Einsparungen beim Gebäudeunterhalt von Förderschulen sowie mit Blick auf Transportwege von Schülern zu erwarten seien (Klemm, 2009, S. 15ff). Dadurch würden die Länder als Arbeitgeber zwar be-, die Kommunen als Träger der Schulen aber entlastet. Aufgrund der Schwierigkeit zu erwartende Veränderungen in den Bereichen nicht-lehrendes Personal (Bsp.: Integrationshilfen), Umrüstung und Bewirtschaftung von Schulgebäuden sowie Schülerfahrtkosten 
seriös zu ermitteln, beziehen sich die Kostenannahmen bei Klemm lediglich auf das Lehrpersonal, wobei er nicht zwischen Förder- und Regelschullehrkräften differenziert, sondern von Vollzeitlehrereinheiten spricht. Das Gutachten von Dohmen und Fuchs wiederum geht von der notwendigen Einstellung zusätzlicher Regelschullehrkräfte aus, da u.a. ein kleinerer Klassenteiler als notwendig angenommen wird. Sie differenzieren insgesamt zwischen Investitionen in den Bereichen Ganztagsschule, Integration von Förderschülern in Regelschulen, Teilhabekosten und Kosten von Lernangeboten für Schulabbrecher und wählen damit einen Ansatz, der von einem weiteren Inklusionsverständnis ausgeht. Die Annahme der Deutschen UNESCOKommission, dass es „weniger kostenintensiv [ist] Schulen einzuführen und zu erhalten, die alle Kinder gemeinsam unterrichten, als ein komplexes System unterschiedlicher Schultypen zu errichten, die jeweils auf verschiedene Gruppen spezialisiert sind.“ (Deutsche UNESCOKommission, 2010, S. 9, zitiert n. Speck-Hamdan, 2015, S. 13f) lässt sich mit Blick auf beide Gutachten nicht aufrechterhalten.

Ein weiteres Gutachten wurde durch Schwarz et al. (2013) am Beispiel der Städte Essen und Borken für das Land NRW erstellt. Es kommt zu folgendem Schluss:

Im Ergebnis zeigt sich, dass selbst bei einer eingeschränkten Ausstattung der Schulen, die zwar oberhalb der derzeitigen Ausstattung der allgemeinen Schulen, aber deutlich unterhalb der aktuellen Standards an Förderschulen liegt, und konservativer Schätzung der laufenden Kosten in beiden betrachteten Kommunen bis zum Schuljahr 2019/20 erhebliche zusätzliche Ausgaben entstehen, die jeweils deutlich oberhalb der Bagatellgrenze im Sinne des Konnexitätsausführungsgesetzes (KonnexAG) liegen. (Schwarz et al., 2013, S. 9)

Abgesehen von den spezifisch für inklusive Bildung zur Verfügung stehenden oder zu stellenden Ressourcen kann darüber hinaus kritisch hinterfragt werden, wie die Ressourcensituation für Schulen, Schulklassen und Unterricht insgesamt aussieht. So wurde schon Anfang der 80er Jahre von Schwartz kritisiert, dass Klassenfrequenzen in Grundschulklassen zu groß seien, zu wenige Lehrerwochenstunden pro Kind zur Verfügung stünden, die Ausstattung mit Lehr- und Lernmitteln bspw. im Vergleich zu der weiterführender Schulen unzureichend sei und die BRD im Primarbereich damit in der Europäischen Gemeinschaft das Schlusslicht bilde (Schwartz, 1982, S. 36). Auch aktuell sind die Bildungsausgaben der BRD im europäischen Vergleich niedriger als in vielen anderen EU-Staaten. Gemäß dem Education und Training Monitor der Europäischen Kommission entsprachen sie im Jahr 2018 einem Anteil am Bruttoinlandsprodukt von 4,2\% im Vergleich zu 6,4\% in Belgien, 6,6\% in Schweden oder 6,9\% in Dänemark (European Commission, 2018, S. 92). Noch deutlicher wird dies, wenn die konkreten Bildungsausgaben je Schüler miteinander verglichen werden:

While the data confirms Nordic countries' relatively high spending, small countries with a low proportion of students, such as Malta and Cyprus, are also at the top of the scale. Compared to these countries, the data reveal relatively low spending on education in the five most populated countries - Germany, France, the UK, Italy and Spain, each accounting for at least around $10 \%$ of the EU population (column 7). (European Commission, 2018, S. 93)

Der Deutsche Städtetag (2011) leitet aus Art 24 Abs 1 und 2 der UN-BRK finanzielle Erfordernisse $\mathrm{ab}$, die „die Beseitigung ökonomischer und räumlicher Hindernisse wie auch die Sicherstellung einer wohnortnahen inklusiven Beschulung“ (Dt. Städtetag, 2011, S. 9), Maßnahmen zur Ermöglichung individueller Unterstützung im Bildungsbereich (Bsp.: auch technische Hilfen) sowie die Einstellung und Fortbildung entsprechend qualifizierter Lehrkräfte betreffen 
(Dt. Städtetag, 2011, S. 5f). Differenziert werden also die Kostenfolgen bei den Ländern (Personalkosten inklusive Aus- und Fortbildungskosten, Entwicklung inklusiver Curricula) und bei den Kommunen (Fahrtkosten, Lehr- und Lernmittel, Barrierefreiheit Schulgebäude, ggf. spezielle Ausstattung mit Blick auf jeweilige Beeinträchtigungen, schulisches Ergänzungspersonal wie Integrationshelfer, Schulpsychologen und Sozialpädagogen). Insbesondere der zunehmende Einsatz von Integrationshelfern stelle für die Kommunen ein „erhebliches Kostenrisiko“ dar (Dt. Städtetag, 2011, S. 15). Hinsichtlich der Bereitstellung technischer Hilfen differenzieren Poscher u.a., dass für deren Finanzierung - je nach Leistungsanspruch - auch die gesetzlichen Krankenkassen zuständig sein können (Poscher et al., 2008, S. 54).

Mögliche Ressourcenvorbehalte der Länder und Kommunen sieht der Deutsche Städtetag kritisch, da die UN-BRK keinen Finanzierungsvorbehalt, sondern lediglich einen Progressionsvorbehalt formuliere, der beinhalte, dass die Vertragsstaaten unter Ausschöpfung ihrer Mittel nach und nach die volle Verwirklichung der Rechte von Menschen mit Behinderung zu erreichen hätten (Dt. Städtetag, 2011, S. 7). Der Städtetag nimmt daher an:

Insbesondere ein in Landesschulgesetzen normierter „starrer“ Haushaltsvorbehalt dürfte daher - jedenfalls auf Dauer - nicht als völkerrechtskonform zu qualifizieren sein. Stellt er doch angesichts der prekären Haushaltssituation der meisten Länder und Kommunen im Regelfall die entscheidende Schranke bzw. Hürde für die inklusive Beschulung dar. (Dt. Städtetag, 2011, S. 8)

Auf der anderen Seite wird aus juristischer Sicht befürchtet, dass die Vorschrift Menschen mit Behinderungen die notwendige individuelle Unterstützung zukommen zu lassen aufgrund ihrer fehlenden „hinreichenden Bestimmtheit“ (Rothfritz, 2010. S. 386) in der Praxis kaum Auswirkungen haben könnte (Rothfritz, 2010, S. 386). So sieht die Einschätzung des Deutschen Instituts für Menschenrechte (2017) auf der Grundlage der Bemerkung des UN-Ausschusses (2016) mit Blick auf die finanziellen Anstrengungen der Bundesrepublik Deutschland für inklusive Bildung ungünstig aus und greift die Forderung des Ausschusses auf, der den Vertragsstaaten empfiehlt „die Finanzierung ihres Bildungssystems zu überdenken und die Mittel nur noch in die Entwicklung von inklusiver Bildung fließen zu lassen“ (Dt. Institut für Menschenrechte, 2017, S. 4) sowie „ausreichende Finanz- und Personalzusagen zur Entwicklung eines inklusiven Bildungswesens“ (Dt. Institut für Menschenrechte, 2017, S. 5) zu tätigen.

Gute materielle Rahmenbedingungen ${ }^{17}$ werden in sonderpädagogischen Forschungsveröffentlichungen zu schulischer Integration zunächst allgemein als wesentliche qualitative Voraussetzung für integratives Arbeiten in Schulen benannt (Bächthold et al., 1990; Feyerer, 1998; Bless, 2003; Speck, 2010). Forschungsergebnisse zu finanziellen Rahmenbedingungen von Schulen können den in diesen Veröffentlichungen angenommen Zusammenhang zwischen Ressourcenausstattung und Qualität der (integrativen) unterrichtlichen Arbeit allerdings nicht eindeutig belegen. Nach Hattie hat die finanzielle Ausstattung von Schulen insgesamt eine geringe Effektstärke (mit Blick auf Schülerleistungen) von $\mathrm{d}=0,23^{18}$ (Hattie, 2013, S. 87), wobei Kosten, die einen direkten unterrichtlichen Bezug haben (Bsp.: Unterrichtsmaterialien, Lehrergehälter) nach Childs und Shakeshaft (1986) eine höhere Effektstärke aufweisen als Bildungsausgaben allgemein. Hanushek (1989) und Rolle (2004) gehen davon aus, dass es vielmehr darum gehen müsse bestehende Ressourcen besser auszunutzen (Hattie, 2013, S. 87). Hanushek et al.

17 Zentrale Subkategorien werden als Orientierungshilfe und der Übersichtlichkeit halber im Text fett markiert.

18 Das Differenzmaß d kennzeichnet das Maß von Mittelwertsunterschieden. $A b \mathrm{~d}=0,20$ spricht man von kleinen Effekten, bei Werten zwischen $\mathrm{d}=0,4$ bis 0,6 von moderaten Effekten und bei Werten größer als $\mathrm{d}=0,8$ von großen Effekten. s. dazu https://www.spektrum.de/lexikon/psychologie/effektstaerke/3796 
weisen außerdem darauf hin, dass finanzielle Anreize wirksamer sein könnten, wenn diese sich direkt auf die Lehrkräfte, nicht auf die Schulen beziehen würden (Hanushek et al., 2002, 2003). Hedges, Laine und Greenwald (1994, 1996) wiederum haben kleine Effekte zwischen finanziellen Ausgaben der Schulen und Lernleistungen der Schüler feststellen können $(d=0,15)$. Zusammenfassend folgert Hattie, dass präziser untersucht werden müsse, in welcher Hinsicht Geld entscheidend sei, da die Fragestellung, inwiefern Geld allgemein Auswirkungen auf Schulen und Unterricht habe, nicht Ziel führend sei (Hattie, 2013, S. 89). Auch Benner gibt in diesem Zusammenhang zu bedenken, dass Bildungsinvestitionen, die sich auf Lernen als einen individuellen Vorgang beziehen, „auch individuell sinnvoll sein [müssen]. Dies sind sie jedoch nur dann, wenn die pädagogische Praxis Lernfortschritte bewirkt, die auf Lernprozesse in pädagogischen Institutionen zurückgeführt werden können und den Aufenthalt in ihnen lohnend erscheinen lassen." (Benner, 2015, S. 208).

Im Rahmen der PISA-Studie wurden mögliche Auswirkungen der materiellen Ausstattung von Schulen indirekt untersucht, indem Schulleiter dazu befragt wurden. Diese sehen kaum Auswirkungen einer möglicherweise mangelhaften Ausstattung auf das Lernen der Schüler. Allerdings weisen die Untersuchungsergebnisse an sich darauf hin, dass „,on den Schulleiterinnen und Schulleitern als schlecht eingeschätzte unterrichtsbezogene Ausstattungsbedingungen mit einem niedrigeren mittleren Leistungsstand der Schülerinnen und Schüler einhergehen." (Dt. PISA-Konsortium, 2001, S. 434). Ob diese Ergebnisse einen direkten Zusammenhang aufweisen oder mit anderen Faktoren konfundieren, blieb zum Zeitpunkt der Veröffentlichung ungeklärt. Mit Blick auf die Kosten, die durch Integration entstehen, thematisieren Innerhofer und Klicpera (für Südtirol) die Frage nach der gesellschaftlichen Wertsetzung des Zusammenlebens behinderter und nichtbehinderter Menschen, d.h. sie gehen davon aus, dass erst eine andere Stellung der integrativen Unterrichtung in der Wertehierarchie dazu führen könnte, dass von den Bürgern erwirtschaftetes Geld in größeren Ausmaß dafür zur Verfügung gestellt werden würde (Innerhofer \& Klicpera, 1991, S. 348f). Sie formulieren genauso wie Bächthold et al. (1990), Feyerer (1998), Bless (2003) und Speck (2010), dass gute Rahmenbedingungen und damit verknüpfte zusätzliche Investitionen eine grundlegende Bedingung für qualitativ hochwertige schulische Integration darstellen (Innerhofer \& Klicpera, 1991, S. 338). Brugger-Paggi (2003) wiederum konkretisiert in ihrer Qualitätsübersicht zu schulischer Integration (in Südtirol), dass im Haushalt der Schulen spezifische Mittel für die Umsetzung vorhanden sein müssen, die ausschließlich zum Zweck des Ankaufs von Lehr- oder Lernmitteln oder anderer Hilfen verwendet werden dürfen. Weitere Veröffentlichungen aus der Integrationspädagogik sowie Kriterienkataloge für die Qualität schulischer Integration belegen darüber hinaus, dass eine barrierefreie Gestaltung von Schulgebäuden (Innerhofer \& Klicpera, 1991; Heyer et al., 1997; Boban \& Hinz, 2003; Brugger-Paggi, 2003; Specht et al., 2006; Wember \& Prändl, 2009; Speck, 2010), sanitären Einrichtungen (Boban \& Hinz, 2003; Brugger-Paggi, 2003; Wember \& Prändl, 2009) und Schulgelände (Boban \& Hinz, 2003; Wember \& Prändl, 2009) als selbstverständliche Grundvoraussetzung für inklusive Schulen angesehen werden. Bauliche Veränderungen sollten nach Brugger-Paggi (2003) vorgenommen werden, bevor Schülerinnen oder Schüler mit Beeinträchtigungen eine Schule besuchen. Die Forderung nach einer barrierefreien Gestaltung bezieht sich dabei vorwiegend auf Schülerinnen und Schüler mit körperlichen Beeinträchtigungen. Boban und Hinz (2003) erwähnen darüber hinaus auch die Relevanz der Zugänglichkeit für gehörlose bzw. blinde und sehbehinderte Menschen. Mit Blick auf die barrierefreie Gestaltung von (Klassen-) Räumen werden in einigen Arbeiten sehr ausdifferenzierte Indikatoren formuliert, die bspw. die Raumakustik, elektronische wie nicht elektronische Hilfen, eine spezifische Ar- 
beitsplatzgestaltung für bestimmte Beeinträchtigungen o.ä. betreffen (Brugger-Paggi, 2003; Leonhardt, 2009; Thiele, 2009; Wember \& Prändl, 2009).

Über die Barrierefreiheit von Gebäude und Schulgelände bzw. die barrierefreie Gestaltung von Klassenräumen hinaus werden als Qualitätskategorie für integrative Schulen auch flexible Raumnutzungsmöglichkeiten benannt, die zum einen ausreichend große Klassenräume (Wember \& Prändl, 2009), zum anderen spezifische Förder- oder Differenzierungsräume umfassen (Heese, 1977; Innerhofer \& Klicpera, 1991; Holzinger et al., 2011), die bspw. besondere therapeutische Angebote für Schüler mit schweren Beeinträchtigungen oder psychomotorische Förderung ermöglichen (Wember \& Prändl, 2009). Diese Raumnutzungsmöglichkeiten bzw. zusätzlichen Räume sollen wiederum unterschiedliche Organisationsformen integrativen Unterrichts wie individuelles Arbeiten oder das Arbeiten in Kleingruppen unterstützen (Innerhofer \& Klicpera, 1991, S. 338). Die Gestaltung des Klassenraums als Gegenstand didaktischer Überlegungen im Zusammenhang mit schulischer Integration war schon Bestandteil von Modellversuchen in den 70er und 80er Jahren (Romey, 1977; Meiers, 1982, S. 205; Wyosnick, 1987, S. 192). ${ }^{19}$ Die (Klassen-) Räume sollten klar strukturiert und als „Lebens-, Handlungs- und Erfahrungsraum“ (Wember \& Prändl, 2009) gestaltet sein sowie die gleichzeitige Unterrichtung mehrerer Gruppen möglich machen (Innerhofer \& Klicpera, 1991). Eine wichtige Rolle spielt offensichtlich auch die Ausstattung der Räume mit Differenzierungsmaterial (Boban \& Hinz, 2003; Brugger-Paggi, 2003; Holzinger et al., 2011) wie bspw. „Spielzeug und Medien, die Interaktion und Kooperation erleichtern" (Mühl, 1987), Medien für unterstützte Kommunikation (Wember \& Prändl, 2009) oder Medien für blinde bzw. sehbehinderte Kinder (Boban \& Hinz, 2003; Wember \& Prändl, 2009).

Mit Blick auf die Einschätzung dieser Rahmenbedingungen kommt Eberl zu dem Ergebnis, dass die räumliche Situation und Materialausstattung von Lehrkräften und Schulleitungen sehr negativ beurteilt werden (Eberl, 2000, S. 280). Amrhein gelangt zu dem Ergebnis, dass knapp $40 \%$ der von ihr befragten Lehrkräfte in weiterführenden Schulen in NRW die räumlichen Voraussetzungen in ihren Schulen für ungünstig halten, über $40 \%$ gehen davon aus, dass auch die Materialausstattung für integrative Beschulung nicht ausreichend gegeben ist (Amhrein, 2011, S. 149). Schwarz et al. (2013) weisen in ihrem Gutachten für kommunale Folgekosten darauf hin, dass eine Senkung des Klassenteilers für Inklusionsklassen auf 25 SuS in NRW zu einem hohen Mehrbedarf an Klassen führen würde (etwa 3000 Klassenräume), was bei einem Investitionsbedarf von 120.000 Euro je Klassenraum erhebliche Kosten verursachen könnte (Schwarz et al., 2013, S. 32f).

\subsubsection{Leitung einer Schule}

Insgesamt wird mit Blick auf die Leitung einer Schule grob zwischen Führung und Management unterschieden (Buchen \& Rolff, 2016, S. 6). Das Management bezieht sich auf alle Abläufe, die die „Schule als Betriebseinheit“ betreffen, und damit u.a. auf die Gestaltung von Verwaltungsabläufen oder Projekten und den Umgang mit Ressourcen (Bsp.: Budgetierung). Führung wieder-

19 „bauliche Voraussetzungen (z.B. mobile Wände für äußere Differenzierungsformen, Sprachlabors, Fahrstühle - z.B. für Körperbehinderte - weitere behindertenspezifische Einrichtungen), genügende Materialien und Medien“ (Romey, 1977, S. 123f)

„Der Klassenraum muß den vielfältigen Funktionen der Klasse für Schüler mit Verhaltensstörungen gerecht werden. Er muß den Schülern eine Art Unterstützungsbasis während des Schultages bieten, in dem sie sich willkommen und sicher fühlen können, er muß die verschiedenen Aktivitäten, die bei der Vielschichtigkeit des Unterrichts entstehen, auf eine übersichtliche Weise ermöglichen [...]. (Wyosnick, 1987, S. 192) 
um bezieht sich auf das Personal in der Schule (Bsp.: Personaleinstellung, - beurteilung, - entwicklung) (Buchen \& Rolff, 2016, S. 6). Auch wenn Überschneidungen zwischen den beiden Bereichen vorliegen, sprechen Buchen \& Rolff daher von struktureller und personeller Leitung (Buchen \& Rolff, 2016, S. 3). Die Schulleitung trägt darüber hinaus einen hohen Teil der Verantwortung für das Gelingen von Schulentwicklungsprozessen (Bryk et al., 2010; Dyson, 2010; Scheer \& Laubenstein, 2018). Schulleitungen und Steuerungsteams spielen hinsichtlich der Gestaltung (inklusiver) Schulentwicklungsprozesse eine entscheidende Rolle und haben die Aufgabe, Veränderungen zu initiieren, zu implementieren und zu evaluieren (Köker, 2012, S. 17; Baum, Bondorf \& Ullrich, 2010; zitiert nach Serke et al., 2014, S. 255). Gemäß einer Studie von Bonsen (2002) ergaben sich als vier wesentliche Handlungsdimensionen einer guten Schulleitung, die „zielbezogene Führung, Innovationsförderung, Partizipation in der Entscheidungsfindung, Organisationskompetenz" (Bonsen, 2010, S. 200). Schulleitungen, die die Entwicklung ihrer Schulen positiv beeinflussen, legen Wert auf die Unterstützung von Lehrerkooperation und die Professionalisierung der Lehrkräfte (Bonsen, 2010, mit Bezug auf Rosenholtz, 1991) sowie auf die Fokussierung des Unterrichts (Bonsen, 2010, mit Bezug auf Leithwood, 1992; Scheerens et al., 2003). Bryk et al. belegen in ihrer Längsschnittstudie die zentrale Bedeutung der Schulleitung für die Entwicklung einer Schule:

Our framework of organizational supports for student learning starts with leadership as the driver for change. These school leaders focus their attention on four domains of work. They reach out to parents and communities to repair the long-standing disconnect between urban schools and the children and families they are intended to serve. Simultaneously, they work to enhance the professional capacity of their school through a deliberate focus on staff quality, strengthening the processes supporting faculty learning and a continuous improvement ethos. Likewise, they aim to expand teachers' capacity to work together to align curriculum and strengthen the overall instruction system. Central to making this work for children is an overall normative environment where students feel safe and are pressed and supported to engage (and succeed) with more ambitious intellectual activity. (Bryk et al., 2010, S. 64f)

Die Schulleitung wiederum kann Veränderungen also nur in enger Kooperation mit allen anderen am Schulleben beteiligten Personen bewirken. So kamen Bryk et al. zu dem Ergebnis, dass es starke Zusammenhänge zwischen der Leitung einer Schule und der Einbeziehung von Eltern gibt $(\mathrm{d}=0,64)$ sowie der Einbindung in Gemeinde oder Stadt $(\mathrm{d}=0,47)$. Darüber hinaus hat die Schulleitung maßgeblichen Einfluss auf die professionellen Kapazitäten einer Schule $(\mathrm{d}=$ 0,77 auf die professionelle Gemeinschaft, $d=0,75$ auf die Arbeitsorientierung and $d=0,45$ auf die qualitätsvolle professionelle Entwicklung) (Bryk et al., 2010, S. 127). Bryk et al. stellen darüber hinaus eindeutige Zusammenhänge zwischen „School Leadership“ und Schülerleistungen fest: „For example, the probability of substantial improvement in math ist seven times higher among schools with strong school leadership than among those with weak leadership (42 percent, compared with 6 percent).“ (Bryk et al., 2010, S. 85). Insgesamt weisen Aktivitäten der Schulleitung eine Effektstärke von $d=0,36$ auf (Hattie, 2013, S. 98). Am effektivsten agieren gemäß dieser Unterscheidung Schulleitungen, „die sich auf die Schülerleistung und auf Unterrichtsstrategien konzentrieren“ (Hattie, 2013, S. 99) (Effektstärken zwischen d = 0,54 und d $=0,66$ (je nach Variable)). Neben der Unterstützung der Lehrenden spielen u.a. die Zielorientierung von Schulleitungen und die Kontrolle der „Effektivität der Schulpraktiken und ihre Auswirkungen auf das Lernverhalten der Lernenden" (Hattie, 2013, S. 100) eine wichtige Rolle. Obwohl der Schulleitung im Rahmen von Schulentwicklungsprozessen und damit auch mit Blick auf die Schulentwicklung inklusiver Schulen eine zentrale Rolle zugemessen wird, die durch Forschungsergebnisse wie die soeben erläuterten belegt werden kann, werden Leitungs- 
aufgaben in der sonderpädagogischen Forschung nur selten als Qualitätskategorien untersucht $^{20}$. Die Kategorienübersicht (im Anhang) zeigt, dass diese bis 2011 vor allem von Innerhofer \& Klicpera (1991) und Lienhard-Tuggener et al. (2011) thematisiert wurden (Autoren aus Südtirol und Österreich). So sehen Innerhofer und Klicpera Schulleitungen aufgrund ihrer Erhebung als „Promoter der Integration“ (Innerhofer \& Klicpera, 1991, S. 339), die auf die Haltungen von Lehrkräften und die Umsetzung der schulischen Integration einen erheblichen Einfluss haben. Aufgabengebiete beträfen vor allem beratende, vernetzende, koordinierende und unterstützende Leistungen für Eltern und Lehrkräfte (bspw. bei der Entscheidung über eine geeignete Beschulungsform) sowie Beiträge zur Individualisierung des Unterrichts sowie zu klassenübergreifenden Unterrichtsformen (Innerhofer \& Klicpera, 1991, S. 339, Herv. im Orig.). Dafür seien die Schulleitungen in integrativen Schulen auf spezielle Fortbildungen und Beratung durch die Schulaufsicht angewiesen (Innerhofer \& Klicpera, 1991, S. 339f). Aufgaben von Schulleitungen im Zusammenhang mit schulischer Integration werden bei der Koordination der Beteiligten sowie bei der Beratung von Eltern und Lehrkräften gesehen (Innerhofer \& Klicpera, 1991; Lienhard-Tuggener et al., 2011). Innerhofer \& Klicpera beschreiben darüber hinaus insbesondere die Verantwortung der Schulleitung für die Unterstützung von Unterrichtsentwicklungsprozessen. Schulleitungen sollten auf der einen Seite Fortbildungen von Lehrkräften ermöglichen und sich auf der anderen Seite selbst fortbilden bzw. beraten lassen (Innerhofer \& Klicpera, 1991; Lienhard-Tuggener et al., 2011).

Dyson kommt aufgrund von Studien in Großbritannien - ähnlich wie Bryk et al. für die Schulleitungen von Grundschulen - zu dem Schluss, dass sich Schulleitungen guter inklusiver Schulen dadurch auszeichnen, dass sie die Steuerung kooperativer Prozesse ermöglichen und unterstützen:

2. Leadership and decision-making. The collaborative nature of school cultures in these studies implied a particular form of leadership and decision-making. Strong school leaders, committed to inclusive values, are crucial to the development of more inclusive schools, but such leaders must recognize the importance of collaborative processes. The leaders of inclusive schools are not autocrats, but, rather, supporters and enablers of their staffs who were engaged in a collaborative process of school development. (Dyson, 2010, S. 3; Herv. im Orig.)

Serke et al. (2014) erläutern, dass auch Harazd \& Drossel (2011, S. 157) belegen konnten, dass „Schulleitungen durch wertschätzendes Verhalten gegenüber kooperativ arbeitenden KollegInnen und v.a. durch Schaffung verbindlicher Strukturen, wie feste Zeiten oder kooperationsfreundliche Stundenpläne, Kooperation nachhaltig positiv beeinflussen können." (Serke et al., 2014, S. 255f). Als Beispiel führen Serke u.a. in diesem Zusammenhang die Grundschule Berg Fidel auf, in der feste Teamzeiten pro Woche, ein Klausurtag im Halbjahr sowie die Unterstützung durch regelmäßige Supervision eine enge Zusammenarbeit der Lehrkräfte ermöglichen (Serke et al., 2014, S. 256). Joller-Graf und Tanner (2011) kommen in einer Interviewstudie mit Eltern, schulischen Heilpädagogen und Lehrkräften in der Schweiz zu dem Ergebnis, dass die befragten Akteure der Schulleitung eine zentrale Rolle mit Blick auf deren Haltung und die Außenkommunikation zuschreiben (Joller-Graf \& Tanner, 2011). Scheer \& Laubenstein (2018) beziehen sich in ihren Ausführungen zur Bedeutung von Schulleitungen für inklusive Schulentwicklungsprozesse u.a. auf Hillenbrand, Metzger \& Hagen (2013) sowie Heimlich (2016), die diese zentrale Funktion thematisieren und theoretische Annahmen dazu formulieren. For-

20 Veröffentlichung einer Dissertation von Scheer, D. zum Thema erscheint im Springer-Verlag erst nach Fertigstellung der vorliegenden Arbeit 
schungsergebnisse dazu, inwiefern diese Annahmen tatsächlich zutreffen, liegen laut dieser Veröffentlichung allerdings nur für Schulleitungshandeln insgesamt vor (nach Leithwood et al., 2008). In Rheinland-Pfalz sind es Schulaufsichtsbeamte, die Schulleitungen von Schwerpunktschulen als wesentlich für „das Gelingen von inklusiver Schule“ (Laubenstein et al., 2015, S. 108) einschätzen. Diese würden nicht nur als „Türöffner“ agieren, sondern hätten auch die Aufgabe Personalbedarfe zu überblicken, organisatorische Rahmenbedingungen (bspw. Zeitfenster für Teamarbeit) zu schaffen und individuelle Lösungen für einzelne Schülerinnen und Schüler in Kooperation mit der Schulaufsicht zu entwickeln (Laubenstein et al., 2015, S. 108f).

\subsubsection{Schule als sozialer Raum}

Unter die Kategorie „Schule als sozialer Raum“ werden alle Qualitätsbausteine gefasst, die die Schule als soziale Gemeinschaft der in ihr tätigen Personen kennzeichnen sowie ihre Einbindung in ein soziales Umfeld (Bsp.: Stadtteil, Kommune) darstellen. Dazu gehören nach eingehender Analyse vorliegender pädagogischer wie sonderpädagogischer Forschungsveröffentlichungen vor allem die Subkategorien Schulklima, Integration als gemeinsame Aufgabe, Partizipation, Zusammenarbeit mit den Eltern und Schülern sowie Vernetzung mit außerschulischen Institutionen.

Die Schule als sozialer Raum wird geprägt durch eine spezifische Kommunikationskultur und die Umgangsformen der in ihr tätigen Menschen. Das Schulklima wird in diesem Zusammenhang definiert

[...] als eine von den Betroffenen (Schüler, Eltern und Lehrer) wahrgenommene spezifische Konfiguration wesentlicher Merkmale des erzieherischen Verhältnisses zwischen Lehrern und Schülern, des Verhältnisses von Schülern untereinander sowie erzieherisch bedeutsamer kollektiver Einstellungen und Verhaltensbereitschaften von Lehrern und Schülern innerhalb der jeweiligen Lernumwelt. (Eder, 1998, S. 424)

Das Wohlbefinden der Beteiligten wird dabei als „psychologisches Konstrukt verstanden, das aus emotionalen und kognitiven Anteilen besteht“ (Hascher, 2017, S. 70). Hascher konnte mit Blick auf dieses Konstrukt sechs Komponenten identifizieren, die „positive Einstellungen zur Schule, Freude in der Schule, schulisches Selbstkonzept als positive Indikatoren, Sorgen wegen der Schule, körperliche Beschwerden wegen der Schule sowie soziale Probleme in der Schule“ (Hascher, 2017, S. 71) umfassen. Der Autor erkennt eine enge Wechselbeziehung zwischen dem Wohlbefinden von Schülern und ihrer Lern- und Leistungsbereitschaft. Innerhalb der Schulqualitätsforschung wird das Schulklima ,sowohl als eigenständige Qualitätsdimension des Erfahrungs- und Lebensraums Schule als auch als Bedingungsvariable für Lernleistungen" (Dt. PISA-Konsortium, 2001, S. 448) aufgefasst. In diesem Zusammenhang stellt die PISA-Studie fest, dass die Beeinträchtigung von Lernprozessen durch die Schülerschaft (fehlende elterliche Unterstützung, Unterrichtsstörungen, Schwänzen, mangelnder Respekt vor Lehrkräften, sozial benachteiligte Verhältnisse) in Hauptschulen signifikant ungünstiger ist als in Gymnasien (Dt. PISA-Konsortium, 2001, S. 448). Effekte hat das Schulklima nach PISA u.a. auf von sozialen Normen abweichendes Verhalten von Schülern: „Jugendliche, die das Schulklima positiv einschätzen, nehmen regelmäßiger als andere am Unterricht teil [...] und neigen seltener zu körperlichen Gewalthandlungen." (Dt. PISA-Konsortium, 2001, S. 493). Dagegen können andere Veröffentlichungen (Fend, 1998; Tillmann, 1995; Ulich, 1991; zitiert n. Dt. PISA-Konsortium, 2001, S. 490) keine durchgängigen Zusammenhänge zwischen Schulklima und Schülerleistungen feststellen. 
Schon 1984 kommen Reiser et al. in ihrer Studie „Sonderschullehrer in Grundschulen“ wiederum zu dem Schluss, dass das Schulklima und die Zusammenarbeit zwischen den Sonderschulund den Grundschullehrern sowie die Lehrerpersönlichkeiten selbst sich stärker auf Lern- und Verhaltensstörungen auswirken als spezifische von Sonderschullehrern getroffene Maßnahmen (Reiser et al., 1984, S. 46). Auch neuere Untersuchungen wie die von Dyson (2010) dargestellten stützen den Befund, dass eine gemeinsame Schulkultur wesentlich für inklusives Arbeiten ist:

[...] an acceptance and celebration of difference, a commitment to offering educational opportunities to all students, a high level of staff collaboration, a willingness of teachers to learn together, and the promotion of collaboration between students and between school staff and parents. These cultural characteristics appeared much more important than specific forms of organization and practice. (Dyson, 2010, S. 3)

Insgesamt werden in diesem Zusammenhang sowohl Faktoren des Schulklimas allgemein als auch diejenigen, die den Umgang mit Aufgaben der schulischen Integration kennzeichnen, als qualitätsrelevant eingestuft. Wesentlich sei, dass das Schulklima von Wertschätzung und Akzeptanz geprägt werde (Boban \& Hinz, 2003; Wember \& Prändl, 2009), was zum einen auf den Umgang der Kollegen untereinander bezogen wird (Schley et al., 1992; Boban \& Hinz, 2003), zum anderen auf den Umgang zwischen Lehrkräften und Schülern (Schley et al., 1992; Boban \& Hinz, 2003; Thiele, 2009; Wember \& Prändl, 2009, Lienhard-Tuggener et al., 2011). Darüber hinaus wird auch der respektvolle Umgang mit den Eltern als wirksam benannt sowie Rituale, die im schulischen Alltag zu einem guten Schulklima beitragen können (Boban \& Hinz, 2003). Speck (2010) und Dyson (2010) weisen darauf hin, dass auch Einstellungen im Umfeld der Schule einen Einfluss auf das Schulklima haben können:

Even where schools develop, inclusive internal culture, they cannot divorce themselves from the policy and wider social contexts in which they are located. Policies which are broadly pro-inclusive support the development of inclusion at school level, and those which are hostile to inclusion or privilege other educational values make the task of inclusive schools significantly more complex. (Dyson, 2010, S. 4)

Die Art und Weise, wie eine Schule sich den Aufgaben stellt, die mit der Beschulung und Unterrichtung von Kindern und Jugendlichen mit Behinderungen verknüpft sind und damit Integration als gemeinsame Aufgabe wahrnimmt, wirkt sich ebenfalls auf die Qualität der schulischen Integration aus. Dabei werden Aspekte benannt, die sich auf die gemeinsame Verantwortungsübernahme für alle Schüler beziehen (Feyerer, 1998; Boban \& Hinz, 2003; Holzinger et al., 2011), oder solche, die den Konsens der Beteiligten zu Fragen der gemeinsamen Unterrichtung betreffen (Bächthold et al., 1990). Mühl (1987) und Boban und Hinz (2003) erwähnen darüber hinaus die Offenheit und Vorbereitung aller Beteiligten hinsichtlich der Aufnahme von Schülern mit Behinderungen als wesentlich.

Die Schule als Gemeinwesen ist auch gekennzeichnet durch die Möglichkeiten aller Beteiligten zur Partizipation. Insbesondere die Längsschnittstudie von Bryk et al. benennt Mitbestimmungsmöglichkeiten als effektvolle Einflussgröße für schulische Entwicklung (Bryk et al., 2010, S. 12). In sonderpädagogischen Veröffentlichungen nimmt dieser mögliche Qualitätsbaustein für inklusive Schulentwicklung zwar wenig Raum ein, er wird jedoch mit unterschiedlichen Aspekten von einer ganzen Reihe von Autoren zumindest aufgegriffen (Mühl, 1987; Innerhofer \& Klicpera, 1991; Bächthold et al., 1990; Hug, 1994b; Bless, 2003; Boban \& Hinz, 2003; Brugger-Paggi, 2003; Lienhard-Tuggener et al., 2011). Boban und Hinz (2003) legen in diesem 
Zusammenhang Wert auf die Einbeziehung der Schülerinnen und Schüler sowie der Eltern in Schulentwicklungsprozesse, während Brugger-Paggi (2003) aufführt, dass Eltern von Kindern mit sonderpädagogischem Förderbedarf in schulischen Gremien vertreten sein sollten. Regelmäßige Informationen für Eltern werden als Qualitätsindikatoren von Hug (1994b), Bless (2003) und Lienhard-Tuggener et al. (2011) benannt.

Die Bedeutung der engen Zusammenarbeit mit den Eltern wird als Qualitätsbaustein schulischer Entwicklung vor allem im englischsprachigen Raum thematisiert:

Engaging families in the education of their children at home and at school is increasingly viewed as an important means to support better learning outcomes for children. When schools and families work together, children have higher achievement in school and stay in school longer (Henderson \& Mapp, 2002; Jeynes, 2005; Pomerantz, Moorman, \& Litwack, 2007; Reynolds \& Clements, 2005). (Berthelsen \& Walker, 2008, S. 34f)

Werning (2013) erläutert diese Gewichtung der Elterneinbindung und verweist darauf, dass in England bspw. so genannte „Extended Schools“ „Kurse zur Kindererziehung, Lernangebote für Familien, in denen Eltern mit ihren Kindern lernen können, aber auch die Vermittlung an außerschulische Experten wie Sprachtherapeuten, kinder- und jugendpsychologische Beratung, Gesundheits- und Sexualberatung “ anbieten (Werning, 2013, S. 25). Wild und Lütje-Klose (2017) benennen in Anlehnung an eine Veröffentlichung der Vodafone-Stiftung, die sich wiederum an den „Standards for Family-School Partnerships“ der Parent Teacher Association aus den USA orientiert, vier Eckpfeiler der Zusammenarbeit zwischen Elternhaus und Schule:

- Schaffung einer Willkommenskultur, die es den Eltern erleichtert einen Zugang zur Schule zu finden,

- regelmäßiger, produktiver und effizienter Informationsaustausch, der nicht nur problembezogen, sondern auch präventiv erfolgt,

- gegenseitige Abstimmung von Lerninhalten und -zielen in Verknüpfung mit der Entwicklung und Umsetzung von Förderplänen, die eine hohe Beratungskompetenz des schulischen Personals voraussetzt,

- Partizipation aller Eltern an schulischen Entwicklungsprozessen (Wild \& Lütje-Klose, 2017, S. 136f).

Die Effekte der elterlichen Einbindung auf die Lernerfolge von Jugendlichen, auf die sich Werning bezieht, haben vor allem Jeynes (2005a und 2005b) sowie Pushor (2007) untersucht. Jeynes kommt diesbezüglich u.a. zu dem Ergebnis, dass subtile Formen der elterlichen Unterstützung wie Kommunikation und Familienstruktur ("an educationally oriented ambiance", Jeynes, 2005b, S. 262) die Lernerfolge von Kindern stärker beeinflussen können als die Bereiche, die von Schulen normalerweise für wichtig erachtet werden wie der Besuch von Elternabenden oder die Begleitung von Hausaufgaben (Jeynes, 2005a, S. 113f; 2005b, S. 262). Dazu fasst Pushor mit Bezug auf Jeynes zusammen:

He found it was not particular parent actions, such as attending school functions, establishing household rules, or checking student homework, which yielded the statistically significant effect sizes in the research in relation to student academic achievement. Instead, it was things which created (p. 262) - an attitude or an atmosphere which formed for the child a sense of standards or support - which produced the strongest results. It seems that such an ambiance may be influenced more greatly by parental engagement in the core work of teaching and learning than by parental involvement in such activities as fundraising efforts or in support tasks such as photocopying. (Pushor, 2007, S. 5) 
Das Verhältnis des Einflusses der elterlichen Einbindung auf die Lernerfolge der Schüler sei erheblich:

Overall, parental involvement's relationship to urban student academic outcomes was about seven tenths to three fourths of a standard deviation. This is close to what Rosnow and Rosenthal (1996) described as a large effect size (about 0.8). (Jeynes, 2005b, S. 259)

Aus diesem Grund sei es für Schulen wichtig Eltern auf unterschiedliche Art und Weise und mit Hilfe verschiedener Programme zu einer stärkeren schulbezogenen Unterstützung ihrer Kinder zu motivieren und sie ins Schulleben einzubeziehen (Jeynes, 2005b, S. 260f). Zu ähnlichen Ergebnissen gelangt Hattie in seiner Meta-Studie. Diese belegt, dass der sozioökonomische Status des Elternhauses eine Relevanz für schulisches Lernen hat $(\mathrm{d}=0,57)$. Besondere Bedeutung kommt in diesem Zusammenhang dem häuslichen Anregungsniveau $(\mathrm{d}=0,57)$ sowie der Elternunterstützung beim Lernen zu $(\mathrm{d}=0,51)$ (Hattie, 2013, S. 73-84). Pushor (2007) führt zum „Parent Engagement“ aus, dass Eltern dazu in die Lage versetzt werden sollten so etwas wie „Nebenlehrer“ ihrer Kinder zu werden, die gemeinsam mit den Lehrern ihre Erziehungsverantwortung übernehmen: „The implication is that the person ,engaged' is an integral and essential part of a process, brought into the act because of care and commitment." (Pushor, 2007, S. 3). Diese Einbeziehung von Eltern in die schulische Arbeit verändert nach Pushor die Schulen weg von einem „Protektorat“, in dem Lehrkräfte ihre Schüler „schützen“ hin zu einer echten Schulgemeinschaft:

No longer are educators working alone to design and enact policies, procedures, programs, schedules and routines for the sole benefit of the children of the community. Instead, educators are entering a community to create with parents a shared world on the ground of school - a world in which "parent knowledge" and teacher knowledge both inform decision-making, the determination of agendas, and the intended outcomes of their efforts for children, families, the community and the school. Both educators and parents wear badges which mark their knowing and their expertise. (Pushor, 2007, S. 3).

Mit Bezug auf Reynolds und Clements (2005) stellen Berthelsen und Walker (2008) heraus, dass die Einbindung der Eltern durch unterstützende schulische Programme sogar stärkere Effekte auf das schulische Lernen habe als bspw. kleinere Klassen. Die Lehrkräfte sollten in diesem Bereich professionalisiert werden, da vor allem die Qualität der Elternkontakte ausschlaggebend für den Erfolg sei (Berthelsen \& Walker, 2008, S. 40f). Auch Bryk et al. (2010) belegen anhand ihrer Längsschnittstudie an 22 Grundschulen, dass die Verankerung von Schulen in ihrem sozialen Umfeld und damit auch in den Familien ihrer Schüler eine wesentliche Rolle für Schulentwicklungsprozesse spielt:

Their change efforts focus on: Encouraging new relations with parents and local communities to repair the long-standing disconnect between urban schools and the children and families they are intended to serve. Through active outreach efforts, staff members seek to make the school a more hospitable and welcoming environment for parents and strengthen the connections to other local institutions concerned with the care and well-being of children and their families. (Bryk et al., 2010, S. 46)

Sie stellen darüber hinaus Zusammenhänge zwischen Elternzusammenarbeit und der mangelnden Verbesserung von Schülerleistungen fest: „Of 19 schools, where parent involvement reports were weak [...], not a single school improved in maths.“ (Bryk et al., 2010, S. 91).

In Forschungsarbeiten und Kriterienkatalogen der Sonderpädagogik wird die Zusammenarbeit mit den Eltern ebenfalls als qualitätsbedeutsam erkannt (Bächthold et al., 1990; Innerhofer \& 
Klicpera, 1991; Hug, 1994b; Feyerer, 1998; Boban \& Hinz, 2003). Innerhofer und Klicpera gehen aufgrund ihrer Untersuchungsergebnisse davon aus, dass die Mitarbeit der Eltern bei der Gestaltung integrativer Schulen noch stärker einbezogen werden sollte. Dafür müssten die Eltern allerdings entsprechende externe Ausbildungsangebote erhalten, damit sie eine aktive Rolle übernehmen können (Innerhofer \& Klicpera, 1991, S. 345f). Schulen sollten Transparenz hinsichtlich ihrer integrativen Arbeit herstellen (Wocken, 1987; Hug, 1994b; Brugger-Paggi, 2003), und Gesprächsabende (Mühl, 1987), regelmäßige Beratungstermine (Boban \& Hinz, 2003) sowie Informationsveranstaltungen (Hug, 1994b) anbieten. Mit Blick auf die konkrete Förderung ihrer Söhne bzw. Töchter sollten Eltern regelmäßig informiert und in Vereinbarungen einbezogen werden (Brugger-Paggi, 2003; Lienhard-Tuggener et al., 2011). Darüber hinaus sollten Eltern beraten werden, wie sie das Lernen ihrer Kinder zu Hause unterstützen können (Boban \& Hinz, 2003). Nach Auffassung von Wocken haben Eltern in dreierlei Hinsicht eine besondere Bedeutung für integrative Schulen: eine geschichtliche Bedeutung (Elterninitiativen), eine politische Bedeutung (als Lobby) sowie eine pädagogische Bedeutung (Wocken, 1987, S. 135-141). Mit Blick auf die Hamburger Integrationsklassen erläutert er als Ergebnis der empirischen Bestandsaufnahme:

In jedem Falle ist es eine bleibende Aufgabe der Schule, mit den Eltern über Ziele, Wege und Bewertungsmaßstäbe einer integrativen Schularbeit im Gespräch zu bleiben und ihnen die neuartigen Probleme und Aufgaben gemeinsamen Lernens in der Schule offenzulegen. (Wocken, 1987, S. 140)

Untersucht wurden im Rahmen des Modellversuchs an der Fläming-Grundschule (Munder, 1988 (Erhebung von 1988)) sowie im Rahmen des Hamburger Integrationsversuchs (Wocken, 1987) u.a. die Motivstrukturen der Eltern mit Blick auf die Entscheidung für eine integrative Schule, die sehr vielfältig sind und sich nur grob drei Motivgruppen zuordnen lassen: dem Integrationsmotiv an sich, der Annahme der Vorteile von Integrationsklassen (verbesserte Lernbedingungen, soziales Lernen, kleinere Klassen, mehr Pädagogen) und dem Wunsch nach wohnortnaher Beschulung (Munder, 1988, S. 293f; Wocken, 1987, S. 152-158). Im Sinne pädagogischer Qualität hoffen die Eltern auf eine bessere Leistungsförderung: „Für den größeren Teil der Eltern ist eine Schule ohne Leistung nicht die bessere, attraktivere Grundschule, für den kleineren Teil kann eine leistungsorientierte Grundschule keine integrative Schule sein.“ (Wocken, 1987, S. 157). Die Eltern in den Hamburger Integrationsklassen zeigen sich sehr zufrieden mit der integrativen Qualität des Unterrichts, der Förderung behinderter wie nicht behinderter Kinder sowie der Förderung von Selbständigkeit und Arbeitsdisziplin (Wocken, 1987, S. 158-162). Sie schätzen die Entwicklung ihrer eigenen Kinder, deren Situation in den Klassen, Motivation, Lern- und Leistungsentwicklung positiv ein (Wocken, 1987, S. 162-165). Die Erhebung an der Fläming-Grundschule ergibt, dass die außerschulischen Kontakte von Eltern und Kindern der Integrationsklassen umfangreich sind. Dabei ist die „Kontaktdichte der behinderten Kinder und ihrer Eltern allerdings geringer als die der nichtbehinderten Kinder und ihrer Eltern" (Munder, 1988, S. 295). Als Ergebnis der Befragung von Eltern im Rahmen des Modellversuchs in Hamburg lässt sich außerdem festhalten, dass Eltern behinderter Kinder häufiger Kontakt zu Sonderschullehrern und Erziehern haben, Eltern nichtbehinderter Kinder häufiger zu Grundschullehrkräften (Wocken, 1987, S. 165-167). 80 \% der Eltern im Modellversuch nehmen an Elternabenden teil (Wocken, 1987, S. 168-172).

In einer Studie zur Bewertung der schulischen Integration durch Eltern (290 Fragebögen an einer Grund- und einer weiterführenden Schule) eruierten Dumke et al. (1989) zunächst, dass $45 \%$ der Eltern behinderter Kinder positive Veränderungen im Leistungsbereich erwarten. 
Höhere Erwartungen stellen sie an den Einfluss schulischer Integration auf das soziale Verhalten $(81 \%) .70 \%$ der Eltern erwarten innerhalb der Regelschule eine spezielle Förderung ihrer Kinder. Insgesamt konnten diese Erwartungen der Befragung nach erfüllt werden, allerdings in geringerem als dem erwarteten Maß (individuelle Förderung für $22 \%$ der Eltern erfüllt, Sozialverhalten zu 20\%) (Dumke et al., 1989, S. 26f). Auch die Unterrichtsgestaltung (gewünscht waren Montessori-Pädagogik, freie Arbeit, freies Spiel, mehr Projektunterricht und weniger Leistungsdruck) änderte sich nicht erwartungsgemäß (30\% der Erwartungen erfüllt). Probleme ergaben sich nach Einschätzung der Eltern vor allem bei der Leistungsdifferenzierung (27\%) und aufgrund der Belastung durch verhaltensauffällige Schüler (35\%) (Dumke et al., 1989, S. 27). Um die Ergebnisse zu verbessern schlugen Eltern vor, die Zusammensetzung der Klassen $(27 \%)$ und die Unterrichtsorganisation $(27 \%)$ zu verändern sowie die individuelle Förderung zu stärken (22\%). Gemäß dieser Studie schätzen Lehrkräfte die Zusammenarbeit mit den Eltern insgesamt als positiv ein, sind allerdings dennoch damit nicht besonders zufrieden (Dumke et al., 1989, S. 148). Die größten Unterschiede finden sich mit Blick auf die Schulformen. So ist bspw. die Zufriedenheit mit dem Engagement der Eltern bei Grundschullehrkräften mit $60 \%$ hoch, während sie bei Hauptschullehrkräften mit nur $10 \%$ vergleichsweise niedrig ist (Dumke et al., 1989, S. 148).

Die Vernetzung von Schulen innerhalb ihres Umfeldes kann durch die Zusammenarbeit mit außerschulischen Institutionen gestaltet werden. Die Zusammenarbeit innerhalb von Netzwerken sollte dabei nach Ahrens (2009) gekennzeichnet sein durch kooperative Handlungen, die auf Freiwilligkeit, Verlässlichkeit und Selbstverpflichtung beruhen sowie auf ein gemeinsames Ziel ausgerichtet sind. Diese Handlungen müssen wiederum durch das Management der damit verknüpften Abläufe professionell organisiert werden (Ahrens, 2009, S. 50f). Ein großer Teil der kooperativen Vernetzung von Schulen richtet sich nach Holtappels et al. (2007, S. 88) mit Bezug auf Ganztagsschulen auf den Freizeitbereich sowie auf Förderangebote mit Unterrichtsbezug. Nach Schnitzer (Abschlussbericht KOSA ${ }^{21}$ ) dient die Vernetzung aus Sicht der Schulen vor allem der Unterstützung der schulischen Arbeit durch außerschulische Partner, „die von der als bereichernd wahrgenommenen fachlichen Unterstützung bis zur additiven Entlastung reicht“ (Schnitzer, 2008, S. 184). Als unterstützend erleben die Schulen in diesem Zusammenhang vor allem Angebote der Jugendhilfe, die sich auf die Arbeit mit verhaltensauffälligen Schülerinnen und Schülern beziehen (Bsp.: Tagesgruppen). Die außerschulischen Vernetzungspartner wiederum haben über die Schulen einen vereinfachten Zugang zu den Kindern und Jugendlichen und profitieren ggf. von einer besseren Auslastung der Einrichtungen (Schnitzer, 2008, S. 184f). Diese Vorteile haben allerdings nach Holtappels et al. (2007) nicht zur Folge, dass es tatsächlich zu einer verstärkten Kooperation zwischen Schule und Jugendhilfe kommen würde (Holtappels et al., 2007, S. 126). Dies kann nach Schnitzer wiederum damit zusammenhängen, dass diese Form der Kooperation verzahnte pädagogische Konzepte zwischen Schulen und Jugendhilfe notwendig mache, während die Zusammenarbeit mit Sportvereinen oder Musikschulen bspw. als additive Modelle umsetzbar seien (Schnitzer, 2008, S. 197).

Die Bedeutung dieser Vernetzung haben auch Bryk et al. herausgestellt (Bryk et al., 2010). Weitere Studien aus den USA (Bsp.: Morgan \& Sorensen, 1999, auf der Basis von NELS = National Educational Longitudinal Study) kommen zu dem Ergebnis, „dass die Wirksamkeit sozialen Kapitals nicht nur in der lernfördernden Durchsetzung von Normen ihren Ausdruck finden kann, sondern auch in der Schaffung zusätzlicher Lerngelegenheiten („Horizonterweiterung“) durch die Pflege intensiver Außenkontakte." (Dt. PISA-Konsortium, 2001, S. 439).

21 Kooperation von Schule mit außerschulischen Akteuren 
Schon 1981 entwickelte Preuss-Lausitz aus sonderpädagogischer Perspektive eine umfassende Übersicht zu möglichen Vernetzungspartnern integrativer Schulen wie Hort, Vorschulbereich, Belegschaften und Betriebsräte größerer Betriebe, Eltern, Sportvereine, kirchliche oder gewerkschaftliche Jugendgruppen und Einrichtungen, Kommunalpolitiker, Gemeindeparlament, lokale Behindertengruppen oder Bürgerinitiativen (Preuss-Lausitz, 1981, S. 215). Qualitätssubkategorien zur Kategorie Vernetzung umfassen aus sonderpädagogischer Forschung heraus die Zusammenarbeit mit außerschulischen Institutionen (Bächthold et al., 1990; Innerhofer \& Klicpera, 1991; Boban \& Hinz, 2003; Brugger-Paggi, 2003; Specht et al., 2006; Feyerer, 2007; Lienhard-Tuggener et al., 2011), Vereinbarungen mit außerschulischen Partnern (Brugger-Paggi, 2003) oder die Koordination von Unterstützungsmaßnahmen (Boban \& Hinz, 2003). Obwohl die Zusammenarbeit mit außerschulischen Partnern als qualitativ bedeutsam angesehen wird, zeigen Untersuchungen wie $\mathrm{GeSchwind}{ }^{22}$, dass diese längst nicht an allen Schulen erfolgt. So kamen Laubenstein et al. (2015) zu dem Ergebnis, dass nur 60 von 133 Schwerpunktschulen mit dem Schulpsychologischen Dienst zusammenarbeiten und nur etwa 68 mit der Jugendhilfe (Laubenstein et al., 2015, S. 138). De Boer und Spies (2014) belegen anhand von 40 leitfadengestützten Interviews mit Schulleitungen und Lehrkräften an 25 Grundschulen im Raum Koblenz, dass das Jugendamt neben den Erziehungsberatungsstellen und dem schulpsychologischen Dienst der wichtigste außerschulische Kooperationspartner ist (De Boer \& Spies, 2014, S. 189). Sie schlussfolgern für die weitere Schulentwicklung, dass diese Kooperation zwar Entlastung bietet, allerdings weiteren Handlungs- und Strukturierungsbedarf sowohl mit Blick auf die Sicherung von Ressourcen als auch hinsichtlich gemeinsamer Konzepte von Schule und Jugendhilfe sowie gleichwertiger Arbeitsbeziehungen erfordert (De Boer \& Spies, 2014, S. 195f).

Beim Pilotversuch „Inklusive Grundschule“ im Land Brandenburg werden als Netzwerkpartner vor allem Kindertagesstätten (alle Schulen) und weiterführende Schulen (etwa $75 \%$ der Schulen) sowie schulpsychologische Beratungsstellen (mehr als $75 \%$ ) und therapeutische Einrichtungen (rund 60\%) benannt. Fast alle Grundschulen arbeiten mit den ortsansässigen Sportvereinen (knapp 90\%) zusammen. Weitere wichtige Kooperationspartner sind die Musikschulen (mehr als $80 \%$ der Schulen), Museen und Theater (etwas mehr als $40 \%$ der Schulen), Kirchengemeinden, Wohlfahrtsverbände und Wirtschaftsunternehmen (etwa $40 \%$ der Schulen) (Stäbler et al., 2015, S. 82).

\subsubsection{Organisationsstrukturen einer Schule}

Grundlegende Organisationsstrukturen von Schulen werden selten in Frage gestellt, wie bspw. folgende Auflistung von Andersen (1987) verdeutlicht, die beliebig verlängert werden könnte:

- Warum soll eine Unterrichtsstunde immer 45 Minuten dauern und eine danach folgende Pause 10 Minuten?

- Warum soll ein Schultag immer in eine Menge unzusammenhängender Aktivitäten (Fächer) aufgeteilt sein?

- Warum soll immer der eine Lehrer in Deutsch unterrichten, der andere in Mathematik, der dritte in Zeichnen, der vierte in Musik usw.?

- Warum soll jede Pause immer die gleiche Länge haben?

- Warum sollen immer alle Fächer dieselbe Unterrichtsdauer haben? (Andersen, 1987, S. 94)

Solche Fragen können daher am Beginn der Veränderung von Organisationsstrukturen innerhalb einer Schule stehen und bspw. zu einer anderen Rhythmisierung des Schulalltags, zu einer

22 Gelingensbedingungen des gemeinsamen Unterrichts an Schwerpunktschulen in Rheinland-Pfalz 
flexiblen Form von Klassenbildung, zu einem veränderten Lehrereinsatz usw. führen. Diese Veränderungen stehen wiederum in einem engen Zusammenhang zur Unterrichtsentwicklung. So stellte Meiers (1982) im Rahmen eines Modellversuchs zu differenzierendem Anfangsunterricht in der Grundschule fest, dass die Organisationsstrukturen der Schule hinsichtlich Pausenzeichen, Schulbeginn, Klassenraumgestaltung, Größe der Klassenräume/Schule, Anordnung von Arbeitsmaterialien zu verändern seien (Meiers, 1982, S. 204). Thematisiert wurden und werden veränderte Organisations-strukturen für Schulen vor allem in der Allgemeinen Pädagogik (Bsp.: Reformpädagogik, Grundschulpädagogik).

Im Rahmen der Modellversuche wurden unterschiedliche organisatorische Möglichkeiten der integrativen Beschulung erprobt (Muth, 1977; Sander, 2003, S. 36). Diese reichen von der Regelklasse ohne oder mit Beratung, Förderunterricht bzw. Ambulanzlehrersystem über Regelklassen mit Resource Room oder Doppelbesetzung, klassenübergreifenden Förderunterricht bis hin zu kooperativen Sonderklassen innerhalb der Regelschulen. Dazu zählt Muth Förderunterricht in Kleingruppen oder Einzelunterricht sowie behinderungsspezifische Förderangebote und auch Angliederungen von Gruppen behinderter Kinder oder ganzer Sonderschulformen, für die eine Teilintegration ermöglicht wird (Muth, 1977, S. 99).

Kleinklassen in Regelschulen wurden bspw. von der KMK (1960, S. 44) für Kinder gefordert, die den Unterricht stören, die aber nach entsprechender Förderung in die Regelklassen zurückgeführt werden könnten. In Studien deutscher Autoren (Preuss-Lausitz, 1981, S. 117f) werden diesbezüglich eher negative Erfahrungen geschildert. Die Unterrichtung in Kleinklassen habe zu Ablehnung und sozialer Außenseiterstellung von Schülern geführt, so dass diese zum Teil in stundenweise Einzelförderung nach dem Modell der schwedischen Schulkliniken überführt worden sei (Preuss-Lausitz, 1981, S. 117). Als Beispiel dafür nennt Preuss-Lausitz den Abbruch des Schulversuchs der IGS Obere Aar (Preuss-Lausitz, 1981, S. 118) und schlussfolgert insgesamt:

[...] die Hamburger und Kölner haben berichtet, daß es sowohl im Sinne des schulischen Lernens als auch der Persönlichkeitsentwicklung der Kinder fruchtbarer ist, mit dem zusätzlichen Personal den Regelunterricht zu verändern, die 'sozialpädagogische' Schule also nicht im Getto einer Klasse, sondern für alle einzurichten. (Preuss-Lausitz, 1981, S. 120)

Schinnen benennt mit Blick auf den Modellversuch zu Integrationsklassen an der FlämingSchule mögliche Grenzen binnendifferenzierter Angebote und erläutert die organisatorische Möglichkeit klassenübergreifenden Förderunterrichts:

Auch in Integrationsklassen wird es immer wieder vorkommen, daß irgendein Kind trotz innerer Differenzierung des Unterrichts in bestimmten Lernbereichen nicht so vorankommt, wie Eltern, Pädagogen und das Kind selbst sich dies wünschen. Manchmal kann dann eine zeitlich begrenzte Herausnahme des Kindes aus dem Unterricht helfen. Die Frage von Notwendigkeit, Zeitpunkt und Dauer solcher $\mathrm{Maßnahmen} \mathrm{bedarf} \mathrm{genauer} \mathrm{kontinuierlicher} \mathrm{Überprüfung.} \mathrm{Immer} \mathrm{wieder} \mathrm{werden} \mathrm{sie} \mathrm{auch} \mathrm{als} \mathrm{Abkehr}$ von der Integrationsidee mißverstanden. Trotzdem haben wir mit dem klassenübergreifenden Förderunterricht gute Erfahrungen gemacht. (Schinnen, 1988, S. 120)

Zusammenfassend weist sie darauf hin, dass klassenübergreifender Förderunterricht dann sinnvoll ist, wenn er kognitives und soziales Lernen in der Klasse unterstützt bzw. ergänzt, Binnendifferenzierung nicht ersetzt, sich an den Bedürfnissen der Schüler orientiert, in enger Zusammenarbeit mit den Klassenlehrern erfolgt und als Ziel die Hereinnahme der Förderung in den gemeinsamen Unterricht hat (Schinnen, 1988, S. 129). Woolfolk differenziert dahin- 
gehend, dass im Auge zu behalten sei, inwiefern Schüler mit Behinderungen tatsächlich einen Nutzen aus gemeinsamem Unterricht ziehen können:

Zum Beispiel berichten Naomi Zigmond und ihre Kollegen (1995) in ihrer Untersuchung mit sechs Inklusionsklassen der Grundschule, dass nur ungefähr die Hälfte der Schüler mit Behinderung von Inklusionsklassen profitierte. Ein ganzes Spektrum von unterstützenden Maßnahmen muss eingeführt werden, damit der Unterricht tatsächlich in einer effizienten Umgebung stattfinden kann; dies kann bedeuten, dass in der praktischen Umsetzung alles von einer teilweisen bis zur vollständigen Inklusion und zu Sonderschulen vorgesehen ist. (Woolfolk, 2008, S. 160)

Einige Studien befassen sich nach Haeberlin u.a. mit der Wahrnehmung äußerer Differenzierung durch die Kinder bzw. Jugendlichen selbst (Haeberlin et al., 2003). Moser (1989) kommt diesbezüglich zu dem Schluss, dass Förderunterricht allgemein positiv bewertet wird, vor allem mit Blick auf Aspekte, die den Unterrichtsprozess als solchen kennzeichnen wie spielerisches Lernen, gute Erklärungen der Förderlehrer oder das Erleben von Hilfe (Haeberlin et al., 2003, S. 50f). Der Förderunterricht wird individuumorientierter erlebt als der Klassenunterricht. Auch der Klassenunterricht werde positiv erlebt. In diesem Zusammenhang stehe allerdings das informelle Unterrichtsgeschehen (bspw. der Kontakt mit den Klassenkameraden) im Vordergrund der Wahrnehmung durch die Schüler (Haeberlin et al., 2003, S. 52). Als Problem äußern die befragten Schüler die Sorge im Klassenunterricht während des Förderunterrichts etwas zu verpassen (Haeberlin et al., 2003, S. 53). Gegen drohende Stigmatisierung formulieren einige der Schüler den Vorschlag auch Kinder ohne schulische Schwierigkeiten am Förderunterricht teilnehmen zu lassen (Haeberlin et al., 2003, S. 55). Demo hat aufgrund einer Befragung von 1877 Menschen mit Behinderung in Italien eruieren können, dass eine Mischform zwischen Klassenunterricht und Förderunterricht außerhalb der Klasse die häufigste Form der Umsetzung integrativer Unterrichtung darstellt (Demo, 2011, S. 131f).

Innerhofer \& Klicpera (1991) fordern aufgrund ihrer Studie in Südtirol Modellpläne für die Durchführung klassenübergreifenden Unterrichts, während Lienhard-Tuggener et al. (2011) zusätzliche schulinterne Unterstützungsangebote für erforderlich halten.

Jahrgangsübergreifender Unterricht wird im Zusammenhang mit inklusiver Unterrichtung vor allem in der Eingangsstufe (1 und 2) der Grundschule als hilfreich und wirksam eingeschätzt, damit dort eine individuelle Verweildauer (Bsp.: FLEX ${ }^{23}$ Brandenburg) ermöglicht werden kann (Kucharz, 2010, S. 340f). Als Organisationsstruktur hat er weitreichende Auswirkungen auf die Personalentwicklung (Bsp.: Zusammenarbeit von Lehrkräften, Kooperations- und Teamzeiten) und die Unterrichtsentwicklung (Bsp.: Binnendifferenzierung/Individualisierung). Auch eine veränderte Raumgestaltung kann notwendig sein. Grundsätzlich kann jahrgangsgemischtes oder jahrgangsübergreifendes Lernen in Klassen erfolgen, in denen zwei, drei oder vier Jahrgänge zusammengefasst sind (Kucharz, 2010, S. 333). Kucharz u.a. formulieren in ihren Veröffentlichungen verschiedene Zielsetzungen jahrgangsübergreifenden Unterrichts. So könnten Kinder sich untereinander zum Lernen anregen, jüngere Kinder von älteren Kindern lernen (u.a. durch Nachahmung), ältere Schüler durch das Erklären von Sachverhalten Lerninhalte tiefer durchdringen und Kinder im Verlauf mehrerer Schuljahre unterschiedliche Rollen in der Lerngruppe einnehmen. So würden bspw. auch schwächere Schüler altersbedingt in die Rolle von Helfern gelangen können (Kucharz, 2010, S. 333; Kucharz et al., 2009, S. 11f). Kritisch wird von diesen Autoren gesehen, dass Erstklässler hinsichtlich des Beziehungsaufbaus benachteiligt sein könnten, ein Ausbalancieren der altersentsprechenden Bedürfnisse schwierig 
sei, ein jährlicher Wechsel der Zusammensetzung der Lerngruppe erfolge, jüngere Kinder durch die Vielfalt der Lern- und Angebotssituation überfordert sein könnten, ältere Kinder sich zu sehr auf das Helfen, statt auf ihren eigenen Lernprozess konzentrieren würden. Für Lehrkräfte stelle diese Unterrichtsform eine hohe Anforderung dar, da fachliche Inhalte vielfach differenziert angeboten werden müssten. Darüber hinaus seien insbesondere Kinder mit besonderen Förderbedürfnissen auf strukturierte, lehrergelenkte Lernsituationen angewiesen (Kucharz et al., 2009, S. 12f). Eine empirische Überprüfung der Wirksamkeit jahrgangsübergreifenden Unterrichts sei bislang nur in wenigen Studien mit kleinen Stichproben erfolgt. Ein Problem vieler Studien liege auch darin, dass die Wirksamkeit nicht unabhängig von weiteren Faktoren der Unterrichtsgestaltung untersucht werden könne (Kucharz, 2010, S. 334). Die bisherigen Studien zeigten, „dass keine eindeutigen Vorteile der einen gegenüber der anderen Form übereinstimmend zu finden sind“ (Kucharz, 2010, S. 334) und es für jahrgangsübergreifende Lerngruppen sehr niedrige negative bis keine Effekte im Leistungsbereich im Vergleich zu Jahrgangsklassen gebe. Leichte Vorteile der gemischten Klassen seien im sozialen Bereich u.a. mit Blick auf die Qualität sozialer Lernprozesse festgestellt worden (Kucharz et al., 2009, S. 13-15; Kucharz, 2010, S. 334). Positiv sei in qualitativen Studien erfasst worden, dass Lehrkräfte differenzierter unterrichten als in nicht altersgemischten Gruppen (Kucharz 2010, 334). Was die Öffnung des Unterrichts im Rahmen jahrgangsübergreifenden Arbeitens angehe, zeige ein Überblick von Lipkowsky (1999, 2002), dass der Erfolg offenen Unterrichts u.a. abhängig von den Lernvoraussetzungen der Schüler sei: beispielsweise könnten Kinder mit besseren Lernvoraussetzungen ihre Lernzeit in offenen Situationen besser nutzen und stärker aufgabenorientiert arbeiten als Schüler mit ungünstigeren Lernvoraussetzungen (Kucharz et al., 2009, S. 16f). Eine Metaanalyse von Giaconia und Hedges (1982) habe ergeben, dass der traditionelle Unterricht eine leichte Überlegenheit hinsichtlich kognitiver Lernzieldimensionen aufweise, während offener Unterricht die Persönlichkeit und eine positive Einstellung zur Schule fördere (Kucharz et al., 2009, S. 17).

Insgesamt beziffert Hattie die Effektstärke jahrgangsübergreifender Klassen mit nahe Null ( $\mathrm{d}=$ 0,04) und zwar sowohl mit Blick auf kognitive als auch auf affektive Lernleistungen von Schülern. Seine Metaanalyse belegt allerdings für kooperatives Lernen, dass dieses effektiver ist als individuelles Lernen $(\mathrm{d}=0,41)$ : „Lernende sind besser in der Lage, kollektiv Fehler zu realisieren und aus ihnen zu lernen. Ihre Gespräche untereinander können außerdem dabei helfen, Lernintentionen und Erfolgskriterien für alle zu verdeutlichen." (Hattie, 2013, S. 253). Auch das so genannte „Peer-Tutoring“, bei dem bspw. ältere Schüler als Co-Lehrende agieren, wirkt sich nach Hattie mit einer Effektstärke von $\mathrm{d}=0,55$ positiv auf das Lernen aus: „Wenn also Lernende für andere zu Lehrenden werden, so lernen die Unterrichtenden ebenso viel wie die Unterrichteten." (Hattie, 2013, S. 223). Diese Ergebnisse können - kombiniert mit den Ergebnissen zu Effekten jahrgangsübergreifender Klassen allgemein - zu der Annahme führen, dass das kooperative Lernen in diesen Klassen stärker umgesetzt werden müsste um zu positiven Effektstärken zu führen. Ähnlich wie bei anderen organisatorischen Variablen auch (Bsp.: Klassengröße) verändert nicht die Variable an sich die Qualität von Lernprozessen, sondern deren praktische Umsetzung bzw. Verknüpfung mit anderen qualitativen Aspekten. Zu dieser Schlussfolgerungen gelangen - trotz unterschiedlicher Untersuchungsergebnisse - auch Veenman, Mason und Burns (n. Hattie, 2013, S. 111):

Sie stellen alle fest, dass Lehrpersonen kaum die besondere Zusammensetzung in jahrgangsübergreifenden Klassen nutzen, um kooperative Lernprozesse zu fördern. Stattdessen neigen Lehrpersonen dazu, deutlich unterschiedliche Curricula zu unterrichten, die Klassenstufung im Grunde genommen beizubehalten und separate Lektionen für jede Gruppe einer Klassenstufe zu erteilen. (Hattie, 2013, S. 111) 
Darüber hinaus sprechen mehrere andere Forschungsergebnisse der Hattie-Studie indirekt für eine Umsetzung jahrgangsübergreifenden Unterrichts. So hat das Sitzenbleiben - das im jahrgangsüber-greifenden Unterricht vermieden werden kann - mit Blick auf die Lernleistung von Schülern eine negative Effektstärke $(d=-0,16)$, während beschleunigter Unterricht (acceleration) einen großen Effekt $(\mathrm{d}=0,88)$ auf die Lernleistung von Schülern hat. Die Vermittlung von Lerntechniken, die notwendig sind um selbständiges Arbeiten in einer jahrgangsübergreifenden Lerngruppe zu ermöglichen, hat eine Effektstärke auf das Lernen von $d=0,69$ (Hattie, 2013, S. 116, S. 119, S. 225).

Jahrgangsübergreifender Unterricht ist zunächst also „nur“ eine Organisationsform, die als solche weder wesentlich schadet noch nützt. Die Organisationsform kann ihre Wirksamkeit dann entfalten, wenn Lehrkräfte ihren Unterricht tatsächlich verändern und kooperative Lernformen innerhalb der gemischten Gruppe systematisch gefördert werden.

In sonderpädagogischen Veröffentlichungen spielt der jahrgangsübergreifende Unterricht lediglich mit Blick auf die flexible Gestaltung der Eingangsstufe (Klassen 1 und 2) eine Rolle. Bei der PInG-Studie (Pilotprojekt „Inklusive Grundschule“ in Brandenburg) wurde erhoben, dass $40 \%$ der Schulen jahrgangsübergreifenden Unterricht in der Schuleingangsphase anbieten. Mit Blick auf die Ganztagsbeschulung benennen nur wenige Forschungsveröffentlichungen der Sonderpädagogik diese als Qualitätsmerkmal integrativer Schulen (Heyer et al., 1997; Wember \& Prändl, 2009). Während nach Stäbler et al. mehr als die Hälfte der inklusiven Schulen in Brandenburg keinen Ganztagsunterricht anbietet, arbeiten $30 \%$ als verlässliche Halbtagsgrundschulen und 15\% haben ein offenes Ganztagsangebot (Stäbler et al., 2015, S. 80). Anstelle des Ganztagsunterrichts wird hinsichtlich der Veränderungen von Organisationsstrukturen von Schulen vor allem die Gestaltung von Übergängen als Qualitätsbaustein integrativer Schulen beschrieben (Übergang Kindergarten - Grundschule, Grundschule - Weiterführende Schule, Weiterführende Schule Berufliche Bildung). In diesem Zusammenhang werden vorbereitende Prozesse als wesentlich erachtet wie bspw. die Vorbereitung durch integrative Kindergärten (Bless, 2003), das Verhaltenstraining mit behinderten Kindern zur Vorbereitung auf die Regelschule (Mühl, 1987), die rechtzeitige Vorbereitung seitens der Schule (Mühl, 1987; Berges, 1996; Feyerer, 1998; Brugger-Paggi, 2003) und seitens der Lehrkräfte (Hug, 1994b). Es sollte eine Verzahnung von Frühförderung bzw. Kindergärten zur Schule sowie von der Schule aus zu Berufsberatung und beruflichen Schulen erfolgen (Boban \& Hinz, 2003; Wember \& Prändl, 2009). Die Studie von Innerhofer und Klicpera (1991) hat außerdem ergeben, dass eine Begleitung dieser Übergänge wichtig ist. Stoellger formuliert aufgrund der positiven Erfahrungen an der Fläming-Grundschule die Empfehlung, dass die sozialen Strukturen aus der Integrationsklasse der Grundschule beim Übergang in die Sekundarstufe in Form einer Kernklasse erhalten bleiben sollten (Stoellger, 1988, S. 336). Nach Brugger-Paggi (2003) sind auch Übergänge innerhalb einer Schule so zu gestalten, dass eine erzieherische und didaktische Kontinuität für die Schülerinnen und Schüler gegeben ist.

\subsubsection{Ergebnisse im Bereich der Organisationsentwicklung}

Als Ergebnisse der Organisationsentwicklung an Schulen werden unter anderem Leitbilder sowie Schulprogramme benannt. Als Teilbereich des Organisationsmanagements zählt zu den Ergebnissen von Entwicklungsprozessen in diesem Bereich auch die Dokumentation von Zielen, Abläufen, Verfahren, Strukturen und Entwicklungsprozessen (ähnlich der Normenreihe DIN EN ISO $\left.9000 \mathrm{ff}^{24}\right)$.

24 Unter: https://www.din.de/de/mitwirken/normenausschuesse/nqsz/normen/wdc-beuth:din21:235671064 
Leitbilder beinhalten nach Steiner-Löffler (2008) bzw. Holtappels (2010e) Formulierungen zur pädagogischen Grundhaltung einer Schule und bestehen aus etwa zehn Leitsätzen, die „das Wunschbild aus der Vision in prägnante Worte fassen“" (Steiner-Löffler, 2008, S. 211). Zech formuliert dazu:

Ein Leitbild ist eine gemeinsame Selbstbeschreibung der Organisation Schule durch deren Beschäftigte. Es soll die Handlungen der Organisation beziehungsweise der Organisationsmitglieder anleiten und an den gemeinsamen Zielen ausrichten. Das Leitbild muss von außen als Profil der Organisation erkennbar und von innen erlebbar sein. Das Leitbild ist ein Ausweis des eigenen Selbstverständnisses und enthält auch Aussagen über die allgemeinen pädagogischen Ziele der Schule. Es ist ein Leistungsversprechen gegenüber den Schülerinnen und Schülern, deren Eltern und gegenüber den weiterführenden bzw. abnehmenden Einrichtungen. Das Leitbild ist ein Maßstab zur Bewertung des Verhaltens der Schulleitung und aller Beschäftigten. Mit Hilfe eines Leitbildes kann eine Organisation zielorientiert geführt und verändert werden. (Zech, 2009, S. 1)

In Schulprogrammen geht es um die Ausformulierung und Konkretisierung dieser Leitbilder (Steiner-Löffler, 2008, S. 215; Holtappels, 2010e, S. 267f).

Ein Schulprogramm muss dabei zumindest eine Zielorientierung, eine Bestandsaufnahme und eine Entwicklungsperspektive umfassen. Damit verbunden sind entsprechende Maßnahmeplanungen und ein inhaltlich und zeitlich ausgewiesenes Arbeitsprogramm [...]. (Holtappels, 2010e, S. 267)

Bei den Erläuterungen werden Entwicklungsschritte so detailliert wie möglich benannt. Darüber hinaus werden Akteure festgelegt, damit die Schritte auch tatsächlich umgesetzt werden. Als Qualitätskriterien für inklusive Schulen werden Schulprogramme, in denen die schulische Integration von Schülerinnen und Schülern mit sonderpädagogischem Förderbedarf inhaltlich verankert ist, seit Ende der 90er Jahre aufgeführt (Berges, 1996; Boban \& Hinz, 2003; BruggerPaggi, 2003; Wember \& Prändl, 2009). Die österreichischen Richtlinien für integrative Schulen benennen außerdem Förderkonzepte als Qualitätsmerkmal (BMBWF Österreich, 2008). Zur Erarbeitung von Schulprogrammen können Hilfsinstrumente wie der Index für Inklusion genutzt werden. Werning (2013) stellt einen internationalen Zuwachs hinsichtlich der Verwendung dieser Hilfsmittel fest, während Laubenstein u.a. die Verwendung des Indexes für Inklusion in Rheinland-Pfalz nur an $18 \%$ der Schwerpunktschulen (20 von 133) erfassen können (Laubenstein et al., 2015, S. 142).

Schulprogramme sind nach Holtappels zugleich Entwicklungs- wie Steuerungsinstrumente (Holtappels, 2010, S. 266f). Obwohl sie also in dieser Arbeit als Qualitätskategorie im Bereich Ergebnisse aufgeführt werden, haben sie gleichzeitig auch während ihrer Erstellung eine Wirkung auf die Entwicklung einer Schule, wie 94\% der Schulleitungen in einer Längsschnittstudie in Niedersachsen angaben. Die Lehrkräfte berichten von einer positiven Wirkung auf Kooperation und Arbeitsklima. Auswirkungen der Schulprogrammarbeit auf die Unterrichtsqualität sowie auf kognitive oder psychosoziale Lernergebnisse bei den Schülern konnten allerdings nach 27 Monaten nicht festgestellt werden (Holtappels, 2010e, S. 271).

Im Rahmen der PISA-Studie wurden Daten zu Maßnahmen der Qualitätssicherung und -entwicklung erhoben. Diese zeigten 2001, dass in den Schulen eine hohe Sensibilität für Fragen der Qualität vorhanden ist, viele Schulen sich aber diesbezüglich in einer noch eher explorativen Phase befanden. Über ein Leitbild oder Schulprofil verfügten zu Beginn der 2000er Jahre $55,3 \%$ der Hauptschulen $(n=62)$, 46,8\% der Realschulen $(n=45)$ und 67,8\% der Gymnasien $(\mathrm{n}=37$ ), über ein Schulprofil 42,9\% (HS), 48,7\% (RS) und 40,5\% (GYM) (Dt. PISAKonsortium, 2001, S. 445). Kritisch sehen die PISA-Autoren die Tatsache, dass die Schulen 
bislang noch nicht in ganz so hohem Maß Leistungsstandards schriftlich festlegen (zwischen $23,8 \%$ und 38,4\%) bzw. standardisierte Leistungstests einsetzen (zwischen 6,8\% und 20,2\%) (Dt. PISA-Konsortium, 2001, S. 445).

Im Zusammenhang mit der wissenschaftlichen Begleitung des Modellprojekts „Inklusive Grundschule“ in Brandenburg wurden 55 Schulprogramme analysiert und mit möglichen Qualitätsmerkmalen inklusiver Schulen verglichen. Als Analysegrundlage dienten neben den Schulprogrammen u.a. Datenbögen von Schulleitungen und Schulleiterfragebögen, Leistungsdaten zu VERA $3^{25}$ und ZVA $6^{26}$ sowie Visitationsergebnisse aus der Zeit des Modellversuchs. Für diese Analyse zugrunde gelegt wurde ein Kategoriensystem, das sich am Index für Inklusion orientiert (Friedrich, 2015, S. 199). Friedrich gelangt aufgrund ihrer detaillierten und umfangreichen Analyse zu folgendem Schluss:

Nicht in allen Schulprogrammen der Pilotschulen ist das Vorhaben „Inklusion“ bereits fest verankert und zum zentralen Bezugspunkt der gesamten pädagogischen Arbeit geworden, wie man hätte annehmen können. Die Analyse der 55 eingereichten Schulprogramme nach dem Index für Inklusion zeigte, dass $45 \%$ der Schulprogramme erkennbar inklusive Ziele bzw. inklusive Maßnahmen und Praktiken enthalten und 55\% so gut wie keine. Dies ist abhängig vom Aktualisierungsgrad der Schulprogramme. (Friedrich, 2015, S. 228)

In den 25 Schulen, die über ein als inklusiv erkennbares Schulprogramm verfügen, werden in den Programmen (zu $40 \%$ bis $90 \%$ ) folgende Qualitätsmerkmale benannt: gute Zusammenarbeit mit Eltern und Mitarbeitern, Anerkennung der Heterogenität der Schülerschaft, respekt- und vertrauensvoller Umgang, binnendifferenzierte Unterrichtsangebote, Mobilisierung von Ressourcen im Umfeld der Schule, Ziel der Kompetenzerweiterung des Kollegiums durch Fortbildungen, enge Zusammenarbeit innerhalb des Kollegiums und Kooperation zwischen Regel- und Förderschullehrkräften (Friedrich, 2015, S. 228). Allerdings fehlen in den Programmen häufig Formulierungen zu konkreten Maßnahmen für die Umsetzung dieser Ziele (Friedrich, 2015, S. 229). Zum Teil konnte durch die Schulvisitationen bestätigt werden, dass an den Schulen mit einem inklusiven Schulprogramm eine bessere Planung der Schul- und Unterrichtsorganisation vorgenommen wird (Bsp: Vertretungspläne ohne Unterrichtsausfall), Schüler und Eltern intensiver am Schulleben beteiligt werden und eine Öffnung und Vernetzung der Schulen nach außen erfolgt. Darüber hinaus „erfassen und dokumentieren die Lehrkräfte der Schulen mit einem inklusiven Schulprogramm verstärkt die Lernentwicklung der Schülerinnen und Schüler.“ (Friedrich, 2015, S. 229). Dagegen konnte nicht belegt werden, „dass es den inklusiven Schulprogrammschulen besser gelingt, die Schülerinnen und Schüler entsprechend ihrer individuellen Voraussetzungen zu fördern und den Unterricht an die individuellen Lernvoraussetzungen anzupassen." (Friedrich, 2015, S. 229). So ergab die Studie keinen Zusammenhang zwischen den Inhalten der Schulprogramme und Schülerleistungen in Deutsch und Mathematik und zwar weder in VERA 3 noch in ZVA 6. Insgesamt waren die Schülerleistungen in den Schulen mit inklusiven Programmen stattdessen etwas schwächer als in den Vergleichsschulen mit weniger inklusiven Schulprogrammen, was mit einem möglicherweise höheren Anteil an Schülern mit spF bzw. anderem Unterstützungsbedarf in diesen Schulen begründet wird (Friedrich, 2015, S. 229). In Schulen mit wenig inklusiven Schulprogrammen wird nach Friedrich vor allem „der Umgang mit sozial und emotional gestörten Schülerinnen und Schülern häufig als Hauptschwierigkeit genannt" (Friedrich, 2015, S. 230).

25 VERA = Vergleichsarbeiten

$26 \mathrm{ZVA}=$ Zentrale Vergleichsarbeiten 
Problematische Effekte von Leitbild- oder Schulprogrammarbeit in Schulen können nach Zech (2013) dann entstehen, wenn diese sehr idealisierte Vorstellungen (einer humanen Schule) beinhalten, die den Fokus auf der einen Seite zu stark auf schwierige Bedingungsfaktoren lenken und auf der anderen Seite dadurch nicht ausreichend bearbeitbar scheinen und als Folge in einem Kollegium zu Resignation führen können (Zech, 2013, S. 210).

Auch die Dokumentation und Außendarstellung von Schulen spielen nach Auffassung einiger sonderpädagogischer Autoren eine wichtige Rolle (Innerhofer \& Klicpera, 1991; Boban \& Hinz, 2003; Brugger-Paggi, 2003). Informationen zu schulischer Integration sollten für Außenstehende barrierefrei zugänglich sein (Boban \& Hinz, 2003) und mit anderen Schulen ausgetauscht werden (Brugger-Paggi, 2003).

\subsubsection{Zusammenfassung zum Bereich der Organisationsentwicklung}

Mit Blick auf die Organisationsentwicklung und damit das organisationale Lernen an (inklusiven) Schulen spielen aus sonder- wie aus allgemeinpädagogischer Sicht die zur Verfügung stehenden Ressourcen, die professionelle Leitung, Bausteine der Schule als sozialer Raum (Bsp.: intensive Elternarbeit und enge Vernetzung im Umfeld) sowie Organisationsstrukturen eine zentrale Rolle. Diese übergeordneten Kategorien sind sowohl für schulische Entwicklungsprozesse allgemein wie für die Qualität schulischer Integration relevant. Als Ergebnisse der Organisationsentwicklung an Schulen werden u.a. Leitbilder und Schulprogramme benannt.

Forschungsveröffentlichungen gehen davon aus, dass die Ressourcenausstattung von Schulen einen Einfluss auf die Qualität der unterrichtlichen Arbeit haben kann (Dt. PISA-Konsortium, 2001; Hattie, 2013). Im europäischen Vergleich bewegen sich die Bildungsausgaben der Bundesrepublik Deutschland im Mittelfeld. Die Problematik unzureichender finanzieller Ressourcen für schulische Integration wurde bereits in den 70er Jahren thematisiert. Autoren der 2000er Jahre (Sander, 2003; Speck, 2010) weisen auf Finanzierungsvorbehalte der Länder hin, die teilweise in den Landesgesetzen zur schulischen Integration festgeschrieben wurden. Für die Umsetzung der schulischen Inklusion in der BRD belegen zwei Gutachten (Klemm, 2009; Dohmen \& Fuchs, 2009), dass diese Kosten verursachen könnte, die - je nach Berechnungsgrundlage zwischen 0,24 und 4,3 Milliarden Euro jährlich betragen. Auch ein weiteres Gutachten für zwei Kommunen in NRW belegt, dass mit der Einführung inklusiver Bildung in den Regelschulen Investitionskosten verknüpft sind (Schwarz et al., 2013). Diese Investitionskosten werden von der Bildungspolitik nur unzureichend thematisiert. Gute materielle Rahmenbedingungen allgemein sowie solche, die die bauliche Gestaltung und Ausstattung (zentrale Kategorien: Barrierefreiheit, räumliche Flexibilität, differenziertes Material) von Schulen betreffen, werden in der sonderpädagogischen Forschung als qualitativ notwendige Rahmenbedingungen für integratives schulisches Arbeiten herausgestellt. Die räumliche Flexibilität bezieht sich dabei zum einen auf genügend große Klassenräume, die eine flexible Unterrichtsgestaltung ermöglichen, sowie auf zusätzliche Förder- oder Differenzierungsräume. Für Schülerinnen und Schüler mit spF werden zum Teil sehr spezifische Hilfen benötigt (Bsp.: elektronische Hilfen, spezifische Arbeitsplatzausstattung). Gleichzeitig wird befürchtet, dass eine mangelnde Ressourcenausstattung dazu führen könnte, dass Eltern von Kindern oder Jugendlichen mit sonderpädagogischem Förderbedarf sich für den Besuch einer Förder- anstelle einer Regelschule entscheiden könnten, was Textor (2015) als „Gefahr indirekter Steuerung“ bezeichnet.

Schulleitungen beeinflussen - wie sowohl allgemein- als auch sonderpädagogische Veröffentlichungen belegen - die Entwicklung von Schulen in hohem Maß (Bonsen, 2002; Bryk et al., 2010; Dyson, 2010; Scheer \& Laubenstein, 2018). Ihr professionelles Management kann sich 
auf die Professionalisierung und die Kooperation der Lehrkräfte untereinander, auf die Zusammenarbeit mit den Eltern, den Grad der Einbindung in die Kommune, die Qualität des Unterrichts sowie die Leistungen der Schülerinnen und Schüler auswirken (Bryk et al., 2010; Hattie, 2013). Trotz dieser Annahme spielt die Funktion der Leitung im Rahmen der Forschung zu schulischer Integration bislang nur eine untergeordnete Rolle (Innerhofer \& Klicpera, 1991; Dyson, 2010; Lienhard-Tuggener et al., 2011; Joller-Graf \& Tanner, 2011; Scheer \& Laubenstein, 2018). Aus sonderpädagogischer Sicht können Schulleitungen als „Promoter der Integration" (Innerhofer \& Klicpera, 1991) gesehen werden, die Einfluss auf die Haltungen von Lehrkräften und die Umsetzung der Integration in den Schulen haben. Sie übernehmen beratende, vernetzende, koordinierende sowie unterstützende Leistungen für Eltern und Lehrkräfte und leisten Beiträge zur Unterrichtsentwicklung. Nach Serke u.a. (2014) haben sie eine wichtige Funktion mit Blick auf die Schaffung verbindlicher organisatorischer Strukturen (Bsp.: Berücksichtigung fester Kooperationszeiten bei der Stundenplanung). Unabhängig von den Aufgaben der Schulleitungen, die mit schulischen Entwicklungsprozessen bzw. schulischer Integration im engeren Sinn zu tun haben, ist relevant, wie Schulleiterinnen und Schulleiter die „Schule als Betriebseinheit" insgesamt führen (Bsp.: Gestaltung von Abläufen und Strukturen, Verwaltung, Kommunikation etc.). Zu diesem Bereich geben die Forschungsergebnisse keine Auskunft. Ressourcen für die o.g. Tätigkeiten können aber nur dann vorhanden sein, wenn die anderen Bereiche der Schulleitungsarbeit effizient umgesetzt werden.

Die Schule als sozialer Raum ist als Einrichtung zu verstehen, deren Schulklima von einer spezifischen Kommunikationskultur und den Umgangsformen aller Beteiligten geprägt wird, die wiederum das emotionale und kognitive Wohlbefinden beeinflussen (Hascher, 2017). Nach Auffassung der PISA-Studie stellt das Schulklima auf der einen Seite eine eigene Qualitätsdimension, auf der anderen Seite eine Bedingungsvariable für die Leistungen von Schülerinnen und Schülern dar (Dt. PISA-Konsortium, 2001). Auch sonderpädagogische Autoren gehen davon aus, dass das schulische Klima sich auf Lernen und Verhalten auswirkt (Reiser et al., 1984; Dyson, 2010). Entscheidend seien in diesem Zusammenhang Wertschätzung, Respekt und Akzeptanz zwischen allen am Schulleben beteiligten Personen. Um Integration als gemeinsame Aufgabe einer Schule wahrzunehmen, sei es notwendig, dass die Beteiligten eine übereinstimmende offene Haltung an den Tag legen (Bächthold, 1998; Mühl, 1987; Boban \& Hinz, 2003) sowie bereit sind gemeinsam Verantwortung zu übernehmen (Feyerer, 1998; Boban \& Hinz, 2003; Holzinger et al., 2011). Mit Blick auf die Partizipation fordern sonderpädagogische Veröffentlichungen u.a. die Einbeziehung von Schülern und Eltern in schulische Entwicklungsprozesse.

Ein entscheidendes Qualitätsmerkmal innerhalb der Schule als sozialem Raum ist die Zusammenarbeit mit den Eltern. Veröffentlichungen der Allgemeinen Pädagogik belegen dabei hohe Effekte der elterlichen Einbeziehung in die Schule durch einen gegenseitigen Austausch vor allem mit Blick auf die Entwicklung der Schülerinnen und Schüler (Jeynes, 2005; Pushor, 2007; Berthelsen \& Walker, 2008; Bryk et al., 2010). Familienstruktur und elterliche Unterstützung hätten in diesem Zusammenhang stärkere Effekte auf schulische Erfolge als bspw. die Einbindung der Eltern durch Elternabende etc. (Jeynes, 2005a u. 2005b). Wesentlich sei, dass Erziehung und Förderung von Schule und Eltern als gemeinsame Aufgabe verstanden werden würden. Nicht die Quantität, sondern die Qualität der Elternkontakte sei wichtig (Berthelsen \& Walker, 2008). Ein hohes Maß an Übereinstimmung hinsichtlich der inhaltlichen Aussagen findet sich diesbezüglich in den sonderpädagogischen Ausführungen zu diesem Thema. Die Einbeziehung und Unterstützung von Eltern der Schülerinnen und Schüler mit sonderpädago- 
gischem Förderbedarf durch Information, Austausch, Beratung oder Fortbildungsangebote für Eltern ist eine Qualitätskategorie integrativer Schulen, die nicht nur häufig benannt, sondern inhaltlich auch differenziert dargestellt wird. Die tatsächliche Umsetzung der Zusammenarbeit mit den Eltern wird in Forschungsveröffentlichungen sehr unterschiedlich beschrieben (Wocken, 1987; Dumke, 1989; Wild \& Lütje-Klose, 2017).

Die Vernetzung mit außerschulischen Einrichtungen bzw. Kooperationspartnern stellt ebenfalls sowohl in der allgemeinpädagogischen als auch in der sonderpädagogischen Forschung ein Qualitätsmerkmal dar, ist allerdings - wie eine Studie an Schwerpunktschulen in RLP belegt in vielen integrativ arbeitenden Schulen kein Standard (Laubenstein et al., 2015). Inwiefern die Kooperation mit außerschulischen Institutionen professionell organisiert ist (Ahrens, 2009) oder noch Handlungs- und Strukturierungsbedarf vorliegt (De Boer \& Spies, 2014) bzw. die Zusammenarbeit von beiden Seiten als unterstützend und bereichernd eingeschätzt wird, lässt sich anhand der sonderpädagogischen Veröffentlichungen nicht belegen. Wichtige Kooperationspartner der Schulen sind die Jugendämter, der schulpsychologische Dienst sowie Vereine, Musikschulen, Kirchengemeinden etc.. Zwischen einzelnen Schulen scheinen große Unterschiede mit Blick auf die Kooperation mit unterschiedlichen Kooperationspartnern zu bestehen. Mögliche positive Effekte der vernetzenden Kooperation (Bryk et al., 2010) sind bislang kaum erfasst.

Veränderungen von Organisationsstrukturen wie bspw. die Rhythmisierung des Schultags, die Nutzung unterschiedlicher organisatorischer Möglichkeiten der integrativen Beschulung (Bsp.: Kleinklassen), die Einrichtung klassenübergreifender Fördergruppen, die Umsetzung jahrgangsübergreifenden Unterrichts oder die Gestaltung eines Ganztagsangebots werden in integrativen Schulen nur teilweise vorgenommen (Laubenstein et al., 2015; Stäbler et al., 2015). Sie sind vor allem ein vielfältiges (Forschungs-) Thema der Allgemeinen Pädagogik, während ihre Thematisierung im Zusammenhang mit schulischer Integration eher ein Randthema darstellt. Von besonderer Bedeutung aus sonderpädagogischer Sicht ist die Gestaltung von Übergängen zwischen Kindertagesstätten und Grundschulen bzw. zwischen Grundschulen und weiterführenden Schulen sowie weiterführenden Schulen und beruflicher Bildung. Jahrgangsübergreifender Unterricht wird aus sonderpädagogischer Sicht für die Eingangsstufe als hilfreich eingeschätzt (Bsp.: FLEX Brandenburg), ansonsten aber als Organisationsform kaum thematisiert. Aus empirischer Perspektive ist dieser nur dann mit Blick auf die Kompetenzentwicklung der Schüler wirksam, wenn er mit anderen qualitativen Ansätzen (Bsp.: kooperatives Lernen) verknüpft wird. Auch die Ganztagsbeschulung spielt als Qualitätssubkategorie so gut wie keine Rolle. Dass Transformationen im Bereich der Organisationsstrukturen insgesamt wenig aufgegriffen bzw. empirisch untersucht werden, erscheint ungewöhnlich, da u.a. Möglichkeiten äußerer Differenzierung, jahrgangsübergrei-fender Lerngruppen oder ganztägiger pädagogischer Angebote im Zusammenhang mit schulischer Integration wichtig erscheinen. Bis auf die Förderschulen Sprache, Lernen und soziale und emotionale Entwicklung sind Förderschulen normalerweise als gebundene Ganztagsschulen konzipiert, in denen innere und äußere Differenzierung organisatorisch häufig in vielfältiger Form ermöglicht werden. Entsprechend naheliegend wäre eine Adaption flexibler organisatorischer Strukturen in integrativen Schulen. Klassenübergreifender Förderunterricht könnte in diesem Zusammenhang nicht nur für Schülerinnen und Schüler mit sonderpädagogischem Förderbedarf angeboten werden, sondern für zeitlich begrenzt zusammengestellte Lerngruppen für unterschiedliche Förderschwerpunkte (Bsp.: jahrgangsübergreifende Gruppe, in der ältere SuS jüngeren SuS Sachverhalte erklären; Gruppen, in denen gezielt unterrichtliche Themen auf verschiedenen Kompetenzniveaus bearbeitet werden etc.). 
Zu den Ergebnissen der Organisationsentwicklung von Schulen wie Leitbildern und Schulprogrammen sowie deren Effekten liegen nur wenige empirische Erkenntnisse vor, obwohl diese sowohl in pädagogischen wie in sonderpädagogischen Veröffentlichungen häufig als Qualitätsmerkmale guter (integrativer) Schulen aufgeführt werden. Studien konnten bislang keine Zusammenhänge zwischen (inklusiven) Schulprogrammen und Schülerleistungen feststellen. Dagegen hat die gemeinsame Erstellung von Leitbildern oder Schulprogrammen offensichtlich Wirkungen auf Kooperation und Arbeitsklima. Gegebenenfalls sind also die Prozesse der Entwicklung Ausschlag gebender als deren Ergebnisse. Während Leitbilder an (integrativen) Schulen noch relativ weit verbreitet sind, liegen Schulprogramme nach PISA in etwa $40 \%-49 \%$ der untersuchten Schulen vor. Für Rheinland-Pfalz belegen die Untersuchungsergebnisse von Laubenstein u.a. (2015), dass $18 \%$ der Schwerpunktschulen Hilfsinstrumente für ihre Schulentwicklung (Bsp.: Index für Inklusion) genutzt haben (Laubenstein et al., 2015, S. 142f).

\subsection{Personalentwicklung}

Welche personellen Ressourcen sind in integrativ arbeitenden Schulen vorhanden bzw. werden als notwendig erachtet? Wie arbeiten Kolleginnen und Kollegen in diesen Schulen insgesamt zusammen? Wie gestaltet sich die Zusammenarbeit zwischen den Regel- und Förderschullehrkräften? Welche Formen der gemeinsamen Unterrichtsvorbereitung, -durchführung und -nachbereitung werden genutzt? Welche Rollen und Aufgaben werden von den Regel- bzw. Förderschullehrkräften übernommen? Welche Aspekte spielen mit Blick auf die Professionalisierung von Lehrkräften in diesen Schulen eine Rolle? Welche Haltungen vertreten die Lehrerinnen und Lehrer in diesen Schulen? Wie können Ergebnisse von Personalentwicklung in integrativen Schulen aussehen? Diesen Fragen widmet sich das vorliegende Unterkapitel. Aufgrund der sonderpädagogischen Forschungsliteratur ergeben sich für diesen Bereich die zwei Prozesskategorien Kooperation und Professionalisierung. In der Systematisierung dieser Arbeit werden also anders als bei Rolff - unter Personalentwicklung auch alle Qualitätsbausteine gefasst, die die Kooperation der Lehrkräfte und anderen pädagogischen Mitarbeiter untereinander betreffen. Diese Entscheidung beruht auf der Annahme, dass auch Maßnahmen, die bspw. Teamarbeit und Co-Teaching fördern, die professionelle Weiterentwicklung des Personals stark beeinflussen können. All diese Maßnahmen können wiederum dazu beitragen, dass die Weiterentwicklung einer Schule überhaupt erst möglich wird, da der Austausch über Unterricht und pädagogische Ziele der Schule im Kollegium sowie gemeinsam mit anderen Fachkräften stattfindet. Andererseits spielen die von Rolff (2010) benannten Möglichkeiten der direkten Personalentwicklung wie Mitarbeitergespräche oder Coaching in der sonderpädagogischen Forschungsliteratur zu schulischer Integration keine Rolle, so dass sie an dieser Stelle nicht beschrieben werden.

Personalentwicklung umfasst als Schulentwicklungsbereich die „Personalfortbildung, Personalführung und Personalförderung“ (Rolff, 2010, S. 33) in Schulen. Sie wird definiert als „Inbegriff aller Maßnahmen, die der individuellen beruflichen Entwicklung der Mitarbeiter dienen und ihnen unter Beachtung ihrer persönlichen Interessen die zur optimalen Wahrnehmung ihrer jetzigen und künftigen Aufgaben erforderlichen Qualifikationen vermitteln." (Mentzel, 1997, S. 15). Unterschieden werden kann dabei zwischen direkter Personalentwicklung wie Mitarbeitergesprächen, Beratungen, Coaching, Supervision sowie Fortbildungen und indirekter Personalentwicklung wie der Gestaltung von „Lernsituationen“ durch Veränderung von Settings in der Schule (Bsp.: Teamsitzungen) (Wunder, 2000, S. 32f). Etwas anders umschreiben dies Harazd und Drossel (2011, S. 149ff, zitiert n. Lütje-Klose \& Urban, 2014b, S. 291), die mit Hilfe 
einer Online-Befragung feststellen konnten, dass direkte Einflussnahme seitens Schulleitungen mit Blick auf Kooperation darin bestehen kann, dass diese strukturelle Rahmenbedingungen wie gemeinsame Zeiten für die Zusammenarbeit von Jahrgangsteams im Stundenplan o.̈. schafft, während indirekte Einflussnahme so aussehen kann, dass die Schulleitung Wertschätzung für die Kooperation der Lehrkräfte zum Ausdruck bringt (Lütje-Klose \& Urban, 2014b, S. 291). In dieser Arbeit wird auch die Kooperation von Lehrkräften unter den Bereich der Personalentwicklung subsumiert, da diese mit Bezug auf die einzelne Lehrperson Dimensionen von Professionalisierung und der Verminderung von Belastungen umfasst sowie mit Blick auf Schulentwicklung einen Indikator für die Organisationsqualität und eine Schlüsselvariable für die Weiterentwicklung von Schulen darstellt (Baum et al., 2012b, S. 9f).

Eine wesentliche Begründung der Bedeutung der Personalentwicklung in Schulen ist darin zu sehen, dass es die Lehrkräfte sind, die das Lernen von Schülern maßgeblich beeinflussen, wie Hattie in seiner Metaanalyse darlegt:

1 Lehrpersonen gehören zu den wirkungsvollsten Einflüssen beim Lernen.

2 Lehrpersonen müssen direktiv, einflussreich, fürsorglich und aktiv in der Leidenschaft des Lehrens und Lernens engagiert sein.3 Lehrpersonen müssen wahrnehmen, was Lernende denken und wissen, um Bedeutung und sinnstiftende Erfahrungen im Lichte dieses Wissens zu konstruieren. Zudem müssen sie ein kompetentes Wissen und Verständnis vom Stoff ihres Fachs besitzen, um sinnvolles und angemessenes Feedback geben zu können [...]. (Hattie, 2013, S. 218)

Hattie stellt daher das Handeln der Lehrkräfte in den Mittelpunkt seiner Überlegungen:

What matters are conceptions of teaching, learning, assessment, and teachers having expectations that all students can progress, that achievement for all is changeable (and not fixed), and that progress for all is understood and articulated. It is teachers who are open to experience, learn from errors, seek and learn from feedback from students, and who foster effort, clarity and engagement in learning. (Hattie, 2009, S. 35)

Pfrang und Viehweger bestätigen diese Sichtweise aufgrund von Ergebnissen im Rahmen einer Studie in einer ersten Klasse, die belegt, dass Schulanfänger Wert darauf legen, wie eine Lehrkraft sich persönlich einbringt und welche Qualitäten sie zeigt (geduldig sein, gut erklären können, Gefühle zeigen, Respekt, Zuneigung, Nähe, Lob, Anerkennung vermitteln, Unterstützung beim Lernen bieten). Das Verhalten des Lehrers beeinflusse die Lernfreude der Erstklässler (Pfrang \& Viehweger, 2015, S. 294f):

Besonders wichtig ist es, dass sich Lehrkräfte bewusst sind, dass ihre Schüler vielseitige Bedürfnisse haben und über ganz unterschiedliche Kompetenzen verfügen. Dies macht eine individuelle Förderung der Kinder in der Gruppe nötig. Die Ergebnisse zeigen weiterhin auf, dass sich Lehrer darüber im Klaren sein müssen, dass die Schüler ihr Verhalten und Handeln im Unterrichtsalltag bewusst erfahren. Dies verweist darauf, dass das Verhalten und Handeln von Lehrern Lernprozesse herausfordern bzw. fördern, aber auch beeinträchtigen kann. Nur wenn im konkreten Fall eruiert wird, wie Schüler das Verhalten und Handeln ihres Lehrers erfahren und wie es sich aus ihrer Sicht auf ihr Lernen auswirkt, können Lehrer selbst ihr Verhalten und Handeln reflektieren und gegebenenfalls auch ändern. (Pfrang \& Viehweger, 2015, S. 295)

Marzano \& Marzano (2003) berichten aufgrund einer Interviewstudie von Chiu \& Tulley (1997) mit 700 Schülerinnen und Schülern in den Klassen 4-7, dass diejenigen Lehrkräfte bevorzugt werden, die eine klare Klassenführung umsetzen, nicht so sehr die Lehrkräfte mit einem eher permissiven Erziehungsstil (Marzano \& Marzano, 2003, S. 163). Die genannten Grundvo- 
raussetzungen mit Blick auf die Handlungskompetenzen von Lehrkräften wiederum scheinen in der BRD im internationalen Vergleich nicht genügend entwickelt zu sein:

Es besteht weitgehend Übereinstimmung darüber, dass bei den deutschen Lehrkräften die didaktischen Fähigkeiten und die allgemeinen Unterrichtskompetenzen weniger stark entwickelt sind als ihr Fachwissen und sie nicht hinreichend darauf vorbereitet sind, die Lernkompetenzen der Schülerinnen und Schüler zu entwickeln, den Unterricht auf individuelle Bedürfnisse abzustimmen, das selbstregulierte Lernen zu fördern, die Schüler durch Anwendung aktiver Methoden zu motivieren, komplexe Projekte für das Lernen zu initiieren oder kooperatives Lernen in Gruppen zu organisieren. (OECD, 2004, S. 33)

Wie Untersuchungen von Terhart (2001) sowie Allemann-Ghionda und Terhart (2006) belegen, führen die erste und zweite Phase der Lehrerausbildung sowie die ersten Berufsjahre in Deutschland zunächst dazu, dass die zentrale Kompetenz zur Gestaltung von Unterrichtssituationen erworben wird, während komplexere Kompetenzen, die bspw. die Lernstandsdiagnostik, Binnendifferenzierung oder spezifische Auswahl von Methoden betreffen, erst im Anschluss entwickelt werden (Terhart, 2010, S. 238f). Aus diesen Gründen spielen die berufsbegleitende weitere Professionalisierung sowie die Kooperation der Lehrkräfte untereinander, die diese Professionalisierung begünstigen kann, eine entscheidende Rolle in Schulentwicklungsprozessen. Problematisch kann im Berufsfeld Schule mit Blick auf die Kooperation von Lehrkräften darüber hinaus sein, dass der Lehrerberuf nach Auffassung der Pädagogik durch „einen Mythos der Individualität“ (Schley, 2002, S. 3) bzw. eine „hohe Autonomie der einzelnen Lehrpersonen gekennzeichnet ist, die sich vor allem aus der räumlichen Trennung der Lehrkräfte untereinander ergibt." (Fussangel \& Gräsel, 2012, S. 33). Der Einsatz von Förderschullehrern in Regelschulen stellt daher sowohl die Förderschullehrkräfte selbst als auch die Regelschullehrkräfte vor weitere Herausforderungen. Während die sonderpädagogisch ausgebildeten Lehrkräfte sich mit einem anderen Berufsbild auseinandersetzen müssen, als sie es in Förderschulen ausüben, geht es für beide Seiten darum, effektive Formen der Kooperation und Arbeitsteilung zu entwickeln. Dazu sind ebenfalls Professionalisierungsprozesse notwendig, die in den Schulalltag integriert werden müssen.

\subsubsection{Ressourcen im Bereich der Personalentwicklung}

An Schulen sind neben der Schulleitung und den Regelschullehrkräften auch Personen beschäftigt, die vom Schulträger eingestellt werden, wie Hausmeister, Beschäftigte im Sekretariat oder Schulsozialarbeiter. Darüber hinaus werden über die Eingliederungs- oder Jugendhilfe auch Integrationshelfer für Schülerinnen und Schüler mit hohem Unterstützungsbedarf (Bsp.: körperliche Beeinträchtigung, drohende seelische Behinderung) in den Schulen eingesetzt. An integrativ arbeitenden Schulen sind außerdem Förderschullehrkräfte oder Pädagogische Fachkräfte als Landesbedienstete tätig. In den folgenden Ausführungen wird die Personalsituation allerdings lediglich mit Blick auf die Lehrkräfte dargestellt. Diese war an bundesdeutschen Schulen in den vergangenen Jahrzehnten größeren Schwankungen ausgesetzt, u.a., da keine zentrale Steuerung der Studierendenzahlen für die unterschiedlichen Lehrämter erfolgt. Ende der 2010er Jahre und statistisch betrachtet voraussichtlich bis 2022 kann für die Bundesrepublik Deutschland von einem Mangel an Lehrkräften gesprochen werden, der sich nach Auffassung des Deutschen Lehrerverbands 2018 mit fast 40.000 fehlenden Pädagogen dramatisch darstellt. Laut Meidinger, Präsident des Verbands, seien zu Beginn des Schuljahres 2018/19 etwa 10.000 Lehrerstellen nicht besetzt, weitere 30.000 Stellen würden durch Seiteinsteiger, Studierende etc. ausgefüllt. Dies ginge soweit, dass in Berlin „70 Prozent der neu eingestellten Lehrer im Grundschulbereich 
Seiteneinsteiger ohne jegliche pädagogische Vorbildung [seien]. (Meidinger, H.-P. in der Tagesschau vom 25.12.2018 ${ }^{27}$ ). Die Zahlen des statistischen Bundesamts weisen für das Jahr 2018 11.510 fehlende Lehrer aus, für die Folgejahre bis 2022 zwischen 1.980 und 3.580 Personen (Statistisches Bundesamt, 2018, o.S.). Die personelle Versorgung von Regelschulen mit Förderschullehrkräften ist statistisch schwer zu erfassen, vor allem wenn es um die konkrete Anzahl von Förderschullehrerstunden für einzelne Klassen bzw. Schüler und Schülerinnen mit spF geht. Das hängt u.a. damit zusammen, dass sich Vorgaben in den einzelnen Bundesländern voneinander unterscheiden. Für Rheinland-Pfalz halten Laubenstein et al. (2015) dazu fest, dass die pauschalierte Zuweisung von FSL-Stunden an Schwerpunktschulen 37 Wochenstunden umfasst. Dazu kommen 0,2 Wochenstunden je Schüler der Schule. Die komplette Zuweisung wird allerdings nur bei „Vollausbau“ (2-3 Kinder mit spF je Klasse) vorgenommen. Es erfolgt also keine stundengenaue Zuweisung zu Schülerinnen oder Schülern mit spF, auch da normalerweise jeweils halbe oder ganze Lehrerstellen zugewiesen werden (Laubenstein et al., 2015, S. 104).

In den inklusiven Pilotschulen des Landes Brandenburg zeigt sich, dass die Form der Budgetierung je nach Größe der Schule ( 48 bis 602 Schülerinnen und Schüler) dazu führt, dass zwischen einer Förderschullehrkraft und elf Förderschullehrkräften an einzelnen Schulen beschäftigt sind (Stäbler et al., 2015, S. 81). Inwiefern sich personelle Ressourcen auf die Qualität des Unterrichts auswirken, ist allerdings nicht umfassend belegt:

Von den Schulleiterinnen und Schulleitern der Hauptschulen und der Realschulen sehen immerhin jeweils 40 Prozent das Lernen der 15-Jährigen an ihren Schulen durch einen Mangel an Lehrkräften oder fachfremd erteilten Unterricht etwas oder sehr beeinträchtigt [...]. (Dt. PISA-Konsortium, 2001, S. 437)

Ausreichendes Personal für integrativ arbeitende Schulen wird bereits seit den 70er Jahren gefordert (Prell \& Link, 1977; Romey, 1977; Speck et al., 1978). Die Autorinnen und Autoren meinten damit nicht nur Regel- bzw. Sonderschullehrer, sondern auch Sozialpädagogen, Psychologen oder Therapeuten. Schon 1977 formulierten Prell und Link zum Modellversuch „Aktion Sonnenschein“ in München diesbezüglich:

Als besonders schwerwiegend erweist sich hier das Fehlen von Sprach-, Physio- und Verhaltenstherapeuten sowie von entsprechenden zusätzlichen Sonderschullehrern für spezielle Behinderungsarten, so z.B. für stark schwerhörige, sehbehinderte, lernbehinderte und körperbehinderte Kinder. (Prell \& Link, 1977, S. 308)

Auch der damalige Personalschlüssel wird kritisch bewertet:

Es ist allerdings fraglich, ob die Zeit, die der Lehrer hierfür [gemeint ist Beschäftigung mit Lernbehinderten Kindern in freien Arbeitsphasen, Anm. d. Verf.] für 20 Kinder zur Verfügung hat, wobei ein Viertel der Kinder behindert ist, ausreicht, um allen gerecht zu werden. (Prell \& Link, 1977, S. 316)

Speck et al. gehen 1978 davon aus, dass für eine effektive Arbeit in sozialpädagogisch-therapeutisch orientierten Gruppen (im Rahmen des Modellversuchs Integration lern- und verhaltensgestörter Kinder) ein zusätzlicher Bedarf von einer Sonderschullehrkraft und einem Sozialpädagogen mit halber Stundenzahl notwendig sei. Außerdem solle ein klinisch ausgebildeter Psychologe an der Schule eingesetzt werden. Die Erzieherinnen und Erzieher, die in den angegliederten Tagesheimen tätig seien, sollten einen Teil ihrer Stunden im Unterricht der Klassen 
einbringen (Speck et al., 1978, S. 71). Im Modellversuch der Hamburger Integrationsklassen arbeiteten ebenfalls drei Pädagogen zusammen: eine Grundschullehrkraft als Klassenleitung, eine pädagogische Unterrichtshilfe (Erzieher) mit 30 Stunden (3/4-Stelle) und eine Sonderschullehrkraft $(11+4=$ Modell 10 Stunden wöchentlich, $18+2=$ Modell 2 Stunden wöchentlich), so dass in jeder Stunde mindestens zwei Pädagogen anwesend waren (Wocken, 1987, S. 69).

Die in sonderpädagogischen Studien oder Qualitätsübersichten am häufigsten benannte Subkategorie für notwendige Personalressourcen an integrativen Schulen ist die so genannte Doppelbesetzung in Integrationsklassen, womit normalerweise ein Zwei-Lehrer-System gemeint ist (Innerhofer \& Klicpera, 1991; Köbberling, 1994; Heyer et al., 1997; Bless, 2003; Specht et al., 2006; BMBWF Österreich, 2008; Thiele, 2009; Textor, 2009). Diese Doppelbesetzung sollte auch in Vertretungssituationen nicht aufgelöst werden müssen (Boban \& Hinz, 2003; Holzinger et al., 2011), weshalb es sinnvoll sei einen Vertretungspool vorzuhalten (Boban \& Hinz, 2003). Sonderpädagogen sollten - abgesehen von Beratungsangeboten - ausschließlich in integrierten Klassen eingesetzt sein (Brugger-Paggi, 2003). Die Anzahl der in Regelschulen beschäftigten Sonderpädagogen (quantitative Ausstattung mit Personal) sollte sich an der Anzahl der Schüler mit sonderpädagogischem Förderbedarf orientieren, ein Sonderpädagoge sollte nicht mehr als sechs Klassen betreuen müssen (Bless, 2003). Wie Arndt und Werning anhand einer qualitativen Einzelfallstudie an einer Integrierten Gesamtschule belegen, gehen die Förderschullehrkräfte davon aus, dass diese Anzahl schon zu hoch sei, da es aufgrund der Vielzahl der Fachlehrer (bspw. innerhalb einer Jahrgangsstufe einer weiterführenden Schule) nicht möglich sei, mit all diesen Lehrkräften intensiv zu kooperieren (Arndt \& Werning, 2013, S. 21). Als Qualitätsmerkmale werden außerdem benannt, dass Klassenleitungen mindestens die Hälfte ihrer Unterrichtszeit in einer integrativen Klasse einbringen (BMBWF Österreich, 2008) und an Integration beteiligte Lehrkräfte Entlastungsstunden erhalten sollten, bspw. für die Teilnahme an schulinternen Arbeitsgruppen oder für Fortbildungen (Köbberling, 1994; Berges, 1996; Brugger-Paggi, 2003). Die Forschungsergebnisse belegen außerdem, dass es günstig sei, wenn Lehrerteams in Integrationsklassen nicht zu viele Lehrkräfte umfassen (Köbberling, 1994; Hug, 1994b; Husinsky, 1994; BMBWF Österreich, 2008; Holzinger et al., 2011).

Damit schulische Integration mit Hilfe der Personalausstattung wirksam umgesetzt werden kann, fordern die österreichischen Richtlinien, dass eine Bündelung sonderpädagogischer Ressourcen an Schulen umgesetzt werden sollte, statt wohnortnaher Integration den Vorrang zu geben (BMBWF Österreich, 2008), während Brugger-Paggi betont, dass Förderschullehrer den Schülern mit sonderpädagogischem Förderbedarf gemäß ihrer fachlichen Qualifikation zugewiesen werden sollten (Brugger-Paggi, 2003). Diesbezüglich gehen die Auffassungen innerhalb der Sonderpädagogik also auseinander. Brugger-Paggi hält darüber hinaus die Gewährleistung der Kontinuität bei der Betreuung von Integrationsmaßnahmen als Qualitätskategorie fest (Brugger-Paggi, 2003).

Neben der personellen Ausstattung an sich sind für die Qualität integrativer Arbeit nach LütjeKlose und Urban (2014b) fest einzurichtende Kooperations- und Teamzeiten eine zentrale Gelingensbedingung (Lütje-Klose \& Urban, 2014b, S. 290). Im von Speck et al. 1978 beschriebenen Modellversuch zur Integration lern- und verhaltensgestörter Kinder war die wöchentlich stattfinden-de Wochenplankonferenz die festgelegte Teamzeit für unterrichtsbezogene Kooperation. Dabei wurden an einem Nachmittag drei bis vier Stunden eingebracht, um Nachbesprechungen, Auswertungen, die Auswahl von Unterrichtsthemen, methodische Planungen sowie die Festlegung von Lernzielen zwischen Regel- und Förderschullehrkräften abzustimmen (Speck et al., 1978, S. 73). Sind solche Teamzeiten nicht in der Schule festgelegt, sieht Preuß die 
Gefahr übermäßiger zeitlicher Beanspruchungen (Preuß, 1985, S. 84). Nach Dumke, Krieger und Schäfer (1989) haben regelmäßige Teambesprechungen wesentlich zu positiver Kooperation beigetragen: „Das bedeutet, daß für eine gedeihliche Kooperation auch Gelegenheit zum Lernen eingeräumt werden muß." (Dumke et al., 1989, S. 135).

Auch Fussangel und Gräsel (2012) weisen auf Ressourcen bzw. Rahmenbedingungen hin, die für eine Ermöglichung bzw. Unterstützung kooperativer Prozesse in Schulen notwendig seien, wie eine entsprechende Unterstützung durch die Schulleitung sowie die Schaffung räumlicher und zeitlicher Strukturen, „die es den Lehrpersonen erlauben, sich regelmäßig zu treffen und zusammenzuarbeiten“" (Fussangel \& Gräsel, 2012, S. 34). Und Serke et al. formulieren:

Die Verankerung von Strukturen systematischer, z.B. im Stundenplan verankerter Zusammenarbeit im Kollegium gilt als eine zentrale Bedingung, um die inklusive Kooperation der Lehrkräfte voranzutreiben (vgl. Lütje-Klose \& Urban 2014b; Werning \& Lohse 2011, S. 11). (Serke et al., 2014, S. 254)

Nach Arndt und Werning (2013) spielen gemeinsame Zeitfenster aus Sicht der Lehrer an der von ihnen untersuchten Schule eine wesentliche Rolle, seien aber nicht institutionalisiert. Teamzeiten müssten selbst organisiert werden und würden nicht auf die Unterrichtszeit angerechnet (Arndt \& Werning, 2013, S. 20). Die Lehrkräfte vertreten die Auffassung, dass zu wenige Stunden in Doppelbesetzung umgesetzt werden können (Arndt \& Werning, 2013, S. 21). Für die Zusammenarbeit zwischen Förder- und Regelschullehrkräften werden feste Zeiten im Stundenplan als Qualitätssubkategorie mehrfach eingefordert (Preuß, 1981; Köbberling, 1994; Boban \& Hinz, 2003; Brugger-Paggi, 2003; Wember \& Prändl, 2009; Holzinger et al., 2011; Lienhard-Tuggener et al., 2011). Gemäß Laubenstein et al. (2015) geben die meisten Schwerpunktschulen in Rheinland-Pfalz an, Teambesprechungen ein bis zwei Mal wöchentlich durchzuführen, an etwa einem Viertel der befragten Schulen würden diese täglich bzw. nach Bedarf stattfinden. Insbesondere an den Schulen, die sich hinsichtlich ihrer „Inklusivität“ als fortschrittlich einschätzen, seien feste Teamzeiten für die Zusammenarbeit von Regel- und Förderschullehrern vorgesehen (Laubenstein et al., 2015, S. 147f).

Empirisch bleibt die Frage nach dem prüfbaren Zusammenhang bspw. der quantitativen Versorgung einer Schule mit Förderschullehrerstunden und der Qualität der unterrichtlichen und kooperativen Arbeit weitgehend offen. So behaupten Lütje-Klose und Urban (2014b), dass „die zur Verfügung stehenden Ressourcen der Förderlehrkräfte von entscheidender Bedeutung für die Qualität der Kooperation [seien]“ (Lütje-Klose \& Urban, 2014b, S. 284). Modelle mit sonderpädagogischer Grundversorgung hätten belegt, dass bei geringer Stundenausstattung Probleme häufig an Sonderpädagogen delegiert und äußere Differenzierung praktiziert werden würden (Lütje-Klose \& Urban, 2014b, S. 284; Studien von Lütje-Klose u.a., 2005; Bischoff, 2011; Werning \& Lohse, 2011). Gleichzeitig weisen sie darauf hin, dass der Ausgleich „einer relativ geringen sonderpädagogischen Ressourcenausstattung pro Klasse“ (Lütje-Klose \& Urban, 2014b, S. 284) erfolgen könne, „sofern die Schule ein vom gesamten Kollegium getragenes Förderkonzept entwickelt hat, das die Kooperation aller beteiligten Professionen (neben der Sonderpädagogik auch die Sozialpädagogik und die Eltern) systematisch berücksichtigt und unterstützt (z.B. durch Jahrgangsteams, gemeinsame Planungszeiten, Förderplankonferenzen, Supervisionsangebote).“ (Lütje-Klose \& Urban, 2014b, S. 284). Kuper und Kapelle (2012) differenzieren, dass insbesondere intensive Kooperationsformen wie die bei Little (1990) beschriebene direkte Zusammenarbeit (s. 3.4.2) von zeitlichen Ressourcen beeinflusst werden und es daher nicht verwunderlich sei, „dass intensive, die gesamte Schule einbeziehende Formen der Kooperation eher zur Ausnahme gehören“" (Kuper \& Kapelle, 2012, S. 47). 


\subsubsection{Kooperation}

„Lehrerkooperation gilt als zentrale Gelingensbedingung für die Entwicklung von Schule und Unterricht sowie pädagogischer Professionalität im Zuge sich verändernder bildungspolitischer Rahmungen und Ansprüche." (Baum et al., 2012, S. 9). Gleichzeitig weisen Baum et al. darauf hin, dass der Begriff der Kooperation im Rahmen schulpädagogischer Diskussionen „häufig normativ stark aufgeladen und mit vielfältigen Erwartungen befrachtet“ (Baum et al., 2012, S. 9) ist, so dass zu prüfen sei, inwiefern empirische Belege für die o.g. Aussage vorhanden sind bzw. ob auch kritische Erfahrungen von Beteiligten in Forschungsergebnissen thematisiert werden:

Zumeist wird angenommen, dass ausgeprägte Lehrerkooperation einen positiven Effekt auf die Bewältigung von Organisations- und Entwicklungsprozessen sowie wichtige Dimensionen schulischer Qualität hat, wenngleich empirische Forschungen diesen Effekt bislang noch nicht eindeutig zu bestätigen vermochten (vgl. Halbheer et al., 2008). (Baum et al., 2012, S. 10)

Grundsätzlich wird zwischen der intraprofessionellen Kooperation unter Lehrkräften und der interprofessionellen Kooperation mit anderen Berufsgruppen unterschieden (Baum et al., 2012, S. 9f). Darüber hinaus kann aus theoretischer Perspektive zwischen der organisationspsychologischen Sicht, der organisationssoziologischen Sicht und der professionstheoretischen Sicht auf Kooperation differenziert werden. Die organisationspsychologische Sicht definiert Kooperation als

[...] gekennzeichnet durch den Bezug auf andere, auf gemeinsam zu erreichende Ziele bzw. Aufgaben. Sie ist intentional, kommunikativ und bedarf des Vertrauens. Sie setzt eine gewisse Autonomie voraus und ist der Norm von Reziprozität verpflichtet. (Spieß, 2004, S. 199)

Die organisationssoziologische Sicht beschreibt Kooperation als „Übergang von einer isolierten, individuellen Bemühung um die Verfolgung eines Zieles zu einer kollektiven Anstrengung im Interesse einer gesteigerten Leistungsfähigkeit (vgl. Bonazzi, 2008, S. 65ff).“ (Kuper \& Kapelle, 2012, S. 41). Sie fragt nach Motiven und Koordinationsleistungen innerhalb von Organisationen, die dazu beitragen, dass effektives Arbeiten möglich ist, und geht davon aus, dass die Motivation durch Belohnungen beeinflusst werden kann (Kuper \& Kapelle, 2012, S. 42). Folgende Strukturdimensionen spielen in diesem Zusammenhang eine Rolle: die Arbeitsteilung (funktionale Gliederung von Abläufen), der Bedarf an Koordination, der sich daraus ergibt, die Hierarchien in der Organisation, die Delegation von Aufgaben sowie die Formalisierung der Kommunikation (Kuper \& Kapelle, 2012, S. 42f). Diese Sicht vermittelt also einen engen Zusammenhang zur Organisationsentwicklung. Aus professionstheoretischer Perspektive wiederum heißt es:

Mit der Kooperation in der professionellen Gemeinschaft sind ein kommunikatives Format und ein sozialer Ort gekennzeichnet, in dem die Verständigung über die theoretischen, praktischen und moralischen Aspekte der professionellen Tätigkeit erfolgt. [...] Die Professionellen bilden eine „Community of Practice“, in der sie das ihnen exklusiv zugängliche Wissen konstruieren und im Interesse der Verbesserung professioneller Praxis kommunizieren. Die Kooperation ist ein Katalysator dieser Kommunikation, der eine gewisse Eigenständigkeit gegenüber den konkreten organisatorischen Umständen der professionellen Arbeit hat [...]. (Kuper \& Kapelle, 2012, S. 44)

Eine sonderpädagogische Definition benennt Werteorientierung und Aushandlungsprozesse als entscheidende Merkmale: 
Kooperation wird als auf demokratischen Werten basierendes, auf der Gleichwertigkeit und gegenseitigem Vertrauen der Kooperationspartner/innen beruhendes, zielgerichtetes und gemeinsam verantwortetes Geschehen interpretiert, in dem aufgrund von Aushandlungsprozessen die Schaffung bestmöglicher Entwicklungsbedingungen aller Kinder angestrebt wird. (Lütje-Klose \& Urban, 2014a, S. 115)

In der Organisation Schule ist nach Kuper und Kapelle (2012) beides vorhanden: aufgrund der flachen hierarchischen Struktur Anteile einer Organisation von Professionellen, gleichzeitig aber auch Anteile einer starren bürokratischen Organisationsstruktur mit Blick auf Hierarchisierung, Standardisierung und Fremdkontrolle (S. 45f); zwischen beiden bestehe lediglich eine lose Kopplung (Kuper \& Kapelle, 2012, S. 48):

Da sowohl die professionelle als auch die organisationale Kommunikation eigenen Logiken und Regeln folgen, lässt sich die Kooperation unter Professionellen nicht durch organisatorische Strukturmaßnahmen herstellen; ebenso wenig kann der Bedarf an Kommunikation in einer professionellen Gemeinschaft nicht umstandslos in eine Organisationsstruktur übersetzt werden. (Kuper \& Kapelle, 2012, S. 48f)

Die Aussage: „Kooperation ist in der Organisation Schule nicht per se vorgesehen oder institutionalisiert und muss bewusst gestaltet werden." (Fussangel \& Gräsel, 2012, S. 33) ist dabei mit Blick auf Schulen als Organisationseinheiten nicht ganz zutreffend, da verschiedene Kooperationsformen (z.B. Gesamtkonferenzen, Fachkonferenzen, Jahrgangsteams nach dem Team-Kleingruppen-Modell) durchaus verbindlich und damit „verordnet“ sind bzw. sein können (Idel \& Ullrich, 2013, S. 57f). Idel und Ullrich (2013) führen dazu an, dass neuere Kooperationsformen wie bspw. Steuergruppen in Schulen ,in der Regel selbstgewählt und interessensmotiviert sind und insofern häufig über einen motivationalen Vorschuss verfügen " (Idel \& Ullrich, 2013, S. 58), so dass in der Forschung grundsätzlich zwischen diesen beiden unterschiedlichen Kooperationsformen unterschieden werden kann. Kooperation im Kollegium (Heyer et al., 1997; Deutsches Schulamt, 2004; Thiele, 2009; Speck, 2010) wird als Qualitätskategorie auch in der Sonderpädagogik benannt, normalerweise beziehen sich die Ausführungen allerdings spezifisch auf die Kooperation zwischen Regel- und Förderschullehrkräften im engeren Sinn.

Zwei zeitlich weit auseinanderliegende Zitate zeigen, dass die enge Kooperation zwischen Regel- und Förderschullehrern mit Blick auf die erfolgreiche Förderung heterogener Schülergruppen über mehrere Jahrzehnte hinweg als wesentlich eingeschätzt wird:

Wie fast alle Berichte [zu Modellschulen, Anm. d. Verf.] sichtbar machen, ist eine weitere Voraussetzung erfolgreicher Förderarbeit die enge Kooperation von Förder- und Fachlehrer (bzw. Klassenlehrer/ Spezialisten), am günstigsten durch die Einführung einer regelmäßigen Fallbesprechung (IGS Göttingen-Geismar; G Essen-Vogelheim). (Preuss-Lausitz, 1981, S. 122)

Die Kooperation der professionell Handelnden stellt für die Umsetzung des Rechts auf eine inklusive Unterrichtung und Förderung gemäß der UN-Konvention über die Rechte von Menschen mit Behinderungen (Art. 24) eine zentrale Bedingung dar, denn die Schaffung entwicklungsfördernder Bedingungen für eine sehr heterogene Gruppe von Schülerinnen und Schülern kann vielfach nicht von einer Lehrkraft allein umgesetzt werden. (Lütje-Klose \& Urban, 2014a, S. 113)

Auch die Eltern stufen gemäß einer Befragung von Fahrni (1989) die Kooperation zwischen Regelschullehrern und schulischen Heilpädagogen als sehr wichtig ein, damit die Förderung der Schüler erfolgreich verlaufen könne (zitiert n. Bless, 2003, S. 37).

Mit Bezug auf die Frage nach der Qualität von Kooperation wurden verschiedene Niveaustufenmodelle entwickelt. Während Little (1990) zwischen den Stufen Storytelling, gegenseitige 
Hilfe und Unterstützung, Entwicklung von Routinen und direkter Zusammenarbeit (Kuper \& Kapelle, 2012, S. 47) unterscheidet, differenzieren Marvin (1990) bzw. in der Folge Lütje-Klose und Willenbring (1999) zwischen Co-Activity (getrennte Durchführung von Unterricht/Förderangeboten durch Regelschullehrkraft (RSL) und Förderschullehrkraft (FSL)), Cooperation (Absprachen zu Stundenplänen und allgemeinen Zielsetzungen), Coordination (Koordination der pädagogischen Angebote von FSL und RSL, klare Absprachen zu Verantwortungsbereichen der einzelnen Lehrkräfte mit Blick auf den Förderprozess) und Collaboration (gleichberechtigte Zusammenarbeit, geteilte Verantwortung, mögliche Rollenwechsel in Unterricht/Förderung) (Lütje-Klose \& Willenbring 1999, nach Lütje-Klose \& Urban, 2014a, S. 118).

Auf der höchsten Niveaustufe

[...] kommt es zunehmend zu einem flexiblen Tausch in den Rollen und Aufgaben, gemeinsame Ziele sind klar abgesprochen und die KooperationspartnerInnen sind in der Lage, sich empathisch in die andere Person hineinzuversetzen. Basis ist eine grundsätzliche Offenheit gegenüber Kooperation und eine hohe Wertschätzung für die Sichtweisen der anderen KollegInnen (vgl. Lütje-Klose \& Willenbring 1999). (Serke et al., 2014, S. 254)

Für das Erreichen der höheren Niveaustufen sind nach Lütje-Klose (1997, S. 436) Wertschätzung und Vertrauen notwendig (Lütje-Klose \& Urban, 2014a, S. 117).

Fussangel und Gräsel (2006) wiederum differenzieren zwischen Austausch, Kooperation und Ko-Konstruktion (Fussangel \& Gräsel, 2012, S. 31). Dieses Modell bezieht sich klar auf die unterrichtsbezogene Kooperation von Lehrkräften. Dabei umfasst die höchste Niveaustufe der Ko-Konstruktion eine intensive inhaltliche Zusammenarbeit, einen hohen reflexiven Anteil, einen Bedarf an Ressourcen (Zeit, Vertrauen) und gemeinsame Ziele (Weiterentwicklung der Lehrkräfte im Sinne von Professionalisierung sowie Weiterentwicklung von Unterricht) (Fussangel \& Gräsel, 2012, S. 32). Diesbezüglich gehen die Autorinnen nicht davon aus, dass eine unterschiedliche Intensität der Zusammenarbeit gleichzeitig ausgeprägtere Wirkung zeige, vielmehr würden die unterschiedlichen Formen von Kooperation im schulischen Alltag verschiedene Funktionen erfüllen (Fussangel \& Gräsel, 2012, S. 31). Keller-Schneider \& Albisser (2013) differenzieren die dritte Niveaustufe der Ko-Konstruktion in Folge einer Studie noch weiter aus, indem sie diese in drei Formen aufgliedern: gemeinsame Unterrichtsplanung, Diskussion pädagogischer Fragen und gemeinsame Verantwortung bei der Unterrichtsdurchführung (S. 44). Neben den Niveaustufen von Kooperation lassen sich nach Spieß (2004) oder Lütje-Klose und Urban (2014a) verschiedene Ebenen von Kooperation unterscheiden wie die individuelle Ebene (Einstellungen, Bereitschaften und Kompetenzen), die interaktionelle Ebene (Gestaltung kooperativer Beziehungen), die Sachebene (Klärung von Rollen und Aufgaben) sowie die institutionelle Ebene (Kooperation im Kontext inklusiver Schulentwicklung) (LütjeKlose \& Urban, 2014b, S. 283ff).

Eine wichtige Rolle mit Blick auf die Umsetzung von Kooperation im Schulalltag spielen auf der individuellen Ebene also zunächst Einstellungen zu und Erwartungen an Kooperation. Pröbstel und Soltau legen den Fokus in ihrer Studie auf Individuum und Lehrergruppe sowie auf Entscheidungen von Lehrkräften für oder gegen Kooperation (individuelle Bedürfnisse, Motive, Einstellungen, die auch dann bedeutsam bleiben, wenn schulstrukturelle Voraussetzungen für Kooperation gut sind - damit auch Teil der Professionalisierung s.u.) (Pröbstel \& Soltau, 2012, S. 55f). Mit Blick auf Teamorientierung und Einstellungen zur Lehrerkooperation $(\mathrm{n}=233)$ kommen sie im Rahmen einer Online-Untersuchung in Bremen zu dem Ergebnis, dass Lehrkräfte Kooperation grundsätzlich positiv gegenüberstehen und im Schnitt Stufe 6 von 
9 möglichen Profilstufen erreichen, diese allerdings selten umsetzen (Pröbstel \& Soltau, 2012, S. 63f). Hinsichtlich des Zusammenhangs zwischen beruflicher Sicherheit und Lehrerkooperation $(\mathrm{n}=377$, Kollegien aus 16 Bremer Schulen) stellen sie fest, dass durchschnittlich betrachtet ein positiver beruflicher Selbstwert vorliegt, so dass Angst vor negativer Bewertung durch Kollegen nur eine geringe Rolle spielt. Die Hypothese der Verhinderung von Kooperation zwischen Lehrkräften durch berufliche Unsicherheit oder Ängste finden sie daher nicht bestätigt (Pröbstel \& Soltau, 2012, S. 64f). Auch das Autonomiebedürfnis sei nicht so hoch wie antizipiert, „vielmehr sind Lehrkräfte teamorientierter als meist angenommen wird“ (Pröbstel \& Soltau, 2012, S. 71). Im Mittelpunkt stehe dagegen eine gemeinsame Zielorientierung:

Wenn die Zusammenarbeit an Schulen gefördert werden soll, erscheint es auf Basis der vorliegenden und anderer Studien (vgl. Friend und Cook, 1990; Rosenholz, 1991) zunächst wichtig, dass gemeinsame Ziele definiert werden. Eine seltene Lehrerkooperation hängt häufig damit zusammen, dass den Lehrkräften nicht klar ist, was überhaupt gemeinsam erreicht werden soll (vgl. Pröbstel, 2008). (Pröbstel \& Soltau, 2012, S. 71)

Lehramtsstudierende verschiedener Lehrämter ( $n=977$, 40,8 \% Gymnasium; 32,5\% Grundschullehramt, 19,7\% Haupt- und Realschule; Rest Berufs- und Förderschulen) messen der Kooperation insgesamt eine hohe Bedeutung zu (Rothland, 2013, S. 94f). Studierende für die Sekundarstufe II schätzen die Bedeutung allerdings geringer ein als die der Primarstufe (Rothland, 2013, S. 98f). Einschränkungen ergeben sich insbesondere für anspruchsvollere Kooperationsformen. So stimmen lediglich $47 \%$ der Studierenden der Aussage zu, dass sie in ihrem eigenen Berufsleben im Team unterrichten möchten, wenn dies möglich ist (Rothland, 2013, S. 99).

Keller-Schneider \& Albisser (2013) belegen in ihrer Untersuchung einen Zusammenhang zwischen Einstellungen und der Umsetzung von Kooperation (statistisch bedeutsame Zusammenhänge mittlerer Stärke): „Je stärker die Einstellung ausgeprägt ist, desto eher wird in der entsprechenden Form kooperiert, [...]“ (Keller-Schneider \& Albisser, 2013, S. 45). Diese Zusammenhänge klären jedoch lediglich einen Anteil von $25 \%$, so dass davon ausgegangen werden muss, dass weitere Faktoren die Häufigkeit der Umsetzung beeinflussen. Hinsichtlich der Auswirkung individueller Merkmale auf Kooperation stellen sie fest: „Hohe Ausprägungen der Berufsmotive, durch den Beruf herausgefordert zu werden und den Beruf mit Gestaltungsspielraum ausüben zu können, wirken sich positiv auf die Häufigkeit von Austausch aus.“ (KellerSchneider \& Albisser, 2012, S. 46), während Selbstwirksamkeit, kognitives Belastungserleben und emotionale Erschöpfung keine bedeutsamen Auswirkungen zeigen (Keller-Schneider \& Albisser, 2013, S. 47).

Grundvoraussetzungen für die professionelle Kooperationsbereitschaft zwischen Regel- und Sonderpädagogen sind für Preuß (1985) die Kommunikationsfähigkeit mit anderen Berufsgruppen, allgemeine sowie fachspezifische Beratungskompetenzen und „umfassende Institutionskenntnisse und Handlungserfahrungen in Institutionen mit sonderpädagogischen Aufgabenstellungen“ (Preuß, 1985, S. 77). Sie benennt als Indikatoren für die erfolgreiche Kooperation zwischen Sonder- und Regelschullehrern eine direkte Initiierung des Kooperationsprozesses durch ein Mitglied einer Institution, den Konkretheitsgrad des Kooperationszieles, die Transparenz der Erwartungshaltung aller am Kooperationsprozeß Beteiligten, die Festlegung der Zeitkomponente sowie die Kenntnis der professionellen Kompetenzstruktur (Preuß, 1985, S. 80). Die Qualitätsmerkmale „Konkretheitsgrad des Kooperationszieles“ und „Transparenz der Erwartungshaltungen“ ständen dabei in einem engen Zusammenhang, da ersteres die inhaltliche Ebene betreffe und zweiteres die Beziehungsebene (Preuß, 1985, S. 81). Bereits 
1978 formulierten auch Speck et al. als Ergebnis einer Studie zur Integration lern- und verhaltensgestörter Kinder klare Anforderungen an die Zusammenarbeit zwischen den beiden Professionen, die präzise und selbstkontrolliert arbeiten und die Zusammenarbeit unter dem Aspekt der gemeinsamen Ziele sehen sollten. Interpersonelle Probleme, die sich negativ auf die Arbeit auswirken, sollten angegangen werden und es sei auf die Abgrenzungen der Kompetenzbereiche zu achten (Speck et al., 1978, S. 130f).

Im Verlauf eines mehrjährigen Schulversuchs an Grundschulen verteilte sich die Kooperationsbereitschaft so, dass etwa ein Drittel der Lehrkräfte ein hohes Maß an Offenheit zur Zusammenarbeit zeigte, ein weiteres Drittel sich ablehnend verhielt und ein weiteres Drittel von einer schwankenen Bereitschaft gekennzeichnet war (Reiser et al., 1984, S. 310). Im Rahmen von Vorversuchen äußerten Lehrkräfte an Versuchsschulen zwar ein signifikant höheres Maß an Kooperationsbereitschaft, dieses schlug sich allerdings nicht in einem höheren Grad an Umsetzung der Zusammenarbeit nieder (Reiser et al., 1984, S. 6).

Ergebnisse zur Kooperationsbereitschaft von Regelschullehrkräften wurden auch von Dumke, Krieger und Schäfer (1989) erhoben: mehr als die Hälfte und zum Teil bis zu zwei Drittel aller Lehrkräfte an Hauptschulen, Gymnasien, Gesamtschulen und Sonderschulen seien sehr bzw. grundsätzlich bereit zu Zusammenarbeit, maximal ein Viertel zeigte sich ablehnend (Dumke et al., 1989, S. 130). Von den Grundschullehrkräften war allerdings nur etwa ein Drittel bereit zu Zusammenarbeit, etwa die Hälfte formulierte mehr oder weniger starke Ablehnung (Dumke et al., 1989, S. 130). Von Bedeutung war zumindest für Integrationsmodelle die Erfahrung der Lehrkräfte mit integrativem Unterricht (Dumke et al., 1989, S. 132). Die Mitarbeit der Regelschullehrer sei auch von der Einhaltung bestimmter Rahmenbedingungen wie der ausreichenden Versorgung mit Sonderschullehrerstunden oder der kleinen Klassengröße abhängig. Während sich die Vermeidung von Aussonderung als konsensfähig erwies, ergaben sich Probleme bei der Umsetzung dieses Ziels in der Praxis (Dumke et al., 1989, S. 83). Die Bereitschaft von Regel- und Förderschullehrern zur Arbeit in einem „Zwei-Lehrer-System“ variierte in dieser Untersuchung je nach Schulform zwischen 55 und $75 \%$ der befragten Lehrkräfte Die Lehrkräfte an Gymnasien wiesen in diesem Zusammenhang ein höheres Maß an Bereitschaft auf (60\%) als die an Grundschulen (55\%) (Dumke et al., 1989, S. 133). Mit Blick auf die Hamburger Integrationsklassen kam Antor ebenfalls zu kritischen Ergebnissen hinsichtlich der Kooperationsbereitschaft von Grundschullehrkräften:

Kaum ein Zweifel besteht nach den Diskussionsergebnissen daran, daß die beteiligten Grundschulpädagogen die Qualität der Kooperation jedenfalls in sachlicher Hinsicht negativer einschätzen als die Sonderpädagogen. Ihre Unzufriedenheit geht soweit, daß sie sich - im Gegensatz zu Sonderpädagogen und pädagogischen Unterrichtshilfen - mehrheitlich für eine sofortige Ablösung des Drei-PädagogenModells durch ein Zwei-Pädagogen-Modell aussprechen. (Antor, 1987, S. 98)

Haeberlin et al. halten als Ergebnis der qualitativen Befragung einer kleinen Stichprobe fest, dass die wichtigste Voraussetzung für eine erfolgreiche Zusammenarbeit von Regel- und Sonderpädagogen darin bestehe eine gemeinsame Vision für eine humane Schule zu teilen. Wesentlich seien darüber hinaus die Gleichberechtigung der Kooperationspartner sowie Kreativität, Flexibilität und Offenheit aller Beteiligten (Haeberlin et al., 1992, zitiert n. Bless, 2003, S. 40). Weitere Voraussetzungen für eine gelingende Kooperation zwischen Regel- und Förderschullehrkräften sind nach Auffassung von Sonderpädagogen neben den ausreichenden Ressourcen der Förderlehrkräfte (Lütje-Klose \& Urban, 2014b, S. 284) eine Verständigung auf gemeinsam geteilte Werte (Bonsen \& Rolff, 2006, bzw. Köker, 2012, zitiert n. Serke et al., 2014, S. 253), 
die allgemein als wesentliche Voraussetzung von Kooperation angesehen wird (Boban \& Hinz, 2003). Werning und Arndt bewerten die Frage nach der Entstehung geteilter Werte noch als ungeklärt (Werning \& Arndt, 2013, S. 13, in Serke et al., 2014, S. 253). Studien zu diesen Voraussetzungen (Lütje-Klose et al., 2005, S. 86; Werning \& Lohse, 2011) haben u.a. ergeben, „dass sich die sonderpädagogischen Lehrkräfte z.T. einseitig für die Herstellung einer inklusiven Grundhaltung und die Gestaltung der kooperativen Prozesse verantwortlich fühlen." (Serke et al., 2014, S. 254). Serke et al. (2014) schlussfolgern daraus die Entstehung möglicher Ungleichgewichte in kooperativen Beziehungen und die Erschwerung inklusiver Prozesse durch die Delegation von Verantwortlichkeiten (Serke et al., 2014, S. 254).

Amrhein kommt vor Einführung des integrativen Unterrichts in weiterführenden Schulen in NRW zu dem Ergebnis, dass $30 \%$ der Regelschulehrkräfte sich den Einsatz in diesem Unterricht vorstellen können, während ein weiteres Drittel unentschieden ist und 35,3\% nicht zu diesem Unterricht bereit sind (Amrhein, 2011, S. 135). Eine niedersächsische Lehrerbefragung von 700 Lehrkräften ergibt, dass sich Grundschullehrkräfte mehr Kooperation wünschen, „denn sie geben an, dass sie mehr Förderlehrkräfte (71\%), mehr Tandemunterricht (68\%) und eine „Kollegiale Hospitation“ (39\%) benötigen, damit eine Individualisierung gelingen kann (vgl. Behrensen, Sauerhering \& Solzbacher 2012, S. 9)“ (Serke et al., 2014a, S. 251).

Mit Blick auf die konkrete Umsetzung von Kooperation auf der interaktionellen Ebene kommen Dieckmann et al. (2008) (nach Fussangel \& Gräsel (2012, S. 35f)) in einer Studie zur Entwicklung von Ganztagsschulen $\left(\mathrm{StEG}^{28}\right)$ zu dem Schluss, dass die Kooperation unter Lehrkräften nur wenig ausgeprägt sei. Fussangel u.a. gelangen $2010 \mathrm{zu}$ dem Ergebnis, dass Austausch häufiger praktiziert werde als gemeinsame Arbeitsplanung oder gemeinsames Unterrichten (Fussangel \& Gräsel, 2012, S. 35), was auch Untersuchungsergebnisse von Soltau und Mienert bestätigen (2013, S. 78f). Die Chancen für Kooperation würden an Ganztagsschulen diesen Ergebnissen zufolge nicht besser genutzt als an Halbtagsschulen. Unterschiede ergeben sich dort mit Blick auf die interprofessionelle Kooperation, die bedarfsorientiert und wenig institutionalisiert schülerbezogen erfolgt (Fussangel \& Gräsel, 2012, S. 36).

Im Rahmen der DESI-Studie ${ }^{29}$ wurde nach Helmke (2010) festgestellt, dass die unterrichtsbezogene Kooperation von Lehrkräften an weiterführenden Schulen sich nach Fächern und nach Schulform unterscheidet. Während eine gemeinsame Unterrichtsvorbereitung im Fach Englisch nur von 3 bis $4 \%$ aller Lehrkräfte umgesetzt wird, geben im Fach Deutsch bspw. 16\% der Hauptschullehrer bzw. $21 \%$ der IGS-Lehrer an diesen zumindest gelegentlich gemeinsam vorzubereiten. Eine gemeinsame Unterrichtsdurchführung erfolgt über alle Schulformen hinweg bei insgesamt $2 \%$ der Lehrer gelegentlich.

Scheerens \& Bosker (1997) (n. Fussangel \& Gräsel, 2012, S. 29) stellten fest, dass in effektiven Schulen, also Schulen, die bezüglich der Schülerleistungen als erfolgreich bezeichnet werden, mehr Kooperation als in anderen Schulen erfolgt. Steinert (2006) (zitiert n. Baum et al., 2012, S. 12) kommt zu dem zentralen Ergebnis, „dass die meisten Kollegien innerhalb der Fächer und Jahrgangsstufen kooperieren und die Stufe der Differenzierung nicht überschreiten“ (Baum et al., 2012b, S. 13). Hohe Einschätzungen der kollektiven Ressourcen des Kollegiums beeinflussen die Häufigkeit von Kooperation (Keller-Schneider \& Albisser, 2013, S. 47). Je häufiger Lehrkräfte wiederum kooperieren, „desto höher werden die Ausprägungen der Qualitätsmerkmale des Kollegiums eingeschätzt (Keller-Schneider \& Albisser, 2013, S. 48f). Baum et al. (2012b) halten diese Ergebnisse für erklärungsbedürftig (S. 14), da ein Widerspruch zwischen

28 Unter: https://www.projekt-steg.de/

29 Deutsch-Englisch-Schülerleistungen-International; DESI-Konsortium (2008) 
dem hohen Grad an Erwünschtheit von Kooperation in Schulen und dem Grad der Umsetzung bestehe. Dabei seien folgende Erklärungsansätze denkbar: auf der einen Seite könnten die Organisationsstruktur von Schule (Verweis auf „zelluläre Organisationsstruktur von Schule als „loosely coupled system“ (Weick, 1976, Terhart, 1986) nach Baum et al., 2012b, S. 14) und Aspekte der Rekontextualisierung (Fend, 2008) in diesem System dazu führen, dass die Umsetzung nicht umfassend erfolge, auf der anderen Seite könne die enge Kooperation mit Blick auf Unterricht ggf. als Autonomieeinbuße wahrgenommen werden (Reh, 2008) und dadurch intensivere Zusammenarbeit verhindern. Nach Lütje-Klose et al. werde Ko-Konstruktion bzw. Collaboration in allgemeinen Schulen eher selten umgesetzt, Kooperation sei stattdessen häufig auf Materialaustausch beschränkt (Gräsel et al., 2006; Kolbe \& Reh, 2008; n. Lütje-Klose \& Urban, 2014a, S. 118).

Auch die Umsetzung der Kooperation zwischen Regel- und Förderschullehrkräften erfolgt nicht so, wie es die weiter oben dargestellten überwiegend positiven Einstellungen erwarten lassen. Nach Serke et al. (2014a) verdeutlichen neuere qualitative Studien, dass ,nur an wenigen inklusiven Schulen produktive Formen der Zusammenarbeit verankert sind (vgl. Lütje-Klose 2005), u.a. angesichts unzureichender Kooperationszeiten und - strukturen (vgl. Werning \& Arndt 2013)“ (Serke et al., 2014a, S. 252). Zwar wird die Kooperation als positiv und entlastend wahrgenommen, die Regelschullehrkräfte sehen Vorteile in der Zusammenarbeit auch für die Regelschüler und Regel- wie Förderschullehrer sehen Vorteile für ihre eigene Arbeit und ihre Lernprozesse (Arndt \& Werning, 2013, S. 14), es fehlen aber gemeinsame Zeitfenster für die Zusammenarbeit, so dass Teamstunden nur freiwillig umgesetzt werden können (Arndt \& Werning, 2013, S. 19). In der Bewertung der Kooperation zwischen Regel- und Förderschullehrkräften spielen die personellen Ressourcen also eine wichtige Rolle (s. dazu 3.4.1). Hinderliche Bedingungen für Kooperation werden vor allem in größeren Systemen wie weiterführenden Schulen beschrieben (Bsp.: Fachlehrersystem) (Amrhein, 2011, S. 44f). So ergab eine Studie von Gebhardt et al. (2013), bei der 174 Regel- und 126 Förderschullehrer in Österreich befragt wurden, dass Grundschullehrerinnen zur zeitlichen Dauer der Zusammenarbeit im Team gute bis sehr gute Werte angaben, während die Angaben der Sekundarstufenlehrerinnen lediglich befriedigend waren (Gebhardt et al., 2013, S. 60). Dies hing damit zusammen, dass die Grundschullehrkräfte fast ihr gesamtes wöchentliches Stundendeputat (23 Stunden) über mit einer Sonderpädagogin im Team arbeiten konnten, während der Stundenanteil für die Sekundarstufenlehrerinnen aufgrund des Fachlehrerprinzips sehr viel geringer war (Gebhardt et al., 2013, S. 60). Mangelnde zeitliche Ressourcen wiederum können nach Serke u.a. dazu führen, dass die Förderung von Schülerinnen und Schülern mit spF gehäuft in Form äußerer Differenzierung umgesetzt wird (Serke et al., 2014a, S. 252).

Aus sonderpädagogischer Sicht betonen Prell und Link zunächst die Bedeutung von Konferenzen als „wichtiges Instrument für den Informationsaustausch und die Weiterbildung. (Prell \& Link, 1977, S. 321). In diesen Konferenzen sei Zeit sich über organisatorische wie pädagogische Fragen ausführlich auszutauschen (Prell \& Link, 1977, S. 321f). Im Schulversuch „Sonderschullehrer in Grundschulen “ (an zwei Frankfurter Grundschulen; Reiser et al., 1984) sollten Sonderschullehrer und Grundschullehrer gleichberechtigt gemeinsam arbeiten, wobei Sonderschullehrer am Anfang vor allem Heilpädagogischen Gruppenunterricht praktizierten. Zielsetzung des Schulversuchs war es, dass sich die Grundschule verhaltensauffälligen Kindern besser anpassen können sollte. Die Studie kam zu dem Ergebnis, dass Ko-Unterricht und Kooperation stärker gewichtet werden müssen, da sich die Kooperation der Lehrer untereinander als schwierigster Problembereich herausstellte. Die Sonderschullehrer wurden bspw. teilweise als Bedrohung er- 
lebt. Als Aufgabenbereiche für die Kooperation ergab sich eine Fülle von Einzelbausteinen wie bspw. die Kooperation im Jahrgangsteam, zwischen Klassenleitung und Förderschullehrkraft, die Gestaltung von Ko- bzw. Förderunterricht, die Durchführung von Elterngesprächen, die Anschaffung von Ausstattung und Materialien, Lernstandsdiagnostik, Beratung, Supervision und didaktische Fortbildung (Reiser et al., 1984, S. 8-14). Problematisierend stufen Reiser et al. die Ergebnisse der Schülerbefragungen ein, die ergeben, dass Ko-Unterricht und Kleingruppenunterricht sich nicht positiv auf das Wahrnehmen des Klassenunterrichts auswirken. Stattdessen werden Spielstunden und Spielgruppen besser eingeschätzt und erinnert (Reiser et al., 1984, S. 93-121). Die Bewertung durch die Grundschullehrer ergibt positive Antworten vor allem für die Einzelfallarbeit von Sonderschullehrern (80\%), die Zusammenarbeit im Team (65\%), die gemeinsame Planung und Durchführung von Förderkursen (54\%) sowie die Reflexion der eigenen Unterrichtstätigkeit (50\%). Aufgrund der Schwierigkeiten hinsichtlich der Umsetzung der Kooperation zwischen Regel- und Förderschullehrkräften fasst Reiser zusammen, dass ein mittleres Kooperationsniveau zwischen einfachen Maßnahmen (Bsp.: Einzelförderung) mit geringer integrativer Wirksamkeit und komplexen Maßnahmen (Bsp.: Ko-Unterricht) angestrebt werden sollte (Reiser, 1987, S.144f). Inhaltlich tauschten sich die Lehrkräfte vor allem über die Förderarbeit an sich, die Kooperation untereinander sowie die Rollenproblematik mit Blick auf die Förderschullehrer aus (Reiser et al., 1984, S. 139-142). Schwierigkeiten bei der Kooperation ergaben sich dahingehend, dass die Sonderschullehrer häufig die Rolle einer Hilfskraft im Unterricht einnahmen und sie mit Blick auf den Rollentausch Ängste hatten im Klassenunterricht bewertet zu werden. Darüber hinaus wurde die Intensität der Kooperation und der Grad „des Sich-Einlassen-Könnens“ sehr unterschiedlich bewertet (Reiser et al., 1984, S. 152f). Positiv geschätzt werden gemeinsame Kooperationsstunden:

In den Kooperationsstunden wurden alle Lehrinhalte, Unterrichtsinhalte und -vorhaben geplant, arbeitsteilig erarbeitet, ausgewertet und ausgetauscht, sowie die didaktisch-methodischen Probleme gemeinsam erörtert. Auch die Entwicklungen einzelner Kinder bzw. Schwierigkeiten und Erfahrungen mit Schülern wurden hier besprochen und der Arbeitseinsatz der Sonderschullehrer geplant. (Reiser, 1984, S. 9)

Köppel verweist grundsätzlich darauf, dass die Tätigkeit von Förderschullehrern in Regelschulen als entlastend wahrgenommen werde: „In der Tat geben Lehrer an Schulen mit Beratungslehrer signifikant weniger hohe Belastungen an als Lehrer an Schulen ohne Beratungslehrer (auf dem 5\% Niveau gesichert).“ (Köppel, 1987, S. 168). An diesen Schulen sei auch eine höhere Kooperationsbereitschaft messbar (Köppel, 1987, S. 168).

Arndt und Werning kommen aufgrund ihrer Einzelfallstudie in zwei Klassen einer Integrierten Gesamtschule zu einer eher kritischen Bewertung der Umsetzung der Kooperation zwischen Regel- und Förderschullehrkräften. Zwar gebe es eine große Variationsbreite unterrichtsbezogener Kooperation (Bsp.: Nutzung eines Förderraums) und eine positive Bewertung der Beratung und der flexiblen Einsatzmöglichkeiten der Förderschullehrer sowie der gemeinsamen Unterstützung von Schülern in Arbeitsphasen, allerdings erfolge die Unterrichtsvorbereitung weitgehend unabhängig durch die Regelschullehrkraft, aufgrund von Zeitmangel sei häufig ein Austausch per Mail notwendig und es fehle gemeinsame Kooperationszeit für die Unterrichtsvorbereitung (Arndt \& Werning, 2013, S. 19f). Problematisch sei diesbezüglich, dass die Kooperation nicht institutionalisiert sei (Bsp.: fehlende im Stundenplan verankerte gemeinsame Beratungsstunden), die Kontinuität bei der Personalisierung teilweise nicht gegeben sei, es keine Supervision gebe und die Förderschullehrkräfte an mehreren Schulen eingesetzt seien. 
Teilweise gebe es Passungsprobleme bezüglich didaktisch-methodischer Vorstellungen und Unklarheiten über die Rollen- und Aufgabenverteilung (Arndt \& Werning, 2013, S. 14f). Arndt und Werning schlussfolgern, dass aufgrund der mangelnden Zeit zur Kooperation häufig Formen äußerer Differenzierung umgesetzt werden. In gemeinsamen Unterrichtssituationen wird den Förderschullehrkräften eine nachrangige Rolle zugewiesen und ihre beratende Rolle werde teilweise nicht akzeptiert (Arndt \& Werning, 2013, S. 16). Auch die GeSchwind-Studie kommt zu dem Ergebnis, dass feste Teamstrukturen im Sinne von regelmäßigen Teamzeiten maßgeblich dafür sind, dass sonderpädagogische Förderung nicht primär in Form von äußerer Differenzierung erfolgt (Laubenstein et al., 2015, S. 299). Positiver scheint dagegen das Ergebnis der GeSchwind-Studie zur Bewertung der Kooperation zwischen Regel- und Förderschullehrkräften. Demnach wird die Qualität der Zusammenarbeit zwischen Regel- und Förderschullehrkräften von einem Großteil der beteiligten Schulen als gut (21\%) bis sehr gut (64\%) eingeschätzt (Laubenstein et al., 2015, S. 149).

Als Subkategorie der Umsetzung der Kooperation zwischen Lehrkräften wird aus sonderpädagogischer Sicht vor allem die Kooperation zwischen der Klassenleitung und einer Förderschullehrkraft aufgeführt (Feyerer, 1998; Bürli et al., 2009). Vorgeschlagen werden darüber hinaus gegenseitige kollegiale Hospitationen (Berges, 1996; Boban \& Hinz, 2003; Wernstedt \& Ohnesorg, 2010), sowie kollegiale Beratung und Erfahrungsaustausch (Innerhofer \& Klicpera, 1991; Wember \& Prändl, 2009). Im Rahmen der unterrichtsbezogenen Zusammenarbeit von Regel- und Förderschullehrkräften spielen insbesondere Formen des Co-Teaching ${ }^{30}$ eine wichtige Rolle, stellen aber auch eine hohe Anforderung an die beteiligten Lehrkräfte:

Kooperation [wird] seltener und schwieriger - aber nach Einschätzung der Lehrkräfte auch wertvoller [...] je ,näher" man dem Unterricht als ihrem Kerngeschäft" kommt. (Kolbe \& Reh, 2008, S. 801, zitiert nach Lütje-Klose \& Urban, 2014a, S. 120)

Dabei umfasst das Co-Teaching eine gemeinsame Unterrichtsplanung, -durchführung und -nachbereitung. Hinsichtlich der Ressourcen (s. 3.4.1) setzt Co-Teaching zunächst voraus, dass Unterrichtsstunden für zwei Lehrkräfte parallel im Stundenplan verankert sind. Mit Blick auf die unterrichtsbezogene Zusammenarbeit im engeren Sinn ergeben sich vielfältige Fragestellungen: Wie kann das funktionieren - zwei Lehrkräfte in einem Klassenraum? Wen nehmen die Schüler dann als „Leitung“ der Klasse wahr? Wie kann dafür gesorgt werden, dass beide Lehrkräfte gleichermaßen ins Unterrichtsgeschehen eingebunden sind und Verantwortung dafür übernehmen? Wie ändert sich das Rollenverständnis von Lehrkräften, wenn sie gemeinsam unterrichten? Nehmen Regel- und Förderschullehrer im Co-Teaching unterschiedliche Rollen ein oder wechseln diese regelmäßig ab? Profitieren Schüler davon, wenn zwei Lehrer in einer Klasse unterrichten, und wenn ja, inwiefern? Wirkt sich Co-Teaching konkret auf Lernergebnisse von Schülern aus? Wie kann gemeinsame Unterrichtsvorbereitung und -nachbereitung aussehen? Wie häufig und in welcher Form setzen Lehrer Co-Teaching-Formen um, wenn sie in integrativen Schulen arbeiten?

Halfhide, die den für den deutschsprachigen Raum gebräuchlicheren Begriff „Teamteaching“ verwendet, definiert dieses als „eine Form der Zusammenarbeit von mindestens zwei kooperierenden Lehrpersonen, bei der die gemeinsame Verantwortung für das Unterrichten und die gemeinsame Unterrichtsentwicklung im Zentrum stehen“" (Halfhide, 2009, S. 103). Das Team-

30 Dieser Begriff wird verwendet, da er den Oberbegriff für verschiedene Formen des gemeinsamen Unterrichtens zweier Lehrkräfte darstellt, bei dem das Team-Teaching (= in Deutschland verwendeter Begriff) nur eine Unterform darstellt. 
teaching sei „im Sinne einer optimalen Förderung eng an den Lernbedürfnissen der Schülerinnen und Schüler auszurichten, um den Unterricht zugunsten gleicher Lern- und Partizipationschancen für alle Schülerinnen und Schüler zu optimieren." (Halfhide, 2009, S. 103; Herv. im Orig.). Zu Kennzeichen des Teamteaching gehören nach Halfhide u.a. eine gemeinsame Unterrichtsplanung und -durchführung, eine flexible Aufteilung von Verantwortung, die grundsätzlich gemeinsam getragen wird, sowie differenziertes und individualisierendes Arbeiten mit den Schülern (Halfhide, 2009, S. 106). Als Voraussetzungen von Teamteaching werden klare Ziele, verbindliche Abmachungen, Rollenklarheit, Gleichberechtigung, soziale und fachliche Kompetenzen, Diskussion von methodischen und didaktischen Fragen, Reflexionsfähigkeit, Zeit- und Raumressourcen benannt (Feuser \& Meyer, 1987, S. 169-S. 171; Halfhide, 2009, S. 106).

Schon 1969 beschrieb Correll die Theorie und Praxis des Teamteaching als „selbstverständliche Fortsetzung der neuen Grundschullehrerausbildung “ (Correll, 1969; zitiert n. Dorow, 1982, S. 71):

Wenn innerhalb eines Lehrerteams Unterricht nicht nur gemeinsam geplant, sondern auch durchgeführt und schließlich ausgewertet werden soll (Team-Teaching), setzt dies u.a. voraus, daß der einzelne Lehrer bereit sein muß, seine Unterrichtsaktivitäten von den anderen Mitgliedern beobachten, diskutieren und kritisieren zu lassen, sein vorhandenes Wissen und Können preiszugeben und zu erfahren, wie er auf andere wirkt. (Correll, 1969; zitiert nach Dorow 1982, S. 71)

Friend \& Cook (2010) beleuchten das Themenfeld „Co-Teaching“ und damit auch mögliche Niveaustufen von Kooperation vorsichtig, da ihrer Einschätzung nach - mit Blick auf die Zusammenhänge zwischen Co-Teaching und inklusiver Förderung sowie Schülerleistungen - bislang nur wenige Studien vorliegen. Für die konkrete Umsetzung des Co-Teaching können nach Friend \& Cook (2010) unterschiedliche Formen genutzt werden:

- One teach, one observe (one teacher leads, the other gathers data),

- One teach, one assist (one teacher leads, the other circulates among the students),

- Station teaching (instruction is divided into nonsequential parts),

- Parallel teaching (class is divided in two groups),

- Alternative Teaching (support teacher implements supplemental activities for individuals or groups),

- Teaming (both teachers teach simultaneously). (Friend \& Cook, 2010, S. 12)

Friend \& Cook gehen davon aus, dass Lehrkräfte, die im Co-Teaching arbeiten, sowohl individuelle Ziele für Schülerinnen und Schüler mit spF als auch für die gesamte Lerngruppe berücksichtigen können. Die Rollenverteilung zwischen den Lehrkräften könne mit Blick auf die möglichen Co-Teaching Formen fließend sein und eine flexible Aufgabenteilung beinhalten (Friend \& Cook, 2010, S. 13). Auch nach Lütje-Klose \& Urban (2014b) ist ein hohes Kooperationsniveau von Lehrkräften „durch einen flexiblen und situationsangemessenen Umgang mit den verschiedenen Formen“" (Lütje-Klose \& Urban, 2014b, S. 287) gekennzeichnet.

Erste Forschungsergebnisse zum Co-Teaching von Regelschullehrern aus den 60er Jahren kamen zu Daten, „die keine signifikanten Unterschiede zwischen Schulleistungen im herkömmlichen Unterricht und im Team Teaching-Verfahren ermittelten“" (Winkel, 1974, S. 137). Nach Winkel konnten in dieser Zeit auch weitere Motive, die Schulen und Lehrkräfte in Verbindung mit dem Team Teaching hatten, nicht erfüllt werden wie bspw. mehr Zeit für Schüler oder eine bessere finanzielle Ausstattung (Winkel, 1974, S. 137). Österreichische Pädagogen, die sich vor allem im Rahmen von Schulversuchen mit dem Co-Teaching befasst haben, kritisieren zu Beginn der 90er Jahre die Beibehaltung einer klaren Trennung von Aufgabengebieten zwischen 
Förder- und Regelschullehrkräften trotz Co-Teaching (Mayer, 1994, S. 10) oder die für das CoTeaching fehlende Zeit für gemeinsame Unterrichtsvorbereitung (Sertl, 1994, S.50). Neuere Forschungsergebnisse zum Co-Teaching allgemein belegen nach Cook et al. (2011) moderate bis hohe Effekte auf Lernleistungen sowie soziale und verhaltensorientierte Wirkungen auf Schüler (Effektgröße d = 0,40) (Murawski \& Swason (2001)), die Verbesserung von Noten von Schülern mit Lernproblemen (Effekt von $\mathrm{d}=0,81$ in Englisch, $\mathrm{d}=0,40$ in Mathe) (Fontana, 2005) oder die Verbesserung der Rechtschreibleistung $(d=1,15)$ bzw. des Leseverständnisses ( $=0,62$ ) (Cook et al., 2011). Teilweise ergeben sich in Studien allerdings auch negative Effekte $(d=-0,49$ auf mathematische Leistungen und $d=-0,51$ auf Vokabellernen sowie $d=-0,95$ auf Freies Schreiben) (Cook et al., 2011) (n. McDuffie, 2012, S. 6). Wenn Unterricht von Förderund Regelschullehrkräften gemeinsam gestaltet wird, interagieren Schüler mit Behinderungen weniger mit dem Regelschullehrer, erhalten aber signifikant mehr individuelle Unterstützung in Form individueller Instruktion durch die Förderschullehrer (Magiera \& Zigmond, 2005). Die am häufigsten genutzten Formen des Co-Teaching waren in den untersuchten Unterrichtsstunden die der gemeinsamen Betreuung von Einzelarbeit sowie die des „One leads - one supports“ - Modells (Magiera \& Zigmond, 2005) (n. McDuffie, 2012, S. 9). Serke u.a. weisen mit Bezug auf eine Meta-Analyse von Scruggs u.a. (2007, S. 392, S. 409) ebenfalls darauf hin, dass Regelschullehrkräfte normalerweise die Unterrichtsverantwortung innehaben, während Förderschullehrkräfte vor allem differenzierende Angebote machen (Serke et al., 2014a, S. 252).

Zusammenfassend kommt Mc Duffie zu dem Schluss, dass Co-Teaching typischerweise das „one leads-one supports“-Modell beinhaltet, die Förderschullehrer sich unausgelastet fühlen, der Unterricht nur selten individualisiert sei und nicht auf wissenschaftlich basierten Konzepten beruht sowie die Interaktion zwischen Schülern und Lehrern durch das Co-Teaching zum Teil nicht verstärkt wird. Die Forschungsergebnisse seien hinsichtlich der fachlichen und verhaltensbezogenen Verbesserungen auf Schülerseite nicht einheitlich. Er fordert daher, dass Schulen dafür sorgen müssten, dass Regel- und Förderschullehrer im Co-Teaching-Prozess tatsächlich zu echten Partnern werden (Mc Duffie, 2012). Nach Gebhardt et al. ergibt auch die Befragung von 71 Lehrern und Lehrerinnen inklusiver Klassen in New York (Kilanowski-Press, Foote und Rinaldo (2010)), dass die häufigste Tätigkeit nur bei acht Lehrkräften Co-Teaching umfasst, und zwar im Schnitt 52.20 Minuten pro Tag, während Kleingruppenunterricht (17 Lehrkräfte) oder Einzelunterricht (16 Lehrkräfte) als Formen sehr viel häufiger umgesetzt werden (Gebhardt et al., 2013, S. 56).

Die von Halfhide angenommenen positiven Auswirkungen des Teamteaching wie die Wahl zwischen zwei Bezugspersonen, mehr Zeit für Unterstützung und Beratung, schnelleres Feedback, leichtere Arbeit in unterschiedlichen Lernformen oder Aufrechterhaltung der Konzentration (Schülerseite) bzw. Innovationsförderlichkeit durch gemeinsame Anregung und Reflexion, Entlastung, Erleichterung des Umgangs mit unvorhergesehenen Situationen, Erhöhung der Objektivität der Leistungsbeurteilung und Erweiterung der Möglichkeiten zu Differenzierung und Individualisierung (Lehrerseite) (Halfhide, 2009, S. 112) können also in der Umsetzung offensichtlich nur teilweise erfahren werden. Zwar fordert Halfhide ihrerseits, dass sich die „Qualität des Unterrichts von im Teamteaching kooperierenden Lehrpersonen [...] daran messen lassen [muss], welche Konsequenzen für die Schülerinnen und Schüler resultieren." (Halfhide, 2009, S. 103). Dazu liegen - vor allem im deutschsprachigen Raum - allerdings nur wenige Forschungsergebnisse der Sonderpädagogik vor. Die vorliegenden Ergebnisse beruhen in erster Linie auf Beschreibungen von Erfahrungen mit Co-Teaching durch Interviews und Beobachtungen. Feuser und Meyer äußern sich dazu Ende der 80er- Jahre vorsichtig dahingehend, dass 
„in einer Anfangsphase die Bedingung der ,kleineren Klasse‘ (16 Schüler) und der ,Kooperation mehrerer Lehrer im Unterricht' nicht auch direkt und sozusagen automatisch zu einer besseren und erforderlichen Unterrichtsqualität (hinsichtlich der Lehrerstrukturen) für die Schüler“ (Feuser \& Meyer, 1987, S. 166) führen müsse. Sie stellten im Gegenteil fest:

Das Problem der Einigung der Lehrer (wie auch des Gesamtteams) sowohl auf das gemeinsame Ziel wie auf den gemeinsamen Gegenstand [...] wie das noch größere Problem der Einigung über die Verfahrensweisen, wie dieses Ziel zu erreichen sei, bedingte für die Schüler z.T. ambivalente bis widersprüchliche Interaktions- und Kommunikationsstrukturen, die das Lernen im Sinne eines wesentlichen Faktors beeinflußten und z.T. auf die Schüler neurotisierend wirkten. (Feuser \& Meyer, 1987, S. 165, Herv. im Orig.)

Als Vorteile des gemeinsamen Unterrichtens schätzen befragte Lehrkräfte nach Dumke u.a. den Erfahrungsaustausch, die gegenseitige fachliche Anregung (36\% der Lehrkräfte), kollegiale Unterstützung, gegenseitige Ergänzung und Teilung der Verantwortung (33\%), bessere Möglichkeiten differenzierten und individuellen Lernens (26\%) sowie eine insgesamt bessere Betreuung (22\%) ein. Nachteile wurden in unterschiedlichen pädagogischen und didaktischen Vorgehensweisen (13\%), vermehrtem Arbeitsaufwand (11\%), persönlichen Problemen (15\%) sowie Auswirkungen mangelnder Harmonie auf die Schüler (7\%) gesehen (Dumke et al., 1989, S. 134).

Nach Müller-Stump \& Rossi Marty bewerten die meisten Schülerinnen und Schüler in heilpädagogisch begleiteten Klassen der Oberstufe es positiv zwei Lehrer zu haben:

Der Heilpädagoge habe eine langsamere Erklärungsweise und gebe mehr Hilfe. Auch biete dieser Unterrichtsstil mehr Abwechslung. Als Nachteile wurden etwa genannt: Es gebe viel Wechsel und Unruhe im Schulzimmer. Es sei auch oft schwierig, zwei Lehrern gleichzeitig zuzuhören und die unterschiedlichen Erklärungsarten verwirrten mehr, als dass sie helfen. (Müller-Stump \& Rossi Marty, 1994, S. 50)

Auch in der Zusammenstellung von zwei Einzelstudien (Gieschen, 2011, sechs Schülerinterviews; Arndt \& Werning, 2011, 16 Schülerinterviews) an zwei Integrierten Gesamtschulen wird deutlich, dass Schüler und Schülerinnen den gemeinsamen Unterricht von Regel- und Förderschullehrkräften insgesamt positiv einschätzen (Arndt \& Giesche, 2013, S. 48). Als günstig bewerten die Schüler die zusätzliche bzw. schnellere individuelle Unterstützung, die Leistungsdifferenzierung, eine bessere Arbeitsatmosphäre und einen verbesserten Lernzuwachs. Unentschieden zeigen sie sich dahingehend, inwiefern das Co-Teaching zu mehr Ruhe bzw. mehr Unruhe führe. Ein negativer Aspekt bestehe darin, dass teilweise Unklarheiten dadurch entstehen, dass zwei Lehrkräfte sprechen (Arndt \& Giesche, 2013, S. 48ff).

Als wesentliches Ergebnis ihres Schulversuchs kommen Reiser u.a. zu dem Schluss, dass der Einsatz der Sonderschullehrer nicht zu den erwarteten Veränderungen auf die Variablen „Schulleistung, Quote der Schulleistungsschwachen, Quote der Schulversager (Reiser et al., 1984, S. 85) geführt hat. Holtappels wiederum leitet aus seiner Studie ab, dass intensive Kooperation auf Schülerseite leistungsfördernd sein kann (Holtappels, 2013, S. 55, zitiert n. Serke et al., 2014a, S. 252). Grundschullehrkräfte, die in fest eingerichteten Jahrgangs- oder Klassenteams arbeiten, neigen dazu ,adaptivere Unterrichtsformen zu implementieren“ (Holtappels, 2013, S. 44, zitiert nach Serke et al., 2014, S. 252).

Durch kontinuierliche Teamgespräche, wie sie zwischen Grundschullehrkräften und Lehrkräften für sonderpädagogische Förderung durchgeführt werden, werden intensivere Reflexionen des professionellen Wissens und Handlungsrepertoires sowie letztlich ein Kompetenztransfer angeregt. (Steinert et al., 2006; Scruggs, Mastropieri \& Mc Duffie, 2007, S. 401; zitiert n. Serke et al., 2014a, S. 252) 
Dieses Ergebnis kann mit Blick auf allgemeinen Kompetenztransfer durch die Untersuchung von Laubenstein et al. (2015) bestätigt werden, bei der $88 \%$ der teilnehmenden rheinland-pfälzischen Schwerpunktschulen angeben, dass die Aussage, dass ein solcher Kompetenztransfer durch kollegialen Austausch stattfinde, eher (50\%) bzw. voll und ganz (38\%) zutreffe (S. 154). $60 \%$ der Kollegien vertreten außerdem die Auffassung, dass die Arbeit in einem Team für die unterrichtliche Arbeit entlastend sei (Laubenstein et al., 2015, S. 293). Amrhein (2011) kommt in ihrer Studie zu Einstellungsveränderungen von Lehrkräften in weiterführenden Schulen zu dem Ergebnis, dass die Arbeit der Förderschullehrkräfte in den Regelschulen insgesamt ein hohes $\mathrm{Maß}$ an Wertschätzung genießt und die Regelschullehrkräfte anerkennen, dass diese „das Kollegium mit sonderpädagogischer Kompetenz bereichern“ (Amrhein, 2011, S. 207).

Aus sonderpädagogischer Sicht spielen die gemeinsame Unterrichtsvorbereitung, - durchführung und -reflexion eine entscheidende Rolle mit Blick auf die Qualität schulischer Integration (Heese, 1977; Mühl, 1987; Husinsky, 1994; Berges, 1996; Boban \& Hinz, 2003; Deutsches Schulamt, 2004; Bürli et al., 2009; Holzinger et al., 2011). Dabei sollten zum einen das sonderpädagogische Fachpersonal (Lienhard-Tuggener et al., 2011) als auch Integrationshelfer (Holzinger et al., 2011) in die Planung und Gestaltung des Unterrichts einbezogen werden. Um Lernprozesse zu evaluieren und zu reflektieren sollten regelmäßige Fallbesprechungen „zur Erstellung und Weiterentwicklung individueller Förderpläne und zur kollegialen Abstimmung des geplanten sonderpädagogischen Prozesses“ (Preuß, 1985, S. 76) durchgeführt werden.

Teamarbeit lässt sich als begriffliches Konstrukt natürlich nicht von „Kooperation“ als solcher trennen. Sie wird in diesem Unterkapitel dennoch gesondert aufgeführt und erläutert, da sie als eine spezifische Unterform von Lehrerkooperation aufgefasst werden kann. Kooperation in Teams verlangt ein verändertes Arbeitszeitmodell von Lehrkräften (Terhart, 2006, S. 164; Werning \& Arndt, 2013, S. 36; n. Serke et al., 2014, S. 256). Im Team finde die „notwendige Professionalisierung der anspruchsvollen Lehrertätigkeit [...] einen hoch wirksamen Lernort." (Schley, 2002, S. 23) Teams können in diesem Verständnis als Träger von Entwicklungsprozessen im Bereich der Organisation von Schulen aufgefasst werden, „denn aus der Summe einzelner inhaltlicher Vorstellungen erwächst noch kein Konzept, das von einem gemeinsamen überindividuellen Horizont an Werten und Leitgedanken getragen wird." (Schley 1990, S. 149). Wesentliches Ergebnis im Rahmen der Schulentwicklungsforschung von Bryk et al. (2010) ist in diesem Zusammenhang, dass „relational trust“, verstanden als beziehungsgebundenes Vertrauen bzw. Vertrauensverhältnis zwischen Lehrkräften und Schulleitung, zwischen Lehrkräften untereinander sowie zwischen allen Personen einer Schulgemeinschaft, einen sehr hohen Einfluss auf das Schulentwicklungspotenzial einer Schule hat:

Some of the most powerful relationships found in our data are associated with relational trust and how it operates as both a lubricant for organizational change and a moral resource for sustaining the hard work of local school improvement. (Bryk, 2010, S. 27)

Eine intensive wissenschaftliche Auseinandersetzung mit so genannten „Unterrichtsteams“ erfolgte an der Universität Zürich. Unterrichtsteams seien u.a. ein Erfordernis, das mit heterogenen Lerngruppen einhergehe:

Die Individualisierung des Unterrichts ist in allen Schulen zu einer wichtigen Entwicklungsaufgabe geworden, welche die Lehrpersonen vor große Herausforderungen stellt. Die mit der gewachsenen Heterogenität einhergehenden Probleme können von einzelnen Lehrpersonen allein kaum bewältigt werden. Diese Individualisierung kann nur gelingen, wenn Lehrpersonen in Unterrichtsteams zusammenarbeiten und konkrete alltagstaugliche Lösungen für den Umgang mit der Vielfalt entwickeln. (Direktion für Bildung und Kultur Zug, 2012, S. 3) 
Unterrichtsteams bestehen aus drei bis ca. sieben Lehrpersonen aus mindestens zwei Klassen der gleichen Schule (oder aus mehreren benachbarten kleinen Schulen). Die Lehrkräfte dieser Teams verantworten ihren Unterricht im Idealfall während mehrerer Schuljahre gemeinsam und verstehen ihr Team als Arbeits-, Lern- und Qualitätsteam. Unterrichtsteams werden als Organisationseinheiten innerhalb der Schule aufgefasst (Direktion für Bildung und Kultur Zug, 2012, S. 12). Die Arbeitszeit, die Lehrkräfte in Unterrichtsteams einbringen, zählt als Teil der (jährlichen) Gesamtarbeitszeit (Direktion für Bildung und Kultur Zug, 2012, S. 28). Aufgaben für Unterrichtsteams bestehen u.a. im Austausch über Fragen zur Unterrichtsqualität, in der Erarbeitung von Unterrichtsplanungen und Materialien, der gemeinsamen Auswertung der Wirkung des Unterrichts und der Materialien, der gemeinsamen Reflexion der Lernerfolge der Schülerinnen und Schüler und der gemeinsamen Reflexion der Zusammenarbeit der Lehrkräfte (Direktion für Bildung und Kultur Zug, 2012, S. 12).

Ein Forschungsprojekt von Arnold et al. (2011), bei dem zehn Interviews sowie eine OnlineBefragung mit 48 Schulleitungen sowie 145 Leitungen von Unterrichtsteams durchgeführt wurden, kommt zu dem Fazit, dass

[...] die Zusammenarbeit in den Unterrichtsteams aus Sicht der Lehrpersonen positive Auswirkungen haben kann. Viele Teams tauschen sich über pädagogische Fragen aus oder bereiten gemeinsam Unterricht vor. Die Arbeit und der Austausch im UT führen nach Ansicht von vielen Lehrpersonen zu höherer Sicherheit und grösserem Selbstvertrauen, sie berichten vom Erwerb von mehr Wissen, reflektieren ihr Tun öfter und nicht zuletzt optimieren und erweitern sie dadurch ihr Unterrichtsrepertoire. Die Arbeit und der Austausch im UT bringt jedoch erst für ein gutes Drittel der Befragten eine Entlastung mit sich. (Arnold et al., 2011, S. 42)

Bedeutsam ist mit Blick auf die Ergebnisse zu der Tätigkeit in Unterrichtsteams, dass diese sich offensichtlich nicht nur auf die Zusammenarbeit an sich auswirkt, sondern auch auf die Lernleistungen von Schülerinnen und Schülern. So stellen $48 \%$ der Befragten positive Auswirkungen auf die unterrichtliche Arbeit fest. Die Wahrnehmung dieser Auswirkungen bezieht sich auf die Selbständigkeit der Schüler (35\%), die Lernmotivation (32\%), das Unterrichtsklima (31\%), Aufmerksamkeit und Leistungen (jeweils 38\%) sowie Konzentration der Schüler (19\%) (Arnold et al., 2011, S. 29f). Die Lehrkräfte selbst nehmen Wirkungen auf die eigenen Unterrichtskompetenzen wahr sowie „auf die Bereiche Wissen, Reflexionsbereitschaft, Selbstsicherheit und Wohlbefinden" (Arnold et al., 2011. S. 40).

Klippert wiederum weist darauf hin, dass Teamsitzungen gut vorbereitet werden, verbindlichen Charakter aufweisen und ziel- und ergebnisorientiert sein müssten, damit sie als produktiv wahrgenommen werden (Klippert, 2006, S. 140; zitiert n. Serke et al., 2014, S. 255). Die Lehrerbelastungsforschung zeigt, dass Teams, die in angenehmer und produktiver Atmosphäre kooperieren, als sozial-emotionale Entlastungs-/Rückzugsorte fungieren können und einen „Schutzschild gegen Arbeitsunzufriedenheit und Burnout (Gräsel et al., 2006, S. 205) darstellen. Auf diese Weise können auch individuelle Ressourcen und die (Arbeits-) Motivation z.B. für schulinterne Innovationen stabilisiert werden (vgl. Johnson \& Johnson 2003; Bondorf 2012).“ (Serke et al., 2014a, S. 253). In der GeSchwind-Studie gelangen auch Laubenstein u.a. zu dem Ergebnis, dass in den Schwerpunktschulen die Erwartung, dass Teamarbeit in der Schule eine Entlastung darstellen kann, in knapp 50 \% der SPS im Grundschulbereich und bei 34,5\% der Schulen im Sek I Bereich erfüllt wurde (Laubenstein et al., 2015, S. 155). Nach Serke et al. (2014) können Teamstrukturen sehr unterschiedlich sein, bspw. je nachdem, ob es sich um ein kleines Schulsystem („Dorfgrundschule“) oder ein größeres Schulsystem (Bsp.: Gemeinschafts- 
schule, Berufsbildende Schule) handelt (Serke et al., 2014, S. 255). Je größer das Schulsystem, desto wesentlicher seien „differenzierte Teamstrukturen“ (Serke et al., 2014, S. 255). Die Vielfalt unterschiedlicher Teams innerhalb gerade größerer Schulen ergebe einen hohen Grad an Komplexität kooperativer Strukturen für die Beteiligten (Bsp.: Schulleitungs-, Steuerungs-, Fach-, Jahrgangs-, Klassen- oder Projektteams (Köker, 2012, S. 17f, zitiert n. Lütje-Klose \& Urban, 2014b, S. 289). Gemeinsame Teamsitzungen können u.a. der Förderplanung dienen (Serke et al., 2014, S. 255) (Bsp.: kooperative Lernbegleitung in der Laborschule Bielefeld nach Werning, 2005), während Jahrgangsteams „Unterrichtsentwicklung mit Blick auf schulinterne Curricula, Beurteilungskriterien und der Entwicklung von Lehr- und Lernmaterial“" (Serke et al., 2014, S. 255) vornehmen könnten.

Nach Lütje-Klose \& Urban (2014b) konnten Böhm-Kasper u.a. (2013) mit Blick auf die Kooperation zwischen Lehrkräften und Beschäftigten im Ganztagsschulbereich herausarbeiten, dass ein Zusammenhang zwischen Kooperation und institutionellen Rahmenbedingungen vorhanden ist (Lütje-Klose \& Urban, 2014b, S. 285). Darüber hinaus sei eine regelmäßige und zeitlich ausgedehnte Präsenz von Förderschullehrern in Regelschulen allgemein wesentlich, damit diese in kollegiale Gespräche sowie die zentralen Gremien der Schule eingebunden werden können (Lütje-Klose \& Urban, 2014a, S. 119). Erschwerend kann sich an weiterführenden Schulen das Fachlehrerprinzip auswirken. Auch in diesem Zusammenhang können Jahrgangsteams (Werning \& Lohse, 2011, S. 11; Arndt \& Werning, 2013, S. 21) bzw. die Umsetzung des Team-Kleingruppen-Modells unterstützende Wirkung mit Blick auf die Gestaltung von Kooperation haben.

In sonderpädagogischen Veröffentlichungen, in denen Teamarbeit vor allem die Zusammenarbeit zwischen Regel- und Förderschullehrkräften meint, wird sie explizit als Qualitätskategorie für integrative Schulen aufgeführt (Hug, 1994b; Berges, 1996; Boban \& Hinz, 2003; Specht et al., 2006). Die Bildung eines Teams sollte bereits ein Jahr vor der Entstehung einer Integrationsklasse erfolgen (Hug, 1994b). Als wesentlich erachtet werden organisatorische Aspekte, rechtzeitige Terminabsprachen, Vorbereitung und zielführende Moderation (Brugger-Paggi, 2003), inhaltliche Aspekte wie Kommunikations- und Entscheidungsprozesse des Teams, die der Komplexität von Aufgaben gerecht werden (Feyerer, 2007) oder eine gemeinsame Problembearbeitung (Bsp.: Fallbesprechungen) (Bächthold et al., 1990; Hug, 1994b; Feyerer, 1998; Boban \& Hinz, 2003). Kooperationsziele in Teams sollten möglichst konkret sein, Erwartungshaltungen der Beteiligten transparent (Preuß, 1981). Getroffene Vereinbarungen sollten verbindlich gehandhabt werden (Brugger-Paggi, 2003).

Darüber hinaus spielt im Zusammenhang mit inklusiven Schulen auch die Zusammenarbeit in multiprofessionellen Teams eine Rolle:

Für das Gelingen eines inklusiven Schullebens, eines differenzierenden und kooperativen Unterrichts stellt die Kooperation der Lehrkräfte eine entscheidende Bedingung und zugleich eine Entwicklungsaufgabe dar, denn individuelle Fördermaßnahmen können - wie Wocken (2013) herausstellt - nur in einem engagierten multiprofessionellen Team koordiniert und im Sinne einer Belastungsreduktion bewältigt werden. (Serke et al., 2014a, S. 250)

Lütje-Klose \& Urban gehen davon aus, dass multiprofessionelle Teams „aufgrund ihrer unterschiedlichen Berufskulturen mit ihren spezifischen Perspektiven und Kompetenzen [...] besonders voneinander profitieren und gemeinsam innovative Problemlösungen finden [können]“ (Lütje-Klose \& Urban, 2014b, S. 286). Einer Studie von Böhm-Kasper u.a. (2013) zufolge betonen Lehrkräfte, die in solchen Teams arbeiten, das Gefühl von Entlastung, 
[...] das sich aus der Möglichkeit des Austauschs mit anderen Fachpersonen über einzelne Kinder ergibt. Vor allem die Übernahme basaler Fördermaßnahmen wie psychomotorische Förderung, Konzentrations- oder sozial-emotionale Förderung, die z.T. im Nachmittagsbereich von den Ganztagskräften angeboten werden, und auch deren Kooperation mit den Eltern wird von Lehrkräften positiv eingeschätzt. (zitiert n. Lütje-Klose \& Urban, 2014a, S. 120)

Als Qualitätsindikatoren für die multiprofessionelle Zusammenarbeit werden der Austausch der Heilpädagogen mit anderen Fachpersonen benannt (Bless, 2003), die Einbindung aller an schulischer Integration Beteiligten in pädagogische Maßnahmen (Brugger-Paggi, 2003) oder Interdisziplinarität als solche (Bürli et al., 2009).

Wenn Lehrkräfte in Schulen kooperieren, ist grundsätzlich eine Klärung von Aufgaben und Rollen wichtig: „Im integrativen Unterricht verlieren die GL und SoL die Eindeutigkeit [...] ihrer Funktionen und damit zunächst ein Stück ihrer Anerkennung." (Feuser \& Meyer, 1987, S. 174). Antor formuliert schon 1987 die Annahme, dass die Klärung von Aufgabenbereichen kein spezifisches Problem zwischen Regel- und Förderschullehrern sei, sondern in der „Struktur pädagogischen Handelns" (Antor, 1987, S. 99) begründet liege:

Sein ganzheitlicher Charakter widerstrebt dem Versuch, zwischen einzelnen pädagogischen Aufgabenbereichen verbindliche Grenzen zu ziehen, wie sie formeller Kompetenz und Zuständigkeit entsprechen. Die Konturen zwischen den verschiedenen Ausbildungsprofilen verschwimmen vollends in solchen Teams, in denen sich alle Mitglieder durch ein sozialpädagogisches Verständnis der Erziehungs- und Unterrichtsarbeit auszeichnen, [...] Aus solchen Überlegungen wird ersichtlich, welche spezifischen Leistungen Teams erbringen müssen, um erfolgreich zusammenzuarbeiten. Sie haben einerseits die Aufgabenbereiche so weit voneinander abzugrenzen, daß jeder eine feste Position im Team und professionelle Anerkennung erhält. Zugleich muß ihnen aber auch daran gelegen sein, flexibel auf besondere Anforderungen der Situation und Bedürfnisse der Mitglieder (etwa des Sonderpädagogen nach Klassenunterricht) einzugehen. Eine starre Addition formal unterschiedlicher Kompetenzen würde das Prinzip der Ganzheitlichkeit pädagogischen Handelns eher pervertieren als einlösen. (Antor, 1987, S. 99)

Reiser (1998) geht davon aus, dass sich im Rahmen schulischer Integration zwei unterschiedliche Rollenbilder entwickelt haben: das des Sonderpädagogen in der Funktion der Doppelbesetzung einer Klasse sowie das der Förderschullehrkraft, die als „Spezialistin für die Lösung von Unterrichts- und Erziehungserschwernissen“ schulische Erziehungshilfe und Beratung leistet (Reiser, 1998, S. 50). Mit Bezug auf Wocken nimmt er an, dass die „Service-Leistung der Sonderpädagogik“ sich stärker in Richtung einer systembezogenen Beratung weiterentwickeln müsse (Reiser, 1998, S. 50).

Serke et al. (2014) weisen auf die besondere Bedeutung einer institutionalisierten Klärung von Rollen und Aufgaben zwischen Regel- und Förderschullehrkräften hin: „Verschiedene Studien stellen die Problematik fehlender Rahmenkonzepte in Bezug auf die Rollenklärung heraus, was Konflikte nach sich ziehen kann und nicht selten zur Wahl von äußeren Differenzierungsformen in der Praxis führt (vgl. Lütje-Klose et al. 2005; Werning \& Arndt 2013).“ (Serke et al., 2014, S. 255). Allerdings bliebe auf theoretischer Ebene weitgehend ungeklärt, wie diese Rollenverteilung günstiger Weise auszusehen habe (Reiser, 1998; Schwager, 2011; n. Serke et al., 2014, S. 255). Gemäß Lütje-Klose \& Urban (2014b) unterscheiden sich Aufgabenformen in den dazu vorhandenen theoretischen Modellen. So vertrete Schwager (2011) das Modell vorwiegend gemeinsamer Unterrichtung, während Reiser et al. (2007) auf Seiten der Förderschullehrkräfte in erster Linie Beratung und individuelle Förderung als Aufgabenschwerpunkte ansehen. Als Grund für das Vorhandensein der unterschiedlichen Modelle führt Reiser bereits Mitte der 80er Jahre an: 
Die Gefühle der professionellen Abwertung durch die bei Grundschullehrern vorherrschende Meinung, Klassenführung, Klassenunterricht seien doch viel anstrengender als Kleingruppenarbeit und Ko-Unterricht, Psychologen wären schließlich doch eine bessere Hilfe bei Problemkindern, führen zu immer wiederkehrenden Diskussionen darüber, wie man als Sonderschullehrer an der Grundschule seine Arbeit verstehen und organisieren soll: $\mathrm{Ob}$ als kindzentrierte Beratung mit eigener Klassenführung oder als klassenzentrierte Zusammenarbeit mit dem Grundschullehrer. (Reiser et al., 1984, S. 154, Herv. im Orig.)

Beide Modelle können sich nach Lütje-Klose \& Urban ungünstig auswirken. Bei Modell eins bestehe die Gefahr, dass Förderschullehrer zu selten oder zu wenig auf spezifische Unterstützungsbedarfe von Schülerinnen und Schülern eingehen, während es bei Modell zwei so sein könne, dass kein ausreichender Bezug zum Unterricht in der Gesamtgruppe hergestellt werde (Lütje-Klose \& Urban, 2014b, S. 287). Speck et al. sehen als zentralen Aspekt den, dass die unterschiedlichen Professionen jeweils über Kompetenzen in ihren eigenen Bereichen verfügen. Dabei sei keine hierarchische Ordnung der Aufgabenbereiche und Rollen der einzelnen Mitarbeiter gegeben, sondern diese seien ,in einem Team mit gemeinsamer Zielsetzung koordiniert“ (Speck et al., 1978, S. 131). Für Flexibilität in der Rollen- und Aufgabenverteilung sprechen dagegen die Ergebnisse der Studie von Arndt und Werning (2013), in der sowohl Förder-als auch Regelschullehrkräfte diese als positiv bewerten, gleichzeitig aber eine zu große Unklarheit bei der Verteilung von Rollen und Aufgaben als belastend einschätzen (Arndt \& Werning, 2013, S. 35). Raum zum Aushandeln von Aufgaben- und Rollenverteilungen halten auch Serke et al. für wichtig (Serke et al., 2014, S. 255).

Die Tätigkeit von Förderschullehrern in Regelschulen kann Unklarheiten und Unsicherheiten auslösen, wie Reiser et al. (1984) im Modellversuch an zwei Frankfurter Grundschulen feststellten. So äußerten sich die Sonderschullehrer dahingehend, dass ihnen häufig unklar sei, was im integrativen Unterricht bewirkt werden solle, es gebe Undeutlichkeit bezüglich der Vorgehensweise an der Schule, Unsicherheiten bezüglich des Vertretens eigener Vorstellungen von Schule und eine fehlende Offenheit bezüglich direkter Rückmeldungen gegenüber Grundschullehrern (Reiser et al., 1984, S. 153). Bach (1987) geht aufgrund von Erfahrungen im Rahmen eines Schulversuchs in Rheinland-Pfalz davon aus, dass das Rollenverständnis der Förderschullehrer (und die Kooperation mit den Regelschullehrern) das zentrale Problem mit Blick auf das Einbringen von Fachkompetenz in die Regelschulen sei (Bach, 1987, S. 108). Notwendig sei daher eine „partnerschaftliche Kooperation [...], die durch Aufnehmen, Fragen, gemeinsames Sehen und Lernen, Zuwenden, Zur-Verfügung-Stehen, Anregen, Sich-Einbringen, Unterstützen gekennzeichnet ist" (Bach, 1987, S. 108). Aus sonderpädagogischer Perspektive wird der „Kenntnis der professionellen Kompetenzstruktur“ (Preuß, 1985, S. 80) sowie einer klaren Rollendefinition der Aufgaben von Regel- und Förderschullehrkräften (Bächthold et al., 1990) eine wichtige Bedeutung zugemessen. Nach Brugger-Paggi (2003) sollen die unterschiedlichen Kompetenzen der Lehrkräfte optimal genutzt und eingesetzt werden. Als Voraussetzung dafür sind nach Preuß (1985) „umfassende Institutionskenntnis und Handlungserfahrungen in Institutionen mit sonderpädagogischen Aufgabenstellungen“" (S. 63) hilfreich. Insgesamt wird das Aufgabengebiet von Förderschullehrern in integrativen Schulen in den sonderpädagogischen Studien als sehr umfassend beschrieben. So differenzieren Reiser et al. aufgrund ihrer Studie etwa ein Dutzend unterschiedlicher Aufgabenbereiche (Bsp.: Ko-Unterricht, Förderunterricht, Vermittlung und Beratung, Beschaffung von Ausstattung und Material, Supervision) (Reiser et al., 1984, S. 8-14): 
Die Aufgaben der Sonderschullehrer umspannten ein weites Feld: von der Kooperation im Jahrgangsteam, der Lehrer- und Elternberatung über den Ko-Unterricht mit dem Klassenlehrer, den eigenen Kleingruppen- und Spielgruppenstunden bis zur Zusammenarbeit mit außerschulischen Institutionen. (Reiser et al., 1984, S. 8)

Preuß nennt als weitere Aufgabe von Förderschullehrern die der Koordination notwendiger Hilfen wie bspw. schulischer und therapeutischer Maßnahmen (Preuß, 1985). Als weitere spezifische Aufgaben werden von der PH Steiermark (2011) die Kenntnis der Lernausgangslage der Schülerinnen und Schüler mit spF, die Festlegung der Förderziele, die methodische-didaktische Umsetzung der Förderung, das Schreiben der Förderpläne und die Dokumentation der Förderung sowie die Information der Regelschullehrkräfte über die Förderschwerpunkte genannt. Eine Förderschullehrkraft kann als Tutor oder Tutorin für neue Förderschullehrkräfte an einer Schule eingesetzt werden, Fortbildungen für Regelschullehrkräfte leiten oder in der Arbeitsgruppe Integration auf Schulebene mitwirken (Brugger-Paggi, 2003). Eine weitere Aufgabe umfasst die Zusammenarbeit mit externen Diensten (Wember \& Prändl, 2009) oder die Kooperation mit Integrationskräften (Bürli et al., 2009).

Als Positivbeispiel für eine klare Definition der Aufgaben für Förderschullehrkräfte benennen Lütje-Klose und Urban (2014b, S. 289) die Laborschule Bielefeld, in der diese als „Begleiter von Entwicklungsprozessen“" (Begalke u.a., 2011, S. 76; zitiert n. Lütje-Klose \& Urban, 2014b, S. 289) bezeichnet werden und Aufgabenbereiche im Schulkonzept verankert sind. In diesem Modell bleibe die hauptsächliche Unterrichtsverantwortung bei den Regelschullehrkräften und die Aufgaben der Förderschullehrkräfte umfassten hauptsächlich kooperative Beratung, Unterstützung bei der Unterrichtsvorbereitung und Differenzierung. Diese Aufgaben könnten auch abhängig vom Alter der Schüler variieren (Bsp.: Eingangsphase: Kooperation Kindertagesstätten, Förderdiagnostik; Übergang Schule - Beruf: Beratung zu Berufsorientierung, Kooperation mit Betrieben oder Arbeitsagentur; in Begalke u.a., 2011, zitiert n. Lütje-Klose \& Urban, 2014b, S. 290).

Geiling und Simon (2014) können anhand einer Befragung von 506 Regel- und Förderschullehrkräften, die in der flexiblen Eingangsstufe Brandenburger Grundschulen tätig sind, belegen, dass die Förderschullehrkräfte sich in erster Linie für die Unterstützung von Kindern mit vermutetem spF sowie langsam lernende Kinder zuständig fühlen (88,0\% bzw. 80,2\%), während die Grundschullehrkräfte auf die Unterstützung der Lernfortschritte aller Kinder fokussiert sind (86,8\%) (Geiling \& Simon, 2014, S. 69). Gemeinsam erleben die beiden Gruppen sich als zuständig für individuelle Lernplanung (55,6\% Grundschullehkräfte, 56,3\% Förderschullehrkräfte) und die Zusammenarbeit mit den Eltern (47,4\% bzw. 48.5\%) (Geiling \& Simon, 2014, S. 70). Insgesamt schlussfolgern sie:

Die von uns befragten Lehrerinnen haben sich in FLEX eingerichtet, indem sie klassische Zuständigkeiten aufrechterhalten, bestimmte Zuständigkeiten jedoch teilen, womit es zum Aufweichen tradierter Orientierungen, aber nicht zu deren Überwindung kommt. (Geiling \& Simon, 2014, S. 72)

Auch Moser, Schäfer und Kropp (2014) kommen zu dem Ergebnis, dass Klassenunterricht in erster Linie durch die Regelschullehrkräfte erfolgt, während Förderschullehrkräfte einen Schwerpunkt auf individuelle Förderung legen (Moser et al., 2014, S. 134). In den rheinlandpfälzischen Schwerpunktschulen wird die Rolle der Förderschullehrkräfte so eingeschätzt, dass diese vor allem wechselnde Aufgaben im Klassenteam wahrnehmen (103 von 123 Schulen) oder Einzel- bzw. Gruppenförderung umsetzen (85 bzw. 102 Nennungen) (Mehrfachnennungen waren möglich) (Laubenstein et al., 2105, S. 149f). 


\subsubsection{Professionalisierung}

Professionelle Kompetenzen im Lehrberuf werden in Deutschland durch ein universitäres Studium (1. Ausbildungsphase) und einen Vorbereitungsdienst (2. Ausbildungsphase) erworben, durch Erfahrungen im Rahmen der eigenen unterrichtlichen Tätigkeit sowie durch Fortbildungsangebote, die schulintern (Bsp.: Pädagogische Tage) oder extern (Bsp.: Fortbildungsinstitute) besucht werden können. Darüber hinaus können bspw. auch kollegiale Fallbesprechungen oder die Teilnahme an Supervisionsangeboten zur Professionalisierung von Lehrkräften beitragen.

Problematisch erscheint nach Hattie zunächst allgemein, dass die derzeitige Lehrerbildung in der ersten Ausbildungsphase lediglich eine Effektstärke von $\mathrm{d}=0,11$ mit Blick auf Schülerleistungen aufweist. Er bezieht sich dabei auf Levine (2006, S. 109), der Lehrerbildung als „das Dodge City der Bildungswelt [beschreibt]. Wie die sagenumwobene Stadt im Wilden Westen ist sie von Ungewissheit und Desorganisation geprägt. Es gibt keinen Standardansatz, wo und wie Lehrpersonen ausgebildet werden sollen." (n. Hattie, 2013, S. 131). Insgesamt sei die Effektivität der Lehrerbildung bislang nur in wenigen Studien überhaupt untersucht worden (Hattie, 2013, S. 133). Die Ergebnisse einer anderen Untersuchung (50 Staaten der USA, 1993/1994, Darling-Hammond 2000) zeigten dagegen,

[...] dass die Lehrerqualifikation - ausgewiesen über entsprechende Studienabschlüsse und über die Tatsache, dass sie ihr Schwerpunktfach auch unterrichten konnten - und die Schülerleistungen zusammenhingen. Die festgelegten Maße für den Vorbereitungsgrad der Lehrer und ihre Abschlusszeugnisse waren bei Weitem die besten Prädikatoren für die Schülerleistungen im Lesen und in Mathematik, [...]. (Woolfolk, 2008, S. 3)

Auch Kane et al. gelangten in ihrer Längsschnittstudie zu dem Ergebnis, dass es einen statistisch messbaren Zusammenhang zwischen Schülerleistungen und dem unterrichtlichen Kompetenzniveau der Lehrkräfte (basic, proficient, distinguished) gibt (Kane et al., 2010, S. 28). Für die positive Entwicklung der Schülerleistungen seien umfassende Kompetenzen in Unterrichtspraxis und Klassenführung („classroom environment“) relevant, wobei die Fähigkeiten im Bereich des Classroom-Managements nach Kane u.a. die höchste Relevanz aufweisen (Kane et al., 2010, S. 29). Teilweise zeigen spezifische Lehrerkompetenzen Einfluss nur auf bestimmte Unterrichtsfächer. So führe die pädagogische Fähigkeit des Nachfragens und Diskutierens zu besseren Schülerleistungen beim Lesen, nicht aber in Mathematik (Kane et al., 2010, S. 29).

Besonders bedeutsam für Schülerleistungen schätzen Bryk et al. als Haltung der Lehrkräfte darüber hinaus die so genannte „Work orientation“ ein. Diese habe beispielsweise hohen Einfluss auf die Lesekompetenzen der Schüler: „Of 18 schools having chronically weak reports about teachers' work orientation, not one improved in reading." (Bryk et al., 2010, S. 90f).

Sonderpädagogische Autorinnen und Autoren haben bereits in den 70er- Jahren darauf hingewiesen, dass Kompetenzen, die im Zusammenhang mit schulischer Integration stehen, eine hohe Anforderung darstellen. Die Meinungen darüber, ob diese Kompetenzen bereits in der ersten Ausbildungsphase vermittelt werden können, gehen weit auseinander. So konstatieren Prell und Link:

Die Forderung behinderte Kinder mit nichtbehinderten Kindern zugleich zu beschulen mit der Intention bestmöglicher individueller Förderung und sozialer Integration erfordert Fähigkeiten, die die Lehrer sich erst erarbeiten müssen, da sie an den Pädagogischen Hochschulen nicht vermittelt werden. (Prell \& Link, 1977, S. 323) 
Dagegen geht Heese davon aus, dass Integration strukturiert „lehrbar“ sei:

Die Integration muss durch Abklärung ihrer Bedingungsfaktoren zu einem differenzierten lehrbaren System von praktikabler Schulorganisation und Methodik gemacht werden. Auch der methodisch durchschnittlich befähigte Lehrer, der nicht voll und ganz nur in seinem Beruf aufgeht, muss in den Stand gesetzt werden, integrierten Unterricht mit bestimmten behinderten Kindern zu leisten. Hier liegen wichtige Aufgaben für die sonderpädagogischen Studienstätten, entsprechendes Erfahrungswissen zu erarbeiten. (Heese, 1977, S. 104)

Antor weist 1987 allerdings auch kritisch darauf hin, dass die Sonderpädagogik noch kein Professionalisierungskonzept entwickelt habe, „das den Erfordernissen von Integrationsklassen Rechnung tragen könnte" (Antor, 1987, S. 102). Eine zu berücksichtigende erschwerende Rahmenbedingung im sonderpädagogischen Studium sei dabei die Ausdifferenzierung in sonderpädagogische Fachrichtungen (Antor, 1987, S. 109). Schon Anfang der 80er Jahre standen auch Forderungen im Raum Ausbildungsschwerpunkte in der Grundschullehrerausbildung zu verändern und u.a. folgende inhaltliche Bausteine zu vermitteln: Grundlagen zur Schülerbeobach-tung, zur Lernausgangs- und Lernfortschrittsdiagnostik, zu Binnendifferenzierung und Individualisierung, zu spezifischen Problemen schulschwacher Kinder sowie zu grundschulbezogenen sonderpädagogischen Inhalten (Reinartz \& Sander, 1982, S. 23f). Sander setzte in diesem Zusammenhang bereits Ende der 70er Jahre durch, dass Studenten für das Lehramt an Hauptschulen an der Universität Saarbrücken vier Semesterwochenstunden „Entwicklungsgestörtenpädagogik“ zu studieren hatten (Preuss-Lausitz, 1981, S. 198).

Auch Mutzeck forderte in den 80er Jahren, dass Grundschullehrkräfte eine heilpädagogische Weiterbildung „in den Fachrichtungen Verhaltensgestörtenpädagogik und Lernbehindertenpädagogik sowie im Bereich Allgemeine Heilpädagogik einschließlich notwendiger Kenntnisse der Sprachheilpädagogik“ (Mutzeck, 1987b, S. 25) erhalten sollten. Dumke, Krieger und Schäfer stellen diesbezüglich einen Zusammenhang dahingehend dar, dass die Bereitschaft der Lehrkräfte zu integrativem Unterricht eng mit ihrer positiven Haltung gegenüber schulischer Integration insgesamt verknüpft sei (Dumke et al., 1989, S. 135, S. 150). Dem spreche entgegen, dass sich die Regelschullehrkräfte nicht als genügend qualifiziert für die Umsetzung integrativen Unterrichts einschätzen würden (Dumke et al., 1989, S. 143) und mehr als „80 \% der Regelschullehrer [...] weder in der Aus- noch in der Fortbildung Gelegenheit [hatten], sich mit sonderpädagogischen Fragestellungen auseinanderzusetzen." (Dumke et al., 1989, S. 143). Dumke et al. führen dies auf zum damaligen Zeitpunkt wenig veränderte Studien- und Prüfungsordnungen zurück (Dumke et al., 1989, S. 144). Feyerer und Fragner fordern zu Beginn der 90er Jahre, dass die Lehrerbildung allgemein Inhalte zur Differenzierung in heterogenen Gruppen, zur prozessorientierten Förder- und Lerndiagnostik, zu neuen Formen der Leistungsbeurteilung oder zur interdisziplinären Zusammenarbeit vermitteln müsse (Feyerer \& Fragner, 1994, S. 45f). Berges stellte in seiner Untersuchung zu Dimensionen der Integration bei kooperativem Unterricht fest, dass die Lehrkräfte mit Blick auf das soziale Lernen der Schülerinnen und Schüler mit und ohne Behinderung Wissens-, Informations- und Erfahrungsmangel zeigten, bspw. zu den Behinderungen an sich oder aber zur Arbeitsweise der Regelschulen (Berges, 1996, S. 247):

Die Kooperationspädagogen fühlen sich auf ihre Arbeit schlecht vorbereitet und zeigen Unsicherheiten im Umgang mit den Schülern der jeweils anderen Schulform, sowie in der Planung und Gestaltung gemeinsamen Unterrichts. [...] Es darf als Tatsache betrachtet werden, daß fachkundiges Personal bei der Implementation pädagogischer Reformen eine entscheidende Bedingung für den Erfolg von kooperativen Begegnungen darstellt. (Berges, 1996, S. 264) 
In Berlin wurde 1999 eine Pflichtlehrveranstaltung mit zwei Semesterwochenstunden zum Gemeinsamen Unterricht in der Berliner Prüfungsordnung für das Erste Staatsexamen festgeschrieben (Demmer-Dieckmann, 2007, S. 153). In Sachsen-Anhalt wurden sonderpädagogische Fachinhalte in das Regelschulstudium integriert (Demmer-Dieckmann, 2007, S. 154). Anfang der 2000er Jahre gehen Eberwein und Knauer insgesamt jedoch davon aus, dass diesbezüglich wenig Fortschritte erzielt worden seien und die Vermittlung von Grundlagenkenntnissen lediglich in der Lehrerfortbildung Fuß gefasst habe, obwohl „das Problem der integrationspädagogischen Ausbildung von Lehrern seit vielen Jahren bekannt und immer wieder angemahnt worden [sei]“ (Eberwein \& Knauer, 2002, S. 15). Inzwischen haben sich tieg greifende Veränderungen zumindest an einzelnen Studienstandorten der BRD ergeben. So kann das Lehramt für Sonderpädagogik an der Universität Bielefeld nur noch in Verbindung mit dem Lehramt an Grund-, Haupt- und Realschulen erworben werden. Der „Umgang mit Heterogenität“ wird dabei bspw. als Nebenfach im Bereich Erziehungswissenschaft angeboten ${ }^{31}$.

Mit Blick auf die Qualität der schulischen Integration ist aus sonderpädagogischer Perspektive entscheidend, dass die in den Regelschulen eingesetzten Förderschullehrkräfte über eine abgeschlossene Ausbildung verfügen (Innerhofer \& Klicpera, 1991; Berges, 1996; Bless, 2003; Wember \& Prändl, 2009). Schon 1977 fordert Heese, dass im Studium eine integrative Methodik gelehrt werden solle. Innerhofer und Klicpera (1991), Hug (1994b) und Bürli et al. (2009) fordern darüber hinausgehend eine Reform der sonderpädagogischen Lehrerbildung; Hug (1994b), Deutsches Schulamt (2004), Specht et al. (2006) sowie Wernstedt \& Ohnesorg (2010) eine sonderpädagogische Bildung für alle Lehramtsstudierenden. Weitreichend sind die Zielsetzungen, die mit Blick auf die Ausbildung von Förderschullehrkräften verfolgt werden. Diese umfassen eine Kompetenzvermittlung in einer Fülle von Bereichen wie Beratungskompetenz, Konfliktlösungsstrategien (Preuß, 1985), Kenntnisse zu spezifischen (technischen) Anforderungen im Unterrichten von Kindern mit unterschiedlichen sonderpädagogischen Förderschwerpunkten usw. (Wember \& Prändl, 2009).

Hinsichtlich der Fortbildung von Lehrkräften betonen Fussangel et al. die Wirksamkeit langfristig angelegter Fortbildungen, die eine Begleitung der Lehrkräfte beinhalten (Fussangel, Rürup \& Gräsel, 2010, n. Jäntsch et al., 2015, S. 156). Wesentlich sei außerdem, dass die Fortbildungen den Lehrkräften direkte Anknüpfungsmöglichkeiten für ihren Unterricht bieten (Haenisch, 1994, n. Jäntsch et al., 2015, S. 156f). Hellmich und Görel (2014) weisen in Anlehnung an Leyser u.a. (1994), Praisner (2003) sowie Avramidis und Kalyva (2007) darauf hin, dass Lehrkräfte, die inklusionsbezogene Fortbildungen wahrgenommen haben, positivere Einstellungen zum Thema entwickeln. Dies konnten sie in einer eigenen Studie ebenfalls nachweisen, wobei sie einschränkend darauf hinweisen, dass möglicherweise die Lehrkräfte Fortbildungen in diesem Bereich wahrnehmen, die bereits über eine positive Grundeinstellung dazu verfügen. Zwischen Lehrkräften ohne bzw. mit Fortbildungsbesuch konnten keine Unterschiede zu Auffassungen zur Inklusion oder zu inklusiver Schul- und Unterrichtsentwicklung festgestellt werden (Hellmich \& Görel, 2014, S. 50). Das Angebot berufsbegleitender Fortbildungen wird im Zusammenhang mit Schulentwicklung allgemein und auch mit Blick auf die Schulentwicklung an integrativ arbeitenden Schulen als wichtige Qualitätskategorie eingeschätzt (Innerhofer \& Klicpera, 1991; Hug, 1994b; Husinsky, 1994; Boban \& Hinz, 2003; BruggerPaggi, 2003; Deutsches Schulamt, 2004; Specht et al., 2006; Bürli et al., 2009; Thiele, 2009; Wember \& Prändl, 2009; Arndt \& Werning, 2013; Lütje-Klose \& Urban 2014b). Gefordert 
werden in diesem Zusammenhang Fortbildungen für einzelne Lehrer (Brugger-Paggi, 2003), die Fortbildung von Schulaufsichtsbeamten und von Schulleitungen (Hug, 1994b), der gemeinsame Besuch von Fortbildungen für Regel- und Förderschullehrer (Bless, 2003) sowie eine Abstimmung von Weiterbildungsmaßnahmen aufeinander (Innerhofer \& Klicpera, 1991). Nach Innerhofer \& Klicpera (1991) sollten Fortbildungsveranstaltungen hinsichtlich ihrer Effizienz überprüft werden. Darüber hinaus sollten Untersuchungen zum Fortbildungsbedarf erfolgen. Aufgrund von Erfahrungen im Rahmen eines Modellversuchs wird allerdings deutlich, dass die in Fortbildungen zu vermittelnden Inhalte als sehr umfassend und komplex einzustufen sind. So formulieren Speck et al. aufgrund ihrer Erfahrungen in einem Modellversuch bereits 1978 rund ein Dutzend Themenkreise, die bspw. mit Blick auf die konkrete Umsetzung integrativen Unterrichts bearbeitet werden müssten (Bsp.: Grundkenntnisse zu sonderpädagogischen Fachrichtungen, Kenntnisse zum Ablauf von Etikettierungsprozessen und psychologischen Konsequenzen, Kenntnisse über Sozialisationsprozesse, Elternarbeit etc.) (Speck et al., 1978, S. 137f). Am häufigsten wünschen sich befragte Lehrkräfte nach Dumke et al. (1989), Berges (1996) oder Deutschem Schulamt (2004) Fortbildungen zu einzelnen Behinderungen sowie in Sonderpädagogik allgemein (Dumke et al., 1989, S. 145). Als weiterer Schwerpunkt werden direkte Kontakte zu bereits integrativ arbeitenden Einrichtungen bspw. in Form von Hospitationen oder Praktika gewünscht (Dumke et al., 1989, S. 145). Feuser und Meyer benennen als Fortbildungsinhalte für die Grundschullehrkräfte behindertenpädagogische, humanbiologische, medizinische und neuropsychologische Fragen sowie Informationen zu Entwicklungsbeeinträchtigungen, für die Sonderschullehrkräfte Grundschulpädagogik und - didaktik, darunter vor allem Fachdidaktiken (Erstunterricht Lesen, Schreiben, Rechnen) (Feuser \& Meyer, 1987, S. 181). Boban und Hinz (2003) halten Fortbildungen zur Planung und Durchführung effektiver Zusammenarbeit für wichtig.

Auch die Vorteile schulinterner Fortbildungen werden von Mutzeck schon in den 80er Jahren herausgestellt: „Schulinterne Fortbildung im Sinne eines gemeinsamen Handeln und Tragen von Verantwortung, einer produktiven und innovativen Mitarbeit in der unterrichtlichen und erzieherischen Gestaltung und Weiterentwicklung einer Schule trägt auch zu dem notwendigen pädagogischen Grundprinzip von Geborgenheit und Vertrauen bei." (Mutzeck, 1987b, S. 27). Für integrative Schulen werden in diesem Zusammenhang Studientage (Thiele, 2009) oder innerschulische Fortbildungen allgemein (Boban \& Hinz, 2003) als Qualitätsindikatoren benannt.

Lütje-Klose und Urban fordern als Konsequenz aus Forschungsergebnissen zur Kooperation zwischen Lehrkräften Fortbildungsangebote, die sich an Teams oder ganze Kollegien richten sowie eine integrierte Ausbildung von Regel- und Förderschullehrkräften im Rahmen der ersten Ausbildungsphase an den Universitäten (Lütje-Klose \& Urban, 2014b, S. 291f). Ähnlich formulieren es Beraterinnen und Berater für Inklusion im Rahmen der GeSchwind-Studie in Rheinland-Pfalz, die einen hohen Beratungsbedarf von Schwerpunktschulen auf der Team- und Organisationsebene wahrnehmen. Darüber hinaus stellen sie fest, dass

[...] ein Bedarf an externer Beratung vor allem hinsichtlich der allgemein pädagogischen und didaktischen Herausforderungen im Umgang mit der Diversität der Lern- und Leistungsvoraussetzungen und Bildungsbiographien der Schülerinnen und Schüler und der Kooperation zwischen den unterschiedlich ausgebildeten Lehrern bzw. in multiprofessionellen Teams besteht. (Laubenstein et al., 2015, S. 283)

In der Untersuchung an rheinland-pfälzischen Schwerpunktschulen kommen Laubenstein et al. (2015) zu dem Ergebnis, dass etwa $90 \%$ der Grundschulen und weiterführenden Schulen Fortbildungsangebote nutzen, die das Themenfeld Heterogenität bzw. Inklusion bearbeiten 
(S. 161). Wenig untersucht sind bislang allerdings die Auswirkungen von Fortbildungen auf die Unterrichtsgestaltung von Lehrkräften. Reiser fasst diesbezüglich als ein Ergebnis des hessischen Modellversuchs zusammen:

Obwohl unsere Angebote an Fortbildungen direkt auf die Wünsche der Grundschullehrer reagierten und qualifizierte unterrichtspraktische Anregungen boten, konnten sowohl in der direkten Unterrichtsbeobachtung wie in den Lehrerinterviews kaum mehr Spuren festgestellt werden. Etwas anders stellt sich das Ergebnis dort dar, wo Lehrerteams selbständig Entwicklungen in Angriff nahmen und wo Anregungen mit persönlichen Begegnungen und Selbsterfahrungen verbunden waren. (Reiser, 1987, S. 139)

Auch die Begleituntersuchung des Landes Brandenburg an den inklusiven Pilotschulen liefert in diesem Zusammenhang kritische Ergebnisse:

Insgesamt besteht kein eindeutiges Bild darüber, inwieweit nach Auskunft der Befragten die Anwendung der Fortbildungsinhalte erfolgte bzw. Auswirkungen der Anwendung beobachtbar waren. Leicht im positiven Bereich lag noch der Mittelwert zur Anwendung der Fortbildungsinhalte $(M=2.61$, $\mathrm{SD}=0.62$ ). Die Auswirkungen in Bezug auf die Unterrichtsgestaltung positionierten sich im Schnitt nahezu exakt auf dem theoretisch möglichen Mittel $(\mathrm{M}=2.52, \mathrm{SD}=0.67)$. Beobachtbare Auswirkungen auf die Schülerinnen und Schüler wurden insgesamt durch die Lehrerinnen und Lehrer vergleichsweise weniger stark wahrgenommen $(M=2.42, S D=0.61)$. (Jäntsch et al., 2015, S. 168f)

Die Lehrkräfte erläuterten, dass sich Transferschwierigkeiten für sie u.a. dadurch ergaben, dass bei den Fortbildungen zwar theoretische Grundlagen zu einzelnen Förderschwerpunkten vermittelt wurden, konkrete Lösungsansätze für die Förderung im Unterricht allerdings fehlten. Gleichzeitig würden schulische Rahmenbedingungen wie Klassengröße, die zur Verfügung stehende Zeit oder die Räumlichkeiten die Umsetzung der Einbindung von Fortbildungsinhalten in den Unterricht zum Teil erschweren. Als besonders schwierig wurde der Transfer von Fortbildungsinhalten in den Alltag von den Lehrkräften beschrieben, die die kollegiale Zusammenarbeit sowie die Kooperation mit Eltern in ihren Schulen als unzureichend einstuften (Jäntsch et al., 2015, S. 173). Positiv bewerteten die Lehrkräfte eine begleitende Unterstützung durch Beraterinnen und Berater, die „erreichbar waren und als Ansprechpersonen in konkreten Problemlagen zur Verfügung standen“" (Jäntsch et al., 2015, S. 176). Die Anzahl bzw. Langfristigkeit der Fortbildungsveranstaltungen zeigte allerdings keine Effekte mit Blick auf deren Erfolg (Jäntsch et al., 2015, S. 175f).

Die begleitende Unterstützung von Lehrkräften, die im Rahmen schulischer Integration tätig sind, wird als Supervision auch in weiteren sonderpädagogischen Forschungsveröffentlichungen aufgeführt (Hug, 1994b; Husinsky, 1994; Specht et al., 2006; Bürli et al., 2009; Wember \& Prändl, 2009).

\subsubsection{Ergebnisse im Bereich der Personalentwicklung}

Wie wird Personalentwicklung in deutschen Schulen konkret umgesetzt und was sind die Ergebnisse, die durch Personalentwicklung in Schulen erreicht werden können? In Gegenüberstellung zu den umfassenden theoretischen Ansätzen zur Beschreibung einer (gelingenden) Kooperation und Professionalisierung von Lehrkräften sind Forschungsergebnisse in diesem Qualitätsbereich sehr begrenzt. Nach Buhren (2010) ist derzeit zunächst einmal eine „systematische Personalentwicklung und Professionalisierung, die sowohl die individuelle Entwicklung der Lehrerinnen und Lehrer umfasst als auch die gemeinsame Entwicklung des Kollegiums einer Schule, [...] in den meisten Schulen nicht existent." (Buhren, 2010, S. 226). 
Die Erfassung von Ergebnissen der Kooperation von Lehrkräften ist in mehrfacher Hinsicht schwierig, u.a. da sich die „Zusammenhänge zwischen Rahmenbedingungen einer Schule, Kooperation unter den Lehrpersonen, Schulklima, Unterrichtsqualität und Schulleistungen [...] als mehrteilige Wirkungskette [erweisen] " (Reichenbach 2011, 266f)“ (zitiert n. Albisser et al., 2013, S. 15). Sie ist also, wie alle anderen dargestellten Qualitätsbereiche auch, nicht isoliert zu untersuchen, sondern immer als Teil des komplexen Bedingungsgefüges Schule. In Deutschland lassen sich laut Baum et al. (2012b) verschiedene Forschungsansätze unterscheiden, die sich allerdings in erster Linie mit der Umsetzung der Kooperation von Lehrkräften in den Schulen, nicht aber mit deren Auswirkungen befassen (Baum et al., 2012b, S. 10f). Aus diesen Ansätzen schlussfolgern die Autoren:

Kooperation ist - in welchem Ausmaß, in welcher Form und auf welcher Stufe auch immer - nicht per se schon qualitativ gut. Sie kann für verschiedene Zwecke eingesetzt werden: Sie ermöglicht Lehrpersonen sowohl die positiven Erfahrungen der Steigerung von gemeinschaftlicher Verbundenheit und intrinsisch motivierter Kreativität als auch die negativen zunehmender Belastung durch Gruppenzwänge und externe Anforderungen der Organisation. (Baum et al., 2012b, S. 13)

Während sich direkte Auswirkungen der Kooperation von Lehrkräften wie bspw. die auf das Schulklima gegebenenfalls noch gut untersuchen lassen, verhält sich dies bei den indirekten Auswirkungen auf die Lernergebnisse von Schülerinnen und Schülern anders:

Zunächst ist festzustellen, dass im deutschsprachigen Raum ein Forschungsdesiderat in Bezug auf die Wirkung von Kooperation auf eine inklusive Schul- und Unterrichtsgestaltung sowie auf die Lernerfolge von Schülerinnen und Schülern mit und ohne Lernschwierigkeiten vorliegt; [...]. (Serke et al., 2014a, S. 251)

Deshalb sprechen sich Albisser et al. (2013) dafür aus, mit Hilfe von Mehrebenen-Analysen im Rahmen der Schulentwicklungsforschung Auswirkungen auf Schülerleistungen zu prüfen und demnach mit Blick auf mögliche qualitätsförderliche Bereiche übergreifend zu erheben, welche Maßnahmen der Schulentwicklung insgesamt als zielführend bzw. entbehrlich einzustufen sind (Albisser et al., 2013, S. 15). Bryk et al. (2010) erläutern in diesem Zusammenhang beispielsweise, dass eine vertrauensvolle Zusammenarbeit zwischen Lehrkräften nicht automatisch positive Auswirkungen auf das Lernen von Schülern hat:

In pulling this altogether, it is important to recognize that relational trust among the adults in a school community does not directly affect student learning. Rather, it creates the basic social fabric within which school professionals, parents and community leaders can initiate and sustain efforts at building the essential supports for school improvement. In short, trust facilitates core organizational change processes that instrumentally contribute to improving academic productivity. (Bryk et al., 2010, S. 140)

Bryk et al. konnten im Rahmen ihrer Längsschnittstudie feststellen, dass eine professionelle Entwicklung von Lehrkräften dann Auswirkungen auf Lernergebnisse von Schülerinnen und Schülern haben kann, wenn diese sich im Kontext einer professionellen Gemeinschaft bewegt und mit einer positiven Arbeitsorientierung kombiniert ist:

Taken alone, reports about poor-quality professional development were not especially predictive of differences in academic productivity rates [...] However, high-quality professional development in the context of a supportive professional community and where teachers were oriented toward improvement appears powerfully related to gains in academic productivity [...] Over half of all schools improved in reading and math when a strong faculty work orientation combined with quality professional development. (Bryk et al., 2010, S. 113) 
Mit Blick auf die persönlichen Überzeugungen im Sinne der „Beliefs“32 von Lehrkräften im Sinne von Einstellungen oder Haltungen ${ }^{33}$, gehen Moser u.a. aufgrund ihrer inhaltsanalytischen Auswertung sonderpädagogischer Fachliteratur davon aus, dass für inklusiven Unterricht v.a. eine individuell-förderbezogene-förderdiagnostische Orientierung, eine biografische, lebenslagenorientierte Sicht auf Schüler sowie eine Orientierung auf soziales Lernen maßgeblich seien (Moser et al., 2011, S. 145ff; zitiert n. Lütje-Klose \& Urban, 2014b, S. 285).

Amrhein, die Einstellungen von Lehrkräften sowie deren Veränderungen nach der Einführung „Integrativer Lerngruppen“ in weiterführenden Schulen in NRW untersucht hat, kann nach einem Jahr feststellen, dass diese sich leicht in Richtung einer größeren Akzeptanz des gemeinsamen Unterrichts verschoben haben, gleichzeitig hat sich allerdings die Bereitschaft der Lehrkräfte, die noch nicht im gemeinsamen Unterricht eingesetzt sind, zum Einsatz in dieser Unterrichtsform verringert (Amrhein, 2011, S. 209f). Sowohl Eberl (2000) als auch Amrhein konnten erheben, dass Grundschul- bzw. Sekundarschullehrkräfte sowie auch Förderschullehrkräfte davon ausgehen, dass integrativer Unterricht eine höhere zeitliche Belastung mit sich bringt (Eberl: GS-Lehrkräfte $97 \%$, FSL 56\%; Amrhein: 76\%) und auch nervlich belastender sein kann (Eberl: GS-Lehrkräfte 89\%, FSL $32 \%$; Amrhein: SEK-I-Lehrkräfte 59,8\%) (Eberl, 2000, S. 212; Amrhein, 2011, S. 139).

Opalinski (2014) fasst bislang vorliegende Forschungsergebnisse dahingehend zusammen, dass Lehrkräfte schulischer Inklusion als Konzept gegenüber grundsätzlich positiv eingestellt sind (Avramidis \& Norwich, 2002; Scruggs \& Mastropieri, 1996; n. Opalinski, 2014, S. 79), der praktischen Umsetzung allerdings skeptisch gegenüberstehen (de Boer, 2012; Hwang \& Evans, 2011; Dumke et al., 1998; n. Opalinski, 2014, S. 80). Häufig hänge die Skepsis mit unzureichenden Rahmenbedingungen zusammen (Janz et al., 2012; Hwang \& Evans, 2011; Meyer, 2011; Scruggs \& Mastropieri, 1996). Bei ihrer eigenen Befragung von Thüringer Grund- und Förderschullehrkräften gelangt Opalinski zu dem Ergebnis, dass diese schulische Inklusion sehr kritisch bewerten, vor allem hinsichtlich der gegebenen Rahmenbedingungen (Opalinski, 2014, S. 83f). Hellmich und Görel (2014) gehen aufgrund bisheriger Studien davon aus, dass die Haltungen von Lehrkräften zu inklusiver Unterrichtung sich positiv verschieben, wenn diese bereits über Erfahrungen mit gemeinsamem Unterricht verfügen (Hellmich \& Görel, 2014, S. 49f). Gleichzeitig können sie mit Hilfe ihrer eigenen Studie mit 201 Grundschullehrkräften zwar nachweisen, dass Lehrkräfte mit Erfahrungen aus dem Unterricht mit Kindern mit sonderpädagogischem Förderbedarf positivere Einstellungen zur Inklusion zeigten als Kolleginnen und Kollegen ohne entsprechende Vorerfah-

32 Kuhl, Moser, Schäfer und Redlich (2013) definieren für den deutschen Sprachraum den Begriff der „Beliefs“, als „gegenstandsbezogenes, wertebasiertes, individuelles, in Clustern verankertes Überzeugungssystem, das teils bewusst, teils unbewusst das eigene Handeln steuert." (S. 6), wobei sie davon ausgehen, dass Beliefs von Lehrkräften wie unter anderem Hattie (2009) und Blömeke et al. (2008) belegt haben - entscheidend für die Umsetzung von (Veränderungen im) Unterricht sind (Kuhl et al., 2013, S. 6).

33 In der Systematik dieser Arbeit werden Einschätzungen von Lehrkräften normalerweise inhaltlichen Bausteinen zugeordnet und bilden eine Akteursperspektive auf einen Qualitätsbaustein o.ä. ab. Viele andere Arbeiten thematisieren die Einstellungen und Haltungen von Lehrkräften allerdings auch als eigenen Qualitätsbaustein (Eberl, 2000; Amrhein, 2011; Moser et al., 2011). An dieser Stelle werden nur die Einschätzungen und Haltungen erläutert, die sich auf inklusiven Unterricht bezogene Grundeinstellungen beziehen. Die Einordnung von Haltungen, die Lehrkräfte im Lauf ihrer Ausbildung bzw. ihres Berufslebens entwickeln, unter den Bereich der Ergebnisse ist auf der einen Seite möglich, da davon ausgegangen werden kann, dass ein Zusammenhang zwischen bisherigen beruflichen Erfahrungen und Einstellungen gegeben ist (Hellmich \& Görel, 2014, S. 49f), auf der anderen Seite kann angenommen werden, dass Haltungen sich auch außerhalb der beruflichen Situation entwickeln und in ihrer Entwicklung nie als Ergebnis „abgeschlossen“ sind, so dass die Einordnung an dieser Stelle auch problematisiert werden kann. 
rungen, dieser Unterschied war für Grundschullehrkräfte, die zum Zeitpunkt der Untersuchung inklusiv arbeiteten, allerdings nicht feststellbar (Hellmich \& Görel, 2014, S. 58). Auch Bosse u.a. (2015) können keinen Zusammenhang zwischen Unterrichtserfahrungen von Grundschullehrkräften mit Schülerinnen und Schülern mit spF und positiven Einstellungen (in diesem Fall Selbstwirksamkeitserwartungen) eruieren (Bosse et al., 2015, S. 147).

\subsubsection{Zusammenfassung zum Bereich der Personalentwicklung}

Haltungen und Handeln von Lehrkräften (Hattie, 2013) stehen letztendlich im Mittelpunkt aller Bemühungen im Bereich der Personalentwicklung. Diese kann in Schulen in direkter (Bsp.: Mitarbeitergespräche durch die Schulleitung) oder indirekter Form (Bsp.: Möglichkeiten zu Kooperation, Teamarbeit, Fortbildung) erfolgen und unterliegt der Annahme, dass sie sich auf das Lernen der Schüler und Schülerinnen auswirken kann. Problematisch erscheinen in diesem Zusammenhang Untersuchungsergebnisse, die belegen, dass die fachdidaktischen und methodischen Kompetenzen der Lehrkräfte in Deutschland nicht so gut ausgebildet werden wie in anderen Ländern und solche, die zeigen, dass die unterrichtsbezogene Zusammenarbeit nur selten praktiziert wird. Damit stehen die zwei großen Qualitätsbausteine Kooperation und Professionalisierung im Fokus der Personalentwicklung in Schulen.

Für die Entwicklung beider Bausteine sind Ressourcen notwendig, die vor allem in sonderpädagogischen Forschungsveröffentlichungen erfasst werden. Dazu gehört zunächst ausreichendes Personal in integrativ arbeitenden Schulen (Romey, 1977; Prell \& Link, 1977; Speck et al., 1978) sowie die Möglichkeit zur Doppelbesetzung von Klassen mit Regel- und Förderschullehrkräften (Innerhofer \& Klicpera, 1991; Köbberling, 1994; Heyer et al., 1997; Bless, 2003; Boban \& Hinz, 2003; Brugger-Paggi, 2003; BMBWF Österreich, 2008; Specht et al., 2006; Thiele, 2009; Textor, 2009; Holzinger et al., 2011). Lehrerteams in integrativen Klassen sollten nicht zu viele Lehrkräfte umfassen (Köbberling, 1994; Hug, 1994b; Husinsky, 1994; BMBWF Österreich, 2008; Holzinger et al., 2011). Zum Teil wird eine schulbezogene Bündelung sonderpädagogischer Ressourcen anstelle des Vorrangs einer wohnortnahen Beschulung gefordert (BMBWF Österreich, 2008). Darüber hinaus wird Kontinuität (gleich bleibende Förderschullehrkräfte in Regelschulen) als wichtig erachtet (Brugger-Paggi, 2003). Vielfach gefordert werden im Stundenplan fest verankerte Kooperationszeiten der Regel- und Förderschullehrkräfte (Preuß, 1981; Köbberling, 1994; Boban \& Hinz, 2003; Brugger-Paggi, 2003; Wember \& Prändl, 2009; Holzinger et al., 2011; Lienhard-Tuggener et al., 2011; Arndt \& Werning, 2013).

Auch wenn die Effekte der quantitativen Versorgung von Schulen mit Lehrkräften auf Unterricht und Lernleistungen von Schülern nicht hinreichend geklärt sind, gehen Lütje-Klose und Urban (2014b) davon aus, dass diese Ressourcen auf jeden Fall Einfluss auf die Möglichkeiten der Kooperation von Lehrkräften untereinander haben. Sind diese Ressourcen nicht gegeben, bestehe die Gefahr, dass keine echte Kooperation erfolge, sondern Probleme bspw. von Regel- an Förderschullehrkräfte delegiert werden könnten sowie häufiger Formen äußerer Differenzierung praktiziert werden würden. Eine Möglichkeit dieser Gefahr entgegenzuwirken könne in der Entwicklung schulischer Förderkonzepte bestehen, die von der Schulgemeinschaft getragen werden würden.

Statistische Daten bzw. Forschungsergebnisse belegen, dass die oben beschriebenen notwendigen Ressourcen derzeit in der BRD in verschiedener Hinsicht nicht oder nur in Teilen gegeben sind. In den 2010er Jahren ist die Grundversorgung von Schulen mit einer ausreichenden Anzahl an Lehrkräften teilweise nicht gesichert, da ein Mangel an ausgebildeten Lehrerinnen und Lehrern vorliegt (Meidinger, 2018). Dieser Mangel bezieht sich sowohl auf Regel- wie auf Förderschullehrkräfte. Die Zuweisung von Förderschullehrkräften an die Regelschulen erfolgt 
in der Regel als Budget von Stunden, das im bundesdeutschen Schnitt etwa zwei Unterrichtsstunden je Regelschulklasse vorsieht. Je nach Schwere der Beeinträchtigung der unterrichteten Schülerinnen und Schüler kommen zu diesem Budget so genannte „Rucksackstunden“ hinzu. Diese Form der Zuweisung entspricht nicht der Anzahl der Stunden, die Förderschullehrkräften für die Schülerinnen und Schüler in den Förderschulen zur Verfügung steht. Die Unterrichtsverpflichtung von Regel- und Förderschullehrkräften hat sich im Zug der Einführung bzw. Umsetzung schulischer Integration/Inklusion nicht verändert. Eine Aufteilung der Arbeitszeit in Zeiten der Unterrichtsverpflichtung und gemeinsame, im Stundenplan verankerte Kooperationszeiten für die Vor- und Nachbereitung von Unterricht ist nicht vorgesehen. Nach Serke et al. (2014a) sowie Arndt und Werning (2013) sind diese Zeiten an den von ihnen untersuchten inklusiv arbeitenden Schulen nicht vorhanden.

Mit Blick auf die Kooperation von Lehrkräften allgemein wird angenommen, dass diese sich positiv auf die Entwicklung und Qualität einer Schule sowie die pädagogische Professionalität auswirken kann. Effekte auf den Unterricht oder die Leistungen von Schülerinnen und Schülern sind allerdings kaum durch Forschung belegt. In der Institution Schule ist Kooperation in erster Linie im Rahmen von Konferenzen oder Dienstbesprechungen vorgesehen bzw. - in neuerer Form - bspw. innerhalb von Steuergruppen. Aus sonderpädagogischer Sicht unterstützen diese Formen den Austausch über organisatorische und pädagogische Fragen in integrativ arbeitenden Schulen (Prell \& Link, 1977). Darüber hinaus sind keine weiteren Kooperationsformen institutionalisiert, weshalb diese häufig in einem informellen Rahmen stattfinden. Kooperation zwischen Lehrkräften kann auf unterschiedlichen Niveaustufen (Bsp.: Co-Activity - Cooperation - Coordination (Lütje-Klose \& Willenbring, 1999); Austausch - Kooperation - Ko-Konstruktion (Fussangel \& Gräsel, 2006)) und auf verschiedenen Ebenen stattfinden (Spieß, 2004; Fussangel \& Gräsel, 2012; Keller-Schneider \& Albisser, 2013; Lütje-Klose \& Urban, 2014a u. 2014b), denen wiederum unterschiedliche Funktionen im schulischen Alltag zukommen. Formen des Austauschs werden in diesem Zusammenhang häufiger praktiziert als Formen, die sich auf eine unterrichtsbezogene Zusammenarbeit im engeren Sinn beziehen.

Studien belegen, dass der Wunsch nach stärkerer Kooperation von Lehrkräften untereinander sehr viel intensiver ausgeprägt ist als deren tatsächliche Umsetzung (Dieckmann et al., 2008; Fussangel \& Gräsel, 2012; Pröbstel \& Soltau, 2012; Arndt \& Werning, 2013; Soltau \& Mienert, 2013; Serke et al., 2014a). Einer Studie von Pröbstel und Soltau (2012) zufolge stehen Lehrkräfte Kooperation grundsätzlich positiv gegenüber, zeigen wenig Ängste vor Bewertungen durch andere Lehrkräfte und verfügen über teamorientierte Grundeinstellungen. Behrensen, Sauerhering \& Solzbacher (2012) konnten ebenfalls belegen, dass Lehrkräfte (in diesem Fall an Grundschulen) sich mehr Kooperation mit Förderschullehrkräften wünschen. Nach eher kritischen Ergebnissen in den 80er Jahren (Reiser et al., 1984; Antor, 1987; Dumke et al., 1989) scheint diesbezüglich also eine positive Entwicklung stattgefunden zu haben.

Baum (2012), Fend (2008) oder Reh (2008) gehen mit Blick auf die Umsetzung der Kooperation zwischen Lehrkräften davon aus, dass es sowohl organisatorische Strukturen in den Schulen als auch Rekontextualisierungsprozesse, die Sorge vor Autonomieeinbußen oder eine unzureichende Professionalisierung sind, die dieser im Wege stehen, während Pröbstel \& Soltau (2012) aufgrund ihrer Studie annehmen, dass den Lehrkräften häufig eine gemeinsame Zielorientierung fehle. Darüber hinaus sind die oben benannten mangelnden Ressourcen (v.a. fehlende gemeinsame Teamzeiten sowie unzureichende Zeiten der Doppelbesetzung) Ausschlag gebend für mangelnde Umsetzungsmöglichkeiten (Arndt \& Werning, 2013; Serke et al., 2014a). Die Kooperation in großen Systemen wie Gesamtschulen stellt sich für Regel- und Förderschullehrkräfte schwieriger dar als in 
kleineren Systemen (Bsp.: kleine Grundschulen) (Wessel, 2005; Amrhein, 2011; Gebhardt et al., 2013), da dort weniger Unterrichtsstunden von den Klassenleitungen erteilt werden und somit die Anzahl der Kooperationspartner (Bsp.: Fachlehrer) größer ist.

Als wesentliche Qualitätsmerkmale der unterrichtsbezogenen Zusammenarbeit werden Transparenz (Bsp.: Erwartungen), die Konkretheit gemeinsamer Ziele, eine gleichberechtigte Tätigkeit auf Augenhöhe sowie Offenheit, Flexibilität und Kreativität benannt (Haeberlin et al., 1992). Mit Blick auf die unterrichtsbezogene Kooperation besteht eine große Bandbreite an Formen der Ausgestaltung (Bsp.: Austausch von Material, gemeinsame Förderung im Klassenraum bzw. Einzel- oder Gruppenförderung in Differenzierungsräumen). Häufig wird die unterstützende Tätigkeit von Förderschullehrkräften in den Regelschulen als Entlastung wahrgenommen (Köppel, 1984; Arndt \& Werning, 2013). Aufgrund der genannten Budgetierung der Stunden der Förderschullehrkräfte (im Schnitt zwei Unterrichtsstunden je Klasse) können Doppelbesetzung und Co-Teaching-Formen allerdings nur selten umgesetzt werden (Gebhardt et al., 2013). Eine gemeinsame Unterrichtsvor- und -nachbereitung und -durchführung sind kaum möglich, obwohl diese aus sonderpädagogischer Sicht eine wichtige Qualitätskategorie für schulische Integration darstellen (Heese, 1977; Mühl, 1987; Husinsky, 1994; Berges, 1996; Boban \& Hinz, 2003; Deutsches Schulamt, 2004; Bürli et al., 2009; Holzinger et al., 2011). Aus diesem Grund erfolgt die Rollenverteilung zwischen Regel- und Förderschullehrkraft in den meisten Fällen nach dem one teach - one observe - bzw. nach dem one teach - one assist Modell (Magiera \& Zigmond, 2005; Mc Duffie, 2012), so dass den Förderschullehrkräften im gemeinsamen Unterricht häufig eine nachrangige Funktion zukommt (Arndt \& Werning, 2013). Forschungsergebnisse zu den Effekten des Co-Teaching sind nicht einheitlich. Die Vorteile dieser Unterrichtsform können offensichtlich nur dann zum Tragen kommen, wenn CoTeaching effektiv und in Kombination mit weiteren Bestandteilen guten Unterrichts umgesetzt wird (Murawski \& Swason, 2001; Fontana, 2005; Cook et al., 2011).

Insgesamt wird auch der Kooperation in Teams ein hoher Stellenwert zugemessen (Bächthold et al., 1990; Hug, 1994b; Berges, 1996; Boban \& Hinz, 2003; Brugger-Paggi, 2003; Specht et al., 2006; Feyerer, 2007). Diese können die Verantwortung für Unterricht - bspw. als professionelle Lerngemeinschaften - teilen, indem sie ihn gemeinsam vorbereiten und reflektieren (Direktion für Bildung und Kultur, 2012), und darüber hinaus eine wichtige Funktion als Träger schulischer Entwicklungsprozesse übernehmen (Schley, 1990; Bryk et al., 2010). Ähnlich wie die unterrichtsbezogene Zusammenarbeit eines Lehrerteams (Bsp.: Klassenleitung - Förderschullehrkraft) sind für die Arbeit dieser Teams andere Arbeitszeitmodelle notwendig (Terhart, 2006; Arnd \& Werning, 2013; Serke et al., 2014). Die Arbeit in Teams kann zur Entlastung der beteiligten Lehrkräfte beitragen (Fussangel \& Gräsel, 2006; Serke et al., 2014a; Laubenstein et al., 2015) und sich - zumindest aus der Wahrnehmung der Lehrkräfte heraus - positiv auf Unterricht und Lernleistungen auswirken (Arnold et al., 2011).

Flexibilität auf der einen und Klarheit auf der anderen Seite sind die wesentlichen Anforderungen an die Rollen- und Aufgabenverteilung zwischen Regel- und Förderschullehrkräften (Preuß, 1981; Antor, 1987; Bächthold et al., 1990; Reiser et al., 2007; Schwager, 2011; Serke et al., 2014; Lütje-Klose \& Urban, 2014b). Unterschiedliche Modelle fokussieren als Aufgaben für die Förderschullehrkräfte im Rahmen schulischer Integration u.a. Beratung, individuelle Förderung, gemeinsamen Unterricht, Materialvorbereitung, Diagnostik, Förderplanung und Dokumentation, die Koordination von Hilfen oder auch Supervision (Preuß, 1985; Reiser et al., 1984; Brugger-Paggi, 2003; Bürli et al., 2009; Wember \& Prändl, 2009; Holzinger et al., 2011). Gleichzeitig ändern sich auch Aufgabenfelder der Regelschullehrkräfte. 
Eine Fülle unterschiedlicher Kompetenzen der Lehrkräfte wird im Zusammenhang mit dem Qualitätsbereich der Professionalisierung thematisiert. Während Heese in den 70er Jahren davon ausgeht, dass Kompetenzen im Zusammenhang mit schulischer Integration im Rahmen des Studiums „lehrbar“ seien (Heese, 1977), weist Antor in den 80er Jahren darauf hin, dass noch kein Professionalisierungskonzept für diesen Bereich vorhanden sei (Antor, 1987). Ansätze zur Veränderung der Grundschullehrerausbildung aus den 80er Jahren (Preuss-Lausitz, 1981; Reinartz \& Sander, 1982; Dumke et al., 1989) wurden - von wenigen Ausnahmen abgesehen - kaum umgesetzt. Auch Anfang der 2000er Jahre sah die Situation nach Eberwein \& Knauer (2002) nicht besser aus. Erst im Lauf der 2010er Jahre ergeben sich diesbezüglich an einzelnen Universitäten Entwicklungsfortschritte (Lütje-Klose \& Urban, 2014b). Neben der Erstausbildung werden berufsbegleitende Fortbildungen mit Blick auf die Qualität schulischer Integration als relevant eingestuft (Innerhofer \& Klicpera, 1991; Hug, 1994b; Husinsky, 1994; Boban \& Hinz, 2003; Brugger-Paggi, 2003; Deutsches Schulamt, 2004; Specht et al., 2006; Bürli et al., 2009; Thiele, 2009; Wember \& Prändl, 2009; Arndt \& Werning, 2013; Lütje-Klose \& Urban 2014b). Diese können eine große Bandbreite unterschiedlicher Inhalte umfassen (Bsp.: Methodik, Didaktik, Förderschwerpunkte, Kooperation, Beratung) und richten sich sowohl an Förder- wie an Regelschullehrkräfte. Während Fortbildungen, die sich an einzelne Lehrkräfte richten, wenige direkte Effekte auf die Unterrichtsgestaltung zu haben scheinen (Jäntsch et al., 2015), werden Fortbildungsangebote für Kollegien oder die längerfristige Beratung von Schulen als vergleichsweise effizient eingeschätzt (Lütje-Klose \& Urban, 2014b; Laubenstein et al., 2015).

Ergebnisse schulischer Personalentwicklung sind bislang wenig untersucht worden. Sie sind auch deshalb schwer messbar, da sie - wie die anderen Qualitätsbereiche bzw. -faktoren auch Teil eines sehr komplexen schulischen Wirkungsgefüges sind. Vereinzelte Forschungsergebnisse liegen dahingehend vor, dass das Vertrauen zwischen Kolleginnen und Kollegen die Weiterentwicklung einer Schule als Organisation erleichtern kann. Die Kombination des Engagements von Lehrkräften (work-orientation) mit qualitätsvoller professioneller Weiterentwicklung kann zu besseren Lernergebnissen der Schülerinnen und Schüler führen (Bryk et al., 2010). Bisherige Studien haben ergeben, dass Förderschullehrkräfte grundsätzlich diagnostisch-förderbezogene Einstellungen verinnerlicht haben, die auf der einen Seite auf die individuelle Wahrnehmung der Schülerinnen und Schüler hin orientiert sind und auf der anderen Seite soziale Lernprozesse in den Klassen in den Blick nehmen.

\subsection{Unterrichtsentwicklung}

Im Mittelpunkt der qualitativen Weiterentwicklung von Schulen steht der Unterricht als Kernstück schulischer Arbeit. Welche Ressourcen werden für (inklusiven) Unterricht benötigt? Wie wird Unterricht in integrativ arbeitenden Schulen gestaltet? Wie gelingt es Lehrkräften in ihren Klassen ein lernförderliches Klima herzustellen und welche Bedeutung hat dieses Klima mit Blick auf die Lernergebnisse von Schülern? Welche Rolle spielen Klassenführung und Classroom-Management in diesen Schulen? Welche didaktischen und methodischen Kategorien kennzeichnen die Qualität gemeinsamen Unterrichts? Wie werden Differenzierung bzw. Individualisierung in diesen Schulen umgesetzt? Und welche Ergebnisse erzielt gemeinsamer Unterricht im kognitiven, emotionalen und sozialen Bereich? Die Fragen an den gemeinsamen Unterricht sind zügig gestellt, ihre Beantwortung verlangt jedoch eine präzise Analyse jedes einzelnen Bausteins.

Im Unterricht wird ein lebensweltliches Thema im Rahmen einer pädagogischen Einrichtung inhaltlich reduziert und sequenziert (Arnold, 2009, S. 15; zitiert n. Arnold \& Lindner-Müller, 2010, 
S. 313). Die verantwortliche Lehrkraft steuert dabei die Tätigkeiten des Lehrens und Lernens, die den Erwerb von Kenntnissen, Fähigkeiten und Fertigkeiten seitens der Lernenden ermöglichen, und zwar sowohl geplant als auch intuitiv (Klingberg, 1995, n. Kiel, 2012, S. 21). Die Lehrkraft gliedert dabei Tätigkeiten und Prozesse als Abfolge von Situationen (Klingberg, 1995, S. 5). Aufgrund des hohen Komplexitätsgrads unterrichtlicher Prozesse sind sowohl die Analyse derselben als auch deren Weiterentwicklung auf verschiedenen Ebenen und in unterschiedlichen Bereichen möglich. So geht Stiller davon aus, dass Veränderungen sich auf der Persönlichkeitsebene der Beteiligten, der Inhalts- und Vermittlungsebene, der Beziehungsebene sowie der institutionellen Ebene vollziehen können (Stiller, 2008, S. 10). Eine Weiterentwicklung kann die Veränderung von Organisationsformen des Unterrichts (Bsp.: jahrgangsübergreifende Lerngruppen) genauso umfassen wie die Erarbeitung pädagogischer, (fach-) didaktischer oder methodischer Konzepte. Anstöße dafür können bspw. Probleme im Unterricht, die Unzufriedenheit mit Lernergebnissen, neue Herausforderungen durch eine veränderte Schülerschaft oder die Auseinandersetzung mit neuen Lehr-Lern-Theorien sein. Veränderungen auf unterrichtlicher Ebene verlangen eine entsprechende Steuerung durch die Schulleitung sowie eine enge Kooperation der Schulgemeinschaft. Sie sind zeitintensiv und benötigen - aufgrund der Komplexität und Dauer der Veränderungsprozesse eine grundsätzliche Veränderungsbereitschaft sowie ein hohes Engagement aller Beteiligten über einen längeren Zeitraum hinweg. Hattie geht in diesem Zusammenhang davon aus, dass für die Weiterentwicklung von Unterricht das Lernen selbst wieder stärker in den Fokus rücken muss:

Visible teaching and learning occurs, when learning is the explicit goal, when it is appropriately challenging, when the teacher and the student both (in their various ways) seek to ascertain whether and to what degree the challenging goal is attained, when there is deliberate practice aimed at attaining mastery of the goal, when there is feedback given and sought, and when there are active, passionate, and engaging people (teacher, student, peers, and so on) participating in the act of learning. (Hattie, 2009, S. 22)

Um Unterricht verändern zu können, sind Konzepte notwendig, die Schulen und ihren Lehrkräften theorie- und wissenschaftsbasierte Rahmungen mit klar definierten Qualitätsbausteinen bieten. Die in Deutschland verbreitete unterrichtliche „Ratgeberliteratur“ erleichtert den Schulen eine Konzeptauswahl daher eventuell weniger als die im angloamerikanischen Sprachraum verbreiteten Veröffentlichungen zum „Effective Teaching“ (Muijs \& Reynolds, 2011), die zwar stark outputorientiert sind, dabei aber gut strukturierte und wissenschaftlich fundierte Kenntnisse und Kompetenzen für qualitätsvollen Unterricht zusammenfassen. Für den deutschen Sprachraum ist in Anlehnung an den Ansatz von Stiller (2008) davon auszugehen, dass eine Verknüpfung lernpsychologischer und konstruktivistischer Theorien (Arnold et al., 1999; von Glasersfeld, 2005; Hüther, 2011), empirischer Unterrichtsforschung (Meyer, 2004; Brophy, 2010; Helmke, 2010; Hattie, 2013), didaktischer Traditionen (Klafki, 2003; Terhart, 2009) und fachdidaktischer Erkenntnisse (u.a. Moser Opitz, 2007; Wildemann, 2010; Schenk, 2012; Wartha \& Schulz, 2013; Sandfuchs \& Menzel, 2018) notwendig ist um einen ganzheitlichen Ansatz für Unterrichtsqualität und ihre Weiterentwicklung zu gestalten. Dafür sind wiederum auf der einen Seite die theoretischen Grundlagen (inklusiven) Unterrichts Bestandteil der Auseinandersetzung, auf der anderen Seite die Perspektiven der empirischen Unterrichtsforschung der Allgemeinen Pädagogik wie der Sonderpädagogik.

Die allgemeinpädagogische empirische Unterrichtsforschung versucht das Unterrichtsgeschehen sowie seine Bedingungen und Wirkungen zu erfassen und dabei fachübergreifende Wirkprinzipien und Qualitätsmerkmale zu erschließen (Helmke, 2010, S. 323f). Mit Blick auf inklusive Unterrichtsentwicklung stehen also zunächst nicht spezifisch sonderpädagogische Fragestellungen im 
Mittelpunkt, sondern Kernfragen, die die Qualitätsentwicklung von Unterricht allgemein (an Regel- und Förderschulen) betreffen. Natürlich ist Unterricht vor allem abhängig davon, wie Lehrkräfte ihn gestalten („what teachers do matters“ (Hattie, 2009, S. 22)), so dass ein enger und natürlicher Zusammenhang zwischen der Personal- und der Unterrichtsentwicklung an Schulen besteht:

When these professionals see learning occurring or not occuring, they intervene in calculated and meaningful ways to alter the direction of learning to attain various shared, specific and challenging goals. In particular, they provide students with multiple opportunities and alternatives for developing learning strategies based on the surface and deep levels of learning some content or domain matter, leading to students building conceptional understanding of this learning which the students and teachers then use in future learning. (Hattie, 2009, S. 23, Herv. im Orig.)

Von Unterrichtsentwicklung wird gesprochen, wenn Lehrkräfte diese Prozesse nicht nur individuell durchlaufen, sondern beispielsweise in Teams und bei schulinterner systematischer Steuerung gestalten (Bsp.: Entscheidungsfindung für einen fachdidaktischen Ansatz, Arbeit an schulinternen Kompetenzrastern). Hattie betont in diesem Zusammenhang das reflexive Handeln von Lehrern und Schülern:

The remarkable feature of the evidence is that the biggest effects on student learning occur when teachers become learners of their own teaching, and when students become their own teachers. When students become their own teachers they exhibit the self-regulatory attributes that seem most desirable for learners (self-monitoring, self-evaluation, self-assessment, self-teaching). Thus, it is visible teaching and learning by teachers and students that makes the difference. (Hattie, 2009, S. 22)

Während Hattie sich auf 800 Metastudien beruft, die insgesamt mehrere Tausend Studien zusammenfassen, spielte die wissenschaftliche Untersuchung von Unterricht in Deutschland bis zur PISA-Studie eine eher untergeordnete Rolle:

Im deutschen Sprachraum ist - vor allem bedingt durch die in der Pädagogik vorherrschende geisteswissenschaftliche Orientierung - empirische Unterrichtsforschung immer noch Mangelware. Es gibt zwar zahlreiche Praxisberichte, theoretische Abhandlungen, Modellversuchsberichte und Ratgeberliteratur zum Unterricht, aber nur wenige empirische Untersuchungen, deren Stichprobenplan, Design und statistische Auswertung methodischen Standards entspricht und denen belastbare Daten zugrunde liegen. (Helmke, 2015, S. 15)

Erst seit den 2000er Jahren wurden verschiedene Merkmalskataloge „guten“ Unterrichts veröffentlicht, die versuchen, die Ergebnisse der empirischen Unterrichtsforschung zusammenzu-fassen (Brophy, 2000; Meyer, 2004; Hattie, 2009; Helmke 2010). Bei diesen bleibt nach Eikenbusch und Heymann (2010) allerdings „meist ungeklärt, wie die untersuchten Bereiche oder Aspekte mit anderen zusammenwirken und wie die herausgefundenen Merkmale einander beeinflussen“" (Eikenbusch \& Heymann, 2010, S. 8):

Denn die Frage, was Unterricht zu „gutem Unterricht“ macht, ist nur zu beantworten, wenn geklärt ist: Wozu soll der Unterricht gut sein? Was ist für die Qualität des Unterrichts wichtig und wertvoll? Zu bestimmen, was guter Unterricht ist, erfordert also normative Entscheidungen: Er lässt sich nur in Bezug auf Vorgaben und Ziele definieren und ist nicht „objektiv“ und eindeutig feststellbar. Die Entscheidung hängt ab von Qualitätskriterien, die gemäß ihrer pädagogischen Intentionalität unterschiedlich gesetzt und gewichtet werden können, die aber auch mit bestimmten fachlichen Qualitätsansprüchen sowie mit gesellschaftlichen und institutionellen Rahmenbedingungen und Vorgaben (z.B. Lehrplänen) verträglich sein müssen. (Eikenbusch \& Heymann, 2010, S. 9) 
Zu berücksichtigen ist bei der Darstellung der für schulische Integration relevanten Kategorien guten Unterrichts, dass diese sich in erster Linie auf Unterrichtsprozesse an sich und erst in zweiter Linie auf die Wirkungen von Unterricht im Sinne von messbaren Lernergebnissen der Schülerinnen und Schüler beziehen (s. dazu auch Helmke, 2015, S. 21f). Bei der Darstellung dieser Kategorien ist weiterhin von Bedeutung, dass sie zwar als mögliche Variablen guten Unterrichts erforscht und beschrieben werden können, sie allerdings nicht unabhängig von Personen (mit Blick auf die Schüler und Schülerinnen wie auf die Lehrkräfte) sind (Helmke, 2015, S. 24). Die wesentlichen Aussagen zu gutem Unterricht beruhen nach Brophy (2010) auf Studien zum Verhältnis von Unterrichtsprozessen und Schülerleistungen (Prozesse werden durch Beobachtung erschlossen, Leistungen über standardisierte Tests), Erkenntnissen über Prozesse der Unterrichtsgestaltung und Gestaltung von Lernumgebungen (instructional design) sowie aktuellen Theorien über Lehr- und Lernprozesse (Bsp.: konstruktivistische Theorien) (Brophy, 2010, S. 77). Folgende Übersicht gibt einen Überblick zu den von den unterschiedlichen Autoren forschungsbasiert abgeleiteten jeweils zehn wichtigsten Merkmalen guten Unterrichts:

Tab. 6: Qualitätsmerkmale guten Unterrichts (erstellt durch Verf.)

\begin{tabular}{|c|c|c|c|c|}
\hline & Bropl & Meyer 2004 & Helmke 2010 & Hattie 2009 \\
\hline & $\begin{array}{l}\text { US-amerikanische } \\
\text { Lehr-Lern-For- } \\
\text { schung }\end{array}$ & $\begin{array}{l}\text { Schulpädagogische } \\
\text { Sicht }\end{array}$ & $\begin{array}{l}\text { Sicht der Pädagogi- } \\
\text { schen Psychologie }\end{array}$ & $\begin{array}{l}\text { Analyse von } 800 \text { Meta- } \\
\text { studien zu einflussreichen } \\
\text { Faktoren des Lernerfolgs } \\
\end{array}$ \\
\hline 1 & $\begin{array}{l}\text { Unterstützendes } \\
\text { Klima in der Klasse }\end{array}$ & $\begin{array}{l}\text { Klare Strukturierung } \\
\text { des Unterrichts }\end{array}$ & $\begin{array}{l}\text { Effiziente Klassen- } \\
\text { führung }\end{array}$ & $\begin{array}{l}\text { Vertrauen der Schüler in } \\
\text { die eigene Leistung }\end{array}$ \\
\hline 2 & $\begin{array}{l}\text { Hoher Anteil echter } \\
\text { Lernzeit }\end{array}$ & $\begin{array}{l}\text { Hoher Anteil echter } \\
\text { Lernzeit }\end{array}$ & $\begin{array}{l}\text { Lernförderliches } \\
\text { Unterrichtsklima } \\
\end{array}$ & $\begin{array}{l}\text { Entwicklungsgerechtes } \\
\text { Unterrichten }\end{array}$ \\
\hline 3 & $\begin{array}{l}\text { Stimmigkeit des } \\
\text { Lehrplans }\end{array}$ & $\begin{array}{l}\text { Lernförderliches } \\
\text { Klima }\end{array}$ & $\begin{array}{l}\text { Vielfältige Motivie- } \\
\text { rung }\end{array}$ & $\begin{array}{l}\text { Fortlaufende Überprüfung } \\
\text { des Unterrichtserfolgs }\end{array}$ \\
\hline 4 & $\begin{array}{l}\text { Aufbau von Lern- } \\
\text { Orientierung }\end{array}$ & Inhaltliche Klarheit & $\begin{array}{l}\text { Strukturiertheit und } \\
\text { Klarheit }\end{array}$ & $\begin{array}{l}\text { Training von methodischen } \\
\text { Fertigkeiten der Lehrer }\end{array}$ \\
\hline 5 & $\begin{array}{l}\text { Stimmige Unter- } \\
\text { richtsinhalte }\end{array}$ & des Kom- & $\begin{array}{l}\text { Wirkungs- und Kom- } \\
\text { petenzorientierung }\end{array}$ & $\begin{array}{l}\text { Möglichkeit beschleunig- } \\
\text { ten Lernens für begabte } \\
\text { Schüler }\end{array}$ \\
\hline 6 & $\begin{array}{l}\text { Sinn } \\
\text { richt }\end{array}$ & Meth & erung, & $\begin{array}{l}\text { derliches Klima } \\
\text { lare Klassenführung }\end{array}$ \\
\hline 7 & $\begin{array}{l}\text { Üben und } \\
\text { Anwenden }\end{array}$ & ern & $\begin{array}{l}\text { Förderung aktiven, } \\
\text { selbständigen Lernens }\end{array}$ & $\begin{array}{l}\text { Förderung von Schülern } \\
\text { mit Lernbehinderung durch } \\
\text { Instruktion und Vermitt- } \\
\text { lung von Lernstrategien }\end{array}$ \\
\hline 8 & $\begin{array}{l}\text { Unterstützung einer } \\
\text { guten Arbeitshaltung }\end{array}$ & Intelligentes Üben & $\begin{array}{l}\text { Angemessene Varia- } \\
\text { tion von Methoden } \\
\text { und Sozialformen } \\
\end{array}$ & Klarheit der Lehrperson \\
\hline 9 & $\begin{array}{l}\text { Vermittlung von } \\
\text { Lernstrategien }\end{array}$ & $\begin{array}{l}\text { Klare Leistungserwar- } \\
\text { tungen }\end{array}$ & $\begin{array}{l}\text { Konsolidierung, } \\
\text { Sicherung, Intelligen- } \\
\text { tes Üben }\end{array}$ & $\begin{array}{l}\text { Training kognitiver Strate- } \\
\text { gien - Schüler als Lehrer } \\
\text { einsetzen }\end{array}$ \\
\hline 10 & Kooperatives Lernen & $\begin{array}{l}\text { Vorbereitete Umge- } \\
\text { bung }\end{array}$ & Passung & $\begin{array}{l}\text { Feedback (zwischen Leh- } \\
\text { rern und Schülern) }\end{array}$ \\
\hline
\end{tabular}


Übereinstimmend wird von allen Autoren der Qualitätsbaustein lernförderliches Klima benannt. Während Brophy u.a. die Stimmigkeit von Lehrplan und Unterrichtsinhalten als wesentlich erachtet, stellt Meyer die klare Strukturierung in den Vordergrund. Helmke stuft die effiziente Klassenführung und Hattie das entwicklungsgerechte Unterrichten als besonders wichtig ein. Als sonderpädagogi-schen Beitrag zur Unterrichtsforschung verweist Preuss-Lausitz (2010) auf eine Zusammenfassung von Meijer (2003) und eine Studie von Podlesch (2003):

Meijer (2003) fasst die in 17 europäischen Staaten gemachten best-practice-Erfahrungen im integrativen Unterricht so zusammen: Optimaler integrativer Unterricht stellt differenzierte Leistungsansprüche, hat vielfältige Sozialformen, verwendet zahlreiche Medien, praktiziert individualisierende und zugleich kooperative Übungsformen und führt Bewertungsformen ein, die den einzelnen Lernprozess genauer dokumentieren. Die individuellen Förderpläne bzw. Entwicklungspläne werden mit dem allgemeinen Curriculum verbunden, die Teamarbeit erstreckt sich auf die ganze Klasse und es bestehen klare Verhaltensnormen und Erwartungen. In Bezug auf die internationale Forschung über „guten“, d.h. lernwirksamen und zugleich sozial befriedigenden Unterricht (vgl. Meyer 2004, Helmke 2007) können diese Elemente als Teil guten Unterrichts angesehen werden. Podlesch (2003) hat auf der Grundlage langjähriger wissenschaftlicher Begleitung integrierter lern-, geistig-, körper- und sinnesbehinderter Kinder die Komponenten Lernen durch Kommunikation (insbesondere der Schüler untereinander, peer tutoring), Lernen mit allen Sinnen, Lernen durch Handeln und Lernen durch (mehr) Selbst- und Mitbestimmung in Form von Wahlmöglichkeiten ergänzt. (Preuss-Lausitz, 2010, S. 47)

Dyson schlussfolgert als Sonderpädagoge aus einer Studie an englischen Schulen, dass in diesen Schulen keine spezielle „inklusive Unterrichtsentwicklung“ praktiziert wird, sondern der Anspruch der Lehrkräfte offensichtlich grundsätzlich darin besteht hervorragenden Unterricht gestalten zu wollen:

[...] their commitment was not so much to inclusion per se as to the principle of ,doing the best they could' for all of their students. ,Doing the best' in this sense did not necessarily carry with it any commitment to educating students in particular settings, and was entirely compatible with the kind of mixed, flexible provision we have just described. (Dyson, 2010, S. 6, Herv. im Orig.)

Tatsächlich ergibt sich bei einer Analyse sonderpädagogischer Forschungsveröffentlichungen zu Qualitätsmerkmalen gemeinsamen Unterrichts ein sehr hohes Maß an Übereinstimmung mit den Qualitätsbereichen guten Unterrichts allgemein. Neben den Bereichen Ressourcen und Ergebnisse werden in diesem Unterkapitel folgende Bereiche dargestellt: Klassenführung, Schulklasse als sozialer Raum, Didaktik, Methodik sowie Differenzierung und Individualisierung.

\subsubsection{Ressourcen im Bereich der Unterrichtsentwicklung}

Allgemein- wie sonderpädagogische Veröffentlichungen gehen mit Blick auf die Gestaltung qualitativ hochwertigen Unterrichts davon aus, dass dieser im Zusammenhang mit der Klassengröße sowie der Zusammensetzung der Klasse steht. Diese Kategorien werden unter den Bereich Ressourcen gefasst, da sie in zweierlei Hinsicht mit der Ressourcensituation an Schulen verknüpft sind. Sie hängen sowohl mit der grundsätzlichen Personalausstattung einer Schule sowie mit ressourcenbezogenen Rahmenvorgaben der Bildungspolitik und Schulaufsicht zusammen (Bsp.: vorgegebener Klassenteiler, Schüler-Lehrer-Relation, vorgesehene Ressourcen für die Unterrichtung von Schülerinnen und Schülern mit sonderpädagogischem Förderbedarf in Regelschulen). Beide Bereiche sind dabei auch Bestandteil der Organisationsentwicklung an Schulen, da vor allem die Schulleitung über die Bildung von Klassen und über deren Zusammensetzung entscheidet. 
Seit den 70er Jahren wird in Forschungsveröffentlichungen der Sonderpädagogik eine Klassengröße in integrativen Regelklassen gefordert, die eine Anzahl von 20-22 Schülern nicht überschreiten sollte (Heese, 1977; Weigt, 1977; Speck et al., 1978; Mühl, 1987; Dumke et al., 1989; Köbberling, 1994; Brugger-Paggi, 2003; Thiele, 2009; Wember \& Prändl, 2009; Speck, 2010). Abgesehen von Berichten zu den Modellversuchen zu integrativem Unterricht, in denen diese Größe aufgrund der Erfahrungswerte als günstig eingeschätzt wird, ist die Anzahl der Forschungsveröffentlichungen zur Frage nach einem möglicherweise günstigen Klassenteiler allerdings nicht sehr umfassend. Eine Ausnahme bildet die Zusammenfassung zu diesem Thema von von Saldern (2011), der den Forschungsstand für diesen Einflussfaktor mit Blick auf Regelklassen zusammengefasst und eine eigene Erhebung dazu durchgeführt hat. Klassengrößen in Deutschland liegen nach von Saldern im internationalen Mittel (von Saldern, 2011, S. 115), eine genaue Analyse unter Heranziehung internationaler Vergleichsdaten (TALIS-Studie ${ }^{34}$ ) zeige allerdings, dass Deutschland innerhalb der EU überdurchschnittlich große Klassen habe (von Saldern, 2011, S. 117f). Die Größe der Klassen habe - abgesehen von Grund- und Hauptschulen - in den vergangenen Jahren durchschnittlich zugenommen und liege in Realschulen, Gymnasien oder integrierten Gesamtschulen bei 25-27 Schülern (von Saldern, 2011, S. 114). Die häufig aufgestellte Behauptung, dass die Klassengröße keine Bedeutung für das Verhalten von Lehrern wie Schülern habe, stuft von Saldern aufgrund seiner Analyse als sachlich falsch ein. Allerdings seien die Zusammenhänge zwischen beiden Faktoren komplex und könnten empirisch nicht unabhängig von der Qualität des Unterrichts erfasst werden (von Saldern, 2011, S. 157). Von Saldern zieht aus seiner Analyse daher den Schluss, dass „die Klassengröße nur in Wechselwirkung mit anderen Variablen ihre Wirkung entfaltet.“ (von Saldern, 2011, S. 135). In der bisherigen Forschung - so von Saldern - gab es allerdings fast ausschließlich einfache Gegenüberstellungen von kleinen und großen Klassen sowie punktuellen Lernergebnissen von Schülern (von Saldern, 2011, S. 119-122). Zu dem Ergebnis, dass Lernleistungen von Schülern nicht wesentlich mit der Klassengröße korrelieren, kommt auch die PISA-Studie. Allerdings bezieht sich dieses Ergebnis darauf, dass es in vielen Ländern große Klassen (durchschnittlich 25-30 Schüler) gibt, die Leistungen aber unabhängig von dieser Größe sehr weit streuen (Dt. PISA-Konsortium, 2001, S. 423f).

Eine Ausnahme von dieser „Regel“ bilden die Länder mit durchschnittlichen Klassenfrequenzen von weniger als 20 Schülerinnen und Schülern: Ihre 15-Jährigen haben zumindest in Mathematik, zum Teil aber auch im Lesen überdurchschnittliche Leistungen gezeigt. (Dt. PISA-Konsortium, 2001, S. 424)

Hinweise auf den Zusammenhang zwischen Klassengröße und Schülerleistungen seien zwar teilweise in Modellen erfasst (von Saldern, 2011, S. 128f), bisherige Untersuchungen könnten allerdings nur geringe Effekte nachweisen. Während die Studien zur Klassengröße hinsichtlich des Leistungsverhaltens der Schüler sowie mit Bezug auf sozio-emotionale Merkmale der Gruppen keine hochsignifikanten Zusammenhänge nachweisen können, spielt die Klassengröße in der Wahrnehmung der Lehrer eine wichtige Rolle (von Saldern, 2011, S. 149). Von wesentlicher Bedeutung für die Ergebnisse von Forschungsarbeiten zur Klassengröße scheint daher zu sein, wer zur Wahrnehmung der Vor- und Nachteile sowie Auswirkungen von kleinen bzw. großen Klassen befragt wird. So zeigt eine Studie von Haselbeck (Befragung von 800 Hauptschülern und 400 Hauptschullehrern), dass kleinere Klassen aus Sicht von Lehrkräften ein günstigeres Lernumfeld bieten und diese sich positiv auf die Gestaltung des Unterrichts, das Lern- und

34 TALIS $=$ The OECD Teaching and Learning International Survey 
Arbeitsverhalten der Schüler sowie auf soziale Beziehungen auswirken, während größere Klassen vor allem die Gefahr von Störfaktoren beinhalten, die das Selbstverständnis von Lehrern und Schülern negativ prägen können (Haselbeck, 2015, S. 7f). Lehrer bspw. empfinden kleinere Klassen als ruhiger, störungsfreier, weniger belastend sowie von der Stimmung her besser als größere Klassen. Gleichzeitig gebe es in diesen Klassen mehr Zeit für die Hinwendung zu einzelnen Schülern. Die Beziehungen zwischen Lehrern und Schülern seien intensiver, so dass Konflikte auch leichter lösbar seien (Haselbeck, 2015, S. 9). Lehrer in kleineren Klassen tragen weniger vor und sprechen Schüler stattdessen öfter individuell an, die Interaktion zwischen Lehrer und Schülern sei direkter und die Arbeit verlaufe insgesamt ruhiger. Schülerleistungen könnten genauer bewertet und Fragen intensiver beantwortet werden. Die dargestellten Effekte seien allerdings für kleinere Klassen nicht stark ausgeprägt (von Saldern, 2011, S. 139). Nach der umfassenden Forschungsanalyse durch von Saldern ergibt sich bei vielen Untersuchungen die Schlussfolgerung, „dass in kleinen Gruppen durch die Kontaktdichte auch die Kommunikationsrate erhöht wird" (von Saldern, 2011, S. 127) und dadurch auch die interpersonelle Attraktivität von Mitgliedern der Gruppe steige. Gleichzeitig könne es dadurch aber auch vermehrt zu Spannungen innerhalb der Gruppe kommen (von Saldern, 2011, S. 127). Effekte von kleineren Klassen - auch auf Schülerleistungen - hat darüber hinaus die amerikanische STAR - Studie (Student/Teacher Achievement Ratio) erschlossen:

STAR liefert Anhaltspunkte dafür, dass kleinen Klassen im Vergleich zu durchschnittlich großen Klassen (im Mittel 15 vs. 24 Schüler) ein leistungsfördernder und nachhaltig wirksamer Einfluss zukommt. Für die Probanden in den kleinen Klassen wurden bessere Ergebnisse in den Leistungsmessungen ermittelt. Zudem wurden in den kleinen Klassen eine verbesserte Lernhaltung der Schüler und weniger Disziplinprobleme festgestellt. Überwiegend waren die Effekte für Kinder aus Minderheitenfamilien und für Kinder aus einkommensschwachen Familien stärker ausgeprägt. Hinweise finden sich auch darauf, dass die positiven Effekte bis zu siebten Klasse nachweisbar bleiben und damit über die Phase des Experiments hinaus. (Avenarius, 2003, S. 113; zitiert n. von Saldern, 2011, S. 136) ${ }^{35}$

Die Ergebnisse von STAR weisen also darauf hin, dass insbesondere benachteiligte Schüler und Schülerinnen von kleinen Klassen profitieren, während die Klassengröße für leistungsstärkere Schüler weniger entscheidend ist, was ihre Lernerfolge angeht.

Als ungünstige Effekte großer Klassen beschreibt von Saldern u.a. die fehlende Flexibilität der Raumgestaltung, eine höhere zeitliche Belastung der Lehrkräfte (Bsp.: Korrekturaufwand) sowie mehr Lärm bzw. Unruhe (von Saldern, 2011, S. 151-156). Insgesamt kommt von Saldern zu dem Ergebnis, dass die Klassengröße einen positiven Effekt auf die Qualität des Unterrichts haben kann, aber nicht muss (von Saldern, 2011, S. 161). Die Einrichtung von Klassen sollte flexibler erfolgen als dies derzeit der Fall ist und Rahmenbedingungen (Bsp.: Migrantenanteil etc.) sollten stärker in die Planung einbezogen werden, auch was die Zurverfügungstellung weiterer Ressourcen angeht.

Hattie gelangt bei seiner Meta-Analyse mit Blick auf die Relevanz der Klassengröße für Lernprozesse insgesamt nur zu einer Effektstärke von $\mathrm{d}=0,21 \mathrm{bzw}$. - basierend auf Vergleichsuntersuchungen, bei denen eine Reduktion der Klassenstärke erfolgte - sogar nur zu einer Effektstärke von $\mathrm{d}=0,13$. Daraus schlussfolgert er zunächst ähnlich wie von Saldern, dass kleine Klassen vor allem Auswirkungen auf die Arbeitsbedingungen von Lehrkräften und Schülern zu haben schei-

35 Originalquelle: Finn, J.D.; Achilles, C.M. (1999): Tennessee's Class Size Study: Findings, Implications, Misconceptions. In: Educational Evaluation and Policy Analysis, Vol. 21, No. 2, Special Issue: Class Size: Issues and New Findings (Summer, 1999), S. 97-109 Unter: https://www.jstor.org/stable/1164294; Stand: 11.09.2018 
nen und nicht so sehr auf die Lernleistungen an sich (Hattie, 2013, S. 102f). Als Begründung für diese geringe Effektstärke nimmt Hattie in Anlehnung an Finn (2002) allerdings darüber hinaus an, „dass Lehrpersonen kleinerer Klassen dieselben Lehrmethoden wie die Lehrpersonen großer Klassen verwenden und dass sie damit die Chancen, die sich ihnen durch die geringere Zahl von Lernenden bieten, ungenutzt lassen“" (Hattie, 2013, S. 103).

Die Vorgaben der einzelnen Bundesländer für die Klassenbildung in der Bundesrepublik Deutschland unterscheiden sich zum Teil erheblich voneinander. Für die Primarstufe liegen sie bei zwischen 13 (Untergrenze) und 28 Schülerinnen und Schülern (Obergrenze), für die Sekundarstufe I (Hauptschul-zweig) bei zwischen 13 (Untergrenze) und 30 (Obergrenze) Kindern und Jugendlichen. Die Stadt-staaten Bremen und Hamburg haben diesbezüglich die für die Bildung vergleichsweise kleiner Klassen günstigsten Vorgaben (zwischen 17 und 24 (Primarstufe), zwischen 20 und 25 (Sekundarstufe I, Hauptschulzweig bzw. gemischte Klassen) und ermöglichen darüber hinaus die Bildung besonders kleiner Klassen in aus sozialer Sicht besonders belasteten Stadtteilen (KMK, 2018b).

Die Zusammensetzung von Klassen wird vor allem in sonderpädagogischen Veröffentlichungen immer wieder thematisiert (Wocken, 1987; Innerhofer \& Klicpera, 1991; Schley et al., 1992; Husinsky, 1994; Feyerer, 1998; Bless, 2003; Brugger-Paggi, 2003; BMBWF Österreich, 2008; Holzinger et al., 2011). Sie bezieht sich zum einen auf den Anteil von Schülerinnen und Schülern mit spF an der Gesamtzahl der Schüler in einer Klasse, zum anderen auf die „Verteilung“ der sonderpädagogischen Förderschwerpunkte innerhalb einer Klasse. Dazu wurden im Rahmen von Modellversuchen unterschiedliche Modelle erprobt, bspw. im Schulversuch der Hamburger Integrationsklassen die Modelle „11 + 4“ sowie „18+2“ (11 bzw. 18 Schülerinnen und Schüler ohne Förderbedarf + vier bzw. zwei mit sonderpädagogischem Förderbedarf) (Wocken, 1987, S. 68). Wocken bewertet nach vier Versuchsjahren das „18 +2 “ - Modell als günstiger, da dies eine größere soziale Vielfältigkeit biete und mehr Möglichkeiten für die Schüler Freunde und Arbeitspartner zu finden. Die Stabilität der Gruppe sei größer (Bsp.: Umzug von nichtbehinderten Kindern in der kleinen Gruppe kann zu erheblichem Ungleichgewicht führen) und die Zusammensetzung entspreche eher der Realität (Anteilen von Behinderten an Nichtbehinderten) (Wocken, 1987, S. 83). Darüber hinaus seien es „keineswegs die behinderten Kinder, die Sorgen machen, sondern eher die sogenannten nichtbehinderten Kinder" (Wocken, 1987, S. 84). Deshalb differenziert er seine Auffassung dahingehend, dass eine

[...] unverhältnismäßige Häufung von Kindern mit Lern- und Verhaltensschwierigkeiten [...] die Arbeitsproduktivität und das Lernklima einer Klasse erheblich belasten und auch von Integrationsklassen pädagogisch nicht mehr bewältigt werden [kann]. [...] Jede Integrationsklasse braucht gleichsam als ruhenden Pol eine Kerngruppe stabiler, emotional gefestigter Kinder, damit das soziale Gleichgewicht in der gesamten Kindergruppe nicht aus den Fugen gerät. (Wocken, 1987, S. 84)

Die Richtlinien für integrative Unterrichtung des BMBWF Österreichs (2008) geben entsprechend an, „dass die Zusammensetzung der Schüler in Integrationsklassen in etwa repräsentativ für den gesamten Schülerjahrgang an der Schule sein soll“" (BMBWF Österreich, S. 3).

Heimer (1992) berichtet zu den Hamburger Integrationsklassen in Gesamtschulen, dass diese nach einem „17 + 3“- Modell zusammengesetzt waren (Heimer, 1992, S. 117). Er kommt u.a. zu dem Ergebnis, dass es in derart zusammengesetzten Klassen insbesondere Kinder im Grenzbereich zwischen normaler und schwacher Begabung sind, die im integrativen Unterricht „zu kurz“ kommen. Diese haben einen hohen Übungs- und Betreuungsbedarf, stehen aber oft nicht im Fokus. Trotz gleich bleibender Zusammensetzung müsse davon ausgegangen werden, dass der Förderbedarf kog- 
nitiv eingeschränkter Schüler mit zunehmender Jahrgangsstufe zunimmt und sich mit zunehmendem Alter bspw. in der Pubertät divergierende Interessen ergeben könnten (Heimer, 1992 S. 123). Brugger-Paggi nennt als Indikator in diesem Zusammenhang, dass nur ein Schüler oder eine Schülerin mit schweren Beeinträchtigungen einer Klasse zugewiesen werden solle (Brugger-Paggi, 2003). Eine besondere Rolle bei der Klassenzusammensetzung spielt nach Auffassung mehrerer Autoren die (ausgewogene) Streuung der Leistungen innerhalb einer Klasse (Feyerer, 1998; Bleidick, 1999; Bless, 2003; Holzinger et al., 2011). Bless schlussfolgert aus seiner Studie in Schweizer Integrationsklassen, dass eine heterogene Zusammensetzung mit großer Streuung des IQ für die Lernfortschritte von Schülern förderlich sei (Bless, 2003, S. 160f) und empfiehlt, dass die Zusammensetzung einer Klasse vor Schuljahresbeginn geplant wird (Bless, 2003, S. 174f). Bleidick vertritt dagegen die Auffassung, dass eine zu große Streuung genauso leistungsabträglich sein könne wie eine zu starke Homogenität innerhalb einer Gruppe (Bleidick, 1999, S. 134, zitiert nach Ellinger und Stein, 2012).

Huber (2009) sieht mit Blick auf das soziale Klima in integrativen Klassen kritisch, dass Feuser (1999) oder Hinz (1995) ohne entsprechende umfassende empirische Befunde davon ausgehen, dass der Anpassungsdruck aufgrund der großen Heterogenität innerhalb der Klassen gleichmäBig auf die Schüler verteilt werde (Huber, 2009, S. 242). Zwar habe die Begleitforschung zu Modellversuchen (Wocken, 1987; Dumke \& Schäfer, 1993; Feuser \& Meyer, 1987; Preuss-Lausitz, 1991) positive Ergebnisse dahingehend beschrieben, dass Schüler mit sonderpädagogischem Förderbedarf in diesen Klassen nicht übermäßig von sozialer Ausgrenzung betroffen sind, er selbst geht allerdings aufgrund von Ergebnissen einer eigenen Studie mit 650 Viertklässlern (darunter 110 Schüler mit spF) davon aus, dass es „keine signifikante Wirkung der Heterogenität der Lerngruppen auf die soziale Integration von Schülern mit Sonderpädagogischem Förderbedarf gab“" (Huber, 2009, S. 244). Stattdessen kommt er aufgrund seiner Werte zu dem Schluss, dass Schüler mit spF „in Klassen mit hoher Streuung der Intelligenz signifikant seltener für soziale Interaktionen gewählt [werden] als in (kognitiv) homogenen Lerngruppen (Huber, 2008, 10)“ (Huber, 2009, S. 244). Seine Befunde belegen außerdem, dass der Beliebtheitsgrad der Schüler mit spF (15,6\%) geringer ist als der von Schülern ohne $\operatorname{spF}(33,5 \%)$ und dass knapp $50 \%$ der SuS mit spF in den integrativen Klassen abgelehnt werden (Huber, 2009, S. 245).

In mehreren Veröffentlichungen wird außerdem darauf hingewiesen, dass eine Problemhäufung in den Klassen vermieden werden sollte (Brugger-Paggi, 2003; BMBWF Österreich, 2008; Holzinger et al., 2011). So fordert das BMBWF Österreich, dass eine „Überrepräsentation erzieherisch schwieriger, verhaltensauffälliger, lernschwacher Schüler“ (BMBWF Österreich, 2008, S. 3) zu vermeiden sei und die PH Steiermark spricht sich gegen eine „vermehrte Ansammlung von Kindern mit besonderen Erziehungsbedürfnissen im Vergleich zu den Parallelklassen“ (Holzinger et al., 2011, S. 17) aus. Forschungsergebnisse dazu, inwiefern sich eine solche Häufung negativ auf den Unterricht auswirken kann, werden aus sonderpädagogischer Sicht nicht referiert. Müller u.a. konnten allerdings in einer Querschnittstudie nachweisen, dass sich Verhaltensauffälligkeiten innerhalb einer Klasse auf einzelne Schüler auswirken können:

In einer Stichprobe von 493 Siebt- bis Neuntklässler/innen zeigt sich, dass das Niveau an Verhaltensproblemen in der persönlichen Klassenumgebung (Klassenmittelwert ohne eigenen Wert) ein signifikantes Erklärungspotenzial für die Ausprägung des individuellen aggressiv-oppositionellen und delinquentkriminellen Verhaltens hat. (Müller et al., 2012, S. 111)

Sie interpretieren diesen Befund vor dem Hintergrund, dass dissoziales Verhalten sich in sozialen Zusammenhängen entwickle (Warr, 2002) und daher einer Schulklasse, in der Peers eine 
wichtige Bedeutung haben, eine zentrale Rolle zukomme. Ähnliche Effekte seien in der Grundschulforschung belegt (Kellam et al., 1998; Barth et al., 2004; Warren et al., 2005; WerthamerLarsson et al., 1991; Thomas et al., 2011; Thomas et al., 2006) (Müller et al., 2012, S. 121). Ergebnisse der PInG-Studie in Brandenburg zur Zusammensetzung inklusiver Klassen konnten einen Zusammenhang zwischen dem Bildungshintergrund der Eltern und dem Anteil an Kindern mit festgestelltem oder vermutetem $\mathrm{spF}$ feststellen:

Je höher der Anteil an Kindern in einer Klasse war, deren Mütter oder Väter die Hochschulreife besaßen, desto weniger Kinder mit einem festgestellten bzw. vermuteten Förderbedarf besuchten die Klasse. Gleiches galt für sozioökonomische Merkmale der Familie. Je höher der Anteil an Kindern aus sozial begünstigten Elternhäusern in einer Klasse war, desto weniger Kinder wiesen einen festgestellten bzw. vermuteten sonderpädagogischen Förderbedarf auf. (Stäbler et al., S. 84)

Darüber hinaus zeigt die Studie eine große Variationsbreite bezüglich der Anzahl der Schülerinnen und Schüler mit festgestelltem oder vermutetem spF in diesen Klassen auf (Stäbler et al., S. 83f).

\subsubsection{Schulklasse als sozialer Raum}

Mit Blick auf die Schulklasse als sozialem Raum spielt das Klassenklima eine entscheidende Rolle, das in engem Zusammenhang zum sozialen Lernen innerhalb der Klasse steht (von Saldern, 1987; Brophy, 2010; Muijs \& Reynolds, 2011; Helmke, 2015; Huber, 2017). Bezüglich der schulischen Integration bzw. der Qualitätsentwicklung von Unterricht sind daher Qualitätsindikatoren relevant, die die Gestaltung eines lernförderlichen Klimas beschreiben, sowie Indikatoren, die das soziale Lernen in diesen Klassen betreffen. Hinsichtlich der Einordnung von Forschungsergebnissen zeigt sich, dass es einen Überlappungsbereich zwischen diesen Indikatoren als prozessbeschreibenden Bausteinen und zwischen denen, die Ergebnisse erfassen, gibt, da bspw. bei Antworten der Schüler nicht unterschieden werden kann, inwiefern bspw. ein gutes soziales Klima in einer Klasse Voraussetzung oder Ergebnis (integrativer) schulischer Arbeit ist. Ähnlich wie das Schulklima (s. 3.3.2) wird also auch das Klassenklima in der Unterrichtsforschung als ein Qualitätsbaustein beschrieben (Brophy, 2010; Muijs \& Reynolds, 2011; Helmke, 2015). Von Saldern definiert in diesem Zusammenhang den Begriff „Sozialklima“:

Das Sozialklima von Schulklassen als hypothetisches Konstrukt ist ein auf die Schulklasse bezogenes, differenzierendes, relativ überdauerndes, molares und mehrdimensionales Aggregat subjektiver Wahrnehmung und kognitiver Verarbeitung von situationalen Reizen, das sich in der Beschreibung von Umwelten, Strukturen und Verhalten in der Schulklasse bzw. in einem ihrer Subsysteme (z.B. Cliquen) durch das Individuum widerspiegelt und die Bildung von Einstellungen zu Lernsituation sowie individuelles Verhalten beeinflusst. (von Saldern, 1987, S. 17; zitiert n. Huber, 2017, S. 70)

Gemäß Muijs und Reynolds handelt es sich beim lernförderlichen Klima insgesamt um ein weit gefasstes Konzept, das sowohl die in einer Klasse geltenden Regeln, als auch die Lehrer-SchülerInteraktion sowie die physische Raumgestaltung umfasst (Muijs \& Reynolds, 2011, S. 127). Das Lehrerverhalten ist laut Brophy in diesen Klassen geprägt durch eine positive Grundeinstellung, Freundlichkeit, emotionale Reife, Ehrlichkeit und die Sorge der Lehrkräfte um ihre Schüler als Individuen wie als Lernende (Brophy, 2000, S. 8). Nach Helmke ist mit lernförderlichem Klima „eine Lernumgebung gemeint, in der das Lernen der Schülerinnen und Schüler erleichtert, begünstigt oder auf andere Weise positiv beeinflusst wird." (Helmke, 2010, S. 220) und er benennt als Kennzeichen eines solchen Klimas den konstruktiven Umgang mit Fehlern, eine entspannte 
Lernatmosphäre, eine überraschungsoffene Grundhaltung, den Abbau hemmender Leistungsangst, die Passung des Unterrichtstempos und das Einhalten von Wartezeiten (bspw. nach Schülerantworten) (Helmke, 2010, S. 220-229). Grundlage für einen konstruktiven Umgang mit Fehlern ist nach Hattie die vertrauensvolle Zusammenarbeit von Lehrkräften und Schülern (Hattie, 2013, S. 26). Insbesondere bei Schülern mit psychischen Problemen scheint eine vertrauensvolle Beziehung zu Lehrkräften gemäß einer Studie von Hamre und Pianta (2001) von hoher Relevanz zu sein. Nach Woolfolk (2008) belegt diese Studie, dass „Schüler mit bedeutsamen Verhaltensproblemen in der frühen Kindheit anscheinend später weniger Probleme [haben], wenn ihre Lehrer auf ihre Bedürfnisse eingehen und häufig und konsistent Rückmeldungen geben. (Woolfolk, 2008, S. 3). Bless beschreibt neben der Beziehungsebene zwischen Lehrkräften und Schülern weitere förderliche Faktoren:

[...] das Erleben guter sozialer Beziehungen zwischen allen am Unterricht Beteiligten, das Erfahren eines höheren Masses an Eigenständigkeit und Eigenverantwortung bei unterrichtlichen Aktivitäten, die Möglichkeit zur Mitgestaltung des Unterrichtsverlaufs, die Aussicht, für Anstrengungen auch belohnt zu werden und schließlich das Empfinden, dass der Unterricht verständlich und gut organisiert ist. (Bless, 2003, S. 170)

Mit Blick auf eine entspannte Lernatmosphäre spielen auch Humor und die Begeisterung der Lehrkräfte für Unterrichtsinhalte eine wichtige Rolle (Helmke, 2015, S. 231). Neben der Vorbildfunktion und dem Engagement der Lehrkräfte für ihre Schüler ist der Schlüsselfaktor für ein positives Lernklima nach Hattie der Klassenzusammenhalt und damit das Gefühl, „dass alle (Lehrperson und Lernende) gemeinsam für positive Lernerfolge arbeiten." (Hattie, 2013, S. 123). So heißt es auch bei Brophy „Schüler lernen am besten in intakten und sozial rücksichtsvollen Lerngemeinschaften." (Brophy, 2010, S. 79). Er geht aufgrund von Forschungsergebnissen von Brophy \& Good (2000) sowie Sergiovanni (1994) davon aus, dass günstige Lernumgebungen durch eine rücksichtsvolle Haltung der Beteiligten geprägt werden und zwar sowohl der Schüler untereinander, als auch der zwischen Lehrkräften und Schüler.

Hinsichtlich der schulischen Integration von Schülern mit Behinderungen in Regelklassen ist die Frage nach einem lernförderlichen Klima auch aus Sicht der Sonderpädagogik relevant, u.a. da Forschung zu der Fragestellung zum Selbstkonzept und zur Wahrnehmung der sozialen Integration durch diese Schüler - vor allem mit Blick auf Schüler mit Verhaltensauffälligkeiten zum Teil zu ungünstigen Ergebnissen kommt (s. dazu 3.5.7; Venetz \& Tarnutzer, 2012) und die Frage „wie Schülerinnen und Schüler mit besonderem Förderbedarf den schulischen Alltag im natürlichen Lernkontext - also im unmittelbaren Unterricht - subjektiv erleben, empirisch noch kaum systematisch untersucht worden [ist]. (Venetz \& Tarnutzer, 2012, S. 105). Feyerer (1998) gelangt zu der Schlussfolgerung, dass Integrationsschüler in allen Dimensionen ein signifikant positiveres Klassenklima erleben, sowohl im Bereich der Lehrer-Schüler-Beziehungen als auch im Bereich des Unterrichtsklimas. Sie erführen weniger sozialen Druck, die Anwendung individualnormorientierter Bewertungsmaßstäbe, mehr Vertrauen gegenüber Lehrern, die Verständnis für sie aufbringen, mehr Schülerzentriertheit, ein höheres Ausmaß an aktiver Mitgestaltung und eigenständiger Mitarbeit in interessanterem und anschaulicherem Unterricht sowie weniger Leistungsdruck (Feyerer, 1998, S. 165). Preuss-Lausitz führt dagegen schon 1977 aus, dass sich Schüler mit spF „nach den zahlreichen Mißerfolgserlebnissen und Diskriminierungsformen in der Grund- oder Volksschule in der Sonderschule wohler fühlen, selbstsicherer werden und häufig wachsende Leistungen zeigen“ (Preuss-Lausitz, 1977, S. 47), da sie dort in geringerem Ausmaß dazu gezwungen seien zu kontrollieren, dass ihre ,abweichenden Merkma- 
le“ (Bsp.: Kleidung, Gesundheitszustand, Sprachmuster) entdeckt werden könnten und weniger unangenehme soziale Situationen managen müssten (Preuss-Lausitz, 1977, S. 47).

Als Qualitätsindikatoren für das Klassenklima werden in der sonderpädagogischen Forschung bzw. in Qualitätsübersichten eine positive Lehrer-Schüler-Beziehung (Wember \& Prändl, 2009), das Erleben guter sozialer Beziehungen zwischen allen am Unterricht Beteiligten (Bless, 2003), ein unterstützendes Lernklima mit einem individualisierenden und differenzierenden Unterricht (Bächthold et al., 1996) oder auch die Lehrereinschätzung eines (positiven) Klassenklimas (Bless, 2003) benannt.

Während die Zusammenhänge zwischen einer entspannten Lernatmosphäre und der Leistungsentwicklung von Schülern durch Unterrichtsforschung nur schwach nachgewiesen werden können (Helmke, 2015, S. 231), sind die leistungsbeeinträchtigenden Auswirkungen von Leistungsangst empirisch gut belegt. Da der Abbau von Leistungsangst über das Schaffen von Sicherheit sowie ein vertrauensvolles und kooperatives Lernklima erfolgen kann, liefern entsprechende Forschungsergebnisse entsprechend indirekte Hinweise auf die Bedeutung eines lernförderlichen Klimas für positive Lernerfolge von Schülern (Helmke, 2015, S. 233). Nach einer umfassenden Meta-Analyse von Studien durch Wang u.a. (1997) wiederum ist das Klassenklima einer der einflussreichsten Faktoren mit Blick auf von Schülern erreichte Leistungen (Muijs \& Reynolds, 2011, S. 128). Darüber hinaus kann sich ein gutes Klassenklima auch positiv auf die Selbstwertschätzung von Schülern (Fraser, 1994) sowie auf die Partizipation von Schülern sowie deren Demokratieverständnis auswirken (Cotton, 1997) (n. Muijs \& Reynolds, 2011, S. 128).

Eine wichtige Rolle mit Blick auf das Klima in einer Klasse spielt das soziale Lernen, das informell erfolgt, aber auch formell ermöglicht werden kann: durch kooperative Lernformen (s. 3.5.5) oder durch soziale Lernangebote wie kooperative Spiele, Klassenrat, erlebnispädagogische Projekte o.ä. Keller und Hafner (1999) vertreten eine breite Auffassung von sozialem Lernen, wenn sie als Bausteine humane Kommunikation, die Übertragung von Verantwortung, Spiel und Entspannung, Lernförderung, schüleraktive Unterrichtsformen, konstruktive Konfliktlösung, Medienerziehung und Medienkontrolle oder Elterninformation und Elterntraining benennen. Ein sozialer Verhaltenskodex solle darüber hinaus soziale Verhaltenserwartungen einer Schulgemeinschaft transparent machen, eine kompensatorische Betreuung setze die Einbindung der Freiwilligen Ganztagsschule, der Vereine, der Jugendhilfe etc. voraus (Keller \& Hafner, 1999, S. 30-38). Hattie geht davon aus, dass das soziale Lernen dazu beitragen kann den Zusammenhalt in einer Schulklasse zu stärken. Am nachhaltigsten seien in diesem Zusammenhang die Auswirkungen erlebnispädagogischer Angebote:

Lernen, wie man einer Herausforderung begegnet, wie man Feedback bekommt, wie man sich dem kooperativen Lernen anpasst, sowie die verbesserte Selbstregulation bezüglich der eigenen Fähigkeiten und Stärken scheinen über das Naturerlebnis hinaus eine andauernde Wirkung zu haben. (Hattie, 2013, S. 187)

Eckermann \& Heinzel (2013) führen nach einer Analyse des Forschungsstands zu „Peer-Interaktionen, Peer-Status und Schüler-Schüler-Kooperation“" (Eckermann \& Heinzel, 2013, S. 188) aus, dass „Schule und Unterricht [...] Grundschulkindern nicht nur die Aneignung und den Erwerb von Wissen [ermöglichen], sondern [...] vor allem einen bedeutsamen Ort für Sozialerfahrungen in der Schulklasse dar[stellen] (Bennewitz/Meier 2010; Breidenstein/Kelle 2002; Krappmann/Oswald 1995; Petillon 1993)““ (Eckermann \& Heinzel, 2013, S. 189). Im Alltag einer Schulklasse würde sich eine „Peer-Kultur“ etablieren, „die jenseits des Unterrichts Lernprozesse initiiert und maßgeblich zur Persönlichkeitsentwicklung der Schülerinnen und Schü- 
ler beiträgt (de Boer/Deckert-Peaceman 2009)“ (Eckermann \& Heinzel, 2013, S. 189). In der Interaktion zwischen Schülern werden nach Eckermann und Heinzel dabei durchaus hierarchische Ordnungen herausgebildet, bei denen die Kinder nach Differenzkriterien wie schulischen Leistungen oder sozialer Herkunft unterscheiden (Breidenstein 2008; Eckermann et al. 2010) (Eckermann \& Heinzel, 2013, S. 189). Die „Kontinuitätshypothese“ der Peer-Forschung bezieht sich in diesem Zusammenhang darauf, dass sich „Bildungsambitionen und -orientierungen von der Eltern- an die Kindergeneration fortschreiben und in schulischen und außerschulischen Peergroups eine Distinktionsfunktion übernehmen“ (Eckermann \& Heinzel, 2013, S. 189), d.h. dass Schüler häufig Peer-Groups auswählen, „deren Mitglieder eine hohe Kongruenz zu den Werten der Eltern aufweisen“" (Eckermann \& Heinzel, 2013, S. 189):

Für Kinder aus dem bildungsfernen Milieu beinhaltet dies jedoch die Gefahr einer doppelten bzw. kumulativen Benachteiligung, da diese oftmals aufgrund fehlender kultureller Ressourcen schlechtere schulische Leistungen erzielen und gleichzeitig von ihren Peers sozial ausgegrenzt werden und damit weniger vom „Peer-Kapital“ (Du Bois-Reymond 2000) profitieren können. Demzufolge lassen sich in Schulklassen häufig Kinder identifizieren, die aufgrund ihres ,Peer-Status ' [...] dauerhaft benachteiligt werden. (Eckermann \& Heinzel, 2013, S. 190)

In der Sonderpädagogik wird das Thema gemeinsamen Lernens nach Reiser et al. (1990, S. 323) häufig mit Bezug auf die individuelle Förderung der Kinder hin differenziert, weniger mit Blick auf die Förderung des Gruppenzusammenhalts. Für den Unterricht scheinen beide Aspekte gleich wichtig (Berges, 1996, S. 37). Berges schlägt diesbezüglich vor:

Da in didaktischen Modellen auch Fragen nach den grundlegenden Wert- und Zielentscheidungen zu diskutieren sind, also die Notwendigkeit wertegeleiteter Entscheidungen [...], sind didaktische Modelle um Kategorien integrativer Prozesse zu ergänzen. Diese integrativen Prozesse sind nicht nur auf integrativen Unterricht von behinderten und nichtbehinderten Schülern, sondern z.B. auch auf interkulturelle und koedukative Erziehung bezogen. Insofern gehören Begriffe wie Integration bez. Partizipation zu grundlegenden Kategorien einer allgemeinen Pädagogik, einer vertiefenden Pädagogik. (Berges, 1996, S. 249, Herv. im Orig.).

Für soziale Reaktionen im gemeinsamen Unterricht sind seiner Auffassung nach folgende Merkmale bedeutsam: Unterrichtsstrukturen und -methoden, pädagogische Kompetenzen, Persönlichkeits-merkmale der nichtbehinderten Schüler und der behinderten Schüler sowie die Haltungen der Eltern und Elternarbeit (Berges, 1996, S. 254). Damit benennt er mögliche dynamisch aufeinander wirkende Faktoren. Ziemen (2011) hält gemeinschaftlich zu bearbeitende Aufgaben in inklusiven Klassen für wesentlich, damit gegenseitige Anerkennung und gleichberechtigte Teilhabe erfolgen können (Ziemen, 2011, S. 16). Huber (2009) vertritt in diesem Zusammenhang die Position, dass mögliche Wirkungen und Wirkrichtungen, die zu sozialer Akzeptanz oder Ablehnung in integrativen Klassen führen, noch systematisch zu untersuchen seien (Huber, 2009, S. 245).

Um soziale Lernprozesse zu optimieren sind nach Berges institutionelle Rahmenbedingungen und die Qualität unterrichtlicher Prozesse (kooperative Handlungsmöglichkeiten und Methoden, Vorbereitungs- und Reflexionsphasen, kommunikative Verständigungsprozesse) weiterzuentwickeln (Berges, 1996, S. 254f). Speck (1973) hält es für notwendig, soziale Integration als verbindliches Lernziel in den Curricula für vorschulische und schulische Einrichtungen zu verankern, da „raumzeitliches Zusammensein“ diese nicht automatisch garantiere (zitiert n. Prell \& Link, 1977, S. 310). Von besonderer Bedeutung sind nach Prell \& Link (1977) pädagogische Kompetenzen der Lehrkräfte: 
Es geht darum, eine Polarisierung zwischen behinderten und nichtbehinderten Schülern gar nicht erst aufkommen zu lassen. Mitglieder aus beiden Gruppen sind zu gemeinsamen Aktivitäten anzuregen. Das erfordert vom Lehrer soziale Wahrnehmungsfähigkeit und Sensibilität im Aufspüren und Analysieren von Situationen und das Umsetzen dieser Situationsanalysen in Unterrichtsthemen. Es erfordert auch weiterhin, daß sich die Lehrer ihrer Abwehrmechanismen gegenüber Schülern mit abweichendem Verhalten bewußt werden. (Prell \& Link, 1977, S. 303)

Es gehe darum, dass Lehrkräfte über sozialpsychologische Kenntnisse und Fähigkeiten verfügen müssten um eine Schulklasse als soziales System analysieren und ein „Wir“-Gefühl vermitteln zu können (Prell \& Link, 1977, S. 316).

Für die Qualitätskategorie soziales Lernen (Holzinger et al., 2011) werden in sonderpädagogischen Veröffentlichungen folgende Qualitätssubkategorien aufgeführt: das gezielte Beobachten der Klassensituation (Leonhardt, 2009), der Wechsel von individuellem und gemeinsamem Lernen (Köbberling, 1994; Berges, 1996, Wocken, 1987), die Abstimmung von Leistungslernen und sozialem Lernen (Speck, 2010), Maßnahmen zur sozialen Integration von Kindern mit Behinderungen in den Unterricht (Innerhofer \& Klicpera, 1991), die Herstellung strukturierter Kontakte (Mühl, 1987) bspw. durch Rollenspiele, das Training individueller Konfliktlösungsstrategien (Wember \& Prändl, 2009) oder die Durchführung des Klassenrats (Boban \& Hinz, 2003). Darüber hinaus spielen auch Indikatoren eine Rolle, die sich auf stärkere Partizipationsmöglichkeiten von Schülern an unterrichtlichen Prozessen beziehen wie das Erfahren eines höheren Maßes an Eigenständigkeit und Eigenverantwortung bei unterrichtlichen Aktivitäten (Bless, 2003), eine zunehmende Eigenverant-wortung der Lernenden (Heyer et al., 1997), die Beteiligung der Lernenden an Entscheidungen über Unterrichtsinhalte und Lernwege (Boban \& Hinz, 2003), eine größere Bedeutung der Aktivität der Schüler (Innerhofer \& Klicpera, 1991) oder lehrerunabhängiges Arbeiten (Köbberling, 1994).

\subsubsection{Klassenführung}

Unterricht bedeutet für die allermeisten Lehrpersonen, vor allem die (Lern)Aktivitäten einer Gruppe von zwanzig bis dreißig Schülerinnen und Schülern in festen Rhythmen vorausplanend, prozessbegleitend und nachsorgend zu organisieren, zu unterstützen und zu regulieren. Die dahinter stehende Fähigkeit, eine Klasse zu managen oder zu führen, zählt heutzutage zu den basalen Bestandteilen beruflichen Wissens und Könnens von Lehrpersonen und wird in zahlreichen Veröffentlichungen als wichtiger Bestandteil des pädagogischen Wissens bzw. als essentieller Aspekt von Unterrichtsqualität angesehen (vgl. z.B. Klieme, Schümer \& Knoll 2001; Baumert \& Kunter 2006; Helmke 2008). (Haag et al., 2015, S. 9)

Wie dieses Zitat verdeutlicht, hat Klassenführung als Qualitätsmerkmal von Unterricht eine entscheidende Bedeutung. Veröffentlichungen zum Themenfeld „Classroom-Management“ bzw. „Klassenführung“ erscheinen dabei im deutschen Sprachraum insbesondere seit den 2000er Jahre (Eikenbusch, 2010; Keller, 2012; Kiel et al., 2013) erneut in größerem Umfang, nachdem diese bereits in den 70er Jahren (Kounin, 1976) eine Rolle spielten. Insgesamt führt das Thema allerdings nach Helmke „in Deutschland ein Schattendasein, sowohl in der Lehrerausbildung als auch in der Forschung" (Helmke, 2015, S. 175). Anders verhält es sich im angloamerikanischen Bereich (Muijs \& Reynolds, 2005; Borich, 2007; Evertson \& Weinstein, 2006). Dabei legen Forschungsergebnisse nahe, dass eine effiziente Klassenführung ein „Schlüsselmerkmal der Unterrichtsqualität“ (Helmke, 2015, S. 173) ist:

Die internationale Forschung zeigt, dass kein anderes Merkmal so eindeutig und konsistent mit dem Leistungsniveau und dem Leistungsfortschritt von Schulklassen verknüpft ist wie die Klassenführung (Walberg und Paik, 2000; Anderson, Ryan, Shapiro, 1989; Emmer \& Stough, 2001; Brophy, 2006; 
Einsiedler, 1997). So befindet sich Classroom Management nach den kognitiven Schülerkompetenzen an der zweiten Stelle der Rangliste in der Metaanalyse zu Bedingungsfaktoren schulischer Leistungen von Wang et al. (1993, S. 93). Auch bei PISA 2003 zeigte sich wieder die Relevanz einer wirksamen Klassenführung für die Leistungsentwicklung im Fach Mathematik. (Helmke, 2010, S. 174f)

Bis zu den von Helmke genannten neueren Veröffentlichungen orientierte sich die deutsche Diskussion vor allem an den Erkenntnissen von Kounin (Kounin, 1976). Kounin stellte bei Felduntersuchungen in Schulen und Camps fest, dass viele (Disziplinierungs-)Maßnahmen nicht zum gewünschten Erfolg führten und untersuchte daraufhin in einer videobasierten Folgestudie, welche Lehrerhandlungen welches Schülerverhalten zur Folge haben (Kounin, 2006 (Reprint des Originals), S. 11-13). Dabei gelangte er zu dem Ergebnis, dass nicht Zurechtweisungen durch Lehrkräfte für eine gute Klassenführung relevant sind, sondern folgende Prinzipien:

- Withitness (Allgegenwärtigkeit/Dabeisein): Lehrer ist über alle Vorgänge der Klasse im Bilde,

- Overlapping (Überlappung): Lehrer kümmert sich um mehrere Vorgänge gleichzeitig,

- Momentum (Zügigkeit/Reibungslosigkeit): Lehrkraft gestaltet Unterricht reibungslos, gut geplant und ohne unnötige Unterbrechungen,

- Smoothness (Geschmeidigkeit): Unterricht hat roten Faden, keine inhaltlichen Brüche,

- Group Focus (Gruppenaktivierung): alle Schüler sind im Blick,

- Managing Transitions (Übergangsmanagement): Phasenübergänge sind gut gestaltet,

- Avoiding Mock Participation (Vermeidung von Überdruss und vorgetäuschter Teilnahme): Lehrkraft zeigt sich sensibel für vorgetäuschte Mitarbeit von Schülern (Kounin, 2006, S. 89-143).

Dieses Lehrerverhalten wirke sich auf alle Schüler aus: auf die gesamte Klasse wie auch auf die als „emotional gestört“ diagnostizierten Kinder (Kounin, 2006, S. 85). Zwar wurde der Fokus durch Kounin weg von „Disziplinierungsstrategien“ hin auf Lehrerverhalten und Unterrichtsablauf gelegt, problematisch ist daran nach Eikenbusch (2010) allerdings, dass Lehrer durch diese Erkenntnisse erheblich unter Druck geraten, weil sie sozusagen alleine für Struktur und Disziplin in der Klasse verantwortlich sind, nach dem Motto: „Die Lehrperson macht den Unterschied.“ (Eikenbusch \& Heymann, 2010, S. 16-19). Erst in den letzten Jahren wird das so genannte „Classroom Management“ nicht mehr als Einzelaufgabe von Lehrern gesehen, sondern als Aufgabe von Teams bzw. der ganzen Schule. Auch die Schüler selbst spielen bei der Organisation und Führung der Klasse eine bedeutende Rolle, wie vor allem Evertson \& Weinstein (2006) darstellen.

Marzano und Marzano kommen aufgrund einer umfangreichen Metaanalyse von 100 Studien zu dem Ergebnis, dass von allen Schulleistungsfaktoren die Klassenleitung oder das Klassenmanagement die einflussreichste Variable darstellt (Marzano \& Marzano, 2003). Die Beziehung zwischen Lehrkraft und Schülern sei wiederum der Schlüssel für alle anderen Aspekte des Klassenmanagements (Marzano \& Marzano, 2003, S. 163):

In fact, our metaanalysis indicates that on average, teachers who had high-quality relationships with their students had 31 percent fewer discipline problems, rule violations, and related problems over a year's time than did teachers who did not have high-quality relationships with their students. (Marzano \& Marzano, 2003, S. 163)

Hier zeigt sich also der enge Zusammenhang zwischen der Führung einer Klasse und der Gestaltung einer Klasse als sozialem Raum (3.5.2). Eine Lehrkraft muss gleichzeitig in der Lage sein die 
Lernerfolge der Schüler über ein gutes Klassenmanagement positiv zu beeinflussen und gruppendynamische Prozesse in der Klasse sensibel zu steuern. Nach Hattie sind „Lehrer-Schüler-Beziehungen [...] starke Moderatorvariablen des Klassenmanagements ( $d=0,87$ )“ (Hattie, 2013, S. 122):

Die Merkmale von Lehrpersonen, die den größten Einfluss auf die Sicherstellung gut geführter Klassen und die Reduzierung von Störungen haben, sind eine angemessene innere Einstellung $(d=1,29)$ oder ein „bei-der-Sache-Sein“ der Lehrperson $(\mathrm{d}=1,42)$, d.h. die Lehrperson hat die Fähigkeit, potenzielle Verhaltensprobleme zu erkennen und schnell darauf zu reagieren, und sie bewahrt eine emotionale Objektivität $(\mathrm{d}=0,71)$. (Hattie, 2013, S. 122)

Für diese Beziehung ist es nach Marzano und Marzano wichtig, dass die Lehrkräfte ein angemessenes Maß von Dominanz auf der einen und von Kooperation auf der anderen Seite an den Tag legen und Schüler mit speziellen Bedürfnissen im Blick haben (Marzano \& Marzano, 2003, S. 163). Angemessene Dominanz könne dadurch hergestellt werden, dass Lehrkräfte klare Erwartungen an Verhaltens- und Lernziele formulieren (Bsp.: Regeln und Abläufe sowie Konsequenzen) und gewünschtes Verhalten verstärken (Marzano \& Marzano, 2003, S. 163). Die Berücksichtigung von Schülern mit speziellen Bedürfnissen gelinge Lehrern aufgrund eines Repertoires an unterschiedlichen Strategien wie der Gestaltung sicherer Interaktionen für passive Schüler, konsistenter Beziehungen zu aggressiven Schülern, der Unterstützung aufmerksamkeits-beeinträchtiger Schüler (bspw. durch Rückzugsmöglichkeiten) oder sozialen Trainings für Schüler mit Schwierigkeiten in diesem Bereich (Marzano \& Marzano, 2003, S. 166). Aufgrund ihrer Ergebnisse halten Marzano und Marzano es für wichtig, dass Lehrkräfte die Beziehungen zu ihren Schülern bewusst gestalten, dadurch die Dynamik in ihrer Klasse beeinflussen und damit bessere Leistungen von Schülern ermöglichen (Marzano \& Marzano, 2003, S. 167). Die Effekte betragen laut Hattie $d=0,52$ auf die Lernleistung von gut organisierten Klassen und $d=0,62$ auf ein erhöhtes Engagement (Hattie, 2013, S. 122). Dieser Bereich ist in Hatties Metastudie also einer derjenigen, die eine starke Auswirkung auf die Lernleistung von Schülern zeigen.

Muijs und Reynolds erläutern ebenfalls, dass es ein gutes Classroom-Management sei, das effektive von weniger effektiven Lehrkräften unterscheide (Muijs \& Reynolds, 1999), und für ein hohes Maß an ,time on task' sorgen könne (Muijs \& Reynolds, 2011, S. 102). Helmke hält zusammenfassend fest:

Eine effiziente Klassenführung ist kein Selbstzweck, sondern unabdingbare Voraussetzung für die Sicherung anspruchsvollen Unterrichts, indem sie einen geordneten Rahmen für die eigentlichen Lehr- und Lernaktivitäten schafft und insbesondere die aktive Lernzeit steuert, das heißt diejenige Zeit, in der sich die Schüler mit den zu lernenden Inhalten engagiert und konstruktiv auseinandersetzen können. (Helmke, 2015, S. 173)

Ein hoher Anteil echter Lernzeit wiederum sorgt für bessere Lernergebnisse: „Schüler lernen mehr, wenn so viel Unterrichtszeit wie möglich auf die im Lehrplan vorgesehenen Themen verwendet wird und wenn die Klasse gezielt zu den dafür erforderlichen Aktivitäten angehalten wird." (Brophy, 2010, S. 80), ein Ergebnis, zu dem neben Brophy (1983) auch Muijs und Reynolds (2011) gelangen (Muijs \& Reynolds, 2011, S. 102).

Im Rahmen seines Modells zur Klassenführung weist Helmke u.a. auf die Bedeutung von Regeln, Routinen und Ritualen, vorausplanendem Handeln sowie dem Umgang mit Störungen für das Classroom Management hin (Helmke, 2015, S. 179f). Regeln und Routinen dienen dabei als ,verbindliche[n] Abmachungen für das Verhalten in der Schule“ (Helmke, 2015, S. 179), deren Wirksamkeit auf den Erfolg von Lernprozessen von Hattie mit $\mathrm{d}=0,76$ beziffert wird 
(Hattie, 2009, S. 102). Mit Blick auf Klassenmanagement als präventivem und vorausplanendem Handeln sind nach Evertson \& Emmer (2009) zehn Punkte relevant, die sich als Ergebnis umfassender Forschung in diesem Bereich (Evertson \& Weinstein, 2006) ergeben haben:

- Vorbereitung des Klassenraums (S. 1-18),

- Einführung von Klassenregeln und Abläufen (S. 19-42),

- Abläufe zum Management von Schüleraktivitäten (S. 43-60),

- Organisation von Aktivitäten zum Schulbeginn (Förderung des Zusammengehörigkeitsgefühls) (S. 61-94),

- Planung und Durchführung von instruktivem Unterricht (S. 95-120),

- Managen kooperativer Lerngruppen (S. 121-142),

- Aufrechterhaltung angemessenen Schülerverhaltens (S. 143-160),

- Kommunikationstechniken für den Unterricht (S. 161-182),

- Umgang mit Verhaltensproblemen (S. 183-208) und

- Umgang mit speziellen Gruppen (Bsp.: Kinder mit Behinderungen). (Evertson \& Emmer, 2009, S. 209-241)

Für den konkreten Umgang mit Unterrichtsstörungen scheinen bspw. der „Low-Profile“ oder „LEAST“-Ansatz ${ }^{36}$ Erfolg versprechend zu sein (Borich, 2007; Muijs \& Reynolds, 2011, S. 119f; Helmke, 2015), bei denen der Unterrichtsfluss bei geringfügigen Störungen möglichst nicht unterbrochen werden sollte. Stattdessen können frühzeitiges deeskalierendes Eingreifen, nonverbale Signale oder das diskrete Ansprechen und Unterbinden von Störungen genutzt werden (Helmke, 2015, S. 187).

Auch in der sonderpädagogischen Literatur findet das Thema Klassenführung sowohl in den 70er Jahren wie erneut seit den 2000er Jahren Berücksichtigung (Prell \& Link, 1977; Boban \& Hinz, 2003; Textor, 2009; Wember \& Prändl, 2009). So wird die Einschätzung, dass die Klassenführung verantwortlich für das Erreichen von Lernzielen ist, 1977 von Prell und Link mit Blick auf die Modellversuchsschule „Aktion Sonnenschein“ in München thematisiert:

Der Lehrer ist in der Klasse die Führungsperson. Er ist verantwortlich für die Erreichung der Lehrplanziele und für den Lernerfolg seiner größtmöglichen Anzahl von Schülern (vgl. mastery-learning, Carroll 1979). Das beinhaltet, daß der Lehrer seine Schüler motivieren und zur Erreichung eines Ziels hinsteuern muß. (Prell \& Link, 1977, S. 315, Herv. im Orig.)

Für die Umsetzung dieser Form der Klassenführung könne ein Regelmodell hilfreich sein, das „soziale Regeln für die Arbeitsdisziplin, für Sozialverhalten und Selbständigkeit im Unterricht“ (Prell \& Link, 1977, S. 318) beinhalte. Insgesamt spielt der Bereich der Klassenführung in der sonderpädagogischen Forschung zu schulischer Integration allerdings im Vergleich zur allgemeinen Unterrichtsforschung lange Zeit eine geringe Rolle. Erst seit Beginn der 2000er Jahre wird eine erfolgreiche Klassenführung als ,zentrale Wirkvariable für den Lern- und Fördererfolg von Kindern und Jugendlichen“" (Casale et al., 2016, S. 122) aufgeführt:

So kann eine effektive Klassenführung zum einen sozial-emotionale Kompetenzen auf- und zum anderen Verhaltensstörungen abbauen (Hennemann \& Hillenbrand, 2010; Hennemann, Hövel, Casale, Hagen \& Fitting-Dahlmann, 2015; Reiber \& McLaughlin, 2004). Weiterhin gilt eine effektive Klassenführung als eine der vielversprechendsten Methoden zur Reduktion von externalisierenden Verhaltensproblemen, wie z.B. aggressiven oder unaufmerksamen Verhaltens in der Schule (z.B. Hennemann \& Hillenbrand, 2010; Reiber \& McLaughlin, 2004). Auch für die Förderung von Kindern und Jugendlichen mit Lernstörungen gelten Methoden, die den Kriterien einer effektiven Klassenführung in der 
Schule entsprechen, gemeinhin als hilfreich (Grünke, 2006). Aus diesen Gründen gilt eine effektive Klassenführung als eine wichtige Kernkompetenz von Lehrkräften hinsichtlich des Umgangs mit Schülerinnen und Schülern mit zusätzlichem Förderbedarf vor allem im Kontext inklusiver Settings (Oliver \& Reschley, 2010). (Casale et al., 2016, S. 122f)

In der Regelklasse seien u.a. der Geräuschpegel, Strafen und Ängste problematisch für schulleistungsschwache Schüler (Haeberlin et al., 2003, S. 54). Als Qualitätssubkategorien werden feste Regeln und Rituale innerhalb der Klasse (Boban \& Hinz, 2003; Wember et al., 2009), eine klare Kommunikation von Verhaltensanforderungen (Textor, 2009) sowie klare Vorgehensweisen als Reaktion auf störendes Verhalten benannt. Kopmann und Zeinz (2015) weisen darauf hin, dass der Unterricht in inklusiven Klassen besonders hohe Anforderungen an die Klassenführung stelle, da diese u.a. auch Schüler mit umfassenden Verhaltensproblemen einbeziehen müsse (Kopmann \& Zeinz, 2015, S. 25).

Hennemann, Hillenbrand und Wilbert (2014) können im Rahmen ihrer wissenschaftlichen Begleitung inklusiver Schulen im Kreis Mettmann für den Qualitätsbereich der Klassenführung feststellen, dass sich deutliche Unterschiede zwischen SuS mit und ohne spF hinsichtlich des Gefühls des Angenommenseins durch die Lehrkräfte ergeben: „In allen drei Förderbereichen [gemeint sind L, S und SE] fühlen die Kinder sich im Mittel weniger angenommen durch Ihre Lehrkraft als ihre Peers ohne Förderbedarf.' (Hennemann et al., 2014, S. 34).

\subsubsection{Didaktik}

Allgemeindidaktische wie fachdidaktische Fragestellungen und damit die nach der Auswahl von Lerninhalten, ihrer inhaltlichen Reduktion, ihrer Strukturierung und Sequenzierung sind diejenigen, mit denen sich Lehrkräfte in ihrer Unterrichtspraxis täglich auseinandersetzen müssen. Sie stehen in einem sehr engen und untrennbaren Zusammenhang mit methodischen Überlegungen (3.5.5) sowie Entscheidungen zur Binnendifferenzierung bzw. Individualisierung (3.5.6). Allgemeine didaktische Modelle (u.a. Klafki, 1963; Heimann et al., 1965; Peterßen 1974) bieten in diesem Kontext zwar übergeordnete theoretische Konzepte an, stellen aber zunächst keine konkrete Hilfe mit Blick auf Entscheidungsprozesse für den Unterricht dar. Diese Entscheidungen können zum Teil nur fachdidaktisch getroffen werden, indem bspw. eine Auswahl bestimmter fachdidaktischer Ansätze für das Erstlesen- und -schreiben oder Erstrechnen erfolgt. Obwohl (fach-) didaktische Entscheidungen sich möglicherweise sehr zentral auf die Lernfortschritte und Kompetenzen von Schülerinnen und Schülern auswirken, spielen diese in der allgemeinpädagogischen und sonderpädagogischen Unterrichtsforschung so gut wie keine Rolle. Auch bei den Qualitätsmerkmalen für guten Unterricht werden sie gar nicht oder kaum aufgeführt, was damit zusammenhängt, dass die Ansätze der Autoren sich auf fachübergreifende Merkmale beziehen. Brophy, der die „Stimmigkeit des Lehrplans“ als Qualitätsmerkmal von Unterricht benennt, meint damit die Abstimmung der einzelnen Bestandteile des Lehrplans dahingehend, dass Unterrichtsziele erreicht werden können (Brophy, 2010, S. 83). Stimmige Unterrichtsinhalte werden dann erzielt, wenn Inhalte erklärt sowie Strukturen und Zusammenhänge herausgestellt werden (Brophy, 2010, S. 86) - beides also Aussagen und Merkmale, die sehr allgemein verfasst sind. Müller et al. (2002) sehen aufgrund der Untersuchungsergebnisse von TIMSS ${ }^{37}$ und PISA einen konkreten Zusammenhang zwischen der Abstimmung von stufenübergreifenden Lehrplänen für den Fachunterricht und der positiven Entwicklung von Schülerkompetenzen und äußern sich dazu mit Blick auf Deutschland kritisch: 
Das ist in der Bundesrepublik keine Selbstverständlichkeit, denn in der Regel werden in allen Bundesländern Lehrpläne von verschiedenen Kommissionen entwickelt, die jeweils ihren eigenen stufenbzw. schulformbezogenen Vorstellungen folgen. Das Problem wird noch dadurch verschärft, dass es in Deutschland keine stufen- und schulformübergreifenden Fachlehrerverbände gibt, in denen sich eine Gesamtvorstellung vom Fachunterricht herausbilden könnte. Was z.B. die Mathematik anbelangt, formulieren - von punktuellen Kooperationen abgesehen - der Grundschulverband, die Gewerkschaft Erziehung und Wissenschaft (GEW), der Verband Bildung und Erziehung (VBE), der Realschullehrerverband, der Philologenverband, der Förderverein für den mathematisch-naturwissenschaftlichen Unterricht (MNU), die Gesellschaft für Didaktik der Mathematik (GDM) und die Deutsche Mathematikervereinigung (DMV) ihre jeweils eigenen Vorstellungen. Gemeinsame Denkschriften zum Fachunterricht von Klasse 1 bis 12, die von allen Verbänden getragen würden, geschweige denn konzertierte Aktionen zur stufenübergreifenden Entwicklung des Fachunterrichts kann man sich unter den zersplitterten Verhältnissen in Deutschland gegenwärtig gar nicht vorstellen. (Müller et al., 2002, S. 38f)

Neben innovativen Lehrplänen bedürfe es einer reformorientierten Einstellungsänderung bei Mitarbeitern und Schulleitungen (Müller et al., 2002, S. 47f). Zu ähnlichen Ergebnissen wie TIMSS und PISA kommen Bryk et al. (2010) in ihrer langfristigen Schulentwicklungsstudie. Die Ausrichtung einer Schule an einem einheitlichen Curriculum für Deutsch bzw. Mathematik führte zu Verbesserungen der Leseleistungen von Schülern (45\% der Schulen) bzw. der mathematischen Leistungen (40\%) (Bryk et al., 2010, S. 115). Noch stärker wirkt sich diese Ausrichtung in Kombination mit einer starken Arbeitsorientierung der Lehrkräfte aus: in zwei Dritteln der Schulen, in denen diese beiden Faktoren Berücksichtigung fanden, verbesserten sich die Leseleistungen, in $43 \%$ dieser Schulen die Mathematikleistungen (Bryk et al., 2010, S. 126). An dieser Stelle zeigt sich ein starker Zusammenhang zwischen diesem Bereich der Unterrichtsentwicklung und der unterrichtsbezogenen Kooperation von Lehrkräften (3.4.2):

Key here is that the practices and routines of instruction are shared across a faculty, rather than being purely matters of individual choice. This is essential if social learning regarding instructional improvement is to occur. Effecting this collective cognition requires a common language for instruction accompanied by a reservoir of teacher experiences in confronting the same work tasks in their classroom. Minus this resource, improvement conversations would resemble a Tower of Babel. (Bryk et al., 2010, S. 205)

Der wissenschaftsfundierte Austausch über fachdidaktische Fragestellungen und der erfahrungsbasierte Austausch über „sichtbares Lernen“ von Schülern sind also die Grundlage für didaktische Weiterentwicklungen an einer Schule. Zu diesem Schluss kommt auch Moser Opitz, die davon ausgeht, dass „Differenzierungsangebote im inklusiven Unterricht [...] nur realisiert werden können, wenn fachdidaktische Erkenntnisse berücksichtigt werden" (Moser Opitz, 2014, S. 63). Die Auseinandersetzung mit fachlichen und fachdidaktischen Überlegungen könne Lehrkräfte dabei unterstützen Lerninhalte nicht nur quantitativ, sondern auch qualitativ zu differenzieren. So hätten Krauthauser und Scherer (2014, S. 27ff) festgestellt,

[...] dass die Negierung der Bedeutung des Fachs und seiner Spezifika dazu geführt hat, dass viele Vorschläge zur Differenzierung entweder auf einer organisatorischen Ebene und dadurch allgemein bleiben, oder aber dass es in der Praxis zu einem ,unverhältnismäßig hohen Einsatz selbstgebastelter Materialien“ sowie einer „,erdrückenden Flut von Arbeitsblättern“ (ebd., S. 31) kommt, um für jedes Kind ein individuelles Lernangebot bereitzustellen [...]. (Moser Opitz, 2014, S. 63)

Ohne den Blick auf fachdidaktische Entscheidungen zu richten, könnten die Qualitätsbausteine von Unterricht so missverstanden werden, dass sie wie die Zutaten eines Rezepts nur richtig dosiert und vermengt werden müssen, um qualitativ hochwertigen Unterricht zu ergeben. Da 
empirische Befunde zur Didaktik bzw. Fachdidaktik, deren grundlegende Fragestellungen sich nicht im Hinblick auf Schüler mit oder ohne Behinderung unterscheiden, in Veröffentlichungen zur schulischen Integration selten sind, und die Darstellung fachdidaktischer Ansätze an dieser Stelle aufgrund des dafür nötigen Umfangs nicht möglich ist, wird zunächst ein kurzer Überblick zu so genannten Ansätzen inklusiver Didaktik gegeben um im Anschluss daran kritisch zu hinterfragen, inwiefern diese inhaltlich über allgemein- und fachdidaktische Ansätze hinausgehen. Die Fragestellung, inwiefern fachdidaktische Aspekte eine sehr viel größere Rolle mit Blick auf die Qualität (inklusiven) Unterrichts spielen als allgemeindidaktische oder inklusive Ansätze und inwiefern sie eine wichtige Bedeutung für die Kompetenzentwicklung von Schülerinnen und Schülern mit und ohne sonderpädagogischen Förderbedarf haben, kann in diesem Zusammenhang nicht geklärt werden (s. dazu Kap. 5).

Während sich nach Seitz die Grundschulforschung in den vergangenen Jahren innerhalb der Allgemeinen Didaktik verstärkt der Frage nach dem Umgang mit Heterogenität gewidmet habe (Verweise auf Faust-Siehl, Garlichs, Ramseger, Schwarz, Warm, 1996; Graumann, 2002; Heinzel \& Prengel, 2002), seien didaktische Neuerungen im Rahmen der Integrations- bzw. Inklusionsforschung wenig diskutiert worden, so dass bspw. auch der „Index für Inklusion“ kaum Hinweise auf diesen Bereiche enthalte (Seitz, 2006, S. 3). Übereinstimmend äußert sich Wocken:

Didaktik, Methodik und Unterrichtsentwicklung haben in der integrativen Pädagogik ein Mauerblümchendasein geführt. Der Inklusion sei dringend nahegelegt, sich ganz konkret mit dem Innenausbau der inklusiven Schule zu beschäftigen und das handwerkliche Rüstzeug für einen inklusiven Unterrichtsalltag zu entwickeln. (Wocken, 2009b, S. 218)

Und auch Ziemen (2011) weist auf diese fehlende Auseinandersetzung hin:

Die didaktische Diskussion in erforderlicher Breite und Tiefe ist bislang ausgeblieben, ebenso die Diskussion um theoretische Verortungen und Bezüge. Für die in der Praxis vor die Frage nach Umsetzung der inklusiven Idee gestellten Lehrpersonen und Teams ist sowohl die didaktische Frage als auch die der Hintergründe und Bezüge äußerst relevant und kann nicht beliebig sein. (Ziemen, 2011, S. 12)

Im Anschluss an ihre Erfahrungen in den integrativen Klassen der Fläming-Grundschule gelangen Maikowski und Podlesch zu einer ähnlichen Schlussfolgerung. Sie stellen in ihren Ausführungen zwar mögliche Bausteine integrativen Unterrichts vor (Bsp.: Klassenklima, Raumaufteilung, Arbeitsformen etc.), formulieren anschließend aber, dass ihrer Einschätzung nach noch keine integrative Didaktik entwickelt worden sei (Maikowski \& Podlesch, 1988, S. 130-151). Diese sehen sie als eine mögliche notwendige Vorform einer wiederum allgemeinen Didaktik:

Letztlich kann eine integrative Didaktik nichts anders sein als die Vermittlungsform einer allgemeinen Pädagogik, die notwendig auch immer integrativ sein muß, weil sie sich auf alle Kinder bezieht. (Maikowski \& Podlesch, 1988, S. 151)

Theoriegeleitete didaktische Ansätze aus der Sonderpädagogik wurden im Anschluss u.a. von Feuser (1989, 1995, 1998), Wocken (2009, 2012), Reich (2002, 2004, 2014), Seitz (2006) sowie Amrhein et al. (2014) entwickelt. Die entwicklungslogische Didaktik nach Feuser orientiert sich an dem Modell Vygotskijs, bei dem im Unterricht jeweils die „Zone der aktuellen Entwicklung“ und die „Zone der nächsten Entwicklung“ zu berücksichtigen sind. Das Modell will sowohl der Sache (dem Lerngegenstand) als auch den Schülerinnen und Schülern mit ihren unterschiedlichen Kompetenzen und Dispositionen gerecht werden und muss daher unterschiedliche Anforderungsniveaus, die ein Thema bereithält, im Blick haben. Der Unterricht soll 
einen Möglichkeitsraum schaffen, in dem alle Schüler entlang eines gemeinsamen Gegenstandes auf ihrem Niveau lernen und gleichzeitig durch die verschiedenen Zugänge der Mitschüler andere Perspektiven als Ergänzung zu ihrem eigenen Lernen kennen lernen. Die Schüler sollen als Individuen wahrgenommen werden, gleichzeitig sollten Angebote kooperativ bzw. dialogisch ausgerichtet sein, damit sie die Einbeziehung von Schülern mit Behinderungen unterstützen können (Feuser, 1989). Bei der Individualisierung von Lernzielen seien die drei Ebenen Tätigkeits-, Handlungs- und Sachstruktur zu berücksichtigen, wobei die Tätigkeitsstruktur sich auf die individuellen Voraussetzungen der Schüler bezieht, die Handlungsstruktur auf den Aneignungsprozess und die Sachstruktur auf die differenzierte Auseinandersetzung mit dem Lerngegenstand (Moser Opitz, 2014, S. 55f). Feuser versteht seinen Ansatz nicht als eine „inklusive Didaktik“, sondern als ein Gesamtkonzept inklusiver Pädagogik (Feuser, 1989, S. 32).

Wocken wiederum kritisiert diesen Ansatz Feusers und fordert eine Balance von gemeinsamen und differentiellen Lernsituationen, indem er postuliert:

Die Didaktik der integrativen Schule ist eine Didaktik der Vielfalt: Vielfalt der Ziele und Vielfalt der Inhalte. Und eine Vielfalt von gemeinsamen und individuellen Lernsituationen. Die Didaktik der Vielfalt will gleichermaßen der Verschiedenheit der Einzelnen gerecht werden als auch die Gemeinsamkeit der Verschiedenen fördern. (Wocken, 2009, S. 218)

Dementsprechend definiert er inklusiven Unterricht als Unterricht, in dem sich alle Schüler mit pädagogischer Unterstützung allgemeine Bildung aneignen können (Wocken, 2012, S. 116), wozu es aufgrund der breiten Streuung an Lernvoraussetzungen notwendig sei gemeinsame und unterschiedliche Lernsituationen in ein ausgewogenes Verhältnis zu bringen (Wocken, 2012, S. 125). Ähnlich wie bei Feuser entsprechen diese Ausführungen also grundlegenden Äußerungen zum Thema Unterricht und lassen sich eher dem Qualitätsbaustein der Binnendifferenzierung und Individualisierung als dem der Didaktik zuordnen. Moser Opitz geht dagegen davon aus, dass das Spannungsfeld zwischen möglichst effektiver Förderung und gemeinsamen Lernsituationen didak-tisch nicht aufzulösen sein wird (Moser Opitz, 2014, S. 65).

Die inklusive Didaktik nach Reich wiederum orientiert sich an der Theorie des Konstruktivismus und fordert daher, dass inklusiver Unterricht Möglichkeiten zu aktivem und selbstbestimmten Lernen bieten sollte, der den Schülern experimentelles Vorgehen, Entdecken, Planen, Reflektieren und Konstruieren von Wirklichkeiten ermöglicht. Folgende vier Grundpostulate hält Reich fest:

- Unterricht ist ein konstruktiver Ort,

- Unterricht hat die Aufgabe „konstruktive Akte des Aufklärens und der Reflexion“ an die Schüler und die Lehrer „,in möglichst hoher Selbsttätigkeit“ zurückzugeben,

- die Themen, Inhalte und Wege sollten mit dem Ziel der Selbst- und Mitbestimmung gemeinsam durch alle am Unterricht Beteiligten ausgehandelt werden,

- die Beziehungen zwischen Lehrenden und Lernenden sowie zwischen den Lernenden haben Vorrang vor der Vermittlung von Inhalten (Reich, 2002, S. 70-80).

Reich benennt seinen Ansatz inzwischen als den einer inklusiven Didaktik (Reich, 2014). Amrhein und Reich (2014) wiederum postulieren, dass dieser allgemeindidaktische Ansatz hin zu dem einer inklusiven Fachdidaktik weiterentwickelt werden müsse.

Seitz geht es bei ihrem Ansatz zur inklusiven Didaktik darum, dass Unterricht nicht entwicklungslogisch, sondern fraktal gedacht werden und sich jeweils mit dem „Kern der Sache“ auseinandersetzen solle: „Selbst in der komplexesten, fraktal aufgebauten Figur finden sich [...] Grundmuster („Fraktale“) wieder, die zugleich alle Aspekte der komplexen Ausformung enthal- 
ten." (Seitz, 2006a, o.S.). Der Einstieg in ein Lernfeld sei daher als offenes Handlungsangebot zu gestalten, in dem Kinder ihre Motivationen entwickeln und zeigen können (Diagnostik). Von diesem Einstieg aus könnten

[...] dann offene Unterrichtsstrukturen entwickelt werden, die sich an den Einzelpersönlichkeiten entlang , anschmiegen' können und für die Kinder ein motiviertes Lernen möglich machen. Aus Lehrer/ innen werden dann Wegbegleiter/innen, deren eigenes fachliches Interesse an der ,Sache` sich über die Neugier auf das unterschiedliche Lernen der Kinder mit all seinen Überraschungen herausbildet und speist. (Seitz, 2006a, o.S.).

Besonders kritisch erscheint nach Moser Opitz (2014) bei allen vorgestellten so genannten inklusiven didaktischen Ansätzen aus der Sonderpädagogik, dass diese die entscheidende Frage nach Differenzierung und Individualisierung im inklusiven Unterricht gar nicht oder nur unzureichend beantworten ${ }^{38}$. Reich erläutere diese Fragestellung nicht näher, Seitz konkretisiere ihren Ansatz zwar am Beispiel des Sachunterrichts, kläre allerdings nicht, wie es zur Auswahl von Lernanlässen komme. Feusers Modell sei mit Blick auf die Differenzierung am ehesten ausgearbeitet, umfasse allerdings auch keine expliziten Ausführungen zur Analyse der Sachstruktur gemeinsamer Unterrichtsgegenstände (Moser Opitz, 2014, S. 62). Als problematisch stuft Moser Opitz darüber hinaus ein, dass die didaktischen Ansätze Ergebnisse der Unterrichtsforschung außer Acht lassen und „Fragen zur Tiefenstruktur im Sinne von spezifischen Qualitätsmerkmalen von Unterricht“ (Moser Opitz, 2014, S. 62) nicht diskutieren würden.

Gleichzeitig lässt sich Unterricht ohne Prüfung dahingehend, ob inhaltliche Ziele (bei einzelnen Schülerinnen und Schülern) erreicht worden sind, nicht qualitativ bewerten: „Unterricht wird auf Ziele hin strukturiert. Das Erreichen dieser Ziele ist letztlich ein Kriterium, ohne das die Qualität von Unterricht kaum festgestellt werden kann.“ (Kiel, 2012, S. 21). Inhaltliche Anknüpfungsmöglichkeiten ergeben sich für Schüler, wenn diese bereits über ein gewisses Maß an Vorwissen verfügen (Kiel, 2012, S. 25). Dabei sind - wie Hattie aus dem Erfolg erlebnispädagogischer Projekte schlussfolgert - folgende Merkmale zur Einbettung inhaltlicher Ziele wesentlich: die Betonung der unmittelbaren Qualität von Erfahrungen und die Ermöglichung eines geplanten und absichtsvollen Transfers, das Setzen anspruchsvoller und spezifischer Ziele, „die den Lernenden bekannt sind. Aufgaben werden so strukturiert, dass die Teilnehmenden diese Ziele erreichen können“, die Verstärkung von Umfang sowie Qualität von Feedback und das Bewusstsein der Lehrenden, „dass sie die Bewältigungsstrategien der Lernenden verstehen, sowie, wenn notwendig, umwerten und neu ausrichten müssen." (Hattie, 2013, S. 30).

Als Subkategorien in der Kategorie Didaktik werden aus sonderpädagogischer Perspektive die Erweiterung didaktischer Modelle um Kategorien integrativer Förderung (Berges, 1996), methodische und fachliche Innovationen (Brugger-Paggi, 2003), ein größerer Freiheitsspielraum bei der

38 Moser Opitz (2014) führt auch den RTI-Ansatz (Response-to-Intervention) als didaktischen Ansatz auf. Dieser sei „ein gestuftes Modell zur Unterstützung von Schülerinnen und Schülern einerseits und Prävention andererseits (Rechley \& Bergstrom, 2009), das insbesondere in den USA als Unterrichtskonzeption für inklusiven Unterricht propagiert wird (z.B. Brownell, Smith, Crocket \& Griffin, 2012).“ (S. 58), aber auch in Deutschland im Rahmen des Rügener Inklusionsmodells (Mahlau et al., 2011; Voß et al, 2014) eine Umsetzung erfährt. Bei diesem Ansatz wird ein dreistufiges Modell verfolgt: 1 . Förderung im regulären Unterricht verknüpft mit regelmäßigen Leistungsüberprüfungen (Curriculum-Based-Measurement), 2. bei Bedarf zeitlich begrenzte Förderung im Klassenunterricht, 3 . spezifische Einzelförderung über einen längeren Zeitraum (Moser Opitz, 2014, S. 58f). Der RTI-Ansatz enthalte allerdings keine genaueren Ausführungen dazu, ,wie Lehrpersonen die passenden Lernziele und Fördermaßnahmen auswählen können, was "qualitativ hochstehenden Unterricht" ausmacht sowie Hinweise, wie innerhalb des Unterrichts differenziert werden kann“ (Moser Opitz, 2014, S. 62). 
Unterrichtsgestaltung (für die Lehrer) (Bless, 2003), eine mittelfristige Themenverteilung (Berges, 1996) sowie curriculare Vereinbarungen und Absprachen zur Auswahl der Lerninhalte (Wember \& Prändl, 2009) bzw. die Verbindung von Curricula und individuellen Förderprogrammen (Bsp.: Orientierung in der Umwelt, basale Fähigkeiten Mathe und Deutsch, Selbstversorgung) (Wember \& Prändl, 2009) aufgeführt. Darüber hinaus spielen bestimmte didaktische Prinzipien eine Rolle wie handelndes Lernen (Hug, 1994b; Berges, 1996; Thiele 2009), ganzheitliches Lernen (Hug, 1994b; Berges, 1996), die Anschaulichkeit des Unterrichts (Wember \& Prändl, 2009), die Nähe zwischen schulischer und außerschulischer Lebenswelt (Heyer et al., 1997), die Bündelung von Lerninhalten zu Vorhaben (Mühl, 1987), Schülerorientierung (Berges, 1996) bzw. Schülerorientierung anstelle der Orientierung an Lehrplänen (Boban \& Hinz, 2003). Weitere didaktische Prinzipien stehen in engem Zusammenhang mit der Ausgewogenheit individueller und gemeinsamer Lernanlässe und beinhalten die Organisation von Tätigkeiten mit gemeinsamen Zielen (Mühl, 1987), die Herstellung gemeinsamer Lernsituationen (Köbberling, 1994), die Ermöglichung sinnvoller Teilhabe an gemeinsamen Lernsituationen (Mühl, 1987) oder das Ausrichten der Arbeit auf eine gemeinsame Erlebens-, Verständigungs- und Verhaltensebene (Feyerer, 2007). Weitere Autorinnen und Autoren formulieren als Qualitätssubkategorie, dass Unterricht von einer gemeinsamen Erfahrung ausgehen solle, die in unterschiedlicher Weise entfaltet werden kann (Köbberling, 1994; Boban \& Hinz, 2003). Wesentlich sei außerdem eine gemeinsame Rückbindung von Ergebnissen (Köbberling, 1994). Mehrfach erwähnt werden auch spezifische Konzepte zur Berufs- und Lebensvorbereitung (Brugger-Paggi, 2003; Feyerer, 2007; Wember \& Prändl, 2009), die in die Curricula integriert sein sollten (Wember \& Prändl, 2009).

Der Qualitätsbaustein klare Unterrichtsstruktur steht quasi als verbindendes Element in engem Zusammenhang zur Klassenführung sowie zu didaktischen und methodischen Unterrichtsentscheidungen, wie u.a. die Auffassungen von Meyer (2004), Kiel (2012) und Helmke (2015) belegen. Nach Meyer, der dieses Merkmal an die erste Stelle seiner Merkmalsliste setzt, zeigt sich eine klare Strukturierung des Unterrichts in der Stimmigkeit von Zielen, Inhalten und Methoden, in der Folgerichtigkeit des methodischen Gangs, im methodischen Grundrhythmus (Einstieg, Erarbeitung, Ergebnissicherung), in der Aufgabenklarheit, in der Regelklarheit sowie in der Rollenklarheit (Meyer, 2004, S. 26-29).

Unterricht ist dann klar strukturiert, wenn das Unterrichtsmanagement funktioniert und wenn sich ein für Lehrer und Schüler gleichermaßen gut erkennbarer „roter Faden“ durch die Stunde zieht. (Meyer, 2004, S. 26)

Sie führt zu einem hohen Anteil echter Lernzeit, zu hoher Schüleraufmerksamkeit, zur Reduzierung und Prävention von Störungen und zur Transparenz der Leistungserwartungen (Meyer, 2004, S. 31). Eine klare Strukturierung des Unterrichts umfasst nach Helmke u.a. die Mitteilung von Unterrichts- und Lernzielen sowie Leistungserwartungen, die ausdrückliche Verknüpfung neu vermittelter Informationen mit Vorwissen oder das Anbieten von „Gerüsten“ in Form übergreifender Ideen (Helmke, 2015, S. 198). Er bezieht sich neben Aspekten der unterrichtlichen Strukturierung vor allem auf die sprachliche Klarheit und Verständlichkeit als bedeutsame Faktoren für erfolgreiche Lernprozesse (Helmke, 2010, S. 191), die sich gemäß Hatties Metastudie mit $\mathrm{d}=0,75$ auswirken (Hattie, 2009, S. 126). Nach Brophy geht es in diesem Zusammenhang vor allem um den Aufbau von Lern-Orientierung mit Hilfe von „Advance Organizern“:

Lehrer können Schüler auf das Lernen vorbereiten, indem sie in der Einstiegsphase Strukturierungshilfen geben und erläutern, welche Ergebnisse erreicht und welche Lernstrategien angewendet werden sollen. (Brophy, 2010, S. 85) 
Hilfreich können nach Helmke und Kiel in diesem Zusammenhang Strukturmodelle bzw. Artikulationsschemata des Unterrichts sein (Helmke, 2010, S. 198; Kiel, 2012, S. 29), die u.a. auf Herbart zurückgehen, der bezüglich der Strukturierung von Unterricht zwischen „Klarheit (Informationsvermittlung), „Assoziation“ (Vernetzung), „System“ (Zusammenfassen) und „Methode“ (üben, umsetzen, anwenden)“ (Kiel, 2012, S. 27) unterscheidet. Bekannt ist auch das Artikulationsschema „AVIVA“ (Ankommen und Einstimmen, Vorwissen aktivieren, Informieren, Verarbeiten, Auswerten) nach Städeli et al. (2010) (n. Kiel, 2012, S. 30f). Forschung zu Artikulationsschemata von Lehrern (Baumert et al., 1997) ergibt, dass diese häufig wenig Abwechslung aufweisen, sondern bspw. folgende typischen Handlungsschritte umfassen: Wiederholung, Hausaufgabenbesprechung, Einführung in ein neues Thema, Durcharbeitung anhand von Aufgaben, neue Hausaufgaben (Kiel, 2012, S. 33):

Dieses u.a. in der TIMS-Studie empirisch gefundene Artikulationsmuster ist häufig mächtiger als die anderen [...] Muster. Aus der Perspektive der ursprünglich von Schank und Abelson (1977) entwickelten Skripttheorie werden solche Handlungsverläufe, die entweder beobachtet, selbst durchgeführt oder von Kollegen vermittelt werden, mental gespeichert und führen zur Routinisierung strukturell ähnlich verlaufender Unterrichtsschritte (Blömeke/Eichler/Müller 2003, Kollar/Fischer 2008). (Kiel, 2012, S. 33)

Nach Helmke und Schrader (1998) ist Strukturiertheit vor allem in den Fächern ein wichtiges Merkmal erfolgreichen Unterrichts, die inhaltlich eine hierarchische Struktur aufweisen (Bsp: Mathematik). Erfolgreiche Lehrkräfte in Mathematik wiesen durchweg hohe Ausprägungen mit Blick auf die Merkmale Klassenführung und Klare Strukturierung auf, während die DESIStudie ähnliche Ergebnisse für Deutsch oder Englisch nicht bestätigen konnte (Helmke et al., 2008). Besonders bedeutsam scheint eine klare Strukturierung des Unterrichts für lernschwache Schüler zu sein (Campbell, Kyriakides, Muijs \& Robinson, 2004; Wellenreuther, 2005) (Helmke, 2010, S. 200).

In sonderpädagogischen Forschungsveröffentlichungen wird die klare Strukturierung von Unterricht dagegen nur selten thematisiert (Feuser \& Meyer, 1987; Bless, 2003; Wember \& Prändl, 2009). So gehen Feuser \& Meyer davon aus, dass Struktur, Transparenz und Orientierung im Unterricht wichtig sind, damit die Schüler erkennen können ,in welcher Weise und an was sie handeln sollen." (Feuser \& Meyer, 1987, S. 167). Lehrkräfte in Schwerpunktschulen (RLP) geben zu $22 \%$ (GS) bzw. 19\% (Sek I) an, dass der Unterricht in Richtung einer stärkeren Strukturierung weiterentwickelt habe (Laubenstein et al., 2015, S. 170).

\subsubsection{Methodik}

Methoden „beschreiben den Prozess der Vorgehensweise bei der Bewältigung einer Aufgabe [...] strukturieren die Vorgehensweise, unterteilen den Lernprozess in Phasen und empfehlen eine zeitliche Abfolge.“ (Matthes, 2011, S. 10). Die Unterrichtsmethodik befasst sich daher mit Fragen nach dem „Wie“ des Unterrichtens: Welche Sozialformen werden im Unterricht genutzt? Welche Makromethoden (Bsp.: problemorientiertes Lernen, projektorientiertes Arbeiten) und welche Mikromethoden (Bsp.: Aufgabenarten wie Gruppenpuzzle) werden ausgewählt (Meyer, 2004; Helmke, 2010; Matthes, 2011)? Die Kompetenzentwicklung der Schüler hat neben der inhaltlichen also immer auch eine methodische Ebene. Matthes (2011) unterscheidet mit Blick auf die Auswahl von Sozialformen fünf Basisformen von Unterricht: Frontalunterricht, Einzel-, Partner- und Gruppenarbeit sowie Schülerpräsentationen (Matthes, S. 12). Diese Basisformen können innerhalb der Sozialformen differenziert werden (Bsp.: Lehrervortrag, Diskussion, individuelles Üben oder Entwickeln von Lernprodukten, Partner- oder Gruppenpuzzle, Schüler- 
vortrag). Makromethoden wie Wochenplanarbeit, Projektunterricht etc. wiederum enthalten jeweils mehrere einzelne Methoden, die miteinander kombiniert werden (Matthes, 2011, S. 17). Ähnlich wie bei didaktischen Fragestellungen ist die Wirksamkeit verschiedener Sozialformen des Unterrichts bislang kaum erforscht worden (Schwartz, 1982, S. 71). Hattie (2013) berichtet über Metastudien zur direkten Instruktion (S. 242f), zum reziproken Lernen (S. 240f) und zum kooperativen Lernen (S. 250f). Wenige Studien (Hage, 1985; Wiechmann, 2006) befassen sich mit der anteiligen Häufigkeit des Einsatzes der Methoden im Unterricht. Die folgenden Ausführungen vermitteln einen kurzen Überblick zu Methoden und Forschungsstand.

Bei der Methode des Frontalunterrichts (auch Klassenunterricht genannt) vermittelt die Lehrkraft einen Inhalt vorwiegend sprachlich für eine größere Lerngruppe (Klasse) und steuert den Unterricht durch Fragen (Matthes, 2011, S. 90-93). Er ist ein Sammelbegriff für verschiedene Unterrichtsformen, zu denen die direkte Instruktion aber auch der fragend-entwickelnde Unterricht gehören (Wiechmann, 2006, S. 21ff; Matthes, 2011, S. 90-133). Unter direkter Instruktion wird wiederum eine „hoch-strukturierte, lehrergesteuerte, lernerzentrierte, schrittweise, feedback- und redundanzreiche Förderung verstanden, in der die zu lernenden Fähigkeiten vom Lehrer präsentiert, in Anleitung mit dem Schüler modelliert und letztendlich so lange einschleifend geübt werden, bis sich ein hoher Automatisierungsgrad zeigt" (Grosche, 2011, S. 148; mit Bezug auf Watkins \& Slocum, 2003):

Three main components enable Direct Instruction to accomplish the goal of teaching all children effectively and efficiently: (a) program design that identifies concepts, rules, strategies, and „big ideas“ to be taught and clear communication through carefully constructed instructional programs to teach these; (b) organization of instruction, including scheduling, grouping, and ongoing progress monitoring to assure that each student receives appropriate and sufficient instruction; and (c) student-teacher interaction techniques that assure that each student is actively engaged with instruction and masters the objectives of each lesson. (Watkins \& Slocum, 2003, S. 75f)

Effekte der direkten Instruktion wurden bislang vor allem im angloamerikanischen Raum untersucht. Vergleichsstudien zu Lernergebnissen in verschiedenen Unterrichtsfächern zwischen Gruppen, in denen direkte Instruktion intensiv genutzt wurde, im Vergleich zu Gruppen, in denen dies nicht geschah, kamen nach Muijs und Reynolds (2011) übereinstimmend zu dem Ergebnis, dass Schüler, die direkt instruktiv unterrichtet wurden, bessere Ergebnisse erreichen konnten (Good \& Grouws, 1979; Fitzpatrick, 1982; Nye et al., 2004). Andere Studien unterscheiden zwischen der Effektivität der direkten Instruktion in unterschiedlichen Fächern (Bsp.: positive Ergebnisse in Mathematik und Sprache, aber nicht beim Lesen; Croll, 1996; Galton \& Croll, 1980) oder differenzieren stärker mit Blick auf die Elemente der Lehreraktivität, mit Hilfe derer die Instruktion als solche gestaltet wurde:

Significant positive relationships were found with such factors as structured sessions, use of higherordered questions and statements, frequent questioning, restricting sessions to a single area of work, involvement of pupils and the proportion of time utilized in communicating with the whole class. Negative relationships were found with teachers spending a high proportion of their time communicating with individual pupils (Mortimore et al., 1998) [...]. (Muijs \& Reynolds, 2011, S. 37)

Auch Muijs und Reynolds selbst kommen aufgrund ihrer Untersuchung (100 Mathematiklehrer in England und Wales) zu dem Schluss, dass Schüler von Lehrkräften, die stärker mit der ganzen Klasse als mit einzelnen Schülern arbeiteten, bessere Lernzuwächse im Fach Mathematik zeigten (Muijs \& Reynolds, 1999, 2001, 2002). Als mögliche Gründe für die Effektivität der 
direkten Instruktion führen sie u.a. an, dass es für Lehrkräfte einfacher sei diese Unterrichtsform $\mathrm{zu}$ gestalten als beispielsweise komplexere Situationen wie Gruppenarbeiten, bei denen innerhalb der Gruppen unterschiedliche Inhalte bearbeitet werden (Brophy \& Good, 1986; Rosenshine \& Stevens, 1986). Darüber hinaus stelle gute direkte Instruktion entsprechend der gängigen Lerntheorien den schnellsten Weg dar um neues Wissen zu vermitteln (Kirschner et al., 2006). Studien kämen außerdem zu dem Ergebnis, dass die direkte Instruktion vor allem für Schüler mit Lernschwächen und für Schüler aus schwierigen sozialen Verhältnissen sehr hilfreich sein kann (Muijs \& Reynolds, 2006; Schippen et al., 2005). Diese Annahme wird auch durch eine Metaanalyse von Grünke (2006) bestätigt, der aufgrund der Datenlage von 26 Studien zu dem Gesamtergebnis kommt,

[...] dass im Hinblick auf die allermeisten Lernziele ein eher lehrkraftgesteuertes und gut geplantes Vorgehen angebracht ist, bei dem die Inhalte oder die Strategien explizit, redundanzreich und schrittweise vermittelt werden. Hierbei sind die Schüler ständig zu einer aktiven Beteiligung sowie zum ausgiebigen Üben aufgefordert. Und sie erhalten für alle Leistungen und Antworten eine sofortige und konkrete Rückmeldung. (Grünke, 2006, S. 251)

Nach Muijs \& Reynolds kann konstruktiver Unterricht nur in Kombination mit anderen Methoden seine positive Wirkung entfalten und funktioniert darüber hinaus nur unter bestimmten Bedingungen, die für den Erfolg dieser Unterrichtsmethode entscheidend sind wie klare Strukturierung, klare und gut strukturierte Präsentationen, ,Modelling' (Modellieren im Sinne des strukturierten Vormachens von Prozeduren oder Fähigkeiten), interaktives Fragen, gute Vorbereitung der mit der Instruktion verknüpften Stillarbeit (differenzierte oder nicht differenzierte Einzel-, Partner- oder Gruppenarbeit) und Feedback zu dieser Arbeitsphase (Muijs \& Reynolds, 2011, S. 39-46). Instruktive Unterrichtsphasen zählen auch nach Hattie (2013) zu den Methoden, die Lernprozesse von Schülern sehr erfolgreich unterstützen. Gleichzeitig sei eine Kombination mit schülerzentrierten Lernphasen wesentlich (Hattie, 2013, S. 31). Grenzen der Effizienz der direkten Instruktion werden darin gesehen, dass diese nicht für die Vermittlung aller möglichen Sachverhalte geeignet ist (bspw. wenig geeignet für Diskussionsthemen) und nicht zu einer zu hohen Schülerpassivität führen darf (Muijs \& Reynolds, 2011, S. 50).

Als Einzelarbeit werden Arbeitsphasen bezeichnet, in denen Schüler sich individuell - und häufig still - mit einer Aufgabe befassen. Sie kann unterschiedliche Mikromethoden beinhalten (Matthes, 2011, S. 44). Für diese Methode benötigen die Schüler passende Unterstützungsformen wie Strategien der Informationsverarbeitung (z.B. Vereinfachung von Inhalt), Kontrollstrategien (z.B. Planung) sowie Stützstrategien (z.B. Zeitmanagement, Lernumgebungsgestaltung) (Merkens, 2010, S. 164). Obwohl zeitliche Anteile dieser Sozialform im Unterricht vergleichsweise umfangreich zu sein scheinen (Hage et al., 1985), wird sie weder in theoretischen Beiträgen noch in Forschungsveröffentlichungen der Sonderpädagogik gezielt thematisiert.

Bei der Partnerarbeit wird eine Aufgabe von zwei Schülern bearbeitet, bei der Gruppenarbeit von einer Kleingruppe. Als kooperative Lernformen werden diese Methoden nur dann bezeichnet, wenn sie eine individuelle Förderung aller Mitglieder einer Gruppe beinhalten und die Kooperationspartner gleichberechtigt miteinander arbeiten (Matthes, 2011, S. 20f; Brüning \& Saum, 2015), da es auch möglich ist, dass „Schüler zu einer bestimmten Zeit etwas zusammen erledigen, sie können dabei kooperieren, müssen es aber nicht." (Woolfolk, 2008, S. 508). Nach Slavin (1995) sowie Johnson \& Johnson (1994) ist kooperatives Lernen gekennzeichnet durch positive Interpendenz, individuelle Verantwortlichkeit, förderliche Interaktionen, kooperative Arbeitstechniken und reflexive Prozesse (Helmke, 2010, S. 212). Die Orientierung an diesen 
Prinzipien gibt den Schülern Sicherheit im Handeln durch einen vordefinierten Rahmen, übergibt „individuelle Verantwortung für das Lernergebnis“ (Brüning \& Saum, 2015, S. 18), vertieft kognitive Prozesse durch kommunikativen Austausch zwischen den Schülern und fördert kommunikative Fähigkeiten (Brüning \& Saum, 2015, S. 18). Eine Aufgabe ist so zu stellen, dass die Schüler und Schülerinnen nur gemeinsam zu einem Arbeitsergebnis gelangen können. Das Grundprinzip des Kooperativen Lernens folgt dabei dem Dreierschritt „Think-Pair-Share“, d.h. dass die Schüler zunächst individuell eine Arbeitsaufgabe, einen Text o.ä. erschließen, bevor sie sich in einer Gruppe dazu austauschen und das Arbeitsergebnis ihrer Gruppe im Anschluss der Gesamtgruppe vorstellen (Brüning \& Saum, 2015, S. 15ff).

Modelle kooperativer Kompetenzen, die bei einer solchen Form der Gruppenarbeit relevant sind, gehen davon aus, dass diese eine Reihe von abgrenzbaren Teilkompetenzen umfassen, die bspw. kognitive und emotionale Prozesse, Arbeitsorganisation und Kommunikation beinhalten (Kiper \& Mischke, 2008, S. 87f). Die Schülerinnen und Schüler lernen beim kooperativen Arbeiten, dass sie positiv voneinander abhängen, da sie nur dann zu einem gemeinsamen Arbeitsergebnis gelangen können, wenn jedes Gruppenmitglied sich selbstverantwortlich einbringt (Green, 2004, S. 1). Dabei sind sie auf unterstützende kommunikative Prozesse angewiesen:

Kooperatives Lernen ist komplexer als konkurrierendes und individuelles Lernen, weil die Schülerinnen und Schüler sich gleichzeitig mit aufgabenbezogener Arbeit (auf den Unterrichtsstoff bezogenes Lernen) und personenbezogener Arbeit (als Gruppe effektiv wirken) beschäftigen müssen. Soziale Fertigkeiten in Bezug auf effektives kooperatives Arbeiten erscheinen nicht magisch von alleine, wenn kooperativer Unterricht gemacht wird. Im Gegenteil, soziale Fertigkeiten müssen den Schülerinnen und Schülern ebenso zweckgerichtet und präzise beigebracht werden wie fachliche Fähigkeiten. Die Fähigkeit zu Führen, Entscheiden, Vertrauensaufbau, Konfliktmanagement und Kommunikation versetzen die Schülerinnen und Schüler in die Lage, sowohl Teamarbeit als auch Aufgabenarbeit erfolgreich zu bewältigen. (Green, 2004, S. 2)

Slavin konnte für kooperative Lernformen, die Übungsprozesse umfassten und die mit Belohnungen für positive Ergebnisse der Gruppenmitglieder verknüpft waren, feststellen, dass diese in $78 \%$ der Fälle effektiver waren als lehrerzentrierter Unterricht; beim Gruppenpuzzle galt dies nur für $27 \%$, während sich ebenfalls $27 \%$ weniger effektiv als der lehrerzentrierte Unterricht erwiesen (Slavin, 1995; n. Huber, 2010, S. 355). Auch Muijs und Reynolds stellen positive Ergebnisse mit Blick auf die Effektivität kooperativer Lernformen vor, die bspw. belegen, dass diese Schüler dabei unterstützen können ihre eigenen Denkprozesse zu restrukturieren, indem sie diese mit anderen Schülern besprechen (O'Donnell, 2006), und auch bessere Lernergebnisse zu erreichen, wenn sie sich gegenseitig Lerninhalte erklären (Webb \& Mastergeorge, 2003) (n. Muijs \& Reynolds, 2011, S. 65). Für die Effizienz der Methoden des kooperativen Lernens sind neben dem Feedback für die Gruppen eine Aufgabenspezialisierung, die Unterstützung aufgabenspezifischer Interaktionen und einer klaren Aufgaben- und Rollenverteilung innerhalb einer Gruppe sowie die Unterstützung der Gruppenprozesse an sich notwendig (Huber, 2010, S. 355f; Muijs \& Reynolds, 2011, S. 65-75). Gruppenarbeit ist u.a. dann ineffektiv, wenn Schüler nicht über die sozialen Kompetenzen verfügen um sich respektvoll miteinander auszutauschen (Battistich et al., 1993; Linn \& Burbules, 1993; n. Muijs \& Reynolds, 2011, S. 65f). Daher müssen nach Muijs und Reynolds ,sharing skills,, participation skills,, ,communication skills' und ,listening skills' vermittelt werden, wenn diese sozialen Kompetenzen fehlen oder nicht in ausreichendem Maß vorhanden sind (Muijs \& Reynolds, 2011, S. 65f). Darüber hinaus sei - wie weitere Studien belegen (Webb, 1991; Nattiv, 1994; Webb, 2009) - wesentlich, dass Schüler auf der einen Seite wissen, wie sie präzise Nachfragen stellen können, und auf der ande- 
ren Seite, wie sie Hilfe effektiv gestalten können (bspw. nicht durch das Vorgeben der richtigen Antwort, sondern durch inhaltlich angemessene Erläuterungen) (s. dazu Hinweise zum jahrgangsübergreifenden Unterricht) (Muijs \& Reynolds, 2011, S. 67). Insgesamt schätzen Muijs und Reynolds kooperative Arbeit als hocheffiziente Unterrichtsform ein, die - wie bspw. Good et al. (1995) vorgeschlagen haben - gut in instruktiven Unterricht integriert werden könne (Muijs \& Reynolds, 2011, S. 71).

Kooperative Lernformen sind nach Eckermann \& Heinzel (2013) allerdings nicht für alle Schüler gleichermaßen geeignet bzw. effektiv:

Statusniedrigere Schülerinnen und Schüler werden [...] häufig beim kooperativen Lernen von den statushöheren Schülerinnen und Schülern dominiert und die Gruppe arbeitet insgesamt weniger effektiv zusammen (Cohen et al. 1990). Die entsprechenden empirischen Befunde sprechen dafür, dass statusniedrigere Kinder sich beim kooperativen Lernen weniger einbringen können, da ihnen Gruppenmitglieder oder auch sie sich selbst weniger Kompetenzen zuschreiben (Huber 2007). (Eckermann \& Heinzel, 2013, S. 190)

Mit Blick auf den Zusammenhang zwischen Statusunterschieden und der Interaktion zwischen den Schülern gelangen sie aufgrund ihrer eigenen Studie (132 Drittklässler) zu dem Ergebnis, „dass in den Gruppen mit einer größeren Statusdifferenz unter den Schülerinnen und Schülern weniger wertschätzende Rückmeldungen den Mitschülerinnen und Mitschülern entgegengebracht werden ( $\mathrm{r}=-.29)$ und auch die Haltung gegenüber den Mitschülerinnen und Mitschülern weniger zugewandt ist ( $\mathrm{r}=-.23)$.“ (Eckermann \& Heinzel, 2013, S. 197).

Interessant im Zusammenhang mit Kleingruppenarbeit erscheinen Ergebnisse, die laut Helmke (2010) Dann, Diegritz und Rosenbusch (2002) bei Lehrern im Anschluss an Unterrichtsstunden festgestellt haben. Diese halten Gruppenarbeit zwar für wichtig, geraten durch die Gruppenarbeit allerdings auch in Rollenkonflikte (Strukturierung - Unübersichtlichkeit; Steuerung - Zurücknahme) und greifen - den Autoren nach - zu schnell ein. Diese schlussfolgern:

Qualitativ hochwertigen Gruppenunterricht leisten schließlich diejenigen Lehrkräfte, die auf ein reichhaltiges, differenziertes und gut organisiertes didaktisch-methodisches Wissen zurückgreifen können. Lehrkräfte, denen guter Gruppenunterricht gelingt, haben also auch eine entsprechende handlungswirksame Wissensbasis aufgebaut. Ohne ein solches Expertenwissen sind Lehrkräfte nicht in der Lage, einen qualitativ hochwertigen Gruppenunterricht zu praktizieren. Versuchen sie es dennoch, kann es leicht zu Misserfolgen kommen, die dann zur vorschnellen Ablehnung dieser Sozialform des Unterrichts führen. (Helmke, 2010, S. 213, Zitat: Dann et al., 2002, S. 14).

Zu diesem Ergebnis gelangen auch Bryk et al., die im Rahmen ihrer Längsschnittstudie festgestellt haben, dass es gerade mit Blick auf Klassenführung unsichere Lehrkräfte sind, die individuelle Projektarbeit oder Gruppenarbeit in ihren Klassen mit der Begründung: „My kids cannot do this kind of work." (Bryk et al., 2010, S. 105) nicht durchführen. Die Organisation kooperativer Lernformen hängt also sehr eng mit den oben genannten Qualitätsmerkmalen der Klassenführung und klaren Strukturierung des Unterrichts zusammen.

Seit den 80er Jahren haben sich die Anteile verschiedener Methoden im Unterricht zwar verändert, allerdings nimmt der frontale Unterricht nach wie vor einen hohen Stellenwert ein. Eine Studie von Hage u.a. (1985) belegt, dass durchschnittlich knapp $77 \%$ des beobachteten Unterrichts (114 Stunden, Sek I) über die verschiedenen Schulformen hinweg als Frontalunterricht erfolgten, etwa $10 \%$ als Einzelarbeit, knapp 3\% als Partnerarbeit und etwas mehr als $7 \%$ als Gruppenarbeit (Hage et al., 1985, n. Götz et al., 2005, S. 345). Bei einer Folgeuntersuchung 
durch Götz u.a. verteilten sich die Unterrichtsmethoden wie folgt: etwa $47 \%$ Frontalunterricht, $18 \%$ Einzelarbeit, knapp 16\% Partnerarbeit und 13\% Gruppenarbeit (Götz et al., 2005, S. 350). Wiechmann, der 2004 eine Wiederholungsstudie in der Primar- und Sekundarstufe I durchführte (927 Unterrichtsstunden), zeigte, dass „der Anteil sprachzentrierter Unterrichtselemente nur noch bei 45 Prozent [liegt], während der Anteil der Schülertätigkeiten von 24 auf 38 Prozent angestiegen ist [...]. 9,2 Prozent der Unterrichtszeit werden für außerunterrichtliche Aufgaben genutzt" (Wiechmann, 2010, S. 15). Was die Umsetzung von Gruppenunterrichtsphasen angehe, kommen Muijs und Reynolds zu dem Ergebnis, dass weniger als $10 \%$ der Klassenunterrichtszeit in Primarschulen darauf verwendet wurde (Muijs \& Reynolds, 1999, 2002) (Muijs \& Reynolds, 2011, S. 65). Weitere Ergebnisse liegen bspw. aufgrund der Erhebungen zum Mathematik-, Deutsch- und Englischunterricht vor (MARKUS, 2002; DESI, 2008), bei denen Lehrkräfte danach gefragt wurden, welche der Unterrichtsmethoden sie mindestens ein paar Mal pro Monat einsetzen.

Tab. 7: Einsatz von Unterrichtsmethoden (Anteile in Prozent) monatlich

\begin{tabular}{lccc}
\hline Lehrkräfte HS, RS, IGS, GY & Deutsch & Mathematik & Englisch \\
\hline Arbeit mit kleinen Schülergruppen & $54 \%$ & $70 \%$ & $35 \%$ \\
Freiarbeit & $17 \%$ & $30 \%$ & $10 \%$ \\
Wochenplan & $14 \%$ & $12 \%$ & $5 \%$ \\
Projektlernen & $10 \%$ & $7 \%$ & $5 \%$ \\
Lernzirkel/Stationslernen & $5 \%$ & $20 \%$ & $5 \%$ \\
Gem. Unterrichtsvorb. & $8 \%$ & $10 \%$ & $3 \%$ \\
Gem. Unterrichtsdurchf. & $2 \%$ & $1 \%$ & $1 \%$ \\
\hline
\end{tabular}

(Helmke et al., 2002, S. 51; Helmke, 2015, S. 269)

Wie die Übersicht zeigt, wird Kleingruppenarbeit vor allem im Fach Mathematik häufig eingesetzt. Sie stellt neben Frontalunterricht und Einzelarbeit die am häufigsten praktizierte Sozialform in den untersuchten Schulen dar. In integrierten Systemen werden vielfältige Methoden stärker eingesetzt als in den Gymnasien. Offene Unterrichtsformen wie Freiarbeit, Wochenpläne, Projektlernen oder Lernzirkel bzw. Stationslernen werden in allen drei Fächern weitaus seltener genutzt. Aus der Vielfalt von Methoden sollte klassenspezifisch nach Helmke eine Auswahl erfolgen. In Anlehnung an die MARKUS-Studie verweist er darauf, dass Klassen „mit einer überschaubaren Anzahl unterschiedlicher Lehr-Lern-Szenarien“ (Helmke, 2010, S. 265) am erfolgreichsten abgeschnitten haben, während Klassen mit einer Fülle unterschiedlicher Unterrichtsformen oder Klassen, in denen vorwiegend frontal unterrichtet wurde, gleichermaßen schlecht abschnitten.

Hinsichtlich der Qualitätskategorie Unterrichtsmethoden kann festgehalten werden, dass diese eher oberflächlich thematisiert werden. Prell und Link thematisieren den Einsatz verschiedener Sozialformen vor allem im Zusammenhang mit sozialen Lernprozessen in integrativen Klassen als wesentlich:

Es [gemeint ist Montessori-Material, Anm. d. Verf.] ist begrenzt was die Erreichung sozialer Lernziele angeht. Hier muß sich der Unterricht in der Klasse Sozialformen bedienen, wie Partner- und Gruppenarbeit, Rollenspiel und Projektarbeit, wofür auch ein Curriculum, das an sozialen Themen ausgerichtet ist, erst noch entwickelt werden muß. (Prell \& Link, 1977, S. 303) 
Dyson et al. führten im Rahmen einer Studie an 16 Primar- und Sekundarschulen Interviews mit dem Schulpersonal durch und ergänzten diese durch Unterrichtsbeobachtungen. Zielsetzung war die Untersuchung des Zusammenhangs zwischen der Inklusivität von Schulen und den Lernleistungen der Schüler (Dyson, 2010, S. 5). Neben der Tatsache, dass diese Schulen ihren Unterrichts insgesamt sehr flexibel organisierten, bspw. was die Unterrichtung von Schülern mit speziellem Förderbedarf im Klassenverband oder in Kleingruppen betrifft, zeigten diese Schulen auch eine hohe Flexibilität hinsichtlich der gewählten Unterrichtsmethoden:

Classroom practices in highly inclusive schools tended to show considerable flexibility in terms of individual planning, individual support, a variety of activities, and a mixture of individual, group, and whole-class work. However, these were features that could probably be found in well-organized classrooms everywhere. There was no indication that there was a distinctive pedagogy that was peculiar to highly-inclusive schools. (Dyson, 2010, S. 6)

Die Vielfältigkeit im methodischen Zugang stellt auch eine Qualitätskategorie der Sonderpädagogik dar (Boban \& Hinz, 2003; Brugger-Paggi, 2003; Wember \& Prändl, 2009). Bei einer Online-Befragung von 104 Lehrkräften aus unterschiedlichen Schulformen im Rahmen des ERINA-Schulversuchs ${ }^{39}$ gaben $38 \%$ an regelmäßig Stationenarbeit und offenen Unterricht anzubieten, 23\% Tages- bzw. Wochenplanarbeit sowie 19\% Projektarbeit. Während Klassenunterricht zu $88 \%$ angewendet wird, liegt der Anteil für die Gruppenarbeit bei $41 \%$ (Liebers et al., 2014, S. 237). Insgesamt sind die Qualitätskategorien und -subkategorien der Sonderpädagogik im Bereich der Methodenauswahl ausschließlich auf offene Unterrichtsformen konzentriert. Schüler aus Integrationsklassen berichten nach Feyerer in sehr viel höherem Ausmaß über offene Lernformen (Kleingruppenarbeit, Wochenplanarbeit, Projektunterricht) als Schüler aus Regelklassen und können häufiger bestimmen, was und wie sie lernen (Feyerer, 1998, S. 165). Als Qualitätssubkategorie werden offene Unterrichtsformen allgemein von Berges (1996), dem Deutschen Schulamt (2004), Specht et al. (2006), Thiele (2009) Wember \& Prändl (2009) oder der PH Steiermark (2011) aufgeführt, der projektorientierte Unterricht von Köbberling (1994), Berges (1996) sowie Bürli et al. (2009), die Wochenplanarbeit von Köbberling (1994). Neben kooperativen Unterrichtsformen (Berges, 1996; Specht et al., 2006; Textor, 2009) messen sonderpädagogische Autoren der eigenständigen Aktivität der Schüler (Innerhofer \& Klicpera, 1991; Köbberling, 1994; Heyer et al., 1997; Bless, 2003) und der Mitgestaltung des Unterrichts durch die Schüler (Bless, 2003; Boban \& Hinz, 2003) eine wichtige Bedeutung zu.

\subsubsection{Differenzierung und Individualisierung}

Die Qualitätsbausteine Differenzierung und Individualisierung sind mit Abstand diejenigen, die in sonderpädagogischen (Forschungs-) Veröffentlichungen am häufigsten genannt und entsprechend ausführlich beschrieben werden (Mühl, 1987; Innerhofer \& Klicpera, 1991; Hug, 1994b; Köbberling, 1994; Heyer et al., 1997; Bless, 2003; Boban \& Hinz, 2003; Brugger-Paggi, 2003; Specht et al., 2006; BMBWF Österreich, 2008; Bürli et al., 2009; Wernstedt \& Ohnesorg, 2010; Lienhard-Tuggener et al., 2011). Auch bei diesem Thema stammen Umsetzungsideen mit Blick auf integrativen Unterricht bereits aus den 70er Jahren: „Vor allen denkbaren Möglichkeiten sollte die innere Differenzierung (Binnendifferenzierung) des Unterrichts praktiziert werden. Damit ist eine Differenzierungsform gemeint, bei der die Klassen oder Schülergruppen bei

39 „Erprobung von Ansätzen zur inklusiven Beschulung von Schülern mit sonderpädagogischem Förderbedarf in Modellregionen" in Sachsen; Unter: https://www.inklusion.bildung.sachsen.de/schulversuch-erina-4190.html; Stand: 28.09.2016 
gleicher Zusammensetzung in allen Fächern unterrichtet werden." (Muth, 1977, S. 97). Helmke weist darauf hin, dass Fragen der Individualisierung noch weiter zurückreichen und bereits 1850 eine Rolle spielten:

Die Individualität der Kinder ist freilich auch darin sehr verschieden, indem die Einen ein lebendiges Ehrgefühl, die Anderen ein stumpfes und unempfängliches von Natur oder durch die häuslichen Verhältnisse erhalten haben. Deßhalb ist es durchaus eine der ersten Forderungen an den denkenden und wachsamen Lehrer, in dieser, wie in anderer Beziehung die Kunst des Individualisierens zu üben, und daher auch auf Geschlecht, Temperament, geistige Begabtheit, Alter und Bildungsstufe der Schüler verständige Rücksicht zu nehmen. (Schnell, 1850, S. 84, zitiert n. Helmke, 2015, S. 249)

Der Unterschied zwischen Differenzierung auf der einen und Individualisierung auf der anderen Seite ist nicht trennscharf. Es lässt sich allerdings grob trennen zwischen differenzierenden Maßnahmen im Unterricht insgesamt und Maßnahmen, die tatsächlich individuell, d.h. auf einzelne Schüler hin, abgestimmt sind. Unter Differenzierung verstehen Bohl et al.

[...] alle Maßnahmen [...], die für einzelne Lernende bzw. Gruppen von Lernenden unterschiedliche Lernangebote bereitstellen. [...] Innere Differenzierung bzw. Binnendifferenzierung meint unterschiedliche Lernangebote innerhalb einer Lerngruppe, wobei sich die Unterschiede u.a. auf das Ziel, die Inhalte, die Materialien, die Arbeitsmethoden, die gegebenen Hilfen, den Grad der Schwierigkeit und Wahlfreiheit sowie die eingeräumte Zeit beziehen können. (Bohl et al., 2010, S. 329, Herv. im Orig.)

Diese differenzierenden Maßnahmen werden oft auf Gruppen von Schülerinnen und Schülern zugeschnitten. Individualisierung geht darüber hinaus, da sie von Individualität als Basis ausgeht und folgende Zielsetzung verfolgt:

Jeder einzelne Schüler soll individuell maximal gefordert und damit optimal gefördert werden. Das individuelle Leistungsvermögen und das Lernverhalten sind Grundlage für differenzierende Maßnahmen auf der inhaltlichen, didaktischen, methodischen, sozialen und organisatorischen Ebene. (Paradies \& Linser, 2001, S. 9)

Differenzierungsmöglichkeiten bestehen in verschiedenen Dimensionen wie Schulorganisation, Didaktik, Unterrichts- bzw. Kompetenzen (Saalfrank, 2012, S. 65) oder nach Zugängen, Art der Aufgabe und Form der Lehrerzuwendung (Klafki \& Stöcker, 1976). In der Unterrichtspraxis lässt sich außerdem grundsätzlich zwischen quantitativer Differenzierung (Anpassung von Anzahl oder Umfang von Aufgaben) und qualitativer Differenzierung (Anpassung des Niveaus der Aufgaben) unterscheiden. Helmke geht in seinen Ausführungen zum Thema Binnendifferenzierung zunächst von Schülerorientierung allgemein als Grundkonzept aus, für das Lernermerkmale wie Vorwissen, Migrationshintergrund, Entwicklungsstand oder Lernstile relevant seien (Helmke, 2010, S. 248f). Es beinhalte, dass Schüler als Personen wertgeschätzt werden sollten, was für den Unterricht auch bedeute, dass dieser sich konsequent an den Lernvoraussetzungen der Schüler auszurichten habe (Helmke, 2010, S. 230-234) und diese an der Auswahl von Lerninhalten, Hausaufgaben, der Reihenfolge von Lerninhalten etc. beteiligt werden sollten. Damit eine Orientierung an den Schülern erfolgen könne, benötigen Lehrkräfte nach Helmke diagnostische Kompetenzen (Helmke, 2010, S. 255). Rossbach und Wellenreuther betonen laut Helmke, dass Leistungsdifferenzierung „lediglich einen Rahmen für wirksame pädagogische Maßnahmen [schaffe]“ (Rossbach \& Wellenreuther, 2002, S. 53) und es nicht auf die Quantität, sondern die Qualität der damit verbundenen Angebote ankomme, die bspw. auch durch das diagnostische Professionswissen der Lehrkräfte beeinflusst werde (Helmke, 2010, S. 258). Im Zusammenhang mit der Anforderung von Binnen- 
differenzierung oder Individualisierung werden verschiedene Strategien beschrieben. Helmke verwendet in diesem Zusammenhang den Begriff der Passung und unterscheidet in Anlehnung an Weinert (1997) vier Reaktionsmöglichkeiten: das Ignorieren der Lern- und Leistungsunterschiede, die Anpassung der Schüler an die Anforderungen des Unterrichts, die Anpassung des Unterrichts an die lernrelevanten Unterschiede zwischen den Schülern sowie die gezielte Förderung der einzelnen Schüler durch adaptive Gestaltung des Unterrichts (Helmke, 2015, S. 250f). Wember differenziert aus sonderpädagogischer Sicht zwei Anpassungsstrategien im inklusiven Unterricht: die remediale Strategie, bei der fehlende Voraussetzungen zum Lernen durch direkte Förderung bearbeitet werden, und die kompensatorische Strategie, bei der mangelnde Lernvoraussetzungen im Unterricht durch andere Angebote ausgeglichen werden (Bsp.: andere Lerninhalte, methodische Zugänge, Ziele o.a.) (Wember, 2001, S. 166f). Während es bei der remedialen Strategie bspw. darum gehe mit einem Kind, das Schwierigkeiten beim Schriftspracherwerb habe, die Wahrnehmung lautlicher Strukturelemente der Sprache zu trainieren, könne eine kompensatorische Strategie auch so aussehen, dass

[...] fehlende oder unzureichend ausgebildete Kenntnisse und Fertigkeiten durch andere Qualifikationen ersetzt oder ergänzt werden; dies ist z.B. dann der Fall, wenn blinde oder hochgradig sehbehinderte Menschen die Punktschrift statt der Schwarzschrift erlernen [...] oder wenn Schwerhörige das Ablesen von den Lippen und die Gebärdensprache erlernen. (Wember, 2009, S. 94)

Trautmann und Wischer gehen in Anlehnung an Weinert und Helmke davon aus, dass mit Hilfe eines binnendifferenzierten Unterrichts nicht alle Lernausgangslagen ausgeglichen werden können:

Diese „pädagogische Utopie“ - so resümiert Weinert (2001, S. 73) den Forschungsstand - habe sich [...] „in allen Ländern als pure psychologische Illusion erwiesen“. Es überwiegen vielmehr die Hinweise, dass bessere Ausgangsbedingungen auch weiterhin einen höheren Gewinn von schulischen Lernangeboten begünstigen. Mit diesem Phänomen - dem sog. Matthäus-Effekt („Wer hat, dem wird gegeben“) - wird der bereits genannte Zielkonflikt angesprochen, der sich einstellt, wenn zwischen individuellen (auf den einzelnen Schüler bezogenen) und kollektiven (auf die Verteilung von Merkmalen innerhalb einer Lerngruppe bezogenen) Unterrichtszielen differenziert wird (Helmke 2003, S. 22). Sollen beide Ziele berücksichtigt werden, dann stellt sich die Frage, wie ein möglichst großer individueller Lernzuwachs für alle erreichbar ist, ohne dass die Leistungsunterschiede immer größer werden. (Trautmann \& Wischer, 2011, S. 72, Herv. im Orig. $)^{40}$

Sie fassen daher zusammen, dass die Schere zwischen den Erwartungen an differenzierten Unterricht und dessen Einlösung weit auseinandergehe (Trautmann \& Wischer, 2011, S. 73) und berufen sich in diesem Zusammenhang erneut auf Weinert, der schon 1997 kritisch formuliert habe, dass in Unterrichtsmodellen

[...] die Notwendigkeit der Individualisierung abgeleitet [werde], ohne im Einzelnen anzugeben, welche differenziellen Unterrichtsformen, Lehrmethoden und soziale Interaktionsmodi unter welchen klassenspezifischen Bedingungen und im Hinblick auf welche pädagogischen Zielsetzungen zu praktizieren sind, um die erwünschten Effekte zu erzielen, unerwünschte Nebenwirkungen zu vermeiden und den Lehrer nicht heillos zu überfordern. (Weinert, 1997, S. 50)

40 siehe dazu auch Originaltexte von Helmke sowie Weinert, F.E. (2001): Qualifikation und Unterricht zwischen gesellschaftlichen Notwendigkeiten, pädagogischen Visionen und psychologischen Möglichkeiten. In: Melzer, W.; Sandfuchs, U. (Hrsg.): Was Schule leistet. Funktionen und Aufgaben von Schule. Weinheim, München: Beltz Juventa Verlag, S. 65-86 
Die Umsetzung differenzierten Unterrichts setzt nach Paradies und Linser (2001) voraus, dass die Schüler ein hohes Maß an eigener Verantwortung für ihr Lernen wahrnehmen (können). Die Fähigkeiten zum eigenverantwortlichen Lernen sind im Lauf der Schulzeit Schritt für Schritt aufzubauen. Je nach Lernvoraussetzungen benötigen die Schüler ein sehr unterschiedliches Maß an Unterstützung durch Lehrer, Mitschüler oder andere am Unterricht beteiligte Personen. Binnendifferenzierter Unterricht hat keine eigene Didaktik oder Methodik. Bestimmte Lernformen oder Methoden bieten sich allerdings besonders gut für binnendifferenziertes Arbeiten an (Beispiele: Freiarbeit, Wochenplanarbeit, Werkstattarbeit, Stationenarbeit, Lernzirkel, Lerntheken, Projekte, Gruppenarbeit, kooperatives Lernen, aber auch Übungssequenzen mit differenzierten Aufgabenstellungen bzw. Materialien) (Paradies \& Linser, 2001, S. 89; Saalfrank, 2012, S. 65-97).

Spannend ist in diesem Zusammenhang, wie die folgenden Ausführungen belegen, dass im Gegensatz zu den vielfältig vorgetragenen Forderungen nach differenzierten bzw. individualisierenden Vorgehensweisen im Unterricht Studien, die sich explizit mit der Verbreitung oder Effizienz solcher Maßnahmen für die Lernergebnisse von Schülern und Schülerinnen auseinandersetzen, nur vereinzelt vorliegen. Bereits in den 70er Jahren befasste sich ein Schulversuch in Reutlingen mit dem Thema „Differenzierung im Anfangsunterricht“ (Meiers, 1982). Meiers kam dabei zu folgenden Ergebnissen:

- Die Durchführung des differenzierenden Unterrichts muss Methode haben, damit dieser effektiv ist.

- Neben Voraussetzungen bei Kindern sind weitere Bedingungen wie Gruppenbildung, Arbeitstechniken für verschiedene Sozialformen, Verhaltensformen etc. zu berücksichtigen.

- Der Umfang der Differenzierungsmaßnahmen schwankte in Klassen je nach Voraussetzungen von Kindern bzw. Gruppe. (Meiers, 1982, S. 197f)

Er hält zusammenfassend fest:

Unterrichtsverlauf und Lernergebnisse haben deutlich werden lassen, daß es den differenzierenden Unterricht nicht gibt, vor allem nicht den, der qua differenzierender Unterricht bereits erfolgreich ist. An den unsteten Lernverläufen einzelner Kinder im Klassenverband und im Stütz- und Förderkurs ist zu erkennen, daß nichtlernbereichsspezifische Faktoren sich stark auswirken. Auf diese besonderen Bedingungen muß der Lehrer ebenfalls angemessen reagieren - nicht mit einem gesonderten Arbeitsblatt, sondern mit einem freundlichen, aufmunternden Wort und pädagogisch begründeten organisatorischen Maßnahmen. Insofern ist der differenzierende Unterricht immer eingebettet in die gesamte unterrichtliche Situation, in die gesamte Unterrichtsatmosphäre und wird von daher relativiert. (Meiers, 1982, S. 198, Herv. im Orig.)

Aufgrund der Ergebnisse des ersten Durchgangs dieses Schulversuchs geht Meiers davon aus, dass eine rein lehrergesteuerte Lehrgangssystematik (,Atomisierung des Lehrgangs in kleinste Lernschritte') Kinder auch davon abhalten könne, ihre eigenen Lernprozesse zu gestalten. Differenzierter Unterricht dürfe also nicht nur kognitive Lernziele verfolgen, sondern müsse auch zur Lernmotivation, Aktivierung und Förderung der Ich-Identität etc. beitragen (Meiers, 1982, S. 199): „Leitziel sollte sein, daß Kind so zu fördern, daß es möglichst bald seinen Lernprozeß kompetent mitgestalten kann; d.h. es sollte in die Lage versetzt werden, selbst zum Agenten seines Lernprozesses zu werden." (Meiers, 1982, S. 199). Aktives und selbständiges Lernen sollten also immer feste Bestandteile eines differenzierenden Unterrichts sein:

Dieser Unterricht ist differenzierender Unterricht in einem anderen Sinne als bisher. Er wurde mit Erfolg erweitert durch die Dimension ,aktives Lernen durch den Schüler“; d.h. Differenzierung bestand nicht mehr ausschließlich aus einer Summe vom Lehrer gesteuerter didaktischer Zuteilungspraktiken, sondern auch aus dem Schüler gesteuerten motivationsgeladenen „Selbstbedienungspraktiken“. (Meiers, 1982, S. 204) 
Eine ähnliche Verknüpfung stellen Feuser und Meyer mit Blick auf den Modellversuch der Bremer Grundschule Robinsbalje her:

Wir erkennen [...], daß,Projektorientierung und ,Individualisierung in gleicher Weise Voraussetzung wie Kernstücke der ,Inneren Differenzierung integrativen Unterrichts sind. Über das Moment der Projektorientierung wird die Vielschichtigkeit des Unterrichts erreicht, ohne daß seine innere Kohärenz und Logik verloren gingen, und über die Individualisierung wird die Verzahnung von Subjekt- und Objektseite (verkürzt gesagt: von Schülern und Sache) erreicht [...]. (Feuser \& Meyer, 1987, S. 38)

Sie sehen in der Selbständigkeit und Selbsttätigkeit, die Kindern durch diese Form von Unterricht ermöglicht wird, einen Vorteil integrativen Unterrichts (Feuser \& Meyer, 1987, S. 84). Feyerer stellt in integrativen Klassen ein größeres Ausmaß an innerer Differenzierung hinsichtlich Umfang, Zeitdauer und/oder Schwierigkeitsgrad der Aufgabenstellungen fest (Feyerer, 1998, S. 165).

Der Großteil der empirischen Untersuchungen zu Fragen bezüglich einer homogenen bzw. heterogenen Zusammensetzung von Lerngruppen bezieht sich nach Trautmann und Wischer darauf, dass Auswirkungen auf „fachliche Leistungen (etwa Lesekompetenz, Mathematikkompetenz) wie auch auf Persönlichkeitsaspekte (etwa das Selbstkonzept oder soziale Orientierungen)“ (Trautmann \& Wischer, 2011, S. 81) geprüft werden, und zwar sowohl aus Sicht der Bildungsforschung allgemein wie auch aus Sicht der Integrationsforschung. Woolfolk (2008) kommt aufgrund ihrer Analyse zu Forschungsergebnissen im Bereich Differenzierung zu dem Schluss, dass Leistungsdifferenzierungen sehr effizient zu sein scheinen, dass davon allerdings begabtere Schüler stärker profitieren würden als schwache Schüler (Castle, Deniz \& Tortora 2005, Garmon, Nystrand, Berends \& Le Pore 1995; Oakes and Wells, 1998; Robinson \& Clinkenbeard, 1998; n. Woolfolk, 2008, S. 150f). Hochbegabte Schüler profitieren allerdings auch von einer homogeneren Klassenzusammensetzung mehr als ebenso begabte Schüler in Regelklassen (Kulik \& Kulik, 1997, S. 230ff). Hattie versteht in seiner Metaanalyse interne Differenzierung als Praxis der Bildung von möglichst leistungshomogenen Kleingruppen innerhalb von Schulklassen. Für die Bildung dieser Kleingruppen an sich kann lediglich eine Effektstärke von $\mathrm{d}=0,16$ belegt werden, wobei auch er feststellt, dass leistungsstarke Schüler von dieser Maßnahme stärker profitieren könnten als leistungsschwache Schüler $(\mathrm{d}=0,30 \mathrm{im}$ Vergleich zu $\mathrm{d}=$ 0,16). Stärkere Effekte $(d=0,49)$ stellen sich nach Hattie nur dann ein, wenn innerhalb dieser Kleingruppen echte kooperative Aufgabenstellungen bearbeitet werden (Hattie, 2013, S. 113). Darüber hinaus ist wesentlich, dass „die Stoffe und der Lehrstil vielfältig und in angemessenem Maß anspruchsvoll sein [müssen], um den Bedarfen der Lernenden mit ihren unterschiedlichen Fähigkeitsniveaus gerecht zu werden." (Hattie, 2013, S. 113). Helmke wiederum zeichnet ein kritisches Bild von der Wirksamkeit leistungsdifferenzierter Angebote:

Soweit Formen der Individualisierung und Differenzierung empirisch untersucht worden sind, ergibt sich ein eher ungünstiges Bild von deren Wirksamkeit. Lüders und Rauin (2004) stellen bei ihrer vergleichenden Übersicht eine leichte Überlegenheit offener Unterrichtsformen beim sozialen Lernen und klare Nachteile bei der Kompetenzentwicklung fest. Letzteres berichtet auch Gruehn (1998) [...]; als Ursache vermutet sie den erhöhten Organisationsbedarf und die suboptimale Zeitnutzung, die mit den durch individuelle Lerntempi bedingten potenziellen Leerlaufphasen für einzelne Schüler(gruppen) zusammenhängt. (Helmke, 2010, S. 257)

Im Schulalltag sind Differenzierung oder Individualisierung Forschungsergebnissen nach nur wenig verbreitet. So ergab die IGLU-Studie, dass zwei Drittel der Schüler einen nicht differenzierten Unterricht erleben (Helmke, 2010, S. 255; Bos et al., 2003, S. 257f). Als Ergebnis 
der DESI-Studie stellte sich heraus, dass $46 \%$ der Lehrkräfte im Fach Deutsch bei Stillarbeiten verschiedene leistungsdifferenzierte Aufgaben vergeben. Bei der Gruppenbildung bilden die Deutschlehrer vor allem leistungsheterogene Gruppen (70\%) und würden eher nicht inhaltlich differenzieren (Klieme et al., 2008, S. 325). Trautmann und Wischer weisen allerdings darauf hin, dass die empirische Grundlage für die Einschätzung der Verbreitung von Differenzierung insgesamt sehr gering sei (Trautmann \& Wischer, 2011, S. 71).

Sonderpädagogische Forschungsergebnisse befassen sich - neben der übergeordneten Qualitätskategorie Differenzierung (s.o.) - mit verschiedenen Teilaspekten binnendifferenzierten Arbeitens im Unterricht. Befürwortet wird aufgrund der Erfahrungen aus den Modellversuchen eine Bevorzugung der inneren vor der äußeren Differenzierung (s. OE) bzw. zumindest ein regelmäßiger Wechsel zwischen beiden Formen (Feyerer, 1998; Thiele, 2009). Andere Autoren sprechen von einem vielfältigen Maßnahmenangebot für unterschiedlich begabte Schüler (Brugger-Paggi, 2003), Einzelmethoden der Differenzierung (Innerhofer \& Klicpera, 1991) oder der Differenzierung von Leistungsanforderungen (Textor, 2009; s. dazu nächster Abschnitt). Präzisere Ausführungen - wie sie zum Teil in der Allgemeinen Pädagogik erfolgen (s.o.) - fehlen allerdings weitgehend.

Neben dieser grundsätzlich positiven Ausrichtung der Sonderpädagogik auf das Thema Differenzierung werden ähnlich wie bei Helmke (2010) oder Trautmann und Wischer (2011) auch mögliche Grenzen dieser Arbeitsweise im Unterricht erfasst. Romey beschrieb bspw. das Prinzip der Leistungsdifferenzierung mit Blick auf die soziale Integration von Schülern mit Lernbehinderungen schon 1977 als Dilemma, vor allem, da es einen engen Zusammenhang zwischen dem Sozial- und dem Leistungsstatus von Schülern gebe. Es sei davon auszugehen, dass „,soziale Integration als schichtunabhängige Voraussetzung für Schulerfolg auf einen Teil der Schüler nicht zutrifft" (Romey, 1977, S. 117) und sie schichtabhängige Sozialisationseinflüsse nicht kompensieren könne. Darüber hinaus könnten Bereiche wie die Entfaltung der eigenen Identität oder die Fähigkeit zur Selbstreflexion nicht über Leistungsdifferenzierung erreicht werden (Romey, 1977, S. 117).

Prell und Link erfassen das Problem der sich vergrößernden Unterschiedlichkeit aufgrund der Alterszunahme ebenfalls:

Es gibt auch noch keine befriedigende Lösung für das Problem, daß sich der Abstand zwischen schwerer behinderten und überdurchschnittlich befähigten Schülern mit zunehmendem Alter extrem vergrößert und lernbehinderte Schüler von dem gemeinsamen Unterricht kaum einen Lerngewinn haben. (Prell \& Link, 1977, S. 303)

Mit Bezug auf differenzierte Förderung zitiert Berges Antor (1992, S. 38), der hinterfragt, ob es irgendwann eine Spanne innerhalb einer Klasse gibt, die nicht mehr durch differenzierten Unterricht überbrückt werden kann, und zu dem Schluss kommt, dass ,irgendwann der Zeitpunkt erreicht ist, an dem kaum mehr als eine abstrakte Einheitsformel von diesem Postulat übrig bleibt." (zitiert n. Berges, 1996, S. 37).

Liebers et al. konnten bei einer Befragung von 104 Lehrkräften im Rahmen des ERINASchulversuchs erheben, dass ein Viertel der befragten Lehrkräfte mit Blick auf mögliche Differenzierungsformen passive Reaktionen wählt, d.h. dass Leistungsunterschiede zwischen den Schülerinnen und Schülern ignoriert werden und alle SuS die gleichen Aufgaben erhalten. Drei weitere Viertel begegnen heterogenen Lernvoraussetzungen mit der Nutzung substitutiver Reaktionsformen (Bsp.: Förderunterricht, Bildung temporärer Lerngruppen o.ä.) (Liebers et al., 2014, S. 237). Jeweils knapp ein Viertel der Befragten gibt an, innere Differenzierung „durch 
variierende Zeitvorgaben, unterschiedliche Aufgabenniveaus sowie verschiedene Materialien“ (Liebers et al., 2014, S. 237f) umzusetzen.

Untrennbar mit differenziertem Unterricht verknüpft ist die Frage nach der differenzierten Bewertung von Schülerleistungen. Wie unter 2.2 dargestellt, stellt die Bewertung von Schülerleistungen grundsätzlich eine Anforderung dar, die im auf Selektion und Allokation ausgerichteten Schulsystem begründet ist. Schulen, die inhaltlich differenzierten und damit auch leistungsdifferenzierten Unterricht anbieten, stehen in diesem Zusammenhang vor der besonderen Herausforderung einer Leistungsbewertung, die sich einerseits an allgemein „verbindlichen“ Anforderungen (Bsp.: Rahmenpläne, Lehrpläne etc.) und andererseits an den individuellen Leistungen von Schülern orientieren sollen, was ein klassisches Dilemma darstellt:

Der Sonderpädagoge mit expliziter Zuständigkeit für sonderpädagogische Probleme benötigt für die Realisierung der individuellen Lernprozeßorientierung einen erweiterten Handlungs- bzw. Entscheidungsrahmen: Die für Schule typische wettbewerbsorientierte und lerngruppenvergleichende Bewertung schulischer Lernleistungen muß durch eine individuelle, nicht an objektiven Gruppennormen orientierte lern- bzw. förderdiagnostische individuelle Beschreibung von Lernprozessen ergänzt werden. Unser schulisches Benotungssystem, an der Normalverteilung orientiert, ist wenig nützlich für die Beschreibung individueller Lernfortschritte und deren Bewertung. (Preuß, 1985, S. 75)

Integrative Schulen nutzen für die Bewältigung dieses Dilemmas unterschiedliche Wege: den kompletten Verzicht auf Notengebung in Form von Ziffernnoten zugunsten verbaler Beurteilungen (Mühl, 1987; Husinsky, 1994; Köbberling, 1994), kombinierte Varianten von Verbalund Ziffernbeurteilungen (Mühl, 1987) oder die Orientierung an in den Förderplänen oder in den Zeugnissen benannten Kompetenzstufen (Brugger-Paggi, 2003), bei denen Leistungen innerhalb dieser Kompetenzstufen und nicht orientiert an einer Klassennorm bewertet werden. So gestaltet die Modellschule „Aktion Sonnenschein“ ihren Unterricht mit Lehrzielen für die Schüler, deren Bewertung orientiert am Curriculum und nicht an einer Durchschnittsnorm erfolgt. Der Lernzielstand und die Lernfortschritte werden an großen Übersichtstafeln in der Klasse dokumentiert. Dargestellte Fortschritte beziehen das Arbeits-, Sozial- und Leistungsverhalten ein. Das Profil der Lernergebnisse kann für individuelle Diagnostik wie für Unterrichtsplanung insgesamt genutzt werden (Prell \& Link, 1977, S. 319). Nach Rheinberg ist die Herstellung einer individuellen Bezugsnormenorientierung statt der Orientierung an sozialen Vergleichen hilfreich für die Motivationsförderung der Schüler:

Zunächst wird unter dieser Bezugsnorm der Lernzuwachs jeden Lerners besonders gut sichtbar. Das fördert Vertrauen in das eigene Lernpotenzial und die Zuversicht, sich weiterentwickeln zu können (Wirksamkeitserwartungen). Dabei zeigt die Vergleichsperspektive („besser oder schlechter als beim letzten Mal“) zudem sehr sensibel die je aktuellen Veränderungen an, die man dann mit den vorangegangenen Lehraktivitäten in Zusammenhang bringen kann: Man sieht genauer, welche Lehraktivitäten zu welchen Lernzuwächsen führen. (Rheinberg, 2008, S. 182)

Forschungsergebnisse und Kriterienkataloge enthalten eine ganze Reihe von Qualitätsindikatoren, die im Zusammenhang mit einer leistungsdifferenzierten Bewertung eine Rolle spielen. Dazu gehört das Angebot differenzierender Klassenarbeiten (Heyer et al., 1997) oder differenzierter Leistungsfeststellung allgemein (Heyer et al., 1997; Holzinger et al., 2011), die Nutzung alternativer Formen der Leistungsbeurteilung (Holzinger et al., 2011) wie bspw. Beobachtungen, Experimente, Präsentationen o.ä. (Boban \& Hinz, 2003; Brugger-Paggi, 2003). Je nach Einschränkung eines Schülers sollten Leistungsmessungen auch eine andere Form haben können (Bsp.: Multiple-Choice-Aufgaben für Schüler mit einer körperlichen Beeinträchti- 
gung) (Thiele, 2009). Prüfungszeitpunkte sollten von Schülern flexibel gewählt werden können (Boban \& Hinz, 2003). Bewertungskriterien können gemeinsam mit den Schülern festgelegt werden (Brugger-Paggi, 2003) und individuelle Maßstäbe nutzen (Deutsches Schulamt, 2004), bspw. die Zielsetzungen individueller Förderpläne (Brugger-Paggi, 2003). Nach Innerhofer \& Klicpera (1991) könnten in diesem Zusammenhang Schemata und Hilfen für eine effiziente Lernzielüberprüfung hilfreich sein.

Ein wichtiges Qualitätsmerkmal im Zusammenhang mit differenzierter Leistungsbewertung sind individuelle Rückmeldungen für Schüler (und Eltern) (Brugger-Paggi, 2003; Wember \& Prändl, 2009). Wichtig ist diesbezüglich die Herstellung von Transparenz von Leistungserwartungen und Leistungsbewertung (Brugger-Paggi, 2003, Feyerer, 2007). Die Möglichkeiten des Nachteilsausgleichs ${ }^{41}$ (Wember \& Prändl, 2009) sollten genauso genutzt werden wie die gültigen gesetzlichen Bestimmungen (Brugger-Paggi, 2003). Schüler können ihre Leistungen auch selbst einschätzen (Boban \& Hinz, 2003). Mühl (1987) nennt als Qualitätsindikator integrativen Unterrichts eine Abmilderung des Leistungsdrucks, während Bless (2003) es für wichtig hält, dass die Schüler die Aussicht haben für Anstrengungen auch belohnt zu werden.

Um differenziert unterrichten und bewerten zu können sind eine präzise Lernstandsdiagnostik und Förderplanung notwendig (Preuß, 1985; Innerhofer \& Klicpera, 1991; Bless, 2003; Boban \& Hinz, 2003; Brugger- Paggi, 2003; Specht et al., 2006; Feyerer, 2007; Wember \& Prändl, 2009; Bürli et al., 2009; Holzinger et al., 2011). Diese beiden Qualitätskategorien sind inhaltlich nicht $\mathrm{zu}$ trennen. Sie beobachten und beschreiben die Lernvoraussetzungen von Schülern in verschiedenen Bereichen (Bsp.: Arbeits- und Sozialverhalten, emotionale Situation, motorische Entwicklung, Leistungen in den Kernfächern etc.) sowie entsprechende Möglichkeiten der individuellen Förderung der Lernentwicklung. Preuß fordert in diesem Zusammenhang schon 1985:

Da geplante Lernprozesse, die Gesamtsituation des Schülers und der Lerngruppe sich gegenseitig beeinflussen, ist eine ganzheitliche Sichtweise des behinderten und von Behinderung bedrohten Kindes oder Jugendlichen unabdingbar, wobei nicht nur die kognitive, sondern gleichgewichtig auch die emotionale und soziale Dimension von Lernen mitreflektiert werden muß. (Preuß, 1985, S. 70)

Eine solche ganzheitliche Sichtweise stellt nach Lienhard-Tuggener u.a. (2011) beispielsweise das bio-psycho-soziale Modell der ICF dar (s. 2.3). Dieses Modell ist nicht in erster Linie auf ein Kind selbst konzentriert, sondern nimmt auch Wechselwirkungen zwischen Kind und Umwelt in den Blick (Lienhard-Tuggener et al., 2011, S. 98-103).

Hattie fordert aufgrund seiner Forschungsergebnisse eine genaue Analyse der Lernfortschritte, die ein Schüler erfolgreich absolviert:

The act of teaching requires deliberate interventions to ensure that there is cognitive change in the student: thus the key ingredients are awareness of the learning intentions, knowing when a student is successful in attaining those intentions, having sufficient understanding of the student's understanding as he or she comes to task, and knowing enough about the content to provide meaningful and challenging experiences in some sort of progressive development. (Hattie, 2009, S. 23)

Lienhard-Tuggener et al. beschreiben, dass ein Förderplanungszyklus sich innerhalb zweier miteinander verknüpfter Kreisläufe bewege, die verschiedene Schwerpunkte beinhalten. Der eine Kreislauf umfasse eine regelmäßige Standortbestimmung (Zusammenführung von Einschätzungen, Vereinbarung und Evaluation von Förderzielen), der andere betreffe die Gestaltung von Unterricht und Förderung (Erstellung bzw. Aktualisierung eines Förderplans, Anpassung

41 s. KMK (2011), S. 10f; InkVO Saarland (2015), \$15 
des Unterrichts, Durchführung und Reflexion der Förderung) (Lienhard-Tuggener et al., 2011, S. 103f).

Als Qualitätsmerkmale für Lernstandsdiagnostik und Förderplanung erfasst Preuß (1985) die Ermittlung der individuellen Lernvoraussetzungen, die Erstellung individueller Lernziele, eine flexible Lernorganisation mit Blick auf das Erreichen der Lernziele und eine „kontinuierliche Lernprozeßdokumentation unter Einbeziehung lerndiagnostischer Verfahren zur Bereitstellung von speziellen Lernhilfen und zur Rückmeldung des Lernprozessverlaufs für Schüler und Lehrer bei Relativierung der vergleichenden Bewertung durch Zensuren“" (Preuß, 1985, S. 76). Darüber hinaus sollten Fallbesprechungen der kollegial abgestimmten Evaluation und Reflexion von Lernprozessen sowie der Erstellung und Weiterentwicklung individueller Förderpläne dienen (Preuß, 1985, S. 76). Auch Schuck beschreibt, dass Lernstandsdiagnostik pädagogische Prozesse als komplexe Lehr- und Lernprozesse begreift, Entwicklungen des Schülers berücksichtigt und qualitativ orientiert ist (Schuck, 1990, S. 103). Weitere sonderpädagogische Beiträge, die die Förderdiagnostik als Qualitätskategorie aufgreifen, erwähnen zunächst die grundlegende sonderpädagogische Diagnostik, die zur Anerkennung eines sonderpädagogischen Förderbedarfs führt (Wember \& Prändl, 2009; Speck, 2010). Eine individuelle Diagnostik bezieht sich auf die Lernausgangslage eines Schülers (Wember \& Prändl, 2009; Holzinger et al., 2011), sollte defizitäre Beschreibungen vermeiden (Preuß, 1985) und sorgfältig Kompetenzen, Bedürfnisse und Umfeld der Schüler erfassen (Lienhard-Tuggener et al., 2011). Ausführlicher als die Förderdiagnostik wird die Qualitätskategorie der Förderplanung differenziert. Die am häufigsten erfasste Subkategorie betrifft dabei die Erstellung individueller Entwicklungs- oder Förderpläne (Preuß, 1985; Innerhofer \& Klicpera, 1991; Boban \& Hinz, 2003; Brugger-Paggi, 2003; Specht et al., 2006; Feyerer, 2007; Bürli et al., 2009; Lienhard-Tuggener et al., 2011). Diese sollten ganzheitliches Lernen und eine Zieltransparenz für Schüler und Eltern gewährleisten (Preuß, 1985), individualisierte Lernziele (Bürli et al., 2009), konkrete Fördervorschläge (Bless, 2003) sowie organisatorische und therapeutische Maßnahmen (Brugger-Paggi, 2003) beinhalten oder gemeinsam mit Schülern und Eltern erstellt werden (Boban \& Hinz, 2003; Lienhard-Tuggener et al., 2011). Für Südtirol benennt Brugger-Paggi den Qualitätsindikator, dass die individuellen Förderpläne vom Klassenrat zu erstellen, regelmäßig zu überprüfen und bei Bedarf an veränderte Situationen angepasst werden sollten (Brugger-Paggi, 2003). Auch Wember und Prändl (2009) sprechen von kooperativer Entwicklung von Förderplänen. Eine begleitende Diagnostik, die Kontinuität des Förderprozesses und die Evaluation des Erreichens der Förderziele werden als weitere Qualitätsindikatoren aufgeführt (Preuß, 1985; Feyerer, 2007; Wember \& Prändl, 2009). Nach Innerhofer \& Klicpera (1991) ist festzulegen, wer für die Durchführung der Förderung Verantwortung übernimmt und wie das Erreichen von Zielen kontrolliert werden kann. Preuß (1985) ergänzt, dass lerndiagnostische Verfahren einbezogen werden sollten. Die Schüler sollten regelmäßig Rückmeldungen zu Entwicklungsfortschritten erhalten (Wember \& Prändl, 2009). Zur Besprechung der Förderpläne mit Eltern und Schülern schlagen Lienhard-Tuggener et al. (2011) Standortgespräche vor, die zu schriftlichen Vereinbarungen führen sollten. Von einer schul- oder bundeslandübergreifenden Systematik seien beide Qualitätsbereiche weit entfernt (Lienhard-Tuggener et al., 2011, S. 95). Zur Effizienz von Förderdiagnostik und -planung liegen bislang keine Forschungsergebnisse vor. Müller, Venetz und Keiser gestalten in der Schweiz derzeit ein Forschungsprojekt zur Frage des Nutzens individueller Förderpläne, das allerdings noch nicht abgeschlossen ist ${ }^{42}$.

42 s. dazu https://www.hfh.ch/de/forschung/projekte/nutzen_und_effektivitaet_individueller_foerderplaene_in_inklusiven_schulsettings/; Stand: 04.05.2019 
Der Ansatz der Individualisierung geht, wie die Definition von Paradies und Linser (2011) zum Einstieg in dieses Unterkapitel gezeigt hat, über den der Differenzierung noch hinaus:

Während dabei [bei Binnendifferenzierung] üblicherweise Lernende mit vergleichbarer Ausgangslage oder ähnlichen Lernbedürfnissen dasselbe Lernangebot erhalten, verlangt die weitergehende Form des individualisierten Lernens (Individualisierung) ein Lernarrangement, das für jede Schülerin und jeden Schüler ein spezifisches Lernangebot bereithält bzw. es ihr oder ihm ermöglicht, einen eigenen Lernweg zu beschreiten. (Bohl et al., 2010, S. 329, Herv. im Orig.)

Individualisierung von Lerninhalten im Unterricht meint also, dass diese sehr spezifisch auf den aktuellen Lernstand eines Schülers angepasst werden. Wie bei der Differenzierung auch, kann eine Individualisierung des Unterrichts mit Blick auf die zu erreichenden Ziele, die Methodenauswahl oder die Auswahl von Medien bzw. didaktischem Material, das Maß der Unterstützung durch die Lehrkräfte, Zeitvorgaben oder die Leistungsbewertung erfolgen (Holzinger \& Wohlhart, 2009, S. 126). Klippert weist als Allgemeinpädagoge in diesem Zusammenhang auf die möglichen unterrichtspraktischen Grenzen einer kompletten Individualisierung hin:

Zwar ist es richtig, dass alle Schüler/innen auf spezifische Weise verschieden sind und mehr oder we-
niger unterschiedliche Leistungspotenziale haben. Daraus jedoch den Schluss abzuleiten, dass jedem
Schüler sein eigenes Lernpaket geschnürt werden muss, ist ebenso utopisch wie pädagogisch fatal. Uto-
pisch deshalb, weil die schon jetzt hohe Vorbereitungsbelastung vieler Lehrkräfte nachgerade ins Un-
ermessliche gesteigert werden müsste, wenn man diesen Ansatz hierzulande ernsthaft zu Ende denkt:
Konsequente Individualisierung setzt nämlich Unmengen an unterschiedlichen Materialien und Aufga-
ben voraus, die bislang aber weder da sind, noch mit vertretbarem Zeit- und Arbeitsaufwand hergestellt
werden könnten. (Klippert, 2008, S. 252)

Vergleichsweise neutral und sachlich beschreibt Helmke die Gelingensbedingungen von Individualisierung wie den dazu notwendigen Einstellungswandel bei Lehrkräften oder die erforderlichen diagnostischen Kompetenzen. Er geht davon aus, dass für die erfolgreiche Umsetzung der Individualisierung vor allem die Orientierung an Kompetenzmodellen hilfreich sein könnte, die anstelle der derzeitigen Regelstandards auch Minimalstandards inhaltlich erfassen. Darüber hinaus seien Ressourcen in Form von Räumen für differenziertes Arbeiten sowie Zeit von Lehrkräften (zusätzliche Stellen) bereitzustellen (Helmke, 2015, S. 257-259).

Wie schon zur Kategorie Differenzierung dargestellt belegen Forschungsergebnisse, dass Individualisierung im schulischen Alltag bislang wenig verbreitet ist (Wischer, 2007, S. 425f). In Hatties Meta-Studie sind die Effekte individualisierten Unterrichtens in etwa so gering wie die interner Binnendifferenzierung und liegen bei $d=0,23$ (Hattie, 2013, S. 234f). Allerdings berichtet er einen großen Effekte für Akzeleration, also beschleunigtes Lernen $(\mathrm{d}=0,88)$, was ein Hinweis darauf ist, dass dieses Angebot, das im jahrgangsübergreifenden Unterricht vereinfacht umgesetzt werden könnte, für gut begabte Lernende entsprechend geeignet ist (Hattie, 2013, S. 119f). Autoren wie bspw. Muth (1977) oder Preuß (1985) gehen schon vor mehreren Jahrzehnten davon aus, dass individualisierte Förderangebote in integrativen Settings unabdingbar sind um den Lernvoraussetzungen von Kindern mit sonderpädagogischem Förderbedarf gerecht werden zu können. Ähnlich wie die Differenzierung ist auch das Thema der Individualisierung in den sonderpädagogischen Studien und Kriterienkatalogen sehr häufig aufgegriffen worden (Muth, 1977; Heese, 1977; Wocken, 1987; Mühl, 1987; Innerhofer \& Klicpera, 1991; Husinsky, 1994; Heyer et al., 1997; Boban \& Hinz, 2003; Specht et al., 2006; Feyerer, 2007). Als Qualitätsindikator für diesen Bereich werden u.a. individuelle sonderpädagogische und therapeutische Maßnahmen für spezifische Förderbedürfnisse (Wocken, 1987) im Wechsel mit 
gemeinsamen Arbeitszeiten, die soziales gruppenbezogenes Lernen umfassen (Wocken, 1987; Köbberling, 1994; Berges, 1996), aufgeführt.

Hinsichtlich des Modellversuchs „Integration lern- und verhaltensgestörter Kinder“ in München werden allerdings auch aus sonderpädagogischer Sicht schon früh mögliche Grenzen für individualisierte Stütz- und Fördermaßnahmen festgehalten:

- bei Kindern aus sehr anregungsarmen Milieu ohne jegliche Frühförderung, die bereits überaltert in die Differenzierte Grundschule kommen und nicht die Eingangsstufe durchlaufen haben

- bei Kindern mit organischen Schädigungen, die sich auf ihre Lernfähigkeit auswirken

- bei stark emotional gestörten Kindern, deren Problematik aus einer negativen Mutterbeziehung resultiert und die nicht durch die Bezugspersonen in Schule und Tagesheim kompensiert werden kann

- bei Kindern, die bereits einige Jahre in speziellen Einrichtungen wie heilpädagogischen Kindergärten oder Sprachheilschule gefördert wurden, aber trotzdem sehr schwache Leistungen zeigen

- Hirnorganiker mit normaler Intelligenz, die aufgrund ihrer erhöhten Reizempfindlichkeit nicht in großen Gruppen arbeiten können. (Speck et al., 1978, S. 132)

Auch wenn die Formulierung dieser Grenzen in Relation zu setzen ist mit den Realisierungsmöglichkeiten und Ressourcen in diesem spezifischen Modellversuch, werden darin doch Hinweise dahingehend gegeben, dass Individualisierung allein unter Umständen nicht ausreicht um integrative Beschulung zu gestalten - gerade dann, wenn die Gruppengröße der Regelschulklasse (aus Mangel an Ressourcen) nicht flexibel gestaltet werden kann. Demgegenüber heißt es bei Preuß:

Individuelle Lernprozeßplanung im Sinne einer sonderpädagogischen Intervention heißt, Lernen bzw. Fördermaßnahmen in allen Strukturelementen - Ziele, Inhalte, Methoden, Medien - für die Erfordernisse des Lernprozesses jedes einzelnen Schülers aufzubereiten, d.h. konsequent auf den individuellen Lernvoraussetzungen des Schülers aufzubauen. (Preuß, 1985, S.70f)

Sie definiert die individuelle Lernprozessorientierung als sonderpädagogisches Handlungsprinzip und plädiert dementsprechend für eine flexible Lernorganisation, die individuelle Lernzeit, Lernart und Lerninhalt zu berücksichtigen habe (Preuß, 1985, S. 76). Bleidick geht 1990 davon aus, dass zieldifferentes Lernen, das sowohl auf Schüler mit geistigen Behinderungen oder Lernbehinderungen wie auf zukünftige Gymnasiasten ausgerichtet ist, hohe Anforderungen an die Lehrkräfte stellt (Bleidick, 1990, S. 28). Holzinger formuliert etwa 20 Jahre später:

Alle Kinder sollen sich auf individuelle Weise mit demselben Lerninhalt befassen und ihren Teil der Auseinandersetzung als Beitrag zum gemeinsamen Bildungsprozess der Klasse erkennen können. Nur durch Kooperation an einem gemeinsamen Gegenstand wird soziale Integration möglich. (Holzinger \& Wohlhart, 2009, S. 104)

Kritisch äußert sich aus sonderpädagogischer Sicht dagegen vor allem Speck:

Die Vorstellung, dass alle Schüler in unbegrenzter Vielfalt, von den hochbegabten bis zu den schwerund mehrfach behinderten, in einer Schule bzw. Klasse nicht nur willkommen sein sollen, sondern auch das Optimum ihrer Lernleistungen erreichen können, ist geradezu mit der Quadratur des Kreises zu vergleichen. (Speck, 2011, S. 89)

Er verknüpft mit dieser Annahme die Sorge, dass spezifische Bedürfnisse von Kindern in einer „Schule für alle“ gegebenenfalls nicht berücksichtigt werden können und geht davon aus, dass eine fachliche Förderung über den Ansatz einer pädagogischen Individualisierung hinausgehe (Speck, 2010, S. 87). Klippert formuliert dazu: 
Pädagogisch fatal ist die skizzierte Individualisierung insofern, als damit auch der Anspruch auf Integration, Kooperation und gemeinsames Lernen über Gebühr aufgegeben wird. Bildung zielt nicht nur auf individuelle kognitive Potenzförderung, sondern auch und zugleich auf das Erlernen von Sozialkompetenz, Solidarität, Empathie, Mitmenschlichkeit, Demokratiekompetenz. (Klippert, 2008, S. 103)

Wenn es einen sehr hohen Anteil an individualisierten Unterrichtsangeboten gebe, könne dies nach Stevenson und Stigler zu einem verstärkten Einsamkeitsempfinden bei Kindern führen (Helmke, 2010, S. 257).

Wenn man eine enge Verknüpfung des Anspruchs individualisierten Unterrichts mit der Formulierung „allen Schülern besser gerecht zu werden“ (Laubenstein et al., 2015, S. 157) voraussetzt, kommen die Forscher des GeSchwind-Projekts zu dem Schluss, dass diese positive Vorerwartung weder im Primar- noch im Sekundarbereich erfüllt werden konnte: während 70\% der Grundschulen diese Vorerwartung angeben, sehen sie diese nach Einführung der Schwerpunktschule nur zu $53 \%$ als erfüllt an, bei den Schulen im Sekundarbereich liegt dieses Verhältnis bei 34\% positiver Vorerwartungen im Vergleich zu 20\% erfüllter Erwartungen (Laubenstein et al., 2015, S. 156f). Eine Spezifizierung der Qualitätskategorie Individualisierung erfolgt aus Sicht der Sonderpädagogik für die unterschiedlichen sonderpädagogischen Förderschwerpunkte. Aufgrund der Fülle der Modellversuche und Veröffentlichungen kann diese Kategorie an dieser Stelle allerdings nicht förderbedarfsspezifisch dargestellt werden ${ }^{43}$. Erläutert wird lediglich, wie die Möglichkeiten für die schulische Integration mit Blick auf die unterschiedlichen sonderpädagogischen Förderbedarfe eingeschätzt werden. Effekte der integrativen Unterrichtung für Schüler mit verschiedenen Förderschwerpunkten werden darüber hinaus unter 3.5.7 (Ergebnisse) beschrieben. Wesentlich für integrativen Unterricht ist zunächst die Unterscheidung zwischen zielgleicher und zieldifferenter Unterrichtung:

Sonderpädagogische Förderung für Schülerinnen und Schüler mit besonderem pädagogischem Förderbedarf orientiert sich an den Bildungsstandards der Allgemeinen Schulen, welche die angestrebten Kompetenzen und Qualifikationen als Leistungsstandards formulieren. [...] Ausgenommen von der Verpflichtung auf Kerncurricula und Bildungsstandards sind die Schülerinnen und Schüler mit sonderpädagogischem Förderbedarf in den Schwerpunkten Lernen und Geistige Entwicklung, die zieldifferent und nach eigenen curricularen Vorgaben zu unterrichten sind, um Überforderung zu vermeiden und individuell geeignete Förderung zu ermöglichen. (Wember \& Prändl, 2009, S. 45)

Wenn von schulischer Integration oder Inklusion gesprochen wird, werden oft Unschärfen hinsichtlich der erheblichen Unterschiede, die bspw. bei der integrativen Unterrichtung eines gehörlosen im Vergleich zur integrativen Förderung eines Kindes mit emotional-sozialen Problemen bestehen, in Kauf genommen:

Der Begriff Behinderung steht für sehr komplizierte und obendrein unterschiedliche Sachverhalte, die sich für simplifizierendes Über-einen-Kamm-scheren nicht eignen. Wenn es um den Grad der möglichen Integration geht, wird man nicht kategorial (d.h. nach der jeweiligen Behinderungsart) entscheiden können, sondern man wird Entscheidungen treffen müssen, die ganz auf den einzelnen behinderten Schüler zugeschnitten sind. (Heese, 1977, S. 105)

43 s. dazu u.a. Wember \& Prändl (2009); Schriber, S. (2010): Mit dem Rollstuhl durch die Baustelle „Schule für alle“. In: Jennessen, S.; Lelgemann, R.; Ortland, B.; Schlüter, M. (Hrsg.) (2010): Leben mit Körperbehinderung. Perspektiven der Inklusion. Stuttgart: Kohlhammer, S. 107-119; Jennessen, S. (2010): Spezifik in einer Pädagogik der Vielfalt - Schulische Inklusion körperbehinderter Kinder und Jugendlicher. In: Jennessen, S.; Lelgemann, R.; Ortland, B.; Schlüter, M. (Hrsg.) (2010): Leben mit Körperbehinderung. Perspektiven der Inklusion. Stuttgart: Kohlhammer, S: 120-134; Berges, 1996; Wilken, 1996; Wagner, M. (2013): Sind sie der Rest? Kinder und Jugendliche mit schwerer Behinderung in einem inklusiven Schulsystem. In: Zeitschrift für Heilpädagogik 64, 12, S. 496-501; Theunissen et al., 2009, S. $315 f$ 
Während Feuser und Meyer (1987) davon ausgehen, dass „von den behinderten Schülern, unabhängig von Art und Schweregrad ihrer Behinderung, keine spezifischen Probleme ausgehen, die nicht auch von anderen Schülern in einer spezifischen und zu lösenden Wiese ausgehen würden." (Feuser \& Meyer, 1987, S. 176; Herv. im Orig.), vertritt Preuss-Lausitz die Auffassung, dass Kinder mit unterschiedlichen Behinderungen den Unterricht unterschiedlich stark beeinflussen:

Zunächst einmal meine ich, dass die Heterogenität von Kindern mit Sinnes- und Körperbeeinträchtigungen keine Heterogenität ist, die den Unterricht belastet. Diese Formen der Beeinträchtigung sind das geringste Problem in einer inklusiven Klasse. Dem gegenüber stehen die Kinder und Jugendlichen, deren Lernentwicklung durch mangelnde soziale Kompetenz, Verhaltensauffälligkeiten oder auch mangelnde Sprachkenntnisse massiv beeinträchtigt wird. Das hat einen viel massiveren Einfluss auf den Unterricht. Nur ist diese Form der Heterogenität für Regelschulen eigentlich nichts Neues. Das sind Beeinträchtigungen, die heute in vielen großstädtischen Regelschulklassen Alltag sind und den Unterricht bestimmen. (Preuss-Lausitz, 2011, S. 48)

Besondere Empfehlungen zur spezifischen Förderung von Kindern mit unterschiedlichen Beeinträchtigungen bzw. Behinderungen sind auch von der Allgemeinen Pädagogik im Zusammenhang mit den Modellversuchen der 70er und 80er Jahre aufgegriffen und bspw. für Grundschullehrkräfte formuliert worden (Reinartz \& Sander, 1982). Schon zum damaligen Zeitpunkt wurde problematisiert, dass eine individuumzentrierte, defizitorientierte Sicht auf diese Kinder nicht angemessen sei: „Aus der Perspektive der Pädagogen sollten Lernschwierigkeiten, Verhaltensauffäl-ligkeiten und Leistungsminderungen vor allem auch als Schwierigkeiten erkannt werden, welche die Schule verursacht, verstärkt oder aufrechterhält." (Schwartz, 1982, S. 35). Nach Dumke, Krieger und Schäfer (1989) halten die meisten Lehrkräfte eine integrative Unterrichtung von Schülern mit Behinderungen dann für unproblematisch, wenn diese zielgleich erfolgen kann (Dumke et al., 1989, S. 138): „Am günstigsten wird die Möglichkeit der Integration leicht Körperbehinderter eingeschätzt. [...] Es folgen die Sprachbehinderten, Sehbehinderten und Hörgeschädigten." (Dumke et al., 1989, S. 138). Dagegen erfährt die mögliche Integration von Kindern mit Lernbehinderungen oder geistigen Behinderungen leichten und mittleren Grades, die eine zieldifferente Unterrichtung notwendig macht, weniger Zustimmung (möglich bei lernbehinderten Schülern 70\%, nicht möglich bei geistigen Behinderungen mittleren Grades 72\%) (Dumke et al., 1989, S. 139). Ähnlich schwierig wird auch die Integration von Schülern eingeschätzt, die zwar zielgleich unterrichtet werden können, deren Behinderungsgrad allerdings als mittel oder schwer eingestuft wird (Dumke et al., 1989, S. 151). Für Schüler mit Verhaltensproblemen liegt der Anteil der Lehrkräfte, die diese als mittel oder schwer bewerten bei $28 \%$ (Dumke et al., 1989, S. 141).

Bleidick formuliert in diesem Zusammenhang, dass sonderpädagogischer Förderbedarf ,immer auch in Abhängigkeit von den Aufgaben, den Anforderungen und den Fördermöglichkeiten der jeweiligen Schule zu definieren [sei]" (Bleidick, 2008, S. 34):

Er hat Konsequenzen für die Erziehung und für die didaktisch-methodischen Entscheidungen und die Gestaltung der Lernsituationen im Unterricht. Er ist damit eine didaktisch-methodische Bedingung der Erziehung und Unterrichtung, die nur individuell bestimmt werden kann und die in jedem neuen Lernzusammenhang eigens bedacht werden muss. (Bleidick \& Ellger-Rüttgardt, 2008, S. 34)

Verschiedene Studien kommen zu dem Schluss, dass es die verhaltensauffälligen Schüler sind, die die höchsten Anforderungen an integrativen Unterricht stellen (Feuser \& Meyer, 1987, S. 165; Dyson, 2010, S. 6; Ellinger \& Stein, 2012, S. 97ff; Laubenstein et al., 2015, S. 211). So 
führt Dyson aus: „Students with ,behaviour difficulties' in particular were seen as being difficult to include in mainstream schools and classrooms, and even the most committed teachers found these challenges hard to meet." (Dyson, 2010, S. 6). Von Amrhein befragte Lehrkräfte gaben dagegen an, dass sie die integrative Unterrichtung von Schülerinnen und Schülern mit dem Förderschwerpunkt geistige Entwicklung (77,6\%) sowie von denen mit Sinnesbeeinträchtigungen (Sehen, 65,8\%, Hören, 60,1\%) für besonders schwierig halten. 53,7\% standen einer integrativen Unterrichtung von Schülern mit dem Förderschwerpunkt soziale und emotionale Entwicklung ablehnend gegenüber (Amrhein, 2011, S. 147).

\subsubsection{Ergebnisse im Bereich der Unterrichtsentwicklung}

Ergebnisse des Unterrichts sind nicht präzise von Prozessen zu trennen, da sich Ergebnisse fortlaufend während der Prozesse verändern und Kompetenzen von Schülern und Schülerinnen sich permanent weiterentwickeln. Dennoch ist die Fokussierung dessen, was (qualitativ hochwertiger) Unterricht mit Blick auf die Lernergebnisse von Kindern und Jugendlichen erreichen kann, von hoher Relevanz für eine kritische Bewertung von Schulentwicklungsprozessen, nicht zuletzt, weil diese als Zielsetzung letztlich im Mittelpunkt aller Einzelmaßnahmen stehen: „Over the long term, the primary standpoint for judging any school reform is very clear: have substantial improvements in student learning occured?" (Bryk et al., 2010, S. 17). Die Einordnung von Lernergebnissen von Schülern und Schülerinnen im Rahmen von wissenschaftlichen Untersuchungen ist nach Bryk et al. (2010) allerdings aus verschiedenen Gründen problematisch. Eine Begründung dafür liegt darin, dass diese Ergebnisse in fast allen Studien bezugsgruppenorientiert erfasst und dargestellt werden, d.h. dass bspw. schulische Leistungen im Fach Mathematik im Vergleich zu einer Schulklasse, zu weiteren Schulklassen bzw. in einem nationalen oder internationalen Kontext erhoben und analysiert werden. Nur sehr vereinzelt wird bislang versucht, die individuelle Lern- und Kompetenzentwicklung von Schülern im Lernverlauf zu erfassen (Lütje-Klose et al., 2018), so wie es für die Qualitätsmessung vor allem mit Blick auf Schüler mit umfassendem Förderbedarf wesentlich wäre.

Erheblichen Einfluss auf die Verbesserung der Lesekompetenz haben nach Bryk et al. u.a. eine positive Arbeitsorientierung der Lehrkräfte (an $47 \%$ der beteiligten Schulen haben sich die Lesekompetenzen aufgrund dieses Bereichs verbessert), eine Anpassung des Curriculums (an Lernstufen und an Bedürfnisse von Schülern) (45\%) und die Schulleitung einer Schule (43\%). Einfluss auf die Verbesserung der mathematischen Kompetenzen haben die Schulleitung sowie die Einbindung der Eltern (je 42\%) und die Anpassung des Curriculums (40\%) (Bryk et al., 2010, S. 108-115). Das systemische und empirisch auf guten Grundlagen basierende Modell von Bryk et al. (2010) verdeutlicht sehr viel stärker als andere im Folgenden dargestellte Ergebnisse die Zusammenhänge, innerhalb derer schulische Leistungen von Schülern analysiert werden können. Es ist also grundsätzlich fraglich, ob die Ansätze bspw. schulische Leistungen von Schülern in integrativen bzw. nicht integrativen Klassen zu vergleichen ausreichen, um diese Leistungen tatsächlich als vergleichbar einstufen zu können. Allein die Anzahl der möglichen Einflussgrößen (schulische Faktoren, klassenbezogene Faktoren, Faktoren, die die Lehrkräfte betreffen) macht deutlich, dass Ergebnisse multifaktoriell bedingt und nicht allein auf die (Nicht-) Anwesenheit von Schülern mit Behinderung rückführbar sind.

Als Qualitätsmerkmale für Ergebnisse allgemein werden aus sonderpädagogischer Perspektive ein subjektiv wahrnehmbarer Prozess der Entwicklung der Schüler (Schley et al., 1992), Lerngewinne für alle Kinder (Speck, 2010) sowie objektivierbare Lernerfolge und Wissensinhalte (Schley et al., 1992) aufgeführt. Nach Brugger-Paggi beziehen sich die Ziele nicht 
nur auf den kognitiven, sondern gleichwertig auch auf den kommunikativen, sozialen und affektiven Bereich (Brugger-Paggi, 2003). Es überwiegen Untersuchungen zu den Ergebnissen schulischer Integration von Schülern mit Lernbehinderungen, Verhaltensauffälligkeiten oder geistigen Behinderungen. Dargestellt werden Auswirkungen schulischer Integration auf kognitive, emotionale und soziale Kompetenzen. Die meisten Studien, die sich auf die Ergebnisebene beziehen, sind - wie unter 3.2 dargestellt - Begleituntersuchungen zu Modellversuchen oder Erhebungen mit kleinen Stichproben, so dass Wocken resümiert: „Trotz erheblicher Nachfrage hat die Schulleistungsthematik in der wissenschaftlichen Begleitung von Integrationsversuchen erstaunlicherweise wenig Beachtung gefunden." (Wocken, 1987, S. 280). Ellinger \& Stein (2012) weisen in diesem Zusammenhang darauf hin, dass die sonderpädagogisch-fachliche Diskussion insgesamt vorwiegend von konzeptionellen Beiträgen bestimmt werde:

Grundsätzlich stellt sich die Forschungslage als sehr schwierig dar. Zum einen hat sich die deutsche Integrationsforschung offensichtlich, um mit Goetze (2008) zu sprechen, „einer soliden, methodischen Standards genügenden, gut kontrollierten Forschung gegenüber abstinent verhalten“ (ebd., 32). Zum anderen weist Huber $(2008,4)$ zurecht darauf hin, dass deutsche Forscher im Gegensatz zur international üblichen Sichtweise (vgl. Bless, 1995; Gasteiger-Klicpera \& Klicpera, 2001; Haeberlin et al., 1999) den Fokus eher auf Einzelfallanalysen setzen und damit häufig sehr positive Effekte insbesondere hinsichtlich der sozialen Integration zu berichten wissen. (Ellinger \& Stein, 2012, S. 89f)

Aufgrund seines Forschungsüberblicks geht Bless Anfang der 2000er Jahre davon aus, dass

[...] mit bestimmten Unterrichtsprogrammen und -strategien die Lernfortschritte behinderter Kinder in Integrationsklassen noch optimiert werden können. Sie sind jenen wenigen Forschungsarbeiten zuzuordnen, die die Frage zu beantworten versuchen, wie Integration sinnvoll zu bewerkstelligen ist. (Bless, 2003, S. 46)

Auch er weist also darauf hin, dass zum damaligen Zeitpunkt keine umfassenden empirischen Erhebungen zur Lern- und Leistungsentwicklung von Schülern mit Behinderungen in integrativen Schulen vorliegen. Ab den 2000er Jahren wird der Fokus auf die Ergebnisse schulischer Integration von der Anzahl der Veröffentlichungen her umfassender. Ein Überblick über die relevante Forschungsliteratur nach Venetz und Tarnutzer (2012) vermittelt die Einsicht, dass eine große Fülle an Studien existiert, die sich allgemein mit den Effekten schulischer Integration befasst haben, dass die Befundlage sich seit den Untersuchungen von Bless und Haeberlin allerdings nicht wesentlich verändert habe (Schüler in integrierten Schulformen erzielen größere Lernfortschritte als solche in segregierenden Schulformen, fühlen sich jedoch weniger gut integriert) und sich vor allem auf Schüler mit Verhaltensauffälligkeiten und Schulleistungsschwächen beziehe (Venetz \& Tarnutzer, 2012, S. 105). Gleichzeitig weisen sie darauf hin, dass „die Frage, wie Schülerinnen und Schüler mit besonderem Förderbedarf den schulischen Alltag im natürlichen Lernkontext - also im unmittelbaren Unterricht - subjektiv erleben, empirisch noch kaum systematisch untersucht worden [ist]“" (Venetz \& Tarnutzer, 2012, S. 105). LütjeKlose et al. haben im Rahmen der „Bielefelder Längsschnittstudie zum Lernen in inklusiven und exklusiven Förderarrangements" (BiLieF) die psychosoziale und die Leistungsentwicklung bei Kindern mit spf Lernen in inklusiven Klassen und in Förderschulen untersucht (3. bis 5 . Klasse). Im Wesentlichen kommen sie zu dem Ergebnis, dass „vor allem proximale Faktoren auf der Ebene der Einzelschule - weniger die Form der Beschulung - für die Entwicklung der Kinder relevant sind“ (Lütje-Klose et al., 2018, S, 109). 


\section{Kognitive Kompetenzen}

Hinsichtlich der Leistungsentwicklung von Schülerinnen und Schülern mit sonderpädagogischem Förderbedarf hat eine Reihe sonderpädagogischer Studien im deutschen Sprachraum diese vor und nach einer zeitlichen Phase schulischer Integration zu messen versucht (Bachmann, 1974; Speck et al., 1978; Wocken, 1987; Tent et al., 1991; Bless, 2003; Haeberlin et al., 2003; Kocaj et al., 2014; Textor, 2014; Spörer et al., 2015; Lütje-Klose et al., 2018). Dabei wurde vor allem geprüft, inwiefern diese sich bspw. in einer integrativen Klasse und einer Klasse in der Sonderschule voneinander unterscheiden oder Unterschiede zwischen integrativen und nicht integrativen Klassen innerhalb von Regelschulen vorliegen. Selbst wenn die untersuchten Gruppen hinsichtlich ihrer Lernvoraussetzungen vergleichbar waren, wurden andere Kriterien, die bspw. Unterrichtsmethodik, - didaktik etc. betreffen, nicht gleichzeitig kontrolliert bzw. umfassend in die Untersuchungen einbezogen. Die Untersuchungen beziehen sich ausschließlich auf Schüler mit den Förderschwerpunkten Lernen und sozial-emotionale Entwicklung.

Mit Blick auf Förderunterricht für so genannte "Grenzfälle" zwischen Sonder- und Gesamtschule im Rahmen des Modellversuchs an der Gesamtschule „Obere Aar" kam Bachmann (1974) zu dem Schluss, dass keine Verbesserungen bzw. Annäherungen an die Kontrollgruppe im Rechtschreiben, hinsichtlich der Leseleistungen bzw. im Fach Mathematik erzielt werden konnten, d.h. dass der beabsichtigte kompensatorische Effekt der Förderung nicht erreicht wurde (Bachmann, 1974, S. 277). Auch Speck kommt aufgrund des Modellversuchs Differenzierte Grundschule München zu dem Schluss, dass schulische Maßnahmen zur Reduktion Lernbeeinträchtigungen nicht ausreichen um diese komplett abzubauen (Speck et al., 1978, S. 162). Einer Studie von Reiser et al. (1984) zufolge haben sich durch die Tätigkeit von Sonderschullehrern weder der Anteil schulleistungsschwacher Schüler in einem Jahrgang noch das mittlere Leistungsniveau signifikant verändert (Reiser et al., 1984, S. 82-92).

Wocken (1987) beschreibt im Rahmen des Modellversuchs der Integrationsklassen in Hamburg - allerdings gegründet auf eine kleine Stichprobe (7 erste und 13 zweite Klassen) - signifikant bessere Leistungsergebnisse für Integrationsklassen im Vergleich zu Parallelklassen, vermutet insgesamt allerdings eine Pattsituation, also keine bedeutsamen Leistungsunterschiede der Schüler (Wocken, 1987, S. 79). Diese Schlussfolgerung begründet er damit, dass die Schüler in den Integrationsklassen zwar im Gesamtvergleich bessere Ergebnisse beim Zahlenrechnen, der Lesefertigkeit und dem Leseverständnis aufweisen, die Unterschiede allerdings zwischen den Integrationsklassen untereinander erheblich sind, so dass aus den Ergebnissen nicht verallgemeinernd geschlussfolgert werden könne, dass der Unterricht in einer Integrationsklasse im Vergleich zu einer "normalen“ Klasse ausschlaggebend für die Leistungsunterschiede sei (Wocken, 1987, S. 294ff):

Die eigentlichen Unterschiede sind nämlich den Untersuchungsergebnissen zufolge nicht $\mathrm{z}$ w is c h en den Systemen „Integrationsklasse“ und „Regelklasse“, sondern in n e r h a l b der Systeme zu finden. Vom System selbst geht kein entscheidender Einfluß auf die Leistungsentwicklung nichtbehinderter Kinder aus. (Wocken, 1987, S. 301, Herv. im Orig.)

Unterschiedliche Schulleistungen seien zu 20\% auf die Schulklasse und nur zu 5\% auf das Schulsystem zurückzuführen (Wocken, 1987, S. 301f). Auch Fend hat nach Wocken bereits 1980 herausgestellt, dass die Unterschiede zwischen einzelnen Gesamtschulen größer sind als zwischen Schulsystemen (Wocken, 1987, S. 302f). Und Bleidick hält fest:

Die wichtigste Erkenntnis lautet jedoch: Klasseneffekte sind allemal größer als Systemeffekte. Das gilt sowohl für die Leistungsentwicklung als besonders auch für die soziale Position der benachteiligten Kinder 
in integrativen Regelklassen. Es gibt in allen drei Versuchsgruppen gute und schlechte Klassen. Der Schulerfolg und die emotional-soziale Befindlichkeit eines Kindes hängen nicht in erster Linie von der Zugehörigkeit zu einem System - Integrative Regelklasse versus übliche Grundschulklasse - ab. Die jeweilige Bezugsgruppe des Lernorts entscheidet. (Bleidick, 1999, S. 131 zitiert n. Ellinger \& Stein, 2012, S. 103)

Wocken weist mit Blick auf die Hamburger Integrationsklassen darauf hin, dass Kinder mit kognitiven Einschränkungen in Regelklassen die schwierige Erfahrung machen, dass ein Lernabstand zwischen ihnen und ihren Mitschülern besteht, der mit zunehmendem Alter trotz Anstrengung größer wird (Wocken, 1987, S. 80).

Mit Blick auf die Fläming-Modellschule untersuchte Hetzner (1988) die Schulleistungen der Schüler in den Integrationsklassen und stellte fest, dass die Leistungen bei Mathematikarbeiten in den vier Klassen 3-6 besser als in nicht integrativen Klassen waren, während sie beim Rechtschreiben zwei Mal bessere und zwei Mal schlechtere Ergebnisse als die Vergleichsklassen erzielten (Hetzner, 1988, S. 251-254). In einer von Dumke veröffentlichten Teilstudie stellt dieser dar, dass sich in den fünf Integrationsklassen (Klasse 1-5) im Vergleich zu den Parallelklassen in den Lernbereichen Mathematik, Lesen und Rechtschreiben keine signifikanten Unterschiede in den T-Werten der genutzten Verfahren ergeben, allerdings eine größere Streuung der Werte vorliege (Dumke, 1991, S. 33-42). Untersucht an einer noch kleineren Stichprobe von drei Integrationsim Vergleich zu Parallelklassen kam Unterleitner (1990) zu dem Ergebnis, dass die Schüler in den Integrationsklassen signifikant mehr Rechenaufgaben in derselben Zeit lösen konnten, signifikant weniger Rechtschreibfehler machten und eine bessere Konzentration zeigten (Unterleitner, 1990, S. 9-16). Haeberlin, der die Lernentwicklung von lernbehinderten Schülern über 11,5 Jahre hinweg untersuchte, gelangt in seiner Studie (1990) zu dem Ergebnis, dass die Leistungssteigerung von lernbehinderten Schülern in Regelklassen höher sei als in Hilfsklassen, in Mathematik etwas deutlicher als in Deutsch (Haeberlin et al., 2003, S. 229ff). Auch Tent et al. kommen 1991 nach dem Vergleich der Unterrichtung von Förderschülern mit Lernbehinderungen in Sonderschulen im Vergleich zu ihrer Unterrichtung in gewöhnlichen Hauptschulen zu dem Schluss, dass diese Förderschüler in den Regelschulen vergleichsweise bessere Schulleistungen erbringen: „Sog. Lernbehinderte werden trotz objektiv günstigerer Lernbedingungen an der SfL (Schule für Lernbehinderte) nicht wirksamer gefördert, als dies an den Grund- und Hauptschulen der Fall wäre, wenn man sie dort beließe." (Tent et al., 1991). Nach Dumke und Schäfer (1993), die sowohl Leistungstests durchführten als auch Einschätzungen von Lehrern zu Schulleistungen der Schüler einbezogen, erzielten Schüler in integrativen Klassen in Mathematik und Englisch bessere Schulleistungen, während die Leistungen in Deutsch vergleichbar waren.

Bless (2003) fasst in seiner Veröffentlichung zunächst den Forschungsstand zur schulischen Integration zusammen und verweist zum damaligen Zeitpunkt darauf, dass - abgesehen von der Untersuchung durch Haeberlin et al. (1991) - kaum Ergebnisse zu Auswirkungen auf schulische Leistungen vorliegen. Die positive Wirkung integrativer Beschulung auf Schulleistungen im Vergleich zur Sonderbeschulung sei bislang für Schüler mit Lernbeeinträchtigungen, leichten geistigen Behinderungen und Hörbehinderung nachgewiesen worden (Bless, 2003, S. 44f). Bless interessiert sich dafür, welche Determinanten schulische Leistungen insgesamt beeinflussen, verweist zum Zeitpunkt seiner Veröffentlichung allerdings auf Helmke, der davon ausgehe, dass „geeignete theoretische Modelle des schulischen Lernens und seiner Bedingungen, die der Vielfalt der Determinanten und ihrer Verknüpfungen gerecht werden (Helmke 1992, S. 596602).“ (Bless, 2003, S. 144) nach wie vor fehlen würden. Bless nimmt an, dass eine Vielfalt von Determinanten schulischer Leistungsentwicklung mit Blick auf schulische Integration existiert, für die er insgesamt 47 Faktoren analysiert, zu denen u.a. Rahmenbedingungen organisatorisch- 
struktureller Art sowie Vorgehensweisen bei der pädagogischen Arbeit gehören (Bless, 2003, S. 145f). In seiner eigenen Untersuchung vergleicht er 24 "gewöhnliche“ Regelklassen mit 24 Regelklassen, in denen heilpädagogische Schülerhilfe umgesetzt wird und nimmt bei diesem Vergleich, der mit Hilfe des CFT $1^{44}$ sowie eines Mathe- und eines Sprachtests umgesetzt wird, schulleistungsschwache Schüler im zweiten Schuljahr in den Blick (Bless, 2003, S. 93-142). Sein Hauptergebnis:

Schulleistungsschwache Kinder in Regelklassen mit Heilpädagogischer Schülerhilfe des zweiten Schuljahres, welche zusätzlich zum Regelschulunterricht heilpädagogische Stützmaßnahmen erhalten, weisen bezüglich der Mathematik- und Sprachleistungen einen größeren Lernfortschritt auf als vergleichbare schulleistungsschwache Kinder in gewöhnlichen Regelklassen. (Bless, 2003, S. 135)

Im Forschungsprojekt „Soziale Integration, Schulzufriedenheit und Schulleistungen in Klassen mit und ohne Integration von Schülern mit dem Förderschwerpunkt emotionale und soziale Entwicklung" (SISSI), in dem 19 Klassen mit und 17 Klassen ohne Einzelintegration in 14 Grundschulen in NRW untersucht wurde, kommt Textor (2014) zu folgendem zentralen Ergebnis:

Kinder in Klassen mit Integration steigern ihre Leistungen weder langsamer noch schneller als Kinder in Klassen ohne Integration, und auch die Anzahl der doppelt besetzten Stunden in der Klasse oder der Kinder mit Verhaltensauffälligkeiten hat keinen Einfluss auf die Steigerung der Leseleistung. (Textor, 2014, S. 8)

Für die inklusiven Pilotgrundschulen in Brandenburg halten Dumont et al. (2015) fest, dass die Kompetenzen der Schülerinnen und Schüler in inklusiven Klassen im Vergleich zur Normstichprobe zunächst niedriger waren, was die Autorinnen und Autoren auf die Zusammensetzung der Klassen zurückführen. Im Verlauf von zwei Jahren ergab sich als zentrales Ergebnis,

[...] dass die Positionsverschiebungen in Relation zur Normstichprobe - d.h. einer repräsentativen Stichprobe von Schülerinnen und Schülern des gleichen Alters - relativ gering ausfallen und grundsätzlich von ähnlichen Zuwächsen wie in der Normstichprobe ausgegangen werden kann. (Dumont et al., 2015, S. 105)

Bei einer Schätzung von Effekten einer inklusiven Beschulung auf Kinder mit den spF Lernen, Sprache sowie soziale und emotionale Entwicklung anhand der Querschnittsanalyse einer bundesweit durchgeführten Large-Scale-Assessment-Studie (IQB-Studie 2011 ${ }^{45}$ ) mit vergleichsweise großem Stichprobenumfang, gelangen Kocaj et al. (2014) zu dem Ergebnis, dass ein positiver Einfluss der Regelbeschulung festzustellen sei (Kocaj et al., 2014. S. 184). Die Schüler mit $\mathrm{spF}$ in den untersuchten Regelschulen zeigten in den untersuchten Bereichen Lesen, Zuhören und Mathematik durchschnittlich höhere Leistungen als Schüler dieser Jahrgangsstufe (4) in den entsprechenden Förderschulen.

Eine nach Förderschwerpunkten differenzierte Betrachtung ergab, dass insbesondere Schüler mit dem Förderschwerpunkt Lernen vom Unterricht in Regelschulen zu profitieren scheinen. Für Kinder mit dem Förderschwerpunkt Sprache waren die Leistungsunterschiede in den sprachlichen Kompetenzen in Abhängigkeit von der Art der Beschulung hingegen geringer ausgeprägt. Für das Fach Mathematik konnte kein bedeutsamer Leistungsunterschied zwischen Regel- und Förderschülern mit dem Förderschwerpunkt Sprache nachgewiesen werden. (Kocaj et al., 2014, S. 181).

44 CFT $1=$ Grundintelligenztest Skala 1, Hogrefe Verlag 45 IQB-Studie = Studie des Instituts zur Qualitätsentwicklung im Bildungswesen, Humboldt-Universität, Berlin 
Einschränkend weisen die Autoren mit Blick auf diese Ergebnisse allerdings darauf hin, „dass die Schätzungen der Lernzuwächse nicht anhand von Leistungsentwicklungen einzelner Schüler sondern durch querschnittliche Vergleiche von Alterskohorten ermittelt wurden" (S. 181). Darüber hinaus wurde der Schweregrad der Beeinträchtigung der Schüler mit spF nicht erhoben (S. 183), so dass der direkte Vergleich mit Schülerinnen und Schülern in Förderschulen nicht zulässig sei.

In ihrer Längsschnittstudie BiLief untersuchten Lütje-Klose et al. (2018) die Lese- Rechtschreibentwicklung von Schülerinnen und Schülern mit spF Lernen in inklusiven Grundschulklassen, in Grundschulen mit Unterstützung durch ein Sonderpädagogisches Kompetenzzentrum sowie in Förderschulen (in einem Zeitraum von der dritten bis zur fünften Klasse). Die Teilstichproben in den inklusiven Grundschulen und in den Förderschulen wurden hinsichtlich der Variablen sozioökonomischer Status des Elternhauses und Migrationshintergrund operationalisiert (Lütje-Klose et al., 2018, S. 112). Grundsätzlich konnte die Studie feststellen, dass Schülerinnen und Schüler in allen Settings ihre Lese- und Rechtschreibkompetenzen ausweiten. Ähnlich wie in anderen Studien auch verfügen SuS mit dem spF Lernen in Regelschulen über Leistungsvorsprünge im Vergleich zu den SuS, die Förderschulen besuchen. Das Autorenteam führt dies jedoch auf Selektions- bzw. Allokationseffekte zurück, „da sich die Kompetenzentwicklung in beiden Gruppen nicht bedeutsam und konsistent unterscheidet" (Lütje-Klose et al., 2018, S. 114). Während die Drittklässler in inklusiven Klassen eine etwas schnellere Entwicklung ihrer Lesekompetenzen aufweisen, trifft dies für SuS in Förderschulen mit Blick auf die Entwicklung der Schreibkompetenzen zu (Lütje-Klose et al., 2018, S. 114).

Untersuchungen zur integrativen Beschulung von Schülern mit dem Förderschwerpunkt soziale und emotionale Entwicklung kommen nach Ellinger und Stein (2012) zu unterschiedlichen Befunden. Willmann (2007) belegt für ein integratives Setting in NRW (Schulversuch Verbundschule (L,S,E)), dass Schüler mit dem Förderschwerpunkt emotional-soziale Entwicklung sich mit geringeren Leistungen zufriedengeben, als sie diese in der Förderschule mit dem Schwerpunkt soziale und emotionale Entwicklung hätten erzielen können. Nach Lindsay (2007) seien positive Effekte inklusiver Beschulung auf akademische Leistungen noch nachzuweisen. Gemäß Gasteiger-Klicpera und Klicpera (2001, S. 12) würden aus Sicht der Lehrer schwächere Leistungen mit sozial problematischen Verhaltensweisen korrelieren. Huber und Wilbert (2012) gelangen zu dem Ergebnis, dass der Zusammenhang zwischen erhöhtem Förderbedarf und schlechten Schulleistungen unklar sei. Insgesamt lässt sich zusammenfassen, dass Leistungssteigerungen bei Kindern mit dem Förderbedarf Lernen erkennbar, dagegen bei Kindern mit dem spF soziale und emotionale Entwicklungen in integrativen Lernumgebungen nicht feststellbar sind.

Mit Blick auf die Leistungsentwicklung nicht behinderter Kinder in integrativen Klassen fassen Feuser und Meyer (1987) hinsichtlich des Modellversuchs Robinsbalje in Bremen zusammen:

Unsere Ergebnisse verweisen darauf, daß in der Tendenz eher nichtbehinderte Schüler mehr vom integrativen Unterricht profitieren als behinderte Schüler [...]. Die Gründe liegen darin, daß das hoch differenzierte Unterrichtsangebot Schüler, denen viele und differenzierte Denk- und Handlungsmöglichkeiten zur Verfügung stehen, intensiver nutzen können als Schüler, die nicht so umfassende Möglichkeiten und Strategien des Selbst-Tuns entwickelt haben. (Feuser \& Meyer, 1987, S. 203)

Wocken schlussfolgert aus der Studie in den Hamburger Integrationsklassen für die nichtbehinderten Schüler, dass diese genauso viel lernen wie in regulären Grundschulklassen: „Bei allen Leistungsprüfungen zeigen sich keine Unterschiede zwischen Integrationsklassen und Grundschulklassen." (Wocken, 1987, S. 79). Feyerer (1998) erhob Daten über Schulleistungen von 
nichtbehinderten Kindern nach 8 Jahren Integrationserfahrung (S. 102f) in neun Integrationsklassen (139 Schüler) mit 23 Parallelklassen ( 512 Schülern) als Kontrollgruppe. Es ergaben sich keine signifikanten Unterschiede in Deutsch, Mathematik und Englisch (Feyerer, 1998, S. 135f). Daraus schlussfolgert er, dass die Anwesenheit behinderter Kinder auf nichtbehinderte Kinder weder positive noch negative Auswirkungen habe (Feyerer, 1998, S. 177ff).

Nachteilige Auswirkungen schulischer Integration auf die Leistungsmotivation wurden vor allem für Schüler mit Verhaltensauffälligkeiten festgestellt (Preuss-Lausitz \& Textor, 2006, S. 5) und zwar sowohl in Regelklassen als auch in Verbundklassen mit sprach- und lernbehinderten Schülern (Willmann, 2007).

Ein Schüler kann sich dann als leistungsmotivational integriert einschätzen, wenn er sich den in der Schule gestellten Aufgaben gewachsen fühlt und diese demzufolge motiviert in Angriff nimmt. Leistungsmotivation ist eine von der Schule in besonderem Maße erwartete und positiv bewertete Eigenschaft eines Schülers. Sie spielt für die Wertorientierung in den schulischen Bezugsgruppen eine zentrale Rolle. (Haeberlin et al., 1989, S. 14)

Genau darin sehen die von Dumke et al. (1989) befragten Lehrkräfte ein Problem, da Schüler mit (Lern-) Behinderungen in Regelklassen überfordert sein bzw. nicht ausreichend gefördert werden könnten (Dumke et al., 1989, S. 136).

Schon 1974 gelangte Bachmann aufgrund der Ergebnisse des Schulversuchs Obere Aar (s. dazu 3.5.8.2) (keine kompensatorischen Effekte von Förderunterricht auf Kinder mit Lernbeeinträchtigungen) zu der Schlussfolgerung, dass Schüler mit Lernbeeinträchtigungen bei der damaligen Struktur und Organisation der Grundschule nicht zu Leistung und Geltung kommen können und stellte daraufhin die Frage:

[...] inwieweit die „Leistung“ als

a) Schulische Forderung an den Schüler,

b) Tätigkeit der Schüler,

c) Ergebnis der Tätigkeit des einzelnen Schülers,

d) Besonderer Beitrag der Schule für Gesellschaft, Staat und Wirklichkeit (vgl. Furck, S. 118)

nicht der auslösende bzw. ursächliche Faktor dessen ist, was mit teils emotionaler und wenig reflektierter Stereotypienbildung als sog. „Separierung“, „Segregation“ bzw. Abwärtsdrängung ins (Sonderschul-) Ghetto usw. angesprochen und neuerdings von Hellbrügge sarkastisch als „Soziose“ bezeichnet wird. (Bachmann, 1974, S. 279f, Herv. im Orig.)

Als mögliche konkrete Ergebnisse kognitiver Entwicklung werden in den sonderpädagogischen Veröffentlichungen Lern- und Entwicklungsfortschritte (lebenspraktische Fertigkeiten, Einhalten sozialer Regeln, sprachliche Entwicklung) (Wocken, 1987), Kommunikation und Sprache (Wember \& Prändl, 2009), Kommunikationsfähigkeit (Speck, 2010), die Vermittlung grundlegender Kenntnisse in den Kulturtechniken sowie die Vermittlung spezifischer Schrift- und Kommunikationstechniken (z.B. Brailleschrift, Gebärdensprache) (Wember \& Prändl, 2009), das Ausschöpfen aller individuellen Entwicklungsmöglichkeiten, die Akzeptanz der eigenen Behinderung und ein bewusster Umgang mit ihr, eine realistische Selbsteinschätzung sowie die Kenntnis von Arbeits- und Lernstrategien benannt (Wember \& Prändl, 2009).

\section{Emotionale Kompetenzen/Selbstkonzept}

Emotionale Kompetenzen oder ein tragfähiges Selbstkonzept werden als Ergebnisse schulischer Integration von Wember \& Prändl (2009) sowie Speck (2010) aufgeführt. Mit Blick auf diese 
Kompetenzen ist zu berücksichtigen, dass die Ergebnisanalyse - wie oben bereits angemerkt - in Studien jeweils bezugsgruppenorientiert vorgenommen wird, d.h. meistens in Anbetracht der Situation eines einzelnen Schülers innerhalb einer (Jahrgangs-) Klasse erfolgt. In Anlehnung an die Bezugsgruppentheorie gehen die entsprechenden Autoren davon aus, dass „Wertorientierungen, Einstellungen und Handlungsmotive einer Person von der sozialen Bezugsgruppe abhängen (Runicman 1968, 69)“ (Haeberlin et al., 1989, S. 11). Außen vor bleiben also andere Bezugsgruppen wie bspw. die Familien oder Peers außerhalb der Klasse bzw. Schule. Haeberlin et al. unterscheiden in ihren Untersuchungen zwischen Dimensionen der sozialen, der emotionalen und der leistungsmotivationalen Integration (Haeberlin et al., 1989, S. 12f).

Haeberlin et al. (1989) gehen davon aus, dass die Emotionalität von Schülern (mit spF in integrativen Klassen) eine zentrale Rolle spielt:

Das emotionale Wohlbefinden gehört zu den Grundbedingungen einer pädagogisch verantwortbaren und fruchtbaren Förderung von Schülern, insbesondere im Falle einer vorliegenden oder drohenden Behinderung (Bach 1987, 738). (Haeberlin et al., 1989, S. 13)

Die Befunde zum emotionalen Wohlbefinden von Schülern mit Beeinträchtigungen in integrativen Klassen sind nicht einheitlich. Während Haeberlin et al. (1991) sowie Tent et al. (1991) aufgrund ihrer Forschungsergebnisse zu dem Schluss kommen, dass Schüler mit dem Förderbedarf Lernen sich in sonderschulischen Settings häufig besser fühlen als in Regelschulen, in denen negative Wirkungen auf das Selbstkonzept messbar seien, kommt Feyerer (1998) zu dem Ergebnis, dass das Leistungsselbstkonzept und das Selbstwertgefühl integrativ unterrichteter Schüler signifikant positiver und deren soziales Selbstkonzept vergleichbar sei (Feyerer, 1998, S. 140). Schülerinnen und Schüler in Integrationsklassen fühlen sich nach Feyerer in der Schule hoch signifikant wohler und sind bedeutsam zufriedener mit Unterricht und Lehrern (Feyerer, 1998, S. 146) ${ }^{46}$. Gasteiger-Klicpera und Klicpera (1997a, 1997b) weisen darauf hin, dass eine schlechte soziale Integration in den Regelschulen negative Folgen auf das Selbstkonzept haben können (n. Ellinger \& Stein, 2012). Insgesamt erläutern Ellinger und Stein (2012) aufgrund eines von ihnen erstellten Forschungsüberblicks, dass die Befundlage zum emotionalen Wohlbefinden uneinheitlich sei (Ellinger \& Stein, 2012, S. 92f).

Dabei besteht nach Green u.a. ein reziproker Zusammenhang zwischen Leistungen bzw. Leistungsrückmeldungen und Selbstkonzept:

Put simply, improved academic self-concepts and motivation will lead to better academic achievement, and improved achievement will lead to better academic selfconcept and academic motivation. (Green et al., 2006, S. 543)

Das Begabungskonzept von Schülern mit Lernbeeinträchtigungen ist nach Bless (2003) in Integrationsklassen niedrig, was am Ende der Schulzeit allerdings auch in der Förderschule Lernen der Fall sei. Ähnlich schwierig verhalte sich dies für gehörlose Schüler, weniger problematisch sei dies für Schüler mit dem Förderschwerpunkt körperlich-motorische Entwicklung (Bless, 2003, S. 42ff). Insgesamt belegen Untersuchungen aus der Schweiz (Haeberlin et al., 2003; Bless, 2003; Venetz \& Tarnutzer, 2012), dass die Selbsteinschätzung von Schülern mit Lernbehinderungen - zumindest bis zur siebten, achten Klasse - in den so genannten Hilfsklassen besser ist als in Regelschulklassen (Haeberlin et al., 2003, S. 215f). Unabhängig von der Be-

46 Feyerers Studie umfasst 9 I-Klassen mit 139 Schülern und 23 Parallelklassen mit 512 Schülern als Kontrollgruppe (nach 8 Jahren Integrationserfahrung). 
schulungsart haben Lernbehinderte und Schwerhörige ein tieferes Selbstbild als andere Schüler, besonders schlechte Werte liegen diesbezüglich bei Schülern vor, die heilpädagogische Unterstützung erhalten (Bsp.: Einzelförderung), in L-Klassen ist das Begabungskonzept besser (Bless, 2003, S. 22ff). Lindsay (2007) ermittelt durch zwei Studien keine Unterschiede zwischen integrativem und inklusivem Setting (n. Ellinger \& Stein, 2012). Preuss-Lausitz und Textor (2006) stellen fest, dass sich integrativ beschulte Schüler mit dem Förderschwerpunkt emotionale und soziale Entwicklung hinsichtlich ihres Wohlbefindens nicht signifikant von ihren Mitschülern (Klasse 1-6) unterscheiden. Venetz und Tarnutzer (2012) haben in ihrer Studie 719 Kinder mit und ohne Verhaltensauffälligkeiten oder Schulleistungsschwächen aus 40 Regelklassen befragt und halten fest:

Die Vergleiche zeigen, dass Kinder mit einer Schulleistungsschwäche ihre schulischen Fähigkeiten zwar deutlich tiefer einschätzen, sich ihre Befindlichkeit im Unterricht aber dennoch durch eine hohe positive Aktivierung kennzeichnen lässt. Kinder mit einer Verhaltensauffälligkeit beurteilen die sozialen Beziehungen zu ihren Mitlernenden deutlich weniger positiv und erleben im Unterricht mehr negative Befindenszustände wie Stress oder Ärger. (Venetz \& Tarnutzer, 2012, S. 102)

Das Fähigkeitsselbstkonzept hat nach Venetz und Tarnutzer generell betrachtet keinen Einfluss auf das durchschnittliche Befinden im Unterricht, bei Kindern mit Verhaltensauffälligkeiten spiele es allerdings eine wichtige Rolle (Venetz und Tarnutzer, 2012, S. 115). Hennemann et al. stellen Unterschiede hinsichtlich des akademischen Selbstkonzepts zwischen Schülerinnen und Schülern mit und ohne spF fest. Negative Abweichungen seien diesbezüglich insbesondere bei Kindern mit den spF Lernen und Sprache deutlich (Hennemann et al., 2014, S. 34).

Lütje-Klose et al. stufen die Ergebnisse ihrer Längsschnittstudie zur psychosozialen Entwicklung von Grundschulkindern (Wohlbefinden und Selbstkonzept) positiv ein, da sich - anders als in den o.g. Studien - durchschnittlich keine Unterschiede zwischen Kindern ergeben, die in Regel- bzw. in Förderschulen unterrichtet werden. Sie führen diese Ergebnisse mit Blick auf die Schülerinnen und Schüler in inklusiven Klassen darauf zurück, dass möglicherweise stigmatisierend wirkende Vergleichserfahrungen in diesem Alter ggf. noch nicht so stark greifen wie bei älteren Schülern. Einer Analyse von Gorges et al. nach scheine „die Einschätzung des akademischen Selbstkonzeptes in inklusiven Settings von der tatsächlichen Leistungsentwicklung „entkoppelt““ (Lütje-Klose et al., 2018, S. 114). Zu berücksichtigen sei allerdings, dass es sich bei dem Ergebnis um Durchschnittswerte handele. Innerhalb der Schulen sei aber eine hohe Streuung der Werte gegeben, was ein Hinweis auf die Relevanz der einzelnen Schule bzw. Klasse sei.

\section{Soziale Integration und soziale Kompetenzen}

Gelungene soziale Integration bedeutet als Idealvorstellung, daß zwischen allen Schülern befriedigende soziale Kontakte möglich und Abgrenzungen zwischen unterschiedlichen Schülerkategorien wie gescheit/dumm, leistungsschwach/leistungsstark, behindert/nicht behindert aufgelöst werden (Kobi 1983, 203). (Haeberlin et al., 1989, S. 12)

Soziale Integration und der Erwerb sozialer Kompetenzen werden als mögliche Ergebnisse schulischer Integration thematisiert (Wember \& Prändl, 2009; Speck, 2010). Modellversuche und die sie begleitenden Forschungsprojekte befassten sich in diesem Zusammenhang u.a. mit der Integration von Schülern in Kleinklassen, die in Regelschulen eingerichtet wurden. Diese Einrichtung beruhte auf einem Vorschlag der KMK von 1960 hinsichtlich der Unterrichtung von Kindern, die nachhaltig stören, bei denen aber erwartet werde, dass „durch eine stärkere 
Einzelbetreuung die Fehlhaltung gemindert oder beseitigt und damit eine Rückführung in eine allgemeine Klasse ermöglicht...werden kann.“ (KMK, 1960, S. 44). In sonderpädagogischen Untersuchungen werden als Ergebnisse eher negative Erfahrungen wie eine soziale Außenseiterstellung und Ablehnung geschildert. Aus dieser Erfahrung heraus erfolgte teilweise ein Übergang zu stundenweiser Einzelförderung nach dem Modell der schwedischen Schulkliniken (Preuss-Lausitz, 1981, S. 117). So wurden der Versuch der IGS Obere Aar nach 5 Jahren integrierter schulischer Erziehungshilfe in Köln-Bocklemünd sowie der Versuch mit Kleinklassen in Hamburg abgebrochen (Preuss-Lausitz, 1981, S.118) und durch Förderung, die teilweise in den Unterricht der Stammgruppe einbezogen war, ersetzt. Als Schlussfolgerung aus diesen Versuchen wurde festgehalten: „[...] die Hamburger und Kölner haben berichtet, daß es sowohl im Sinne des schulischen Lernens als auch der Persönlichkeitsentwicklung der Kinder fruchtbarer ist, mit dem zusätzlichen Personal den Regelunterricht zu verändern, die 'sozialpädagogische' Schule also nicht im Getto einer Klassen, sondern für alle einzurichten." (Preuss-Lausitz, 1981, S. 120). Tardif und Wiener (2004) konnten nach Ellinger und Stein (2012) belegen, dass Kinder mit klasseninterner Unterstützung von Lehrkräften als weniger verhaltensauffällig beurteilt wurden als Kinder, die in einem gesonderten Raum unterrichtet wurden (Ellinger \& Stein, 2012, S. 92).

Für den Schulversuch Obere Aar stellt Bachmann (1974) dar, dass soziometrische Tests mit 329 Schülern aus 11 Grundschulklassen belegen, dass Schüler, die an Förderkursen teilnehmen zu sozialer Randständigkeit neigen und selten als „Freund“ begehrt werden (Bachmann, 1974, S. 270f). Nach Reiser et al. (1984) ergaben Soziogramme im Rahmen eines Schulversuchs an zwei Frankfurter Grundschulen, dass geförderte Schüler nicht stigmatisiert wurden. Allerdings wurden zehn Schüler aus diesen Klassen herausgenommen, bei denen es zu einer destruktiven Interaktion zwischen Klassenlehrer und Kind gekommen war, erhebliche Leistungsrückstände vorlagen und die Kinder sich aufgrund dessen unwohl und belastet gefühlt hatten. Reiser et al. führen diese Situation darauf zurück, dass es einige für das emotionale Wohlbefinden entscheidende Faktoren gibt, die zum damaligen Zeitpunkt nicht durch die Sonderschullehrer beeinflusst werden konnten wie das Schulklima, Erfahrungen aus den Elternhäusern, das soziale Umfeld der Kinder insgesamt, die Haltungen der Lehrkräfte sowie unberechenbare Dynamiken, die zwischen Personen entstehen können (Reiser et al., 1984, S. 45f). Nach Wocken (1987) liegen behinderte und nichtbehinderte Kinder mit Blick auf Wahl- bzw. Ablehnungsstatus in den Hamburger Integrationsklassen gleichauf (Wocken, 1987, S. 236f). Behinderte und nichtbehinderte Kinder sind auf die sechs soziometrischen Typen der Untersuchung allerdings ungleich verteilt: so gibt es einen geringeren Anteil an positiv und einen höheren Anteil an neutral oder negativ besetzten Rollen (Wocken, 1987, S. 243ff). Auffällige Unterschiede ergeben sich dahingehend, dass Kinder mit körperlichen oder geistigen Behinderungen eher gleichermaßen gewählt werden, während Kinder mit „unsichtbaren Behinderungen “ (Lernen, Sprache, Verhalten) weniger in positive Rollen gewählt werden (Wocken, 1987, S. 246). Der Beziehungsaspekt (emotionaler Austausch) innerhalb der Gruppen kann als ausgeglichen eingestuft werden (Wocken, 1987, S. 247). Maikowski und Podlesch (1988) untersuchten von 1984-1986 die soziale Entwicklung behinderter und nicht behinderter Kinder in drei Integrationsklassen der Fläming-Grundschule. Sie können insgesamt eine positive Sozialentwicklung feststellen (Bsp.: Modellfunktion der Schüler füreinander, gegenseitige Rücksichtnahme, gutes Lernklima). Aufgrund der soziometrischen Befragungen ergibt sich das Ergebnis, dass zu den besonders abgelehnten Kindern hyperaktive und aggressive Kinder ohne spF zählen (Maikowski \& Podlesch, 1988, S. 232-250). Goetze berichtet dies aufgrund eines Forschungsreviews für Schüler mit Verhaltensstörungen: 
Die Zielgruppe verhaltensgestörter Schüler bietet aufgrund ihres Erscheinungsbildes die denkbar ungünstigsten Voraussetzungen zur integrativen Unterrichtung mit nicht gestörten Regelschülern. Sie sind in der Integrationsklasse von sozialer Ablehnung betroffen, sehen sich selbst sozial- und leistungsbezogen in ungünstigem Licht und neigen unter der Bedingung der Hyperaktivität dazu, negativ modellierend zu wirken. Damit scheint die Annahme, dass ihre Platzierung im Regelschulmilieu positive Effekte bei ihnen auslösen und Verhaltensänderungen erleichtern könnte, nicht haltbar zu sein. (Goetze, 2008, S. 50).

Dumke et al. (1989) berichten von positiven Lehrereinschätzungen zu sozialem Lernen und sozialer Entwicklung nichtbehinderter Schüler im gemeinsamen Unterricht (Dumke et al., 1989, S. 135). Vorteile in Form von Gefühlen der Akzeptanz und des Dazugehörens werden auch für Kinder mit Behinderung gesehen (Dumke et al., 1989, S. 136). Allerdings befürchten Lehrkräfte auch „negative Auswirkungen auf das Selbsterleben und Probleme in der Auseinandersetzung mit den nichtbehinderten Schülern. So werden Frustrationen beim Erleben der behinderungsspezifischen Grenzen erwartet." (Dumke et al., 1989, S. 137). Kontakte mit behinderten Kindern finden nach Elternangaben (Dumke et al., 1989, S. 37) zu 25\% häufig und zu $75 \%$ gelegentlich statt. Während die Kommunikation und Interaktion mit geistig behinderten Schülern aufSeiten der nichtbehinderten Schüler in der Untersuchung von Berges unproblematisch erfolgt, ist der Umgang von nichtbehinderten Schülern mit schwer behinderten Schülern nicht einfach:

Eine abweichende Reaktion zeigen die Schüler gegenüber bestimmten (schwerbehinderten) Ss. Die anfänglich neutrale/negative Bewertung gegenüber diesen Ss wird aufrechterhalten, da die kognitive Situationsanalyse immer wieder auf die ungelösten Situationsmerkmale, wie aversive/aggressive Kontakte, Kommunikationsabbruch oder Anderssein, stößt. Es erfolgt keine oder unbefriedigende Informationsverarbeitung hinsichtlich jener Ss, und trotz entspannter Unterrichtsatmosphäre kommt es zu einer konnotativen affektiven Änderung. Eine zurückhaltende, unsichere, auch ablehnende Reaktion ist die Folge. (Berges, 1996, S. 260)

Die Unbeliebheit bestimmter Schüler hängt nach Berges mit individuellen Merkmalen des Sozialverhaltens zusammen, während die Beliebtheit sich eher an allgemeinen Faktoren wie Geschlecht oder ähnlichen Interessen bzw. ähnlicher Kleidung festmachen lasse (Berges, 1996, S. 258).

Für Heyer et al. (1997) ergeben sich bei einer Lehrer-, Eltern-, Schülerbefragung in Brandenburg zwar insgesamt positive Ergebnisse bezüglich Einstellungsveränderungen, nicht aber mit Blick auf soziometrische Kenndaten (S. 123-204). Bless unterscheidet in seinem Forschungsüberblick zwischen verschiedenen Förderschwerpunkten und erläutert, dass sich die niedrige soziometrische Stellung von Kindern mit den Förderschwerpunkten Lernen oder Verhalten in Regelklassen bestätigt, während Schüler mit den Förderschwerpunkten körperlich-motorische oder geistige Entwicklung eine vergleichsweise positive Stellung innehaben (Bless, 2003, S. 18-21; S. 41f). Auch Haeberlin et al. (2003) äußern sich diesbezüglich sehr kritisch:

Alle uns bekannten soziometrischen Untersuchungen [...] zeigen, daß das Ziel der sozialen Integration im Sinne der skizzierten normativen Vision bezüglich schwacher und verhaltensauffälliger Kinder in keinem der bisherigen europäischen und amerikanischen Integrationsmodelle auch nur annähernd erreicht worden ist. (Haeberlin et al., 2003, S. 153)

Textor (2008) schlussfolgert aus einer Studie in einem Berliner Integrationsprojekt, dass Schülerinnen und Schüler mit dem spF soziale und emotionale Entwicklung „meist einen relativ 
ungünstigen Sympathiestatus haben“ (Textor, 2008, S. 102) und ein Drittel dieser SuS eine Außenseiterposition in der Klasse einnehme (Textor, 2008, S. 102).

Ähnlich wie Venetz und Tarnutzer (2012) weist Ahrbeck (2011) darauf hin, dass Bezugsgruppe und Lehrperson unter Umständen stärkere Effekte haben als integrative oder separierende Beschulung (Ahrbeck, 2011, S. 39f; n. Ellinger und Stein, 2012, S. 103). Huber (2009) geht davon aus, dass die Hypothese der „egalitären Differenz" (nach Prengel 1999) in Deutschland durch Modellversuchsergebnisse aus den 80er- und 90er-Jahren insofern als bestätigt gilt, dass „die Heterogenität von Lerngruppen einen günstigen Einfluss auf die soziale Integration von Schülern mit Sonderpädagogischem Förderbedarf hat. Seither zähle die „integrationsfähige" Schule nicht mehr als pädagogische Utopie, sondern als real existierendes Modell." (Huber, 2009, S. 243). Bislang sei eine direkte empirische Überprüfung dieser Annahme in Deutschland allerdings nicht unternommen worden. In einer Studie mit 650 Viertklässlern in Nordrhein-Westfalen, die an Grundschulen mit Gemeinsamem Unterricht durchgeführt wurde, gelangte Huber zu folgenden Befunden: Zur Grundannahme 1: „je heterogener eine Lerngruppe, desto besser die zu erwartende soziale Integration von Schülern mit Sonderpädagogischem Förderbedarf“ (in dieser Studie L, S und SE) kann Huber keine Anhaltspunkte für eine solche Wirkung erheben, so dass er schlussfolgert, dass es „keine signifikante Wirkung der Heterogenität der Lerngruppen auf die soziale Integration von Schülern mit Sonderpädagogischem Förderbedarf gab“ (Huber, 2009, S. 244). Mit Blick auf die zweite Grundannahme, dass „soziale Integration von Schülern mit Sonderpädagogischem Förderbedarf im Gemeinsamen Unterricht“ günstig sei (abgeleitet aus Modellversuchen Hamburg, Bremen, Berlin und Bonn), erläutert er: „Die Befunde zeigen, dass sich die soziale Integration von Schülern mit und ohne sonderpädagogischen Förderbedarf im Gemeinsamen Unterricht signifikant unterscheidet [...]“ (Huber, 2009, S. 245), d.h. dass Schüler mit Förderbedarf signifikant seltener „beliebt“ oder „durchschnittlich integriert“ sind. So erfahre fast jeder zweite Schüler mit Sonderpädagogischem Förderbedarf Ablehnung (Huber, 2009, S. 245) Darüber hinaus stellt Huber in seiner Studie heraus, dass „mit der Schulleistung (.721) und der Leistungsmotivation (.672) eindeutig leistungsbezogene Eigenschaften den stärksten Einfluss auf die soziale Integration von Schülern mit Sonderpädagogischem Förderbedarf im Gemeinsamen Unterricht“ (Huber, 2009, S. 245) haben.

Danach folgen mit Belastbarkeit (.378), Selbstreflexion (.285) und Selbstständigkeit (-.250) drei Variablen des Sozialverhaltens, für die zumindest ein schwacher Einfluss auf die Entstehung sozialer Ausgrenzungsprozesse bei Schülern mit Sonderpädagogischem Förderbedarf im Gemeinsamen Unterricht angenommen werden kann. Beim letztgenannten Koeffizienten steht der negative Wert für den überraschenden Umstand, dass eine möglichst geringe Ausprägung der Selbstständigkeit zu einer höheren sozialen Integration von Schülern mit Sonderpädagogischem Förderbedarf führt. (Huber, 2009, S. 245)

Huber grenzt mit Blick auf seine Ergebnisse ein, dass diese Befunde lediglich beschreibenden Charakter haben und keine Aussagen zu Wirkrichtungen zwischen Einflussfaktoren erlauben: „Demnach bleibt offen, ob die soziometrische Position die Leistung bzw. die Leistungsmotivation beeinflusst oder tatsächlich von der hier unterstellten, umgekehrten Wirkrichtung ausgegangen werden kann, nach der Leistung und Leistungsmotivation einen Einfluss auf die soziale Integration haben.“ (Huber, 2009, S. 245). Für das Erschließen der Wirkrichtungen seien weitere Studien notwendig.

Auch Hennmann et al. weisen aufgrund der Ergebnisse ihrer Studie in NRW darauf hin, dass Schüler mit Förderbedarf im Verhalten: 
[...] seltener als Sitznachbarn gewählt wurden und deutlich häufiger die Mitschüler und Mitschülerinnen sie als Sitznachbar explizit ablehnten. Ebenso werden diese Schülerinnen und Schüler in hohem $\mathrm{Maß}$ als gemein angesehen und, im Rahmen eines kleinen bis mittleren Effektes als wenig hilfsbereit. (Hennemann et al., 2014, S. 33)

\subsubsection{Zusammenfassung zum Bereich der Unterrichtsentwicklung}

Obwohl für die Konzeptentwicklung im Bereich Unterricht neben den Ergebnissen der empirischen Unterrichtsforschung aus pädagogischer und sonderpädagogischer Perspektive auch die Orientierung an lernpsychologischen und konstruktivistischen Theorien sowie (fach-) didaktischen Ansätzen notwendig erscheint, verdeutlichen die in diesem Unterkapitel vorgestellten Qualitätsbausteine schon sehr deutlich die Komplexität möglicher Wirkfaktoren. Ziel der folgenden Zusammenfassung ist vor allem die Beantwortung der eingangs gestellten Forschungsfragen sowie eine kritische Einschätzung der dargestellten Ergebnisse. Dabei können nur zentrale Problemstellungen in den Blick genommen werden. Querverbindungen zu den Qualitätsbausteinen der Personal- und Organisationsentwicklung werden in der Gesamtzusammenfassung beleuchtet.

Hinsichtlich der in der Einleitung zu diesem Unterkapitel vorgestellten Qualitätsmerkmale guten Unterrichts nach Brophy (2000), Meyer (2004), Hattie (2009) und Helmke (2010) lässt sich zunächst zusammenfassend festhalten, dass die Qualitätskategorien, die sich für so genannten inklusiven Unterricht im Lauf der Analyse von Forschungsarbeiten der Sonderpädagogik ergeben haben, mit diesen weitgehend übereinstimmen. Sie stellen also keine spezifisch „inklusiven“ Qualitätsmerkmale dar, sondern kennzeichnen qualitativ hochwertigen Unterricht insgesamt, legen allerdings bestimmte Schwerpunkte (s. Kategorien, Subkategorien und Indikatoren) fest, die aus sonderpädagogischer Perspektive eine hohe Relevanz für die Qualität gemeinsamen Unterrichts haben. Daraus lässt sich schlussfolgern, dass qualitativ guter oder sehr guter Unterricht auch qualitativ guter oder sehr guter gemeinsamer Unterricht ist. Nicht zulässig ist allerdings der Umkehrschluss, dass ein solcher Unterricht ausreicht um spezifischen Förderbedürfnissen - wie sie unter der Kategorie Individualisierung angerissen sind - gerecht werden zu können. Darüber hinaus ist naheliegender Weise davon auszugehen, dass auch weitere - in dieser Arbeit nicht näher analysierte - Merkmale guten Unterrichts, die aufgrund der Ergebnisse der allgemeinen Unterrichtsforschung für bedeutsam gehalten werden, für den gemeinsamen Unterricht relevant sein können.

Mit Blick auf die Ressourcen für den Unterricht in integrativen Klassen können Klassengröße (Heese, 1977; Weigt, 1977; Speck et al., 1978; Mühl, 1987; Dumke et al., 1989; Köbberling, 1994; Brugger-Paggi, 2003; Thiele, 2009; Wember \& Prändl, 2009; Speck, 2010; von Saldern, 2011) und Klassenzusammensetzung (Wocken, 1987; Innerhofer \& Klicpera, 1991; Schley et al., 1992; Husinsky, 1994; Feyerer, 1998; Bless, 2003; Brugger-Paggi, 2003; BMBWF Österreich, 2008; Holzinger et al., 2011) eine Rolle spielen. Untersuchungsergebnisse für diese Qualitätskategorien belegen allerdings, dass nicht Größe oder Zusammensetzung allein entscheidend sind, sondern diese nur im Zusammenwirken mit weiteren Qualitätsbausteinen guten Unterrichts Effekte zeigen (Hattie, 2013). Während Auswirkungen auf die Arbeitsbedingungen der Lehrkräfte messbar sind, gilt dies bislang in geringerem Maß mit Blick auf die Verbesserung von Lernleistungen der Schüler. Eine wichtige Bedeutung haben Größe und Zusammensetzung von Klassen in der Wahrnehmung von Lehrkräften und Schülern. Lehrkräfte schätzen u.a. das höhere Maß an Ruhe in diesen Klassen und die Möglichkeit Schüler individuell anzusprechen. In der schulischen Realität liegen die Klassenteiler im Grundschulbereich in Deutschland zwischen 
23 und 28 Schülerinnen und Schülern, in den weiterführenden Schulen zwischen 25 und 30 (KMK, 2018). Um die aufgrund der vorliegenden Forschungsergebnisse empfohlene Klassengröße von 20 Kindern bzw. Jugendlichen zu erreichen, ist aus politischer Sicht also zunächst eine Veränderung von Rahmenvorgaben in den Bundesländern notwendig, da die Obergrenzen für den Primar- und den Sekundarstufenbereich entsprechend gesenkt werden müssen. Vorgaben für die Anteile von Schülern mit sonderpädagogischem Förderbedarf in den Klassen sind nicht gegeben. Qualitätsmerkmalen wie einer ausgewogenen Leistungsheterogenität oder der Vermeidung von Problemhäufungen in einzelnen Klassen (Feyerer, 1998; Bleidick, 1999; Bless, 2003; Huber, 2009; BMBWF Österreich, 2008; Holzinger et al., 2011) kann auf diese Art und Weise nicht entsprochen werden. Die Idee der Bündelung mehrerer Schüler mit spF in einer so genannten Integrationsklasse (je Jahrgang) wird in manchen Schulen nicht deshalb praktiziert, weil diese als sinnvoll eingeschätzt wird, sondern um die wenigen Ressourcen, die Förderschullehrkräfte in die Schulen einbringen können (s. 3.3.1), bündeln zu können. Auf diese Art und Weise ist es Lehrkräften möglich eine größere Anzahl an Unterrichtsstunden in Doppelbesetzung zu gestalten, was Einfluss auf die Kooperation der Lehrkräfte untereinander haben kann (3.3.2). Darüber hinaus sinkt auf diese Art und Weise die Anzahl der Klassen, in denen eine Förderschullehrkraft tätig ist, und damit auch die Anzahl der Kooperationspartner, mit denen sie sich abstimmen muss.

Für die Unterrichtsgestaltung in integrativ arbeitenden Schulen scheinen vor allem die Bausteine Schulklasse als sozialer Raum, Klassenführung, Didaktik und Methodik sowie Differenzierung bzw. Individualisierung qualitativ prägend zu sein.

Hinsichtlich der Schulklasse als sozialem Raum lassen Forschungsergebnisse den Schluss zu, dass das lernförderliche Klima durch vielfältige Faktoren mitbestimmt wird (Brophy, 2000; Muijs \& Reynolds, 2011; Helmke, 2015). Eine besonders wesentliche Rolle spielt die Beziehung zwischen Lehrkraft und Schülern, die durch Freundlichkeit und Vertrauen geprägt sein sollte (Hamre \& Pianta, 2001; Bless, 2003; Woolfolk, 2008; Wember \& Prändl, 2009; Muijs \& Reynolds, 2011). Um das Lernen zu fördern werden vor allem ein konstruktiver Umgang mit Fehlern und die Herstellung einer entspannten Lernatmosphäre durch Humor und eine positive Grundeinstellung der Lehrkräfte als Qualitätsmerkmale benannt (Feyerer, 1998; Brophy, 2000; Hattie, 2013; Helmke, 2015). Auch die Möglichkeit der Partizipation von Schülern beeinflusst das Klassenklima (Heyer et al., 1997; Bless, 2003; Boban \& Hinz, 2003). Tendenziell wirkt sich ein lernförderliches Klima positiv auf Leistungen, Selbstwertschätzung von Schülern und deren soziale Integration aus (Muijs \& Reynolds, 2011; Helmke, 2015). Für das soziale Lernen (Keller \& Hafner, 1999; Eckermann \& Heinzel, 2013) innerhalb der Klassen haben neben der Beziehungsgestaltung zwischen Lehrkraft und Schülern vor allem Peer-Status, Peer-Interaktionen und die Kooperation zwischen den Schülern eine zentrale Bedeutung. Soziale Erfahrungen können in diesem Rahmen informell gesammelt werden, Lehrkräfte können diese aber auch formell - bspw. durch moderierte Klassengespräche oder die Organisation kooperativer Lernsituationen - ermöglichen. Problematisch kann vor allem bezüglich der Peer-Prozesse sein, dass Schüler und Schülerinnen mit schlechteren Leistungen einen geringeren Beliebtheitsgrad aufweisen und seltener für soziale Interaktionen ausgewählt werden. Aus Sicht der Sonderpädagogik scheint es daher wesentlich soziale Lernprozesse in den Klassen zu optimieren, bspw. durch eine gezielte Analyse der Klassensituation, den Wechsel von individuellen und gemeinsamen Lernphasen sowie die Ausgewogenheit von leistungsbetonten und sozialen Lernmöglichkeiten (Prell \& Link, 1977; Berges, 1996; Bless, 2003; Huber, 2009; Leonhardt, 2009; Holzinger et al., 2011; Ziemen, 2011). 
Die Kategorie Klassenführung scheint aufgrund der messbaren Effekte auf Lernaktivität und Lernleistung im Zusammenhang mit der Qualität (inklusiven) Unterrichts eine entscheidende Rolle zu spielen (Kounin, 1976; Marzano \& Marzano, 2003; Muijs \& Reynolds, 2005; Borich, 2007; Evertson \& Weinstein, 2006; Eikenbusch, 2010; Keller, 2010; Kiel, 2012; Hattie, 2013; Helmke, 2015). Von besonderer Relevanz sind als Voraussetzungen für das professionelle Führen einer Klasse - ähnlich wie mit Blick auf das lernförderliche Klima - die Gestaltung der Beziehungsebene zwischen Lehrern und Schülern sowie die Fähigkeit der professionellen Steuerung individueller wie gruppendynamischer Prozesse innerhalb von Schulklassen durch die Lehrkräfte (Marzano \& Marzano, 2003; Hattie, 2013). Insbesondere aufgrund der Forschungsergebnisse aus dem allgemeinpädagogi-schen Bereich ist anzunehmen, dass das Führen und Managen einer Klasse als sozialdynamischer Gruppe eine Grundvoraussetzung für störungsfreien Unterricht darstellt und damit einen hohen Anteil aktiver Lernzeit ermöglichen kann (Brophy, 2010; Muijs \& Reynolds, 2011; Hattie, 2013; Helmke, 2015). Dabei scheint es wesentlich zu sein, dass Lehrkräfte ein gesundes Maß zwischen Dominanz und Kooperation finden und ein hohes Maß an Präsenz („,bei der Sache sein“) in ihrer Klasse zeigen (Marzano \& Marzano, 2003). Der hohe Anteil aktiver Lernzeit sorgt wiederum für eine deutliche Verbesserung von Lernergebnissen der Schüler. Qualitätsindikatoren für den Bereich der Klassenführung sind u.a. Regeln, Routinen (für Abläufe), Rituale, die Klassenraumgestaltung sowie der Umgang mit Verhaltensproblemen von Schülern mit hohem Unterstützungsbedarf im emotionalen und sozialen Bereich (Evertson \& Weinstein, 2006; Helmke, 2015). Für den Umgang mit Unterrichtsstörungen können Ansätze genutzt werden, die geringfügige Störungen übergehen, gleichzeitig aber Verhaltensanforderungen klar formulieren und konsequent auf massivere Störungen reagieren. Aus sonderpädagogischer Sicht wird Klassenführung ebenfalls als relevant für das Erreichen von Lernzielen und der Reduktion von auffälligem Verhalten eingestuft (Prell \& Link, 1977; Boban \& Hinz, 2003; Textor, 2009; Wember \& Prändl, 2009; Hennemann \& Hillenbrand, 2010; Casale et al., 2016), allerdings bislang nicht so umfangreich erforscht, wie dies in der Allgemeinen Pädagogik der Fall ist.

Während die klare Strukturierung als Qualitätsmerkmal in der allgemeinen Pädagogik eine wichtige Rolle spielt (Meyer, 2004; Kiel, 2012; Helmke, 2015), findet sie aus sonderpädagogischer Sicht wenig Berücksichtigung in Forschungsveröffentlichungen (Feuser \& Meyer, 1987; Bless, 2003; Wember \& Prändl, 2009). Dabei ist anzunehmen, dass sowohl übergreifende Aspekte unterrichtlicher Strukturierung (Nutzung von Advance Organizern, Tagesablauf- sowie Stundenablaufplänen/Artikulationsschemata) wie die konkrete Strukturierung des Unterrichts durch klare und verständliche Sprache insbesondere für Schüler mit kognitiven Schwächen in Regelklassen hilfreich sein können.

Zu Beginn der 2000er Jahre waren fachdidaktische Veröffentlichungen, die im Zusammenhang mit Anforderungen des integrativen Unterrichts stehen, kaum vorhanden, während diese im Lauf der 2010er Jahre gehäuft erscheinen (Bsp.: Amrhein \& Dziak-Mahler, 2014; Trumpa et al., 2014) und verdeutlichen, dass Fragen der inhaltlichen Aufbereitung von Lerninhalten (Bsp.: Leselernprozess, Erfassung eines Zahlenraums) und der Wirkung dieser Aufbereitung auf Leistungen und Kompetenzen der Schüler ein Theorie- und Forschungsdesideratum sowohl in der allgemeinen Pädagogik wie in der Sonderpädagogik darstellen. Aufgrund der Tatsache, dass die Ansätze sich auf den gesamten Fächerkanon in Primar- und Sekundarstufe beziehen und somit ein umfangreiches Inhaltsfeld darstellen, konnten sie in den vorliegenden Ausführungen nicht aufgegriffen werden. Gleichzeitig bieten die in Kürze dargestellten didaktischen Ansätze so genannter „inklusiver Didaktik“ (Feuser, 1989; Reich, 2004; Seitz, 2006; Wocken, 2009), in 
Anlehnung an die Kritik von Moser Opitz keine Modelle an, die im integrativen Unterricht direkt und konkret nutzbar sind. Sonderpädagogische Autorinnen und Autoren thematisieren mit Blick auf die Unterrichtsqualität weniger didaktische Ansätze als didaktische Prinzipien (Hug, 1994b; Köbberling, 1994; Berges, 1996; Heyer et al., 1997; Mühl, 1987; Boban \& Hinz, 2003; Brugger-Paggi, 2003; Feyerer, 2007; Wember \& Prändl, 2009).

Die Flexibilität im Einsatz unterschiedlicher unterrichtlicher Methoden (Matthes, 2011) wird als weiterer Qualitätsbaustein beschrieben. Der so genannte Frontalunterricht ist dabei die in deutschen Schulen anteilsmäßig am häufigsten eingesetzte Unterrichtsmethode, die als Sozialform normalerweise in der Gesamtgruppe (= Klasse) erfolgt, während schüleraktive Arbeitsphasen (wie Einzel-/Partner- und Gruppenarbeit) einen geringeren Anteil einnehmen (Hage et al., 1985; Götz et al., 2005; Wiechmann, 2010). Der frontale Unterricht umfasst ein weites Feld methodischer Ansätze (Bsp.: fragend-entwickelndes Unterrichtsgespräch, Gruppendiskussionen etc.). Positive Effekte auf Lernleistungen von Schülern können im Rahmen der Unterrichtsforschung allerdings vor allem für die spezifische Methode der direkten Instruktion festgestellt werden, die als hochstrukturiert, kleinschrittig und feedbackreich gekennzeichnet wird, und sich insbesondere für leistungsschwache Schülerinnen und Schüler als unterstützend erweist (Grünke, 2006; Grosche, 2011; Muijs \& Reynolds, 2011). Weiterhin belegen Studien, dass eine Kombination dieser Methode mit aus Schülersicht noch stärker eigenaktiv geprägten Methoden wie Einzelarbeit oder kooperativen Lernformen positive Effekte auf die Kompetenzentwicklung hat (Muijs \& Reynolds, 2011). Für kooperative Lernformen ist mit Blick auf heterogen zusammengesetzte Lerngruppen allerdings festzuhalten, dass diese zum Teil hohe kognitive Ansprüche sowie Ansprüche an soziale Kompetenzen von Schülerinnen und Schülern stellen und sich nur dann als effektiv erweisen, wenn grundlegende Fähigkeiten auf Schülerseite gegeben sind (Huber, 2010; Muijs \& Reynolds, 2011; Eckermann \& Heinzel, 2013). Ähnlich wie bei Untersuchungen zum sozialen Lernen zeigt sich für diese Methode, dass Statusdifferenzen zwischen Peers Auswirkungen auf den sozialen Umgang und in diesem Fall bspw. auf wertschätzende Rückmeldungen bei kooperativen Gruppenarbeiten haben. In sonderpädagogischen Forschungsveröffentlichungen finden methodische Fragestellungen wenig Berücksichtigung. Kennzeichnend für gute (inklusive) Schulen scheint eine hohe Flexibilität mit Blick auf die Auswahl der gewählten Unterrichtsmethoden (Boban \& Hinz, 2003; Brugger-Paggi, 2003; Wember \& Prändl, 2009) zu sein, eine Qualitätssubkategorie, die in mehreren Veröffentlichungen konkret benannt wird. Darüber hinaus belegen sonderpädagogische Studien, das so genannte offene Unterrichtsformen und damit bspw. Methoden wie Wochenplan- oder Stationenarbeit bzw. Projekte in integrativen Schulen gehäuft umgesetzt werden (Köbberling, 1994; Berges, 1996; Feyerer, 1998; Deutsches Schulamt, 2004; Specht et al., 2006; Bürli et al., 2009; Wember \& Prändl, 2009; Holzinger et al., 2011; Liebers et al., 2014). Nicht belegt ist wiederum die Effizienz dieser Methoden hinsichtlich der Lernleistungen der Schülerinnen und Schüler.

Differenzierung und Individualisierung beinhalten verschiedene Anpassungsstrategien, die das Ziel verfolgen Unterrichtsangebote an die unterschiedlichen Lernvoraussetzungen von Schülerinnen und Schülern zu adaptieren (Weinert, 1997; Wember \& Prändl, 2009; Helmke, 2015). Diese Passung setzt auf der einen Seite voraus, dass Lehrkräfte Unterrichtsgegenstände inhaltlich durchdringen und didaktisch erschließen sowie geeignete Methoden für die Vermittlung der Inhalte auswählen, auf der anderen Seite, dass sie über professionelle Kompetenzen zur Lernstandsdiagnostik und Förderplanung verfügen. Insofern geht es bei 
der Darstellung der Qualitätsbausteine Differenzierung und Individualisierung ähnlich wie bei den didaktischen Ansätzen zunächst vordergründig um Prinzipien, die im Unterricht berücksichtigt werden sollten, nicht um konkrete fachliche Differenzierungsmaßnahmen. Diese Prinzipien beziehen sich bspw. auf die Berücksichtigung unterschiedlicher Lernzugänge, unterschiedlicher Grade inhaltlicher Komplexität, unterschiedlicher Zeitspannen für die Bearbeitung von Aufgaben oder eines unterschiedlich hohen Maßes an individueller Unterstützung, das die Schülerinnen und Schüler benötigen (Paradies \& Linser, 2001; Saalfrank, 2012). Grenzen ergeben sich in diesem Zusammenhang daher bei dem Anspruch an eigenverantwortliches Arbeiten der Schüler: Nicht immer reicht die Vorbereitung differenzierter Arbeitsmaterialien für individuelle Förderung aus, in vielen Situationen ist eine 1:1-Ansprache oder die Ansprache in einer sehr kleinen Gruppe notwendig (Beispiele: gezieltes Einüben von Lautgebärden, gezielte Förderung in den Bereichen Wahrnehmung/Motorik). Insofern ist es wenig erstaunlich, dass Forschungsergebnisse zur Wirksamkeit differenzierter Lernangebote nicht einheitlich sind. Während leistungsstärkere Schüler eher von einer möglichst homogenen Klassenzusammensetzung in Verbindung mit leistungsdifferenzierten Aufgabenstellungen profitieren, gilt dies nicht gleichzeitig für andere Schüler (Woolfolk, 2008). Individualisierte Angebote führen mit Blick auf die Leistungsentwicklung nur zu schwach messbaren Effekten (Helmke, 2010; Hattie, 2013). Darüber hinaus ist eine differenzierte oder sogar individualisierte Unterrichtsgestaltung in deutschen Schulen nur wenig verbreitet (Bos et al., 2003; Klieme et al., 2008; Helmke, 2010). Auch wenn im integrativen Unterricht also - wie vor allem die sonderpädagogischen Veröffentlichungen belegen - Differenzierung eine (hohe) qualitative Anforderung darstellt und aufgrund der Heterogenität der Klassenzusammensetzung und der individuellen Unterstützungsbedarfe (nicht nur) der Schülerinnen und Schüler mit spF notwendig ist, erfolgt derzeit offensichtlich einerseits keine flächendeckende bzw. in Unterrichtsanteilen umfangreich messbare Umsetzung im Unterricht. Auch die Individualisierung wird im Unterrichtsalltag nicht umfassend umgesetzt (Wischer, 2007; Laubenstein et al., 2015). Individualisierende Lernangebote im Rahmen der integrativen Unterrichtung werden dabei mit Blick auf unterschiedliche sonderpädagogische Förderschwerpunkte als unterschiedlich anspruchsvoll eingeschätzt. Während die zielgleiche Unterrichtung von Schülerinnen und Schülern mit den spF Sprache, körperliche und motorische Entwicklung oder leichten Sinnesbeeinträchtigungen (Bsp.: Schwerhörigkeit) als vergleichsweise unproblematisch eingestuft wird, sehen Lehrkräfte größere Probleme vor allem bei der zieldifferenten Unterrichtung (spF Lernen, geistige Entwicklung) bzw. bei der Unterrichtung von Schülerinnen und Schülern mit Verhaltensauffälligkeiten oder schwer wiegenden Sinnesbeeinträchtigungen (Bsp.: Blindheit) (Feuser \& Meyer, 1987; Dumke et al., 1989; Dyson, 2010; Amrhein, 2011; Ellinger \& Stein, 2012; Laubenstein et al., 2015). Besonders hohe Anforderungen an inklusiven Unterricht stellen Schülerinnen und Schüler mit schweren Mehrfachbehinderungen (Wagner, 2013).

Die differenzierende Bewertung von Schülerleistungen ist eine Qualitätskategorie, die sich aus differenzierenden Lernangeboten heraus ergibt, und in sonderpädagogischen Veröffentlichungen entsprechend vielfältig thematisiert wird (Mühl, 1987; Innerhofer \& Klicpera, 1991; Husinsky, 1994; Köbberling, 1994; Heyer et al., 1997; Boban \& Hinz, 2003; Brugger-Paggi, 2003; Thiele, 2009; Wember \& Prändl, 2009; Holzinger et al., 2011). Grundlage für differenziertes Arbeiten im Unterricht sind außerdem die Qualitätsbausteine Lernstandsdiagnostik und Förderplanung als weitere Qualitätsmerkmale (Preuß, 1985; Innerhofer \& Klicpera, 1991; Bless, 2003; Boban \& Hinz, 2003; Brugger- Paggi, 2003; Specht et al., 2006; Feyerer, 2007; 
Wember \& Prändl, 2009; Bürli et al., 2009; Lienhard-Tuggener et al., 2011; Holzinger et al., 2011).

Forschungsergebnisse zu den Ergebnissen gemeinsamen Unterrichts liegen für kognitive, emotionale und soziale Aspekte vor. Für den kognitiven Bereich lässt sich trotz zum Teil nicht übereinstimmender Forschungsergebnisse zusammenfassend festhalten, dass der gemeinsame Unterricht an sich weder positive noch negative Effekte auf die Lernleistungen von Schülerinnen und Schülern zu haben scheint und zwar weder auf diejenigen mit noch auf diejenigen ohne sonderpädagogischen Förderbedarf (Bachmann, 1974; Speck et al., 1978; Wocken, 1987; Tent et al., 1991; Bless, 2003; Haeberlin et al., 2003; Venetz \& Tarnutzer, 2012; Kocaj et al., 2014; Textor, 2014; Dumont et al., 2015; Lütje-Klose et al., 2018). Einschränkend muss hinzugefügt werden, dass Forschungsergebnisse sich vorwiegend auf Schüler mit dem spF Lernen beziehen und keine umfassende Längsschnittuntersuchung für Schüler mit noch schwerwiegenderen kognitiven Einschränkungen vorliegt. Problematisch ist darüber hinaus, dass auch künftige Studien zu Effekten gemeinsamen Unterrichts mit einer Fülle von Wirkfaktoren konfrontiert sind, die den Unterricht insgesamt kennzeichnen, und mit der Schwierigkeit vergleichbare Gruppen (Bsp.: Klassen ohne und mit Schülerinnen und Schüler mit spF) untersuchen zu können. Für andere Förderschwerpunkte liegen kaum differenzierte Ergebnisse zu dieser Fragestellung vor. Auch hinsichtlich des emotionalen bzw. sozialen Bereichs bezieht sich das Gros der Forschungsveröffentlichungen auf die zwei Förderschwerpunkte Lernen und emotionales und soziales Verhalten. Ungünstig kann sich schulische Integration auf die Leistungsmotivation von Schülern mit diesen Förderschwerpunkten auswirken. Für kognitiv eingeschränkte sowie hörbeeinträchtigte Schüler können sich negative Auswirkungen auf das Begabungs- und damit auch Fähigkeitsselbstkonzept ergeben (Haeberlin et al., 1989; Tent et al., 1991; Gasteiger-Klicpera \& Klicpera, 1997a, 1997b; Haeberlin et al., 2003; Bless, 2003; Ellinger \& Stein, 2012). Mit Bezug auf die soziale Integration sind die Befunde nicht einheitlich, geben zum Teil aber Hinweise darauf, dass die soziale Akzeptanz von Schülern mit niedrigen Leistungen, einem niedrigen sozialen Status und/oder unangepassten sozialen Verhaltensweisen in integrativen Klassen erschwert ist (Bachmann, 1974; Preuss-Lausitz, 1981; Reiser, 1984; Wocken, 1987; Maikowski \& Podlesch, 1988; Berges, 1996; Heyer et al., 1997; Bless, 2003; Haeberlin et al., 2003; Huber, 2009).

\subsection{Zusammenfassung mit Blick auf die forschungsleitenden Fragestellungen}

Aufgrund der umfassenden Analyse sonderpädagogischer Forschungsveröffentlichungen und der Verknüpfung und Ergänzung der dadurch entstandenen Kategorien mit Ergebnissen der Schulentwicklungs- und Unterrichtsforschung ist ein umfassendes Kategoriensystem entstanden. Die Qualitätskategorien, - subkategorien und -indikatoren, die in den drei Bereichen Organisations-, Personal- und Unterrichtsentwicklung als relevant für die Qualität schulischer Integration eingestuft werden, wurden im vorliegenden dritten Kapitel dieser Arbeit voneinander getrennt und seriell dargestellt. Diese Darstellungsweise ist ein zwar erfahrungsbasiertes, da auf empirischen Ergebnissen beruhendes, dennoch aber theoretisches Konstrukt, da das System einer Einzelschule in der Realität komplexer ist und alle aufgeführten Wirkfaktoren Teil eines dynamischen Gefüges sind. Wie in den Unterkapiteln bereits mehrfach erwähnt, sind Überlappungen zwischen den Bereichen gegeben und Kategorien nicht trennscharf. Folgende grafische Übersicht veranschaulicht die Haupt- und Subkategorien als Ergebnis der Forschungsanalyse: 


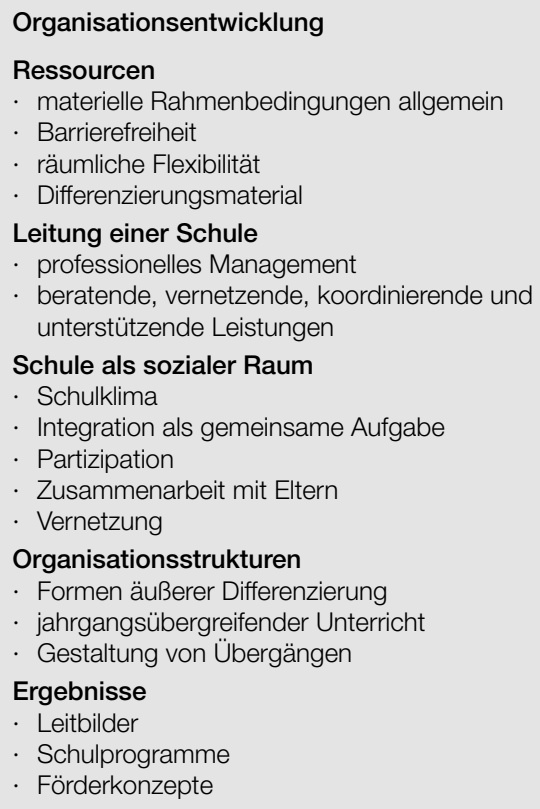

\section{Personalentwicklung}

\section{Ressourcen}

- Grundversorgung mit Lehrkräften

- Doppelbesetzung

- Kontinuität der Zuweisung

- Bündelung sonderpäd. Ressourcen

- fest eingerichtete Kooperations-/ Teamzeiten

\section{Kooperation von Lehrkräften}

- Voraussetzungen/Erwartungen/Bereitschaft

- Niveaustufen

- Kooperation im Gesamtkollegium

- Kooperation in Teams (multiprofessionell)

- Kooperation zwischen Regel- und

Förderschullehrkräften

- unterrichtsbezogene Zusammenarbeit/ CoTeaching

- Aufgaben- und Rollenklärung

Professionalisierung

- Studium

- Vorbereitungsdienst

- Fortbildungen (schulintern/schulextern)

- Supervision

\section{Ergebnisse}

- Auswirkungen Schulklima?

- Auswirkungen Lernergebnisse SuS?

- Haltungen

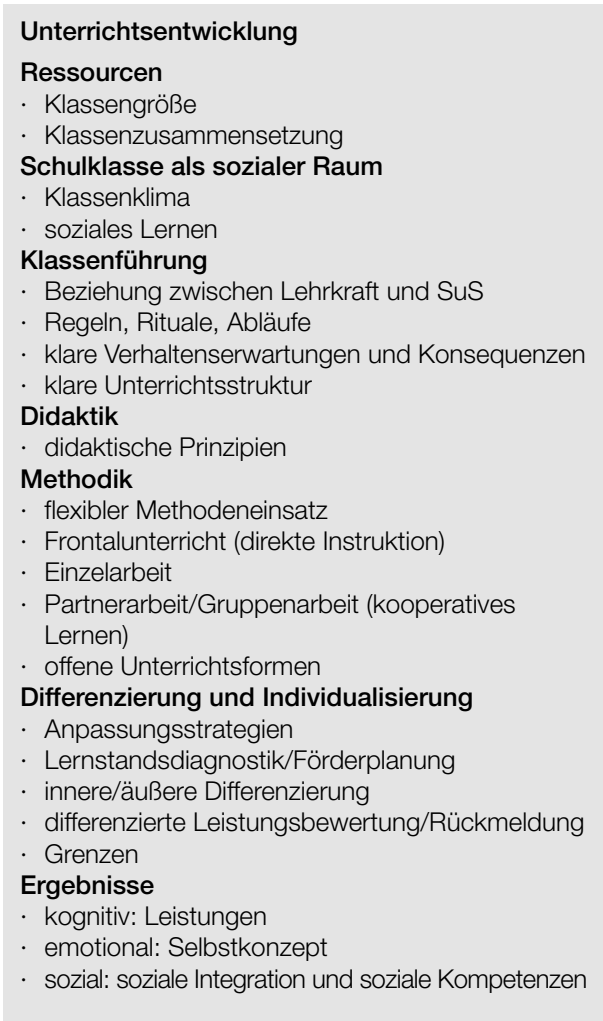

Abb. 4: Übersicht über das Kategoriensystem schulische Integration mit Subkategorien 


\section{Hypothesen}

Nach einer umfassenden Sichtung der Forschungsliteratur zur integrativen Beschulung von Kindern und Jugendlichen mit Behinderungen wurden Übersichten mit Qualitätskategorien, -subkategorien und -indikatoren erstellt (s. oben und Tabellen im Anhang). In Anlehnung an die Strukturierung von Schulentwicklung nach Rolff wurden diese Kategorien, Subkategorien und Indikatoren sortiert und gewichtet. Das dadurch entstandene Kategoriensystem wird für den eigenen Forschungsansatz anschließend in zweifacher Hinsicht genutzt: Zunächst dienen sie für die qualitative Erhebung als Ansatzpunkt für die Auswahl von Fragestellungen für die strukturierten Interviews. Nach der Auswertung der Interviews, bei denen entlang der Fragestellungen im ersten Schritt eine induktive Vorgehensweise gewählt wird, dienen sie wiederum als Strukturierungshilfe für die Zuordnung und Erweiterung der von den Beteiligten genannten Qualitätsbereiche und -anzeiger. Mit Hilfe der derart veränderten und erweiterten Kategorien und Indikatoren werden dann wiederum die Fragebögen für die einzelnen Personengruppen Schüler, Eltern, Regel- und Förderschullehrkräfte sowie Regelschulschulleitungen und Leitungen der Förderzentren entwickelt. Aufgrund des Forschungsüberblicks können Hypothesen für die Kategorien und Subkategorien der Bereichte Organisations-, Personal- und Unterrichtsentwicklung formuliert werden.

\section{Organisationsentwicklung}

Aufgrund der dargestellten Ergebnisse ist zunächst grundlegend davon auszugehen, dass Schulen, an denen Schülerinnen und Schüler mit spF unterrichtet werden, sich organisatorisch weiterentwickelt und verändert haben. Für die Kategorien im Entwicklungsfeld der Schule als Organisation lassen sich aus den vorangegangenen Ausführungen folgende Hypothesen ableiten:

\section{Ressourcen}

Wenig Einfluss haben Schulen auf den Bereich der Ressourcen. Wie die Erläuterungen zu den Ressourcen belegen, ist nicht davon auszugehen, dass die Schulen barrierefrei gestaltet sind und über flexible Raumnutzungsmöglichkeiten bzw. eine ausreichende Anzahl an Differenzierungsräumen verfügen. Auch die Materialausstattung wird - u.a. mit Blick auf die möglicherweise sehr spezifischen Anforderungen einzelner Schüler oder Schülerinnen mit spF - in diesen Schulen nicht adäquat sein.

\section{Leitung einer Schule}

Hinsichtlich der Schulleitungen kann angenommen werden, dass diese integrative Prozesse an ihren Schulen unterstützen. Da die Funktion von Schulleitungen allerdings insgesamt in der bisherigen Forschung nicht im Vordergrund steht, könnte eine weitere Hypothese lauten, dass diese auch in der Wahrnehmung der Beteiligten zunächst keine wichtige Rolle spielt.

\section{Schule als sozialer Raum}

Für das Schulklima an inklusiven Schulen kann vermutet werden, dass dieses von Respekt, Wertschätzung und Akzeptanz aller Beteiligten untereinander geprägt ist und diese sich in ihren Schulen wohlfühlen. Darüber hinaus kann davon ausgegangen werden, dass Integration in diesen Schulen als gemeinsame Aufgabe aufgefasst wird, der die Beteiligten offen gegenüberstehen, und dass auch Eltern und Schülerinnen wie Schüler in die Weiterentwicklung der Schulen einbezogen sind. Die Zusammenarbeit mit den Eltern wird in inklusiven Schulen intensiv gestaltet und zwar sowohl in quantitativer wie in qualitativer Hinsicht. Es gibt entsprechend häufige und qualitätsvolle Kontakte vor allem in Form gemeinsamer regelmäßiger Gesprächstermine. Inklusive Schulen sind in ihrer Umgebung sehr gut vernetzt und kooperieren professionell u.a. mit den Jugendämtern, dem schulpsychologischen Dienst sowie Vereinen etc. 


\section{Organisationsstrukturen}

Grundlegende organisatorische Strukturen werden an inklusiven Schulen wenig verändert. In Eingangsklassen werden zum Teil jahrgangsübergreifende schulische Angebote gestaltet. Gegebenenfalls sind klassenübergreifende Fördergruppen eingerichtet. Die Beschulung von Schülerinnen und Schülern erfolgt teilweise in Form äußerer Differenzierung. Die Übergänge zwischen Kindergärten - Grundschule - weiterführender Schule - berufsbildenden Angeboten werden bewusst gestaltet.

\section{Ergebnisse}

Schulen, in denen Schülerinnen und Schüler mit spF unterrichtet werden, verfügen zum Großteil über Leitbilder und Schulprogramme. Allerdings steht das Thema schulische Integration/ Inklusion dabei nicht unbedingt im Vordergrund.

\section{Personalentwicklung}

Obwohl zur Personalentwicklung in integrativ arbeitenden Schulen, und dabei insbesondere zu den Qualitätsbereichen Kooperation und Professionalisierung, eine Vielzahl von (Forschungs-) Veröffentlichungen vorliegt, sind die konkreten Effekte auf die schulische Entwicklung insgesamt und den Unterricht sowie die Lernleistungen von Schülerinnen und Schülern bislang kaum untersucht worden. Aufgrund des Querschnittcharakters der geplanten Erhebungen wird dies auch in der vorliegenden Arbeit nicht möglich sein. Für diese Erhebungen ist also zunächst einmal interessant, in welcher Form die zuvor beschriebenen Aspekte der Personalentwicklung umgesetzt werden. Diesbezüglich lassen sich folgende Hypothesen formulieren:

\section{Ressourcen}

Die Ressourcenausstattung in den inklusiven Schulen (ausreichende Anzahl ausgebildeter Regel- und Förderschullehrkräfte, ausreichende Anzahl an FSL-Stunden, die den Regelschulen für die schulische Integration zugewiesen werden) sieht nicht so aus, dass eine Doppelbesetzung im gemeinsamen Unterricht durchgängig bzw. in den Kernfächern möglich ist. Eine Kontinuität der Zusammenarbeit ist nur teilweise gegeben, da Förderschullehrkräfte bestimmten Schulen nicht immer über längere Zeiträume (mehrere Jahre) hinweg zugewiesen sind.In den meisten Schulen sind fest eingerichtete Team- bzw. Beratungszeiten nicht vorhanden, so dass die konkrete Umsetzung der Förderung von Schülerinnen und Schülern mit spF häufig an die Förderschullehrkräfte delegiert wird und äußere Differenzierung erfolgt.

\section{Kooperation}

Konferenzen und Dienstbesprechungen werden in den Schulen genutzt um Fragen schulischer Integration zu thematisieren. Die Kooperation zwischen Regel- und Förderschullehrkräften findet auf verschiedenen Niveaustufen statt, wobei die Niveaustufen Austausch bzw. Co-Activity und Kooperation häufiger praktiziert werden als die Stufen Coordination, Collaboration bzw. Ko-Konstruktion. Ein hohes Maß an Befürwortung und Bereitschaft zu unterrichtsbezogener Kooperation auf Seiten der Lehrkräfte steht einem geringen Maß an Umsetzung derselben gegenüber. Wenn Co-Teaching umgesetzt wird, werden vor allem die Formen one teach - one observe bzw. one teach - one assist genutzt. Unterrichtsverantwortung wird also nur selten tatsächlich gemeinsam wahrgenommen. Rollen und Aufgaben sind zwischen Regel- und Förderschullehrkräften so verteilt, dass die RSL va. die Klassen- und Unterrichtsverantwortung übernehmen, während die FSL Diagnostik, Beratung und Förderung gestalten.Teilweise werden Rollen- und Aufgabenverteilung flexibel gehandhabt, so dass Rollenwechsel möglich sind. 
Zielsetzung und Klärung der Aufgabenbereiche zwischen FSL und RSL sind nicht umfassend gegeben, woraus sich berufliche Unsicherheiten ergeben können.

\section{Professionalisierung}

Im Studium der Regelschullehrkräfte fehlen sonderpädagogische Inhalte bzw. Inhalte, die schulische Inklusion (Heterogenität, Differenzierung) thematisieren, weitgehend. Das Empfinden mangelnder Kompetenzen aufgrund unzureichender Ausbildung kann vor allem bei den Regelschullehrkräften zu einer ablehnenden Haltung gegenüber schulischer Integration führen. Fortbildungsangebote für Regel- wie Förderschullehrkräfte im Zusammenhang mit dem Themenfeld schulischer Integration sind vielseitig und werden umfassend genutzt und zwar sowohl Fortbildungen für einzelne Lehrkräfte wie für ganze Kollegien. Einstellungen und Haltungen von Regel- wie Förderschullehrkräften, Schulleitungen, Eltern und Schülerinnen und Schülern gegenüber schulischer Integration decken ein breites Meinungsspektrum (zwischen umfassender Ablehnung bzw. Befürwortung) ab.

\section{Unterrichtsentwicklung}

Für den Bereich der Unterrichtsentwicklung in integrativen Schulen ergeben sich folgende Hypothesen:

\section{Ressourcen}

Die Klassengröße in den integrativ arbeitenden Schulen übersteigt häufig die empfohlene Anzahl von 20-22 Schülerinnen und Schülern. Bei der Klassenzusammensetzung wird in diesen Schulen grundsätzlich darauf geachtet, dass der Anteil an Schülerinnen und Schülern mit spF nicht zu hoch ist.

In manchen Schulen werden Schüler mit spF in bestimmten Klassen gebündelt, damit sonderpädagogische Ressourcen in diesen Klassen gebündelt genutzt werden können.

\section{Schulklasse als sozialer Raum}

Integrative Klassen sind von einem lernförderlichen Klima geprägt. Neben sozialen Lernprozessen auf informeller Ebene gestalten Lehrkräfte ihren Unterricht so, dass soziales Lernen möglich ist (Bsp.: gemeinsame Lernsituationen).

\section{Klassenführung}

Die Klassenführung hat für Lehrkräfte in integrativen Klassen eine hohe Relevanz. Die Gestaltung der Beziehungen zwischen Lehrkräften und Schülerinnen und Schülern spielt dabei eine wesentliche Rolle. In integrativen Klassen werden Regeln und Rituale genutzt. Klare Verhaltensanforderungen sind kommuniziert. Bei Regelverstößen erfolgen entsprechende Konsequenzen. Integrativer Unterricht weist ein hohes Maß an Strukturierung auf.

\section{Didaktik}

Die didaktische Umsetzung integrativen Unterrichts ist bislang wenig entwickelt. Von Bedeutung für die Lehrkräfte ist die Berücksichtigung didaktischer Prinzipien wie handelndes, ganzheitliches, anschauliches und schülerorientiertes Lernen sowie den Wechsel zwischen individuellen und gemeinsamen Lernangeboten.

\section{Methodik}

Im integrativen Unterricht werden unterschiedliche unterrichtliche Methoden flexibel genutzt. Anteile am Frontalunterricht sind geringer als in anderen Regelklassen. Schüleraktive Arbeits- 
phasen (Einzel-, Partner-, Gruppenarbeiten) nehmen prozentual einen höheren Anteil ein. Offene Unterrichtsformen wie Stationenarbeit, Lerntheken oder Wochenplanarbeit werden häufig umgesetzt.

\section{Differenzierung/Individualisierung}

Binnendifferenzierter oder individualisierter Unterricht im engeren Sinn wird selten angeboten. Angebote äußerer Differenzierung (Einzel- oder Gruppenförderung) überwiegen. Schülerleistungen werden leistungsdifferenziert bewertet. Lernstandsdiagnostik und Förderplanung sind selbstverständlich und spielen eine wichtige Rolle. Grenzen der Möglichkeiten der pädagogischen Bearbeitung von Differenz durch angepasste Unterrichtsangebote werden von Lehrkräften wahrgenommen. Diese werden mit Blick auch unterschiedliche sonderpädagogische Förderbedarfe hin differenziert.

\section{Ergebnisse}

Die Lernentwicklung von Schülerinnen und Schülern mit spF in integrativen Schulen verläuft positiv.

Schülerinnen und Schüler mit spF sind in ihren Klassen grundsätzlich sozial gut integriert. Schwierigkeiten können sich bezüglich der sozialen Integration für Schülerinnen und Schüler ergeben, die niedrige Leistungen zeigen, einen niedrigen sozialen Status innehaben und/oder durch unangepasste soziale Verhaltensweisen auffallen. 



\section{Empirischer Teil: Studie zur Qualität schulischer Integration im Saarland}

Die vorliegende Arbeit verfolgt das Ziel die Qualität des Untersuchungsgegenstands „schulische Integration " in Theorie und Forschung wie innerhalb der schulischen Realität zu erfassen. Dazu wurde zunächst ein theoretischer Rahmen gesteckt, der geschichtliche Eckdaten und Begriffe analysiert. Im Anschluss wurde - anhand der vorliegenden Forschungsliteratur - ein Kategoriensystem erstellt, das Qualitätskategorien, -subkategorien und -indikatoren in den drei Bereichen Organisations-, Personal- und Unterrichtsentwicklung umfasst. Im empirischen Teil dieser Arbeit geht es nun darum zu untersuchen, welche Bestandteile dieses Kategoriensystems in der schulischen Realität eine Rolle spielen und in welcher Form diese Qualitätsbausteine umgesetzt werden bzw. inwiefern sie nicht umgesetzt werden. Es geht dieser Untersuchung um eine in erster Linie explorative Herangehensweise und Zielsetzung, da in vorangegangenen Studien weder von einem solchen Kategoriensystem ausgegangen wurde, dieses also in dieser Form zum ersten Mal überprüft wird, noch im ausgewählten Untersuchungsraum, dem Saarland, eine vergleichbare Erhebung zur Umsetzung der schulischen Integration bzw. Inklusion vorliegt. ${ }^{47}$ Mit Blick auf die Typologie von Untersuchungszielen nach Diekmann (2011) spielen neben dieser explorativen Zielsetzung allerdings auch deskriptive und Hypothesen prüfende Bestandteile eine Rolle (Diekmann, 2011, S. 339). Beschreibend soll erschlossen werden, in welcher Häufigkeit einzelne Qualitätsbausteine umgesetzt werden und wie bestimmte Aspekte der Qualität schulischer Integration von unterschiedlichen Beteiligten bewertet werden. Darüber hinaus sollen das theoretisch erschlossene Kategoriensystem und damit verknüpfte Vorannahmen und Hypothesen mit Hilfe der empirischen Forschung überprüft werden. Um diesen Zielsetzungen zu entsprechen wird die Triangulation eines qualitativen mit einem quantitativen Zugang gewählt. Darüber hinaus werden verschiedene Akteure einbezogen: Schulleitungen von Sonderpädagogischen Förder- und Beratungszentren, Schulleitungen von Grund- und Gemeinschaftsschulen, Regel- und Förderschullehrkräfte, die in Regelschulen tätig sind, sowie Eltern von Schülerinnen und Schülern mit sonderpädagogischem Förderbedarf, die in Regelschulen integrativ unterrichtet werden, und nicht zuletzt diese Schüler selbst.

Das vorliegende Kapitel ist folgendermaßen strukturiert: zu Beginn erfolgt eine Beschreibung der Ausgangssituation für die Untersuchung im Saarland. Im Anschluss folgen methodische Vorüberlegungen für das Forschungsprojekt sowie eine Darstellung des geplanten Forschungsdesigns. Danach erfolgt eine ausführliche Darstellung der qualitativen und der quantitativen Teilstudie (methodische Überlegungen, Untersuchungsinstrumente, Vorgehensweise bei der Vorbereitung und bei der Auswertung der Erhebung, Stichprobe, Darstellung der Ergebnisse, Interpretation), bevor eine Gesamtinterpretation der Untersuchungsergebnisse vermittelt wird.

47 Begleituntersuchungen von Sander u.a. befassen sich durchaus mit einzelnen Bestandteilen, nicht aber mit einer umfassenden Bestandsaufnahme. 


\subsection{Schulische Integration bzw. Inklusion im Saarland}

Nach Sander ${ }^{48}$ (1971) erfolgte - ähnlich wie im Bundesgebiet insgesamt - nach dem zweiten Weltkrieg zunächst der Wiederaufbau und Ausbau des Sonderschulwesens an der Saar. Für die Ausbildung von Hilfsschullehrern wurden „behelfsmäßige Ausbildungskurse“ (Sander, 1971, S. 136) eingerichtet. Die Anzahl der Hilfsschulen im Saarland stieg zwischen 1950 und 1956 von 10 auf 17. Diese hatten allerdings im Schnitt nur jeweils 2,5 Klassen (Sander, 1971, S. 147). Im Vergleich zu anderen Bundesländern bezeichnet Sander die „Entwicklung des saarländischen Sonderschulwesens als Ganzes [...] in allen untersuchten Epochen rückständig gegenüber dem Aufbau der Behindertenbeschulung im übrigen Deutschland“"(Sander, 1971, S. 190). Bereits im Oktober 1974 stellte Sander (zu diesem Zeitpunkt Professor an der Pädagogischen Hochschule des Saarlandes) bei einer Jahrestagung sonderpädagogischer Studienstätten das so genannte "Saarbrücker Modell“ vor, das zum Ziel hatte sonderpädagogische Studieninhalte in den Studiengängen aller Lehrämter zu verankern (AK des Saarlandes, 2016, S. 341). Das Saarland hat 1985 nach einem Regierungswechsel von der CDU zur SPD unter Bildungsminister Diether Breitenbach als erstes Bundesland der Bundesrepublik Deutschland die schulische Integration in das Schulordnungsgesetz aufgenommen, die damit ab 1986 eine gesetzliche Grundlage hatte (Schulordnungsgesetz (SchOG) 1986; Integrationsverordnung (IVO), 1987).

Dort heißt es: „Der Unterrichts- und Erziehungsauftrag der Schulen umfasst grundsätzlich auch die behinderten Schüler." (SchoG vom 04.06.1986, \$4 Abs. 1). Zum ersten Mal wurde damit in der Bundesrepublik ,in einem Landesschulgesetz ein grundsätzliches Recht behinderter Kinder auf gemeinsamen Unterricht in den Regelschulen verankert“ (Hinz, 1993, S. 98). Ergänzt wurde das Schulgesetz im Jahr 1987 durch die so genannte „Integrationsverordnung“ (IVO), die es den Eltern von Schülerinnen und Schülern mit festgestelltem sonderpädagogischen Förderbedarf ermöglichte einen Antrag auf integrative Unterrichtung in einer Regelschule zu stellen, unabhängig von Behinderungsart oder Schulform, allerdings unter dem Vorbehalt, dass die Schüler ,in der Schule der Regelform die erforderliche sonderpädagogische Förderung erhalten." (IVO, $₫ 1$ Abs. 1). Die schulgesetzlichen Regelungen im Saarland waren zum damaligen Zeitpunkt damit „weitreichender und eindeutiger als in allen anderen Bundesländern, die diese Integration trotz positiver Erfahrungen immer noch im Status des Schulversuchs belassen hatten" (AK des Saarlandes, 2016, S. 341). Die IVO sah unterschiedliche mögliche abgestufte Organisationsformen integrativer Unterrichtung vor (Bsp.: Regelklasse mit Beratung, Regelklasse mit Ambulanzlehrkraft bis hin zur Kooperation einer Schule für Behinderte mit einer Schule der Regelform) (IVO, $\$ 2$ Abs. 1). Wurde der Antrag auf integrative Unterrichtung durch die Eltern gestellt, beriet ein Förderausschuss auf der Basis einer umfassenden Kind-Umfeld-Diagnose über den möglichen passenden Förderort. Stimmberechtigt waren in diesem Ausschuss die Elternteile, der Klassenleiter der künftigen Regelschulklasse, die Förderschullehrkraft, die das sonderpädagogische Fördergutachten erstellt hat, sowie die Schulleitung der Regelschule als Vorsitz (IVO, §8). Die Entscheidung über eine mögliche integrative Beschulung wurde auf der Grundlage der Empfehlung des Förderausschusses durch die Schulaufsichtsbehörde getroffen

48 Sander, der selbst zunächst als Grund- und Hauptschul- sowie Förderschullehrer tätig war, lehrte als Professor für Erziehungswissenschaft und Sonderpädagogik an der Universität des Saarlandes. In seiner Forschung setzte er sich kritisch mit Förderschulen auseinander, vor allem mit der „Schule für Lernbehinderte“, und begleitete Untersuchungen zur integrativen Förderung im Saarland. Damit nahm er im Saarland eine zentrale Rolle mit Blick auf die Unterstützung der Entwicklung schulischer Integration ein. Aufgrund der Anforderungen, die sich in den Regelschulen hinsichtlich des Umgangs mit Heterogenität ergeben, bemühte er sich wie eingangs erwähnt sehr früh um die Einbindung sonderpädagogischer Inhalte in die universitäre Ausbildung von Regelschullehrern. 
(IVO, §9). Besondere Bedeutung wurde also der Kind-Umfeld-Diagnose zugemessen, die von Sander mit Bezug auf Hobbs (Sander, 1993) entwickelt wurde und die es ermöglichen sollte weg von einer Kategorisierung der Schüler nach Behinderungen hin zu einer Umfeld orientierten Beschreibung zu kommen:

Die Kind-Umfeld-Diagnose wurde dann auch in der saarländischen Integrationsverordnung (IVO) vorgeschrieben und war über 20 Jahre lang die Grundlage für schulische Entscheidungen. Es wurde also nicht mehr klassifiziert „das Kind ist blind“ oder „das Kind ist lernbehindert“, sondern es wurde geprüft, in welche konkrete Schule dieses Kind denn gehen soll und wie dort die Bedingungen sind. Im Rahmen der Umfeld-Diagnose wurde und wird dann in der Regelschule geprüft: Wie groß wird die Klasse sein? Wie steht die Lehrerin oder der Lehrer der Regelschule dazu, solch ein Kind aufzunehmen? Welche Unterstützung kann vonseiten der Eltern gebracht werden, ohne sie zu überfordern? Und so weiter. All diese Umfeldmerkmale spielen also eine wichtige Rolle für die abschließende Empfehlung über die Platzierung und Unterstützung, eine ebenso wichtige Rolle wie die behinderungsbezogenen Merkmale, die in klassischer sonderpädagogischer Diagnostik erhoben werden. Das war das praktische Ergebnis dieser Dekategorisierungskampagne. (Sander \& Müller, 2018, S. 13f)

In den ersten Schuljahren nach Einführung des Gesetzes bzw. der Integrationsverordnung entwickelten sich die Anzahlen der integriert unterrichteten Schüler und Schülerinnen im Saarland von 223 im Jahr 1989 hin zu 374 im Schuljahr 1993/1994 (Sander, 1994, S. 12). Eine Begrenzung des Zuwachses lag - laut Sander - u.a. im Haushaltsvorbehalt der IVO begründet. So äußerte die Bildungsministerin Marianne Granz sich 1991 dahingehend, dass ein weiteres Anwachsen der integrativen Maßnahmen aufgrund der Haushaltslage ausgeschlossen sei, vor allem mit Blick auf zieldifferent zu unterrichtende Schüler (Sander et al., 1994, S. 26). Sowohl der Abschnitt im Schulordnungsgesetz von 1986 als auch die Integrationsverordnung von 1987 sind mehrfach verändert worden, laut Sander und Schnell dienten diese Veränderungen allerdings nicht einer Erleichterung oder Verbesserung der Integration, sondern „anderen Verwaltungszielen“ (Sander \& Schnell, 2006). So lautet der Passus im Schulordnungsgesetz 1996:

Der Unterrichts- und Erziehungsauftrag der Schulen der Regelform umfasst grundsätzlich auch die Schüler mit sonderpädagogischem Förderbedarf. Daher sind im Rahmen der vorhandenen schulorganisatorischen, personellen und sächlichen Möglichkeiten geeignete Formen der gemeinsamen Unterrichtung von Behinderten und Nichtbehinderten zu entwickeln; das Nähere regelt die obere Schulaufsichtsbehörde durch Rechtsverordnung. (SchoG 1996, \$4 Abs. 1)

Sehr früh wurden im Saarland auch erste Sonderpädagogischen Förderzentren (SFZ) eingerichtet. Seit 1988 waren dort zwei Sonderpädagogische Förderzentren aktiv: das SFZ Blieskastel (angegliedert an eine Schule für Lernbehinderte) und das SFZ Sulzbach-Neuweiler (angegliedert an eine Schule für Sprachbehinderte), die zwischen 1991 und 1994 an einem Modellversuch der Bund-Länder-Kommission mit dem Titel „Sonderpädagogische Förderzentren als Weiterentwicklung der Organisation sonderpädagogischer Arbeit für behinderte und nichtbehinderte Schülerinnen und Schüler" teilnahmen (Sander et al., 1995, S. 25). Während der Zeit des Modellversuchs entwickelten die zwei beteiligten SFZ Konzepte, die bspw. die Beratung an den allgemeinbildenden Schulen und die integrationsorientierten Anteile sonderpädagogischer Arbeit thematisierten (Sander et al., 1995, S. 11). Kritisch bewertet und diskutiert wurde allerdings der „Erfolg“ der SFZ. Während von den Antragstellern des Modellversuchs angenommen wurde, dass die Sonderschulen, denen die integrativ arbeitenden SFZ angegliedert wurden, sich im Zeitraum des Modellversuchs zu einer „Schule ohne Schüler“ oder zumindest zu „Sonderschulen mit deutlicher Integrationsorientierung und entsprechendem Interesse an einem Rück- 
gang der sonderschulinternen Schüler/innenzahl“ (Franck-Weber et al., 1990, S. 139ff, zitiert n. Sander et al., 1995, S. 13) entwickeln würden, stieg stattdessen die Anzahl der Schüler mit diagnostiziertem sonderpädagogischen Förderbedarf in den Einzugsgebieten insgesamt, während die Anzahl der Schülerinnen und Schüler in den Sonderschulen unverändert gleich blieb bzw. sogar anstieg (Sander, 1995b, S. 277ff).

Die Organisationsanalyse dokumentiert auf verschiedenen Systemebenen gravierende Hürden und Barrieren (Sander 1995). Angesichts der Vorgabe der Kostenneutralität, der ungenauen Zielübereinstimmung auf den verschiedenen Systemebenen, der sichtbaren Ausgrenzung schwerer beeinträchtigter Kinder und der Gefahr der Zweckentfremdung der Mittel wurde grundlegend diskutiert, inwieweit Förderzentren überbaupt einen Weg zur Integration ebnen (können). (Schumann, 2006, o.S., Herv. im Orig.)

So forderte Sander (1995), dass die Einrichtung von SFZ an die strenge Auflage zu binden sei, dass die Anzahl der Schüler, die in die Sonderschule aufgenommen werden, zurückgeht und die Bezeichnung SFZ wieder entzogen werden solle, wenn das nicht der Fall sei (Sander, 1995, S. 287). Ungeachtet dieser Kritik erfolgte im Jahr 1998 die flächendeckende Einrichtung von sechs regionalen Sonderpädagogischen Förderzentren per Erlass. Diese Förderzentren wurden fachrichtungsübergreifend personalisiert und sollten die sonderpädagogische Förderung in den Regelschulen der Landkreise koordinieren und leisten (Sander \& Schnell, 2006, S. 1). Nach Sander \& Schnell (2006) wurde dieser Erlass ohne Anhörung dahingehend verändert, dass sechs Förderzentren weiterhin in regionaler Zuständigkeit für die Schülerinnen und Schüler mit spF tätig sind, während die Förderzentren Sehen und Hören diese Aufgabe im Saarland für die entsprechenden Förderschwerpunkte überregional erfüllen (Sander \& Schnell, 2006, S. 1). Aus dem Länderbericht von Sander und Schnell aus dem Jahr 2006 geht hervor, dass sich die Entwicklung der schulischen Integration zu dieser Zeit im Saarland eher ungünstig dargestellt hat: „der erreichte Stand [wird] quantitativ nicht abgebaut. In qualitativer Hinsicht muss die Integrationspraxis jedoch seit Jahren an vielen Stellen Einbußen hinnehmen [...], offiziell begründet durch Sparzwänge“ (Sander \& Schnell, 2006, S. 1). Mit Blick auf den Inklusionsanteil von SuS mit spF in den Regelschulen sei zwischen 2003 (24,9\%) und 2006 (26,0\%) ein leichter Aufwärtstrend zu verzeichnen (Sander \& Schnell, 2006, S. 1). Problematisch sei, dass die Förderschullehrerstunden, die in Regelschulen eingebracht werden, seit Ende der 90er Jahre im Saarland nicht mehr statistisch erfasst werden würden:

[...] Auskünfte von der Schulbehörde sind auch auf Anfrage nicht zu erhalten. Die für Sonderschulen geltende Schüler-Lehrer-Relation, die seit Mitte der 90er Jahre als Grundlage für die stundenmäßige Ausstattung der Einzelintegration oder auch der Integrationsklassen galt und die Berechnungsbasis für den Stunden-Pool der Förderzentren bildete, wird jetzt immer häufiger ignoriert, so dass auch Förderzentren mit großer Bereitschaft zur Integration Schwierigkeiten haben, Kinder mit schwereren Behinderungen integrativ zu unterstützen. (Sander \& Schnell, 2006, S. 2)

Von Bedeutung für die Umsetzung der schulischen Integration im Saarland ist auch die Einführung der Gemeinschaftsschule, die ab dem Schuljahr 2012/2013 in Klasse 5 aufbauend erfolgte und seit 2017 die Gesamtschulen und Erweiterten Realschulen ersetzt hat. Diese bietet vom Hauptschulabschluss bis zum Abitur nach 9 Jahren alle Abschlüsse an. Pädagogische Ziele dieser Schulform sind Individualisierung, selbstständiges, fächerübergreifendes und projektorientiertes Lernen und eine Versetzungsentscheidung erst am Ende der Klassenstufe 8. Die Stufen 5 und 6 werden im Klassenverband unterrichtet, ab Klassenstufe 7 werden Differenzierungskurse in Deutsch, Mathematik, erster Fremdsprache und Naturwissenschaften angeboten. Für zieldif- 
ferent unterrichtete Schüler ist die Einführung der zweiten Fremdsprache in Klassenstufe 7 als schwierige Anforderung einzustufen (Ministerium für Bildung des Saarlandes, 2011a).

In den Schuljahren 2011/12 bis 2013/14 ${ }^{49}$ fand im Saarland an sieben Grundschulen und vier weiterführenden Schulen (2 ERS, 2 GS) der „Modellversuch inklusive Schule“ als Pilotprojekt statt. („Erlass zur Einrichtung des Pilotprojekts zur Entwicklung eines inklusiven Förderkonzepts an Regelschulen im Saarland" vom 14.06.2011). Die beteiligten Schulen sollten ein inklusives Förderkonzept für Regelschulen entwickeln (Lange, 2015, S. 9). Als Eckpunkte dieses Förderkonzepts wurden von der Expertengruppe Inklusion u.a. folgende Aspekte angedacht:

- Einleitung von Fördermaßnahmen in den Bereichen Lernen, Sprache und emotionale und soziale Entwicklung durch die Regelschulen in Kooperation mit den an diesen Schulen tätigten Sonderpädagogen, Beratung im Rahmen von Förderkonferenzen,

- Anträge auf Überprüfung des spF nur bei Wunsch der Erziehungsberechtigten nach Beschulung in einer Förderschule oder bei schweren Beeinträchtigungen,

- individuelle Förderpläne als Grundlage für die Beschulung, Möglichkeit Leistungsanforderungen (zeitlich befristet) zu senken,

- bei leistungsdifferenzierter Unterrichtung Vermerk auf dem Zeugnis, dass der betreffende Schüler nach einem individuellen Förderplan unterrichtet wurde,

- Klassenstufen 1 und 2 als pädagogische Einheit,

- Erstellung einer Arbeitsplatzbeschreibung für Förderschullehrkräfte in Regelschulen,

- Ressourcenzuweisung entsprechend dem Förderstundenbedarf in den Förderschulen Lernen, Sprache sowie emotionale und soziale Entwicklung + zusätzliche Förderstunden in den Eingangsklassen 1 bzw. 5 (Ministerium für Bildung des Saarlandes, 2011c, S. 2-6) (Ministerium für Bildung des Saarlandes, 2011b; Minsterium für Bildung des Saarlandes, 2011c).

Im Mittelpunkt standen dabei strukturelle Veränderungen (Bsp.: diagnostische Überprüfung, befristete Senkung des Anforderungsniveaus möglich, Leistungsbeurteilung (Ministerium für Bildung des Saarlandes, 2011c, S. 2). Der Modellversuch wurde von der Universität des Saarlandes unter Federführung von Herrn Prof. Dr. Stark und seines wissenschaftlichen Mitarbeiters Herr Schön wissenschaftlich begleitet (s. Forschungsergebnisse weiter unten).

Am 25. Juni 2014 beschloss der Landtag des Saarlandes mit der Änderung des Schulordnungsgesetzes einstimmig eine neue Schulgesetzgebung, bei der der Begriff der „inklusiven Schule“ aufgegriffen wurde. Dort heißt es: „Die öffentlichen Schulen der Regelform sind inklusive Schulen. Sie ermöglichen grundsätzlich allen Schülerinnen und Schülern einen gleichberechtigten und ungehinderten Zugang." (SChoG 2016, \$4 Abs. 1). Dieses Schulgesetz beinhaltet anders als die Integrationsverordnung - keinen Haushaltsvorbehalt (Lange, 2015, S. 11).

Statt eines Integrationsantrages auf Regelbeschulung muss nun ein Antrag auf Sonderbeschulung gestellt werden. Konkret bedeutet dies, dass seit dem Schuljahr 2014/2015 grundsätzlich alle Kinder im Einzugsbereich einer Grundschule in die erste Jahrgangsstufe aufgenommen werden. Ab dem [...] Schuljahr 2016/2017 finden die Regelungen auch auf die allgemeinbildenden weiterführenden Schulen beginnend mit der fünften Jahrgangsstufe Anwendung. Mit Beginn des Schuljahres 2018/2019 sollen schließlich die beruflichen Schulen einbezogen werden. (AK des Saarlandes, 2016, S. 341)

In den Regelschulen kann besondere pädagogische Förderung von den Erziehungsberechtigten individuell beantragt werden. Anschließend werden Umfang und Art der Unterstützung

49 Nach der Schulgesetzänderung vom 25.06.2014 wurden der Modellversuch verlängert und weitere Schulen aufgenommen. 
geplant. Nur wenn Eltern dies wünschen, kann - in Kooperation mit einem Förder- und Beratungszentrum - entschieden werden, ob die Förderung ggf. an einer Förderschule stattfindet (AK des Saarlandes, 2018, S. 378). Die die Integrationsverordnung ablösende Inklusionsverordnung trat zu Beginn des Schuljahres 2015/2016, am 3. August 2015, in Kraft.

„Die in den vergangenen Jahren getätigten Anpassungen der rechtlichen Rahmenbedingungen führen nicht zwingend dazu, dass sich der Schulalltag hin zu einer inklusiven Schulgemeinschaft verändert." (AK des Saarlandes, 2016, S. 342). Zumindest in quantitativer Hinsicht lässt sich für das Saarland festhalten, dass zwischen der Ratifizierung der UN-BRK im Jahr 2009 und dem Jahr 2015/2016 bzw. 2016/2017 der Inklusionsanteil gestiegen ist, gleichzeitig allerdings auch die Förderquote, so dass sich die Exklusionsquote kaum verändert hat. So verdeutlichen die landesbezogenen statistischen Daten, dass die Förderquote sich um 2,54\% erhöht hat (2008/2009: 6,16\%; 2015/2016: 8,7\%) und der Inklusionsanteil von 31,22 \% im Jahr 2008/2009 auf 48,6\% im Jahr 2015/2016. Die Exklusionsquote blieb konstant bei 4,2\%. Die Anzahl der Schülerinnen und Schüler, die in Förderschulen unterrichtet werden, sank in diesem Zeitraum von 3365 auf 3359, also lediglich um 6 Schüler (Klemm, 2018, S. 18; Lange, 2017, S. 14-20). ${ }^{50}$

2014/2015 standen an den 162 saarländischen Grundschulen 123 Stellen für Förderschullehrer zur Verfügung. Die Zuweisung erfolgt nicht mehr orientiert an der Anzahl der Schüler mit spF, sondern als „Schulbudget“, das sich an der Größe der Schule, der Anzahl der Klassen und dem Einzugsgebiet orientiert (AK des Saarlandes, 2016, S. 343). Seit 2015/2016 erfolgt diese Budgetierung auch an den weiterführenden Schulen. Auf den Stellenplan der Regelschullehrer hat die Einführung der inklusiven Beschulung im Saarland keine Auswirkungen. Als Mindestvoraussetzung in den weiterführenden Schulen benennt die Arbeitskammer gemeinsam mit der GEW eine Förderschullehrkraft je Jahrgangsstufe als Mindestversorgung (AK des Saarlandes, 2016, S. 344). Ähnlich wie bundesweit fehlen auch im Saarland nach Angaben der Arbeitskammer ausgebildete Lehrkräfte, im Grundschul- wie im Förderschulbereich.

Mit Blick auf die Professionalisierung der Lehrkräfte ist der „Umgang mit Heterogenität“ als Wahlpflichtmodul im Studiengang Lehramt für Primarstufe an der Universität Saarbrücken eingerichtet worden. Für die Lehrkräfte an weiterführenden Schulen sind Fortbildungen möglich. Von Seiten der saarländischen Fortbildungsinstitute (LPM und ILF) aus werden neben den Fortbildungsveranstaltungen auch Pädagogische Tage für ganze Kollegien, Hospitationen an den Pilotschulen oder die Unterstützung durch Inklusionsberater angeboten (AK des Saarlandes, 2016, S. 345; Lange \& Sastges-Schank, 2017, S. 22).

Nach Lange (2015) gibt es im Saarland - anders als in anderen Bundesländern - keine ausdrückliche Möglichkeit „einer finanziellen Unterstützung baulicher Maßnahmen nach Maßgabe des Landeshaushalts" (Mißling \& Ücker, 2014, S. 27; zitiert n. Lange, 2015, S. 18f). Das Ministerium für Bildung und Kultur des Saarlandes habe zwar 2014 angekündigt, Gespräche mit den Schulträgern mit Blick auf eine barrierefreie Gestaltung von Schulen zu führen, finanzielle Unterstützungsmaßnahmen seien diesbezüglich aber nicht geplant (Lange, 2015, S. 19). Die Verzahnung von Kostenträgern benennt die AK des Saarlandes als Anforderung, die neben baulichen Maßnahmen vor allem mit Blick auf die „bedarfsgerechte pflegerische und therapeutische Versorgung von Kindern und Jugendlichen mit hohem Unterstützungsbedarf in einem ganzheitlichen Bildungs- und Versorgungskonzept verankert werden [sollte] " (AK des Saarlandes, 2016, S. 345). Tatsächlich belegt bspw. der Koalitionsvertrag im Saarland (2012), dass keine

50 Da der sonderpädagogische Förderbedarf im Saarland nur noch dann diagnostiziert und festgestellt wird, wenn eine Umschulung in eine Förderschule erfolgt, können Inklusionsanteil und Förderquote nach Klemm seit dem Schuljahr 2016/2017 nicht mehr statistisch erfasst werden (Klemm, 2018, S. 18). 
„Anschubinvestitionen“ mit Blick auf das Personal geplant sind. Mit Blick auf die Einhaltung der grundgesetzlichen Schuldenbremse formulieren CDU und SPD zwar, dass „die „demografische Rendite“, die sich aufgrund rückläufiger Schülerzahlen und nach Gegenrechnung der deswegen sinkenden Einnahmen des Landes aus dem bundesstaatlichen Finanzausgleich ergibt, im System belassen [werden soll]“ (Koalitionsvertrag Saarland CDU \& SPD, 2012, S. 7), im Klartext bedeutet dies allerdings, dass keine zusätzlichen Ausgaben für Lehrkräfte vorgesehen sind. Der Koalitionsvertrag aus dem Jahr 2017 enthält lediglich die Aussage, dass „die Landesregierung gemeinsam mit den Schulträgern und Leistungsträgern im Rahmen einer stärkeren Sozialraumorientierung die Entwicklung von Kriterien einer passgenauen Ressourcenzuweisung [prüft]“ (Koalitionsvertrag Saarland CDU \& SPD, 2017, S. 47). Im Gegensatz zum Koalitionsvertrag 2012 werden keine konkreten Zahlen (Bsp.: 25 einzustellende Lehrkräfte für GMS, 27 für GYM) mehr genannt.

Über die vergangenen Jahrzehnte hinweg hat im Saarland ein intensiver und lebhafter Diskurs zu Frage schulischer Integration bzw. Inklusion stattgefunden. In diesen Diskurs waren und sind vielfältige unterschiedliche Akteure eingebunden, darunter vor allem die Bildungspolitik, die Schulverwaltung (v.a. Referatsleitungen Förderschule, Grundschule und weiterführende Schulen), Herr Prof. Dr. Sander und Frau Dr. Schnell als wissenschaftliche Vertreter, die Schulträger, die GEW, der Verein „Miteinander leben lernen e.V“, der Vds, die Gesamtlandeselternvertretung des Saarlandes, die Arbeitskammer des Saarlandes sowie die Leitungen der Förder- und Beratungszentren.

Sander bezeichnet mit Blick auf den Diskurs und die Umsetzung schulischer Integration im Saarland rückblickend vor allem „die Auseinandersetzung mit der Schulverwaltung, nicht mit der Ministeriumsspitze, aber mit den mittleren Chargen; und auch auf der Ebene der Schulräte, die ja regional zuständig waren“ (Sander \& Müller, 2018, S. 12) als Herausforderung. Im Einzelfall sei es oft schwierig gewesen, „Integrationsmaßnahmen durchzusetzen, und zwar so durchzusetzen, dass nicht das betroffene Kind darunter hätte leiden müssen." (Sander \& Müller, 2018, S. 12). Darüber hinaus habe vor allem das Sonderschulreferat im Kultusministerium häufig ablehnend auf die Vorschläge der Arbeitseinheit Sonderpädagogik der Universität reagiert und diese nicht unterstützt. Auch die Gründung einer „Landeskommission für Integration“ unter Vorsitz von Herrn Christ hätte schulpolitisch nur wenig Einfluss gehabt. Als die Haushaltsvorbehalte im Zusammenhang mit der Umsetzung schulischer Integration zunahmen, habe man sich daher zunehmend mit dem Landesinstitut für Pädagogik und Medien (LPM) sowie mit der GEW vernetzt (Sander \& Müller, 2018, S. 12). Problematisch sei aus wissenschaftlicher Sicht im Nachhinein zu werten, dass die bildungspolitische, strategische Arbeit und die Netzwerkarbeit viel Zeit beansprucht hätten und die „Politikberatung“ immer wieder im Vordergrund stand (Sander \& Müller, 2018, S. 17). Zu Beginn der 2000er Jahre ist es nach Einschätzung von Sander und Schnell bildungspolitisch

[...] eher still geworden. Die Landesregierung, gestützt auf absolute CDU-Mehrheit im Landtag, ergreift keine Initiative zur Weiterentwicklung von gemeinsamem Leben und Lernen - weder im Vorschul- noch im Schulbereich. Auch die parlamentarische Opposition schweigt. Allerdings wird der erreichte Stand quantitativ nicht abgebaut. In qualitativer Hinsicht muss die Integrationspraxis jedoch seit Jahren an vielen Stellen Einbußen hinnehmen (s. Punkt 3), offiziell begründet durch Sparzwänge. (Sander \& Schnell, 2006, S. 1)

Wie u.a. der Internetauftritt, die gesetzlichen Veränderungen und die Diskussionspapiere des Ministeriums für Bildung des Saarlandes zeigen, ist die Gesamthaltung der Bildungspolitik inzwischen offensichtlich im Vergleich „inklusionsfreundlicher“, auch wenn diese Einschätzung 
von einigen Diskursbeteiligten im Saarland (Bsp.: Arbeitskammer, MLL) anders eingeschätzt wird.

Besonders aktiv sei dagegen weiterhin der Elternverein „Miteinander Leben Lernen e.V:, der die Beratung und Unterstützung von Integration in Kindergärten, Schulen und nachschulischen Einrichtung vornehme und sich immer wieder in Diskussionen mit Politik und Verwaltung einbringe (Sander \& Schnell, 2006, S. 1). Insbesondere dieser Verein ist in keiner Weise mit dem neuen Schulgesetz und der Inklusionsverordnung des Saarlandes aus den Jahren 2014 und 2015 einverstanden, da diese gesetzliche Grundlage weder einen einklagbaren Rechtsanspruch für Kinder und Jugendliche mit Behinderung auf eine wohnortnahe inklusive Beschulung beinhalte noch dazu führe, dass die bestehenden Förderschulen abgeschafft werden würden ${ }^{51}$ (MLL, 2014, S. 1). Der Verein hat daher gemeinsam mit der Kanzlei Latham \& Watkins einen eigenen Gesetzentwurf erarbeitet, der u.a. weitreichende gesetzliche Veränderungen im Schulordnungsgesetz vorsieht (MLL \& Latham \& Watkins, 2014). Darüber hinaus befürwortet der Verein orientiert am Institut für Menschenrechte in Berlin ${ }^{52}$ - die Abschaffung des Elternwahlrechts und kritisiert die indirekte Steuerung der Fortführung der Beschulung in Förderschulen, die darin begründet sei, dass Eltern ihre Kinder in Förderschulen unterrichten lassen wollen, da diese besser ausgestattet seien (AK Saarland, 2016, S. 346), während Kiefer (2010) zur Abschaffung des Elternrechts verfassungsrechtliche Bedenken anführt:

Es besteht [...] eine Verpflichtung des Staates, ein ausreichend differenziertes Schulangebot zur Verfügung zu stellen. Das daraus resultierende Wahlrecht der Eltern zwischen den vom Staat zur Verfügung gestellten Schulformen darf nicht mehr als zulässig begrenzt werden. Die Grenze des verfassungsrechtlich Zulässigen wird dann überschritten, wenn das Wahl- und Bestimmungsrecht der Eltern angesichts nur noch einer einzigen vorhandenen obligatorischen Schulform mit einem vom Staat einseitig festgelegten Bildungsziel obsolet wird und praktisch leerläuft. Von daher wäre der Versuch, die Gemeinschaftsschule als einzige Schulform (im Sinne einer Einheitsschule) etablieren zu wollen, von vornherein zum Scheitern verurteilt. (Kiefer, 2010, S. 135)

Die Arbeitskammer des Saarlandes zieht 2018 dahingehend Bilanz, das bildungspolitische Interessenverbände, Lehrerverbände und Gewerkschaften die Umsetzung der Inklusion als „durchwachsen“ bewerten und dies in erster Linie mit der mangelnden Ressourcenausstattung begründen. Auch die Arbeitskammer des Saarlandes selbst positioniert sich dahingehend, dass die schulpolitischen Vorgaben im Saarland nicht den Vorgaben der UN-BRK für den Bereich der schulischen Bildung entsprechen und verweist ebenfalls auf das Institut für Menschenrechte, das die Aufrechterhaltung eines Parallelsystems von Förderschulen als widersprüchlich zu Artikel 24 der UN-BRK auffasst (Dt. Institut für Menschrechte, 2017; AK Saarland, 2018, S. 378). Kritisch bewertet die Arbeitskammer auch den Koalitionsvertrag von 2017, in dem bei steigenden Schülerzahlen sogar die Einrichtung weiterer Förderschulen angekündigt werde. Begrüßt werden dagegen die Einrichtung eines Kompetenzzentrums Inklusion sowie die geplante Analyse und Bewertung der Inklusion im Saarland durch das Institut für Menschenrechte (AK Saarland, 2018, S. 379).

51 s. dazu auch Saarbrücker Zeitung vom 11.12.2014. Unter: https://www.saarbruecker-zeitung.de/saarland/saarbruecken/saarbruecken/saarbruecken/saarbruecker-verband-fordert-neues-gesetz-fuer-inklusion_aid-1407225; Stand: 25.08.2015

52 Nimmt man die Vorgaben ernst, existiert laut Valentin Aichele von der Monitoring-Stelle am Deutschen Institut für Menschenrechte gar kein Elternwahlrecht, denn „das Recht auf Inklusion ist das Recht der Person mit Behinderung. Die Eltern haben bei der Ausübung ihrer elterlichen Sorge den Leitgedanken der Inklusion zu beachten und ggf. zu erklären, warum sie keine inklusiven Bildungsangebote wahrnehmen“. (AK Saarland, 2016. S. 346) 
Begleitet wurde die schulische Integration im Saarland immer wieder auch durch wissenschaftliche Veröffentlichungen zur Thematik, die jährlich oder alle zwei Jahre federführend von Herrn Prof. Dr. Sander herausgegeben wurden (Institut Erziehungswissenschaft, Arbeitseinheit Sonderpädagogik an der Universität des Saarlandes, Saarbrücker Beiträge zur Integrationspädagogik, insgesamt 12 Bände). Darüber hinaus erfolgte über diese Arbeitseinheit auch ein bundesweiter Austausch mit anderen Begleitgruppen, die sich wissenschaftlich mit schulischer Integration befassten (Sander \& Müller, 2018, S. 10). Bereits 1988 wurden bspw. Qualitätsanforderungen an schulische Integration formuliert, die eine Klassengröße von 20 Schülern und Schülerinnen, eine Doppelbesetzung sowie zwei Beratungsstunden bei sechs Förderstunden fordern (Fuchs, 1988, S. 147f), Integration auch im Elementarbereich, Informationen und Lehrerfortbildungen, spezifische Vorgehensweisen für Leistungsbeurteilung und Versetzung oder die Einrichtung sonderpädagogischer Zentren zur Sicherung der wohnortnahen integrativen Beschulung durch Ambulanzlehrer (Beispiele für Empfehlungen der KIBS (Kommission für die Integration behinderter Schüler und Schülerinnen ${ }^{53}$ ); Sander, 1988, S. 215-270).

Der „Modellversuch inklusive Schule“ wurde ebenfalls wissenschaftlich begleitet. Stark und Schön $(2012$, 2014) befassten sich im Rahmen ihrer Studie mit Fragestellungen, die den Stand der Schulen sowie die Einstellungen der Beteiligten (Schulleitungen, Lehrkräfte, Eltern und Schüler) mit Blick auf inklusive Bildung betreffen. Als den Schulentwicklungsbereichen übergeordnetes Problem beschreiben sie aufgrund ihrer Befragung den mangelnden Informationsfluss und zwischen dem Ministerium für Bildung, den Schulträgern und den Schulen (Schön \& Stark, 2012, S. 24 und S. 30), der wiederum zu mangelnder Transparenz und teilweise zu schwierigen Verhandlungen mit den Kommunen hinsichtlich der Bereitstellung von Sachmitteln geführt habe (Schön \& Stark, 2012, S. 30f).

Ihre zentralen Ergebnisse beziehen sich im Bereich der Organisationsentwicklung darauf, dass die Pilotschulen nach Auffassung der Befragten mit unzureichenden Rahmenbedingungen umgehen müssen (Bsp.: wenig flexible Räumlichkeiten, zu wenige Differenzierungsräume, Personalmangel, zu große Klassen (s.u.)) (Schön \& Stark, 2012, S. 29f). Ein Problem stelle für die Pilotschulen vor allem die Anschaffung differenzierter Materialien für den Unterricht dar (Schön \& Stark, 2014, S. 8), bspw. da die Schulbuchausleihe für inklusives Arbeiten neu geregelt werden müsse (Schön \& Stark, 2012, S. 29). Das soziale Lernen in den Schulen wird positiv erlebt (Schön \& Stark, 2014, S. 8), Akzeptanz und gegenseitige Hilfestellung seien zwischen den Schülern selbstverständlich und eine Bereicherung für den schulischen Alltag (Schön \& Stark, 2012, S. 25). Hinsichtlich der Organisationsstrukturen wird von einigen beteiligten Grundschulen bemängelt, dass ihre Schulkindergärten weggefallen sind (Schön \& Stark, 2012, S. 29).

Auch im Bereich der Personalentwicklung werden die Ressourcen kritisch eingeschätzt. Da für Schüler mit sonderpädagischem Förderbedarf im Pilotversuch keine Gutachtentanträge mehr gestellt werden, wird die Sorge geäußert, dass zukünftig ggf. keine weiteren Förderschullehrerstunden mehr hinzukommen könnten (Schön \& Stark, 2012, S. 29). Der vorhandene Personalschlüssel reiche nicht aus für gemeinsame Besprechungen und Unterrichtsplanung (Schön \& Stark, 2014, S. 8). Auch Teamteaching sei nur vereinzelt möglich (Schön \& Stark, 2012, S. 26). Positiv berichtet wird, dass die Förderschullehrkräfte an den Pilotschulen ein sehr hohes Maß an Akzeptanz erfahren und die Diagnostik sonderpädagogischen Förderbedarfs stark vereinfacht werde:

53 Diese Kommission wurde 1985 durch den damaligen Kultusminister Prof. Dr. Breitenbach einberufen und setzte sich aus Vertretern von Verbänden, Vereinen, der Landeselternvertretung, des schulpsychologischen Dienstes, des schulärztlichen Dienstes, der Sonderpädagogik (Schule und Universität), der Referate im Kultusministerium und weiteren Experten zusammen (Sander, 1988, S. 216f). 
Förderschullehrkräfte werden von ihren Regelschulkollegen und -kolleginnen als Bereicherung gesehen, vor allem auf Grund der Tatsache, somit fachlich kompetente Ansprechpartner für spezifische sonderpädagogische Fragestellungen direkt vor Ort zu haben und sich jederzeit bei Problemen an sie wenden zu können. [...] Darüber hinaus ergibt sich dadurch ein weitaus schnelleres und effektiveres Arbeiten innerhalb der Schule. Durch die internen Prozesse, Meldeprogramme und Diagnosen kann Förderbedarf wesentlich schneller erkannt und flexibler auf diesen Bedarf reagiert werden als dies durch die bisherigen aufwändigen, zeitintensiven und als zu bürokratisch empfundenen Meldeverfahren möglich war. (Schön \& Stark, 2012, S. 24 und S. 26)

Das Fortbildungsangebot wird von den befragten Lehrkräften als wenig hilfreich und unzureichend empfunden, da es zu wenig konkrete Unterstützung für die praktische Umsetzung biete. Die Lehrkräfte wünschen sich fachlich kompetente Ansprechpartner und anwendungsbezogene Konzepte, die für die Umsetzung inklusiver Bildung genutzt werden können (Schön \& Stark, 2012, S. 28 und 30). Die Einstellungen der Beteiligten (Lehrkräfte, Eltern und Schüler) gegenüber der Inklusion seien grundsätzlich positiv, was zum Teil mit jahrzehntelangen Erfahrungen der Schulen im Rahmen schulischer Integration zu tun habe. Gleichzeitig seien allerdings vielfältige Ängste präsent, die mit den unzureichenden Rahmenbedingungen zusammenhängen (Schön \& Stark, 2012, S. 23). Als besondere Belastung wird die Unterrichtung von SuS mit dem spF soziale und emotionale Entwicklung thematisiert (Schön \& Stark, 2012, S. 24). Mit Bezug auf die Unterrichtsentwicklung wird die Klassenstärke in den Pilotschulen als zu hoch eingeschätzt um differenzierend mit den Schülern arbeiten zu können (Schön \& Stark, 2012, S. 29). Diesbezüglich sei der Klassenteiler im Saarland nicht für inklusives Arbeiten geeignet (Schön \& Stark, 2014, S. 8).

\subsection{Forschungsmethodik}

In Anlehnung an Moser (2004) wird übergeordnet von einem konstruktivistischen Forschungszugang und damit einem systemtheoretischen Modell qualitativer und quantitativer Methoden (Moser, 2004, S. 7) ausgegangen, welches „das Bewusstsein um die Vernetztheit von Problemlagen und die Komplexität wissenschaftlicher Beobachtungsfelder“ (Moser, 2004, S. 8) berücksichtigt. Konstruktionen werden in diesem Zusammenhang als „spontane Ordnungsbildungen“ (Moser, 2004, S. 10) verstanden und als dynamische Systeme, „die in Relation zu Umwelten ihre Wirkungspotenziale entfalten und Wirklichkeiten als Verhaltensspielräume hervorbringen" (Moser, 2004, S. 10). Bei der Analyse von Konstruktionen spielen die Intentionalität und Funktionalität von Handlungen eine Rolle. Die konstruktivistische Methodologie vertritt eine prozessuale, relationale und kontextualisierende Auffassung wissenschaftlicher Tätigkeit. Sie widmet sich komplexen Beschreibungen und bettet diese Beschreibungen kontextuell erklärend ein (Moser, 2004, S. 10f):

Wissenschaftlichen Methoden kommt hier sozusagen die Rolle operativer Schnittstellen zu, welche anhand von Messoperationen einen selektiven Kontakt mit den theoretisch definierten Gegenständen ermöglichen und die Komplexität theoretischer Beschreibungen auf einfachere Beschreibungen („Indikatoren“) reduzieren. Die Ergebnisse dieses methodisch kontrollierten Umgangs mit der Wirklichkeit werden im Kontext des theoretischen Begriffsnetzes („Informationsmusters“) interpretiert. (Moser, 2004, S. 19f)

Neben der Untersuchung des im Theorieteil erarbeiteten Kategoriensystems in der schulischen Praxis geht es also auch darum die Ergebnisse in inhaltliche Zusammenhänge einzubetten und mögliche dynamische Wechselbeziehungen aufzuzeigen. 
Mit Hilfe der geplanten Studien soll es - in Anlehnung an das Verständnis von Lüders \& Reicherz (1986, S. 93f) - gelingen soziales Handeln mit Blick auf schulische Integration zu beschreiben. Die Untersuchung zur Qualität schulischer Integration im Saarland hat explorativen Charakter (Diekmann, 2011, S.33f), da derzeit keine aktuellen Forschungsergebnisse zum Thema in diesem Bundesland vorliegen, und verfolgt eine in erster Linie eine deskriptive Zielsetzung (Diekmann, 2011, S. 35f) in dem Sinn, dass grundlegende qualitative wie quantitative Daten zur Umsetzung schulischer Integration erhoben werden sollen, mit Hilfe derer die oben aufgeführten Hypothesen überprüft werden können. Aufgrund des explorativen Charakters des Forschungsprojekts soll eine Triangulation der Daten dazu dienen über zwei methodische $\mathrm{Zu}$ gänge - qualitativ orientierte Interviews sowie eine quantitativ orientierte Befragung - einen möglichst intensiven Einblick in das Forschungsfeld der Qualität der schulischen Integration im Saarland zu gewinnen:

Durch die Triangulation (etwa verschiedener Methoden oder verschiedener Datensorten) sollte ein prinzipieller Erkenntniszuwachs möglich sein, dass also bspw. Erkenntnisse auf unterschiedlichen Ebenen gewonnen werden, die damit weiter reichen, als es mit einem Zugang möglich wäre. (Flick, 2011b, S. 12)

Die Triangulation dient nach Denzin (1970) der Validierung von Ergebnissen, der Steigerung der Reliabilität von Vorgehensweisen sowie der Fundierung der Theoriebildung durch die verschiedenen Triangulationsformen. Kritisch ist an dieser Auffassung nach Flick einzuschätzen, dass „jede Methode den Gegenstand, der mit ihr erforscht bzw. abgebildet werden soll, auf spezifische Weise konstituiert" (Flick, 2011b, S. 17), so dass die Verwendung unterschiedlicher Methoden nicht automatisch dazu führe, dass ähnliche Ergebnisse zustande kommen bzw. die Ergebnisse der einen Studie durch die der anderen widerlegt werden. Hammersley (1996) geht gemäß Flick (2011) davon aus, dass die Verknüpfung der Verwendung eines qualitativen und eines quantitativen Zugangs allerdings dazu führen kann, dass die Ansätze unterstützend wirken, „bspw. liefert der eine Ansatz Hypothesen und Denkansätze für die Weiterführung der Analysen mit dem anderen Ansatz" (Flick, 2011, S.76), außerdem könnten die Ansätze als „komplementäre Forschungsstrategien kombiniert werden“ (Flick, 2011, S. 76). Dabei liegt der Schwerpunkt der Kombination nach Hammersley (1996, S. 167f) auf der Möglichkeit der wechselseitigen Überprüfung der Ergebnisse (n. Flick 2011, 75f).

Da für die eigenen Studien aufgrund der theoretischen Vorarbeit ein komplexes Kategoriensystem vorliegt, dient die qualitative Studie in Form von Experteninterviews zunächst dazu mit Hilfe von offenen Fragestellungen zu eruieren, inwiefern diese Kategorien im Forschungsfeld tatsächlich relevant sind. Welche dieser Kategorien finden in der praktischen Umsetzung der schulischen Integration Berücksichtigung? Welche Bereiche werden zustimmend oder kritisch eingeschätzt? Wie nehmen unterschiedliche Akteure die Umsetzung schulischer Integration in ihren Schulen qualitativ wahr? Die Aussagen der Expertinnen und Experten und die oben erwähnte mögliche Einbettung dieser Aussagen in Zusammenhänge ermöglichen eine inhaltlich tief gehende Analyse. Dagegen folgt die quantitative Teilstudie einem eher geschlossenen Forschungsansatz. Ziel der Verschränkung der beiden Forschungsansätze ist eine mehrperspektivische Prüfung des Kategoriensystems.

Für die quantitative Erhebung wird das Kategoriensystems dann zunächst mit Hilfe der Ergebnisse der Experteninterviews überarbeitet. Darüber hinaus wird mit Blick auf die Anzahl der Subkategorien bzw. Indikatoren aus pragmatischen Gründen eine Auswahl der Kategorien für die quantitative Erhebung ermöglicht. In Anlehnung an Bryman dienen die Ergebnisse beider 
Untersuchungen der „Herstellung eines allgemeineren Bildes des untersuchten Gegenstandes" (Flick, 2011b, S. 76). Zielsetzung der Kombination ist nicht, dass die Ergebnisse aus den Interviews durch die Fragebogenerhebung generalisierbar werden, sondern dass eine größere Menge an Erfahrungsaussagen beteiligter Personen gewonnen werden kann. Der Stellenwert beider Forschungsansätze wird als gleichberechtigt eingestuft, auch wenn diese unterschiedliche Arten von Daten liefern. Die Fragebogenerhebung dient also vor allem dazu die qualitativen Ergebnisse der ersten Erhebung durch die weiteren Ergebnisse mit einer größeren Anzahl von Befragten zu ergänzen, so dass ein quantitativ (wie ggf. auch qualitativ) umfassenderes Bild des Forschungsgegenstandes hergestellt werden kann (Flick, 2011b, S. 76), was Bryman als „completeness“ bezeichnet: „the researcher can bring together a more comprehensive account oft he area" (Bryman, 2012, S. 633). Da auch die quantitativ orientierten Fragebögen qualitative Daten erheben werden (teilweise offenen Fragen), ist keine strenge methodische Trennung zwischen rein qualitativer Studie und rein quantitativer Studie möglich. Wesentlich ist die Zusammenschau und Interpretation der Ergebnisse beider Teilstudien vor dem Hintergrund des umfassenden theoretischen Modells und die Unterstützung derselben durch die Darstellung von (möglichen) Zusammenhängen zwischen einzelnen Variablen.

Somit ergänzen sich qualitative und quantitative Ansätze gegenseitig und konkurrieren nicht miteinander. Jeder liefert eine Art von Information, die sich nicht nur von der anderen unterscheidet, sondern auch für deren Verständnis wichtig ist (...) Die Anwendung einer bestimmten Methode kann man also nicht mit seinem „Paradigma“ oder seinen Neigungen begründen, sondern sie muss von der Eigenart des jeweiligen Forschungsproblems ausgehen." (Wilson 1982, S. 501; zitiert n. Flick, 2011b, S. 53)

Die Vorgehensweise lässt sich dabei in folgenden Phasen darstellen:

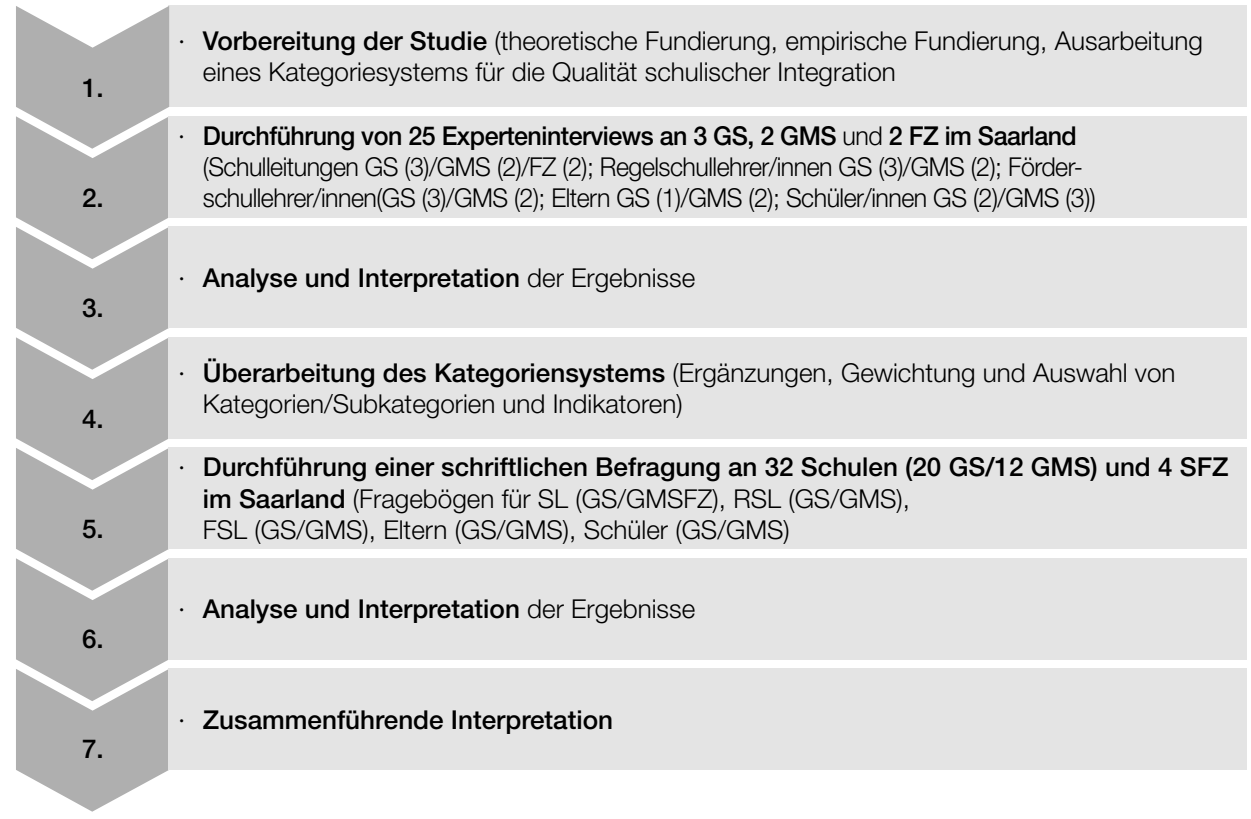

Abb. 5: Phasen des Forschungsprojekts 
$\mathrm{Zu}$ berücksichtigen ist in diesem Zusammenhang, dass es sich bei der Phasendarstellung um eine idealisierte lineare Vorgehensweise handelt:

A further point to bear in mind is that the deductive process appears very linear - one step follows the other in a clear, logical sequence. However, there are many instances where this is not the case: a researcher's view of the theory or literature may have changed as a result of the analysis of the collected data for a theory may become apparent after the data have been collected. (Bryman, 2012, S. 25, Herv. im Orig.)

Deduktive und induktive Zugänge bewegen sich vielmehr in einem Kreislauf von der Theorie zu empirischen Ergebnissen und umgekehrt:

However, just as deduction entails an element of induction, the inductive process is likely to entail a modicum of deduction. Once the phase of theoretical reflection in a set of data has been carried out, the researcher may want to collect further data in order to establish the conditions in which a theory will and will not hold. Such a general strategy is often called iterative: it involves a weaving back and forth between data and theory. (Bryman, 2012, S. 26, Herv. im Orig.)

\subsection{Qualitative Studie}

\subsubsection{Forschungsmethodik der qualitativen Studie}

Die Begründung zur Erfassung qualitativer Daten hängt damit zusammen, dass nach Vester „weiche Daten“ eine wesentliche Rolle mit Blick auf das Verständnis von Systemabläufen spielen (Vester 1999, S. 20f). Qualitative Forschung orientiert sich am Alltagsgeschehen und versucht die Perspektiven beteiligter Personen zu erfassen. Ihre Leitgedanken bestehen darin Kontextualität herzustellen und Verstehen als Erkenntnisprinzip zu nutzen. Ihre Ziele sind Theoriebildung auf der einen und Entdeckung auf der anderen Seite (Flick, Kardoff u. Steinke 2005, S. 24, zitiert n. Kuckartz, 2012, S. 17). Das qualitative Forschungsparadigma setzt daher auf „Kontextspezifität, Situationsadäquanz und Subjektrelevanz“ (Bogner et al., 2009, S. 16).

In der vorliegenden Studie wird zur Erhebung dieser Daten ein systematisches Vorgehen verfolgt. Dieses sieht so aus, dass leitfadenstrukturierte Experteninterviews mit unterschiedlichen Beteiligten geführt werden. Nach der Transkription dieser Interviews werden die Aussagen der Beteiligten zunächst unabhängig vom bereits bestehenden Kategoriensystem gelesen und kategorienorientiert markiert, bevor sie in das zuvor erstellte Kategoriensystem eingefügt werden (EXCEL-Tabellen). Die Analyse und Interpretation des Datenmaterials wird theoriegeleitet vorgenommen (Mayring, 2010, S. 13). Damit verknüpft ist eine Mischung zwischen induktiver und deduktiver Vorgehensweise (iterativ nach Bryman, s.o.), da das Material einerseits nach Kategorien ,sortiert“ wird, andererseits aber auch neue Kategorien, Subkategorien oder Indikatoren der Qualität schulischer Integration hinzukommen können. Zwar werden - ähnlich wie im Theorieteil - Qualitätsbausteine vorwiegend „isoliert“ erfasst, mit Hilfe des Erschließens kausaler Zusammenhänge können ggf. aber auch komplexe Verknüpfungen zwischen diesen Bausteinen analysiert werden (Mayring, 2010, S. 19).

Die Experteninterviews ermöglichen in diesem Zusammenhang eine „konkurrenzlos dichte Datengewinnung“"gegenüber anderen Erhebungsformen (Bogner et al., 2009, S. 8). Das Verständnis von „Experteninterviews“ ist in der Wissenschaftstheorie unterschiedlich (Bogner et al., 2009, S. 17). In der vorliegenden Arbeit werden diese mit Personen durchgeführt, die als Experten für den Bereich der schulischen Integration eingestuft werden, da sie als Schulleitungen von Förder- und Beratungszentren, Grundschulen oder weiterführenden Schulen, 
als Regel- oder Förderschullehrkräfte in diesen Schulen bzw. als Eltern oder Schülerinnen und Schüler mit spF über eine Fülle persönlicher bzw. auch beruflicher Erfahrungen verfügen, auch wenn ihr „Expertenstatus“ unterschiedlich ist. Während Schulleitungen und Lehrkräfte aufgrund diskursiver Auseinandersetzung wahrscheinlich über ein höheres Maß an explizitem und organisationalem Wissen verfügen, weisen Eltern und Schüler aufgrund ihres direkten Erfahrungszugangs gegebenenfalls ein höheres $\mathrm{Maß}$ an emotionaler Nähe zu den Fragestellungen auf. Grundlegend für die Annahme, dass alle befragten Personen als Experten angesehen werden können, ist dabei das Verständnis nach Meuser und Nagel (2009), die als Kriterium für Expertise nicht nur eine spezifische berufliche Rolle, sondern die aktive Partizipation an einer Sache an sich ansehen (S. 42ff) und formulieren:

Eine Person wird im Rahmen eines Forschungszusammenhangs als Experte angesprochen, weil wir wie auch immer begründet annehmen, dass sie über ein Wissen verfügt, das sie zwar nicht notwendigerweise alleine besitzt, das aber doch nicht jedermann in dem interessierenden Handlungsfeld zugänglich ist. Auf diesen Wissensvorsprung zielt das Experteninterview. (Meuser \& Nagel, 2009, S. 37)

Die Interviews erfolgen gestützt auf einen offen gehaltenen Leitfaden, der situativ bei Bedarf flexibel gehandhabt werden kann. Bei der Analyse des Datenmaterials im Anschluss ist davon auszugehen, dass das Expertenwissen zum Teil inhaltlich rekonstruiert werden muss:

Der Prozesscharakter und die Nicht-Expliziertheit eines großen Teils auch des Expertenwissens haben zur Konsequenz, dass dieses, große Anteile von vortheoretischem Erfahrungswissen beinhaltende, Wissen nicht einfach bzw. nur teilweise "abgefragt“ werden kann. Nimmt man die Giddenssche Unterscheidung von praktischem und diskursivem Bewusstsein auf, so lässt sich diese Form des Expertenwissens, das sich auf habitualisierte Formen des Problemmanagements bezieht, zwischen den beiden Polen verorten. Es ist kein völlig vorreflexives Wissen auf der Ebene von Basisregeln bzw. Ethnomethoden im Sinne Cicourels (1973), nicht vergleichbar dem grammatikalischen Regelwissen, das die meisten zwar intuitiv beherrschen, aber nur in Teilen explizieren können; es ist aber auch kein Wissen, das die Experten ohne weiteres einfach „abspulen“ können. Sie können über Entscheidungsfälle berichten, auch Prinzipien benennen, nach denen sie verfahren; die überindividuellen, handlungs- bzw. funktionsbereichsspezifischen Muster des Expertenwissens müssen jedoch auf der Basis dieser Daten rekonstruiert werden. (Meuser \& Nagel, 2009, S. 51f)

Damit die „Relevanzstrukturen der Beteiligten“ (Meuser \& Nagel, 2009, S. 54) in den Interviews zu Tage treten können, wird bei der Formulierung der Interviewfragen darauf geachtet, dass diese zwar inhaltliche Kategorien aus dem Theorieteil dieser Arbeit aufgreifen, gleichzeitig aber so offen wie möglich gestellt werden (Bsp.: Welche Qualitätsmerkmale kennzeichnen Ihrer Meinung nach den gemeinsamen Unterricht?) und sich „auf das Wie des Entscheidens und Handelns" (Meuser \& Nagel, 2009, S. 54) der Beteiligten konzentrieren.

Bei der Konstruktion der Interviewleitfäden wurden Hinweise von Dresing \& Pehl (2012) genutzt, die u.a. die Verwendung textgenerierender, offener sowie kurzer und verständlicher Fragen empfehlen. Die Auswahl der Inhaltsbereiche erfolgte zum einen orientiert am Theorieteil, zum anderen wurde versucht Fragen zu Erfahrungshorizonten auszuwählen, die sich für alle beteiligten Personengruppen zumindest in großen Teilen überschneiden. Da die Interviews nicht länger als eine halbe Stunde dauern sollten, wurden zwischen zwölf und vierzehn Fragen ausgewählt. Beim Interviewleitfaden für die Schülerinnen und Schüler mit sonderpädagogischem Förderbedarf in Regelschulen war bei den Vorüberlegungen wesentlich, dass damit sowohl Schüler und Schülerinnen in Grundschulen wie in integrierten Gesamtschulen befragt werden sollten. Nach einleitenden gesprächsmotivierenden Fragen zum Schul- und Klassenklima (Was gefällt dir an 
deiner Schule und in deiner Klasse? Hast du Freunde oder Freundinnen in der Klasse/an der Schule, die du auch nachmittags triffst?) sowie zur zeitlichen Dauer der Unterstützung durch eine Förderschullehrkraft und zu Lieblingsfächern folgen neun offene Fragen, die auf ausführlichere Antwortmöglichkeiten zielen. Dazu gehören eine Frage zu bisherigen Erfahrungen mit der Unterstützung durch eine weitere Lehrerin bzw. einen weiteren Lehrer (PE), eine Frage zur Organisationsform der Differenzierung (OE) und sieben Fragen zum Qualitätsbereich der Unterrichtsentwicklung. Die Schülerinnen und Schüler können sich dazu äußern, wie sie in der Schule gefördert werden (Bsp.: Einzelförderung, Förderung innerhalb der Klasse), wie ihr besonderer Förderbedarf berücksichtigt wird (Bsp.: Arbeitsmaterial, Lerntempo o.ä.), wie sie ihre Lernentwicklung (Lernfortschritte) in verschiedenen Bereichen einschätzen (Bsp.: Deutsch, Mathematik, weitere Unterrichtsfächer, sozialer Bereich, persönliche Entwicklung), bei welcher Form des Unterrichts sie besonders gut lernen können (Bsp.: wenn der Lehrer/die Lehrerin etwas ausführlich erklärt, wenn sie selber etwas erarbeiten), welche Sozialformen ihr Lernen besonders gut unterstützen (Bsp.: alleine arbeiten, mit einem Partner/einer Partnerin arbeiten, in einer Gruppe arbeiten), welche Rückmeldung sie zu ihren Lernfortschritten erhalten (Bsp.: persönliche Gespräche, schriftliche Berichte) und was sie sich im Unterricht bzw. in ihrer Klasse wünschen, damit sie noch besser lernen können. Neben der Qualitätskategorie der wahrgenommenen Unterstützung durch eine Förderschullehrkraft (PE) geht es bei den Fragen insgesamt also um die Abdeckung der Qualitätskategorien: Schulklima und Organisationsform der Förderung (äußere oder innere Differenzierung) (OE), Klassenklima, Klassenführung, Methoden (Sozialformen), Differenzierung, Leistungsbeurteilung (Form der Rückmeldung) und Ergebnisse (kognitiv, emotional, sozial). Es wird davon ausgegangen, dass dies die wesentlichen Kategorien bzw. Subkategorien sind, die von den Schülerinnen und Schülern aufgrund ihrer Erfahrungen beschrieben werden können.

Auch der Interviewleitfaden für Eltern von Schülerinnen und Schüler mit sonderpädagogischem Förderbedarf in Regelschulen sollte sowohl für Eltern von Grundschulkindern als auch für Eltern von Schülern der Sekundarstufe I geeignet sein. Als Einstiegsfragen dienen Fragen zur Dauer der integrativen Unterrichtung und zu bisherigen Erfahrungen damit. Es folgen acht weitere Fragen zum Bereich der Personalentwicklung (Zusammenarbeit mit den Regelschullehrkräften bzw. mit den Förderschullehrkräften, Haltung der Lehrkräfte im Hinblick auf die Entwicklung inklusiver Bildungsangebote) und zur Unterrichtsentwicklung: Formen der Auseinandersetzung der Schule mit den Anforderungen des gemeinsamen Unterrichts, organisatorische Umsetzung der integrativen Unterrichtung (Bsp.: Einzelförderung, Förderung innerhalb der Klasse), Form der Berücksichtigung des besonderen Förderbedarfs (Bsp.: Arbeitsmaterial, Lerntempo), Einschätzung der Lernentwicklung in verschiedenen Bereichen (Bsp.: kognitive Entwicklung, soziale Entwicklung, emotionale Entwicklung), Verbesserungsmöglichkeiten hinsichtlich der Förderung der Lernentwicklung sowie Tendenzen, die hinsichtlich der Weiterentwicklung des gemeinsamen Unterrichts gesehen werden. Eine letzte offene Frage richtet sich darauf, welche Probleme und welche Chancen die Eltern mit Blick auf die Zukunft inklusiver Bildungsangebote erkennen. Diese Fragen berücksichtigen also die Qualitätskategorien Organisationsform der Förderung (äußere oder innere Differenzierung) (OE), Zusammenarbeit zwischen Eltern und Lehrkräften, Haltung der Lehrkräfte (PE), Unterrichtsentwicklung allgemein, Differenzierung und Ergebnisse (kognitiv, emotional, sozial). Im Vergleich zu den Interviewleitfragen für die Schüler sind die Fragen an die Eltern offener gehalten und geben damit Raum zur sprachlichen „Ausarbeitung “ eigener Vorstellungen und Sichtweisen. Auch bei der Auswahl der Fragen für die Eltern wurde berücksichtigt, dass diese mit deren Erfahrungshorizont beantwortet werden können. 
Die Interviewleitfäden für Regelschullehrkräfte und Förderschullehrkräfte sowie für die Schulleitungen an Grund- und weiterführenden Schulen weisen ein hohes Maß an Übereinstimmung auf, d.h. sie enthalten jeweils 14 (RSL/FSL) bzw. 13 (SL) inhaltlich übereinstimmende Fragen, bei denen lediglich die Perspektive (Bsp.: eigener Unterricht, Unterricht der RSL) bei Bedarf sprachlich berücksichtigt wird. Nach einer einleitenden Frage zur Dauer der Tätigkeit mit Blick auf die Begleitung (SL) bzw. Unterrichtung (RSL/FSL) von Schülern mit sonderpädagogischem Förderbedarf werden zwei Fragen zur Wahrnehmung von Veränderungen in der Schule aufgrund der integrativen Unterrichtung und zur Form gestellt, in der sich die Schule mit Anforderungen des gemeinsamen Unterrichts befasst hat (Bsp.: pädagogische Konferenzen, pädagogische Tage, Fortbildungen, Schulprogramm o.ä.). Im Anschluss geht es in den Fragen um Qualitätsmerkmale, die nach Auffassung der Lehrkräfte den gemeinsamen Unterricht kennzeichnen, die organisatorische Umsetzung der integrativen Unterrichtung (Bsp.: Einzelförderung, Förderung innerhalb der Klasse), die Veränderungen, die sich im Unterricht zeigen, seit Schüler mit spF in der Klasse sind, und die Einschätzung der Lernentwicklung der Schülerinnen und Schüler mit sonderpädagogischem Förderbedarf(Bsp.: kognitive Entwicklung, soziale Entwicklung, emotionale Entwicklung). Darüber hinaus werden Fragen zur Zusammenarbeit mit den Eltern (der Schülerinnen und Schüler mit spF) und zur Kooperation mit den Regel- bzw. Förderschullehrkräften gestellt. Die Lehrkräfte werden gefragt, welche Aufgaben sie im Zusammenhang mit dem gemeinsamen Unterricht übernehmen (Bsp.: Förderung, Teamteaching, Beratung, Vernetzung) (diese Frage fehlt entsprechend bei den Schulleitungen) und sowohl Lehrkräfte wie Schulleitungen sollen sich dazu äußern, in welchen Bereichen sie an ihrer Schule bzw. für sich persönlich Fortbildungsbedarf im Zusammenhang mit integrativem Unterricht sehen. In weiteren Fragen geht es um die Einschätzung der Haltung der Lehrkräfte an der Schule bzw. dem Förderzentrum im Hinblick auf die Entwicklung inklusiver Bildungsangebote und um Tendenzen, die an der Schule hinsichtlich der Weiterentwicklung des gemeinsamen Unterrichts gesehen werden. Wie bei dem Interviewleitfaden für die Eltern geht es in der letzten Frage um Probleme und Chancen, die die Lehrkräfte mit Blick auf die Zukunft inklusiver Bildungsangebote erkennen. Die Fragen für die Lehrkräfte und Schulleitungen umfassen also die Kategorien bzw. Subkategorien Schule als sozialer Raum (Zusammenarbeit mit Eltern, Vernetzung), organisatorische Umsetzung der Förderung, Ergebnisse (OE), Kooperation (zwischen RSL und FSL, Rollen- und Aufgabenklärung) und Professionalisierung (Konferenzen, Pädagogische Tage, Fortbildungen, Fortbildungsbedarf, Haltungen) (PE) sowie Qualitätsmerkmale des gemeinsamen Unterrichts und Ergebnisse der Lernentwicklung der SuS mit spF (UE).

Auch der Interviewleitfaden für Schulleitungen an Förderzentren enthält einige übereinstimmende Fragen, allerdings auch einige, die sich speziell auf die Arbeit an den Förderzentren beziehen. Nach der einleitenden Frage, seit wann die Leitungen an ihrem Förderzentrum die Unterrichtung von Schülern mit spF organisieren, geht es zunächst darum, welche Veränderungen in der Arbeit der Förderschullehrer in integrativen Maßnahmen wahrgenommen werden. Übereinstimmende Fragen beziehen sich darauf, in welcher Form sich die Förderzentren inhaltlich mit Anforderungen des gemeinsamen Unterrichts befasst haben (Bsp.: pädagogische Konferenzen, pädagogische Tage, Fortbildungen, Schulprogramm o.ä.), wie die integrative Unterrichtung an den Regelschulen organisatorisch umgesetzt wird (Bsp.: Einzelförderung, Förderung innerhalb der Klasse), welche Veränderungen an den Regelschulen beobachtet werden, in denen Schüler mit spF unterrichtet werden, welche Qualitätsmerkmale den gemeinsamen Unterricht auszeichnen und wie die Lernentwicklung der Schülerinnen und Schüler mit sonderpädagogischem Förderbedarf an den Regelschulen (Bsp.: kognitive Entwicklung, soziale Entwicklung, emotionale Entwicklung) sowie 
die Kooperation zwischen den Regelschul- und den Förderschullehrkräften eingeschätzt werden. Mit Blick auf die Förderzentren wird nachgefragt, wie diese die Zusammenarbeit der Förderschullehrerinnen und -lehrer untereinander organisieren, in welchen Bereichen Fortbildungsbedarf im Zusammenhang mit integrativem Unterricht gesehen wird und wie die Haltung der Förderschullehrkräfte im Hinblick auf die Entwicklung inklusiver Bildungsangebote eingeschätzt wird. In den zwei abschließenden sehr offenen Fragen geht es erneut um Tendenzen hinsichtlich der Weiterentwicklung des gemeinsamen Unterrichts sowie um Probleme und Chancen, die mit Blick auf die Zukunft inklusiver Bildungsangebote gesehen werden. Abgefragt werden also die Kategorien bzw. Subkategorien organisatorische Umsetzung der Förderung und Ergebnisse der Schulentwicklung (OE), Kooperation (zwischen RSL und FSL, Veränderung der Aufgaben der FSL, Organisation der Zusammenarbeit der FSL untereinander) und Professionalisierung (Konferenzen, Pädagogische Tage, Fortbildungen, Fortbildungsbedarf, Haltungen) (PE) sowie Qualitätsmerkmale des gemeinsamen Unterrichts und Ergebnisse der Lernentwicklung der SuS mit spF (UE).

Die Durchführung der Interviews erfolgte in den Räumlichkeiten der fünf beteiligten Schulen. Die Schulleitungen sorgten für die Organisation der Termine, die jeweils an einem Schultag stattfanden, sowie für die Bereitstellung möglichst ruhiger und ungestörter Räume. Vor Beginn der Interviews erfolgten eine möglichst persönliche Ansprache der Interviewpartner und eine kurze Information zum Forschungsprojekt (Dresing \& Pehl, 2012, S. 14). Im Rahmen der Interviews erhielten die Befragten soweit wie möglich ein „monopolisches Rederecht“ (Dresing \& Pehl, 2012, S. 12f).

Die Transkription der Interviews orientiert sich an den einfachen Transkriptionsregeln nach Dresing und Pehl (2012) bzw. Kuckartz et al. (2008). In den Transkripten wird wörtlich transkribiert, ohne para- oder nonverbale Äußerungen bzw. Äußerungen wie „mmh“, „aha“ o.ä. miteinzubeziehen. Dialekte werden übersetzt und die Texte dadurch geglättet. Syntaktische Fehler werden beibehalten, die Interpunktion erfolgt gemäß den Sinneinheiten, Pausen werden durch drei Auslassungspunkt in Klammern kenntlich gemacht. Eine Zeitmarkierung erfolgt lediglich am Ende des Interviews.

Für die Auswertung der Experteninterviews wird die inhaltlich strukturierende qualitative Inhaltsanalyse nach Kuckartz (2012) genutzt. Diese ist gekennzeichnet durch die zentrale Rolle der Kategorien für die Analyse, eine systematische Vorgehensweise mit einem klar festgelegtem Regelsystem für die einzelnen Schritte, die Klassifizierung und Kategorisierung des gesamten Materials sowie den Einsatz von Techniken der Kategorienbildung am Material (Kuckartz, 2012, S. 39):

Von großer Bedeutung sind die mit der Kategorienentwicklung und -modifizierung befassten Phasen des Arbeitsablaufs, in denen ein fortschreitendes Arbeiten am Material stattfindet. Selbst dann, wenn eine qualitative Inhaltsanalyse theoriebasiert und auf der Basis von Hypothesen unternommen wird was keineswegs ausgeschlossen ist - werden die Kategorien üblicherweise während des Analyseprozesses verfeinert und ausdifferenziert und ggf. werden auch neue Kategorien hinzugefügt, weil die intensive Beschäftigung mit dem Material dies nahe legt. (Kuckartz, 2012, S. 52)

Die inhaltlich strukturierende qualitative Inhaltsanalyse ermöglicht ein „mehrstufiges Verfahren der Kategorienbildung und Codierung“ (Kuckartz, 2012, S. 77), indem eine Vorgehensweise in zwei Phasen verfolgt wird:

1. Phase: Codierung entlang von etwa 10 bis 20 Hauptkategorien: leitfadenorientierte Auswertung,

2. Phase: Weiterentwicklung und Ausdifferenzierung der Kategorien durch eine erneute Codierung im zweiten Materialdurchlauf: kategorienbasierte Auswertung. (Kuckartz, 2012, S. 77) 
Nachdem im Theorieteil eine umfassende deduktive Kategorienbildung mit Hilfe sonderpädagogischer Forschungsveröffentlichungen erfolgt ist und eine Systematisierung der Kategorien, Subkategorien und Indikatoren im Rahmen des Schulentwicklungsmodells nach Rolff vorgenommen wurde, werden die Interviews im Anschluss an die Transkription zunächst leitfadenorientiert grob ausgewertet (Markierung von Textstellen), bevor eine am vorliegenden Kategoriensystem orientierte Zuordnung des auf diese Weise codierten Materials in EXCEL-Tabellen erfolgt ${ }^{54}$. Bei dieser Vorgehensweise werden zum einen Textstellen deduktiv zugeordnet, zum anderen werden neue Kategorien, Subkategorien oder Indikatoren induktiv hinzugefügt. Diese Vorgehensweise beschreibt Kuckartz folgendermaßen:

In der ersten Phase wird eher grob entlang von Hauptkategorien codiert, die beispielsweise aus dem bei der Datenerhebung eingesetzten Leitfaden stammen [...] In der nächsten Phase werden die Kategorien am Material weiterentwickelt und ausdifferenziert. Das gesamte Datenmaterial wird anschließend in einem zweiten Materialdurchlauf erneut codiert, im Folgenden kategorienbasiert ausgewertet und für den zu schreibenden Forschungsbericht aufbereitet [...] Durch Vergleichen und Kontrastieren von interessierenden Subgruppen gewinnt die kategorienbasierte Auswertung und Darstellung an Differenziertheit, Komplexität und Erklärungskraft. (Kuckartz, 2012, S. 77)

Diese Strukturierung anhand der Fragestellungen aus den Interviewleitfäden erfolgt, damit sich die grobe Kategorienbildung im ersten Analyseschritt direkt am Material und nicht am zuvor bereits erstellten Kategoriensystem orientiert. Im Anschluss erfolgt für die Vorbereitung und Entwicklung der Fragebögen (quantitativer Teil der Untersuchung) eine Ausdifferenzierung der Qualitätskategorien, - subkategorien und - indikatoren sowie eine Gewichtung und Auswahl der - aus den verschiedenen Perspektiven der Beteiligten heraus - besonders relevant erscheinenden Kategorien. Diese werden in den EXCEL-Tabellen entsprechend farbig markiert. Bei der qualitativen Auswertung geht es neben der kategorienorientierten Vorgehensweise auch um Analysen von (Begründungs-) Zusammenhängen innerhalb von oder zwischen Kategorien (Kuckartz, 2012, S. 94). Kommen Antworten gehäuft vor, werden diese auch quantitativ ausgewertet. Auch bei dieser sehr strukturierten Vorgehensweise bleibt als Problem der Kategorienbildung, dass Kategorien nicht immer vollkommen trennscharf erfasst werden können bzw. dass Aussagen gleichzeitig mehreren Kategorien zugeordnet werden können (Kuckartz, 2012, S. 61).

Die qualitative Teilstudie sollte den grundlegenden Gütekriterien qualitativer Forschung nach Steinke (2004) und Mayring (2002) genügen. Sie ist intersubjektiv nachvollziehbar (Steinke, 2004, S. 324f), indem das Vorverständnis zum Untersuchungsgegenstand, die Interviewleitfäden, die Transkriptionsregeln, die Transkripte selbst und die einzelnen Auswertungsschritte erläutert bzw. zur Verfügung gestellt werden (s. Anhang) (s. auch Mayring, 2002, S. 144f, Kriterium der Verfahrensdokumentation). Das Kriterium der Indikation im Sinne der Angemessenheit des qualitativen Vorgehens, der Methodenwahl (Steinke, 2004, S. 326ff) bzw. der Nähe zum Gegenstand (Mayring, 2002, S. 146) wird u.a. dahingehend berücksichtigt, dass den Äußerungen der Befragten genügend Raum gegeben wird, die Forscherin über Erfahrungen und Kenntnisse im Forschungsfeld verfügt und die Methode (Experteninterviews) passend zum Untersuchungsgegenstand ausgewählt wurde. Die empirische Verankerung (Steinke, 2004, S. 328f) wird mit Hilfe der Verknüpfung einer eher induktiven mit einer eher deduktiven Vorgehensweise hinsichtlich der Erarbeitung von Kategorien und Subkategorien im Rahmen der Datenanalyse gewährleistet sowie durch eine strukturierte Vorgehensweise (strukurierte qualitative Inhaltsanalyse nach Kuckartz, 2012), während sich die Limitation der Studie (Steinke, 
2004, S. 329f) dadurch ergibt, dass sich die Ergebnisse zunächst genau auf die Schulen beziehen, an denen die Interviews durchgeführt werden. Da allerdings davon ausgegangen werden kann, dass sich die Rahmenbedingungen in den Schulen des Saarlandes grundsätzlich ähneln, sind weiterführende Rückschlüsse auf die Qualität schulischer Integration in diesem Bundesland als möglichem Geltungsbereich zulässig. Das Gütekriterium der reflektierten Subjektivität (Steinke, 2004, S. 330f) erfährt dadurch Berücksichtigung, dass sowohl die Reflexion des Forschungsgegenstands selbst als auch der Forschungsmethode in ständiger Wechselwirkung zwischen wissenschaftlicher Theoriebildung und schulischen Erfahrungshorizonten vollzogen wird. Inwiefern das Forschungskonstrukt kohärent ist (Steinke, 2004, S. 330), wird iterativ dadurch geprüft, dass die Interviewinhalte zur Generierung von Kategorien und Subkategorien genutzt und mit dem bereits generierten Kategoriensystem abgeglichen werden. Mögliche Widersprüche werden gegebenenfalls aufgegriffen. Die Relevanz der Teilstudie und damit ihr möglicher pragmatischer Nutzen (Steinke, 2004, S. 330) sollte dadurch gegeben sein, dass die Aussagen der Interviewten Einschätzungen bzw. Erklärungen zur Qualität schulischer Integration umfassen, die theorieprüfend bzw. -generierend überschaubar zusammengefasst werden können. Die Interpretationsabsicherung erfolgt argumentativ und schlüssig im Rückbezug auf das durch bereits vorliegende Forschungsergebnisse erstellte Kategoriensystem (Mayring, 2002, S. 145) sowie regelgeleitet (Mayring, 2002, S. 145f).

\subsubsection{Stichprobe}

Für die Durchführung der Interviews werden fünf Schulen im Saarpfalz-Kreis ausgewählt, darunter drei Grundschulen und zwei Gesamtschulen (inzwischen Gemeinschaftsschulen), die über mehrjährige Erfahrung im Zusammenhang mit schulischer Integration verfügen. An jeder Schule sollen ein Mitglied der Schulleitung, eine Regel- wie eine Förderschullehrkraft, ein Elternteil und ein Schüler/eine Schülerin mit sonderpädagogischem Förderbedarf befragt werden. Darüber hinaus werden zwei Leitungen sonderpädagogischer Förder- und Beratungszentren angefragt. Die Terminabsprachen zu den Interviews erfolgen im September und Oktober 2012. Die Interviewtermine finden zwischen November 2012 und Januar 2013 statt. Für die Interviews mit den Schulleitungen, Lehrkräften bzw. Eltern werden jeweils etwa 30 Minuten Durchführungsdauer eingeplant, für die mit den Schülern etwa 20 Minuten. Im Anschreiben zu den Interviewanfragen wird die Zielsetzung kurz erläutert, indem darauf hingewiesen wird, dass Einschätzungen und Erfahrungen zur integrativen Unterrichtung (alle Beteiligten) sowie Möglichkeiten zur Weiterentwicklung (Lehrkräfte und Schulleitungen) erhoben werden sollen. Zusagen zu den Anfragen ergeben folgende Zusammensetzung der Stichprobe:

Tab. 8: Zusammensetzung der Stichprobe $(n=25)$ für die qualitativen Interviews

\begin{tabular}{lcccc}
\hline & Grundschulen & $\begin{array}{c}\text { Gemeinschafts- } \\
\text { schulen }\end{array}$ & Förderzentren & insgesamt \\
\hline Schulleitungen & 3 & 2 & 2 & 7 \\
Regelschullehrer & 3 & 2 & - & 5 \\
Förderschullehrer & 3 & 2 & - & 5 \\
Eltern & 1 & 2 & - & 3 \\
Schüler & 2 & 3 & - & 5 \\
Gesamtzahl & 12 & 11 & 2 & 25 \\
\hline
\end{tabular}


Zur Sicherung der Anonymität der Beteiligten wurden im Rahmen der Durchführung der Interviews keine personenbezogenen Eckdaten (Alter, Geschlecht o.ä.) erfasst.

\subsubsection{Darstellung der Ergebnisse zur Organisationsentwicklung}

\subsubsection{Ressourcen im Bereich der Organisationsentwicklung}

Zur materiellen Ausstattung bzw. den materiellen Rahmenbedingungen allgemein äußern sich Schulleitungen, Regel- und Förderschullehrkräfte mit insgesamt 13 Aussagen. Äußerungen zu den materiellen Rahmenbedingungen werden vor allem bei der Frage nach Problemen und Chancen schulischer Integration getätigt. Sie greifen die unzureichenden Rahmenbedingungen für integrative bzw. inklusive Maßnahmen auf. FSL 4 und FSL 2 thematisieren in diesem Zusammenhang die Tatsache, dass notwendige Investitionen nicht in ausreichendem Maß erfolgen:

Das sind auf jeden Fall die Ressourcen und dass es nicht so aussieht, dass in diesen Bereich investiert werden würde, also bundesweit. Das soll zwar umgesetzt werden, aber bis jetzt sehe ich noch nicht, dass da wirklich Investitionen stattfinden, im Gegensatz zu anderen Bereichen, da wird ja auch Geld locker gemacht und da ist dann Geld da. Also wenn dieser Bereich wichtig wäre, würde man vielleicht mehr investieren. Da, muss ich sagen, sehe ich etwas schwarz. (FSL 4, Z. 182-187)

[...] inklusive Bildungsangebote machen mir etwas Bauchschmerzen, weil die Rahmenbedingungen keiner inklusiven Beschulung oder keinen inklusiven Bildungsangeboten gerecht werden kann. Also dann muss sich definitiv an unserem Schulsystem einiges ändern um inklusive Bildungsangebote gezielter einsetzen zu können. (FSL 2, Z. 139-142)

Auch Schulleitung 1 betont, dass gute Rahmenbedingungen notwendig sind um inklusive Bildungsangebote zu ermöglichen (SL 1, Z. 377-379), „sonst ist es nur ein Stück auf einem Blatt Papier und man wird am Schluss keinem gerecht.“ (Z. 378f). Die Schulleitung eines Förderzentrums äußert in diesem Zusammenhang die Sorge, dass die integrative Arbeit aufgrund mangelnder Ressourcenausstattung von den Regelschulen hinterfragt werden könnte:

[...] nur wird also zunehmend der Erfolg der integrativen Maßnahmen wegen fehlender Ressourcen und Unterstützungsmöglichkeiten von den Regelschulen in Frage gestellt. Das heißt nicht, dass man was gegen Integration hat, im Gegenteil, man findet das ungeheuer positiv, nur man sieht also den Effekt, den man eigentlich beabsichtigt, den sieht man dort nicht und kann ihn auch, muss ich de facto sagen, auch nicht wahrnehmen. (SLFZ 1, Z.93-97)

Ähnlich formuliert es eine Förderschullehrkraft aus dem Grundschulbereich:

Ich fände es schade, wenn diese, diese ganze Inklusionsarbeit, die schon geleistet wurde, im Sande verläuft. Einfach nur, weil es ein Ressourcenproblem gibt. [...] und ich habe im Moment so das Gefühl, dass wir wieder auf den Stand von vor 10 Jahren kommen, als ich hier angefangen habe. (FSL 5, Z. 207-214)

Die Äußerungen lassen sich also dahingehend zusammenfassen, dass Lehrkräfte wie Schulleitungen auf der einen Seite nicht davon ausgehen, dass sich finanzielle Rahmenbedingungen der integrativen Arbeit zum Positiven ändern werden, und auf der anderen Seite befürchten, dass nicht ausreichende Rahmenbedingungen sich negativ auf die Umsetzung der Integration sowie auf die Wahrnehmung der Umsetzung durch die Regelschulen auswirken können.

Barrierefreiheit mit Blick auf die bauliche Gestaltung der Schulen sowie das Schulgelände sowie räumliche Flexibilität in den Klassenräumen werden in keinem der Interviews thematisiert. Eine wichtige Bedeutung messen die Interviewpartner allerdings Differenzierungsräumen und 
deren Ausstattung innerhalb der Schulen zu. SL 1, SL 3 und SL 5 verweisen darauf, dass die Förderschullehrerinnen nicht über genügend eigene Räumlichkeiten verfügen (SL 1, Z. 63-64; SLGS 3, Z. 110-113; SL 5, Z. 132-134), so dass teilweise der Flur mitbenutzt werden müsse (SL 2, Z. 113). Auch Förderschullehrkraft 2 bemängelt, dass es in der Regelschule nicht „genug Ausweichmöglichkeiten“ (Z. 147f) gebe und andere räumliche Voraussetzungen für gemeinsamen Unterricht notwendig seien (Z. 149-151). Förderschullehrkraft 3 befasst sich ebenfalls mit dieser Problematik und führt aus ihrer Perspektive anschaulich aus:

Räumlichkeiten für die Förderung. Also das ist katastrophal. (FSL 3, Z. 128); Es gibt keine Fördermöglichkeiten, keine Förderräume. Man kann dann im Flur lernen, aber ich kann nicht im Flur lernen und Kinder, die visuell, auditive Wahrnehmungsprobleme haben, für die ist es nicht unbedingt von Vorteil, wenn sie auf dem Flur sitzen (FSL 3, Z.31-134); für mich wäre es einfach ein kleiner Raum, in dem man Unterrichtsmaterialien hinlegen kann und mal nicht tütenweise an die Schule kommen kann und man kann etwas rausnehmen, mal ein Spiel greifen kann und nicht sagen muss, das habe ich jetzt nicht dabei, schade. Und deswegen finde ich, für mich wäre das so der Traum. (FSL 3, Z. 136-139)

Schulleitung 5 macht sich darüber hinaus Gedanken über die Ausstattung eines Differenzierungsraums:

Die hat dann manchmal drei Kinder, die XY, und dann geht die in diesen Raum. (Z. 121-124); wir müssen diesen Raum jetzt entsprechend auch ausstatten mit Regalen, wo sie immer ihr Material hinlegen kann für die einzelnen Klassenstufen. Ich meine die haben zum Teil ja auch ganz andere Bücher (Z. 128-130); Da habe ich jetzt mal den Bürgermeister gefragt, weil wir ja auch Kinder haben, die Probleme haben mit dem Gehör, dass wir bei dem Raum, obwohl der klein ist, der schallt fürchterlich, werden wir wahrscheinlich einen Teppichboden reinlegen. (SL 5, Z. 132-134)

Insbesondere mit Blick auf die Möglichkeiten differenzierter Förderung bemängeln die Interviewpartner die Tatsache, dass dafür häufig keine spezifisch ausgewiesenen Räume zur Verfügung stehen. Stattdessen müsse diese Form der Förderung oft auf den Schulfluren stattfinden. Thematisiert wird von den Interviewpartnern auch die Ausstattung der Schulen mit Differenzierungsmaterial. Schulleitung 5 setzt sich dafür ein passendes Material für Förderschüler in seiner Schule anzuschaffen:

Wir müssen uns halt weiter fortbilden, entsprechendes Material - wir sichten dann auch entsprechend kaufen, anschaffen, dass wir auf dem neuesten Stand immer sind. (SL 5, Z. 150-152) Das sind ja, im Grunde genommen, die Schwächsten von den Schwachen und die dürfen wir nicht jetzt hängenlassen und sagen, nein, wir investieren nur die Zahl X, das ist bei mir gibt es nach oben keine Grenze und was die brauchen, kriegen sie auch. Da müssen wir auch in der nächsten Zeit, in der Zukunft, eine Schaufel zulegen. (SL 5, Z. 155-158),

Dagegen weisen FSL1 und E 2 darauf hin, dass Material zum Großteil selbst organisiert werden muss:

[...] ich hab hier kein einziges Material für meine geistig behinderten Kinder. Alles, was wir haben, haben wir selbst angeschafft. (FSL 1, Z. 196f)

Das Arbeitsmaterial, ja, besorgen wir uns hauptsächlich, wenn nötig, selbst, aber meistens nach Information der Förderlehrer, was fehlt. (E 2, Z. 24f)

Diese Aussagen verdeutlichen, dass Material für differenziertes Arbeiten in den integrativen Schulen nicht oder nicht in ausreichendem Maß vorliegt. 


\subsubsection{Leitung einer Schule}

Die Bedeutung der Schulleitungen im Zusammenhang mit schulischer Integration wird in den Interviews lediglich von einem Elternteil thematisiert. Dieser weist darauf hin, dass es auf die Schulleitung ankomme, wie Integration an einer Schule umgesetzt werde (E 3, Z. 9) und dass es an der Schule ihres Kindes eine „sehr gute direktorische Führung“ (E 3, Z. 64) gebe. Abgesehen von dieser Aussage wird die Bedeutung der Schulleitung für die Prozesse schulischer Integration durch die interviewten Personen nicht thematisiert.

\subsubsection{Schule als sozialer Raum}

Das Schulklima an ihrer Schule mit Blick auf schulische Integration wird von zwei Regelschulschulleitungen erwähnt. SL 1 verweist darauf, dass die Integration aus ihrer Sicht kein Thema mehr sei, „da es so viele zu integrierende Schüler gibt“ (Z. 12-19), während SL 3 darüber hinausgehend keine Gefahr von Ausgegrenzt-Sein sieht, da die Kinder selbstbewusst mit dem Thema umgingen (Z. 11-13). Auch die Lehrkraft RSL 1 betont, dass Kinder mit Einschränkungen ein hohes Maß an Akzeptanz erfahren und erläutert dazu:

Ich war vorher an einer anderen Schule im sozialen Brennpunkt. Da waren diese Schüler halt oft auch ausgegrenzt. Das muss man schon sagen. ...Aber hier ist es eigentlich so, dass, äh, die Kinder, also die werden genauso akzeptiert wie die anderen auch. Ich hab' jetzt zum Beispiel das eine Kind, das konnte überhaupt gar nicht lesen. Und wie der dann die ersten zwei Sätze gelesen hat, hat die ganze Klasse geklatscht. Und ich denk mal, dass so dieses Sozialverhalten, das ist ja schon bemerkenswert, denn Kinder können ja teilweise auch einfach grausam sein. (RSL 1, Z. 98-103)

RSL 5 weist darauf hin, dass bspw. auch Klassenwiederholungen für Schüler kein Thema mehr sind (Z. 69-73). RSL 2 und RSL 5 betonen den respektvollen Umgang der Schüler und Schülerinnen untereinander, der damit zusammenhänge, dass die Schüler lernen aufeinander Rücksicht zu nehmen und sich gegenseitig zu helfen (RSL 2, Z. 10f, Z. 54f; RSL, 5, Z. 64-67).

Die Eltern haben differenzierte Perspektiven mit Blick auf das Schulklima, da sie in verschiedenen Schulen unterschiedliche Erfahrungen gemacht haben. So äußert sich E 1 dahingehend, dass die Entwicklung des Sohnes in der Grundschule ungünstig verlaufen sei, da diese keine Erfahrung mit Integration gehabt habe (Z. 13f):

[...] anfangs dachte ich, es läuft gut, es, ja, ich war froh, dass mein Sohn aufgenommen wurde in die Grundschule. (E 1, Z.14f)

Ich merkte dann, dass die Lehrer überfordert waren. (E 1, Z. 23f)[...] dass halt nur die Kinder gefördert wurden, die halt, ja, die was leisteten (E 1, Z. 24)

Ich merkte halt dann, dass mein Sohn in der Schule so ein bisschen [...] nicht als Außenseiter, aber er wurde nicht richtig integriert. (E 1, Z. 29)

Mein Sohn merkte das auch. Er merkte das, indem er sich zum Beispiel unter den Tisch oder ja, aggressiv, und er ist ein sehr sensibles Kind und er spürt auch, er ist halt so fit, dass er auch merkt, dass er anders ist und er merkt auch, wenn die Kinder, ja wenn er auch anders behandelt wird. (E 1, Z. 32-35)

Grundsätzlich hänge der Erfolg der Integration mit einzelnen Personen zusammen, die bereit seien die mühevolle Arbeit mit einem Kind zu übernehmen (E 1, Z.69-71). Elternteil 3 erläutert zur Grundschule in ähnlicher Form:

Wir haben auch die Grundschule gewechselt, weil es diese Probleme gab, dass man damit überhaupt nicht klarkam [...]. Nach dem Wechsel dann auf eine andere Grundschule hat das sehr, sehr gut geklappt [...] (E 3, Z. 9-14) 
Die neue Schule lege ein hohes Engagement an den Tag: „also die tun schon viel und gucken auch, dass die Kinder gefördert werden“ (E 3, Z. 101f). Ähnlich positiv äußern sich die Schüler über das Klima in ihrer Schule. S 1 (Z.2) und S 2 (Z. 2f) formulieren, dass die Leute freundlich und nett seien. $S 3$ weist darauf hin, dass er/sie akzeptiert werde und einige Schulfreunde habe (Z. 6f) und S 4, dass es in der Schule nur wenige Streitereien gebe (Z. 2).

In den Interviews finden sich keine Aussagen, die sich präzise den Subkategorien schulische Integration als gemeinsame Aufgabe bzw. Partizipation zuordnen lassen.

Die Zusammenarbeit mit den Eltern allgemein wird in den Interviews mit insgesamt 18 Nennungen auf vielfältige Art und Weise thematisiert. SL 1 weist darauf hin, dass allen Kollegen bewusst sei, „dass sie auch mit den Elternhäusern mehr zusammenarbeiten müssen“ (Z. 18f). SL 2 betont, dass die Eltern der Schule sehr wichtig seien, es aber von der Lehrperson abhänge, wie eng eine Klasse mit den Eltern zusammenarbeite (Z. 174ff). Von besonderer Bedeutung sei dabei der „Informationsfluss zwischen Schule und Elternhaus“ (Z. 184f). Auch SL 4 erläutert, dass es einen intensiven Kontakt zwischen Schulen und Eltern gebe: „Also ständig heißt wirklich ständig. Mit manchen fast täglich, weil täglich Gesprächsbedarf besteht.“ (Z. 61f). Ähnlich bewertet es RSL 4 (Z.87). Einen offenen Umgang verknüpft mit positiven „Rückmeldungen von Elternseite“ (Z.75f) sieht SL 3. SL 1 verweist neben den gewöhnlichen Elternabenden auf

[..] regelmäßige Stammtische, alle vier bis sechs Wochen, wo die Elternvertretung und jeder interessierte Lehrer - außerhalb der Schule findet das dann statt - kommen kann und wo über das, was in der Schule so passiert, informiert wird. (SL 1, Z.239-241)

SL 2 erkennt Unterstützungsbedarf im Bereich der Elternarbeit (Z. 271-273).

Die Zusammenarbeit mit den Eltern der Schüler und Schülerinnen mit sonderpädagogischem Förderbedarf wird besonders ausführlich beschrieben (35 Nennungen). SL 3 sieht in diesem Zusammenhang vor allem die intensiven Kontakte zwischen Klassenleitungen und Eltern (Z. 81f). RSL 1 vergleicht den Aufwand der Zusammenarbeit mit diesen Eltern im Vergleich zu den Eltern von Schülern ohne Förderbedarf: „es ist schon mehr Aufwand, aber es gibt auch nichtintegrative Schüler, bei denen der Aufwand größer ist." (Z. 222f). Probleme in der Kooperation mit den Eltern beschreiben die Regelschullehrkräfte:

Was die Förderschüler angeht, ist der Kontakt doch schon intensiver, weil häufiger halt auch Probleme auftreten, allerdings wäre eine bessere Zusammenarbeit schon wünschenswert. Das habe ich gerade bei den Förderschülern erlebt, dass die Eltern einfach, ja, bis auf eine Mutter, die sehr engagiert ist, überhaupt kein Interesse haben oder wenig. (RSL 2, Z. 89-92)

Ähnlich erläutert dieses Phänomen RSL 5:

Bei den Kindern mit Integrations- oder mit Förderbedarf muss ich halt leider sagen, dass es oft die Eltern sind, die relativ wenig Interesse an ihrem Kind vielleicht haben. (Z. 108-110); Ja, oder es ist halt einfach nur der Wunsch: mein Kind ist auf der ganz normalen Schule und gut ist, der Rest ist egal. Die registrieren das oder möchten es auch einfach nicht wahrhaben, habe ich manchmal so den Eindruck. (Z. 112-114)

Die Förderschullehrkräfte nehmen das Interesse der Eltern unterschiedlich wahr und betonen gleichzeitig, dass sie sich darum bemühen die Eltern kooperativ einzubeziehen (FSL 1, Z. 135f; FSL 2, Z. 106-109; FSL 3, Z. 41f; FSL 4, Z. 138-140; FSL 5, Z. 155-158). So sagt FSL 4: „Wenn mir etwas auffällt, biete ich mich immer an und ich möchte und bin da auch hartnäckig, also ich will da Antworten, lade die auch ein und so weiter." (Z 138-140) oder FSL 5 führt aus: 
Wir versuchen relativ regelmäßig, entweder telefonisch oder hier an der Schule Gespräche zu machen. Das gelingt nicht immer, weil nicht immer alle Eltern so kommen, das Problem haben wir hier auch, aber im Großen und Ganzen läuft's eigentlich ganz gut, also wir haben schon so ein bis zwei Mal im Halbjahr mindestens ein Gespräch mit den Eltern auch. (Z. 155-158)

FSL 1 erwähnt in diesem Zusammenhang, dass die Zusammenarbeit „gerade bei den Kindern mit geistiger Behinderung natürlich stärker“ (Z. 135f) sei und FSL 3, dass die Zusammenarbeit mit den Eltern im Team (gemeint ist die Klassenleitung) erfolge: „Da sprechen wir uns immer ab. Also da treten wir immer als Team eigentlich auf. Manche Sachen übernehme ich dann, manche übernehmen die." (Z. 41f)

Die befragten Elternteile bewerten die Zusammenarbeit durchweg als positiv (E 1, Z. 169-171, Z. 176f; E 2, Z. 52-54; E 3, Z. 91-93). E 1 äußert sich zu dieser Frage folgendermaßen:

Sehr gut, ja, sehr gut, also ich hab immer wieder Rückmeldung, wenn ich Fragen habe, immer ein offenes Ohr, sowohl Integrationshelfer, Integrationslehrerin, Schulleitung habe ich ein sehr, sehr gutes Gefühl. (Z. 169-171) XY ist ja noch nicht so lange an dieser Schule, was ich hier merke, ja, dass, wie ich vorhin schon erwähnt habe, dass man immer reden kann (Z. 176f)

Elternteil 2 erklärt, ebenfalls positiv bewertend, dass eine Regelschullehrkraft die Probleme von Schülern mit Förderbedarf nicht einfach kenne, sondern sich speziell damit auseinandersetzen müsse (Z. 52-54). Dieser Elternteil fühlt sich daher ,in dieser Integration hier richtig gut aufgehoben“" (Z. 52). Elternteil 3 ist froh darüber, dass er/sie sofort angerufen werde, wenn der Sohn/ die Tochter Probleme habe (Z.91-93).

Insgesamt $13 \mathrm{Mal}$ werden regelmäßige Beratungstermine erwähnt. Die Schulleitungen beschreiben in diesem Zusammenhang sowohl spontane Kontakte: „Es gibt auch Eltern, die ihre Kinder nach dem Unterricht abholen und da kommt immer wieder so ein Türschwellengespräch zustande." (SL 3, Z. 82-84) wie geplante Termine: „Und wenn Eltern kommen und wollen ein Gespräch - auch mit mir - also, wie gesagt, das ist voll jeden Mittag.“ (SL 1, Z. 246f). Ein Schulleiter weist darauf hin, dass die Förderschullehrkraft ihn zu diesen Gesprächen hinzubitte:

Die werden regelmäßig, was ich so höre und sehe, eingeladen, wenn was ansteht kurzfristig mal, ansonsten sagt XY mir dann immer, wir müssten mal wieder ein Gespräch führen mit der Frau oder mit den Eltern von dem und dem Kind, da gibt es die und die Schwierigkeiten. Die werden dann einbestellt. (SL 5, Z. 69-72)

Ähnlich wie die Regelschullehrkräfte verweisen auch die Schulleitungen darauf, dass die Bereitschaft der Eltern von Schülerinnen und Schülern zu regelmäßigen Beratungsterminen unterschiedlich sei: „Bei manchen muss man eben ständig nachlegen und bei anderen läuft es einfach.“ (SL 4, Z. 64f) oder: „Es kommt natürlich dann auch vor, es sind immer dieselben, die muss man dann drei Mal einladen, bis da einer kommt." (SL 5, Z.72f). Manche Gespräche finden nach Auskunft der Schulleitungen als gemeinsame Gespräche zwischen Eltern, Klassenleitung und Förderschullehrkraft statt (SL 3, Z. 84-86), in Form von Förderausschüssen (SL 4, Z. 62) oder als Runde Tische, bei denen auch die Vernetzung mit außerschulischen Kooperationspartnern relevant ist:

Bei den Down-Kindern haben wir ja fest installiert alle paar Wochen hier einen Runden Tisch mit allen, die an der Integration etwas zu tun haben, also Eltern, manchmal auch ein Logopäde, Integrationshelferin, die Fachlehrer, insbesondere die Fachlehrer der Hauptfächer, wenn sie eine Ganztagsklasse sind auch noch die Erzieherinnen, Schulleitung selbstverständlich und, wenn es nötig ist, vom schulpsychologischen Dienst jemand und auch manchmal war schon die Schulärztin mit. (SL 1, Z. 262-268) 
[...] da hatten wir das Autismuszentrum geladen, wir hatten eine Kollegin aus dem Förderzentrum, [...] es war der Herr XY da vom schulpsychologischen Dienst, der Krankenhausunterricht. Das hat Wellen gezogen und wir hatten alle am Tisch gesessen um zu gucken, wie, was muss ein Lehrer machen bei Klassenarbeiten, bei was auch immer um diesen Nachteilsausgleich zu geben. Das war eigentlich, fand ich das sehr toll, dass man sich einfach nicht sagt: können wir gar nicht leisten, dass man die Waffen streckt, sondern dass man einfach sagt wir setzen uns zusammen und gucken, wie wir ihnen helfen können. (SL 1, Z. 301-308)

SL 3 bezeichnet die Förderausschüsse als formale „Förderkonferenzen“, die auch von Eltern angefragt werden (SL 3, Z. 79-81), während SL 1 anspricht, dass diese häufiger als vorgeschrieben stattfinden (SL 1, Z. 260f). Mit Blick auf Klassenkonferenzen, die vor allem im Zusammenhang mit schwierigem Schülerverhalten stattfinden, führt dieser Schulleiter aus: „auch die Klassenkonferenzen sind bei uns nicht Tribunale, sondern dass man sich um einen Tisch sitzt und guckt, wie man das Problem für ein Kind löst." (SL 1, Z. 248-250).

Sieben von zehn Lehrkräften erwähnen Beratungsgespräche mit den Eltern der Schülerinnen und Schüler mit sonderpädagogischem Förderbedarf, die sie teilweise alleine (RSL 3, Z. 87f) oder im Team (RSL 2, Z. 92-94) führen. Sowohl Regel- wie Förderschullehrkräfte verweisen in diesem Zusammenhang auf die unterschiedliche Häufigkeit von Gesprächen: „bei den einen alle sechs Wochen, bei den anderen alle zwei bis drei Mal pro Halbjahr Gespräche“ (FSL 1, Z. 136f). Dazu erläutert FSL 2:

[...] es finden auch mindestens einmal im Halbjahr persönliche Gespräche statt und bei neuen Schülern mache ich es immer so, dass ich nach wenigen Wochen auch die Eltern anrufe und zum persönlichen Gespräch einlade, damit man ein Gesicht hat zu der Person. (Z. 103-106)

Ebenso verweisen die Eltern auf regelmäßige Gesprächskontakte (E 3, Z.91) und heben dabei insbesondere den Kontakt zu den Förderschullehrkräften hervor: „Ich habe halt mehr Kontakt mit der Integrationslehrerin." (E 1, Z. 178f) oder

Auch sehr gut. Das kommt natürlich darauf an, für mich als Mutter, für unseren Sohn jetzt eher nicht, das klappt immer prima, menschlich kann das mal besser, mal weniger gut sein. Was mich aber ja überhaupt nicht interessiert, wenn es in der Schule gut läuft und ich sehe unser Sohn bekommt die Führung, die er braucht. In der Regel sind wir auch sonderpädagogisch wirklich sehr, sehr gut beraten. (E 2, Z. 68-72)

Als Gesprächsinhalte werden von zwei Förderschullehrkräften Zeugnisgespräche (FSL 3, S. 82-84) sowie Gespräche zum IST-Stand eines Schülers oder einer Schülerin aufgeführt

[...] wo wir dann einfach im Prinzip den Ist-Stand kurz festhalten, wie sieht es aus, wo beide Seiten, also Eltern und Schule dann Probleme anbringen können, wo es gerade ihrer Meinung nach hakt und wo wir gemeinsam versuchen mal wieder die Richtlinien festzulegen wie es die nächste Zeit dann weitergeht. Ja und die sind eigentlich ganz konstruktiv, hat halt den Vorteil, dass man wirklich auch reagieren kann, wenn was ist oder auch was, was wir hier nicht unbedingt mitkriegen, was zu Hause dann aktuell ist, da können wir dann entsprechend reagieren. (FSL 1, Z 137-142)

RSL 3 benennt als Gesprächsinhalte neben Lernstand und Arbeitshaltung auch die Einführung neuer Unterrichtsmethoden (RSL 3, Z. 84-86), RSL 4 den Austausch über medizinische Belange:

Wir müssen uns zum Beispiel auch über Arzt- und Kliniktermine ganz oft austauschen. Sie fragt dann immer nach, wie es mit den medikamentösen Einstellungen aussieht, ob das klappt, da muss es schon eine Rückmeldung geben. (RSL 4, Z 79-81) 
Als andere Kontaktform wird von einer Förderschullehrkraft ein Mitteilungsheft erwähnt: „die Kinder haben ein Mitteilungsheft, wo ich einfach auch nicht nur Negatives, sondern auch positive Sachen reinschreibe, gerade in den kleineren, also erste und zweite Klasse." (FSL 3, Z. 7981) Darüber hinaus werden von neun Interviewpartnern telefonische Kontakte beschrieben. So verweist SL 1 darauf,

[...] dass bei uns also grundsätzlich die Lehrer von sich aus, wenn irgendetwas ist, die Eltern anrufen, aber auch Telefonate, die von der anderen Seite kommen, definitiv, wenn die sich gemeldet haben und die Sekretärin gibt das Telefonat an die Fachlehrer weiter, zurückrufen. Ja, dass diese Kommunikation stattfindet und dass sie ernst genommen wird. (SL 1, Z. 242-246)

Sie beschreibt dies als hohe Anforderung für die Förderschullehrkräfte (SL 1, Z. 190f). Eine Regelschullehrkraft hebt die Flexibilität mit Blick auf Telefonate hervor:

[...] es wird auch ganz flexibel mal gestaltet, dass ich einfach mal kurz anrufe, und sage, das und das ist oder wie ist das und das und das Thema ist dann gleich abgehakt, also man braucht dann nicht eine Riesenkonferenz immer wegen jedem Bisschen, also ich denke, da reicht auch teilweise mal ein Anruf. (RSL 1, Z.216-220)

Für RSL 3 ist es selbstverständlich, dass die Eltern über ihre Telefonnummer und Mailadresse verfügen (RSL 3, Z. 86-87) und RSL 4 und 5 geben an, dass sie häufig mit den Eltern telefonieren (RSL 4, Z. 76) bzw. dies anbieten (RSL 5, Z. 137). FSL 3 nutzt den telefonischen Kontakt zu den Eltern bedarfsorientiert (FSL 3, Z. 79) und FSL 2 hebt die Selbstverständlichkeit von Elterntelefonaten hervor:

Also telefonischer Kontakt besteht eigentlich zu allen Eltern regelmäßig bzw. die Eltern und ich haben immer die Absprache: wenn wir nichts voneinander hören, ist alles gut, sobald eine von beiden Seiten was auf dem Herzen hat, meldet sie sich. (FSL 2, Z. 102-104)

Dies thematisiert auch E 3: „Die rufen auch direkt an. Also nicht direkt, aber wenn sie Bedarf haben, wenn sie sagen, das und das ist mir aufgefallen, dann rufen sie schon an und sagen, man müsste mal wieder ein Gespräch führen." (E 3, Z 95-97).

Die Nutzung von Mailkontakt wird von einer Förderschullehrkraft aufgeführt: „Es gibt Eltern, mit denen ich mich maile, also da ist ein Austausch einfach, vor kurzem hat mir eine Frau zwei DIN-A4-Seiten gemailt, nur mal so als Austausch." (FSL 4, Z. 134f).

Die Vernetzung mit außerschulischen Diensten wird mit fünf Nennungen vergleichsweise selten aufgeführt. Von Seiten der Schulleitungen äußert sich lediglich SL 2 mit Blick auf „ein Bewusstsein von allen Lehrern, welche Hilfestellung kann ich mir irgendwo holen" (SL 2, Z. 205f). Ansonsten werden die Zusammenarbeit mit der Schulärztin (RSL 1, Z. 21f), mit dem Autismuszentrum (RSL 1, Z. 22-24) mit Psychologen (RSL 1, Z. 30) sowie mit einer Schoolworkerin erwähnt:

[...] und das ist hier auch ganz gut geregelt. Einmal die Woche ist hier eine Schoolworkerin in der Schule, die dann wenigstens ein bisschen was auffängt. [...] wenn das zu viel ins Detail geht, dann muss ja sowieso das Jugendamt eingeschaltet werden und dieses Verbindungsstück zwischen Kind, Lehrer, Eltern, Jugendamt, das [...] funktioniert auch ganz gut. Also, wie gesagt, es geht nur mit Kooperation. (RSL 2, Z. 266-271)

Weitere Austauschpartner sind Pädagogen einer Einrichtung der Therapeutischen Schülerhilfe (RSL 4, Z. 35-37) und Familienhelfer: 
Ich kommuniziere mit den Familienhelfern allgemein und versuche manchmal auch über Umwege Informationen zu kriegen und Informationen weitergeben zu lassen, weil, ja, aber, ich versuche sie mit ins Boot zu holen. (FSL 4, Z. 140-142)

\subsubsection{Organisationsstrukturen einer Schule}

$\mathrm{Zu}$ den Organisationsstrukturen einer Schule wird in den 25 Interviews nur folgende Aussage durch eine Förderschullehrkraft getroffen, die von einer schulorganisatorischen Variante berichtet, bei der klassenübergreifende Fördergruppen eingerichtet worden sind, von denen sie eine übernommen habe:

Und wir haben jetzt neu hier so übergreifende Fördergruppen gemacht also aus Klassenstufen. Und da habe ich dann im dritten Schuljahr fünf L-Kinder, die nehme ich jetzt in Deutsch und Mathe im Grunde zusammen raus. Und habe dann ab und zu noch andere Kinder mit dabei je nach Thema, also so, die dann auch keinen Förderbedarf haben, also speziell jetzt in Mathe, wo wir dann einfach nochmal Zahlaufbau oder solche Dinge dann machen. Die Kinder bekommen dann zusätzlich eben für die anderen Stunden, die sie drin sind, Förder-, Arbeitsplan nochmal. Aber diese Stunden sind grundsätzlich abgesprochen. Die sind dann auch in den Deutsch- und Mathestunden hauptsächlich bei mir, so dass sie in den anderen Stunden dann eigentlich komplett in der Klasse sind. (FSL 5, Z. 24-32)

Auf die Frage, welche Veränderungen sich in den Schulen aufgrund der integrativen Unterrichtung von SuS mit spF ergeben haben, werden also keine organisatorischen Entwicklungen aufgezeigt.

\subsubsection{Ergebnisse im Bereich der Organisationsentwicklung}

Am differenziertesten äußern sich die Schulleitungen der Förderzentren zum Thema der Konzeptentwicklung. Die SLFZ führen dazu sowohl Entwicklungselemente der SFZ selber an wie auch Entwicklungsbausteine (Förderkonzepte) der Regelschulen und nehmen dazu ausführlicher Stellung als andere Interviewpartner. SL FZ1 beschreibt dazu die Entwicklung seines Förderzentrums über zwei Jahrzehnte hinweg und stellt fest:

Wir wurden mit der Integration konfrontiert, muss man so sagen, das war 1988. Die Zeit war sehr gespalten. Es wurde sogar einmal unserer Schule untersagt Integrationsmaßnahmen zu personalisieren, vom Ministerium selbst. Und dann mussten wir halt erkennen, also ich war einer der ersten, der mit in der Integration draußen war, dass Integration an und für sich ein Förderelement war, das der Schule bis dahin gefehlt hat. Und insofern war sie für uns sehr wichtig, weil wir wollten in diesem Zusammenhang bereits mit der benachbarten Grundschule ein gemeinsames Förderkonzept Regelschule-Förderschule, wollten wir entwickeln, wir hatten einen Modellversuch beantragt beim Ministerium, wurde abgelehnt, auch die Grundschule wollte nicht mitmachen an der Stelle, obwohl das konzeptionell, ich denke, genau dort stand, wo die heute hinmöchten. Und man muss überlegen, das ist schon über 20 Jahre her, als wir diese Konzeption hatten, wovon man heute träumt. (SLFZ 1, Z. 32-42)

Zwischen 1992 und 1994 habe das Förderzentrum im Rahmen eines Modellversuchs der BundLänder-Kommission grundlegende Strukturen für die integrative Arbeit entwickelt, die im Anschluss zwar „in die Schublade geschoben“ (Z.49), an dem betreffenden Förderzentrum allerdings weiterhin genutzt wurden (SLFZ 1, Z.42-51). Als die Förderzentren letztendlich 1998 per Erlass eingerichtet worden seien, sei diese strukturelle Vorarbeit von Vorteil gewesen. Positiv sei an diesem Prozess zu bewerten, dass die Schulaufsicht diese Arbeit mitgetragen habe, ohne selbst Vorgaben zu machen:

Wir können frei entwickeln und haben auch diese Spielräume, denke ich, bis zum Anschlag haben wir die also ausgenutzt und ausgereizt. Man muss sagen mit einem sehr, sehr langem Atem um auf den heutigen Stand zu kommen. (SLFZ 1, Z. 63-65) 
SLFZ 2 äußert sich zu konzeptionellen Entwicklungsbausteinen engagierter Regelschulen, „die dann auch in ihrem Schulkonzept Sachen machen, die, sagen wir mal, fortschrittlich oder dem entgegen kommend sind.“ (SLFZ 2, Z.70-72). Dazu gehören aus seiner Sicht die Gestaltung eines didaktischen Rahmens sowie mit Blick auf die Zusammenarbeit zwischen Förderzentrum und Regelschulen die „Mitwirkung an Arbeitskreisen zur Erstellung von Konzepten zur Schulabwesenheit, zu Textdetektiven und so Sachen“ (SLFZ 2, Z. 75f), „Methodentraining“ (SLFZ 2, Z. 79) sowie gemeinsame Fachkonferenzen (SLFZ 2, Z. 78). Diese Schulen:

[...] gingen auch schon länger davon aus, dass wir eigentlich keine Schüler abweisen und auch versuchen alle, die zu ihnen kommen, irgendwie weiterzubringen, außer die, die in ihrem Verhalten so schwierig waren, dass sie sich und andere gefährdet haben, ansonsten behalten die alle. (SLFZ 2, Z. 79-82)

SL 2 und SL 3 benennen ihre Schulprogramme, wobei SL 2 darauf verweist, dass im Schulprogramm explizit erwähnt werde, dass die Schule eine Schule für alle Kinder sei, während SL 3 erläutert, dass im Schulprogramm das Recht jedes Kindes auf Bildung erwähnt sei, und somit ja behinderte wie nicht-behinderte Kinder eingeschlossen seien, wenn auch nicht „expressis verbis“ (SL 2, Z. 49f; SL 3, Z. 18-21).

\subsubsection{Darstellung der Ergebnisse zur Personalentwicklung}

\subsubsection{Ressourcen im Bereich der Personalentwicklung}

Obwohl in den Leitfadeninterviews keine Fragen enthalten sind, die sich explizit auf die Ressourcensituation in den Schulen beziehen, werden Aussagen dazu vor allem mit Blick auf die personelle Ausstattung zahlenmäßig häufig und inhaltlich differenziert getätigt. Äußerungen verschiedener Personengruppen finden sich dazu sowohl als Antworten auf die Frage nach den in den Schulen wahrgenommenen Veränderungen als auch auf die Frage nach möglichen Problemen, die sich mit Blick auf die Zukunft inklusiver Bildungsangebote ergeben.

\section{Ressourcen Personal}

Insgesamt 58 Aussagen thematisieren in den Interviews direkt oder indirekt die personelle Ausstattung der Schulen. Besonders häufig werden eine Erhöhung der Förderschullehrerstunden (9) bzw. eine Doppelbesetzung mit Regel- und Förderschullehrkraft (8) gefordert. Insgesamt 20 Bemerkungen beziehen sich darüber hinaus auf die mit der Personalisierung sowie weiteren Rahmenbedingungen zusammenhängende Qualität der Förderung in den Regelschulen. Neun Mal wird darauf Bezug genommen, dass die Entscheidung für den Förderort Förderschule auch mit den dort vorhandenen personellen Ressourcen (personelle Ausstattung, kleine Lerngruppen, bessere Möglichkeiten individueller Förderung) zusammenhängt. Wie die quantitative Auswertung belegt, findet eine intensive Auseinandersetzung mit dem Thema der personellen Ausstattung der Regelschulen bei allen Personengruppen, abgesehen von den Schülern, statt.

Die Wünsche nach einer Doppelbesetzung von Klassen mit Förder- und Regelschullehrkräften sowie nach einer Erhöhung der Stundenzahlen der Förderschullehrer werden unter anderem mit den Anforderungen der Differenzierung des Unterrichts bzw. der Individualisierung von Lernangeboten begründet. Die Doppelbesetzung wird sowohl von Schulleitungen, von Regelwie von Förderschullehrkräften wie auch von einem Elternteil gewünscht, damit Teamteaching im schulischen Alltag umgesetzt werden kann (SL 3, Z. 107f u.Z. 110) und damit auch eine fachliche Zusammenarbeit mit Blick auf eine bestmögliche Förderung der Schülerinnen und Schüler möglich wird, wie folgende Aussagen beispielhaft belegen: 
Wir bündeln die Maßnahmen schon immer, dass wir mehrere Maßnahmen in einer Klasse haben und der Anteil so insgesamt etwas erhöht wird, aber eigentlich müsste in einer Klasse mit vier, fünf Förderkindern eine Doppelbesetzung sein, eine feste Doppelbesetzung, die Doppelbesetzung vielleicht sogar als Tutor in der Klasse, dass eben auch der Förderschullehrer als voll-, vollwertige Lehrkraft“. (SL 2, Z. 262-271) Es ist viel zu wenig. Gerade in Mathematik, also gut ich unterrichte halt Mathematik, von daher kann ich das nur in dem Fach sagen, ist es zu wenig. Da bräuchte man eigentlich einen Förderlehrer parallel dazu. Das geht nicht mit drei Stunden die Woche, das ist noch zu wenig. Es sind einfach, die Unterschiede sind zu groß. Es muss zu viel abgedeckt werden. (RSL 1, Z. 74-77)

Eine Förderschullehrkraft wünscht sich diesbezüglich „einfach Stunden, damit man auch wirklich zusammen arbeiten kann. Wenn ich zwei Mal in der Woche komme, kann ich kein Thema erarbeiten. Das funktioniert nicht.“ (FSL 2, Z. 59f), während eine andere FSL betont, dass die integrative Arbeit in der Schule nur aufgrund der Unterstützung einiger Schülerinnen und Schüler durch Integrationshelfer gelingt:

[...] wobei man dazusagen muss, dass das im Moment bei uns nur gelingt, weil Integrationshelfer auch da sind. Es ist einfach sehr schwierig, wenn dann ein Lehrer mit 26 Kindern und vier Integrationskindern in der Klasse, dann ist das alleine eigentlich fast nicht machbar so ein gemeinsamer Unterricht, weil man viel zu wenig auf die Kinder eingehen kann oder auf die achten kann. (FSL 4, Z. 128-132); Einfach organisatorische Unterstützung, denke ich, weil man das sonst organisatorisch nicht umsetzen kann. Ich meine inhaltlich das Planen das geht gut und das funktioniert auch vom, vom wie die Kinder das machen, das funktioniert unheimlich gut, aber dieses Organisatorische, weil man eben doch den Blick dann nicht so speziell auf die Kinder mehr richten kann. (FSL 4, Z. 134-137)

FSL 5 macht sich Sorgen mit Blick auf die geplante Budgetierung der Förderschullehrerstunden in den saarländischen Grundschulen und stellt plastisch dar:

[...] ohne die Ressourcen kann es nicht funktionieren, weil zum Beispiel die Klassen 25, 26 Kinder haben und mittlerweile alle Klassen 2 bis 3 Integrationskinder haben und, ich denke, wenn man jetzt die Stunden pauschalisiert, dann sind das am Schluss einfach viel zu wenige Stunden schon allein für die Integrationskinder und präventive Arbeit, wie wir sie ja eigentlich leisten wollen mit Klassenstufen übergreifenden Fördergruppen, die ist dann einfach nicht mehr möglich. (FSL 5, Z. 199-204)

In Einzelfällen wird auch die Belastung der Regelschullehrkräfte angesprochen:

Die Probleme haben wir ja schon angesprochen. Das größte Problem ist für uns, dass ich sage, dass die Kollegen das irgendwann nicht mehr bewältigen können. Manche sind jetzt schon nah an der Wand, also ich sage immer zur XY, weil sie die meisten hat, ich bewundere die, wie sie das noch durchhält. Ich weiß nicht, ob die alle so geartet sind, so standfest wie die Frau XY“" (SL 5, Z. 145-149)

Auch die Schulleitungen befassen sich in ihren inhaltlichen Aussagen mit der ihrer Auffassung nach zu geringen Stundenzahl der Förderschullehrkräfte:

Wir haben ja doch einen gewissen Prozentsatz, aber wenn das ganz in die Grundschule reingeht, da haben wir große Fragezeichen und Bedenken, denn die wenigen Stunden, die die Förderlehrer hier mit den Kindern zubringen, wird ein inklusives Unterrichten, glaube ich, nicht erfolgreich gehen. Die Grundschullehrer andererseits haben auch nicht die intensive Ausbildung, haben sich vieles angeeignet, aber sie sind eben nicht die Fachfrauen. (SL 3, Z. 94-99) [...] wir bräuchten dringend viel mehr Förderschullehrer, die momentan nicht vorhanden sind. Es kann nicht sein, dass wir Kinder mit 1,...-Stunden unterrichten und fördern. Da langt die Zeit überhaupt nicht aus [...]. Wenn der mit zwei Stunden in eine Klasse springt, der bekommt niemals den Überblick über das, was in der Klasse läuft, sowohl vom Sozialen her als auch vom Fachlichen. Der weiß überhaupt nicht, wo das Kind steht. (SL 2, Z. 262-266) 
SL 5 formuliert ihre Stellungnahme verknüpft mit der Sorge, dass diese sowie andere Lehrerstunden für besondere Projekte in Zukunft gekürzt werden könnten:

So viele Stunden hat sie [gemeint ist die Förderschullehrkraft, Anm. d. Verf.] ja gar nicht. Das ist ja das Bedauerliche, dass man da...aber das kostet ja alles Geld. Und die prekäre Frage ist hier noch lange nicht gelöst. Im Gegenteil, ich glaube hier wird man in Zukunft den Rotstift ansetzen und irgendwo wieder Einsparungen vornehmen. Das wird wahrscheinlich anfangen mit dem Kindergarten, dieses tolle Projekt, das wir hier jetzt, die Verbindung zwischen Kindergarten - Grundschule. Da habe ich die Befürchtung, dass man hier die Stundenzahl reduziert und das Ganze wieder abbläst, leider, denn das war ja auch ganz, ganz, ganz wichtig. Seit vielen Jahren plädieren wir dafür, dass so was passiert, jetzt kommt es und jetzt sehe ich schon, dass da wahrscheinlich wieder der Rotstift angesetzt wird. Und hier hoffentlich ja nicht! Hoffentlich kriegen wir noch ein paar Stunden mehr, aber das ist eine Frage des Geldes. (SL 5, Z. 101-110)

Auch die Eltern (E 1, E 3) haben im Blick, dass zum Zeitpunkt der Befragung etwa zwei bis drei Stunden wöchentlich von einer Förderschullehrkraft für ihren Sohn oder ihre Tochter eingebracht werden und halten dies ebenfalls für zu wenig:

Das größte Problem sind halt die Integrationsstunden. Man müsste die Stunden halt auf jeden Fall erhöhen, also dass ein Kind mit geistiger Behinderung, sage ich jetzt mal, drei Stunden in der Woche hat, das ist zu wenig, ja Unterstützung, da müsste man an erster Stelle halt dieses Problem lösen und ich denke dann kann Inklusion wirklich laufen. (E 1, Z. 197-200)

[...] was dann halt auch wichtig ist, ist, die Kinder, die Integrationskinder wie gesagt im Unterricht begleiten und ja, dabei sein können und dass eine Lehrerin halt für ein Kind, ein Integrationskind halt eine Integrationslehrerin, ja, nur für das eine Kind da wäre. (E 1, Z. 200-203)

Komplett im Unterricht, dass die Kinder halt komplett wie möglich am Unterricht teilnehmen, ja, was die anderen Kinder auch machen, so, so nach ihren Fähigkeiten unterrichtet werden. (E 1, Z. 210-212)

SL 2 fordert in diesem Zusammenhang, dass es Beratungsstunden für Regel- und Förderschullehrkräfte geben (SL 2, Z. 295) und „die Unterrichtsverpflichtung einfach auch herabgesetzt werden“" (SL 2, Z. 297f) müsste.

Mit Blick auf die Budgetierung von Ressourcen berichtet SLFZ 1, dass die Anzahl der zu betreuenden Kinder mit sonderpädagogischem Förderbedarf gestiegen sei und er deshalb „keine Stunden-Personen-Zuweisung mache, sondern Kollegen an Schulen direkt einsetze und die dort vor Ort gucken, welche Maßnahme sie wie betreuen können." (SLFZ 1, Z.7-9). Auch SLFZ 2 erläutert,

[...] dass die Zuweisung von Stunden zu Fällen keinesfalls mehr haltbar ist, weder aus pädagogischen noch anderen Gründen, insofern ist eine wie auch immer Freiheit in dem Bereich der Budgetierung, wenn es denn eine wird, von mir aus positiv gesehen, dass zunächst mal alle Kinder an einer Grundschule eingeschult werden sollen außer die, die eine medizinische Indikation haben, halte ich eigentlich auch für sinnvoll, weil dann kann man nämlich im Schulbezug klären, ob tatsächlich die Dinge, die im Kindergarten so schwierig waren, auch so sind. (Z. 232-237)

Darüber hinaus erwähnt SLFZ 1 die Notwendigkeit systemischer Arbeit, die ebenfalls damit zusammenhänge, dass personelle Ressourcen nicht mehr in ausreichendem Maß vorhanden seien und sieht die Verantwortung dafür bei der Schulverwaltung:

Die Veränderungen, die ich wahrgenommen habe, ist zunächst von der Einzelbetreuung der Kinder mehr hin auch zu versuchen systemisch zu arbeiten, da die Rahmenbedingungen sich so verschlechtert haben, dass man nicht mehr 1:1 mit den Kindern arbeiten kann. (SLFZ 1, Z. 6-8) [...] diese Ent- 
wicklung, gerade im sonderpädagogischen Förderbedarf wurde seitens der Schulverwaltung, sprich Bildungsministerium, in dieser Form nicht wahrgenommen und man hat es also verabsäumt, als diese Entwicklung einsetzte, das zur Kenntnis zu nehmen und entsprechend zu reagieren, so dass man jetzt mittlerweile dann, wenn ich mal so beschreiben darf, in dieser desolaten Situation gelandet ist. (SLFZ 1, Z 14-19)

Als problematisch sieht SLFZ 2 diesbezüglich die Tatsache an, dass „der Erfolg der integrativen Maßnahmen wegen fehlender Ressourcen und Unterstützungsmöglichkeiten von den Regelschulen in Frage gestellt" werde (Z.93-97). Während Integration insgesamt als positiv eingeschätzt werde, würden deren Effekte aufgrund der ungünstigen personellen Versorgung nicht wahrgenommen (Z.93-97).

Hinsichtlich der passenden Zuweisung von Förderschullehrern an Regelschulen äußern sich die Leitungen der Förderzentren sowohl kritisch wie differenziert:

[...] das ist ein ganz komplexes System. Ich meine Leute unterschätzen das. Sie sagen dann, man teilt einer Lehrkraft mit an die und die Schule gehst du und kümmerst dich um die und die Kinder, aber es gibt noch andere Parameter. Das heißt da, jede Schule und jede Schulleitung hat so ihre Eigenarten, die sind bei dieser Planung mit zu berücksichtigen und man muss auch die Eigenarten und die Persönlichkeitsmerkmale, wenn man das mal so nennen darf, der Förderschullehrkräfte, die dort arbeiten, muss man ebenfalls mit ins Kalkül ziehen. Man braucht aufgrund der Aktenunterlage, der Akteneinsicht, die man hat, muss man sich mit den Besonderheiten und den Bedarfen der Kinder auseinandersetzen, was sie brauchen, das, denke ich, daraus wird ersichtlich, dass es an für sich eine ungeheuer komplexe Angelegenheit ist alle Beteiligten so zusammenzuführen, dass da ein positives Ergebnis oder zumindest kein negatives Ergebnis rauskommt. (SLFZ 1, Z. 167-177)

Als Beispiele benennt dieser Schulleiter eine Schule, die eine Systemumstellung plant und daher für den Einstiegsjahrgang auf passende Lehrkräfte angewiesen ist: „[...] ich weiß von einer Schule, die beginnen jetzt mit dem 5er-Jahrgang und dieser 5er-Jahrgang soll schon mit passenden Lehrkräften auf ein neues System umgestellt werden“ (SLFZ 1, Z. 245-247). Auch der Schulleiter des anderen Förderzentrums weist auf die notwendige Passung von Lehrkräften und Schulen hin: „Insofern mache ich einen relativ transparenten Einsatzplan mit je nachdem auch Wünschen, was so persönliche, private Angelegenheiten angeht, wo man hinfährt, was so funktioniert und was nicht funktioniert." (SLFZ 2, Z. 158-160).

Beide Schulleitungen der sonderpädagogischen Förder- und Beratungszentren sind um Kontinuität hinsichtlich der Personalisierung der Regelschulen bemüht: „[...] wir haben also versucht eine gewisse Personenkonstanz in Regelschulen zu erhalten, soweit das organisatorisch und strukturell möglich war, und dann hat man natürlich Beratungskompetenzen in den Regelschulen drin. Und die werden sehr, sehr gerne angenommen.“ (SLFZ 1, Z. 90-92); „[...] ich versuche von Jahr zu Jahr die Kontinuität zu wahren, soweit sie irgendwie möglich ist“" (SLFZ 2, Z. 157f). SLFZ 1 beschreibt, dass es einige Jahre dauern kann, bis ein Förderschullehrerteam sich in einer Schule eingearbeitet hat:

[...] es verlangt einen erhöhten Zeitaufwand, zumindest mal in der Startphase, in den ersten zwei, drei Jahren, bis man sich dort eingefunden hat und da bedarf es auch der Abstimmung der Lehrkräfte, das heißt man könnte, dürfte dann in diesem Fall die Beziehung dort nicht ändern, sondern muss diese Teams bestehen lassen, mit der Hoffnung, dass die Arbeit dieses Teams auf andere Lehrkräfte in der Regelschule ausstrahlt und gegebenenfalls sukzessive übertragen wird. Ich denke, das ist ein ganz, ganz langer Prozess. Ich denke, der hat jetzt im Moment begonnen, ist beobachtbar und das kann einen dann wieder so ein bisschen optimistisch stimmen. (SLFZ 1, Z. 118-125) 
Was das spezifische Personal in Regelschulen betrifft, äußert sich eine Schulleitung zum Bedarf an Schoolworkern „[... ] auch die Schulsozialarbeit ist bei uns nicht gut genug ausgebaut (Z 271); Schoolworker ist bei uns zweimal in der Woche, das langt auch nicht." (SL 2, Z. 273) und eine andere dahingehend, dass auch Erzieher in den Schulen benötigt werden würden:

Wir bräuchten eigentlich noch Erzieher, die den Klassen zugeteilt sind, auch nicht ein Erzieher für eine ganze Schule, sondern vielleicht einen Erzieher für einen Jahrgang mit vier, fünf Klassen, der dann Elternarbeit eben auch einfach noch machen kann und öfter mal zum Hörer greifen kann und sagen kann, Kind ist heute nicht gekommen, nicht da, schon so und so viele Fehltage oder Hausaufgaben klappen nicht. (SL 2, Z. 273-278)

Ausführlich thematisieren vor allem die Förderschullehrkräfte sowie eine Schulleitung den möglichen Zusammenhang zwischen einer ungünstigen personellen Ressourcensituation und deren Auswirkungen auf die Umsetzung schulischer Integration. So erläutert FSL 4, dass sie den Ansatz inklusiver Beschulung auf der einen Seite für sehr wichtig halte, „weil wir eigentlich, ja, im Grunde keine Gesellschaft haben, die wirklich inklusiv ist, sondern eher auf Auslese " (FSL 4, Z. 187), verweist aber gleichzeitig darauf, dass dies nur positiv enden und in der praktischen Umsetzung funktionieren könne, wenn die entsprechenden Ressourcen gegeben seien (FSL 4, Z. 162-164 u.Z. 187-190). FSL 2 beschreibt ihre positiven Erfahrungen mit inklusivem Unterricht in einer Schule mit dem Förderschwerpunkt körperlich-motorische Entwicklung, in der sie „quasi alle Schüler - Grundschüler, Schüler mit Beeinträchtigung Lernen, geistig behinderte Schüler und auch schwerstmehrfachbehinderte Schüler - in einer Klasse unterrichtet [habe]“ (FSL 2, Z. 144f). Dies sei allerdings nur mit einem passenden Personalschlüssel möglich (FSL 2, Z. 144-149). Und FSL 1 und FSL 5 beschreiben plastisch:

[...] wenn man sieht, dass das mit teilweise noch weniger Stunden als jetzt geplant werden soll, dann, denke ich, sehen die meisten Kollegen und ich auch es eben so, dass es eben so nicht funktionieren kann, und dass das eben auf Kosten der Kinder dann halt auch geht (FSL 1, Z. 183-185)

Ich glaube aber, dass das nicht unbedingt nur mit der Inklusion zu tun hat, sondern mit den momentanen Arbeitsbedingungen und dass keiner damit rechnet, dass die Inklusion umgesetzt werden kann, also vor allen Dingen personell so umgesetzt werden kann, dass sie, dass sie gut durchführbar ist und zwar für alle Beteiligten und zwar sowohl vom Arbeitsaufwand und vom Kräfteverzehr bei den, beim Lehrkörper, als auch bei den Schülern, weil die einfach durch diese wenigen Stunden, die wenige Zuwendung, die man für sie hat, einfach auch zu kurz kommen. Also ich denke, dass grundsätzlich schon ein positiver Gedanke da ist, aber dass alle Probleme haben mit der Umsetzung im Moment. (FSL 5, Z. 171-178) [...] konkret im Alltag würde viel mehr Zeit, viel mehr Ressourcen und so was, das würde wirklich helfen und sicher auch wieder Kreativität freisetzen und Experimentierfreude (FSL 4, Z. 150-152)

\subsubsection{Kooperation}

Hinsichtlich der Kooperation von Lehrkräften innerhalb der Regelschulen erwähnen SL 2 und 3 Dienstbesprechungen im Kontext mit Integration: „[...] bei Dienstbesprechungen sind wir jetzt häufiger darauf eingegangen, welche Voraussetzungen die Integration braucht, also da geht es um die Kooperation mit den Förderschullehrern, das waren aber allerdings eher kurze Einheiten“" (SL 2, Z. 42-44). SLFZ 1 spricht mit Blick auf die Kooperation in den Regelschulen allgemein von einer „Zusammenarbeit auf Augenhöhe mit den Leuten, die halt dort arbeiten, egal welche, auch Schulsozialarbeiter, andere von Vereinen oder sonst woher, ein Platz in den Beratungsgremien, in den Konferenzen." (SLFZ1, Z. 88-90) An einer Regelschule sind stufenbezogene Förderkonferenzen eingeführt worden: 
Wir haben jetzt eine pädagogische Förderkonferenz für Jahrgang 5 jetzt gerade gemacht, haben in Anwesenheit von zwei Förderschullehrern haben wir alle Kinder besprochen, die in der Jahrgangsstufe drin sind und haben uns auch den Rat dann der Förderschullehrer geholt und haben bei dem einen festgestellt, den und den werden wir jetzt wohl melden und bei anderen haben wir andere Möglichkeiten gefunden, die wir jetzt zuerst einmal angehen wollen. (SL 2, Z. 44-49)

Eine Regelschullehrkraft erwähnt mit Blick auf die Kooperation die in den Schulen stattfindenden Förderausschüsse (RSL 3, Z. 120-140).

Die Schulleitungen der Förderzentren erläutern, dass für die Kooperation der Förderschullehrkräfte innerhalb der Förderzentren Arbeitskreise (SLFZ 1, Z. 180-184; SLFZ 2, Z. 165167) und Kollegiumstage (SLFZ 2, S. 167-169) angeboten werden.

Es gibt schon, noch aus Zeiten als ich dort anfing, also in den 90er Jahren einen Arbeitskreis mit je nachdem anderer Gewichtung, je nachdem, wer der Schulleiter war. Den gibt es aber kontinuierlich und wir haben uns dort circa sechs Mal im Jahr getroffen und verschiedene Sachen aktueller, aber auch organisatorischer Art, aber auch gesetzliche Veränderungen und Besuche anderer Einrichtungen gemacht. (Z. 20-24); Der Arbeitskreis muss sich dann nächste Woche quasi für dieses Jahr noch mal organisieren, ob wir mit der Psychologin noch ein, zwei Sachen weiterarbeiten, welche Termine wir uns für den Arbeitskreis geben und welche Themen dann wichtig sind. (SLFZ 2, Z. 165-167)

Die Teilnahme an diesen Arbeitskreisen beruht auf Freiwilligkeit, so dass laut SLFZ 1 nicht alle Förderschullehrkräfte eines Förderzentrums regelmäßig daran teilnehmen:

Wir haben diese regelmäßigen Treffen, aber sie werden, ich denke, in einer Größenordnung von 30$40 \%$ wahrgenommen. Ich denke das ist ein Faktor, der ist noch verbesserungswürdig, aber wenn man dann auch berücksichtigt, welche Arbeit teilweise vor Ort geleistet wird, welche Belastung es ist, ist es auch verständlich, dass nicht immer an den regelmäßigen Treffen teilgenommen werden kann. (SLFZ 1, Z. 180-184)

Dies begründet SLFZ 2 damit, dass die Förderschullehrer terminlich sowohl in den Regelschulen als auch am Förderzentrum durch Dienstbesprechungen, Konferenzen etc. eingebunden sind:

Das sind fast 10 Termine außerhalb und die Leute müssen ja auch noch zu den Besprechungen, Zeugniskonferenzen und sonstigem an den Schulen, an denen sie sind, neben Elterngesprächen und den anderen Sachen. Und da denke ich sind sechs Termine Arbeitskreis und drei, vier Dienstbesprechungen, eine Gesamtkonferenz, wo wir alle da sind, schon ein üppiger Rahmen, zumal es dafür ja keine Zeit frei gibt, wie an sonstigen sozialen Einrichtungen. (SLFZ 2, Z. 173-177)

Die Schulleitungen der Förderzentren beschreiben die Kooperation zwischen den Regel- und Förderschullehrkräften auf einer eher übergeordneten, systemischen Ebene und thematisieren diesbezüglich die Abhängigkeit der Kooperation von der Offenheit der Einzelschule:

Die Kooperation dort, wo sie gewünscht wird, dort wird sie auch stattfinden (SLFZ 1, Z. 152); dort, wo diese Sichtweise nicht vorhanden ist, ich meine, da sind wir auf einem Stand von, ganz optimistisch von 1990. (SLFZ 1, Z. 163f)

SLFZ 1 verweist in diesem Zusammenhang auch auf die Kooperation zwischen den Regelschulen und dem Förderzentrum, die er - abhängig von der Einzelschule - als eng beschreibt (Z. 155-163). 
[...] dort, wo wir länger zusammengearbeitet haben und wo wir auch systemisch arbeiten konnten, konnten Kompetenzen in die Regelschulen übertragen, was ja auch eine unserer Aufgaben ist, diese Kompetenz in die Regelschulen zu bringen. Dort, wo die Schulen aufgeschlossen waren, dort läuft das, da ist eine ganz starke Entwicklung erkennbar und dort ist auch die Kooperation zwischen Regelschule und Förderzentrum, die ist ziemlich eng, nicht. Das muss man wirklich sagen, es wird nachgefragt, es wird bestätigt, man tauscht sich aus. Und diese Schulen sind auch bemüht unseren Kolleginnen und Kollegen vor Ort in der Regelschule die Bedingungen zu schaffen, die sie für ihre Arbeit brauchen. (SLFZ 1, Z. 155-163);

Die Kontinuität, die schon mit Blick auf die Personalisierung der Regelschulen (Ressourcen) Erwähnung gefunden hat, wird von SLFZ 2 an dieser Stelle als wesentliche Voraussetzung kooperativen Arbeitens dargestellt:

[...] wenn eine Kontinuität gewahrt ist, und wenn die Kollegen dazu bereit sind, andere Menschen in ihrem Umfeld wahrzunehmen, und dann ist die Kooperation meistens gut, zumindest auf dem sozialen und emotionalen Bereich im Austausch untereinander. (Z. 136-138); An den weiterführenden Schulen ist das nicht so, aber da spielt halt der langjährige Kontakt eine Rolle und dann funktioniert das auch gut (Z. 141-143); wenn auf beiden Seiten Akzeptanz da ist und dann auch eine Kontinuität über ein, zwei, drei Jahre und dann kann man recht gut zusammen arbeiten. (Z. 152f)

SLFZ 1 bezieht sich auf das besondere Engagement der Förderschullehrkräfte an den Regelschulen:

Dann laufen sehr, sehr viele Dinge außerhalb der regulären Arbeitszeit und ich denke, das kann man mit einzelnen, mit Einzelfällen allgemein für alle bestätigen, dass so gearbeitet wird, nämlich ohne dieses Engagement, den Einsatz unserer Lehrkräfte vor Ort, wäre die Sache schon gestorben. (SLFZ 1, Z. 187-190)

Die Schulleitungen der Regelschulen haben im Blick, dass die Kooperation mit den Förderschullehrern Offenheit und Absprachen erfordert sowie eine Notwendigkeit darstellt:

[...] dass diese Offenheit da ist, ein Problem kann man, bevor man lang drüber jammert, auch einfach mal wegarbeiten, und so kooperieren die aus meiner Sicht eigentlich sehr positiv mit den Förderlehrern, die bei uns an der Schule sind (SL 1, Z. 36-38)

Veränderungen insofern auch, dass man weiß, dass man sich in Anführungszeichen eigentlich mit den Förderschullehrern immer kooperativ auseinandersetzen muss und bei vielen Kollegen auch das Bewusstsein, bei allen Kollegen, dass sie auch mit den Elternhäusern mehr zusammenarbeiten müssen und dass man auch versucht, oder ich versuche das zumindest, Hilfestellung von außen immer mehr reinzubekommen. (SL 2, Z. 16-20)

Die Regelschullehrkräfte sprechen von einer engen Kooperation mit den Förderschullehrkräften (RSL 1, Z.71; RSL 3, Z 11; RSL 5, Z. 33-35), wobei RSL 5 erwähnt, dass die „Integrationslehrerin über das Übliche hinaus stets Ansprechpartnerin [sei]“ (RSL 5, Z. 10f). RSL 1 und FSL 4 erwähnen in ihren Ausführungen gemeinsam geführte Elterngespräche als Kooperationselement (RSL 1, Z. 85-89; FSL 4, Z. 49f).

Bewertet wird die Kooperation von Regel- und Förderschullehrkräften vorwiegend positiv. So äußern sich vier der fünf Schulleitungen dahingehend, dass diese „optimal“ (SL 1, Z. 20), „in keinster Weise verbesserungswürdig“ (SL 3, Z. 67), „mehr als optimal“ (SL 3, Z. 67) oder „sehr gut" (SL 4, Z. 50; SL 5, Z.61) sei. SL 1 erwähnt in diesem Zusammenhang, dass die Förderschullehrkräfte an der Schule „alle offen aufgenommen“ (SL 1, Z. 227) werden und SL 4 sowie SL 5 erklären, dass die positive Bewertung der Zusammenarbeit vor allem auf das hohe Engagement der Förderschullehrkräfte zurückzuführen sei: 
Also hier bei uns im Haus, würde ich sagen, klappt das sehr gut, weil wir einfach auch eine super engagierte Kollegin hier haben, immer gesprächsbereit, der ist auch kein Telefonanruf zu viel. Die ist wirklich top engagiert, also das klappt wirklich gut. (SL 4, Z. 50-52)

Also die ist sehr gut. Das ist halt unser Vorteil, dass wir hier die XY haben, die schon jahrelang hier ist, sich hier gut auskennt, die Schüler alle kennt, die Probleme der Schüler bis ins Detail fast kennt, viele Gespräche auch mit Eltern hat, die ich für wichtig erachte, in jedem Förderausschuss sitzt sie dabei und die Absprache auch jeden Morgen. Ich finde es ganz toll, es läuft ganz prima. Da kann ich nur sagen, kann man nicht viel verbessern. Und alle Förderlehrer, die hier sind, sind sehr kooperativ. (SL 5, Z. 61-66)

Ähnlich wie die Schulleitung eines Förderzentrums (s.o.) betont auch die Schulleitung einer Regelschule dabei die Bedeutung der Zusammenarbeit der Beteiligten ,auf Augenhöhe“:

Ob ich in der Lage bin auf Augenhöhe mit anderen zu sprechen, was der zu sagen hat oder die zu sagen hat, ernst nehme, wichtig nehme, so wichtig nehme, dass ich es in meinen Unterricht miteinbinde. Das, glaube ich, ist gegeben. Also, eh, dass die sich auch gegenseitig als Hilfe ansehen. Partner, die am selben Strang ziehen. (SL 1, Z. 229-232)

SL 2 bewertet die Kooperation im Vergleich etwas kritischer und führt mögliche Probleme darauf zurück, dass die Regelschullehrkräfte den Förderschullehrkräften teilweise nicht „auf Augenhöhe“ begegnen:

Das kommt sehr auf die Personen an, sowohl im Regelschul- als auch im Förderschulbereich. Es gibt Lehrkräfte der Regelschule, die ein Bewusstsein entwickeln für Kinder mit diesem speziellen Förderbedarf, die sich auch auf die Hilfe der Förderschullehrer verlassen, nachfragen, sich Hilfe holen und sich absprechen, es gibt aber auch den Fall, dass Regelschullehrer gar kein Bewusstsein dafür haben und sich selbst eben als den wissenschaftlich ausgebildeten Menschen sehen und dem jetzt auch der Förderschullehrer, der alles vielleicht ein bisschen gemacht hat, aber eben nicht auf dieser wissenschaftlichen Ebene war, nicht gerecht werden kann. Und an der Stelle kann es eben etwas schwierig werden oder ist es etwas schwierig. Also das unterscheidet sich sehr von Lehrer zu Lehrer und wir haben etwa 60 Lehrkräfte, also da ist von allem etwas auch gegeben. (SL 2, Z. 163-171)

Sehr ähnlich sieht die Bewertung der Zusammenarbeit bei den Regelschullehrkräften aus, deren Aussagen von „Das klappt ganz gut [...] richtig gut.“ (RSL 1, Z. 205) über „Ich denke gut.“ (RSL 2, Z. 81), „Wunderbar, also das läuft super. Also das kann ich nicht anders sagen. Hatte ich bisher auch noch nie Schwierigkeiten." (RSL 3, Z. 83f) bis zu „Die ist sehr gut.“ (RSL 4, Z.71) oder „Super. Super. Bei uns hier wirklich.“ (RSL 5, Z. 94) reichen. RSL 2 formuliert an dieser Stelle vorsichtiger und begründet ihre Wahrnehmung ähnlich wie SL 2 damit, dass die Regelschullehrkräfte der Kooperation unterschiedlich offen gegenüberstehen:

[...] das hängt vom Kollegen auch einfach ab. Das klappt wohl in manchen Klassen besser als in anderen auch, weil manche Kollegen flexibler sind, andere sind da weniger flexibel. Die können das nicht so gut ertragen, wenn da noch jemand im Unterricht mit dabei ist, das ist für die schon mal dann schwierig. (RSL 2, Z. 99-102)

Auch die Förderschullehrkräfte äußern sich - von einer Ausnahme abgesehen (FSL 2, Z. 88) mit Blick auf die Kooperation mit den Regelschullehrkräften positiv: „[...] die ist hier wirklich super“ (FSL 1, Z. 115), „Gut. Hier gut.“ (FSL 3, Z. 76) oder „Ich finde es insgesamt ganz gut.“ (FSL 4, Z. 121) sowie „die sind eigentlich offen“ (FSL 4, Z. 129). FSL 5 gibt an, dass die Zusammenarbeit sehr von der jeweiligen Lehrkraft abhänge (FSL 5, Z. 36) und führt die Qualität der Kooperation insgesamt auf die Kontinuität der Zusammenarbeit zurück: 
Hier an der Schule läuft es sehr gut. Ich bin ja jetzt auch schon ein paar Jahre hier und wir kennen uns alle gut und das ist sehr schulabhängig. Da habe ich schon andere Erfahrungen auch gemacht, aber hier halten sich alle an Absprachen, es wird nachgefragt, wenn Probleme da sind. Es werden gemeinsame Absprachen getroffen, also hier läuft es wirklich gut. (FSL 5, Z. 149-152)

Eine Förderschullehrkraft weist darauf hin, dass die Regelschullehrkräfte zum Teil erst lernen müssen, dass sie unterstützend tätig werden kann:

Ich merke zum Beispiel in der Grundschule, da bin ich noch nicht so lange, dass die dann teilweise schon gewöhnt sind Sachen selber zu machen und mich nicht unbedingt mit einzubeziehen, aber das bringe ich ihnen noch bei. Nein, dass ich dann einfach sage, das kann ich auch übernehmen und würde auch gerne daran teilhaben. (FSL 4, Z. 121-125)

Die Schulleitungen der Förderzentren äußern sich hinsichtlich der konkreten Umsetzung der unterrichtsbezogenen Kooperation sehr kritisch und beschreiben u.a. mögliche Ängste der Regelschullehrkräfte, die sich durch Förderschullehrkräfte im Unterricht beobachtet und damit auch kontrolliert fühlen können:

Dann das ganze Feld von persönlichen Spannungen und Ängsten, wenn da jemand dabei ist, guckt der mich an oder guckt der nach den Kindern? Erklärt der Sachen so, dass die nachher denken, ich wäre irgendwie...Angst um ihre Position, ihre Rolle in den Klassen und das sind halt Felder, da kommt man wenig bei an. (SLFZ 2, Z. 149-152)

Darüber hinaus kann die Anwesenheit der Förderschullehrkraft bzw. weiterer Kräfte wie Integrationshelfer auch als Störung wahrgenommen werden:

[...] allerdings müssen unsere Kolleginnen, wenn sie mit in der Klasse sind, in der Regel den Mund halten, ruhig sein und sich still auf dem Platz verhalten, damit der Unterricht der Regelschule nicht gestört wird. Das sind ganz miserable Voraussetzungen. (SLFZ 1, Z. 103-105)

Man muss sehen es ist schon ein Problem, wenn man in einer Klasse sitzt und dann mehrere Integrationshelfer hat und der Lehrer dabei. Das bringt Unruhe in die Klasse und es ist für viele Kollegen nicht so einfach, weil man doch sich auch beobachtet fühlt. Es kommt immer auch die Chemie, denke ich auch an, welche Leute man dabei hat, also mich hat es bis jetzt noch nie groß gestört, aber ich könnte mir schon vorstellen, wenn da jemand wäre, mit dem ich nicht arbeiten könnte, dass das ein großes Problem wäre. (RSL 2, Z. 110-115)

Durch Regelschulschulleitungen und - lehrkräfte erfolgen allerdings auch positivere Aussagen zu diesem Bereich. SL 1 bezieht sich dabei auf die Entlastung der Regel- durch die Förderschullehrkräfte:

Weil diese Hilfe, die die Förderlehrer den Stammlehrern geben, ist natürlich auch, ist ja auch eine Entlastung. Man nimmt ihnen ja auch einiges ab und das sehen die Kollegen auch. Sie sehen also nicht nur, oh, so viele Integrationskinder, was bedeutet das für mich?, sondern sie sehen natürlich ja auch, da ist einer, der unterstützt mich, das macht mir die Arbeit in dieser Binnendifferenzierung ja auch nochmal leichter. (SL 1, Z. 105-110); [...] die Binnendifferenzierung ja auch durch die Förderlehrerin mit entsprechenden Arbeitsblättern und so weiter auch gefördert wird, diese Last der Binnendifferenzierung dem Lehrer ja auch genommen ist (SL 1, Z. 113-115)

SL 2 erläutert, dass die Schule Wert auf paralleles Arbeiten in den Jahrgangsstufen legt, wodurch die unterrichtsbezogene Kooperation der Förderschullehrkraft, die für einen Jahrgang zuständig ist, mit den Regelschullehrkräften erleichtert werden könne (SL 2, Z.90f) und führt als Beispiel aus: 
Im Moment sind alle sechsten Klassen beim Thema Fabel, alle besprechen die gleichen Fabeln und dann ist es für die Förderschullehrer auch einfacher und wir legen im Moment sehr viel Wert auf Vergleichsarbeiten, unter anderem auch, weil ich sage, die Förderschullehrer müssen es einfacher haben und wir haben aber auch die Möglichkeit, wenn es zu einem Fachlehrerausfall kommt zu sagen, ok, Klassenstufe 6 ist da und da und hundert Schüler haben im Moment dieses Thema und dann wäre es das auch einfacher, also daran arbeiten wir. (SL 2, Z. 93-99)

Dass die unterrichtsbezogene Zusammenarbeit sich nicht nur auf die Schüler und Schülerinnen mit sonderpädagogischem Förderbedarf, sondern auf alle Schüler bezieht, hebt RSL 5 hervor:

Wir klären im Team die Sachen ab. Wir tauschen auch aus, Unterrichtsmaterialien und Erfahrungen, die wir damit haben, allerdings nicht mit Schwerpunkt Fördern, also nicht für die Integrationskinder, sondern alle. (RSL 5, Z. 38-40)

Hospitationen als Baustein der Kooperation werden von einer Regel- und einer Förderschullehrkraft erwähnt:

[...] die Integrationslehrerin kommt dann trotzdem auf mich zu und sagt dann: Wie ist das in den Nebenfächern? Und sie kommt auch zwischen durch mal in ein Nebenfach um zu gucken, ob das da läuft oder nicht läuft. (RSL 2, Z. 74-76)

Ich habe viele Stunden, wo ich einfach mehrere Klassen auf einer Stunde liegen habe und wo ich dann ganz gezielt in gewissen Stunden reingehe. (FSL 2, Z. 36-38)

Das Kooperationselement Beratung wird von insgesamt elf Interviewpartnern aufgegriffen. Während SL 2 davon spricht, dass Regel- wie Förderschullehrkräfte „das Bewusstsein [haben], dass sie sich absprechen müssen.“ (SL 2, Z. 102f) betont SL 1 die Selbstverständlichkeit gegenseitiger Beratung:

Man hat leichter den Kontakt zu den Förderlehrern oder -lehrerinnen gefunden, man spricht sich ab. Es ist heute eine Selbstverständlichkeit, dass man sich berät, in den Pausen oder auch in den Stunden, wo sie zusammen sind, dass man kurz was macht oder dass sich auch nach dem Unterricht zusammensitzt und das ein oder andere berät, die Kinder profitieren davon und letztlich die Lehrkraft auch. (SL 1, Z. 101-105)

Schulleitung 3 kann an ihrer Schule beobachten, dass mögliche Ängste der Regelschullehrkräfte abgebaut werden und diese inzwischen Beratung annehmen:

Kolleginnen, die noch eher ängstlich sind und die Tür zu haben, die haben zum Beispiel furchtbar Angst, wenn da jetzt noch ein zweiter Lehrer auftaucht, und ich beobachte, dass die sich allgemein öffnen. Ja, also auch offener sind, jetzt nicht mehr diese Ängste haben, sich dann Rat holen. Und sich aber nicht nur Rat holen bezogen auf ein Kind, das Förderbedarf hat, sondern sie fragen dann auch die Lehrerin um andere Kinder. Also das Spektrum ist jetzt halt ausgeweitet. Für die Klassenlehrer wunderbar, für die Förderlehrer natürlich zusätzlich Aufwand und Arbeit. (SL 3, Z. 36-42)

Eine weitere Schulleitung macht deutlich, dass das Einbringen einer anderen Perspektive durch die Förderschullehrkräfte hilfreich sein kann:

[...] wenn sich für uns Probleme aufgetan haben und wir konnten das nicht richtig einschätzen, da waren die Förderkollegen immer bereit da mal einen Blick darauf zu werfen und eine Einschätzung aus ihrer Situation vorzunehmen (SL 4, Z. 56-58)

Aus den Aussagen der Regelschullehrkräfte geht die Selbstverständlichkeit und Häufigkeit der kollegialen Beratung hervor: 
Immer. Wir machen das eigentlich täglich, also wir treffen uns meistens schon vor der Schule auf dem Parkplatz, wenn dann irgendwas ist, wird besprochen. Wir treffen uns dann in der Pause nochmal und wenn möglich nach der Schule auch nochmal. (RSL 1, Z. 85-89); über die Schule hinaus telefonieren wir teilweise auch abends noch (RSL 1, Z. 205f)

Also wir sind wirklich fast täglich in regem Austausch. (RSL 3, Z. 71); über Mail (RSL 3, Z. 73); Ich denke es ist zu wenig, aber wie gesagt, sie nimmt sich zum Glück immer die Zeit. Man kann sie auch anrufen, wenn es ganz brennt und so. (RSL 3, Z. 40f)

Auf Inhalte der Beratungsgespräche geht Regelschullehrkraft 4 ein, die beschreibt, dass ein regelmäßiger Austausch über Arzt- und Kliniktermine wichtig sei (RSL 4, Z.79-81) und der Austausch sich auch nicht nur auf die Integrationsschüler beziehe (RSL 4, Z. 42f). Während Förderschullehrkraft 2 äußert, dass Beratung im schulischen Alltag im Vordergrund stehe (FSL 2, Z. 35) und FSL 5 ebenfalls auf einen erhöhten Beratungsaufwand hinweist, „weil die Lehrer da mehr interessiert sind, was sie mit den Kindern machen können, wie sie die Kinder mit Förderbedarf einbinden können. (FSL 5, Z. 66-68), gehen die Förderschullehrkräfte in ihren Aussagen ansonsten vor allem auf die Problematik fehlender bzw. unzureichender fester Beratungszeiten ein und stellen damit eine Verknüpfung zum Bereich der Ressourcen her. So beschreibt Förderschullehrkraft 1 ihren Beratungsalltag folgendermaßen:

[...] es muss eigentlich ganz flexibel sein, da durch diesen Ganztagsschulbetrieb die Kollegen oft auch nachmittags dann noch hier eingebunden sind und das ist schon nicht so einfach, weil man nicht sagen kann, also wir treffen uns jetzt montags und dienstags immer nach der sechsten, weil man dann auch Nachmittagsbetreuung hat und es findet meist eigentlich in den Pausen statt. Ich habe jetzt das Glück, dass ich komplett hier bin und bin eigentlich meistens in den großen Pausen unterwegs einmal die Runde machen im Kollegium und dann diese organisatorischen Dinge klären. Oder eben auch, wenn ich in der Stunde mit drin bin und wir Stillarbeit machen, dass wir dann solche Dinge klären. Oder halt vor Elterngesprächen, falls da kritische Elterngespräche anstehen, dass wir uns dann gezielt dann irgendwann nochmal zwischendrin treffen um dann halt bestimmte Dinge zu klären, damit wir dann von schulischer Seite eine einheitliche Linie fahren können. (FSL 1, Z. 123-132)

Neben der Beratung während des Schultags erfolgen auch Telefonate oder Mailverkehr außerhalb dieser Zeit, wie FSL 5 berichtet:

Das heißt, da telefonieren wir auch sehr viel nachmittags oder abends noch, da läuft sehr viel E-Mailverkehr, da wir ja den Stoff dann im Grunde wieder absprechen müssen, der dann als Nächstes drankommt. (FSL 5, Z. 68-70)

Und FSL 2 erwidert auf die Rückfrage, ob für Beratung mehr Zeit notwendig sei: „Definitiv. Mehr Beratungszeit, mehr Zeit in der Klasse, mehr Zeit im Unterricht, ja, vielleicht auch mal für denjenigen sich die Zeit zu setzen und seine Klasse mal zu beobachten." (FSL 2, Z. 96f). FSL 5 sieht sich nur deshalb in der Lage Beratungen auch während des Schulvormittags umzusetzen, weil sie dafür Stunden einsetzen kann, die ihr als Inklusionsberaterin zur Verfügung stehen:

Ich mache trotzdem Beratungsstunden. Das mache ich halt deswegen, weil ich die Deputatsstunden für die Inklusionsberatungen habe. Dann laufen diese Beratungen. Die laufen aber eigentlich zusätzlich, also im Rahmen der Stunden, die ich für die Kinder habe, wäre das nicht möglich. (FSL 5, Z. 77-80)

Nur eine Förderschullehrkraft erwähnt explizit, dass den Regelschullehrkräften an ihrer Schule Beratungsstunden zur Verfügung stehen (FSL 4, Z. 47). FSL 5 hat diese Beratungsstunden teilweise so umfunktioniert, dass die Regelschullehrkräfte in diesen Stunden gezielt Zeit mit den Schülerinnen und Schülern mit sonderpädagogischem Förderbedarf verbringen: 
Bei den anderen Lehrern habe ich teilweise die Beratungsstunden ersetzt durch Arbeit, also die Regelschullehrer arbeiten jetzt mit den Förderkindern in dieser Stunde. Das hat sich vor allem bei den Kindern bewährt, die einen Wochenplan haben, weil sich die Regelschullehrer dann mit dem Wochenplan befassen müssen. Die haben dann den Auftrag in dieser Stunde, in der Beratung wäre, mit den Kindern am Wochenplan zu arbeiten und dafür zu sorgen, dass die Kinder den auch führen. Sie schreiben, was sie gemacht haben, und dadurch, habe ich festgestellt, dass sie sehr viel mehr wissen, was die Kinder eigentlich so tun. (FSL 5, Z. 70-77)

Kooperative Unterrichtsplanung wird von Regel- und Förderschullehrkräften u.a. so beschrieben, dass diese sich die Vorbereitung vor allem mit Blick auf das zu erstellende oder auszuwählende Material teilweise aufteilen:

Ich bereite selbst Material vor, hol mir aber immer, wenn es um die Förderkinder geht Hilfe von der Förderlehrerin. Weil es halt doch wichtig ist, gerade $a b$ und zu hänge ich dann einfach, weil ich nicht weiß, wie ich gerade besonders schwache Kinder gut fördern kann. (RSL 2, Z. 32-34)

Das machen wir gemeinsam. Mal hat sie Unterlagen oder sie nimmt meine Unterlagen und verfeinert das ganze oder ich bereit was vor und sie guckt nochmal drüber (RSL 1, Z. 92-94)

[...] ich kann eigentlich zu jedem Kollegen gehen, dem, also der meine Kinder betrifft und sagen: wie sieht es aus? Wann wird die Arbeit geschrieben? Was kommt darin vor? Hast du dieses und jenes Arbeitsblatt? Kann ich das abändern? Also da bekomme ich eigentlich immer eine Antwort oder die Sachen auch zugeschickt. Je nach Organisation des Kollegen früher oder später, aber das ist überhaupt kein Problem. Ich kann auch jederzeit selbst hinkommen und Vorschläge machen, wie ich es gerne hätte und wenn es irgendwie machbar ist, wird es eigentlich auch umgesetzt. (FSL 2, Z. 115-121)

Insbesondere hinsichtlich der zu planenden Differenzierung von Arbeitsaufträgen für Schülerinnen und Schüler mit sonderpädagogischem Förderbedarf wird die unterrichtsbezogene Kooperation von den Förderschullehrkräften auch unterschiedlich bzw. kritisch bewertet:

Es gibt Kollegen, mit denen funktioniert die Absprache mittlerweile eigentlich auch schon ohne viele Absprachen. Es gibt aber allerdings auch Kollegen, die sind da beratungsresistent und das ist hier immer so ein Kämpfen gegen Windmühlen, sage ich mal. Also jeden Tag aufs Neue wieder zu sagen: achte bitte darauf, denkst du daran, er braucht mehr Zeit, er hat eigentlich einen anderen Lehrplan, wir müssen die Anforderungen reduzieren. Ja, also es ist schon, man muss sich immer wieder ins Gedächtnis bringen. Zwar sind die Kollegen größtenteils offen, wenn man dann das wieder ins Gedächtnis gerufen hat, aber die Zusammenarbeit ist doch immer wieder schwer, holprig. (FSL 3, Z. 88-94)

Gut, wenn wir Beratungsstunden zusammen haben zum Thema, dann besprechen wir das und ich übernehme halt Teile oder mache halt Vorschläge inhaltlich, wie noch mehr differenziert werden kann. Das ist ganz unterschiedlich und hängt vor allem auch von der Zeit der Kollegen ab und sicher auch von der Offenheit damit auch. (FSL 5, Z. 42-45)

[...] die Kollegen, die offen arbeiten, kennen die Kinder sehr gut, also auch die Förderkinder, aber dann einfach diese Umsetzung, dieses: wie fördere ich das Kind, das wird dann mir überlassen. (FSL 3, Z. 48f)

Während eine Regelschullehrkraft davon spricht, dass es eher die Förderschullehrkraft sei, die die Vorbereitung differenzierender Angebote übernehme (RSL 5, Z. 36f), führt FSL 4 aus, dass es ihr um eine gemeinsame Unterrichtsplanung gehe, die sich auf alle Schülerinnen und Schüler einer Klasse beziehe:

Und da sehe ich meine Aufgabe nicht nur darin bei diesem einen Kind oder so, sondern auch für die Klasse. Also ich versuche Regelschullehrer schon auch insgesamt zu entlasten, wenn das geht. (FSL 4, Z. 47-49); bei zwei Lehrerinnen haben wir eine Klassenliste und gehen wirklich dann jedes Kind durch: was steht an? Was passiert? Wo sind wir? Was haben wir jetzt gemacht und so? Was kann ich machen? (FSL 4, Z. 50-52) 
Auch zur gemeinsamen Unterrichtsdurchführung werden einige Aussagen getätigt. Eine Regelschullehrkraft beschreibt die Vorteile der Co-Teaching-Form „one teach - one observe“ folgendermaßen:

[...] dass man, wenn man zusammen arbeitet, nochmal schaut. Also man sieht, wenn man alleine unterrichtet, auch jetzt mal frontal oder wie auch immer, mit Wochenplan oder so, dann sieht der zweite doch viel eher, Moment, da war ein Problem, da hakt es noch und kann genauer hinschauen. Auch wenn ich mit drin bin und schaue mir eine Stunde an, dann kann ich Kinder auch im Verhalten einfach mal sehen, ja? Kommen die klar? Wie arbeiten die? Was müsste ich da ändern? Also das ist für mich so ein Riesenvorteil, den man hätte. (RSL 4, Z. 54-59)

Eine weitere verweist darauf, dass die Förderschullehrkraft ihr aufgrund der Anwesenheit von vier bis fünf Stunden wöchentlich ,auch bei den anderen Kindern immer wieder wertvolle Tipps geben“ (RSL 5, Z. 45) könne und formuliert mit Blick auf mögliches Co-Teaching: „Also ich denke, das wäre etwas Tolles für die Zukunft." (RSL 5, Z. 45f). Sie hält die Anwesenheit einer Förderschullehrkraft auch deshalb für hilfreich, da sie dadurch manchmal Freiräume gewinne: „,...] es ist halt ein Erwachsener da und dadurch kann ich mal mich vor die Tür setzen und was machen, also das ist schon von Vorteil.“ (RSL 5, Z. 106f). Die Förderschullehrkräfte berichten, dass sie teilweise Unterricht in den Regelschulen übernehmen können: „Ja, es ist auch so, dass ich Kollegen habe, mit denen es recht gut funktioniert, wo ich Teile vom Unterricht übernehme." (FSL 2, Z. 38f); „[... wenn ich darf, unterrichte ich auch mal. Dann besprechen wir, dass wir einen Perspektivwechsel machen. Das ist aber schon selten, muss man sagen." (FSL 4, Z. 40-42).

Die konkreten Ausführungen der Lehrkräfte zum Thema „Co-Teaching“ sehen sehr unterschiedlich aus. Die Möglichkeiten des Co-Teaching an sich werden in diesem Zusammenhang zwar sehr positiv bewertet und vielfach wird Co-Teaching als Bestandteil integrativen Unterrichts - vor allem von den Förderschullehrkräften - gefordert (FSL 1, FSL 3, FSL 5, RSL 3, RSL 5), Möglichkeiten der aktuellen Umsetzung werden dagegen sehr kritisch eingeschätzt, wie folgende Aussagen belegen:

Teamteaching gibt es da, wo ich in den Klassen drin bin. Da wechselt das, da ist das auch für die Kinder ganz egal, wer von uns gerade vorne steht oder wer von uns jetzt gerade mit den Kindern irgendwas macht oder das werden wir auch immer beide gleichzeitig angesprochen. Das ist auch, da ist auch keine große Absprache vorher notwendig, das läuft eigentlich ganz gut. (FSL 5, Z. 63-66)

Ich denke, wenn wir wünsch dir was spielen könnten und sagen, wir machen Doppelbesetzung in allen Klassen mit Förderschul- und Regelschullehrern mit vielleicht noch einer Pädagogischen Fachkraft bei großen Klassen, dann wäre sicherlich keiner von uns dagegen. (FSL 1, Z. 180-183)

Es gab schon mal Teamteaching, es hat auch ganz toll geklappt, funktioniert nicht bei allen Kollegen und ist natürlich auch davon abhängig mit wie vielen Stunden, wie viele Kinder ich in der Klasse habe. (FSL 3, Z. 36-38)

[...] aufgrund der Stundenzahl eigentlich fast gar nicht (FSL 1, Z. 40)

[...] weil im Moment auch einfach die Zeit nicht da ist, dass ich viele Stunden im Teamteaching einbringen kann (FSL 2, Z. 35f)

Teamteaching gibt es nicht. (RSL 5, Z.33)

Wunsch und Wirklichkeit gehen an dieser Stelle aus Wahrnehmung der Lehrkräfte heraus also weit auseinander.

Als weiterer Aspekt der Kooperation wird die kooperative Leistungsbewertung genannt:

[..] bei Klassenarbeiten, teilweise die Klassenarbeiten von den Kindern, die [...] zieldifferent unterrichtet werden, nehmen Integrationslehrer mit und korrigieren die. Wir haben aber einen gemeinsamen Fehlerschlüssel. (RSL 1, Z. 207-209) 
Problematisiert wird auch die Übernahme veränderter Rollen und Aufgaben durch Förderschullehrkräfte in den Regelschulen. So nimmt SLFZ 2 die Perspektive der Förderschullehrkräfte ein, die in einer Förderschule arbeiten und für die die Vorstellung der Tätigkeit in einer Regelschule mit Sorgen verbunden sein kann:

Die Kollegen an der Schule sind noch mehr davon überzeugt, dass die Arbeit Sinn macht und sie vermissen Räume und Orte für sich und für das, was sie darstellen und was sie gerne machen würden in der Inklusion mehr als jetzt schon. Für die ist der Schritt meilenweit und ich weiß nicht, wie sie ihn gehen werden. (SLFZ 2, Z. 213-216)

Das ist schwierig zu sagen, weil einige Leute, denke ich mal, so aus ihrem tiefsten Herzen mir auch nicht sagen würden, dass sie doch viel lieber eine Klasse hätten oder in ihrem Fach mit einzelnen Kindern arbeiten wollen. Ich weiß, dass einige das vermissen. (SLFZ 2, Z. 200-202)

Darüber hinaus bezieht er sich auf Ängste der Förderschullehrkräfte, die bereits in Regelschulen arbeiten:

Manche befürchten auch, dass die Budgetierung für sie Nachteile bringt, nämlich das so nicht mehr gestalten können und es gab voriges Jahr schon ein paar Vorfälle, indem Regelschulleiter von Kollegen Sachen verlangt haben, wo sie gefragt haben: was soll das? (SLFZ 2, Z. 221-223)

Gleichzeitig beschreibt er auch Lehrkräfte, die ihre Arbeit in den Regelschulen als sinnvoll wahrnehmen:

[...] nichtsdestotrotz habe ich viele Leute, die gerne vernetzt arbeiten und die das von mir jetzt auch über Jahre schon gewohnt sind und das auch mitgehen, obwohl sie lieber vielleicht eine Sprachheilklasse hätten oder sonst etwas, aber das ist jetzt halt so und man kann da auch sinnvoll tun und sich da so einbringen. (SLFZ 2, Z. 202-205)

Beide SLFZ sprechen das erschwerte Rollenverständnis der Förder- wie der Regelschullehrkräfte an:

Und ich nehme auch dann wahr, dass einfach die, das Selbstverständnis der Lehrer zu ihrem Beruf ins Wanken geraten ist, weil viele sagen einfach: das, wofür ich ausgebildet wurde, was eigentlich der Grund war, diesen Beruf zu wählen, heute nicht mehr umsetzbar ist. Und das führt zu vielen Verunsicherungen und vielen Fragezeichen in dieser Hinsicht. (SLFZ 1, Z. 8-12)

Des Rollenverständnisses und des gesamten Bildungssystems. Ich meine da muss ein totaler Umbau stattfinden. Ich denke damit hat das einfach was zu tun. Ich denke, wie Sie schon gesagt haben, ich muss von dem Rollenverständnis ausgehen. Das erste: ich muss meine Rolle als Lehrer oder Lehrerin neu wahrnehmen. In manchen Fällen sogar, wenn man schon ein bisschen länger im Geschäft ist sogar neu erfinden, weil man diese Denkweise einfach nicht hat und ich gehe mal davon aus, aus meiner Beobachtung her, dass an der Stelle Förderschullehrerinnen flexibler sind, weil sie schon immer diese Freiräume hatten und in diesen Freiräumen auch arbeiten konnten und die Schwierigkeit liegt dort im Regelschulbereich, obwohl man sie diese Verantwortung heute in den Förderschulbereich verschiebt und ich denke das ist eine etwas schräge Sichtweise, die die Arbeit in Zukunft sicher nicht erleichtern wird. (SLFZ 2, Z. 209-218)

[...] aber es gibt sowohl bei Förderschullehrern als auch bei Regelschullehrern, eigentlich ist das nicht das, was ich machen will und auch nicht das, was ich haben will, und bei den Förderschullehrern entweder deswegen, weil sie keine nähere Bindung zu einem einzelnen Kind aufbauen können und therapeutisch arbeiten können, was sie gerne würden und bei den Regelschullehrern, weil sie sagen, ich bin zum Beispiel Französischlehrerin für SEK I Realschule und was soll ich bitteschön damit? Und was soll ich bitteschön mit Ihnen und was wollen Sie in meinem Unterricht. (SLFZ 2, Z. 143-149)

Auch eine Förderschullehrkraft äußert diesbezüglich ihre Ängste: 
[...] es ist einfach eine große Angst vor der Aufgabe, weil die Aufgabe und unser Arbeitsumfeld sich total ändern wird und es nicht unbedingt das ist, was wir machen wollen, so dass auch einige Kollegen schon nach Alternativen am Suchen sind, weil sie einfach am Kind arbeiten wollen und nicht nur Beratungstätigkeit übernehmen möchten. (Z.97-100)

SLFZ 1 geht davon aus, dass die FSL aufgrund der schwierigen Voraussetzungen in den Schulen berechtigte Ängste dahingehend haben könnten, ihre Aufgabe zu bewältigen:

[...] aufgrund der Erfahrung mit der Integration, wie sich das entwickelt hat, möchte ich mal sagen, positiv formuliert: skeptisch. Weil man einfach nicht davon ausgehen kann, dass die Voraussetzungen geschaffen werden und ich denke, dass hier auch den Förderschullehrkräften bewusst wird, dass man ihnen hier eine Aufgabe aufhalst und auflädt, die sie gar nicht erfüllen können, weil das Problemfeld auf der Gegenseite ist so riesig, dass das ein einzelner an seiner Schule gar nicht, wo er arbeitet, oder selbst wenn es ein größeres System ist und wir sind mit mehreren Leuten dort vor Ort, dass die wirklich die Angst haben und ich denke die Angst ist wirklich berechtigt, dass sie diese Aufgabe nicht leisten können. (SLFZ 1, Z. 222-229)

Die Förderschullehrkräfte geben vor allem individuelle Förderung (FSL 1, FSL 4) und Vernetzung (FSL 4) als ihre Aufgaben an:

Also grundsätzlich biete ich immer an Vernetzung, dass ich die - gerade bei Kindern mit Erziehungshilfebedarf - den Kontakt zu Ärzten, Kliniken, Therapeutinnen, den Eltern halt sehr eng pflege und oft maile ich halt auch und da sind auch die Institutionen offen. Jugendamt, also wenn die Eltern die Schweigepflichtsentbindung geben, ist selten ein Problem. Das empfinden die Lehrer auch dann als Entlastung. (FSL 4, Z. 36-40)

\subsubsection{Professionalisierung}

Eine Regelschullehrkraft erläutert, dass sie sich aufgrund ihrer Ausbildung nicht ausreichend auf die Anforderungen integrativer Beschulung vorbereitet fühlt:

Was uns halt alle oder die meisten, und da zähle ich mich auch dazu, ärgert oder - es kann so nicht gehen. Es ändert sich halt gar nichts. Wir kriegen diese Kinder einfach dazu, wir haben die Ausbildung nicht. Ok, man kann mal auf ein Seminar gehen. Passt es dann auch zu dem Kind? Es ist unwahrscheinlich, finde ich, mehr, also mehr Arbeit halt. (RSL 5, Z. 123-126)

Professionalisierung unter dem Aspekt der Fortbildung von Lehrkräften greifen die Schulleitungen der Förderzentren sowohl mit Blick auf die Förderschulkollegen wie auch hinsichtlich der Regelschulkollegen auf. Beide Schulleitungen erwähnen in diesem Zusammenhang Fortbildungsangebote in Form von Pädagogischen Tagen, die zum Teil als Fortsetzungsveranstaltungen stattfinden (SLFZ 1, Z. 23f; SLFZ 2, Z. 24-28), und sich bspw. mit dem Thema „Kommunikation im gemeinsamen Unterricht" (SLFZ 2, Z. 24-28) auseinandersetzen. SLFZ 1 stellt bezüglich des Fortbildungsbedarfs an Regelschulen plastisch dar:

Es gibt ein zwei Schulen, die haben das geschnallt, ich meine, das sind keine Grundschulen, sondern es sind größere Systeme, die unter diesen Missständen und gerade mit der Vielzahl der Problemkinder verstärkt zu leiden haben. Das ist also im SEK I-Bereich. Ich denke, das sind so die ersten Schulen, die jetzt so langsam wach werden und sagen: hier am System muss sich was verändern, was ich auch aus Gesprächen herausgehört habe, die dann einfach sagen, unsere Lehrerschaft ist noch gar nicht dort, dass wir an den Start gehen können, sondern die muss man erst mal abholen und zur Startlinie hinführen (SLFZ 1, Z. 231-239) 
Während sich Pädagogische Tage an den Förderzentren mit den Fragen integrativer Beschulung befassen, sind diese an den Regelschulen gemäß der Äußerungen von zwei Schulleitungen und zwei Lehrkräften nicht spezifisch auf diese Thematik hin orientiert (SL 1, Z. 30; SL 4, Z. 13), beschäftigen sich zum Teil aber mit Themen, die mit Blick auf inklusiven Unterricht eine Rolle spielen wie ,individuelle Förderung“ (SL 4, Z. 13f), „Unterrichtsmethoden“ (RSL 3, Z. 1820), KESS erziehen (RSL 5, Z. 85-87) oder Regeln:

Es gab einen Pädagogischen Tag bzw. mehrere Pädagogische Tage schon zum Thema, zum Beispiel mit Herrn Grüner, wo es einfach darum ging allgemeine Regeln aufzufassen. Dann war jetzt Fortbildung Klippert. Man ist schon bemüht sich in die Richtung zu öffnen. Ja, wobei viele Regelkollegen sich schwer tun einfach von den alten Unterrichtsformen sich zu trennen und sich zu öffnen für neue. (FSL 2, Z. 15-19)

Die Förderschullehrkräfte weisen darauf hin, dass es bislang keine spezifischen schulinternen Fortbildungsangebote zum Thema schulischer Integration gab (FSL 3, Z. 21; FSL 5, Z. 11-13), bzw. diese eine „Randerscheinung“ darstellen (FSL 4, Z. 17f). Eine Förderschullehrkraft berichtet von einem Pädagogischen Tag, an dem sich das Kollegium mit dem Thema „Förderplanung“ auseinandergesetzt hat:

Ich hatte halt so gehofft, dass man das Kollegium als Ganzes dazu bringt, dann auch an einem Pädagogischen Tag was zu machen. Das war jetzt hier so, dass die sich aufgeteilt haben. Also es gab Lehrer hier, die zum Beispiel Förderpläne entworfen haben hier für die Schule, die also, wo sie gesagt haben, die sind für uns praktikabel. Die anderen, wie die LENI-Hefte, die finden die einfach zu aufwändig, die sagen, das können wir nicht leisten für die vielen Kinder und die, also eine Gruppe hier, die beschäftigt sich damit und da bin ich dann auch immer wieder beteiligt. Also so in einzelnen Bereichen geht es voran, aber leider nicht als gesamtes Kollegium und die sind auch noch nicht wirklich so weit, dass sie sagen, gut, wir hätten jetzt gern mal einen Pädagogischen Tag, weil das sowieso auf uns zukommt. Ja, ich denke so steter Tropfen höhlt den Stein. (FSL 5, Z. 49-58)

Fortbildungen, die von einzelnen Lehrkräften besucht werden, finden insgesamt drei Mal Erwähnung, wobei in einem Fall kein direkter Bezug zum Thema schulische Integration hergestellt wird (SL 5, Z. 16) und in einem anderen Beispiel bemängelt wird, dass die Ideen der Fortbildungsveranstaltungen nicht zum schulischen Alltag passen (RSL 5, Z. 138-143). Positiv äußert sich eine Förderschullehrkraft:

[...] in der Gemeinschaftsschule da machen die schon seit 20 Jahren Integration, da ist es aufgenommen und es werden Fortbildungen besucht, aber von einzelnen Personen, die dann ihr Wissen wieder ins Kollegium reintragen. Also das ist wesentlich integrierter auch im Konzept. Es ist Teil des Konzepts der Schule. (FSL 4, Z. 19-22)

Zwei Schulleitungen berichten vom Besuch von Tagungen zum Thema Inklusion (SL 1, Z. 283; SL 3, Z. 17f). Eine Regelschullehrkraft verweist darauf, dass sie viel zum Thema liest, da ihre Ausbildung nicht ausreichend sei (RSL 1, Z. 226f).

Während der Besuch bzw. die Durchführung inklusionsspezifischer Fortbildungsangebote in den Interviews keine besondere Rolle spielen, wird der Fortbildungsbedarf der Lehrkräfte von allen Beteiligten als hoch eingeschätzt. Dabei sieht der Schulleiter eines Förderzentrums eine vorrangige Aufgabe darin, dass Fortbildungen den systemischen anstelle des einzelfallbezogenen Aspekts schulischer Integration bei den Kollegen verankern:

Die müssen auch lernen, dass man jetzt von dem wirklich einzelfallbezogenen Unterricht in die systemische Arbeit mit reingeht und dass man einfach wahrnimmt, dass aufgrund der zur Verfügung ste- 
henden Ressourcen man gar nicht, wie die Kollegen das so nennen, am Kind arbeiten kann, sondern ich muss tatsächlich wieder zu dem eigentlichen Gedanken der Integration zurückgehen, wie er ganz am Anfang stand. Da hieß es nämlich unsere Aufgabe ist es die Regelschule so zu unterstützen, dass sie mit den Kindern mit zusätzlichem Förderbedarf arbeiten kann. Und man hat das damals so in eine Art Nachhilfeunterricht, unterstützenden Unterricht umgemünzt und ich denke an diesen ursprünglichen Gedanken der Integration muss man zurückkehren. Und dort ist natürlich der Fortbildungs- und Ausbildungsbedarf immens hoch, da das ja im Bewusstsein der Regelschullehrerinnen und der Förderschullehrerinnen verankert sein muss. Dort muss das ankommen. Und ich denke da liegt an und für sich der Bedarf: einmal die Bereitschaft sich unterstützen zu lassen und diese Unterstützung anzunehmen und umzusetzen und auf der anderen Seite die Fähigkeit zu haben genau dieses Wissen als Förderschullehrerin systemisch weiterzuvermitteln und zu begleiten. (SLFZ 1, Z. 194-207)

Derweil zwei Regelschulleitungen offensichtlich einen als sehr hoch einzustufenden Fortbildungsbedarf für Inklusion sehen und dies folgendermaßen formulieren: „[...] wenn ich so das Wort Inklusion so in den Raum stelle, denke ich, da brauchen wir noch eine Zauberformel. Also da sind wir auch mit 25.000 Fragezeichen bestückt.“ (SL 3, Z. 92f) oder „[...] auf breiter Ebene muss da eine Weiterschulung erfolgen“ (SL 4, Z. 70), sieht SL 5 keinen speziellen Fortbildungsbedarf bzw. formuliert, dass man diesen bei den Regelschullehrkräften erfragen müsste (SL 5, Z. $93 \mathrm{f})$.

SLFZ 2 beschreibt wiederum den fachlichen Ausbildungsbedarf der Förderschullehrkräfte, die aufgrund ihres Studiums nicht in allen sonderpädagogischen Fachrichtungen ausgebildet sind:

Den Bereich emotionale Auffälligkeiten, Autismus, der einige Leute immer beschäftigt. (SLFZ2, Z. 180); Es gibt halt den Austausch im Grunde ganz allgemein, der Fachlichkeit angeht, weil immer irgendeiner irgendein Kind betreut mit Förderbedarf, was derjenige dann gerade nicht hat. Bei uns hat die absolute Mehrheit $S$ und $L$ und es gibt E-Leute, aber nur wenige, es gibt nur zwei mit $\mathrm{K}$ und ein paar, drei, vier mit G, so dass die dann immer gefragt sind, was man da tun kann. (SLFZ 2, Z. 180-184); [...] und natürlich fachdidaktische Sachen, wo die Leute denken, eigentlich bin ich ja Sprachheillehrer und es ist mal ganz schön, da mal noch etwas zu hören (SLFZ 2, Z. 187f)

Darüber hinaus thematisiert er den Fortbildungsbedarf hinsichtlich sonderpädagogischer Diagnostik:

Diagnostik ist immer auch ein Thema und es gibt leider immer noch Leute, die Gutachten schreiben, die den Namen nicht so ganz wert ist. Und das kann man, glaube ich, jedes Jahr, das ist so ein Kontinuum: Zeugnis, Differenzierungsbemerkung, Gutachten, was kommt darein, wie schreibe ich das - eine endlose Geschichte. (SLFZ 2, Z. 193-196)

Der sonderpädagogisch fachspezifische Fortbildungsbedarf wird auch von zwei Regelschullehrkräften (RSL 1, Z. 233f; RSL 5, Z. 104-106) sowie von zwei Regelschulschulleitungen gesehen:

[...] je nachdem besuchen, wir haben ja auch Autisten hier an der Schule, das Autismuszentrum oder Autismustagungen (SL 1, Z. 290f) Ich denke, dass die Lehrer sensibilisiert werden müssten für die Besonderheiten, also zum Beispiel, wenn ein Kind schlecht hört, hört es ja nicht einfach nur schlecht, sondern das hat Auswirkungen auf, und da bin ich eben auch nicht genug geschult, auf Konzentration, auf Arbeitstempo. (SL 2, Z. 194-197); Ganz kritisch sehe ich im Moment die Autisten, weil sie schlecht zu greifen sind und weil es da immer wieder zu Missverständnissen kommt, wo ich dann sag, ok, er hat jetzt dann seine Sachen nicht geschrieben, aber es ist keine Bösartigkeit euch gegenüber, sondern ein Erscheinungsbild dieser Krankheit und ich glaube an der Stelle, ja, was bedeutet das? (SL 2, Z. 200-204) 
Eine Förderschullehrkraft wünscht sich aus ihrer Perspektive dagegen mehr fachspezifisches Wissen in Unterrichtsfächern: „[...] also wenn ich jetzt zum Beispiel lernbehinderte Schüler in der achten und neunten Klasse habe und in Mathe" (FSL 1, Z. 166-168), eine weitere Inhalte zur Umsetzung schulischer Integration (FSL 3, S. 89f). Fortbildungswünsche beziehen sich darüber hinaus auf Bausteine der Unterrichtsentwicklung wie „Binnendifferenzierung“ oder „Unterrichtsmethoden“. Der Fortbildungsbedarf im Bereich Binnendifferenzierung wird dabei von einer Schulleitung sowie zwei Förderschullehrkräften anschaulich beschrieben:

Allerdings merke ich auch, das Problem, weil, ja, ich nenn es jetzt mal anders, heute nennt man es vielleicht Binnendifferenzierung, weil nicht jedes Kind, das gefördert wird, oder das extra behandelt werden muss oder auf das individuell Rücksicht genommen werden muss, hat gleich einen attestierten Förderbedarf, nicht? Also von daher ist das Problem etwas, das den Lehrern auch auf den Nägeln brennt, ja, besuchen sie Stück für Stück Fortbildungen, haben natürlich jetzt auch diese Fortbildung, was die neue Lernkultur angeht, so dass man binnendifferenziert wirklich in der Lage ist mehr zu arbeiten. (SL 1, Z. 38-45)

[...] im differenzierenden Unterricht, im offenen Unterricht. Ich denke da herrscht noch ganz viel Unkenntnis, Unwissen vor und deshalb sind da auch ganz viele Ängste, dass man einfach diesen Unterricht nicht bewältigen kann, dass er zu arbeitsintensiv ist (FSL 5, Z. 161-163)

[...] wie schaffe ich es zum Beispiel eine Werkstatt zu erstellen, die jetzt Regelschülern und geistig behinderten Schülern gerecht wird? (FSL 1, Z. 173-175)

Hinsichtlich des Erwerbs methodischer Kompetenzen werden in den Antworten neben methodischen Fragen allgemein (FSL 3, Z. 92) das selbstorganisierte Lernen (SL 1, Z. 45-47) sowie Kenntnisse zu Lerntechniken oder Methoden wie Lerntheke bzw. Wochenplan erwähnt:

[...] spezielle Lerntechniken. Ich habe gerade in Mathematik festgestellt, dass ich bei einigen Schülern

[...] nicht an die ran komme, weil ich nicht verstehe, was die Schüler nicht verstehen, wenn sie ganz schwach sind. Gerade wenn es um so einfache Grundrechenarten geht, da weiß ich irgendwann nicht mehr, was ich noch machen soll um es ihnen zu erklären. Und da sind doch, wo ich dann merke, wo ich neue Methoden entdecke, wie man das an die Leute ran bringt. Das wird ja in der normalen Lehrerausbildung nicht angesprochen. (RSL 2, Z. 101-106)

Wenn ich denen dann komme mit Lerntheke oder, na gut, Stationenarbeit kennen sie meistens noch, aber bei Lerntheke oder Wochenplan, haben die, ist das für die ein Buch mit sieben Siegeln, also dass wirklich einfach solche Methoden auch mal bekannt wären. (FSL 1, Z. 147-150)

Zwei Förderschullehrkräfte sehen Fortbildungsbedarf mit Blick auf die Öffnung von Unterricht:

[...] für die Schule gesehen einfach so diese Sachen, die in unserem Studium, sage ich jetzt mal, grundlegend sind. Also wirklich offene Unterrichtsformen (FSL 1, Z. 145f)

[...] für die Schule auf jeden Fall weiter an dem Thema: Wie öffne ich meinen Unterricht? (FSL 2, Z. 113f)

Zwei weitere Förderschullehrkräfte sehen das Problem von Fortbildungen in diesem Bereich eher darin, dass teilweise das Wissen für eine flexiblere Gestaltung von Unterricht vorhanden, die Umsetzung in der Praxis dann allerdings problematisch sei:

[...] wenn man mit Lehrern redet, die wissen sehr viel, die haben eigentlich auch viel gelernt, aber die Sachzwänge führen dazu, dass der Unterricht eben teilweise doch nicht so interessant oder so flexibel oder experimentierfreudig ist, wie er sein müsste. (FSL 4, Z. 152-154)

Letztendlich denke ich, dass man im Bereich der Unterrichtsumgestaltung mehr machen kann und ich da auch viel mehr lernen könnte, aber in der Praxis habe ich gar keine Zeit dafür. (FSL 4, Z. 145-147) 


\subsubsection{Ergebnisse im Bereich der Personalentwicklung}

Besonders intensiv thematisiert wird in den Interviews die Haltung der Lehrkräfte zur schulischen Integration. In den Aussagen zeigt sich eine sehr große Bandbreite von Auffassungen, die von umfassender Zustimmung bis zu erheblicher Skepsis reichen.

Erschwerend kommt aus Sicht der Leitung eines Förderzentrums hinzu, dass Regelschullehrkräfte wie Schulleitungen Schüler und Schülerinnen mit Förderbedarf zum Teil nicht als „ihre“ Schüler ansehen würden:

Nämlich leider, leider trifft man es noch sehr oft, dass die sagen, Kinder mit Förderbedarf sind Schüler des Förderzentrums und der Förderschule, nicht Regelschüler und ich denke dieser Gedanke ist also erschreckend, wie nach 25 Jahren Integration in den Köpfen der Lehrkräfte und auch der Schulleitung immer noch dieser Gedanke herumspukt, dass das gar nicht ihre Schüler sind. Das ist eins der größten Hemmnisse, die es gilt also möglichst rasch abzubauen. (SLFZ 1, Z. 145-149)

Auch eine Förderschullehrkraft thematisiert diesen Aspekt:

[...] Chance sehe ich einfach, dass sich die Kollegen auch mehr öffnen für dieses Thema, also dass sie vielleicht selber auch - also es heißt ja immer in der Inklusion sind es ja auch nicht mehr unsere Schüler, also Schüler der Förderschullehrer, sondern Schüler der Regelschule. Und diese Einstellung, selbst bei den extrem positiven Lehrern, ist also momentan noch nicht da. (FSL 1, Z. 207-210); dass über die Inklusion das mehr in den Blickpunkt rückt, dass die Kinder hier nicht zu Gast sind, sondern wirklich in die Klasse gehören und Kinder der Regelschullehrer sind. (FSL 1, Z. 213f)

Während SL 4 ihre eigene Haltung zum Ausdruck bringt: „Das kann eine wunderbare Sache sein, das kann ein Selbstläufer werden. Das kann für Kinder, die integrativ unterrichtet werden, ein Segen sein." (SL 4, Z. 108f), beschreibt SL 1 in ihrer Aussage die Selbstverständlichkeit und den Pragmatismus der Haltung der Lehrkräfte an ihrer Schule:

Wir sind eigentlich Stück für Stück in dieses Problem hineingewachsen mit dem großen Glück, dass wir, ich glaube mal einfach in der Zusammensetzung unserer Lehrerschaft Leute haben, die nicht groß fragen: „Belastet mich das mehr?"“ sich da jetzt: „Kommt das auch noch auf mich zu?“ oder, sondern dass man einfach sagt, das ist jetzt ein Problem, dem Kind muss geholfen werden und dazu muss ich kooperieren mit dem und dem, ich krieg ja Hilfe an die Hand und man macht es einfach. (SL 1, Z. 30-35)

Aus den Äußerungen der anderen Schulleitungen lassen sich eher Skepsis bzw. Ablehnung schulischer Integration gegenüber entnehmen. So beschreibt SL 2 die Haltung ihres Kollegiums knapp als „eher ablehnend“ (SL 2, Z. 211); SL 3 erläutert: „[...] wir sind ganz offen für solche Formen, aber wir erhoffen uns eine andere Struktur." (SL 3, Z. 103) und SL 5 bringt auf den Punkt: „[... ] ich glaube die wären alle froh, wenn die Förderkinder in die entsprechende Schule gehen dürften, in die Förderschule nämlich, denn es belastet nicht nur die Lehrkräfte, sondern auch die Klasse. Insofern sind sie glaube ich nicht so ganz glücklich mit dem Zustand, wie er ist." (SL 5, Z. 113-115)

Vorteile der schulischen Integration werden u.a. darin gesehen, dass die Wahrnehmung der Probleme der Schüler sich verändert:

Bei uns ist es so, dass seit 2008 die Anzahl der integrativen Schüler stark angestiegen ist. Ich denke, dann man den Blick auch auf in Anführungszeichen normale Kinder mit Schwächen eher hat und vielleicht auch früher erkennt, dass Schüler Probleme haben, die nicht nur aufgrund von, sagen wir mal, Faulheit zurückzuführen sind. Also ich denke, dass dieser Blick auf die schwachen Kinder etwas geschärft wird. (SL 3, Z. 12-16) 


\section{SL 1 bezieht sich auf das Menschenrecht und sagt dazu:}

Grundsätzlich denke ich, dass es ein richtiger Weg ist. Dass man einfach die Menschen nicht voneinander trennt, nicht mehr selektiert. Also, ich denke, dass das wirkliches Grundmenschenrecht ist, das es auch zu fördern gilt und das es auch von allen Seiten positive Seiten abgewinnen kann. (SL 1, Z. 374-376)

Schulleitung 2 stellt vor allem soziale Aspekte schulischer Integration in den Blickpunkt:

[...] ich finde sehr gut, wenn zum Beispiel körperbehinderte Schüler ganz selbstverständlich die Möglichkeit haben an der Regelschule unterrichtet zu werden (SL 2, Z. 239f); weil einfach alle davon profitieren und, ja, die Regelschulkinder den gemeinsamen Umgang erlernen und nicht einfach peinlich berührt irgendwie auf die Seite gucken, sondern das sind ganz normale, ganz normale Kinder, die irgendeine Beeinträchtigung haben und es ist ganz wichtig, dass man miteinander umgeht, lernt miteinander umzugehen. (SL 2, Z. 241-245)

Ich denke auch, dass Lehrer diesen Blick haben müssen. Es gibt nicht nur den Schülertyp XY, sondern ganz verschiedene Menschen mit unterschiedlichen Stärken und Schwächen und dass man sich auch einfach darauf einlassen muss, weil, ja, es handelt sich um Menschen, die man so behandeln muss, wie sie eben, wie sie eben sind. Und man muss sich mehr Gedanken darüber machen, über das einzelne Kind. (SL 2, Z. 245-249)

\section{Schulleiter 5 sieht dagegen keine Vorteile:}

Wenn ich jetzt Vorteile aufzählen müsste, müsste ich lügen. Da gibt es ja keine Vorteile, für uns ist es nur mehr Belastung und jeder versucht ja das Beste herauszuholen für die Kinder, was er machen kann. (SL 5, Z. 148-150)

Regel- wie Förderschullehrkräfte sprechen - von zwei Ausnahmen abgesehen - von einer überwiegend offenen Haltung der Lehrkräfte gegenüber schulischer Integration bzw. gegenüber Schülerinnen und Schülern mit sonderpädagogischem Förderbedarf. So äußern sich die Regelschullehrkräfte folgendermaßen: „[...] hier in der Schule ist es eigentlich so, dass die meisten offen sind für Integrationskinder“ (RSL 1, Z. 239f); „[...] an unserer Schule ist das sehr gut bewältigt worden bisher, aber es gibt auch ein, zwei Kollegen, die vielleicht hin und wieder negative Sachen auch mal sehen. (RSL 3, Z. 114f); „[...] sehr offen. Natürlich weiß man nicht, was einen erwartet, aber wir sind ein junges Kollegium, sehr motiviert und engagiert und ich denke, das ist, sind wir alle sehr offen." (RSL 4, Z. 93f) und „[...] ich glaube nicht, dass es eine Ablehnung von Inklusion gibt, bestimmt nicht. Generell nicht.“ (RSL 5, Z. 122f). FSL 1 bezeichnet die Haltung der Regelschullehrkräfte grundsätzlich als positiv (FSL 1, Z. 178), FSL 2 als „mit Sicherheit nicht negativ“ (FSL 2, Z. 121) und FSL 4 beschreibt ähnlich wie SL 2 eine pragmatisch-offene Haltung der Regelschullehrkräfte:

Also ich denke, dass alle Lehrkräfte, die hier arbeiten, das sowieso schon machen. Also sie sind immer in den Klassen auch für andere Kinder zuständig oder kriegen das alles mit. Sie sind oder wir sind alle offen (FSL 4, Z. 162-164)

Auch zwei Elternteile schätzen die Haltung der Kollegien in den Schulen zu schulischer Integration positiv ein:

Ich denke, dass sich das hier an der Schule weiterentwickelt. Also weil die Schule hier sehr offen ist und ich habe das Gefühl, dass das hier, ja, dass das, dass die da breit sind und engagiert sind. (E 1, Z. 188f)

[...] nachdem diese Schule sich Integration auf die Fahne geschrieben hat, gehe ich doch ganz stark davon aus, dass die Schule eine Vorreiterschaft eventuell gerne haben möchte in Sachen Integration, was sie auch schaffen kann (E 2, Z. 110-112); Ja, das sind die Voraussetzungen für Inklusion. Dass man bereit und engagiert ist und sagt, wir nehmen das Kind, so wie es ist, und wir gucken, was man besser machen kann und wo die Probleme liegen. Das ist hier an dieser Schule, ja, möglich, ja. (E 2, Z. 191-193) 
RSL 2 wie FSL 5 bringen zum Ausdruck, dass die Lehrkräfte eine kritische (RSL 2, Z. 110) bzw. durchwachsene (FSL 5, Z. 171) Einstellung gegenüber schulischer Integration zeigen.

Differenziert werden diese allgemeinen Aussagen dahingehend, dass „die Kolleginnen und Kollegen auch anderen schwächeren Schülern gegenüber offener werden, also, dass sie mehr merken, wo es auch bei den Regelschülern fehlt (FSL 1, Z. 9f), dass allerdings das Verständnis dahingehend, dass schulische Integration auch Veränderungen des Unterrichts erfordere, noch weitgehend fehle:

Ich habe die Kollegen als offen den Kindern gegenüber erlebt, aber jetzt weniger offen im Hinblick auf unterrichtsbezogene Veränderungen. Also schon, Sie haben die Kinder angenommen auch mit ihrem besonderen Förderbedarf, aber eben nicht darin den Unterricht entsprechend, ihren eigenen Unterricht entsprechend anzupassen. (FSL 1, Z. 14-17)

Ich hab noch von keinem Lehrer hier erlebt, dass er gesagt hat, was will der denn hier oder so. [...] Aber es fehlt halt wirklich dieser Gedanke, dass man, dass die Schüler hier positiv sich entfalten können, wirklich was ändern muss. (FSL 1, Z. 192-194)

[...] die Tendenz ist auf jeden Fall da, dass man sich der Integration weiter öffnet und auf jeden Fall auch, so ist mein Empfinden, für gemeinsamen Unterricht öffnen will, allerdings den Gegebenheiten oder an den Gegebenheiten noch einiges ändern muss, dass man sich so öffnen kann (FSL 2, Z. 132-134)

Positiver schätzen dies mit Blick auf die Zukunft die drei anderen Förderschullehrkräfte sowie die Leitung eines Förderzentrums ein:

Ich glaube, das sieht gut aus. Also ich bin von einer Fortbildung gekommen, habe dann noch mal gute Ideen, werde das jetzt demnächst mit neuen Kollegen, also mit einigen Kollegen besprechen, mit denen das einfach machbar ist und da einfach im neuen Schuljahr nochmal neue Sachen angehen. (FSL 3, Z. 107-110)

[...] ich sehe das grundsätzlich auch an den Grundschulen, vielleicht auch angeregt durch Rückmeldungen von Förderschullehrerinnen, was auch noch möglich wäre. Also eine Offenheit ist da, auf jeden Fall. (FSL 4, Z. 170-172)

[...] ich denke die Bereitschaft wächst, einfach auch, weil im Moment sehr viele Kinder kommen, viele Kinder hier sind, wo die Kollegen sehen, dass die zwar keinen sonderpädagogischen Förderbedarf haben, aber ganz große Probleme selbst haben oder im Unterricht Probleme aufwerfen, also gerade die jetzigen ersten Klassen hier sind wirklich, kann man schon sagen, Problemklassen. Da sind sehr viele Kinder mit, mit irgendeiner Art von Förderbedarf und insofern merken die Lehrer natürlich, dass sie an ihre Grenzen stoßen und so ganz langsam wächst eben diese Bereitschaft beziehungsweise einfach die Erkenntnis, dass irgendwas passieren muss. (FSL 5, Z. 181-188)

[...] intern selbst gab es eine konstante Weiterentwicklung, da wir das unwahrscheinliche Glück hatten, dass also alle Kolleginnen und Kollegen bei uns interessiert waren ihren Arbeitsplatz an für sich mitzugestalten, so dass ich denke, dass das ein kontinuierlicher Prozess ist, der schulintern bewältigt wurde oder angestoßen wurde beziehungsweise auch von externen Kräften begleitet wurde. Immer waren die Motivation hierzu, der Anstoß hierzu in der Regel immer aus dem Kollegium, aus dem Förderzentrum selbst. (SLFZ 1, Z. 24-29)

Zwei Förderschullehrkräfte sowie drei Regelschulschulleitungen äußern Verständnis bzw. formulieren Begründungen für eine mögliche ablehnende Haltung der Regelschullehrkräfte, die damit zusammenhängen könne, dass diese sich überfordert fühlen:

Also dass sich viele Kollegen nicht darauf einlassen. Das habe ich hier auch schon öfter gehört, dass sie auch sehr eingespannt sind mit den Nachmittagen und mit AGs oder solche Sachen und das ist dann einfach noch zu viel verlangt, dass wenn sie solche offenen Sachen machen sollen und die Arbeit dann. Das, denke ich, ist das größte Problem. (FSL 1, Z. 226-229)

[...] problematisch sehe ich auch einfach, dass zu viele Aufgaben an die Regelschullehrer weitergegeben werden, die einfach nicht dafür ausgebildet sind und sie einfach überfordert sind. Weil es sind nicht nur 
die Förderkinder, die da sind, da sind so viele auffällige Kinder, also von Wahrnehmungsgeschichten ist es einfach zu viel. Also ich denke mal, wenn es ein Tandem geben würde oder gerade Hauptfächer, wenn es da einfach noch eine zusätzliche Kraft geben würde, würde die Sache anders aussehen. Es ist einfach für die Regelschullehrer zu viel. Das können die nicht leisten. (FSL 3, Z. 119-125)

Auch die Schulleitungen äußern ausführlich und plastisch ihre Sorgen mit Blick auf eine mögliche Überforderung der Lehrkräfte aufgrund der Aufgabenfülle, die nicht nur auf den Bereich der schulischen Integration zurückzuführen ist:

Ja, im Moment geht das noch, aber ich merke natürlich auch, wir haben viele Konferenzen, im Sinne, ja, letztlich sind all diese Elterngespräche dafür da, dass es besser im Unterricht läuft. Nur, wissen Sie, da kamen jetzt die Fünfer und die Klassenlehrer haben irgendwann, nach dem zehnten oder elften Fragebogen, den sie von irgendeiner Stelle dann kriegen, den man auch zur Unterstützung dann ausfüllen muss. Ob das jetzt die Kinder- und Jugendpsychiatrie, ob das irgendein Therapeut ist, was auch immer. Da kommen Fragen, die auch ein Lehrer total überfordert ist zu beantworten. Aber all diese Dinge, man stellt sich gar nicht vor, was das Zeit schluckt. Und diese Zeit geht derzeit ab, indem man sich für einen schönen Unterricht richtig vorbereiten kann. Das muss man halt einfach auch so sehen. Irgendwann wird das grenzwertig und momentan, denke ich einfach mal oder aus meiner Sicht denke ich einfach mal, es geht noch. Sehr stark ansteigen kann das nicht mehr. Dafür haben wir einfach schon sehr viel. Da stimmt die Mischung dann endgültig nicht mehr. (SL 1, Z. 343-353)

Schulleitung 2 begründet ihre Sorge mit der steigenden Anzahl der Schülerinnen und Schüler mit sonderpädagogischem Förderbedarf in der Regelschule, vor allem der Kinder mit sozialemotionalem Förderbedarf:

Wir haben viele Integrationskinder, wir haben sie auch gerne, wir haben aber auch eine Grenze. Wir schulen auch schon um, das Wort Inklusion ist schon so etwas bedrohlich, auch für mich, ehrlich gesagt, weil ich mir eigentlich darüber im Klaren für mich persönlich bin, dass wir als Regelschule mit 700 Kindern, die auch zum Abitur führt, zum mittleren Bildungsabschluss, nicht jedem gerecht werden können, vor allem bei der Stundenzahl, die wir haben. Ganz große Angst haben wir vor den E-Fällen, da kann ein Kind den ganzen Unterricht und die halbe Schule schmeißen und da hilft unter Umständen ja auch nicht ein Integrationshelfer, wenn das Kind ausrastet. Kinder, die ganz schlecht gefördert sind von zu Hause, wenn die Elternhäuser nicht zugänglich sind, an die kommen wir nicht dran. Die Kinder gehen dann entweder einfach unter, im günstigsten Falle, oder stören noch den Unterricht der restlichen Kinder und das ist ein Problem. Also wenn wir trotz aller Versuche und Bemühungen nicht von der Stelle kommen und wenn wir bei einem Kind keine Persönlichkeitsentwicklung im positiven Sinne sehen, dann sehe ich darin auch ein riesengroßes Problem und es ist auch schwer den Kollegen die Ängste und die Vorurteile, da sind auch viele Vorurteile drin, zu nehmen. Manche sind aber berechtigt. (SL 2, Z. 212-221)

Auch SL 4 äußert sich mit Blick auf die Haltung des Kollegiums vorsichtig und stellt vor allem einen Zusammenhang zu den Ressourcen sowie zu unterschiedlichen Förderbedarfen her:

[...] ich schätze mal viele sind skeptisch, eben auch weil wir dazu nicht ausgebildet sind (Z. 80); weil die Förderschulzentren auch viele Schülermaßnahmen nicht ausreichend personalisieren können, weil der Punkt Integrationshelfer mit einem großen Fragezeichen versehen werden muss (Z. 80-82); dann kommt es ja auch auf das Kind selbst an, auf die Behinderung an, die das Kind mitbringt, also das muss man einfach, man kann es einfach nicht verallgemeinern, man muss es immer vom Kind abhängig machen. Bei manchen ist es überhaupt kein Problem und bei anderen, gut schwierig ist es halt bei Kindern, die Bedarf haben beim sozial-emotionalen Lernen, die können ja wirklich den ganzen Laden aufmischen, nicht? Also ich habe ja dann eigentlich auch die Pflicht, die anderen Kinder auch zu schützen. Ich kann ja nicht zulassen, dass da irgendwelche Verletzungen oder so was passieren. (Z. 85-91) 
Die Sorge hinsichtlich einer möglichen Zunahme verhaltensauffälliger Schüler äußern auch eine Regelschul- und eine Förderschullehrkraft:

Ich sehe auch in der Zunahme der E-Schüler ein großes Problem. Noch kann man lernschwache und körperbehinderte Schüler ausreichend fördern, aber bei Schülern, die Unterrichtsstörungen verursachen, ist es nicht mehr eigentlich abzufangen. (RSL 2, Z 114-118)

[...] mit Sicherheit mit Sorgen behaftet, also das geht uns hier ja auch so, weil man eigentlich denkt, die Entwicklung, so wie sie ist, die ist ja schon bedenklich. Also, wenn ich sehe, was wir immer im neuen Fünferjahrgang an Potenzial haben, dann frage ich mich, in welche Richtung sich unsere Gesellschaft entwickelt, dass wir dahin kommen, dass wir so viele Kinder mit Förderbedarf vor allem im emotional-sozialen Bereich haben. Man sieht das schon auch im Förderzentrum. Man sieht das schon mit Sorge, mit welchem Päckchen so mancher Grundschüler schon belastet ist, ja, auf jeden Fall. (FSL 2, Z. 123-129)

RSL 1 geht ebenfalls davon aus, dass eine zu hohe Anzahl an Schülerinnen und Schülern mit sonderpädagogischem Förderbedarf erreicht werden könnte und sieht darin eine mögliche Grenze schulischer Integration in der Regelschule:

Aber wenn auch mal verschiedene Grenzen erreicht sind, muss man das auch ganz strikt sehen und sagen: bis hierhin geht es und dann muss man auch teilweise die übrige Klasse [...] sehen[...] Man kann nicht einfach sagen, wir machen jetzt Integration und [...] die übrige Klasse fällt hinten runter. [...] Wenn dann ein gewisses Niveau erreicht ist, denke ich, muss man auch ehrlich sagen, also bis hierhin können wir es tragen, jetzt geht es nicht mehr. (RSL 1, Z. 240-245)

Dass die Regelschullehrkräfte mit individueller Förderung zum Teil Schwierigkeiten haben, begründet SLFZ 1 damit, dass diese eine andere unterrichtliche Ausrichtung hätten als die Förderschullehrkräfte ${ }^{55}$ :

Das ist einfach, sind die unterschiedlichen Sichtweisen. Man muss einfach sehen hier an der Förderschule und so ist es auch in der Integration zwangsläufig, arbeiten stark personenzentriert und auf den einzelnen Schüler mit seinen Bedarfen fixiert, während die Regelschulklasse, die unterliegt ganz bestimmten Regularien und das erste ist also der Lehrplan, das sind die Lehrplanaufgaben, die sie erfüllen müssen, so dass also selbst, wenn Regelschullehrerinnen und -lehrer das wollen, dass aufgrund der Rahmenbedingungen gar nicht in dieser gewünschten Form umsetzen können. (SLFZ 1, Z.73-78)

Die Schulleitung eines Förderzentrums begründet ihre skeptische Haltung mit übergeordneten Argumenten, die sich auf die Bildungspolitik und Diskurse über mehrere Jahrzehnte hinweg beziehen:

Ich würde mal sagen, wenn man seitens der Bildungspolitik in den bisherigen Kategorien denkt, denke ich wird das genauso scheitern von einem qualitativen Ergebnis, was man anstrebt, wie die Integration, wo ich sagen muss, leider Gottes qualitativ ebenfalls gescheitert ist. Sagen wir mal für mich ist auf jeden Fall erkennbar, da ich schon lange genug im Geschäft bin, dass ich die gleichen Argumente für die Inklusion höre, wie ich sie damals für die Integration gehört habe und ich hatte das Vergnügen die ganze Entwicklung zu begleiten über die ganzen Jahre und das zu sehen. Das ist ein Totschlagargument, weil man auch damals die ganzen Hintergründe dieser Entwicklung nicht wahrhaben wollte, ja? Und ich befürchte das gleiche für die Zukunft. Sagen wir mal, wenn, das war auch damals unser Ansatz hier in dem Schulversuch, habe ich gesagt dann muss sich auch in der Regelschule etwas ändern. [...] Das ist in den ganzen 20 Jahren, über 20 Jahren oder fast 20 Jahren jetzt mit den Förderzentren nichts passiert. Da hat sich nichts getan. Und es ist im Moment auch nicht erkennbar, dass sich hier im Regelschulbereich hier etwas tun soll. (SLFZ 1, Z. 274-286)

55 An dieser Stelle wird deutlich, dass Aussagen zu bestimmten inhaltlichen Fragestellungen nicht trennscharf zu den Haltungen von Lehrkräften eingeordnet werden können. 
Sie führt aus, dass für weitere Veränderungen zunächst eine Bestandsaufnahme erfolgen müsse, um dann zu prüfen, mit welchen Mitteln diese Veränderungen erreichbar seien (SLFZ 1, Z. 288-301). Aus den kritischen Begründungsansätzen für eine eher ablehnende Haltung heraus (Personalisierung, Klassenstärke, fehlende Möglichkeiten zur Individualisierung) äußern einzelne Befragte sich auch zur Frage nach dem geeigneten Förderort für die Schüler. Zwei Regelschullehrkräfte sprechen von Schülern, die in kleineren Gruppen besser lernen könnten (RSL 1, Z. 162f; RSL 3, Z. 75 f und Z. 80f), darunter RSL 1: „Die brauchen einfach diese kleineren Klassen wie an den Sonderschulen. Es ist so. Die brauchen dieses Engere, dieses Kleinere, und [...] mehr Förderung einfach.“ (RSL 1, Z. 162f). RSL 3 begründet ihre Auffassung dabei folgendermaßen:

Es kommt immer auf das Kind einfach auch an, also ob das möglich ist. Also, im Normal-, also in der Klasse integrativ zu fördern, es gibt Kinder, die können das gut, die können da arbeiten vielleicht mit ihrer Mappe, die sie da bekommen, es gibt aber auch Kinder, die schaffen das nicht. Und ich denke für diese, oder die schaffen es generell auch nicht die Aufmerksamkeit dann nur auf ihre Arbeit zu lenken und mit dem Thema, das sie dann machen, gerade wieder für den Fall des Kindes, das jetzt hier verblieben ist und da fände ich es durchaus sinnvoll die Förderschulen eben zu haben. Mehr Lehrkräfte und mehr Stunden in kleineren Gruppen zur Verfügung haben. Also ich sehe das ein bisschen zwie- gespalten auch. Ich bin generell dafür, dass man das versucht, aber wenn man sieht, aus irgendeinem Grund, es funktioniert einfach bei dem Kind nicht und dann, dass man dann sagt, sollte man umschulen. (RSL 3, Z. 152-161)

Sehr ähnlich führt eine Schulleitung dazu aus:

Also ich persönlich von meiner Erfahrung her, bin der Meinung, dass die Förderschulen extrem gute Arbeit leisten. Dass es nicht unbedingt jetzt ein einseitiges Inkludieren geben soll, dass kann, glaube ich, auf Dauer wird es wieder zurückgenommen werden, wenn es denn überhaupt kommt. Manche Kinder brauchen dann ganz kleine Gruppen, in denen sie leben können und arbeiten. Die sind in einer Gruppe von 25 total überfordert, weil sie den persönlichen Anspruch, also die Ansprache einer erwachsenen Person, brauchen um zu lernen. Das kann die Grundschule so nicht leisten. Da haben wir auch schon in zwei Fällen auf jeden Fall, ich erinnere mich, auch Kinder zur Förderschule schon mal zurückgegeben, wo wir gesagt haben, das ist für das Kind - nicht für uns, sondern für das Kind - nicht ab- nicht zuträglich. (SL 3, Z. 128-136); die Idee einer inklusiven Schule ist eine wunderbare Idee, von der Theorie her. Von der Praxis, wie sie im Moment sind, halte ich sie für kein Erfolgsmodell. (SL 3, Z. 138f)

Die Leitung eines Förderzentrums wiederum argumentiert, dass sich auch Förderschulen im Zuge der Entwicklung inklusiver Bildungsangebote weiterentwickeln müssen:

Dann kämen wir zur nächsten Frage: Förderschulen ja oder nein? Ich würde ganz kategorisch sagen: die Förderschulen, wie sie bisher bestehen, noch bestehen, die in Kategorien eingeteilt sind, nein. Ich denke, die haben keine Zukunft mehr, wenn ich jetzt an Inklusion und solche Dinge denke, sondern ich brauche dann eine Förderschule, die für alle Kinder definitiv offen ist, [...] die dann einfach multiprofessionell arbeitet, wo jeder Zugang hat, meinetwegen ein Kind mit Down-Syndrom genauso wie jemand mit gymnasialer Reife, Autisten und wie auch immer, dass die hier ihre persönliche Möglichkeit haben zu lernen, sich in unsere Gesellschaft, sich in unser gesellschaftliches Schulsystem einzufinden, vorbereitet werden, und eine solche Schule, denke ich, wäre auf Augenhöhe mit anderen und wir brauchen diesen ganzen Diskriminierungsblödsinn brauchen wir nicht mehr zu reden, weil nämlich dann das Anderssein normal wäre [...]. (SLFZ 1, Z.315-331)

\subsubsection{Darstellung der Ergebnisse zur Unterrichtsentwicklung}

\subsubsection{Ressourcen im Bereich der Unterrichtsentwicklung}

Der Klassenteiler wird von insgesamt 12 Personen in den Interviews thematisch aufgegriffen und zwar häufig in Verknüpfung mit dem Aspekt der Klassenzusammensetzung. Dabei weist 
eine SL darauf hin, dass in einer 5er-Klasse 29 Schüler sind, von denen fünf Kinder einen sonderpädagogischem Förderbedarf haben, darunter eines den spF geistige Entwicklung (SL 2, Z. 249-251). Lehrkräfte erfassen Zusammenhänge zwischen Klassengröße, Klassenzusammensetzung und differenziertem Arbeiten. Eine Regelschullehrkraft und eine Förderschullehrkraft erläutern, dass es in Klassen mit mehr als 20 bzw. mit 26 Schülern schwierig sei, „den Anforderungen des einzelnen Kindes mit sonderpädagogischem Förderbedarf [...] gerecht zu werden“ (RSL 5, Z. 43-35) bzw. gemeinsamen Unterricht umzusetzen, „weil man viel zu wenig auf die Kinder eingehen kann oder auf die achten kann" (FSL 5, Z 128-132). Eine Regelschullehrkraft präzisiert:

Wenn ich eine Klasse habe mit 28 Kindern, dann kann ich keine halbe Stunde für ein Kind in der Klasse aufbringen. Es geht manchmal. Das kriegt man mal organisiert, dass die anderen Kinder mal eine Stillarbeit machen oder eine Partnerarbeit, wo sie dann länger dran arbeiten. Aber ich kann das nicht täglich machen. Und es gibt Kinder, die brauchen das eigentlich. (RSL 1, Z. 163-167)

Eine Verringerung des Klassenteilers in den Regelschulen wird in den Interviews insgesamt acht Mal gefordert, sieben Mal wird die zunehmende Komplexität von (sonderpädagogischem) Förderbedarf in den Schulen thematisiert (mit Bezug auf verhaltensauffällige sowie schwer behinderte Schüler oder die Zunahme von Unterstützungsbedarf insgesamt).

FSL 5 stellt einen Zusammenhang zwischen den zur Verfügung stehenden Ressourcen budgetierter Förderschullehrerstunden sowie Klassengröße wie - zusammensetzung her und kritisiert, dass bei Klassengrößen von 25 oder 26 Kindern, darunter jeweils zwei bis drei Kinder mit spF „präventive Arbeit, wie wir sie ja eigentlich leisten wollen mit Klassenstufen übergreifenden Fördergruppen“, nicht mehr möglich sei (FSL 5, Z. 203f). Die Schulleitung einer Gesamtschule äußert ihre Ängste in diesem Zusammenhang folgendermaßen:

[...] es ist nur irgendwann die Frage: wo ist die Grenze, wann man das bei dieser Schülerzahl nicht mehr packt? Ja, dass man dem Ganzen nicht mehr gerecht wird und das ist die einzige Angst, die ich eigentlich habe, dass man einfach nicht mehr weiß, wann mach ich noch was? Wann habe ich für die noch Zeit? Weil es geht, wenn du den Kindern Zeit widmest. Und dazu brauchst du aber auch Ruhe und darfst nicht gestresst sein. Und das ist halt, irgendwo, dann wird das grenzwertig. Also selbst ein 25erKlassenschlüssel ist bei so vielen Integrationskindern, ist, wenn du dich jedem Kind wirklich widmen möchtest und willst da binnendifferenzierten Unterricht machen. Den machst du nicht, indem du das Buch aufschlägst und sagst, heute rechnen wir mal die Aufgabe, sondern da musst du dir ja vorher auch Gedanken machen. (SL 1, Z. 329-337)

Und FSL 4 präzisiert noch weitergehend:

Ansonsten ist es ja eine Bestätigung, dass es keinen Sinn macht und dass eben schwierige Schüler den Unterricht eher wählen und dann fragt keiner mehr: waren denn die Voraussetzungen auch da? Also so wird im Moment, finde ich, das ad Absurdum geführt und führt dazu, dass die nicht etwa sagen: Ja, wir haben kleine Klassen und Doppelbesetzung und so, wir können das und können auch schwierige Kinder aufnehmen und weiter fördern, sondern es führt dazu, ganz realistisch am Alltag gemessen: Jo, wir haben eben keine Stunden, die Klassen sind zu groß, wir wollen, dass bestimmte Schüler umgeschult werden. Also die Tendenz sehe ich ein bisschen, ist mir auch selbst schon im Alltag widerfahren, dass und da sehe ich eine große Gefahr. Also dass die Idee als gut propagiert wird und wenn die Mittel fehlen, geschieht eigentlich das Gegenteil. (Z. 190-199)

Eine Klassengröße von etwa 20 Schülerinnen und Schülern stellt für manche Befragten offensichtlich ein Maß dar, das im Rahmen schulischer Integration sinnvoll sein könnte. Eine För- 
derschullehrkraft verweist darauf, dass sie in ihrer Anfangszeit in einem anderen Bundesland Klassen unterrichtet hat, die zwischen 15 und 20 Schülern hatten („Das waren traumhafte Bedingungen." (RSL 2, Z. 138-140), während sie im Saarland Klassen mit einer Größe von 30 bis 33 Schülern vorfindet. Ein Elternteil schildert die Erfahrung, dass Sohn bzw. Tochter anfangs in einer Klasse mit 21 oder 22 Kindern war, aufgrund einer Klassenwiederholung dann allerdings in einer Klasse mit 29 Kindern unterrichtet wurde. Aufgrund der Lautstärke habe ihr Kind „dann auch irgendwann abgeschaltet und hat gar nichts mehr mitgekriegt. Das fand ich dann schon ziemlich heftig." (E 3, Z. 134-137). Eine Schulleitung stellt eine Verknüpfung zwischen der Klassengröße und den Räumlichkeiten her:

Die Zwanziger könnte ich mir vorstellen, dass das gut ist. Bei 26 ist eigentlich schon die normale Unterrichtung nicht einfach. Da ist der Raum zu klein. Da ist es schwierig Gruppen zu stellen. Wenn da noch eine Sitzecke, wie das bei uns so üblich ist, diese Erzählecken hat, dann ist auch ein sehr engagierter Lehrer irgendwann am Ende. (SL 3, Z. 121-124)

Von den Schulleitungen und Lehrkräften wird hinsichtlich der Klassenzusammensetzung vor allem auf die zunehmende Komplexität der Anforderungen Bezug genommen, die sich durch die Zunahme von Schülern mit sonderpädagogischem Förderbedarf bzw. Unterstützungsbedarf allgemein oder durch Schüler mit komplexen Problemen (schwere Behinderungen, Verhaltensauffälligkeiten) im Unterricht ergeben. Eine Schulleitung berichtet von einer 5er-Ganztagsklasse mit 28 Kindern, in der sechs oder sieben „Integrationskinder“, darunter ein Schüler mit Down-Syndrom, unterrichtet werden würden. Da in diesen Klassen auch „ADHSKinder“ oder Kinder mit anderen „Handicaps“ dazukämen, auf die man Rücksicht nehmen müsse (Bsp.: „schiefe Seelenlage“), könne die Mischung irgendwann nicht mehr stimmen (SL 1, Z. 118-123). Diese Komplexität wird auch von einer Regelschullehrkraft derselben Schule sehr differenziert beschrieben:

Wir als Lehrkräfte sind sowieso sehr flexibel, bis man an die Schmerzgrenze gelangt, aber [...] man muss auch die Klasse einfach sehen. Also ich sage mal so viele Kinder, die keine Integration haben und trotzdem Probleme haben, es nicht unbedingt, von Lernproblemen angefangen über Rechtschreibprobleme, und familiäre Probleme, das ist [...] ein ganz wichtiger Faktor, das kann man sich nicht vorstellen, dass da auch teilweise mal ein Unterricht dran hängt, wenn es mal einem Kind ganz, ganz schlecht geht, weil ja, ich finde auch die Kinder, ja, die haben sich unheimlich geändert. Ich sage mal, wenn ich jetzt an meine Schulzeit denke, ich wär nie zum Lehrer gegangen, wenn ich zu Hause Probleme gehabt hätte, und jetzt kommen auch Nicht-Integrationskinder und ja, dann tun sich jetzt manchmal Welten auf. (RSL 1, Z. 258-266)

Und eine Förderschullehrkraft, die ebenfalls davon ausgeht, dass es nicht mehr nur um „klassische“ Behinderungen wie eine „Lernbehinderung“ gehe, sondern zum Beispiel auch um Schüler mit Wahrnehmungsproblemen, fasst zusammen, dass sie immer „weniger Zeit für immer mehr Schüler [...]“ (FSL 3, Z. 6-9) habe. Die Annahme, dass die Schülerschaft aus Sicht der Schulleitungen und Förderschullehrkräfte in den Regelschulen insgesamt heterogener wird und dies ein Problem darstellen kann, wird mit Blick auf die Klassenzusammensetzung der Regelschulklassen (Anzahl der Kinder mit sonderpädagogischem Förderbedarf, Schwere der Beeinträchtigungen) auch von den Eltern geäußert (Verknüpfung Individualisierung etc.). Ein Elternteil verweist auf die Ressourcenproblematik und geht davon aus, dass integrativer Unterricht schwierig wird, wenn die Anzahl der Kinder mit spF in den Regelschulen steigt, ohne dass mehr Lehrkräfte zur Verfügung stehen. Sie hält einen zu hohen Anteil von Schülern mit spF in einer Klasse nicht für günstig, da diese dann nicht mehr die Gelegenheit hätten sich mit „,normalen" Schülern zu vergleichen (E 3, Z. 121-126). Ein anderer Elternteil geht auch deshalb 
von möglicherweise steigenden Zahlen von Kindern mit Behinderungen aus, da der medizinische Fortschritt dafür sorge, dass frühgeborene Kinder überleben könnten (E 2, Z. 76-79). Ein weiterer Elternteil hält die Beschulung in einer Regelschule für Kinder, die sehr schwere Behinderungen haben, für ungünstig und befürwortet für diese Kinder die Beschulung in einer Förderschule (E 1, Z. 224-226).

Die Ressourcenproblematik spielt auch bei Argumenten für die Bündelung von Schülern mit spF in einzelnen Klassen eine Rolle, die von der Schulleitung eines Förderzentrums und der Schulleitung einer Grundschule thematisiert wird. Während die Grundschulschulleitung darauf hinweist, dass eine Förderschullehrkraft sich bei Bündelung von bspw. drei Kindern in einer Grundschulklasse "gleichzeitig um mehrere kümmern kann“" (SL 5, Z. 98-100), führt SLFZ 1 aus:

Das heißt, wenn ich Kinder unterschiedlicher Behinderung in einer Klasse zusammenführe, sage ich nicht, ich mache eine Förderschule in der Regelschule auf. Das soll nicht der Fall sein, sondern es soll möglich sein, dann möglichst viele Stunden in diese Klassen hineinzubringen, dass man gemeinsam halt mit den Kindern arbeiten kann. Das ist also die Zielsetzung. Nämlich wenn meinetwegen eine Schule ist vier- oder fünfzügig, verteile die auf fünf Klassen und dann ist das organisatorisch von der Schule unheimlich schwer zu bewerkstelligen aufgrund der Ressourcensituation, die adäquat zu betreuen, also fasst man die zusammen, soweit es möglich ist, allerdings in Absprache mit den Eltern. (SLFZ 1, Z.261-268)

\subsubsection{Schulklasse als sozialer Raum}

Das Klassenklima wird in den Aussagen einer Regelschullehrkraft und in den Aussagen von vier Schülern vor allem mit Blick auf die soziale Akzeptanz beschrieben, so dass diese Beschreibungen redundant auch im Bereich der Ergebnisse dargestellt werden könnten. Die Regelschullehrkraft achtet in ihrem Unterricht darauf, „dass die Integrationsschüler es kaum merken, dass die Integrationskinder sind“ (RSL 1, Z. 132f), da sie davon ausgeht, dass man diesen keinen „Sonderstatus" geben darf (RSL 1, Z. 138-140). Die Schülerinnen und Schüler fühlen sich in ihren Klassen gut akzeptiert, auch dann, wenn sie beispielsweise zieldifferent arbeiten. So weist S 2 darauf hin, dass alle anderen Schüler wissen, dass er/sie ein „Integrationskind“ ist und sowohl er/sie selbst als auch die Mitschüler gut damit umgehen können (S 2, Z. 40-42). S 4 erläutert, dass er/sie Mitschüler lediglich bei Hausaufgaben oder der Verwendung von Schulbüchern oft darauf hinweisen müsse, dass er/sie „nicht mitmache“ (S 4, Z. 135f), wofür diese Verständnis zeigen. $S 3$ fasst zusammen:

Also gefallen tut mir, dass, äh, meine Klasse mich so akzeptiert wie ich bin, also dass ich nicht ausgeschlossen werde. Dass ich so akzeptiert werde, wie ich bin, und das ich nicht gemobbt werde. Das gefällt mir eigentlich am besten. (S 3, Z. 2-4)

Dass sie sich in ihrer Klasse wohl fühlen, hänge auch damit zusammen, dass sie Freunde haben (S 2, Z. 14-16; S 4, Z. 2f). Drei Interviewpartner, eine Schulleitung und zwei Regelschullehrkräfte, erwähnen, dass das soziale Lernen in Unterrichtsprozessen positiv beeinflusst werde. Während RSL 4 Chancen darin sieht, dass Schüler „in beide Richtungen“voneinander lernen können (RSL 4, Z. 115f) sieht FSL 1 (oder RSL 1) eine Chance darin, „dass das soziale Miteinander gestärkt wird, [...], dass leistungsstärkere Kinder [...] nicht nur vom sozialen Verhalten her, sondern auch so vom Lernverhalten her den andern Kindern was weitergeben können (RSL 1, Z. 278-281)“. Nach SL 2 wirkt es sich auf das Wohlbefinden der Schülerinnen und Schüler mit spF aus, dass die Kinder mit Gleichaltrigen zusammenarbeiten und in Fächern, in denen sie keine Schwierigkeiten haben, von den anderen lernen können (SL 2, Z. 48-50). Ansonsten wird 
das soziale Lernen nicht explizit als Qualitätsmerkmal gemeinsamen Unterrichts beschrieben, sondern als Ergebnisaspekt der Lernentwicklung thematisiert (s. folgender Abschnitt).

Die Schüler wurden auch danach gefragt, inwiefern sie auch am Nachmittag soziale Kontakte zu Mitschülern haben. Schüler 1 verweist darauf, dass dies manchmal wegfalle, weil daheim viel zu tun sei (S 1, Z. 7), während Schüler 3 dies darauf zurückführt, dass er nicht so sehr auf andere zugehe:

Kaum. Also eigentlich gar nicht. Einen habe ich, das ist der XY, den sehe ich aber auch nur ab und zu. (S 3, Z. 10f); Das kann stark daran liegen, das fällt mir auch selbst auf, dass ich nicht auf einen zugehe, das heißt ich ziehe mich eher zurück. Und ich bin auch eher so der Einzelgänger. Ja, daran denke ich. (S 3, Z. 13f)

Schüler 4 und 5 sprechen davon, dass diese nur selten stattfinden (S 4, Z. 6f; S 5, Z. 11, 13, 15).

\subsubsection{Klassenführung}

Bis auf eine Regelschullehrkraft äußern sich zu diesem Bereich ausschließlich die befragten Schüler ${ }^{56}$. Die Lehrkraft merkt an, dass der Unterricht schwieriger sei, „weil es oft laut ist“ (RSL 4, Z. 43). Zwei Schüler antworten auf die Frage, ob es in ihren Klassen Regeln gebe mit ja (S 1, Z. 105, S 2, Z. 120-122). Eine Schülerin führt dazu anschaulich aus:

Also so etwas wie Regeln das wurde schon versucht. Das ist komplett in die Hose gegangen. Dann also bei den Klassenlehrern ist das gut gegangen, aber in den Nebenfächern war die Klasse manchmal furchtbar. (S 3, Z. 120-122)

[...] mit den Murmelbechern in unserer Klasse wird es auch immer besser, weil, wenn XY sagt „Murmelzeit“, dann sind alle ganz leise um Murmeln zu bekommen, weil, wenn man Murmeln hat, bekommt man was aus der Schatzkiste und deswegen ist unsere Tischgruppe im Moment sehr leise. Ich kann mich dann gut konzentrieren und wenn wir doch laut sind, dann sage ich immer, dass sie leise sein sollen. (S 3, Z. 89-93)

Danach gefragt, inwiefern es in Verbindung mit den Regeln auch Konsequenzen gebe, antworten drei Schüler mit ja (S1, Z. 109; S 2, Z. 126-128; S 4, Z. 127f). Zwei Schüler beschreiben ergänzend, „dass manche Lehrer entweder nicht konsequent genug waren oder dass der Lehrer einfach etwas unterrichtet hat und das den Kindern nicht gepasst hat" (S 2, Z. 126-128) bzw. dass eine Änderung der Sitzordnung nötig wäre, „weil sich keiner richtig konzentriert" (S 4, Z. 127f). Einer der Schüler greift in diesem Zusammenhang offensichtlich auf längere Erfahrung zurück, wenn er plastisch darstellt:

Genau, also die Lehrer sollen halt darauf achten, dass es in der Klasse ruhig ist. Bei manchen Lehrer ist es halt gut, und so, nur halt bei, sage ich einmal bei Referendaren oder halt bei neuen Lehrern, wo man halt noch nicht so kennt und so, wo von einer anderen Schule gekommen sind, die können da halt noch nicht so richtig durchgreifen, sage ich jetzt mal so, nicht? Aber das kommt dann halt mit den Jahren [...]. (S 2, Z. 133-137)

Auf die Frage, was im Unterricht anders sein müsse, damit sie besser lernen können, antworten alle befragten Schüler übereinstimmend, dass es in den Klassen leiser sein müsse, damit sie sich besser auf die Arbeit konzentrieren können. $S 1$ ist es in der Klasse zu laut (S 1, Z. 112) und er erklärt: „wenn es im Raum ganz ruhig ist, dann kann ich viel mehr denken“ (S 1, Z. 101). S 2 sagt, dass man gar nicht lernen könne, da es „wirklich echt zu laut“ (S 2, Z. 125f) sei und S 3 formuliert übereinstimmend: „Weil an gewissen Stellen ist es mir einfach zu laut und da kann

56 Die Schüler wurden zu dieser Thematik als einzige gezielt befragt. Als Qualitätsmerkmal gemeinsamen Unterrichts wird die Klassenführung von den Lehrkräften bzw. Schulleitungen nicht aufgeführt. 
ich mich nicht voll konzentrieren." (S 3, Z. 114f). S 4 führt aus, dass eine der Tischgruppen in der Klasse meistens laut sei, so dass er/sie sich daher nicht konzentrieren könne (S 4, Z. 110f) und verdeutlicht: „Und das habe ich doch nicht so gerne, wenn Kinder so quatschen, weil, egal wie ich versuche mich anzustrengen, mich auf meine Aufgaben zu konzentrieren in der Klasse, packe ich manchmal nicht." (S 4, Z. 123-127). S 5 stört die Lautstärke in seiner/ihrer Klasse, da er/sie dann nicht so viel hören könne (S 5, Z. 116).

\subsubsection{Didaktik}

Zur Didaktik werden zwei Aussagen getätigt, die die Umsetzung eines spezifischen Rechtschreibkonzepts an einer Schule in Kooperation zwischen Förder- und Regelschullehrkräften betreffen. Dazu führt die Regelschullehrkraft (RSL 5, Z. 164-166) aus, dass sie das „Silben Lernen" für gut halte und die Förderschullehrkraft erklärt:

Wir haben das in der Klasse 2, in der Stufe 2 eigentlich, geschafft, dass wir so ein neues Rechtschreibkonzept eingeführt haben. Da machen jetzt alle mit. Also es gibt jetzt auch nur lauttreue Diktate, die es vorher nur für die Förderkinder gab, die gibt es jetzt für die ganze Klassenstufe. Wir haben Elternabende gemacht zu diesem Thema, wie der Rechtschreibunterricht hier jetzt funktioniert und da haben sich zum Beispiel jetzt alle aus der zweiten Klasse angeschlossen. Jetzt hoffe ich, dass das dann halt hochwächst. Die Drittklässler haben sich beim Diktat angeschlossen, noch nicht unbedingt beim Rechtschreibunterricht. Beim Diktat haben die jetzt, gehen die jetzt auch ein bisschen in die Richtung. (FSL 5, Z. 36-45)

Ansonsten werden didaktische Aspekte nicht als Qualitätsmerkmal gemeinsamen Unterrichts aufgegriffen. In keinem der Interviews wird Bezug auf den Aspekt der klaren Unterrichtsstruktur genommen.

\subsubsection{Methodik}

Sehr viel ausführlicher als zur Didaktik äußern sich die Interviewpartner zur Methodik des gemeinsamen Unterrichts. Eine Lehrkraft erläutert dazu, dass sie inzwischen im Unterricht eher situativ vorgehe:

Ja, ich hab' also vorher habe ich immer mein festes Konzept gehabt. Also schrittweise, wo ich dann sage, das mache ich dann, dann und dann. Jetzt habe ich ein Thema, ich überleg mir, so will ich es machen und meistens wird es dann ganz anders, weil es einfach, ja, man guckt von der Situation her wie es passt. Ich habe dann zuhause meistens drei vier Möglichkeiten, wie ich es dann machen könnte und es ist dann so eine Mischung zwischen allem. (RSL 1, Z. 107-111)

Eine weitere Lehrkraft macht den gemeinsamen Unterricht nicht an Lernformen fest, weil jede Lernform im gemeinsamen Unterricht ihre Berechtigung habe (RSL 3, Z. 60-62). Die Methode des Frontalunterrichts wird von einer Schulleitung, einer Regel- und einer Förderschullehrkraft sowie drei Schülern thematisiert. Die Schulleitung geht davon aus, dass diese Unterrichtsform anspruchsvoll und daher mit Blick auf gemeinsamen Unterricht schwierig sei:

[...] so dass dieser darbringende Unterricht oder wo der Lehrer halt im Frontalunterricht vorne steht, dass da muss einer schon eine wirklich starke Lehrerpersönlichkeit sein um da lückenlos bei den Kindern anzukommen, dass die sich nicht abmelden und da braucht man noch gar nicht mit Förderkindern anzufangen, die sich vielleicht gar nicht erreicht fühlen und die vielleicht gar nicht rankommen an das, was der Lehrer vielleicht sagt. (SL 1, Z. 146-151)

Die Lehrkräfte sprechen an, dass diese Form den gemeinsamen Unterricht erschwere, da die Schüler mit spF dabei „untergehen“ würden (FSL 3, Z. 47f) oder die individuelle Förderung während frontaler Phasen als störend empfunden werde: 
[...] größtenteils wird nur Frontalunterricht gemacht und wenn ich dann natürlich hinten mit irgendwelchen Montessori-Materialien rumklappere oder wenn ich nicht da bin, der Integrationslehrer dann macht, das stört natürlich dann vorne. Also da, wie gesagt, vielleicht kommt über die neue Lernkultur da ein bisschen Bewegung in die Sache. (RSL 1, Z. 200-204)

Auf die Frage, bei welcher Unterrichtsform sie besonders gut lernen können, äußern sich drei der befragten Schüler dahingehend, dass sie diese Unterrichtsform am ehesten beim Lernen unterstützt. S 1 findet es besser „wenn der Lehrer vorne steht (S 1, Z. 65), weil er alleine ,anfange falsch zu lernen“ (S 1, Z. 67). Nur wenn die Lehrkräfte ihm sagen würden, was er falsch gemacht habe, könne er dies merken und es richtig machen (S 1, Z.67-69). S 2 weist darauf hin, dass er/sie zwar bei manchen Lehrern noch ein zweites Mal nachfragen müsse, diese ihm/ihr das dann aber auch so erklären würden, dass er/sie es verstehen könne (S 2, Z. 100f). S 3 begründet seine Auffassung, dass er/sie bei Frontalunterricht am besten lernen könne (S 3, Z. 91) folgendermaßen:

Also wenn Tafelbilder angeschrieben werden, der Lehrer was erklärt, das finde ich eigentlich am allerbesten für mich. Da habe ich das Tafelbild, kann das auswendig lernen und dann fällt mir das eigentlich ganz leicht und die Lehrer hier auf der Schule, die erklären gut und deswegen finde ich den Frontalunterricht eigentlich am besten. (S 3, Z. 91-94)

Offene Lernformen werden von Schulleitungen, Regel- wie Förderschullehrkräften als Qualitätsmerkmal gemeinsamen Unterrichts charakterisiert. Da die Schüler im Zusammenhang mit offenen Lernformen selbständiger arbeiten und sich untereinander helfen würden, sei es im gemeinsamen Unterricht dadurch einfacher sich mit einzelnen Schülern zu befassen (RSL 1, Z. 56f; RSL 2, Z. 48f). Als offene Lernformen, die im gemeinsamen Unterricht praktiziert werden, werden konkret benannt: Projekt und Portfolios (SLFZ 2, Z. 87f), Stationen (RSL 2, Z. 105; RSL 5, Z. 46), Lerntheke (RSL 2, Z. 105; SL 1, Z. 157), Wochenpläne (SL 2, Z. 103; RSL 2, Z. 106; RSL 3, Z. 46; E 3, Z. 46) bzw. Tagespläne (RSL 2, Z. 106; RSL 5, Z. 46). Dabei bemerkt eine Regelschullehrkraft, dass für sie eine Mischform zwischen frontalem Unterricht und offenen Lernformen wichtig sei (RSL 2, Z. 54-57). Die offene Form der Lerntheke wird von einer Regelschulschulleitung folgendermaßen beschrieben:

[...] Lerntheke aufgebaut mit Aufgaben, die einzuüben waren, und da gab es leichte und mittlere und schwere Aufgaben und das Down-Kind hatte ein Extrablatt zum selben Arbeitsgebiet, allerdings Aufgaben für das eigene Niveau und die lernbehinderten Kinder hatten auch ein Extrablatt. Ja, da wusste jedes Kind, jedes Kind arbeitet auf einem anderen Niveau. Welches Niveau interessiert die untereinander nicht, jeder das, mit dem er klarkommt und der Lehrer ist dann halt moderierend und Hilfe stellend, jedenfalls in der Stunde war es dann meine Aufgabe, bin ich dann durchgegangen um zu gucken, dass jeder punktuell einen Schritt weiterkommt. (SL 1, Z. 157-164)

Zwei Regelschullehrkräfte arbeiten mit Wochenplänen (RSL 2, Z. 46-49; RSL 3, Z. 51). RSL 2 begründet die Arbeit mit Wochenplänen damit, dass sie sich durch diese Unterrichtsform gezielter mit einzelnen Kindern befassen könne:

Das hat sich für mich als sehr praktisch gezeigt, weil wenn die Kinder auch am Arbeiten sind, kann ich mich immer mal wieder zu einem Kind setzen und mit ihm gezielt arbeiten. Weil viele sehr selbständig auch arbeiten und sehr gut klarkommen und dann kann ich wirklich schauen, wo hakt's, wo ist ein Problem und nochmal mit den einzelnen erfahren. (RSL 2, Z. 46-49)

Ein Elternteil bewertet die Wochenplanarbeit als gut, da die Schüler sich selbst einteilen können, wann sie welche Aufgaben machen (E 3, Z. 108-110). 
Kritisch mit Blick auf die konkrete Umsetzung offener Unterrichtsformen im gemeinsamen Unterricht äußern sich eine Schulleitung, eine Regel- und eine Förderschullehrkraft. Die Schulleitung geht davon aus, dass es nur einzelne Regelschullehrkräfte sind, „die sich gut darauf einlassen“, die Unterrichtsmaterialien für die Förderschullehrkräfte frühzeitig bereithalten und mit Wochenplänen arbeiten. Ansonsten sei dies eher eine „Wunschvorstellung“" (SL 2, Z. 103-105). RSL 5 beschreibt „kleine Veränderungen“ dahingehend, dass inzwischen auch mit Tagesplänen, Lernzirkeln oder Stationenarbeit zu Sachthemen gearbeitet werden würde. Dies seien allerdings Veränderungen, die nur sehr langsam stattfinden würden (RSL 5, S. 45-47). FSL 1 kritisiert die Regelschullehrkräfte:

Das kommt ihnen einfach nicht in den Sinn, dass es hier mal eine offene Methode sein müsste oder nicht nur zehn mal zehn, sondern das offener gestalten, auch mal Material zur Verfügung stellen (FSL 1, Z. 194-196); [...] weil vielleicht einige Kollegen auch nicht unbedingt bereit sind sich auf diese offenen Methoden einzulassen, weil sie zumindest anfangs dadurch mehr Arbeit haben, also zum Beispiel so eine Werkstatt vorzubereiten, das ist natürlich gerade, wenn man diese zum ersten Mal vorbereitet sehr viel Bastel-, Schnipsel- und was weiß ich für Arbeit. (FSL 1, Z. 219-222)

Partner- und Gruppenarbeit werden als Methoden von einer Regelschullehrkraft erwähnt (RSL 2, Z. 105). Von den Schülern wird der Einsatz verschiedener Sozialformen differenziert bewertet. Vier der fünf Schüler geben an, dass sie Einzelarbeit bevorzugen (S 2, Z. $94 \mathrm{f}$; S 3, Z. 98; S 4, Z. 88; S 5, Z. 84). Schüler 3 begründet dies folgendermaßen: „Aber am liebsten arbeite ich alleine, da kann ich machen, worauf ich fixiert bin, was ich in meinem Kopf habe und kann es niederschreiben." (S 3, Z. 98-100), während Schüler 4 anführt, dass er sich dann besser konzentrieren könne, da die anderen Schüler nicht so laut seien (S 4, Z. 88). Zwei Schüler merken an, dass die Bevorzugung der Einzelarbeit fächerabhängig sei (S 3, Z. 92; S 2, Z. 92-94) und S 2 erklärt dies damit, dass er/sie in manchen Fächern nicht gerne alleine arbeite, weil er/ sie nicht wisse, was zu tun sei (S 2, Z.92-94), während er/sie in anderen Fächern Ruhe zum Arbeiten brauche (S 2, Z. 94f).

Auch mit Partnerarbeit kommen die Schüler nach eigener Auffassung zurecht (S 1, Z. 72; S 2, Z. 88-90 und Z. 108; S 3, Z. 98; S 5, Z. 89 und Z. 92). Schüler 1 begründet dies damit, dass eine Partnerin in Ruhe erklären könne, wie etwas funktioniert (S 1, Z. 75f). Schüler 2 verweist darauf, dass Partnerarbeit besser klappe als Gruppenarbeit (S 2, Z. 88-90). Die Sozialform Gruppenarbeit wird von einem Schüler positiv bewertet, da dort auch mal diskutiert werden könne (S 3, Z. 100f), von zwei Schülern allerdings eher kritisch gesehen:

Weil in einer Gruppe dann sprechen die ganz viel durcheinander und manche haben etwas anderes und die anderen haben auch etwas anderes und dann streiten wir, welche richtig ist. (S 1, Z.74f)

Wenn er jetzt halt sagt eine Gruppe, fällt es mir halt schon manchmal schwer, nämlich weil die einen sagen doch was anderes, weil wie halt andere und dann passt dem das nicht und dann sind wir uns halt alle unterschiedlich und können uns dann halt nicht auf ein Thema einigen. (S 2, Z. 86-88)

Eine Regelschullehrkraft führt Gruppenarbeiten in leistungsheterogenen Kleingruppen durch, „wo ich dann halt leistungsstärkere Kinder in die Gruppe setze und die ziehen dann halt die Leistungsschwächeren schon mit. Es funktioniert aber nicht immer." (RSL 1, Z. 80-82). FSL 5 nimmt Partner- oder Gruppenarbeit in leistungsgemischten Zusammensetzungen positiv wahr:

[...] wenn eben Gruppen- oder Partneraufgaben gestellt werden, an denen die Kinder dann auch teilnehmen können, weil das unsere Erfahrung ist, dass das auch nicht schlimm ist, wenn die Kinder nicht das gleiche machen, sondern die Kinder finden in der Gruppe mittlerweile im zweiten Schuljahr selbständig Aufgaben auch für das andere Kind. Die sagen dann von sich aus, ach ja, gut, du kannst noch 
nicht so gut lesen, aber du kannst schon abschreiben. Möchtest du jetzt schreiben? Oder du kannst besonders gut malen, dann darfst du auf dem Plakat malen. Und wenn die so offen gestellt sind die Aufgaben, dann finde ich das eigentlich sehr positiv im gemeinsamen Unterricht (FSL 5, Z. 122-128)

\subsubsection{Differenzierung und Individualisierung}

Obwohl sich nur eine Interviewfrage explizit auf die organisatorische Umsetzung der Differenzierung bezieht, stellt der Qualitätsbereich der Differenzierung und Individualisierung mit Abstand denjenigen dar, der quantitativ und qualitativ am umfangreichsten beschrieben wird, und zwar in Form von Antworten auf die Fragen nach den Veränderungen, die in Schule bzw. Unterricht aufgrund der integrativen Unterrichtung wahrgenommen werden bzw. nach den Qualitätsmerkmalen integrativen Unterrichts. Zwei Schulleitungen und drei Regelschullehrkräfte bezeichnen das binnendifferenzierte Arbeiten im gemeinsamen Unterricht als Notwendigkeit (SL 1, Z. 145 f und Z. 151f; SL 2, Z. 105-109 und Z. 121-126; RSL 1, Z. 10-13; RSL 3, Z. 43f; RSL 5, Z. 50-52). RSL 1 beschreibt dies so, dass „früher [...] eher für ein Mittelmaß unterrichtet“ wurde, während man jetzt versuche „weiterzugehen“" (RSL 1, Z. 10-13). RSL 3 und RSL 5 erläutern, dass sie selbst mehr differenzieren müssen und zwar sowohl "qualitativ als auch quantitativ" (RSL 3, Z.43f), nicht nur aufgrund der "Integrationsschüler“, sondern aufgrund dessen, dass sich das „Spektrum“ insgesamt verändert habe (RSL 5, Z. 50-52). Besonders ausführlich äußert sich SL 2 zu den notwendigen Veränderungen Richtung Differenzierung und Individualisierung:

Ich denke, der Unterricht muss sich so verändern, dass er individualisierter ist, dass man mit Stationen arbeitet, mit Wochenplänen arbeitet. Das ist bei uns an der Schule durchaus auch der Fall. Es kommt auf die Kollegen an. Manche jüngere Kollegen machen das von sich aus und das hat auch nichts eigentlich mit Integration zu tun. Wir haben ja insgesamt sehr viele Schüler mit unterschiedlichen Kompetenzen, Fähigkeiten. (SL 2, Z. 105-109) [...] es wäre notwendig, dass für alle Kinder Unterrichtsangebote vorhanden sind und diese auch im Großen und Ganzen im selben Raum zu bewerkstelligen wären und dass sowohl die Schwachen ihren Anteil haben können als auch die Mittelstarken und die Starken und auch miteinander sozial profitieren könnten, aber eben auch auf der Lernebene, weil stärkere Schüler ja durchaus auch den schwächeren helfen können und selbst dann Kompetenzerwerb eben erhalten könnten. (SL 2, Z. 121-126)

RSL 4 bezieht in ihren Gedanken den Aspekt mit ein, dass es bei individuell angepassten Lerngruppen auch darum gehe, dass tatsächlich alle Kinder so gut wie nur möglich gefördert werden würden, Kinder mit spF wie die anderen Kinder der Klasse auch (RSL 4, Z. 49-53). Auch FSL 5 legt Wert darauf, dass der Unterricht auf die Teilhabe aller Schüler ausgerichtet ist:

[...] der Unterricht sollte eben Anteile enthalten für alle Kinder, dass die wirklich auch zusammenarbeiten können, dass die Kinder auch nicht das Gefühl haben, sie sind immer auch ausgegrenzt und sollte halt trotzdem Anforderungen haben für alle Kinder. Das gelingt eigentlich mit solchen Stationen relativ gut, wenn sie mit Partnern arbeiten. (FSL 5, Z. 115-118)

Eine Förderschullehrkraft erkennt an, dass einige Regelschullehrkräfte versuchen differenzierte Lernangebote zu gestalten:

Und manche, denen kann man das sagen und die machen das auch, was weiß ich, ihre Sprache, deutlicher sprechen und deutlicher schreiben und langsamer schreiben und verschiedene Anforderungsniveaus auch anbieten oder auch Texte auswählen, das habe ich jetzt auch mitgekriegt. Einfach Lektüren angepasst halt nicht so an das Alter, sondern ans Niveau und dass, die Kinder sind oft nicht mehr gewöhnt zu lesen, dass die dann halt kürzere Texte wählen und so weiter. So, sagen wir, gerade in der Gesamtschule würde ich sagen, gucken die schon sehr genau, versuchen die schon alle mitzunehmen. Das geht, man passt sich an. (FSL 4, Z. 61-67) 
Differenziertes Arbeiten kann sich - nach Auffassung von SL 5-positiv auf den gesamten Unterricht auswirken:

[...] manches vielleicht dadurch bedingt, dass Integrationskinder dabei sind, Dinge auch vertiefender dargestellt werden und in Gänsefüßchen vielleicht auch manchmal vereinfacht noch, so dass auch die etwas schwächeren, nicht Integrationskinder, die Schwächeren manches doch besser verstehen, dadurch, dass man sich extrem viel Mühe gibt und das auf ein anderes Niveau noch mal bringt. Und da hat der ein oder andere, der normal schon schwach ist, Vorteil davon. (SL 5, Z. 47-51)

Eine Schulleitung und eine Förderschullehrkraft befassen sich inhaltlich mit der Selbstverständlichkeit differenzierter Lernangebote. SL 1 spricht in diesem Zusammenhang davon, dass die Schülerinnen und Schüler selbst keine Probleme damit haben an unterschiedlichen Aufgaben zu arbeiten:

[...] also von daher irgendwie auch für die Kinder ist das nicht wirklich ein Problem. Die wissen halt, jeder arbeitet an seinem Aufgabenspektrum. Und wie hoch und wie tief das ist, das interessiert die Kinder nicht. Die sagen auch nicht: „Ach Gott, hat der leichte Aufgaben!“ Das interessiert die nicht. (SL 1, Z. 165-168)

Einen Zusammenhang zwischen offenen Unterrichtsformen und der Selbstverständlichkeit von Differenzierung stellt FSL 1 her:

[...] dass der Unterricht so weit geöffnet wird, dass eben nicht auffällt, wenn meine Schüler dann eben das andere Buch oder Arbeitsblatt rausholen, weil es irgendwann hoffentlich mal normal geworden ist, dass jeder mal was anderes vor sich hat. (FSL 1, Z.215-217)

Einen weiteren Zusammenhang sehen die Leitungen der Förder- und Beratungszentren zwischen Differenzierung und selbstorganisiertem Lernen. Während SLFZ 2 betont, dass geprüft werden müsse ,in welchen Bereichen entweder selbst organisiert lernen oder modulorientiertes Lernen, inwiefern das möglich ist“" (SLFZ 2, Z. 85-87), nimmt SLFZ 1 eine systemorientierte Perspektive ein:

[...] das ist eine systemische Arbeit, die man hier aufbauen will, wirklich dann die Regelschullehrerin unterstützt mit diesen Kindern zu arbeiten. Das eine Projekt, das ich eingangs erwähnte, ist also ein landesweites Projekt zum selbstorientierten Lernen und da sieht man das, selbst wenn dann die Arbeit differenzierend wird, sie ist also Kind bezogen, dann sieht man, dass es dort möglich ist, so ein Hinweis, in welche Richtung das gehen muss, aber das sind nur einzelne Klassen oder eine Schule, die sich auf den Weg machen. (SLFZ 1, Z. 251-256)

Dass der Arbeitsaufwand im Zusammenhang mit Differenzierung zunehme, beschreiben drei Regelschullehrkräfte. RSL 2 sieht, dass die Unterrichtsvorbereitung schwieriger werde und mehr Arbeit verursache (RSL 2, Z. 43) und fügt erläuternd hinzu:

Also ich muss mir viel mehr Gedanken machen, wie ich die ganze Bandbreite abdecke. Ich habe jetzt in meiner Klasse vom L-Schüler bis zu potenziellen Abiturienten gehabt und das ist schon sehr schwer, wenn man sich sehr Gedanken machen muss, wie man die alle abdeckt. Die einzelnen Schüler müssen mit Themen schon weiter sein, während andere Schüler besondere Hilfe brauchen. Also hier ist es sehr schwer vorzubereiten. (RSL 2, Z. 44-48)

RSL 3 hält den Aufwand für enorm hoch, da eine starke qualitative wie quantitative Differenzierung notwendig sei (RSL 3, Z. 43f). Auch sie erläutert: 
[...] die Schere bei mir in der Klasse zum Beispiel ist sehr weit offen. Ich habe, ich würde sagen, zwei mindestens hoch begabte Kinder drin oder sehr gut begabte Kinder. Das ist schon nicht immer so zu händeln, wie man das möchte, aber ich schaue schon, dass ich das machen kann. (RSL 3, Z. 37-39)

Ähnlich beschreibt dies auch RSL 5, die davon ausgeht, dass „in jeder Klasse auch Kinder sind, die Extrafördermaßnahmen brauchen, auch wenn sie keinen sonderpädagogischen Förderbedarf haben. (RSL 5, Z. 54f) [...] Auch einfach schwächere Schüler vielleicht." (RSL 5, Z. 60). Ihre Erläuterung ähnelt den vorangegangenen:

[...] der Schwierigkeitsgrad steigt für die Lehrerinnen natürlich enorm. Das ist ja klar, weil die Heterogenität nimmt ja zu durch solche Schüler und das erhöht dann doch noch mal das Anforderungsprofil an die Lehrkraft. Also sie muss dann noch mehr individualisieren und noch mehr differenzieren, als das bisher schon der Fall war. (SL 1, Z. 24-27)

Problematisch werde der Vorbereitungsaufwand laut einer FSL dann, wenn bei der Konzeption des Unterrichts Aufgabenstellungen in bis zu vier verschiedenen Fassungen zu gestalten seien. Das würde dazu führen, dass viele Kollegen sich nicht auf binnendifferenzierten Unterricht einlassen (FSL 1, Z. 222-226). Auch die Schulleitung einer Regelschule nimmt Bezug auf den erhöhten Vorbereitungsaufwand, ergänzt allerdings, dass binnendifferenzierte Unterrichtsformen den Unterricht selbst stressfreier machen würden (SL 1, Z. 326-340).

Eltern, Schüler und eine Regelschullehrkraft weisen darauf hin, dass im Unterricht teilweise mit differenzierten Materialien gearbeitet wird. Der Elternteil eines Kindes mit dem spF G berichtet von differenziertem Material in Mathematik, Deutsch und naturwissenschaftlichen Fächern (E 1, Z. 114-117 und 119-121), E 3 von leichteren Lernangeboten (E 3, Z. 46-48) und davon, dass ihr Kind die Möglichkeit erhält langsamer zu arbeiten (E 3, Z. 46). Die Schüler nennen eigene Bücher und Arbeitshefte bzw. Aufgaben (S 2, Z. 54f; S 4. Z. 29; S 5, Z. 44 und Z. 46), eine Regelschullehrkraft spricht von einfacheren und wenigeren Aufgaben (RSL 5, Z. 22f).

Mit Blick auf eine differenzierte Leistungsbewertung nennt FSL 1 Absprachen mit den Regelschullehrkräften bei der Vorbereitung sowie Bewertung und Notenfindung zu Klassenarbeiten von Schülern mit spF (FSL 1, Z. 35-38) und E 3 erklärt, dass bspw. Textaufgaben bei Mathearbeiten „dann vielleicht einfacher gehalten oder ganz weggelassen, durch andere Aufgaben ersetzt“ (E 3, Z. 54f) werden. Als problematisch empfindet FSL 4 den Leistungsdruck, mit dem die Grundschulen konfrontiert seien:

Also gerade in den Grundschulen, der Leistungsdruck und dann mit G8 und diesen Vergleichsarbeiten, der ist sehr hoch. Die Eltern sind im Nacken. Die machen dann schon Vorschläge, was sie inhaltlich wollen, habe ich gerade von einer Grundschullehrerin wieder erfahren, also das eine geht gar nicht und sie soll doch lieber was anderes unterrichten. Also ich denke die Zwänge, in denen die Lehrer heute sind, bei den großen Klassen und immer mehr Kindern mit Förderbedarf, die ersticken oft Weiterentwicklung. (FSL 4, Z. 172-179)

Die Schulleitung eines Förderzentrums und eine Förderschullehrkraft bemängeln, dass die rechtlichen Voraussetzungen für eine differenzierte Leistungsbewertung noch nicht ausreichend seien:

Die rechtlichen Voraussetzungen, ich erinnere mal an Zeugnis- Versetzungsordnung, Notengebung, Vergleichbarkeit der Notengebung, all diese Dinge sind nicht möglich. Insofern ist der Rahmen äußerst begrenzt, dass man wirklich integrativ arbeiten kann. Wir haben das natürlich an einzelnen Schulen, nicht viele, ich würde mal sagen vier, fünf, vielleicht vier, fünf Klassen, wo es machbar ist, wo die Lehrer einfach den Mut haben sich darauf einzulassen, aber die Mehrzahl der Kolleginnen und Kollegen der Regelschule ist nicht in der Lage das zu leisten, weil die Voraussetzungen nicht gegeben sind. (SLFZ 1, Z. 78-85) 
[...] wobei man auch dazu [gemeint ist das binnendifferenzierte Arbeiten] sagen muss, dass das auch ein Problem der momentanen Notengebung ist. Im Moment haben die halt ein Problem, weil die sagen, ja, wie bewerte ich das dann anschließend? Ich muss ja irgendwie eine Bewertung abgeben, wenn ich das Kind bei dir im Förderunterricht die Klassenarbeiten mitschreiben lasse. Dann habe ich ja keine Regelnoten und das lässt im Moment die Gesetzgebung ja eigentlich noch gar nicht zu. Also wenn das wegfallen würde, dann wären durchaus viel mehr Kollegen bereit auch Differenzierung zu machen. Im Moment ist immer noch die Angst, dass sie Probleme bekommen, weil das Kind dann nicht das gleiche gemacht hat im Unterricht. Das ist schon noch ein großes Problem. (FSL 5, Z. 100-110)

Auch die Gewährung von Nachteilsausgleichen bspw. für Kinder mit einer Körperbeeinträchtigung kann nach Einschätzung einer Regelschullehrkraft zu Problemen führen, da andere Kinder „sich dann ungerecht behandelt fühlen, weil jetzt das Integrationskind [...] mehr Zeit hat [...] in der Klassenarbeit" (RSL 2, Z. 148-151), wobei dies mittlerweile akzeptiert werde. Ein Elternteil ist für die Möglichkeit der Gewährung eines Nachteilsausgleichs dankbar:

Bei unserem Kind war es zum Beispiel ganz von Nöten, dass die Fremdsprache abgesetzt wurde. XY hat nämlich Wortfindungsstörungen, jetzt einfach als Beispiel mal gesagt. Es war nicht so einfach, aber wurde auch hier vom Personal sehr gut eingeschätzt. Denn wenn jemand in Deutsch schon Wortfindungsstörungen hat, wie soll er eine Fremdsprache lernen? Das war ein Weg, den ist die Schule mit uns auch wirklich mitgegangen, dass das durchgehen konnte, denn das hätte XY nicht geschafft. (E 2, Z. 41-46)

Neben SL 2 (s.o.) sprechen der Schulleiter eines Förderzentrums sowie zwei Förderschullehrkräfte die Anforderung der Individualisierung an (SLFZ 2, Z. 85; FSL 2, Z. 60 und 62f; FSL 4, Z. 69-76). FSL 2 glaubt, dass dies die Grundlage für gemeinsamen Unterricht sei (FSL 2, Z. 62f) und deshalb „groß geschrieben“ werden solle (FSL 2, Z. 60). Und SL 2 schildert die aktuelle Situation mit Blick auf Individualisierung an ihrer Schule wie folgt:

[...] dass wir nicht da sind, wo wir sein sollen, wobei ich auch ehrlich sagen muss, ich habe auch Integrationsschüler in einer Klasse, zwei L-Kinder, bei einem kombiniert mit E, ein S-Kind, es fällt einem unheimlich schwer im Unterrichtsgeschehen vornedran immer die Unterrichtsmaterialien vollständig beim Förderschullehrer zu haben und dass der Förderschullehrer die Möglichkeit hat das runterzubrechen in allen Fächern wirklich abzusprechen, aber eigentlich müssten wir dahin kommen. Nur da langen die Stundenzahlen nicht aus. (SL 2, Z. 73-79); das ist ein zeitliches Problem, weil die Beratungsstunden nicht da sind. Es ist ein zeitliches Problem, weil ein Lehrer mit 27 Stunden im Unterricht drin ist und vielleicht dann noch ein zwei Vertretungsstunden ab und zu hat oder ein Elterngespräch oder wie auch immer und wirklich dann nur von Stunde a zu Stunde b hetzt und ganz viel Wissen auch vermitteln muss. (SL 2, Z. 81-84)

Ein Elternteil nimmt positiv Bezug auf die Umsetzung der individualisierten Förderung, da er davon ausgeht, dass sein Kind im Unterricht „nach seinem Niveau“ gefördert werden (E 1, Z. 60f). Die qualitative Umsetzung eines individualisierten Lernangebots stellt eine Förderschullehrkraft mit Bezug auf einen autistischen Schüler sehr anschaulich dar:

[...] bei dem autistischen Kind [...] Wir haben Klassenarbeit geschrieben, Brief, und ich hab dann so'n rechteckiges Kästchen aufgemalt (ja) und das war der Briefumschlag und das konnt' er nicht akzeptieren. Da muss man dann schon flexibel sein [...] (RSL 1, Z. 54-57); Und dann bin ich irgendwann hin, hab 'nen Briefumschlag aufs Blatt geklebt und dann konnt er das beschriften. (RSL 1, Z. 60f); In Biologie $[\ldots]$ haben wir jetzt vor den Ferien Test geschrieben und er war total blockiert, konnte es einfach im Klassenverband an diesem Tag nicht schreiben und dann [...] bin ich mit ihm raus, die Integrationslehrerin blieb dann bei der Klasse. Ich bin mit ihm raus, hab' ihn mündlich geprüft und dann war's in Ordnung. (RSL 1, Z. 61-64) 
FSL 4 beschreibt zusammenfassend eine mögliche Idealsituation individualisierten Unterrichts:

[...] im Prinzip wäre das im Idealfall jedem Kind gerecht zu werden, egal auf welchem Niveau es sich befindet und entsprechende Angebote zu machen auch, je nach Grad der Selbständigkeit des Kindes. Dass Kinder halt wirklich lernen selber zu arbeiten, sich Sachen zu erarbeiten, wenn möglich, und, ja, alles im Blick zu haben. Hilfe anzunehmen, abzugeben, inhaltliche, also Werke auszusuchen und zu ergänzen an Schulbüchern und Materialien, die passen. Auch Sachen anzunehmen, dass Sachen halt anschaulicher dargestellt werden müssen, gerade bei Einführungen. Das ist je nach Lehrer nicht so selbstverständlich. Ja, flexibles Reagieren auf die Klasse, die vor einem sitzt. (FSL 4, Z. 69-76)

Mögliche Grenzen der Differenzierung bzw. Individualisierung werden allerdings ebenfalls ausführlich thematisiert. Ähnlich wie unter dem Qualitätsbaustein Ressourcen (Klassenteiler und -zusammensetzung) heißt es auch hier, dass es die Größe der Klasse sei, die diese Arbeitsform erschwere:

Es wird probiert, wobei das Klientel und vor allem die Klassengröße es schwierig machen diese Unterrichtsformen wirklich konsequent durchzusetzen. Also, in Fächern, in denen es möglich ist, wird es schon teilweise eingesetzt, aber bei 30 Schülern in der Klasse ist es einfach schwer so differenziert zu planen, zu unterrichten und vor allem diese Korrekturarbeit auch zu leisten, ob auch jeder von seiner individuellen Arbeit profitiert. Das bringt ja nichts, wenn sie individuell arbeiten, aber profitieren nicht davon. (FSL 2, Z. 52-57)

Schulleitung 2 hält es für schwierig, bei „so vielen Kindern dann aber den Blick für den einzelnen zu behalten und zu überlegen: was braucht dieses Kind jetzt in meiner Stunde? In einer Stunde von 30 in der Woche" (SL 2, Z. 255-257), wodurch manche Lehrkräfte Überforderung empfinden würden, bspw. wenn es darum gehe vom Bildungsgang Lernen über den Bildungsgang Hauptschule bis zum Bildungsgang Gymnasium alles anzubieten (SL 2, Z.111-115). RSL 4 fügt an, dass Individualisierung in einer Klasse dann problematisch werde, wenn die Leistungsschere zu weit auseinandergehe (RSL 4, Z. 112-115). Eine weitere Schulleitung merkt an, dass gemeinsamer Unterricht für Kinder mit spF „eine totale Überforderung sein [kann], weil wir ihnen nicht gerecht werden können." (SL 4, Z. 109), was allerdings vom einzelnen Kind abhänge (SL 4, Z. 112). Einen anderen Aspekt bringt RSL 5 ins Spiel, die davon berichtet, dass auch stärkere Schüler im integrativen Unterricht zu kurz kommen könnten:

Das Problem sehe ich da, auch wenn man sagt starke Schüler können alleine arbeiten, sind eindeutig die, die zu kurz kommen. Die kriegen dann was, was anspruchsvoller ist und ein Mädchen hat mal zu mir gesagt: Kommst du auch mal zu mir? Und das ist, so soll es ja auch nicht sein. (RSL 5, Z. 172-175); Die fallen dann hinten raus. Gut, die brauchen diese Hilfen nicht so, aber sie möchten diese Hilfe vielleicht auch und eigentlich möchte ich gerne auch mit denen mal was machen. Nur, wenn die Klassen so groß bleiben, dann werden es immer die sein, die zu kurz kommen. (RSL 5, Z. 178-182)

Bei der Bewertung spielen zum Teil auch die unterschiedlichen Förderbedarfe eine Rolle. Während die Unterrichtung von Schülern mit Körperbeeinträchtigungen von einer FSL positiv eingeschätzt wird, da diese ihrer Meinung nach an der Schule sehr gut integriert seien (FSL 2, Z. 130f), sieht die Auffassung zur Integration von Schülern mit dem sonderpädagogischen Förderbedarf soziale und emotionale Entwicklung anders aus, wie eine RSL und eine Schulleitung ausführen:

Probleme sehe ich vor allem bei den E-Schülern, also bei Klassengrößen von 30 Kindern nicht zu schaffen ist noch mehr schwierige Schüler zu integrieren, wenn man dann als Lehrer alleine davorsteht. (RSL 2, Z. 132-134) 
Wenn eine Integration nicht gelingt, ist es in der Regel nicht die Integration L, sondern es ist die EIntegration. Da kann dir auch ein Schüler den ganzen Klassenverband sprengen. (Z 203f); Die anderen Kinder waren es satt, den Namen des Schülers zu hören, dass man versucht hat ihn zurechtzuweisen, die Förderlehrerin war überfordert, das ging gar nicht, ja, und dann war man irgendwann froh, als wir umgeschult hatten, weil das konnte man der Klasse nicht mehr zumuten, weil an die anderen muss man auch denken. (SL 1, Z.215-218)

Mit Sorge sieht eine Schulleitung auch die Zunahme der Anzahl von Kindern mit Down-Syndrom in ihrer Schule:

Ich kann nicht noch 20, Down-Kindern sage ich jetzt mal, dieselbe Fürsorge bieten, wie wir jetzt den Dreien bieten können. Ich weiß, dass im nächsten Jahr mindestens noch ein zwei kommen und dann wird das alles irgendwann, die anderen bleiben ja auch, die sollen ja weiterhin betreut werden. Das wird nicht weniger. (SL 1, Z. 366-369)

Eine Schulleitung nimmt in ihrer Aussage mehrere Förderschwerpunkte in den Fokus:

[...] grundsätzlich bin ich da, bin ich da schon offen (SL 5, Z. 95); Wir könnten zum Beispiel von heute auf morgen hier ein körperbehindertes Kind integrativ unterrichten, wäre überhaupt kein Problem. Wir haben die Möglichkeiten, wir würden umziehen, wir würden alles umstellen. Das wäre gar kein Thema. Das geht in anderen Bereichen auch so. (SL 5, Z. 97-100); Im L-Bereich sehe ich auch wenige Schwierigkeiten. Schwierig wird es halt, wenn spezielle Behinderungen vorliegen, eine starke Hörbehinderung oder Sehbehinderung, wo man einfach technisches Know-How auch braucht, das haben wir einfach nicht. Und, wie gesagt dann habe ich halt ein Riesenproblem im Bereich E. Das weiß ich nicht, wie sich das entwickelt und wie wir dann bestimmte Kinder hier integrieren sollen. Das ist so das größte Problem, das ich habe. (SL 5, Z. 100-105)

Mit Blick auf die organisatorische Umsetzung differenzierter Lernangebote in den Regelschulen ${ }^{57}$ wird von einer großen Bandbreite berichtet. Eine Schulleitung der Förderzentren geht davon aus, dass es „von Förderraum 1:1 bis immer überall“ (SLFZ 2, Z. 17) alles gebe und dies „stark schulabhängig“" sei (SLFZ 2, Z. 43). Dies bestätigen auch die Schulleitungen der Regelschulen sowie die Förderschullehrkräfte. So vertritt Schulleitung 1 die Auffassung: „[...] das hängt ja ganz stark wieder von dem Individuum ab, um das es geht (SL 1, Z. 60); Schulleitung 3 beschreibt die organisatorische Umsetzung als „sehr wechselhaft“ (SL 3, Z. 27); Schulleitung 5 sagt: „Das ist ganz unterschiedlich. Das kommt darauf an, was gerade das Problem ist." (SL 5, Z 26) und Schulleitung 4 geht davon aus, dass die differenzierte Förderung sowohl inner- als auch außerhalb des Klassenraums erfolge (SL 4,Z. 19-21). Drei Förderschullehrkräfte bestätigen die große Bandbreite (FSL 1, Z. 27; FSL 4, Z. 26; FSL 5, Z. 19) und begründen die Vielfalt mit dem Förderbedarf der Kinder (FSL 4, Z. 26f) oder mit der jeweiligen Klasse (FSL 5, Z. 19f) bzw. mit dem Unterricht in dieser Klasse (FSL 5, Z. 25). Das gemeinsame (zielgleiche oder zieldifferente) Arbeiten in der Klasse stellt dabei insgesamt - auch aufgrund der Ressourcensituation - quantitativ einen hohen Anteil dar.

Die Schüler bleiben meistens in der Klasse drin, geht ja gar nicht anders. (RSL 5, Z. 17f); machen auch teilweise ja mit. Also es ist ja nicht so, dass sie in allen Bereichen nicht am Regelunterricht teilnehmen können. Und da gucken wir dann schon, dass sie bei Einführungssachen und so weiter immer mit dabei sind (RSL 5, Z. 20-22)

57 Angebote im Zusammenhang mit äußerer Differenzierung sind im Kategoriensystem unter dem Bereich Organisationsentwicklung aufgeführt. Dort passen sie aber nur dann hin, wenn die äußere Differenzierung bspw. für einen ganzen Jahrgang oder eine ganze Schule organisiert ist (bspw. in Form von jahrgangsübergreifendem Förderunterricht o.ä.). Im Zusammenhang hier ist sie Folge unterrichtsbezogener und teilweise situativer Entscheidungen einzelner Lehrkräfte. 
Zwei Schulleitungen verweisen darauf, dass die Förderlehrer - abgesehen von individuellen Fördersituationen außerhalb des Klassenraums - ,auch im Unterrichtsgeschehen drin“ (SL 3, Z. 30) seien und dies bedarfsabhängig sei (SL 3, Z. 30) bzw. versucht werde „das Kind im Verband, im Klassenverband zu belassen“ (SL 5, Z. 32f). Positiv sieht es auch der Leiter eines Förderzentrums: „An den Grundschulen gibt es viele Leute im gemeinsamen Unterricht.“ (SLFZ 2, Z. 47). Eine Regelschullehrerin achtet gemeinsam mit der Förderschullehrkraft darauf, dass eine Schülerin „möglichst viele Stunden im Klassenverband mit dabei“" sein kann (RSL 1, Z. 43-46), eine weitere gibt an, dass bedarfsorientiert entschieden wird (RSL 3, Z. 19f) und zwei Elternteile berichten, dass ihr Kind nur wenige Stunden in Einzelförderung unterrichtet werde und alle anderen Stunden in der Klasse verbringe (E 1, Z. 82; E 2, Z. 18-20): „Hier wird sehr darauf geachtet, was möglich ist: schafft er es im Klassenverband, kann er im Klassenverband mit dabei sein?“ (E 2, Z. 18-20).

Die Lehrkräfte erläutern, dass die individuelle Förderung innerhalb der Klasse vor allem bei Schülerinnen und Schülern üblich sei, die zielgleich unterrichtet werden (Bsp.: Schülerin mit dem spF Sprache, RSL 3, Z. 31-33). Für diese seien lediglich punktuell Einzelfördersituationen notwendig (FSL 3, Z. 28-30). Darüber hinaus gebe es Kinder, bei denen eine individuelle Förderung außerhalb der Klasse bei manchen Themen nicht notwendig sei bzw. über Wochenplanarbeit gut organisiert werden könne. FSL 1 geht diesbezüglich so vor, dass sie für Schüler mit den spF Lernen oder Sprache Aufgaben situativ im gemeinsamen Unterricht umgestalte, damit diese soweit wie möglich am selben Stoff mitarbeiten können (FSL 1, Z. 29-31). SL 1 erklärt, dass es Kinder gebe, bei denen die Unterstützung innerhalb der Klasse aufgrund des Austauschs zwischen Förder- und Fachlehrkraft möglich sei: „das reicht dann aus, dass über ein gewisses Stoffgebiet ein Schüler überhaupt keinen Extrabedarf hat, sondern die Unterstützung in der Klasse, also wirklich integriert in der Klasse stattfinden kann." (SL 1, Z. 60-65). FSL 2 berichtet, dass viele Schüler mit spF Lernen in den Grundkursen (der Gemeinschaftsschule) unterrichtet werden. Dort sei es möglich, dass Unterrichtsinhalte so reduziert werden, „dass es für den Schüler ok ist“. Die Umsetzung erfolge über Wochenpläne, da nicht genügend Zeit für Einzelförderung vorhanden sei (FSL 2, Z. 26-29).

Auch drei Schüler äußern sich zur Förderung innerhalb bzw. außerhalb der Klasse dahingehend, dass diese sich abwechsle (S 1, Z. 30f; S 2; Z. 47f; S 4, Z. 46), je nachdem, ob die Mitarbeit in der Klasse möglich sei (S 1, Z. 30f) oder auch je nach Unterrichtsfach (S 4, Z. 58f). S3 wird zielgleich unterrichtet und erhält Unterstützung durch eine Integrationshilfe, die ihm bspw. beim Zeichnen hilft, da er kein räumliches Sehvermögen hat (S 3, Z. 53-55), ansonsten legt er Wert darauf, dass er genauso behandelt wird wie die anderen Schüler auch:

Eigentlich nicht und das finde ich auch gut so, weil ich bin behindert, gut, ok, aber ich will, dass man mich so akzeptiert so wie ich bin und nicht sagt, ah, der ist behindert und der kriegt jetzt die und die Vorteile. (S 3, Z. 50-52)

Die Schulleitungen der Förderzentren sowie die Schulleitung einer Regelschule gehen davon aus, dass individuelle Förderung außerhalb der Klasse häufig umgesetzt werde. SLFZ 1 denkt, dass Einzelförderung den „Löwenanteil“ in den Regelschulen darstellt, da dies so gewünscht werde (SLFZ 1, Z. 69), wogegen die Förderung innerhalb der Klasse zu einem prozentualen Anteil umgesetzt werde, „den man fast vernachlässigen kann“ (SLFZ 1, Z. 70f). SLFZ 2 geht von vielen „individuellen Maßnahmen“ aus, da viele Förderschullehrkräfte gerne im „Einzelbezug“ arbeiten (SLFZ 2, Z. 13f), was vielleicht mit „besser strukturierten Nachhilfe" vergleichbar sei (SLFZ 2, Z. 47f). Auch SL 2 spricht davon, dass „Einzelförderung überwiegt“ (SL 2, Z. 62), wobei ihre Wunschvorstellung anders aussieht: 
Ich denke, dass die Einzelförderung, also die Förderung im Klassenverband orientiert an den Lernzielen der Klasse, dass die eigentlich stärker sein müsste, weise auch darauf immer wieder hin, weise darauf hin, dass sich die Förderschullehrer mit den Fachlehrern absprechen müssen und umgekehrt und man eigentlich die Inhalte des Unterrichts runterbrechen muss soweit es geht auf die Bedürfnisse der Schüler. Also ich spreche jetzt von den L-Kindern, bei den K-Kindern ist es ja noch einmal, bei den zieldifferenten eine andere Sache. (SL 2, Z. 68-73)

SL 5 nimmt dagegen an, dass es sich bei der Einzelförderung außerhalb des Klassenraums nur um „Einzelfälle“ handele und spricht von einem Anteil von maximal $30 \%$ (SL 5, Z. 33f). Zwei Schulleitungen und zwei Förderschullehrkräfte erklären in diesem Zusammenhang, dass eine solche Einzelförderung je nach Förderschwerpunkt eines Schülers notwendig und hilfreich sein könne. So nutzt FSL 1 ihre Stunden bei „den Kindern mit geistiger Behinderung [...] wirklich um sie außerhalb der Klasse zu fördern um genau dort ansetzen zu können, wo die Kinder wirklich stehen“ (FSL 1, Z. 27-29), während FSL 4 diese manchmal benötigt, um „Soforthilfe“ für Kinder mit dem Förderbedarf soziale und emotionale Entwicklung anbieten zu können (FSL 4, Z. 27f). Die beiden Schulleitungen bringen diese Form der Einzelförderung auch damit in Verbindung, dass der Unterricht innerhalb der Klasse dann nicht „gestört“ werde (SL 1, Z. 65-69; SL 3, Z. 27-29) (s. dazu auch Sozialform frontaler Unterricht oben):

[...] je nachdem, wie stark jetzt auch die, ich sage jetzt mal Behinderung ist, ist es natürlich einfach so, dass ein Zuflüstern und stille Betreuen in der Klasse, andere Schüler auch wiederum nicht zu stören, da muss man ja auch Waage halten, dass das nicht ausreicht, Schüler herausnehmen muss, damit sie auch nochmal den Anschluss an ein neues Lernthema für die Klasse nochmal schaffen. Und da macht es dann auch mal Sinn sie aus dem Unterricht rauszunehmen. (SL 1, Z. 65-69)

Den von den Schulleitungen aufgeführten Aspekt, dass der Klassenunterricht durch Einzelförderung gestört werden könne, da bspw. vorwiegend frontaler Unterricht stattfinde, spricht auch eine Förderschullehrkraft an:

Dann gibt es Klassen, wo fast nur die Kinder rausgeholt werden, weil eben noch sehr viel Frontalunterricht gemacht wird und es eigentlich mehr stört, wenn man drin dann irgendwas machen will. (FSL 5, Z. 22-24)

Eine andere Förderschullehrkraft wiederum beschreibt, dass sie die Einzelförderung außerhalb des Klassenraums durchführe, da es dafür in der Klasse zu laut sei:

Es kommt immer auf den Stoff an, ob ich mit dem Kind etwas erarbeite. Dann nehme ich die Kinder eigentlich immer raus. Also ich könnte es auch im Klassenverband nicht machen. Mir ist das einfach zu laut. Mich macht das ganz durcheinander. (FSL 3, Z. 25-28)

Insgesamt wird die Einzelförderung außerhalb des Klassenraums offensichtlich häufig dazu genutzt um Unterrichtsinhalte individuell zu erarbeiten, wenn diese bspw. im Klassenunterricht nicht verstanden wurden oder die Schülerinnen und Schüler mit sonderpädagogischem Förderbedarf an anderen Inhalten arbeiten (SL 5, Z. 26-32; RSL 1, Z. 46f; RSL 2, Z. 2-28; RSL 3, Z. $20 \mathrm{f}$ und Z. 29-31; RSL 5, Z. 18; FSL 2, Z. 25f; FSL 4, Z. 30f). So schildert eine Schulleitung:

Manchmal wird das Kind herausgenommen, wir haben extra einen kleinen Raum oben so einen kleinen Klassensaal, den kleinen Raum oder in den Konferenzraum. Dann geht der Integrationslehrer dann in einen von den beiden Räumen, weil, wie ich gerade eben gesagt habe, vielleicht was ansteht, was das Kind so nicht begreifen kann. Es braucht diese individuelle Förderung und es ist oft, merke ich dann oder höre ich im Gespräch, nach ein zwei Stunden zusätzlich durch den Integrationslehrer hat das Kind das dann gerafft. Also das ist schon eine gute Sache. (SL 5, Z. 26-32) 
Das kann in Einzelfällen dazu führen, dass Schüler mit dem spF Lernen in einem Fach komplett außerhalb der Klasse gefördert werden:

Bei mir ist es jetzt halt so, dass beide im Bereich Mathematik ganz draußen sind. Das geht dann jetzt nur, wenn wir mal wieder ein Geometriethema machen, das sie da vielleicht mit einsteigen, aber ansonsten halt ganz allein. (RSL 5, Z. 23-25)

Während zwei Elternteile von individueller Förderung außerhalb der Klasse berichten und zwar in allen „drei Integrationsstunden“ (E 1, Z. 81f) oder teilweise (E 3, Z. 32), weist ein Elternteil darauf hin, dass dies sehr flexibel gehandhabt werde (E 2, Z. 18-21).

Teilweise wird die differenzierte Förderung nach Aussagen der Befragten auch als Gruppenförderung außerhalb der Klasse organisiert (SLFZ 1, Z. 69f; SLFZ 2, Z. 45f; RSL 5, Z. 19; FSL 4, Z.31f). Eine Schulleitung begründet dies damit, dass - neben pragmatischen Aspekten - die Schüler mit sonderpädagogischem Förderbedarf so auch untereinander Kontakte haben können:

Erstens Mal haben die dann mehr Spaß und man kann gleich, und dann sind sie doch nicht ganz allein und isoliert, so dass man da versucht auch noch den sozialen Kontakt zu Mitschülern in der Klasse oder Klassenstufe einfach zu nehmen und je mehr Kinder wir haben umso mehr gelingt das dann auch. (SL 1, Z. 69-75)

Die Bewertung der individuellen Förderung außerhalb der Klasse stellt sich bei den Befragten vorwiegend positiv dar. Diesbezüglich betont der Elternteil eines Schülers, dass es auf der einen Seite so sei, „dass er wirklich integriert unterrichtet wird, auf der anderen Seite differenziert, so wie es für ihn wirklich von Nöten ist“. (E 2, Z. 108-110). Ein weiterer Elternteil schildert die Vorteile dahingehend, dass ihr Kind während der Einzelförderung noch einmal gesonderte Erklärungen durch die FSL erhält (E 3, Z. 36-41). Ein Elternteil bringt kritisch ein, dass die Einzelförderung außerhalb der Klasse „keine, in Anführungsstrichen, man sagt ja Integration“ sei (E 1, Z. 95). Ihr Kind würde durch die Herausnahme aus dem Unterricht in einen anderen Raum ja auch selber spüren, dass dies anders sei als bei anderen Kindern (E 1, Z. 104-108). Die Förderschullehrkraft habe aber erklärt, dass die Einzelstunden eine intensivere Förderung ermöglichen würden (E 1, Z. 95-98) . Auch die Schüler selbst nehmen zu dieser Organisationsform Stellung. S 2 führt aus, dass die Förderschullehrkraft in diesen Situationen Aufgaben und Übungen mit ihm gemacht und er dadurch Hilfe erhalten habe (S 2, Z. 45-47). S 3 ist es egal, dass die Förderung in dieser Form erfolgt (S 3, Z. 51). S 4 bewertet die Einzelförderung außerhalb der Klasse als positiv, da er/sie daran gewöhnt sei alleine in einem anderen Raum gefördert zu werden (S 4, Z. 34-38) und diese als gut empfinde (S 4, Z. 38), u.a. da es „draußen so ruhig ist" (S 4, Z. 40).

FSL 5 beschreibt sehr ausführlich, warum es manchmal schwierig ist, die Einzelförderung als Maßnahme bei den Regelschullehrkräften zu begründen:

Sehr selten. Also es gibt hier so zwei drei Kolleginnen, die auch sehr viel Missionsarbeit leisten bei den anderen, die dann, also, gerade heute hatten wir jetzt den Fall, da kam eine Lehrerin und hat gesagt: Guck mal diesen Aufsatz hat das Kind geschrieben, das wir letztes Jahr drei Monate lang aus dem Lese-Rechtschreiblehrgang rausgenommen haben, weil es das nicht geschafft hat. Und das zeige ich jetzt jedem, damit man sieht, dass das Kind aufholen kann und dann irgendwann wieder das machen kann, was die anderen auch machen, aber das ist einfach noch ganz, ganz schwer und das wird einfach noch nicht akzeptiert. (FSL 5, Z. 94-100); Also es gibt einzelne Lehrer, die sagen, ich habe da kein Problem damit, wenn du mir als Förderschullehrerin sagst, ich soll das Kind mal ein bisschen rausnehmen und Grundlagen schaffen, dann mache ich das. Ja, das ist maximal ein Drittel vom Kollegium, denke ich, [...]. (FSL 5, Z. 94-102) 


\subsubsection{Ergebnisse im Bereich der Unterrichtsentwicklung}

Nach der Lernentwicklung der Schüler mit sonderpädagogischem Förderbedarf an ihren Schulen gefragt, weisen zwei Schulleitungen darauf hin, dass sie dazu keine allgemeine Aussage treffen können, da dies nur aus das einzelne Kind bezogen beschrieben werden könne (SL 2, Z. 138; SL 4, Z. 39f), während eine andere Schulleitung diese als gut kennzeichnet: „[...] ich kann nur sagen, dass die sich gut entwickeln (Z 57); die Fortschritte doch verhältnismäßig gut sind. Ich sage immer, was ihr da in der kurzen Zeit noch bewerkstelligen könnt, Hut ab." (SL 5, Z. 58f). Hinsichtlich der kognitiven Lernentwicklung von Schülerinnen und Schülern mit sonderpädagogischem Förderbedarf differenzieren die Befragten teilweise nach Förderschwerpunkten. So weist Schulleitung 3 darauf hin,

[...] dass die Kinder in ihrer, also jetzt rede ich mal von lernbehinderten Kindern, wegen ihrer Behinderung andere Kriterien bei Klassenarbeiten haben, kriegen sie auch andere Noten und sind dann mehr motiviert auch zu arbeiten, das glaube ich schon. Es ist ein Unterschied, ob ich immer da ein mangelhaft stehen habe oder ein ungenügend, wenn dann da mal ein befriedigend steht. So dass sich auch das Öffnen für Lernen halt besser ist. (SL 3, Z. 56-60)

Mit Blick auf ein Kind mit dem Förderschwerpunkt geistige Entwicklung nimmt eine andere Schulleitung dessen künftige mögliche Entwicklung in den Blick:

Wir kriegen das hin und er fühlt sich wohl, aber wie seine Entwicklung ist, wenn die Kinder 13, 14 sind und wenn er vielleicht auch das Bewusstsein hat, Mensch, ich bin ganz anders, ganz anders in dem Fall, das weiß ich nicht und ob wir dann den lebenspraktischen Bereich so schulen können, wie das vielleicht an einer Förderschule der Fall ist, das ist fraglich, aber wir bemühen uns. (SL 2, Z. 158-161)

Eine Schulleitung und zwei Förderschullehrkräfte stellen die kognitiven Entwicklungsmöglichkeiten in der Regelschule positiv dar. In einem Fall habe ein Kind mit einer „relativ pessimistischen Prognose [...] sich [...] toll entwickelt" und werde das Klassenziel erreichen (SL 4, Z. 4043), in anderen Fällen könnten Kinder durch die Unterstützung von Förderschullehrkräften oder Jugendamt so gut sozial-emotional gefestigt werden, dass eine positive Entwicklung möglich sei (FSL 5, Z. 194-198). Zwei Förderschullehrkräfte sehen Chancen darin, „dass die Kinder einfach mehr mitnehmen, gerade kognitiv und sozial-emotional“ (FSL 3, Z. 117-119) oder dass sich auch ganz schwache Kinder sehr gut entwickeln können, wenn die Rahmenbedingungen wie Lehrerstunden, Integrationshilfe etc. gegeben sind (FSL 5, Z. 194-198).

Sieben Interviewpartner nehmen Stellung zur sozialen Lernentwicklung der Schüler mit sonderpädagogischem Förderbedarf. Darunter beschreiben zwei Schulleitungen den Aspekt der Rücksichtnahme als Lernergebnis (SL 1, Z. 195; SL 4, Z. 30-33). Eine davon fasst zusammen:

[...] positiv ist auf jeden Fall, dass man lernt aufeinander Rücksicht zu nehmen, dass man lernt Schwächere zu akzeptieren, dass man lernt, dass man helfen kann, Respekt, Achtung auch vor Kindern, die bestimmte Dinge nicht so gut können. Empathiefähigkeit, man lernt sich auch in die Rolle mal reinzuversetzen. (SL 4, Z. 30-33)

Die soziale Integration allgemein stellen fünf Interviewpartner als positiv dar (SL 1, Z. $186 \mathrm{f}$, Z. 188f; SL 2, Z. 130 f (mit Blick auf „K- und L-Kinder“); SL 4, Z. 61; RSL 2, Z. 130-132; RSL 5 , Z. 187-191), wobei zwei Regelschullehrkräfte von möglichen Einstellungsveränderungen sprechen:

Positive Chancen sind natürlich gegeben, wenn man schon merkt, dass gerade wenn es bei körperbehinderten Kindern, die ja oft ausgegrenzt werden, wenn so ein Schüler länger in der Klasse ist, ändern sich die Kinder und das, diese Einstellung finde ich sehr positiv. (RSL 2, Z. 130-132) 
[...] ich sehe sie halt einfach in dem „als normal akzeptieren“. Dass es nicht so wie bei uns früher war oder am Anfang, als ich gelernt habe, hat jemand zu mir gesagt: „Muss ich dann auf die Dummenschule?". Ja, dass das sich einfach endlich mal erledigt. Dass es einfach normal ist: manche lernen langsamer oder manche lernen nichts und die können halt andere Sachen toll oder die sind trotzdem wertvoll, dass das auch ankommt. (RSL 5, Z. 187-191)

Eine Förderschullehrkraft und ein Elternteil thematisieren das positive soziale Miteinander:

Gerade in den Klassen, wo jetzt eben wirklich die sehr schwachen G-Schüler drin sind, merkt man wirklich, dass die Kinder sich mehr kümmern. Gucken, jetzt gerade bei den Fünftklässlern, kommen die mit? Wissen die, in welchen Saal es jetzt geht? Sind sie dabei oder halt auch, wenn Sport angesagt ist, denk dran deinen Turnbeutel mitzunehmen und solche Sachen. Das kommt auf jeden Fall vor. Auch wenn Gruppenarbeiten gemacht werden, dass die Kinder dann auch speziell diesen Kindern nochmal erklären, was denn eigentlich die Aufgabenstellung war. Also im sozialen Bereich würde ich da deutlich positive Entwicklungen schon sehen. (FSL 1, Z. 58-64)

[...] eine Chance ist, vor allem, dass das soziale Miteinander gestärkt wird, also ich denke das ist ein ganz wesentlicher Faktor, dass leistungsstärkere Kinder [...] nicht nur vom sozialen Verhalten her, sondern auch so vom Lernverhalten her den andern Kindern was weitergeben können (E 1, Z. 278-281)

Die emotionale Entwicklung von Schülerinnen und Schülern mit sonderpädagogischem Förderbedarf greifen drei Schulleitungen auf. Dabei wird ein positives Beispiel erfasst: „Das, was ein kleines, ängstliches Mäuschen war, ist jetzt eine selbstbewusste junge Frau geworden." (SL 3, Z. 55f). In zwei Beispielen erfolgt dagegen eine Problematisierung:

Emotionale Entwicklung ist jetzt etwas schwierig, ich denke jetzt auch gerade an die E-Kinder. Ich bin mir nicht sicher, ob wir die Kinder immer zielgerecht wirklich fördern können oder ob wir manchmal auch Kinder überfordern, sowohl kognitiv als auch vielleicht emotional. Wenn wir zum Beispiel Autisten haben, fällt es den Fachlehrern sehr, sehr schwer sich auf Autisten einzulassen, weil man schwer erklären kann, was ist jetzt mit diesem Schüler. Das kann man auch ganz schlecht in einem einzigen Buch nachlesen und von daher wird vielleicht diesen Kindern manchmal ein Unrecht zuteil. (SL 2, Z. 132-137)

[...] wir haben aber auch einen Schüler, da haben wir bis jetzt überhaupt keinen Fortschritt festgestellt, dass er in irgendeiner Weise eine Weiterentwicklung hier gemacht hätte. (SL 4, Z. 43f); Das ist der Förderbedarf E, sozial-emotionale Entwicklung. Also das stagniert. Es funktioniert auch mit der Integrationshelferin nicht so gut, also da ist kein Fortschritt zu erkennen. (SL 4, Z. 466)

Und ein Elternteil beschreibt im Zusammenhang mit der Entwicklung ihres Kindes insgesamt ganz persönliche Erfahrungen:

Aber die Problematik ist natürlich wirklich da, dass man nicht abschätzen kann, welche Kinder dann tatsächlich in einer Regelschule gut aufgehoben sein werden, welche besser in eine Förderschule gingen. Und wir hatten Phasen, als es unserem Kind nicht gut ging, wo wir wirklich gesagt haben, also, jetzt warten wir noch. Das halbe Jahr schaffen wir mit dem noch durch, aber dann wird XY an die K-Schule gehen müssen. Und, was heißt gehen müssen, ich sah einfach, dass es nicht mehr geht, dass XY überfordert war, dass XY keine Freizeit mehr für sich hatte um dieses Pensum hier, was ein normales, gesundes Kind schafft, zu schaffen. Und da denke ich muss man ganz ganz viel Feingefühl haben, auch von Politikseite, um zu erkennen, was für Kinder wirklich richtig und wichtig, vielleicht die Entscheidung tatsächlich bei den Eltern zu belassen und wenn die Eltern sagen, das geht nicht, wir müssen unser Kind in eine andere Schulform stecken, aber dafür muss die Option auch da sein. Gibt es diese Schulform nicht mehr, hat man diese Option nicht. Ob man dann tatsächlich froh ist, wenn ein Kind nicht mehr weiter kommt und nur noch krank und kränker wird durch diesen Druck, das ist die Frage. (E 2, Z. 87-100) 


\subsubsection{Interpretation der Ergebnisse zur Organisationsentwicklung}

\subsubsection{Ressourcen im Bereich der Organisationsentwicklung}

Die Problematik begrenzter finanzieller Ressourcen, die in der sonderpädagogischen Forschung seit den 70er Jahren thematisiert wird, wird von 13 Interviewpartnern und -partnerinnen aufgegriffen, die die Rahmenbedingungen für inklusive Bildungsangebote als unzureichend bezeichnen. Dieses Ergebnis ist mit Blick auf die Interviewaussagen vor allem deshalb als relevant einzuschätzen, da nicht explizit nach der Bedeutung materieller Rahmenbedingungen in den Schulen gefragt wurde. Während Gutachten zur inklusiven Bildung (Dohmen \& Fuchs, 2009; Klemm, 2009; Schwarz et al., 2013) und Autoren der Sonderpädagogik (Evers-Meyer, 2010; Textor, 2015) darüber hinaus von der Notwendigkeit erheblicher Anschubinvestitionen ausgehen, sind diese für die befragten Schulleitungen und Lehrkräfte im Saarland nicht feststellbar. Dabei wird auch die von Textor (2015) beschriebene „Gefahr der indirekten Steuerung“ (S. 52) von mehreren Personen aufgegriffen, allerdings nicht mit Blick auf mögliche Elternentscheidungen in Richtung der Beschulung in einer Förderschule, sondern dahingehend, dass die integrative Arbeit aufgrund der mangelnden Ressourcen von den Regelschulen in Frage gestellt werden könnte (SLFZ 1, Z. 93) oder „im Sande verläuft“ (FSL 5, Z. 208). Damit könnte wiederum indirekt die Bestätigung verknüpft sein, „dass es keinen Sinn macht“ (FSL, Z. 190), so dass inklusive Bildung aus Ressourcengründen als gescheitert eingeschätzt werden könnte. Auf Grundlage der getätigten Aussagen ist also insgesamt nicht davon auszugehen, dass die von Bächthold et al. (1990), Innerhofer \& Klicpera (1991) Feyerer (1998) oder Speck (2010) gefasste Qualitätskategorie guter materieller Rahmenbedingungen aus Sicht der Interviewpartner gegeben ist.

Während Barrierefreiheit sowie die räumliche Flexibilität mit Blick auf die Größe der Klassenräume als Qualitätskategorien inklusiver Schulen in der Forschung wie in Kriterienkatalogen eine wichtige Rolle spielen, werden diese von keinem der Interviewpartner aufgegriffen. Stattdessen erläutern die Befragten vor allem die Bedeutung des Vorhandenseins von Differenzierungsräumen. Während verschiedene Autoren (Heese, 1977; Innerhofer \& Klicpera, 1991; Brugger-Paggi, 2003; Wember \& Prändl, 2009; Holzinger et al., 2011) Differenzierungsräume als wichtiges Qualitätsmerkmal integrativer Schulen benennen, beschreiben Schulleitungen wie Förderschullehrkräfte das Problem, dass diese zum Teil gar nicht bzw. nicht in ausreichender Anzahl zur Verfügung stehen (SL 1, SL 2, SL 3, SL 5, FSL 3), so dass häufig der Flur als Arbeitsraum genutzt werden müsse. Flexible Organisationsformen des Unterrichts (Bsp.: Arbeit in Kleingruppen, individualisierende Angebote (Innerhofer und Klicpera, 1991)), können also offensichtlich nicht uneingeschränkt angeboten werden. Die Bedeutung der Differenzierungsräume für die Beteiligten kann damit zusammenhängen, dass differenziertes Arbeiten vorwiegend in Form äußerer Differenzierung umgesetzt wird, d.h. dass die sonderpädagogische Förderung als Einzel- oder Kleingruppenförderung außerhalb des Klassenraums stattfindet (s. 4.3.5.6).

Differenzierungsmaterial und spezifische Hilfsmittel, die in sonderpädagogischen Qualitätsübersichten sehr umfassend aufgeführt werden (Boban \& Hinz, 2003; Brugger-Paggi, 2003; Leonhardt, 2009; Thiele, 2009; Wember \& Prändl, 2009; Holzinger et al., 2011), werden in den Schulen teilweise von den Förderschullehrkräften selbst oder von den Eltern angeschafft. Ein Schulleiter hat für die Ausstattung seiner Schule mit passendem Material für die Schüler mit sonderpädagogischem Förderbedarf vom Träger zusätzliche Mittel angefragt, was ein Hinweis darauf ist, dass für Arbeits- bzw. Hilfsmittel für SuS mit spF in den Schulen kein gesonderter Etat von den Schulträgern zur Verfügung gestellt wird. Besondere therapeutische Angebote (Wember \& Prändl, 2009) werden von den Interviewpartnern nicht erwähnt. 
Obwohl in den Interviews nicht explizit nach den finanziellen und räumlichen Rahmenbedingungen gefragt wird, werden diese Aspekte von den Interviewpartnerinnen und -partnern thematisiert. Grundtenor der Aussagen ist, dass die Rahmenbedingungen in den Regelschulen zum Zeitpunkt der Befragung nicht so gestaltet sind, dass bspw. gut ausgestattete Räumlichkeiten für differenzierende Förderangebote vorhanden sind und die Umsetzung der Integrativen Arbeit unterstützt wird. Es werden stattdessen Sorgen dahingehend formuliert, dass die mangelhaften Rahmenbedingungen zu einer sinkenden Akzeptanz der schulischen Integration in den Regelschulen führen könnten.

\subsubsection{Leitung einer Schule}

Bis auf einen Elternteil, der die Erfahrung gemacht hat, dass es bei der Umsetzung integrativer Arbeit auf die Schulleitung ankomme, wird die Bedeutung der Schulleitung für inklusive Prozesse von den Befragten nicht aufgegriffen. Hinweise zu dieser Bedeutung ergeben sich im Rahmen der Interviews allerdings indirekt durch die Ausführungen der Schulleitungen der Regelschulen wie der Förderzentren, da diese Themen inklusiver Beschulung und Unterricht nicht in erster Linie auf die Situation in einer Klasse oder einzelfallbeogen analysieren, sondern über Überblickswissen verfügen. Insbesondere die Interviews mit den Schulleitungen der Sonderpädagogischen Förder- und Beratungszentren machen deutlich, dass deren Erfahrungswerte noch stärker für die Weiterentwicklung schulischer Integration genutzt werden könnten. SLFZ 1 und 2 geben in diesem Zusammenhang Hinweise darauf, dass bspw. ein Austausch auf Schulleitungsebene zwischen FZ und Regelschulen erfolgt (s. dazu 4.3.7.3). Aus den Aussagen der Schulleitungen der Regelschulen wiederum geht hervor, dass diese den mit der Thematik zusammenhängenden Entwicklungsaufgaben vorwiegend aufgeschlossen und unterstützend gegenüberstehen, gleichzeitig aber auch mögliche Probleme bzw. Grenzen gezielt kennzeichnen können.

\subsubsection{Schule als sozialer Raum}

Die als Indikatoren für ein gutes Schulklima an inklusiven Schulen aufgeführten Aspekte Respekt, Akzeptanz sowie gegenseitige Rücksichtnahme und Hilfestellung von und zwischen Schülern mit und ohne sonderpädagogischen Förderbedarf (Boban \& Hinz, 2003; Wember \& Prändl, 2009; Thiele, 2009: Lienhard-Tuggener et al., 2011) werden von drei Schulleitungen, zwei Regelschullehrkräften sowie den fünf befragten Schülern und Schülerinnen als vorhanden benannt. Eine Schulleitung betont in diesem Zusammenhang den selbstbewussten Umgang der Schüler mit diesem Thema (SL 3, S. 11-13). Zwei Elternteile stellen aufgrund ihrer Erfahrungen fest, dass die Akzeptanz ihrer Söhne in den von diesen zunächst besuchten Grundschulen nicht gegeben war, was sie darauf zurückführen, dass die Schulen bzw. Lehrkräfte große Schwierigkeiten mit integrativer Arbeit hatten und damit zum Teil überfordert waren. Der Erfolg der Integration hängt nach Auffassung eines Elternteils letztlich an einzelnen Personen und deren Bereitschaft sich in der Arbeit mit Kindern mit sonderpädagogischem Förderbedarf zu engagieren. Ein Elternteil beschreibt diesbezüglich eine enge Wechselwirkung zwischen der mangelnden Akzeptanz des Sohnes und dessen Lern- und Arbeitsverhaltens (E1, Z. 13-35). Diese Aussage stellt eine Querverbindung zu der von Hascher (2007) oder dem deutschen PISA-Konsortium (2001) festgestellten Wechselbeziehung zwischen dem Wohlbefinden eines Schülers und seiner Lern- und Leistungsbereitschaft dar.

Mit Blick auf die zum Zeitpunkt der Befragung besuchten Schulen äußern sich die drei Elternteile übereinstimmend dahingehend, dass Offenheit und Engagement der Lehrkräfte (Mühl, 1987; Boban \& Hinz, 2003) für integratives Arbeiten gegeben sind. Die in den Aussagen der 
Eltern beschriebenen großen Unterschiede zwischen einzelnen Schulen können als Hinweis auf die dieser Arbeit zugrunde gelegte Annahme der Bedeutung der Entwicklung der Einzelschule für die inklusive Arbeit aufgefasst werden (dazu auch Lütje-Klose et al., 2018).

Interessant erscheint mit Blick auf die Interpretation der Daten der Zusammenhang, den die Befragten zwischen dem Engagement der Lehrkräfte und einem positiven Schulklima herstellen.

Zusammenfassend lässt sich festhalten, dass die Interviewpartner eher wenige Aussagen zum Schulklima allgemein treffen. Die Förderschullehrkräfte tätigen keine Aussagen zu dieser Thematik. Deutlich wird, dass die Eltern das Klima an einer Schule mit dem speziellen Blick auf ihre Kinder mit Behinderung analysieren und daher auch ihre emotionale Beteiligung thematisieren. Die in den zugrundeliegenden Forschungsarbeiten aufgeführten Subkategorien Integration als gemeinsame Aufgabe (Bächthold et al., 1990; Feyerer, 1998; Boban \& Hinz, 2003; Holzinger et al., 2011) sowie Partizipationsmöglichkeiten von Eltern und Schülern in integrativ arbeitenden Schulen (Mühl, 1987; Bächthold et al., 1990; Innerhofer \& Klicpera, 1991; Hug, 1994b; Bless, 2003; Boban \& Hinz, 2003; Brugger-Paggi, 2003; Lienhard-Tuggener et al., 2011) werden von den Befragten nicht direkt aufgegriffen. Während der Bereich der Integration als gemeinsame Aufgabe zumindest indirekt angesprochen wird, indem ein Elternteil bspw. das hohe Engagement einer Schule insgesamt beschreibt, stehen Partizipationsmöglichkeiten als Qualitätsmerkmal integrativer Schulen für die Befragten also nicht im Fokus.

Den mit Abstand größten inhaltlichen Raum innerhalb der Kategorie Schule als sozialer Raum nehmen dagegen Aussagen zur Zusammenarbeit der Schulen und Lehrkräfte mit den Eltern allgemein (18 Nennungen) sowie mit den Eltern von Schülern mit sonderpädagogischem Förderbedarf (35 Nennungen) ein. Die Aussagen der Interviewpartner beziehen sich in diesem Zusammenhang vor allem auf den Informationsfluss (Telefonate, Mitteilungshefte, Mails), Beratungsgespräche, Runde Tische oder Förderkonferenzen - also den kommunikativen Austausch zwischen den Beteiligten - und damit auf die Kategorien, Subkategorien und Indikatoren, die auch in der sonderpädagogischen Forschung als bedeutsam erfasst sind (Mühl, 1987; Hug, 1994b; Boban \& Hinz, 2003; Brugger-Paggi, 2003). Alle Aussagen belegen, dass dieser kommunikative Austausch von schulischer Seite aus quantitativ intensiv gepflegt wird. Die Aussagen einer Schulleitung zu den Runden Tischen belegen darüber hinaus die Bedeutung der Einbindung außerschulischer Vernetzungspartner in die Zusammenarbeit mit den Eltern. Vereinzelt werden Elterngespräche im Team von Klassenleitung und Förderschullehrkraft thematisiert, eine neue Subkategorie, die im Kategoriensystem nicht enthalten ist. Ebenfalls neu werden als Subkategorie Gespräche zwischen Schulleitungen und Eltern von SuS mit spF benannt. Problematisiert wird auf der anderen Seite, dass eine Reihe von Eltern der Schüler mit sonderpädagogischem Förderbedarf zum Teil nur geringes Interesse an Beratungsgesprächen zeigt (SL 4, SL 5, RSL 2, RSL 5, FSL 5).

Darüber hinausgehend fokussiert die Forschung allerdings auch die stärkere Einbindung der Eltern hinsichtlich der Lernprozesse der Schüler (Brugger-Paggi, 2003; Jeynes, 2005; Pushor, 2007; Lienhard-Tuggener et al., 2011; Werning, 2013), das sogenannte „Parent Engagement“, und die damit verbundenen positiven Auswirkungen (Hattie, 2013). In den Interviews findet sich dazu lediglich in der Aussage einer Förderschullehrkraft (FSL 1, Z. 137-142) ein Hinweis darauf, dass es zum Beispiel Gespräche zum „IST-Stand“ und zur möglichen Weiterentwicklung von Schülern - also Förderplan- oder Zeugnisgespräche - sein können, die eine solche Einbindung ermöglichen, da sie auf den Lernprozess eines Kindes oder Jugendlichen ausgerichtet sind. Anregungen wie die von Berthelsen \& Walker (2008) oder Innerhofer \& Klicpera (1991), die thematisieren, dass Eltern bezüglich ihrer Ressourcen und Aktivitäten durch die Schulen direkt gestärkt werden sollten - auch durch Fortbildungsangebote für die Eltern - um dadurch Effekte auf die Lernerfol- 
ge der Schüler zu erzielen, werden als Qualitätsmerkmale nicht erwähnt. Während die Häufigkeit und die Intensität der Elternkontakte in den Ausführungen der Interviewten eine wichtige Rolle spielen, lassen sich aufgrund der Ergebnisse kaum Schlussfolgerungen mit Blick auf die Qualität der Elternkontakte (Bsp.: Gesprächsinhalte) (Berthelsen \& Walker, 2008) ziehen.

Nur fünf Nennungen der Befragten beziehen sich auf die Qualitätssubkategorie der Vernetzung (Vernetzungspartner Schulärztin, Autismuszentrum, Psychologen, Schoolworker, Therapeutische Schülerhilfe, Familienhelfer). Dieses Ergebnis kann - wie die Ergebnisse aus der GeSchwind-Studie (Laubenstein et al., 2015) - ein Hinweis darauf sein, dass Unterstützungsnetzwerke in den Schulen noch nicht umfassend ausgebaut sind und genutzt bzw. professionell ausgestaltet werden (Ahrens, 2009). Es ist also nicht davon auszugehen, dass die in der Studie von Bryk et al. (2010) aufgeführten möglichen positiven Effekte der Vernetzungsarbeit in den Schulen der Interviewpartner greifbar sind oder umfassend zur Entlastung (Schnitzer, 2008) beitragen.

\subsubsection{Organisationsstrukturen einer Schule}

Schulleitungen, Regel- wie Förderschullehrkräfte sowie Eltern wurden in den Interviews nach Veränderungen innerhalb der Schulen durch die integrative Arbeit befragt. Die Veränderungen übergeordneter schulischer Organisationsstrukturen wie Zeit- und Raumkonzepte Meiers, 1982), unterschiedliche organisatorische Möglichkeiten der integrativen Beschulung (Muth, 1977; Sander, 2003) oder jahrgangsübergreifender Unterrichtskonzepte (Kucharz et al., 2009; Kucharz, 2010; Stäbler et al., 2015) werden allerdings nicht benannt. Aufgeführt wird lediglich die Einführung klassenübergreifender Fördergruppen (Schinnen, 1988). Auf organisatorischer Ebene scheint in den befragten Schulen also keine strukturierte Weiterentwicklung stattgefunden zu haben. Auch Übergänge zwischen Einrichtungen werden nicht fokussiert (abgesehen von der Aussage eines Schulleiters, der befürchtet, dass ein Übergangsprojekt zwischen Kindergärten und Schulen aus finanziellen Gründen gestrichen werden könnte).

\subsubsection{Ergebnisse im Bereich der Organisationsentwicklung}

Zu den Ergebnissen der konzeptionellen Entwicklung ihrer Schulen in Form von Konzeptbausteinen oder Schulprogrammen (Berges, 1996; Boban \& Hinz, 2003; Brugger-Paggi, 2003; Wember \& Prändl, 2009) bzw. Förderkonzepten (BMBWF Österreich, 2008) äußern sich lediglich die zwei Schulleitungen der Förderzentren sowie SL 2 und SL 3. Während die Leitungen der Förderzentren mögliche gemeinsame Entwicklungsbausteine mit den Regelschulen als Qualitätssubkategorien benennen (Bsp.: gemeinsames Förderkonzept Grundschule - Förderschule, Schulabwesenheit, Methodentraining, fachdidaktische Bausteine), führen die Schulleitungen der Regelschulen ihre Schulprogramme an, die sich allerdings nicht spezifisch mit dem Thema schulischer Integration befassen. Während also aus den Interviews insgesamt sehr deutlich hervorgeht, dass sich alle Beteiligten intensiv mit Fragen der Qualität der integrativen Arbeit auseinandersetzen, scheinen sie sich - ähnlich wie in der PISA-Studie (2001) belegt oder für den Bereich der schulischen Integration bei Laubenstein u.a. (2015) - nicht unbedingt strukturiert und im Sinne einer schriftlichen Dokumentation programmatisch damit zu befassen. Die von den SLFZ erwähnte Konzeptarbeit, die sich konkret auf bestimmte (fachliche) Arbeitsschwerpunkte bezieht, könnte ggf. ausgebaut werden.

\subsubsection{Interpretation der Ergebnisse zur Personalentwicklung}

\subsubsection{Ressourcen im Bereich der Personalentwicklung}

Das Thema Ressourcen mit Blick auf das Personal hat aus Sicht der Interviewpartner eine hohe Relevanz für die Qualität der schulischen Integration, was sich daran zeigt, dass dazu insge- 
samt 50 Aussagen getätigt werden. Die in Modellversuchen und der damit verbundenen sonderpädagogischen Forschung für qualitativ wesentlich gehaltenen personellen Ressourcen integrativ arbeitender Schulen wie die Doppelbesetzung von Klassen (Innerhofer \& Klicpera, 1991; Köbberling, 1994; Heyer et al., 1997; Bless, 2003; Boban \& Hinz, 2003; Brugger-Paggi, 2003; BMBWF Österreich, 2008; Specht et al., 2006; Thiele, 2009; Textor, 2009; Holzinger et al., 2011) oder die Anteile an Förderschullehrerstunden je Klasse bzw. Schüler (Bless, 2003; BMBWF Österreich, 2008; Sander \& Schnell, 2006) werden von den Interviewpartnern in insgesamt 17 Aussagen thematisiert. Die Befragten sehen - deckungsgleich mit den in Studien zur Kooperation belegten Ergebnissen (Dumke et al., 1989; Arndt \& Werning, 2013; Serke et al., 2014a; Lütje-Klose \& Urban, 2014b) - einen engen Zusammenhang zwischen diesen Ressourcen und der Qualität der sonderpädagogischen Förderung sowie der Qualität der Kooperation zwischen Förder- und Regelschullehrkräften in den Regelschulen (insgesamt 20 Aussagen). Eine Doppelbesetzung bzw. allgemeiner formuliert „Stunden, damit man auch wirklich zusammen arbeiten kann“ (FSL 2, Z. 59) werden als Grundvoraussetzung für unterrichtsbezogene Zusammenarbeit gesehen. Ist diese nicht umfassend vorhanden, wird dies als Belastung empfunden (FSL 4, Z. 128-132; SL 5, Z. 145-149).

Insbesondere der angenommene Zusammenhang zwischen der Doppelbesetzung einer Klasse mit einer Regel- und einer Förderschullehrkraft und den Möglichkeiten zum differenzierten Arbeiten mit den Schülerinnen und Schülern wird mehrfach hervorgehoben (RSL 1, Z. 74-77; FSL 4, Z. 128-137; SL 2, Z. 262-266; SL 3, Z. 94-99; E 1, Z. 210-212), auch mit Blick auf die lernstandsdiagnostische Arbeit von FSL in den Klassen, die bei geringer Stundenzahl und großen Klassen als unmöglich beschrieben wird (SL 2, Z. 262-266). Dieser von den Befragten angenommene enge Zusammenhang wird in der Forschung in Ansätzen im Zusammenhang mit Co-Teaching-Formen hergestellt (Prell \& Link, 1977; Halfhide, 2009). Während empirisch gesehen also die Untersuchung der Auswirkung der personellen Ausstattung einer inklusiven Schule auf die Kooperation der Lehrkräfte an sich im Vordergrund steht bzw. Forderungen nach einer Doppelbesetzung nicht umfassend mit erweiterten Möglichkeiten zu binnendifferenziertem Arbeiten verknüpft werden, stellen die befragten Personen die Annahme in den Raum, dass mit heterogen zusammengesetzten Lerngruppen besser gearbeitet werden kann, wenn zwei Personen sich diese Arbeit teilen. Die Schulleitung einer Gemeinschaftsschule verweist diesbezüglich darauf, dass die Schule dazu übergegangen ist, Schülerinnen und Schüler mit sonderpädagogischem Förderbedarf in einer Klasse zu bündeln, damit eine Doppelbesetzung in einer größeren Anzahl von Stunden gewährleistet werden kann - eine Vorgehensweise, die den sonderpädagogischen Ansätzen zur Klassenzusammensetzung (s.u.; Wocken, 1987; Innerhofer \& Klicpera, 1991; Schley et al., 1992; Husinsky, 1994; Feyerer, 1998; Bless, 2003; Brugger-Paggi, 2003; BMBWF Österreich, 2008; Holzinger et al., 2011) entgegen läuft. Einen möglichen Ausweg aus den Nachteilen einer mangelnden Personalausstattung in den Regelschulen sieht der Leiter eines Förder- und Beratungsteams (SLFZ 1, Z. 14-19) - ähnlich wie Lütje-Klose und Urban (2014b) - in einem systemischen Arbeitsansatz, in dem ein ganzes Kollegium/eine ganze Schule ein Konzept für die sonderpädagogische Förderung in einer Regelschule entwickelt und umsetzt. Die von Textor mit Blick auf die unzureichenden Rahmenbedingungen für individuelle Förderung in den Regelschulen in Zusammenhang gebrachte „Gefahr der indirekten Steuerung“ (Textor, 2015) hin zur Beschulung in Förderschulen wird von neun der interviewten Personen benannt. Sie gehen davon aus, dass es u.a. die dort vorhandenen personellen Ressourcen sind, die die Entscheidung der Eltern beeinflussen.

Die neun Aussagen zu den Anteilen von Förderschullehrerstunden je Klasse bzw. Schüler problematisieren übereinstimmend die Tatsache, dass im Schnitt je Schüler mit sonderpäda- 
gogischem Förderbedarf nur etwa zwei Förderschullehrerstunden wöchentlich zur Verfügung stehen. Auch die geplante Budgetierung der Förderschullehrerstunden an Regelschulen wird kritisch eingeschätzt, da diese für präventives und inklusives Arbeiten keine ausreichende Stundenzahl vorsehen würde. Diese Budgetierung sonderpädagogischer Ressourcen im Sinne der gebündelten Zuweisung von FSL-Stunden an die Regelschulen, zu der in der sonderpädagogischen Forschung keine einheitliche Auffassung besteht (BMBWF Österreich, 2008; BruggerPaggi, 2003), wird von den zwei saarländischen Schulleitungen der Förder- und Beratungszentren wiederum positiv gesehen. Anders als in den sonderpädagogischen Veröffentlichungen geht es diesen Schulleitungen allerdings vor allem darum, dass die sogenannte „Budgetierung von Regelschulen mit Förderschullehrerstunden“ nicht in „Kopf-zu-Kopf“-Zuordnung zu Kindern mit sonderpädagogischem Förderbedarf erfolgt, sondern Förderschullehrkräfte Regelschulen möglichst kontinuierlich fest zugewiesen werden (SLFZ 1, Z. 7-9; SLFZ 2, Z. 232-237). Die von den beiden Schulleitungen angesprochene Kontinuität einer solchen Zuweisung, die als Subkategorie für die Qualität schulischer Integration lediglich in einer Veröffentlichung genannt wird (Brugger-Paggi, 2003), wird unter anderem damit begründet, dass für die Entwicklung der Kooperation der Förder- und Regelschullehrkräfte größere Zeiträume benötigt werden (Verknüpfung zum Thema Kooperation: Aufbau von Vertrauen - höhere Niveaustufe der Zusammenarbeit) und die Einarbeitung gemischter Teams mehrere Jahre dauern könne (SLFZ 1, Z. 118-225), bevor die positive Zusammenarbeit gegebenenfalls auf andere Teams ,ausstrahlt“ (SLFZ 1, Z.123). Anders als in der bisherigen Forschungsliteratur wird dieser Subkategorie damit eine stärkere Gewichtung zugeordnet.

Eine besondere Rolle spielt für die Schulleitungen der Förderzentren auch die Frage nach der passenden Zuweisung von einzelnen Förderschullehrern an bestimmte Regelschulen, eine Kategorie, die in der rezipierten Forschungsliteratur nicht benannt wird und somit eine neue Subkategorie darstellt. Diesbezüglich weisen die Schulleitungen der Förder- und Beratungszentren darauf hin, dass sowohl die Eigenheiten der Lehrkräfte als auch die spezifischen Eigenheiten einer einzelnen Schule aufeinander abgestimmt werden sollten, wenn das möglich ist, bspw. indem Lehrkräfte, die Interesse an einer systemischen Umstellung der inklusiven unterrichtlichen Arbeit in einem neuen fünften Jahrgang zeigen, auch an dieser Schule und in diesem Jahrgang eingesetzt werden (SLFZ 1, Z. 167-177).

Die Vorteile einer multiprofessionellen Personalisierung innerhalb der Regelschulen werden in den Interviews nur von einer Schulleitung mit Blick auf den Einsatz von Erziehern (SL 2, Z. 273-278) sowie den Bedarf an Schulsozialarbeitern in den Schulen (SL 2, Z. 271-273) angesprochen. Auch die Einbindung von Integrationshelfern spielt hinsichtlich des Auffangens der schwierigen personellen Situation eine Rolle. Die seit den 70er Jahren von sonderpädagogischer Seite geforderte multiprofessionelle Zusammensetzung von Unterstützungsteams (Romey, 1977; Prell \& Link, 1977; Speck et al., 1978; Klemm \& Preuss-Lausitz, 2008) haben die Interviewpartner im Zusammenhang mit der Qualität schulischer Integration zunächst nicht im Blick.

Auch die Schaffung passender räumlicher und zeitlicher Rahmenbedingungen für kooperatives Arbeiten, bspw. in Form fester im Stundenplan verankerter Kooperationszeiten, die von Fussangel \& Gräsel (2012), Kuper \& Kapelle (2012) sowie einer ganzen Reihe sonderpädagogischer Autoren als Qualitätskategorie benannt werden (Preuß, 1981; Köbberling, 1994; Boban \& Hinz, 2003; Brugger-Paggi, 2003; Wember \& Prändl, 2009; Holzinger et al., 2011; LienhardTuggener et al., 2011; Arndt \& Werning, 2013), werden in den Interviews thematisiert und zwar mit Blick auf die Tatsache, dass solche festgelegten Zeiten (FSL 1, FSL 2, FSL 4, FSL 5) in den 
integrativ arbeitenden Schulen der Befragten (weitgehend) fehlen. Die Förderschullehrkräfte sprechen in diesem Zusammenhang allerdings nicht von fehlenden Team-, sondern fehlenden Beratungszeiten auch für einzelne Lehrkräfte. Um diese Beratungszeiten zu installieren sei eine Reduktion der Unterrichtsverpflichtung der kooperierenden Lehrkräfte (SL 2, Z. 297f) notwendig $^{58}$ (Köbberling, 1994; Berges, 1996; Brugger-Paggi, 2003).

\subsubsection{Kooperation}

Mit Blick auf die allgemeine Kooperation von Lehrkräften innerhalb der Regelschulen werden in den Interviews Dienstbesprechungen (SL 2; SL 3), Beratungsgremien, Konferenzen (SLFZ 1, Z. 88-90), stufenbezogene Förderkonferenzen (SL 2, Z. 44-49) sowie Förderausschüsse (RSL 3., Z. 120f) als institutionalisierte Formen der Kooperation (Idel \& Ullrich, 2013) erwähnt, die sich mit Fragestellungen der schulischen Integration befassen. Diese stellen also eine systemische Voraussetzung für die strukturierte Auseinandersetzung mit der Thematik dar. Die Nennung der Einbindung von Förderschullehrkräften in diese oder ähnliche Gremien als Qualitätskategorie für integratives Arbeiten wird auch in der sonderpädagogischen Forschung benannt (Prell \& Link, 1977; Mühl, 1987; Boban \& Hinz, 2003; Thiele, 2009; Lütje-Klose \& Urban 2014a, 2014b). Sie steht allerdings - weder in der Theorie, noch in der Praxis - bei Weitem nicht ähnlich intensiv im Fokus wie die spezifische Kooperation zwischen Regel- und Förderschullehrkräften.

In der sonderpädagogischen Forschung nur einmal aufgegriffen wird die Zusammenarbeit von Förderschullehrkräften untereinander als mögliche Qualitätssubkategorie inklusiver Schulentwicklung (Bürli et al., 2009). Diese wird laut Auskunft der Schulleitungen der Förder- und Beratungszentren im Saarland in Form von internen Arbeitskreisen als freiwilliger Kooperationsform ermöglicht, die mehrmals im Jahr angeboten werden (SLFZ 1, Z. 180-184; SLFZ 2, Z. 20-24). Als entsprechend stärker zu gewichtende Subategorie, die im Überlappungsbereich von Kooperation und Professionalisierung einzuordnen ist, kann also die intraprofessionelle Kooperation zwischen Förderschullehrkräften aufgefasst werden. Diese findet in den FZ des Saarlandes sowohl in institutionalisierter Form statt (bspw. bei Dienstbesprechungen, Gesamtkonferenzen oder schulinternen Fortbildungen der Förder- und Beratungszentren) als auch in freiwilliger Form (Arbeitskreise, Runde Tische mit Vernetzungspartnern (s. 4.3.6.3).

Darüber hinaus beschreibt die Schulleitung eines SFZ die Kooperation auf Schulleitungsebene, und zwar zwischen der Schulleitung eines Förderzentrums und den Schulleitungen der umliegenden Regelschulen, als Qualitätsbaustein (SLFZ 1, Z. 155-163). Diese Kooperationsform spielt in sonderpädagogischen Forschungsveröffentlichungen bislang keine Rolle und kann daher als neue Subkategorie aufgegriffen werden.

Alle weiteren Aussagen in den Interviews beziehen sich auf die spezifische Kooperation zwischen Förder- und Regelschullehrkräften. Als notwendige Voraussetzung für kooperatives Arbeiten beschreiben die Interviewpartner ähnlich wie Haeberlin et al. (1992) vor allem die Offenheit der Regelschule für die interprofessionelle Zusammenarbeit zwischen Regel- und Förderschullehrern (SLFZ 1, SL 1, SL 2), die - wie die Bewertung der Kooperation durch die Interviewpartner zeigt - in den Schulen offensichtlich insgesamt in sehr positiver Hinsicht vorhanden ist, was den Ergebnissen von Behrensen et al. (2012), Pröbstel und Soltau (2012) sowie

58 Vor Einführung der Inklusionsverordnung waren Beratungsstunden für zieldifferente Integrationsmaßnahmen vorgesehen. Diese wurden im Zuge der Inklusionsverordnung mit der Begründung abgeschafft, dass durch die Budgetierung der Stunden der Lehrkräfte und die feste Zuordnung an bestimmte Regelschulen keine gezielt ausgewiesenen Beratungsstunden mehr notwendig seien. 
Serke et al. (2014) entspricht, die belegen, dass (Grundschul-) Lehrkräfte sich Kooperation mit Förderschullehrkräften wünschen. Die Bewertung der Kooperation zwischen Förder- und Regelschullehrkräften erfolgt in den Interviews - von drei Ausnahmen abgesehen - durchweg positiv (SL 1, SL 3, SL 4, SL 5, RSL 1, RSL 2, RSL 3, RSL 5, FSL 1, FSL 2, FSL 3, FSL 4) Diese positive Bewertung hängt auch damit zusammen, dass eine Zusammenarbeit der Beteiligten zumindest teilweise auf Augenhöhe erfolgt (s. auch dazu Haeberlin et al., 1992), eine Subkategorie, die in den Interviews eine stärkere Gewichtung erfährt als im vorliegenden Kategoriensystem. Eine positive Bewertung der Zusammenarbeit hängt nach Auffassung von SL 1 u.a. damit zusammen, dass durch die Unterstützung der Förderschullehrer eine Entlastung der Regelschullehrkräfte erfolgt, v.a. mit Blick auf die notwendige Binnendifferenzierung im Unterricht; ein Aspekt, der auch von Köppel (1984) sowie Arndt \& Werning (2013) aufgeführt wird bzw. von Laubenstein u.a. (2015) für die Kooperation allgemein. Von drei interviewten Schulleitungen wird darüber hinaus - als neue Subkategorie - die Bedeutung des besonderen Engagements der Förderschullehrkräfte betont.

Einige Aussagen der befragten Personen geben Hinweise darauf, dass der in der sonderpädagogischen Forschung untersuchte Zusammenhang zwischen der Offenheit zu Kooperation und den vorhandenen Rahmenbedingungen wie Ressourcen der Förderlehrkräfte und gemeinsamen Zeitfenstern für die Kooperation (Zeit für Absprachen, Beratung, Unterrichtsplanung etc.) (Dumke et al., 1989; Lütje-Klose et al., 2012; Arndt \& Werning, 2013; Serke et al., 2014a) auch von den Interviewten gesehen wird. So beschreiben FSL 1 und FSL 5 explizit den Mangel an im Stundenplan festgelegten Beratungszeiten mit den Regelschullehrkräften, während FSL 4 darauf hinweist, dass diese an ihrer Schule verankert seien.

Darüber hinaus wird ein Zusammenhang dahingehend beschrieben, dass die Kontinuität der Zusammenarbeit eine weitere wichtige Grundlage für funktionierende und qualitativ hochwertige Kooperation darstellt (SLFZ 2, FSL 5), was in Übereinstimmung mit Arndt \& Werning (2013) steht, die mangelnde Kontinuität als mögliches Problem mit Blick auf die Kooperation beschreiben (s. dazu auch Kontinuität der Zuweisung von FSL weiter oben). Als besonders wesentliche Grundlage für die Kooperation benennen drei Schulleitungen (SFLZ 2, Z. 152f; SL 4, Z. 50-52; SL 5; Z. 61-66) sowie eine Regelschullehrkraft (RSL 5, Z. 10f) das besonders hohe Engagement der Förderschullehrkräfte in den Regelschulen. Ähnlich wie beim Qualitätsbereich Schulklima (s.o.) spielt also auch hier die von Bryk u.a. (2010) beschriebene „work orientation“ eine wichtige Rolle.

Ein möglicher Baustein der interprofessionellen Kooperation wird darin gesehen, dass ein Kompetenztransfer aus den Förderzentren in die Regelschulen hinein erfolgen kann (SLFZ 1, Z. 155-163), was - ähnlich wie der intraprofessionelle Kompetenztransfer innerhalb der Förderund Beratungszentren - als weitere Subkategorie im Überlappungsbereich zwischen Kooperation und Professionalisierung eingestuft werden kann. Erwähnt wird diese Form des Kompetenztransfers bislang u.a. durch Laubenstein u.a. (2015), die für die Schwerpunktschulen in RLP feststellen, dass $38 \%$ der Schulen angeben, dass dieser durch kollegialen Austausch erfolge.

Was die konkrete Umsetzung der Kooperation betrifft, beschreiben allerdings die Schulleitungen der Förderzentren (SLFZ 1, SLFZ 2), eine Schulleitung (SL 3) sowie eine Regelschullehrerin (RSL 2) auch die in der Kooperationsforschung beschriebene Angst der Regelschullehrkräfte vor Autonomieverlust (Reiser, 1984) oder - vorsichtiger formuliert - vor Beobachtung, die nach Wellendorf (1967), Schley (2002), Fussangel \& Gräsel (2012) oder Reh (2008) damit zusammenhängen kann, dass der Lehrerberuf grundsätzlich von einem hohen Maß an Autonomie und Individualität geprägt ist. 
Unter 3.4.2 wurde beschrieben, dass Kooperation zwischen Lehrkräften auf unterschiedlichen Niveaustufen stattfinden kann. Mit Blick auf diese Niveaustufen lässt sich die Umsetzung nach Aussagen der Befragten in ihren Schulen vor allem den Stufen „Austausch“ und „Kooperation“ (Fussangel \& Gräsel, 2012) bzw. „Co-Activity“, „Cooperation“ und „Coordination“ (Lütje-Klose \& Willenbring, 1999) zuordnen. Dabei nimmt nach Auffassung mehrerer Interviewpartner (SL 1, SL 3, SL 4, RSL 1, RSL 3, RSL 4) vor allem die Beratung der Regel- durch die Förderschullehrkräfte einen hohen Stellenwert ein und wird als selbstverständlich bezeichnet. Darüber hinaus wird - ähnlich wie in der Studie von Dumke et al. (1989) auch der Austausch von Material oder Erfahrungen als Kooperationselement erwähnt (RSL 5).

Die unterrichtsbezogene Zusammenarbeit im engeren Sinn umfasst hinsichtlich der gemeinsamen Unterrichtsplanung nach Aussagen dreier Lehrkräfte ebenfalls vor allem die Vorbereitung differenzierter Materialien bzw. Arbeitsaufträge durch die Förderschullehrkräfte (RSL 1, RSL 2, RSL 5, FSL 2, FSL 4), nicht aber die u.a. von Halfhide (2009) beschriebene gemeinsame Unterrichtsplanung im engeren Sinn, für die gemeinsame Vorbereitungszeiten vorhanden sein müssten (s. dazu Ressourcen).

Bei den Ausführungen zur gemeinsamen Unterrichtsdurchführung wird deutlich, dass diese vor allem durch die Co-Teaching-Formen „One teach - one observe“ (RSL 2, FSL 2) bzw. „One teach - one assist“ geprägt ist, so wie auch Magiera \& Zigmond (2005) und Mc Duffie (2012) dies in ihren Studien beschrieben haben. Aus den Aussagen der Interviewpartner geht dies vor allem indirekt hervor, da Förderschullehrkräfte zwar einen Teil ihrer Unterrichtszeit in den Regelschulen im gemeinsamen Unterricht verbringen, für eine gemeinsame Vorbereitung allerdings keine Zeit vorgesehen ist. Das gemeinsame Unterrichten im engeren Sinn wird zwar gewünscht (RSL 3, RSL 5, FSL 1, FSL 2, FSL 3) und in Einzelfällen - verknüpft mit positiven Erfahrungen - auch praktiziert (FSL 1, FSL 3, FSL 5, RSL 3), wird aber u.a. aufgrund der Ressourcensituation als nicht üblich beschrieben, ein Ergebnis, das sich mit dem von Gebhardt et al. (2013) deckt. Gleichzeitig entspricht dieses Ergebnis auch denen der Studien, die belegen, dass der Wunsch nach stärkerer Kooperation von Lehrkräften untereinander sehr viel intensiver ausgeprägt ist als deren tatsächliche Umsetzung (Dieckmann et al., 2008; Fussangel \& Gräsel, 2012; Pröbstel \& Soltau, 2012; Arndt \& Werning, 2013; Soltau \& Mienert, 2013; Serke et al., 2014a). Ebenso wird die von Arndt und Werning (2013) aufgeführte nachrangige Funktion von Förderschullehrkräften im gemeinsamen Unterricht von einer Förderschullehrkraft auf den Punkt gebracht, die formuliert: „[...] wenn ich darf, unterrichte ich auch mal“ (FSL 4). Im Abgleich zu bisherigen Forschungsveröffentlichungen als neu einzustufen ist der Hinweis, dass die Anwesenheit von Förderschullehrkräften den Unterricht stören kann, wenn diese individuelle Förderung umsetzen wollen (SLFZ 1, Z. 103-105; RSL 2, Z. 110-115). Erwähnung findet darüber hinaus auch die Entlastung, die für die RSL durch die Unterstützung durch die FSL (bspw. durch die Erstellung differenzierter Arbeitsblätter) entsteht (SL 1, Z. 105-110, Z. 113115). Als entlastender Moment wird von einer Regelschullehrkraft wahrgenommen, dass sie die Unterrichtsverantwortung auf eine FSL übertragen kann und dann Zeit habe sich außerhalb des Klassenraums anderen Aufgaben zu widmen (RSL 5, Z. 106f), was sie als Gewinnung von Freiraum kennzeichnet.

Die kooperative Bewertung von Schülerleistungen wird von einer Regelschulehrkraft als Qualitätsmerkmal und damit neue Qualitätssubkategorie erwähnt (RSL 1).

Eine Möglichkeit der Vereinfachung unterrichtsbezogener Kooperation, die in Forschungsveröffentlichungen bislang nicht aufgeführt ist, stellt das inhaltlich parallele Arbeiten innerhalb einer Jahrgangsstufe als neue Subkategorie dar, das von einer Schulleiterin beschrieben wird 
und der Organisationsentwicklung zugeordnet werden kann. Dieses kann eine Erleichterung der Arbeitsorganisation sowohl für die RSL wie für die FSL darstellen. Eine wichtige Rolle spielt für die Interviewpartner gegenseitige Beratung als kooperatives Element. Diese normalerweise eher ausschließlich den Förderschullehrkräften zugeschriebene Aufgabe (Reiser et al., 1984; Dumke et al., 1989) wird mit insgesamt 11 Nennungen häufig aufgegriffen und als selbstverständlich und - mit Blick auf das gemeinsame pädagogische Arbeiten mit den Schülerinnen und Schülern - Gewinn bringend beschrieben. Inhalte der gegenseitigen Beratungsgespräche würden sich dabei nicht nur auf Schüler mit spF beziehen. Auch Hospitationen werden als Baustein von Kooperation zwischen RSL und FSL erwähnt (RSL 2, Z. 74-76), ähnlich wie bei Berges (1996), Boban und Hinz (2003) oder Wernstedt und Ohnesorg (2010).

Die zwei Bestandteile der gemeinsamen Unterrichtsnachbereitung (Halfhide, 2009), die Erwähnung finden, betreffen die Beratung im Anschluss an Beobachtungssituationen (SL 4, Z. 56-58) sowie die kooperative Leistungsbewertung von Klassenarbeiten (RSL 1, Z. 207209). Sie beziehen sich allerdings nicht auf eine gemeinsame Reflexion von Unterrichtssituationen im engeren Sinn. Diese u.a. von Hattie so intensiv geforderte Tätigkeit (2013) bleibt also offensichtlich weitgehend aus.

Zusammengefasst formuliert findet die in sonderpädagogischen Veröffentlichungen vielfach aufgeführte Qualitätskategorie einer gemeinsamen Unterrichtsvor- und -nachbereitung sowie -durchführung (Heese, 1977; Müh, 1987; Husinsky, 1994; Berges, 1996; Boban \& Hinz, 2003; Deutsches Schulamt, 2004; Bürli et al., 2009; Holzinger et al., 2011) in der Praxis der Interviewpartner kaum Berücksichtigung. Insgesamt legen die Ausführung zur gemeinsamen Unterrichtsgestaltung nahe, dass Kooperation von Lehrkräften in den beteiligten Schulen tatsächlich - wie von Kolbe \& Reh (2008) erfasst - „seltener und schwieriger - aber nach Einschätzung der Lehrkräfte auch wertvoller - wird je ,näher" man dem Unterricht als ihrem Kerngeschäft“ kommt (zitiert nach Lütje-Klose \& Urban, 2014a, S. 120). Eine mögliche Ursache dafür kann in den unzureichenden personellen Ressourcen gesehen werden (geringe Anzahl von Förderschullehrerstunden je Klasse, fehlende gemeinsame Beratungszeiten). Zur Kooperation in Teams werden keine Aussagen getätigt.

Kaum Erwähnung findet in den Interviews die in der sonderpädagogischen Forschung häufig thematisierte Rollen- und Aufgabenklärung zwischen Regel- und Förderschullehrkräften. Aspekte, die diesbezüglich angesprochen werden, betreffen vor allem den (verunsichernden) Wandel des Berufsbildes der Förderschullehrer, ähnlich wie er auch von Reiser (1998) beschrieben wurde. So greift SLFZ 2 die Perspektive von Förderschullehrkräften auf, die gerne als Klassenleitungen tätig sind und die Sorge haben, diese Aufgabe im Rahmen schulischer Integration nicht mehr ausüben zu können. Da sie ihre Tätigkeit in einer Förderschule als sinnvoll einstufen würden, stelle sich der Schritt hin zu integrativem Arbeiten in einer Regelschule für diese Kollegen als „meilenweit“ dar. Dagegen hätten Lehrkräfte, die gerne vernetzt arbeiten, weniger Probleme sich auf diese Arbeitsweise einzustellen (SLFZ 2). Das Problem, das durch schulische Integration für Förderschullehrkräfte ein neues Tätigkeitsfeld entstanden ist, kann also zu Problemen im beruflichen Selbstverständnis führen (SLFZ 1), die - wie SLFZ 1 und SLFZ 2 zu bedenken geben - allerdings auch die Regelschullehrkräfte betreffen. Die in sonderpädagogischen Veröffentlichungen als Qualitätssubkategorie aufgeführte Flexibilität (Preuß, 1981; Antor, 1987; Bächthold et al., 1990; Reiser et al., 2007; Schwager, 2011; Serke et al., 2014; Lütje-Klose \& Urban, 2014b) wird von einer der befragten Schulleitungen der Förder- und Beratungszentren trotz der oben angesprochenen Einschränkungen eher den Förderschullehrkräften zugesprochen (SLFZ 2), da diese gewohnt seien mit Freiräumen zu arbeiten. Anstelle der ebenfalls ge- 
forderten Klarheit mit Blick auf die Aufgabenklärungen geben die Aussagen der Interviewten eher Hinweise darauf, dass diese Klärung mit Aushandlungsprozessen verknüpft ist, die von der Offenheit und dem Engagement einzelner Lehrkräfte abhängen, so dass die Förderschullehrkräfte zum Teil sehr unterschiedliche Aufgaben wahrnehmen (Bsp.: vorwiegend Beratung, Einzel- oder Gruppenförderung, Unterstützung einzelner Schüler im Unterricht etc.). Dies entspricht zwar der Vielfalt der Aufgaben, die auch in bisherigen sonderpädagogischen Studien erfasst werden konnte (Preuß, 1985; Reiser et al., 1984; Brugger-Paggi, 2003; Bürli et al., 2009; Wember \& Prändl, 2009; Holzinger et al., 2011), verdeutlicht aber, dass eine klare Strukturierung der Aufgaben für die Beteiligten nicht immer gegeben ist. Ein sehr spezifisches Problem spricht SLFZ 1 an, der formuliert, dass den Förderschullehrkräften mit dem Aufgabenfeld schulischer Integration eine Aufgabe „aufgehalst und aufgeladen“werde, die sie aufgrund der Größe des Problemfelds gar nicht bewältigen könnten. Inhaltlich lässt sich hier eine Querverbindung zu Wenning (2013) herstellen, der eine unzulässige Zuschreibung vonVerantwortung an pädagogisch Handelnde kritisch beschreibt.

\subsubsection{Professionalisierung}

Die bereits seit Ende der 70er Jahren geforderte (Prell \& Link, 1977; Reinartz \& Sander, 1982) um sonderpädagogische Inhalte erweiterte universitäre Ausbildung der Regelschullehrkräfte wird von einer Regelschullehrkraft als nicht vorhanden kritisiert. Auf die Frage, in welcher Form sich die Schulen bereits mit Anforderungen des gemeinsamen Unterrichts befasst haben (Bsp.: pädagogische Konferenzen, Pädagogische Tage, Fortbildungen) bzw. welcher Fortbildungsbedarf gesehen wird, werden dagegen vielfältige Aussagen getätigt. Pädagogische Tage für ganze Kollegien, die in der sonderpädagogischen Forschung für die Entwicklung inklusiver Schulen als wesentlich angesehen werden (Erbring \& Amrhein, 2009; Arndt \& Werning, 2013; LütjeKlose \& Urban, 2014b), werden laut Aussagen der zwei Schulleitungen an den sonderpädagogischen Förder- und Beratungszentren durchgeführt, nicht aber mit einer eindeutig auf integrative Unterrichtung hin orientierten Schwerpunktsetzung an den Regelschulen, an denen die Interviews durchgeführt worden sind. Drei Personen weisen darauf hin, dass an solchen Tagen Themen im Mittelpunkt standen, die zumindest mit dieser Unterrichtung zusammenhängen, wie ,individuelle Förderung“ (SL 4), „Unterrichtsmethoden“ (RSL 3), „Regeln“ (FSL 2) oder „KESS erziehen“59 (RSL 5). Drei Förderschullehrkräfte äußern sich in der Hinsicht kritisch zu diesem Thema, dass sie gemeinsame Fortbildungen mit einem ganzen Kollegium für wichtig halten, diese aber entweder von der Schulleitung oder vom Kollegium bislang nicht gewünscht werden (FSL 3, FSL 4, FSL 5). Die bspw. von Mutzeck (1987), Reiser (1987) oder Lütje-Klose \& Urban (2014b) beschriebenen Vorteile der Fortbildung von Teams oder ganzen Kollegien mit Blick auf die Umsetzung der Fortbildungsinhalte im Unterrichtsalltag greifen derzeit an den befragten Schulen also nur teilweise. Ein in der Forschung wenig beachteter Teilbereich bezieht sich auf den Kompetenztransfer, der zwischen Regel- und Förderschullehrkräften, zwischen den Schulleitungen der Förderzentren bzw. Regelschulen in deren eigenen Reihen bzw. auch zwischen den Schulleitungen der Förderzentren und Regelschulen untereinander (SLFZ 1) erfolgen kann (s. dazu Kooperation weiter oben).

Während der konkrete Besuch von Fortbildungen durch einzelne Lehrkräfte lediglich drei Mal erwähnt wird (SL 5, RSL 5, FSL 4), wird der Fortbildungsbedarf von einer ganzen Reihe der Interviewpartner beschrieben und insgesamt als hoch eingeschätzt. Dieser bezieht sich auf systemische Aspekte der Inklusion (SLFZ 1), auf die Thematik der Inklusion insgesamt (SL 3,

59 s. dazu https://www.kess-erziehen.de/ 
SL 4), auf den fachrichtungsbezogenen Ausbildungsbedarf der Förderschullehrkräfte (SLFZ 2), auf Diagnostik (SLFZ 2), auf sonderpädagogisch fachspezifischen Fortbildungsbedarf der Regelschullehrkräfte (SL 1, SL 2, RSL 1, RSL 5), fachdidaktischen Fortbildungsbedarf (FSL 1), Bausteine der Unterrichtsentwicklung wie Binnendifferenzierung (SL 1, FSL 1, FSL 2, FSL 5), Methoden (SL 1, RSL 2, FSL 1, FSL 3) oder die Öffnung von Unterricht allgemein (FSL 1, FSL 2). Er ist also so vielseitig, wie dies auch in bisherigen sonderpädagogischen Veröffentlichungen beschrieben wurde (Feuser \& Meyer, 1987; Dumke et al., 1989; 2003; Deutsches Schulamt, 2004; Specht et al., 2006; Bürli et al., 2009; Thiele, 2009; Wember \& Prändl, 2009; Speck, 2010; Arndt \& Werning, 2013; Lütje-Klose \& Urban 2014b) und umfasst sowohl allgemein- wie sonderpädagogische Inhalte. Möglicherweise besteht allerdings - worauf die unterschiedliche Anzahl von Aussagen zum Besuch von Fortbildungen vs. Fortbildungsbedarf hinweist - ein Unterschied zwischen der Erkenntnis, dass Fortbildungen in diesen Bereichen hilfreich seien und der konkreten Nutzung des Fortbildungsangebots. Die Schulleitung eines Förderzentrums hebt als besonderen Fortbildungsbedarf hervor, dass systemische anstelle einzelfallbezogener Sichtweisen in den Kollegien verankert werden müssten. Dabei gehe es nicht in erster Linie darum, dass Förderschullehrkräfte weiterhin „unterstützenden Unterricht “ mit Schülern mit spF umsetzen sollten, sondern dass sie gemeinsam mit den Regelschullehrkräften Unterricht dahingehend zu verändern hätten, dass diese Schüler innerhalb des Unterrichts gefördert werden könnten. Eine Förderschullehrkraft äußert sich kritisch bezüglich der in Ausund Fortbildung zwar erlernten, in der Praxis aber nur schwer umsetzbaren flexiblen Gestaltung des Unterrichts (FSL 4), eine Aussage, die mit den Forschungsergebnissen von Reiser (1987) übereinstimmt. Die von Jäntsch et al. (2015) im Rahmen der PING-Studie erhobenen Transferschwierigkeiten von Fortbildungsinhalten in die unterrichtliche Praxis werden von einer Förderschullehrkraft aufgegriffen.

\subsubsection{Ergebnisse im Bereich der Personalentwicklung}

Die interviewten Personen beschreiben in ihren Stellungnahmen sehr ausführlich und umfassend die unterschiedlichen Haltungen von Lehrkräften in den integrativen Schulen. Deutlicher als die mit Blick auf die Kooperation von Lehrkräften geschilderte große Offenheit der Beteiligten weisen diese Aussagen eine eher skeptisch-differenzierte Haltung der Regelschullehrkräfte, aber auch der Förderschullehrkräfte, zur Arbeit in einer inklusiven Schule auf.

Eine offene Haltung von Lehrkräften bzw. Kollegien wird mit Motivation und Engagement begründet oder damit, dass neue Ideen aus Fortbildungen aufgegriffen werden. Mit Blick auf eine Schule wird von einer Vorreiterschaft in Sachen Integration gesprochen, an einem Förderund Beratungszentrum führt die positive Einstellung der Förderschulkollegen zu einer kontinuierlichen inhaltlichen Weiterentwicklung der Arbeit. Manche der Befragten sehen Vorteile schulischer Integration wie die Schärfung des Blicks auf Kinder mit Schwächen insgesamt bzw. die Umsetzung eines Menschenrechts. Kinder würden - unter sozialem Aspekt - lernen Unterschiedlichkeiten zu akzeptieren und miteinander selbstverständlich umzugehen. Teilweise wird auch eine pragmatische Einstellung dahingehend vertreten, dass Schülern eben geholfen werden müsse und man gemeinsam mit den Förderschullehrkräften schaue, wie das möglich sei. Eine skeptische bzw. ablehnende Haltung wird ebenfalls beobachtet. Begründungen werden in der unzureichenden Ressourcensituation, dem Zeitaufwand für Vernetzungsarbeit sowie darin gesehen, dass die Aufgabe für die Regelschullehrkräfte eine weitere Zusatzaufgabe darstelle und daher als belastend wahrgenommen werde. Auch nach 25 Jahren Integrationserfahrung im Saarland würden RSL und SL SuS mit SpF zum Teil noch nicht als „ihre“ Schüler ansehen. 
Offenheit sei zwar den Kindern gegenüber gegeben, nicht aber mit Blick auf unterrichtsbezogene Veränderungen. Sorgen bereitet die steigende Anzahl der SuS mit spF in Regelschulen. Ein Schulleiter einer Grundschule geht davon aus, dass alle Kollegen froh wären „wenn die Förderkinder in die entsprechende Schule gehen dürften “. Eine Reihe von Beteiligten sieht einen engen Zusammenhang zwischen dem Mangel an personellen Ressourcen und einer ablehnenden Haltung der Kollegien. Die Ergebnisse mit Blick auf diese ablehnende Haltung im Zusammenhang mit Rahmenbedingungen stimmen mit denen überein, die Opalinski (2014) in ihrem Forschungsüberblick sowie aufgrund ihrer eigenen Befragung von Thüringer Lehrkräften erheben konnte. Vereinzelt wird die Meinung vertreten, dass die Förderschule für einzelne Schüler ein günstiger Förderort sein könne, da es dort kleinere Klassen gebe (RSL 1, RSL 3), kein so hohes Maß an selbständigem Arbeiten erwartet werde (RSL 3) und eine intensivere Ansprache und Unterstützung durch Erwachsene möglich sei (SL 3). SLFZ 1 spricht davon, dass sich auch Förderschulen zu inklusiven Schulen weiterentwickeln müssten. Die Haltungen der befragten Personen schließen also eine große Bandbreite unterschiedlicher Positionen ein. Positive Einstellungen werden nach Auffasssung einiger interviewter Personen insbesondere mit dem grundlegenden und zum Teil pragmatischen Engagement von Lehrkräften in Verbindung gebracht, ähnlich wie Dyson (2010) dies für gute (inklusive) Schulen beschreibt. Anders als bei Amrhein (2011) können aufgrund des Querschnittscharakters der Erhebung keine Schlussfolgerungen auf Veränderungsprozesse hinsichtlich der Einstellungen aufgrund von Erfahrungen mit schulischer Integration gezogen werden.

\subsubsection{Interpretation der Ergebnisse zur Unterrichtsentwicklung}

\subsubsection{Ressourcen im Bereich der Unterrichtsentwicklung}

Die Qualitätskategorie Klassengröße wird in den Interviews insgesamt zwölf Mal aufgegriffen, was insofern bemerkenswert ist, als dass danach nicht explizit gefragt wurde. Inhaltlich ergibt sich in den Aussagen ein sehr hohes Maß an Überschneidungen mit den Klassengrößenangaben der Sonderpädagogik. So weisen zwei Schulleitungen, ein Förder- und zwei Regelschullehrer (SL 1, SL 2, RSL 1, RSL 5, FSL 5) darauf hin, dass die im Saarland zum Teil üblichen Klassen mit bis zu 29 Schülerinnen und Schülern für integratives Arbeiten zu groß seien. Wünschenswert seien dagegen Klassen mit 20-22 Schülern (E 3, SL 3, RSL 2). Die genannte Anzahl entspricht damit exakt der von verschiedenen sonderpädagogischen Autoren geforderten Klassengröße von etwa 20 Schülern für integrative Klassen (Heese, 1977; Weigt, 1977; Speck et al., 1978; Mühl, 1987; Dumke et al., 1989; Köbberling, 1994; Brugger-Paggi, 2003; Thiele, 2009; Wember \& Prändl, 2009; Speck, 2010), während die tatsächliche Klassengrößen mit der im Saarland verbindlichen Obergrenze für die Klassenbildung (im SEK I-Bereich) übereinstimmen.

Auch die inhaltlichen Begründungen, die für kleinere Klassen angeführt werden, stimmen mit Forschungsergebnissen überein, wobei sie diese quasi indirekt bestätigen, indem sie ungünstige Voraussetzungen in großen Klassen beschreiben. So problematisieren sie die von Haselbeck (2007) benannte Möglichkeit in kleinen Klassen mehr Zeit für die Hinwendung zu einzelnen Schülern zu haben, indem sie beschreiben, dass diese Form der individuellen Zuwendung in großen Klassen nicht ausreichend möglich sei (FSL 5, RSL 1, RSL 5, SL 1). Thematisiert wird dadurch ein Zusammenhang zwischen der Größe der Klassen und Möglichkeiten präventiven bzw. binnendifferenzierten Arbeitens (SL 1, RSL 5, FSL 5). Während - wie Avenarius (2003), Haselbeck (2007) und v. Saldern (2011) durch ihre Studien belegen - Lehrkräfte in kleineren Klassen ein höheres Maß an Ruhe und damit Störungsfreiheit schätzen, verweisen eine Schul- 
leitung (SL 1) und ein Elternteil (E 3) auf die Lautstärke in großen Klassen, durch die es sowohl Lehrkräften wie auch Schülern schwer falle sich auf das Unterrichtsgeschehen zu konzentrieren. Darüber hinaus fehle - wie in der Studie durch von Saldern (2011) beschrieben - die Möglichkeit der Flexibilität der Raumgestaltung (s. dazu Ressourcen OE) (SL 3). Außerdem verweist eine Schulleitung auf den höheren Vorbereitungsaufwand einer Lehrkraft in heterogen zusammengesetzten Klassen (SL 1), einen Aspekt, den von Saldern (2011) als größere zeitliche Belastung von Lehrkräften in diesen Klassen benennt (s. dazu Differenzierung/Individualisierung). Die Ausführungen zur Klassenzusammensetzung problematisieren die in den sonderpädagogischen Veröffentlichungen geforderte Heterogenität der Gesamtklasse (Feyerer, 1998; Bleidick, 1999; Bless, 2003; Holzinger et al., 2011). Insbesondere die Elternteile äußern diesbezüglich Sorgen hinsichtlich der Integration von Kindern mit schweren Behinderungen (E 1 und E 2) sowie mit Blick auf einen zu hohen Anteil von Kindern mit sonderpädagogischem Förderbedarf in einer Klasse (E 3, SL 1, RSL 1). Diese Aussagen beinhalten also eine Verbindung zur Forderung von Brugger-Paggi (2003), dass lediglich ein Schüler mit schweren Beeinträchtigungen einer Klasse zugewiesen werden solle, und zu den Forderungen nach der Vermeidung der "Ansammlung von Problemkindern in einer Klasse“ (Innerhofer \& Klicpera, 1991; ähnlich u.a. bei Brugger-Paggi, 2003; Holzinger et al., 2011) bzw. nach einer „ausgewogenen Klassenzusammensetzung “" (Wocken, 1987; Feyerer, 1998). Zwei Lehrkräfte (RSL 1, FSL 3) sowie eine Schulleitung (SL 1) beschreiben in diesem Zusammenhang die ihrer Einschätzung nach gestiegene Komplexität der Anforderungen an Lehrkräfte in den Klassen allgemein, d.h. unabhängig von den Schülerinnen und Schülern mit sonderpädagogischem Förderbedarf (Bsp.: „ADHSKinder“ „momentane schiefe Seelenlage“ (SL 1), „Lernprobleme“, „Rechtschreibprobleme“, „familiäre Probleme“ (RSL 1); „Wahrnehmungsgeschichten“ (FSL 3)). Insbesondere an diesen Beispielen zeigt sich das von Trautmann und Wischer (2011) beschriebene mögliche Dilemma zwischen institutionellen Anforderungen und Individualisierung in Schulklassen, das durch eine sehr heterogene Zusammensetzung von Gruppen verstärkt werden kann. Insgesamt belegen die Ausführungen der Interviewpartner zu den Qualitätssubkategorien Klassengröße sowie Klassenzusammensetzung die von von Saldern (2011) aufgeführte Bedeutung derselben in der Wahrnehmung der Lehrkräfte bzw. in diesem Fall auch der Schulleitungen.

Die Bündelung von Kindern oder Jugendlichen mit sonderpädagogischem Förderbedarf in einzelnen Klassen stellt in diesem Zusammenhang nach Aussage von zwei Schulleitungen lediglich eine pragmatische Möglichkeit dar mit den unzureichenden personellen Ressourcen (Förderschullehrerstunden) in den Regelschulen umzugehen. Sie entspricht nicht den u.a. von Wocken (1987) oder Feyerer (1998) geforderten Qualitätsansprüchen einer vielfältigen und mit Blick auf die Anteile der Schüler mit Behinderungen realitätsnahen Klassenzusammensetzung.

\subsubsection{Schulklasse als sozialer Raum}

Auch zum Klassenklima und zum sozialen Lernen gab es in den Interviews keine explizite Fragestellung. Deutlich wird allerdings in den Aussagen von drei Schülern und Schülerinnen, dass diese sich offensichtlich in ihren Klassen wohlfühlen und die Mitschüler sowohl ihr Anderssein akzeptieren als auch die Tatsache, dass sie teilweise an anderen Aufgabenstellungen arbeiten (S 2, S 3, S 4). Während Klassenklima und soziales Lernen v.a. aus Sicht der Allgemeinen Pädagogik eine zentrale Rolle spielen (von Saldern, 1987; Brophy, 2010; Muijs \& Reynolds, 2011; Helmke, 2015; Huber, 2017), werden diese als Qualitätsmerkmale integrativen Unterrichts von den befragten Lehrkräften und Schulleitungen nicht aufgegriffen. Indikatoren für diese Subkategorien wie das Erleben guter sozialer Beziehungen (Bless, 2003) oder die Vermittlung positi- 
ver sozialer Erfahrungen (Eckermann \& Heinzel, 2013) werden allerdings durchaus benannt. Während Freundschaften in der Schule von allen SuS als vorhanden benannt werden, geben sie bis auf $S 2$ übereinstimmend an, dass nachmittägliche Kontakte aus unterschiedlichen Gründen selten bis gar nicht stattfinden. Dies stimmt etwa mit dem Ergebnis von Dumke et al. (1989) überein, das belegt, dass die Kontakte zwischen behinderten und nichtbehinderten Kindern nach Auskunft von Eltern zu $25 \%$ häufig und zu $75 \%$ nur gelegentlich gegeben sind.

\subsubsection{Klassenführung}

Das Thema Klassenführung wird fast ausschließlich von den befragten Schülern und Schülerinnen thematisiert, die auf die Frage, was im Unterricht anders sein müsse, damit sie besser lernen können übereinstimmend (S 1, S 2, S 3, S 4, S 5) formulieren, dass es leiser sein müsse, damit sie konzentrierter und damit besser arbeiten könnten, so dass als neue Subkategorie die Ruhe in den Klassen aufgefasst werden kann. Ihre Aussagen stützen sozusagen als Annahme die Forschungsergebnisse, die belegen, dass eine wirksame Klassenführung in sehr hohem Maß mit den Leistungen der Schüler korreliert (u.a. Brophy, 2006; Helmke, 2010; Hattie, 2013), u.a. da sie den Anteil echter Lernzeit erhöht (Brophy, 2010; Muijs \& Reynolds, 2011). Ein Schüler bezieht sich in seinen Aussagen auf Regeln in der Klasse, wobei er durch die Einführung von Regeln einerseits eine positive Veränderung mit Blick auf ruhiges Arbeiten erkennt, gleichzeitig aber differenziert, dass dies nur bei den Klassenlehrern funktioniert habe (S 3). Dies kann wiederum als Hinweis auf die vor allem von Hattie (2013) belegte Bedeutung der Beziehungsebene zwischen Lehrkräften und Schülern aufgefasst werden und zeigt gleichzeitig auf, dass die u.a. von Booth \& Ainscow (2003), Wember \& Prändl (2009) oder Helmke (2015) als Qualitätssubkategorie benannten Regeln in einer (inklusiven) Klasse nur dann Auswirkungen auf den Unterricht haben, wenn deren Einhaltung konsequent eingefordert wird. In diesem Zusammenhang richtet sich eine Schüleraussage auf die Annahme, dass diese Konsequenz vor allem „bei Referendaren oder [...] bei neuen Lehrern“ (S 2, Z. 133-137) noch nicht gegeben sei und sich erst „mit den Jahren“ entwickle. Er weist darüber hinaus darauf hin, dass Unruhe dadurch entstehen kann, dass der Unterricht nicht auf die Schüler ausgerichtet ist (S 2, Z. 126-128). Der von den Schülern angenommene Zusammenhang zwischen einer ruhigen Arbeitsatmosphäre und ihren Lernmöglichkeiten ist deshalb interessant, da damit eine Voraussetzung für Lernprozesse im (inklusiven) Unterricht benannt wird, die in der sonderpädagogischen Forschung nur eine untergeordnete Rolle spielt (außer bspw. bei Textor, 2009; Hennemann \& Hillenbrand, 2010; Casale et al., 2016). Die von einem Schüler benannte Konsequenz von Lehrkräften lässt sich mit dem von Marzano und Marzano (2003) als günstig beschriebenen angemessenen Maß von Dominanz und Kooperation sowie der Beziehungsabhängigkeit von Schülerverhalten in Verbindung bringen. Ähnlich wie das Klassenklima oder das soziale Lernen steht also auch die Klassenführung für die befragten Lehrkräfte oder für die Schulleitungen als möglicher Qualitätsbereich inklusiven Unterrichts nicht im Fokus, obwohl dieser in der allgemeinen Unterrichtsforschung als Schlüsselmerkmal bezeichnet wird (Muijs \& Reynolds, 2005; Borich, 2006, 2007; Evertson \& Weinstein, 2006; Helmke, 2010; Hattie, 2013).

\subsubsection{Didaktik}

Didaktische Fragestellungen scheinen für die Interviewpartner ebenfalls nicht in dem Bereich zu liegen, der die besondere Qualität integrativen Unterrichts kennzeichnet. Lediglich zwei Lehrkräfte, die an derselben Schule tätig sind, beschreiben die gemeinsame Einigung auf die Umsetzung eines einheitlichen Rechtschreibkonzepts, das positive Auswirkungen auf den Rechtschreibunterricht hat. Sie greifen damit als einzige Lehrkräfte einen fachdidaktischen 
Aspekt auf. Die Auswahl von Lerninhalten, ihre inhaltliche Reduktion, Strukturierung und Sequenzierung wird ansonsten nicht weiter thematisiert. Curriculare Fragen, die als Folge der UN-BRK bspw. mit Blick auf die Entwicklung „inklusiver“ Curricula (Dt. Städtetag, 2011) oder von Bryk et al. (2010) als hochrelevant hinsichtlich ihrer Auswirkungen auf Lese- und Mathematikleistungen beschrieben werden, werden von den Interviewpartnern nicht als qualitätsrelevant erwähnt. Zum Teil werden didaktische Aspekte eher im Zusammenhang mit binnendifferenzierten Lernangeboten (s. 4.3.8.6) problematisiert, ein Ansatz der auch bei Feuser (1989, 1995, 1998), Wocken $(2009,2012)$ oder Seitz (2006) eine Rolle spielt.

Das Thema der klaren Strukturierung von Unterricht als Qualitätskategorie (Bless, 2003; Meyer, 2004; Wember \& Prändl, 2009; Helmke, 2015) wird von keinem der Interviewpartner aufgegriffen.

\subsubsection{Methodik}

Die von drei Interviewpartnern benannte notwendige „Mischung zwischen allem“ (RSL 1, Z. 111) bzw. Mischung zwischen frontalen und offenen Unterrichtsphasen (RSL 2, RSL 4) greift die u.a. von Matthes (2011) geforderte Abwechslung verschiedener Methoden bzw. die von Hattie (2013) als effizient eingestufte Kombination frontaler Phasen mit schülerzentrierten Phasen im (inklusiven) Unterricht auf. Eine Lehrkraft thematisiert darüber hinausgehend die Notwendigkeit im inklusiven Unterricht flexibel und situationsorientiert vorgehen zu können (RSL 1).

Interessant sind mit Blick auf die Interpretation der Interviewaussagen zunächst die unterschiedlichen Sichtweisen von Schülern und Lehrkräften bzw. Schulleitungen auf den Frontalunterricht. Während die drei Schüler, die sich zu dieser Methode äußern, vor allem die Vorteile dieser Methode für ihre eigenen Lernprozesse beschreiben, da sie mit Hilfe guter Erklärungen und Visualisierungen der Lehrkräfte in die Lage versetzt werden Inhalte zu verstehen und im Anschluss korrekt für sich zu verinnerlichen (S 1, S 2, S 3), problematisieren zwei Lehrkräfte und eine Schulleitung diese Methode dahingehend, dass individualisierte Lernangebote während frontaler Phasen nicht gut angeboten werden können, da diese Geräusche verursachen (RSL 1), bzw. es hohe Anforderungen an eine Lehrkraft stellt, dass diese eine Instruktionsphase so gestaltet, dass deren Inhalte bei Schülern mit sehr unterschiedlichen Lernvoraussetzungen überhaupt „ankommen“ (SL 1, FSL 3). Die Aussagen der Schüler verweisen also auf die in der Forschung belegten möglichen positiven Effekte der Methode (Kirschner et al., 2006; Muijs \& Reynolds, 2011), während die von den Lehrenden erwähnten Probleme instruktiven Unterrichtens als Hinweis darauf aufgefasst werden können, dass dieser einen hohen Anspruch an die Fähigkeiten von Lehrkräften darstellt Themen sachlich und inhaltlich klar für eine heterogene Lerngruppe aufzubereiten und darzustellen.

Die in der sonderpädagogischen Forschung aufgeführte Qualitätskategorie offene Unterrichtsformen (u.a. Berges, 1996; Feyerer, 1998; Specht et al., 2006; Thiele, 2009; Wember \& Prändl, 2009) wird von den Interviewpartnern in vielfältiger Form aufgegriffen, und zwar sowohl in positiver Hinsicht mit Blick auf das dadurch erhöhte Maß an selbständigem bzw. auch kooperativem Arbeiten der Schüler (RSL 1, RSL 2), als auch in negativer Hinsicht bezüglich der nur selten erfolgenden Umsetzung bzw. Erkenntnis der Notwendigkeit offenen Unterrichts in den Schulen der Befragten (SL 2, RSL 5, FSL 1). Der von Hage (1985), Wiechmann oder Helmke (2010) festgestellte hohe Anteil des Frontalunterrichts im Vergleich zu offenen Unterrichtsformen scheint sich also insgesamt auch in den beteiligten integrativen Schulen zu bestätigen.

Als offene Lernformen konkret und mit mehr als einem Dutzend häufig als Qualitätsmerkmal integrativen Unterrichts benannt werden in den Interviews die in der sonderpädagogischen Forschung aufgeführten Mikromethoden wie bspw. Lerntheken, Stationen oder Wochenplanarbeit. 
Die Wochenplanarbeit wird dabei als besonders geeignete Methode beschrieben um sich einzelnen Kindern stärker zuwenden zu können (RSL 2). Dagegen bezieht sich nur eine Lehrkraft auf Partner- oder Gruppenarbeiten als Unterrichtsmethode (RSL 2). Interessant sind mit Blick auf die Verknüpfung mit Forschungsergebnissen wiederum die Aussagen der Schüler, die konkret dazu befragt wurden, welche Arbeitsform (Einzel-, Partner- oder Gruppenarbeit) sie für ihr eigenes Lernen bevorzugen. Dabei wird die Einzelarbeit von vier der fünf Schüler als bevorzugte Methode benannt. Auch die Partnerarbeit wird - ebenfalls von vier der fünf Schüler - als positiv bewertet, während die Gruppenarbeit zweifach kritisch gesehen wird, da diese für die Schüler irritierend sein kann, wenn unterschiedliche inhaltliche Aussagen anderer Schüler sich gegenüberstehen und die Arbeit dadurch aus Schülerperspektive nicht gut strukturiert erscheint (S 1, S 2). Die Organisation kooperativer Lernformen dahingehend, dass diese tatsächlich effiziente Lernprozesse auf Schülerseite ermöglichen, stellt im integrativen Unterricht also aus Sicht dieser einzelnen Schüler eine hohe Anforderung dar, die in übereinstimmender Form Ergebnis pädagogischer Forschung dazu ist (Huber, 2010; Helmke, 2010; Muijs \& Reynolds, 2011). Nicht die reine Einbeziehung kooperativen Lernens in (inklusiven) Unterricht (Berges, 1996; Specht et al., 2006; Textor, 2009) scheint also als Qualitätskategorie entscheidend, sondern die Sicherung der Voraussetzungen (u.a. soziale Kompetenzen der Schüler (Muijs \& Reynolds, 2011)) sowie die Art ihrer Umsetzung (u.a. Art der Aufgabenstellung, klare Aufgaben- und Rollenverteilung (Huber, 2010)).

Wockens Aussage, dass „Didaktik, Methodik und Unterrichtsentwicklung [...] in der integrativen Pädagogik ein Mauerblümchendasein geführt [haben]“(Wocken, 2009, S. 218) trifft mit Blick auf die in den Interviews benannten Qualitätskategorien also nur zum Teil zu. Während die Didaktik tatsächlich lediglich von zwei Lehrkräften aufgegriffen wird, spielen methodische Fragestellungen vor allem mit Blick auf die für diesen Unterricht notwendige Öffnung und Flexibilisierung sowie die Vielfältigkeit im methodischen Zugang (Boban \& Hinz, 2003; BruggerPaggi, 2003; Wember \& Prändl, 2009) für die Befragten eine wichtige Rolle. Spezifische Vorteile der Methoden der Direkten Instruktion (Grosche, 2011; Watkins \& Slocum, 2003) und des kooperativen Lernens (Muijs \& Reynolds, 2011) werden nicht benannt.

\subsubsection{Differenzierung und Individualisierung}

Die in der sonderpädagogischen Integrationsforschung vielfach beschriebene Notwendigkeit differenzierten Arbeitens (u.a. Innerhofer \& Klicpera, 1991; Bless, 2003; Boban \& Hinz, 2003; Bürli et al., 2009 etc.) wird auch von den befragten Lehrkräften und Schulleitungen betont und stellt - wie die Ausführlichkeit der Ausführungen belegt - für diese offensichtlich das Kernthema hinsichtlich der Umsetzungs schulischer Integration dar. Diese Notwendigkeit wird dabei nicht nur mit den Lernvoraussetzungen der Schüler mit sonderpädagogischem Förderbedarf begründet, sondern allgemein auf heterogene Lern- und Leistungsvoraussetzungen in den Klassen zurückgeführt (SL 1, SL 2, SL 5, RSL 1, RSL 3, RSL 5). In diesem Kontext unterscheiden die Befragten - ähnlich wie in der theoretischen Grundlagenliteratur (Bohl et al., 2010; Paradies \& Linser, 2001) - zwischen differenzierenden Maßnahmen im weiteren (s.o.) und individualisierenden Maßnahmen im engeren Sinn (SLFZ 2, RSL 4, FSL 2, FSL 4), wobei die individuell abgestimmte Förderung von einer Lehrkraft als „Idealfall“ (FSL 4) benannt wird. Unterschieden wird auch zwischen quantitativer und qualitativer Differenzierung (RSL 3). Vergleichbar den Aussagen von Paradies \& Linser (2001), Bohl et al. (2010) oder Saalfrank (2012) wird von einer Schulleitung und einer Förderschullehrkraft erwähnt, dass offene Lernformen wie Wochenplan- oder Stationenarbeit sich gut für differenziertes Lernen anbieten, zumal das Arbeiten an unterschiedlichen Aufgabenstellungen dadurch für alle Schüler „normal“ werde (SL 2, FSL 1). Darüber hinaus wird - wie bei Paradies \& Linser (2001) - auch der Zusammenhang zwischen differenziertem 
und selbstorganisiertem Lernen aufgegriffen (SLFZ 1, SLFZ 2). Das Bearbeiten differenzierter Aufgaben durch einzelne Schüler ist nach Meinung der Leitungen der Förderzentren dann leichter, wenn alle Schüler mit selbstorganisiertem Lernen vertraut sind (SLFZ 1, SLFZ 2).

Eine eher geringe Rolle spielt in den Ausführungen der Befragten dagegen die Beschreibung der unterschiedlichen Ebenen, auf denen sich differenziertes Arbeiten im Unterricht abspielen kann. Anders als in den komplexen Übersichten von Klafki und Stöcker (1976) oder Saalfrank (2012) wird lediglich die Unterscheidung der Differenzierung auf sozialer und kognitiver Ebene von einer Schulleitung getroffen (SL 2). Eine Förderschullehrkraft spricht diesbezüglich verschiedene Differenzierungsaspekte wie die Anpassung sprachlicher Ausführungen oder die Auswahl an die Fähigkeiten der Schüler angepasster Texte oder Lektüren an (FSL 4). Eine Fülle von Aussagen der Befragten bezieht sich auf die organisatorische Umsetzung differenzierter Lernangebote in den integrativen Schulen, die - wie übereinstimmend berichtet wird eine große Bandbreite umfasst und sehr flexibel gehandhabt wird (u.a. SLFZ 2, SL 1, SL 3, SL 4, SL 5, FSL 1, FSL 4, FSL 5). Dabei wird zunächst darauf hingewiesen, dass die Schülerinnen und Schüler - auch aus personellen Gründen (s. Ressourcen) (RSL 5) - einen großen Anteil des Unterrichts innerhalb der Klasse verbringen (SLFZ 2, RSL 1, RSL 5, SL 5). Die Entscheidung, ob eine Förderung innerhalb oder außerhalb des Klassenunterrichts erfolge, werde bedarfsorientiert getroffen (SL 3, RSL 3, E 1, S 1, S 4) und richte sich u.a. danach, ob Schüler zielgleich oder zieldifferent unterrichtet würden (RSL 3, RSL 5, FSL 3) oder ob sie nur bei bestimmten Themen differenzierte Ansprache oder differenziertes Material benötigen (SL 1, FSL 1, FSL 2) und die Mitarbeit im Unterricht der Klasse bspw. über einen Wochenplan organisiert werden kann (FSL 2).

Differenziertes Material erhalten die vier zieldifferent unterrichteten Schüler (E 1, E 3, S 2, S 4). Für Mitschüler ist es nach Auffassung von zwei Befragten inzwischen selbstverständlich, wenn an differenzierten Aufgabenstellungen gearbeitet wird (SL 1, FSL 1). Die in der sonderpädagogischen Forschung benannte Ermöglichung der Teilhabe an gemeinsamen Lernsituationen (Mühl, 1987; Hug, 1994b; Boban \& Hinz, 2003) findet nach Aussage der Interviewpartner also dann statt, wenn diese - mit Blick auf das Unterrichtsthema und dessen inhaltlichen Anspruch - für die Schüler individuell möglich ist. Eine Besonderheit stellt die individuelle Unterstützung durch Integrationshilfen ${ }^{60}$ im Unterricht dar (S 3). Der Anteil von Einzelförderung außerhalb des Klassenraums überwiegt nach Auffassung einiger Befragter, wenn ein Förderschullehrer vor Ort ist (SLFZ 1, SLFZ 2, SL 2), was darauf zurückgeführt wird, dass dies sowohl von den Förderschullehrern (SLFZ 2) als auch von den Regelschullehrern (SLFZ 1, SL 2) so gewünscht werde.

Während die sonderpädagogische Forschung die innere Differenzierung insgesamt als günstiger bewertet als die äußere Differenzierung in Form von Einzel- oder Gruppenunterricht (Bächthold et al., 1990; Feyerer, 1998; Bless, 2003; Boban \& Hinz, 2003; Thiele, 2009), erläutern die Interviewpartner mögliche Vorteile der Einzelförderung außerhalb des Klassenraums im Gesamtrahmen des integrativen Unterrichts vor allem bei Schülern mit kognitiven Schwierigkeiten, da diese dann noch einmal individuell angepasste Erklärungen oder unterrichtliche Angebote erhalten könnten (SL 1, SL 3, SL 5, RSL 1, RSL 2, RSL, RSL 5, FSL 1, FSL 2, FSL 4). Dies sei bspw. dann notwendig, wenn Unterricht in frontaler Form erfolge, so dass eine persönliche Ansprache diesen Unterricht akustisch störe (FSL 5), es in einer Klasse zu laut sei (FSL 3) oder Schüler mit sozial-emotionalem Förderbedarf Soforthilfe benötigen (FSL 4). Von zwei Förderschullehrkräften wird also ein Zusammenhang zwischen der Klassenführung bzw.

60 Kinder und Jugendliche mit einer (drohenden) seelischen Behinderung haben nach $₫ 35$ a SGB VIII einen Anspruch auf eine Integrationshilfe. Kinder und Jugendliche mit Bedarf an pflegerischen, medizinischen und therapeutischen Hilfen haben Anspruch auf eine Eingliederungshilfe nach $\$ \$ 5354$ SGB XII. 
dem Umgang mit störendem Verhalten und differenziertem Arbeiten hergestellt. Unterricht in Kleingruppen (SLFZ 2, SL 1, RSL 5, FSL 4, FSL 5) ermögliche Schülern mit sonderpädagogischem Förderbedarf den Kontakt untereinander (SL 1) oder führe zur Einrichtung von gemischten Fördergruppen (aus Schülern mehrerer Klassen, aus Schülern einer Stufe, aus Schülern mit und ohne Förderbedarf) (SLFZ 1, RSL 5, FSL 4, FSL 5). Zum einen scheint es dabei also um die Bildung möglichst homogener Untergruppen zu gehen (Hattie, 2013), zum anderen stellt die Bildung klassenübergreifender Fördergruppen eine Maßnahme dar, die sich im Bereich der Organisationsentwicklung verorten lässt, da sie übergeordnete schulorganisatorische Maßnahmen betrifft. Insgesamt verweist die Auseinandersetzung der Beteiligten mit der Frage nach innerer und äußerer Differenzierung darauf, dass binnendifferenziertes Arbeiten zwar als Wunsch formuliert wird (s. dazu unterrichtsbezogene Zusammenarbeit), Formen äußerer Differenzierung allerdings - auch aus pragmatischen Gründen - bevorzugt werden, da sie aufgrund der unzureichenden Rahmenbedingungen (Bsp.: zu wenige Stunden in Doppelbesetzung) oder fehlender unterrichtlicher Veränderungen (Bsp.: Anteil Frontalunterricht im Vergleich zu offenen Unterrichtsformen) leichter umsetzbar sind.

Die Befragten heben bei der Bewertung der individuellen Förderung außerhalb des Klassenraums in positiver Hinsicht insbesondere die mögliche Intensität und den individuellen Zuschnitt in Einzelsituationen hervor (SL 5, RSL 3, E 1, E 2, E 3), auch wenn ein Elternteil bemerkt, dass dies ja dann keine „Integration“ sei (E 1). Die Schulleitung eines Förderzentrums problematisiert, dass diese Form der Förderung die Gefahr beinhalte, als „besser strukturierte Nachhilfe“ angesehen zu werden (SLFZ 2). Eine Förderschullehrkraft erläutert, dass die Regelschullehrkräfte teilweise Probleme damit haben, dass Kinder aus ihrem Unterricht herausgenommen werden und erst dann überzeugt sind, wenn diese durch Einzelunterricht in bestimmten Bereichen tatsächlich Lernfortschritte machen oder zum Inhalt des Klassenunterrichts aufschließen können (FSL 5). Vier der fünf befragten Schüler werden zum Teil außerhalb des Klassenraums unterrichtet und sind mit dieser Form der Unterrichtung einverstanden bzw. bewerten diese günstig (S 1, S 2, S 3, S4).

Eine differenzierte Leistungsbewertung (Heyer et al., 1997; Holzinger et al., 2011) erfolgt zwar im Rahmen angepasster Klassenarbeiten (FSL 1, E 3), führt aber auch zu Schwierigkeiten aufgrund des in den Regelschulen vorhandenen Leistungsdrucks der Eltern (FSL 4), der von Mühl bereits 1987 problematisiert wurde, und der rechtlichen Voraussetzungen (SLFZ 1, FSL 5). Während die Bewertung zieldifferent unterrichteter SuS mit spF geregelt ist, sei v.a. die Bewertung differenziert unterrichteter Schüler ohne anerkannten sonderpädagogischen Förderbedarf problematisch (FSL 5), da ein passender gesetzlicher Rahmen (Notengebung etc.) fehle ${ }^{61}$. Von einer Lehrkraft und einem Elternteil wird in diesem Zusammenhang auch der Nachteilsausgleich (Wember \& Prändl, 2009) erwähnt, der zwar rechtlich geregelt, aber nicht immer einfach zu handhaben sei, u.a. da sich andere Kinder dadurch ungerecht behandelt fühlen können (RSL 2).

Im Vordergrund der Aussagen zu Fragen der Differenzierung steht also die organisatorische Umsetzung, während qualitativ-inhaltliche Fragestellungen, die bspw. fachdidaktische Entscheidungen (Reduktion von Unterrichtsinhalten etc.) betreffen, nicht angesprochen werden. Verschiedene Anpassungsstrategien, die thematisiert werden, beziehen sich vor allem auf die remediale Strategie, bei der mit direkter Förderung gearbeitet wird (Wember, 2001), sowie die Anpassung des Unterrichts an Unterschiede zwischen den Schülern bzw. die gezielte Förderung durch eine adaptive Unterrichtsgestaltung (Weinert, 1997; Helmke, 2015).

61 Die Zeugnis- und Versetzungsordnungen für Grundschulen und Gemeinschaftsschulen im Saarland wurden zwar 2015 bzw. 2016 geändert, allerdings nicht so weitreichend, dass damit ein hohes Maß an Flexibilität mit Blick auf differenzierte Leistungsbewertung gegeben wäre. 
Der von FSL 4 formulierte Anspruch „im Idealfall jedem Kind gerecht zu werden“ wird von mehreren Interviewten als Anforderung erwähnt (SL 2, SLFZ 2, FSL 2, FSL 4). Besonders umfassend und präzise werden gleichzeitig Erklärungen zu Schwierigkeiten bezüglich umfassender unterrichtlicher Veränderungen in den Regelschulen hin zu stärker differenzierten bzw. individualisierten Angeboten formuliert. Dazu gehören: Individualisierung versus Lehrplanorientierung (SLFZ 1), Individualisierung versus (vermeintlicher) Gleichschrittigkeit (FSL 5), ein erhöhter Arbeitsaufwand (RSL 2, RSL 3, RSL 5, SL 1), der die Lehrkräfte teilweise überfordere (SL 2, FSL 1) (s. dazu Klippert, 2008), zu große und heterogene Klassen (FSL 2) sowie fehlende Zeit für gemeinsame Unterrichtsvorbereitung und die damit verknüpfte erschwerte differenzierte Materialvorbereitung für Förderschullehrkräfte (SL 2). Problematisch sei neben fehlenden gemeinsamen Beratungsstunden auch die mit 27 Stunden als hoch eingeschätzte Unterrichtsverpflichtung der Förderschullehrkräfte. Zudem könnten die Schüler selbst (SL 4, E 2) durch diese Unterrichtsform überfordert sein. Die fehlende Möglichkeit der individuellen Zuwendung für leistungsstärkere Schüler (RSL 5) und das immer stärker werdende Auseinanderklaffen der Leistungsschere in den Klassen (RSL 4), das auch Romey (1977) thematisiert hat, beschäftigen zwei Regelschullehrkräfte. Die Benennung dieser möglichen Grenzen greift die kritische Haltung von Weinert (2001), Helmke (2003), Klippert (2008) oder Trautmann und Wischer (2011) auf, die nicht davon ausgehen, dass das Ideal der Individualisierung im Klassenunterricht erreichbar sei. Die kritischen Auffassungen entsprechen den Ergebnissen von Laubenstein et al. (2015), die im Rahmen des GeSchwind-Projekts zum Schluss gelangten, dass positive Vorerwartungen an Individualisierung in den beteiligten Grundschulen nur zu $53 \%$, in den weiterführenden Schulen sogar nur zu $20 \%$ erfüllt werden konnten.

Inwiefern differenzierende oder individualisierende Unterrichtsformen anteilsmäßig tatsächlich umgesetzt werden, kann anhand der Aussagen insgesamt nur vermutet werden. Die Förderung außerhalb des Klassenraums stellt nach Auffassung der Schulleitungen der Förderzentren sowie von zwei Schulleitungen der Regelschulen allerdings den Löwenanteil dar (SLFZ 1, SLFZ 2, SL 2, SL 5). Die Feststellung, dass differenzierter Unterricht seltener erfolgt als bspw. undifferenzierter Unterricht (Bos et al., 2003; Wischer, 2007; Helmke, 2010), lässt sich also nur indirekt für die beteiligten Schulen annehmen, da aufgrund der Vielzahl der Ausführungen zu Formen äußerer Differenzierung (Einzel- oder Gruppenförderung) davon ausgegangen werden kann, dass zwar Differenzierungsangebote häufig sind, diese allerdings oft außerhalb des Klassenunterrrichts stattfinden. Die in sonderpädagogischen Veröffentlichungen aufgeführten Qualitätssubkategorien des Wechsels von individuellen und gemeinsamen Lernsituationen (Feyerer, 1998; Thiele, 2009) sowie deren Vielfalt insgesamt (Innerhofer \& Klicpera, 1991; Brugger-Paggi, 2003) sind laut Aussagen der Interviewpartner gegeben. Mögliche Effekte der Binnendifferenzierung (Trautmann \& Wischer, 2011; Woolfolk, 2008; Helmke, 2010) werden nicht angesprochen.

Übereinstimmend mit den Ergebnissen von Feuser \& Meyer (1987), Dyson (2010), Ellinger \& Stein (2012), Amrhein (2011) oder Laubenstein et al. (2015) halten einzelne Interviewpartner binnendifferenzierte Angebote bzw. inklusiven Unterricht allgemein vor allem mit Blick auf Schüler mit den spF geistige Entwicklung (SL 1, FSL 1), soziale und emotionale Entwicklung (SL 1, SL 5, RSL 2) bzw. Hören und Sehen (SL 5) für gegebenenfalls nicht ausreichend. Ein Elternteil spricht die mögliche Überforderung eines Kindes mit dem spF körperlich-motorische Entwicklung in der Regelschule an (E 2).

Lernstandsdiagnostik und Förderplanung, die als Qualitätsmerkmale in sonderpädagogischen Forschungsveröffentlichung als sehr relevant eingestuft werden (Preuß, 1985; Innerhofer \& Klicpera, 1991; Bless, 2003; Boban \& Hinz, 2003; Brugger- Paggi, 2003; Specht et al., 2006; 
Feyerer, 2007; Wember \& Prändl, 2009; Bürli et al., 2009; Holzinger et al., 2011), spielen in den Aussagen der Interviewpartner keine Rolle.

\subsubsection{Ergebnisse im Bereich der Unterrichtsentwicklung}

Die Wahrnehmung der Lernentwicklung ist für die Befragten letztendlich nur individuell möglich (SL 2, SL 4), stellt sich allerdings aufgrund der Aussagen insgesamt positiv dar (SL 4, SL 5, FSL 3, FSL 5). Eine Förderschullehrkraft (FSL 3) und ein Elternteil (E 1) verweisen in diesem Zusammenhang darauf, dass die Schüler in der Regelschule sowohl mit Blick auf ihre kognitiven Kompetenzen als auch hinsichtlich ihrer sozial-emotionalen Fähigkeiten von leistungsstärkeren Schülern profitieren können, da diese ihre Kompetenzen „weitergeben“. Diese Aussagen stimmen mit denen von Schley u.a. (1992), die als Indikator für diesen Bereich subjektiv wahrnehmbare Entwicklungsprozesse aufführen, oder Speck (2010), der von Lerngewinnen für alle Kinder spricht, überein.

Bezüglich ihrer kognitiven Lernentwicklung (Schley et al., 1992: objektivierbare Lernerfolge und Wissensinhalte) könnten Schüler mit dem spF Lernen durch zielzieldifferente Lernangebote (auch Klassenarbeiten) motiviert werden und sich dadurch für das Lernen öffnen (SL 3), während eine gute emotionale Festigung durch vernetzte Unterstützung bei Schülern mit dem spF soziale und emotionale Entwicklung zu einer guten kognitiven Entwicklung führen könne (FSL 5). Bei Schülern mit dem spF geistige Entwicklung werden Sorgen hinsichtlich der Möglichkeiten lebenspraktischer Schulung innerhalb der Regelschule aufgeführt (SL 2). Wenn die Rahmenbedingungen gut sind, sei aber auch für diese Schüler insgesamt gute Förderung möglich (FSL 5). Probleme sieht eine Schulleitung (SL 2) mit Blick auf mögliche Grenzerfahrungen eines Schülers mit dem spF geistige Entwicklung in der Zeit der Pubertät, eine Sorge, die als Erwartung von Frustrationen beim Erleben der behinderungsspezifischen Grenzen auch von befragten Lehrkräften in der Studie von Dumke et al. (1989) geäußert wurde. Die Ergebnisse lassen sich nicht in Bezug setzen zu Längsschnittuntersuchungen der Sonderpädagogik, wenig thematisiert werden Lernabstandserfahrungen (Wocken, 1987). Mit Blick auf die Entwicklung in bestimmten Unterrichtsfächern (Schulleistungsorientierung) erfolgen von Seiten der Interviewpartner keine Ausführungen.

Mit Bezug auf die emotionalen Entwicklung sind die Aussagen sehr unterschiedlich. Eine Schülerin konnte viel Selbstbewusstsein entwickeln (SL 3). Dagegen zeigen sich bei einem Schüler mit dem spF soziale und emotionale Entwicklung in diesem Kompetenzbereich keine Fortschritte (SL 4). Besonders schwierig sei die Unterstützung der emotionalen Entwicklung bei autistischen Schülern, da die Fachlehrer häufig nicht wissen, wie sie diese angemessen fördern können (SL 2). Während das emotionale Wohlbefinden in den Klassen von Seiten der befragten Schüler gegeben ist (s. Schulklasse als sozialer Raum), ein Ergebnis, das mit dem von Feyerer (1998) übereinstimmt, verweisen die an dieser Stelle getätigten Aussagen darauf, dass anders als bezugsgruppenorientierte Untersuchungen (Bsp.: Haeberlin et al., 1991; Tent et al., 1991) - für die weitere Forschung die Beobachtung individueller Entwicklungsverläufe eine Rolle spielen könnte (Bryk et al., 2010; Lütje-Klose et al., 2018).

Besonders positiv stellen die Interviewpartner die soziale Entwicklung dar und zwar mit Blick auf alle Schüler und Schülerinnen. Diese hätten aufgrund der integrativen Unterrichtung die Möglichkeit Rücksichtnahme, Respekt, Achtung und Empathiefähigkeit zu erlernen und Vielfalt „als normal zu akzeptieren“ (SL 1, SL 4, RSL 2, RSL 5, FSL 1, E 1) und werden als sozial integriert beschrieben bzw. beschreiben sich selbst so (S 1, S 2, S 3, S 4, S 5). Die von Wember und Prändl (2009) aufgeführte soziale Integration bzw. der von Speck (2010) benannte Erwerb sozialer Kompetenzen (Speck, 2010) scheinen also gegeben zu sein. Dies stimmt auch mit den Ergebnissen von Dumke (1989) überein, der positive Lehrereinschätzungen zu sozialem Lernen und sozialer Entwicklung nichtbehinderter Schülerinnen und Schüler berichtet. Keine 
Aussagen sind aufgrund der Interviews zum soziometrischen Status der SuS mit spF möglich (Haeberlin et al., 2003, Huber, 2009).

\subsubsection{Zusammenfassung und Weiterentwicklung des Kategoriensystems mit Hilfe der Ergebnisse sowie Konsequenzen für die inhaltliche Gestaltung der Fragebögen}

Das im Theorieteil erarbeitete Kategoriensystem hat sich für die Einordnung der Ergebnisse der qualitativen Erhebung insofern bewährt, dass die Aussagen der Interviewpartnerinnen und -partner sich alle den vorhandenen Hauptkategorien zuordnen lassen. Gleichzeitig wird durch Analyse und Interpretation der Daten deutlich, dass einige Subkategorien von den Befragten stärker gewichtet werden, als dies in bisherigen Forschungsveröffentlichungen der Fall war, bzw. einige Subkategorien neu hinzukommen. Diese Veränderungen werden in den folgenden Übersichten zu den Qualitätsbereichen Organisations-, Personal- und Unterrichtsentwicklung veranschaulicht $^{62}$. Für die Fragebogenerhebung werden die Kategorien oder Subkategorien nicht erfragt, die für die interviewten Personen keine oder eine sehr untergeordnete Rolle zu spielen scheinen. Auch wenn es interessant sein könnte genau diese Aspekte in die quantitative Erhebung aufzunehmen, wird die qualitative Gewichtung also insofern genutzt, dass eine Auswahl von Fragebogenitems und damit eine inhaltliche Beschränkung vorgenommen werden kann.

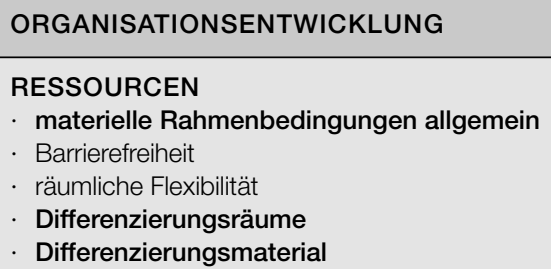

\section{LEITUNG EINER SCHULE}

professionelles Management

beratende, vernetzende, koordinierende und unterstützende Leistungen

Kooperation auf Schulleitungsebene

\section{SCHULE ALS SOZIALER RAUM}

Schulklima

Integration als gemeinsame Aufgabe

Partizipation

Zusammenarbeit mit Eltern (Elterngespräche im Team RSL - FSL, Elterngespräche SL - E)

Vernetzung

\section{ORGANISATIONSSTRUKTUREN}

Formen äußerer Differenzierung - wird in die Kategorie Differenzierung/Individualisierung verschoben inhaltlich paralleles Arbeiten innerhalb einer Jahrgangsstufe jahrgangsübergreifender Unterricht Gestaltung von Übergängen

\section{ERGEBNISSE}

Leitbilder

Schulprogramme

Förderkonzepte

Abb. 6: Qualitätskategorien und -subkategorien im Bereich der Organisationsentwicklung

62 Gewichtung von Subkategorien wird durch fette Markierung derselben veranschaulicht, neu hinzugekommene Subkategorien sind weiß hinterlegt. 


\section{PERSONALENTWICKLUNG}

\section{RESSOURCEN}

- Grundversorgung mit Lehrkräften

Senkung der Unterrichtsverpflichtung

Doppelbesetzung

Kontinuität der Zuweisung

zu Einzelschulen passende Zuweisung (pädagogische Anforderungen)

Bündelung sonderpäd. Ressourcen

fest eingerichtete Kooperations-/ Teamzeiten

\section{KOOPERATION VON LEHRKRÄFTEN}

- Voraussetzungen/Erwartungen/Bereitschaft

Niveaustufen

Kooperation im Gesamtkollegium

Kooperation in Teams (multiprofessionell)

intraprofessionelle Kooperation der Förderschullehrkräfte untereinander

Kooperation zwischen Regel- und Förderschullehrkräften (Zusammenarbeit auf Augenhöhe)

unterrichtsbezogene Zusammenarbeit/ Co-Teaching

Entlastung der Regel- durch die Förderschullehrkräfte

Aufgaben- und Rollenklärung

\section{PROFESSIONALISIERUNG}

- Studium und Vorbereitungsdienst

Fortbildungen (schulintern/schulextern)

Supervision

\section{ERGEBNISSE}

Auswirkungen Schulklima?

Auswirkungen Lernergebnisse SuS?

Haltungen

Abb.7: Qualitätskategorien und -subkategorien im Bereich der Personalentwicklung

\section{UNTERRICHTSENTWICKLUNG}

RESSOURCEN

Klassengröße

Klassenzusammensetzung

SCHULKLASSE ALS SOZIALER RAUM

Klassenklima

soziales Lernen

\section{KLASSENFÜHRUNG}

- Beziehung zwischen Lehrkraft und SuS

Regeln, Rituale, Abläufe

Ruhe in der Klasse

klare Verhaltenserwartungen und Konsequenzen

klare Unterrichtsstruktur

\section{DIDAKTIK}

didaktische Prinzipien

\section{METHODIK}

flexibler Methodeneinsatz

Frontalunterricht (direkte Instruktion)

Einzelarbeit

Partnerarbeit/Gruppenarbeit (kooperatives Lernen)

offene Unterrichtsformen 


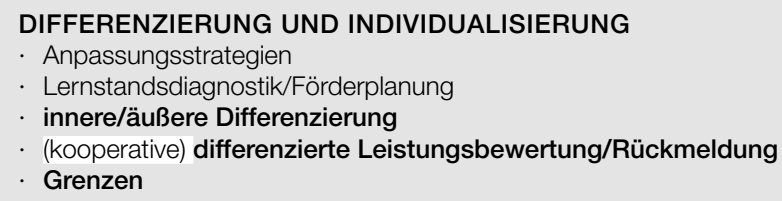

Abb. 8: Qualitätskategorien und -subkategorien im Bereich der Unterrichtsentwicklung

In die Fragebogenerhebung werden alle Subkategorien aufgenommen, die in den Übersichten als stark gewichtet gekennzeichnet sind. Darüber hinaus werden - in Verbindung mit einfachen, wenig differenzierten Fragestellungen - einzelne auch weniger stark gewichtete Aspekte aufgegriffen, die leicht abgefragt werden können (Bsp.: Leitbilder/Schulprogramme als Ergebnisse). Bei mehreren Subkategorien scheint aufgrund der in den Interviews erhobenen Bedeutung und Komplexität eine Ausdifferenzierung der Fragestellungen in mehrere Items sinnvoll. Diese wird im Bereich der Organisationsentwicklung mit Blick auf die soziale Integration der SuS mit spF vorgenommen (teilweise redundant zu Unterrichtsentwicklung - Ergebnisse) sowie hinsichtlich der Zusammenarbeit mit den Eltern, im Bereich der Personalentwicklung für die Zusammenarbeit zwischen RSL und FSL, das dadurch empfunde Maß an Entlastung sowie die Qualitätssubkategorie Fortbildung und im Bereich der Unterrichtsentwicklung für die Subkategorien Klassenführung, Differenzierung sowie Lernentwicklung.

\subsection{Quantitative Studie}

\subsubsection{Forschungsmethodik der quantitativen Studie}

Wie unter 4.2 bereits erwähnt, geht es bei der quantitativen Erhebung vor allem darum, eine größere Menge an Erfahrungsaussagen von Expertinnen und Experten zu sammeln. Im Vergleich zur Durchführung der Interviews nimmt die forschende Person bei der Vorbereitung der Fragebögen eine wichtige Funktion ein und legt die Untersuchungsinhalte stärker fest, ist aber während der Erhebung selbst naturgemäß distanzierter. Eine Fragebogenerhebung verläuft statisch - nicht prozessorientiert - und strukturiert. Ihre Auswertung geht generalisierend vor und orientiert sich an "harten“ statt „weichen" Daten (Bryman, 2012, S. 408). Gleichzeitig ist das Ziel einer quantitativen Erhebung - ähnlich wie bei einer qualitativen Studie - Daten zu reduzieren (Bsp.: Antwortverhalten zu bestimmten Fragestellungen) und Variationen in Wahrnehmungen und Einschätzungen der Befragten zu erfassen (Bryman, 2012, S. 209).

Die Fragebogenerhebung versucht den drei wesentlichen Gütekriterien quantitativer Forschung Objektivität, Reliabilität und Validität gerecht zu werden. Aufgrund der Struktur der Fragebögen sollten zumindest die geschlossenen Fragen in hohem Grad zu objektiven Ergebnissen führen, die „unabhängig sind von der jeweiligen Person, die das Messinstrument anwendet." (Diekmann, 2011, S. 249). Dass die Messergebnisse im Sinne der Reliabilität reproduzierbar sind (Diekmann, 2011, S. 250), lässt sich für die vorliegende Studie nur annehmen, da sie nur zu einem Durchführungszeitpunkt erfolgt ist. Ähnliches gilt für den Grad der Genauigkeit, also die Validität des Testinstruments. Aufgrund der präzisen Vorbereitung und Konstruktion der Fragestellungen und 
Skalierungen sollte der Fragebogen das messen „das [...] er messen soll oder zu messen vorgibt“ (Diekmann, 2011, S. 257) und damit Gültigkeit für den Untersuchungsgegenstand gewährleisten. Zielsetzung der Fragebogenerhebung ist die weitergehende Analyse der Qualität der integrativen Beschulung im Saarland mit Hilfe des im Theorieteil erstellten und durch die qualitativen Ergebnisse aus den Interviews überarbeiteten Kategoriensystems. Ähnlich wie bei den Interviews sollen die Perspektiven unterschiedlicher beteiligter Personengruppen systematisch erfasst werden.

\section{Konstruktion der Fragebögen}

In den Fragebögen werden zunächst soziale Merkmale der Befragten erfasst (Alter, Geschlecht, etc.). Etwa zwei Drittel der Fragen erfassen Einstellungen und Bewertungen mit Hilfe von Likert-Skalen, teilweise ergänzt durch offene Fragestellungen (s. Übersicht S. 316), mit Hilfe derer diese Einschätzungen begründet werden können (s. dazu Diekmann, 2011, S. 471). Als Skalierungen werden solche ohne mögliche „neutrale Mitte“, d.h. vorwiegend in vierstufiger Form ausgewählt (Bsp.: stimme sehr zu, stimme eher zu, stimme eher nicht zu, stimme gar nicht zu). Bei ausgewählten Fragestellungen werden Einstellungsdimensionen multipel erfasst (Diekmann, 2011, S. 472), bspw. bei der Frage zur Bewertung individueller Förderung im Rahmen äußerer Differenzierung (Fragebogen RSL Fragen 46 sowie 84) oder bei der Frage zur gemeinsamen Unterrichtsdurchführung von RSL und FSL (Fragebogen RSL Fragen 43 sowie 89), damit Einstellung und Umsetzung zugleich erfasst werden können.

Damit für einige Qualitätskategorien bzw. -subkategorien erweiterte inhaltliche Informationen bzw. kontextualisierende Begründungen der Befragten erfasst werden können, werden einige Items mit offenen Fragestellungen verknüpft:

\section{Organisationsentwicklung}

- Hilfsmittel der SuS mit spF

- Kennzeichen der Zusammenarbeit mit den Eltern der SuS mit spF (SL)

- Bewertung der Zusammenarbeit zwischen Eltern und SL/RSL/FSL

- Inhalte der Gespräche mit Eltern (RSL/FSL)

- ergänzende Aussagen zur Klasse bzw. zu Regelschulen bzw. zu Förderzentren

- weitere Vernetzungspartner

\section{Personalentwicklung}

- Themen der inhaltlichen Auseinandersetzung mit schulischer Integration (Fortbildungen, Fortbildungsbedarf)

- Bewertung der Zusammenarbeit zwischen SL/RSL - FSL

- Begründung für empfundenes Maß der Unterstützung durch FSL

- Formen der Zusammenarbeit und Gesprächsinhalte RSL - FSL

- Begründungen zur Haltung gegenüber schulischer Integration

3 Unterrichtsentwicklung

- Bewertung der unterschiedlichen Sozialformen (nur SuS)

- Bewertung der individuellen Förderung inner- bzw. außerhalb des Klassenraums

- Begründungen für wahrgenommene Lernfortschritte von SuS mit spF

- Vorschläge für verbesserte Lernmöglichkeiten (nur SuS)

- Einschätzung der Förderung insgesamt und Vorschläge für Verbesserungsmöglichkeiten

4 übergreifend:

- Verbesserungsvorschläge Klasse/Schule (nur SuS)

- Wünsche für die Zukunft inklusiver Bildung

- Bereiche für die Weiterentwicklung der (sonderpädagogischen) Professionalität 
Die Auswahl der Kategorien, Subkategorien bzw. Indikatoren für die Fragebögen ist - wie unter 4.2.2 dargestellt und unter 4.3.9 anhand der qualitativen Erhebung gewichtet und ergänzt sehr systematisch erfolgt. Bei der konkreten Auswahl von Fragestellungen muss berücksichtigt werden, dass die verschiedenen Personengruppen (Schulleitungen GS,GMS, SFZ, RSL, FSL, Eltern und Schüler) unterschiedliche Formen von Fragebögen erhalten werden. Diese richten sich nach deren möglichen Erfahrungshorizonten sowie sprachlichen Voraussetzungen. In diesem Zusammenhang muss insbesondere für die Schülerinnen und Schüler mit sonderpädagogischem Förderbedarf ab Klasse 2 auf sehr verständliche Formulierungen und kurze Fragestellungen geachtet werden (Kirchhoff et al., 2008, S. 27). Das bedeutet, dass für dieselben Kategorien nicht immer identische Fragestellungen möglich sind, wodurch sich - streng methodologisch betrachtet - Schwierigkeiten mit der Vergleichbarkeit der Antworten ergeben können. Aufgrund der Anzahl der ausgewählten Kategorien ist die Länge der Fragebögen durchaus anspruchsvoll (bis zu zehn Seiten mit bis zu 93 Items). Geachtet wird bei der Konstruktion auf eine Mischung offener und geschlossener Fragen, damit neben einer rein quantitativen Auswertung auch qualitative Aussagen gewonnen werden, die zum Teil Begründungsaspekte zu Fragestellungen oder auch offene Antworten liefern können. Diese Vorgehensweise wird gewählt, damit auch hier ggf. eine induktiv ableitbare Gewichtung bzw. inhaltliche Struktur sichtbar wird und keine zu starke Vorfestlegung auf die Relevanz von Themen erfolgt, auch wenn diese durch die Gestaltung der Fragebögen natürlich gegeben ist. Neben der inhaltlichen und sprachlichen Gestaltung wird auch auf Klarheit und Struktur beim Layout der Fragebögen geachtet (Kirchhoff et al., 2008, S. 25f). Damit alle Beteiligten die gleichen Ausgangsvoraussetzungen mit Blick auf die Möglichkeit der Bearbeitung der Fragebögen haben, werden die Bögen per Post an die Schulen gesendet. Bei einer Online-Befragung könnten Probleme (vor allem für Eltern oder Schüler) bezüglich des Zugangs zu PCs oder Internet entstehen. Die Entwürfe der Fragebögen wurden - mit mehrfacher Überarbeitung - zwischen Dezember 2013 und August 2014 erstellt. Die Anschreiben und Einverständniserklärungen ${ }^{63}$ wurden für die insgesamt elf angesprochenen Personengruppen (SLFZ, SLGS, SLGmS, RSL GS, FSL GS, RSLGMS, FSLGMS, Eltern GS, ElternGMS, Schüler GS, SchülerGMS) entwickelt. Für die Ermöglichung der schulbezogenen Durchführung wurden die Schulleitungen der in der Zufallsstichprobe ausgewählten Schulen (s.u.) vorab per Mail angeschrieben (Oktober 2014) sowie im Anschreiben darum gebeten die Anschreiben, Einverständniserklärungen, Fragebögen und Rückumschläge an die jeweiligen Personen, die sich für die Befragung bereit erklären, weiterzugeben. Der Versand der Fragebögen an insgesamt 78 Schulen, darunter 48 Grundschulen und 30 Gemeinschaftsschulen sowie 4 SFZ, erfolgte Anfang November 2014. Eine Erinnerungsmail zur ersten Mail bzw. zum Anschreiben wurde Anfang Dezember 2014 versendet. Die Rücklauffrist endete am 31. Januar 2015. In Form einer tabellarischen Übersicht wurde der Rücklauf der Fragebögen erfasst.

Die Kodierung und Auswertung der Fragebögen erfolgte mit Hilfe der Software SPSS. Die Erstellung der Datenmaske, die Dateneingabe sowie die Fehlerbereinigung wurden mit der Unterstützung studentischer Hilfskräfte durchgeführt. Zielsetzung der Datenanalyse war eine deskriptive, gruppenorientierte Auswertung, wobei nicht zwischen den Schulformen Grund- und weiterführenden Schulen unterschieden wurde. Fehlende Werte wurden nur dann einbezogen, wenn sie in ihrer Häufigkeit auffällig waren. Im Rahmen dieser Arbeit werden die deskriptiven Ergebnisse vorwiegend in Form übersichtlich strukturierter Tabellen vorgestellt. Diagramme werden dann genutzt, wenn eine komplexere Datenlage (Bsp.: Vergleich Sozialformen, Ver-

63 Die Fragebögen und Anschreiben wurden vor allem hinsichtlich des Datenschutzes vom Ministerium für Bildung des Saarlandes (Herr Endlich) eingehend geprüft und mehrfach überarbeitet. 
gleich Antwortverhalten unterschiedlicher Gruppen) dies sinnvoll erscheinen lässt. Qualitative Aussagen wurden mit Hilfe einer strukturierenden inhaltlichen Analyse (Kuckartz, 2012) kategorienorientiert zusammengefasst.

\subsubsection{Stichprobe}

Bei der Auswahl der 48 Grundschulen und 30 Gemeinschaftsschulen, die für die Fragebogenerhebung angeschrieben wurden, handelt es sich um eine Zufallsauswahl. Es wurde darauf geachtet, dass zu gleichen Anteilen Schulen aus den fünf Landkreisen bzw. dem einen Stadtverband des Saarlandes einbezogen wurden. Nicht angeschrieben wurden Schulen, die sich in diesem Zeitraum am Pilotprojekt Inklusion beteiligt haben. An jede Schule wurden insgesamt jeweils neun Fragebögen verschickt (1 SL, 2 RSL, 1-2 FSL, 2 Elternteile, 2 SuS mit spF). Mit Blick auf die Sonderpädagogischen Förderzentren wurden die vier fachrichtungsübergreifenden Förderzentren angeschrieben, deren Schulleitungen nicht in die Interviews einbezogen waren. Zusammen mit den vier Schulleitungen der SFZ hätten sich also insgesamt höchstens 706 Personen an der Befragung beteiligen können.

Tatsächlich ist ein Rücklauf von 193 Fragebögen erfolgt. Das entspricht einem prozentualen Anteil von 27,34\%. Mit Blick auf die Beteiligung der Schulen haben von den 78 angeschriebenen Schulen 32 an der Befragung teilgenommen, also ein Anteil von 41 \%, darunter 20 Grundschulen und 12 Gemeinschaftsschulen. Dazu kamen die Fragebögen der vier Schulleitungen der Förderzentren. An den Grundschulen haben 19 Schulleitungen, 34 Regelschullehrkräfte, 24 Förderschullehrkräfte, 19 Elternteile und 20 Schülerinnen und Schüler mit spF Fragebögen bearbeitet, an den Gemeinschaftsschulen 12 SL, 19 RSL, 15 FSL, 14 Elternteile und 11 SuS. Auch die vier Schulleitungen der SFZ haben ihre Fragebögen ausgefüllt. Insgesamt haben sich also 35 Schulleitungen, 53 Regelschullehrkräfte, 38 Förderschullehrkräfte, 34 Elternteile und 31 Schülerinnen und Schüler mit spF an der Befragung beteiligt. Der Befragungszeitraum erstreckte sich von November 2014 bis Februar 2015.

Tab. 9: Rücklauf der Fragebögen - einzelne Personengruppen

\begin{tabular}{lrr}
\hline SL GS & 19 & $9,8 \%$ \\
SL GMS & 12 & $6,2 \%$ \\
SL FZ & 4 & $2,1 \%$ \\
RSL GS & 34 & $17,6 \%$ \\
RSL GMS & 19 & $9,8 \%$ \\
FSL GS & 23 & $11,9 \%$ \\
FSL GMS & 15 & $7,8 \%$ \\
Eltern GS & 19 & $9,8 \%$ \\
Eltern GMS & 15 & $7,8 \%$ \\
S GS & 21 & $10,9 \%$ \\
S GMS & 12 & $6,2 \%$ \\
\hline Gesamtsumme & $\mathbf{1 9 3}$ & $\mathbf{1 0 0 , 0 \%}$ \\
\hline
\end{tabular}

Mit Blick auf mögliche Begründungen dafür, dass der Grad der Beteiligung nicht höher ausgefallen ist, können nur Vermutungen angestellt werden. Einige Schulleitungen äußerten sich am Telefon dahingehend, dass mit der Verteilung und dem Einsammeln der Anschreiben, Einwil- 
ligungserklärungen und Fragebögen ein hoher organisatorischer Aufwand verbunden sei, da diese sich an verschiedene Personengruppen richten und der Rücklauf v.a. von Eltern und Schülerinnen wie Schülern mehrfach angefordert werden musste. Auch das Interesse am Thema der Befragung wurde als unterschiedlich hoch beschrieben. Möglicherweise haben sich engagierte Schulen mit ihren Schulleitungen anteilsmäßig häufiger an der Erhebung beteiligt als Schulen, die mit dem Thema - ggf. auch aufgrund ungünstiger Rahmenbedingungen in den Schulen keine positive Entwicklung verbinden.

\section{Altersspanne und Tätigkeiten Schulleitungen}

73,1 \% der Befragten sind weiblich (141), 24,4\% männlich (47). Fünf Personen haben zu ihrem Geschlecht keine Angaben gemacht. 186 Personen haben Altersangaben gemacht. Das Alter der Teilnehmer liegt zwischen 7 und 64 Jahren, das Durchschnittsalter bei 36,34 Jahren. Die Schulleitungen der Grundschulen sind zwischen 38 und 61 Jahre alt. Fünf dieser Schulleitungen arbeiten erst seit wenigen Monaten in dieser Funktion, vier haben etwa ein Jahr Erfahrung, sieben haben drei bis sieben Jahre Erfahrung und drei arbeiten bereits seit mehr als 10 Jahren als Schulleitung. Die Altersspanne der Schulleitungen der Gemeinschaftsschulen erstreckt sich von 33 bis 64 Jahren. Drei Schulleitungen haben diese Funktion seit einigen Monaten inne, drei seit etwa einem Jahr, zwei seit sechs Jahren und vier weitere zwischen 10 und 33 Jahren. Für die Schulleitungen an den Grund- und Gemeinschaftsschulen insgesamt ergibt sich damit ein Mittelwert von 47,63 ( $s=9,14)$.

Eine ganze Reihe der Schulleitungen an den Grund- und weiterführenden Schulen verfügt über langjährige Erfahrung mit der schulischen Integration von SuS mit spF:

Tab. 10: Dauer der Erfahrung mit Unterrichtung von SuS mit spF SL ( $\mathrm{n}=31)$

\begin{tabular}{lr}
\hline 1-3 Jahre & 4 \\
4-7 Jahre & 3 \\
8-12 Jahre & 6 \\
13-20 Jahre & 13 \\
mehr als 20 Jahre & 5 \\
fehlend & 1 \\
\hline gesamt & 31 \\
\hline
\end{tabular}

\section{Altersspanne und Tätigkeiten Regelschul- und Förderschullehrkräfte}

Die Regelschullehrkräfte sind zwischen 27 und 64 Jahre $(M=40,23, s=12,16)$, die Förderschullehrkräfte zwischen 30 und 60 Jahre alt $(M=41,50 ; s=7,69)$.

Ein Großteil der Regel- und Förderschullehkraft ist vollzeitbeschäftigt, wie folgende Tabelle zeigt:

Tab. 11: Lehrkräfte Vollzeit - Teilzeit $(\mathrm{n}=91)$

\begin{tabular}{lcc}
\hline & RSL $(\mathbf{n}=\mathbf{5 3})$ & FSL $(\mathbf{n}=\mathbf{3 8})$ \\
\hline Vollzeit & $79,2 \%$ & $73,7 \%$ \\
Teilzeit & $29,8 \%$ & $26,3 \%$ \\
\hline
\end{tabular}


Der größte Teil der RSL arbeitet seit mehr als einem Jahr in der Grundschule, in der er gerade tätig ist (29), während drei dort seit etwa einem Jahr und zwei erst seit einigen Monaten arbeiten. In den Gemeinschaftsschulen sind 19 Lehrkräfte bereits seit mehr als einem Jahr tätig, 17 seit mehr als einem Jahr und zwei seit etwa einem Jahr. Der überwiegende Teil der Regelschullehrkräfte verfügt also über mehrjährige Erfahrung in der eigenen Schule und - wie folgende Tabelle zeigt - auch über mehrere Jahre Erfahrung in der Unterrichtung von SuS mit spF:

Tab. 12: Dauer der Erfahrung mit Unterrichtung von SuS mit spF RSL ( $\mathrm{n}=53$ )

\begin{tabular}{lc}
\hline einige Monate & 2 \\
etwa ein Jahr & 7 \\
mehr als ein Jahr & 44 \\
\hline gesamt & $\mathbf{5 3}$ \\
\hline
\end{tabular}

In den Grundschulen sind 12 FSL seit mehr als einem Jahr, vier seit etwa einem Jahr und sieben erst seit einigen Monaten beschäftigt, in den Gemeinschaftsschulen sind es zehn (mehr als ein Jahr), zwei (etwa ein Jahr) und drei (seit einigen Monaten). Mehr als die Hälfte aller Förderschullehrkräfte hat also zumindest mehr als ein Jahr lang kontinuierlich in einer Regelschule gearbeitet.

\section{Klassenstufen und Unterrichtsfächer Regel- bzw. Förderschullehrkräfte}

Die Regel- und Förderschullehrkräfte sind in folgender Verteilung in den verschiedenen Klassenstufen tätig:

Tab. 13: Tätigkeit der RSL und FSL in verschiedenen Klassenstufen ( $\mathrm{n}=53$ )

\begin{tabular}{ccccc}
\hline KIassenstufe & Anzahl RSL & $\begin{array}{c}\text { Prozentangabe } \\
\text { RSL }\end{array}$ & Anzahl FSL & $\begin{array}{c}\text { Prozentangabe } \\
\text { FSL }\end{array}$ \\
\hline 1 & 5 & $9,4 \%$ & 19 & \\
2 & 6 & $11,3 \%$ & 20 & \\
3 & 7 & $13,2 \%$ & 17 & \\
4 & 14 & $26,4 \%$ & 19 & \\
5 & 4 & $7,5 \%$ & 11 & Angabe nicht \\
6 & 5 & $9,4 \%$ & 8 & möglich, da \\
7 & 1 & $1,9 \%$ & 11 & Mehrfachangaben \\
8 & 4 & $7,5 \%$ & 8 & \\
9 & 3 & $5,7 \%$ & 3 & \\
10 & 1 & $1,9 \%$ & 0 & \\
fehlend & 3 & $5,7 \%$ & 0 & \\
\hline
\end{tabular}

Alle Förderschullehrkräfte geben an im Rahmen ihrer Tätigkeit in den Regelschulen Deutsch als Fach zu unterrichten, 97,4\% geben dies auch für Mathematikunterricht an. Darüber hinaus unterrichten FSL nach eigenen Angaben auch in den Fächern Englisch (4), Französisch, Naturwissenschaften (4), Gesellschaftswissenschaften (4), Sachunterricht (2) und Sport. 


\section{Anzahl der Schüler und Schülerinnen (je Schule/Klasse/nach spF)}

Die Grundschulen haben zwischen 115 und 272 Schülerinnen und Schüler, die Gemeinschaftsschulen zwischen 180 und 761 Schüler. Der Mittelwert liegt für die Schüleranzahl in beiden Schulformen bei 238,79, die Standardabweichung bei 167,78.

Die Klassengröße liegt in 33,9\% \% der Klassen zwischen 15 und 20 SuS, in weiteren $26 \%$ der Klassen zwischen 21 und $25 \mathrm{SuS}$ sowie in $22 \%$ der Klassen zwischen 26 und 30 SuS. Die Regelschullehrkräfte der Grundschulen geben an, dass sie in ihren Klassen zwischen 15 und $29 \mathrm{SuS}$ unterrichten, an den Gemeinschaftsschulen sind dies zwischen 15 und $30 \mathrm{SuS}$.

Tab. 14: Anzahl Schüler je Klasse (Angaben der SL, n=31)

\begin{tabular}{ccc}
\hline Anzahl SuS & Anzahl Klassen & Prozent \\
\hline 15 & 1 & $1,9 \%$ \\
16 & 4 & $7,5 \%$ \\
17 & 2 & $3,8 \%$ \\
18 & 4 & $7,5 \%$ \\
19 & 6 & $11,3 \%$ \\
20 & 1 & $1,9 \%$ \\
21 & 7 & $13,2 \%$ \\
22 & 8 & $15,1 \%$ \\
23 & 1 & $1,9 \%$ \\
25 & 2 & $3,8 \%$ \\
26 & 3 & $5,7 \%$ \\
28 & 2 & $3,8 \%$ \\
29 & 5 & $10,6 \%$ \\
30 & 1 & $1,9 \%$ \\
\hline
\end{tabular}

Die Anzahl der Schülerinnen und Schüler mit Blick auf die verschiedenen sonderpädagogischen Förderbedarfe in den Grund- und weiterführenden Schulen stellt sich wie folgt dar:

Tab. 15: SuS nach Förderbedarfen - Antworten der Schulleitungen GS ( $\mathrm{n}=19)$

\begin{tabular}{|c|c|c|}
\hline \multicolumn{2}{|c|}{ SL Grundschulen } & \multirow[b]{2}{*}{ Prozent } \\
\hline $\mathrm{spF}$ & Anzahl & \\
\hline spf Lernen & 74 & $35 \%$ \\
\hline spF Verhalten & 53 & $25 \%$ \\
\hline spF Sprache & 51 & $24 \%$ \\
\hline spF Motorik & 16 & $8 \%$ \\
\hline spF Sehen & 4 & $2 \%$ \\
\hline spF Hören & 6 & $3 \%$ \\
\hline spF geistige Entwicklung & 7 & $3 \%$ \\
\hline gesamt & 211 & $100 \%$ \\
\hline
\end{tabular}


Tab. 16: SuS nach Förderbedarfen - Antworten der Schulleitungen GMS ( $\mathrm{n}=12)$

\begin{tabular}{lcc}
\hline \multicolumn{1}{c}{ spF } & SL GMS & \\
\hline spf Lernen & Anzahl & Prozent \\
spF Verhalten & 94 & $53 \%$ \\
spF Sprache & 35 & $20 \%$ \\
spF Motorik & 37 & $21 \%$ \\
spF Sehen & 5 & $3 \%$ \\
spF Hören & 2 & $1 \%$ \\
spF geistige Entwicklung & 4 & $2 \%$ \\
gesamt & 0 & $0 \%$ \\
\hline
\end{tabular}

\section{Dauer der sonderpädagogischen Förderung der Schüler}

Der überwiegende Teil der SuS mit spF wird bereits seit mehr als einem Jahr sonderpädagogisch gefördert.

Tab. 17: Dauer der sonderpäd. Förderung Sohn/Tocher (Angaben der Eltern, $n=34$ )

\begin{tabular}{lcc}
\hline & \multicolumn{2}{c}{ Eltern } \\
& Anzahl & Prozent \\
\hline weiß ich nicht & 1 & $2,9 \%$ \\
seit weniger als einem Jahr & 3 & $8,8 \%$ \\
seit einem Jahr & 4 & $11,8 \%$ \\
seit mehr als einem Jahr & 25 & $73,5 \%$ \\
fehlend & 1 & $2,9 \%$ \\
\hline
\end{tabular}

Die Verteilung der 33 Schülerinnen und Schüler mit spF, die sich an der Erhebung beteiligt haben, auf die verschiedenen Klassenstufen sieht folgendermaßen aus:

Tab. 18: Verteilung der SuS mit spF auf die verschiedenen Klassenstufen $(\mathrm{n}=33)$

\begin{tabular}{lcc}
\hline & Anzahl & Prozent \\
\hline Klasse 1 & 1 & $4,8 \%$ \\
Klasse 2 & 5 & $23,8 \%$ \\
Klasse 3 & 4 & $19,0 \%$ \\
Klasse 4 & 11 & $52,4 \%$ \\
Klasse 5 & 1 & $8,3 \%$ \\
Klasse 6 & 1 & $8,3 \%$ \\
Klasse 7 & 4 & $33,3 \%$ \\
Klasse 8 & 3 & $25,0 \%$ \\
Klasse 9 & 3 & $25,0 \%$ \\
gesamt & 33 & $\mathbf{1 0 0} \%$ \\
\hline
\end{tabular}


Der Förderbedarf der Schülerinnen und Schüler wurde erfragt, indem diese ankreuzen sollten, in welchem Bereich/in welchen Bereichen sie Unterstützung benötigen, so dass Mehrfachnennungen möglich waren. Dabei ergibt sich folgende Verteilung:

Tab. 19: Förderbedarfe der $\operatorname{SuS}$ mit spF $(\mathrm{n}=33)$

\begin{tabular}{lcc}
\hline & $\begin{array}{c}\text { Anz.d. } \\
\text { Nennungen }\end{array}$ & Prozent \\
\hline Lernen & 27 & $50 \%$ \\
Verhalten & 9 & $16,7 \%$ \\
Sprache & 10 & $18,5 \%$ \\
Bewegung & 4 & $7,4 \%$ \\
Sehen & 1 & $1,9 \%$ \\
Hören & 3 & $5,5 \%$ \\
\hline
\end{tabular}

\subsubsection{Darstellung der Ergebnisse zur Organisationsentwicklung}

\subsubsection{Ressourcen im Bereich der Organisationsentwicklung Ressourcen allgemein}

$\mathrm{Zu}$ den Ressourcen allgemein wurde keine spezifische Frage gestellt, allerdings äußern einige Befragte sich mit Blick auf ihre Wünsche für die Zukunft inklusiver Beschulung dahingehend, dass eine bessere finanzielle Ausstattung und mehr Ressourcen oder ein höheres Budget notwendig seien. Ein Schulleiter formuliert: „Damit Inklusion keine „Sparmaßnahme“ ist und nicht auf ein „Abwälzen“ von Aufgaben an Regelschulen hinausläuft, erhoffe ich mir die Bereitstellung der notwendigen Ressourcen.. Eine Lehrkraft vertritt die Auffassung: „Ich wünsche mir vernünftige Regelungen im Sinne der bestmöglichen Förderung der Kinder. Es muss hier Geld investiert werden, Sparmaßnahmen sind hier kontraproduktiv“.. Die Bereitstellung weiterer Ressourcen wird in diesem Zusammenhang sowohl mit Blick auf die bauliche Ausstattung der Schulen als auch hinsichtlich des Personals gewünscht (s. dazu 4.5.4.1). Drei Personen sprechen im Zusammenhang mit der Ressourcenausstattung von schlechten oder unzureichenden Rahmenbedingungen, die „nicht optimal“ bzw. „bisher schlecht“ seien. Weitere wünschen sich eine Verbesserung der materiellen (und personellen) Versorgung der Schulen $(4)^{64}$ sowie einen größeren finanziellen Spielraum. Ein weiterer Wunsch mit Blick auf die Schulträger betrifft das Wegfallen von „Bürokratie“ zur Erleichterung der Förderung der Schülerinnen und Schüler mit spF.

\section{Barrierefreiheit}

Eine barrierefreie Gestaltung der Schulen für Schüler mit körperlichen Beeinträchtigungen ist nach Auskunft der Schulleitungen nur in vier der 32 Schulen gegeben, auch geplant wird diese Umgestaltung nur in vier weiteren Schulen. Sieben Schulen sind teilweise barrierefrei. Zwei Drittel der teilnehmenden Schulen sind also nicht barrierefrei gebaut bzw. umgebaut worden. Allerdings ist eine barrierefreie Gestaltung nach Einschätzung der Schulleitungen und Lehrkräfte auch nur für etwa $3 \%$ der integrativ unterrichteten Schüler notwendig.

64 Qualitative Aussagen, die mehrfach getätigt werden, werden in den Klammern mit ihrer Anzahl angegeben. 
Tab. 20: Barrierefreiheit Schulgebäude

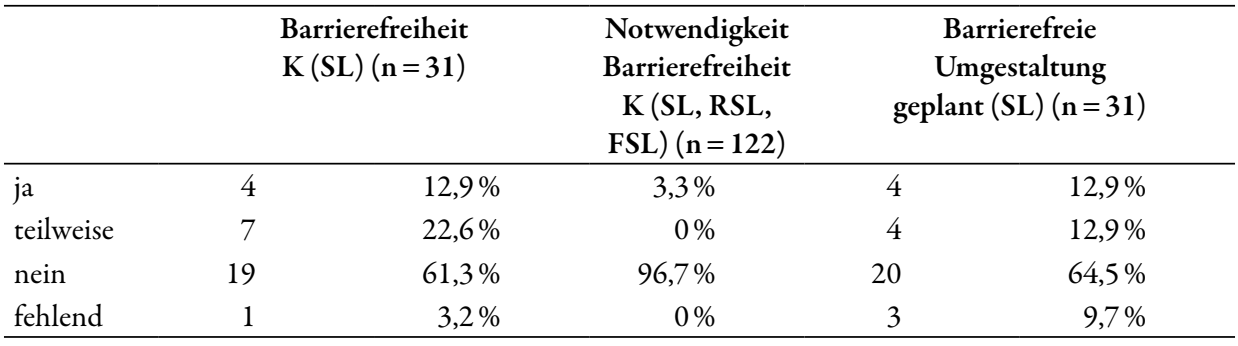

Diese Angaben lassen sich in Beziehung setzen zur Anzahl der Schülerinnen und Schüler mit spF körperlich-motorische Entwicklung in den Schulen. Wie folgende Tabelle zeigt, werden in fast $60 \%$ der Schulen keine SuS mit diesem spF unterrichtet. An knapp $20 \%$ der Schulen wird ein Schüler bzw. eine Schülerin mit diesem spF integriert, an knapp $13 \%$ der Schulen sind dies zwei Schüler und an 6,5\% der Schulen drei Schüler.

Tab. 21: Anzahl der SuS mit spF körperlich-motorische Entwicklung im Verhältnis zur Anzahl der Schulen (Angaben der SL, $\mathrm{n}=31$ )

\begin{tabular}{crr}
\hline 0 & 18 & $58,1 \%$ \\
1 & 6 & $19,4 \%$ \\
2 & 4 & $12,9 \%$ \\
3 & 2 & $6,5 \%$ \\
\hline gesamt & 31 & $100 \%$ \\
\hline
\end{tabular}

\section{Flexibilität der Räume}

Etwa ein Drittel der Schulleitungen und Lehrkräfte an den beteiligten Schulen gibt an, dass die Größe der Klassenräume an die Größe der Klassen angepasst ist. Flexible Gestaltungsmöglichkeiten sind allerdings nur in 16,5\% der Klassenräume möglich.

Tab. 22: Größe und Flexibilität der Klassenräume

\begin{tabular}{lcc}
\hline & $\begin{array}{c}\text { Größe der Klassenräume passend zur } \\
\text { Größe der Klassen } \\
\text { (SL, RSL, FSL) (n= 122) }\end{array}$ & $\begin{array}{c}\text { flexible Gestaltungsmöglichkeiten } \\
\text { Klassenräume } \\
(\text { SL, RSL, FSL) (n= 122) }\end{array}$ \\
\hline ja & $32,5 \%$ & $16,5 \%$ \\
teilweise & $45,0 \%$ & $47,1 \%$ \\
nein & $21,7 \%$ & $34,7 \%$ \\
\hline
\end{tabular}

\section{Differenzierungsräume}

Die beteiligten Schulen, die sich in ihrer Größe erheblich voneinander unterscheiden (was an dieser Stelle aber nicht in Beziehung gesetzt wird), sind sehr unterschiedlich mit Differenzierungsräumen ausgestattet. Drei Schulen verfügen über gar keinen Differenzierungsraum, wäh- 
rend die anderen zwischen einem (sechs Schulen) und bis zu zehn (eine Schule) Räumen nutzen können. Der größte Teil der Schulen (36,7\%) hat zwei bis drei Differenzierungsräume. Knapp die Hälfte der Regel- und Förderschullehrkräfte gibt an, dass sie für ihren Unterricht Differenzierungsräume nutzen können, für ein Drittel der Lehrer ist dies allerdings nur teilweise und für etwa ein Fünftel gar nicht möglich.

Tab. 23: Anzahl und Nutzungsmöglichkeiten von Differenzierungsräumen

\begin{tabular}{|c|c|c|c|c|c|}
\hline \multicolumn{3}{|c|}{$\begin{array}{l}\text { Anzahl Differenzierungsräume in den Schulen } \\
\qquad(\mathrm{SL})(\mathbf{n}=\mathbf{3 1})\end{array}$} & \multicolumn{3}{|c|}{$\begin{array}{l}\text { Nutzungsmöglichkeiten Differenzierungsräum } \\
\text { (RSL, FSL) }(\mathbf{n}=91)\end{array}$} \\
\hline 0 & 3 & $10 \%$ & ja & 44 & $48,9 \%$ \\
\hline 1 & 6 & $20 \%$ & teilweise & 28 & $31,1 \%$ \\
\hline 2 & 5 & $16,7 \%$ & nein & 17 & $18,9 \%$ \\
\hline 3 & 6 & $20 \%$ & & & \\
\hline 4 & 3 & $10,0 \%$ & & & \\
\hline 5 & 2 & $6,7 \%$ & & & \\
\hline 6 & 2 & $6,7 \%$ & & & \\
\hline 8 & 2 & $6,7 \%$ & & & \\
\hline 10 & 1 & $3,3 \%$ & & & \\
\hline
\end{tabular}

\section{Wünsche für die Zukunft}

Hinsichtlich der räumlichen Ausstattung der Schulen wünschen sich die Befragten insgesamt bessere räumliche Voraussetzungen (2), mehr Räume (2), größere und besser ausgestattete Klassensäle (auf dem neuesten technischen Stand), Räume, die Platz für Stationen etc. bieten, je einen Förderraum pro Klassensaal oder zusätzliche Räume zum Bewegen oder zum Zurückziehen (4). Eine Lehrkraft stellt dabei einen Zusammenhang zwischen den räumlichen Rahmenbedingungen und den Möglichkeiten zu differenziertem Arbeiten her:

Räumlich müsste sich in den Schulen etwas ändern; wenn man keine Bereiche schafft, in denen sich die Schüler auch in der Klasse einmal getrennt von den anderen hinsetzen können, wird eine Differenzierung innerhalb der Klasse erschwert.

Mehrere der befragten Personen sprechen räumliche Umbaumaßnahmen an, darunter die Neugestaltung bzw. Einrichtung von Förderräumen, „die angemessen bezüglich Größe und Ausstattung sind“ bzw. gut ausgerüstet („mit Tafel, Computer + Differenzierungsmaterialien“) (7). Eine Lehrkraft bemängelt, dass „leider alle Förderräume, der Gymnastikraum, Spielraum, Förderräume, der Nachmittagsbetreuung als Gruppenräume „geopfert“ werden mussten“.

\section{Differenzierungsmaterial Schule und Klassenräume}

Die Ausstattung mit Differenzierungsmaterial ist nach Einschätzung von $10 \%$ der Schulleitungen und Lehrkräfte in den Schulen gar nicht gegeben. In den Klassen sieht dies so aus, dass ein Viertel der Befragten angibt, dass in den Klassenräumen kein Differenzierungsmaterial vorhanden ist. Auf der anderen Seite gibt mehr als ein Drittel an, dass die beteiligten Schulen über Differenzierungsmaterial verfügen und etwas mehr als $15 \%$ können dies für die Klassenräume bestätigen. 
Tab. 24: Differenzierungsmaterial

\begin{tabular}{lcc}
\hline & $\begin{array}{c}\text { Differenzierungsmaterial Schule vorhan- } \\
\text { den (SL, RSL, FSL) }(\mathbf{n}=\mathbf{1 2 2})\end{array}$ & $\begin{array}{c}\text { Differenzierungsmaterial Klassenräume } \\
\text { vorhanden (SL, RSL, FSL) (n= 122) }\end{array}$ \\
\hline ja & $34,7 \%$ & $16,7 \%$ \\
teilweise & $54,5 \%$ & $55,8 \%$ \\
nein & $9,9 \%$ & $26,7 \%$ \\
\hline
\end{tabular}

\section{Hilfsmittel}

Mit Blick auf spezifische Hilfsmittel weisen die befragten Schulleitungen und Lehrkräfte darauf hin, dass ein großer Teil der Schülerinnen und Schüler mit sonderpädagogischem Förderbedarf andere Bücher und Arbeitshefte sowie weitere Hilfsmittel im Unterricht benötigt. Die Angaben verteilen sich dabei wie folgt:

Tab. 25: Hilfsmittel für $\mathrm{SuS}$ mit spF a)

\begin{tabular}{lccc}
\hline $\begin{array}{c}\text { SuS benötigen andere } \\
\text { Bücher (SL, RSL, FSL) } \\
(\mathbf{n = 1 2 2})\end{array}$ & $\begin{array}{c}\text { SuS benötigen andere Ar- } \\
\text { beitshefte (SL, RSL, FSL) } \\
(\mathbf{n = 1 2 2})\end{array}$ & $\begin{array}{c}\text { SuS benötigen handlungs- } \\
\text { orientiertes Material (SL, } \\
\text { RSL, FSL) (n= 122) }\end{array}$ \\
\hline $\mathrm{ja}$ & $58,3 \%$ & $62,5 \%$ & $67,5 \%$ \\
nein & $41,7 \%$ & $37,5 \%$ & $32,5 \%$ \\
\hline
\end{tabular}

Tab. 26: Hilfsmittel für $\mathrm{SuS}$ mit spF b)

\begin{tabular}{lccc}
\hline & $\begin{array}{c}\text { SuS benötigen Mikroport- } \\
\text { anlage (SL, RSL, FSL) } \\
(\mathbf{n = 1 2 2})\end{array}$ & $\begin{array}{c}\text { SuS benötigen großen } \\
\text { Bildschirm (SL, RSL, } \\
\text { FSL) }(\mathbf{n}=\mathbf{1 2 2})\end{array}$ & $\begin{array}{c}\text { SuS benötigen besondere } \\
\text { Möbel (SL, RSL, FSL) } \\
(\mathbf{n}=\mathbf{1 2 2})\end{array}$ \\
\hline $\mathrm{ja}$ & $10,8 \%$ & $4,2 \%$ & $2,5 \%$ \\
nein & $89,2 \%$ & $95,8 \%$ & $97,5 \%$ \\
\hline
\end{tabular}

\section{Weitere Hilfsmittel}

In ihren qualitativen Aussagen geben die Befragten an, dass spezifische Hilfsmittel für notwendig erachtet werden wie bspw. Bildschirmlesegeräte, Lupen, Monokulare, Punktschriftmaschinen oder eine bessere Beleuchtung für Schülerinnen und Schüler mit Sehbehinderungen bzw. die Ausstattung geräuscharmer Klassenräume (besondere Schallschutzdecken; Mittel zur Reizreduktion (z.B. Gehörschutz) und Fokussierung (z.B. Aufpass-Punkt)) für Schülerinnen oder Schüler mit Hörbehinderungen. Auch ein Schüler (ohne Hörbeeinträchtigung) wünscht sich in diesem Zusammenhang leisere Klassenräume. Mit Blick auf Differenzierung seien neben anderen Büchern oder Heften andere Linienvorlagen, individuell erstellte Arbeitsblätter, handlungsorientiertes Material, Übungsmaterial für die PC-Nutzung, Handlungspläne sowie zur Förderung im sozial-emotionalen Bereich - Verhaltensrückmeldepläne, positive Verstärker, erlebnispädagogische Angebote und Sozialkompetenztrainings hilfreich. Für die Zukunft wünschen die befragten Lehrkräfte sich die Bereitstellung bzw. Optimierung von Förder- und An- 
schauungsmaterial (in den Klassenräumen) sowie eine entsprechende finanzielle Unterstützung (Vorschläge: bestimmtes Budget für jeden Schüler (2)). Mehrere Lehrkräfte gehen davon aus, dass die Förderung der Schülerinnen und Schüler mit spF verbessert werden könnte, wenn mehr differenziertes Material angeschafft werden könnte (8), ggf. als Grundausstattung für die Klassenräume. Drei Lehrkräfte schlagen vor, dass eine Materialsammlung zusammengestellt werden sollte, also „ein Pool an Arbeits-/Diff.-/Fördermaterial gebildet wird, auf den jeder Zugriff hat“.

\subsubsection{Leitung einer Schule}

Die Aussage, dass die Schulleitung offen für die Integration von Kindern und Jugendlichen mit Förderbedarf sei, erfährt von RSL, FSL und Eltern übereinstimmend ein sehr hohes Maß (75,5\%, 76,3\%, 73,5\%) bzw. ein eher hohes Maß (20,8\%, 21,1\%, 23,5\%) an Zustimmung. Drei der vier Schulleitungen der SFZ schätzen dies mit Blick auf die Schulleitungen der Regelschulen insgesamt vorsichtiger mit einem eher hohen Maß an Zustimmung ein.

Tab. 27: Offenheit der Schulleitung für die Integration von $\mathrm{SuS}$ mit spF

\begin{tabular}{lccr}
\hline & RSL $(\mathbf{n}=\mathbf{5 3})$ & FSL $(\mathbf{n}=\mathbf{3 8})$ & $\mathbf{E}(\mathbf{n}=\mathbf{3 4})$ \\
\hline stimme sehr zu & $75,5 \%$ & $76,3 \%$ & $73,5 \%$ \\
stimme eher zu & $20,8 \%$ & $21,1 \%$ & $23,5 \%$ \\
stimme eher nicht zu & $1,9 \%$ & $0 \%$ & $0 \%$ \\
stimme gar nicht zu & $0 \%$ & $0 \%$ & $0 \%$ \\
fehlend & $1,9 \%$ & $2,6 \%$ & $2,9 \%$ \\
\hline
\end{tabular}

Eine kritische qualitative Aussage einer Regelschullehrkraft zur Schulleitung bezieht sich darauf,

[...] dass die Schulleitung die Kollegen nur wenig unterstützt, ihnen Fö-Kinder in die Klassen setzt, ohne Mögl. aufzuzeigen, wie man Unterricht dahingehend verändern müsste. Die Kollegen fühlen sich überfordert, belastet und „ohnmächtig“. Dennoch versuchen viele im Rahmen ihrer Ressourcen der Integration gerecht zu werden.

Für die Zukunft wünscht sich eine Lehrkraft eine „Schulleitung, die „vorlebt“ und Tipps zur Umsetzung ans Kollegium weiter gibt“.

\subsubsection{Schule als sozialer Raum}

Insgesamt wird das Schulklima in den Schulen von allen befragten Beteiligten vorwiegend als sehr gut bzw. gut beurteilt und zwar sowohl mit Blick darauf, wie die SuS mit spF sich in den Schulen fühlen als auch hinsichtlich der sozialen Beziehungen untereinander, der gegenseitigen Akzeptanz - zwischen SuS mit und ohne spF wie zwischen RSL und SuS mit spF - sowie bezüglich des Themas Freundschaften innerhalb der Schulen. Auffällig ist, dass die Einschätzungen der RSL sowie der FSL etwas vorsichtiger sind als die der anderen Befragten. Besonders positiv ist das Antwortverhalten bei den Eltern sowie bei den Schülerinnen und Schülern selbst. Lediglich ein Bereich scheint nicht genauso gut ausgeprägt zu sein, wie die anderen, und zwar der der nachmittäglichen Kontakte von SuS mit spF zu anderen SuS.

In einem offenen Feld, in dem weitere Aussagen zur Schule möglich waren, findet sich eine Fülle von Äußerungen dazu, dass es sich bei den beteiligten Schulen zum Teil um Schulen handelt, die über langjährige Integrationserfahrung verfügen und stark auf die individuelle Förderung von Kindern und Jugendlichen ausgerichtet sind. So gibt es eine Schule mit einem eigenen Un- 
terrichts- und Erziehungskonzept (bspw. zu offenen Arbeits- und Lernmethoden), eine Schule, die von den Stärken der Kinder ausgeht, eine weitere Schule mit einem inklusionsbejahenden, individualisierenden Förderkonzept, eine Schule, die als „familiär“ bezeichnet wird sowie eine Schule, bei der integrativer Unterricht Teil des Leitbilds ist. Zum Teil weisen die Aussagen darauf hin, dass es den Schulen insgesamt darum geht ihre erzieherische und unterrichtliche Arbeit kontinuierlich zu verbessern. Eine weitere Aussage bezieht sich auf das problematische Einzugsgebiet der Schule: „Das Einzugsgebiet der Schule ist geprägt von wenig renovierten Altbauten, in denen vorwiegend Immigranten und sozialschwache Familien leben... Zwei Befragte wünschen sich mit Blick auf die Zukunft des Schulklimas, dass Lehrer den Schülern gegenüber Respekt zeigen, so dass die „Chemie zwischen Lehrer und Schüler“ stimme, ein „sozialeres Verhalten gegenüber oder durch Kinder mit Defiziten“ an den Tag gelegt werde bzw. schlicht „mehr Toleranz von Eltern und Mitschülern“.

\section{Wohlbefinden der Schülerinnen und Schüler mit spF}

Über $90 \%$ der SuS mit spF geben an sich in ihren Schulen gut bzw. sehr gut zu fühlen. Übertroffen werden diese Werte noch von der Einschätzung der SL der Regelschulen (insgesamt 93,5\%), während sie bei den RSL, FSL und den Eltern etwas niedriger liegen. Zwischen 5,3\% (FSL) und $11,8 \%$ (Eltern) geben an, dass die Sus sich weniger gut fühlen.

Tab. 28: Wohlbefinden der SuS mit spF in der Schule

\begin{tabular}{lccccc}
\hline & SL $(\mathbf{n}=\mathbf{3 1})$ & RSL $(\mathbf{n}=\mathbf{5 3})$ & FSL $(\mathbf{n}=\mathbf{3 8})$ & $\mathbf{E}(\mathbf{n}=\mathbf{3 4})$ & $\mathbf{S}($ alle $)(\mathbf{n}=\mathbf{3 3})$ \\
\hline sehr gut & $25,8 \%$ & $20,8 \%$ & $10,5 \%$ & $41,2 \%$ & $48,5 \%$ \\
gut & $67,7 \%$ & $60,4 \%$ & $73,7 \%$ & $35,3 \%$ & $42,4 \%$ \\
weniger gut & $0 \%$ & $7,5 \%$ & $5,3 \%$ & $11,8 \%$ & $3,0 \%$ \\
gar nicht gut & $0 \%$ & $1,9 \%$ & $0 \%$ & $2,9 \%$ & $0 \%$ \\
fehlend & $6,5 \%$ & $9,4 \%$ & $10,5 \%$ & $8,8 \%$ & $6,1 \%$ \\
\hline
\end{tabular}

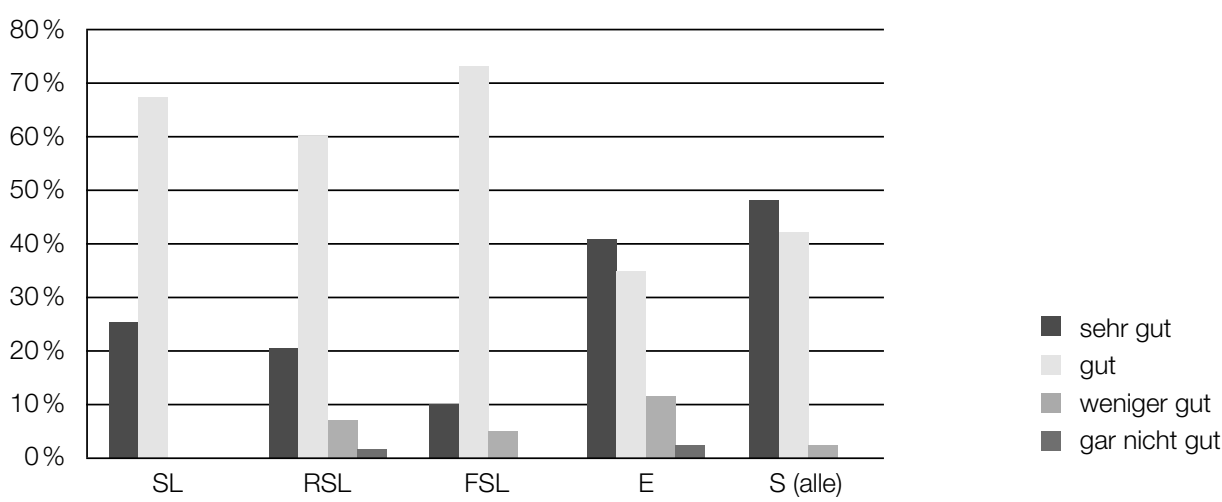

Abb. 9: Wohlbefinden der SuS mit spF in den Regelschulen

\section{Qualitative Begründungen zum Wohlbefinden der Schülerinnen und Schüler}

Schulleitungen, Regel- und Förderschulehrkräfte führen vielfältige Begründungen dafür an, dass die Schülerinnen und Schüler mit spF sich in ihren Schulen wohlfühlen. Eine Reihe von 
Aussagen gibt aber auch kritische Meinungen wieder. Die Schulleitungen begründen die Tatsache, dass die SuS mit spF sich in ihren Schulen gut bzw. sehr gut fühlen, damit, dass „die Schule sehr großen Wert legt auf Toleranz, Gerechtigkeit, Akzeptanz von Andersartigkeit und ein lebendiges Schulleben hat“ oder es „,normaler Alltag ist, an unserer Schule gemeinsam unterrichtet zu werden“ bzw. dass „die Schule als Ganztagsschule ein gutes soziales Klima hat“. Auch zwei Lehrkräfte verweisen auf das gute bzw. familiäre Klima der Schule, das sich positiv auswirke. Eine Schulleitung erläutert, dass die Lehrkräfte „viel Kompetenztraining durchführen und auf das entspr. Miteinander größten Wert legen“. Eine Lehrkraft bezieht sich auf das gute Verhältnis aller beteiligten Personen untereinander. Drei weitere Lehrkräfte betonen, dass die Schüler mit spF zur Schule gehören „und das für sie selbst und alle anderen „normal“/selbstverständlich ist“, „sie genauso gern kommen, wie die Regelschüler, sich aber auch genauso über Stundenausfälle freuen“ oder „sie motiviert sind und gerne zur Schule gehen“. Eine Förderschullehrkraft erwähnt, dass die $\mathrm{SuS}$ mit spF sich wohlfühlen, „weil sie auf keinen Fall in eine Sonderschule wollen!“. 23 Schulleitungen und Lehrkräfte führen als Begründung an, dass die Schülerinnen und Schüler akzeptiert werden bzw. sich angenommen fühlen. Zwei weitere Befragte sprechen davon, dass die SuS „sich geborgen und aufgehoben fühlen“ bzw. „sozial dazu gehören“. Auf die gute soziale Integration in die Schulgemeinschaft verweisen acht Schulleitungen, Regelund Förderschullehrkräfte. Weitere Aussagen beziehen sich auf ein gutes Klassenklima (2), die gute Integration im Klassenverband (11) sowie Freundschaften in der Schule (3). Von wichtiger Bedeutung für das Wohlbefinden der SuS mit spF ist nach Auffassung der Schulleitungen und Lehrkräfte auch die Unterstützung durch die Regel- und Förderschullehrer mit Blick auf an die Lernvoraussetzungen angepasste Anforderungen (12). Diese wird dahingehend beschrieben, dass „sich die Förderlehrerinnen und die Lehrerinnne intensiv um sie kümmern und sie jederzeit unterstützen“, „sie dort abgeholt werden, wo sie stehen“, „sie ihrem Lernniveau entsprechend ohne großen Druck lernen können“, „die Förderung individuell auf sie abgestimmt ist“, „sie in ihrer Individualität angenommen werden und Lernfortschritte erzielen können“ oder „weil sie in ihrer Individualität erkannt werden und das Mögliche getan wird, sie zu begleiten, zu fördern und stark zu machen, selbstbewusst zu sein“. Weitere Schulleitungen und Lehrkräfte begründen ihre Auffassung damit, dass die SuS mit spF „keinen erkennbaren Unterschied zu ihren Mitschülern spüren“, „nicht ,in eine spezielle Schublade gesteckt“ werden bzw. „noch nicht spüren, dass sie ,anders“ sind“.

Auch die Eltern erwähnen das gute Schulklima, dass die Kinder akzeptiert werden bzw. sich gut aufgehoben fühlen (6). Sie erwähnen, dass ihr Kind „Spaß am Lernen und am Spielen in der Betreuung “ habe, „gerne lernt, sich interessiert“, „die Lehrer mag, sich „gut“ mit seinen Klassenkameraden versteht, er gerne lernt + wissbegierig ist, man sich um ihn bemüht, ihn pusht wo es notwendig ist“ bzw. „Kinderhilfshausmeister“ sei.

Kritische Ausführungen beziehen sich darauf, dass das Wohlbefinden individuell sehr unterschiedlich sei und auch vom Grad der Behinderung abängen könne. Während die Integration von SuS mit den spF Lernen oder Sprache gut gelinge, seien verhaltensauffällige Kindern nicht gut in den Schulalltag integriert. Je nach Klassengröße sei der Lärmpegel zu hoch, so dass SuS mit SE dann in Abseiterpositionen geraten könnten. Diesen SuS fehle „sofortige Rückmeldung“ oder „erzieherische Hilfe“. Andere Aussagen thematisieren Abstandserfahrungen und erläutern, dass SuS sich nicht wohlfühlen, „weil manche ständig vor Augen haben, dass andere „besser“ sind und darunter leiden“ oder es nicht spurlos an ihnen vorbeigehe, „dass sie andere Arbeiten schreiben und anders bewertet werden“. Ungünstige Lernerfahrungen könnten zu „Motivationsverlust“ führen. Teilweise würden Schüler „merken, dass sie eine Sonderrolle einnehmen 
und häufig an ihre Grenzen stoßen und es oft zu Problemen mit Mitschülern kommt“. Zwei Förderschullehrkräfte verweisen auf leistungs- bzw. sozialbezogene Randständigkeit von SuS mit spF. Eine führt dazu aus: „keine soziale Integration, werden ausgeschlossen, sind immer die „Störenfriede“, haben ein schlechtes Selbstkonzept, werden nicht zum Spielen eingeladen, bekommen keine ind. Förderung“. Da die Klassen unterschiedlich seien und „Klassenkameraden unterschiedlich reagieren“, könne die Integration unterschiedlich gut bzw. weniger gut verlaufen. Einige Schüler würden in ihren Klassen gehänselt. Einzelne, weniger gut integrierte Schüler, würden sich daher entsprechend weniger gut fühlen. Vier Elternteile äußern sich kritisch mit Bezug darauf, dass ihre Kinder geärgert werden (2), in einer großen, lauten Klasse keine Freunde hätten bzw. die Anforderungen sehr hoch seien und „nicht alle Lehrpersonen ausreichend auf ihre Behinderung eingehen“.

\section{Einschätzung der sozialen Beziehungen der SuS an der Schule untereinander}

Insgesamt werden die sozialen Beziehungen aller SuS untereinander in den Schulen vorwiegend positiv eingeschätzt. Lediglich die FSL sehen dies in Teilen kritischer: 15,8\% schätzen diese Beziehungen als weniger gut ein.

Tab. 29: Einschätzung der sozialen Beziehungen der SuS an der Schule untereinander

\begin{tabular}{lccc}
\hline & SL $(\mathbf{n}=\mathbf{3 1})$ & RSL $(\mathbf{n}=\mathbf{5 3})$ & FSL $(\mathbf{n}=\mathbf{3 8})$ \\
\hline sehr gut & $16,1 \%$ & $11,3 \%$ & $2,6 \%$ \\
gut & $80,6 \%$ & $75,5 \%$ & $73,7 \%$ \\
weniger gut & $0 \%$ & $11,3 \%$ & $15,8 \%$ \\
gar nicht gut & $0 \%$ & $1,9 \%$ & $0 \%$ \\
\hline
\end{tabular}

\section{Verbesserung des sozialen Miteinanders durch schulische Integration ${ }^{65}$}

Dabei gehen abgesehen von knapp $10 \%$ der SL und zwischen knapp $18 \%$ bzw. $20 \%$ der Eltern, RSL und FSL davon aus, dass die schulische Integration das soziale Miteinander in den Schulen verbessert. Die Schulleitungen schätzen die Wirkung aus ihrer Perspektive offensichtlich positiver ein.

Tab. 30: Verbesserung des sozialen Miteinanders der SuS in der Schule durch die integrative Unterrichtung

\begin{tabular}{lcccc}
\hline & SL (n=31) & RSL (n=53) & FSL (n=38) & E (n=34) \\
\hline stimme sehr zu & $48,4 \%$ & $24,5 \%$ & $26,3 \%$ & $38,2 \%$ \\
stimme eher zu & $35,5 \%$ & $49,1 \%$ & $52,6 \%$ & $38,2 \%$ \\
stimme eher nicht zu & $9,7 \%$ & $18,9 \%$ & $21,1 \%$ & $17,6 \%$ \\
stimme gar nicht zu & $0 \%$ & $3,8 \%$ & $0 \%$ & $0 \%$ \\
\hline
\end{tabular}

\section{Akzeptanz der SuS mit spF durch andere SuS}

Die Aussage, dass die Schülerinnen und Schüler mit Förderbedarf von den anderen Schülern akzeptiert werden, erfährt von etwa bzw. etwas mehr als der Hälfte der Schulleitungen der Regelschulen, der Regelschullehrkräfte wie der Eltern ein sehr hohes Maß an Zustimmung, wäh- 
rend die Förderschullehrkräfte dies nur zu 26,3\% angeben. Besonders positiv wird das Maß der Akzeptanz durch die $\mathrm{SuS}$ selbst eingeschätzt.

Tab. 31: Akzeptanz der SuS mit spF durch andere SuS

\begin{tabular}{lrrrrr}
\hline & SL (n=31) & RSL (n= 53) & FSL (n=38) & E (n=34) & $\begin{array}{r}\text { S GMS } \\
(\mathbf{n}=\mathbf{1 2})\end{array}$ \\
\hline stimme sehr zu & $58,1 \%$ & $54,7 \%$ & $26,3 \%$ & $50,0 \%$ & $66,7 \%$ \\
stimme eher zu & $41,9 \%$ & $41,5 \%$ & $65,8 \%$ & $38,2 \%$ & $33,3 \%$ \\
stimme eher nicht zu & $0 \%$ & $3,8 \%$ & $2,6 \%$ & $8,8 \%$ & $0 \%$ \\
stimme gar nicht zu & $0 \%$ & $0 \%$ & $0 \%$ & $0 \%$ & $0 \%$ \\
\hline
\end{tabular}

\section{Freunde der Schülerinnen und Schüler mit spF}

Ähnlich sieht das Antwortverhalten bei der Aussage dazu aus, dass die Schüler mit Förderbedarf Freunde in der Schule haben, bei der 61,3\% der SL und 49,1\% der RSL in sehr hohem Maß zustimmen. Hier schätzen die FSL mit nur $23,7 \%$ sehr hoher Zustimmung sowie die Eltern mit $38,2 \%$ dies vorsichtiger ein, während die SuS dies zu $100 \%$ befürworten.

Tab. 32: SuS mit spF haben Freunde in der Schule

\begin{tabular}{lrcccc}
\hline & SL (n=31) & RSL (n=53) & FSL (n=38) & E (n=34) & $\begin{array}{r}\text { S GMS } \\
(\mathbf{n}=\mathbf{1 2})\end{array}$ \\
\hline stimme sehr zu & $61,3 \%$ & $49,9 \%$ & $23,7 \%$ & $38,2 \%$ & $100 \%$ \\
stimme eher zu & $35,5 \%$ & $37,7 \%$ & $65,8 \%$ & $52,9 \%$ & $0 \%$ \\
stimme eher nicht zu & $3,2 \%$ & $22,6 \%$ & $55,3 \%$ & $8,8 \%$ & $0 \%$ \\
stimme gar nicht zu & $0 \%$ & $3,8 \%$ & $21,1 \%$ & $0 \%$ & $0 \%$ \\
\hline
\end{tabular}

\section{Nachmittagskontakte spF}

Am stärksten unterscheidet sich das Antwortverhalten zum Bereich Schulklima bei der Frage danach, inwiefern die SuS mit spF auch nachmittags Kontakte zu anderen SuS haben. Dies schätzen die SuS selbst am positivsten ein, allerdings wurden dazu nur die SuS der GMS befragt, bei denen die Stichprobe mit $12 \mathrm{SuS}$ sehr klein ist. Etwa $10 \%$ der Schulleitungen, ein Fünftel der RSL und FSL sowie knapp $40 \%$ der Eltern geben an, dass diese Nachmittagskontakte eher nicht bestehen.

Tab. 33: Nachmittagskontakte der SuS mit spF

\begin{tabular}{lrcccr}
\hline & SL (n=31) & RSL (n=53) & FSL (n=38) & E (n=34) & $\begin{array}{r}\text { S GMS } \\
(\mathbf{n}=\mathbf{1 2})\end{array}$ \\
\hline stimme sehr zu & $35,5 \%$ & $34,0 \%$ & $13,2 \%$ & $26,5 \%$ & $58,3 \%$ \\
stimme eher zu & $51,6 \%$ & $39,6 \%$ & $55,3 \%$ & $26,5 \%$ & $33,3 \%$ \\
stimme eher nicht zu & $9,7 \%$ & $22,6 \%$ & $21,1 \%$ & $38,2 \%$ & $8,3 \%$ \\
stimme gar nicht zu & $0 \%$ & $3,8 \%$ & $0 \%$ & $8,8 \%$ & $0 \%$ \\
\hline
\end{tabular}




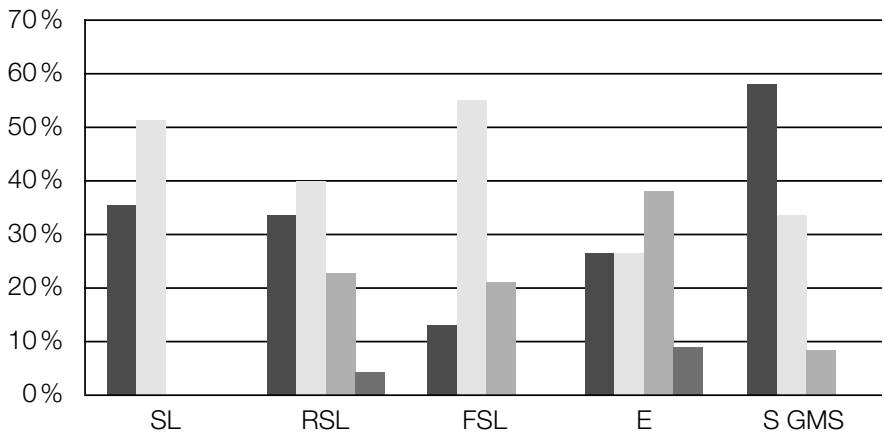

stimme sehr zu stimme eher zu stimme eher nicht zu stimme gar nicht zu

Abb. 10: Nachmittagskontakte von SuS mit spF in den Regelschulen

Akzeptanz der Schüler mit sonderpädagogischem Förderbedarf durch die Regelschullehrkräfte Die Aussage, dass die Schüler mit spF von den Lehrerinnen und Lehrern der Schule akzeptiert werden, erfährt - abgesehen von den FSL - von allen Befragten vorwiegend ein sehr hohes Maß an Zustimmung, wobei die Werte von RSL, E und S fast deckungsgleich sind. Insbesondere die Schulleitungen der Regelschulen schätzen diese Akzeptanz mit $83 \%$ als sehr positiv ein.

Tab. 34: SuS mit spF werden durch die Lehrkräfte der Regelschulen akzeptiert

\begin{tabular}{lrrrrr}
\hline & SL (n=31) & RSL (n=53) & FSL (n=38) & E (n=34) & $\begin{array}{r}\text { S GMS } \\
(\mathbf{n}=\mathbf{1 2})\end{array}$ \\
\hline stimme sehr zu & $67,7 \%$ & $83,0 \%$ & $31,6 \%$ & $64,7 \%$ & $66,7 \%$ \\
stimme eher zu & $32,3 \%$ & $15,1 \%$ & $63,2 \%$ & $32,4 \%$ & $33,3 \%$ \\
stimme eher nicht zu & $0 \%$ & $1,9 \%$ & $0 \%$ & $2,9 \%$ & $0 \%$ \\
stimme gar nicht zu & $0 \%$ & $0 \%$ & $0 \%$ & $0 \%$ & $0 \%$ \\
\hline
\end{tabular}

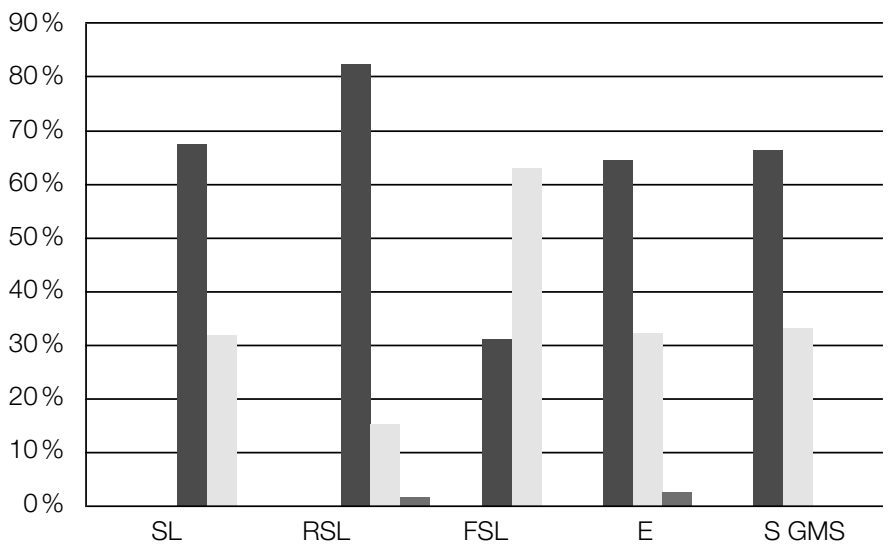

stimme sehr zu stimme eher zu stimme eher nicht zu

Abb. 11: SuS mit spF werden durch die Lehrkräfte der Regelschulen akzeptiert 


\section{Zusammenarbeit mit Eltern}

Eine ganze Reihe von Items befasst sich im Rahmen des Fragebogens mit der Zusammenarbeit zwischen Eltern und Schule allgemein sowie mit der zwischen Eltern von SuS mit spF und den RSL bzw. FSL im Speziellen.

\section{Elternwahlrecht}

Grundlegend spielt zunächst die Frage nach dem Erhalt des Elternwahlrechts zwischen der Förderung in einer Regel- oder Förderschule eine Rolle. Der Erhalt des Elternwahlrechts dahingehend, ob als Förderort eine Regel- oder eine Förderschule ausgewählt wird, erfährt das höchste $\mathrm{Maß}$ an Zustimmung durch die Eltern selbst (insgesamt $97 \%$ positive Antworten). Differenzierter sieht das Antwortverhalten bei den SL, RSL und FSL aus, bei denen zwischen 16,1\% (SL), 26,4\% (RSL) und 15,8\% (FSL) dieser Aussage eher nicht bzw. gar nicht zustimmen.

Tab. 35: Das Elternwahlrecht sollte erhalten bleiben

\begin{tabular}{lcccc}
\hline & SL $(\mathbf{n}=\mathbf{3 1})$ & RSL $(\mathbf{n}=\mathbf{5 3})$ & FSL $(\mathbf{n}=\mathbf{3 8})$ & $\mathbf{E}(\mathbf{n}=\mathbf{3 4})$ \\
\hline stimme sehr zu & $41,9 \%$ & $35,8 \%$ & $57,9 \%$ & $73,5 \%$ \\
stimme eher zu & $32,3 \%$ & $35,8 \%$ & $23,7 \%$ & $23,5 \%$ \\
stimme eher nicht zu & $12,9 \%$ & $13,2 \%$ & $10,5 \%$ & $2,9 \%$ \\
stimme gar nicht zu & $3,2 \%$ & $13,2 \%$ & $5,3 \%$ & $0 \%$ \\
\hline
\end{tabular}

\section{Bewertung der Zusammenarbeit zwischen Schulleitungen und Eltern}

Die Zusammenarbeit mit den Schulleitungen, die auf Seiten der Eltern abgefragt wurde, wird sehr positiv bewertet. So gibt etwa die Hälfte der Eltern $(52,9 \%)$ an, dass diese sehr gut sei und $38,2 \%$ stimmen dieser Aussage eher zu.

Tab. 36: Bewertung der Zusammenarbeit zwischen Eltern und SL (Angaben Eltern, $n=34$ )

\begin{tabular}{lr}
\hline & Eltern \\
\hline sehr gut & $52,9 \%$ \\
gut & $38,2 \%$ \\
weniger gut & $5,9 \%$ \\
gar nicht gut & $0 \%$ \\
\hline
\end{tabular}

Begründungen der Bewertungen der Zusammenarbeit zwischen Schulleitungen und Eltern Nur zwei Aussagen von Elternteilen beziehen sich darauf, dass die Zusammenarbeit mit den Schulleitungen als weniger gut eingestuft wird: „weil die Direktorin neu ist und noch nicht wirklich viel Kontakt mit mir hatte“; „weil keine Rückfragen, Infos von Schule, sondern nur nach Anfrage, Anregung durch uns“. Alle anderen Aussagen beschreiben die positive Unterstützung, die Eltern von SuS mit sonderpädagogischem Förderbedarf durch die Schulleitungen erfahren. Sie beschreiben, dass „die Schulleitung immer auf uns zukommt und ich mit ihnen über alles reden kann, was meine Tochter betrifft", in der Schulleitung immer ein Ansprechpartner vorhanden sei, der Informationsfluss regelmäßig und zügig erfolge, ein hohes Maß an Unterstützung vorhanden sei, lösungsorientierte bzw. pragmatische Hilfe bei Problemen erfolge (4) bspw. bei 
schulischen Leistungen, aber auch im sozialen Bereich, die Schulleitungen gut auf Kinder und Eltern eingehen und sich für die Kinder mit spF interessieren und engagieren. Ein Elternteil hebt die emotionale und soziale Kompetenz der Schulleitung hervor, die „außerhalb des Lehrplanes“ viele Angebote für die Kinder veranstalte.

Bewertung der Zusammenarbeit zwischen Eltern und Klassenleitung

Ähnlich positiv wird die Zusammenarbeit mit den Klassenleitungen durch die Eltern bewertet:

Tab. 37: Bewertung der Zusammenarbeit zwischen Eltern und Klassenleitung (Angaben Eltern, $\mathrm{n}=34$ )

\begin{tabular}{lr}
\hline & Eltern \\
\hline sehr gut & $63,6 \%$ \\
gut & $30,3 \%$ \\
weniger gut & $6,1 \%$ \\
gar nicht gut & $0 \%$ \\
\hline
\end{tabular}

Begründungen für die Bewertungen der Zusammenarbeit zwischen Eltern und Klassenleitungen

Auch die Begründungen der Eltern zu den Bewertungen der Zusammenarbeit mit den Klassenleitungen fallen sehr positiv aus und thematisieren die Regelmäßigkeit der Gespräche, den (zügigen) Informationsfluss (4), bspw. zum Leistungsstand, das hohe Engagement der Lehrkräfte (4): „der Klassenlehrer macht viele Überstunden für „seine“ Schüler“, „die neue Klassenleitung sich sehr für die Kinder einsetzt und sich sehr bemüht", die Tatsache, dass die Klassenleitungen gut auf die Kinder eingehen, von sich aus auf die Eltern zugehen, bei Problemen geholfen wird (3), die Eltern unterstützen, ein offenes Ohr haben, das Interesse am Kind hoch ist oder „sehr viel Ahnung von Integration" gegeben sei. Lediglich ein Elternteil bemängelt, dass die Kommunikation immer von Elternseite aus erfolgen müsse.

Bewertung Zusammenarbeit Eltern - Förderschullehrkräfte

Die Bewertung der Zusammenarbeit mit den Förderschullehrkräften unterscheidet sich nur geringfügig von der mit den RSL:

Tab. 38: Bewertung der Zusammenarbeit zwischen Eltern und FSL (Angaben Eltern, $\mathrm{n}=34$ )

\begin{tabular}{lr}
\hline & Eltern \\
\hline stimme sehr zu & $59,4 \%$ \\
stimme eher zu & $28,1 \%$ \\
stimme eher nicht zu & $12,5 \%$ \\
stimme gar nicht zu & $0 \%$ \\
\hline
\end{tabular}

Begründungen für die Bewertungen der Zusammenarbeit zwischen Eltern und Förderschullehrkräften

Fünf Elternteile begründen ihre weniger positive Bewertung der Zusammenarbeit mit den Förderschullehrkräften damit, dass entweder noch keine Gespräche stattgefunden haben (3), 
der Kontakt weniger intensiv sei bzw. gar nicht erfolge. Die anderen Elternteile begründen die gute Zusammenarbeit u.a. damit, dass diese hervorragend funktioniere, sie regelmäßig über den Stand der Förderung ihrer Kinder informiert werden, dass die Lehrkräfte Interesse an den Kindern zeigen, sich sehr engagieren (3), immer Ansprechpartner sind und sich auch mit anderen Professionen (Bsp.: Ergotherapeutin, Arzt, Integrationshelferin) sehr gut austauschen.

\section{Formen der Zusammenarbeit mit Eltern}

Elterabende und Gespräche finden - von einer Ausnahme abgesehen - in allen befragten Schulen statt. Zwei Drittel der Schulleitungen nutzen E-Mails zur Kommunikation mit den Eltern.

Tab. 39: SL - Formen der Zusammenarbeit mit Eltern $(\mathrm{n}=31)$

\begin{tabular}{lccc}
\hline & Elternabende & Gespräche & E-Mails \\
\hline ja & $96,8 \%$ & $100 \%$ & $61,3 \%$ \\
nein & $3,2 \%$ & $0 \%$ & $38,7 \%$ \\
\hline
\end{tabular}

17 Schulleitungen erwähnen weitere Formen der Zusammenarbeit wie die Kommunikation über das Hausaufgabenheft bzw. Mitteilungshefte (3), Elternbriefe (2), Elternstammtische bzw. ein Elterncafé (3), Elternsprechtage (2), Feste oder andere Veranstaltungen (Bastelnachmittage, Wanderungen, Grillfest) (4), Projekte (Schulobst, Klasse 2000) (2), Umfragen, „feste Entwicklungsgespräche in Friedenszeiten“, Hospitationen, eine Elternschule, eine „Elternsprecherkonferenz (alle Elternvertreter + Schulleitung + Nachbetreuung, ca. alle 2 Monate)“, Elternstunden „best. Themen, 2x/Jahr“, Förderausschusssitzungen, Klassenkonferenzen, runde Tische mit allen Therapeuten oder den Austausch über Logbuch, SMS oder Whatsapp. Die Formen der Zusammenarbeit zwischen RSL und Eltern sowie zwischen FSL und Eltern unterscheiden sich anteilsmäßig nur sehr geringfügig voneinander. Am häufigsten finden Telefonate (RSL $51 \%$ (sehr häufig + häufig); FSL 63,2\%) und Gesprächstermine (RSL 71,7\%; FSL 71,1\%) statt.

Tab. 40: RSL - Formen der Zusammenarbeit mit den Eltern $(n=53)$

\begin{tabular}{lcccc}
\hline & Telefonate & E-Mails & Gesprächstermine & Mitteilungshefte \\
\hline sehr häufig & $3,8 \%$ & $5,7 \%$ & $1,9 \%$ & $30,2 \%$ \\
häufig & $47,2 \%$ & $15,1 \%$ & $69,8 \%$ & $34,0 \%$ \\
eher selten & $35,8 \%$ & $37,7 \%$ & $24,5 \%$ & $22,6 \%$ \\
gar nicht & $1,9 \%$ & $0 \%$ & $1,9 \%$ & $3,8 \%$ \\
fehlend & $11,3 \%$ & $41,5 \%$ & $1,9 \%$ & $9,4 \%$ \\
\hline
\end{tabular}

Tab. 41: FSL - Formen der Zusammenarbeit mit den Eltern $(\mathrm{n}=38)$

\begin{tabular}{lcccc}
\hline & Telefonate & E-Mails & Gesprächstermine & Mitteilungshefte \\
\hline sehr häufig & $21,1 \%$ & $7,9 \%$ & $7,9 \%$ & $28,9 \%$ \\
häufig & $42,1 \%$ & $15,8 \%$ & $63,2 \%$ & $34,2 \%$ \\
eher selten & $26,3 \%$ & $18,4 \%$ & $23,7 \%$ & $23,7 \%$ \\
gar nicht & $0 \%$ & $0 \%$ & $0 \%$ & $0 \%$ \\
fehlend & $10,5 \%$ & $57,9 \%$ & $5,3 \%$ & $10,5 \%$ \\
\hline
\end{tabular}


Die Eltern der SuS mit spF schätzen die Häufigkeit der persönlichen Kontakte über Telefonate oder Gesprächstermine - abgesehen von den Telefonaten zwischen Eltern und FSL - offensichtlich geringer ein (Telefonate E - KL 26,4\%; E - FSL 63,2\%; Gesprächstermine E - KL $38,3 \%$; E - FSL 32,4\%).

Tab. 42: E - Formen der Zusammenarbeit mit der Klassenleitung ( $\mathrm{n}=34)$

\begin{tabular}{lcccc}
\hline & Telefonate & E-Mails & Gesprächstermine & Mitteilungshefte \\
\hline sehr häufig & $8,8 \%$ & $8,8 \%$ & $5,9 \%$ & $14,7 \%$ \\
häufig & $17,6 \%$ & $17,6 \%$ & $32,4 \%$ & $32,4 \%$ \\
eher selten & $35,3 \%$ & $11,8 \%$ & $55,9 \%$ & $20,6 \%$ \\
gar nicht & $8,8 \%$ & $0 \%$ & $0 \%$ & $8,8 \%$ \\
fehlend & $29,4 \%$ & $61,8 \%$ & $5,9 \%$ & $23,5 \%$ \\
\hline
\end{tabular}

Tab. 43: E - Formen der Zusammenarbeit mit den Eltern - FSL $(\mathrm{n}=34)$

\begin{tabular}{lcccc}
\hline & Telefonate & E-Mails & Gesprächstermine & Mitteilungshefte \\
\hline sehr häufig & $21,1 \%$ & $0 \%$ & $5,9 \%$ & $0 \%$ \\
häufig & $42,1 \%$ & $0 \%$ & $26,5 \%$ & $23,5 \%$ \\
eher selten & $26,3 \%$ & $5,9 \%$ & $35,3 \%$ & $11,8 \%$ \\
gar nicht & $0 \%$ & $17,6 \%$ & $8,8 \%$ & $14,7 \%$ \\
fehlend & $10,5 \%$ & $76,5 \%$ & $23,5 \%$ & $50 \%$ \\
\hline
\end{tabular}

Als weitere Formen der Zusammenarbeit mit den Eltern werden Mitteilungshefte bzw. ein Logbuch (3), Elternbriefe, SMS, WhatsApp-Nachrichten, Verstärkerpläne, außerschulische Veranstaltungen wie Projekte, Bastelnachmittage, Wanderungen oder Stammtische, Elternschulen, Elternsprechtage, Elternsprecherkonferenzen, Runde Tische, Förderausschusssitzungen, Entwicklungsgespräche, Klassenkonferenzen sowie Hausbesuche benannt.

\section{Kennzeichen der Zusammenarbeit mit Eltern}

Mit Blick auf Kennzeichen der Zusammenarbeit zwischen Schule und Eltern betonen die Schulleitungen der Regelschulen ein reges Interesse, ein hohes Maß an Offenheit, die Bedeutung kurzer Wege, eine schnelle Erreichbarkeit sowie einen ausgeprägten Informationsfluss. Eine Schulleitung fügt hinzu, dass bei SuS mit spF eine schnellere und engere Zusammenarbeit als bei Regelschülern notwendig sei. Der Austausch finde häufiger statt als mit Eltern anderer Kinder. Eine Schulleitung weist darauf hin, dass die FSL einen besonders engen Kontakt zu den Eltern halten. Häufig hervorgehoben werden die Häufigkeit, Regelmäßigkeit und Intensität von Kontakten und gemeinsamen Gesprächen zwischen Eltern und Schulleitungen bzw. Lehrkräften (14). Die Zusammenarbeit mit diesen Eltern finde häufiger bzw. „schneller und enger" statt als bei Regelschülern (3). Der Informationsfluss sei ausgeprägt, die Gespräche seien intensiv (3), eine Rückmeldung für die Eltern erfolge zum Teil täglich. Wichtig seien offene und vertrauensvolle Gespräche, die am Kind orientiert sein und auf Augenhöhe stattfinden sollten, so dass hierarchische Strukturen vermindert werden könnten. Eine Schulleitung weist darauf hin, dass es sich dabei manchmal um schwierige Gespräche handele, „die aber auch nach Tränen oder Konflikt stets sachlich und gut zu Ende gebracht werden“. Als bedeutsam heben zwei Schulleitungen Zielvorgaben bzw. die Arbeit hin auf spezifische Ziele hervor (Bsp.: Schulverein- 
barung), eine andere spricht in diesem Zusammenhang von Zuverlässigkeit und dem Einhalten von Absprachen. Als institutionalisierte Form der Zusammenarbeit erwähnt eine Schulleitung jährliche Förderkonferenzen, eine Lehrkraft die regelmäßigen Treffen in den Förderausschüssen. Eine weitere Schulleitung hebt die Bedeutung langfristigen Arbeitens mit den Eltern hervor. Prägnant fasst eine Schulleitung zusammen: „Je besser die enge Zusammenarbeit mit den Eltern gelingt, desto fruchtbarer und förderlicher gestaltet sich die integrative Beschulung." Zwei der Befragten weisen darauf hin, dass die Eltern Interesse an der Zusammenarbeit zeigen, bzw. in der Regel gut mitarbeiten würden. Zwei andere differenzieren, dass die Kommunikation mit manchen Eltern gut sei (Bsp.: Rückmeldung über Hausaufgaben, Entwicklungen), während andere kein Verständnis für Tipps zeigen und angeratene schulische Maßnahmen nicht umsetzen würden. Dann wiederum seien auch kaum Verbesserungen eines Schülers in der Schule möglich. Eine Schulleitung bemängelt, dass „oft nur ein geringes Interesse an einer sinnvollen Zusammenarbeit“ gegeben sei, eine andere beschreibt die Zusammenarbeit als schwierig, „da die Eltern viele Problematiken, gerade im E-Bereich, nicht sehen wollen“.

Vier Schulleitungen sprechen das Interesse von Eltern an der Zusammenarbeit an, vier weitere thematisieren aber auch Probleme in der Zusammenarbeit, da die Eltern mangelndes Interesse zeigen oder kein Verständnis für Tipps, angeratene außerschulische Maßnahmen nicht umsetzen würden, so dass kaum Verbesserungen in der Schule möglich seien oder Probleme im Verhaltensbereich nicht sehen wollen.

Tab. 44: Gesprächsinhalte in Elterngesprächen FSL - E ( $\mathrm{n}=38)$

\begin{tabular}{lcc}
\hline & Rückmeldung Lernfortschritte & Rückmeldung Probleme der SuS \\
\hline ja & $92,1 \%$ & $100 \%$ \\
nein & $7,9 \%$ & $0 \%$ \\
\hline
\end{tabular}

Neben den abgefragten Gesprächsinhalten (Rückmeldung zu Lernfortschritten und zu Probleme der Schülerinenn und Schüler) sind die Inhalte, die in Gesprächen mit den Eltern thematisiert werden, sehr vielfältig und beziehen sich aus Sicht der Schulleitungen und Lehrkräfte insgesamt auf die vier größeren Themenblöcke Organisation der integrativen Förderung, Förderplanung, Kind-Umfeld-Diagnostik sowie Zusammenarbeit mit außerschulischen Partnern. Mit Blick auf organisatorische Fragen geht es in den Gesprächen bspw. um den Ablauf und die Umsetzung der Integration bzw. Förderung, Absprachen bezüglich der Einleitung von Meldungen zur sonderpädagogischen Überprüfung, die Regelung des Nachteilsausgleichs, die Anschaffung von und Versorgung mit Hilfsmitteln (Bsp.: Bildschirmlesegeräte, Lupen) oder andere zusätzliche Hilfen im Unterricht, bspw. Verstärkerpläne.

Hinsichtlich der Förderplanung für die Schülerinnen und Schüler werden Lernstand (3) und Lernfortschritte thematisiert, allgemeine Entwicklung und Perspektiven. Es erfolgen Rückmeldungen zu Problemen allgemein sowie zu Problemen beim Lernen, zu Problemen im Bereich des Sozialverhaltens (5), der Konzentration oder bei Regelverstößen. Evaluation bzw. Rückmeldungen (3) erfolgen zum Teil täglich (z.B. Smiley-System). Besprochen werden die Förderziele, die individuelle Bewertung bzw. Notengebung in Klassenarbeiten oder Fragen, die mit der Zukunft der SuS zusammenhängen, und sich auf Abschlussmöglichkeiten, die weitere Schullaufbahn, eine mögliche Rückstufung in den Regelschulbereich oder Zukunftsprognosen allgemein beziehen. Darüber hinaus werden die Eltern über aktuelle Sachverhalte aufgeklärt oder um Mithilfe in bestimmten Bereichen gebeten. Eine Lehrkraft gibt an, dass auch Widerstände der Eltern gegenüber 
geplanten Veränderungen aufgegriffen werden. Inhalte sind darüber hinaus Maßnahmen der Schule bzw. Lehrer oder das Vermitteln in Konflikten zwischen RSL und Eltern bzw. FSL und Eltern. Lehrkräfte klären die Eltern über hilfreiche Strukturen im Alltag, angemessene Kleidung, Übungs- oder Fördermöglichkeiten innerhalb und außerhalb der Schule auf oder geben Tipps für die Hausaufgaben und für die Vorbereitung auf Klassenarbeiten. Eine Lehrkraft betont, dass es in den Elterngesprächen darum gehe, das Verhältnis zwischen Kind und Eltern zu stärken und den Eltern zu einer positiven Sicht auf das Kind zu verhelfen. In Gesprächen mit den Eltern werde auch versucht zu einer umfassenderen Kind-Umfeld-Diagnostik zu gelangen, indem ein Austausch zur Freizeit, dem Privatleben etc., der Eltern-Kind-Beziehung, den familiären Hintergründen, der häuslichen Situation bzw. Lebenssituation und dem Umfeld der Schülerinnen und Schüler erfolgt. In einem Fall wurde geklärt, welche körperlichen Möglichkeiten ein Kind mit spF in diesem Bereich habe. Bezüglich der möglichen Unterstützung der Eltern durch außerschulische Dienste geben die Lehrkräfte Empfehlungen (Bsp.: Logopädie) (4), beraten zu Therapien oder achten auf Absprachen mit Ärzten.

\section{Vernetzung}

Aufgrund des Antwortverhaltens zur Vernetzung der Schule nach außen durch die Zusammenarbeit mit außerschulischen Diensten lässt sich erkennen, dass die Kooperation mit dem Jugendamt häufiger erfolgt als die mit dem Schulpsychologischen Dienst, gefolgt von der mit dem Schulärztlichen Dienst. Sowohl auf Schulleitungsebene als auch auf Ebene der Lehrkräfte wird deutlich, dass etwa ein Drittel aller Befragten eher selten mit dem Jugendamt kooperiert. Die Schulleitungen arbeiten offensichtlich etwas intensiver mit dem Schulpsychologischen Dienst zusammen als die Lehrkräfte, die dies etwa zur Hälfte eher selten tun. Die Kooperation mit dem Schulärztlichen Dienst erfolgt von Seiten der Lehrkräfte aus zu zwei Dritteln selten bis gar nicht. Das Antwortverhalten der Regel- und Förderschullehrkräfte unterscheidet sich im Vergleich bezüglich aller drei außerschulischen Dienste nur geringfügig voneinander. Lediglich die Arbeit mit dem Jugendamt ist bei den befragten FSL stärker ausgeprägt als bei den RSL ( $52,6 \%$ häufig bzw. sehr häufig FSL; $34 \%$ RSL).

\section{Zusammenarbeit mit außerschulischen Diensten}

Tab. 45: Zusammenarbeit mit Jugendamt

\begin{tabular}{lccc}
\hline & SL $(\mathbf{n}=\mathbf{3 1})$ & RSL $(\mathbf{n}=\mathbf{5 3})$ & FSL $(\mathbf{n}=\mathbf{3 8})$ \\
\hline sehr häufig & $12,9 \%$ & $5,7 \%$ & $18,4 \%$ \\
häufig & $48,4 \%$ & $28,3 \%$ & $34,2 \%$ \\
eher selten & $32,2 \%$ & $35,8 \%$ & $31,6 \%$ \\
gar nicht & $0 \%$ & $18,9 \%$ & $2,6 \%$ \\
fehlend & $6,5 \%$ & $11,3 \%$ & $13,2 \%$ \\
\hline
\end{tabular}

Tab. 46: Zusammenarbeit mit Schulpsychologischem Dienst

\begin{tabular}{lccc}
\hline & SL $(\mathbf{n}=\mathbf{3 1})$ & RSL $(\mathbf{n}=\mathbf{5 3})$ & FSL $(\mathbf{n}=\mathbf{3 8})$ \\
\hline sehr häufig & $19,4 \%$ & $3,8 \%$ & $10,5 \%$ \\
häufig & $45,2 \%$ & $30,2 \%$ & $34,2 \%$ \\
eher selten & $25,8 \%$ & $52,8 \%$ & $50,0 \%$ \\
gar nicht & $0 \%$ & $5,7 \%$ & $0 \%$ \\
fehlend & $9,7 \%$ & $7,5 \%$ & $5,3 \%$ \\
\hline
\end{tabular}


Tab. 47: Zusammenarbeit mit Schulärztlichem Dienst

\begin{tabular}{lccc}
\hline & SL $(\mathbf{n}=\mathbf{3 1})$ & RSL $(\mathbf{n}=\mathbf{5 3})$ & FSL $(\mathbf{n}=\mathbf{3 8})$ \\
\hline sehr häufig & $0 \%$ & $0 \%$ & $0 \%$ \\
häufig & $38,7 \%$ & $9,4 \%$ & $5,3 \%$ \\
eher selten & $38,7 \%$ & $34,0 \%$ & $50,0 \%$ \\
gar nicht & $3,2 \%$ & $26,4 \%$ & $10,5 \%$ \\
fehlend & $19,4 \%$ & $30,2 \%$ & $34,2 \%$ \\
\hline
\end{tabular}

Darüber hinaus benennen die Schulleitungen und Lehrkräfte der FZ, GS und GMS eine große Anzahl weiterer Dienste, mit denen sie außerschulisch zusammenarbeiten. Dazu gehören mit Blick auf sonderpädagogische Förderbedarfe das Autismuszentrum (9) sowie andere Förderschulen (Bsp.: L, SE, Blindenschule) (2). Für den Bereich der ärztlichen bzw. psychologischen oder psychiatrischen Versorgung werden Hausärzte, die Uniklinik, das Sozialpädiatrische Zentrum (3), Kinder- und Jugendpsychologen (6) sowie kinder- und jugendpsychiatrische Abteilungen (5) aufgeführt. Außerdem arbeiten die Schulen mit Logopäden (3) und Ergotherapeuten (3) zusammen. Weitere Netzwerkpartner sind Freie Träger bzw. Vereine wie die Lebenshilfe, die Caritas (2) oder der Verein „Miteinander leben lernen e.V.“ (2) sowie Anbieter von Eingliederungshilfen bzw. die Eingliederungs- oder Integrationshelfer selbst (2). Hinsichtlich der Kooperation mit der Jugendhilfe werden vor allem die Schoolworker benannt (8). Darüber hinaus wird mit Schulsozialarbeitern zusammengearbeitet (3), mit dem Familienhilfezentrum (7), Einrichtungen der Therapeutischen Schülerhilfe (5) sowie Wohngruppen und Kinderheimen (2). Für den Nachmittagsbereich erfolgt die Vernetzung mit der Ganztagsschule, der Nachmittagsbetreuung durch Freie Träger oder Nachhilfeinstitutionen (5). Darüber hinaus erfolgt eine Kooperation mit der Agentur für Arbeit (3) bzw. den Berufseinstiegsbegleitern (2). Ein Förderzentrum kooperiert mit der Schulverweigerungsstelle und dem Kriminaldienst.

Die Befragten nennen in diesem Zusammenhang außerdem den konkreten Austausch in Form von Treffen mit dem schulpsychologischen Dienst (8), dem Jugendamt (3), dem schulärztlichen Dienst, dem Schoolworker, der Autismus-Ambulanz, der Lebenshilfe, dem Verein „Miteinander Leben Lernen e.V., der AWO, dem Förder- und Beratungszentrum, dem Inklusionsberater, der Hochbegabtenstelle, Ärzten, Psychologen, Therapeuten, der Frühförderstelle, dem „Sprungbrett“ - Übergang Kiga-Schule (Caritas) sowie der Familienhilfe. Eine Lehrkraft wünscht für die Zukunft eine „verbesserte Vernetzung der Schule mit Therapeuten \& entsprechender Ressourcen bei Bedarf".

Wie diese Einzelangaben zeigen, spielt die Vernetzung mit sehr unterschiedlichen Kooperationspartnern für einzelne Schule eine sehr wichtige Rolle. Sie machen auch deutlich, dass es gerade im Zusammenhang mit schulischer Integration eine ganze Reihe von Institutionen, Vereinen und Ansprechpartnern gibt, die sich mit Fragestellungen in diesem Themenfeld befassen und dies teilweise in hochspezialisierter Form vornehmen (Bsp.: Hochbegabtenstelle oder Autismus-Ambulanz).

Angaben zu Organisationsstrukturen wurden in den Fragebögen nicht erhoben, eine einzelne Aussage bezieht sich diesbezüglich auf die Einrichtung von Förderbändern in den Klassen 2 bis 4 . Die Schülerinnen und Schüler wünschen sich eine Mischung der Klassenstufen im Rahmen der Ganztagsschule, längere Pausen, die Aufhebung des Handyverbots, mehr Technik in der Schule und ein härteres Durchgreifen bei Gewalt. 


\subsubsection{Ergebnisse im Bereich der Organisationsentwicklung}

Während Leitbilder und Konzepte für den Umgang mit Unterrichtsstörungen jeweils an etwa zwei Dritteln der Schulen vorhanden bzw. bekannt sind, gibt es nach Auskunft der Schulleitungen nur zu $43 \%$ Schulprogramme, in denen die integrative Unterrichtung von SuS mit spF eine Rolle spielt. Besonders selten wurde - nach Angaben der befragten Schulleitungen nur mit etwas mehr als 10\% - eine Evaluation der schulischen Arbeit vorgenommen.

Tab. 48: Ergebnisse der Schulentwicklung an integrativen Schulen

\begin{tabular}{lcccc}
\hline & $\begin{array}{c}\text { Leitbild } \\
(\mathbf{S L}, \mathbf{n}=31)\end{array}$ & $\begin{array}{c}\text { Schulprogramm, in } \\
\text { dem Integration von } \\
\text { SuS mit spF Thema } \\
\text { ist (SL, } \mathbf{n}=31)\end{array}$ & $\begin{array}{c}\text { Konzept zum } \\
\text { Umgang mit } \\
\text { U-Störungen } \\
(\mathbf{S L}, \mathbf{n}=31)\end{array}$ & $\begin{array}{c}\text { Evaluation der } \\
\text { integrativen Arbeit } \\
(\mathbf{S L}, \mathbf{n}=31)\end{array}$ \\
\hline ja & $67,8 \%$ & $43,0 \%$ & $58,1 \%$ & $12,9 \%$ \\
nein & $19,5 \%$ & $39,5 \%$ & $38,7 \% \%$ & $87,1 \%$ \\
weiß ich nicht & $12,7 \%$ & $17,5 \%$ & & \\
\hline
\end{tabular}

\section{Zukunft der Organisationsentwicklung}

Interessant sind die Ausführungen der Befragten hinsichtlich der offenen Frage zu Wünschen für die Zukunft der inklusiven Beschulung. Die Schulleitungen richten ihren Fokus teilweise auf die Makroebene der Schulentwicklung wie den gesellschaftlichen Diskurs zum Thema, bessere Vorleistungen durch die Politik und das Bildungsministerium, die „sich grundsätzlich Gedanken über notwendige Voraussetzungen der Inklusion machen und die notwendigen strukturellen Maßnahmen ergreifen“ bzw. die Schulträger, die mehr Vorgaben erhalten sollten. Die SLFZ erwarten außerdem eine „realistische Kleinschrittigkeit“, Zeit zur Entwicklung und eine grundsätzliche Öffnung der Regelschulen. Regel- wie Förderschullehrkräfte wünschen sich gleiche Strukturen in allen Bundesländern sowie Professionalität und Entschlossenheit statt Sparprogrammen, politischer Halbherzigkeit und Abschaffung der Förderschulen. Offizielle und greifbare Richtlinien werden als hilfreich eingeschätzt. Gewünscht sind realistische Arbeitsbedingungen, die an den tatsächlich vor Ort vorhandenen und bekannten Förderbedarfen orientiert sind. Drei Lehrkräfte fordern, dass beide Förderorte - Regel- wie Förderschulen - erhalten bleiben sollten, was für das Wohl des Kindes bedeutsam sein könne. Eine andere Lehrkraft schlägt vor, dass Förderschulzentren den Regelschulen angegliedert werden sollten. Dem Elternwillen sollte weiterhin eine große Bedeutung zukommen, da diese ihr Kind am besten kennen würden. Kinder sollten weiterhin eine Förderschule besuchen dürfen, wenn deren Eltern es explizit wünschen. Die Feststellung des sonderpädagogischen Förderbedarfs solle beibehalten werden, ohne dass eine Stigmatisierung erfolge, von der Meldung der Sus bis zur Förderung (durch Lehrer oder Integrationshelfer) dürfe nicht mehr als ein halbes Jahr vergehen. Schülerinnen oder Schüler, bei denen die Inklusion nicht möglich sei, müssten an passende Schulen vermittelt werden können.

Eine ganze Reihe von Aussagen konzentriert sich in diesem Zusammenhang auf die Offenheit und Akzeptanz inklusiver Konzepte in den Schulen: „Inklusion sollte selbstverständlich werden - im Rahmen der Möglichkeiten“ formuliert eine SLGS und eine Lehrkraft greift in ihrer Aussage auf das Zitat eines betroffenen Elternteils zurück: „Wer Inklusion will, findet Wege, wer sie nicht möchte, findet Gründe!“. Die Lehrkräfte wünschen sich Offenheit für „Neues“ im Kol- 
legium, bei Eltern, Schülern und bei den Schulleitungen, so dass Weiterentwicklung möglich ist. Dabei sollten positive Aspekte hervorgehoben werden, statt einfach nur von einer „Verpflichtung" auszugehen. Eltern sollten neben ihren Rechten (z.B. Wahlrecht) auch auf ihre Pflichten hinsichtlich integrativer Beschulung hingewiesen werden. Lehrkräfte fordern darüber hinaus eine gemeinsame Schule für alle, dass alle von inklusiver Beschulung profitieren, dass diese in den Köpfen ankomme, da sie nur dann realisierbar sei, dass anstelle einer Ideologie der Blick auf das Individuum geschärft werden solle und dass es keine Vorgabe von Vorgehensweisen zu behinderten Schülern geben solle, damit jede Schule gemäß ihren Möglichkeiten und gezielt individuell jedem einzelnen Förderschüler gerecht werden kann. Mehrere Aussagen der Lehrkräfte thematisieren den Wunsch nach (Förder-) Konzepten für die Schulen, zumal die RSL nicht für die Förderbereiche ausgebildet seien. Problematisch sei, dass Strukturen und Konzepte noch fehlen würden. Zwei Lehrkräfte wünschen sich vorgegebene Förderkonzepte für die Schulen (erstellt durch das Ministerium für Bildung). Die Eltern formulieren ihre Wünsche persönlicher dahingehend, dass die Arbeit so toll oder erfolgreich weitergeführt werden solle wie bisher (4). Ein Elternteil formuliert den Wunsch, eine weiterführende Schule zu finden, die genauso gut auf die Bedürfnisse eingehe wie die Grundschule.

\subsubsection{Darstellung der Ergebnisse zur Personalentwicklung}

\subsubsection{Ressourcen im Bereich der Personalentwicklung}

Die Ausstattung mit Personalressourcen ist offensichtlich ein Thema, das vor allem die befragten Schulleitungen und Lehrkräfte intensiv beschäftigt, was sich daran zeigt, dass diese auf zwei offene Fragen im Fragebogen sehr viele Aussagen (137) dazu formulieren. Bei der offenen Frage nach Wünschen für die Zukunft der inklusiven Beschulung richten sich dabei insgesamt 17 Anmerkungen von Schulleitungen, Regel- und Förderschullehrkräften auf die bessere personelle Ausstattung der integrativ arbeitenden Regelschulen und zwar sowohl mit Blick auf „Personal“ allgemein, als auch auf die Versorgung mit RSL und FSL bzw. weiterem Schulpersonal. Integrationshelfer sollten rechtzeitig beantragt werden, die Bewilligungszeit solle verkürzt werden (RSL/FSL). Zwei Lehrkräfte wünschen sich, dass die Klassenleitungen möglichst viele Stunden und damit Unterricht in ihren eigenen Klassen haben können.

Auf die Frage danach, wie die Förderung in den Regelschulen verbessert werden könnte bzw. bei Begründungen für Aussagen zur Lernentwicklung von SuS mit spF, befassen sich weitere 120 Aussagen - vorwiegend von Schulleitungen und Lehrkräften - mit den personellen Ressourcen. Eine befragte Person fasst diese Auffassungen zusammen, indem sie formuliert, dass die Förderung dadurch verbessert werden könnte, dass das „Konzept „Inklusion“ entsprechend angepasst wäre. Auf den Punkt: Es liegt am Geld, an zu wenig FÖ-Lehrern u. Integrationskräften (guten) u. zu vollen Klassen. Mehr Personal = mehr Förderung“. Eine andere spitzt zu, „dass die Schüler insgesamt alle Verlierer sind! Die Klassen sind zu groß (29 S.) und an ausgebildeten Integrationslehrern fehlt es völlig (z.Z. ein Realschullehrer, direkt nach seiner Ausbildung!!!)“.

Einige der Befragten wünschen sich allgemein eine Verbesserung der Rahmenbedingungen (Finanzen, Ausstattung, Personal), dass diese sich nicht so oft verändern und dass eine größere Anzahl von Förderschullehrkräften an den Regelschulen eingesetzt wird (18), u.a. um die Regelschullehrkräfte entlasten und eine bessere individuelle Förderung der Schüler gewährleisten zu können. Eine befragte Person wünscht sich, dass die „Mehrbelastung von Kollegen beachtet wird“, eine andere, dass „die Förderstunden nicht für Vertretungsstunden genutzt werden“. Eine weitere bemängelt, dass sich „die Theorie gut anhört, aber die nötigen (Personal-)Ressourcen fehlen. Die Kinder werden gefördert und die Lehrer überfordert., eine andere hält für ungüns- 
tig, dass die „Förderung nur durch die Förderschullehrer stattfindet, wenn diese dem Unterricht beiwohnen“. Zwei Personen halten die sonderpädagogische Förderung in den Regelschulen aufgrund mangelnder Lehrerstunden für nicht ausreichend effektiv.

Mit Blick auf weiteres Personal, das in den Schulen eingesetzt werden sollte, nennen zehn befragte Personen (kompetente) Eingliederungs- bzw. Integrationshelfer, eine weitere spricht von „mehr Fachpersonal“ und wieder eine andere erwähnt die Möglichkeit Bundesfreiwilligendienstler zu beschäftigen. Drei Personen wünschen sich eine schnellere Bewilligung von Integrationshelfern, „nicht erst nach Monaten, wenn überhaupt!“. Eine Lehrkraft wünscht sich „gut ausgebildete Erzieherinnen als feste Kräfte an der Schule“. Neben den allgemeinen Wünschen für eine bessere Personalisierung beziehen sich 16 Nennungen auf den Wunsch nach Doppelbesetzung in integrativen Klassen. Weitere fünf Aussagen benennen den Wunsch nach einer Doppelbesetzung zumindest in den Hauptfächern. Eine Lehrkraft vertritt in diesem Zusammenhang die Auffassung:

Eine inklusive Unterrichtung kann dann nur sinnvoll sein, wenn eine ständige Präsenz einer Förderschullehrkraft in der Regelschule gewährleistet ist, d.h. die Klassen müssten doppeltbesetzt sein und die beiden Lehrkräfte gleichberechtigt zusammenarbeiten (Problem: unterschiedl. Bezahlung!).

Und eine weitere Lehrkraft formuliert:

Inklusive Unterrichtung erfordert sehr viel Kraft, daher wünsche ich mir mehr Unterstützung in Form von Lehrerstunden; Doppelbesetzung pro Kind wäre optimal!

Auf die Frage danach, wie die Förderung in den Regelschulen verbessert werden könnte, sprechen 13 Personen den Wunsch nach (mehr) Doppelbesetzung aus. Darunter wünscht sich eine Lehrkraft, „dass die Klassenlehrerin durch eine zweite Lehrkraft entlastet würde. Es gibt zunehmend mehr förderbedürftige Kinder. (z.B. Doppelbesetzung: mehr Entlastung, Reflexion und bessere Hilfen)“. Weitere sechs wünschen sich Doppelbesetzungen mindestens in den Hauptfächern. Eine befragte Person schlägt vor, Schülerinnen und Schüler mit spF in „Inklusionsklas-

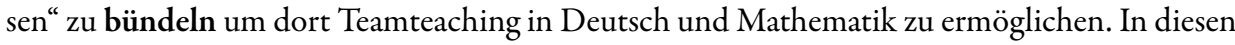
Klassen sollten dann besonders engagierte und kooperative Kollegen eingesetzt werden. Hinsichtlich der Anzahl der Förderschullehrerstunden, die wöchentlich für einen Schüler oder eine Schülerin zur Verfügung stehen, gibt es eine sehr große Bandbreite zwischen den einzelnen Schulen, wie folgende Tabelle verdeutlicht:

Tab. 49: Anzahl der FSL-Stunden wöchentlich je SuS mit spF $(n=156)$

\begin{tabular}{ccc}
\hline Anzahl der Stunden pro Woche & Anzahl der Nennungen & Anteil in Prozent \\
\hline 1,0 & 26 & $17,7 \%$ \\
1,5 & 3 & $2,0 \%$ \\
1,9 & 0,5 & $0,7 \%$ \\
2,0 & 59 & $40,1 \%$ \\
2,5 & 2 & $1,4 \%$ \\
3,0 & 20 & $13,6 \%$ \\
4,0 & 7 & $4,8 \%$ \\
5,0 & 4 & $2,7 \%$ \\
6,0 & 2 & $1,4 \%$
\end{tabular}




\begin{tabular}{ccc}
\hline Anzahl der Stunden pro Woche & Anzahl der Nennungen & Anteil in Prozent \\
\hline 7,0 & 1 & $0,7 \%$ \\
8,0 & 1 & $0,7 \%$ \\
12,0 & 2 & $1,4 \%$ \\
20,0 & 1 & $0,7 \%$ \\
36,0 & 1 & $0,7 \%$ \\
weiß ich nicht & 17 & $11,6 \%$ \\
fehlend & 9 & $6,2 \%$ \\
\hline
\end{tabular}

Etwa ein Fünftel der befragten SL, RSL, FSL und E (19,7\%) gibt demnach an, dass für einen $\mathrm{SuS}$ mit spF wöchentlich im Durchschnitt lediglich 1 bis 1,5 Stunden zur Verfügung stehen, bei weiteren $42,2 \%$ bewegt sich die angegebene Stundenzahl zwischen 1,9 und 2,5 Stunden wöchentlich, 18,4\% der Angaben gehen von 3 bis vier Stunden wöchentlich aus. In Einzelfällen (je Angabe jeweils 1-2) stehen auch mehr Stunden zur Verfügung. Knapp zwei Drittel der SuS mit $\operatorname{spF}(61,9 \%)$ werden wöchentlich im Durchschnitt also mit 1 bis 2,5 Stunden versorgt. Diese Angaben stimmen mit denen der Schulleitungen der Förderzentren überein ${ }^{66}$, die angeben, das SuS mit spF rechnerisch zwischen 1,9 und 2,0 FSL-Stunden pro Woche zur Verfügung stehen.

Tab. 50: Stunden der FSL je Schüler mit spF pro Woche

\begin{tabular}{cccccc}
\hline & SL $(\mathbf{n}=\mathbf{3 1})$ & RSL $(\mathbf{n}=\mathbf{5 3})$ & FSL $(\mathbf{n}=\mathbf{3 8})$ & Eltern $(\mathbf{n}=\mathbf{3 4})$ & Schüler (n=33) \\
\hline 1,0 & $16,1 \%$ & $24,5 \%$ & $15,8 \%$ & $8,8 \%$ & $15,2 \%$ \\
1,5 & $9,7 \%$ & $0 \%$ & $5,3 \%$ & $0 \%$ & $0 \%$ \\
2,0 & $61,3 \%$ & $43,4 \%$ & $47,4 \%$ & $23,5 \%$ & $18,2 \%$ \\
2,4 & $0 \%$ & $0 \%$ & $2,6 \%$ & $0 \%$ & $0 \%$ \\
2,5 & $0 \%$ & $1,9 \%$ & $5,3 \%$ & $2,9 \%$ & $0 \%$ \\
3,0 & $3,2 \%$ & $9,4 \%$ & $7,9 \%$ & $2,9 \%$ & $39,4 \%$ \\
3,5 & $0 \%$ & $0 \%$ & $5,3 \%$ & $0 \%$ & $0 \%$ \\
4,0 & $0 \%$ & $5,7 \%$ & $0 \%$ & $11,8 \%$ & $0 \%$ \\
5,0 & $3,2 \%$ & $1,9 \%$ & $5,3 \%$ & $5,9 \%$ & $0 \%$ \\
6,0 & $0 \%$ & $3,8 \%$ & $0 \%$ & $0 \%$ & $0 \%$ \\
7,0 & $0 \%$ & $1,9 \%$ & $0 \%$ & $0 \%$ & $0 \%$ \\
8,0 & $0 \%$ & $1,9 \%$ & $0 \%$ & $0 \%$ & $0 \%$ \\
12,0 & $0 \%$ & $1,9 \%$ & $0 \%$ & $2,9 \%$ & $0 \%$ \\
20,0 & $0 \%$ & $0 \%$ & $0 \%$ & $2,9 \%$ & $0 \%$ \\
27,0 & $0 \%$ & $0 \%$ & $2,6 \%$ & $0 \%$ & $0 \%$ \\
36,0 & $0 \%$ & $0 \%$ & $0 \%$ & $2,9 \%$ & $0 \%$ \\
weiß ich nicht & $3,2 \%$ & $0 \%$ & $0 \%$ & $20,6 \%$ & $27,3 \%$ \\
\hline
\end{tabular}

Die sehr unterschiedliche personelle Ausstattung wird auch durch folgende zwei qualitative Aussagen beispielhaft deutlich:

Der Förderbedarf der Kinder an der Schule ist erheblich größer als das von der Schule bereitstellbare Angebot (zu wenig Förderlehrer); Die Anzahl von 2 Stunden pro Schüler mit Förderbedarf ist zu we-

66 Diese verfügen aufgrund ihres Überblicks zur Anzahl der SuS mit spF in den Regelschulen sowie zur Anzahl der Stunden der FSL in den Regelschulen über eine verlässliche statistische Basis. 
nig! Nur im Saarland sind die Bedingungen so schlecht! Die Regelschullehrer bekommen zu wenig praktische Unterstützung durch Fö-Lehrer. Besonders im Bereich „E“. Die Rahmenbedingungen, um fördern zu können, sind an der Regelschule nicht gegeben. Klassenziel zu groß!

Seit 2014/15 (Inklusion) hat sich die Stundenzahl des Integrationslehrers erhöht. Seitdem ist eine bessere Zusammenarbeit möglich.; Mein Kind wird von allen Seiten sehr gut betreut; Integrationslehrerin + Integrationshelferin $\otimes 20$ Std pro Woche Lehrerin.

In Anbetracht dieser Wochenstundenangaben äußern ebenfalls knapp zwei Drittel der Befragten, dass sie dies für zu wenig halten, während 32,7\% davon ausgehen, dass dies genug sei.

Tab. 51: Einschätzung der Anzahl der FSL-Stunden je SuS/Woche: Ich finde, das ist...

\begin{tabular}{lccccc}
\hline & SL $(\mathbf{n}=\mathbf{3 1})$ & RSL $(\mathbf{n}=\mathbf{5 3})$ & FSL $(\mathbf{n}=\mathbf{3 8})$ & Eltern $(\mathbf{n}=\mathbf{3 4})$ & Schüler $(\mathbf{n}=\mathbf{3 3})$ \\
\hline zu viel & $0 \%$ & $0 \%$ & $0 \%$ & $0 \%$ & $3,0 \%$ \\
genug & $6,5 \%$ & $17,0 \%$ & $7,9 \%$ & $50,0 \%$ & $57,6 \%$ \\
zu wenig & $90,3 \%$ & $83,0 \%$ & $92,1 \%$ & $32,4 \%$ & $30,3 \%$ \\
weiß ich nicht & $0 \%$ & $0 \%$ & $0 \%$ & $11,8 \%$ & $6,1 \%$ \\
fehlend & $3,2 \%$ & $0 \%$ & $0 \%$ & $5,9 \%$ & $3,0 \%$ \\
\hline
\end{tabular}

Ein Elternteil äußert sich dahingehend, dass „die integrative Unterrichtung mit 2 Stunden in der Woche mit einer(m) Integrationslehrer/in [...] deutlich zu gering [sei]!“.

Zwei der Befragten beschreiben das Problem, dass bei Ausfall einer Förderschullehrkraft („auch über einen längeren Zeitraum“) kein Ersatz erfolgt bzw. Förderstunden „aus organisatorischen Gründen im Unterrichtsalltag [...] anderweitig verwendet [werden]“, zum Beispiel für die Vertretung von Regelschullehrkräften. Eine Lehrkraft beschreibt als Problem: „Integrationslehrer gehören oft Förderzentrum an und pendeln - sind also nicht fester Bestandteil der Schule“. Zwei Lehrkräfte äußern den Wunsch, dass die Anzahl der Schulen je Förderschullehrkraft verringert werden sollte und zwar auf eine bzw. zwei feste Einsatzschulen.

Auch bei den Wünschen für die Zukunft inklusiver Beschulung bzw. für die Verbesserung der Förderung spielt die Zuweisung von Förderschullehrerstunden an die Regelschulen eine wichtige Rolle, was sich daran zeigt, dass zu diesem Themenfeld insgesamt knapp 80 Aussagen getätigt werden. 78 Personen wünschen sich eine Erhöhung der Stundenanzahl der Förderschullehrkräfte in den Regelschulen, so dass Unterstützung in „ALLEN Stunden der Hauptfächer“ möglich sei, die Förderschullehrkräfte mehr Zeit für individuelle Förderung hätten bzw. den Schülerinnen und Schülern mit spF in den Regelschulen genauso viele Lehrerstunden zur Verfügung stehen wie in den entsprechenden Förderschulen. Zwei der Befragten äußern, dass die Förderschullehrkräfte mehr als zwei Mal in der Woche bzw. sogar jeden Tag für die Förderung da sein sollten. Eine Person weist darauf hin, dass keine „Helfer“ mehr notwendig seien, wenn es „mehr Förderlehrerstunden“ gäbe. Sieben Lehrkräfte legen Wert darauf, dass die Förderschullehrkräfte an einer bzw. höchstens an zwei Regelschulen beschäftigt sind. Drei Lehrkräfte schlagen vor, dass die Stunden der Förderschullehrkräfte flexibel zugewiesen und eingesetzt werden sollten.

Eine Reihe von Aussagen thematisiert die Kontinuität bzw. feste Zuweisung von Förderschullehrkräften an Regelschulen als Möglichkeit zur Verbesserung der (Konstanz der) Förderung (9). Eine befragte Person wünscht sich in diesem Zusammenhang, dass „die Lehrer und Förderlehrer möglichst über viele Jahre zusammen arbeiten“. Eine Person geht davon aus, „dass bei konstanter Anwesenheit eines Förderschullehrers eine konzeptionelle und effektive auf den Punkt bringende 
Förderung möglich ist“. Als Ideal beschreibt eine weitere eine Förderschullehrkraft, die individuelle Förderpläne erstellt, Lernerfolgskontrollen gemeinsam mit den Regelschullehrkräften bewertet, immer da ist und als Bindeglied zwischen Schülern, Eltern, Lehrkräften und Schulleitung fungiert. Eine Regelschullehrkraft äußert den Wunsch, „dass Förderlehrer der Gemeinschaftsschule fest als Kollegen zugewiesen werden, welche dann als Teil der Schulgemeinschaft gezielt beraten und unterstützen können“. Eine kontinuierliche Zusammenarbeit mit ausgebildeten Fachkräften wird von einer anderen befragten Person für notwendig gehalten, damit ein Kollegiumden schwierigen und umfangreichen Aufgaben gerecht werden kann. Vier der Befragten weisen darauf hin, dass ihre Schulen personell besser mit qualifizierten, gut ausgebildeten Förderschullehrkräften besetzt werden sollten. Eine weitere Lehrkraft legt Wert darauf, „dass mehr auf die Auswahl der Lehrer geachtet wird, [da] nicht alle [...] bestens ausgebildet [sind]“. Eine Lehrkraft bemängelt, dass Kontinuität nicht $100 \%$-ig gegeben sei, da Förderschullehrkräfte zum Teil wechseln würden.

Mehr als zwei Drittel der FSL sowie jeweils knapp über $80 \%$ der SL und RSL geben an, dass für die Beratung zwischen FSL und RSL keine festen Zeiten in den Stundenplänen der Schulen verankert sind. Zwischen 9,7\% (SL) und 15,8\% (FSL) der Angaben sagen aus, dass eine Stunde vorhanden sei.

Tab. 52: Beratungszeiten zwischen RSL und FSL, die im Stundenplan fest verankert sind

\begin{tabular}{lccc}
\hline & SL $(\mathbf{n}=\mathbf{3 1})$ & RSL $(\mathbf{n}=\mathbf{5 3})$ & FSL $(\mathbf{n}=\mathbf{3 8})$ \\
\hline 0 Stunden & $80,6 \%$ & $81,1 \%$ & $73,7 \%$ \\
1 Stunde & $9,7 \%$ & $11,3 \%$ & $15,8 \%$ \\
2 oder mehr Stunden & $0 \%$ & $1,9 \%$ & $10,5 \%$ \\
\hline
\end{tabular}

Vier Regelschullehrkräfte und drei Förderschullehrkräfte bemängeln, dass oft zu wenig Beratungszeit dafür vorhanden ist um sich miteinander auszutauschen. Einmal wird dies damit begründet, dass die Förderschullehrkraft noch an anderen Schulen eingesetzt sei, ein anderes Mal damit, dass die Stundenzeiten der FSL schwanken. Für die Zukunft wünschen sich insgesamt 13 Lehrpersonen mehr Zeit für Beratung, Austausch und Absprachen (4), mehr Zeit für Beratung und gemeinsame Planung, feste Zeiten für Beratung (5) (Bsp.: gemeinsame Beratungsstunden (1-3h) mit Klassenlehrerin), mehr Zeit für Zusammenarbeit mit Kollegen allgemein und für Gespräche sowie Zeit zum Austausch mit Lehrern, Integrationshelfern, Eltern, Jugendamt etc.. Eine Lehrkraft beschreibt die enge Zusammenarbeit zwischen RSL und FSL als schwierig, „weil diese oft unterschiedliche Präsenzzeiten haben“.

Bei der Frage danach, wie die Förderung in den Regelschulen verbessert werden könnte, formulieren vier Befragte den Wunsch nach festen Teamzeiten, wobei eine diesbezüglich ihren Wunsch dahingehend konkretisiert, „dass die weggefallene Beratungsstunde zwischen Regelund Förderlehrkraft wieder eingeführt wird“. Sieben der Befragten benennen Forderungen, die eine Erhöhung der Anzahl an Beratungsstunden aufgreifen. Diese sollten zur Verfügung stehen, damit „sich Klassenlehrer und Förderlehrer zusammensetzen können und gemeinsam planen können“ und so ein „regelmäßiger Austausch“ möglich wird. Sie sollten nach Auffassung von zwei Lehrkräften als Deputatsstunden ausgewiesen werden.

Die Reduktion der Unterrichtsverpflichtung von RSL, die in integrativen Klassen unterrichten, wird insbesondere von SL und RSL in sehr hohem Maß befürwortet (zu etwa je zwei Dritteln), während die FSL dies nur zur Hälfte so sehen. Mit etwa 15\% ist der Anteil derjenigen Personen, die diese Reduktion gar nicht befürworten, bei den RSL am höchsten. 
Tab. 53: Reduktion der Unterrichtsverpflichtung von RSL in integrativen Klassen

\begin{tabular}{lccc}
\hline & SL $(\mathbf{n}=\mathbf{3 1})$ & RSL $(\mathbf{n}=\mathbf{5 3})$ & FSL $(\mathbf{n}=\mathbf{3 8})$ \\
\hline stimme sehr zu & $67,7 \%$ & $60,4 \%$ & $50,0 \%$ \\
stimme eher zu & $22,6 \%$ & $13,2 \%$ & $36,8 \%$ \\
stimme eher nicht zu & $3,2 \%$ & $7,5 \%$ & $7,9 \%$ \\
stimme gar nicht zu & $0 \%$ & $15,1 \%$ & $2,6 \%$ \\
\hline
\end{tabular}

Die Schulleitung eines Förder- und Beratungszentrums begründet ihre Forderung nach einer Reduktion der Unterrichtsverpflichtung damit, dass die „Vielfalt an Aufgaben, die heute Schulen und ihre Lehrpersonen zu bewältigen haben, [...] immens [sei]“. Sechs Lehrkräfte äußern den Wunsch nach einer Reduktion der Unterrichtsverpflichtung im Zusammenhang mit den Wünschen für die Zukunft inklusiver Beschulung.

Eine Querverbindung zwischen den personellen Rahmenbedingungen und ihrer Haltung gegenüber schulischer Integration stellt eine Regelschullehrkraft her, indem sie bemängelt,

dass es viel zu wenige Förder-/Integrationslehrer gibt, die die Schüler im notwendigen Umfang betreuen. Dadurch resultiert ein erheblicher Mehraufwand und z.T. eine Überforderung für die Klassen- u. Fachlehrer. Grundsätzlich finde ich die integrative Beschulung von Schülern mit sonderpäd. Förderbedarf absolut sinnvoll und notwendig, jedoch sind häufig keine ausreichenden Rahmenbedingungen geschaffen, um diese umzusetzen. Jedes Kind mit sonderpäd. Förderbedarf bedeutet für den Klassenlehrer einen erhöhten Aufwand. Ich bin gerne bereit, diesen zu leisten. Ich verstehe jedoch auch die Kollegen, die dies nicht gerne tun.

\subsubsection{Kooperation}

\section{Dienstbesprechungen und Gesamkonferenzen}

Innerhalb institutionalisierter Kooperationsgremien wie Dienstbesprechungen und Gesamtkonferenzen findet in den Regelschulen auch eine Auseinandersetzung mit Fragen der schulischen Integration statt, wie die folgenden Werte belegen, wobei diese Fragen häufiger in Dienstbesprechungen als in Konferenzen aufgegriffen werden.

Tab. 54: Dienstbesprechungen zum Thema Integration von $\mathrm{SuS}$ mit spF

\begin{tabular}{lcccc}
\hline & $\mathbf{S L}(\mathbf{n}=\mathbf{3 1})$ & RSL $(\mathbf{n}=\mathbf{5 3})$ & FSL $(\mathbf{n}=\mathbf{3 8})$ & SLFZ $(\mathbf{n}=\mathbf{4})$ \\
\hline ja & $93,5 \%$ & $92,5 \%$ & $81,6 \%$ & $100 \%$ \\
nein & $6,5 \%$ & $7,5 \%$ & $18,4 \%$ & $0 \%$ \\
fehlend & $0 \%$ & $0 \%$ & $0 \%$ & $0 \%$ \\
\hline
\end{tabular}

Tab. 55: Gesamtkonferenzen zum Thema Integration von $\mathrm{SuS}$ mit spF

\begin{tabular}{lcccc}
\hline & SL (n=31) & RSL (n=53) & FSL (n=38) & SLFZ (n=4) \\
\hline ja & $64,5 \%$ & $52,8 \%$ & $52,6 \%$ & $100 \%$ \\
nein & $35,5 \%$ & $47,2 \%$ & $47,4 \%$ & $0 \%$ \\
fehlend & $0 \%$ & $0 \%$ & $0 \%$ & \\
\hline
\end{tabular}




\section{Zusammenarbeit zwischen Regel- und Förderschullehrkräften}

Die Zusammenarbeit zwischen Regel- und Förderschullehrkräften wird von SL, RSL und FSL in deutlich überwiegendem Maß als sehr gut bzw. gut bewertet. Dabei schätzen die SL und die RSL diese positiver ein als die FSL.

Tab. 56: Bewertung der Zusammenarbeit (bei FSL mit Blick auf RSL)

\begin{tabular}{lccc}
\hline & SL $(\mathbf{n}=\mathbf{3 1})$ & RSL $(\mathbf{n}=\mathbf{5 3})$ & FSL $(\mathbf{n}=\mathbf{3 8})$ \\
\hline sehr gut & $54,8 \%$ & $54,7 \%$ & $23,7 \%$ \\
gut & $32,3 \%$ & $30,2 \%$ & $55,3 \%$ \\
weniger gut & $6,5 \%$ & $9,4 \%$ & $7,9 \%$ \\
gar nicht gut & $3,2 \%$ & $0 \%$ & $0 \%$ \\
fehlend & $3,2 \%$ & $5,7 \%$ & $13,2 \%$ \\
\hline
\end{tabular}

Zwei Schulleitungen begründen die sehr gute Zusammenarbeit mit dem Argument, dass „alle Beteiligten sehr aufgeschlossen und engagiert zusammenarbeiten“ bzw. „partnerschaftlich auf Augenhöhe zusammenarbeiten“. Eine Schulleitung argumentiert sehr ähnlich: „weil beide Seiten profitieren: aus Sicht der Schulleitung WIR sind EIN Kollegium und arbeiten GEMEINSAM“. Eine andere Schulleitung erläutert, dass „in wöchentlichen Gesprächen mit der Schulleitung die Zusammenarbeit organisiert wird“. Drei weitere Schulleitungen der GS führen als Begründung die Kompetenz, die Offenheit und das Engagement sowie den Erfolg der an den Schulen tätigen Förderschullehrkräfte an. Eine weitere Schulleitung honoriert, dass die Förderschullehrkräfte „eine Sprechstunde anbieten und über ihr Stundenkontingent arbeiten“ und eine weitere kommentiert die gute Zusammenarbeit damit, dass „ein ständiger Informationsaustausch stattfindet und eine gegenseitige Wertschätzung praktiziert wird“.

Auch aus Sicht der Regelschullehrkräfte und Förderschullehrkräfte werden vielseitige Argumente dafür aufgeführt, warum die Kooperation mit den FSL als gut bzw. sehr gut eingeschätzt wird. Fünf Förderschullehrkräfte beschreiben die Kooperation als positiv, da die RSL der Zusammenarbeit offen und aufgeschlossen gegenüber stehen (3), Kooperation und Kommunikation möglich seien und „weil die meisten Lehrkräfte dazu bereit sind und ich als Förderschullehrer mit meinem Schwerpunkt voll akzeptiert und wertgeschätzt werde“ bzw. „weil kooperative, engagierte Lehrer mit viel Herz, Methoden, Interesse am Kind an der Schule sind“. Weitere Lehrkräfte formulieren als positiv, „dass z. Bsp. auch ich alle Möglichkeiten und Freiheiten habe, meine Förderideen anzuwenden. Das Vertrauen der Kollegen begleitet mich in meiner Arbeit, der Suche nach dem individuellen Zugang zum Kind“, dass die Förderung gut funktioniere, da Förder- und Regelschullehrkräfte gerne und vertrauensvoll zusammenarbeiten und das Thema „Toleranz“ ernst genommen werde, dass alle Schüler in ihrer Heterogenität willkommen seien (nicht nur die Förderschüler), die verschiedenen Berufsgruppen auf Augenhöhe zusammenarbeiten und es starke Bemühungen um individualisiertes Lernen gebe. Eine Lehrkraft fühlt sich von vielen ihrer Kollegen in ihrer Arbeit unterstützt. Drei Lehrkräfte sprechen von positiver Teamfähigkeit, Akzeptanz und intensiver kollegialer Zusammenarbeit, auch zwischen Förderschullehrkräften und Schulleitung. Zwei Lehrkräfte wünschen sich „Beratungen (ex-/intern), Netzwerken, Teams (Klassenstufen)“ und „dass die Förderung auch durch Hospitation unterstützt“.

Eine Reihe von RSL äußert sich allerdings auch kritisch mit Blick auf die Kooperation mit den FSL und nimmt dabei fehlende Beratungszeiten bzw. mangelndes Engagement von FSL in den Blick. Vier Lehrkräfte wünschen sich, dass Kollegen offener für Team-Teaching bzw. gemein- 
schaftliches Arbeiten sind (2), den Unterricht im Sinne des Team-Teaching gemeinsam strukturieren und durchführen bwz. „sich auch die weniger „offenen“ Kolleginnen mehr um Hilfe und Beratung bemühen“. Zwei Regelschullehrkräfte wünschen sich Möglichkeiten für eine intensivere Zusammenarbeit mit den Förderschullehrkräften durch eine Erhöhung der Förderschullehrerstunden in einzelnen Klassen.

Insgesamt 15 Aussagen der RSL greifen mit Blick auf die Niveaustufen der Kooperation die Tatsache auf, dass Absprachen und Austausch mit den FSL gut und regelmäßig (teilweise täglich) erfolgen. Weitere neun Aussagen beziehen sich auf höhere Stufen der Kooperation wie das „Vertrauensverhältnis“, den offenen Gedankenaustausch, die Möglichkeit zu privatem Kontakt oder die gute Zusammenarbeit. Eine Regelschullehrkraft formuliert, dass „die Kollegin gut in unser Lehrerteam passt, engagiert ist und gleiche Vorstellungen von Schule und Unterricht hat“, eine weitere spricht von "gleichen Zielen“. Vier der neun Aussagen beziehen sich auf die Zusammenarbeit im Team und beschreiben die Kooperation als gut, weil die Beteiligten gleicher Meinung seien und viel Verständnis gegeben sei, weil sie als Team zusammengewachsen seien und sich gegenseitig mögen und respektieren, weil sie ständig in Gespräch und Entwicklung seien oder ihre Arbeit als gemeinsame Arbeit auffassen. Von einem regelmäßigen und guten Austausch sprechen auch fünfFSL. Fünf weitere Förderschullehrkräfte berichten von einer höheren Stufe der Kooperation, da eine Arbeit „auf Augenhöhe“ erfolge, eine gleichberechtigte Arbeit möglich sei, es eine gute Zusammenarbeit gebe bzw. da die Lehrkräfte über die Jahre hinweg zu einem guten Team zusammengewachsen seien.

Positiv lässt sich festhalten, dass die Tätigkeit der RSL und FSL überwiegend so eingeschätzt wird, dass diese „auf Augenhöhe“ erfolgt. Dieser Aussage stimmen zwei Drittel der SL und RSL sowie knapp die Hälfte der FSL in sehr hohem Maß und zwischen 29\% und 36,8 \% der Befragten eher zu. Etwa ein Zehntel der FSL kann dieser Aussage eher nicht zustimmen.

Tab. 57: Tätigkeit auf Augenhöhe

\begin{tabular}{lccc}
\hline & SL $(\mathbf{n}=\mathbf{3 1})$ & RSL $(\mathbf{n}=\mathbf{5 3})$ & FSL $(\mathbf{n}=\mathbf{3 8})$ \\
\hline stimme sehr zu & $67,7 \%$ & $67,9 \%$ & $47,4 \%$ \\
stimme eher zu & $29,0 \%$ & $28,3 \%$ & $36,8 \%$ \\
stimme eher nicht zu & $3,2 \%$ & $3,8 \%$ & $13,2 \%$ \\
stimme gar nicht zu & $0 \%$ & $0 \%$ & $0 \%$ \\
\hline
\end{tabular}

Für die Zukunft der Kooperation im Rahmen der inklusiven Beschulung wünschen sich die RSL und FSL „eine selbstverständliche und regelmäßige Zusammenarbeit zwischen Regel- und Förderschullehrkräften“, „mehr Zeit für Beratung und gemeinsame Planung“, „Teamarbeit, dass alle an einem Strang ziehen“ und die „Zusammenarbeit mit einem Co-Lehrer oder einer festen Förderlehrkraft, die möglichst viele Stunden in der Klasse verbringt - abhängig vom jeweiligen Förderbedarf der Schüler“. Eine Lehrkraft führt als Wunsch aus:

Ein echtes Team-Teaching und gemeinsamer Unterricht, dass die Kinder je nach Bedarf gefördert werden können. Dadurch sollte die Möglichkeit bestehen, die Lerngruppe zu teilen, um neue Inhalte je nach Lernstand einzuführen. Gleichzeitig sollten Rückzugsmöglichkeiten bestehen bleiben um Kindern eine ruhige Lernatmosphäre zu schaffen.

Eine weitere Lehrkraft stellt eine Verknüpfung zur Ressourcensituation her und argumentiert: 
Inklusive Unterrichtung ist nur begrenzt möglich. Eine individuelle Förderung aller Schüler wird nicht mehr möglich sein. Daher wünsche ich mir eine Unterrichtung im Team, von der alle Schüler profitieren.

\section{Bedeutung der Kontinuität der Tätigkeit von Förderschullehrkräften}

Insbesondere die Schulleitungen stimmen der Aussage in sehr hohem Maß zu, dass die Kontinuität der Tätigkeit der FSL in den Regelschulen zur Akzeptanz der integrativen Unterrichtung beitragen kann (83,9\%), während die Anteile bei den RSL mit 54,7\% und den FSL mit 57,9\% geringer sind.

Tab. 58: Kontinuität der Tätigkeit von FSL in Regelschulen trägt zur Akzeptanz der integrativen Unterrichtung bei

\begin{tabular}{lccc}
\hline & SL $(\mathbf{n}=\mathbf{3 1})$ & RSL $(\mathbf{n}=\mathbf{5 3})$ & FSL $(\mathbf{n}=\mathbf{3 8})$ \\
\hline stimme sehr zu & $83,9 \%$ & $54,7 \%$ & $57,9 \%$ \\
stimme eher zu & $12,9 \%$ & $39,6 \%$ & $31,6 \%$ \\
stimme eher nicht zu & $3,2 \%$ & $5,7 \%$ & $7,9 \%$ \\
stimme gar nicht zu & $0 \%$ & $0 \%$ & $0 \%$ \\
\hline
\end{tabular}

Die gewünschte Kontinuität der Zuweisung der FSL an die Regelschulen wird von insgesamt fünf Lehrkräften auch bei der Frage nach Wünschen für die Zukunft der inklusiven Beschulung aufgegriffen. Drei nennen die „feste Zuteilung“ als Wunsch, eine die „Kontinuität bzgl. der Schulzuweisung“ und eine sagt aus: „Meiner Meinung nach sollten die Integrationslehrer konstant an einer Schule eingesetzt werden und nicht im jährlichen Wechsel.“ Mit Blick auf die Formen der Zusammenarbeit zwischen SL, RSL und FSL erfolgt ein reger Austausch zwischen Schulleitungen und FSL im Rahmen von Gesprächsterminen (77,4\% sehr häufig bzw. häufig) sowie Pausengesprächen (83,8 \% häufig bzw. sehr häufig).

Tab. 59: SL Formen der Zusammenarbeit zwischen SL und FSL $(\mathrm{n}=31)$

\begin{tabular}{lcccc}
\hline & Telefonate & E-Mails & Gesprächstermine & Pausengespräche \\
\hline sehr häufig & $6,5 \%$ & $3,2 \%$ & $38,7 \%$ & $54,8 \%$ \\
häufig & $16,1 \%$ & $22,6 \%$ & $38,7 \%$ & $29,0 \%$ \\
eher selten & $22,6 \%$ & $22,6 \%$ & $12,9 \%$ & $6,5 \%$ \\
gar nicht & $12,9 \%$ & $9,7 \%$ & $0 \%$ & $3,2 \%$ \\
fehlend & $41,9 \%$ & $41,9 \%$ & $9,7 \%$ & $6,5 \%$ \\
\hline
\end{tabular}

Tab. 60: RSL Formen der Zusammenarbeit mit den FSL $(n=53)$

\begin{tabular}{lcccc}
\hline & Telefonate & E-Mails & Gesprächstermine & Pausengespräche \\
\hline sehr häufig & $0 \%$ & $5,7 \%$ & $31,0 \%$ & $58,5 \%$ \\
häufig & $13,2 \%$ & $20,8 \%$ & $33,3 \%$ & $28,3 \%$ \\
eher selten & $15,1 \%$ & $9,4 \%$ & $31,0 \%$ & $7,5 \%$ \\
gar nicht & $20,8 \%$ & $24,5 \%$ & $4,8 \%$ & $0 \%$ \\
fehlend & $50,9 \%$ & $39,6 \%$ & $20,8 \%$ & $5,7 \%$
\end{tabular}


Tab. 61: FSL Formen der Zusammenarbeit mit den RSL $(\mathrm{n}=38)$

\begin{tabular}{lcccc}
\hline & Telefonate & E-Mails & Gesprächstermine & Pausengespräche \\
\hline sehr häufig & $7,9 \%$ & $23,7 \%$ & $28,9 \%$ & $86,8 \%$ \\
häufig & $5,3 \%$ & $18,4 \%$ & $42,1 \%$ & $10,5 \%$ \\
eher selten & $44,7 \%$ & $31,6 \%$ & $18,4 \%$ & $2,6 \%$ \\
gar nicht & $5,3 \%$ & $5,3 \%$ & $2,6 \%$ & $0 \%$ \\
fehlend & $36,8 \%$ & $21,1 \%$ & $7,9 \%$ & $0 \%$ \\
\hline
\end{tabular}

Die meisten Gespräche zwischen RSL und FSL finden offensichtlich „zwischen Tür und Angel“ in den Pausen statt (RSL 86,8 \% sehr häufig bzw. häufig; FSL 97,3\%). Gesprächstermine werden insgesamt intensiver genutzt als der Austausch über Telefon oder E-Mails (RSL 64,3\% gegenüber 13,2\% bzw. 26,5\%; FSL 71,0\% gegenüber 13,2\% bzw. 42,1\%). Die Angaben der RSL und FSL weisen für den Bereich der Formen des Austauschs ein hohes Maß an Übereinstimmung auf.

Die Schulleitungen der Grundschulen und weiterführenden Schulen tauschen sich mit den FSL über „Inklusion und deren Umsetzung als aktuelles Thema“ aus, über organisatorische Fragen (Bsp.: Raumorganisation, Termine für Elterngespräche) sowie über die Zusammenarbeit mit Vernetzungspartnern wie Jugendamt, schulpsychologischem Dienst oder den Eingliederungshelfern. Sie sprechen mit ihnen über Förderkonzepte, „alternative Modelle, um auf Verhaltensprobleme einzugehen“ oder die Beurteilung von Klassenarbeiten. Weitere Themen sind Probleme mit Fachlehrern oder die Gestaltung von Förderausschusssitzungen.

Tab. 62: Austausch zu verschiedenen Themen RSL-FSL a)

\begin{tabular}{lcccc}
\hline & \multicolumn{2}{c}{ Unterrichtsplanung } & \multicolumn{2}{c}{ Bereitstellung Material SuS mit spF } \\
& RSL $(\mathbf{n}=\mathbf{5 3})$ & FSL $(\mathbf{n}=\mathbf{3 8})$ & RSL $(\mathbf{n}=\mathbf{5 3})$ & FSL $(\mathbf{n}=\mathbf{3 8})$ \\
\hline ja & $50,9 \%$ & $76,3 \%$ & $60,4 \%$ & $92,1 \%$ \\
nein & $49,1 \%$ & $23,7 \%$ & $39,6 \%$ & $7,9 \%$ \\
\hline
\end{tabular}

Tab. 63: Austausch zu verschiedenen Themen RSL-FSL b)

\begin{tabular}{lcccc}
\hline & \multicolumn{2}{c}{ Verhaltensprobleme SuS allgemein } & \multicolumn{2}{c}{ Verhaltensprobleme SuS mit spF } \\
& RSL $(\mathbf{n}=\mathbf{5 3})$ & FSL $(\mathbf{n}=\mathbf{3 8})$ & RSL $(\mathbf{n}=53)$ & FSL $(\mathbf{n}=\mathbf{3 8})$ \\
\hline ja & $67,9 \%$ & $94,7 \%$ & $77,4 \%$ & $97,4 \%$ \\
nein & $33,1 \%$ & $5,3 \%$ & $22,6 \%$ & 2,6 \\
\hline
\end{tabular}

Tab. 64: Austausch zu verschiedenen Themen RSL-FSL c)

\begin{tabular}{lcccc}
\hline & \multicolumn{2}{c}{ Lernschwierigkeiten SuS allgemein } & \multicolumn{2}{c}{ Lernschwierigkeiten SuS mit spF } \\
& RSL (n=53) & FSL (n=38) & RSL & FSL \\
\hline ja & $56,6 \%$ & $89,5 \%$ & $88,7 \%$ & $100 \%$ \\
nein & $43,4 \%$ & $10,5 \%$ & $11,3 \%$ & $0 \%$ \\
\hline
\end{tabular}

Die Regelschullehrkräfte besprechen mit den FSL ebenfalls die „Inklusionsentwicklung“, organisatorische Fragen (Bsp.: Termine), ihre „Unzufriedenheit bezüglich der Stundenzuweisung“ sowie die (gemeinsame) Elternarbeit (4) oder die Zusammenarbeit mit Eingliederungshelfern. 
Mit Blick auf den Unterricht geht es um fallabhängige Absprachen, um „schwache/verhaltensauffällige Schüler, die evtl. getestet werden“, das „Anfertigen bspw. von Arbeitsblättern oder verringerte Anforderung bei Arbeiten“ oder die „Unterrichtsauswertung bzw. Nachbereitung“ sowie diagnostische Fragestellungen. Weitere Gesprächsthemen sind die Optimierung der Förderung der SuS, der Austausch über „Fortschritte einzelner SchülerInnen“ oder Möglichkeiten zur Verbesserung der Zusammenarbeit.

Die Förderschullehrkräfte nennen als weitere Gesprächsthemen mit den RSL Dinge, die den Schulalltag, allgemeine Schulthemen, Fortbildungen oder die Zusammenarbeit mit den Eltern (4) bzw. der Arbeitsagentur betreffen. Thema sei auch der Austausch über die SuS (4) (Bsp.: Freizeitverhalten, Arbeitsmaterialien, Deutsch als Zweitsprache, akute Probleme/Schwierigkeiten, Schulbesuchsverhalten, Hausaufgaben, Verhalten oder sprachliche Auffälligkeiten).

Als weitere Formen der Zusammenarbeit werden von den Schulleitungen Förderkonferenzen, Förderausschüsse, Dienstbesprechungen, die Teilnahme an Teamgesprächen und manchmal an Konferenzen und Dienstbesprechungen sowie regelmäßige Inklusionstreffen aufgeführt. Die Regelschullehrkräfte erwähnen Gespräche in der Klasse (2), das Nutzen gemeinsamer Freistunden sowie Förderkonferenzen, während die Förderschullehrkräfte die Kommunikation per Handy aufführen (Bsp.: SMS, Whatsapp) (4), die Nutzung von Zeiten vor, während oder nach dem Unterricht (2), die gemeinsame Unterrichtsvorbereitung (2) oder "gemeinsame Treffen bei internen kollegialen Veranstaltungen“.

Mit Blick auf die unterrichtsbezogene Kooperation gehe es um die Gestaltung von Klassenarbeiten (3), die Differenzierung von Lerninhalten, differenziertes Fördermaterial, Nachteilsausgleich, Verstärkersysteme, Lern- und Entwicklungsfortschritte, gelungene Förderung, (vermuteten) sonderpädagogischen Förderbedarf oder Hausaufgaben.

Bezüglich der unterrichtsbezogenen Kooperation im engeren Sinn (gemeinsame Unterrichtsvorbereitung, -durchführung, -nachbereitung) sehen die Einschätzungen von SL, RSL und FSL bezüglich der Häufigkeiten sehr unterschiedlich aus. Wie die Diagramme veranschaulichen, gehen die Schulleitungen am ehesten von einer sehr häufigen bzw. häufigen gemeinsamen Unterrichtsvorbereitung (38,7\%), -durchführung $(61,3 \%)$ bzw. -reflexion $(41,9 \%)$ aus. Diese Einschätzung wird von den befragten RSL nicht bestätigt, deren Antworten in diesen drei Bereichen bei 15,1\%, 24,5\% bzw. 47,1 \% liegen. Am häufigsten findet nach Auffassung dieser Gruppe also die gemeinsame Reflexion von Unterrichtssituationen statt. Die FSL wiederum zeigen mit Blick auf die Unterrichtsvorbereitung und -durchführung mit 15,8\% bzw. 21,0\% ein ähnliches Antwortverhalten wie die RSL. Lediglich hinsichtlich der Unterrichtsreflexion äußern sie nur zu 26,3\%, dass diese häufig bzw. sehr häufig stattfinde. Übereinstimmend gehen RSL und FSL in größerem Maß von einer eher seltenen bzw. gar nicht stattfindenden unterrichtlichen Kooperation im engeren Sinn aus, auch wenn die Werte der beteiligten Befragten sich dahingehend unterscheiden, dass die RSL dies zu 79,2\%, 73,6\% bzw. 47,2\% angeben, die FSL zu 84,2\%, 79\% bzw. 73,7\%. Eine Lehrkraft wünscht sich mehr gemeinsame Zeit für gemeinsame Unterrichtsvor- und -nachbereitung.

Tab. 65: Gemeinsame Unterrichtsvorbereitung

\begin{tabular}{lccc}
\hline & SL $(\mathbf{n}=\mathbf{3 1})$ & RSL $(\mathbf{n}=\mathbf{5 3})$ & FSL $(\mathbf{n}=\mathbf{3 8})$ \\
\hline sehr häufig & $3,2 \%$ & $3,8 \%$ & $0 \%$ \\
häufig & $35,5 \%$ & $11,3 \%$ & $15,8 \%$ \\
eher selten & $38,7 \%$ & $50,9 \%$ & $68,4 \%$ \\
gar nicht & $16,1 \%$ & $28,3 \%$ & $15,8 \%$ \\
fehlend & $6,5 \%$ & $5,7 \%$ & $0 \%$ \\
\hline
\end{tabular}




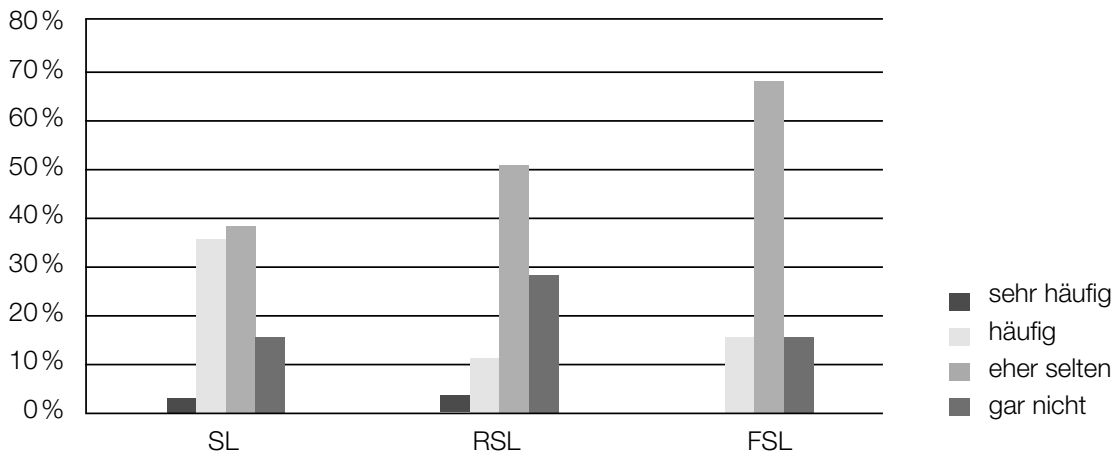

Abb. 12: Häufigkeit der gemeinsamen Unterrichtsvorbereitung von RSL/FSL

Tab. 66: Gemeinsame Unterrichtsdurchführung

\begin{tabular}{lccc}
\hline & SL $(\mathbf{n}=\mathbf{3 1})$ & RSL $(\mathbf{n}=\mathbf{5 3})$ & FSL $(\mathbf{n}=\mathbf{3 8})$ \\
\hline sehr häufig & $9,7 \%$ & $9,4 \%$ & $2,6 \%$ \\
häufig & $51,6 \%$ & $15,1 \%$ & $18,4 \%$ \\
eher selten & $22,6 \%$ & $45,3 \%$ & $71,1 \%$ \\
gar nicht & $9,7 \%$ & $28,3 \%$ & $7,9 \%$ \\
fehlend & $6,5 \%$ & $1,9 \%$ & $0 \%$ \\
\hline
\end{tabular}

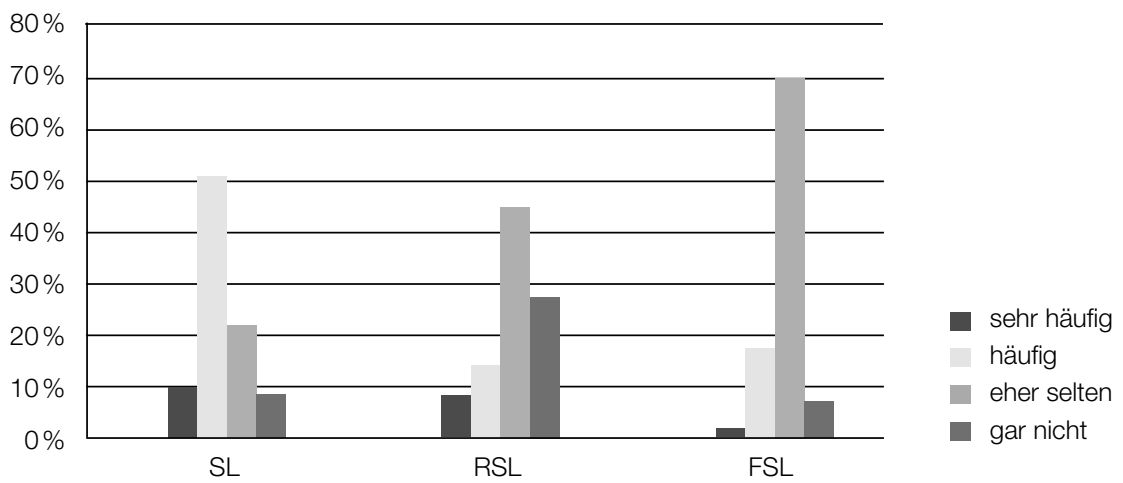

Abb. 13: Häufigkeit der gemeinsamen Unterrichtsdurchführung von RSL/FSL

Tab. 67: Gemeinsame Unterrichtsreflexion

\begin{tabular}{lccc}
\hline & SL $(\mathbf{n}=\mathbf{3 1})$ & RSL $(\mathbf{n}=\mathbf{5 3})$ & FSL $(\mathbf{n}=\mathbf{3 8})$ \\
\hline sehr häufig & $3,2 \%$ & $11,3 \%$ & $2,6 \%$ \\
häufig & $38,7 \%$ & $35,8 \%$ & $23,7 \%$ \\
eher selten & $45,2 \%$ & $18,9 \%$ & $65,8 \%$ \\
gar nicht & $6,5 \%$ & $28,3 \%$ & $7,9 \%$ \\
fehlend & $6,5 \%$ & $5,7 \%$ & $0 \%$ \\
\hline
\end{tabular}




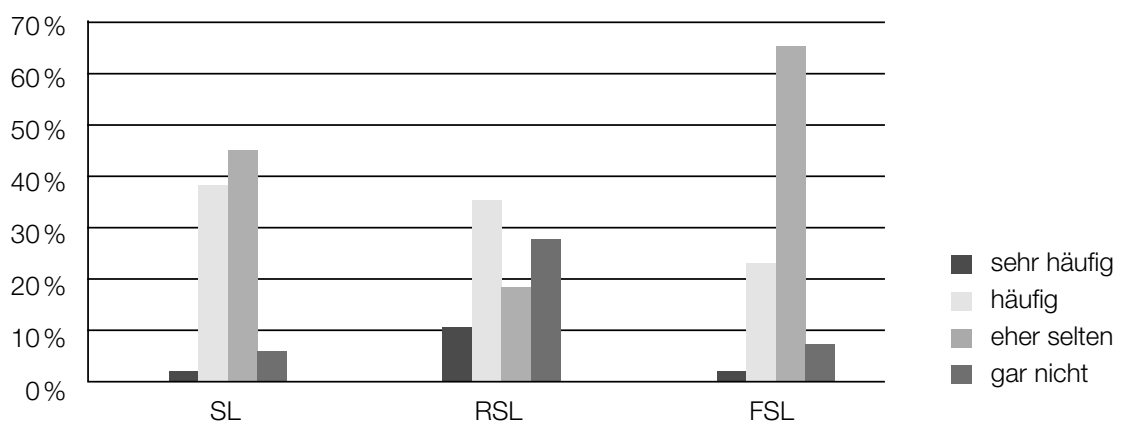

Abb. 14: Häufigkeit der gemeinsamen Unterrichtsreflexion von RSL/FSL

Vier RSL erleben die Kooperation als positiv, da sie mit den FSL unterrichtsbezogen kooperieren können und formulieren dies dahingehend, dass sie Tipps für den Unterricht, „besondere Arbeitsmaterialien und die Erstellung von Arbeitsblättern“ erhalten, „als Regellehrer nochmal die Lernzuwächse/bzw. den Lernstand auch nochmal aus anderer Sicht aufgewiesen bekommt“, sie sich „gegenseitig Rückmeldung geben und den Schüler bestmöglich fördern“ sowie gemeinsam Unterricht planen, durchführen \& uns austauschen“. Drei FSL greifen die unterrichtsbezogene Zusammenarbeit auf, bei der „alle Seiten interessiert sind an Förderung“, ständige Gespräche über Unterrichtsinhalte und Leistungsstand stattfinden bzw. „ein Austausch über Lernfortschritte, Arbeits- und Lernverhalten sowie Probleme besteht“. Als ungünstig wird von fünf FSL bewertet, dass sie „den U. zu häufig nicht auf Schüler mit Förderbedarf Lernen abstimmen“, „die Kooperationsfähigkeit u. -bereitschaft teils sehr gering sind“, „einige Kolleginnen/ Kollegen der Integration gegenüber eher verschlossen sind“, „wenig Interesse für Inklusion + Zusammenarbeit“ vorhanden sei bzw. die Zusammenarbeit „extrem personabhängig“ sei.

Gleichzeitig stimmt die überwältigende Mehrheit der Befragten der Aussage in sehr hohem $\mathrm{Maß} \mathrm{zu,} \mathrm{dass} \mathrm{Teamteaching} \mathrm{(von} \mathrm{RSL} \mathrm{und} \mathrm{FSL)} \mathrm{möglich} \mathrm{sein} \mathrm{sollte.}$

Tab. 68: Teamteaching sollte möglich sein

\begin{tabular}{lccc}
\hline & SL $(\mathbf{n}=\mathbf{3 1})$ & RSL $(\mathbf{n}=\mathbf{5 3})$ & FSL $(\mathbf{n}=\mathbf{3 8})$ \\
\hline stimme sehr zu & $77,4 \%$ & $73,6 \%$ & $65,8 \%$ \\
stimme eher zu & $12,9 \%$ & $18,9 \%$ & $34,2 \%$ \\
stimme eher nicht zu & $3,2 \%$ & $3,8 \%$ & $0 \%$ \\
stimme gar nicht zu & $0 \%$ & $0 \%$ & $0 \%$ \\
\hline
\end{tabular}

Das Teamteaching von Regel- und Förderschullehrkräften steht mit neun Nennungen auch als Wunsch für die Zukunft inklusiver Beschulung für eine Reihe von Lehrkräften im Fokus.

Das Maß an Unterstützung, das SL bzw. RSL durch die FSL bzw. die FSL umgekehrt durch die RSL erfahren, wird differenzierter bewertet als die Kooperation allgemein. Etwa ein Viertel der RSL und FSL empfindet das Maß an gegenseitiger Unterstützung als weniger hoch. Gleichzeitig stimmen allerdings sowohl insgesamt 87,1\% Schulleitungen sowie 96,2\% der RSL der Aussage in sehr hohem bzw. hohem Maß zu, dass die Ressourcen der FSL durch die RSL genutzt werden. Auch $85 \%$ der FSL selbst stimmen dieser Aussage zu. Obwohl dies also der Fall ist, wirkt sich offenbar nur für einen Teil der RSL bzw. umgekehrt auch der FSL diese Ressourcennutzung unterstützend aus. 
Tab. 69: Maß der Unterstützung durch FSL bzw. RSL

\begin{tabular}{lccc}
\hline & SL $(\mathbf{n}=\mathbf{3 1})$ & RSL $(\mathbf{n}=\mathbf{5 3})$ & FSL $(\mathbf{n}=\mathbf{3 8})$ \\
\hline sehr hoch & $25,8 \%$ & $34,0 \%$ & $15,8 \%$ \\
hoch & $48,4 \%$ & $34,0 \%$ & $50,0 \%$ \\
weniger hoch & $16,1 \%$ & $24,5 \%$ & $26,3 \%$ \\
gar nicht hoch & $3,2 \%$ & $3,8 \%$ & $0 \%$ \\
\hline
\end{tabular}

Als Begründungen dafür, dass das Maß der Unterstützung durch die FSL hoch bzw. sehr hoch empfunden wird, führen einzelne Schulleitungen an, dass die FSL ,immer ein offenes Ohr haben“, „unter den gegebenen Voraussetzungen das Beste herausholen“, „kompetente Ansprechpartner sind und sich durch Teamfähigkeit auszeichnen“, oder „sie auf Wunsch der LehrerInnen auch andere schwächere Schüler mit in der Gruppe betreuen“.

Eine Schulleitung weist darauf hin, dass die Kollegen sich regelmäßig zusammensetzen und austauschen. Eine weitere Schulleitung betont, dass sie selbst „bisher in allen wichtigen Fragen kompetente und pragmatische Unterstützung gefunden habe“. Lediglich eine Schulleitung empfindet das Maß an Unterstützung durch die FSL als weniger hoch, weil diese „meist nur als "Nachhilfelehrer" fungieren“.

Aus Sicht der RSL wird das Maß der Unterstützung durch die FSL teilweise als hoch empfunden, da Austausch (4), Beratung und Zusammenarbeit gut funktionieren. „Unterstützung und die gute Kooperation [sind] sehr entlastend und gewinnbringend“, äußert eine RSL und eine weitere fühlt sich gut unterstützt, da sie „bisher in allen wichtigen Fragen kompetente und pragmatische Unterstützung gefunden habe“. Eine befragte Person beschreibt, dass ihre „Arbeit durch die Integrationslehrkraft fortgeführt und unterstützt wird“.

Auch die unterrichtsbezogene Zusammenarbeit wird von vielen RSL als unterstützend wahrgenommen. Hilfreich seien bspw. die Anwesenheit in Mathematik und Deutsch, die Erstellung (und Korrektur) differenzierter Arbeitsblätter oder Klassenarbeiten durch die FSL oder auch die gemeinsame Erstellung derselben. Eine RSL hebt hervor, dass die FSL „viele Materialien aus ihrem privaten Besitz zur Verfügung stellt“, eine weitere, dass „der Austausch von Unterrichtsmaterialien auch schwachen Regelschülern nutzt“. Als Unterstützung beschreiben die RSL darüber hinaus, dass die FSL „die Situation der Förderschüler immer im Auge haben und zum richtigen Zeitpunkt aktiv werden“, dass diese sich bemühen, den Kindern mit spF gerecht zu werden, ihnen passende Hilfestellungen zu geben (3) und auch Elterngespräche führen.

Die RSL empfinden die FSL als unterstützend, da diese sich Zeit für Gespräche nehmen und immer „ein offenes Ohr haben“ (4), auch außerhalb der Schulzeit, sie mitdenken und flexibel sind. Die fachlichen Kompetenzen werden als unterstützend eingestuft, da „in manchen Situationen die Regellehrkräfte nicht mehr weiter wissen“, „die Förderlehrer im Bereich Integration und Inklusion besser ausgebildet sind“, über eine „spezielle Qualifikation“ verfügen, „sich gut auskennen“ und als „kompetente Ansprechpartner“ fungieren.

Ähnlich wie bei der Bewertung der Kooperation werden auch mit Blick auf die als nicht sehr hoch wahrgenommene Unterstützung der RSL durch die FSL fehlende Beratungszeiten sowie mangelnde Qualifikation der FSL aufgeführt. Die fehlende Beratungszeit bzw. eine unzureichende Anzahl von FSL-Stunden in der Regelschule werden in diesem Zusammenhang von 17 RSL thematisiert. Dadurch könne es nicht genügend Absprachen geben (3) und es sei zu wenig Unterstützung im Unterricht möglich. Zwei RSL bewerten das Maß an Unterstützung kritisch, da „die Integrationslehrkraft nur die Rolle des stillen Beobachters einnimmt“ bzw. „es eher so ist, dass der Klassenlehrer der Förderkraft sagt, was sie arbeiten sollte“. 
Die FSL empfinden das Maß an Unterstützung offensichtlich als geringer als die SL bzw. RSL, was sich daran zeigt, dass die kritischen qualitativen Aussagen zu diesem Bereich überwiegen. Positiv heben einige FSL die Offenheit und Flexibilität der RSL (2), gute Absprachen und Austausch sowie die unterrichtsbezogene Zusammenarbeit hervor. Fünf FSL beschreiben die unterrichtsbezogene Kooperation als schwierig und damit auch das Maß an empfundener Unterstützung als gering, weil sie „Aufgabengebiete (Klassenarbeiten, Wochenpläne) unabhängig vom Regelschullehrer durchführe[n]“, „,die Arbeit an den Förderschullehrkräften hängen bleibt “, andere Lehrer kaum differenzieren und Arbeitsblätter oder Klassenarbeiten umgestalten würden und „die Förderung der Integrationsschüler meist auf die Förderschullehrer abgewälzt wird“. Eine FSL erklärt, dass manche Kollegen sie nicht ausreichend über anstehende Unterrichtsthemen informieren oder $\mathrm{SuS}$ mit spF reguläre Klassenarbeiten aushändigen würden, obwohl sie diese zuvor differenziert erstellt hätte. Andere FSL nennen mögliche Gründe dafür, dass die RSL nicht viel Unterstützung leisten können, wie bspw. die sowieso schon vorhandene Belastung oder Überforderung, deren unzureichende Ausbildung oder Zeitmangel.

Mehr Entlastung und Unterstützung wünschen sich auch insgesamt fünf Lehrkräfte und eine Schulleitung mit Blick auf die Zukunft inklusiver Beschulung und zwar sowohl Unterstützung allgemein als auch eine Entlastung der Lehrer und Schulleitungen sowie der Schulleitung zur Konzeptualisierung. Eine Lehrkraft wünscht sich, dass „alle Lehrkräfte (Integrationsl. + Regelschull.) mehr Unterstützung (zeitl. Rahmen + FoBi + Zusammenarbeit mit weiteren Stellen) erhalten“, eine weitere wünscht sich „Unterstützung - sowohl mehr Förderstunden und bessere räumliche Versorgung -, damit uns als Team die Umsetzung der Inklusion noch besser gelingt..”

Nutzung der Ressourcen der Förderschullehrkräfte durch die Regelschullehrkräfte

Gleichzeitig stimmen allerdings die SL und RSL in den Regelschulen zu 58,1 \% bzw. 54,7\% in sehr hohem Maß zu, dass die Ressourcen der FSL durch die RSL genutzt werden, während die FSL dies nicht in geringerem Maß so einschätzen $(32,4 \%)$.

Tab. 70: Nutzung der Ressourcen der FSL durch RSL

\begin{tabular}{lccc}
\hline & SL $(\mathbf{n}=\mathbf{3 1})$ & RSL $(\mathbf{n}=\mathbf{5 3})$ & FSL $(\mathbf{n}=\mathbf{3 8})$ \\
\hline stimme sehr zu & $58,1 \%$ & $54,7 \%$ & $32,4 \%$ \\
stimme eher zu & $29,0 \%$ & $41,5 \%$ & $52,6 \%$ \\
stimme eher nicht zu & $12,9 \%$ & $0 \%$ & $13,2 \%$ \\
stimme gar nicht zu & $0 \%$ & $0 \%$ & $0 \%$ \\
\hline
\end{tabular}

\section{Aufgaben von Förderschullehrkräften in den Regelschulen}

Die Aufgabenfelder von FSL, die in der Erhebung abgefragt wurden, beziehen sich auf Lernstandsdiagnostik und individuelle Förderung, die Erstellung von Material für SuS mit spF, die Beratung von Lehrkräften sowie die Vernetzungsarbeit in den Schulen. Während die FSL zu $71 \%$ angeben, dass sie sehr häufig bzw. häufig lernstandsdiagnostisch in den Regelschulen tätig sind, schätzen die SL dies mit $58 \%$ etwas und die RSL mit $51 \%$ diesen Anteil sehr viel geringer ein. Ein großer Teil der Befragten gibt an, dass die FSL sehr häufig bzw. häufig mit individueller Förderung (von SuS mit spF) befasst sind (SL 80,6\%; RSL 71,7\%; FSL 97,3\%). Bei der Frage nach der Häufigkeit der Materialerstellung für $\mathrm{SuS}$ mit spF durch die FSL differieren die Antworten dahingehend, dass die FSL angeben dies zu 86,8\% häufig bzw. sehr häufig zu tun, während diese Angaben bei den SL und RSL geringer ausfallen (71\%, RSL sogar nur 35,8\%). 
Tab. 71: Lernstandsdiagnostik (s. auch UE)

\begin{tabular}{lccc}
\hline & SL $(\mathbf{n}=\mathbf{3 1})$ & RSL $(\mathbf{n}=\mathbf{5 3})$ & FSL $(\mathbf{n}=\mathbf{3 8})$ \\
\hline sehr häufig & $29,0 \%$ & $3,8 \%$ & $10,5 \%$ \\
häufig & $29,0 \%$ & $47,2 \%$ & $60,5 \%$ \\
eher selten & $29,0 \%$ & $28,3 \%$ & $23,7 \%$ \\
gar nicht & $6,5 \%$ & $15,1 \%$ & $2,6 \%$ \\
fehlend & $6,5 \%$ & $5,7 \%$ & $2,6 \%$ \\
\hline
\end{tabular}

Tab. 72: Individuelle Förderung durch FSL (bei RSL gemeinsam mit FSL)

\begin{tabular}{lccc}
\hline & SL $(\mathbf{n}=\mathbf{3 1})$ & RSL $(\mathbf{n}=\mathbf{5 3})$ & FSL $(\mathbf{n}=\mathbf{3 8})$ \\
\hline sehr häufig & $54,8 \%$ & $22,6 \%$ & $73,7 \%$ \\
häufig & $25,8 \%$ & $49,1 \%$ & $23,7 \%$ \\
eher selten & $9,7 \%$ & $22,6 \%$ & $2,6 \%$ \\
gar nicht & $0 \%$ & $3,8 \%$ & $0 \%$ \\
fehlend & $9,7 \%$ & $1,9 \%$ & $0 \%$ \\
\hline
\end{tabular}

Tab. 73: Erstellung von Material durch FSL

\begin{tabular}{lccc}
\hline & SL $(\mathbf{n}=\mathbf{3 1})$ & RSL $(\mathbf{n}=\mathbf{5 3})$ & FSL $(\mathbf{n}=\mathbf{3 8})$ \\
\hline sehr häufig & $22,6 \%$ & $9,4 \%$ & $36,8 \%$ \\
häufig & $48,4 \%$ & $26,4 \%$ & $50,0 \%$ \\
eher selten & $12,9 \%$ & $39,6 \%$ & $13,2 \%$ \\
gar nicht & $9,7 \%$ & $18,9 \%$ & $0 \%$ \\
fehlend & $6,5 \%$ & $5,7 \%$ & $0 \%$ \\
\hline
\end{tabular}

Die Beratung von RSL erfolgt durch die FSL an den beteiligten Schulen offensichtlich sehr häufig (zwischen 29,0\% und 39,5\%) bzw. häufig (zwischen 45,2\% und 50,0\%).

Tab. 74: Beratung von Lehrkräften durch FSL

\begin{tabular}{lcc}
\hline & SL $(\mathbf{n}=\mathbf{3 1})$ & FSL $(\mathbf{n}=\mathbf{3 8})$ \\
\hline sehr häufig & $29,0 \%$ & $39,5 \%$ \\
häufig & $45,2 \%$ & $50,0 \%$ \\
eher selten & $19,4 \%$ & $7,9 \%$ \\
gar nicht & $0 \%$ & $0 \%$ \\
fehlend & $6,5 \%$ & $2,6 \%$ \\
\hline
\end{tabular}

Etwa ein Drittel der SL und RSL gibt an, dass die FSL häufig bzw. sehr häufig Vernetzungsarbeit mit außerschulischen Diensten übernehmen. Die FSL selbst schätzen diesen Anteil mit $52,6 \%$ sehr viel höher ein. Andererseits gehen die Beteiligten davon aus, dass diese Arbeit zu 47,4\% (FSL), 56,6\% (RSL) bzw. 58,1\% (SL) eher selten bzw. gar nicht erfolgt. Übereinstimmend verdeutlichen diese Ergebnisse also, dass dieser Aufgabenbereich in den beteiligten Schulen nur für einen Teil der FSL relevant zu sein scheint. 
Tab. 75: Vernetzungsarbeit mit außerschulischen Diensten durch FSL (bei RSL gemeinsam mit FSL)

\begin{tabular}{lccc}
\hline & SL $(\mathbf{n}=\mathbf{3 1})$ & RSL $(\mathbf{n}=\mathbf{5 3})$ & FSL $(\mathbf{n}=\mathbf{3 8})$ \\
\hline sehr häufig & $6,5 \%$ & $7,5 \%$ & $15,8 \%$ \\
häufig & $25,8 \%$ & $26,4 \%$ & $36,8 \%$ \\
eher selten & $48,4 \%$ & $30,2 \%$ & $39,5 \%$ \\
gar nicht & $9,7 \%$ & $26,4 \%$ & $7,9 \%$ \\
fehlend & $9,6 \%$ & $9,4 \%$ & $0 \%$ \\
\hline
\end{tabular}

Als weitere Aufgaben der FSL werden von den SLGS und SLGMS die Förderplanerstellung, das Festlegen des Nachteilsausgleichs, die Erstellung von differenzierten Klassenarbeiten, Gespräche mit Ärzten, Gespräche mit Therapeuten, Gespräche mit Trägern sowie Gespräche mit Förderschulen benannt.

Eine Reihe von Aussagen (insgesamt 8) befasst sich damit, dass die Förderschullehrkräfte gute Beratung einbringen, als offen für Anregungen wahrgenommen werden, nach Einschätzung der RSL ihre Aufgaben engagiert erledigen, sich viel Zeit nehmen, immer zu Gesprächen bereit sind bzw. Rückmeldungen geben, Unterstützung bei Problemen anbieten oder außerschulische Ansprechpartner kennen. Eine Aussage bezieht sich darauf, dass die Kooperation mit dem FSL dann gut gelingen könne, wenn Teams „,vorbildlich engagiert und permanent bemüht [seien], den FL/KL zu unterstützen“, während andere Teams nur den Einzelunterricht (der SuS mit spF) im Blick hätten, was die Kooperation erschwere. Fünf Regelschullehrkräfte äußern sich dahingehend, dass „kaum Gespräche über Integrationsschüler stattfinden“ würden und die „Schüler [...] in manchen Fällen zu wenig Rückmeldung von dem Integrationslehrer bzgl. Fortschritt ihres Lernens“ bekommen würden, die FSL wenig Bereitschaft zur Zusammenarbeit zeige, „keinerlei Eigeninitiative oder Unterstützung zur Entwicklung von Förderplänen oder Bereitstellung von Material erfolgt“ und „Arbeitszeiten [...] nie überschritten“" werden, keine Lernstandsdiagnostik und Förderung von Lernprozessen stattfinde oder der FSL kein ausgebildeter Förderschullehrer und daher unsicher sei.

\subsubsection{Professionalisierung}

\section{Hohes Engagement der Lehrkräfte}

Mit Blick auf die Professionalität der Lehkräfte stimmen sowohl Schulleitungen als auch Lehrkräfte und Eltern überwiegend in sehr hohem Maß der Aussage zu, dass die schulische Integration von SuS mit spF ein hohes Engagement der Lehrkräfte voraussetzt.

Tab. 76: Schulische Integration von SuS mit Förderbedarf setzt ein hohes Engagement der Lehrkräfte voraus

\begin{tabular}{lcccc}
\hline & SL (n=31) & RSL (n= 53) & FSL (n=38) & E (n=34) \\
\hline stimme sehr zu & $83,9 \%$ & $77,4 \%$ & $92,1 \%$ & $79,4 \%$ \\
stimme eher zu & $9,7 \%$ & $20,8 \%$ & $7,9 \%$ & $14,7 \%$ \\
stimme eher nicht zu & $0 \%$ & $0 \%$ & $0 \%$ & $0 \%$ \\
stimme gar nicht zu & $0 \%$ & $0 \%$ & $0 \%$ & $0 \%$ \\
\hline
\end{tabular}




\section{Schulinterne Lehrerfortbildungen und Pädagogische Tage}

Mit Blick auf die Durchführung schulinterner nachmittäglicher Fortbildungsveranstaltungen (SCHILF) geben nur ein Fünftel der RSL (20,8\%) sowie etwa ein Viertel der befragten Schulleitungen $(25,8 \%)$ und FSL (23,7\%) an, dass diese in ihren Schulen zu Fragestellungen schulischer Integration durchgeführt worden sind. Für Pädagogische Tage, die sich mit Bausteinen inklusiver Beschulung auseinandersetzen, sehen diese Anteile mit je etwa $50 \%$ der RSL und FSL sowie $75 \%$ der SL günstiger aus. Inhaltlich stehen bei den schulinternen Fortbildungen vor allem die Auseinandersetzung mit der übergeordneten Thematik Inklusion (36 Nennungen), der Umgang mit Heterogenität/Differenzierung/Individualisierung (16 Nennungen), der Umgang mit herausforderndem Verhalten (12 Nennungen), die Entwicklung von (Förder-) Konzepten (9 Nennungen) sowie die Beschäftigung mit methodischen Ansätzen (6 Nennungen) im Blickpunkt.

Tab. 77: SCHILF zum Thema Integration von SuS mit spF (alphabetisch sortiert)67

\begin{tabular}{|c|c|c|c|c|c|}
\hline & $S L(n=31)$ & $\operatorname{RSL}(n=53)$ & FSL $(n=38)$ & $\operatorname{SLFZ~}(n=4)$ & gesamt \\
\hline Autismus & 1 & & 1 & 1 & 3 \\
\hline Differenzierung & & 1 & 2 & & 3 \\
\hline diff. Leistungsbewertung & 1 & & & & 1 \\
\hline Förderkonzepte & 2 & & & & 2 \\
\hline Förderplanerstellung & & & & 1 & 1 \\
\hline Hausordnung & 1 & 1 & & & 2 \\
\hline Herausforderndes Verhalten & & 1 & 1 & & 2 \\
\hline Heterogenität & & & & 1 & 1 \\
\hline Individualisierung & & & 1 & & 1 \\
\hline Inklusion & 1 & 2 & 4 & 1 & 8 \\
\hline Kooperation Lebenshilfe & & 1 & & & 1 \\
\hline Leitbild & 1 & & & & 1 \\
\hline Montessori-Pädagogik & 1 & & 1 & & 2 \\
\hline Morgenkreis & & 1 & & & 1 \\
\hline Mutismus & & & & 1 & 1 \\
\hline Nachteilsausgleich & & & & 1 & 1 \\
\hline Regeln/Konsequenzen & & & & 1 & 1 \\
\hline Wahrnehmungsstörungen & 1 & & & & 1 \\
\hline nicht näher benannt & 2 & 4 & 1 & 1 & 9 \\
\hline gesamt & $8(25,8 \%)$ & $11(20,8 \%)$ & $9(23,7 \%)$ & 3 & 31 \\
\hline fehlend & $23(74,2 \%)$ & $42(79,2 \%)$ & $29(76,3 \%)$ & 1 & 94 \\
\hline
\end{tabular}

An den Förder- und Beratungszentren finden laut Angaben der dortigen Schulleitungen vielfältige schulinterne Fortbildungsveranstaltungen im Zusammenhang mit schulischer Integration statt, die sich an das ganze Kollegium - also alle FSL, die an einem SFZ beschäftigt sind richten. Dazu gehören so genannte nachmittägliche SCHILF-Veranstaltungen (= schulinterne Lehrerfortbildungen), die sich mit Förderplanerstellung, Nachteilsausgleich, Inklusion, Heterogenität, Mutismus, Autismus oder Regeln und Konsequenzen befasst haben.

67 Mehrfachnennungen führen zu abweichenden Prozentzahlen, in SPSS gezählt wurden nur die Personen, die Angaben gemacht haben, nicht die Angaben selbst 
Tab. 78: Pädagogische Tage zum Thema Integration von SuS mit spF (alphabetisch sortiert)

\begin{tabular}{|c|c|c|c|c|c|}
\hline & $\operatorname{SL}(n=31)$ & $\begin{array}{c}\text { RSL } \\
(n=53)\end{array}$ & $\begin{array}{c}\text { FSL } \\
(\mathrm{n}=38)\end{array}$ & $\begin{array}{l}\text { SLFZ } \\
(\mathrm{n}=4)\end{array}$ & gesamt \\
\hline Autismus & & & & 1 & 1 \\
\hline Besichtigung Modellschulen & 1 & & & & 1 \\
\hline Differenzierung & 2 & 1 & 1 & 1 & 5 \\
\hline Differenzierungsmaterial & & 1 & & & 1 \\
\hline diff. Leistungsbewertung & & & & 1 & 1 \\
\hline Disce-Motorik & & 1 & 1 & & 2 \\
\hline Förderplanerstellung & 1 & & & & 1 \\
\hline EVA/SOL & 1 & & 1 & & 2 \\
\hline Förderkonzepte/Schulkonzepte & 3 & 1 & 2 & 1 & 7 \\
\hline Individualisierung & 1 & & 1 & 1 & 3 \\
\hline Inklusion & 3 & 14 & 11 & & 28 \\
\hline JÜ/Familienklassen & 2 & & & & 2 \\
\hline Kooperation Jugendhilfe & & & & 1 & 1 \\
\hline $\begin{array}{l}\text { Kooperation Schule - RSL - } \\
\text { FSL - I-Hilfen }\end{array}$ & & 1 & & & 1 \\
\hline Leitbild & & & & 1 & 1 \\
\hline Lernspiralen & 1 & 1 & & & 2 \\
\hline offener Unterricht & 1 & & & & 1 \\
\hline Schulbücher für Integration & 1 & & & & 1 \\
\hline $\begin{array}{l}\text { Umgang mit herausforderndem } \\
\text { Verhalten }\end{array}$ & 2 & 3 & 3 & 1 & 9 \\
\hline Umgang mit Heterogenität & & 1 & 1 & 1 & 3 \\
\hline Unterrichtsentwicklung & & & & 1 & 1 \\
\hline Werkstattunterricht & 1 & & & & 1 \\
\hline nicht näher benannt & 5 & 4 & 2 & & 11 \\
\hline gesamt & $23(74,2 \%)$ & $27(50,9 \%)$ & $19(50 \%)$ & 4 & 86 \\
\hline fehlend & $8(25,8 \%)$ & $26(49,1 \%)$ & $19(50 \%)$ & 0 & \\
\hline
\end{tabular}

Auch im Rahmen von Pädagogischen Tagen erfolgte an den SFZ eine Auseinandersetzung mit Themen, die die schulische Integration betreffen: Leitbild- oder Konzeptentwicklung, Unterrichtsentwicklung allgemein, heterogene Leistungsmessung, Umgang mit Heterogenität, Differenzierung und Individualisierung, Autismus, E-Problematik im Aufwind“ oder die Kooperation mit dem Jugendamt. Mehrere SFZ veranstalten Runde Tische mit außerschulischen Diensten wie dem Jugendamt (3), dem schulpsychologischen Dienst, dem schulärztlichen Dienst, der Autismusambulanz, der Arbeitsstelle für Integration, dem Verein „Miteinander leben lernen“ oder der Lebenshilfe. Eine SLFZ gibt außerdem an, dass am SFZ zwei Mal jährlich Fachkonferenzen für Integration/Inklusion stattfinden, eine andere, dass kollegialer Austausch in Form von Feedback ermöglicht wird.

Abgesehen von Fortbildungsveranstaltungen im engeren Sinn haben sich die SFZ in den vergangenen Monaten mit folgenden Inhalten befasst: Umsetzung der Inklusion in Verbindung mit der Konzeptentwicklung an Regelschulen, Kooperation zwischen Grund- und Förderschullehrkräften sowie Erstellung einer Arbeitsplatzbeschreibung für diese beiden Professionen, in- 
klusiver Unterricht bzw. Unterrichtsentwicklung, Nachteilsausgleich und Förderplanerstellung sowie unterschiedliche Förderbedarfe. An einem weiteren SFZ wurden nach Angaben der SL Fachgruppen zu einzelnen Förderbedarfen gebildet (SE, L, G, K, Inklusion).

Auch an den Regelschulen gab es SCHILF-Veranstaltungen (Inklusion, Autismus, Förderkonzepte) sowie Pädagogische Tage (Inklusion, Umgang mit herausforderndem Verhalten, Jahrgangsmischung, Förderkonzepte, Individualisierung).

Abgesehen von schulinternen Fortbildungen und Pädagogischen Tagen haben die Schulen sich in vielfältiger Art und Weise mit den Anforderungen der Integration auseinandergesetzt. Dazu gehören eine Veranstaltung zum Thema Übergänge („Sprungbrett“ --> Übergang Kiga-Schule (Caritas)), die Teilnahme an dem Pilotprojekt „Inklusion“, Besprechungen zu unterschiedlichen Themen (Bsp.: Feedback, Inklusionstreffen, Koordinierungs- oder Steuergruppen), Konferenzen (Fach-/Klassen-/Päd. Konferenzen), Förderausschüsse, Treffen mit Vernetzungspartnern (Jugendamt, Psychologen, Therapeutische Schülerhilfe, Tagesgruppen, Arbeitskreis ADHS), Fortbildungsplanung für das Kollegium oder Projekttage.

Viele Schulleitungen und Lehrkräfte geben an bereits externe Fortbildungen besucht zu haben, die mit dem Thema schulische Integration bzw. Inklusion zu tun haben. Darunter nennen neun Personen Veranstaltungen, die sich konkret mit „Integration“ bzw. „Inklusion“ befasst haben. Zwölf weitere Personen schreiben, dass sie viele (3), sehr viele (3), verschiedene (7) oder sogar „alle verfügbaren“ Fortbildungen zu diesem Themenfeld gemacht haben. Drei der Befragten haben zusätzliche Ausbildungen (Montessori-Diplomausbildung, systemische Beraterin, Konfliktmoderation) absolviert.

Eine ganze Reihe der bisher besuchten Fortbildungsveranstaltungen befassen sich mit unterrichtlichen Themen. Dazu gehören u.a. allgemeinere Themen wie "gemeinsam unterrichten“ oder der Umgang mit Unterrichtstörungen. Als Methoden wurden offener Unterricht und Projektarbeit bei Fortbildungen thematisiert sowie die Discemotorik, selbstorganisiertes Lernen (SOL) in heterogenen Klassen oder die Sensorische Integrationstherapie. Zur Didaktik wurden Veranstaltungen besucht, die sich mit der Förderung im Fach Mathematik (3) bzw. mit Blick auf die Teilleistungsschwäche Dyskalkulie beschäftigt haben oder mit der Förderung bei vorliegender Lese-Rechtschreib-Schwäche (5) sowie im Bereich Sprache. Im Bereich Diagnostik und Förderplanung werden insgesamt von acht der Befragten Fortbildungen erwähnt, die u.a. die Themen Lernstandserhebung, Diagnostik (4), Erstellung von Förderplänen (4) sowie Förderung (4) beinhalteten. Auch die Bausteine Differenzierung und Individualisierung sind Inhalte bisheriger Fortbildungen und werden von insgesamt elf der Befragten aufgeführt. Sie befassen sich mit Heterogenität bzw. Binnendifferenzierung allgemein (6), Binnendifferenzierung in Deutsch, Englisch oder Erdkunde bzw. speziell für SuS mit dem spF Lernen, mit Individualisierung (3) und differenzierter Leistungsmessung.

Mehrere der Befragten haben bereits Fortbildungen besucht, die verschiedene Förderschwerpunkte fokussieren, wie die Autismus-Spektrum-Störung (6), herausforderndes Verhalten bzw. sozial-emotionalen Förderbedarf (9), darunter ein Angebot zur konfrontativen Pädagogik, die Förderbedarfe Sehen, Hören, Schwerbehinderung, Mutismus und Sprechangst oder verschiedene Schwerpunkte. Eine Veranstaltung befasste sich mit elektronischen Hilfsmitteln.

Fortbildungsveranstaltungen zu schulischer Integration werden - abgesehen von den FSL mit insgesamt $42,1 \%$ der Antworten - nur von wenigen Befragten häufig bzw. sehr häufig besucht (SL 22,6\% \%, RSL 13,2\%). Im Vergleich zu den Angaben zum Fortbildungsbedarf erscheinen diese Angaben also niedrig. Die Fortbildungen, die besucht werden, sind fast ausschließlich halb- oder ganztägige Veranstaltungen. 
Tab. 79: Häufigkeit des Besuchs von Fortbildungen zu schulischer Integration

\begin{tabular}{lccc}
\hline & SL (n=31) & RSL (n=53) & FSL (n= 38) \\
\hline sehr häufig & $0 \%$ & $0 \%$ & $10,5 \%$ \\
häufig & $22,6 \%$ & $13,2 \%$ & $31,6 \%$ \\
eher selten & $41,9 \%$ & $24,5 \%$ & $36,8 \%$ \\
gar nicht & $12,9 \%$ & $37,7 \%$ & $10,5 \%$ \\
fehlend & $22,6 \%$ & $24,5 \%$ & \\
\hline
\end{tabular}

Tab. 80: Dauer des Besuchs von Fortbildungen zu schulischer Integration

\begin{tabular}{lccc}
\hline & SL $(\mathbf{n}=\mathbf{3 1})$ & RSL $(\mathbf{n}=\mathbf{5 3})$ & FSL $(\mathbf{n}=\mathbf{3 8})$ \\
\hline weniger als 1 Tag & $12,9 \%$ & $15,1 \%$ & $28,0 \%$ \\
1 Tag & $29,0 \%$ & $18,9 \%$ & $68,0 \%$ \\
mehrere Tage & $3,2 \%$ & $1,9 \%$ & $4,0 \%$ \\
fehlend & $54,8 \%$ & $64,2 \%$ & \\
\hline
\end{tabular}

Lediglich SLFZ 1 gibt an selbst Fortbildungsveranstaltungen besucht zu haben und zwar mit den Inhalten systemische Beratung, Konfliktmoderation, Unterrichtsstörungen, offener Unterricht und Projektarbeit. Die Schulleitungen der GMS haben Fortbildungen zu Heterogenität im Unterricht, SOL im heterogenen Klassensaal und Leistungsmessung besucht.

Sechs Lehrkräfte gehen davon aus, dass die Förderung der SuS mit spF durch Fortbildungen verbessert werden könnte, darunter verweisen zwei auf Fortbildungen im Bereich differenzierter Unterrichtsgestaltung und weitere zwei auf die Bereiche Inklusion, rechtliche Belange, Fördermöglichkeiten und Behinderungen.

Eigenen Fortbildungsbedarf sehen die SLFZ beim Förderbedarf Sprache, im Umgang mit verhaltensauffälligen SuS, in der Vernetzung der SFZ mit den Regelschulen und der Verwaltung (Abbau und Vereinfachung). Die Schulleitungen der Grundschulen formulieren Bedarf hinsichtlich der Erstellung eines Leitbilds, des Bereichs „Stärken stärken - Förderkonzepte, Diagnostizieren und Fördern“, pädagogischen Diagnostik, Individualisierung des Unterrichts, Umgang mit verhaltensauffälligen Kinder sowie Autismus/Autismusspektrumstörung. Die Schulleitungen der Gemeinschaftsschulen nennen Bedarf mit Blick auf die Förderschwerpunkte emotionale und soziale Entwicklung sowie Sprache.

Den Fortbildungsbedarf im Kollegium sehen die SLFZ beim Umgang mit schwierigen Schülern bzw. „Querbeet, da im Zuge der Inklusion zuständig für alles“; die SLGS zu verhaltensauffälligen Kindern, Autismus/Autismusspektrumstörung sowie Unterstützungssystemen von außen. Eine weitere SL sieht Bedarf in allen Punkten, eine weitere sieht gar keinen Fortbildungsbedarf, sondern Bedarf an Personal und Räumen. Zwei der Befragten sehen Fortbildungsbedarf zu Integration bzw. Inklusion allgemein, eine weitere mit Blick auf „Unterstützungssysteme von außen“. Darüber hinaus werden Fortbildungen genannt, die sich auf die sonderpädagogischen Förderschwerpunkte beziehen: in allen Bereichen, zur Autismus-Spektrum-Störung, zu sozialemotionalem Förderbedarf (22), zum Förderbedarf Lernen (9), Förderbedarf Sprache (6), geistige Entwicklung (3) und körperliche Entwicklung.

Besonders ausführlich äußern sich die Befragten zu der offenen Frage bezüglich der Weiterentwicklung ihrer eigenen Professionalität im Hinblick auf inklusive Bildung. Ähnlich wie 
bei den Nennungen zum Fortbildungsbedarf lassen sich die Aussagen vor allem den Bereichen der sonderpädagogischen Förderschwerpunkte und darunter verstärkt dem Umgang mit verhaltensauffälligen SuS zuordnen sowie den Qualitätsbausteinen inklusiven Unterrichts und Professionsfeldern wie Beratung und Kooperation. Eine Person hält es in diesem Zusammenhang für wichtig, dass die Förderschullehrkräfte, die als „Inklusionskollegen“ arbeiten zunächst in Förderschulen arbeiten sollten, bevor sie in Regelschulen tätig werden. Sechs der Befragten betonen die Bedeutung sonderpädagogischen Fachwissens für die professionelle Weiterentwicklung. So vertritt eine Lehrkraft die Auffassung, dass an jeder Grundschule alle Fachrichtungen vertreten sein sollen, die in der Schule aufgenommen werden. Eine FSL möchte mehr zu den Fachrichtungen erfahren, die sie nicht studiert hat; eine andere Person möchte sich möglichst viele Merkmale einzelner Förderbedarfe aneignen, eine andere möchte mehr zum „Umgang mit diesen Kindern" erfahren, da sie Sonderpädagogik nicht studiert habe und eine andere möchte mehr über die „Unterrichtung von Integrationskindern“ lernen. Gewünscht werden von einer weiteren Lehrkraft eine „genaue medizinische Beratung bezüglich bestimmter Fördergutachten“ sowie „mehr Einblick in Förderbereiche (Diagnose etc. “. Ganz präzise möchte eine andere Lehrkraft mehr Kenntnisse „im Umgang mit Krankheitsbildern wie beispielsweise Tourette, Autismus“ erwerben. Eine weitere Lehrkraft argumentiert: „Die einzelnen Fälle sind so unterschiedlich, dass man für jedes Kind andere Kompetenzen benötigt. Fachwissen zu den Problemen und dessen Anwendung in der Praxis sind für jeden Fall wichtig."

Neben dem Erwerb von Kenntnissen in Sonderpädagogik bzw. den Förderbereichen allgemein (6), werden auch Kenntnisse zu speziellen Förderbedarfen wie Lernen, Hören, Sehen, Sprache, geistige Entwicklung, körperliche Entwicklung bzw. auch Autismus und DAZ als sinnvoll erachtet. Eine befragte Person hält es für wichtig „sonderpäd. Fachwissen und daraus resultierendes Wissen bzgl. besondere Bedarfe in die Konzept(weiter)entwicklung von Grundschulen miteinzubeziehen“. Zwei weitere Lehrkräfte wünschen sich Professionalisierung mit Bezug auf Konzeptentwicklung bzw. die Umsetzung eines inklusiven Konzepts. Eine weitere möchte Kenntnisse mit Blick auf die „bestmögliche Förderung aller Kinder der Klasse (was ich unter den derzeitigen Bedingungen für unmöglich halte!)“ erwerben.

Einen besonderen Schwerpunkt mit Blick auf die professionelle Weiterentwicklung stellt - wie bei den Fortbildungswünschen auch - die gewünschte Auseinandersetzung mit dem Umgang mit Schülern und Schülerinnen dar, die Unterstützungsbedarf im sozialen und emotionalen Bereich haben (15), darunter u.a. mit den Hintergründen für die Entstehung von auffälligem Verhalten. Eine Lehrkraft begründet dies folgendermaßen: „Die größte Herausforderung der angestrebten Inklusion in der Regelschule sind nicht die L-, S- oder K-Kinder, sondern die zunehmende Zahl der Kinder mit extremen Beeinträchtigungen im sozial-emotionalen Bereich!“. Weitere Kompetenzen möchten die befragten Lehrkräfte in den Bereichen Gesprächsführung, Beratung (10), Diagnostik (4), Lernstandsdiagnostik (3), Erstellung von Förderplänen (5), neue Unterrichtsmethoden (5) (kooperative Lernformen, Wochenplanarbeit, neue Lerntechniken, Selbstkontrolle von SchülerInnen), Didaktik, Mathematik, gemeinsamer Unterricht, „Unterricht in inklusiven Settings“, Differenzierung und Individualisierung (8), Material für binnendifferenzierten Unterricht sowie Kooperation (3) und Teamteaching (6) erwerben. Drei Personen wünschen sich Fortbildungen für ihre Professionalisierung, eine weitere fordert die Vermittlung von Grundkenntnissen zur sonderpädagogischen Förderung in der Ausbildung der Grundschullehrer. Zwei Lehrkräfte verweisen darauf, dass sie dies wegen ihres Alters nicht mehr betreffe. Eine weitere Lehrkraft, die ebenfalls kurz vor ihrem Ruhestand ist, formuliert zum Thema „Professionalisierung “ kritisch und plastisch: 
Die Nerven sind durch die vielen problematischen Kinder einer Dauerreißprobe ausgesetzt. Mehr geht psychisch nicht mehr. Wer vom grünen Tisch aus über Inklusion redet, sollte mindestens ein Jahr lang alleine eine unserer Klassen unterrichten. Ich sehe das rohe Zahnfleisch, auf dem diese Menschen gingen, bildlich vor mir.

Drei weitere Lehrkräfte möchten ihre „nervliche Stärke weiterentwickeln“, ihre Zufriedenheit und ihren „Energiehaushalt“ bzw. ihr Zeitmanagement.

Als Wünsche für die Professionalisierung werden entsprechende Inhalte in Studium und Referendariat sowie inhaltlich bessere und verpflichtende Fortbildungen gefordert (3).

Wie die Aussagen der SLFZ zu schulinternen Fortbildungen sowie zu den Inhalten, die gemeinsam bearbeitet werden, indirekt zeigen, findet zum Teil eine enge inhaltlich-pädagogische Zusammenarbeit der FSL untereinander statt. Dazu führen die Schulleitungen in den Fragebögen ergänzend aus, dass diese zwischen einzelnen Kollegen bzw. Gruppen sehr eng sei, so dass diese untereinander gegenseitige „Stütze und Hilfe“ erfahren könnten (SLFZ 2). Die zunehmende Größe der SFZ erfordere die Entwicklung neuer Kommunikationsstrukturen untereinander (SLFZ 1). SLFZ 1 und SLFZ 3 geben zu bedenken, dass das Aufgabengebiet der SFZ sehr viel umfassender geworden sei und dafür nicht die notwendigen zeitlichen, räumlichen und finanziellen Ressourcen vorhanden seien (SLFZ 1), was SLFZ 3 folgendermaßen plastisch zum Ausdruck bringt:

Die Anrechnung von 2 LWS zur regulären Schulleiterdeputatsstundenzuweisung steht in keinem Verhältnis zu den bei etwa 200 anfallenden Betreuungsaufgaben zzgl. die Betreuung der Lehrer des FZ, Regelschulen. Ein Witz! Aber ein schlechter! (SLFZ 3).

Mit Blick auf die Weiterentwicklung sonderpädagogischer Professionalität aus Sicht der SFZ nennen die SLFZ die Bereiche Kommunikations- und Beratungskompetenz (3), neue Lerntechniken, die Ausweitung der sonderpädagogischen Fachkompetenzen bezüglich der Förderschwerpunkte bzw. eine fachübergreifende Ausrichtung, Kompetenzen im Teamteaching und ein Zeitkontingent für reine Diagnosetätigkeit.

\subsubsection{Ergebnisse im Bereich der Personalentwicklung}

\section{Offenheit Lehrkräfte für SuS mit spF}

Das höchste Maß an Zustimmung zur Aussage, dass die Lehrkräfte an der Schule offen für die Integration von Kindern mit spF sind signalisieren die RSL selbst mit 50,9\%, während die Schulleitungen dieser Aussage mit 45,2\% und vor allem die FSL mit 26,3\% mit geringeren Anteilen zustimmen.

Tab. 81: Offenheit der Lehrkräfte für SuS mit spF

\begin{tabular}{lccc}
\hline & SL (n=31) & RSL $(\mathbf{n}=\mathbf{5 3})$ & FSL $(\mathbf{n}=\mathbf{3 8})$ \\
\hline stimme sehr zu & $45,2 \%$ & $50,9 \%$ & $26,3 \%$ \\
stimme eher zu & $31,6 \%$ & $45,3 \%$ & $65,8 \%$ \\
stimme eher nicht zu & $3,2 \%$ & $3,8 \%$ & $2,6 \%$ \\
stimme gar nicht zu & $0 \%$ & $0 \%$ & $0 \%$ \\
\hline
\end{tabular}

\section{Maß an Akzeptanz der bisherigen Form der Integration}

Auch das Maß an Akzeptanz, das die bisherige Form der schulischen Integration erfährt, wird von den Beteiligten unterschiedlich eingeschätzt. 
Tab. 82: Maß der Akzeptanz der bisherigen Form der Integration

\begin{tabular}{lcccc}
\hline & SL $(\mathbf{n}=\mathbf{3 1})$ & RSL $(\mathbf{n}=\mathbf{5 3})$ & FSL $(\mathbf{n}=\mathbf{3 8})$ & E $(\mathbf{n}=34)$ \\
\hline stimme sehr zu & $35,5 \%$ & $47,2 \%$ & $31,6 \%$ & $61,8 \%$ \\
stimme eher zu & $58,1 \%$ & $41,5 \%$ & $52,6 \%$ & $29,4 \%$ \\
stimme eher nicht zu & $6,5 \%$ & $7,5 \%$ & $10,5 \%$ & $5,9 \%$ \\
stimme gar nicht zu & $0 \%$ & $3,8 \%$ & $0 \%$ & $0 \%$ \\
\hline
\end{tabular}

Die Schulleitungen der SFZ halten es für schwierig dieses Maß an Akzeptanz zu bewerten, da die Erfahrungen mit den einzelnen Schulen sehr unterschiedlich seien (SLFZ 1, SLFZ 2). SLFZ 4 wünscht sich bei dieser Fragestellung eine Unterteilung in Grundschulen und weiterführende Schulen.

Mit Blick auf die Professionalität der RSL hinsichtlich der Förderung der SuS mit spF ergibt sich nach Auffassung der überwiegenden Mehrheit aller Befragten ein positives Bild. Diese geben zu 42,1\% (FSL) bis zu 67,6\% (Eltern) an, dass die RSL diese Schüler so gut wie möglich fördern würden.

Tab. 83: Förderung der SuS mit spF durch RSL so gut wie möglich

\begin{tabular}{lcccrc}
\hline & SL (n=31) & RSL (n= 53) & FSL (n=38) & E (n=34) & S GMS (n= 12) \\
\hline stimme sehr zu & $51,6 \%$ & $64,2 \%$ & $42,1 \%$ & $67,6 \%$ & $50,0 \%$ \\
stimme eher zu & $45,2 \%$ & $30,2 \%$ & $44,7 \%$ & $23,5 \%$ & $33,3 \%$ \\
stimme eher nicht zu & $1 \%$ & $5,7 \%$ & $10,5 \%$ & $5,9 \%$ & $16,7 \%$ \\
stimme gar nicht zu & $0 \%$ & $0 \%$ & $0 \%$ & $0 \%$ & $0 \%$ \\
\hline
\end{tabular}

Sechs Lehrkräfte betonen das hohe Engagement der Unterrichtenden hinsichtlich der Förderung der SuS mit spF. Sie beschreiben, dass alle Beteiligten sehr bemüht sind die Kinder bestmöglich zu fördern (5) bzw. dass „alles ihr Bestes geben“ und sich daher nur wenige Schüler aufgaben und die Hilfe nicht annehmen, sondern sich stattdessen aufgrund der Hilfe sicherer fühlen.

Die Hälfte (RSL) bis drei Viertel (FSL) der Befragten geben an, dass sie davon ausgehen, dass die Haltung der Lehrkräfte inklusiver Bildung gegenüber eher zustimmend sei. Nach Einschätzung der befragten FSL ist die Haltung der FSL in diesem Zusammenhang also vergleichsweise günstig, während etwa ein Fünftel der SL und ein Drittel der RSL davon ausgeht, dass die Haltung der RSL eher ablehnend sei. Unklar bleiben diese Daten, da es bei dieser Fragestellung einen vergleichsweise hohen Anteil fehlender Angaben gibt.

Tab. 84: Die Haltung der Lehrkräfte in unserer Schule ist der zukünftigen inklusiven Bildung gegenüber...

\begin{tabular}{lccc}
\hline & SL $(\mathbf{n}=\mathbf{3 1})$ & RSL $(\mathbf{n}=\mathbf{5 3})$ & FSL $(\mathbf{n}=\mathbf{3 8})$ \\
\hline sehr zustimmend & $0 \%$ & $0 \%$ & $0 \%$ \\
eher zustimmend & $58,1 \%$ & $50,9 \%$ & $73,7 \%$ \\
eher ablehnend & $22,6 \%$ & $34,0 \%$ & $15,8 \%$ \\
ablehnend & $0 \%$ & $0 \%$ & $0 \%$ \\
fehlend & $19,4 \%$ & $15,1 \%$ & $10,5 \%$ \\
\hline
\end{tabular}


Viele der Befragten führen inhaltliche Begründungen für eine zustimmende, unentschiedene oder skeptisch-ablehnende Haltung der Beteiligten an. SLFZ 1 geht davon aus, dass die Lehrkräfte der inklusiven Bildung eher zustimmend gegenüber stehen, „weil sie grundsätzlich die Öffnung der Regelschulen für Kinder mit Beeinträchtigungen für gesellschaftlich notwendig erachten“. Ähnlich begründet dies die Schulleitung einer Grundschule, die ausführt, dass „die Notwendigkeit gesellschaftlich gesehen wird“ sowie die Schulleitung einer Gemeinschaftsschule die über ihre Lehrkräfte sagt, dass dies sich „den Herausforderungen der Gesellschaft verantwortungsbewusst stellen“. Die Schulleitungen der Grund- bzw. Gemeinschaftsschulen begründen die eher zustimmende Haltung auch damit, dass die Schulen „schon viel Erfahrung mit „Integrationen“ haben“, zum Teil seit etwa 20 Jahren, die Lehrkräfte jedem Schüler helfen wollen und eine grundsätzliche Bereitschaft zu integrativem Arbeiten vorhanden sei bzw. sie als „Klassenlehrer Kinder mit sonderpädagogischem Förderbedarf gerne und erfolgreich unterrichten und sie die richtige Haltung zu Inklusion haben“. Die integrative Arbeit sei Alltag und Teil des pädagogischen Selbstverständnisses. Lehrkräfte würden „freiwillig Hilfsmaßnahmen annehmen und sich fort- und weiterbilden“. Eine weitere GSL benennt ihre eigene positive Einstellung zu Inklusion als Chance.

Drei Lehrkräfte begründen die eher zustimmende Haltung des Kollegiums zur Integration mit einer offenen Grundhaltung und dem Menschenbild. Trotz Sorgen, Überforderung und Unsicherheit gäbe es - laut einer weiteren Lehrkraft - Zuversicht, dass Inklusion gelingen könne. Eine Fülle von Aussagen der Lehrkräfte bezieht sich darauf, dass die Schulen bereits seit Jahren integrativ arbeiten und eine zustimmende Haltung daher selbstverständlich sei (15), gute Ergebnisse aus diesen Erfahrungen vorliegen (3) und die Vorteile bekannt sind. Eine Lehrkraft erwähnt, dass „das Schulkonzept sich schon seit vielen Jahren auf individuelle Förderung eingestellt hat“, eine andere, dass „sich die Schule in besonderem Maße als Schule für alle versteht und an pädagogischer Weiterentwicklung arbeitet" und eine weitere, dass die Schulleitung gewechselt habe und dadurch ein „neuer Wind“ zu wehen beginne. Weitere Lehrkräfte erkennen eine positive Haltung der Kollegien daran, dass „alle Kinder gleich behandelt werden“, „die Lehrkräfte [sich] nach ihren Möglichkeiten bemühen, Kinder mit sonderpäd. Förderbedarf in ihrer Klasse zu fördern“ bzw. „auch von ihnen Ideen bzgl. des Umsetzens von Integration/Inklusion kommen“, „sie an kommunikativem und fachl. Austausch sowie Kooperation zur Optimierung der Lern- und Übungsmöglichkeiten für die einzelnen Schüler interessiert sind“ und „erkennen, dass sie durch Förderschullehrkräfte und die Umstrukturierung des Unterrichts viel Hilfe und Erleichterung haben“.

SLFZ 3 gibt die Haltung seines Kollegiums als unentschieden an und geht davon aus, dass dies so ist, da die FSL sich als "Mädchen für alles“, d.h. für alle Problemfälle eingestuft fühlen und dafür nicht die Zeit zur Verfügung steht.“ Eine unentschiedene Haltung gegenüber Inklusion wird von zwei Lehrkräften dadurch erklärt, dass diese sich nicht verallgemeinern lasse, sondern individuell entschieden werden müsse, ob Kinder eher in Förder- oder Regelschulen unterrichtet werden sollten. Eine Lehrkraft erläutert, dass das Kollegium nicht grundsätzlich gegen inklusive Beschulung sei, „die Hospitation an einer Modellschule [aber] auch nicht 100 Prozent überzeugt" habe.

SLFZ 2 und SLFZ 4 schätzen die Haltung der Kollegien an den SFZ als eher ablehnend ein und begründen dies damit, dass „durch die weitgefasste Arbeitsplatzbeschreibung die Förderung der Kinder mit spF zu kurz kommen könnte“ (SLFZ 2) oder „weil es an den Ressourcen fehlt“ (SLFZ 4). Eine ablehnende Haltung wird von einer Lehrkraft damit begründet, dass „unsere Gesellschaft nach wie vor dazu neigt, Menschen in Schubladen einzusortieren, das gilt auch 
für das Schulwesen!“, von einer anderen damit, dass „die Voraussetzungen und Forderungen seitens der Regierung nicht zufriedenstellend umzusetzen sind“ oder von einer weiteren, dass „viele Dinge noch ungeklärt sind. Es gibt zu wenige konkrete Infos von Seiten des Ministeriums“. Eine weitere bemängelt, dass „Aufklärung und Bekanntmachung fehlen“ und „noch keine ausreichenden Voraussetzungen und Rahmenbedingungen zur effektiven und angemessenen inklusiven Unterrichtung bestehen".

Ablehnende Haltungen werden von den Schulleitungen aber auch auf „Ängste, insbesondere vor Überforderung“ (SLGS) oder vor „Überlastung“ (SLGMS) zurückgeführt. Ähnlich führen einige Lehrkräfte die eher ablehnende Haltung des Kollegiums auf Skepsis, Unsicherheit, Befürchtungen, Überforderung oder Ängste zurück. Die Kollegen und Kolleginnen seien „skeptisch, da sie nicht wissen, was auf sie zukommt und wie sie das leisten können, oder ablehnend, weil große Unsicherheit herrscht. Viele fühlen sich schlecht informiert und vorbereitet und ins kalte Wasser geworfen!“. Befürchtungen hingen damit zusammen „nicht genügend Unterstützung zu erfahren“. Teilweise fühlten sich Lehrer mit dem Thema Inklusion allein gelassen „ohne ein Konzept zur Förderung von „oben“““. Bei manchen bestünde die Angst, „dass inklusive Bildung auf Kosten der Kinder durchgeführt wird und nicht alle Kinder bestmöglich gefördert werden können“. Die Angst vor Überforderung könne wiederum damit zusammenhängen, dass die Ausbildung fehle (Bsp.: Grundschullehramt). Das könne zu Ablehnung führen, „weil sie Anforderungen „übergestülpt“ bekommen, ohne ausreichend „Werkzeug “ und „Unterstützung“ zu erhalten. Viele haben Angst, dieser Aufgabe nicht gewachsen zu sein... Darüber hinaus gebe es auch Ängste vor der Mehrarbeit (4), bspw. „weil die Inklusion mit viel Zeitaufwand verbunden ist, durch besondere Zuwendung “. Eine Lehrkraft äußert die Sorge, dass vieles unsicher sei, Probleme sich erst in der Praxis zeigen würden und gegebenenfalls ,,,von oben“ Dinge auferlegt werden, die Lehrern Probleme bereiten“.

Andere Lehrkräfte führen eine ablehnende Haltung darauf zurück, dass „nicht oder kaum Motivation vorhanden ist, sich mit diesem Thema auseinanderzusetzen“, „durch die weitgefasste Arbeitsplatzzuschreibung die Förderung der SchülerInnen mit SoPäFö zu kurz kommen könnte“, „weil FÖLehrer, Integrationshelfer,... trotz aller Bemühungen die anderen Kinder ablenken, Störungen verursachen“ oder die inklusive Beschulung als „Bedrohung“ gesehen würde.

Weitere Aussagen thematisieren den Zusammenhang zwischen einer ablehnenden Haltung auf der einen und mangelhaften personelle Ressourcen auf der anderen Seite (9). Vier Lehrkräfte fügen an, dass die Grundhaltung unter der Bedingung zustimmend sei, dass verbesserte Rahmenbedingungen, eine entsprechende Personalisierung, mehrere zusätzliche Stunden oder „ausreichend Unterstützung und Zusammenarbeit mit Förderschulen“ gegeben seien. Eine Lehrkraft weist in diesem Zusammenhang darauf hin, dass die „Förderlehrerwochenstunden [...] trotz der Erhöhung d. Schüler mit Förderbedarf verringert [wurden]“, eine andere darauf, dass „die Klassen [teilweise] zu groß sind und inklusive Schüler nicht ausreichend gefördert werden können“. Eine Lehrkraft beklagt, dass Inklusion umgesetzt werden solle,

[...] wobei nicht genügend finanzielle Mittel (Material, Teamteaching in jedem Fach...) und räumliche Möglichkeiten vorhanden sind und nur das Konzept steht. So ist keine erfolgreiche Umsetzung möglich. Schüler leiden, Eltern beschweren sich. Wären diese Punkte umgesetzt, wären die Lehrkräfte von diesem Konzept auch überzeugt. Die grundsätzliche Idee überzeugt sie, nur die Art der Umsetzung nicht.

Drei weitere Lehrkräfte sowie die Schulleitung einer Grundschule erwähnen, dass die Haltung des Kollegiums dann ablehnend werde, wenn die Rahmenbedingungen so bleiben bzw. sich weiter verschlechtern würden. 


\subsubsection{Darstellung der Ergebnisse zur Unterrichtsentwicklung}

\subsubsection{Ressourcen im Bereich der Unterrichtsentwicklung}

\section{Klassengröße}

Bis auf die Angaben der Schüler der GMS erfährt die Aussage, dass eine Klasse nicht mehr als $20 \mathrm{SuS}$ haben solle, von einem Großteil der Befragten ein sehr hohes Maß an Zustimmung zwischen $71,0 \%$ bei den SL und übereinstimmend $86,8 \%$ der RSL und FSL.

Tab. 85: Eine Klasse sollte nicht mehr als $20 \mathrm{SuS}$ haben

\begin{tabular}{lccccc}
\hline & SL (n=31) & RSL (n= 53) & FSL (n=38) & E (n=34) & S GMS (n=12) \\
\hline stimme sehr zu & $71,0 \%$ & $86,8 \%$ & $86,8 \%$ & $79,4 \%$ & $45,5 \%$ \\
stimme eher zu & $25,8 \%$ & $9,4 \%$ & $13,2 \%$ & $14,7 \%$ & $9,1 \%$ \\
stimme eher nicht zu & $0 \%$ & $1,9 \%$ & $0 \%$ & $2,9 \%$ & $27,3 \%$ \\
stimme gar nicht zu & $0 \%$ & $0 \%$ & $0 \%$ & $0 \%$ & $0 \%$ \\
\hline
\end{tabular}

Besonders deutlich wird der ausgeprägte Wunsch nach kleineren Klassen auch bei der offenen Formulierung von Wünschen für die inklusive Beschulung bzw. die Verbesserung der Förderung, bei der dieser $28 \mathrm{Mal}$ genannt wird. Kleinere Klassen werden auch von elf Beteiligten als Antwort auf die Frage genannt, wie die Förderung der Schülerinnen und Schüler mit spF verbessert werden könnte. Diese könnten dazu beitragen, dass „die Lehrperson Zeit hat, auf die Kinder einzugehen".

Mit Blick auf die Klassenzusammensetzung erhält die Aussage, dass nur drei bis vier SuS mit $\mathrm{spF}$ in einer Klasse unterrichtet werden sollen, vorwiegend ein hohes bzw. sehr hohes Maß an Zustimmung.

Tab. 86: Es sollte darauf geachtet werden, dass nur drei bis vier SuS mit Förderbedarf in einer Klasse unterrichtet werden

\begin{tabular}{lcccc}
\hline & SL (n=31) & RSL (n=53) & FSL (n=38) & E (n=34) \\
\hline stimme sehr zu & $48,4 \%$ & $60,4 \%$ & $44,7 \%$ & $44,1 \%$ \\
stimme eher zu & $32,3 \%$ & $26,4 \%$ & $36,8 \%$ & $29,4 \%$ \\
stimme eher nicht zu & $6,5 \%$ & $5,7 \%$ & $5,3 \%$ & $11,8 \%$ \\
stimme gar nicht zu & $3,2 \%$ & $0 \%$ & $5,3 \%$ & $5,9 \%$ \\
\hline
\end{tabular}

Gleichzeitig stimmt ein Großteil der Befragten der Aussage zu, dass in einer Klasse möglichst mehrere SuS mit spF unterrichtet werden sollten. Lediglich die Regelschullehrkräfte zeigen diesbezüglich ein geringeres Maß an Zustimmung.

Tab. 87: Wenn möglich sollten in einer Klasse mehrere SuS mit Förderbedarf unterrichtet werden

\begin{tabular}{lccccc}
\hline & SL (n=31) & RSL (n= 53) & FSL (n=38) & E (n=34) & S GMS (n= 12) \\
\hline stimme sehr zu & $29,0 \%$ & $17,0 \%$ & $31,6 \%$ & $35,3 \%$ & $27,3 \%$ \\
stimme eher zu & $35,5 \%$ & $32,1 \%$ & $52,6 \%$ & $41,2 \%$ & $54,5 \%$ \\
stimme eher nicht zu & $16,1 \%$ & $35,8 \%$ & $7,9 \%$ & $1,8 \%$ & $18,2 \%$ \\
stimme gar nicht zu & $9,7 \%$ & $9,4 \%$ & $0 \%$ & $2,9 \%$ & $0 \%$ \\
\hline
\end{tabular}


Eltern und SuS befürworten die Unterrichtung von Schülerinnen und Schülern mit schweren Behinderungen in der Regelschule am stärksten, während RSL und FSL dies übereinstimmend eher nicht tun.

Tab. 88: In der Regelschule sollten auch SuS mit schweren Behinderungen unterrichtet werden.

\begin{tabular}{lccccc}
\hline & SL (n=31) & RSL (n= 53) & FSL (n=38) & E (n=34) & S GMS (n=12) \\
\hline stimme sehr zu & $9,7 \%$ & $1,9 \%$ & $5,3 \%$ & $32,4 \%$ & $50,0 \%$ \\
stimme eher zu & $22,6 \%$ & $9,4 \%$ & $18,4 \%$ & $29,4 \%$ & $25,0 \%$ \\
stimme eher nicht zu & $38,7 \%$ & $52,8 \%$ & $44,7 \%$ & $26,5 \%$ & $16,7 \%$ \\
stimme gar nicht zu & $0 \%$ & $26,4 \%$ & $21,1 \%$ & $5,9 \%$ & \\
\hline
\end{tabular}

\subsubsection{Schulklasse als sozialer Raum}

Die Schülerinnen und Schüler wurden direkt dazu befragt, inwiefern in ihren Klassen ein freundliches Klima gegeben ist, die Lehrkräfte sie gut behandeln und sie Freunde haben. Während die Zustimmung zur Aussage, dass Freunde vorhanden sind, mit über $80 \%$ sehr hoch ist, geben nur etwas zwei Drittel der SuS mit spF an, von ihren Lehrern gut behandelt zu werden. Mehr als die Hälfte stimmte der Aussage, dass in der Klasse alle freundlich zu ihnen sind, nicht zu.

Tab. 89: Fragen zur Klasse an $S(n=33)$

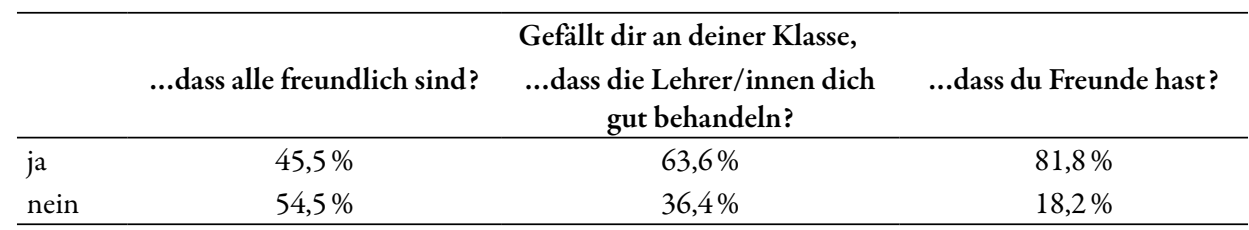

Das soziale Klima in den Klassen scheint mit Blick darauf, inwiefern sie sich untereinander in ihrer Klasse verstehen, nach Angaben des Großteils der Schülerinnen und Schüler (87,8\%) gut bis sehr gut zu sein, während der Umgang mit Streit nur von 60,6\% als gut bzw. sehr gut bezeichnet wird. Das eigene Verhalten schätzt mit 72,4\% der überwiegende Teil der SuS mit spF als gut ein.

Tab. 90: Wie versteht ihr euch in eurer Klasse? $(S, n=33)$

\begin{tabular}{lr}
\hline sehr gut & $33,3 \%$ \\
gut & $54,5 \%$ \\
weniger gut & $9,1 \%$ \\
gar nicht gut & $3,0 \%$
\end{tabular}

Tab. 91: Wie gut geht ihr in deiner Klasse mit Streit um? (S, n= 33)

\begin{tabular}{lc}
\hline sehr gut & $24,2 \%$ \\
gut & $36,4 \%$ \\
weniger gut & $30,3 \%$ \\
gar nicht gut & $6,1 \%$ \\
\hline
\end{tabular}


Tab. 92: Im Umgang mit den anderen $S u S$ verhalte ich mich $(S, n=33)$

\begin{tabular}{lr}
\hline sehr gut & $21,2 \%$ \\
gut & $72,4 \%$ \\
weniger gut & $6,1 \%$ \\
gar nicht gut & $0 \%$ \\
\hline
\end{tabular}

\subsubsection{Klassenführung}

Ein Fragenblock innerhalb der Fragebögen, der sich an SL, RSL, FSL und die SuS mit spF richtet, bezieht sich auf den Qualitätsbaustein der Klassenführung. In diesem Zusammenhang wird abgefragt, wie gut die SuS sich an Regeln halten, wie ruhig es in den Klassen ist, wie gut die Lehrkräfte für Ruhe sorgen, wie konsequent diese mit Fehlverhalten der SuS umgehen und wie gut die SuS sich in ihren Klassen konzentrieren können.

Es sind die SuS selbst, die die Frage nach der Regeleinhaltung in den Klassen am kritischsten beantworten und zu einem Drittel angeben, dass es den $\mathrm{SuS}$ weniger gut gelinge sich an die Regeln zu halten. Ähnlich kritisch sehen dies mit 23,7\% die FSL, während die SL und die RSL diese Auffassung nur zu etwa $15 \%$ vertreten. Der überwiegende Teil aller Befragten nimmt allerdings eine Einschätzung dahingehend vor, dass die Regeleinhaltung den SuS gut gelinge.

Tab. 93: Wie gut halten sich die Schüler an Regeln?

\begin{tabular}{lcccc}
\hline & $\mathbf{S L}(\mathbf{n}=\mathbf{3 1})$ & $\mathbf{R S L}(\mathbf{n}=\mathbf{5 3})$ & FSL $(\mathbf{n}=\mathbf{3 8})$ & $\mathbf{S}(\mathbf{n}=\mathbf{3 3})$ \\
\hline sehr gut & $0 \%$ & $5,7 \%$ & $2,6 \%$ & $3,0 \%$ \\
gut & $80,6 \%$ & $69,8 \%$ & $55,3 \%$ & $60,6 \%$ \\
weniger gut & $16,1 \%$ & $15,1 \%$ & $23,7 \%$ & $33,3 \%$ \\
gar nicht gut & $0 \%$ & $3,8 \%$ & $0 \%$ & $3,0 \%$ \\
\hline
\end{tabular}

Die überwiegende Mehrheit der Befragten geht auch davon aus, dass die Lehrkräfte in gutem Maß für Ruhe in den Klassen sorgen. Hier fällt die Einschätzung der SuS, die zu über 50 \% angeben, dass die Lehrer dies in sehr guter Form tun, im Vergleich besonders positiv aus.

Tab. 94: Wie gut sorgen die Lehrkräfte für Ruhe?

\begin{tabular}{lcccc}
\hline & SL (n=31) & RSL (n= 53) & FSL (n=38) & S (n=33) \\
\hline sehr gut & $9,7 \%$ & $24,5 \%$ & $5,3 \%$ & $54,5 \%$ \\
gut & $83,9 \%$ & $73,6 \%$ & $73,7 \%$ & $42,4 \%$ \\
weniger gut & $3,2 \%$ & $0 \%$ & $7,9 \%$ & $0 \%$ \\
gar nicht gut & $0 \%$ & $0 \%$ & $2,6 \%$ & $0 \%$ \\
\hline
\end{tabular}

Am weitesten auseinander gehen dagegen die Antworten in diesem Fragenblock bei der Frage danach, wie ruhig es in den Klassen tatsächlich sei. Hier sind es die SuS mit spF, die mit über $60 \%$ aussagen, dass es in ihren Klassen weniger ruhig sei, während diese Meinung nur $6 \%$ der SL sowie 20,8\% der RSL und 28,9\% der FSL vertreten. Auch wenn lediglich das Antwortverhalten der Lehrkräfte berücksichtigt werden würde, bedeutet dies, dass es in einem Viertel bzw. einem Fünftel der Klassen, deren Lehrkräfte befragt wurden, weniger ruhig wäre. Nur in einem 
sehr geringen Teil der Klassen (Antworten zwischen 2,6\% und 5,7\%) ginge es sehr ruhig zu und - nach Einschätzung der SL, RSL und FSL - im überwiegenden Teil (zwischen $22 \%$ und $64,2 \%)$ eher ruhig.

Tab. 95: Wie ruhig ist es in den Klassen?

\begin{tabular}{lcccc}
\hline & SL (n=31) & RSL (n= 53) & FSL (n=38) & S (n= 33) \\
\hline sehr ruhig & $3,2 \%$ & $5,7 \%$ & $2,6 \%$ & $3,0 \%$ \\
eher ruhig & $71,0 \%$ & $64,2 \%$ & $50,0 \%$ & $21,2 \%$ \\
weniger ruhig & $19,4 \%$ & $20,8 \%$ & $28,9 \%$ & $63,6 \%$ \\
gar nicht ruhig & $0 \%$ & $1,9 \%$ & $0 \%$ & $12,1 \%$ \\
fehlend & $6,5 \%$ & $7,5 \%$ & $18,4 \%$ & $0 \%$ \\
\hline
\end{tabular}

Trotz ggf. vorhandener Unruhe geben die SuS mit spF zu insgesamt 75,7\% an sich in ihren Klassen gut bzw. sehr gut konzentrieren zu können, Werte, die denen der Antworten der SL etwa vergleichbar sind (84,0\%), während sie bei den RSL und FSL niedriger liegen $(58,5 \%$, FSL 50,0\%). Gleichzeitig geben die SuS, RSL und FSL mit Prozentwerten zwischen 24,2\% und $32,1 \%$ an, dass die SuS sich in den Klassen weniger gut konzentrieren können.

Tab. 96: Wie gut können sich die Schüler in den Klassen konzentrieren?

\begin{tabular}{lcccc}
\hline & SL $(\mathbf{n}=\mathbf{3 1})$ & RSL $(\mathbf{n}=\mathbf{5 3})$ & FSL $(\mathbf{n}=\mathbf{3 8})$ & $\mathbf{S}(\mathbf{n}=\mathbf{3 3})$ \\
\hline sehr gut & $9,7 \%$ & $1,9 \%$ & $2,6 \%$ & $21,2 \%$ \\
gut & $74,2 \%$ & $56,6 \%$ & $47,4 \%$ & $54,5 \%$ \\
weniger gut & $12,9 \%$ & $32,1 \%$ & $28,9 \%$ & $24,2 \%$ \\
gar nicht gut & $0 \%$ & $1,9 \%$ & $0 \%$ & $0 \%$ \\
\hline
\end{tabular}

Insgesamt 26 Schülerinnen und Schüler ergänzen in den Fragebögen die Aussage: „Ich könnte in der Schule noch besser lernen, wenn..... In fünf Aussagen formulieren die SuS, dass sie besser lernen könnten, wenn es in der Klasse leiser sei, bzw. in weiteren neun, wenn sie besser zuhören könnten (3), sich nicht ablenken lassen oder mehr anstrengen würden (4). Jeweils vier SuS weisen darauf hin, dass es besser wäre, wenn nicht so viele Kinder in der Klasse wären. Ein Schüler geht davon aus, dass er besser lernen könne, „wenn ein paar Schüler aus der Klasse wären“. Zwei Schüler schreiben, dass sie mehrfache Erklärungen benötigen, wenn sie etwas nicht verstehen. In weiteren Aussagen geht es um „mehr Pausen“, „öfters Förderunterricht“ oder dass es einfacher sei, „wenn mich meine Mitschüler nicht immer ärgern würden“, „wenn die Lehrerin oder meine Mama neben mir sitzt“ oder „wenn es die Kurse nicht gäbe“. Am Unterricht könne man verbessern, dass es ruhiger sei und die Lehrer, falls notwendig, Sachverhalte mehrmals erklären: „dass es die Lehrer so oft wie möglich erklären können, wenn es einer noch nicht verstanden hat und er sollte sich dann nicht beschweren“. Weitere Wünsche sind differenzierte Lernangebote, mehr Fairness im Umgang mit Schülern, weniger Hausaufgaben bei manchen Lehrern und dass man mehr spielt.

Auf die Frage, die sich auf die Konsequenz der Lehrkräfte bezüglich Fehlverhalten bezieht, antwortet nur ein geringer Teil der SL, RSL und FSL, dass diese weniger konsequent reagieren würden. Jeweils etwa zwei Dritte der Befragten gehen von einer konsequenten Vorgehensweise aus. 
Tab. 97: Wie konsequent reagieren die Lehrkräfte auf Fehlverhalten?

\begin{tabular}{lccc}
\hline & SL $(\mathbf{n}=\mathbf{3 1})$ & RSL $(\mathbf{n}=53)$ & FSL $(\mathbf{n}=\mathbf{3 8})$ \\
\hline sehr konsequent & $19,4 \%$ & $37,7 \%$ & $10,5 \%$ \\
konsequent & $67,7 \%$ & $58,5 \%$ & $65,8 \%$ \\
weniger konsequent & $6,5 \%$ & $1,9 \%$ & $7,9 \%$ \\
gar nicht konsequent & $0 \%$ & $0 \%$ & $0 \%$ \\
\hline
\end{tabular}

Bei der Sicherheit im Umgang mit Verhaltensauffälligkeiten gehen die Meinungen der Befragten sehr weit auseinander, wie folgendes Diagramm veranschaulicht:

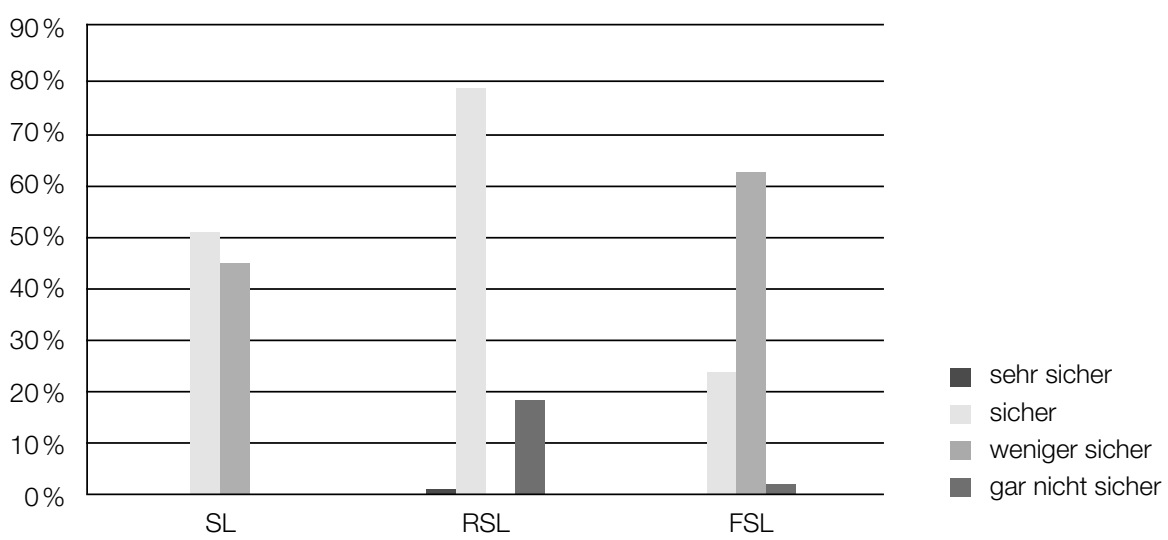

Abb. 15: Sicherheit der RSL im Umgang mit Verhaltensauffälligkeiten

Während die RSL selbst sich hinsichtlich dieser Fragestellung vorwiegend als sicher bzw. sehr sicher einstufen (81,2\%), schätzen die SL dies vorsichtiger ein (51,6\%). Die FSL gehen zu einem überwiegenden Teil davon aus, dass diese Sicherheit eher nicht vorliegt (63,2\%).

Tab. 98: Wie sicher fühlen sich die Lehrkräfte im Umgang mit Verhaltensauffälligkeiten von $\mathrm{SuS} ?^{\text {68 }}$

\begin{tabular}{lccc}
\hline & SL $(\mathbf{n}=\mathbf{3 1})$ & RSL $(\mathbf{n}=\mathbf{5 3})$ & FSL $(\mathbf{n}=\mathbf{3 8})$ \\
\hline sehr sicher & $0 \%$ & $1,9 \%$ & $0 \%$ \\
sicher & $51,6 \%$ & $79,2 \%$ & $24,2 \%$ \\
weniger sicher & $45,2 \%$ & $18,9 \%$ & $63,2 \%$ \\
gar nicht sicher & $0 \%$ & $0 \%$ & $2,6 \%$ \\
\hline
\end{tabular}

Das Maß an klarer Strukturierung des Unterrichts wird von den RSL etwas positiver eingeschätzt als von den FSL.

68 An dieser Stelle wird diese Sicherheit im Zusammenhang mit dem Thema Klassenführung erfasst. Sie lässt sich allerdings auch dem Qualitätsbaustein Professsionalisierung zuordnen. 
Tab. 99: Wie hoch ist das Maß an klarer Strukturierung des Unterrichts?

\begin{tabular}{lcc}
\hline & RSL $(\mathbf{n}=\mathbf{5 3})$ & FSL $(\mathbf{n}=\mathbf{3 8})$ \\
\hline sehr hoch & $22,6 \%$ & $7,9 \%$ \\
hoch & $64,2 \%$ & $52,6 \%$ \\
weniger hoch & $1,9 \%$ & $13,2 \%$ \\
gar nicht hoch & $0 \%$ & $0 \%$ \\
\hline
\end{tabular}

\subsubsection{Methodik}

Mit Blick auf die unterschiedlichen Sozialformen zeigt das Antwortverhalten der Befragten, dass der Frontalunterricht eine sehr häufige bis häufige Unterrichtsform in den integrativ arbeitenden Schulen ist. Insbesondere die SuS, die zu 63,6\% angeben, dass Frontalunterricht sehr häufig erfolge, nehmen dies offensichtlich so wahr.

Tab. 100: Zu welchen Anteilen wird Frontalunterricht umgesetzt?

\begin{tabular}{lcccc}
\hline & SL (n=31) & RSL (n= 53) & FSL (n=38) & S (n=33) \\
\hline sehr häufig & $25,8 \%$ & $20,8 \%$ & $23,7 \%$ & $63,6 \%$ \\
häufig & $54,8 \%$ & $58,5 \%$ & $60,5 \%$ & $36,4 \%$ \\
eher selten & $6,5 \%$ & $18,9 \%$ & $13,2 \%$ & $0 \%$ \\
gar nicht & $0 \%$ & $1,9 \%$ & $0 \%$ & $0 \%$ \\
\hline
\end{tabular}

Bis auf einen Schüler geben alle SuS mit spF an, dass sie es für sehr gut bzw. gut halten, wenn der Lehrer vorne steht und etwas erklärt. Fast alle SuS (84,9\%) vertreten darüber hinaus die Auffassung, dass sie die Ausführungen der Lehrkraft sehr gut bzw. gut verstehen.

Tab. 101: Wenn der Lehrer vorne steht und erklärt etwas, finde ich das... (S, n=33)

\begin{tabular}{lr}
\hline sehr gut & $54,5 \%$ \\
gut & $42,4 \%$ \\
weniger gut & $3,0 \%$ \\
gar nicht gut & $\mathbf{0 \%}$ \\
\hline
\end{tabular}

Tab. 102: Was die Lehrer erklären, verstehe ich meistens... $(S, n=33)$

\begin{tabular}{lr}
\hline sehr gut & $18,2 \%$ \\
gut & $66,7 \%$ \\
weniger gut & $6,1 \%$ \\
gar nicht gut & $0 \%$ \\
\hline
\end{tabular}

Insgesamt formulieren die Schülerinnen und Schüler 19 qualitative Begründungen dafür, dass sie den Frontalunterricht als positiv bewerten. Dabei beziehen sich elf Aussagen darauf, dass man Dinge dann besser verstehe, zwei darauf, dass man etwas erklärt bekomme. Zwei SuS schreiben, dass sie dadurch lernen können und weitere zwei, dass sie dann wissen, was zu tun sei. Für zwei SuS ist es hilfreich die Lehrkraft gut sehen zu können. Nur ein Schüler bewertet den frontalen Unterricht als weniger gut, da er Zeit vom Unterricht koste. 
Die Aussage: „Was die Lehrer/innen erklären, verstehe ich meistens...“ wird von 17 SuS ergänzt. Sechs SuS betonen, dass die Lehrkräfte gut erklären können und ein weiterer formuliert, dass „man vieles genauer dadurch weiß“. Ein Schüler hält es für günstig, dass die Lehrkraft Dinge ein paar Mal erklärt. Zwei SuS verstehen dies gut, da sie gut zuhören, ein weiterer, weil er weit vorne sitzt. Ein Schüler hebt hervor, dass er nach den Erklärungen die Hausaufgaben machen könne. Ein Schüler versteht die Erklärungen, die Aufgaben würden ihm aber in den Arbeiten dennoch schwer fallen. Drei SuS erwähnen, dass sei die Erklärungen mal gut, mal weniger gut verstehen könnten. Ein weiterer versteht die Erklärungen nicht gut, da andere SuS zu laut seien.

Hinsichtlich der Sozialformen geben die SuS an, dass sie im Unterricht am häufigsten in Einzelarbeit tätig sind, gefolgt von Partner- und Gruppenarbeit. Die Wahrnehmung der Anteile von Einzelarbeit im Vergleich zu anderen Arbeitsformen unterscheidet sich dabei zwischen den SuS sowie den RSL und FSL dahingehend, dass die Lehrkräfte jeweils zu zwei Dritteln angeben, dass sie Einzelarbeit häufig einsetzen, während die SuS dies nur zu etwas mehr als $40 \%$ so realisieren.

Tab. 103: Einzelarbeit

\begin{tabular}{lccc}
\hline & RSL $(\mathbf{n}=\mathbf{5 3})$ & FSL $(\mathbf{n}=\mathbf{3 8})$ & $\mathbf{S ~ ( \mathbf { n } = 3 3 )}$ \\
\hline sehr häufig & $26,4 \%$ & $26,3 \%$ & $18,2 \%$ \\
häufig & $62,3 \%$ & $63,2 \%$ & $42,4 \%$ \\
eher selten & $9,4 \%$ & $10,5 \%$ & $27,3 \%$ \\
gar nicht & $0 \%$ & $0 \%$ & $9,1 \%$ \\
\hline
\end{tabular}

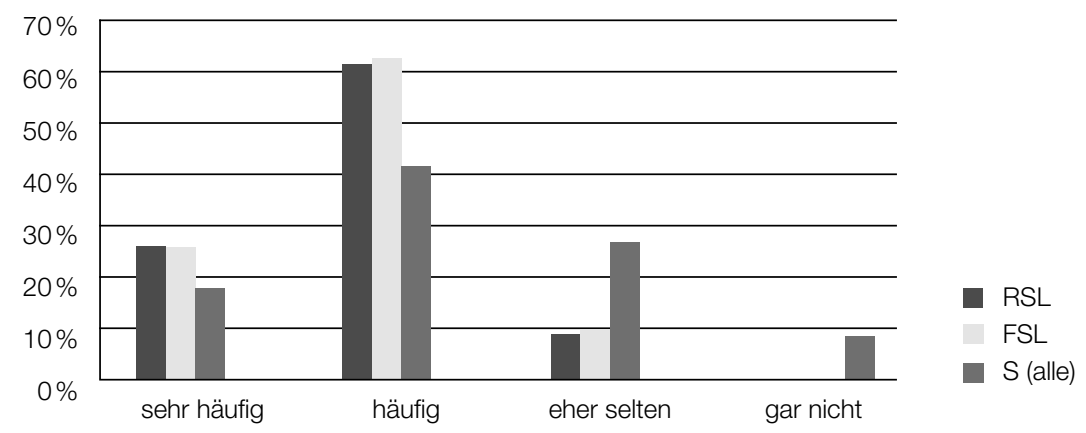

Abb. 16: Anteil Einzelarbeit

Die Einzelarbeit wird von den Schülerinnen zu gleichen Anteilen (jeweils 18,2\%) als sehr gut bzw. weniger gut bewertet. Mehr als die Hälfte bezeichnet diese Sozialform als gut.

Tab. 104: Wenn ich alleine an einer Aufgabe arbeite, finde ich das ...

\begin{tabular}{lc}
\hline & $\mathbf{S}(\mathbf{n}=\mathbf{3 3})$ \\
\hline sehr gut & $18,2 \%$ \\
gut & $51,5 \%$ \\
weniger gut & $18,2 \%$ \\
gar nicht gut & $6,1 \%$ \\
\hline
\end{tabular}


$20 \mathrm{SuS}$ begründen die gute oder weniger gute Bewertung der Einzelarbeit als Sozialform. Sie wird von acht $\mathrm{SuS}$ deshalb positiv bewertet, da sie sich dabei besser konzentrieren können. Weitere Aussagen beziehen sich darauf, dass man bei der Einzelarbeit nicht gestört werden würde, das Arbeiten Spaß mache oder es einfacher sei. Sieben SuS begründen ihre weniger gute Einschätzung damit, dass sie die Aufgaben alleine nicht immer verstehen (4), es alleine nicht können, es ihnen dann schwerer falle oder sie manchmal Hilfe brauchen bzw. nichts lernen können, weil sie keine Hilfe bekommen. Ein Schüler/eine Schülerin bewertet die Einzelarbeit als gar nicht gut, weil er/sie lieber mit jemand anderem zusammen arbeitet.

Die Sozialform Partnerarbeit wird nach Einschätzung der Regelschullehrkräfte zu knapp $80 \%$ häufig bis sehr häufig eingesetzt, während die Förderschullehrkräfte und Schüler diesen Anteil geringer einschätzen, indem sie zu 36,8\% bzw. 45,5\% angeben, dass diese eher selten erfolge.

Tab. 105: Partnerarbeit

\begin{tabular}{lccc}
\hline & RSL $(\mathbf{n}=\mathbf{5 3})$ & FSL $(\mathbf{n}=\mathbf{3 8})$ & $\mathbf{S ~ ( \mathbf { n } = 3 3 )}$ \\
\hline sehr häufig & $13,2 \%$ & $10,5 \%$ & $21,2 \%$ \\
häufig & $66,0 \%$ & $47,4 \%$ & $33,3 \%$ \\
eher selten & $18,9 \%$ & $36,8 \%$ & $45,5 \%$ \\
gar nicht & $0 \%$ & $0 \%$ & $0 \%$ \\
\hline
\end{tabular}

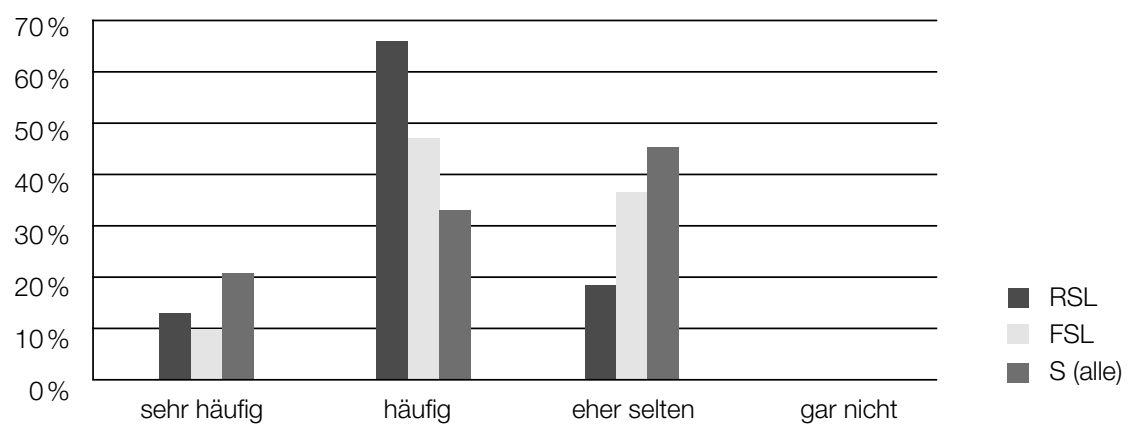

Abb. 17: Anteil Partnerarbeit

Ähnlich wie die Einzelarbeit wird auch die Partnerarbeit vom Großteil der Schülerinnen und Schüler als gut bzw. sehr gut bewertet.

Tab. 106: Wenn ich mit einem Partner an einer Aufgabe arbeite, finde ich das ...

\begin{tabular}{lr}
\hline & $\mathbf{S}(\mathbf{n}=\mathbf{3 3})$ \\
\hline sehr gut & $33,3 \%$ \\
gut & $54,5 \%$ \\
weniger gut & $6,1 \%$ \\
gar nicht gut & $0 \%$ \\
\hline
\end{tabular}


Zur Partnerarbeit als Sozialform äußern sich 21 SuS, wobei sich nur drei Aussagen auf eine kritische Bewertung beziehen, da die $\mathrm{SuS}$ sich dabei nicht so gut konzentrieren können, sie das nur manchmal gerne machen oder sie als nicht hilfreich empfinden. Die anderen $18 \mathrm{SuS}$ begründen ihre positive Bewertung damit, dass man Aufgaben zusammen lösen (4) oder sich gegenseitig helfen könne (7) (Bsp.: „sehr gut, weil mein Partner das erklärt, was ich nicht verstehe“ oder „gut, weil dann jeder Tipps geben kann“). Manche SuS halten die Partnerarbeit für gut, weil sie Spaß mache, weil sie gerne mit jemand anderem zusammenarbeiten oder „mit einem Partner über ein Thema reden“, man sich die Aufgaben aufteilen könne oder weil es einfacher sei.

Die Sozialform Gruppenarbeit wird nach Angaben aller Befragten im Vergleich seltener genutzt als Einzel- oder Partnerarbeit. Wieder sind es die Schülerinnen und Schüler, die diese Arbeitsform zu einem größeren Anteil als die RSL bzw. FSL als eher selten (48,5\%) oder gar nicht $(24,2 \%)$ wahrnehmen.

Tab. 107: Gruppenarbeit

\begin{tabular}{lccc}
\hline & RSL $(\mathbf{n}=\mathbf{5 3})$ & FSL $(\mathbf{n}=\mathbf{3 8})$ & $\mathbf{S ~ ( \mathbf { n } = 3 3 )}$ \\
\hline sehr häufig & $0 \%$ & $2,6 \%$ & $3,0 \%$ \\
häufig & $47,2 \%$ & $31,6 \%$ & $18,2 \%$ \\
eher selten & $45,3 \%$ & $60,5 \%$ & $48,5 \%$ \\
gar nicht & $5,7 \%$ & $0 \%$ & $24,2 \%$ \\
\hline
\end{tabular}

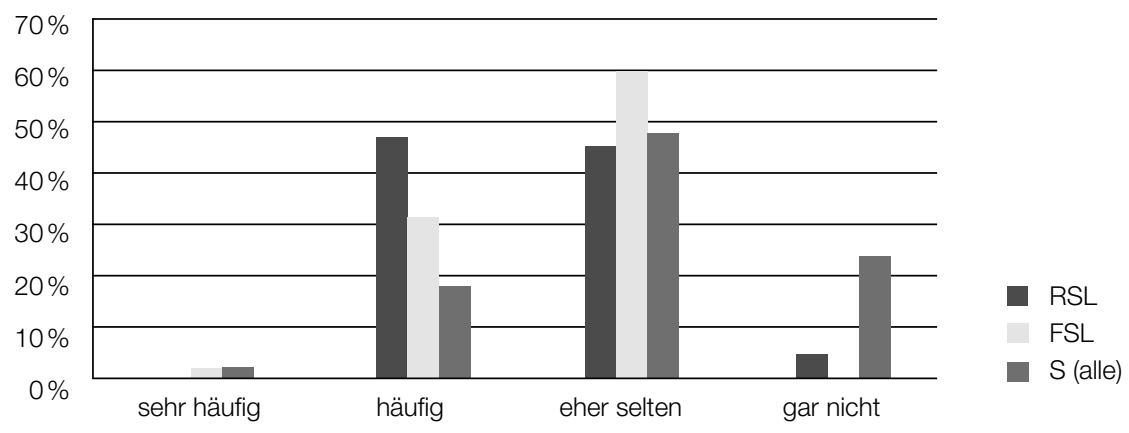

Abb. 18: Anteil Gruppenarbeit

Die Gruppenarbeit als Sozialform wird von den SuS ähnlich gut bewertet wie die Einzelarbeit.

Tab. 108: Wenn ich in einer Gruppe arbeite, finde ich das ...

\begin{tabular}{lr}
\hline & $\mathbf{S}(\mathbf{n}=33)$ \\
\hline sehr gut & $21,2 \%$ \\
gut & $45,5 \%$ \\
weniger gut & $12,1 \%$ \\
gar nicht gut & $9,1 \%$ \\
\hline
\end{tabular}


16 von $20 \mathrm{SuS}$ beschreiben die Gruppenarbeit als positiv, weil sie abwechslungsreich sei, man dabei zusammen Aufgaben bearbeiten könne, man sich untereinander helfen oder Vorschläge machen könne (5), man nicht alleine arbeiten müsse, die Wahrscheinlichkeit hoch sei einen Freund in der Gruppe zu haben, es Spaß mache oder schön sei (5) bzw. es einfacher sei. Vier kritische Anmerkungen beziehen sich darauf, dass man sich bei der Gruppenarbeit nicht gut konzentrieren könne, es zu laut sei, die anderen „fast immer Quatsch machen“ oder „es manchmal Streitereien in der Gruppe geben kann“.

Folgende Tabelle und Grafik veranschaulichen auf einen Blick die Bewertung der unterschiedlichen Sozialformen durch die SuS mit spF:

Tab. 109: Bewertung von Sozialformen durch $\operatorname{SuS}(\mathrm{n}=33)$

\begin{tabular}{lccc}
\hline & Einzelarbeit & Partnerarbeit & Gruppenarbeit \\
\hline sehr gut & $18,20 \%$ & $33,30 \%$ & $21,20 \%$ \\
gut & $51,50 \%$ & $54,50 \%$ & $45,50 \%$ \\
weniger gut & $18,20 \%$ & $6,10 \%$ & $12,10 \%$ \\
gar nicht gut & $6,10 \%$ & $0 \%$ & $9,10 \%$ \\
\hline
\end{tabular}

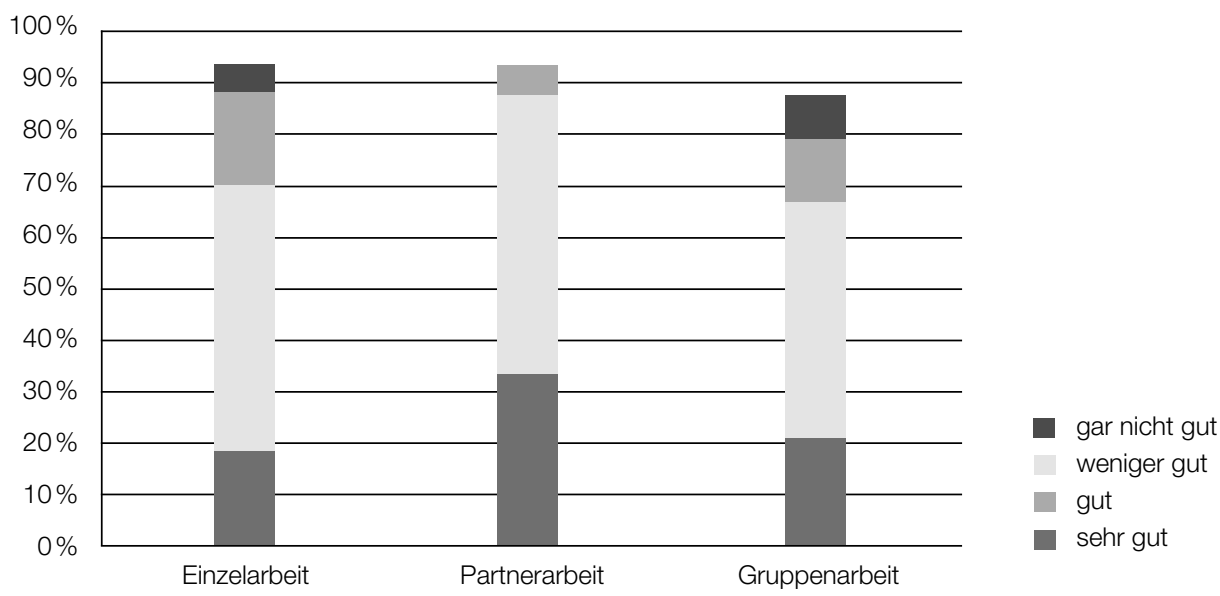

Abb. 19: Bewertung Sozialformen durch SuS mit spF

Am positivsten bewertet wird von den SuS die Partnerarbeit, für die 87,8\% angeben, dass sie diese als gut bzw. sehr gut einstufen. Bei der Bewertung von Einzel- bzw. Gruppenarbeit zeigen sich kaum Unterschiede.

Auf die Frage, zu welchen Anteilen offene Unterrichtsformen umgesetzt werden, ergibt sich zum Teil ein stark voneinander abweichendes Antwortverhalten von RSL und FSL. Während die RSL zu etwa $45 \%$ davon ausgehen, dass Stationenarbeit eine häufige bzw. sehr häufige Arbeitsform darstelle, geben die FSL zu über $76 \%$ an, dass diese eher selten oder gar nicht erfolge. Hinsichtlich der Nutzung von Lerntheken gehen sowohl die RSL (60,4\%) sowie die FSL $(68,4 \%)$ davon aus, dass diese eher selten bzw. gar nicht gegeben sei. Wochenpläne werden von über $60 \%$ der RSL sowie von knapp $50 \%$ der FSL eher selten oder gar nicht eingesetzt. 
Tab. 110: Anteile Sozialformen am Unterricht

\begin{tabular}{lcccccc}
\hline & \multicolumn{2}{c}{ Stationenarbeit } & \multicolumn{2}{c}{ Lerntheke } & \multicolumn{2}{c}{ Wochenplan } \\
& RSL & FSL & RSL & FSL & RSL & FSL \\
& $(\mathbf{n = 5 3 )}$ & $(\mathbf{n = 3 8 )}$ & $(\mathbf{n}=\mathbf{5 3})$ & $(\mathbf{n}=\mathbf{3 8})$ & $(\mathbf{n}=\mathbf{5 3})$ & $(\mathbf{n}=\mathbf{3 8})$ \\
\hline sehr häufig & $5,7 \%$ & $5,3 \%$ & $11,3 \%$ & $2,6 \%$ & $13,2 \%$ & $18,4 \%$ \\
häufig & $39,6 \%$ & $10,5 \%$ & $20,8 \%$ & $18,4 \%$ & $22,6 \%$ & $28,9 \%$ \\
eher selten & $39,6 \%$ & $71,1 \%$ & $34,0 \%$ & $50,0 \%$ & $39,6 \%$ & $26,3 \%$ \\
gar nicht & $15,1 \%$ & $5,3 \%$ & $26,4 \%$ & $18,4 \%$ & $22,6 \%$ & $18,4 \%$ \\
\hline
\end{tabular}

Drei der Befragten gehen davon aus, dass die Förderung verbessert werden könnte, „wenn der Unterricht nicht so stark frontal ausgerichtet ist“ oder offener und freier gestaltet wäre (2).

\subsubsection{Differenzierung und Individualisierung}

\section{Anteile binnendifferenzierten Unterrichts}

Hinsichtlich der Umsetzung binnendifferenzierten Unterrichts unterscheiden sich die Angaben der SL und RSL zum Teil erheblich von denen der FSL bzw. SuS.

Tab. 111: Zu welchen Anteilen wird binnendifferenzierter Unterricht umgesetzt?

\begin{tabular}{lcccrrr}
\hline & SL $(\mathbf{n}=31)$ & \multicolumn{2}{c}{ RSL $(\mathbf{n}=\mathbf{5 3})$} & \multicolumn{2}{c}{ FSL $(\mathbf{n}=\mathbf{3 8})$} & S (n=33) \\
& insgesamt & quant. & \multicolumn{1}{c}{ qual. } & quant. & qual. & \\
\hline sehr häufig & $9,7 \%$ & $24,5 \%$ & $9,4 \%$ & $7,9 \%$ & $7,9 \%$ & $12,1 \%$ \\
häufig & $51,6 \%$ & $52,8 \%$ & $47,2 \%$ & $36,8 \%$ & $28,9 \%$ & $36,4 \%$ \\
eher selten & $25,8 \%$ & $17,0 \%$ & $35,8 \%$ & $47,4 \%$ & $50,0 \%$ & $30,3 \%$ \\
gar nicht & $0 \%$ & $1,9 \%$ & $3,8 \%$ & $2,6 \%$ & $7,9 \%$ & $21,2 \%$ \\
\hline
\end{tabular}

\section{Differenzierte Materialien für Schüler mit sonderpädagogischem Förderbedarf ${ }^{69}$}

Mehr als 40 \% bzw. über die Hälfte der RSL und FSL geben an, dass zieldifferent unterrichtete $\mathrm{SuS}$ mit spF im Fach Deutsch die gleichen Materialien benutzen wie die Regelschüler. Mit Blick auf das Fach Mathematik wird dieser Anteil von Seiten der RSL mit 24,5\% geringer angegeben, während er bei den FSL mit dem Fach Deutsch übereinstimmt. Bei den Angaben zu differenzierten Materialien bzw. Klassenarbeiten, die für diese Schüler in Deutsch bzw. Mathematik eingesetzt werden, weichen die Angaben zum Teil erheblich voneinander ab.

Tab. 112: Deutsch - Materialien SuS (zieldfifferent) wie andere SuS?

\begin{tabular}{lcc}
\hline & RSL $(\mathbf{n}=\mathbf{5 3})$ & FSL $(\mathbf{n}=\mathbf{3 8})$ \\
\hline ja & $41,5 \%$ & $55,3 \%$ \\
nein & $58,5 \%$ & $44,7 \%$ \\
\hline
\end{tabular}

69 Im Fragebogen wurde diese Frage im Zusammenhang mit der Thematik der Differenzierung gestellt, nicht im Zusammenhang mit den vorhandenen Ressourcen in den Klassen. Das hängt damit zusammen, dass erfasst werden sollte, inwiefern die SuS in den Kernfächern tatsächlich mit differenzierten Materialien arbeiten. 
Tab. 113: Andere Materialien etc. im Deutschunterricht

\begin{tabular}{lcccccccc}
\hline & \multicolumn{2}{c}{ andere Bücher } & \multicolumn{2}{c}{ andere Arbeitshefte } & \multicolumn{2}{c}{ andere } & \multicolumn{2}{c}{ andere } \\
& & & & & \multicolumn{2}{c}{ Arbeitsblätter } & \multicolumn{2}{c}{ Klassenarbeiten } \\
& RSL & FSL & RSL & FSL & RSL & FSL & RSL & FSL \\
& $(\mathbf{n}=\mathbf{5 3})$ & $(\mathbf{n}=\mathbf{3 8})$ & $(\mathbf{n}=\mathbf{5 3})$ & $(\mathbf{n}=\mathbf{3 8})$ & $(\mathbf{n}=\mathbf{5 3})$ & $(\mathbf{n}=\mathbf{3 8})$ & $(\mathbf{n}=\mathbf{5 3})$ & $(\mathbf{n}=\mathbf{3 8})$ \\
\hline ja & $24,5 \%$ & $68,4 \%$ & $32,1 \%$ & $78,9 \%$ & $50,9 \%$ & $81,6 \%$ & $47,2 \%$ & $92,1 \%$ \\
nein & $75,5 \%$ & $31,6 \%$ & $67,9 \%$ & $21,1 \%$ & $49,1 \%$ & $18,4 \%$ & $52,8 \%$ & $7,9 \%$ \\
\hline
\end{tabular}

Tab. 114: Mathe - Materialien SuS (zieldfifferent) wie andere SuS?

\begin{tabular}{lcc}
\hline & RSL $(\mathbf{n}=\mathbf{5 3})$ & FSL $(\mathbf{n}=\mathbf{3 8})$ \\
\hline $\mathrm{ja}$ & $24,5 \%$ & $55,3 \%$ \\
nein & $75,5 \%$ & $44,7 \%$ \\
\hline
\end{tabular}

Tab. 115: Andere Materialien etc. im Mathematikunterricht

\begin{tabular}{lcccccccc}
\hline & \multicolumn{2}{c}{ andere Bücher } & \multicolumn{2}{c}{ andere Arbeitshefte } & \multicolumn{2}{c}{ andere } & \multicolumn{2}{c}{ andere } \\
& & & & & Arbeitsblätter & \multicolumn{2}{c}{ Klassenarbeiten } \\
& RSL & FSL & RSL & FSL & RSL & FSL & RSL & FSL \\
& $(\mathbf{n}=\mathbf{5 3})$ & $(\mathbf{n}=\mathbf{3 8})$ & $(\mathbf{n}=\mathbf{5 3})$ & $(\mathbf{n}=\mathbf{3 8})$ & $(\mathbf{n}=\mathbf{5 3})$ & $(\mathbf{n}=\mathbf{3 8})$ & $(\mathbf{n}=\mathbf{5 3})$ & $(\mathbf{n}=\mathbf{3 8})$ \\
\hline ja & $26,4 \%$ & $71,1 \%$ & $37,7 \%$ & $81,6 \%$ & $52,8 \%$ & $86,8 \%$ & $52,8 \%$ & $86,8 \%$ \\
nein & $73,6 \%$ & $28,9 \%$ & $62,3 \%$ & $18,4 \%$ & $47,2 \%$ & $13,2 \%$ & $47,2 \%$ & $13,2 \%$ \\
\hline
\end{tabular}

Die Auffassung, dass Differenzierung zu einer Verbesserung der Förderung beitragen kann, wird von acht Personen vertreten, die eine Außendifferenzierung fordern (2), wünschen, dass „zieldifferent unterrichtete Schüler ebenfalls in den Nebenfächern ein ihnen angepasstes Lernangebot erhalten“, „öfter von der Möglichkeit der Differenzierung (auch Nachteilsausgleich) Gebrauch gemacht würde“, „individuell besser und intensiver auf die Integrationsschüler eingegangen wird und zwar nicht nur durch die Förderschullehrer - vielmehr im regulären Klassenlehrer-Unterricht“, „individueller auf die Förderbedürfnisse eingegangen wird“ oder „,ür die Förderschüler mit Förderbedarf geistige Entwicklung noch ein individuelleres Tageskonzept entwickelt“ wird. Eine Person wünscht sich, dass die Schüler mit spF „nicht aus dem Unterricht geholt werden oder im Unterricht durch die Interaktion von Lehrer und Förderlehrer nicht gestört werden. Es wäre gut, den Schulmorgen in Ruhe zu erleben und danach erst in zusätzliche Förderung zu kommen.", eine weitere spricht sich für differenzierte Hausaufgaben aus.

Für die Zukunft äußern die Lehrkräfte und Schulleitungen für den Bereich der Unterrichtsentwicklung eine Fülle unterschiedlicher Wünsche. Dazu gehört: die Abschaffung der Noten bis mindestens zur 6. Klasse, mehr Zeit für die Schüler (3) bzw. für die Vermittlung sozialer Kompetenzen, Möglichkeiten den Lehrplan zu vernachlässigen und die Anzahl der Klassenarbeiten zu reduzieren. Die Lehrkräfte möchten gerne handlungsorientiert und fächerübergreifend arbeiten, offenere Unterrichtsformen einsetzen und sich verstärkt um spezifische Förderung der $\mathrm{SuS}$ mit spF kümmern können. Sie möchten die Kompetenzen ihrer SuS gerne umfassend und nicht nur fachlich fördern, auch starken Schülern besondere Angebote machen können, einen 
„unverkrampften Blick auf die Persönlichkeit des betroffenen Schülers [bewahren] um die beste Möglichkeit für diesen jungen Menschen zu finden“ und das Augenmerk möglichst individuell auf die Schüler legen können.

Kritische Anmerkungen mit Blick auf den Unterricht beziehen sich auf die „Betreuung von Kindern mit massiven Beeinträchtigungen“, bei denen die inklusive Beschulung eine Einzelfallentscheidung bleiben solle, darauf, „dass nur solche Schüler integriert werden, die davon wirklich profitieren und dem Unterricht auch folgen können (wenn auch mit etwas Hilfe), Bsp.: Seh-, Gehörgeschädigte, Rollstuhlfahrer,...; problematisch sehe ich geistig Behinderte..." und darauf, dass schwer verhaltensauffällige Kinder die Klassen nicht zu lange belasten sollten. Eine Lehrkraft formuliert vorausblickend:

Da ich nach diesem Schuljahr in Pension sein werde, betrifft mich dieses Thema nicht mehr. Meine jungen Kolleginnen u. Kollegen beneide ich allerdings nicht. Wenn zu all den schwierigen Schülern, die wir seit über 20 Jahren an unserer Schule wie selbstverständlich unterrichten, noch Kinder mit körperlicher oder geistiger Behinderung hinzukommen, dann müssten die Kollegen/innen als Supermänner/bzw. -frauen ausgebildet werden.

\section{Unterstützungsempfinden der SuS mit spF mit Blick auf Integrationslehrer}

Als besonders hilfreich scheinen $\mathrm{SuS}$ mit spF im Rahmen der Unterstützung durch Förderschullehkräfte wahrzunehmen, dass die Sachverhalte noch einmal erklärt bekommen (75,8\%), Fragen stellen (63,6\%) bzw. Dinge noch einmal üben können (60,6\%).

Tab. 116: Unterstützung der SuS mit spF durch I-Lehrer $(S, n=33)$

\begin{tabular}{lccccc}
\hline & $\begin{array}{c}\text { ich erhalte } \\
\text { Unterstützung }\end{array}$ & $\begin{array}{c}\text { Hilfe dadurch, } \\
\text { dass ich alleine } \\
\text { mit jemandem } \\
\text { arbeiten kann }\end{array}$ & $\begin{array}{c}\text { Hilfe dadurch, } \\
\text { dass ich Fragen } \\
\text { stellen kann }\end{array}$ & $\begin{array}{c}\text { Hilfe dadurch, } \\
\text { dass ich Dinge } \\
\text { noch einmal er- } \\
\text { klärt bekomme }\end{array}$ & $\begin{array}{c}\text { Hilfe dadurch, } \\
\text { dass ich noch } \\
\text { einmal Sachen } \\
\text { üben kann }\end{array}$ \\
\hline ja & $93,9 \%$ & $33,3 \%$ & $63,6 \%$ & $75,8 \%$ & $60,6 \%$ \\
nein & & $66,7 \%$ & $36,4 \%$ & $24,2 \%$ & $39,4 \%$ \\
weiß ich nicht & $6,1 \%$ & & & & \\
\hline
\end{tabular}

Verhältnis zwischen den Schülern mit sonderpädagogischem Förderbedarf und den Förderschullehrkräften

Das Verhältnis zu den Förderschullehrkräften wird von den Schülerinnen und Schülern mit spF als gut bzw. sehr gut gekennzeichnet.

Tab. 117: Mit dem Integrationslehrer verstehe ich mich... $(S, n=33)$

\begin{tabular}{ll}
\hline sehr gut & $72,7 \%$ \\
gut & $27,3 \%$ \\
\hline
\end{tabular}

Organisatorische Umsetzung der sonderpädagogischen Förderung Hinsichtlich der organisatorischen Umsetzung der sonderpädagogischen Förderung sollten im Fragebogen verschiedene Aussagen bewertet werden. Während die Schulleitungen zu 64,5\% und die SuS mit spF zu 58,3\% der Aussage, dass die Kinder mit spF immer innerhalb der Klasse 
gefördert werden sollten sehr oder eher zustimmen, sehen dies nur 26,4\% der RSL, 31,5\% der FSL bzw. 44,1\% der Eltern genauso.

Tab. 118: Die Kinder mit Förderbedarf sollten immer innerhalb der Klasse gefördert werden

\begin{tabular}{lccccc}
\hline & SL (n=31) & RSL (n= 53) & FSL (n=38) & E (n=34) & S GMS (n= 12) \\
\hline stimme sehr zu & $12,9 \%$ & $3,8 \%$ & $2,6 \%$ & $14,7 \%$ & $33,3 \%$ \\
stimme eher zu & $51,6 \%$ & $22,6 \%$ & $28,9 \%$ & $29,4 \%$ & $25,0 \%$ \\
stimme eher nicht zu & $29,9 \%$ & $50,9 \%$ & $42,1 \%$ & $41,2 \%$ & $33,3 \%$ \\
stimme gar nicht zu & $0 \%$ & $20,8 \%$ & $18,4 \%$ & $8,8 \%$ & $8,3 \%$ \\
fehlend & $6,5 \%$ & $1,9 \%$ & $7,9 \%$ & $5,9 \%$ & $0 \%$ \\
\hline
\end{tabular}

Tab. 119: Meistens lernen die I-SuS mit dem I-Lehrer ...

\begin{tabular}{lcccc}
\hline & SL $(\mathbf{n}=\mathbf{3 1})$ & RSL $(\mathbf{n}=\mathbf{5 3})$ & FSL $(\mathbf{n}=\mathbf{3 8})$ & $\mathbf{S}(\mathbf{n}=\mathbf{3 3})$ \\
\hline innerhalb der Klasse & $35,5 \%$ & $24,5 \%$ & $10,5 \%$ & $9,1 \%$ \\
außerhalb der Klasse & $41,9 \%$ & $41,5 \%$ & $13,2 \%$ & $60,6 \%$ \\
das ist unterschiedlich & $0 \%$ & $34,0 \%$ & $36,8 \%$ & $30,3 \%$ \\
kann ich nicht sagen & $6,5 \%$ & $0 \%$ & $0 \%$ & $0 \%$ \\
fehlend & $16,1 \%$ & $0 \%$ & $39,5 \%$ & $0 \%$ \\
\hline
\end{tabular}

Tab. 120: Wenn die SuS mit spF außerhalb der Klasse gefördert werden, erfolgt diese Förderung meistens ...

\begin{tabular}{lcccc}
\hline & SL $(\mathbf{n}=\mathbf{3 1})$ & RSL $(\mathbf{n}=\mathbf{5 3})$ & FSL $(\mathbf{n}=\mathbf{3 8})$ & $\mathbf{S ~ ( \mathbf { n } = 3 3 )}$ \\
\hline als Einzelförderung & $35,5 \%$ & $28,3 \%$ & $10,5 \%$ & $30,3 \%$ \\
als Gruppenförderung & $38,7 \%$ & $22,6 \%$ & $26,3 \%$ & $30,3 \%$ \\
das ist unterschiedlich & $0 \%$ & $41,5 \%$ & $63,2 \%$ & $39,4 \%$ \\
fehlend & $25,8 \%$ & $7,5 \%$ & $0 \%$ & $0 \%$ \\
\hline
\end{tabular}

Ein sehr hohes Maß an Zustimmung findet die Aussage, dass die Förderung von SuS mit spF bei Bedarf individuell erfolgen solle, vor allem von Seiten der RSL, der FSL sowie der Eltern (zwischen 83,0\% und 76,5\%, während die SL dieser Aussage zu 64,5\% und die SuS selbst nur zu $50 \%$ zustimmen.

Tab. 121: Bei Bedarf sollten Kinder mit Förderbedarf in einzelnen Stunden individuell gefördert werden

\begin{tabular}{lcccrc}
\hline & SL (n=31) & RSL (n= 53) & FSL (n=38) & E (n=34) & S GMS (n=12) \\
\hline stimme sehr zu & $64,5 \%$ & $83,0 \%$ & $78,9 \%$ & $76,5 \%$ & $50,0 \%$ \\
stimme eher zu & $22,6 \%$ & $13,2 \%$ & $15,8 \%$ & $14,7 \%$ & $33,3 \%$ \\
stimme eher nicht zu & $6,5 \%$ & $3,8 \%$ & $5,3 \%$ & $2,9 \%$ & $16,7 \%$ \\
stimme gar nicht zu & $0 \%$ & $0 \%$ & $0 \%$ & $0 \%$ & $0 \%$ \\
fehlend & $6,5 \%$ & $0 \%$ & $0 \%$ & $5,9 \%$ & $0 \%$ \\
\hline
\end{tabular}


Gleichzeitig bewerten die SuS diese Form der individuellen Förderung außerhalb der Klasse mit 54,5\% ähnlich wie ihre Eltern mit $50 \%$ als sehr gut, während die SL und RSL bei dieser Bewertung weniger positive Angaben machen.

Tab. 122: Ich halte die Form der individuellen Förderung außerhalb der Klasse für...

\begin{tabular}{lccccc}
\hline & SL $(\mathbf{n}=\mathbf{3 1})$ & $\mathbf{R S L}(\mathbf{n}=\mathbf{5 3})$ & FSL $(\mathbf{n}=\mathbf{3 8})$ & $\mathbf{E}(\mathbf{n}=\mathbf{3 4})$ & $\mathbf{S}(\mathbf{n}=\mathbf{3 3})$ \\
\hline sehr gut & $16,1 \%$ & $28,3 \%$ & $28,9 \%$ & $50,0 \%$ & $54,5 \%$ \\
gut & $35,5 \%$ & $49,1 \%$ & $52,6 \%$ & $29,4 \%$ & $39,4 \%$ \\
weniger gut & $25,8 \%$ & $13,2 \%$ & $10,5 \%$ & $5,9 \%$ & $3,0 \%$ \\
gar nicht gut & $3,2 \%$ & & $2,6 \%$ & & \\
\hline
\end{tabular}

\section{Bewertung Unterstützung durch Förderschullehrkräfte - Auffassung der Schüler mit sonderpädagogischem Förderbedarf}

Tab. 123: Unterstützung der SuS mit spF durch FSL (S, n=33)

\begin{tabular}{lcccc}
\hline Fragen stellen & $\begin{array}{c}\text { Dinge noch einmal } \\
\text { erklärt bekommen }\end{array}$ & $\begin{array}{c}\text { alleine mit jnd. } \\
\text { arbeiten }\end{array}$ & $\begin{array}{c}\text { noch einmal } \\
\text { Sachen üben }\end{array}$ \\
\hline ja & $63,6 \%$ & $75,8 \%$ & $33,3 \%$ & $60,6 \%$ \\
nein & $36,4 \%$ & $24,2 \%$ & $66,7 \%$ & $39,4 \%$ \\
\hline
\end{tabular}

Sehr viele befragte Personen geben bezüglich ihrer Bewertung der organisatorischen Umsetzung der integrativen Förderung persönliche Begründungen an. Die Schulleitungen der Förderzentren geben an, dass die Entscheidung für die individuelle Förderung sehr abhängig vom Förderbedarf und von der individuellen Situation des Kindes sei. Als weniger gut wird diese Organisationsform von den SLFZ bzw. anderen SL bewertet, weil integrative Unterrichtung den kooperativen Unterricht mit Regel- und Förderschullehrer beinhalte und „dies der INTEGRATION nicht gerecht" werde bzw. nicht ihren Prinzipien entspreche. Eine andere Schulleitung begründet ihre weniger gute Bewertung damit, dass „alle Kinder von zusätzlichen Personen innerhalb des Unterrichts profitieren sollen“, zwei weitere damit, dass ,im Zuge der Inklusion FöKinder nicht separiert werden sollten“ bzw. dies „einen „Nachhilfeunterricht“ darstellt, der allen Bemühungen von Inklusion zuwider handelt“. Ab und an habe äußere Differenzierung aber ihre Rechtfertigung. Die Auffassungen der Schulleitungen der Grund- und Gemeinschaftsschulen decken ein weites Spektrum ab. Diejenigen, die diese Organisationsform als sehr gut oder gut bewerten, begründen dies damit, dass dadurch spezifisch auf den individuellen Förderbedarf eingegangen werden könne (4) bzw. weil sie nur wenige Stunden betreffe und den Kindern oft viel bringe, „weil die Schüler/innen mehr Zeit, eine kleinere Lernumgebung, eine direkte Zuwendung und eine schnelle Rückmeldung über Erfolg haben" oder weil innerhalb der Schule viele räumliche Möglichkeiten für individuelle Förderung mit spezifischen Materialien gegeben seien. Eine andere Schulleitung verweist darauf, dass die Förderung von Schülern in Kleingruppen in ihrer Schule nicht nur mit Blick auf SuS mit spF selbstverständlich sei.

Eine ganze Reihe der Befragten verweist darauf, dass es sich bei der Förderung außerhalb des Klassenraums um eine individuelle Entscheidung handle. Insgesamt 10 der Befragten erläutern 
in diesem Zusammenhang, dass diese Form der Förderung fallabhängig und flexibel erfolge (5), vom Förderbedarf und von der jeweiligen Situation des Kindes abhänge (3) bzw. von der Zielsetzung, den äußeren Bedingungen, dem Thema, der Methode oder den jeweiligen Problemen eines Schülers. Eine Person weist darauf hin, dass sie diese nur umsetze, wenn es nötig sei, dass sie „lieber im Team und mit Gruppen“ arbeite, eine andere darauf, dass die Mischung stimmen müsse, Einzelzuwendung aber teilweise besser helfen könne. Weitere Befragte äußern, die Förderung außerhalb des Klassenraums solle nicht die Regel sein und eine Mischform der „Förderung in \& außerhalb der Klasse wäre erstrebenswert“. Eine Lehrkraft hält die Form der Förderung für sinnvoll, bezeichnet es aber als schade, „dass immer wieder andere Stunden (BK, SU) verpasst werden“. Eine Person formuliert, dass es außerhalb des Klassenraums besser möglich sei auf Schwächen einzugehen, während man ,innerhalb der Klasse [...] in der Klassengemeinschaft [bleibe]“. Eine Lehrkraft erklärt, dass ihrer Meinung nach „bei speziellen Schülern gemeinsames Lernen mit der Klasse nicht immer möglich ist und eine gelegentliche „Auszeit“ beiden Seiten gut tut.

Weitere 35 Personen (teilweise mit Mehrfachnennungen innerhalb der Aussagen) beschreiben, dass ein großer Vorteil der individuellen Förderung außerhalb des Klassenraums das Mehr an Ruhe im Vergleich zum Klassenraum sei (13), verknüpft mit weniger Störfaktoren (3), weniger Ablenkung (7) oder besserer Konzentration (16). Eine Person führt dies dabei so aus, dass „manche Schüler zeitweise einen ruhigen Arbeitsrahmen benötigen, um sich auf schwierige Aufgaben einlassen zu können“, eine weitere erklärt, dass ,individuelle Unterschiede die Konzentration betreffend eine Förderung außerhalb der Klasse manchmal notwendig machen“. Ein Elternteil argumentiert: „Vor allem mein autistisches Kind lernt in der individuellen Förderung viel besser und effektiver... Ein weiteres Argument lautet, dass ein Schüler sich dann „ganz auf die Förderschullehrkraft konzentrieren“ könne.

Dabei wird nicht nur thematisiert, dass SuS in Einzel- oder Gruppenförderung mehr Ruhe zum Arbeiten hätten, sondern auch, dass es dann für andere SuS der Klasse nicht während des Unterrichts zu Störungen komme (10). Häufig sei es bei individueller Förderung notwendig laut zu sprechen (insgesamt 6) (Bsp.: „Sprach-/Sprechförderung“, Leseübungen, Sprechübungen, Lautieren, laut rechnen, Einführung neuer Themen). Eine Lehrkraft weist darauf hin, dass "gerade beeinträchtigte Schüler intensiv kommunizieren müssen“.

Eine Reihe der Befragten nennt die Vorteile der in der Einzelsituation möglichen Konzentration auf das Kind (5) oder des intensiven Eingehens auf die Kinder (5) als sinnvolle Form von Individualisierung. Während der Förderung außerhalb des Klassenraums hätten die SuS mit spF die Möglichkeit individuellen Kontakt zur Förderschullehrkraft herzustellen, die dann die Möglichkeit habe intensiver mit dem Kind zu arbeiten. Einzel- oder Gruppenförderung werden für sinnvoll gehalten um im Unterricht Einführungen zu bestimmten Sachverhalten vorzunehmen (4), bspw. weil ein „Schüler diese 1:1-Situation benötigt, um für ihn schwierige Sachverhalte erklärt zu bekommen“ oder weil "Schüler mit Förderbedarf Lernen nicht am Stoff der Regelschüler arbeiten und eigens Instruktionen benötigen“. Unterrichtsinhalte könnten gezielt noch einmal aufgegriffen und aufgearbeitet werden (4). Bei dieser Form der Förderung habe man mehr Zeit für Nachfragen, zusätzliche Erklärungen (3), vertiefende Wiederholungen oder Übungen. Es könne eine individuelle Förderung (insgesamt 23) nach Lernstand, Lerntempo (3) oder orientiert an den Bedürfnissen der einzelnen Schüler (6) erfolgen. Dies spiele insbesondere bei SuS eine Rolle, die zieldifferent unterrichtet werden, wobei eine Lehrkraft argumentiert, dass diese Kinder in der Klasse untergingen. Im Rahmen der Einzelförderung könne individueller und damit gezielter auf Probleme eingegangen werden (4), „spezifisch auf den individuellen 
Förderbedarf“ oder „speziell auf die Schwächen der Schüler“ und man könne „Arbeitsweisen und Denkweisen“ der SuS besser erkennen sowie kleinschrittiger und strukturierter vorgehen. Besonderes Material könne außerhalb der Klasse besser besprochen werden oder es könne eine spezifische Förderung im Discemotorikraum erfolgen.

Eine ganze Reihe der Befragten bewertet die individuelle Förderung außerhalb des Klassenraums als positiv, da sie davon ausgehen, dass diese die Effizienz der Förderung positiv beeinflusse. So nehmen sie an, dass die „Lernfortschritte effektiver sind“, „sehr gezieltes, effizientes Arbeiten möglich [sei]“ oder diese Form „die beste Möglichkeit, die Schüler entspr. zu fördern“. In Einzel- oder Kleingruppenarbeit sei eine bessere Förderung möglich. Da nur wenige Stunden pro Schüler bzw. Klasse zur Verfügung stünden, könnten diese „dann effektiver und individueller genutzt werden“. Die Zeit könne als effektive Übungszeit genutzt werden oder dafür Hausaufgaben zu besprechen. Gerade „bei Schülern mit massivem Förderbedarf [führe] eine getrennte Förderung zu größeren Lernfortschritten“. Zwei Personen führen aus, dass sie die individuelle Förderung außerhalb der Klasse für gut halten:

[...] weil es für manche Kinder (massive Lernschwäche) die effektivste Möglichkeit der Förderung darstellt. Diese Schüler arbeiten mit komplett anderen Materialien, neuem Lernstoff parallel zum laufenden Klassenunterricht, stört den Rest der Klasse und stellt auch eine große Ablenkung für den Schüler dar. Um Vorfälle mit emotional-sozial geförderten Schülern aufzuarbeiten, eignet sich ebenfalls die indiv. Förderung am besten (Bloßstellung wird vermieden).

[...] weil sich der Schüler je nach Situation und Unterrichtsgegenstand fokussierter mit dem Inhalt beschäftigen kann und somit eine breitere Methodenvielfalt \& die Umsetzung einer individuellen Förderung ermöglicht wird.

Vorteile der Einzel- oder Gruppenförderung werden darüber hinaus in Möglichkeiten der Kommunikation und Zuwendung bzw. deren Schutzfunktion gesehen. So wird darauf hingewiesen, dass „,in diesem Rahmen auch Gespräche möglich sind (Probleme zu Hause, negative Erlebnisse...)“. Im Gespräch könne bspw. das Verhalten der SuS reflektiert werden („Verhaltensprobleme und Regelverstöße“). Die Kommunikation in der Einzelsituation sei „ein wichtiger Teil der Beziehungsarbeit“ und es sei „manchmal sinnvoller [...] mit dem Schüler in Ruhe etwas zu besprechen und die Woche zu planen“. Ein Elternteil hält diese Förderung für hilfreich, da Sohn oder Tochter „die Betreuung in der Kleingruppe genießt, mehr Beachtung findet, auch mal erzählen kann“. Auch zwei Lehrkräfte weisen darauf hin, dass Schüler diese Art der Zuwendung genießen, bzw. eine, dass sie die Förderung für gut hält, „wenn ein Kind die entsprechende Ruhe und Zuwendung braucht." Eine weitere Lehrkraft berichtet, dass „die Kinder sich auf die Förderung freuen und sie einfordern“. Einige der Befragten vertreten die Auffassung, dass es SuS mit spF gut tun könne, wenn sie „in geschütztem Rahmen Erfolgserlebnisse haben können“, „dann die annähernd gleichen Chancen haben“ oder „die L-Schüler/innen dabei nicht ständig die meist erhöhten Anforderungen ihrer Klassenkameraden sehen“ bzw. sich in Deutsch oder Mathe nicht benachteiligt fühlen. Diese Befragten schreiben der Förderung außerhalb des Klassenraums also eine schützende Funktion für eine kleine Gruppe zu. Die Schüler würden diese Zeit nutzen und „trauen sich meist mehr zu fragen als in der Klasse“.

Die Befragten, die eine Ablehnung der individuellen Förderung außerhalb des Klassenraums äußern, gehen u.a. davon aus, dass den SuS mit spF durch diese individuelle Förderung ein Sonderstatus zukomme, der sich negativ auswirken könne, dazu führe, dass diese sich „ausgegrenzt " fühlen und nicht in die Klasse integriert, sondern stattdessen segregiert bzw. separiert oder auch stigmatisiert werden. Aufgrund der Herausnahme aus dem regulären Unterricht 
könne es sein, dass die SuS den Anschluss an die Inhalte des Regelunterrichts verlieren. Durch die individuelle Förderung werde „das Kind aus dem Klassenverband herausgenommen [...], statt dort in dem jeweiligen Unter.fach von der Förderlehrkraft differenziert unterrichtet zu werden“. Weitere Personen argumentieren damit, dass diese Form der Förderung der Integration oder Inklusion nicht gerecht und damit „das Prinzip der Aussonderung zu sehr verfolgt“ werde, wobei zwei dieser Personen einschränkend hinzufügen, dass dies im Einzelfall dennoch sinnvoll sein könne. Ein weiteres Gegenargument bezieht sich darauf, dass ,alle Kinder von zusätzlichen Personen innerhalb des Unterrichts profitieren sollten!“. Durch den Unterricht im Klassenverband könnten „die sozialen Beziehungen erweitert und gefestigt werden“ und es bestünde die Möglichkeit dass „Kinder auch von Kindern lernen!“. Der gemeinsame Unterricht beinhalte die Kooperation zwischen Förder- und Regelschullehrer. Wenn zwei Lehrkräfte in einer Klasse seien, biete das mehr Ressourcen, lauten weitere Argumente. Eine Person weist darauf hin, dass „auch Lernstärkere zusätzl. Aufgaben innerhalb der Klasse bearbeiten - diese Förderung der Lernstarken kommt bei Inklusion teils zu kurz!“, eine weitere erläutert, dass die individuelle Förderung sich an der eigenen Schule in Grenzen halte, da viele Fächer sowieso in Kursen stattfinden würden.

\section{Aussagen zur individuellen Förderung in den Regelschulen mit Blick auf bestimmte Förderschwerpunkte}

Die befragten Personen differenzieren in ihren qualitativen Aussagen zwischen den individuellen Fördermöglichkeiten für bestimmte Förderschwerpunkte. So formuliert eine Person: „Bestimmte Teilbereiche der Förderbedürftigkeit, wie z.B. Sprache, Körper (Schwerhörigkeit, Sehschwäche, Rollstuhl) können vollkommen problemlos in der Regelschule integriert werden. Lernbehinderung oder sozial-emotionale oder geistige Behinderungen zu fördern, sind aber nicht zu leisten... Während sechs Befragte angeben, dass die meisten Schüler mit spF gut integriert werden könnten und gut aufgehoben seien, erläutern ebenfalls fünf Aussagen, dass mit Blick auf inklusive Unterrichtung individuelle Entscheidungen getroffen werden sollten, die bspw. äußere Bedingungen, Arbeitshaltung und Lernstand berücksichtigen. Teilweise würden die Kapazitäten der Regelschullehrkräfte für eine individuelle Förderung nicht ausreichen oder den Lehrkräften würde das nötige Wissen mit Blick auf spezifische Förderbedarfe noch fehlen. Zwei Befragte geben an, dass die Wahlmöglichkeit zwischen Regel- und Förderschule erhalten bleiben sollte, drei weitere gehen davon aus, dass „nicht alle Schüler optimal in der Regelschule gefördert werden können“, „für einige Schüler der Wechsel an eine Förderschule mit umfassenderer und intensiverer Förderung der bessere Weg ist“ bzw. „es trotz aller Bemühungen auch Kinder gibt, die in einer Grundschule überfordert sind (Klassengröße) und die an einer speziellen Förderschule bessere Lernfortschritte erzielen könnten“"

Für besonders problematisch halten sieben Personen die integrative Unterrichtung von Schülern und Schülerinnen mit sozial-emotionalem Förderbedarf. Einer der Befragten begründet dies damit, „dass alle sehr, sehr bemüht sind, jedoch bei „E“ Kindern die Grenze schnell erreicht ist. Zu wenig Personal, zu viele i.d. Klasse. Man kann gut fördern außerhalb der Klasse. Lehrer zweifeln bereits an sich selbst, obwohl diese sich im hohen Maße einsetzen!", eine weitere hält fest: „Hier muss häufig der Rest der Klasse zurückstehen, da diese Kinder einen Großteil der Aufmerksamkeit des L. brauchen...

Zwei Personen problematisieren die Förderung von Schülern mit dem spF Lernen, da diese darunter leiden könnten „,nicht mit den anderen Schülern regulär mithalten zu können“ oder „täglich den Unterschied zu seinen Klassenkameraden vor Augen geführt“ zu bekommen. 


\section{Förderplanung}

Individuelle Förderpläne sind nach Angaben der SL, RSL und FSL überwiegend nur für die zieldifferent unterrichteten $\mathrm{SuS}$ mit spF vorhanden.

Tab. 124: Für die I-SuS gibt es individuelle Förderpläne

\begin{tabular}{lrrrrc}
\hline & SL (n=31) & RSL (n= 53) & FSL (n=38) & E (n=33) & SLFZ (n =4) \\
\hline ja & $38,7 \%$ & $43,4 \%$ & $47,4 \%$ & $35,3 \%$ & 3 \\
nur für die zieldiff. SuS & $48,4 \%$ & $35,8 \%$ & $47,4 \%$ & nicht gefragt & 1 \\
nein & $3,2 \%$ & $11,3 \%$ & $2,6 \%$ & $29,4 \%$ & 0 \\
weiß ich nicht & $3,2 \%$ & $0 \%$ & $0 \%$ & $29,4 \%$ & \\
fehlend & $6,5 \%$ & $9,4 \%$ & $2,6 \%$ & $5,9 \%$ & \\
\hline
\end{tabular}

Rückmeldung zu Lernfortschritten der Schüler mit sonderpägogischem Förderbedarf Insbesondere die FSL geben den SuS mit spF häufig Rückmeldungen zu ihren Lernfortschritten.

Tab. 125: Zu den Lernfortschritten gebe ich den SuS mit Förderbedarf Rückmeldung

\begin{tabular}{lcc}
\hline & RSL $(\mathbf{n}=\mathbf{5 3})$ & FSL $(\mathbf{n}=\mathbf{3 8})$ \\
\hline sehr häufig & $0 \%$ & $0 \%$ \\
häufig & $52,8 \%$ & $89,5 \%$ \\
eher selten & $43,4 \%$ & $10,5 \%$ \\
gar nicht & $3,8 \%$ & $0 \%$ \\
\hline
\end{tabular}

Formen der Rückmeldung zu Lernfortschritten der SuS mit spF

Besonders häufig erfolgen Rückmeldungen im Rahmen persönlicher Gespräche mit den Schülerinnen und Schülern mit spF, auf Seiten der RSL gefolgt von Hinweisen im Mitteilungsheft. Seltener werden Rückmeldungen in schriftlicher Form (Bsp.: als Hinweise in Entwicklungsberichten oder zu Zeugnissen) vorgenommen.

Tab. 126: Zu den Lernfortschritten gebe ich den SuS mit spF Rückmeldung in Form von...

\begin{tabular}{lcccccccc}
\hline & \multicolumn{2}{c}{$\begin{array}{l}\text { Hinweise im } \\
\text { Mitteilungsheft }\end{array}$} & \multicolumn{2}{c}{ Beratungsgespräche } & \multicolumn{2}{c}{ Hinweise in Ent- } & \multicolumn{2}{c}{ Hinweise zum } \\
& RSL & FSL & RSL & FSL & RSL & FSL & RSL & FSL \\
& $(\mathbf{n = 5 3 )}$ & $(\mathbf{n}=\mathbf{3 8})$ & $(\mathbf{n}=\mathbf{5 3})$ & $(\mathbf{n}=\mathbf{3 8})$ & $(\mathbf{n}=\mathbf{5 3})$ & $(\mathbf{n}=\mathbf{3 8})$ & $(\mathbf{n}=\mathbf{5 3})$ & $(\mathbf{n}=\mathbf{3 8})$ \\
\hline ja & $45,3 \%$ & $28,9 \%$ & $77,4 \%$ & $76,3 \%$ & $17,0 \%$ & $34,2 \%$ & $3,8 \%$ & $31,6 \%$ \\
nein & $54,7 \%$ & $71,1 \%$ & $22,6 \%$ & $23,7 \%$ & $83,0 \%$ & $65,8 \%$ & $96,2 \%$ & $68,4 \%$ \\
\hline
\end{tabular}

Zu den Lernfortschritten geben die Regel- und Förderschullehrkräfte den SuS mit spF darüber hinaus auch kurze mündliche Rückmeldungen (11), bspw. in Form von Lob (4), oder führen Gespräche mit den Schülerinnen und Schülern (7) oder auch mit Eltern und Schülern gemeinsam (3), bspw. in Form von Entwicklungsgesprächen. In einem Fall erfolgen Rückmeldungen durch die Integrationshelferin. Darüber hinaus nutzen die Lehrkräfte Verstärkerpläne (4), formulieren Notizen in Heften oder auf Arbeitsblättern (2), unter Tests oder Klassenarbeiten (8) bzw. auf Zeugnissen oder entsprechenden Beiblättern (3). 
Die Schülerinnen und Schüler mit spF nehmen in erster Linie Rückmeldungen in mündlicher Form sowie in ihren Heften wahr.

Tab. 127: Rückmeldung zu Lernfortschritten durch die Lehrkräfte (S, $\mathrm{n}=33)$

\begin{tabular}{lccrc}
\hline & $\begin{array}{c}\text { sagen, wenn ich } \\
\text { Fortschritte beim } \\
\text { Lernen mache }\end{array}$ & $\begin{array}{c}\text { Rückm. zu Lern- } \\
\text { fortschritten im } \\
\text { Gespräch }\end{array}$ & $\begin{array}{c}\text { Rückm. in Beiblät- } \\
\text { tern zum Zeugnis }\end{array}$ & Rückm. in Heften \\
\hline ja & $78,8 \%$ & $41,7 \%$ & $0 \%$ & $53,8 \%$ \\
nein & $12,1 \%$ & $58,3 \%$ & $100 \%$ & $41,7 \%$ \\
weiß ich nicht & $9,1 \%$ & $0 \%$ & $0 \%$ & $0 \%$ \\
\hline
\end{tabular}

Die Eltern erhalten häufiger Rückmeldungen in den Mitteilungsheften oder in Form von Entwicklungsberichten als durch Hinweise zu den Zeugnissen.

Tab. 128: Rückmeldung zu Lernfortschritten durch die Lehrkräfte $(E, n=34))$

\begin{tabular}{lccc}
\hline & $\begin{array}{c}\text { Rückmeldung in Form von } \\
\text { Hinweisen im Mitteilungs- } \\
\text { heft }\end{array}$ & $\begin{array}{c}\text { Rückm. zu Lernfort- } \\
\text { schritten in Form von } \\
\text { Entwicklungsberichten }\end{array}$ & $\begin{array}{c}\text { Rückm. in Beiblättern } \\
\text { zum Zeugnis }\end{array}$ \\
\hline ja & $44,1 \%$ & $29,4 \%$ & $14,7 \%$ \\
nein & $55,9 \%$ & $70,6 \%$ & $85,3 \%$ \\
weiß ich nicht & $0 \%$ & $0 \%$ & $0 \%$ \\
\hline
\end{tabular}

Eine Lehrkraft wünscht sich für die Zukunft eine gemeinsame Förderplanung, ein Elternteil, „dass der Förderplan auf die jeweilige Person abgestimmt ist, mehr Verständnis und mehr Engagement der Lehrer, Berücksichtigung des Nachteilsausgleichs und vieles mehr!!!“.

\subsubsection{Ergebnisse im Bereich der Unterrichtsentwicklung}

Sechs Eltern der Schülerinnen und Schüler mit spF äußern sich im Rahmen ihrer qualitativen Aussagen zu übergeordneten Aspekten der Unterrichtsentwicklung, die vor allem die Ergebnisebene betreffen. Darunter sind vier Elternteile, die sich wünschen, dass ihre Kinder es mit Hilfe sonderpädagogischer Unterstützung schaffen in der Regelschule einen Schulabschluss zu erlangen und damit bessere Chancen im Berufsleben zu haben. Ein Elternteil wünscht sich, dass das „Kind es leichter hat zu lernen und dass sie sich wohl in der Schule fühlt.“

\section{Lernfortschritte der Schüler mit sonderpädagogischem Förderbedarf allgemein}

Tab. 129: Die Lernfortschritte der SuS mit spF halte ich insgesamt für...

\begin{tabular}{lcccr}
\hline & SL (n=31) & RSL (n= 53) & FSL (n= 38) & E (n=34) \\
\hline sehr gut & $9,7 \%$ & $11,3 \%$ & $10,5 \%$ & $41,2 \%$ \\
gut & $64,5 \%$ & $50,9 \%$ & $60,5 \%$ & $41,2 \%$ \\
weniger gut & $19,4 \%$ & $30,2 \%$ & $18,4 \%$ & $8,8 \%$ \\
gar nicht gut & $0 \%$ & $0 \%$ & $0 \%$ & $0 \%$ \\
fehlend & $6,5 \%$ & $7,5 \%$ & $10,5 \%$ & $8,8 \%$ \\
\hline
\end{tabular}


Die Lernfortschritte der SuS mit spF allgemein werden von den Befragten vorwiegend als gut eingeschätzt, auf Elternseite bei mehr als $40 \%$ als sehr gut. Am kritischsten werden die Fortschritte von den Regelschullehrkräften eingestuft, die diese zu etwa $30 \%$ als weniger gut bewerten, während dieser Anteil bei den SL bei 19,4\% und bei den FSL bei 18,4\% liegt.

\section{Lernfortschritte Deutsch/Mathematik}

Unterschiede ergeben sich teilweise in der Einschätzung der Lernfortschritte in den Fächern Deutsch und Mathematik. Eltern wie die Schüler selbst schätzen die Fortschritte in beiden Fächern vorwiegend als sehr gut bzw. gut ein, während die Regelschullehrkräfte insbesondere die Fortschritte in Mathematik zu mehr als $30 \%$ als weniger gut beurteilen.

Tab. 130: Die Lernfortschritte in Deutsch (Lesen/Schreiben) halte ich für...

\begin{tabular}{lcccc}
\hline & RSL (n= 53) & FSL (n= 38) & $\mathbf{E}(\mathbf{n}=34)$ & S (n= 33) \\
\hline sehr gut & $7,5 \%$ & $2,6 \%$ & $16,1 \%$ & $33,3 \%$ \\
gut & $52,8 \%$ & $71,1 \%$ & $64,7 \%$ & $51,5 \%$ \\
weniger gut & $20,8 \%$ & $15,8 \%$ & $11,8 \%$ & $15,2 \%$ \\
gar nicht gut & $5,7 \%$ & $0 \%$ & $0 \%$ & $3,0 \%$ \\
fehlend & $13,2 \%$ & $10,5 \%$ & $7,4 \%$ & $7,0 \%$ \\
\hline
\end{tabular}

Tab. 131: Die Lernfortschritte in Mathe/beim Rechnen halte ich für...

\begin{tabular}{lcccc}
\hline & RSL $(\mathbf{n}=\mathbf{5 3})$ & FSL $(\mathbf{n}=\mathbf{3 8})$ & $\mathbf{E}(\mathbf{n}=\mathbf{3 4})$ & $\mathbf{S}(\mathbf{n}=\mathbf{3 3})$ \\
\hline sehr gut & $9,4 \%$ & $2,6 \%$ & $32,4 \%$ & $33,3 \%$ \\
gut & $47,2 \%$ & $76,3 \%$ & $47,1 \%$ & $48,5 \%$ \\
weniger gut & $30,2 \%$ & $10,5 \%$ & $8,8 \%$ & $18,2 \%$ \\
gar nicht gut & $1,9 \%$ & $0 \%$ & $2,9 \%$ & $0 \%$ \\
fehlend & $0 \%$ & $10,5 \%$ & $8,8 \%$ & $0 \%$ \\
\hline
\end{tabular}

\section{Lernbereitschaft der Schüler mit sonderpädagogischem Förderbedarf}

Die grundsätzliche Lernbereitschaft der SuS mit spF wird insgesamt sehr positiv bewertet auch von den Schülerinnen und Schülern selbst, während auch diesbezüglich die Bewertungen der RSL etwas ungünstiger ausfallen.

Tab. 132: Die Lernbereitschaft halte ich für...

\begin{tabular}{lccccr}
\hline & SL (n=31) & RSL (n= 53) & FSL (n= 38) & E (n=34) & S (n= 33) \\
\hline sehr gut & $12,9 \%$ & $20,8 \%$ & $10,5 \%$ & $23,5 \%$ & $27,3 \%$ \\
gut & $58,1 \%$ & $47,2 \%$ & $73,7 \%$ & $58,8 \%$ & $63,6 \%$ \\
weniger gut & $6,5 \%$ & $18,9 \%$ & $7,9 \%$ & $8,8 \%$ & $9,1 \%$ \\
gar nicht gut & $0 \%$ & $1,9 \%$ & $0 \%$ & $2,9 \%$ & $0 \%$ \\
fehlend & $22,6 \%$ & $11,3 \%$ & $7,9 \%$ & $0 \%$ & $0 \%$ \\
\hline
\end{tabular}


Lernfortschritte der Schüler mit sonderpädagogischem Förderbedarf im sozialen Bereich Ein ähnliches Bild ergibt sich hinsichtlich der Lernfortschritte der SuS mit spF im sozialen Bereich.

Tab. 133: Die Lernfortschritte im sozialen Bereich halte ich für...

\begin{tabular}{lcccc}
\hline & SL (n=31) & RSL (n= 53) & FSL (n=38) & E (n=34) \\
\hline sehr gut & $12,9 \%$ & $17,0 \%$ & $7,9 \%$ & $26,5 \%$ \\
gut & $58,1 \%$ & $47,2 \%$ & $68,4 \%$ & $52,9 \%$ \\
weniger gut & $9,7 \%$ & $24,5 \%$ & $13,2 \%$ & $5,9 \%$ \\
gar nicht gut & $3,2 \%$ & $1,9 \%$ & $5,3 \%$ & $2,9 \%$ \\
fehlend & $16,1 \%$ & $9,4 \%$ & $5,3 \%$ & $\%$ \\
\hline
\end{tabular}

Nach Bewertungen und Begründungen für als gut oder sehr gut eingestufte Lernfortschritte im kognitiven und sozialen Bereich gefragt, beschreiben die Befragten eine vorwiegend positive Wahrnehmung. Sie halten in ihren Aussagen fest, dass eine bestmögliche Förderung stattfinde (4), sie sehr gut gelinge, die SuS mit spF sehr gut betreut seien, dass sie erfolgreich sei bzw. entsprechend der Möglichkeiten gut laufe. Die Förderung werde „als etwas Normales angesehen [...] u. [sei] im Schulalltag fester Bestandteil“, sie sei „wichtig und natürlich“, mit Hilfe der Förderung könnten „viele unterschiedliche Schüler in die Klasse integriert werden“, sie sei notwendig und sinnvoll und ein „zeitintensives System [...], aber „ohne Förderung würden die Kinder nicht da stehen, wo sie jetzt sind. Sie würden wohl in der Großgruppe „verschwinden““. Eine Lehrkraft beschreibt als positiv, dass die Förderung auf einem guten Weg sei: „2 Förderräume in Planung. Beginnende Umsetzung von Inklusion: Info-Termine, Besprechungen, Lernstandsdiagnosen, Anschaffung Fö-Material + Räume“. Zwei Personen weisen auf die Verbesserung der Förderung in den letzten Jahren („dank immer gleicher Integrationslehrkraft“) bzw. weitere Verbesserungsmöglichkeiten hin.

Elf der Befragten stellen Querverbindungen zur Schule bzw. Schulklasse als sozialer Raum her, indem sie erläutern, dass „,in der Schule ein gutes soziales Klima herrscht“, die Kinder integriert seien (4), Freundschaften gebildet werden könnten, ihre Förderung „eine große Bereicherung für alle Beteiligten“ sei und die Schüler sich „höchst sozial entwickeln“bzw. „die Schüler mit der Schulgemeinschaft gut aufgehoben sind und sich nicht als „besonders“ erfahren müssen - trotz ihrer Defizite“. Die Förderung im sozialen Bereich könne als gelungen bezeichnet werden, da sie „von allen Beteiligten getragen wird“ und ein „respektvoller Umgang“ gegeben sei. Soziale Lernfortschritte seien möglich, da in der Schule „auf gutes Benehmen großen Wert gelegt wird. Vorfälle werden sofort besprochen \& aufgearbeitet.“ oder „weil in den einzelnen Klassen stark am sozialen Klima gearbeitet" werde. Durch die schulische Integration und Förderung stehe der Bereich des sozialen Lernens im Vordergrund. Die Förderung sei „ein wichtiger Beitrag zur Integration der Gesellschaft" und sei für die Persönlichkeitswentwicklung förderlich, da „Ausgrenzung bzw. Abstempelung vermieden wird“. Als positiv beschreiben zwei Befragte, dass Schülerinnen und Schüler täglich mit Konflikten konfrontiert werden würden und dadurch lernen könnten Lösungen in solchen Situationen zu finden. Ein Elternteil beschreibt, dass ein Kind „ein wenig offener geworden ist und auf die Mitschüler zugeht. Seine Mitschüler akzeptieren ihn als Mitglied der Klasse, weil klare Strukturen und Regeln sowie konkrete Rückmeldung ihr soziales Verhalten fördern“. Ein Schüler/eine Schülerin selbst schreibt, dass die Integration in seinem/ihrem Fall sehr gut gelinge. 
Weitere Befragte thematisieren die Bedeutung der Didaktik und Methodik des Unterrichts für die Lernfortschritte von Schülerinnen und Schülern mit spF und gehen davon aus, dass „offener Unterricht und freie Arbeit sehr gut dazu beitragen“, das Lernprogramm durch Fach- und Integrationslehrer angepasst werde, die Schüler „behutsam an neue Inhalte herangeführt werden und so viel Zeit erhalten, wie sie benötigen“ und „der Unterricht kleinschrittig und motivierend gestaltet" werde. Als unterstützend wird auch die Verwendung anschaulicher Materialien durch einen Mathematiklehrer erwähnt. Einen wesentlichen Beitrag mit Blick auf die Lernentwicklung der Schüler würden auch Differenzierung und Individualisierung im Unterricht leisten (4), die ,teilweise auch außerhalb der Klasse“ stattfinde. Präventive differenzierende Fördermaßnahmen könnten dazu beitragen den sonderpädagogischen Förderbedarf im Bereich Lernen zu verhindern. Von besonderer Bedeutung sind für die Befragten auch Aktivierung und Motivation der Schülerinnen und Schüler mit spF. Diese würden durch individuelle Leistungsbeurteilung, Lernerfolge in Form von besseren Noten, Erfolgserlebnisse, abwechslungsreiche Übungen, Lob und kleine Fortschritte ermöglicht. Durch eine differenzierte Leistungsbewertung könne Konkurrenzdruck vermieden werden (2). Eine Person weist darauf hin, dass das Selbswertgefühl der Schüler mit spF ohne zusätzliche Förderung nur schwach ausgeprägt wäre. Auch die Ergebnisse der unterrichtlichen Förderung werden positiv bewertet. Äußerungen dahingehend greifen auf, „dass das Gelernte gesichert ist und sie ihr Wissen erweitern“, die Schüler mit spF „zeigen wollen, was sie können“, „durch die individuelle Vorbereitung und die Klassenarbeiten mit Nachteilsausgleich nahe am Niveau der Klasse sind“ bzw. „in der Regel [...] die selbst gesteckten schulischen Ziele erreichen“ oder „vielen die Rückgliederung in den Regelbereich gelingt“ und „es vorkommt, dass Schüler mit sonderpädagogischem Förderbedarf die Schule mit einem Regelabschluss verlassen“. Die Förderung trage dazu bei, dass Schülerinnen und Schüler mit spF „weniger auffällig werden“, darunter auch solche Kinder, „die als extrem verhaltensauffällig [...] ankommen“.

Relevant für die schulische Förderung sei auch die Kooperation der Lehrkräfte (4), u.a. „weil durch die enge Zusammenarbeit zwischen Fachlehrer und Förderlehrer Synergien erzielt werden“. Diese sei wiederum abhängig von Kompetenz und Engagement der Lehrkräfte (3).

Zwölf der Befragten geben an, dass die Förderung noch verbessert werden könne. Problematisch seien u.a. „die vielen nicht gemeldeten Schüler, die Unterstützung benötigen“. Fünf Beteiligte gehen davon aus, dass die Förderung verbessert werden könnte, wenn die Ressourcenausstattung eine andere sei. Es gebe zu wenig Stunden, bspw. für unterrichtsbezogene Zusammenarbeit und Team-Teaching, zu wenig strukturelle Hilfen und Hilfen für Schülerinnen und Schüler mit dem spF soziale und emotionale Entwicklung. Eine weitere Lehrkraft weist darauf hin, „dass die Fachlehrer noch mehr auf die Förderschüler eingehen müssen und deutlicher reduzieren/differenzieren sollten“"

Sechs Personen halten es für wichtig, dass indidividuell geprüft werde, welcher Lernort für ein Kind günstiger sei. Im Einzelfall gebe es Grenzen, die „ausgelotet werden“ müssen. Es müsse „immer genau geschaut werden, welcher Lernort für ein Kind das Beste ist“, da es auch Kinder gebe „die an einer Förderschule besser lernen können“. Eine „Generalisierung“ sei daher „fehl am Platz“. Man müsse sich „im Vorfeld jedes Kind genau anschauen [...], um den besten Weg für das Kind zu finden“. Eine Person geht davon aus, dass „Lernbehinderte [...] besser an Förderschulen gefördert werden [können]“.

Neun Befragte vertreten die Meinung, dass die Lernentwicklung von Schülerinnen und Schülern mit spF nicht verallgemeinernd bewertet werden könne, da diese sehr individuell verlaufe. Die Befragten, die die Förderung und Lernentwicklung der Schülerinnen und Schüler als weniger gut einstufen, begründen dies vor allem mit Verweisen auf mangelnde Ressourcen (Bsp.: 
nicht ausreichende Rahmenbedingungen, mangelhafte räumliche und materielle Ressourcen) und stellen damit klare Querverbindungen zu diesem Bereich her. Darunter bemängeln zwei Personen die Klassengröße. Eine Reihe von Aussagen bezieht sich auf die personelle Ausstattung. Kritisch benannt wird in diesem Zusammenhang, dass ,insgesamt zu wenige Stunden zur Verfügung stehen, um Fö-Kinder zufriedenstellend zu fördern; weil die personelle, räumliche und fachliche Unterstützung/Situation nicht angemessen ist“, „nicht immer eine zweite Lehrkraft vorhanden ist“, einfach zu wenig Zeit vorhanden sei „um die Kinder so zu fördern, wie sie es bräuchten“ bzw. „manche Kinder besser in einer Kleingruppe mit Doppelbesetzung (Schonraum) lernen würden“. Die Förderung könne nur „bruchstückhaft umgesetzt" werden (2), u.a., da viele Fächer nicht einbezogen werden könnten (2). Fachlehrerwechsel würden viele Schülerinnen und Schüler überfordern. Die Förderung müsse konstanter innerhalb der Klasse erfolgen, da „ständiger Wechsel“ den Schülern nicht helfe und es für viele unangenehm sei „wenn sie aus dem Unterricht herausgenommen werden, um in einer Kleingruppe zu arbeiten“. Die Förderung innerhalb der Klasse wiederum sei deshalb erschwert, da dies mit nur einer Förderschullehrkraft an einer Schule nicht umsetzbar sei. Die Zeit für soziale Förderung sei dadurch zu knapp (2) und das bei hohem Lehrplandruck. Eine Lehrkraft weist darauf hin, dass Schülerinnen oder Schüler mit spF soziale und emotionale Entwicklung „mehr Rückzugsmöglichkeiten und individuelle Begleitung brauchen“. Eine weitere konstatiert, dass „die Probleme oft so gravierend sind, dass nur therapeutische Maßnahmen greifen würden“. Zwei Befragte wünschen sich Integrationshelfer für einzelne Kinder. Eine weitere Querverbindung wird dahingehend hergestellt, dass bei der derzeitigen Ressourcensituation keine angemessene Elternarbeit möglich sei (2). Bezüglich der Entwicklung des Selbstkonzepts von Schülerinnen und Schülern mit spF befürchten einige Lehrkräfte ungünstige Auswirkungen der integrativen Förderung. In den Regelklassen müssten

Schüler mit sonderpädagogischem Förderbedarf sich immer an den anderen Schülern messen und stets erfahren [...], was sie nicht können und dass ihre Grenzen schnell erreicht sind! Im sozialen Bereich: ab Klasse 7 (spätestens) werden diese Schüler immer stärker ausgegrenzt. Sie werden durch die Inklusion alles andere als optimal gefordert und gefördert!!!

Diese Schüler würden in den Klassen oft als „Lernversager“ gelten und ihr Leistungsdefizit durch störendes Verhalten kompensieren. Es würde sie wenig motivieren „permanent etwas anderes zu tun - auch am selben Thema“. Einzelne Schüler zeigten „kein Interesse an sozialer Interaktion“. Drei weitere kritische Aussagen zur Förderung beziehen sich darauf, dass „die meisten Kinder Deutsch nicht als Muttersprache haben“, „,von zu Hause wenig Unterstützung bekommen“ oder den Materialeinsatz nicht selbst bewerkstelligen können und dazu Anleitung brauchen. Insbesondere bei verhaltensauffälligen Schülern seien ständig Zuspruch sowie Korrekturen notwendig. Die Hürden zur Anerkennung des Förderbedarfs seien noch zu hoch (Dauer des Prozesses). Eine Mutter wünscht sich zur Verbesserung der Förderung, „dass es regelmäßige Rückmeldung an mich als Mutter gäbe gegebenenfalls mit Vorschlägen, was man wie gezielt mit dem Kind üben kann!“.

\subsubsection{Interpretation der Ergebnisse zur Organisationsentwicklung}

\subsubsection{Ressourcen im Bereich der Organisationsentwicklung}

Im Rahmen von qualitativen Aussagen äußert sich ein großer Anteil der Befragten zu den Ressourcen und damit Rahmenbedingungen schulischer Integration. Besonders häufig werden in diesem Zusammenhang die personellen Ressourcen aufgegriffen (s. 4.5.7.1). Aber auch mit Blick 
auf die (bauliche) Ausstattung der Schulen äußern sich vor allem Schulleitungen und Lehrkräfte dahingehend kritisch, dass diese den Anforderungen, die die Unterrichtung von Schülerinnen und Schülern mit spF stellt, noch nicht genüge. Das Thema Barrierefreiheit spielt in den befragten Schulen dabei - anders als in den Qualitätskategorien der bisherigen Forschung (Innerhofer \& Klicprea, 1991; Heyer et al., 1997; Boban \& Hinz, 2003; Brugger-Paggi, 2003; Specht et al., 2006; Wember \& Prändl, 2009; Speck, 2010) - eine untergeordnete Rolle, was mit dem geringen Anteil der Schülerinnen und Schüler mit körperlichen Beeinträchtigungen in diesen Schulen (Schulleitungen gehen von etwa 3\% aus) zusammenhängen könnte. Stattdessen wird deutlich, dass flexible Raumgestaltungsmöglichkeiten sowie Differenzierungsräume eine zentrale Rolle mit Blick auf die baulichen Voraussetzungen in den Schulen im Zusammenhang mit schulischer Integration innehaben.

Während die Größe der Klassenräume (Wember \& Prändl, 2009; Speck, 2010) nach Aussagen von Schulleitungen und Lehrkräften immerhin zu einem Drittel passend zur Größe der Klassen gegeben ist, geben nur 16,5\% der Personen an, dass die Raumgestaltungsmöglichkeiten flexibel sind. Etwa die Hälfte der Befragten stimmt der Aussage zu, dass Differenzierungsräume genutzt werden können, während dies für ein Drittel nur teilweise und für knapp 20 \% gar nicht möglich ist. Anders als bei Wember \& Prändl (2009) aufgeführt, fehlen teilweise Räume, die für Bewegungsangebote, Rückzug oder spezifische Förderung genutzt werden können. Die Voraussetzungen für differenzierende bzw. individualisierende Förderung von einzelnen Schülern und Schülerinnen oder Schülergruppen (Heese, 1977; Innerhofer \& Klicpera, 1991; Brugger-Paggi, 2003; Wember \& Prändl, 2009; Holzinger et al., 2011) sind also längst nicht in allen Schulen gegeben. Dies ist ein wichtiger Beleg dafür, dass die baulichen Gegebenheiten in den befragten Schulen nur in Teilen den Anforderungen inklusiven Unterrichts entsprechen. Zu vergleichbaren Ergebnissen gelangten Eberl (2000) und Amrhein (2011) mit Bezug auf die von ihnen untersuchten integrativ arbeitenden Schulen. Für die Zukunft inklusiver Schulen und hinsichtlich der Verbesserung der Förderung wünschen sich daher vor allem Lehrkräfte und Schulleitungen eine größere Anzahl an zur Verfügung stehenden Räumen, gut ausgestattete Förderräume sowie spezifische Räumlichkeiten für spezifische Anforderungen.

Auch die Ausstattung der Schulen mit Differenzierungsmaterial entspricht nur in Teilen den in der Forschung oder in Kriterienkatalogen (Innerhofer \& Klicpera, 1991; Boban \& Hinz, 2003; Brugger-Paggi, 2003; Wember \& Prändl, 2009; Holzinger et al., 2011) formulierten Ansprüchen, da dieses - laut Angaben der befragten Personen - nur in etwa einem Drittel der Schulen und in etwa einem Sechstel der Klassenräume vorhanden ist. Gleichzeitig geben die Befragten an, dass die Schülerinnen und Schüler zu bis zu 67,5\% auf differenziertes Material angewiesen sind, darunter handlungsorientiertes Material. Auch die qualitativen Aussagen zu (fehlenden) spezifischen Materialien und Raumausstattungsmerkmalen bzw. Wünsche für die zukünftige Ressourcensituation (Bsp.: Pool an Differenzierungsmaterialien in den Schulen) vermitteln ein deutliches Bild dahingehend, dass der Qualitätsbaustein der sächlichen Ressourcen (Bächthold et al., 1990; Feyerer, 1998; Bless, 2003; Brugger-Paggi, 2003; Speck, 2010) in den an der Befragung beteiligten Schulen nicht oder nur in Teilen gegeben ist. Abgesehen davon, dass in dieser Hinsicht offensichtlich Handlungsbedarf vorliegt, vermitteln also auch die Ergebnisse der quantitativen Erhebung den Eindruck, dass die von Textor (2015) beschriebene „Gefahr der indirekten Steuerung“ durch mangelhafte finanzielle Ressourcen in der Praxis gegeben ist. Trotz der im Saarland zum Zeitpunkt der Befragung kurz bevorstehenden Einführung der Inklusionsverordnung ist dieser Bereich seitens der Befragten mit Sorgen verknüpft. Von Anschubinvestitionen (Innerhofer \& Klicpera, 1991; Dohmen \& Fuchs, 2009; Klemm, 2009; Schwarz et al., 2013) ist keine Rede. 


\subsubsection{Leitung einer Schule}

Die von Seiten der Lehrkräfte und Eltern erfolgende sehr positive Einschätzung der Offenheit der Schulleitungen für schulische Integration (jeweils etwa $75 \%$ ) ist ein Hinweis darauf, dass diese Schulleitungen als „Türöffner“ fungieren können (Innerhofer \& Klicpera, 1991). Mit Bezug auf Bryk et al. (2010), Dyson (2010) oder Scheer \& Laubenstein (2018) ist dieses Ergebnis als relevant einzuschätzen, da die Schulleitungen eine zentrale Rolle bei der Gestaltung von (inklusiven) Schulentwicklungsprozessen spielen. Darüber hinaus bewerten die befragten Eltern die Zusammenarbeit mit den Schulleitungen vorwiegend positiv bzw. sehr positiv. Die von Bryk et al. (2010) als ein maßgeblicher Faktor für die Entwicklung von Schulen benannte Elternarbeit ist also von Seiten der Schulleitungen grundsätzlich gegeben.

\subsubsection{Schule als sozialer Raum}

\section{Schulklima}

Insgesamt ergibt sich aufgrund der Befragungsergebnisse ein sehr positives Bild des Schulklimas in den an der Befragung beteiligten Schulen, wobei ein hohes Maß an Übereinstimmung im Antwortverhalten der unterschiedlichen Gruppen besteht. Die Schulen legen großen Wert auf gegenseitigen Respekt sowie Akzeptanz aller zur Schule gehörenden Personen innerhalb der Schulgemeinschaft. Schülerinnen und Schüler mit sonderpädagogischem Förderbedarf werden von ihren Mitschülerinnen und Mitschülern sowie von den Lehrkräften in hohem bzw. sehr hohem Maß akzeptiert und geben auch an Freundschaften innerhalb der Schule zu pflegen. Positive Auswirkungen auf das Wohlbefinden der Schülerinnen und Schüler mit spF sind vorhanden (Hascher, 2007), wie die sehr guten Rückmeldungen der SuS zu diesem Bereich belegen. Die Vielfalt der positiven Begründungen für das Wohlbefinden der SuS mit spF schließt neben den Aspekten der Akzeptanz, Integration, der Freundschaften von Schülern untereinander und des guten Schul- bzw. Klassenklimas auch den der Unterstützung dieser Schüler durch die Regel- und Förderschullehrkräfte ein. Diese würden darauf achten die Kinder und Jugendlichen „dort abzuholen, wo sie stehen“, sie nicht zu überfordern, sondern individuell zu fördern. Dieser Zusammenhang wurde in der rezipierten Forschungsliteratur bislang nur wenig direkt formuliert. Anschlussmöglichkeiten finden sich allerdings in den Ausführungen zur Bedeutung der Beziehungsebene zwischen Lehrkräften und Schülern (Marzano \& Marzano, 2003; Hattie, 2013) sowie mit Blick auf die Subkategorie Integration als gemeinsame Aufgabe (Feyerer, 1998; Boban \& Hinz, 2003; Holzinger et al., 2011). Etwas kritischer als die anderen befragten Personengruppen bewerten in diesem Zusammenhang die FSL die Akzeptanz der $\mathrm{SuS}$ mit spF durch die Regelschullehrkräfte (über $60 \%$ stimmen der entsprechenden Aussage eher zu, während etwa zwei Drittel der Schulleitungen, Eltern und Schüler sowie über 80 \% der Regelschullehrkräfte sehr zustimmen).

Ungünstigen Einfluss auf das Wohlbefinden der SuS mit spF haben nach Einschätzung der Befragten Lernabstandserfahrungen zu Mitschülern, der Lärmpegel in großen Klassen, Probleme mit Mitschülern oder Erfahrungen sozialer Ablehnung (Außenseiterpositionen). Mehrfach werden diese Aussagen auf verhaltensauffällige Schüler bezogen. Diese - anteilsmäßig selteneren - Aussagen stimmen mit Ergebnissen von Bachmann (1974), Maikowski \& Podlesch (1988), Bless (2003), Goetze (2008) und Huber (2009) überein.

Hohe Anteile der SL, RSL, FSL und E befürworten die Aussage, dass das soziale Miteinander der Schüler in einer Schule durch die integrative Unterrichtung verbessert werde. Auch die sozialen Kontakte zwischen $\mathrm{SuS}$ mit und ohne spF werden vorwiegend als gut eingestuft. Lediglich der Bereich der nachmittäglichen Kontakte erscheint ausbaufähig, wobei die SuS mit 
spF selbst diese sehr viel günstiger einschätzen als alle anderen Beteiligten. Die Ergebnisse sind diebezüglich insgesamt etwas positiver als die von Dumke et al. (1989), die belegen, dass die Kontakte zwischen behinderten und nichtbehinderten Kindern nach Auskunft von Eltern zu $25 \%$ häufig und zu 75\% nur gelegentlich gegeben sind. Werden nur die Angaben der Eltern einbezogen, stimmt das Ergebnis allerdings durchaus überein (26,5\% der Eltern stimmen der Aussage, dass Nachmittagskontakte stattfinden, überein, 64,7\% stimmen eher bzw. eher nicht zu, 8,8\% gar nicht).

Die in der Forschung als relevant eingestufte Qualitätsdimension des Schulklimas (Schley et al., 1992; Dt. PISA-Konsortium, 2001; Boban \& Hinz, 2003; Wember \& Prändl, 2009; Dyson, 2010; Lienhard-Tuggener et al., 2011) ist an den befragten saarländischen Schulen also insgesamt sehr positiv ausgeprägt, was - wie die qualitativen Aussagen belegen - u.a. mit der langjährigen Erfahrung und Entwicklungsgeschichte von Schulen mit schulischer Integration zusammenzuhängen scheint.

\section{Zusammenarbeit mit Eltern}

Die Qualitätskategorie der Zusammenarbeit mit den Eltern wurde in den Fragebögen mit Hilfe mehrerer Fragen erhoben, u.a. da deren Bedeutung sowohl in der (sonder-)pädagogischen Forschung als auch als Ergebnis der qualitativen Studie stark gewichtet wird. Vom überwiegenden Teil der Eltern wird die Zusammenarbeit mit den Schulleitungen, Klassenleitungen und Förderschullehrkräften positiv bewertet. Festhalten lässt sich also zusammenfassend, dass die Qualitätskategorie der Zusammenarbeit mit den Eltern (Bächthold et al., 1990; Innerhofer \& Klicpera, 1991; Hug, 1994b; Feyerer, 1998; Boban \& Hinz, 2003) grundsätzlich in den befragten Schulen gegeben ist.

Interessant ist in diesem Zusammenhang vor allem das hohe Maß an Unterstützungserfahrung, das die Eltern von SuS mit spF mit Blick auf die Kooperation mit den Schulleitungen beschreiben. Dies ist ein Hinweis darauf, dass dieser Zusammenarbeit von Seiten der Eltern ein hoher Wert zugemessen wird und in einem Teil der Schulen selbstverständlich zu sein scheint, während diese Subkategorie in den bisherigen Forschungsveröffentlichungen nicht benannt wurde. Neben Elternabenden und Gesprächsangeboten führen die Schulleitungen vielfältige weitere Formen der Elterneinbindung auf wie bspw. Stammtische, Feste oder Projekte. Ähnlich wie bei Jeynes (2005b) erläutert sind die Schulen also darum bemüht Eltern auf unterschiedliche Art und Weise ins Schulleben einzubeziehen und gestalten eine sogenannte „Willkommenskultur“ (Wild \& Lütje-Klose, 2017).

Die Antworten der Regel- und Förderschullehrkräfte belegen sehr unterschiedliche Formen der Zusammenarbeit mit den Eltern (Bsp.: Telefonate, Mails, Kommunikation über Mitteilungshefte, Gespräche), wobei die Häufigkeit von RSL bzw. FSL und Eltern unterschiedlich wahrgenommen wird. Mit Blick auf die Qualität der Zusammenarbeit, die in der (sonder-)pädagogischen Forschung als besonders relevant eingestuft wird (Mühl, 1987; Hug, 1994b; Boban \& Hinz, 2003; Brugger-Paggi, 2003), ergibt sich daher ein differenziertes Bild bspw. dahingehend, dass Gesprächstermine zwischen Eltern und Klassenleitungen sowie zwischen Eltern und Förderschullehrkräften von einem Viertel der befragten Lehrkräfte (RSL und FSL) sowie von etwa der Hälfte der Eltern (Zusammenarbeit mit KL) bzw. einem guten Drittel (Zusammenarbeit mit FSL) als eher selten bezeichnet werden. Das insbesondere in der englischsprachigen Forschungsliteratur beschriebene qualitätsvolle „Parent Engagement“ (Pushor, 2007; Berthelsen \& Walker, 2008; Bryk et al., 2010) scheint also nicht in der Intensität vorzuliegen, wie es für notwendig erachtet wird, wenn es um Auswirkungen auf das schulische Lernen der Schülerinnen 
und Schüler geht. Eine Reihe der Befragten beschreibt bezüglich der Gesprächsinhalte solche, die einen direkten Zusammenhang mit den Lernprozessen der Schülerinnen und Schüler aufweisen und vorwiegend die vier Themenblöcke Organisation der Förderung, Förderplanung, Kind-Umfeld-Diagnostik und Zusammenarbeit mit außerschulischen Partnern umfassen. Die beschriebenen Gesprächsinhalte entsprechen damit in Teilen den Anforderungen an die inhaltliche Qualität von Elternkontakten, die u.a. von Berthelsen und Walker (2008) formuliert worden sind. In Anlehnung an die „Standards for Family-School Partnerships“ (Wild \& LütjeKlose, 2017), findet also ein regelmäßiger und produktiver Austausch statt und die gegenseitige Abstimmung von Lerninhalten und -zielen (in Verknüpfung mit Förderplanung) erfolgt ebenfalls (teilweise), während die Einbindung der Eltern in schulische Entwicklungsprozesse keine Erwähnung findet. Regelmäßige Informationen und Vereinbarungen zur Förderung (BruggerPaggi, 2003; Lienhard-Tuggener et al., 2011) als Qualitätsindikatoren der Sonderpädagogik sind Kennzeichen der Zusammenarbeit mit den Eltern.

Die Voraussetzungen für eine Intensivierung der Elternarbeit sind aufgrund der Ergebnisse wiederum als gut bis sehr gut einzustufen, da als Kennzeichen der Zusammenarbeit mit den Eltern sowohl Offenheit und Informationsfluss, als auch die Intensität mit Blick auf Kontakte und Gespräche von vielen Beteiligten als bereits vorhanden hervorgehoben werden. Eltern thematisieren in diesem Zusammenhang vor allem das hohe Engagement der Lehrkräfte für die Schülerinnen und Schüler mit sonderpädagogischem Förderbedarf. Die Spezifizierung der Zusammenarbeit als ziel- bzw. förderorientiert erfolgt vor allem durch die Schulleitungen. Von besonderer Bedeutung ist für die Beteiligten eine vertrauensvolle Zusammenarbeit auf Augenhöhe.

Mit Blick auf die - zahlenmäßig eher wenigen - Eltern, die wenig bzw. gar kein Interesse an einer Zusammenarbeit zeigen, werden qualitative Hinweise daraufhin gegeben, dass dadurch auch die Zusammenarbeit mit den Schülerinnen und Schülern erschwert wird, insbesondere mit Blick auf die Arbeit im Verhaltensbereich.

\section{Vernetzung}

Ähnlich wie die Elternarbeit stellt auch die Vernetzung ein Arbeitsfeld integrativer Schulen dar, das aufgrund der Untersuchungsergebnisse ausbaufähig erscheint. Nach Aussagen der an der Befragung beteiligten Schulleitungen bzw. Lehrkräfte findet die Kooperation mit Jugendamt, schulärztlichem Dienst bzw. schulpsychologischem Dienst an etwa einem Drittel der Schulen eher selten statt, ein Ergebnis, das mit den Ergebnissen der GeSchwindstudie für die Schwerpunktschulen in Rheinland-Pfalz (Laubenstein et al., 2015) übereinstimmt. Dennoch entsprechen die Ergebnisse denen von De Boer und Spies (2014), die Jugendämter und schulpsychologischen Dienst als wichtigste Vernetzungspartner inklusiver Schulen benennen. Eine enge Zusammenarbeit erfolgt in einigen Schulen mit den Schoolworkern, die als Bindeglied zwischen Schule und Jugendhilfe tätig sind. Gleichzeitig zeigen die Angaben zu weiteren Kooperationspartnern im Zusammenhang mit der schulischen Integration von Schülerinnen und Schülern mit spF allerdings auch, dass diese eine große Fülle in Teilen hochspezialisierter Angebote umfassen (Bsp.: Autismuszentrum, Kinder- und Jugendpsychiatrie, Logo- und Ergotherapeuten, Familienhilfezentren, Freie Träger, etc.), was damit zusammenhängt, dass viele außerschulische Einrichtungen bzw. Ärzte, Kinder- und Jugendpsychologen oder - therapeuten mit der Unterstützung von SuS mit spF befasst sind. Möglicherweise erfolgt die Zusammenarbeit mit diesen Partnern in erster Linie einzelfallbezogen. Als weitere und neue Qualitätssubkategorie in inhaltlichen Aussagen aufgeführt werden Vernetzungstreffen der Regelschulen bzw. 
Förderzentren mit dem schulpsychologischen Dienst, den Jugendämtern, dem schulärztlichen Dienst oder anderen Vernetzungspartnern. Diese Treffen können für einen fallunabhängigen übergeordneten Austausch zwischen den Beteiligten sorgen, der eine Professionalisierung oder auch stärkere Systematisierung der Zusammenarbeit - wie von Ahrens (2009) oder De Boer und Spies (2014) gefordert - unterstützen bzw. gewährleisten kann.

Während Bryk u.a. (2010) also die Bedeutung einer guten Vernetzung von Schulen in ihrem Umfeld als relevant für die Verbesserung schulischer Leistungen von Kindern und Jugendlichen herausgestellt haben und die Zusammenarbeit mit außerschulischen Institutionen im Rahmen sonderpädagogischer Veröffentlichungen als einer der zentralen Qualitätsbausteine integrativer Schulen erfasst wird (Bächthold et al., 1990; Innerhofer \& Klicpera, 1991; Boban \& Hinz, 2003; Brugger-Paggi, 2003; Specht et al., 2006; Feyerer, 2007; Lienhard-Tuggener et al., 2011), kann für die befragten Schulen festgehalten werden, dass diese in strukturierter Form nur in Teilen umgesetzt und noch nicht umfassend genutzt wird.

\subsubsection{Ergebnisse im Bereich der Organisationsentwicklung}

In etwa $40 \%$ der beteiligten Schulen sind keine Schulprogramme vorhanden, in denen die schulische Integration thematisiert wird. Besonders selten evaluieren die beteiligten Schulen ihre eigene Arbeit (nur etwa 13\%). Die Ergebnisse stimmen wiederum weitgehend mit Ergebnissen der PISA-Studie (2011) überein, die belegen, dass Schulprogramme über die verschiedenen Schulformen (HS, RS, GYM) hinweg in etwa $40 \%$ bis $49 \%$ der Schulen vorhanden sind (an den befragten Schulen zu knapp $40 \%$ ). Werden Schulprogramme als greifbare Ergebnisse schulischer Entwicklungsprozesse aufgefasst, wie dies die sonderpädagogische Forschung nahelegt (Berges, 1996; Brugger-Paggi, 2003; Boban \& Hinz, 2003; Wember \& Prändl, 2009), weisen die Daten auf möglichen Weiterentwicklungsbedarf in dieser Qualitätskategorie hin. Da neuere Ergebnisse von Friedrich (2015) allerdings belegen, dass Schulprogramme nicht zu messbaren Auswirkungen auf Lernergebnisse von Schülerinnen und Schülern führen, ist diese Interpretation nur unter Vorbehalt weiterer Forschung möglich.

Die Leitbildentwicklung scheint in einem großen Teil der Schulen (70\%) selbstverständlich zu sein, so dass mit Vorsicht angenommen werden kann, dass in diesen Schulen eine grundlegende Auseinandersetzung mit pädagogischen Haltungen zu Werten und Zielsetzungen erfolgt ist. Von wichtiger Bedeutung scheinen für die beteiligten Schulen auch Konzepte zum Umgang mit Unterrichtsstörungen zu sein, da diese in $60 \%$ der Schulen als vorhanden benannt werden. In Form einer grafischen Übersicht ergibt sich nun folgendes Bild:

\begin{tabular}{|c|}
\hline ORGANISATIONSENTWICKLUNG \\
\hline $\begin{array}{l}\text { RESSOURCEN } \\
\text { materielle Rahmenbedingungen allgemein } \\
\text {. Barrierefreiheit } \\
\text { räumliche Flexibilität } \\
\text { Differenzierungsräume } \\
\text { Differenzierungsmaterial/ spezifische Hilfsmittel }\end{array}$ \\
\hline $\begin{array}{l}\text { LEITUNG EINER SCHULE } \\
\cdot \text { Offenheit und Engagement } \\
\cdot \text { professionelles Management } \\
\cdot \text { beratende, vernetzende, koordinierende und unterstützende Leistungen } \\
. \quad \text { Kooperation auf Schulleitungsebene }\end{array}$ \\
\hline
\end{tabular}




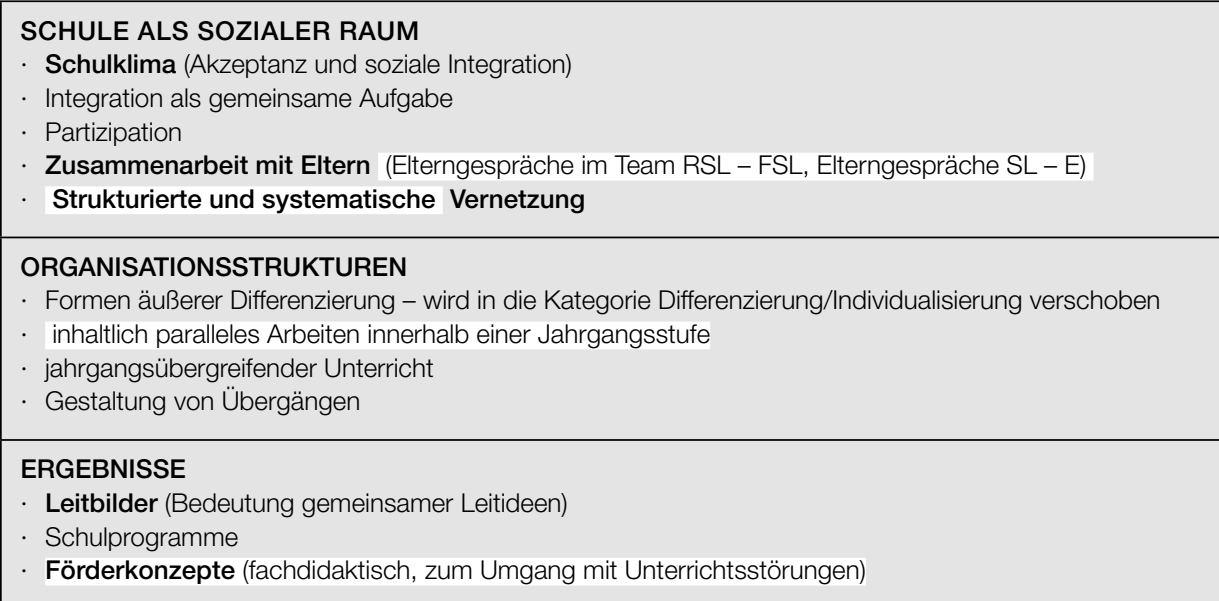

Abb. 20: Qualitätskategorien und -subkategorien im Bereich der Organisationsentwicklung überarbeitet

\subsubsection{Interpretation der Ergebnisse zur Personalentwicklung}

\subsubsection{Ressourcen im Bereich der Personalentwicklung}

Die Ausstattung mit personellen Ressourcen ist für die Schulen, an denen die Befragung durchgeführt wurde, offensichtlich eines der Kernthemen hinsichtlich der Qualität schulischer Integration, was sich daran zeigt, dass zu diesem Qualitätsbereich knapp 140 qualitative Aussagen auf offene Fragen getätigt werden. Diese beziehen sich auf die Subkategorien, die zum Großteil auch in bisherigen Studien als bedeutsam dargestellt worden sind, wie eine bessere personelle Ausstattung allgemein (Romey, 1977; Prell \& Link, 1977; Speck, 1978), eine gröBere Anzahl an Förderschullehrkräften in den Schulen, eine größere Anzahl an Förderschullehrerstunden je Schule (Bless, 2003; BMBF Österreich, 2008), eine geringere Anzahl an Schulen je Förderschullehrkraft, die Kontinuität der Zuweisung von Förderschullehrkräften (Brugger-Paggi, 2003), die Beschäftigung weiteren Personals (v.a. Integrationshilfen) (Romey, 1977; Prell \& Link, 1977; Speck et al., 1978; Klemm \& Preuss-Lausitz, 2008), einen hohen Anteil an Stunden der Klassenleitung in der eigenen Klasse (BMBF Österreich, 2008), Doppelbesetzung (s.u.), die Einrichtung fester Teamzeiten für FSL und RSL (Preuß, 1981; Köbberling, 1994; Boban \& Hinz, 2003; Brugger-Paggi, 2003; Wember \& Prändl, 2009; Holzinger et al., 2011; Lienhard-Tuggener et al., 2011; Arndt \& Werning, 2013) sowie die Reduktion der Unterrichtsverpflichtung von RSL, die SuS mit spF in ihren Klassen unterrichten (Köbberling, 1994; Berges, 1996; Brugger-Paggi, 2003). Die Möglichkeit der Reduktion der Unterrichtsverpflichtung von Regelschullehrkräften im Zusammenhang mit deren Arbeit in integrativen Klassen wird von Schulleitungen, Regel- und Förderschullehrkräften nicht einheitlich befürwortet, aber insgesamt in hohem Maß als Forderung unterstützt. Da qualitative Aussagen zu dieser Möglichkeit im Rahmen der Befragung nicht erhoben wurden, fehlen Begründungen für das unterschiedliche Maß an Zustimmung (bspw. stimmen 15\% der Regelschullehrkräfte der entsprechenden Aussage gar nicht zu). Eine besondere Bedeutung wird der Kontinuität der Zuweisung von Förderschullehrkräften an die Regelschulen zugemessen. Insbesondere die Schulleitungen stimmen der Aussage in sehr hohem Maß zu, dass die Kontinuität der Tätigkeit der FSL in den Regelschulen zur Akzeptanz der integrativen Unterrichtung beitragen kann 
(83,9\%), während die Anteile bei den RSL mit 54,7\% und den FSL mit 57,9\% geringer sind. In der rezipierten Forschungsliteratur wird dieser Qualitätsindikator einmal direkt benannt (Brugger-Paggi, 2003) bzw. einmal indirekt, indem Arndt \& Werning (2013) mangelnde Kontinuität als ungünstige Voraussetzung für Kooperation beschreiben.

Die Fülle der genannten Kategorien belegt indirekt eine differenzierte Auseinandersetzung der Befragten mit diesem Qualitätsbereich, da diese nicht einfach „mehr Personal“ fordern, sondern in ihren Ausführungen in vielen Fällen sehr konkret begründen, warum sie eine bestimmte Forderung als sinnvoll erachten. Begründungsansätze sind in diesem Zusammenhang Möglichkeiten zur Verbesserung der (individuellen) Förderung von Schülerinnen und Schülern mit spF, die Entlastung der Regelschullehrkräfte und die mögliche intensivere Kooperation zwischen RSL und FSL (v.a. mit Blick auf gemeinsame Unterrichtsplanung). Diese Ergebnisse decken sich also mit bisherigen Studien zum Zusammenhang zwischen Ressourcen und sonderpädagogischer Förderung sowie der Qualität der Kooperation von RSL und FSL (Dumke et al., 1989; Arndt \& Werning, 2013; Serke et al., 2014a; Lütje-Klose \& Urban, 2014b). Andererseits vermitteln die Aussagen ebenfalls indirekt mehrfach den Eindruck, dass die Regelschullehrkräfte davon ausgehen, dass die „Förderlehrer“ vor allem für die „Förderung“ der Schülerinnen und Schüler mit spF zuständig seien und diese entsprechend nicht funktionieren könne, wenn sie nicht anwesend sind.

Aufgrund der in der quantitativen Studie erhobenen Daten zur Anzahl der Förderschullehrerstunden je Schülerin bzw. Schüler (etwa 60 \% höchstens 2,5 Stunden wöchentlich, etwa $20 \%$ 3-4 Stunden), ist insgesamt davon auszugehen, dass anteilig nur in wenigen Unterrichtsstunden der befragten Schulen eine Doppelbesetzung mit Regel- und Förderschullehrkraft gegeben bzw. diese nur dann bspw. in den Kernfächern gegeben sein kann, wenn mehrere SuS mit spF in einer Klasse unterrichtet werden. Während die Doppelbesetzung aufgrund bisheriger Forschungsergebnisse eine zentrale Qualitätskategorie schulischer Integration darstellt (Innerhofer \& Klicpera, 1991; Köbberling, 1994; Heyer et al., 1997; Bless, 2003; Boban \& Hinz, 2003; Brugger-Paggi, 2003; BMBF Österreich, 2008; Specht et al., 2006; Thiele, 2009; Textor, 2009; Holzinger et al., 2011), ist sie im gemeinsamen Unterricht der saarländischen Schulen also wenn überhaupt - nur in einzelnen Stunden gegeben. Die personelle Versorgung der Schulen mit Stunden der Förderschullehrkräfte wird vor allem von den Schulleitungen (90,3\%), den Förderschullehrkräften (92,1\%) und Regelschullehrern (83\%) als unzureichend gekennzeichnet. Gleichzeitig sind nach Angaben der Befragten in 74\% bis $80 \%$ der Schulen keine Beratungszeiten zwischen Förder- und Regelschullehrkräften im Stundenplan verankert. Die Voraussetzungen für eine unterrichtsbezogene Kooperation zwischen Regel- und Förderschullehrkräften sind also in mehrfacher Hinsicht nicht vorhanden: zum einen fehlen gemeinsame Zeiten für die Vor- und Nachbereitung von Unterricht, zum anderen besteht auch nur selten überhaupt die Möglichkeit Unterricht im Co-Teaching zu gestalten. Diese Ergebnisse stimmen mit denen von Arndt und Werning (2013) überein.

\subsubsection{Kooperation}

Institutionalisierte Formen der Kooperation in den Regelschulkollegien wie Dienstbesprechungen oder Gesamtkonferenzen (Idel \& Ullrich, 2013) befassen sich in den befragten integrativen Schulen u.a. auch mit Fragen der schulischen Integration, was als Hinweis darauf aufgefasst werden kann, dass Aspekte dieses Themas auch auf einer Metaebene besprochen, beraten und diskutiert werden können.

Insgesamt wird die Kooperation zwischen Regel- und Förderschullehrkräften vorwiegend als gut bis sehr gut bewertet, wobei die Förderschullehrkräfte in ihrer Bewertung vorsichtiger sind 
als die Schulleitungen und Regelschullehrkräfte. Als qualitative Begründung für die positiven Bewertungen werden vor allem die gegenseitige Offenheit der Lehrkräfte für die Zusammenarbeit (Haeberlin et al., 1992) sowie Toleranz und Vertrauen benannt. Aufgrund der Bewertungen können die Ausgangsvoraussetzungen für Kooperation als gut bis sehr gut eingestuft werden. Vor allem die Tatsache, dass die Schulleitungen und Regelschullehrkräfte diese als sehr gut bzw. gut einschätzen, ist ein deutlicher Hinweis darauf, dass deren Wahrnehmung sehr positiv geprägt ist und die Kooperation Wertschätzung erfährt. Dieses Ergebnis steht in Übereinstimmung mit anderen neueren Ergebnissen zur Bewertung der Kooperation zwischen Regel- und Förderschullehrkräften (Behrensen et al., 2012) und ist im Vergleich zu älteren Forschungsergebnissen (Reiser, 1984; Antor, 1987; Dumke et al., 1989) möglicherweise Zeichen einer positiven Entwicklung in diesem Qualitätsbereich.

In diesem Zusammenhang stellt auch das hohe Maß an Zustimmung der Befragten zur Aussage, dass Regel- und Förderschullehrkräfte auf Augenhöhe zusammenarbeiten, ein positives Ergebnis dar (Haeberlin et al., 1992).

Von besonderem Interesse ist auch das Ergebnis, das belegt, dass es an den beteiligten saarländischen Schulen eine enge Zusammenarbeit zwischen den Schulleitungen der Regelschulen und den Förderschullehrkräften gibt, was daraus geschlussfolgert werden kann, dass die Schulleitungen zu sehr großen Anteilen häufig bis sehr häufig Pausengespräche oder Gesprächstermine zum Austausch mit den Förderschullehrkräften nutzen. Diese Qualitätssubkategorie ist bislang nicht Bestandteil vorliegender Forschung und - ähnlich wie die Qualitätssubkategorien Austausch auf Schulleitungsebene oder Austausch zwischen Förderschullehrkräften untereinander - ein Hinweis darauf, dass diese zusätzlichen bzw. erweiterten Austauschoptionen im Rahmen der Qualitätsentwicklung schulischer Integration eine wichtige Rolle spielen können. Während der Austausch auf Schulleitungsebene in bisherigen Studien nicht untersucht worden ist, wird die Zusammenarbeit der FSL untereinander von Bürli et al. (2009) als Qualitätssubkategorie benannt.

Weniger positiv ist das Ergebnis zu werten, dass es sich beim Großteil der Gespräche zwischen Förder- und Regelschullehrkräften um Pausengespräche handelt, während die Vereinbarung von Gesprächsterminen nach Angaben von etwa 35\% der Regelschullehrkräfte eher selten bis gar nicht und nach Angaben der Förderschullehrkräfte zu $21 \%$ selten oder gar nicht stattfindet. In Verknüpfung mit dem Ergebnis zu den an den allermeisten Schulen fehlenden Beratungszeiten bedeutet dies, dass eine professionelle Zusammenarbeit auf einer höheren und im engeren Sinn unterrichtsbezogenen Stufe kaum möglich erscheint. Hinsichtlich der Inhalte des Austauschs zwischen SL und FSL bzw. RSL und FSL stehen die Themenfelder Inklusion und deren Umsetzung, organisatorische Fragen, die Zusammenarbeit mit Vernetzungspartnern, differenzierte Leistungsbewertung, gemeinsame Elternarbeit, die Zusammenarbeit mit Integrationshilfen, fallbezogene Absprachen, diagnostische Fragestellungen und Möglichkeiten der Verbesserung der Förderung sowie der Kooperation im Fokus der Beteiligten. Indirekt kann dies als Hinweis darauf eingeschätzt werden, dass es gegebenenfalls ein Mangel an strukturiertem bzw. systemischem Austausch anstelle des rein einzelfallbezogenen Austauschs vorhanden ist, was eine koordinierte Weiterentwicklung schulischer Integration erschweren könnte. Neben Gesprächen werden als weitere Formen der Zusammenarbeit Teamgespräche, Inklusionstreffen, Förderkonferenzen und Förderausschüsse benannt, so dass die Einbindung von Förderschullehrkräften in kooperativen Austausch auf Gremienebene zumindest in Teilen gegeben ist (Prell \& Link, 1977; Mühl, 1987; Boban \& Hinz, 2003; Thiele, 2009; Lütje-Klose \& Urban, 2014a, 2014b). 
Aufgrund der qualitativen Aussagen zum Qualitätsbaustein Kooperation ist davon auszugehen, dass die Niveaustufe Austausch bzw. Co-Activity (s. dazu auch Formen der Zusammenarbeit) besonders häufig gegeben ist (Lütje-Klose \& Willenbring, 1999; Fussangel \& Gräsel, 2012). Einzelne Lehrkräfte berichten darüber hinaus von echter Kooperation (Lütje-Klose \& Willenbring, 1999; Fussangel \& Gräsel, 2012) bspw. in Form von gegenseitigen Rückmeldungen zu Unterrichtsinhalten oder Lernfortschritten, gemeinsamer Unterrichtsvor- oder -nachbereitung. Als wichtige Kennzeichen solcher Teams beschreiben die Lehrkräfte u.a. das gegenseitige Vertrauensverhältnis (Bryk, 2010), gemeinsame Entwicklungsprozesse, das gleichmäßige Engagement der Beteiligten, gleichberechtigtes Arbeiten sowie gemeinsame Ziele (Preuß, 1985) und damit Bestandteile von Teamarbeit, die einige Lehrkräfte auch als Wünsche für die Zukunft inklusiver Beschulung formulieren.

\section{Unterrichtsbezogene Kooperation}

Hinsichtlich der unterrichtsbezogenen Kooperation werden von einzelnen Lehrkräften die Gestaltung von Klassenarbeiten, die Differenzierung von Lerninhalten, die Vorbereitung differenzierten Materials, die Gestaltung von Nachteilsausgleichen und das Einbringen von Verstärkersystemen als inhaltliche Bausteine benannt. Insgesamt findet die unterrichtsbezogene Kooperation zwischen Regel- und Förderschullehrkräften in den beteiligten Schulen allerdings nur in sehr geringem Umfang statt. Dies bezieht sich sowohl auf die gemeinsame Unterrichtsvorbereitung, die laut Angaben der Lehrkräfte zu 79,2\% (RSL) bzw. 84,2\% (FSL) eher selten bis gar nicht stattfindet, als auch auf die gemeinsame Unterrichtsdurchführung (73,6\% bzw. 79\%) und Unterrichtsreflexion, wobei diese zumindest nach Einschätzung der Regelschullehrkräfte „nur“ zu 47,2 \% eher selten bis gar nicht, nach Auffassung der Förderschullehrkräfte wiederum zu 73,7\% selten bis gar nicht erfolgt. Diese Werte entsprechen den Ergebnissen bisheriger Studien (Reiser et al., 1984; Helmke, 2010; Behrensen et al., 2012; McDuffie, 2012; Arndt \& Werning, 2013; Serke et al., 2014a) und stellen mit Blick auf die Qualität schulischer Integration ein besonders brisantes Ergebnis dar: Der „gemeinsame“ Unterricht ist offensichtlich - auch aufgrund der oben benannten mangelhaften personellen wie organisatorischen Voraussetzungen - nicht das entscheidende gemeinsame Betätigungsfeld von Regel- und Förderschullehrkräften. Eine wesentliche Qualitätskategorie schulischer Integration (Heese, 1977; Mühl, 1987; Innerhofer \& Klicpera, 1991; Hug, 1994b; Husinsky, 1994; Berges, 1996; Feyerer, 1998; Boban \& Hinz, 2003; Dt. Schulamt, 2004; Bürli et al., 2009; Wernstedt \& Ohnesorg, 2010; Holzinger et al., 2011; Lienhard-Tuggener et al., 2011) wird nicht umfassend realisiert. Gleichzeitig wünscht sich eine große Mehrheit der Befragten, dass das Co-Teaching von Förder- und Regelschullehrkräften möglich sein sollte (SL 90,3\%, RSL 92,5\%, FSL 100 \% stimmen sehr oder eher zu). Wunsch und Wirklichkeit liegen bei diesem Qualitätsbaustein also weit auseinander, ein Ergebnis, das sich mit denen der Studien von Dieckmann et al. (2008), Fussangel \& Gräsel (2012), Pröbstel \& Soltau (2012), Arndt \& Werning (2013), Gebhardt et al. (2013), Soltau \& Mienert (2013) oder Serke et al. (2014) deckt.

Die von Halfhide (2009), Friend \& Cook (2010) oder Holtappels (2013) beschriebenen Vorteile der gemeinsamen Unterrichtsvorbereitung und - durchführung sowie die von Hattie (2013) als für Lernprozesse wesentlich eingestufte gemeinsame Unterrichtsreflexion können demnach keine oder nur geringfügige Auswirkungen auf den Unterricht und die Unterrichtsentwicklung in den befragten Schulen haben. Darüber hinaus können auch höhere Niveaustufen der Kooperation wie „Coordination“ oder „Collaboration“ (Lütje-Klose \& Willenbring, 1999) bzw. Ko-Konstruktion (Fussangel \& Gräsel, 2006) kaum oder nur in Einzelfällen (Bsp.: höhere personelle Ressourcen, fest verankerte Teamzeiten) erreicht werden. 


\section{Maß an Unterstützung}

Trotz der ungünstigen Ausgangsvoraussetzungen hinsichtlich der personellen Ressourcen bzw. gemeinsamer Beratungs-/Teamzeiten wird das Maß an Unterstützung, das die Förderschullehrkräfte in die Regelschulen einbringen, vorwiegend als hoch (SL 48,4\%, RSL 34\%) oder sehr hoch (SL 25,8 \%, RSL 34\%) empfunden. Noch höher sind die Werte bei der Frage danach, inwiefern die Ressourcen der Förderschullehrkräfte in den Regelschulen genutzt werden. Dabei nehmen die Regelschullehrkräfte vor allem Austausch und Beratung sowie die Vorbereitung differenzierter Materialien oder Klassenarbeiten - ähnlich wie in den Studien von Köppel (1984), Arndt \& Werning (2013) oder Laubenstein et al. (2015) - als entlastend wahr. Die 24,5\% der Regelschullehrkräfte, die das Maß an Unterstützung als weniger hoch (24,5\%) oder gar nicht hoch $(3,8 \%)$ einstufen, begründen ihre Bewertung u.a. damit, dass zu wenig FSLStunden und keine Beratungszeiten vorhanden seien (17 Aussagen). Etwas kritischer bewerten auch die Förderschullehrkräfte das Maß an Unterstützung, das ihnen durch die Regelschullehrkräfte entgegengebracht wird ( $50 \%$ hoch, $26,3 \%$ weniger hoch). Sie begründen dies ebenfalls damit, dass eine unterrichtsbezogene Kooperation kaum möglich sei und sie dadurch teilweise „unabhängig vom Regelschullehrer“ arbeiten müssen. Die Nutzung der Ressourcen, die die FSL in die Regelschulen einbringen, wird von mehr als $50 \%$ der SL und RSL sehr positiv bewertet, von den FSL selbst mit mehr als $50 \%$ zumindest eher positiv.

\section{Rollen- und Aufgabenklärung}

Auffällig ist zunächst, dass die Einschätzung der Wahrnehmung von Aufgaben durch die Förderschullehrkräfte (alleine bzw. gemeinsam mit den RSL) durch die befragten Personengruppen sehr unterschiedlich erfolgt. Während die Förderschullehrkräfte selbst angeben, dass sie häufig bis sehr häufig lernstandsdiagnostisch tätig sind (71\%), individuell fördern $(97,4 \%)$, differenziertes Material erstellen (86,8\%) oder Lehrkräfte beraten (89,5\%), geben die Schulleitungen und Regelschullehrkräfte zu diesen Bereichen jeweils geringere Werte an. Insgesamt ist aufgrund der Gesamtergebnisse allerdings davon auszugehen, dass vor allem die individuelle Förderung von Schülerinnen und Schülern einen wesentlichen Arbeitsbaustein der Förderschullehrkräfte darstellt. Der Anteil der Förderschullehrkräfte an Lernstandsdiagnostik, der von den FSL selbst ja als sehr hoch angenommen wird, wird von RSL bzw. SL entweder nicht im selben Ausmaß wahrgenommen oder es erfolgt zu wenig Austausch zu diesem Qualitätsbaustein. Trotz unterschiedlicher Einschätzungen entspricht das Tätigkeitsfeld der FSL den in der Forschung bereits beschriebenen Aufgaben (Reiser et al., 1984; Preuß, 1985; Brugger-Paggi, 2003; Bürli et al., 2009; Wember \& Prändl, 2009; Holzinger et al., 2011). Gleichzeitig kann die unterschiedliche Wahrnehmung der Aufgabenbearbeitung durch die verschiedenen Personengruppen ein Hinweis darauf sein, dass klärende Aushandlungsprozesse zwischen den Professionen noch nicht umfassend erfolgt sind.

Das Ergebnis, dass etwa die Hälfte der Befragten angibt, dass die FSL eher selten bzw. gar nicht in Form von Vernetzungsarbeit mit außerschulischen Diensten kooperieren würden, erstaunt, da das Tätigkeitsfeld diese Kooperation in vielerlei Hinsicht erfordert (Jugendamt, schulpsychologischer Dienst, Ärzte und Therapeuten, Erziehungsberatungsstellen usw.) (Preuß, 1985; Wember \& Prändl, 2009).

\subsubsection{Professionalisierung}

Grundsätzlich stimmen zunächst zwischen 77,4\% (RSL) und 92,1 \% (FSL) der befragten Personen der Aussage zu, dass die schulische Integration von Schülerinnen und Schülern mit sonderpädagogischem Förderbedarf ein hohes Engagement der Lehrkräfte voraussetzt. Die von 
Bryk et al. (2010) beschriebene Work orientation oder von Hattie (2013) benannte „Leidenschaft“ von Lehrkräften, die für schulische Entwicklungsprozesse und vor allem mit Blick auf die Förderung der Leistungsentwicklung notwendig ist, werden also auch für diesen Arbeitsbereich als Grundvoraussetzung eingestuft.

Interne Fortbildungsveranstaltungen zur schulischen Integration in Form von SCHILF werden nur von etwa einem Viertel (SL) bzw. Fünftel (RSL/FSL) der Befragten als durchgeführt angegeben, Pädagogische Tage dagegen von $50 \%$ (RSL/FSL) bzw. $75 \%$ (SL). Diese schulischen Veranstaltungen werden vor allem dazu genutzt um sich inhaltlich-konzeptionell mit den Anforderungen der inklusiven Beschulung bzw. Unterrichtung auseinanderzusetzen (Bsp.: „Inklusion“ übergeordnet, Konzepterarbeitung, Differenzierung/Individualisierung, Umgang mit herausforderndem Verhalten). Sie befassen sich also vorwiegend mit den Bereichen Organisations- und Unterrichtsentwicklung, während bspw. der Qualitätsbaustein Kooperation (Personalentwicklung) nur ein Mal als Inhalt benannt wird.

Während die SFZ sich im Rahmen solcher Veranstaltungen regelmäßig und intensiv mit Fragestellungen der Inklusion befassen und damit eine Weiterentwicklung sonderpädagogischer Professionalität ermöglicht wird, findet dies in strukturierter Fortbildungsform also nur in einem Teil der befragten Schulen statt. Dieses Ergebnis steht zunächst teilweise im Widerspruch zu Ergebnissen sonderpädagogischer Forschung, die die Relevanz solcher Fortbildungsmaßnahmen für schulische Integration belegen (Mutzeck, 1987; Reiser, 1987; Erbring \& Amrhein, 2009; Arndt \& Werning, 2013; Lütje-Klose \& Urban, 2014b). Auch das Ergebnis von Laubenstein et al. (2015) für rheinland-pfälzische Schwerpunktschulen (90\% der Grund- und weiterführenden Schulen nutzen Fortbildungsangeobte für ihre Kollegien) fällt günstiger aus. Stattdessen befassen sich die Schulen allerdings in vielfältigen anderen Formen mit dem Themenfeld (Bsp.: Dienstbesprechungen, Konzeptentwicklung, Austausch mit Vernetzungspartnern etc.). Die Vorteile der Fortbildung ganzer Kollegien (Mutzeck, 1987; Reiser, 1987; Lütje-Klose \& Urban, 2014b) werden noch nicht umfassend genutzt.

Insbesondere die SFZ legen Wert auf Runde Tische mit außerschulischen Diensten. Darüber hinaus erarbeiten sie konzeptionelle Grundlagen für die Umsetzung schulischer Integration wie Arbeitsplatzbeschreibungen für RSL und FSL oder befassen sich mit einzelnen sonderpädagogischen Förderschwerpunkten. Von besonderer Bedeutung ist für die FSL also - vielleicht gerade aufgrund ihrer Tätigkeit im Regelschulsystem - die inhaltlich-pädagogische Zusammenarbeit mit anderen FSL und damit der intraprofessionelle Austausch und Kompetenztransfer.

Obwohl Schulleitungen wie Lehrkräfte einen hohen Fortbildungsbedarf (für sich selbst wie für die Kollegien) sehen (Bsp.: Förderbedarfe, Vernetzung, Konzeptarbeit, Diagnostik und Förderplanung, Individualisierung), nimmt der überwiegende Teil (SL 54,8 \%, RSL 62,2\%, FSL 47,3\%) eher selten bis gar nicht Fortbildungsangebote im Zusammenhang mit schulischer Integration wahr. Gleichzeitig äußern sich wiederum sehr viele Befragte differenziert und ausführlich zur offenen Frage bezüglich der Weiterentwicklung ihrer persönlichen Professionalität im Hinblick auf inklusive Bildung (Bsp.: sonderpädagogisches Fachwissen, Beratungskompetenzen, Diagnostik und Förderplanung, Differenzierung und Individualisierung, Co-Teaching).

Insgesamt zeigt die Auswertung der Daten, dass interne wie externe Fortbildungsveranstaltungen, die einen Bezug zu schulischer Integration haben, eine hohe Variationsbreite aufweisen und entspricht damit bisherigen Ergebnissen sonderpädagogischer Forschung (Feuser \& Meyer, 1987; Dumke et al., 1989; Dt. Schulamt, 2004; Specht et al., 2006; Bürli et al., 2009; Thiele, 2009; Wember \& Prändl, 2009; Speck, 2010; Arndt \& Werning, 2013; Lütje-Klose \& Urban, 2014b). Bis auf die Veranstaltungen, die sich übergeordnet mit dem Thema Inklusion oder Kon- 
zeptentwicklung in diesem Bereich bzw. spezifisch mit bestimmten Förderbedarfen oder Kooperationspartnern befassen, beinhalten die weiteren Fortbildungsangebote vor allem Themen der Allgemeinen Pädagogik (Bsp.: Umgang mit Heterogenität, Methoden o.ä.). Die Komplexität der zu vermittelnden Inhalte (Speck et al., 1978) könnte ein Mitgrund dafür sein, dass die Lehrkräfte ihren Fortbildungsbedarf als hoch einschätzen. Mögliche Transferschwierigkeiten, wie von Jäntsch et al. (2015) beschrieben, wurden nicht erfasst.

\subsubsection{Ergebnisse im Bereich der Personalentwicklung}

\section{Haltung der Lehrkräfte}

Interessant ist die Gegenüberstellung der Ergebnisse zu den Fragestellungen zur Offenheit der Lehrkräfte für die Integration von Schülerinnen und Schülern mit sonderpädagogischem Förderbedarf bzw. zum Maß an Akzeptanz der bisherigen Form schulischer Integration mit denen zur Haltung der Lehrkräfte der zukünftigen inklusiven Bildung gegenüber. Während die Offenheit vor allem von den Schulleitungen wie Regelschullehrkräften positiv eingeschätzt wird (SL 45,2\%, RSL 50,9\%) oder das Maß an Akzeptanz von den Regelschullehrkräften wie Eltern als sehr hoch bewertet wird (RSL 47,2\%, E 61,8\%), halten $0 \%$ der Befragten die Haltung des Kollegiums der künftigen inklusiven Bildung gegenüber für sehr zustimmend. Immerhin wird von $58,1 \%$ der SL, 50,9\% der RSL und 73,7\% der FSL eine eher zustimmende Haltung angenommen, die in erster Linie damit begründet wird, dass schulische Integration ja bereits seit langem praktiziert und als gesellschaftlich notwendig erachtet werde. Sorgen und damit eine eher ablehnende Haltung beziehen sich vor allem auf mangelnde personelle Ressourcen und Angst vor möglicher Mehrarbeit und Überlastung (der RSL), Ergebnisse, die denen von Amrhein (2011) sowie Eberl (2000) entsprechen.

Die grafische Übersicht zum Bereich der Personalentwicklung stellt sich aufgrund der Ergebnisse jetzt folgendermaßen dar:

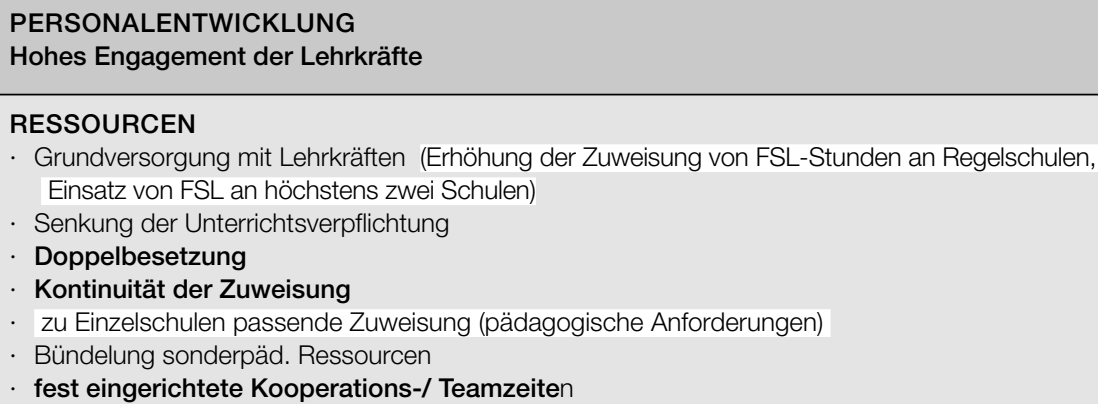


PROFESSIONALISIERUNG

Studium und Vorbereitungsdienst

Fortbildungen (schulintern/schulextern)

Kooperation mit außerschulischen Diensten

Supervision

ERGEBNISSE

Auswirkungen Schulklima?

Auswirkungen Lernergebnisse SuS?

Haltungen

Abb. 21: Qualitätskategorien und -subkategorien im Bereich der Personalentwicklung überarbeitet

\subsubsection{Interpretation der Ergebnisse zur Unterrichtsentwicklung}

\subsubsection{Ressourcen im Bereich der Unterrichtsentwicklung}

\section{Klassengröße}

Die Aussage, dass eine Klasse nicht mehr als 20 Schülerinnen und Schüler haben solle, erfährt insgesamt ein sehr hohes Maß an Zustimmung und stimmt damit als Ergebnis mit der auch in sonderpädagogischen Veröffentlichungen geforderten Größe überein (Heese, 1977; Weigt, 1977; Speck et al., 1978; Mühl, 1987; Dumke et al., 1989; Köbberling, 1994; Brugger-Paggi, 2003; Thiele, 2009; Wember \& Prändl, 2009; Speck, 2010). Gleichzeitig hat der überwiegende Teil der Klassen der Regelschullehrkräfte, die an der Befragung teilgenommen haben (71,3\%), tatsächlich nur bis zu 22 Schüler, 17,1\% der Klassen haben zwischen 23 und 28 Schüler und 12,7\% zwischen 29 und 30 Schüler. Während die Voraussetzungen für differenziertes und leistungsförderndes Arbeiten in Anlehnung an die Forschungsergebnisse von Avenarius (2003), Haselbeck (2007) und von Saldern (2011) im überwiegenden Teil der Klassen hinsichtlich der Größe also bereits günstig sind, ist diese Voraussetzung - vor allem in Klassen der Gemeinschaftsschulen - noch nicht gegeben.

\section{Klassenzusammensetzung und Bündelung von SuS mit spF in einzelnen Klassen}

Der überwiegende Teil der Befragten stimmt den Aussagen eher oder in sehr hohem Maß zu, dass zwar mehrere Schüler mit sonderpädagogischem Förderbedarf in einer Klasse unterrichtet werden sollten, die Anzahl aber nicht mehr als drei bis vier Schüler umfassen sollte. Dieses Ergebnis stimmt mit den sonderpädagogischen Ausführungen zu den Qualitätssubkategorien „ausgewogene Klassenzusammensetzung“ (Wocken, 1987; Feyerer, 1998; Bleidick, 1999; Bless, 2003; Holzinger et al., 2011) bzw. „Vermeidung der Ansammlung von Problemkindern“ (Innerhofer \& Klicpera, 1991; Brugger-Paggi, 2003; Holzinger et al., 2011) überein. Ein differenzierteres Bild ergibt sich bei der Fragestellung, ob in den Regelschulen auch Schüler mit schweren Behinderungen unterrichtet werden sollten. Dieser Frage stehen vor allem die Regel- und Förderschullehrkräfte eher ablehnend gegenüber (RSL 79,2\% stimmen eher nicht oder gar nicht zu; FSL 65,8\%), während Eltern (61,8\%) und Schülerinnen und Schüler der Gemeinschaftsschulen $(75 \%)$ diese Frage sehr oder eher zustimmend beantworten.

\subsubsection{Schulklasse als sozialer Raum}

Insgesamt wird das Klima in den Klassen von den befragten Schülerinnen und Schülern positiv eingeschätzt. So stimmen $87,8 \%$ der SuS mit spF der Aussage zu, dass die SuS sich in der Klasse sehr gut oder gut verstehen. Gleichzeitig geben allerdings über $50 \%$ der Schüler an, dass in der eigenen Klasse nicht alle freundlich zueinander sind. 36,4\% sind der Auffassung, dass sie in den 
Klassen weniger oder gar nicht gut mit Streit umgehen und ebenfalls 36,4\% stimmen der Aussage nicht zu, dass die Lehrer sie gut behandeln. Obwohl diese Antworten zunächst nur ein oberflächliches Bild zur Einschätzung des Klassenklimas vermitteln können und berücksichtigt werden muss, dass es sich um eine kleine Stichprobengröße von SuS handelt, sind die Ergebnisse mit Blick auf soziale Lernprozesse innerhalb der Schulklassen (von Saldern, 1987; Bless, 2003; Brophy, 2010; Muijs \& Reynolds, 2011; Eckermann \& Heinzel, 2013; Helmke, 2015, Huber, 2017) als kritisch einzustufen. Zum einen scheint der freundliche Umgang der Schüler untereinander nicht immer gegeben zu sein, was nach Brophy (2000) ein wesentlicher Faktor für eine unterstützende Lernumgebung ist, zum anderen ist eine "gute Behandlung durch Lehrkräfte" als wichtige Voraussetzung für die Entwicklung einer vertrauensvollen Beziehung zwischen Lehrkräften und Schülern (Hamre \& Pianta, 2001; Marzano \& Marzano, 2003; Hattie, 2013) nur in Teilen vorhanden.

\subsubsection{Klassenführung}

Die Ruhe bzw. Lautstärke in den Klassen ist ein Bereich, der von den befragten Lehrkräften und Schülern sehr unterschiedlich wahrgenommen wird. Es sind in erster Linie die Schüler selbst, die zu 75,7\% angeben, dass es in ihren Klassen weniger oder gar nicht ruhig sei, während die Anteile bei den Lehrkräften geringer sind (RSL 22,7\%, FSL 28,9\%). Gleichzeitig gehen die Lehrkräfte zu etwa einem Drittel und die Schüler zu einem Viertel davon aus, dass die Schüler sich weniger gut konzentrieren können. Insbesondere in Verknüpfung mit dem qualitativen Ergebnis, dass insgesamt 14 Schülerinnen und Schüler mit sonderpädagogischem Förderbedarf angeben noch besser lernen zu können, wenn es in den Klassen leiser sei und sie weniger abgelenkt werden würden, sind dies Hinweise darauf, dass Klassenführung nach Auffassung der SuS als Qualitätsbaustein - wie von Brophy (2006 u. 2010), Helmke (2010), Muijs und Reynolds (2011) oder Hattie (2013) belegt - eine wichtige Funktion für die Erhöhung des Anteils echter Lernzeit haben kann. Obwohl die Lehrkräfte nach übereinstimmenden Angaben aller Befragten gut bis sehr gut für Ruhe sorgen und überwiegend sehr konsequent oder konsequent auf Fehlverhalten reagieren, was nach Marzano und Marzano (2003) als günstig für die Klassenführung einzustufen ist, gelingt es gleichzeitig zwischen einem Sechstel und einem Drittel (Angaben variieren) der Schüler weniger oder gar nicht gut sich an Regeln zu halten. Mit Blick auf herausforderndes Verhalten durch Schülerinnen und Schüler, die Verhaltensauffälligkeiten zeigen, gehen die Regelschullehrkräfte selbst zwar davon aus, dass sie damit zu 81,1\% sicher bis sehr sicher umgehen können, die Förderschullehrkräfte schätzen dies allerdings nur zu 24,2\% so ein.

\subsubsection{Methodik}

Das Maß an klarer Strukturierung des Unterrichts, das u.a. in Anlehnung an Bless (2003), Meyer (2004), Wember \& Prändl (2009) oder Helmke (2015) als Qualitätssubkategorie beschrieben wird, ist nach Auskunft der RSL und FSL vorwiegend in hohem bis sehr hohen Maß gegeben, womit allerdings nicht (wie bei Laubenstein et al., 2015) belegt werden kann, dass dieses Maß auf Veränderungen im Zusammenhang mit integrativer Unterrichtung zurückzuführen ist.

Ähnlich wie in den Studien von Hage et al. (1985) oder Wiechmann (2006) ist der Frontalunterricht in den befragten Schulen die am häufigsten eingesetzte Unterrichtsform: 80,6\% der SL, 79,3\% der RSL, 84,2\% der FSL und 100\% der SuS geben an, dass dieser häufig bzw. sehr häufig erfolge. Zwar werden in den genannten Studien teilweise andere Begriffe und damit Konzepte für die Erhebungen verwendet (Hage: Klassenunterricht; Wiechmann: Lehrervortrag) und anstelle der Einschätzung der Häufigkeiten konkrete Anteile der Sozialformen im Unterricht beobachtet, dennoch lassen sich aufgrund der Ergebnisse zunächst - anders als von Feyerer (1998) für integrative Klassen eruiert - keine offensichtlichen Unterschiede im Vergleich zu 
den in den genannten Studien untersuchten Regelklassen ableiten. Hinsichtlich der Bewertung dieser Unterrichtsform ist interessant, dass diese von den Schülerinnen und Schülern sehr positiv vorgenommen wird. Diese bewerten Lehrervorträge zu 54,5\% als sehr gut und zu $42,4 \%$ als gut. $84,9 \%$ geben darüber hinaus an, dass sie das, was in diesen Vorträgen erklärt wird, gut bis sehr gut verstehen können. Auch die qualitativen Aussagen der Schüler belegen, dass diese zu einem großen Anteil davon ausgehen Sachverhalte aufgrund der Lehrervorträge besser verstehen zu können. Die von Kirschner und anderen (2006) sowie Muijs \& Reynolds (2011) dargestellten positiven Effekte dieser Methode auf die Lernprozesse von Schülern werden also auch von Schülern mit sonderpädagogischem Förderbedarf gesehen.

Die Einzelarbeit wird (erwartungsgemäß) ebenfalls häufig als Sozialform eingesetzt (vergleichbar den Ergebnissen von Götz et al., 2005), wobei die Lehrkräfte diesen Anteil im Vergleich zu den $\mathrm{SuS}$ mit spF als höher wahrnehmen. Der überwiegende Anteil der SuS bewertet diese Sozialform als sehr gut (18,2\%) bzw. gut (51,5\%) und begründet dies damit, dass man sich bei dieser Arbeitsform gut konzentrieren könne und nicht gestört werde. Manche erläutern allerdings auch, dass sie Aufgaben ohne Hilfestellung von anderen nicht gut bearbeiten können. Am positivsten bewerten Schülerinnen und Schüler mit spF die Partnerarbeit als Methode (33,3\% sehr gut, 54,5\% gut) und begründen dies u.a. damit, dass man sich bei dieser Arbeitsform gegenseitig helfen könne und sie Spaß mache. Während Regelschullehrkäfte vorwiegend eine sehr häufige bzw. häufige Umsetzung von Partnerarbeit angeben (79,2\%), schätzen FSL und SuS mit spF diesen Anteil als geringer ein (57,9\% bzw. 54,5\%). Knapp die Hälfte der SuS gibt eine eher seltene Umsetzung an. Am seltensten wird offensichtlich die Sozialform Gruppenarbeit angeboten, was den Ergebnissen von Götz u.a. (2005) und Muijs und Reynolds (2011) entspricht. Auch hier ergeben sich unterschiedliche Einschätzungen der Häufigkeit, vor allem zwischen RSL und SuS. Die Gruppenarbeit wird von den $\mathrm{SuS}$ mit spF etwas kritischer bewertet als die anderen Arbeitsformen. Zwar bestehe auch hier die Möglichkeit sich untereinander zu helfen, allerdings könne es auch vorkommen, dass es in der Gruppe zu laut sei und keine Konzentration auf die Aufgabenstellung erfolge, ein Hinweis, der die hohen Anforderungen kooperativer Aufgabenstellungen kennzeichnet (Huber, 2010; Helmke, 2010; Muijs \& Reynolds, 2011; Brüning \& Saum, 2015) und verdeutlicht, dass die u.a. von Kiper und Mischke (2008) oder Muijs und Reynolds (2011) beschriebenen notwendigen Teilkompetenzen für Arbeitsorganisation und Kommunikation in den Gruppen relevant sind.

Die in der sonderpädagogischen Literatur als Qualitätskategorien benannten offenen Unterrichtsformen (Berges, 1996; Feyerer, 1998; Specht et al., 2006; Wember \& Prändl, 2009; Thiele, 2009; Holzinger et al., 2011) werden in den befragten Schulen nicht in umfassender Form eingesetzt, sondern - abgesehen von der Stationenarbeit, die nach Wahrnehmung der RSL häufig (39,6\%) bis sehr häufig (5,7\%) als Methode genutzt wird - vorwiegend eher selten bis gar nicht. Das sind Ergebnisse, die mit schulartübergreifenden Studien wie MARKUS oder DESI in Teilen übereinstimmen, aber ungünstiger sind als bspw. die im ERINA-Schulversuch eruierten (Liebers et al., 2014).

\subsubsection{Differenzierung und Individualisierung}

Die Angaben zur Umsetzung binnendifferenzierten Unterrichts variieren zwischen den befragten Personengruppen erheblich. Auch bei einer vorsichtigen Gewichtung der unterschiedlichen Angaben lassen diese allerdings den Schluss zu, dass binnendifferenzierte Angebote im gemeinsamen Unterricht keine Selbstverständlichkeit darstellen, sondern zu nicht unwesentlichen Anteilen nur selten oder gar nicht gegeben sind (SL 25,8\% eher selten, RSL 18,9\% eher selten bis gar nicht, FSL $50 \%$ eher selten bis gar nicht, S 52,5\% eher selten bis gar nicht). Dieses Ergebnis erstaunt, da die Angaben zu den SuS mit spF verdeutlichen, dass an den Grundschulen 38\% (74 SuS mit spF Lernen, 3 mit spF geistige Entwicklung) und an den Gemeinschaftsschulen sogar 53\% (94 SuS 
mit spF Lernen) der SuS mit spF zieldifferent unterrichtet werden müssen. Gleichzeitig erfährt die Aussage, dass Kinder mit spF bei Bedarf in einzelnen Stunden individuell gefördert werden sollten, insgesamt ein sehr hohes Maß an Zustimmung (abgesehen von den SuS selbst, die diese Aussage vorsichtiger positiv bewerten). Mit Blick auf die konkrete Umsetzung bewerten Eltern und Schülerinnen und Schüler mit spF die Form der individuellen Förderung außerhalb der Klasse als gut (E 29,4\%, S 39,4\%) bzw. sehr gut (E 50\%, S 54,5\%), während SL und RSL dies etwas kritischer sehen. Sehr viele der Befragten äußern sich darüber hinaus in qualitativen Aussagen zu dieser Form der Förderung. Dabei gehen die Meinungen dazu zwar zum Teil auseinander (Kritik an Separation, „Nachhilfeunterricht“ etc.), befürwortet wird die individuelle Förderung, die zu einem größeren Teil als Gruppenförderung stattfindet, allerdings vor allem mit der Begründung, dass diese ein hohes Maß an Ruhe und Konzentration ermögliche und andererseits auch die Klasse entsprechend nicht akustisch gestört werde. Die Möglichkeit individueller Zuwendung zu den Schülerinnen und Schülern oder die Funktion dieser Förderung als „Schutzraum“ werden genauso hervorgehoben wie das hohe Maß an Effizienz, das dieser Förderform eigen sei.

Offensichtlich ist der Anteil an individueller Förderung außerhalb des Klassenraums in den an der Befragung beteiligten Schulen hoch und wird - anders als in der sonderpädagogischen Forschung, die innere Differenzierung als günstiger einstuft als äußere Differenzierung (Bächthold et al., 1996; Feyerer, 1998; Bless, 2003; Boban \& Hinz, 2003; Thiele, 2009) - gleichzeitig vorwiegend positiv bewertet. Die Befragten beschreiben insgesamt eine flexible Handhabung der Umsetzung der Förderung inner- oder außerhalb des Klassenraums, als Einzel- oder Gruppenförderung. Mit Blick auf die Einschätzung der Fördermöglichkeiten für bestimmte sonderpädagogische Förderbedarfe halten mehrere Befragte die integrative Unterrichtung von SuS mit dem spF soziale und emotionale Entwicklung für besonders problematisch, eine Einschätzung, die mit mehreren Studien übereinstimmt (Feuser \& Meyer, 1987; Dyson, 2010; Ellinger \& Stein, 2012; Laubenstein et al., 2015).

Die teilweise widersprüchlich scheinenden Angaben zu differenzierten Arbeitsmaterialien für zieldifferent unterrichtete SuS mit spF könnten Hinweise darauf sein, dass diese im Klassenunterricht zum Teil dieselben Bücher und Arbeitshefte verwenden wie ihre Mitschülerinnen und Mitschüler, während für die Einzel- und Gruppenförderung andere Materialien durch die FSL angeboten werden, was allerdings nur eine Vermutung darstellen kann. Insbesondere die abweichenden Angaben zu zieldifferenten Klassenarbeiten (Mathematik: RSL 52,8\%, FSL 86,8\%; Deutsch: RSL 47,2\%, FSL 92,1\%) werfen Fragen dahingehend auf, inwiefern diese unterschiedlichen Angaben auf unzureichenden Informationsfluss zwischen RSL und FSL zurückgeführt werden können oder ob die SuS mit spF zum Teil sowohl Klassenarbeiten in der Klasse als auch in Einzel- oder Gruppensituationen mit den FSL schreiben. Fraglich ist in diesem Zusammenhang auch, wie unter diesen Umständen eine differenzierte Bewertung von zieldifferent unterrichteten $\mathrm{SuS}$ vorgenommen wird.

Förderpläne sind nach Angaben der Befragten nur zu 35\% bis 50 \% für die SuS mit spF vorhanden, während diese in der sonderpädagogischen Forschung als wesentliches Qualitätsmerkmal integrativ arbeitender Schulen aufgeführt werden (Preuß, 1985; Innerhofer et al., 1991; Boban \& Hinz, 2003; Brugger-Paggi, 2003; Specht et al., 2006; Feyerer, 2007; Bürli et al., 2009; Lienhard-Tuggener et al., 2011). Rückmeldungen zu Lernfortschritten, die vor allem Hattie (2013) als wirksam mit Blick auf Lernprozesse einstuft, geben FSL den SuS mit spF häufiger als RSL und zwar vorwiegend in mündlicher Form oder als Rückmeldungen in Klassenarbeitsoder Mitteilungsheften. Schriftliches Feedback im Rahmen von Entwicklungsberichten oder Beiblättern zum Zeugnis erfolgen selten. Insgesamt geben diese Ergebnisse Hinweise darauf, 
dass die systematische, schriftliche Dokumentation des Lernstands bzw. der Lernfortschritte und Förderplanung in den integrativen Schulen noch keine Selbstverständlichkeit ist.

\subsubsection{Ergebnisse im Bereich der Unterrichtsentwicklung}

Insgesamt liegen zur Einschätzung der Lernfortschritte der SuS mit spF vorwiegend gute bis sehr gute Einschätzungen vor. Lediglich die Regelschullehrkräfte nehmen diese weniger positiv wahr als die anderen Befragten. Sie bewerten die Lernfortschritte in Mathematik ungünstiger als die im Fach Deutsch und bewerten die Entwicklung im sozialen Bereich zu 25\% als weniger gut. Die Befragten stellen in ihren Begründungen für die Lernfortschritte vielfältige Zusammenhänge her: zur Schule als sozialem Raum (Schulklima), zu offenen Lernangeboten sowie Differenzierung bzw. Individualisierung, zu differenzierter Leistungsbewertung, Aktivierung und Motivierung der SuS mit spF durch die Lehrkräfte, zur (unterrichtsbezogenen) Kooperation der Lehrkräfte untereinander sowie - mit Blick auf Verbesserungsmöglichkeiten - zu Ressourcen (Räume, Personal, feste Kooperationszeiten, Klassengröße).

Aus Ergebnissen und Interpretation heraus ergibt sich folgende grafische Darstellung:

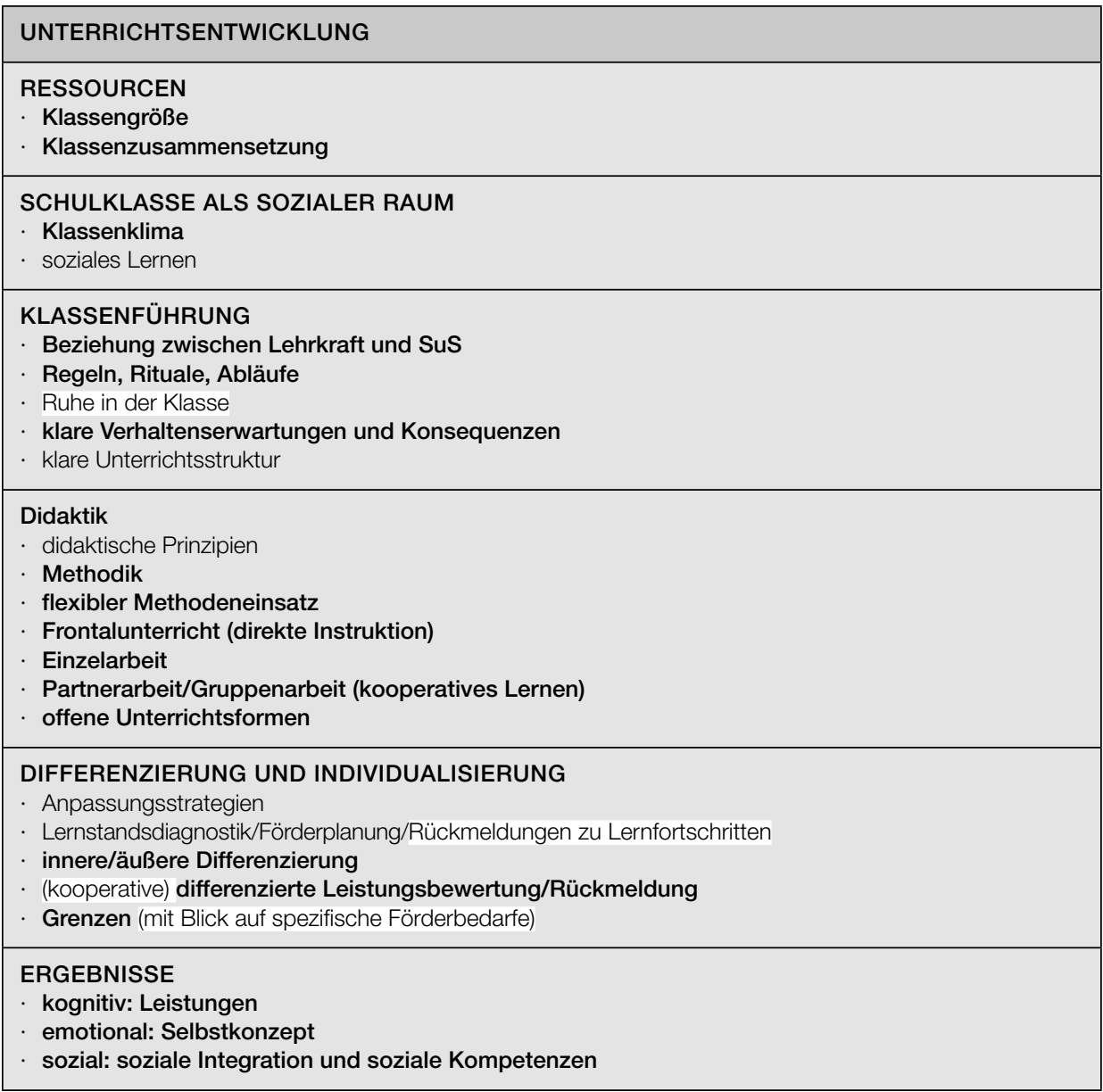

Abb. 22: Qualitätskategorien und -subkategorien im Bereich der Unterrichtsentwicklung überarbeitet 


\subsection{Zusammenführende Darstellung, kritische Einordnung und Perspektiven der zentralen Ergebnisse der qualitativen und der quantitativen Studie zur Qualität der schulischen Integration im Saarland}

\subsubsection{Organisationsentwicklung}

\section{Ressourcen}

Mit Blick auf die Ressourcen, die für die Gestaltung von Rahmenbedingungen in den integrativ arbeitenden Schulen des Saarlandes notwendig sind, zeigt sich sowohl im Rahmen der Interviews als auch der schriftlichen Befragung, dass diesen von den befragten Personen eine zentrale Rolle zugemessen wird. Es überwiegt eine ungünstige Einschätzung dahingehend, dass diese sowohl in sächlicher wie in personeller Hinsicht (s. dazu Ressourcen Personal) nicht in ausreichendem Maß vorhanden sind. Anschubinvestitionen sind für die Beteiligten nicht wahrnehmbar. Es überwiegen Sorgen dahingehend, dass schulische Inklusion aufgrund dieser Rahmenbedingungen scheitern sowie die weitere Entwicklung beschränkt werden könnte und eine indirekte Form der Steuerung dahingehend erfolgt, dass Eltern sich für die Wahl einer Förderschule als Förderort für ihr Kind entscheiden könnten bzw. dass Lehrkräfte und Schulleitungen eine ablehnende Haltung zur inklusiven schulischen Bildung entwickeln. Es lässt sich festhalten, dass gute materielle Voraussetzungen zur Umsetzung schulischer Integration in den an den Befragungen beteiligten Regelschulen fehlen. Anders als in der Forschungsliteratur spielt das Thema der Barriefreiheit von Gebäuden offensichtlich eine geringe Rolle, was mit dem niedrigen Anteil an SuS mit den spF körperliche-motorische Entwicklung bzw. Sinnesbehinderungen zusammenhängen könnte. Gleichzeitig werden angemessene spezifische Hilfen sowie räumliche Bedingungen für SuS mit Schwerhörigkeit oder Sehbehinderungen als wichtig benannt (Akustik, Beleuchtung). Darüber hinaus zeigt die schriftliche Befragung, dass die Größe der Klassenräume nur zu einem Drittel zur Größe der Klassen passt und lediglich zu etwas mehr als $15 \%$ flexible Gestaltungsmöglichkeiten bietet, d.h. dass eine wichtige Voraussetzung für offenes, flexibles und differenziertes Unterrichten innerhalb der Klassen fehlt.

Eine sehr viel wichtigere Rolle spielen - vor allem für die befragten Förderschullehrkräfte - die Nutzungsmöglichkeiten von Differenzierungsräumen für individuelle Förderung, Kleingruppenförderung oder spezifische Anforderungen (Bsp.: Entspannung, Bewegung etc.). Die Situation ist diesbezüglich an den Schulen sehr unterschiedlich. Schulen, in denen gar kein (20\%) bzw. nur teilweise (30\%) Differenzierungsräume von Lehrkräften genutzt werden können, können - auch unabhängig von $\mathrm{SuS}$ mit spF - keine flexiblen Unterrichtsgestaltungsmöglichkeiten (Bsp.: Formen des Co-Teaching, bei denen in zwei Räumen gearbeitet wird, Arbeit in Förderbändern o.ä.) bieten, was in Teilen die sogenannte „Flurpädagogik“ zur Folge hat. Ein Mitgrund dafür, dass die Differenzierungsräume für die Befragten eine wichtige Rolle spielen, könnte darin bestehen, dass ein großer Teil der sonderpädagogischen Förderung in Form äußerer Differenzierung erfolgt (s.u.).

Besonders schwierig ist für die integrativ arbeitenden Schulen offensichtlich die Anschaffung von Differenzierungsmaterial und Hilfsmitteln, da sie diese über ihren gewöhnlichen - nicht auf die spezifischen Bedarfe ausgelegten - Haushalt anschaffen bzw. zum Teil selbst kaufen müssen (Förderschullehrkräfte, Eltern). Nur in einem Drittel der befragten Schulen bzw. einem Sechstel der Klassenräume sind entsprechende Materialien vorhanden. Dieses Problem wurde auch von Schön \& Stark (2012 und 2014) für saarländische Schulen aufgezeigt.

Die Hypothese, dass nicht davon auszugehen ist, dass die Schulen barrierefrei gestaltet sind und über flexible Raumnutzungsmöglichkeiten bzw. eine ausreichende Anzahl an Differenzie- 
rungsräumen verfügen, kann also für einen großen Anteil der befragten Schulen als zutreffend eingestuft werden. Ähnliches gilt für die Materialausstattung, die - u.a. mit Blick auf die zum Teil sehr spezifischen Anforderungen einzelner Schüler oder Schülerinnen mit spF - in diesen Schulen nicht immer adäquat zu sein scheint. Die aufgeführten Subkategorien zur Ressourcenausstattung werden von den Befragten in einen engen Zusammenhang damit gestellt, dass methodisch variantenreiches und differenziertes Unterrichten erschwert sowie Möglichkeiten des Co-Teaching nur eingeschränkt umsetzbar sind.

In Verknüpfung mit den Ausführungen unter 2.1 hängen diese Ergebnisse damit zusammen, dass die Bildungsausgaben in der BRD insgesamt im europäischen Vergleich lediglich im Mittelfeld einzuordnen sind und diese im Vergleich zu anderen Ländern keinen Vorrang vor anderen Ausgaben haben. Das Ziel bestmögliche Voraussetzungen für die (individuelle) Förderung von Schülerinnen und Schülern zu schaffen scheint nicht im Fokus der Bildungspolitik des Bundes bzw. der Länder zu stehen. Vorweggreifend mit Blick auf die Ausführungen zu Ressourcen Personal und Unterricht lässt sich festhalten, dass weder die Unterrichtsverpflichtung der Lehrkräfte (und Schulleitungen) noch die Obergrenzen für Klassen in der Primar- und Sekundarstufe an die Anforderungen in Schulen mit einer sehr heterogenen Schülerschaft und den damit zusammenhängenden erweiterten Aufgabengebieten (Bsp: Vernetzung, Kooperation von Lehkräften, Differenzierung) angepasst worden sind. Während das Deutsche Institut für Menschenrechte, der Deutsche Städtetag, die Verbände bzw. Gutachten für die Entwicklung inklusiver Bildungsangebote (Anschub-) Investitionen zur Einführung und Umsetzung inklusiver Bildung klar benennen, finden diese bspw. in den Koalitionsvereinbarungen des Saarlandes keinen Niederschlag. Auch die Unterschiedlichkeit schulspezifischer finanzieller Unterstützung findet bislang (abgesehen bspw. von angepassten Klassenteilern in Brennpunktstadtteilen Bremens oder Hamburgs bzw. der standortspezifischen Berechnung des Budgets an Förderschullehrkräften im Saarland) keine Berücksichtigung. Wie Schön und Stark für die saarländischen Pilotschulen beschrieben haben, wäre in diesem Zusammenhang eine engere Kooperation zwischen den Bildungs- und Finanzministerien, der Eingliederungshilfe und den Schulträgern sowie gegebenenfalls den Krankenkassen dringend notwendig.

Perspektiven für diesen Bereich können sich Schulen hinsichtlich der Weiterentwicklung inklusiver Bildungsangebote nur in sehr geringem Umfang selbst erschließen, bspw. durch die Umnutzung selten genutzter Räume zu Differenzierungsräumen, die Anschaffung flexibel einsetzbaren Mobiliars oder die Bündelung von Materialien in der Schule (Bsp.: digitaler Pool von Arbeitsmaterialien). Ansonsten stellen die barriefreie Umgestaltung von Schulgebäuden und Schulgeländen oder die Verbesserung der technischen Ausstattung (Bsp.: Raumakustik, Beleuchtung, Digitalisierung) in erster Linie die Träger der Schulen vor Herausforderungen. Die Länder sind dahingehend gefragt eine Änderung der Schulbaurichtlinien mit Blick auf Anforderungen in der Arbeit mit heterogenen Gruppen vorzunehmen. Diese beinhalten bspw. eine Raumgröße für Schulklassen, die pro Schüler einen Raumbedarf von etwa $2 \mathrm{~m}^{2}$ vorsieht $^{70}$, womit den Anforderungen für eine flexible Raumgestaltung nicht entsprochen werden kann. Insbesondere bei Neubauten geht es um eine fachübergreifende nachhaltige Planung von Schulen, die flexible Bildungsangebote bereithalten können sollen.

\section{Leitung einer Schule}

Die Bedeutung der Leitung einer Schule für schulische Integration bzw. die Weiterentwicklung schulischer Prozesse wurde in den Interviews nur von einem Elternteil aufgegriffen und

70 Beispiel: Amtsblatt des Ministeriums für Bildung, Wissenschaft,Jugend und Kultur Rheinland-Pfalz 03/2010, S. 102 
daher - abgesehen von zwei Fragen zur Offenheit der Schulleitung für diesen Bereich bzw. zur Zusammenarbeit zwischen Schulleitungen und Eltern - nicht in die schriftliche Befragung einbezogen. Diese Kategorie stellt also einen ersten „blinden Fleck“ der vorliegenden Arbeit dar. Die Hypothese dazu, dass die Schulleitungen integrative Prozesse an ihren Schulen unterstützen, kann für die beteiligten Schulen in hohem Maß bestätigt werden. Wie in der bisherigen sonderpädagogischen Forschung auch, spielt die Schulleitung allerdings - wie in der zweiten Hypothese zu dieser Kategorie formuliert - mit Blick auf von den Beteiligten wahrgenommene Veränderungen in den Schulen zunächst keine wichtige Rolle.

Entscheidende Hinweise für Perspektiven in diesem Bereich ergeben sich allerdings sowohl in den Interviews als auch bei der quantitativen Erhebung dahingehend, dass einerseits die umfassenden Erfahrungen und tief gehenden Kenntnisse der Leitungen der Förder- und Beratungszentren und andererseits der intra- wie interprofessionelle Austausch auf Schulleitungsebene (Regel- wie Förderschulen und Förder- und Beratungszentren) wesentliche Ansatzpunkte für Kompetenzaustausch, Kompetenztransfer und Weiterentwicklung schulischer Integration hin zu inklusiven Bildungsangeboten darstellen können. Da aus den Aussagen der Schulleitungen der Regelschulen sowie den Einschätzungen der Lehrkräfte und Eltern hervorgeht, dass diese den mit der Thematik zusammenhängenden Entwicklungsaufgaben vorwiegend aufgeschlossen und unterstützend gegenüberstehen (75\%), gleichzeitig aber auch mögliche Probleme bzw. Grenzen gezielt kennzeichnen können, sind Personen in dieser Funktion zentrale Dreh- und Angelpunkte für Transformationsprozesse. Ein im Vergleich zur bisherigen Forschung neuer Qualitätsbaustein kann - wie die vorwiegend positiven Bewertungen der Eltern zeigen - die direkte Kooperation zwischen Schulleitungen und den Eltern von Kindern mit spF sein, wobei sich die Frage stellt, inwiefern diese Kooperation auch in der Zusammenarbeit mit den Eltern von Kindern ohne spF relevant bzw. dann entsprechend zeitlich möglich ist. Neben der persönlichen Haltung und dem Engagement können die Schulleitungen im Vergleich zu anderen Beteiligten einfacher eine „Vogelperspektive“ auf das System der eigenen Schule sowie die Vernetzung in der Kommune und die Zusammenarbeit mit der Schulaufsicht einnehmen. Aufgrund ihrer Funktion sind sie dazu angehalten sich mit bildungspolitischen Entwicklungen in ihrem Bundesland auseinanderzusetzen. Aus dieser Vogelperspektive heraus und in Kooperation mit der Schulgemeinschaft tragen sie die Verantwortung für die Entwicklung ihrer Schule, für Zielorientierung, Strukturen und Abläufe, eine Stundenplangestaltung, die paralleles Arbeiten bzw. Kooperationszeiten ermöglicht, Kooperation und Professionalisierung des Kollegiums, die pädagogisch-didaktisch-methodische Qualität des Unterrichts und eine enge Einbeziehung von Eltern und Schülern. Im Idealfall sind Kompetenzen und Know-How bei den Schulleitungen - mit Blick auf Visionen, aber auch mögliche Grenzen der Umsetzung inklusiver Bildung gebündelt und können - wie oben angemerkt - durch den Austausch zwischen Schulleitungen gleicher und unterschiedlicher Schulformen transferiert und multipliziert werden. Von besonderer Bedeutung kann in diesem Zusammenhang eine transparente und konstruktive Zusammenarbeit von Seiten der Schulverwaltung und der Bildungsministerien mit den Schulleitungen sein, die - zumindest in fachlich-inhaltlicher Hinsicht - auf Augenhöhe statt gedacht in hierarchischen Strukturen stattfinden sollte. Wenn diese Kooperation auch Lehrerbildungseinrichtungen wie Universitäten, Studienseminare und Lehrerfortbildungsinstitute sowie Vertreter der Schulträger involvieren würde, wäre diese auf mehreren Ebenen zukunftsweisend denkbar und würde sich vom durch den geschichtlichen Überblick entstehenden Eindruck einer eher unabhängigen Arbeitsweise positiv-konstruktiv unterscheiden. Ähnlich wie beim Schulversuch in Chicago (Bryk et al., 2010) sind dazu Vertrauen der Kooperationspartner mit Blick auf die 
Aufgabenwahrnehmung und das Gewähren eines hohen Maßes an Autonomie für die Schulleitungen und Schulen relevant.

\section{Schule als sozialer Raum}

Die im Rahmen der übergeordneten Kategorie Schule als sozialer Raum benannten Subkategorien für ein gutes Schulklima wie Respekt, Akzeptanz und soziale Integration sind in den an den Befragungen teilnehmenden Schulen in hohem Maß vorhanden, was als sehr positives Ergebnis für die saarländischen Schulen gewertet werden kann und von den Befragten zum Teil auf die langjährige Integrationserfahrung dieser Schulen zurückgeführt wird. Sowohl die Beziehungen der Schüler untereinander als auch die zwischen Lehrkräften und Schülern werden überwiegend positiv eingeschätzt, auch wenn sich Unterschiede zwischen einzelnen Schulen ergeben. Während Freundschaften innerhalb der Schule offensichtlich gepflegt werden, erscheint der Bereich der nachmittäglichen Kontakte zwischen Schülerinnen und Schülern mit und ohne spF ausbaufähig, ein Bereich allerdings, auf den die Schulen eher wenig Einfluss ausüben können, da „PeerVoraussetzungen" in diesem Zusammenhang entscheidend sind (Müller et al., 2012; Eckermann \& Heinzel, 2013). Mögliche exklusive Prozesse in diesem Zusammenhang können, wie unter 2.2.1 ausführlich beschrieben, unter Umständen auf eine Kumulation von Ausgrenzungsfaktoren bei einzelnen Schülerinnen und Schülern zurückgeführt werden und kennzeichnen damit auch allgemeine gesellschaftliche Entwicklungen.

Während die Hypothese, dass das Schulklima an inklusiven Schulen von Respekt, Wertschätzung und Akzeptanz aller Beteiligten untereinander geprägt ist und diese sich in ihren Schulen wohlfühlen insgesamt bestätigt werden kann, können also kaum Aussagen dahingehend getroffen werden, wie sich dieses innerschulische Klima auf das außerschulische Leben und Erleben der Schülerinnen und Schüler mit und ohne spF auswirkt. Die Hypothese, dass Integration in diesen Schulen als gemeinsame Aufgabe aufgefasst wird, der die Beteiligten offen gegenüberstehen, kann zumindest indirekt in Teilen verifiziert werden. Der zweite Teil dieser Hypothese, dass auch Eltern und Schülerinnen wie Schüler in die Weiterentwicklung der Schulen einbezogen sind, lässt sich aufgrund der Befragungen allerdings nicht positiv belegen, so dass sich für diese Subkategorie eine Perspektive dahingehend aufzeigen lässt, dass eine stärkere Einbindung - aufgrund der Erfahrungswerte und der großen Offenheit der Eltern und Schüler für inklusives Arbeiten - wichtig wäre und hilfreiche Impulse für die Weiterentwicklung der Qualität inklusiver Bildunsangebote ermöglichen kann.

Von besonderem Interesse - ggf. auch für weitere Forschungsvorhaben - sind die Zusammenhänge, die sich nach Analyse der Ergebnisse zwischen verschiedenen anderen Subkategorien mit der des Schulklimas ergeben. Dazu gehört zum einen der Zusammenhang zwischen dem Schulklima und der Offenheit und dem Engagement von Lehrkräften, der von den Befragten hergestellt wird, indem sie u.a. darauf verweisen, dass es die Unterstützung der Lehrkräfte ist, die für die SuS mit spF zu größerem Wohlbefinden innerhalb der Schule beiträgt. Das Wohlbefinden der Schüler wiederum scheint im Zusammenhang mit ihrer Lern- und Leistungsbereitschaft zu stehen. Dabei spielt die Beziehung zwischen Lehrkräften und Schülern eine entscheidende Rolle. Darüber hinaus zeigt sich ein möglicher negativer Zusammenhang zwischen der mangelnden Akzeptanz von Schülern und deren Wohlbefinden sowie Lern- und Arbeitsverhalten. Dazu können Lernabstandserfahrungen oder soziale Probleme mit anderen Schülern beitragen sowie das Erfahren sozialer Ablehnung. Anknüpfungsmöglichkeiten ergeben sich an dieser Stelle zu den bildungssoziologischen bzw. pädagogischen Überlegungen von Trautmann \& Wischer (2011) sowie Tenorth (1994) oder Heitmeyer et al. (2015), die in ihren Arbeiten auf das Dilemma von 
Schulen im Spannungsfeld zwischen institutionellen Anforderungen und Individualisierung, die Bedeutung vorschulischer Sozialisation bzw. gesellschaftlich bedingte Ausgrenzungsrisiken hinweisen. Grundsätzlich ist davon auszugehen, dass Schülerinnen und Schüler negative wie positive Erfahrungen in diesem Bereich in allen Schulen, unabhängig von der jeweiligen Schulform, sammeln und Schulen diese auf gesamtschulischer oder klassenbezogener Ebene mit Blick auf das Schul- oder Lernklima in höherem oder geringerem Maß pädagogisch bearbeiten.

Auch wenn die Subkategorie Integration als gemeinsame Aufgabe von den Befragten kaum direkt thematisiert wird, kann sie doch als Hintergrund eingeschätzt werden, vor dem bestimmte Aussagen getätigt werden, wie bspw. die zum langjährigen und intensiven Engagement einiger Schulen im Zusammenhang mit schulischer Integration.

Die Subkategorie des Schulklimas ist also ein Teilbereich der Qualität schulischer Integration, der an den schon über einen längeren Zeitraum integrativ arbeitenden Schulen positiv ausgeprägt ist, positiv wertgeschätzt wird und keine „Großbaustelle“ darstellt. Die Bedeutung der Schulgemeinschaft kann aufgrund der Gesamtergebnisse der qualitativen und quantitativen Befragung als wesentlich für alle Beteiligten eingeschätzt werden.

Interessant wäre in diesem Zusammenhang zu erfahren, warum FSL die Situation vorsichtiger einschätzen. Darüber hinaus könnte weitere Forschung den Zusammenhang zwischen dem Schuklima sowie einem lernförderlichen Klima in den Klassen und der Leistungsentwicklung von Schülern in (inklusiven) Schulen erschließen, indem Aspekte wie die Unterrichtsgestaltung oder die Kooperation von Lehrkräften in Erhebungen einbezogen werden.

Ein Desiderat für (saarländische) Schulen ist - wie die Ergebnisse nahelegen - eine stärkere Einbindung von Eltern, Schülern und ggf. Netzwerkpartnern in schulische Entwicklung. Wie u.a. die qualitativen Antworten der Eltern und Schüler zeigen, verfügen diese - aufgrund ihrer ebenfalls oft mehrjährigen Erfahrung mit schulischer Integration - über eine differenzierte Wahrnehmung schulischer wie unterrichtlicher Prozesse und formulieren - wie Lehrkräfte und Schulleitungen auch - vielfältige Veränderungsvorschläge, vor allem mit Blick auf das Lernen. Hinsichtlich weiterer Forschung wären wiederum insbesondere die Perspektiven der Schülerinnen und Schüler mit und ohne $\mathrm{spF}$ - nicht nur ihre Lernfortschritte, wie in bisheriger Forschung meistens üblich - aufzugreifen und intensiver zu analysieren. Wie bspw. ihre Wahrnehmung von Ruhe bzw. Unruhe in den Klassen und ihre diesbezüglichen Ideen zeigen, können sie sich sehr gut als Expertinnen und Experten für Unterricht und Schulleben einbringen.

Zur Zusammenarbeit der Schulleitungen, Regel- und Förderschullehrkräfte mit den Eltern der SuS mit spF liefern die beiden Teilstudien - ähnlich wie zum Schulklima - ebenfalls ein positives Bild. Die Eltern werden auf unterschiedliche Art und Weise ins Schulleben eingebunden (neben Elternabenden bspw. durch Stammtische, Feste oder Projekte). Zumindest in quantitativer Hinsicht und mit Blick auf die vielfältigen Formen gegenseitiger Kontakte geben die Ergebnisse Aufschluss darüber, dass die Kooperation offen und auf kurzen Wegen erfolgt - über Mitteilungshefte der Schüler, Telefonate oder E-Mails. Beratungsgespräche, Runde Tische oder Förderkonferenzen finden in regelmäßigen Abständen statt, allerdings nach Angaben einiger Befragten und vor allem aus Sicht der Eltern nicht immer häufig. Elterngespräche finden manchmal im Team, d.h. gemeinsam mit RSL und FSL statt. Teilweise werden dazu außerschulische Vernetzungspartner eingeladen. Gespräche in dieser vernetzten Form sind vor allem hinsichtlich einer umfassenden und systemisch orientierten Kind-Umfeld-Diagnose und entsprechenden Förderplanung sinnvoll (Sander, 1993). Interessant ist - wie unter Leitung der Schule bereits erwähnt - das hohe Maß an Unterstützung, das die Eltern von SuS mit spF aus ihrer Sicht durch die Schulleitungen erfahren. Die Aufschlüsselung der Gesprächsinhalte durch 
die befragten Personen zeigt, dass diese vorwiegend die Organisation der integrativen Förderung, die bereits genannte Kind-Umfeld-Diagnose und Förderplanung sowie die Vernetzung mit außerschulischen Partnern wie Therapeuten etc. thematisieren. Von wichtiger Bedeutung für den Austausch mit den Eltern sind darüber hinaus Verstärkerpläne oder Logbücher im Sinne gemeinsamer Feedbacksysteme.

Die eingangs aufgestellte Hypothese, dass die Zusammenarbeit mit den Eltern in den integrativen Schulen sowohl in quantitativer wie in qualitativer Hinsicht intensiv gestaltet wird und es entsprechend häufige und qualitätsvolle Kontakte vor allem in Form gemeinsamer regelmäßiger Gesprächstermine gibt, kann also in Teilen bestätigt werden. Was die Häufigkeit und Qualität der Gespräche betrifft sowie darüber hinausgehend ein umfassendes „Parent Engagement“ (Pushor, 2007; Berthelsen \& Walker, 2008; Bryk et al., 2010), entsprechen die Ergebnisse allerdings nur eingeschränkt den Erwartungen der bisherigen Forschung, sowohl, was die Daten zur Häufigkeit betrifft, als auch dahingehend, dass die erwähnten Elternkontakte nicht in allen Schulen in der gleichen Intensität und Qualität stattzufinden scheinen. Insbesondere die Tatsache, dass nur in einem Interview eine Aussage dazu erfolgt, dass es gezielt Gespräche zum ISTStand und zu möglichen weiteren Lernprozessen eines Schülers gibt, und qualitative Ergebnisse der quantitativen Erhebung, die belegen dass neben diesen Gesprächsinhalten noch eine Fülle weiterer Aspekte mit Eltern geklärt werden (Bsp.: organisatorische Fragen, Vernetzung mit auBerschulischen Partnern), weisen indirekt darauf hin, dass eine noch stärkere Fokussierung auf die Zusammenarbeit mit Blick auf die Lernprozesse der Schülerinnen und Schüler möglich sein kann.

Die von Brugger-Paggi (2003), Lienhard-Tuggener et al. (2011) sowie Werning (2013) als Qualitätskategorie benannte stärkere Einbindung der Eltern hinsichtlich der Lernprozesse von Schülern könnte aufgrund der vorliegenden Ergebnisse also intensiviert werden, bspw. durch einen regelmäßigen Austausch zum Lernstand der Schülerinnen und Schüler sowie durch eine gemeinsame Förderplanung von Lehrkräften und Eltern bzw. auch den Schülerinnen und Schülern selbst. Die Häufigkeit dieser Gespräche ist dabei - auch mit Blick auf die Arbeitsbelastung der Lehrkräfte oder Berufstätigkeit der Eltern - nicht so entscheidend wie eine (stärkere) Strukturierung und Systematisierung dieser Gespräche hin zu einfachen Zielvereinbarungen für die weiteren Lernprozesse der Schülerinnen und Schüler mit und ohne spF. In diesem Zusammenhang können auch die oben genannten Feedbacksysteme zwischen Schule und Eltern bedarfsorientiert verstärkt genutzt werden (Bsp.: Rückmeldung zu Arbeits- und Sozialverhalten, Verstärkerpläne, etc.). Darüber hinaus können Schulen zu Themen, die die Eltern oder auch Schüler interessieren, Fortbildungen anbieten (Berthelsen \& Walker, 2008; Innerhofer \& Klicpera, 1991).

Die Voraussetzungen für eine qualitative Weiterentwicklung der Zusammenarbeit mit den Eltern sind in den befragten Schulen als gut bis sehr gut einzuschätzen, da die Kooperation auch jetzt schon einen wichtigen Qualitätsbaustein der Schulen darstellt und zwar sowohl aus Sicht der SL wie aus Sicht der Lehrkräfte und Eltern. Schwierig bleibt - wie einzelne Aussagen zu dieser Thematik zeigen - die Einbindung von Eltern, die wenig bis gar kein Interesse an der schulischen Bildung oder Lernentwicklung ihrer Kinder zeigen. In diesem Zusammenhang können oft nur niedrigschwellige Angebote (Bsp.: zwei verpflichtende Austauschtermine im Schuljahr, kurze Telefonate o.̈.) erfolgen. Die Schaffung solcher verbindlicher Strukturen (bspw. durch eine schulische Vereinbarung mit Eltern und Schülern zu Beginn des Schuljahres) kann unterstützend sein. Insbesondere mit Blick auf die Sozialisations- oder Bildungshintergründe von Eltern (s. dazu 2.2.1 und 2.2.2) und die damit verbundenen möglichen Schwierigkeiten des Fin- 
dens einer gemeinsamen Sprache spielt der in den Erhebungen genannte Aspekt der „Arbeit auf Augenhöhe“ eine wesentliche Rolle. Nur wenn die Eltern als gleichberechtigte Partner (Jeynes 2005a und 2005b; Pushor, 2007; Bryk et al., 2010) in die schulische Arbeit einbezogen werden, kann deren Verantwortung für Entwicklungsprozesse ihrer Kinder entsprechend gestärkt werden. Mit Bezug darauf, dass die vorschulische Sozialisation nicht veränderbar ist (Tenorth, 1994), können im Austausch mit den Eltern gegenwärtige Sozialisationsbedingungen der Kinder und Jugendlichen gemeinsam erfasst und ggf. Lösungsvorschläge für problematische Entwicklungen (Bsp.: Medienkonsum) erarbeitet werden.

Nur bei einer solch qualitätsvollen Gestaltung der Zusammenarbeit mit den Eltern ist davon auszugehen, dass positve Effekte auf die Lernentwicklung der Schülerinnen und Schüler mit und ohne spF erzielt werden können (Jeynes 2005a und 2005b; Pushor, 2007; Berthelsen \& Walker, 2008; Bryk et al., 2010).

Die wichtigsten Netzwerkpartner der Schulen sind - wie erwartet - das Jugendamt, der schulpsychologische Dienst sowie der schulärztliche Dienst (in dieser Reihenfolge). An etwa einem Drittel der Schulen findet die Zusammenarbeit mit diesen außerschulischen Einrichtungen allerdings eher selten statt. Eine enge Zusammenarbeit erfolgt in einigen Schulen mit den Schoolworkern, die (im Saarland) als Bindeglied zwischen Schule und Jugendhilfe tätig sind. Ein positives Ergebnis der Erhebungen kann in der großen Bandbreite weiterer Netzwerkpartner der Schulen (schul- und ggf. auch standortspezifisch) gesehen werden, durch die aufgezeigt wird, dass Lehrkräfte und Schulleitungen sich differenziert mit Unterstützungs- und Fördermöglichkeiten für die SuS mit spF auseinandersetzen. Aufgrund der Spezialisierung dieser Vernetzungspartner kann davon ausgegangen werden, dass die Zusammenarbeit mit diesen Partnern in erster Linie einzelfallbezogen erfolgt. Teilweise werden sie - wie die Ausführungen in den vorangegangenen Abschnitten zeigen - zu Runden Tischen für einzelne Schülerinnen und Schüler mit spF in die Schulen eingeladen. Auf übergeordneter Ebene und mit der Zielrichtung einer strukturierteren und systematischeren Ausrichtung der Kooperation (Ahrens, 2009; De Boer und Spies, 2014) werden - sowohl in den Regelschulen als auch an den SFZ - darüber hinausgehend Runde Tische als Vernetzungstreffen mit dem schulpsychologischen Dienst, den Jugendämtern, dem schulärztlichen Dienst oder anderen Vernetzungspartnern veranstaltet und dienen damit einer Professsionalisierung dieser Zusammenarbeit. Vergleichsweise wenig genannt werden im Zusammenhang mit schulischer Integration andere Netzwerkpartner wie Vereine, Musikschulen etc.. Da nach diesen aber nicht gezielt gefragt wurde, ist das Ergebnis nicht aussagekräftig.

Die Hypothese, dass inklusive Schulen in ihrer Umgebung sehr gut vernetzt sind und professionell u.a. mit den Jugendämtern, dem schulpsychologischen Dienst sowie Vereinen etc. kooperieren, kann aufgrund der Erhebungen also nur in Teilen bestätigt werden. Unterstützungsnetzwerke scheinen noch nicht in allen Schulen umfassend ausgebaut bzw. professionell ausgestaltet zu werden (Ahrens, 2009). Demnach kann - wie in der Interpretation der quantitativen Erhebung bereits ausgeführt - nicht davon ausgegangen werden, dass die in der Studie von Bryk et al. (2010) aufgeführten möglichen positiven Effekte der Vernetzungsarbeit in den Schulen der Interviewpartner greifbar sind oder bereits umfassend zur Entlastung (Schnitzer, 2008) beitragen. Zwar darf aus den Ergebnissen, die belegen, dass eine Zusammenarbeit mit den Jugendämtern, dem schulpsychologischen Dienst etc. nicht an allen Schulen im Zusammenhang mit der Integration von $\mathrm{SuS}$ mit spF erfolgt, nicht geschlossen werden, dass diese immer und unbedingt einzelfallbezogen notwendig wäre, sondern stattdessen vor allem dann hilfreich ist, wenn schwierige soziale Hintergründe, psychische oder medizinische Auffälligkeiten bei SuS mit oder 
ohne spF vorliegen. Andererseits könnte die - auch übergeordnete - Vernetzung der Schule im Stadtteil oder der Gemeinde, in Kooperation mit den genannten Einrichtungen sowie Vereinen einen wichtigen Beitrag hinsichtlich der Förderung der gesellschaftlichen Teilhabe aller Schülerinnen und Schüler leisten. Das Lebenslagen-Konzept von Engels (2006) oder das ICF-Modell von Behinderung (2005) bieten in diesem Zusammenhang wichtige Analyseschablonen. Weitere Forschung könnte dazu beitragen Inhalte und Qualität dieser Vernetzung mit Blick auf die pädagogische Bearbeitung von Problemen der Schülerinnen und Schüler zu fokussieren. Zielrichtung bezüglich der Entwicklung inklusiver Bildungsangebote kann für Schulen sein kooperative Handlungen, strukturierte und professionalisierte Abläufe mit Netzwerkpartnern so zu gestalten, dass eine Wechselbeziehung zwischen der Unterstützung und Entlastung der Schulen (Schulleitungen, Lehrkräfte, Eltern) sowie der Schaffung von Zugangsmöglichkeiten zu Kindern und Jugendlichen für die Netzwerkpartner (Schnitzer, 2008) als gegenseitige Bereicherung (auch durch Perspektivwechsel) gegeben sind. Voraussetzung dafür sind - unabhängig von den Möglichkeiten der einzelnen Schulen - eine entsprechende Ressourcenausstattung sowie die Offenheit möglicher Netzwerkpartner. Eine stärkere Vernetzung könnte entlastend sein, Schülerinnen und Schüler systemisch in den Blick nehmen und in Teilen verstärkt durch Förderschullehrkräfte erfolgen. Von wichtiger Bedeutung ist in diesem Zusammenhang die enge Kooperation mit den Schoolworkern als den Bindegliedern zwischen Schule und Jugendhilfe, da die Jugendhilfe insbesondere mit Blick auf die Gestaltung von Unterstützungsangeboten für Schülerinnen und Schüler mit dem sonderpädagogischen Förderbedarf soziale und emotionale Entwicklung eine zentrale Bedeutung hat.

\section{Organisationsstrukturen}

Neben der Funktion der Leitung von Schulen bezüglich inklusiver Bildungsangebote stellen Organisationsstrukturen und deren Veränderung einen zweiten großen blinden Fleck der vorliegenden Forschung dar. Entwicklungsprozesse der Schulen auf übergeordneter organisatorischer Ebene werden - von einer vereinzelten Aussage zu klassenübergreifenden Fördergruppen abgesehen - nicht benannt und scheinen für die beteiligten Schulen, bspw. auch bezüglich der Wünsche und Ideen für die Zukunft inklusiver Bildung, keine Rolle zu spielen. Dabei stellen veränderte Zeit- und Raumkonzepte (Meiers, 1982), unterschiedliche organisatorische Möglichkeiten der integrativen Beschulung (Muth, 1977; Sander, 2003) oder jahrgangsübergreifende Unterrichtskonzepte (Kucharz et al., 2009; Kucharz, 2010; Stäbler et al., 2015) laut Theorie und Forschung geeignete Wege für eine Öffnung und Flexibilisierung der Schulgestaltung dar. Eine strukturierte Weiterentwicklung von integrativen Schulen auf organisatorischer Ebene scheint in den befragten Schulen also nicht stattgefunden zu haben.

Dementsprechend kann die Hypothese, dass grundlegende organisatorische Strukturen an inklusiven Schulen wenig verändert werden, die darauf gründet, dass diese in Schulen grundsätzlich nur wenige Veränderungen erfahren (Meiers, 1982), als bestätigt gelten. Jahrgangsübergreifende Angebote in Eingangsklassen werden allerdings in den Erhebungen - anders als in einer Hypothese vermutet - nicht beschrieben und auch die angenommenen klassenübergreifenden Fördergruppen finden nur einmal Erwähnung.

In umfangreichem Maß kann die Hypothese bestätigt werden, dass die Beschulung von Schülerinnen und Schülern teilweise in Form äußerer Differenzierung erfolgt. Da Entscheidungen zu dieser organisatorischen Maßnahme allerdings nicht auf übergeordneter schulorganisatorischer Ebene, sondern klassen- bzw. schülerbezogen stattfinden, erfolgt die Darstellung dieser Subkategorie unter dem Bereich Unterrichtsentwicklung(Differenzierung/Individualisierung). Auch 
zur bewussten Gestaltung von Übergängen zwischen Kindergärten, Grundschulen, weiterführenden Schulen oder berufsbildenden Angeboten werden keine Angaben gemacht. Da Organisationsstrukturen aufgrund der geringen Relevanz für die Interviewpartner nicht als Item in die quantitative Erhebung aufgenommen wurden, sind die Ergebnisse einerseits vorsichtig zu bewerten, andererseits liegt dennoch die Schlussfolgerung nahe, dass in diesem Bereich ein hohes Maß an noch ungenutztem Veränderungspotenzial vorhanden ist. Vorbehaltlich weiterer Forschung kann die Hypothese formuliert werden, dass im Saarland trotz jahrzehntelanger schulischer Integration keine tiefgreifenden organisatorischen Veränderungen in den Schulen stattgefunden haben, die mit der schulischen Integration an sich zusammenhängen. Diese sind vielmehr aus anderen Gründen geschehen (Bsp.: Ganztagsoffensive der Bundesregierung, Einführung der Gemeinschaftsschule).

Für die Gestaltung zukünftiger inklusiver Bildungsangebote bietet eine systemisch-vernetzte Herangehensweise an organisatorische Veränderungen (Bsp.: jahrgangsübergreifender Unterricht, Ganztagsangebot) in Verbindung mit pädagogisch-didaktischen Veränderungen auf tiefer gehenden unterrichtlichen Ebenen eine hilfreiche Perspektive. Diese komplexen Veränderungen sind in kleinen, weniger komplexen Systemen (Bsp.: kleine Grundschulen) ggf. einfacher umsetzbar als in größeren und damit komplexeren Systemen. Da diese Veränderungen nur in Teilen in der Verantwortung von Einzelschulen umsetzbar sind (Bsp.: Einrichtung kleiner und großer Klassen, paralleles Arbeiten in Jahrgängen, Förderbänder, andere Rhythmisierung), sind darüber hinaus zum Teil zunächst gesetzliche Rahmenbedingungen für solche Gestaltungsspielräume der Schulen zu schaffen.

Während die Leitbildentwicklung und damit die Auseinandersetzung mit (pädagogischen) Grundhaltungen in einem großen Teil der Schulen (70\%) selbstverständlich ist, scheinen Schulprogramme oder eine Evaluation der eigenen Arbeit - wie die Ergebnisse belegen - mit Blick auf schulische Integration kein qualitativer Schwerpunktbereich für saarländische Schulen zu sein. Positiv hervorzuheben sind dagegen Ideen zu Förderkonzepten (SFZ und Regelschulen), die Aspekte der Unterrichtsentwicklung (Bsp.: (Fach-) Didaktik, Methoden) berücksichtigen. Von wichtiger Bedeutung scheinen für die beteiligten Schulen auch Konzepte zum Umgang mit Unterrichtsstörungen zu sein, die in $60 \%$ der Schulen vorhanden sind. Dies kann auch als indirekter Hinweis darauf gedeutet werden, dass das Thema Klassenführung in den befragten Schulen eine wichtige Rolle spielt. Die Hypothese, dass Schulen, in denen Schülerinnen und Schüler mit spF unterrichtet werden, zum Großteil über Leitbilder und Schulprogramme verfügen, das Thema schulische Integration/Inklusion dabei aber nicht unbedingt im Vordergrund steht, kann also in Teilen als bestätigt gelten.

Zur kritischen Einordnung dieser Ergebnisse ist festzuhalten, dass die Teilstudie von Friedrich (2015) im Rahmen von PInG belegt, dass Schulprogramme nicht zu messbaren Auswirkungen auf Lernergebnisse von Schülerinnen und Schülern führen, so dass die tatsächliche Relevanz für die Qualität schulischer Integration nicht ausreichend belegt werden kann und weitere Forschung notwendig wäre um diese Fragestellung näher zu beleuchten. Als Annahmen für weitere Studien können formuliert werden, dass Entwicklungsprozesse in den Schulen ggf. wichtiger sind als die schriftlichen Ergebnisse dieser Prozesse (Holtappels, 2010e) und dass „inklusive" Schulentwicklung in den beteiligten Schulen zunächst nicht automatisch als Bestandteil „allgemeiner" Schulentwicklung in Schulprogrammen aufgegriffen wird bzw. mögliche Verknüpfungen zwischen allgemeinpädagogischen Konzepten (Bsp.: Konzepte zum Umgang mit Unterrichtsstörungen) und der Unterstützung von SuS mit dem spF soziale und emotionale Entwicklung für die Beteiligten nicht unbedingt präsent sind. 


\subsubsection{Personalentwicklung}

\section{Ressourcen}

Das Thema Ressourcen mit Blick auf das Personal hat aus Sicht der befragten Personen (Interviews und Fragebögen) eine sehr hohe Relevanz für die Qualität der schulischen Integration, was insgesamt 190 qualitative Aussagen, die dazu in beiden Teilstudien getätigt wurden, belegen. Diese beziehen sich nicht nur - wie teilweise in anderen Veröffentlichungen üblich auf eine bessere personelle Ausstattung allgemein, sondern differenzieren zwischen folgenden Subkategorien: Anzahl an Förderschullehrkräften je Regelschule, Anzahl an Schulen je Förderschullehrkraft, Doppelbesetzung, Förderschullehrerstunden je Klasse bzw. Schüler, Unterrichtsverpflichtung von RSL und FSL sowie im Stundenplan fest verankerte Kooperationszeiten. Alle Subkategorien werden kritisch beleuchtet und es ergeben sich eindeutige Belege dahingehen, dass die Anzahl an Förderschullehrkräften bzw. die Anteile an Förderschullehrerstunden in den Schulen/Klassen (im Schnitt je Schüler mit sonderpädagogischem Förderbedarf etwa zwei Förderschullehrerstunden wöchentlich; von 83 bis $92 \%$ der Befragten als unzureichend gekennzeichnet) und Möglichkeiten zur Doppelbesetzung in zu geringem Maß vorhanden sind, während die Anzahl der Schulen je Förderschullehrkraft und die Unterrichtsverpflichtung der RSL und FSL als zu hoch eingeschätzt werden. Ein besonders kritisches Ergebnis der quantitativen Befragung ist darin zu sehen, dass $74 \%$ bis $80 \%$ der Schulen keine Beratungszeiten zwischen Förder- und Regelschullehrkräften im Stundenplan vorsehen. Bezüglich der Qualitätssicherung schulischer Integration erscheint die saarländische Maßnahme, die zuvor vorgesehenen Beratungsstunden zwischen Regel- und Förderschullehrkräften im Saarland im Zuge der Einführung der Inklusionsverordnung und der budgetierten Zuweisung von FSL an Regelschulen abzuschaffen, als nicht nachvollziehbar.

Bei der Befragung nicht erhoben wurde der Anteil an Stunden der Klassenleitung in der eigenen Klasse, der von den Interviewpartnern ebenfalls als gute Voraussetzung für schulische Integration eingestuft wird. Aus pragmatischen Gründen und als „Notlösung“ werden Schülerinnen und Schüler mit spF teilweise in bestimmten Klassen gebündelt, damit in diesen Klassen eine größere Anzahl von Förderschullehrerstunden eingebracht werden kann. Als erhebliche Erschwernis für die Qualitätsentwicklung schulischer Integration im Saarland ist also zusammenfassend festzuhalten, dass die Voraussetzungen für eine unterrichtsbezogene Kooperation zwischen Regelund Förderschullehrkräften in mehrfacher Hinsicht fehlen.

Darüber hinaus werden Zusammenhänge (auch als Begründungen für die Verbesserung der Personalsituation) zwischen diesen Ressourcen und der Qualität der sonderpädagogischen Förderung sowie der Qualität der Kooperation zwischen Förder- und Regelschullehrkräften in den Regelschulen beschrieben, u.a. da fest verankerte Zeiten für Kooperation und Beratung sowie die Doppelbesetzung mindestens in den Hauptfächern als Grundvoraussetzungen für unterrichtsbezogene Zusammenarbeit und Lernstandsdiagnostik sowie differenziertes Arbeiten in den Klasse nicht umfassend genug gegeben sind. Systemische Arbeitsansätze, innerhalb derer ganze Kollegien Konzepte für die sonderpädagogische Förderung in einer Regelschule entwickeln und umsetzen (Lütje-Klose \& Urban, 2014b) werden nicht erwähnt. Gleichzeitig kann nicht davon ausgegangen werden, dass diese Ansätze eine Lösung für grundsätzliche personelle Ressourcenprobleme bieten.

Zwei Wirkfaktoren, die nach Auffassung der Befragten mit Blick auf die Zuweisung von Förderschullehrkräften an Regelschulen wesentlich sind, stellen die Kontinuität sowie die Passung dieser Zuweisung dar. Unter der Gewährleistung von Kontinuität verstehen Schulleitungen und Lehrkräfte dabei, dass Förderschullehrkräfte für einen längeren Zeitraum an eine oder mehrere 
bestimmte Regelschulen abgeordnet werden ${ }^{71}$. Die Begründungen dafür lassen sich dem Bereich der Kooperation von Lehrkräften zuordnen, da sie beinhalten, dass diese längeren Zeiträume für die Entwicklung einer vertrauens- und niveauvollen Kooperation der Förder- und Regelschullehrkräfte benötigt werden und die Einarbeitungsphase gemischter Teams mehrere Jahre dauern könne. Außerdem gehen insbesondere die Schulleitungen der Regelschulen davon aus, dass eine Kontinuität in der Zusammenarbeit zwischen Förderschullehrkraft bzw. -kräften und Schule positiv zur Akzeptanz der integrativen Unterrichtung beitragen kann. Unter der passenden Zuweisung von Förderschullehrern an Regelschulen verstehen die Leitungen der Förder- und Beratungszentren die Idee, dass Eigenheiten der Lehrkräfte (Bsp.: systemische Orientierung, Offenheit für neue Unterrichtsansätze) etc. und Eigenheiten der Schulen (Bsp.: Einführung selbstorganisierten Lernens o.ä.) hinsichtlich der Abordnungen Berücksichtigung finden sollten.

Eine multiprofessionelle Personalisierung liegt im Saarland nicht in den Händen der Schulleitungen, da die Einstellung weiteren Personals über die Jugendämter, die Eingliederungshilfe oder die Schulträger erfolgen müsste. Unter anderem aufgrund der unzureichenden personellen Rahmenbedingungen profitieren die Schulen vom Einsatz von Integrations- oder Eingliederungshilfen, der von den Beteiligten vielfach als notwendig und mehrfach als bislang ungünstig organisiert (Bsp.: Dauer von der Antragstellung bis zum konkreten Einsatz) oder unzureichend erachtet wird.

Die eingangs aufgestellten Hypothesen zur Ressourcenausstattung lauteten aufgrund der bisherigen Forschungsergebnisse folgendermaßen:

Die Ressourcenausstattung in den inklusiven Schulen (ausreichende Anzahl ausgebildeter Regel- und Förderschullehrkräfte, ausreichende Anzahl an FSL-Stunden, die den Regelschulen für die schulische Integration zugewiesen werden) sieht nicht so aus, dass eine Doppelbesetzung im gemeinsamen Unterricht durchgängig bzw. in den Kernfächern möglich ist. Eine Kontinuität der Zusammenarbeit ist nur teilweise gegeben, da Förderschullehrkräfte bestimmten Schulen nicht immer über längere Zeiträume (mehrere Jahre) hinweg zugewiesen sind. In den meisten Schulen sind fest eingerichtete Team- bzw. Beratungszeiten nicht vorhanden, so dass die konkrete Umsetzung der Förderung von Schülerinnen und Schülern mit spF häufig an die Förderschullehrkräfte delegiert wird und äußere Differenzierung erfolgt.

Bis auf den Aspekt der Kontinuität der Zuweisung, der nicht konkret erhoben wurde, können damit alle Hypothesen zur Ressourcenausstattung mit Personal bestätigt werden. Hinsichtlich möglicher Perspektiven für die Weiterentwicklung inklusiver Bildungsangebote könnten alternative Einsatzmöglichkeiten im Stundenplan „doppelt gesteckter“ Lehrkräfte sowie die in der Schweiz erprobten Unterrichtsteams eine ressourcenschonende Möglichkeit für Schulen darstellen um unterrichtsbezogene Kooperation zu unterstützen. So könnte eine Jahrgangsstufe eine Förder- oder Regelschullehrkraft, die in den Hauptfächern einer Klasse als Doppelbesetzung vorgesehen ist, auch so einsetzen, dass aus vier Deutsch- oder Mathematikgruppen flexibel und bedarfsorientiert fünf kleinere Gruppen gebildet werden, die aufgrund ihrer geringeren Größe binnendifferenziertes Arbeiten erleichtern. Diese können - ebenfalls je nach Bedarf eher nach homogenisierenden (Bsp.: Phasen direkter Instruktion für Einführungen in Unterrichtsthemen) oder heterogenisierenden (Bsp.: kooperative Aufgabenstellungen) Kriterien für einzelne Stunden oder über längere Zeiträume hinweg gebildet werden.

71 Da die Schulleitungen der Förder- und Beratungszentren die Aufgabe der Abordnung einzelner Förderschullehrkräfte an die Regelschulen dezentral vornehmen und diese nicht über die Schulaufsicht erfolgt, können die Aspekte Kontinuität und Passung berücksichtigt werden. 
Wenn in Jahrgangsstufen bspw. in den Hauptfächern inhaltlich parallel gearbeitet wird, könnte zumindest die grobe Vor- und Nachbereitung von Unterricht innerhalb der Teams erfolgen, so dass nicht eine Beratungsstunde pro Klassenleitung und Förderschullehrkraft, sondern „nur“ ein bis zwei im Stundenplan geblockte Kooperationsstunden je Team eingerichtet werden müssten. Wie weiter oben dargestellt beschreiben die Lehrkräfte u.a. das gegenseitige Vertrauensverhältnis (Bryk, 2010), gemeinsame Entwicklungsprozesse, das gleichmäßige Engagement der Beteiligten, gleichberechtigtes Arbeiten sowie gemeinsame Ziele (Preuß, 1985) als wichtige Kennzeichen solcher Teams, die damit Chancen bieten, die von den befragten Lehrkräften als Zukunftswünsche für inklusive Bildung formuliert werden.

Auch dafür sind allerdings von den Bildungs- bzw. Finanzministerien der Länder Ressourcen zur Verfügung zu stellen (bspw. durch Senkung der Unterrichtsverpflichtung). Darüber hinaus können Schulleitungen bei der Stundenplanung gemeinsame Zeiten der Jahrgangsteams vorsehen. Bei parallelem Arbeiten können binnendifferenzierte Materialien in mehreren Klassen gleichzeitig genutzt werden. Ein Server, auf dem Unterichtsplanungen und Materialien hochgeladen werden können, ist eine sinnvolle Ergänzung für die Arbeit in den Teams bzw. für die Gestaltung eines gemeinsamen Materialpools der Schule. Nur wenn Beratungszeiten zwischen RSL und FSL regelmäßig und nicht zwischen Tür und Angel ermöglicht werden, sind ein Austausch auch auf systemischer Ebene und damit eine nachhaltige Weiterentwicklung der Qualität des Unterrichts möglich. Die punktuelle Teilnahme der Schulleitungen an den Jahrgangsteams bzw. die Weiterleitung von Protokollen können zur schulübergreifenden Koordination beitragen und damit eine Querverbindung zur Organisationsentwicklung darstellen.

Für die Kooperation zwischen Lehrkräften allgemein werden mit Blick auf schulische Integration innerhalb der Regelschulen Dienstbesprechungen, Gesamtkonferenzen, stufenbezogene Förderkonferenzen sowie Förderausschüsse als institutionalisierte Formen der Kooperation und damit systemische Voraussetzung für eine strukturierte Auseinandersetzung mit der Thematik erwähnt. Von wichtiger Bedeutung für die Förderschullehrkräfte ist darüber hinaus der intraprofessionelle Austausch, der über die Förder- und Beratungszentren bereits in Form von Dienstbesprechungen, Gesamtkonferenzen oder Arbeitskreisen organisiert wird (s. dazu auch weitere Ausführungen unter Professionalisierung), während der Kompetenztransfer, der aus den Förderzentren in die Regelschulen und umgekehrt erfolgt, durch interprofessionelle Kooperation noch verstärkt werden könnte.

Die spezifische Kooperation zwischen Regel- und Förderschullehrkräften sowie zwischen Schulleitungen und Förderschullehrern wird an den befragten Schulen als gut bis sehr gut bewertet, auch wenn die Förderschullehrkräfte in ihrer Bewertung vorsichtiger sind als die Schulleitungen und Regelschullehrkräfte, ggf. da sie höhere Erwartungen haben. Entscheidende Voraussetzungen für eine gelingende Kooperation wie Offenheit, Toleranz, die Arbeit auf Augenhöhe und Vertrauen scheinen in den Schulen vorwiegend gegeben. Darüber hinaus wird das besondere Engagement der Förderschullehrkräfte in seiner Bedeutung für eine gute Zusammenarbeit benannt. Die Offenheit für Kooperation steht für die Befragten im Zusammenhang mit den Rahmenbedingungen (Anzahl der Stunden der FSL, Zeitfenster für Kooperation), die dafür in den Schulen vorhanden bzw. nicht vorhanden sind.

Inhalte des Austauschs zwischen FSL und RSL umfassen eine Fülle unterschiedlicher Themenfelder (organisatorische Fragen, Zusammenarbeit mit Vernetzungspartnern, differenzierte Leistungsbewertung, gemeinsame Elternarbeit, Zusammenarbeit mit Integrationshilfen, fallbezogene Absprachen, diagnostische Fragestellungen, Möglichkeiten der Verbesserung der Förderung). Ob mit Blick auf das Zeitproblem auch Analysen schulischer Inklusion auf der Meta-Ebene oder eine 
strukturierte und koordinierte Weiterentwicklung anstelle einzelfallbezogener Fragestellungen aufgegriffen werden können, kann aus den Angaben der Befragten nicht geschlussfolgert werden. Neben Dienstbesprechungen, Konferenzen oder schulinternen Fortbildungen kann ggf. auch der Austausch zwischen den Schulleitungen der Regelschulen und den Förderschullehrkräften zu einer solch übergeordneten Auseinandersetzung mit Fragen schulischer Integration beitragen, so dass die Kompetenzen der FSL besser in die Regelschulen „aufgenommen und eingebaut“ werden können als bisher. In einigen Schulen werden regelmäßig Teamgespräche, Inklusionstreffen oder so genannte Förderkonferenzen (Bsp.: jahrgangsbezogene Konferenz, bei der Kurzbesprechungen zu allen SuS mit spF erfolgen) als über die üblichen Konferenzen oder Fortbildungen hinausgehende Möglichkeiten für Kooperation und Kompetenztransfer durchgeführt.

Problematisch sind insbesondere die Ausführungen zur Umsetzung der Kooperation einzustufen, aus denen hervorgeht, dass häufig Tür- und Angelgespräche (in Pausen, in Zeiten vor oder nach dem Unterricht) für Austausch und Absprachen genutzt werden müssen, während gemeinsam vereinbarte Gespräche für $35 \%$ der Regelschullehrkräfte und etwa 20 \% der Förderschullehrkräfte eher selten bis gar nicht stattfinden. Aufgrund der Ressourcensituation erstaunt das Ergebnis, dass die Kooperation der RSL und FSL an den untersuchten Schulen vorwiegend den Niveaustufen „Austausch“ und „Kooperation“ (Fussangel \& Gräsel, 2012) bzw. „Co-Activity“, „Cooperation“ und „Coordination“ (Lütje-Klose \& Willenbring, 1999) zugeordnet werden kann, wenig. Dabei findet Beratung nicht nur einseitig statt, dahingehend dass FSL die RSL beraten (Reiser et al., 1984; Dumke et al., 1989), sondern es wird auch von gegenseitiger Beratung gesprochen, der ein hoher Stellenwert zugemessen und die als Gewinn bringend beschrieben wird. Seltener erfolgen - aus o.g. Grund - gegenseitige Rückmeldungen zu Unterrichtsinhalten oder Lernfortschritten bzw. gemeinsame Unterrichtsvor- oder -nachbereitung.

Trotz der ungünstigen Ausgangsvoraussetzungen hinsichtlich der personellen Ressourcen bewerten Schulleitungen und Regelschullehrkräfte das Maß an Unterstützung, das die Förderschullehrkräfte in die Regelschulen einbringen, sowie die Nutzung der Ressourcen der FSL vorwiegend als hoch oder sehr hoch, wobei Austausch und Beratung und die Vorbereitung differenzierter Materialien oder Klassenarbeiten als entlastend wahrgenommen werden. Gegebenenfalls hängt diese positive Bewertung damit zusammen, dass FSL schon seit etwa 1998 (mit flächendeckender Einrichtung der Förder- und Beratungszentren) immer stärker in den Regelschulen vertreten sind und sich damit auch die Kooperation zwischen FSL und RSL etabliert hat. Die „Ressourcen“ der FSL werden also wertgeschätzt und genutzt, obwohl diese sich zum Teil nicht in erster Linie als unterrichtende Lehrkräfte im „klassischen Sinn“ in die Regelschulen einbringen (können) und eine direkte Unterstützung der RSL im Rahmen unterrichtsbezogener Kooperation kaum möglich ist. Etwas kritischer bewerten die Förderschullehrkräfte das Maß an Unterstützung, das ihnen durch die Regelschullehrkräfte entgegengebracht wird, führen dies aber darauf zurück, dass eine unterrichtsbezogene Kooperation aufgrund der Ressourcen kaum möglich sei.

Die unterrichtsbezogene Zusammenarbeit umfasst hinsichtlich der gemeinsamen Unterrichtsplanung vor allem die Vorbereitung differenzierter Materialien oder Klassenarbeiten durch die Förderschullehrkräfte, nicht aber die gemeinsame Unterrichtsplanung im engeren Sinn. CoTeaching-Formen, die genutzt werden, lassen sich daher vor allem den Formen „One teach one observe“ bzw. „One teach - one assist“ zuordnen, so dass die Förderschullehrkräfte sich vorwiegend „nachrangig“ in den Unterricht einbringen können. Während ein Großteil der FSL und RSL sich Co-Teaching wünscht (zwischen 90 und $100 \%$ der Befragten), können in den befragten Schulen weder eine gemeinsame Unterrichtsvorbereitung (zu etwa $80 \%$ selten bis 
gar nicht), Unterrichtsdurchführung (etwa $75 \%$ selten bis gar nicht) oder Nachbereitung (zwischen $47 \%$ RSL und $74 \%$ FSL selten bis gar nicht) umfassend umgesetzt werden. Die Brisanz dieser Ergebnisse liegt - wie in der Interpreation weiter oben bereits erwähnt - vor allem darin, dass der sogenannte „gemeinsame“ Unterricht (gemeint ist normalerweise der gemeinsame Unterricht von $\mathrm{SuS}$ mit und ohne spF) nicht als gemeinsamer Unterricht von RSL und FSL durchgeführt werden kann und somit ein ganz wesentlicher Bestandteil der Qualität der schulischen Integration nur in sehr geringem Umfang gegeben ist. Gründe dafür können in den unzureichenden personellen Ressourcen gesehen werden (geringe Anzahl von Förderschullehrerstunden je Klasse, fehlende gemeinsame Beratungszeiten) und können daher durch die Schulen selbst keine Veränderung erfahren. Diese Tatsache ist ggf. auch ein Mitgrund dafür, dass sich der Unterricht an den integrativ arbeitenden Schulen (s. Ausführungen unter Unterrichtsentwicklung) nur wenig von dem anderer Schulen unterscheidet bzw. unterscheiden kann. Insbesondere unter Berücksichtigung der Tatsache, dass schulische Integration im Saarland seit 1987 erfolgt, ist dies mit Blick auf die qualitätsvolle Umsetzung (nach mehr als drei Jahrzehnten) ein schwieriges Ergebnis und ggf. ein schwer zu gewichtendes Transformationsproblem. Mögliche Vorteile des Co-Teachings können - vor allem mit Blick auf differenzierende Unterrichtsangebote - nicht genutzt werden.

Die Rollen- und Aufgabenklärung zwischen RSL und FSL wird in den Erhebungen - anders als in der sonderpädagogischen Forschung - nicht umfassend problematisiert. Die qualitative Auseinandersetzung betrifft vor allem den Wandel des Berufsbildes der Förderschullehrer mit Bezug darauf, dass Förderschullehrkräfte, die in den Förderschulen häufig als Klassenleitungen tätig sind, diese Aufgabe im Rahmen schulischer Integration nicht mehr ausüben können ${ }^{72}$. Lehrkräfte, die gerne vernetzt arbeiten, haben nach Einschätzung der Leitungen der SFZ weniger Probleme sich in ihr integratives Tätigkeitsfeld einzufügen. Wie die Ergebnisse der Interviews zeigen, ist die Klärung von Aufgaben zwischen RSL und FSL mit Aushandlungsprozessen verknüpft, die zum Teil - abhängig von der Offenheit und den Einstellungen der Kooperationspartner - differieren, so dass eine klare Strukturierung der Aufgaben für die Beteiligten nicht immer gegeben ist. Interessant und ggf. durch weitere Forschung zu erschließen sind in diesem Zusammenhang die Unterschiede, die sich mit Blick auf die Einschätzung der Wahrnehmung dahingehend ergeben, welche Aufgaben von den Regel- und Förderschullehrkräften in welchem Umfang übernommen werden. So schätzen die Förderschullehrkräfte ihre Tätigkeiten (Lernstandsdiagnostik, individuelle Förderung, Erstellung differenzierten Materials und Beratung der Lehrkräfte) jeweils von der Häufigkeit her höher ein als SL und RSL. Übereinstimmend sind die Ergebnisse dahingehend, dass die individuelle Förderung von SuS mit spF eine hauptsächliche Tätigkeit der FSL zu sein scheint. Positiv könnte dieses Ergebnis dahingehend ausgelegt werden, dass die individuelle Förderung in den beteiligten Regelschulen einen hohen Stellenwert hat und sich gegebenenfalls günstig auf die Lernentwicklung der Schülerinnen und Schüler auswirkt. Kritischer ist die Auslegung, die aufgrund der oben beschriebenen Ergebnisse zu fehlender unterrichtsbezogener Kooperation im engeren Sinn näher liegt, dass ein großer Teil der unterrichtlichen Arbeit der Förderschullehrkräfte mit einzelnen Schülerinnen und Schülern in der Form äußerer Differenzierung erfolgt - eine Annahme, die durch die Ergebnisse zur konkreten Unterrichtsgestaltung untermauert werden kann (s. dazu Abschnitt zur Differenzierung/Individualisierung weiter unten). Derzeit stehen also - wie auch von Reiser u.a. (2007) belegt - vor allem Beratung und individuelle Förderung als Aufgaben der FSL im Mittelpunkt.

72 Zum Zeitpunkt der Erhebungen gab es für FSL keine Möglichkeit eine Klassenleitung in einer Regelschule zu übernehmen. 
Transparenz bei der Rollen- und Aufgabenklärung und -verteilung scheint aufgrund der Ergebnisse nicht in jeder Hinsicht gegeben zu sein. Eventuell ist dies - neben den unzureichenden personellen Ressourcen - ein Mitgrund dafür, dass - wie Arndt \& Werning (2013) oder LütjeKlose et al. (2015) belegen - Formen äußerer Differenzierung gewählt werden. Die weitere Klärung durch eine qualitative Studie könnte eine Interpretation der abweichenden Einschätzungen ermöglichen. Offensichtlich werden viele unterschiedliche Aufgaben wahrgenommen und die Kooperation wird positiv bewertet, obwohl kaum unterrichtsbezogene Zusammenarbeit und ein klares Aufgabenverständnis sowie eine transparente Rollenverteilung gegeben sind, was für die Flexibilität der Beteiligten spricht. Die Arbeitsplatzbeschreibung für Regelschullehrkräfte und das dazugehörige Rundschreiben des saarländischen Ministeriums für Bildung sind diesbezüglich eine mögliche grobe Arbeitshilfe für die Lehrkräfte (Ministerium für Bildung des Saarlandes, 2016a, 2016b). Eine gemeinsame Planung der Förderung - vor allem mit Blick auf Schülerinnen und Schüler mit hohem Unterstützungs- oder sonderpädagogischem Förderbedarf - kann einen inhaltlichen Anker der Zusammenarbeit darstellen, problematisch ist allerdings aufgrund der Ressourcensituation und des Fehlens konkreter Arbeitshilfen (Bsp.: Anleitungen zur Strukturierung kurzer und effektiver Beratungsgespräche oder Förderkonferenzen, zu kurzen Fallbesprechungen, zur Gestaltung gemeinsamer Schüler-Eltern-Lehrergespräche, zur gemeinsamen Unterrichtsplanung, -gestaltung und -reflexion etc.) die Umsetzung im schulischen Alltag. Interessant ist mit Blick auf mögliche Perspektiven zur Weiterentwicklung das Ergebnis, dass die Förderschullehrkräfte das Tätigkeitsfeld der Vernetzung mit außerschulischen Partnern bislang nicht umfassend nutzen (ggf. aufgrund der Ressourcensituation), obwohl sie aufgrund ihrer als größer einzuschätzenden zeitlichen Flexibilität (normalerweise im Stundenplan nicht für Klassenunterricht eingeteilt) diese Vernetzung einfacher übernehmen könnten als die Regelschullehrkräfte.

Bis auf die Forschung zu den Unterrichtsteams wurden in dieser Arbeit keine Veröffentlichungen referiert, die sich mit den Auswirkungen der Umgestaltung der Arbeitszeit und damit ggf. auch auf Arbeitsplätze von Lehrkräften (Bsp.: schulinterne Teamzeiten, Arbeitsplätze in der Schule, Reduktion der Unterrichtsverpflichtung zugunsten gemeinsamer Unterrichtsvorbereitung) befassen. Einzelne Schulen in Deutschland haben ihre Arbeitsweise bereits dahingehend verändert und berichten von sehr guten Erfahrungen, so dass eine Perspektive der Weiterentwicklung inklusiver Bildungsangebote auch darin bestehen kann Arbeitszeit und Arbeitsplätze von Regel- wie Förderschullehrkräften grundsätzlich zu überdenken und ggf. umzugestalten ${ }^{73}$. Kritisch ist, dass die Notwendigkeit verbesserter personeller Ressourcen im Zusammenhang mit schulischer Integration zwar durch die Aussagen der Befragten und die Annahmen bisheriger Forschung belegt werden kann, bislang allerdings wenige Ergebnisse dahingehend vorliegen, inwiefern Co-Teaching bspw. tatsächlich die schulische Entwicklung von Kindern und Jugendlichen beeinflusst. Kooperative Prozesse in der Schule können, müssen sich aber nicht positiv auf Lernergebnisse auswirken. Ihre Erforschung kann daher nur in Verknüpfung mit Aspekten der Unterrichsgestaltung (siehe Unterrichtsentwicklung) Ziel führend sein.

Alle forschungsbasierten Hypothesen können durch die qualitative und quantitative Studie also weitgehend bestätigt werden, weshalb sie an dieser Stelle noch einmal aufgeführt werden: Konferenzen und Dienstbesprechungen werden in den Schulen genutzt um Fragen schulischer Integration zu thematisieren. Die Kooperation zwischen Regel- und Förderschullehrkräften findet auf verschiedenen Niveaustufen statt, wobei die Niveaustufen Austausch bzw. Co-Activity und

73 s. dazu bspw. Tegge, E. (2015): Die Einrichtung individueller Lehrerarbeitsplätze - eine kleine Umstrukturierung mit großen Folgen. In: Maschke, S. u.a. (2015): Jahrbuch Ganztagsschule 2015, Schwalbach, S. 118-123 
Kooperation häufiger praktiziert werden als die Stufen Coordination, Collaboration bzw. KoKonstruktion. Ein hohes Maß an Befürwortung und Bereitschaft zu unterrichtsbezogener Kooperation auf Seiten der Lehrkräfte steht einem geringen Maß an Umsetzung derselben gegenüber. Wenn Co-Teaching umgesetzt wird, werden vor allem die Formen one teach - one observe bzw. one teach - one assist genutzt. Unterrichtsverantwortung wird also nur selten tatsächlich gemeinsam wahrgenommen. Rollen und Aufgaben sind zwischen Regel- und Förderschullehrkräften so verteilt, dass die RSL v.a. die Klassen- und Unterrichtsverantwortung übernehmen, während die FSL Diagnostik, Beratung und Förderung gestalten.Teilweise werden Rollen- und Aufgabenverteilung flexibel gehandhabt, so dass Rollenwechsel möglich sind. Zielsetzung und Klärung der Aufgabenbereiche zwischen FSL und RSL sind nicht umfassend gegeben, woraus sich berufliche Unsicherheiten ergeben können.

\section{Professionalisierung}

Fragen zur universitären Ausbildung oder zum Vorbereitungsdienst der Regelschullehrkräfte wurden im Forschungsprojekt nicht thematisiert. Stattdessen richteten sich Fragestellungen auf interne und externe Fortbildungsangebote von Lehrkräften, den Fortbildungsbedarf, der in den Schulen im Zusammenhang mit schulischer Integration gesehen wird, sowie auf Möglichkeiten professioneller Weiterentwicklung in diesem Bereich insgesamt.

Ähnlich wie mit Blick auf das Schulklima oder auf die Kooperation zwischen RSL und FSL wird hinsichtlich der professionellen Grundausrichtung von Lehrkräften von den Befragten zunächst angenommen, dass die schulische Integration von Schülerinnen und Schülern mit sonderpädagogischem Förderbedarf ein hohes Engagement der Lehrkräfte voraussetzt $77,4 \%$ RSL, 92,1 \% FSL).

Während halbtägige schulinterne Lehrerfortbildungen (SCHILF) zum Themenfeld schulischer Integration eher selten stattfinden (Angaben variieren zwischen einem Fünftel und einem Viertel der Befragten), haben nach Angaben der RSL 50 \% bzw. der SL $75 \%$ der Schulen bereits Pädagogische Tage durchgeführt, deren Inhalte mit dem Thema in Verbindung stehen (Bsp.: „Inklusion“ übergeordnet, Differenzierung/Individualisierung, Umgang mit herausforderndem Verhalten). In erster Linie werden unterrichtliche Bausteine bearbeitet, während Organisations- oder Kooperationsstrukturen der Schule fast gar keine Rolle im Rahmen kollegialer Fortbildungen spielen. Intraprofessionell erarbeiten die FSL an den SFZ bspw. konzeptionelle Grundlagen für die Umsetzung schulischer Integration (Arbeitsplatzbeschreibungen für RSL und FSL) oder befassen sich mit einzelnen sonderpädagogischen Förderschwerpunkten, so dass diese Form des Austauschs (s. oben bei Kooperation) eine gute Möglichkeit für die Entwicklung bzw. Weiterentwicklung für Konzepten im Rahmen inklusiver Bildung darstellt. Die Frage, inwiefern die Vorteile der Fortbildung ganzer Teams oder Kollegien umfassend genutzt werden, bleibt aufgrund der Erhebungen offen. Fortbildungsreihen zu bestimmten Themen, ggf. auch begleitet durch eine längerfristige Beratung von Schulen, könnten laut Auffassung von Lütje-Klose und Urban (2014b) für die Weiterentwicklung inklusiver Bildung in diesem Rahmen eine Rolle spielen.

Ein widersprüchliches Bild ergibt sich in den Schulen hinsichtlich des beschriebenen umfassenden Fortbildungsbedarfs im Vergleich zu den tatsächlich besuchten Fortbildungsveranstaltungen. Der als hoch eingeschätzte Bedarf richtet sich inhaltlich auf die Thematik der Inklusion insgesamt, spezifische sonderpädagogische Fachrichtungen, Diagnostik, Fachdidaktik, Binnendifferenzierung, Methoden oder die Öffnung von Unterricht, also auf eine Mischung von Themen der Allgemeinen Pädagogik und der Sonderpädagogik. Ein großer Teil der Befragten (zwischen $47 \%$ und 62,2\%) nimmt allerdings eher selten bis gar nicht an Fortbildungsveranstaltungen teil. Dieser Widerspruch lässt sich aufgrund der Forschungsergebnisse nicht aufklä- 
ren und wirft Fragen danach auf, inwiefern die angebotenen Fortbildungen zu den Bedürfnissen der Lehrkräfte und Schulleitungen passen bzw. auf den spezifischen Bedarf einzelner Schulen spezifiziert sind bzw. ob Angebote für ganze Schulen, die ggf. kontinuierlich und damit nachhaltig strukturiert werden können, eine Alternative darstellen können. Da nicht nach dem allgemeinen Fortbildungsbedarf gefragt wurde, könnte es auch sein, dass andere Themen für die Lehrkräfte noch stärker im Vordergrund stehen wie bspw. fachdidaktische Fortbildungen. Auch die Arbeitsbelastung von Lehrkräften und die häufig schwierige Vertretunggsituation an den Schulen könnten im Zusammenhang mit dem seltenen Besuch stehen.

Unabhängig davon, dass keine Gründe für diesen geringen Grad an Teilnahme erhoben wurden, stellt die große Komplexität und Variationsbreite der Themen, die mit inklusiver Bildung zusammenhängen, sicher eine Herausforderung dar, da die Kollegien bzw. einzelne Lehrkräfte inhaltliche Prioritäten setzen und sich zunächst zwischen einer Vielzahl unterschiedlicher Angebote entscheiden müssen. Auch die Tatsache, dass viele Befragte sich sehr differenziert zu ihren Vorstellungen zur Weiterentwicklung ihrer eigenen Professionalität äußern und gerne weitere Kenntnisse in den Bereichen sonderpädagogisches Fachwissen, Beratungskompetenzen, Diagnostik und Förderplanung, Differenzierung und Individualisierung sowie Co-Teaching erwerben wollen, wirft die Frage auf, in welcher Form und in welchem Umfang Fortbildungen in diesen Bereichen gezielt angeboten werden können, die - was nach Jäntsch et al. (2015) nicht immer der Fall ist bzw. so wahrgenommen wird - konkreten Praxisbezug und ggf. eine Begleitung bei der Umsetzung ermöglichen.

Wie unter Kooperation bereits erwähnt, erfährt die intraprofessionelle Kooperation der FSL ein hohes Maß an Wertschätzung. Diese stellt also - aus Perspektive der Beteiligten - einen ganz wesentlichen Bereich für (fachliche) Weiterentwicklung und damit Professionalisierung dar, der in der bisherigen Forschung kaum Beachtung gefunden hat. Neben der Erarbeitung konzeptioneller Bausteine können FSL sich untereinaner fachrichtungsspezifisch beraten, was insbesondere dann relevant ist, wenn sie als einzelne Fachkraft bspw. an einer Grundschule tätig sind, in der Kinder mit spF unterrichtet werden, die nicht Studieninhalt der Lehrkraft waren. Die Organisationsform der Sonderpädagogischen Förder- und Beratungszentren im Saarland eröffnet diese Möglichkeit des intraprofessionellen Austauschs in Form von Arbeitskreisen, Dienstbesprechungen, Gesamtkonferenzen, Runden Tischen, schulinternen Fortbildungen etc., was auch darin begründet ist, dass die FSL diesen SFZ als „Stammschule“ angehören und von dort aus an Regelschulen abgeordnet werden. Die Förder- und Beratungszentren eignen sich daher als „Keimzellen“ für die Weiterentwicklung inklusiver Bildungsangebote, vor allem dann, wenn auch die Schulleitungen der überregionalen und fachspezifischen Zentren wiederum miteinander kooperieren (s.o.).

Die Hypothese, inwiefern sonderpädagogische Inhalte bzw. Inhalte, die schulische Inklusion (Heterogenität, Differenzierung) thematisieren, im Studium der Regelschullehrkräfte weitgehend fehlen, wurde in der Erhebung nicht gezielt aufgegriffen und lediglich von der Aussage einer Regelschullehrkraft im Rahmen der Interviews bestätigt. Einzelne Aussagen geben Hinweise darauf, dass die weitere Hypothese, dass das Empfinden mangelnder Kompetenzen aufgrund unzureichender Ausbildung vor allem bei den Regelschullehrkräften zu einer ablehnenden Haltung gegenüber schulischer Integration führen kann, für einzelne Personen zutrifft. Dies steht allerdings hinsichtlich der Ablehnung inklusiver Bildungsangebote nicht so sehr im Fokus wie die von den Beteiligten bemängelten unzureichenden Rahmenbedingungen. Die letzte Hypothese für diese Kategorie: Fortbildungsangebote für Regel- wie Förderschullehrkräfte im Zusammenhang mit dem Themenfeld schulischer Integration sind vielseitig und werden umfas- 
send genutzt und zwar sowohl Fortbildungen für einzelne Lehrkräfte wie für ganze Kollegien, kann differenziert aufgeschlüsselt werden. Der Fortbildungsbedarf der Lehrkräfte und Schulleitungen ist tatsächlich sehr vielseitig, das vorhandene Fortbildungsangebot wird allerdings von vielen Befragten in individueller Form nur selten genutzt. Hinsichtlich der Auseinandersetzung ganzer Kollegien mit der Thematik hat diese in Form Pädagogischer Tage etwa in der Hälfte bis in zwei Dritteln der Schulen stattgefunden. Eine wichtige Perspektive für die Weiterentwicklung inklusiver Bildungsangebote stellt der intraprofessionelle Austausch von Förderschullehrkräften untereinander dar. Wenn diesbezüglich Verschränkungen erfolgen würden - bspw. eine Ausweitung auf einen interprofessionellen Austausch, eine Erweiterung des Austauschs zwischen Schulleitungen sowie ggf. eine noch engere Zusammenarbeit zwischen den Schulen und den Lehrerfortbildungsinstituten - könnte dies die professionelle Weiterentwicklung der Beteiligten unterstützen. Denkbar ist in diesem Zusammenhang auch eine gezielte Ausweitung des Fortbildungsangebots auf Eltern sowie auf die Schülerinnen und Schüler (Bsp.: Schülervertretungen von Schulen).

\section{Ergebnisse}

Wie in der Hypothese: „Einstellungen und Haltungen von Regel- wie Förderschullehrkräften, Schulleitungen, Eltern und Schülerinnen und Schülern gegenüber schulischer Integration decken ein breites Meinungsspektrum (zwischen umfassender Ablehnung bzw. Befürwortung) ab." formuliert, lässt sich in den integrativ arbeitenden saarländischen Schulen tatsächlich eine Fülle unterschiedlicher Haltungen zu Fragestellungen schulischer Integration erheben. Diese lassen sich grob den Typen offene Haltung, pragmatische Haltung, skeptische Haltung und ablehnende Haltung zuordnen. Während eine offene Haltung - wie oben bereits zu anderen Kategorien vermerkt - mit der Motivation und dem Engagement der Lehrkräfte in Verbindung gebracht wird, hängt die pragmatische Haltung damit zusammen, dass schulische Integration im Saarland über die Jahrzehnte hinweg selbstverständlich geworden ist und die RSL in Kooperation mit den FSL um eine bestmögliche Umsetzung unter den gegebenen Umständen bemüht sind. Skeptische oder ablehnende Haltungen werden nicht - wie oben vermutet - auf mangelnde professionelle Kompetenzen der RSL in diesem Bereich zurückgeführt, sondern auf die unzureichende Ressourcensituation und die dadurch entstehende Belastung der Lehrkräfte. Sorgen bereitet den Befragten in diesem Zusammenhang die steigende Anzahl an Kindern und Jugendlichen mit spF in den Regelschulen. Als problematisch erweist sich eine ablehnende Haltung aus Sicht der FSL dann, wenn RSL die SuS mit spF nicht als „ihre“ Schülerinnen und Schüler ansehen, sondern als die der Förderschullehrkräfte. Vereinzelt wird die Haltung vertreten, dass Förderschulen günstigere Förderorte für SuS mit spF sein könnten, was wiederum mit der dort vorhandenen besseren personellen und materiellen Ausstattung in Verbindung gebracht wird.

Die grundsätzliche Offenheit der Kolleginnen und Kollegen hinsichtlich der Integration von $\mathrm{SuS}$ mit spF in den befragten Schulen und das Maß an Akzeptanz der bisherigen Form werden positiv bewertet. Vom Großteil der Befragten wird auch eine eher zustimmende Haltung der Kollegien der zukünftigen inklusiven Bildung gebenüber angenommen, wobei als Begründung u.a. die langjährige Erfahrung mit Integration angegeben wird. Allerdings geben $0 \%$ der Befragten an, dass sie diese Haltung als sehr zustimmend einstufen, was ggf. mit den Sorgen hinsichtlich der Rahmenbedingungen zusammenhängt.

Interessant ist dabei - wenn auch nur einmal aufgegriffen - der von der Leitung eines Förderund Beratungszentrums eingebrachte Aspekt, dass eine Weiterentwicklung hin zu inklusiver 
Bildung nicht nur in den Regelschulen, sondern auch in den Förderschulen notwendig sei (s. dazu 5.3).

Ein Forschungsdesiderat im Zusammenhang mit den Kategorien Kooperation und Professionalisierung besteht insgesamt weiterhin darin zu untersuchen, welche konkreten Effekte auf die schulische Entwicklung insgesamt und den Unterricht sowie die Lernleistungen von Schülerinnen und Schülern messbar sind.

\subsubsection{Unterrichtsentwicklung}

\section{Ressourcen}

Mit Blick auf die Klassen sind alle Befragten übereinstimmend der Meinung, dass diese im Rahmen schulischer Integration idealerweise zwischen 20 und $22 \mathrm{SuS}$ umfassen sollten. In den befragten Grundschulen kann dieser Richtwert häufig eingehalten werden, während die Klassen in den Gemeinschaftsschulen mit bis zu 30 Schülerinnen und Schülern (Obergrenze normalerweise im Saarland bei 29) als zu groß eingeschätzt werden. Inhaltlich begründen die Befragten ihre Auffassung u.a. damit, dass in kleineren Klassen ein höheres Maß an Ruhe herrsche und mehr Zeit für individuelle Zuwendung gegeben sei. Die Hypothese, dass die Klassengröße in den integrativ arbeitenden Schulen häufig die empfohlene Anzahl von 20-22 Schülerinnen und Schülern übersteigt, kann also für die weiterführenden Schulen bestätigt werden, während die Grundschulen oft mit dieser Klassengröße arbeiten können.

Hinsichtlich der Zusammensetzung der Klassen befürwortet die Mehrheit der Befragten, dass in einer Klasse bis zu vier SuS mit spF unterrichtet werden. Als Schwierigkeit wird in diesem Zusammenhang die gestiegene Komplexität der Anforderungen in den Klassen insgesamt beschrieben, die nicht nur auf die Unterrichtung von SuS mit spF zurückzuführen sei, sondern auf eine ganze Reihe von SuS mit spezifischen Problemlagen (ADHS, familiäre Situation, Teilleistungsschwächen, etc.). Ablehnend stehen vor allem die RSL und FSL der Unterrichtung von $\mathrm{SuS}$ mit schweren Behinderungen gegenüber, während Eltern (mehr als 60\%) und neun der zwölf SuS der Gemeinschaftsschulen sich diese gut vorstellen können. Teilweise werden SuS mit spF gezielt in bestimmten Klassen gebündelt, damit mehr Förderschullehrerstunden in diese Klassen eingebracht werden können. Die Hypothese, dass bei der Klassenzusammensetzung grundsätzlich darauf geachtet wird, dass der Anteil an Schülerinnen und Schülern mit spF nicht zu hoch ist, trifft also nur teilweise zu, während die Hypothese, dass in manchen Schulen Schüler mit spF in bestimmten Klassen gebündelt werden, damit auch sonderpädagogische Ressourcen in diesen Klassen gebündelt genutzt werden können, als zutreffend eingestuft werden kann. Unter bestimmten Voraussetzungen haben einzelne Schulen Möglichkeiten Klassengrößen und -zusammensetzung etwas flexibler zu gestalten, als dies derzeit normalerweise der Fall ist. Zum einen können (vorausgesetzt, dass hinsichtlich der Obergrenzen Spielraum dahingehend gegeben ist, dass diese aufgrund der Anmeldezahlen nicht ausgereizt werden müssen), in einem Jahrgang unterschiedlich große Klassen gebildet werden. Zum anderen können - wenn Lehrkräfte in einzelnen Stunden doppelt gesteckt sind - statt der Doppelbesetzung in einer Klasse bspw. aus vier Gruppen fünf oder sechs kleinere Gruppen gebildet werden (s. Ressourcen Personal). Die spezifische Zusammensetzung von Klassen kann bei Neubildung - was manchmal bereits geschieht - vor Schuljahresbeginn gemeinsam im Jahrgangsteam festgelegt werden. Davon abgesehen sind durch die Bildungspolitik allerdings dringend Landesvorgaben zu Klassenobergrenzen zu ändern (beispielhaft Bremen und Hamburg oder auch differenzierte saarländische Vorgaben bei Klassen mit hohem Anteil an Schülerinnen und Schülern mit unzureichenden Deutschkenntnissen). 


\section{Schulklasse als sozialer Raum}

Ähnlich wie beim Schulklima ergibt sich auch beim Klima in den Klassen in den saarländischen Schulen, die sich an den Teilstudien beteiligt haben, zunächst ein positiver Gesamteindruck, der sowohl durch das Wohlbefinden der SuS mit spF in ihren Klassen, als auch durch Freundschaften mit den anderen SuS geprägt ist. Schwierigere Ergebnisse betreffen die Tatsache, dass das soziale Lernen in den Klassen im Rahmen der Interviews nicht als Qualitätsmerkmal integrativen Unterrichts benannt wurde, und dieses aus Sicht der SuS mit spF nicht in allen Klassen gut ausgeprägt ist (ungünstigere Ergebnisse zu Freundlichkeit der SuS untereinander, zum Umgang mit Streit und dazu, wie die SuS mit spF von den Lehrkräften behandelt werden) sowie dass nachmittägliche Kontakte aus unterschiedlichen Gründen selten bis gar nicht stattfinden (s. Schulklima). Die Hypothese, dass integrative Klassen von einem lernförderlichen Klima geprägt sind, kann also nur in Teilen bestätigt werden. Weitere Forschung müsste zu der Hypothese getätigt werden, inwiefern Lehrkräfte ihren Unterricht - neben sozialen Lernprozessen auf informeller Ebene - so gestalten, dass soziales Lernen möglich ist, da diese aufgrund der vorliegenden Ergebnisse nur unzureichend bestätigt werden kann.

Als Perspektive für die Weiterentwicklung inklusiver Bildung kann aufgrund der Teilstudien also formuliert werden, dass die konkrete Steuerung und Förderung sozialer Lernprozesse durch Lehrkräfte innerhalb der Klassen verstärkt umgesetzt werden kann (Keller \& Hafner, 1999; Hattie, 2013).

\section{Klassenführung}

Die wichtigste neue Subkategorie, die sich aufgrund der Teilstudien für diese Kategorie ergeben hat, ist der Wunsch - vor allem der SuS mit spF - nach mehr Ruhe in den Klassen. Auf diese sind sie nach eigenen Angaben angewiesen um erfolgreich lernen zu können. Die Einheitlichkeit der Aussagen zu diesem Thema überzeugt sowohl in den Schülerinterviews wie in über einem Dutzend qualitativen Aussagen zu Verbesserungsmöglichkeiten der Förderung in den Fragebögen. Die Schülerinnen und Schüler mit spF sind es auch, die zu etwa $75 \%$ angeben, dass es in ihren Klassen weniger oder gar nicht ruhig sei, während die Anteile bei den Lehrkräften sehr viel geringer sind (22,7\% RSL, 28,9\% FSL); ein Hinweis darauf, dass die Wahrnehmug der SuS diesbezüglich anders ausgeprägt ist. Die Aussagen der SuS mit spF sind also ein wichtiger Anhaltspunkt dafür, dass der Qualitätsbaustein der Klassenführung hinsichtlich der Weiterentwicklung inklusiver Bildungsangebote stärker als bisher berücksichtigt werden sollte. Das Maß an Ruhe in den Klassen stellt für die Schüler eine Grundvoraussetzung dar um sich auf das Lernen fokussieren zu können - ein Ergebnis, das der Forschung zur Klassenführung dahingehend entspricht, dass eine wirksame Klassenführung positive Auswirkungen auf den Anteil aktiver Lernzeit und damit die Leistungen der Schülerinnen und Schüler haben kann (Brophy, 2006; Helmke, 2010; Muijs \& Reynolds, 2011; Hattie, 2013). Ursachen dafür können aufgrund der Ergebnisse nicht benannt werden. Regeln und Konsequenzen sind in den Klassen gegeben und die Lehrkräfte sind - auch der Einschätzung der SuS mit spF gemäß - bemüht für Ruhe zu sorgen und auf die Einhaltung eingeführter Regeln konsequent zu achten. Eine stärkere Gewichtung des Qualitätsbausteins der Klassenführung als Bestandteil inklusiver Bildung kann also - nicht nur mit Blick auf herausforderndes Verhalten von SuS, sondern vor allem mit Blick auf die Gestaltung aktiver Lernprozesse - Ziel führend sein. Weitere Forschung zu den tatsächlichen Effekten der Klassenführung in inklusiven Klassen ist allerdings notwendig, auch mit dem Fokus darauf, inwiefern sich Auswirkungen für SuS mit und ohne kognitive Schwierigkeiten oder mit und ohne Verhaltensprobleme u.a. ggf. unterscheiden. Die Hypothese, dass 
die Klassenführung für Lehrkräfte in integrativen Klassen eine hohe Relevanz hat, konnte durch die Studien nicht bestätigt werden. Dass die Gestaltung der Beziehungen zwischen Lehrkräften und Schülerinnen und Schülern dabei eine wesentliche Rolle spielt, wird in den Aussagen teilweise aufgegriffen. Die Hypothesen zur Nutzung von Regeln und Ritualen in den Klassen sowie zur klaren Kommunikation von Verhaltensanforderungen und zu Konsequenzen bei Regelverstößen treffen teilweise zu.

\section{Didaktik}

Neben den Organisationsstrukturen handelt es sich beim Qualitätsbaustein der Didaktik um den dritten großen „blinden Fleck“ der Erhebung. Da didaktische Fragestellungen von den Interviewpartnern nicht als Qualitätsmerkmale integrativen Unterrichts herausgestellt wurden, wurden sie in der zweiten Teilstudie nicht aufgegriffen. Für die Weiterentwicklung inklusiver Bildung sind didaktische und vor allem fachdidaktische Überlegungen allerdings durchaus zentral. Die entwicklungslogische Didaktik nach Feuser bietet dabei insofern Anschlussmöglichkeiten für das grundlegende Prinzip entwicklungsgerechter Lernangebote, als dass Hattie aufgrund seiner Metaanalyse sowohl „accelerated learning“ wie auch „scaffolding“ als wirksam einstufen konnte (Hattie, 2013). Inwiefern es sich dabei um ein didaktisches Prinzip oder eine Grundlage für differenziertes und individualisierendes Lernen handelt, sei dahingestellt. Im Prinzip versuchen alle sonderpädagogischen „didaktischen“ Ansätze die Frage zu beantworten, wie Unterrichtsinhalte in sehr heterogen zusammengesetzten Gruppen so dargeboten werden können, dass jede einzelne Schülerin und jeder einzelne Schüler Lernfortschritte machen kann. Dass, wie Feuser bspw. postuliert, Unterricht einen Möglichkeitsraum schaffen soll, in dem alle Schüler entlang eines gemeinsamen Gegenstandes auf ihrem Niveau lernen und durch die anderen Lernzugänge ihrer Mitschüler eine Perspektiverweiterung erfahren, ist - abgesehen von der oberflächlichen Struktur des Ansatzes - theoretisch zwar nachvollziehbar oder wünschenswert, praktisch allerdings - vor allem im Sekundarstufenbereich und bspw. im mathematischen oder naturwissenschaftlichen Bereich - kaum effektiv umsetzbar. Die von Wocken geforderte Balance zwischen gemeinsamen und individuellen Lernangeboten wiederum stellt zwar ein Prinzip zur Unterrichtsgestaltung dar, aber keinen didaktischen Ansatz im engeren Sinn. Auch Reichs Ansatz stellt keinen spezifisch „inklusiven“ Ansatz dar, sondern orientiert sich an der Theorie des Konstruktivismus (= lerntheoretischer Ansatz). Seine Annahmen stehen im Widerspruch zu den im Methodenteil aufgeführten Vorteilen instruktiven Unterrichtens (gerade für kognitiv schwache Schüler) und können entsprechend nur einzelne Bestandteile des Unterrichts (Bsp.: Einstiegsphasen oder Experimente) abdecken. Seitz bietet zwar mit ihrem Ansatz sich bei der differenzierten didaktischen Aufbereitung von Unterricht jeweils dem „Kern der Sache“ zuzuwenden eine qualitative Herangehensweise an die Auseinandersetzung mit didaktischen Fragestellungen an, allerdings ebenfalls kein umfassendes didaktisches Konzept für inklusiven Unterricht. Zielführender scheinen Auseinandersetzungen mit fächerspezifischen Curricula oder Kompetenzrastern zu sein (Helmke, 2015), die lernstufenübergreifend strukturiert und für die Schulen vereinheitlicht sind. Diese Auffassung kann damit begründet werden, dass für qualitative Differenzierung mit „Tiefenstruktur“ jeweils fundierte fachdidaktische Kenntnisse (Bsp.: Leseaufbau, Stufen der Rechtschreibentwicklung, Stufen der Entwicklung der Mengenvorstellung etc.) notwendig sind.

Die Relevanz des untergeordneten Qualitätsbausteins der klaren Strukturierung kann aufgrund der Teilstudien nicht näher erschlossen werden. Diese ist nach Angaben der RSL und FSL im Unterricht der Regelschulen weitgehend gegeben. Weitere Forschung kann in diesem 
Zusammenhang wichtig sein, da strukturierte Unterrichtsangebote für bestimmte Gruppen von Schülerinnen und Schülern (Bsp.: Schüler mit Autismus-Spektrum-Störung ${ }^{74}$, ADHS, schwerer beeinträchtigte SuS, die bspw. auf Symbole zur Tages- oder Stundenstrukturierung angewiesen sind) eine hohe Relevanz haben. Die Hypothese, dass integrativer Unterricht ein hohes Maß an Strukturierung aufweist, kann anhand der Aussagen der Beteiligten in der quantitativen Teilstudie bestätigt werden. Es bleibt allerdings aufgrund mangelnder qualitativer Aussagen unklar, wie diese umgesetzt wird.

\section{Methoden}

Der Unterricht in den untersuchten integrativ arbeitenden saarländischen Schulen weist grundsätzlich auf eine Vielfalt genutzter Methoden hin. Ähnlich wie in anderen Regelschulen auch, stellt der Frontalunterricht dabei mit großem Abstand die am häufigsten eingesetzte Unterrichtsform dar (zwischen 79,3\% (RSL) und $100 \%$ (S) der Befragten geben an, dass dieser häufig bzw. sehr häufig sei), gefolgt von den Sozialformen Einzelarbeit (nach Angaben von RSL und FSL zu knapp $90 \%$ häufig bis sehr häufig), Partnerarbeit (Angaben zwischen 54,5\% (S) und 79,2\% (RSL)) und Gruppenarbeit (Angaben zwischen 47,2\% (RSL) und 21,2\% (S)). Die Häufigkeitsangaben weichen zwischen den unterschiedlichen befragten Personengruppen stark voneinander $\mathrm{ab}$ und geben keinen Aufschluss über die tatsächlichen zeitlichen Anteile der einzelnen Methoden im Rahmen von Unterrichtsverläufen.

Spannend sind die Bewertungen der verschiedenen Sozialformen durch die Lehrkräfte und die SuS mit spF. Die Schülerinnen und Schüler mit spF bewerten vor allem den Frontalunterricht als Unterrichtsmethode sehr positiv (54,5\% als sehr gut und $42,4 \%$ als gut), da dieser sie dabei unterstütze mit Hilfe guter Erklärungen und Visualisierungen der Lehrkräfte Inhalte zu verstehen und durch entsprechende Übungen im Anschluss für sich zu verinnerlichen. Fast $85 \%$ der $\mathrm{SuS}$ mit spF geben dazu an, dass sie das, was von den Lehrkräften erklärt wird, gut bis sehr gut verstehen können. Damit werden die von Kirschner u.a. (2006) oder Muijs \& Reynolds (2011) dargestellten positiven Effekte dieser Methode aus Schülersicht bestätigt. Einzelne Lehrkräfte problematisieren diese Methode allerdings dahingehend, dass während frontaler Phasen nicht gleichzeitig individualisierend gearbeitet werden könne (Bsp.: aufgrund akustischer Störung des Lehrervortrags) und dass die Gestaltung frontalen Unterricht in heterogenen Klassen aufgrund der unterschiedlichen Lernvoraussetzungen der SuS eine hohe Anforderung darstelle. Nicht untersucht bzw. thematisiert wurde diesbezüglich die Fragestellung, inwiefern die sprachliche Gestaltung von Lehrerdarbietungen im Zusammenhang mit der heterogenen Zusammensetzung von Schulklassen eine Herausforderung darstellen $\mathrm{kann}^{75}$. Einschränkend ist an dieser Stelle forschungsmethodisch zu werten, dass diese Ergebnisse nicht durch Beobachtungen gestützt werden können. Es ist daher weitere Forschung dahingehend notwendig, inwiefern Lehrervorträge tatsächlich „barrierefrei“ erfolgen (bspw. in leichter Sprache) und welche Auswirkungen diese auf Lernprozesse von SuS haben.

Auch die Einzelarbeit wird von etwa $70 \%$ der SuS mit spF als gut (51,5\%) oder sehr gut (18,2\%) bewertet, da man sich dabei gut konzentrieren könne und nicht abgelenkt werde. Am positivsten bewerten Schülerinnen und Schüler mit spF - neben dem Frontalunterricht - die Partnerarbeit als Methode (33,3\% sehr gut, 54,5\% gut), wobei sie als Begründungen die gegenseitige

74 Beispiel: Häußler, A. (2012): Der TEACCH Ansatz zur Förderung von Menschen mit Autismus: Einführung in Theorie und Praxis. Dortmund: Borgmann

75 Das Thema „Leichte Sprache“ (www.leichte-sprache.de) wurde bspw. in keiner der rezipierten Forschungsveröffentlichungen im Zusammenhang mit methodischen Fragen aufgegriffen. 
Hilfestellung und den Spaß an dieser Arbeitsform anführen. Die Gruppenarbeit wird von den $\mathrm{SuS}$ mit spF etwas kritischer bewertet als die anderen Arbeitsformen, da diese zwar auch die Möglichkeit gegenseitiger Unterstützung biete, sie aber dann schwierig werde, wenn es in einer Gruppe zu laut sei, keine Konzentration auf die Aufgabenstellung erfolge oder inhaltliche Aussagen unterschiedlicher Schüler nicht zusammengeführt werden können. Die Schüleraussagen belegen somit indirekt die Bedeutung der Bausteine soziale Kompetenzen (als Voraussetzung) sowie der Strukturierung und inhaltlichen Gestaltung (als Umsetzungsaspekte) kooperativer Aufgabenstellungen (Brüning \& Saum, 2015). Die Organisation kooperativer Lernformen dahingehend, dass diese tatsächlich effiziente Lernprozesse auf Schülerseite ermöglichen, stellt im integrativen Unterricht also aus dieser Perspektive heraus eine hohe Anforderung dar. Nicht die reine Einbeziehung kooperativen Lernens in (inklusiven) Unterricht ist daher als Qualitätskategorie entscheidend, sondern die Sicherung der Voraussetzungen auf Seiten der Schülerinnen und Schülern sowie die Art ihrer Umsetzung.

Während die allgemeine Hypothese, dass im integrativen Unterricht unterschiedliche unterrichtliche Methoden flexibel genutzt werden, für die befragten Schulen zutrifft, sind im Unterricht dieser Schulen weder die Anteile an Frontalunterricht geringer als in anderen Regelklassen, noch nehmen schüleraktive Arbeitsphasen (Einzel-, Partner-, Gruppenarbeiten) einen prozentual höheren Anteil ein.

Als offene Unterrichtsformen werden in den befragten Schulen Lerntheken, Stationen oder Wochenplanarbeit umgesetzt, wobei die Wochenplanarbeit als besonders geeignete Methode für individualisierendes Arbeiten beschrieben wird. Während die Stationenarbeit vergleichsweise häufig als Methode genutzt wird (RSL etwa $45 \%$ häufig bis sehr häufig), geschieht dies bei den anderen beiden Mikromethoden allerdings eher selten bis gar nicht, so dass auch die Hypothese, dass offene Unterrichtsformen wie Stationenarbeit, Lerntheken oder Wochenplanarbeit häufig umgesetzt werden, für den integrativen Unterricht der beteiligten Schulen nicht bestätigt werden kann.

Insbesondere in Verknüpfung mit den Ergebnissen zur unterrichtsbezogenen Zusammenarbeit von Regel- und Förderschullehrkräften und im Abgleich mit bereits vorliegenden Forschungsergebnissen ergibt sich also auch mit Blick auf die Unterrichtsgestaltung allgemein in den befragten integrativ arbeitenden Schulen der Gesamteindruck, dass sich der Unterricht nicht wesentlich von dem in nicht integrativ arbeitenden Schulen unterscheidet. Er erfolgt vorwiegend in frontaler Form in Verbindung mit Einzelarbeit und erfährt nur selten eine Veränderung in Richtung offenerer Lernformen. Eine umfassende Weiterentwicklung des Unterrichts hat in den zurückliegenden Jahrzehnten seit Einführung der Integrationsverordnung in den an der Befragung beteiligten Schulen offensichtlich nicht stattgefunden. Das differenzierende Arbeiten (s. Folgeabschnitt) erfolgt also in einem Rahmen, der - aufgrund der mangelnden Öffnung nur phasenweise (Bsp.: während der Einzel- oder Partnerarbeit) unterschiedliche Aufgabenstellungen zulässt, während der Großteil der unterrichtlichen Arbeit weiterhin als Klassenunterricht erfolgt. Nicht belegen lässt sich, inwiefern diese geringfügigen Veränderungen mit den zur Verfügung stehenden Ressourcen zusammenhängen oder inwiefern tief greifende pädagogischdidaktische Veränderungen als Transformationsaspekt grundsätzlich im alltäglichen Unterricht keine wesentliche Rolle spielen.

Als Perspektive für die Weiterentwicklung inklusiver Bildungsangebote lässt sich aufgrund der Ergebnisse - unabhängig von den Begründungsansätzen - festhalten, dass in methodischer Hinsicht ein Wechsel zwischen Phasen direkter Instruktion (ggf. in homogenisierten Kleingruppen - „entwicklungsgerecht“, wenn Ressourcen dies ermöglichen), gut strukturierter Ein- 
zelarbeit (Bsp.: intelligentes Anwenden und Üben) und kooperativen Arbeitsphasen geeignet scheint, um Unterricht in heterogenen Lerngruppen so zu gestalten, dass die Lernprozesse der Schülerinnen und Schüler positiv unterstützt werden. Die Flexibilität und Situationsangemessenheit des Methodeneinsatzes stellt in diesem Zusammenhang eine Herausforderung für die Lehrkräfte dar, während die methodischen Grundlagen sowohl im Studium als auch in der zweiten Lehrerbildungsphase oder im Rahmen von Fortbildungen aufgrund der unter 3.5 erläuterten theoretischen Grundlagen als gut lehrbar einzustufen sind. Auf Seiten der Schülerinnen und Schüler können methodische Trainingsphasen - wie die Ergebnisse ebenfalls nahelegen zu selbstorganisiertem sowie kooperativem Lernen wesentlich sein. Querverbindungen ergeben sich u.a. zwischen dieser Art der methodischen Unterrichtsgestaltung und den Qualitätsbausteinen Co-Teaching und Binnendifferenzierung.

\section{Differenzierung und Individualisierung}

Differenzierung und Individualisierung stellen neben der Zusammenarbeit mit den Eltern und der Kooperation der Lehrkräfte das Kernthema der Qualität schulischer Integration an den befragten saarländischen Schulen dar. Die Notwendigkeit differenzierten bzw. individualisierenden Arbeitens ergibt sich dabei nach Aussagen der Schulleitungen und Lehrkräfte nicht nur aufgrund der Lernvoraussetzungen der Schüler mit sonderpädagogischem Förderbedarf, sondern allgemein mit Blick auf heterogene Lern- und Leistungsvoraussetzungen in den Klassen. Lernstandsdiagnostik und Förderplanung als qualitative Voraussetzung für differenziertes oder individualisierendes Arbeiten werden in den Interviews allerdings nicht thematisiert. Bei der schriftlichen Befragung ergibt sich das Ergebnis, dass Förderpläne nur zu $35 \%$ bis knapp $50 \%$ für die SuS mit spF vorhanden sind (Angaben variieren bei Eltern, RSL, FSL und Schulleitungen). Auch Formen schriftlicher Rückmeldungen (Bsp.: Entwicklungsberichte, Beiblätter zu Zeugnissen) sind in den befragten Schulen selten, während Rückmeldungen zu Lernfortschritten insgesamt - vor allem in mündlicher Form - in vielfältiger Art und Weise erfolgen. Die Lehrkräfte unterscheiden zwischen quantitativen und qualitativen Formen der Differenzierung und gehen davon aus, dass offene Lernformen wie Wochenplan- oder Stationenarbeit sich gut für differenzierten Unterricht eignen. Aus diesem Grund formulieren sie auch Aussagen zum Zusammenhang zwischen differenziertem und selbstorganisiertem Lernen. Als Anpassungsstrategien werden remediale Strategien wie eine adaptive Unterrichtsgestaltung aufgeführt. Für besonders problematisch halten die Befragten die individuellen Unterstützungsmöglichkeiten der Regelschulen für SuS mit den spF geistige sowie soziale und emotionale Entwicklung und für Kinder und Jugendliche mit starken Sinnesbeeinträchtigungen.

Auch bei vorsichtiger Gewichtung der sehr unterschiedlichen Angaben zur Umsetzung binnendifferenzierten Unterrichts in den Schulen ist davon auszugehen, dass dieser in einem Teil der Schulen eher selten bis gar nicht gestaltet wird (Angaben SL 25,8\% eher selten, RSL 18,9\% eher selten bis gar nicht, FSL $50 \%$ eher selten bis gar nicht, S 52,5\% eher selten bis gar nicht). Stattdessen steht die äußere Differenzierung in der Organisationsform von Einzel- oder Kleingruppenunterricht durch die Förderschullehrkräfte außerhalb des Klassenraums im Vordergrund und wird anteilsmäßig häufig umgesetzt. Diese Organisationsform umfasst nach Aussagen der SL, RSL und FSL sowie Eltern und SuS mit spF eine große Bandbreite, wird flexibel und teilweise situations-/bedarfsorientiert gehandhabt und richtet sich u.a. danach, ob SuS zieldifferent unterrichtet werden. Diese Form der individuellen Förderung wird aus Sicht aller Beteiligter - vor allem von FSL, Eltern und den SuS mit spF selbst - vorwiegend positiv bewer- 
tet (SL 51,6\%, RSL 77,4\%, FSL 81,5\%, E 79,4\%, S 93,9\% gut bzw. sehr gut). Auch wenn einzelne Schulleitungen, Lehrkräfte oder Elternteile kritisieren, dass diese Unterrichtsform Kinder separiere oder die Arbeit der FSL zu „besser strukturiertem Nachhilfeunterricht“ degradiere, überwiegen Begründungen dahingehend, dass äußere Differenzierung ein hohes Maß an Ruhe und Konzentration sowie individueller Zuwendung zu den SuS ermögliche, zeitweise einen „Schutzraum“ im Vergleich zum Klassenverband biete und effizient gestaltet werden könnte. So könnten im Rahmen von Einzel- oder Gruppensequenzen Unterrichtsinhalte noch einmal lernstandsgerecht erläutert werden. Der Unterricht in Kleingruppen biete SuS die Möglichkeit den Kontakt untereinander zu pflegen (Bsp.: SuS mit Down-Syndrom) oder in klassen- oder jahrgangsübergreifenden Fördergruppen zu arbeiten. Zieldifferent unterrichtete SuS könnten im Rahmen der Einzel- oder Kleingruppenförderung gute Lernfortschritte machen oder in Teilen zu Inhalten des Klassenunterrichts aufschließen. Teilweise werden Zusammenhänge zwischen einer unzureichenden Klassenführung und damit einhergehendem störendem Verhalten im Unterricht und der daraus resultierenden Notwendigkeit äußerlich differenzierten Arbeitens hergestellt.

Diese - im Vergleich zur sonderpädagogischen Diskussion und Forschung - sehr positive Wertschätzung der Einzelförderung bzw. des Kleingruppenunterrichts könnte neben den von den Befragten aufgeführten positiven Begründungsansätzen damit zusammenhängen, dass - wie oben dargestellt - selten offener und binnendifferenzierter Unterricht erfolgt, so dass die Förderung lediglich im Rahmen dieser Unterstützung erfolgen kann, und dass zu wenige FSL-Stunden für die unterrichtsbezogene Kooperation vorhanden sind (s. Ressourcen Personal und Kooperation). Wie ein Brennglas fokussieren die Ausführungen also indirekt die vor allem von Trautmann und Wischer (2011) thematisierten Spannungen zwischen den institutionellen Bedingungen und den Ansprüchen an individuelle Förderung. So verweist die Auseinandersetzung der Beteiligten mit der Frage nach innerer und äußerer Differenzierung darauf, dass binnendifferenziertes Arbeiten zwar als Wunsch formuliert wird (s. dazu unterrichtsbezogene Zusammenarbeit), Formen äußerer Differenzierung allerdings - auch aus pragmatischen Gründen - bevorzugt werden, da sie aufgrund der unzureichenden Rahmenbedingungen (Bsp.: zu wenige Stunden in Doppelbesetzung) oder fehlenden unterrichtlichen Veränderungen (Bsp.: Anteil Frontalunterricht im Vergleich zu offenen Unterrichtsformen)) leichter umsetzbar sind.

Da die Angaben der befragten RSL und FSL zur Verwendung differenzierter Arbeitsmaterialien für zieldifferent unterrichtet $\mathrm{SuS}$ mit spF (Bücher, Arbeitshefte und Arbeitsblätter) widersprüchlich sind, lässt sich zur Verwendung in den integrativen Schulen lediglich die Aussage treffen, dass diese nur teilweise eingesetzt bzw. genutzt werden. Auch die Angaben zu zieldifferent gestalteten Klassenarbeiten für diese Schülerinnen und Schüler weichen in den Antworten der RSL und FSL stark voneinander ab (Mathematik: RSL 52,8\%, FSL 86,8\%; Deutsch: RSL 47,2\%, FSL 92,1\%), wobei diese nach Auffassung der FSL für fast alle SuS angeboten werden. Tiefer gehende Ergebnisse zur zieldifferenten Leistungsbewertung wurden nicht erhoben. Einzelne Lehrkräfte problematisieren unzureichende Möglichkeiten zur differenzierten Bewertung von SuS ohne spF (fehlende rechtliche Rahmenbedingungen) oder den Nachteilsausgleich als Möglichkeit, die zwar rechtlich geregelt, dennoch aber nicht immer einfach zu handhaben sei.

Besonders umfassend und präzise werden die Grenzen (binnen-) differenzierten bzw. individualisierenden Arbeitens in den Regelschulen aufgezeigt, die in der Lehrplanorientierung sowie der Orientierung an Gleichschrittigkeit bei den SuS gesehen werden sowie darin, dass dadurch eine zu hohe Arbeitsbelastung von Lehrkräften entstehen kann (Bsp.: Materialvorbereitung) und 
dass die Rahmenbedingungen für diese Arbeitsweise nicht gegeben seien (Bsp.: Größe der Klassen, fehlende Kooperationszeiten der RSL und FSL, zu hohe Unterrichtsverpflichtung). So sei eine individuelle Zuwendung zu allen Schülerinnen und Schülern nicht möglich. Eine weitere Erschwernis bestehe darin, dass die Leistungsschere in den Klassen mit zunehmendem Altern größer werde. Die Ausführungen der Beteiligten geben damit kritische Einschätzungen wieder, die denen von Weinert (2001), Helmke (2003), Klippert (2008), Speck (2010) oder Trautmann und Wischer (2011) entsprechen.

Während die Hypothesen dazu, dass binnendifferenzierter oder individualisierter Unterricht im engeren Sinn selten angeboten wird und stattdessen Angebote äußerer Differenzierung (Einzel- oder Gruppenförderung) überwiegen, entsprechend bestätigt werden können, lässt sich nicht belegen, dass Schülerleistungen umfassend leistungsdifferenziert bewertet werden und dass Lernstandsdiagnostik und Förderplanung selbstverständlich sind und eine wichtige Rolle spielen. Wie in einer weiteren Hypothese angenommen, werden die Grenzen der Möglichkeiten der pädagogischen Bearbeitung von Differenz durch angepasste Unterrichtsangebote von den Lehrkräften wahrgenommen und mit Blick auf unterschiedliche sonderpädagogische Förderbedarfe hin differenziert.

Aufgrund der unzureichenden Umsetzung(smöglichkeiten) binnendifferenzierter bzw. individualisierender Lernangebote lassen sich vielfältige Perspektiven für diesen Qualitätsbereich aufzeigen. Unabhängig von der Ressourcensituation scheint zunächst wesentlich, dass diese Angebote allen Schülerinnen und Schülern gemacht werden sollten, unabhängig davon, ob ein besonderer Unterstützungs- oder Förderbedarf vorliegt. Insbesondere die positiven Ergebnisse Hatties zur Akzeleration können ein Hinweis darauf sein, dass an die Lernvoraussetzungen, das Lerntempo etc. angepasste unterrichtliche Angebote Lernfortschritte - nicht nur begabter Schülerinnen und Schüler - erleichtern können. Nur wenn der grundlegende Ansatz nicht von einer gleichschrittigen Arbeitsweise auszugehen, sondern von einer in vielerlei Hinsicht unterschiedlichen Arbeitsweise insgesamt Berücksichtigung findet, können bspw. die von den Befragten beschriebenen Vorteile von Einzel- oder Kleingruppenfördersituationen allen Kindern und Jugendlichen zugänglich gemacht werden. Mögliche Nachteile dieser Organisationsform (Bsp.: Separierung bestimmter SuS) äußerer Differenzierung könnten so minimiert werden, da alle SuS einer Klasse einbezogen werden können. Voraussetzung dafür sind flexible Möglichkeiten der Bewertung von Schülerleistungen bspw. in Form der Zuordnung zu bestimmten Kompetenzstufen, die sich - je nach Fach - unterscheiden können und Lehrkräften, Eltern wie Schülerinnen und Schülern Transparenz gewähren. Dazu ist nicht in erster Linie eine „Abschaffung “ von Ziffernnoten notwendig, sondern die Flexibilisierung des klassenbezogenen Unterrichts. Bei dieser Art der Flexibilisierung können wiederum doppelt besetzte Stunden effektiver genutzt werden.

Hinsichtlich der nur in Teilen erfolgenden Lernstandsdiagnostik bzw. Dokumentation von Lernfortschritten (Bsp.: Förderpläne, schriftliche Rückmeldungen) ist bspw. als Perspektive denkbar, dass den Schulen einfache und effiziente Hilfsmittel (Bsp.: „klickbare“ Kompetenzübersichten, verlagsunabhängige Förderplanvorlagen etc.) zur Verfügung gestellt werden. In Kombination mit regelmäßig stattfindenden Schüler-Eltern-Lehrergesprächen (s.o.) können diese auch für schriftliche Rückmeldungen und neue Zielvereinbarungen genutzt werden. Der Ansatz der Individualisierung des Unterrichts durch die Arbeit mit Lernleitern ${ }^{76}$ (Bsp.: Lehr-

76 s. dazu Girg, R.; Lichtinger, U.; Müller, T. (2012): Lernen mit Lernleitern. Unterrichten mit der MultiGradeMultiLevel-Methodology (MGML). Immenhausen bei Kassel: Prolog-Verlag 
gänge für fachbezogene „Bausteine“ wie das Verständnis kurzer Texte etc.) kann in diesem Zusammenhang eine Hilfestellung für individuelle Arbeitsphasen (s.o. intelligentes Üben) sein. Auch wenn - wie diese Beispiele zeigen - Möglichkeiten für die Weiterentwicklung inklusiver Bildung hin zu differenzierten unterrichtlichen Angeboten vorhanden sind, bleiben im Zusammenhang mit diesem zentralen Qualitätsbaustein viele Fragen offen und bedürfen weiterer Auseinandersetzung. Selbst wenn differenzierter Unterricht umfassend umgesetzt werden sollte, kann daraus nicht geschlussfolgert werden, dass dieser zu einer Verbesserung von Lernleistungen aller Schülerinnen und Schüler führt. Als Zielsetzung kann entsprechend eher formuliert werden, dass Differenzierung und Individualisierung im integrativen Unterricht dazu beitragen können, dass die Leistungs- und Kompetenzentwicklung vergleichbar der in homogener zusammengesetzten Gruppen erfolgt. Dazu liegen - bis auf Längsschnittstudien wie BiLief - allerdings kaum aussagekräftige Forschungsergebnisse vor. Im Hinblick auf die gemeinsame Unterrichtung von Schülern mit den Förderbedarfen Lernen oder ganzheitliche Entwicklung ist darüber hinaus davon auszugehen, dass die Leistungsunterschiede und damit auch die Leistungsstreuung innerhalb von Schulklassen mit steigender Lernstufe zunehmen. Je intensiver eine differenzierte Unterrichtung umgesetzt wird, desto weiter entfernen sich Schülerleistungen innerhalb einer Gruppe voneinander (Trautmann \& Wischer, 2011). Vor allem im Sekundarbereich stellt diese Tatsache Lehrkräfte vor die hohe Anforderung, bis zum Schulabschluss gemeinsame Lerngegenstände für den Unterricht zu finden, so dass zumindest während eines Teils der Unterrichtszeit gemeinsames Lernen möglich bleibt (problematisch bspw. v.a. in Mathematik und naturwissenschaftlichen Fächern). Zugespitzt könnte also formuliert werden, dass die Verwirklichung des Unterrichtsprinzips der Differenzierung über mehrere Schuljahre hinweg den Anspruch an das Prinzip selbst kontinuierlich erhöht und nicht verringert.

Sobald tatsächlich hochgradig individualisierte Lernangebote notwendig werden (Bsp.: SuS mit Sinnesbehinderungen oder schweren kognitiven Beeinträchtigungen), stellt sich die Frage, inwiefern der Anspruch inklusiver Bildung dem Anspruch auf gemeinsamen Unterricht im engeren Sinn gleichgesetzt werden kann bzw. sollte. Im Rahmen des (sonder-)pädagogischen bzw. auch juristischen Diskurses (s. dazu 2.2) (und der Frage nach der „Unteilbarkeit“ von „Inklusion“) erfolgt diese Gleichsetzung quasi „automatisiert“, obwohl flexible Angebotsstrukturen auch in bereits stärker inklusiv arbeitenden europäischen Ländern vorgehalten werden. Diese Angebotsstruktur schließt nicht zwangsläufig Förderschulen als Schulform ein, sondern kann in einem gemeinsamen Schulgebäude variable Strukturen ermöglichen: Phasen individueller Förderung und Kleingruppenförderung (in unterschiedlichen Zusammensetzungen), zeitlich mehr oder weniger ausgedehnte Phasen der Unterrichtung in „Peer-Gruppen“ (Bsp.: gehörlose Schülerinnen und Schüler) oder ausgedehnte Phasen der Unterrichtung in spezifischen Gruppen (Bsp.: schwer und mehrfach beeinträchtigte Schülerinnen und Schüler) in Kombination mit vielfältigen Kooperationsformen (Bsp.: Beginn und Abschluss eines Schultags, gemeinsames Mittagessen, gemeinsame Aktivitäten im Nachmittagsbereich oder im Rahmen kreativer Projekte, gemeinsame Fahrten etc.).

\section{Ergebnisse}

Als günstiges Ergebnis für die saarländischen integrativen Schulen, die sich an den Erhebungen beteiligt haben, lässt sich werten, dass die Wahrnehmung der Lernentwicklung der SuS mit $\mathrm{spF}$ im kognitiven, emotionalen und sozialen Bereich vorwiegend positiv ist - und zwar auf Seiten der Schulleitungen, Förderschullehrkräfte, Eltern und der SuS mit spF selbst. Nur die 
Regelschullehrkräfte sind in ihren Einschätzungen vorsichtiger als die anderen Befragten (25\% bewerten Fortschritte im sozialen Bereich als weniger gut). Deutlich wird anhand der Aussagen der Beteiligten allerdings auch, dass konkrete Einschätzungen sich letztlich nur auf individuelle Lernverläufe beziehen können, für deren Erhebung wiederum Längsschnitterhebungen wie die von Bryk et al. (2010) oder Lütje-Klose et al. (2018) notwendig sind.

Auch die Aussagen zur emotionalen Entwicklung zeigen, dass die Bewertung von Lernfortschritten letztlich nur qualitativ-einzelfallbezogen möglich ist. So weisen Befragte in den Interviews darauf hin, dass diese von einer sehr weitreichenden Weiterentwicklung bis zu gar keiner feststellbaren Entwicklung reichen. Als besonders anspruchsvoll wird die Unterstützung der emotionalen Entwicklung bei autistischen Schülern eingeschätzt, vor allem, wenn ein Fachlehrersystem den diesbezüglich notwendigen Informationsfluss erschwere. Hinsichtlich sozialer Entwicklungsverläufe äußern sich Interviewpartner dahingehend, dass diese sowohl bei SuS mit als auch bei denen ohne Förderbedarf in den integrativen Schulen vorwiegend positiv bewertet werden, da die SuS die Möglichkeit hätten Vielfalt untereinander zu respektieren und zu akzeptieren.

Die Hypothesen, dass die Lernentwicklung von Schülerinnen und Schülern mit spF in integrativen Schulen positiv verläuft und Schülerinnen und Schüler mit spF sind in ihren Klassen grundsätzlich sozial gut integriert sind, können für die befragten Schulen - unter den genannten Einschränkungen - bestätigt werden. Inwiefern sich Schwierigkeiten bezüglich der sozialen Integration für Schülerinnen und Schüler ergeben können, die niedrige Leistungen zeigen, einen niedrigen sozialen Status innehaben und/oder durch unangepasste soziale Verhaltensweisen auffallen, lässt sich nur anhand weniger Aussagen (bspw. zu negativen Lernabstandserfahrungen oder Ablehnungserfahrungen sozial-emotional auffälliger Schüler) tatsächlich belegen, da keine soziometrischen Daten erhoben wurden.

Naheliegenderweise werden von den Beteiligten vielfältige Zusammenhänge zwischen den Lernergebnissen der SuS mit spF und den Rahmenbedingungen bzw. pädagogischen Voraussetzungen der Schulen hergestellt: zur Schule als sozialem Raum (Schulklima), zu offenen Lernangeboten sowie Differenzierung bzw. Individualisierung, zu differenzierter Leistungsbewertung, Aktivierung und Motivierung der SuS mit spF durch die Lehrkräfte, zur (unterrichtsbezogenen) Kooperation der Lehrkräfte untereinander sowie - mit Blick auf Verbesserungsmöglichkeiten zu Ressourcen (Räume, Personal, feste Kooperationszeiten, Klassengröße).

Hinsichtlich möglicher Weiterentwicklungsperspektiven für diesen Bereich legen Ergebnisse zu kognitiven Entwicklungsverläufen nahe, dass diese pädagogisch-didaktisch gut zu beeinflussen sind. Sehr viel schwieriger stellt sich die Ausgangssituation mit Blick auf pädagogische Einflussmöglichkeiten dahingehend dar, dass soziale Prozesse in einer Klasse sich nur zum Teil durch Lehrerinnen und Lehrer steuern lassen. Je nach Zusammensetzung einer Klasse (Bsp.: Sozialisationshintergründe, deviantes Verhalten) entziehen sich soziale Prozesse in einer Klasse - vor allem mit zunehmendem Alter der Schülerinnen und Schüler - zum Teil dem Einflussbereich der Lehrer (Bsp.: Pausen, informelle Situationen, Kontakte über soziale Medien und im Freizeitbereich). So können sich - vor allem im außerschulischen Bereich - inklusive wie exklusive Prozesse vollziehen, die bspw. aus unterschiedlichen Interessen, materieller Ausstattung, Wohnsituation etc. resultieren. In diesem Qualitätsbereich ergeben sich aus allgemein- wie aus sonderpädagogischer Perspektive also vielfältige Querbezüge zu (bildungs-)soziologischen und (sonder-)pädagogischen Sichtweisen auf inklusive und exklusive Prozesse innerhalb von Schulen oder Klassen, auf die unter 5.2 noch einmal Bezug genommen wird. 


\section{Zusammenhänge zwischen Kategorien und Subkategorien}

Durch die Verknüpfung des Forschungsüberblicks mit den Ergebnissen der qualitativen und quantitativen Teilstudie lassen sich eine ganze Reihe von Zusammenhängen zwischen Subkategorien einer Kategorie bzw. mehrerer Kategorien darstellen. Dazu gehören beispielsweise:

- Ein Zusammenhang zwischen der Größe der Klassenräume, Möglichkeiten der flexiblen Raumgestaltung, dem Vorhandensein von Differenzierungsräumen, Möglichkeiten des CoTeaching, Möglichkeiten der inneren und äußeren Differenzierung,

- Schulklima als Ergebnis des Zusammenwirkens der Leitung einer Schule, des Engagements der Lehrkräfte und der engen Zusammenarbeit mit den Eltern (und ggf. Vernetzung),

- Zusammenhang zwischen personellen Ressourcen (Doppelbesetzung, Anzahl der FSLStunden in den Regelschulen, feste Kooperationszeiten) und Umsetzung der unterrichtsbezogenen Kooperation (Wunsch vs. Wirklichkeit) sowie der Lernstandsdiagnostik und differenzierter bzw. individualisierter Lernangebote (innere vs. äußere Differenzierung),

- Zusammenhang zwischen Kontinuität der personellen Zuweisung und Qualität der Kooperation zwischen RSL und FSL,

- Zusammenhang zwischen personellen Ressourcen und Belastung der Lehrkräfte,

- Zusammenhang zwischen Kooperation und Professionalisierung (intra-/interprofessioneller Kompetenztransfer SL, SLFZ, RSL, FSL),

- Zusammenhang zwischen Engagement der Lehrkräfte und Kooperation sowie Haltungen,

- Zusammenhang zwischen skeptisch-ablehnenden Haltungen und ungünstigen Rahmenbedingungen (personelle Ressourcen, Klassengröße und - zusammensetzung),

- Zusammenhang von Klassengröße und -zusammensetzung mit Möglichkeiten der flexiblen Raumgestaltung, Differenzierung und Individualisierung, Belastungs- vs. Wohlfühlempfinden von Lehrkräften und $\mathrm{SuS}$ sowie möglichen Abstufungen hinsichtlich der Integration von $\mathrm{SuS}$ mit unterschiedlichen $\mathrm{spF}$,

- Zusammenhang zwischen Klassengröße und -zusammensetzung und Klassenführung, Klasse als sozialer Raum, Differenzierung/Individualisierung sowie Lernergebnissen im emotionalen und sozialen Bereich,

- Zusammenhang zwischen Klassenführung, lernförderlichem Klima, Beziehungen zwischen Lehrkräften und SuS sowie Eltern und sozialen Lernprozessen (kooperative Methoden, soziale Projekte, Umgangsformen in Schule/Klasse),

- Zusammenhang zwischen Ruhe in Klassen und Konzentration/Lernorientierung der SuS.

Da nicht alle Zusammenhänge an dieser Stelle auf- und ausgeführt werden können, werden zwei Beispiele (oben fett markiert) beschreibend herausgegriffen. Die Führung einer Klasse beispielsweise wird von Lehrkräften als einfacher beschrieben, wenn in dieser Klasse weniger Schüler sind und der Anteil problematischer Schüler nicht zu hoch ist. Das hängt damit zusammen, dass eine Lehrkraft engere Beziehungen zu diesen Schülern aufbauen kann - ein Faktor, der sich positiv auf das Disziplinmanagement auswirkt. Das Klima in der Klasse profitiert von vertrauensvollen Beziehungen, die insbesondere für Schülerinnen mit erhöhtem emotionalem und sozialem Förderbedarf wichtig sind. Für die Lehrkraft ist es einfacher möglich dynamische soziale Prozesse in der Klasse zu analysieren und zu steuern sowie auf einzelne Schüler individuell einzugehen. Der Lernstand der Schüler kann in kleineren Klassen besser erfasst werden, eine individuelle Förderplanung kann für 20 Schüler präziser erfolgen und umgesetzt werden als für knapp 30 Schüler. Auf die Lernergebnisse im emotionalen und sozialen Bereich kann sich eine solche Vorgehensweise positiv auswirken. Inwiefern die Berücksichtigung dieser verschiedenen Qualitätsbausteine innerhalb einer Klasse auch zu besseren Lernleistungen führen 
kann, ist wiederum abhängig von den pädagogischen, fachlichen und vor allem fachdidaktischen Kompetenzen, die Lehrkräfte in diese Klasse einbringen, und damit von inhaltlichen Bestandteilen des Studiums und Vorbereitungsdienstes sowie von Fortbildungen (schulintern wie -extern) und Erfahrungswissen, das sich während der praktischen Tätigkeit entwickelt. Darüber hinausgehend lassen sich weitere Verknüpfungen zu organisatorischen oder personellen Strukturen einer Schule herstellen: Werden neue Klassenteams bei der Planung der Zusammensetzung einer Klasse beteiligt? Wie werden Übergänge der Schülerinnen und Schüler (bspw. beim Wechsel von der Primar- in die Sekundarstufe) gestaltet? Gibt es ein Team von RSL und FSL, das zumindest in den Hauptfächern gemeinsam in der Klasse arbeiten kann? Verfügt die Schule über ein übergreifendes Konzept zum Umgang mit Unterrichtsstörungen, das dieses Klassenteam für das Classroom-Management nutzen kann? Verfügen die Lehrkräfte über professionelles Know-How zur Durchführung kurzer Fallbesprechungen und falls nicht, werden entsprechende Fortbildungen angeboten? Wie diese kurze Auflistung zeigt, lassen sich diese Fragen im Prinzip durch das komplette Kategoriensystem hinweg stellen und zeigen damit die Unzulänglichkeit der „unvernetzten“ Darstellung der Wirkfaktoren auf, wie auch das folgende Beispiel veranschaulichen kann:

Ist die Doppelbesetzung einer Klasse in den Hauptfächern gegeben und haben die kooperierende RSL und FSL eine gemeinsame Kooperationsstunde pro Woche, können sie den Unterricht für Mathematik und Deutsch zumindest in sehr groben Zügen gemeinsam vorbereiten. Da sie regelmäßig zu zweit in der Klasse tätig sind, können sie verschiedene Co-Teaching-Formen genauso flexibel nutzen wie Formen der inneren bzw. äußeren Differenzierung. Eine prozessorientierte Lernstandsdiagnostik sowie Absprachen zu einer entsprechend differenzierten Unterrichtsvorbereitung erfolgen im Team. Die Umsetzung differenzierter Lernangebote kann umgesetzt werden, da in den Deutsch- und Mathematikstunden nicht nur differenzierte Arbeitsmaterialien für die Schülerinnen und Schüler zur Verfügung stehen, sondern jeweils eine der beiden Lehrkräfte regelmäßig Zeit hat sich auch einzelnen Schüler(gruppen) zuzuwenden um tiefer gehende Erklärungen oder spezifische Übungen anzubieten. Je versierter die Lehrkräfte in fachdidaktischer und methodischer Hinsicht sind, desto einfacher wird es ihnen fallen Phasen direkter Instruktion im Wechsel mit Phasen intelligenten Übens und Phasen kooperativen Lernens in der Gesamtgruppe oder für Teilgruppen zu gestalten. Wie aber verfahren die Lehrkräfte, wenn sie den Eindruck haben, dass es zu einer ungleichen „Lastenverteilung“ innerhalb ihres Teams kommt und die Rollen- und Aufgabenverteilung nicht ausreichend geklärt werden kann? Wie kann Kooperation gestaltet werden, wenn die RSL weiterhin die hauptsächliche Unterrichtsverantwortung tragen möchte? Wie können die Lehrkräfte langfristig binnendifferenziert arbeiten, wenn flexible Möglichkeiten für eine differenzierte Leistungsbeurteilung fehlen? Wie kann ein interprofessioneller Kompetenztransfer erfolgen, wenn RSL und FSL ihre spezifischen Fähigkeiten und Kenntnisse ggf. nicht ausgewogen gegenseitig anerkennen? Auch eine solche Liste kritischer Fragen, die Wirkfaktoren und deren Zusammenhänge „auf den Prüfstand“ stellen, ließe sich beliebig fortführen.

Erst die Analyse und Darstellung solcher Zusammenhänge und Fragestellungen verdeutlicht, dass aus dem Kategoriensystem zur Qualität schulischer Integration heraus keine einfachen Umkehrschlüsse dahingehend möglich sind, dass bspw. eine ausreichende Personalisierung in Form der Doppelbesetzung und das dadurch mögliche Co-Teaching in den Hauptfächern „automatisch“ zu besserem inklusivem Unterricht führen würden, sondern dass - ganz im Gegenteil - die Zusammenhänge zwischen einzelnen möglichen Wirkfaktoren hochkomplex sind. Ein Schaubild kann den soeben dargestellten beispielhaften Zusammenhang in Grundzügen 
darstellen und ansatzweise verdeutlichen, dass die Verbindungen zu anderen Kategorien und Subkategorien sich erweitern und immer wieder neu vernetzen lassen:

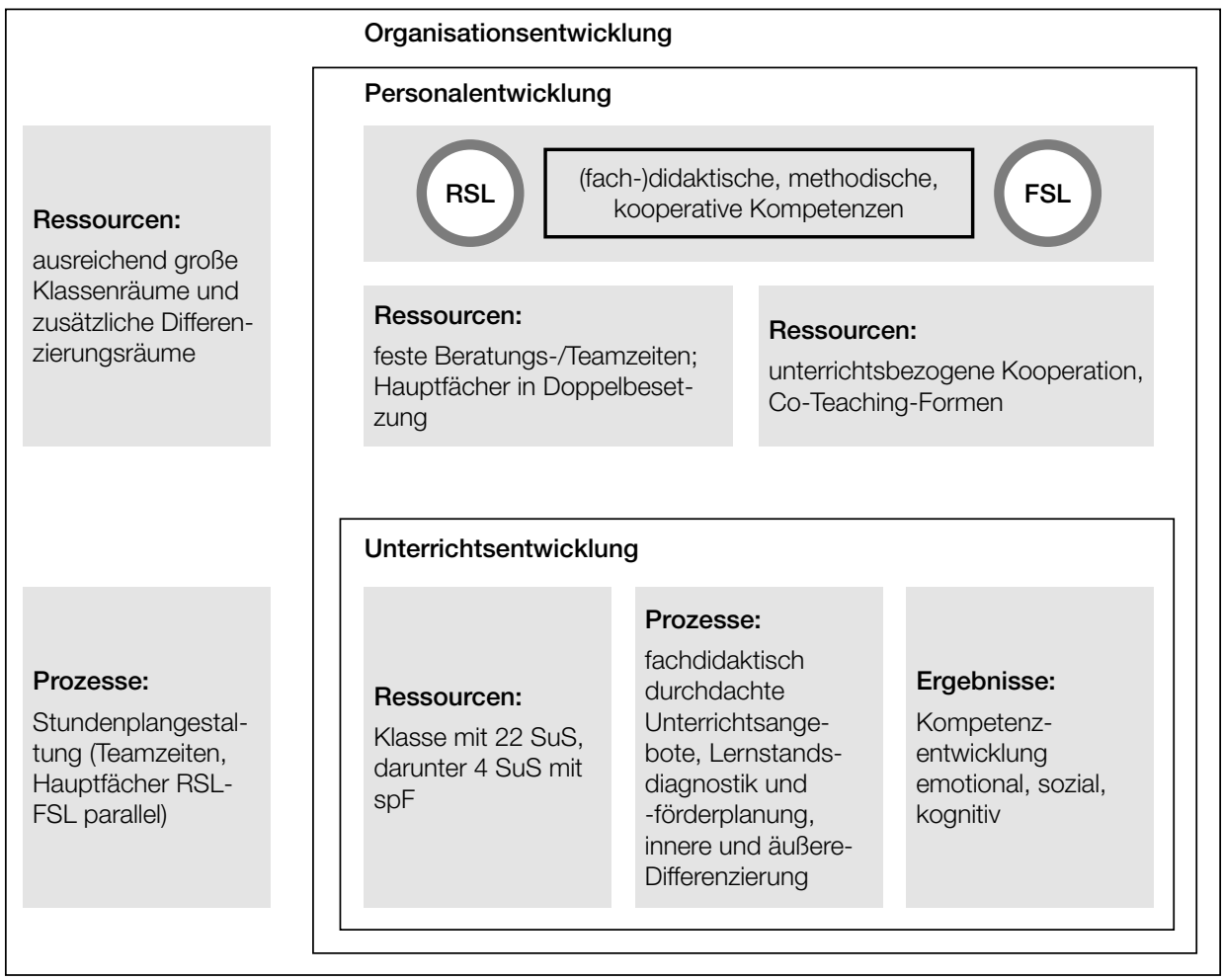

Abb. 23: Beispiel für Zusammenhänge Doppelbesetzung/Co-Teaching

Das Kategoriensystem bietet also eine wissenschaftlich fundierte Übersicht über die Bausteine, die für die Analyse und Umsetzung schulischer Inklusion als relevant eingestuft werden, und kann grundsätzlich auch zur Darstellung möglicher Zusammenhänge zwischen diesen Bausteinen genutzt werden, kann diese allerdings nicht umfassend hinsichtlich ihrer reziproken Wirkungen aufeinander und ihrer möglichen Gewichtung innerhalb eines dynamischen Wirkungsgefüges erschließen. Mit Blick auf mögliche Perspektiven zur Weiterentwicklung inklusiver Bildung bedeutet dies, dass sich Schulen auf der einen Seite, wenn sie Entwicklung anstreben, zunächst auf einzelne Wirkungsgefüge fokussieren können, und sich Forschung auf der anderen Seite ebenfalls auf bestimmte Teilbereiche der Qualität schulischer Inklusion richten kann um diese Wirkungsgefüge tiefer gehend zu analysieren. 


\section{Fazit und Perspektiven für das Bildungssystem}

Nachdem die einzelschulbezogenen Ausführungen zu den Schlussfolgerungen dieser Arbeit bereits im vorangegangenen Teilkapitel dargestellt wurden, geht es im Fazit und der Perspektiveröffnung des letzten Teils dieser Arbeit darum, einen Bogen vor allem zum übergreifenden (theoretischen) Rahmen zu spannen, innerhalb dessen sowohl der im dritten Kapitel dargestellte Forschungsüberblick als auch die im vierten Kapitel erläuterten Ergebnisse der qualitativen und quantitativen Erhebung geschichtlich und begrifflich eingeordnet werden können. Die folgenden Ausführungen sollen also die Frage danach beantworten, welche übergeordneten Schlussfolgerungen die in Kapitel 2 dargestellten theoretischen Grundlagen mit Blick auf die Frage nach der Qualität schulischer Integration bzw. Inklusion unter dem Blickwinkel der Entwicklung inklusiver Bildungsangebote zulassen. Dazu werden die wesentlichen Ergebnisse aus den Unterkapiteln 2.1 und 2.2 zusammengeführt und kritisch eingeordnet.

Gesellschaftliche Veränderungen wie Modernisierung, Individualisierung und Globalisierung führen zu einer starken Ausdifferenzierung des gesellschaftlichen Systems. Die Komplexität dieses Systems stellt einen Möglichkeitsrahmen dar, der beispielsweise individualisierte Lebenswege, lebenslanges Lernen oder Teilhabe in verschiedenen gesellschaftlichen Bereichen beinhalten kann. Gleichzeitig kann dieser Möglichkeitsrahmen aber auch als verunsichernd, überfordernd oder einschränkend erlebt werden, u.a. da Schulabschlüsse, berufliche Perspektiven und gesellschaftliche (Nicht-) Zugehörigkeiten sich begrenzend auf einzelne Personen oder Personengruppen auswirken können. Aus den soziologischen Ausführungen kann geschlussfolgert werden, dass die Begriffe Exklusion und Inklusion in diesem Zusammenhang nicht als simples, dichotom angelegtes Gegensatzpaar gesehen werden können, sondern vielmehr als relationale, mehrdimensionale, graduell unterschiedliche und prozesshaft angelegte Konstrukte zu verstehen sind, die Personen in ihrer "gesellschaftlichen Ganzheit“ betreffen bzw. beschreiben. Wie die Gegenüberstellung der wichtigsten Theoriestränge der Soziologie zeigt, bietet die Theorie der funktionalen Differenzierung in diesem Zusammenhang eine gewissermaßen „neutrale Schablone“, mit Hilfe derer gesellschaftliche Veränderungsprozesse sowie Kommunikationsprozesse zwischen und in ausdifferenzierten Teilsystemen erfasst werden können. Die Sozialstrukturanalyse fokussiert Dimensionen sozialer Gleichheit vs. Ungleichheit, Gerechtigkeit vs. Ungerechtigkeit sowie Teilhabe vs. Ausgrenzung. Auch dieser theoretische Ansatz ist mehrdimensional angelegt. Analytische Zugänge zu Exklusion bzw. Inklusion erfordern dementsprechend die Berücksichtigung differenzierter, prozesshafter und mehrdimensionaler Kriterien.

Die Qualität von Schulen, ihre Ressourcen, Prozesse und Ergebnisse, lassen sich also nicht beschreiben, ohne Schulen als Teil der Gesellschaft in den Blick zu nehmen. Alle an Schule als Institution und Organisation beteiligten Personen (Bildungspolitiker, Schulaufsichtsbeamte, Schulleitungen, Lehrkräfte, Eltern, Schüler usw.) sind Teil der Gesellschaft und durch die Sozialisation in dieser Gesellschaft geprägt. Sie bewegen sich, durchlaufen und gestalten inklusive wie exklusive Prozesse und werden innerhalb der Gesellschaft unterschiedlich adressiert. Diese Aussagen scheinen selbstverständlich, verdeutlichen aber, dass eine soziologische Perspektive und damit auch die Orientierung an ihren Begrifflichkeiten eine wesentliche Ebene für die Analyse schulischer Prozesse darstellt. Bezug nehmend auf Nassehi können dabei die Theorie der funktionalen Differenzierung sowie die Theorie der Sozialstrukturanalyse als zwei Achsen eines Koordinatensystems aufgefasst werden. Anschlussfähig erweist sich diesbezüglich das „Lebenslagen-Konzept“ (Engels, 2006), das - wenn auch ursprünglich für die Sozialberichterstattung 
formuliert - mit Blick auf inklusive bzw. exklusive Prozesse innerhalb von Schulen bspw. verknüpft mit dem ICF-Modell als Instrumentarium analytisch hilfreich erscheint.

Am Beispiel von Schülerinnen oder Schülern mit Behinderungen lässt sich anhand des Theoriestrangs der funktionalen Differenzierung u.a. erfragen, wie diese adressiert werden oder welche Relevanz ihnen in Kommunikationszusammenhängen zugesprochen wird. Anhand der Sozialstrukturanalyse lässt sich fragen, welchen Ausgrenzungsrisiken diese Kinder und Jugendlichen ausgesetzt sind. Foucault (1974), der von der Möglichkeit der „exclusion sur place“ spricht, gibt damit indirekt einen Hinweis darauf, dass auch „eine Schule für alle“ gesellschaftliche Faktoren wie die Ungleichverteilung von Ausgrenzungsrisiken oder die unterschiedliche Relevanz in Kommunikationszusammenhängen nicht „aufheben“ kann. Wenn zutrifft - wie Nassehi (2004) und Zick (2015) annehmen -, dass soziale Ungleichheit eine stabilisierende Funktion für einzelne Personen und deren Identität oder für Gruppen hat, wird diese Stabilisierung auch innerhalb sozialer Systeme wie Schulen eine Rolle spielen. Dabei ist die Kategorie „Behinderung“ - wie die Untersuchungen von Heitmeyer et al. (2015) sowie die Bildungs- bzw. Armuts- und Reichtumsberichte belegen - nicht allein entscheidend für das Risiko sozialer Ausgrenzung, sondern stellt nur eine mögliche Determinante für Exklusionsprozesse dar. Um es an zwei Beispielen zu veranschaulichen: das schwerhörige Kind aus einer Mittelschichtsfamilie unterliegt wahrscheinlich einem geringeren Ausgrenzungsrisiko als das verhaltensauffällige Kind aus einem sogenannten „bildungsfernen“ Haushalt. Aus dem katholischen Mädchen eines Arbeiterhaushalts vom Land ist heute eher der muslimische Junge aus dem Arbeiterhaushalt eines benachteiligten Stadtteils geworden. So können Modernisierung und Globalisierung auch Ausgrenzungslinien verschieben. Während die soeben aufgeführte Schwerhörigkeit ggf. ein geringes Ausgrenzungsrisiko in schulischer oder gesellschaftlicher Hinsicht mit sich bringt, können bei Schülern und Schülerinnen ohne Behinderung höhere Ausgrenzungsrisiken vorliegen, ggf. kumulieren und zu exklusiven Prozessen führen (Bsp.: Geschlecht, Migrationshintergrund, familiärer Hintergrund etc.). Eine wesentliche Rolle spielen - und das haben vor allem die Ergebnisse der PISA-Studie ergeben - diesbezüglich soziale Ungleichheiten.

Andere Kategorien für Differenz haben letztendlich mit Blick auf „Gerechtigkeit“ und somit auch Chancengerechtigkeit also ggf. eine höhere Relevanz für die Lebensgestaltung von Kindern, Jugendlichen und Erwachsenen als die Kategorie „Behinderung“ an sich. Für alle diese Kategorien kann nachgefragt werden, inwiefern die Vermittlung von Bildungsabschlüssen gelingt, wie der Übergang ins Erwerbsleben vollzogen wird, inwiefern sich Lebensverläufe (dis-) kontinuierlich gestalten und wie Personen sich selbst als „Teilhabende an der Gesellschaft“ entwickeln oder nicht entwickeln können. Bedeutsam sind hinsichtlich der Schlussfolgerungen aus den bildungssoziologischen, pädagogischen und sonderpädagogischen Ausführungen diesbezüglich mehrere Ansätze. Die Bildungssoziologie geht davon aus, dass das Bildungssystem in Deutschland Ungleichheiten verstärkt (Dt. PISA-Konsortium, 2001; Kronauer, 2010) bzw. „Ungleichheiten im familiären kulturellen Kapital einer Familie [...] in Bildungsungleichheiten transferiert“ (Solga \& Dombrowski, 2009), indem es diese vor allem als Leistungsdifferenzen wahrnimmt und reproduziert (Budde, 2013; Emmerich \& Hormel, 2013). Die Allgemeine Pädagogik spricht von schulischen „Selektionsprozessen“, die sowohl zu sozialer Akzeptanz wie zu sozialer Diskriminierung führen können (Muth, 1977), oder davon, dass die Schule damit konfrontiert sei, einerseits Bildungsprozesse anzugleichen (vor allem bei Kindern und Jugendlichen mit ungünstigen Sozialisationserfahrungen), andererseits aber dadurch zur Verstärkung von Differenzen beizutragen (Bsp.: Vergabe von Abschlüssen und damit Lebensperspektiven) (Tenorth, 1994). Innerhalb von Schulen bestehe ein Spannungsverhältnis zwischen Ansprüchen der Schule als Institution (Aufgaben und Funk- 
tionen) und denen der Individualisierung von Bildungsprozessen (Wischer, 2013). Bildungserfolge wiederum seien nicht so sehr vom Schulsystem bzw. dem Besuch spezifischer Schulformen abhängig, sondern können „systematischer Zufälligkeit“ (Kronig, 2007) unterliegen, da diese u.a. mit standortabhängigen Faktoren der Einzelschulen zusammenhängen. Aus sonderpädagogischer Sicht wird die Qualität der Ermöglichung gesellschaftlicher Teilhabe von Kindern und Jugendlichen durch das Schulsystem thematisiert (Kronauer, 2013).

Annahmen wie die von Prengel (1993) oder sonderpädagogischen Autoren, die - stark vereinfacht formuliert - davon ausgehen, dass „Inklusion“ der „einen Schule für alle“ entspricht bzw. die zumindest diese begriffliche Gleichsetzung vornehmen, lassen sich daher in mehrfacher Hinsicht nicht aufrecht erhalten. Vielfalt umfasst auch all die Aspekte einer Gesellschaft, die aus pädagogischer oder sonderpädagogischer Sicht zum Teil als problematisch eingestuft werden wie Leistungsorientierung, Ungerechtigkeit, prekäre Lebensverhältnisse, unsoziales Verhalten, Ablehnung und Ausschluss in bestimmten gesellschaftlichen Bezügen. Das sind Anhaltspunkte dafür, warum Schulen den von Wischer beschriebenen Widerspruch zwischen Institutionalisierung und Individualisierung nicht einfach pädagogisch oder sonderpädagogisch auflösen können, indem sie wenn der Gedanke der „einen Schule für alle“ konsequent zu Ende gedacht wird - in einer Einheitsschule und in einem Unterricht versuchen alle Kinder inklusiv zu beschulen. Nicht nur, dass eine „Einheitsschule“ in Deutschland verfassungsgemäß unzulässig wäre ${ }^{77}$, da Eltern ein Wahlrecht zwischen verschiedenen Bildungsangeboten zugebilligt werden muss, sie widerspricht - bei aller notwendigen Kritik am mehrgliedrigen Schulsystem - dem Ansatz eines vielfältigen, differenzierten, offenen und auf die Kinder und Jugendlichen sowie deren Eltern hin ausgerichtetem System. Auch der Ansatz von Tenorth (1994) Bildungs- und damit Sozialentscheidungen zeitlich „nach hinten“" zu verlagern, kann diese Widersprüche nicht auflösen, da sie im schulischen Alltag fortbestehen. Widersprüchlich erscheint in diesem Zusammenhang auch, dass auf der einen Seite äußere Differenzierung in den Kernfächern bspw. für die Gemeinschaftsschulen des Saarlandes ab der siebten Klasse eine selbstverständliche organisatorische Maßgabe darstellt, während diese in sonderpädagogischen Veröffentlichungen für Schülerinnen und Schüler mit sonderpädagogischem Förderbedarf als ungünstig, da möglicherweise diskriminierend, beschrieben wird. Während „besondere" Förderung in Gymnasien oder - darüber hinausgehend - Einrichtungen für Hochbegabte gesellschaftlich akzeptiert bzw. sogar unterstützt wird, werden „andere Förderschulen“ aus dieser Sichtweise ausgenommen und als separierende Institutionen bezeichnet.

Die in der Sonderpädagogik zum Teil stark vereinfachte Gegenüberstellung „Exklusion = Beschulung in ,separaten“ Förderschulen“ - „Inklusion = Beschulung in ,allgemeinen' Schulen“ ist daher aus folgenden Gründen in mehrfacher Hinsicht zu hinterfragen:

- ,Schule ist nur ein geringfügiger Bestandteil gesellschaftlicher Bezüge und Dynamiken (auch in (lebens-) zeitlicher Hinsicht).

- Schüler können als Kinder oder Jugendliche in vielen weiteren gesellschaftlichen Bezügen in- bzw. exkludiert sein. Der Begriff, Schüler' kennzeichnet sie in ihrer zeitlich, räumlich und interaktionistisch begrenzten Zugehörigkeit zu einer gesellschaftlichen Institution unter vielen anderen.

- „Exklusion“ kann auf mehreren Ebenen und als Prozess erfolgen. Sie ist nicht institutionengebunden, sie kann von Schülern (ob mit oder ohne ,Einschränkung') bspw. auch in ,Regelschulen' oder eben in anderen Bezügen erlebt werden; dementsprechend ist die pädagogisch so verstandene ,schulische Inklusion' kein ,Garant' für inklusive Prozesse.

77 s. dazu Kiefer, W. (2010): Verfassungsrechtliche Grenzen einer Schulreform im Saarland. In: Zeitschrift für Landesund Kommunalrecht Hessen, Rheinland-Pfalz, Saarland, 2010, Heft 4, S. $131 \mathrm{ff}$ 
Diese Auffassung entspricht nicht einer rein „pessimistisch-kritischen“ Haltung, sondern versucht Schulen als gesellschaftliche Institutionen pragmatisch-realistisch zu fokussieren. Vor dem Hintergrund dieser „Analyseschablone“ können die pädagogische und sonderpädagogische Qualität von Schulen in einen Gesamtzusammenhang eingeordnet werden.

Eine zu starke Pädagogisierung der beschriebenen Problematik und Widersprüche kann - ähnlich wie von Wenning für die „Pädagogisierung von Heterogenität" formuliert - zu unzulässigen Zuschreibungen von Verantwortung hinsichtlich schulischer Inklusion an pädagogisch handelnde Personen führen. „Verantwortlich“ sind dann sowohl Schulleitungen von Regel- wie von Förderschulen, Förder- wie Regelschullehrkräfte sowie alle anderen in diesem System tätigten Personen (Bsp.: Integrationshelfer) und Eltern von Schülern mit sonderpädagogischem Förderbedarf. Schulleitungen und Lehrkräfte, die an Förderschulen tätig sind, tragen (Mit-) Verantwortung für die „Separierung“ von Schülern. Schulleitungen und Lehrkräfte, die an Regelschulen tätig sind, tragen (Mit-) Verantwortung für unzureichende Förderung von Schülern. Und die Eltern tragen, je nach Entscheidung über einen Förderort, (Mit-)Verantwortung für „Exklusion“ oder „Inklusion“ ihrer Kinder.

Eine erste wesentliche Schlussfolgerung aus den theoretischen Ausführungen zu Begriffen und Diskurs lässt sich also so zusammenfassen, dass ein Kind als Teil hochausdifferenzierter Teilsysteme innerhalb eines komplexen Gesellschaftssystems wahrgenommen werden kann. Schule bildet als institutionelle Einrichtung nur eins dieser Teilsysteme, das wiederum aus unzähligen weiteren Teilsystemen besteht, da sich dort Zugehörige anderer mit weiteren Teilsystemen verknüpfter Personen befinden und Interaktionen auf vielfältigen Ebenen und teilweise in einem langjährigen Prozess erfolgen. Diese Sichtweise erfordert eine Analyse- und Forschungsperspektive, die diese Teilsysteme, Verknüpfungen zwischen den Teilsystemen innerhalb von Schule sowie die Verknüpfung zwischen Schule und außerschulischen Teilsystemen wahrnimmt und als „Hintergrundschablone“ für die Darstellung und Interpretation von Erkenntnissen nutzt.

Als Zeitachse für die Bearbeitung des Themas schulischer Integration bzw. Inklusion wird als Teilausschnitt der Zeitraum von der ersten bis zur höchstens dreizehnten Klasse, also zwischen dem frühestens fünften und spätestens neunzehnten Lebensjahrs gewählt. Die soziale Achse berücksichtigt die Bedeutung der frühkindlichen individuellen Entwicklung, der Herkunftsfamilie sowie des erweiterten privaten/persönlichen Netzwerks (Peers) und die Einbeziehung in ein öffentliches Umfeld (grundsätzlich festgelegt durch Zugehörigkeit zu einer Institution, qualitativ abhängig vor allem von Zuschreibungen innerhalb dieser Institution). Die Dimensionen Inklusion bzw. Exklusion werden aufgefasst als relationale, mehrdimensionale, prozesshafte und graduell möglicherweise abgestufte Konstrukte. Vektoren können innerhalb dieses Koordinationsystems nur individuell bestimmt werden und lassen streng genommen keine Verallgemeinerungen (wissenschaftlicher Aussagen) zu. Prozesse können diskontinuierlich verlaufen, ein idealisierter Verlauf ist nicht festzulegen. Schüler und Schülerinnen bewegen sich in einem komplexen und dynamischen Wirkungsgefüge, das durch vielfältige soziale Prozesse (institutionalisiert und nicht-institutionalisiert), schulisch-unterrichtliche Prozesse (Kompetenzentwicklung auf vielen Ebenen), (dis-)kontinuierliche zeitliche Prozesse (darunter auch die subjektive Erfahrung persönlicher Weiterentwicklung bzw. Stagnation) etc. geprägt ist und keine vereinfachenden Zuschreibungen der Form „Schüler der Regelschule = inkludiert, Schüler der Förderschule $=$ exkludiert“ ${ }^{\text {z }}$ zulässt. Inklusive bzw. exklusive Prozesse können sich innerhalb des dargestellten Wirkungsgefüges auf vielfältige Art und Weise vollziehen und nehmen Schüler und Schülerinnen auch als „unschulische“ Personen in den Blick. 
Zusätzlich zur begrifflich-diskursiven Einordnung der Frage nach der Qualität schulischer Integration/Inklusion lassen auch die Ausführungen zur geschichtlichen Einordnung Schlussfolgerungen mit Blick auf das Forschungsthema zu. Spannend ist in diesem Zusammenhang die Frage, wie ein Bildungssystem mit der hochgradigen gesellschaftlichen Komplexität und ihren ausdifferenzierten Teilsystemen umgeht. Hinsichtlich der äußeren Struktur kann das System so damit umgehen, dass - wie in der BRD - mit dem Eintritt in die Sekundarstufe der Versuch einer leistungsdifferenzierten Homogenisierung in verschiedenen Schulformen erfolgt. Der entgegengesetzte Ansatz versucht der Heterogenität in einer entsprechend leistungsheterogenen Schulform zu begegnen. Der erste diesbezüglich ernsthafte Versuch die Dreigliedrigkeit (bzw. Viergliedrigkeit) des deutschen Bildungssystems in den 70er- und 80er-Jahren durch eine „Gesamtschule " zu durchbrechen, konnte nicht flächendeckend umgesetzt werden, da eine entsprechende bildungspolitische Unterstützung fehlte. Die Gesamtschule ist als Schulform - sowohl in ihrer konzeptionellen Ausrichtung als auch in ihrer praktischen Umsetzung - also nicht zu der heute geforderten „Schule für alle“ geworden, obwohl sie strukturell Möglichkeiten dafür bietet. Die Gesamtschulen kooperativer Form bieten selbst Regelschülern keine „echte“ Integration in bildungsgangübergreifendes Lernen an. Auch die Gesamtschulen in integrierter Form differenzieren ab Klasse 7 in unterschiedliche Leistungsgruppen (Kurse). In den Ausführungen wird deutlich, dass sie ebenfalls dem Leistungsanspruch verschiedener Schulabschlüsse unterliegen. Schon bei ihrer Einrichtung wurden daher Schüler mit Behinderung als „Kategorie“ ausgeblendet, obwohl Kinder mit Lernbehinderungen und Verhaltensauffälligkeiten für kompensatorische Erziehung sozusagen "geeignet" gewesen wären.

Entscheidend ist also mit Blick auf die Fragen nach der Qualität schulischer Integration bzw. Inklusion, dass diese das (nach Schulabschlüssen) gegliederte Schulwesen der BRD in Frage stellen. Denn wenn in einer Hauptschulklasse bspw. auch Kinder mit dem sonderpädagogischen Förderbedarf Lernen zieldifferent unterrichtet werden und einen anderen Schulabschluss (der Förderschule) erlangen können, warum kann dies dann - zumindest theoretisch - nicht auch für Realschüler in einem Gymnasium möglich $\operatorname{sein}^{78}$ ? Eine grundlegende systemische Veränderung des Schulsystems ist in den 2000er Jahren durch die Einführung von Gemeinschaftsschulen und der Schaffung eines zwei- statt dreigliedrigen bzw. drei- statt viergliedrigen (wenn man die Förderschulen als vierte Gliederung hinzunimmt) zwar in einigen Bundesländern erfolgt, ist aber weit davon entfernt eine gemeinsame Schulform für alle Schüler anzubieten. Der Erhalt der Gymnasien wird in keinem Bundesland ernsthaft in Frage gestellt. Diese kurze Darstellung eines offensichtlich mehrere Jahrzehnte alten Problems verdeutlicht sehr anschaulich die zu Beginn des Kapitels 2.1 dargestellte fehlende „Mobilität“, Flexibilität und Transformationskompetenz des deutschen Bildungssystems. Die „Standhaftigkeit“ dieses Systems muss geradezu zwangsläufig hinderlich für den Ansatz einer ,inklusiven“ Regelschule sein.

Wären die Ergebnisse aus den Modell- und Schulversuchen fester Bestandteil der allgemeinen Pädagogik und der Bildungspolitik geworden, hätte die pädagogische Umsetzung des gemeinsamen Unterrichts bei einem großen Teil der Schüler mit Behinderung schon früher erfolgen können. Das von Bleidick angesprochene Beharrungsvermögen traditioneller Bildungsstrukturen (Bleidick \& Ellger-Rüttgardt, 2008, S. 49) zeigt sich dabei sowohl auf der breiten Ebene der Regelschulen, als auch bei bildungspolitischen Entscheidungen sowie der Lehrerausbildung an den Hochschulen. Insbesondere die von Weigt und anderen schon 1977 geforderte „Einbezie-

78 Beispiel: inklusive Beschulung von SuS mit dem spF Lernen in Gymnasien in NRW, die inzwischen wieder aufgehoben wurde; s. dazu https://www.watson.de/deutschland/politik/560297467-immer-mehr-gymnasien-in-nrwsteigen-aus-der-inklusion-aus 
hung sonderpädagogischer Fachinhalte in die allgemeine Lehrerausbildung" ist auch heute erst in wenigen Studiengängen in der BRD umgesetzt.

Die Bildungspolitik der vergangenen Jahrzehnte ist mit Blick auf die schulische Integration von Schülern mit Einschränkungen oder Behinderungen insgesamt als „zögerlich“ einzustufen. Veröffentlichungen der Kultusministerkonferenz hatten jeweils nur empfehlenden Charakter, die Landesgesetze berücksichtigten die gemeinsame Unterrichtung immer unter Ressourcenvorbehalten. Eine kontinuierlich unterstützende Arbeit hinsichtlich der Integration in vorschulischen und schulischen Bildungseinrichtungen ging in den letzten Jahrzehnten (wenn auch teilweise ebenfalls konservativ-vorsichtig) von den Interessenverbänden (Bsp.: Elternverbände, vds, GEW) aus. Kennzeichnend ist, dass die Bildungspolitik als solche in den letzten Jahrzehnten nicht innovativ-verändernd agierte, sondern verwaltungsorientiert-reaktiv. Als pragmatische Konsequenz zur Sicherung einer flächendeckenden Versorgung mit Sonderschulen erfolgte nach Kriegsende ein Ausbau des Sonderschulwesens, ohne dass die Frage nach integrativen Ansätzen wie in anderen europäischen Ländern bildungspolitisch diskutiert wurde. Als Reaktion auf eine prognostizierte Bildungskatastrophe und drohenden Fachkräfte- und Akademikermangel entstand die Gesamtschulidee, die vor allem die Integration von Schülern aus sozial schwachen Familien fördern sollte. Diese konnte sich allerdings nicht flächendeckend durchsetzen, da die bildungspolitische Unterstützung dazu (vor allem in den CDU-regierten Ländern) fehlte. Darüber hinaus konnte sie den Anspruch kompensatorischer Erziehung nicht umfassend einlösen. In den 70er Jahren standen bildungspolitisch die Empfehlungen der KMK zur Ordnung des Sonderschulwesens im Mittelpunkt, während die integrativ orientierten Empfehlungen des Deutschen Bildungsrats keine Wirkkraft entfalten konnten. So gelangte die mögliche Lernortunabhängigkeit der Förderung von Kindern mit Behinderungen zwar in den Fokus, wurde aufgrund des empfehlenden Charakters allerdings nur in Einzelfällen umgesetzt. Während die Ergebnisse der PISA-Studie als Reaktion der Bildungspolitik u.a. die Entwicklung outputorientierter Standards (Stärkung frühkindlicher Bildung, Einführung von Ganztagsschulen, Bildungsstandards, Kompetenzorientierung) als qualitative Merkmale für alle Schulformen zur Folge hatten, führte die UN-BRK bislang in erster Linie zu quantitativen Veränderungen dahingehend, dass ein höherer Anteil an Schülern mit Behinderungen inklusiv unterrichtet wird. PISA hat zu vergleichsweise gut koordinierten bundesweiten Anstrengungen mit Blick auf die Veränderung der Qualität von Unterricht geführt, wohingegen die UN-BRK bildungspolitisch betrachtet einen „inklusiven Flickenteppich“ hervorgebracht hat, in dem Auslegung und Umsetzung in den einzelnen Bundesländern sehr unterschiedlich erfolgen.

Der Überblick über die Geschichte schulischer Integration zeigt, dass bildungspolitische Reformen vor allem dann erfolgen, wenn „Bildungskatastrophen“ gesehen werden (60er-Jahre: fehlende kompensatorische Erziehung innerhalb der BRD und dadurch drohender Mangel an qualifizierten Arbeitskräften, 2000er-Jahre: fehlende Exzellenz der deutschen Schulen im internationalen Vergleich). Da - ähnlich wie bei Wellenbewegungen - auf Phasen versuchter weitreichender Veränderungen des Bildungssystems, die durch diese „Katastrophen“ ausgelöst wurden, restaurative Phasen folgten ${ }^{79}$ (Bsp.: Modellversuche statt struktureller Veränderungen in den 80er-Jahren; Reduktion der PISA-Anstrengungen auf die Durchführung von Vergleichsarbeiten), kehrte zumindest in Teilen immer wieder bildungspolitische „Ruhe“ ins Schulsystem ein. Während die zwei zurückliegenden umfassenden Reformbestrebungen in den 70er- bzw. 2000er-Jahren allerdings in erster Linie durch bildungspolitische und pädagogische Diskussio-

79 zum Teil im Zusammenhang mit Rezessionsphasen wie Mitte der 80er Jahre oder im Anschluss an die Finanzkrise 2008 
nen und damit zusammenhängende Überlegungen ausgelöst wurden, gründet der Versuch der Umsetzung eines inklusiven Bildungssystems in Deutschland auf einer Rechtskonvention der Vereinten Nationen, die in nationales Recht überführt wurde und per Rechtsverordnungen in den einzelnen Bundesländern geregelt wird. Es ist fraglich, ob diese grundsätzlich andere Ausgangssituation tatsächlich dazu führt, dass tief greifende Veränderungen in den Schulen und im Schulsystem stattfinden oder ob auf eine Welle der Reformbestrebungen zunächst einmal wieder restaurative Bemühungen folgen, die - wie bei den vorangegangenen Reformversuchen auch - u.a. aufgrund fehlender finanzieller Ressourcen dazu führen, dass keine grundlegenden qualitativen Veränderungen im Unterricht und der Schulstruktur der Regelschulen erfolgen.

Aufgrund der geschichtlichen Analyse bildungspolitischer Prozesse kann davon ausgegangen werden, dass der Reformschwung, der - zumindest aus struktureller Sicht mit Blick auf landesgesetzliche Rahmenvorgaben - als Folge der Verabschiedung der UN-BRK eingetreten ist, in den kommenden Jahren nachlassen und ähnlich der beschriebenen Wellenbewegung an einen Punkt „zurückschwingen“ wird, an dem gar keine bis geringfügige äußere und innere Schulstrukturreformen erfolgen. Darüber hinaus ist anzunehmen, dass die Grundfunktionen von Schule (Selektion, Allokation, Qualifikation) mit Blick auf die Förderung Deutschlands als Wirtschaftsstandort notwendigerweise unverändert erhalten bleiben. Inklusive Bildungsprozesse lassen sich im beruflichen Bereich nicht einfach fortsetzen, da qualitative Ansprüche an durch Bildung erworbene Kompetenzen im Zeitalter der Digitalisierung nicht niedriger, sondern höher werden.

Auch wenn die Auswirkungen bildungspolitischer Entscheidungen - vor allem im Sinne schulstruktureller Veränderungen - nicht überschätzt werden dürfen (Fend, 2008), ist eine aktive und umfassende politische Unterstützung tief greifender Veränderungen im deutschen Schulsystem in den vergangenen Jahrzehnten und aktuell nicht sichtbar. Diese Feststellung betrifft das Festhalten an einem mehrgliedrigen Schulsystem, auch wenn die Gliedrigkeit jetzt zum Teil in eine Schulform wie die Gemeinschaftsschule „verlegt“ worden ist. Die Bundesländer entwerfen unterschiedliche verfahrensrechtliche Rahmungen (Bsp.: Inklusionsverordnung im Saarland), die keine direkten (qualitativen) Veränderungen von Schule und Unterricht erfordern. Derzeitige Veröffentlichungen in deutschen Tageszeitungen oder dem Deutschlandfunk ${ }^{80}$ sprechen bereits vom „Scheitern der Inklusion“, der u.a. mit dem Mangel an Ressourcen in den Schulen in Verbindung gebracht wird.

Ressourcenknappheit im Bildungswesen ist ebenfalls eine Begleiterscheinung der vergangenen Jahrzehnte. Eine dauerhafte Erschwernis wird in diesem Zusammenhang von Allgemein- wie Sonderpädagogen darin gesehen, dass Schulen nicht ausreichend mit Ressourcen ausgestattet werden um differenziertes pädagogisches Arbeiten in räumlicher und personeller Hinsicht sowie mit Blick auf Unterrichtsmaterial und Hilfsmittel zu gestalten. Schulen stoßen an ihre Grenzen, wenn nicht weitere Regelschul- und Förderschullehrkräfte, Sozialpädagogen und weitere Fachkräfte in ausreichender Anzahl in den Regelschulen beschäftigt werden, von einer barrierefreien und guten materiellen Ausstattung der Schulgebäude ganz zu schweigen. Bezeichnend erscheint in diesem Zusammenhang die Tatsache, dass die Bereitstellung angemessener Ressourcen für integratives Arbeiten in Regelschulen bereits Ende der 70er-Jahre gefordert wur-

80 Bsp.: Hannoversche Allgemeine vom 22.02.2019; Unter: https://www.haz.de/Nachrichten/Der-Norden/Ist-die-Inklusion-gescheitert-Grant-Hendrik-Tonne-im-Interview; Stand: 28.04.2019; Deutschlandfunk vom 20.03.2019; Unter: https://www.deutschlandfunk.de/institut-fuer-menschenrechte-inklusion-vorerst-gescheitert.680.de.html? dram:article_id=444140; Stand: 28.04.2019; Südddeutsche vom 27.04.2019; Unter: https://www.sueddeutsche.de/ bildung/jahre-un-behindertenrechtskonvention-ist-die-inklusion-gescheitert-1.4422777; Stand: 28.04 .2019 
de (Heese, 1977, S. 105). Die Ressourcenausstattung des Bildungssystems und damit auch die Ressourcenausstattung für die Umsetzung inklusiver Bildung im Regelschulsystem werden aller Voraussicht nach unzureichend bleiben.

Die Frage nach qualitativen Veränderungsprozessen in Schulen wurde - wie die Ausführungen unter 2.1 zeigen - auch bei den zurückliegenden Reformversuchen gestellt und es erscheint in diesem Zusammenhang hochinteressant, dass Antwortversuche vor allem aus den 70er- und 80er-Jahren teilweise im Wortlaut identisch zu aktuellen Ausführungen vorgenommen wurden (Bsp.: Bleidick (1974) zur Zukunft des Bildungssystems; Muth oder Heese (1977) zur Ausgestaltung von Gesamtschulen; Weigt (1977) zu Gesamtschulen; Reiser et al. (1984) zu notwendigen Veränderungen in Schulen). Bündelt man die Qualitätsmerkmale, die für integrativ arbeitende Schulen formuliert wurden, sind es folgende Kategorien und Subkategorien, die Allgemein- wie Sonderpädagogen bereits in den 70er Jahren relevant erscheinen: die Früherkennung von Behinderungen und vorschulische Erziehung bzw. Frühförderung im Elementarbereich, die Ausstattung der Schulen mit technischen Medien und behinderungsspezifischen Fördermitteln bzw. auch besonderen Räumlichkeiten, die Senkung und Flexibilisierung von Klassenfrequenzen, der Einsatz therapeutischen Personals in Regelschulen, Flexibilität bei der Schüler-Lehrer-Relation für individualisierte Lernprozesse bspw. für Kleingruppenbildung, Flexibilität im integrativen System insgesamt, der Einsatz von Sonderpädagogen an Regelschulen in dem Umfang, dass Individualisierung durch Teamteaching möglich wird, die Individualisierung des Bildungsangebotes durch innere Differenzierung und (behinderungsspezifischen) Förderunterricht, der Abbau des Leistungsdrucks in den Schulen und die Einbeziehung sonderpädagogischer Fachinhalte in die allgemeine Lehrerausbildung (Dt. Bildungsrat, 1973; Arbeitskreis Grundschule, 1977; Heese, 1977; Muth, 1977; Preuss-Lausitz, 1977; Weigt, 1977). Mehrere Jahrzehnte später werden diese qualitativen Veränderungen in weitgehend identischer Form erneut gefordert (vgl. dazu Kapitel 3), so dass die Annahme naheliegend ist, dass bislang zumindest keine flächendeckende Umsetzung derselben erfolgt ist. Die bereits Ende der 70erJahre von einzelnen (Sonder-) Pädagogen präzise formulierten qualitativen Voraussetzungen für die Ermöglichung der Integration von Schülern mit Behinderungen in Regelschulen stehen heute erstaunlich unverändert auf der Agenda inklusiver Bildungsbestrebungen.

Diese Arbeit wiederum hat gezeigt, dass die Qualitätsbausteine schulischer Inklusion in sehr hohem Maß mit den Qualitätsbausteinen guter Schulen und guten Unterrichts deckungsgleich sind, und lediglich durch wenige Bereiche wie bestimmte Ressourcen, die Kooperation von Regel- und Förderschullehrkräften sowie individualisierende Angebote für Schülerinnen und Schüler mit sehr spezifischen Förderbedürfnissen zu ergänzen sind. Die Qualität schulischer Inklusion kann also in Teilen in einzelnen Schulen pädagogisch bearbeitet werden und befasst sich in diesem Zusammenhang mit Fragestellungen, die in Schulen institutionalisierte Lernprozesse von Kindern und Jugendlichen allgemein betreffen. An den Schulen, an denen sich Schulleitungen, Lehrkräfte, pädagogisches Personal, Eltern sowie Schülerinnen und Schüler in Vernetzung mit dem Umfeld der Schule gemeinsam auf den Weg der Verbesserung ihres Schullebens und ihres Unterrichts begeben, kann eine Weiterentwicklung von (inklusiver) Bildung stattfinden:

Unter glücklichen Bedingungen kann sich dann nicht nur die jetzt alltägliche und nicht selten beliebigunverbindliche Form schulischen Lernens ausbilden, sondern der Stil pädagogischer Arbeit bewußt kultiviert werden, mit einem eindeutigen Anspruchsniveau, einer selbstkritischen Binnenkommunikation, mit professioneller Selbstkontrolle, unter Beteiligung von Schülern, Lehrern und Eltern, und mit Selbstbildern, die auf Zukunft und die Bedeutung des Lernens setzen. (Tenorth, 1994, S. 185) 
Dabei kann davon ausgegangen werden, dass es bereits eine ganze Reihe engagierter Schulen gibt, die pädagogisch in hohem Maß schülerorientiert arbeiten und einen großen Teil der in dieser Arbeit aufgeführten Qualitätsbausteine erfolgreich ausgestalten und umsetzen (s. Preisträgerschulen Jakob-Muth-Preis, Deutscher Schulpreis, private Schulen mit individualisierenden Angeboten wie die Montessori-Schulen etc.).

Während die Frage, wo „innerhalb der pädagogischen Institutionen, wie sie heute beschaffen sind, das Wandlungspotential [liegt]?" (Bernstein 1970, in Bauer et al. 2012, S. 154), anhand des Forschungsüberblicks und der Ergebnisse für einzelne Schulen also in Ansätzen beantwortet werden kann, ist dies für die Frage, wie eine Transformation des deutschen Bildungssystems erfolgen und welche Bestandteile diese umfassen könnte, nicht ohne weiteres möglich. In Anlehnung an Haselbeck (2007) lässt sich aufgrund der Analyse festhalten, dass schulische Entwicklungen mit gesellschaftlichen Entwicklungen einhergehen (gesellschaftlicher Kontext), dass Schulen als Teil eines übergeordneten Schulsystems, das stark durch bildungspolitische Entscheidungen geprägt ist, zentrale Funktionen für Lebensprozesse von Schülerinnen und Schülern übernehmen (institutioneller Kontext) und dass die Interaktionen zwischen Lehrkräften und Schülern spezifischen äußeren Bedingungen und damit zusammenhängenden Bewältigungsansätzen unterliegen (individuell-personaler Kontext) (Haselbeck, 2007, S. 3). Auch wenn einzelne Schulen sich also mit der pädagogischen Bearbeitung der grundlegenden Frage nach dem Umgang mit Heterogenität und der Gestaltung eines qualitätsvollen Unterrichts und Schullebens befassen können, sind sie als Teil eines übergeordneten Schulsystems und im Rahmen der gesellschaftlichen Entwicklung darauf angewiesen, dass sich ein grundlegender Wandel des Bildungssystems - wie vom UN-Ausschuss gefordert (s. S. 9) - vollzieht. Dabei ist nicht in erster Linie eine Auseinandersetzung mit der Unterrichtung von Kindern und Jugendlichen mit Behinderungen in Förder- oder Regelschulen in den Blick zu nehmen, sondern die Frage nach der Qualität von Schulen und Unterricht allgemein.

Dass dieser Prozess geschieht, ist - wie oben dargestellt - in Anbetracht der Analyse der geschichtlichen Eckdaten zu bildungspolitischen Reformprozessen in der BRD als äußerst unwahrscheinlich anzunehmen und er könnte - aus gesellschaftlicher und politischer Perspektive - nur dann Reformschwung erhalten, wenn aufgrund der demografischen Entwicklung in Verbindung mit der wirtschaftlichen Situation die Notwendigkeit entsteht, einen noch höheren Anteil an Schülerinnen und Schülern zur Ausbildungsreife zu bringen. Anzeichen für diese Entwicklung sind derzeit vorhanden (Bundesvereinigung der deutschen Arbeitgeber, 2015, S. 8; Bundesministerium für Wirtschaft und Energie, 2019, S. 46-51), so dass bspw. bildungspolitische Reformforderungen der Bundesvereinigung der deutschen Arbeitgeber den kompletten Bereich von der frühkindlichen bis zur beruflichen Bildung umfassen (BDA, 2015, S. 17-25). Aufgrund eines möglichen Fachkräftemangels postuliert der Bund der deutschen Industrie in diesem Zusammenhang schlicht: „Bildungspolitik ist [...] Standortpolitik“ (BDI, 2019, S.1). Allerdings haben mögliche Reformanstrengungen neben dem Ziel eines höheren Anteils von Jugendlichen mit Schulabschlüssen vor allem die Digitalisierung im Blick und es wird - ähnlich wie hinsichtlich inklusiver Bildung - kein umfassender Reformprozess gestaltet, sondern stattdessen eine Fülle unterschiedlicher Projekte in den einzelnen Bundesländern (BMWI, 2019, S. 103-110). Nur ein Reformanlass aus wirtschaftlichen Gründen, der mit inklusiver Bildung im engeren Sinn zunächst wenig zu tun hat, könnte also ggf. dazu führen, dass die Qualität von Schulen und Unterricht noch stärker als bisher in den Fokus der Bildungspolitik rückt. Sollten Reformanstrengungen unternommen werden, ist es wiederum aufgrund der föderalistischen Struktur der BRD notwendig, dass diese Anstrengungen Möglichkeiten eröffnen ein hochfle- 
xibles Schulsystem zu gestalten, innerhalb dessen Einzelschulen ein höheres Maß an zumindest in Teilen autonomer Entwicklung zugestanden wird. Ohne diese umfassende Umgestaltung der deutschen Schulen durch einen entsprechenden bildungspolitischen Rahmen und verknüpft mit einer hohen bis sehr hohen Eigenverantwortung von Schulen für ihre pädagogische Entwicklung wird es keine tiefgreifende und umfassende Transformation geben. Gleichzeitig erfordert diese Transformation das Engagement des kompletten Systems, das heißt dass gesellschaftliche Entscheidungsträger, Bildungspolitik und -verwaltung, Universitäten bzw. Pädagogische Hochschulen und Studienseminare, Schulleitungen, Lehrkräfte, Eltern, Schülerinnen und Schüler, Netzwerkpartner, die Schulträger und weitere mögliche Beteiligte kooperativ agieren (s. dazu auch Dt. Institut für Menschenrechte, 2017).

Diese Grundvoraussetzung für die Transformation im Sinne der Eignung sowie der „Work Orientation“" (Bryk et al., 2010) bzw. der „Leidenschaft“ (Hattie, 2013) der Lehrkräfte und anderer Beteiligter für ihre Tätigkeit könnte bspw. dadurch gesichert werden, dass Studieninteressierte bzw. zu verbeamtende Lehrkräfte oder Funktionsstellenbewerber ein Auswahlverfahren durchlaufen, das diese Voraussetzung prüft ${ }^{81}$.

Bleibt die Frage zu beantworten, inwiefern schulstrukurelle Reformen in diesem Zusammenhang eine Rolle spielen können und sollen. Wie in Kapitel 2 dargestellt, sind es nicht in erster Linie die unterschiedlichen Schulformen, die Bildungserfolge beeinflussen, sondern vielmehr spezifische Standortfaktoren einer Schule (Kronig, 2007; Bryk et al., 2010). Eine Idee zu einer möglichen strukturellen Reform besteht darin „eine Schule für alle“ in dem Sinn zu schaffen, dass neben den Grundschulen lediglich eine weitere Schulform für die Sekundarstufe I eingeführt wird (Prengel, 1993; Tenorth, 1994; Jennessen \& Wagner, 2012), und erst in der Sekundarstufe II eine Aufgliederung in eine Werkstufe, einen berufsvorbereitenden Bereich, den berufsbildenden Unterricht oder eine gymnasiale Oberstufe vorzunehmen, ähnlich wie dies bspw. in Südtirol der Fall ist. Sowohl innerhalb der Grundschulen als auch innerhalb der Sekundarstufenschulen müsste für einen solchen Reformansatz ein flexibles schulisches Angebot bereitgehalten werden, bei dem es - mit Blick auf die vielfältigen Förderbedürfnisse - nicht ausreichen würde "gemeinsamen Unterricht“ für alle $\mathrm{SuS}$ anzubieten. Stattdessen könnte ein Unterrichtsangebot entwickelt werden, das sowohl gemeinsamen wie auch individualisierten Unterricht in unterschiedlich zusammengesetzten Gruppen oder auch in Einzelförderung vorsieht (s. dazu Ausführungen unter 4.5). Unabhängig davon, dass eine solche strukturelle Reform zunächst keinen Einfluss auf die Qualität von Schulen hat, würde in diesem Rahmen eine „Verschiebung“ der strukturellen Berücksichtigung von Differenz (Bsp.: Schulabschluss) nach „hinten“ erfolgen. Die Frage nach Abschlussberechtigungen und Zugangsmöglichkeiten in das berufliche System ließe sich dadurch also genauso wenig aufösen wie die Frage danach, wie in einem solchen System gewährleistet werden kann, dass auch hochspezifische Förderansprüche von blinden oder gehörlosen, kognitiv bzw. physisch schwer beeinträchtigten oder auch hochbegabten Schülerinnen und Schülern sowie von Kindern und Jugendlichen mit sehr hohem emotionalen und sozialem Unterstützungsbedarf erfüllt werden können. Darüber hinaus würde das Elternwahlrecht auf unzulässige Art und Weise eingeschränkt, wenn nicht mehr zwischen mehreren Schulformen gewählt werden kann (Kiefer, 2010).

Aus diesen Gründen und aufgrund der Annahme, dass eine derartige Schulstrukturreformidee keine breite politische Unterstützung erfahren wird, könnte es Ziel führender sein, die große Bandbreite und Vielfalt des deutschen Bildungssystems positiv zu sehen und die Idee einer flexiblen Weiterentwicklung der bestehenden Schulformen konsequent zu verfolgen. In einem fi-

81 ähnlich wie bspw. in Finnland, s. dazu https://deutsches-schulportal.de/bildungswesen/strenges-auswahlverfahren-fuer-das-lehramtsstudium/; Stand: 13.05.2019 
nanziell gut ausgestatteten bildungspolitischem Rahmen, der Kontinuität über Wahlperioden hinweg ermöglicht, könnten Schulen die pädagogische Aufgabe annehmen, unter Umständen auf sehr individuellen Wegen, ein Lernangebot zu gestalten, das es Schülerinnen und Schülern ermöglicht am Lernen in diesen Schulen teilzuhaben, ohne dass diese dafür gezwungen sind „bestimmte“ Schulformen zu besuchen. Wesentlich wäre bei diesem Weg nicht die Schulform, sondern das pädagogische Innenleben einer Schule. Es würde in diesem Zusammenhang nicht darum gehen, so genannte inklusive Schulen von Förderschulen und Gymnasien abzugrenzen, sondern es könnte darum gehen alle Schulformen zu geeigneten Förderorten (weiter) zu entwickeln. Jede Schule könnte die Möglichkeit nutzen Schüler und Schülerinnen aufzunehmen, denen sie ein besonderes Förderangebot machen kann. So können Gymnasien sich bspw. für die Aufnahme schwerhöriger oder sehbehinderter Schüler spezialisieren bzw. besondere Angebote für Schüler mit körperlichen Beeinträchtigungen oder Autismus-Spektrum-Störungen bereithalten. Beispiele dafür sind das Gymnasium Essen-Nordost ${ }^{82}$ (Integration von $\mathrm{SuS}$ mit spF körperlich-motorische Entwicklung und Sehen), die Stadtteilschule Hamburg-Mitte ${ }^{83}$ (Klassen für hörbeeinträchtigte sowie für gehörlose SuS) oder das Leibniz-Gymnasium in Altdorf bei Nürnberg ${ }^{84}$ (Integration von $\mathrm{SuS}$ mit dem spF körperlich-motorische Entwicklung), die dies bereits seit mehreren Jahrzehnten praktizieren. Grundschulen können inklusive Bildung für $\mathrm{SuS}$ mit unterschiedlichen Förderbedarfen anbieten (Bsp.: Modellversuchsschulen aus den 70er bzw. 80er Jahren wie die Grundschule Robinsbalje, Bremen ${ }^{85}$, oder die Fläming-Grundschule, Berlin $\left.{ }^{86}\right)$. Gemeinschaftsschulen könnten ihr Angebot so ausweiten, dass sie differenzierenden Unterricht für die Förderbedarfe Lernen und geistige Entwicklung in flexibler Klassen-, Gruppen- oder Einzelförderung bzw. in besonderen Klassen gestalten. Schulen für verschiedene Förderschwerpunkte können Schülerinnen und Schüler ohne spF aufnehmen, deren Eltern besonderen Wert auf Förderung im Rahmen dieser Schwerpunkte im weiteren Sinn legen. Auch Förderschulen können sich also hin zu fachrichtungsübergreifenden und abschlussbezogenen Bildungsangeboten entwickeln - ein Weg, auf den sich bereits einige Förderschulen gemacht haben, darunter zum Beispiel die Martinschule in Greifswald, eine Schule zur individuellen Lebensbewältigung, die sich für „Regelschüler“ geöffnet hat, oder die Waldhofschule in Templin ${ }^{87}$. Dafür könnte bspw. auch der Abschluss der Schule für geistige Entwicklung eine Benennung erhalten (Bsp: lebenspraktische berufliche Reife) und Schulen mit dem Förderschwerpunkt Lernen müssten - neben diesem Abschluss für kognitiv schwächere Schüler - grundsätzlich auch den Hauptschulabschluss oder die so genannte Berufsreife anbieten können.

Wichtig wäre für diesen Weg der Transformation die unbedingte Wertschätzung allen Schulformen gegenüber und eine Wertschätzung der Vorstellungen von Eltern und Schülerinnen und Schülern als den „Kunden“ dieser Schulen. Nur die Verbindung eines ein Höchstmaß an Flexibilität gewährenden bildungspolitischen Rahmens mit einer guten finanziellen Ausstattung des Bildungssystems, mit dem kooperativen Engagement von Beteiligten auf allen Ebenen und mit innerschulisch-pädagogischen Entwicklungsprozessen, die zum Ziel haben hervorragende Bildungs- und Lernmöglichkeiten für Kinder und Jugendlichen zu schaffen, kann zu einer tiefgreifenden und umfassenden Transformation von Schulen und Schulsystem führen.

82 Unter: www.gtgeno.de/wir-ueber-uns/schulprogramm. Stand: 11.05 .2019

83 Unter: https://stadtteilschule-mitte.hamburg.de/unsere-hoergeschaedigten-klassen/; Stand: 11.05.2019

84 Unter: https://leibniz-gymnasium-altdorf.de/inklusion/; Stand: 11.05 .2019

85 Unter: https://robinsbalje.schule.bremen.de/willkommen/kurzvorstellung-9642; Stand: 11.05 .2019

86 Unter: http://www.flaeming-grundschule.de/; Stand: 11.05 .2019

87 Unter: https://odebrecht-stiftung.de/schule/; http://www.waldhofschule.de/; Stand: 11.05.2019 



\section{Anhang}

\section{Literaturverzeichnis}

Ahrbeck, B. (2011): Der Umgang mit Behinderung. Stuttgart: Kohlhammer

Ahrens, F. (2009): Kooperation und Netzwerke zwischen Jugendarbeit und Schule - Grundlagen, Rabmenbedingungen und Beispiele der Jugendverbandsarbeit in Niedersachsen. Unter: www.jugendserver-niedersachsen.de/uploads/tx_ sgfilelist/02660-Ahrens_Kooperation_Jugendarbeit_und_Schule.pdf; Stand: 09.02.2019

Aigner, H. (1989): Bericht über Schulleistungen in Integrationsklassen. Schulpsychologische Beratungsstelle Graz-Umgebung (unveröffentlichtes Manuskript)

Ainscow, M.; Both, T.; Dyson, A. (2006): Improving Schools, Developing Inclusion. Abingdon: Routledge

Albisser, S.; Keller-Schneider, M.; Wissinger, J. (2013): Zusammenarbeit in Kollegien von Schulen unter dem Anspruch von Professionalität. In: Keller-Schneider, M.; Albisser, S.; Wissinger, J. (Hrsg.) (2013): Professionalität und Kooperation in Schulen. Beiträge zur Diskussion über Schulqualität. Bad Heilbrunn: Klinkhardt, 9-29

Altrichter, H.; Feyerer, E. (2017): Schulentwicklung und Inklusion in Österreich. In: Lütje-Klose, B.; Miller, S.; Schwab, S.; Streese, B. (Hrsg.) (2017): Inklusion: Profile für die Schul-und Unterrichtsentwicklung in Deutschland, Österreich und der Schweiz. Theoretische Grundlagen - Empirische Befunde - Praxisbeispiele. Münster, New York: Waxmann, $31-42$

Amrhein, B. (2011): Inklusion in der Sekundarstufe. Eine empirische Analyse. Bad Heilbrunn: Klinkhardt

Amrhein, B.; Dziak-Mahler, M. (Hrsg.) (2014): Inklusive Fachdidaktik. Auf der Suche nach didaktischen Leitlinien für den Umgang mit Vielfalt in der Schule. Münster, New York: Waxmann

Amrhein, B.; Reich, K. (2014): Inklusive Fachdidaktik. In: Amrhein, B.; Dziak-Mahler, M. (Hrsg.) (2014): Inklusive Fachdidaktik. Auf der Suche nach didaktischen Leitlinien für den Umgang mit Vielfalt in der Schule. Münster, New York: Waxmann, 31-44

Andersen, V.V. (1987): Weil es immer so gewesen ist - ist es nicht ganz sicher, dass es das Beste ist. In: Mutzeck, W.; Pallasch, W. (Hrsg.): Integration von Schülern mit Verhaltensstörungen. Praktische Modelle und Versuche. Weinheim: Deutscher Studien Verlag, 92-100

Anhut, R.; Heitmeyer, W. (2000): Desintegration, Konflikt und Ethnisierung. Eine Problemanalyse und theoretische Rahmenkonzeption. In: Heitmeyer, W.; Anhut, R. (Hrsg.): Bedrohte Stadtgesellschaft. Konflikt- und Gewaltforschung. Weinheim \& München: Juventa, 17-75

Anken, L. (2010): Konstruktivismus und Inklusion im Dialog. Heidelberg: Carl-Auer-Verlag

Antor, G. (1987): Hamburger Integrationsklassen: ein Schulversuch zwischen System und Lebenswelt. In: Wocken, H.; Antor, G. (Hrsg.) (1987): Integrationsklassen in Hamburg. Erfahrungen - Untersuchungen - Anregungen. SolmsOberbiel: Jarick-Oberbiel, 91-115

Antor, G. (1992): Pluralität der schulischen Normen - Uniformität des Lernorts? Erfahrungen mit der schulischen Integation Behinderter und Nichtbehinderter. In: Lersch, R.; Vernooij, M.A. (Hrsg.) (1992): Behinderte Kinder und Jugendliche in der Schule. Herausforderungen an Schul-und Sonderpädagogik. Bad Heilbrunn: Klinkhardt Verlag, 30-52

Arbeitskammer des Saarlandes (2016): Der mühsame Weg hin zu einem inklusiven Schulsystem. In: Arbeitskammer des Saarlandes (2016): Zur wirtschaftlichen, ökologischen, sozialen und kulturellen Lage der Arbeitnehmerinnen und Arbeitnehmer. Bericht an die Regierung des Saarlandes 2016. Unter: https://www.arbeitskammer.de/fileadmin/ user_upload/ak_download_datenbank/Publikationen/Jahresberichte_und_Datenbaende/Jahresbericht_2016/ AK_-_Bericht_an_die_Regierung_2016.pdf; Stand: 12.03.2018,340-349

Arbeitskammer des Saarlandes (2018): Inklusion an saarländischen Schulen. In: Arbeitskammer des Saarlandes (2016): Zur wirtschaftlichen, ökologischen, sozialen und kulturellen Lage der Arbeitnehmerinnen und Arbeitnehmer. Bericht an die Regierung des Saarlandes 2018. Unter: https://www.arbeitskammer.de/fileadmin/user_upload/ak_download_datenbank/Publikationen/Jahresberichte_und_Datenbaende/Jahresbericht_2018/AK_Bericht_2018_aktiv. pdf; Stand: 12.03.2018, 378-381

Arbeitskreis Grundschule/Gewerkschaft Erziehung und Wissenschaft (1977): Maßnahmen zur Verminderung von Schulschwäche in der Grundschule. Frankfurt: GEW

Arendt, H. (1967, 3. Aufl.): Vita activa oder Vom tätigen Leben. München: Piper

Arndt, A.K.; Gieschen, A. (2013): Von der Verschiedenheit der Lehrkräfte profitieren. Kooperation von Regel- und Förderschullehrkräften aus der Sicht von SchülerInnen. In: Lernchancen 93/94, 22-26

Arndt, A.K.; Werning, R. (2013): Unterrichtsbezogene Kooperation von Regelschullehrkräften und Lehrkräften für Sonderpädagogik. Ergebnisse eines qualitativen Forschungsprojekts. In: Werning, R.; Arndt, A.K. (Hrsg.) (2013): Inklusion: Kooperation und Unterricht entwickeln. Bad Heilbrunn: Klinkhardt, 12-40 
Arnold, R.; Harth, T.; Schüssler, I. (1999): Konstruktivistische Impulse für Lehren und Lernen. In: Außerschulische Bildung, (1999) 4, S. 372-376

Arnold, C.; Bauer, F.; Kunz Heim, D. (2011): Arbeiten in Unterrichtsteams Erfahrungen von Lehrerinnen und Lehrern. Eine qualitativ-quantitative Untersuchung. Unter: http://web.fhnw.ch/plattformen/ressourcenplus/themen/ArbeiteninUnterrichtsteams_Hauptbericht.pdf; Stand: 13.05.2014

Arnold, K.-H. \& Lindner-Müller, C. (2010): Einführung: Unterrichtsentwicklung - Die Perspektive der Unterrichtswissenschaften. In: Bohl, T.; Helsper, W.; Holtappels, H.G.; Schelle, C. (Hrsg.) (2010): Handbuch Schulentwicklung. Bad Heilbrunn: Klinkhardt, 313-315

Bach, H. (1987): Konzept und Praxis schulintegrierter Förderung bei Verhaltensauffälligkeiten. In: Mutzeck, W.; Pallasch, W. (Hrsg.): Integration von Schülern mit Verhaltensstörungen. Praktische Modelle und Versuche. Weinheim: Deutscher Studien Verlag, 101-109

Bachmann, W. (1974): Die Integration von ,Grenzfällen' zwischen Sonderschule und Gesamtschule. In: Pädagogische Rundschau, 9, 1974, 718-736

Bächthold, A.; Coradi, U.; Hildbrand, J.; Strasser, U. (1990): Integration ist lernbar. Erfahrungen mit schulschwierigen Kindern im Kanton Zürich. Luzern: Edition SZH

Bauer, U.; Bittlingmayer, U.H.; Scherr, A. (Hrsg.) (2012): Handbuch Bildungs- und Erziehungssoziologie. Wiesbaden: Springer VS

Baum, E.; Idel, T.-S.; Ullrich, H. (Hrsg.) (2012a): Kollegialität und Kooperation in der Schule. Theoretische Konzepte und empirische Befunde. Wiesbaden: Springer VS

Baum, E.; Idel, T.-S.; Ullrich, H. (2012b): Kollegialität und Kooperation in der Schule - Zur Einleitung in diesen Band. In: Baum, E.; Idel, T.-S.; Ullrich, H. (Hrsg.) (2012): Kollegialität und Kooperation in der Schule. Theoretische Konzepte und empirische Befunde. Wiesbaden: Springer VS, 9-25

Bauman, Z. (2003): Flüchtige Moderne. Berlin: Suhrkamp

Bauman, Z. (2005): Verworfenes Leben. Die Ausgegrenzten der Moderne. Hamburg: Hamburger Edition

Baumert, J.; Gruehn, S.; Heyn, S.; Köller, O.; Schnabel, K.-U. (1997): Bildungsverläufe und psychosoziale Entwicklung im Jugendalter (BIJU). Dokumentation - Band 1. Skalen Längsschnitt I, Welle 1-4. Berlin: Max-Planck-Institut für Bildungsforschung

Baumert, J.; Trautwein, U.; Artelt, C. (2003): Schulumwelten - Institutionelle Bedingungen des Lehrens und Lernens. In: Deutsches PISA-Konsortium (Hrsg.): PISA 2000-Ein differenzierter Blick auf die Länder der Bundesrepublik Deutschland. Opladen: Leske und Budrich, 261-331

Beck, U. (1986): Risikogesellschaft - Aufdem Weg in eine andere Moderne. Berlin: Suhrkamp

Becker, R. (2000): Klassenlage und Bildungsentscheidungen. Eine empirische Anwendung der Wert-Erwartungstheorie. In: Kölner Zeitschrift für Soziologie und Sozialpsychologie, 52, 450-474

Becker, R.; Lauterbach, W. (Hrsg.) (2004): Bildung als Privileg. Erklärungen und Befunde zu den Ursachen der Bildungsungleichheit. Wiesbaden: Springer VS

Behrensen, B.; Sauerhering, M.; Schwer, C.; Solzbacher, C. (2012): Jedem Kind gerecht werden? Sichtweisen und Erfahrungen von Grundschullehrkräften. Köln: Carl Link Verlag

Benner, D. (2015, 8. Aufl.): Allgemeine Pädagogik. Eine systematisch-problemgeschichtliche Einführung in die Grundstruktur pädagogischen Denken und Handelns. Weinheim und Basel: Beltz Verlag Juventa

Berg, K.H. (1975): Separierung der Heilerziehung, eine Chance für die Behinderten. In: Zeitschrift für Heilpädagogik, $11,1975,759-768$

Berges, M. (1996): Dimensionen der Integration bei kooperativem Unterricht. Eine empirische Untersuchung zu Strukturen und Prozessen beim gemeinsamen Unterricht von behinderten und nichtbehinderten Kindern und Jugendlichen. Frankfurt a.M., Berlin, Bern, Bruxelles, New York, Oxford, Wien: Peter Lang

Bernstein, B. (1970): Eine Kritik des Begriffs ,kompensatorische Erziehung. In: Bauer, U.; Bittlingmayer, U.H.; Scherr, A. (Hrsg.) (2012): Handbuch Bildungs- und Erziehungssoziologie. Wiesbaden: Springer VS, 151-159

Berthelsen, D.; Walker, S. (2008): Parents' involvement in their children's education. In: Family Matters 2008, No. 79, 34-42

Bielefeldt, H. (2009, 3. Auflage): Zum Innovationspotenzial der UN-Behindertenrechtskonvention. Berlin: Deutsches Institut für Menschenrechte Unter: https://nbn-resolving.org/urn:nbn:de:0168-ssoar-328138; Stand: 03.10.2012

Bildungskommission NRW (1995): Zukunft der Bildung - Schule der Zukunft. Denkschrift der Kommission „Zukunft der Bildung - Schule der Zukunft“ beim Ministerpräsidenten des Landes Nordrhein-Westfalen. Neuwied, Kriftel, Berlin: Luchterhand

Bleidick, U. (1974, 2. Aufl.): Pädagogik der Behinderten. Grundzüge einer Theorie der Erziehung behinderter Kinder und Jugendlicher. Berlin: Marhold

Bleidick, U. (1978, 3. Aufl.): Pädagogik der Behinderten. Grundzüge einer Theorie der Erziehung behinderter Kinder und Jugendlicher. Berlin: Marhold 
Bleidick, U. (1990): Bildungspolitische Entwicklungslinien zur gesellschaftlichen Integration von Behinderten. In: Schuck, K.D. (Hrsg.) (1990): Beiträge zur Integrativen Pädagogik. Weiterentwicklungen des Konzepts gemeinsamen Lebens und Lernens Behinderter und Nichtbehinderter. Hamburg: Feldhaus Verlag, 9-32

Bleidick, U.; Ellger-Rüttgardt, S. L. (2008): Behindertenpädagogik-eine Bilanz. Bildungspolitik und Theorieentwicklung von 1950 bis zur Gegenwart. Stuttgart: Verlag W. Kohlhammer

Bless, G.; Klaghofer, R. (1991): Begabte Schüler in Integrationsklassen. Untersuchung zur Entwicklung von Schulleistung, sozialen und emotionalen Faktoren. In: Zeitschrift für Pädagogik 37, Heft 2, 215-222

Bless, G. (2003, 4. unv. Auflage): Zur Wirksamkeit der Integration. Forschungsüberblick, praktische Umsetzung einer integrativen Schulform, Untersuchungen zum Lernfortschritt. Bern, Stuttgart, Wien: Haupt Verlag

Blömer, D.; Lichblau, M.; Jüttner, A.-K.; Koch, K.; Krüger, M.; Werning, R. (Hrsg.) (2015): Perspektiven auf inklusive Bildung. Gemeinsam anders lehren und lernen. Jahrbuch Grundschulforschung. Wiesbaden: Springer VS

Boban, I.; Hinz, A. (2003): Index für Inklusion. Lernen und Teilhabe in der Schule der Vielfalt entwickeln. Übersetzt, für deutschsprachige Verhältnisse bearbeitet und herausgegeben von Ines Boban und Andreas Hinz. Halle-Wittenberg: Martin-Luther-Universität. Unter: www.eenet.org.uk/resources/docs/Index German.pdf; 04.03.2013

Böhnke, P. (2006): Soziale Ausgrenzung als allgemeines Lebensrisiko?: Befunde zu einer populären Zeitdiagnose. In: (Rehberg, K.-S. (Hrsg.); Deutsche Gesellschaft für Soziologie (DGS) (Hrsg.): Soziale Ungleichheit, kulturelle Unterschiede. Verhandlungen des 32. Kongresses der Deutschen Gesellschaft für Soziologie in München. Teilband 1 und 2. Frankfurt am Main: Campus Verlag, 4191-4209

Börner, S.; Glink, A.; Jäpelt, B.; Sanders, D.; Sasse, A. (Hrsg.) (2009): Integration im vierten Jahrzehnt. Bilanz und Perspektiven. Bad Heilbrunn: Klinkhardt

Bogner, A. (2009); Littig, B.; Menz, W. (Hrsg.): Experteninterviews. Theorien, Methoden, Anwendungsfehler. Wiesbaden: Springer VS

Bohl, T.; Helsper, W.; Holtappels, H.G.; Schelle, C. (Hrsg.) (2010): Handbuch Schulentwicklung. Bad Heilbrunn: Klinkhardt

Bonsen, M. (2010): Die Bedeutung der Schulleitung für die Schulentwicklung. In: Bohl, T.; Helsper, W.; Holtappels, H.G.; Schelle, C. (Hrsg.) (2010): Handbuch Schulentwicklung. Bad Heilbrunn: Klinkhardt, 199-203

Booth, T.; Ainscow, M. (2002): Index for inclusion. Developing learning and participation in schools. Bristol: Centre for Studies on Inclusive Education (CSIE). Unter: www.eenet.org.uk/resources/docs/Index English.pdf; Stand: 04.03.2013

Borich, G.D. (2007, 6. Aufl.): Efective teaching methods. Research-based practice. Upper Saddle River: Merrill Prentice Hall

Bos, W.; Lankes, E.-M.; Prenzel, M.; Schwippert, K.; Walther, G.; Valtin, R. (Hrsg.) (2003): Erste Ergebnisse aus IGLU. Schülerleistungen am Ende der vierten Jahrgangsstufe im internationalen Vergleich. Münster u. a: Waxmann

Bos, W.; Bonsen, M.; Berkemeyer, N. (2010): Einzelschule und Schülerleistungen; In: Bohl, T.; Helsper, W.; Holtappels, H.G.; Schelle, C. (Hrsg.) (2010): Handbuch Schulentwicklung. Bad Heilbrunn: Klinkhardt, S. 62-65

Bosse, S.; Jäntsch, C.; Spörer, N. (2015): Einschätzungen von Lehrerinnen und Lehrern zum inklusiven Unterricht. In: Landesinstitut für Schule und Medien Berlin-Brandenburg (LISUM), Universität Potsdam, Deutsches Institut für internationale Pädagogische Forschung (DIPF) (2015) (Hrsg.): Inklusives Lernen und Lehren im Land Brandenburg. Abschlussbericht zur Begleitforschung des Pilotprojekts „Inklusive Grundschule“. Potsdam; Unter: http://www. inklusion-brandenburg.de/fileadmin/daten/inklusion_im_land_brandenburg/pilotprojekt_inklusive_grundschule/wissenschaftliche__begleitung/Abschlussbericht_PING.pdf; Stand: 14.03.2018, 137-154

Boudon, R. (1974): Education, Opportunity, and Social Inequality - Changing Prospects in Western Society. New York: John Wiley \& Sons

Bourdieu, P.; Passeron, J.-C. (1971): Die Illusion der Chancengleichheit. Untersuchungen zur Soziologie des Bildungswesens am Beispiel Frankreichs. Stuttgart: Klett

Bourdieu, P.; Champagne, P. (1997): Die intern Ausgegrenzen. In: Bourdieu, P. (Hrsg.) (1997): Das Elend der Welt. Zeugnisse und Diagnosen alltäglichen Leidens an der Gesellschaft. Konstanz: Universitäts-Verlag, 527-534

Brophy, J. (2010): Was wissen wir darüber, wie guter Unterricht gelingt? In: Eikenbusch, G.; Heymann, H.W. (Hrsg.) (2010): Was wissen wir über guten Unterricht? Hamburg: Bergmann und Helbig Verlag, S. 77-104

Brüning, L.; Saum, T. (2015): Erfolgreich unterrichten durch Kooperatives Lernen. Strategien zur Schüleraktivierung. Essen: Neue Deutsche Schule

Brugger-Paggi, E. (2003): Fragebogen zur Qualität der Integration an Schulen. Unter: http://www.blikk.it/angebote/ reformpaedagogik/rp83138.htm, Stand: 04.11.2011

Bryk, A. (2010): Organizing Schools for Improvement. In: Phi Delta Kappan, v91 n7, Apr 2010, 23-30

Bryk, A.; Bender Sebring, P.; Allensworth, E.; Luppescu, S.; Easton, J.Q. (2010): Organizing Schools for Improvement. Lessons from Chicago. Chicago and London: The University of Chicago Press

Bryman, A. (2012, 4. Aufl.): Social research methods. New York: Oxford University Press

Buchen, H.; Rolff, H.-G. (Hrsg.) (2016): Professionswissen Schulleitung. Weinheim und Basel: Beltz Verlag 
Buchen, H.; Rolff, H.-G. (2016): Leitung als Trias von Führung, Management und Steuerung. In: Buchen, H.; Rolff, H.-G. (Hrsg.) (2016): Professionswissen Schulleitung. Weinheim und Basel: Beltz Verlag, S. 3-10

Budde, J. (Hrsg.) (2013): Unscharfe Einsätze: (Re-) Produktion von Heterogenität im schulischen Feld. Wiesbaden: Springer

Bude, H. (2004): Das Phänomen der Exklusion. Der Widerspruch zwischen gesellschaftlicher Erfahrung und soziologischer Rekonstruktion. In: Mittelweg 36, 4/2004, 3-15

Bude, H.; Willisch, A. (Hrsg.) (2007): Das Problem der Exkusion. In: Bude, H.; Willisch, A. (2007): Das Problem der Exklusion. Ausgegrenzte, Entbehrliche, Überflüssige. Hamburg: Hamburger Edition, 7-23

Bude, H.; Willisch, A. (2006): Das Problem der Exklusion. Ausgegrenzte, Entbehrliche, Überflüssige. Hamburg: Hamburger Edition

Bürli, A. (Hrsg.) (1997): Sonderpädagogik international. Luzern: Edition SZH, SPC

Bürli, A.; Strasser, U.; Stein, Anne-Dore (Hrsg.) (2009): Integration / Inklusion aus internationaler Sicht. Heilbrunn: Klinkhardt

Buhren, C. (2010): Einführung: Personalentwicklung, Personalmanagement und Professionalisierung. In: Bohl, T.; Helsper, W.; Holtappels, H.G.; Schelle, C. (Hrsg.) (2010): Handbuch Schulentwicklung. Bad Heilbrunn: Klinkhardt, 225-231

Bundesvereinigung der deutschen Arbeitgeber (2015): Fachkräftemängel bekämpfen - Wettbewerbsfähigkeit sichern. Unter: https://www.arbeitgeber.de/www/arbeitgeber.nsf / id/DE_Fachkraeftemangel_bekaempfen-Wettbewerbsfae_sichern; Stand: 11.05.2019

Bund der deutschen Industrie (BDI) (2019): Deutsche Wirtschaft für weltoffenes Deutschland. Unter: https://bdi.eu/ artikel/news/deutsche-wirtschaft-fuer-weltoffenes-deutschland/; Stand: 11.05 .2019

Bundesministerium der Justiz (1994): Grundgesetz für die Bundesrepublik Deutschland.

Bundesministerium für Arbeit und Soziales (Hrsg.) (2010): Übereinkommen der Vereinten Nationen über die Rechte von Menschen mit Behinderung. Bonn: BMAS

Bundesministerium für Arbeit und Soziales (Hrsg.) (2011): Unser Weg in eine inklusive Gesellschaft. Der Nationale Aktionsplan der Bundesregierung zur Umsetzung der UN-Behindertenrechtskonvention. Bonn: BMAS

Bundesministerium für Arbeit und Soziales (Hrsg.) (2017): Lebenslagen in Deutschland. Armuts- und Reichtumsberichterstattung der Bundesregierung. Der füfte Armuts- und Reichtumsbericht der Bundesregierung. Unter: http://www.bmas.de/SharedDocs/Downloads/DE/PDF-Pressemitteilungen/2017/5-arb-kurzfassung.pdf ? blob=publicationFile\&v=2; Stand: 15.02 .2018

Bundesministerium für Bildung und Wissenschaft (1970): Bildungsbericht 1970. Bericht der Bundesregierung zur Bildungspolitik. Bonn-Bad Godesberg: BMBW

Bundesministerium für Bildung, Wissenschaft und Forschung Österreich (2008): Richtlinien für die Umsetzung und das Monitoring von Qualitätsstandards im integrativen Unterricht von Schülerinnen und Schülern mit sonderpädagogischem Förderbedarf. Wien:BMWF; Unter: https://bildung.bmbwf.gv.at/ministerium/rs/2008_18_beilage_17197. pdf?6cczlq; Stand: 26.12 .2018

Bundesministerium für Wirtschaft und Energie (2019): Nationales Reformprogramm. Unter: https://www.bmwi.de/ Redaktion/DE/Publikationen/Europa/nationales-reformprogramm-2019.pdf?__blob=publicationFile\&v=5; Stand: 11.05 .2019

Bundesregierung (2017): Lebenslagen in Deutschland. Der Fünfte Armuts- und Reichtumsbericht der Bundesregierung. Unter: https://www.armuts-und-reichtumsbericht.de/SharedDocs/ Downloads/Berichte/5-arb-langfassung.pdf? blob= publicationFile\&v=6; Stand: 18.05.2018

Casale, Gino; Strauß, Sarah; Hennemann, Thomas; König, Johannes (2016): Wie lässt sich Klassenführungsexpertise messen? Überprüfung eines videobasierten Erhebungsinstruments für Lehrkräfte unter Anwendung der Generalisierbarkeitstheorie. Empirische Sonderpädagogik (2016) 2, 119-139

CDU und SPD (Saar) (2012): Chancen nutzen. Zusammenhalt bewahren. Eigenständigkeit sichern. Gemeinsam Verantwortung tragen für unser Saarland. Koalitionsvertrag für die 15. Legislaturperiode des Landtags des Saarlandes (20122017); Unter: http://www.zw-rail.de/Docs/Download/2012_KoalitionsvertragSaarland.pdf; Stand: 25.12 .2018

CDU und SPD (Saar) (2017): Für die Zukunft unseres Landes. Solide wirtschaften - mutig gestalten - mehr investieren. Koalitionsvertrag für die 16. Legislaturperiode des Landtages des Saarlandes (2017-2022); Unter: https://www.spdsaar.de/wp-content/uploads/2016/08/Koalitionsvertrag_CDU_SPD_2017-2022.pdf; Stand: 25.12.2018

Dalin, P.; Rolff, H.-G. (unter Mitarbeit von Buchen, H.) (1990): Institutionelles Schulentwicklungsprogramm. Eine neue Perspektive für Schulleiter, Kollegium und Schulaufsicht. Soest: Soester Verlag Kontor

Daoud-Harms, M. (1997): Arbeitsgegenstand oder Subjekt unserer Lebensgeschichte. Erfahrungen und Reflexionen zum Verhältnis von Betreuern/Experten und „Behinderten“. In: Eberwein, H. (Hrsg.) (1997, 4. Aufl.): Handbuch Integrationspädagogik. Kinder mit und ohne Behinderung lernen gemeinsam. Weinheim und Basel: Beltz Verlag, 429-434 
De Boer, H.; Spies, A. (2014): Kooperationssettings im Kontext inklusiver Grundschulentwicklungsprozesse. In: Lichtblau, M.; Blömer, D.; Jüttner, A.-K.; Koch, K.; Krüger, M.; Werning, R. (2014): Forschung zu inklusiver Bildung. Gemeinsam anders lehren und lernen. Bad Heilbrunn: Julius Klinkhardt, 186-198

Demmer, M. (2007): Verwirklichung des Rechts auf Bildung in Deutschland: Die schwierige Rolle von Pädagoginnen und Pädagogen. In: Overwien, B.; Prengel, A. (Hrsg.): Recht auf Bildung: Zum Besuch des Sonderberichterstatters der Vereinten Nationen in Deutschland. Opladen \& Farmington Hills: Budrich, 157-179

Demmer-Dieckmann, J.; Textor, A. (Hrsg.) (2007): Integrationsforschung und Bildungspolitik im Dialog. Bad Heilbrunn: Klinkhardt

Demmer-Dieckmann, J. (2007): „Aus Zwang wurde Interesse.“ Eine Studie zur Wirksamkeit von Seminaren zum Gemeinsamen Unterricht in Berlin. In: Demmer-Dieckmann, J.; Textor, A. (Hrsg.) (2007): Integrationsforschung und Bildungspolitik im Dialog. Bad Heilbrunn: Klinkhardt, 153-162

Demo, H. (2011): Integrazione scolastica und Lebensqualität der Menschen mit Beeinträchtigung. Ergebnisse einer Studie zu Schulkarriere der Menschen mit Beeinträchtigungen in Italien und ihre Auswirkungen auf das Erwachsenenleben. In: Carle, U.; Bräu, K.; Kunze, I. (2011): Umgang mit Heterogenität - Das Bildungswesen in Südtirol als Anstoßfür schulpädagogische Diskussionen. Opladen: Budrich-Verlag, 127-140

Denzin, N.K. (1970): The Research Act. Chicago: Aldine

Deppe-Wolfinger, H.; Prengel, A.; Reiser, H. (Hrsg.) (1990): Integrative Pädagogik in der Grundschule: Bilanz und Perspektiven der Integration behinderter Kinder in der Bundesrepublik Deutschland 1976-1988. Weinheim und Basel: Beltz Verlag

Deppe-Wolfinger, H. (1990): Perspektiven der Integration: Integration im gesellschaftlichen Wandel. In: Deppe-Wolfinger, H.; Prengel, A.; Reiser, H. (Hrsg.) (1990): Integrative Pädagogik in der Grundschule: Bilanz und Perspektiven der Integration behinderter Kinder in der Bundesrepublik Deutschland 1976-1988. Weinheim und Basel: Beltz Verlag, 310-324

Deppe-Wolfinger, H. (2009): Integration in Hessen. In: Börner, S.; Glink, A.; Jäpelt, B.; Sanders, D.; Sasse, A. (Hrsg.): Integration im vierten Jahrzehnt. Bilanz und Perspektiven. Bad Heilbrunn: Klinkhardt, 50-55

DESI-Konsortium (Hrsg.) (2008): Unterricht und Kompetenzerwerb in Deutsch und Englisch Ergebnisse der DESIStudie. Unter: https://www.pedocs.de/ volltexte/2010/3149/pdf/978_3_407_25491_7_1A_D_A.pdf; Stand: 14.03.2013

Deutscher Bildungsrat (1969): Empfehlungen der Bildungskommission: Einrichtung von Schulversuchen mit Gesamtschulen. Stuttgart: Klett

Deutscher Bildungsrat (1972): Empfehlungen der Bildungskommission. Strukturplan für das Bildungswesen. Stuttgart: Klett

Deutscher Bildungsrat (1973): Zur pädagogischen Förderung behinderter und von Behinderung bedrohter Kinder und Jugendlicher. Bonn: Bundesdruckerei

Deutscher Bildungsrat (Hrsg.) (1976): Schulversuche zur Integration behinderter Kinder in den allgemeinen Unterricht: Materialien zur Bildungsplanung. Heft 6. Braunschweig: Westermann

Deutscher Philologenverband (2010): Inklusion durch Kooperation. Unter: https://www.dphv.de/fileadmin/user_upload/positionen/bildungspolitik/standpunkte/Profil_Artikel_Inklusion.pdf; Stand: 18.04.2015

Deutscher Städtetag (2007): Aachener Erklärung des Deutschen Städtetages anlässlich des Kongresses „Bildung in der Stadt“ am 22./23. November 2007. Unter: https:/www.jena.de/fm/1727/aachener_erklaerung.pdf; Stand: 13.11.2009

Deutscher Städtetag (2011): Inklusion in der Bildung. Ein Sachstandsbericht zur Umsetzung der UN-Behindertenrechtskonvention im Elementar- und Schulbereich in Deutschland (Stand 2011). Unter: http://www.staedtetag.de/impe$\mathrm{ria} / \mathrm{md} /$ content/dst/inklusion-in-der-bildung.pdf; Stand: 13.03.2011

Deutsches Institut für Medizinische Dokumentation und Information (DIMDI) (2005): ICF (Internationale Klassifkation der Funktionsfähigkeit, Behinderung und Gesundheit. Unter: https://www.dimdi.de/dynamic/de/klassifikationen/icf/; Stand: 18.05.2013

Deutsches Institut für Menschenrechte (2011): Stellungnahme der Monitoring-Stelle zur Verwirklichung eines inklusiven Bildungssystems (Primarstufe und Sekundarstufe I und II). Empfehlungen an die Länder, die Kultusministerkonferenz (KMK) und den Bund. Unter: https://www.institut-fuer-menschenrechte.de/uploads/tx_commerce/stellungnahme_der_monitoring_stelle_eckpunkte_z_verwirklichung_eines_inklusiven_bildungssystems_31_03_2011.pdf; Stand: 03.10 .2012

Deutsches Institut für Menschenrechte (2017): Das Recht auf inklusive Bildung Allgemeine Bemerkung Nr. 4 des UNAusschusses für die Rechte von Menschen mit Behinderungen. Unter: https://www.institut-fuer-menschenrechte.de/ fileadmin/user_upload/ Publikationen/ Information/Information_12_Das_Recht_auf_inklusive_Bildung.pdf; Stand: 05.04.2019

Deutsches Institut für Menschenrechte (2019): Wer Inklusion will, sucht Wege. Zehn Jahre UN-Behindertenrechtskonvention in Deutschland. Berlin. Unter: https://www.institut-fuer-menschenrechte.de/fileadmin/user_upload/Publikationen/ ANALYSE/Wer_Inklusion_will_sucht_Wege_Zehn_Jahre_UN_BRK_in_Deutschland.pdf; Stand: 05.04.2019 
Deutsches PISA-Konsortium (Hrsg.) (2001): PISA 2000. Basiskompetenzen von Schülerinnen und Schülern im internationalen Vergleich. Hrsg. von J. Baumert, C. Artelt, E. Klieme, M. Neubrand, M. Prenzel, U. Schiefele, W. Schneider, K.J. Tillmann, M. Weiß. Opladen: Leske und Budrich

Deutsches Schulamt (2004): Rundschreiben des Schulamtsleiters Nr. 16: Neuregelung in Bezug auf die Erstellung von Funktionsdiagnosen und Funktionsbeschreibungen. Unter: http://www.provinz.bz.it/schulamt/aktuelles/319. asp?369_page=4; Stand: 24.10 .2012

Diekmann, A. (2011, 5. Aufl.): Empirische Sozialforschung. Grundlagen, Methoden, Anwendungen. Reinbek: Rowohlt Verlag

Dietze, T. (2013): Integration von Schülern mit sonderpädagogischem Förderbedarf in der Grundschule - zur Situation in den 16 Bundesländern. In: Zeitschrift für Grundschulforschung 6 (2013) 1, 34-44

Direktion für Bildung und Kultur Zug (2012): Unterrichtsteams. Zusammenarbeit im Unterrichtsteam - kooperative Unterrichtsentwicklung. Unter: https:/www.zg.ch/behoerden/direktion-fur-bildung-und-kultur/amt-fur-gemeindlicheschulen/inhalte-ags/strukturen-zur-qualitatsentwicklung/unterrichtsteams/downloads/broschuere-unterrichtsteams; Stand: 17.12 .2014

Dlugosch, A. (2013): Inklusion als Mehrebenenkonstellation. In: Behinderte Menschen. Zeitschrift für gemeinsames Leben, Lernen und Arbeiten, Nr. 2/2013, 21-33

Dohmen, D., Fuchs, K. (2009): Kosten und Erträge ausgewählter Reformmaßnahmen: Teilhabe durch qualitativ hochwertige und gut ausgebaute Bildungs- und Betreuungsinfrastruktur sichern (FiBS-Forum, 44). Berlin: Forschungsinstitut für Bildungs- und Sozialökonomie (FiBS).

Dorow, J. (1982): Förderung als unterrichts- und schulorganisatorisches Problem II. In: Reinartz, A.; Sander, A. (Hrsg.) (1982): Schulschwache Kinder in der Grundschule. Pädagogische Maßnabmen zur Vorbeugung und Verminderung von Schulschwäche in der Primarstufe. Weinheim und Basel: Beltz Verlag, 70-79

Dresing, T.; Pehl, T. (2012, 4. Auf.): Praxisbuch Interview und Transkription. Regelsysteme und für qualitative ForscherInnen. Marburg. Unter: www.audiotranskription.de/Praxisbuch (Stand: 17.05.2013)

Dubs, Rolf (2005, 2. Aufl.): Die Führung einer Schule: Leadership und Management. Zürich: Franz Steiner Verlag

Dumke, D.; Krieger, G.; Schäfer, G. (1989): Schulische Integration in der Beurteilung von Eltern und Lehrern. Weinheim: Deutscher Studien Verlag

Dumke, D. (1991): Schulleistungen nichtbehinderter Schüler in Integrationsklassen. In: Zeitschrift für Pädagogische Psychologie, 5, Heft 1, 33-42

Dumke, D.; Schäfer, G. (1993): Entwicklung behinderter und nichtbehinderter Kinder in Integrationsklassen. Einstellungen, soziale Beziehungen, Persönlichkeitsmerkmale und Schulleistungen. Weinheim: Deutscher Studienverlag

Dumont, H.; Stäbler, F.; Henke, T.; Maaz, K. (2015): Kompetenzentwicklung in den Fächern Deutsch und Mathematik in Abhängigkeit des sonderpädagogischen Förderbedarfs. In: Landesinstitut für Schule und Medien Berlin-Brandenburg (LISUM), Universität Potsdam, Deutsches Institut für internationale Pädagogische Forschung (DIPF) (2015) (Hrsg.): Inklusives Lernen und Lehren im Land Brandenburg. Abschlussbericht zur Begleitforschung des Pilotprojekts „Inklusive Grundschule“. Potsdam. Unter: http://www.inklusion-brandenburg.de/fileadmin/daten/ inklusion_im_land_brandenburg/pilotprojekt_inklusive_grundschule/wissenschaftliche_begleitung/Abschlussbericht_PING.pdf; Stand: 14.03.2018, 87-108

Dyson, A. (2010): Developing inclusive schools: three perspectives from England. Unter: www.dds-home.de/v2010/inhalte/, Stand: 09.08.2014

Eberl, D. (2000): Gemeinsamer Unterricht von behinderten und nichtbehinderten Schülern in der Beurteilung von Schulleitern und Lehrern: eine Untersuchung an Grund-und Förderschulen in Nordrhein-Westfalen. Wittschlick und Bonn: Wehle

Eberwein, H.; Knauer, S. (Hrsg.) (2002, 6. Auflage): Integrationspädagogik. Weinheim und Basel: Beltz Verlag

Eckermann, T.; Heinzel, F. (2013): Etablierte und Außenseiter. Wie Kinder beim kooperativen Lernen mit Heterogenität umgehen. In: Budde, J. (Hrsg.) (2013): Unscharfe Einsätze. (Re-) Produktion von Heterogenität im schulischen Feld. Wiesbaden: Springer VS, 187-210

Eder, F. (1998): Schul-und Klassenklima. In: Rost, D.H. (Hrsg.) (1998): Handwörterbuch Pädagogische Psychologie. Weinheim: Psychologie Verlags Union, 424-430

Ehmke, T.; Jude, N. (2010): Soziale Herkunft und Kompetenzerwerb. In: Klieme, E. u.a. (Hrsg.) (2010): PISA 2009. Bilanz nach einem Jahrzehnt. Münster/New York/München/Berlin: Waxmann, 231-254

Eikenbusch, G. (2010): Lernen, sich zu organisieren und zu führen. Was wissen wir über effektive Klassenführung? In: Eikenbusch, G.; Heymann, H.W. (Hrsg.): Was wissen wir über guten Unterricht? Hamburg: Bergmann und Helbig Verlag, 13-22

Eikenbusch, G.; Heymann, H.W. (Hrsg.) (2010): Was wissen wir über guten Unterricht? Hamburg: Bergmann und Helbig Verlag 
Ellger-Rüttgardt, S. L. (2007): Eine europäische Vision seit 200 Jahren: Bildung für alle. In: Zeitschrift für Heilpädagogik 58 (2007), 242-249

Ellger-Rüttgardt, S. L. (2008): Geschichte der Sonderpädagogik. München: Reinhardt UTB

Ellinger, S.; Stein, R. (2012): Effekte inklusiver Beschulung: Forschungsstand im Förderschwerpunkt emotionale und soziale Entwicklung. In: Empirische Sonderpädagogik 4 (2012) 2, 85-109

Emmerich, M.; Hormel, U. (2013): Heterogenität - Diversity - Intersektionalität. Zur Logik sozialer Unterscheidungen in pädagogischen Semantiken der Differenz. Wiesbaden: Springer VS

Engels, D. (2006): Lebenslagen und soziale Exklusion. Thesen zur Reformulierung des Lebenslagenkonzepts für die Sozialberichterstattung. In: Sozialer Fortschritt, Heft 5, 109-117

Europäische Agentur für Entwicklungen in der sonderpädagogischen Förderung (Hrsg.) (2009): Grundprinzipien zur Förderung der Qualität in der inklusiven Bildung. Unter: https://www.european-agency.org/sites/default/files/keyprinciples-for-promoting-quality-in-inclusive-education_key-principles-DE.pdf; Stand: 24.12.2018

European Commission (2018): Education and Training MONITOR 2018. Unter: https://ec.europa.eu/education/sites/education/files/document-library-docs/volume-1-2018-education-and-training-monitor-country-analysis.pdf; Stand: 05.04 .2019

Evers-Meyer, K. (2010): Zur Situation von Menschen mit Behinderung in Deutschland, In: Wernstedt, R.; John-Ohnesorg, M. (Hrsg.): Inklusive Bildung. Die UN-Konvention und ihre Folgen. Berlin: Friedrich-Ebert-Stiftung, 27-31

Evertson, C.M.; Weinstein, C. (2006): Handbook of Classroom Management: Research, practice, and contemporary issues. Mahwah, NJ: Lawrence Erlbaum Associates, Inc.

Evertson, C.M.; Emmer, E.T.; Clements, B.S.; Worsham, M.E. (2009): Classroom Management for Elementary Teachers. Boston: Allyn and Bacon

Fend, H. (1986): „Gute Schulen - schlechte Schulen“. Die einzelne Schule als pädagogische Handlungseinheit. In: Die Deutsche Schule, 78, 3, 275-293

Fend, H. (2008): Schule gestalten. Wie man Schule macht. Wiesbaden: Springer VS

Fend, H.; Berger, F.; Grob, U. (Hrsg.) (2009): Lebensverläufe, Lebensbewältigung, Lebensglück: Ergebnisse der LifEStudie. Wiesbaden: Springer VS

Fend, H. (2012): Drei Reproduktionsfunktionen des Schulsystems. In: Bauer, U.; Bittlingmayer, U.H.; Scherr, A. (Hrsg.) (2012): Handbuch Bildungs- und Erziehungssoziologie. Wiesbaden: Springer VS, 161-165

Feuser, G.; Meyer, H. (1987): Integrativer Unterricht in der Grundschule. Ein Zwischenbericht. Solms: Jarick Oberbiel

Feuser, G. (1989): Allgemeine integrative Pädagogik und entwicklungslogische Didaktik. In: Behindertenpädagogik, 1, 4-48

Feuser, G. (1998): Gemeinsames Lernen am gemeinsamen Gegenstand. Didaktisches Fundamentum einer Allgemeinen (integrativen) Pädagogik. In: Hildeschmidt, A. \& Schnell, I. (Hrsg.) (1998): Integrationspädagogik. Auf dem Weg zu einer Schule für alle. Weinheim: Juventa, 19-36

Feuser, G. (1999): Modelle der Integration. Fortschritt oder Inflationierung des Integrationsanliegens. In: Flieger, P. (1999): Behinderung, Integration in der Schule: Positionen, Dilemma: die Praxis, ungeklärt: die Zukunft. In: Schulheft 94, Wien 1999, 29-42

Feuser, G. (2010): Integration und Inklusion als Möglichkeitsräume. In: Stein, A.-D.; Krach, S.; Niediek, I. (Hrsg.) (2010): Integration und Inklusion auf dem Weg ins Gemeinwesen. Möglichkeitsräume und Perspektiven. Bad Heilbrunn: Klinkhardt, 17-31

Feyerer, E.; Fragner, J. (1994): Lehrer-Bildung als entscheidender Faktor für das Gelingen der schulischen Integration. In: Zeitschrift BEHINDERTE in Familie, Schule und Gesellschaft, 17, Heft 1, 25-50

Feyerer, E. (1998): Behindern Behinderte? Integrativer Unterricht auf der Sekundarstufe I. Innsbruck: StudienVerlag

Feyerer, E. (2009): Ist Integration „,normal“ geworden? In: Erziehung und Unterricht, 159, 241-254

Finn, J.D.; Achilles, C.M. (1999): Tennessee's Class Size Study: Findings, Implications, Misconceptions. In: Educational Evaluation and Policy Analysis, Vol. 21, No. 2, Special Issue: Class Size: Issues and New Findings (Summer, 1999), 97-109 Unter: https://www.jstor.org/stable/1164294; Stand: 11.09.2018

Flick, U.; Kardoff, Ernst von; Steinke, I. (Hrsg.) (2004, 3. Auf.): Qualitative Forschung. Ein Handbuch. Reinbek bei Hamburg: rowohlts enzyklopädie

Flick, U. (2011, 3. Aufl.): Triangulation. Eine Einführung. Wiesbaden: Springer VS

Foucault, M. (1974): La vérité et les formes juridiques. In: Foucault, M. (1974): Dits et écrits, 1954-1988. Bd. 2, 19701975. Paris: Gallimard, 538-646

Friedrich, K. (2015): Inklusives Schulprogramm: Geduldiges Papier oder Gelingensbedingung inklusiver Schulen. In: Landesinstitut für Schule und Medien Berlin-Brandenburg (LISUM), Universität Potsdam, Deutsches Institut für internationale Pädagogische Forschung (DIPF) (2015) (Hrsg.): Inklusives Lernen und Lehren im Land Brandenburg. Abschlussbericht zur Begleitforschung des Pilotprojekts „Inklusive Grundschule“. Potsdam. Unter: http://www. inklusion-brandenburg.de/fileadmin/daten/inklusion_im_land_brandenburg/pilotprojekt_inklusive_grundschule/wissenschaftliche_begleitung/Abschlussbericht_PING.pdf; Stand: 14.03.2018, 197-232 
Friend, M.; Cook, L. (2010): Co-Teaching: An Illustration oft he Complexity of Collaboration in Special Education. In: Journal of Educational and Psychological Consultation, 20, 2010, 9-27

Frühauf, T. (2008): Von der Integration zur Inklusion - ein Überblick. In: Hinz, A.; Körner, I.; Niehoff, U. (Hrsg.) (2008): Von der Integration zur Inklusion. Grundlagen - Perspektiven - Praxis. Marburg: Lebenshilfe-Verlag, 11-32

Fuchs, I. (1988): Integrative Unterrichtung mit unterschiedlicher Zielvorgabe. In: Sander, A. (1988): Behinderte Kinder und Jugendliche in Regelschulen. Jahresbericht 1987 über schulische Integration im Saarland. St. Ingbert: Röhrig Universitätsverlag, S. 123-152

Fuchs, P. (2002): Behinderung und soziale Systeme. Anmerkungen zu einem schier unlösbaren Problem. Unter: http:// ibs-networld.de/altesferkel/fuchs-behinderungen.shtml; Stand: 13.11 .2012

Füssel, H.P.; Kretschmann, R. (1993): Gemeinsamer Unterricht für behinderte und nichtbehinderte Kinder. Pädagogische und juristische Voraussetzungen. Witterschlick: Wehle

Fussangel, K.; Gräsel, C. (2012): Lehrerkooperation aus der Sicht der Bildungsforschung. In: Baum, E.; Idel, T.-S.; Ullrich, H. (Hrsg.) (2012a): Kollegialität und Kooperation in der Schule. Theoretische Konzepte und empirische Befunde. Wiesbaden: Springer VS, 29-40

Gasteiger-Klicpera, B. \& Klicpera, C. (1997a): Die Bedeutung der sozialen Stellung in der Gruppe der Gleichaltrigen für die Entwicklung der Kinder. In: Zeitschrift für Kinder-und Jugendpsychatrie, 25, 234-246

Gasteiger-Klicpera, B. \& Klicpera, C. (1997b): Aggressivität und soziale Stellung in der Klassengemeinschaft. In: Zeitschrift für Kinder-und Jugendpsychatrie, 25, 139-150

Gasteiger-Klicpera, B. \& Klicpera, C. (2001): Der Zusammenhang zwischen Schulleistungen, dem sozialen Status in der Klasse und dem Sozialverhalten. In: Heilpädagogische Forschung, 1, 2-14

Gebhardt, M.; Krammer, M.; Schwab, S.; Gasteiger-Klicpera, B. (2013): Zusammenarbeit zwischen KlassenlehrerIn und SonderpädagogIn in der Integration. Eine Untersuchung in integrativen Klassen in der Steiermark. In: Heilpädagogische Forschung, Band 39, Heft 2, 2013, 54-62

Geiling, U.; Simon, T. (2014): Zuständigkeitserleben und berufliche Zufriedenheit von Grund- und Förderschullehrkräften in inklusionsorientierten Settings der Schuleingangsstufe. In: Lichtblau, M.; Blömer, D.; Jüttner, A.-K.; Koch, K.; Krüger, M.; Werning, R. (Hrsg.) (2014): Forschung zu inklusiver Bildung. Gemeinsam anders lehren und lernen. Bad Heilbrunn: Klinkhardt, 62-75

Georg, W. (Hrsg.): Soziale Ungleichheit im Bildungssystem. Eine empirisch-theoretische Bestandsaufnahme. Konstanz: UVK

GEW (1977): Bildungspolitisches Programm. Gewerkschaftstag 1977. Sonderdruck aus Erziehung und Wissenschaft, 12,1977

GGG (1971): Bericht über Jahrestagung der Gemeinnützigen Gesellschaft Gesamtschule (GGG). In: GesamtschulInformationen 2, 1971, 10-12

Girg, R.; Lichtinger, U.; Müller, T. (2012): Lernen mit Lernleitern. Unterrichten mit der MultiGradeMultiLevel-Methodology (MGML). Immenhausen bei Kassel: Prolog-Verlag

Glasersfeld, E.v. (2005): Was heißt „Lernen“ aus konstruktivistischer Sicht? In: Voß, R. (Hrsg.) (2005, 2. Auflage): Unterricht aus konstruktivistischer Sicht. Die Welten in den Köpfen der Kinder. Weinheim und Basel: Beltz Verlag, 214-223

Götz, G.; Lohrmann, K.; Ganser, B.; Haag, L. (2005): Einsatz von Unterrichtsmethoden - Konstanz oder Wandel? In: Empirische Pädagogik 19 (2005), 4, 342-360

Goetze, D. (2004): Modernisierung. In: Nohlen, D. (Hrsg.): Lexikon der Politik. Bd. 4: Die östlichen und die südlichen Länder. Berlin: Directmedia, 380-384

Goetze, H. (2008): Verhaltensgestörte in Integrationsklassen - Fiktion und Fakten. In: Heilpädagogik online 02/08, 32-52

Green, N. (2004): Der Unterschied zwischen Kooperativem Lernen und Gruppenarbeit besteht in den 5 grundlegenden Elementen. Unter: http://methodenpool.uni-koeln.de/koopunterricht/ger_the_difference.pdf; Stand: 17.03.2018

Green, J.; Nelson, G.; Martin, A.J.; Marsh, H. (2006): The causal ordering of self-concept and academic motivation and its effect on academic achievement. In: International Education Journal, 2006, 7(4), S. 534-546. Unter: https://fles. eric.ed.gov/fulltext/EJ854309.pdf; Stand: 14.03.2013

Grosche, M. (2011): Effekte einer direkt-instruktiven Förderung der Lesegenauigkeit. In: Empirische Sonderpädagogik, 2011, Nr. 2, 147-161

Grünke, M. (2006): Zur Effektivität von Fördermethoden bei Kindern und Jugendlichen mit Lernstörungen. In: Kindbeit und Entwicklung 15 (4) 2006, 239-254

Haag, L.; Kiel, E.; Trautmann, M. (2015): Einführung in den Thementeil ,Klassenmanagement/Klassenführung - Perspektiven, Befunde, Kontroversen. In: Zierer, K.; Lamers, D. (Hrsg.): Jabrbuch für Allgemeine Didaktik 2015. Baltmannsweiler: Schneider Verlag Hohengehren, 10-14 
Haeberlin, U.; Moser, U.; Bless, G.; Klaghofer, R. (1989): Integration in die Schulklasse. Fragebogen zur Erfassung von Dimensionen der Integration von Schülern FDI 4-6. Bern, Stuttgart: Haupt Verlag

Haeberlin, U. (1990): Die Situation von vergleichbar leistungsschwachen Schülern in Regelklassen und in Sonderklassen. Bericht über ein Forschungsprojekt in „Regelklassen mit Heilpädagogischer Schülerhilfe“ in der deutschsprachigen Schweiz und im Fürstentum Liechtenstein. In: Zeitschrift für Pädagogik, 25, Beiheft: Bilanz für die Zukunft: Aufgaben, Konzepte und Forschung in der Erziehungswissenschaft. Weinheim: Beltz Verlag, 246-248

Haeberlin, U.; Bless, G.; Moser, U.; Klaghofer, R. (2003, 4. unv. Auflage): Die Integration von Lernbehinderten. Versuche, Theorien, Forschungen, Enttäuschungen, Hoffnungen. Bern, Stuttgart, Wien: Haupt Verlag

Hänsel, D.; Miller, S. (2014): Kritische Analyse der Ansprüche der inklusiven Sonderpädagogik aus allgemeinpädagogischer und professionstheoretischer Perspektive. In: Lichtblau, M.; Blömer, D.; Jüttner, A.-K.; Koch, K.; Krüger, M.; Werning, R. (2014): Forschung zu inklusiver Bildung. Gemeinsam anders lehren und lernen. Bad Heilbrunn: Julius Klinkhardt, 91-104

Hage, K.; Bischoff, H.; Dichanz, H.; Eubel, K.-D.; Oehlschläger, H.-J.; Schwittmann, D. (1985): Das Methoden-Repertoire von Lehrern. Opladen: Leske und Budrich

Halfhide, T. (2009): Teamteaching. In: Fürstenau, S.; Gomella, M. (Hrsg.) (2009): Migration und schulischer Wandel: Unterricht. Wiesbaden: Springer VS, 103-120

Hamre, B.K.; Pianta, R.C. (2001): Student-Teacher-Relationships. Unter: https://pdfs.semanticscholar.org/83a9/c40f1c110437d3ce114f709bb4ca0cde41e2.pdf; Stand: 13.04.2017

Hanushek, E.A.; Kain, J.F.; Rivkin, S. G. (2002): Inferring program effects for special populations: Does special education raise achievement for students with disabilities? In: Review of Economics and Statistics, 84, 584-599

Hanushek, E.A., Kain, J.F.; Markman, J.M.; Rivkin, S.G. (2003): Does peer ability affect student achievement? In: Journal of Applied Econometrics, 18, 527-544

Hascher, T. (2017): Die Bedeutung von Wohlbefinden und Sozialklima für Inklusion. In: Lütje-Klose, B.; Miller, S.; Schwab, S.; Streese, B. (Hrsg.) (2017): Inklusion: Proflle für die Schul-und Unterrichtsentwicklung in Deutschland, Österreich und der Schweiz. Theoretische Grundlagen - Empirische Befunde - Praxisbeispiele. Münster, New York: Waxmann, 69-79

Haselbeck, F. (2015): Lehren und Lernen im Fokus der Klassengröße. Unter: www.dr-haselbeck.de/files/docs/Klassengroesse_201215.doc, Stand: 30.10.2017

Hattie, J.A.C. (2009): Visible learning. A synthesis of over 800 meta-analyses relating to achievement. London \& New York: Routledge

Hattie, J.A.C. (2013): Lernen sichtbar machen. Überarbeitete deutschsprachige Ausgabe von „Visible Learning“besorgt von Wolfgang Beywl und Klaus Zierer. Baltmannsweiler: Schneiderverlag Hohengehren

Heese, G. (1977): Einbezug oder Sonderung behinderter Schüler. In: Weigt, M. (Hrsg.) (1977): Schulische Integration von Behinderten. Beiträge zum Verhältnis von Sonderschulen zum Regelschulsystem. Weinheim und Basel: Beltz Verlag, 101-106

Heimann, P.; Schulz, O.G.; Blumenthal, A. (Hrsg.) (1965): Unterricht. Analyse und Planung. Hannover, Berlin, Darmstadt, Dortmund: Schroedel

Heimer, W. (1992): Bedingungen für Integrationsklassen in Hamburger Gesamtschulen. In: Schley, W., Boban, I.; Hinz, A. (Hrsg.) (1992, 2. Aufl.): Integrationsklassen in Hamburger Gesamtschulen. Hamburg: Curio, 117-130

Heimlich, U. (Hrsg.) (1999): Sonderpädagogische Fördersysteme. Auf dem Weg zur Integration. Stuttgart/Berlin/Köln: Kohlhammer

Heitmeyer, W. (Hrsg.) (2015, 3. Aufl.): Deutsche Zustände. Folge 10. Berlin: suhrkamp Verlag

Heitmeyer, W. (2015): Gruppenbezogene Menschenfeindlichkeit (GMF) in einem entsicherten Jahrzehnt. In: Heitmeyer, W. (Hrsg.) (2015, 3. Aufl.): Deutsche Zustände. Folge 10. Berlin: suhrkamp Verlag, 15-41

Hellbrügge, T. (1977): Unser Montessori Modell.Erfahrungen mit einem neuen Kindergarten und einer neuen Schule. München: Kindler

Hellmich, F.; Görel, G. (2014): Einstellungen zur Inklusion und Vorerfahrungen aus dem „Gemeinsamen Unterricht“ bei Lehrkräften in der Grundschule. In: Lichtblau, M.; Blömer, D.; Jüttner, A.-K.; Koch, K.; Krüger, M.; Werning, R. (Hrsg.) (2014): Forschung zu inklusiver Bildung. Gemeinsam anders lehren und lernen. Bad Heilbrunn: Klinkhardt, 48-61

Helmke, A.; Weinert, F.E. (1997): Bedingungsfaktoren schulischer Leistungen. In: Weinert, F.E. (Hrsg.): Enzyklopädie der Psychologie, Band 3 (Psychologie der Schule und des Unterrichts). Göttingen: Hogrefe-Verlag, 71-176

Helmke, A.; Jäger, R.S.; Balzer, L.; Hosenfeld, I.; Ridder, A.; Schrader, F.-W. (2002): Mathematik-Gesamterhebung Rheinland-Pfalz: Kompetenzen, Unterrichtsmerkmale, Schulkontext. Kurzbericht. Unter: http://www.lars-balzer. info/publications/pub-balzer_2002-01_MARKUS2002-Kurzbericht.pdf; Stand: 14.03.2013

Helmke, A.; Helmke, T.; Schrader, F.-W.; Wagner, W.; Nold, G.; Schröder, K. (2008): Alltagspraxis des Englischunterrichts. In: Klieme, E. (2008) (Hrsg.): Unterricht und Kompetenzerwerb in Deutsch und Englisch. Ergebnisse der DESI-Studie. Weinheim u.a.: Beltz Verlag, 371-381 
Helmke, A. (2010, 3. Aufl.): Unterrichtsqualität und Lehrerprofessionalität. Diagnose, Evaluation und Verbesserung des Unterrichts. Seelze-Velber: Kallmeyer, Klett

Helmke, A. (2015, 7. Aufl.): Unterrichtsqualität und Lehrerprofessionalität. Diagnose, Evaluation und Verbesserung des Unterrichts. Seelze-Velber: Kallmeyer, Klett

Hennemann, T., Hillenbrand, C. (2010): Klassenführung - Classroom Management. In: Hartke, B.; Koch, K.; Diehl, K. (Hrsg.)(2010): Förderung in der schulischen Eingangsstufe. Stuttgart: Kohlhammer, 255-279

Hennemann, T., Hillenbrand, C; Wilbert, J. (2014): Abschlussbericht „Wissenschaftliche Begleitung im Rahmen der Umsetzung zur inklusiven Schule im Kreis Mettmann“. Unter: https://www.hf.uni-koeln.de/data/e/File/Abschlussbericht\%20Mettmann.pdf; Stand: 14.08.2015

Hensen, G.; Küstermann, B.; Maykus, S.; Riecken, A.; Schinnenburg, H.; Wiedebusch, S. (Hrsg.) (2014): Inklusive Bildung. Organisations- und professionsbezogene Aspekte eines sozialen Programms. Weinheim und Basel: Beltz Verlag Juventa

Hetzner, R. (1988): Schulleistungen der Schüler in Integrationsklassen. In: Projektgruppe Integrationsversuch (Hrsg.): Das Fläming-Modell. Weinheim, Basel: Beltz Verlag, 251-254

Heyer, P.; Preuss-Lausitz, U.; Schöler, J. (1997): „Behinderte sind doch Kinder wie wir!“ Gemeinsame Erziehung in einem neuen Bundesland. Hrsg. vom Ministerium für Bildung, Jugend und Sport des Landes Brandenburg. Berlin: Wissenschaft und Technik Verlag

Hildeschmidt, A.; Sander, A. (1995): Integration behinderter Schüler und Schülerinnen in der Sekundarstufe I. In: Heilpädagogische Forschung, Band XXI, Heft 1, 14-26

Hinz, A. (1993): Heterogenität in der Schule. Unter: http://bidok.uibk.ac.at/library/hinz-heterogenitaet_schule.html; Stand: 13.08 .2013

Hinz, A. (2002): Von der Integration zur Inklusion - terminologisches Spiel oder konzeptionelle Weiterentwicklung? In: Zeitschrift für Heilpädagogik 53, Heft 9, 354-161

Hinz, A. (2004): Vom sonderpädagogischen Verständnis der Integration zum integrationspädagogischen Verständnis der Inklusion!? In: Schnell, I.; Sander, A. (Hrsg.) (2004): Inklusive Pädagogik. Bad Heilbrunn: Klinkhardt, 41-74

Hinz, A. (2009): Inklusive Pädagogik und Disability Studies - Gemeinsamkeiten und Spannungsfelder. Unter: www. zedis-ev-hochschule-hh.de/files/hinz_thesen_inkled_disabstud.pdf; Stand: 13.08.2013

Hinz, A. (2010): Aktuelle Erträge der Debatte um Inklusion - worin besteht der, Mehrwert' gegenüber Integration? Unter: https://www.bdja.org/files/hinz-aktuelle_ertr_ge_der_debatte_um_inklusion.pdf; Stand: 10.05.2014

Hinz, T.; Groß, J. (2006): Schulempfehlung und Leseleistung in Abhängigkeit von Bildungsherkunft und kulturellem Kapital. In: Georg, W. (Hrsg.): Soziale Ungleichheit im Bildungssystem. Eine empirisch-theoretische Bestandsaufnahme. Konstanz: UVK, 199-226

Hirschberg, M. (2011): Behinderung: Neues Verständnis nach der Behindertenrechtskonvention. In: Deutsches Institut für Menschenrechte (Hrsg.): Positionen Nr. 4, 1-4

Holtappels, H.G.; Klieme, E.; Rauschenbach, T.; Stecher, L. (Hrsg.) (2007): Ganztagsschule in Deutschland: Ergebnisse der Ausgangserhebung der „Studie zur Entwicklung von Ganztagsschulen“(StEG). Weinheim: Juventa

Holtappels, H.G.; Feldhoff, T. (2010a): Einführung: Change Management. In: Bohl, T.; Helsper, W.; Holtappels, H.G.; Schelle, C. (Hrsg.) (2010): Handbuch Schulentwicklung. Bad Heilbrunn: Klinkhardt, 159-166

Holtappels, H.G.; Rolff, H.-G. (2010b): Einführung: Theorien der Schulentwicklung. In: Bohl, T.; Helsper, W.; Holtappels, H.G.; Schelle, C. (Hrsg.) (2010): Handbuch Schulentwicklung. Bad Heilbrunn: Klinkhardt, 73-79

Holtappels, H.G. (2010c): Schule als Lernende Organisation. In: Bohl, T.; Helsper, W.; Holtappels, H.G.; Schelle, C. (Hrsg.) (2010): Handbuch Schulentwicklung. Bad Heilbrunn: Klinkhardt, 99-105

Holtappels, H.G. (2010d): Schulentwicklungsforschung. In: Bohl, T.; Helsper, W.; Holtappels, H.G.; Schelle, C. (Hrsg.) (2010): Handbuch Schulentwicklung. Bad Heilbrunn: Klinkhardt, 26-29

Holtappels, H.G. (2010e): Schulprogramm als Entwicklungsinstrument. In: Bohl, T.; Helsper, W.; Holtappels, H.G.; Schelle, C. (Hrsg.) (2010): Handbuch Schulentwicklung. Bad Heilbrunn: Klinkhardt, 266-272

Holtappels, H.G. (2013): Schulentwicklung und Lehrerkooperation. In: McElvany, N.; Holtappels, H.G. (Hrsg.): Empirische Bildungsforschung. Theorien, Methoden, Befunde und Perspektiven. Münster u.a.: Waxmann, 35-61

Holzäpfel, L. (2008): Beratung bei der Einführung von Selbstevaluation von Schulen. Münster, New York, München, Berlin: Waxmann Verlag

Holzinger, A.; Wohlhart, D. (2009): Schulische Integration. Innsbruck: Studien Verlag

Holzinger, A.; Ebner, M.; Kernbichler, G.; Kopp-Sixt, S.; Much, P.; Pongratz, H.J. (2011): Forschungsprojekt Qualität in der Integration: Projektzeitraum WS 2008/09-WS 2010/11. Graz: PH Steiermark. Unter: https://www.ph-online. ac.at/phst/wbLdb2.downloadDocument? pLstNr=282\&pLstSchichtNr=110766\&pDocStoreNr=57785; Stand. 13.12.2012

Hornberg, S. (2010): Schulentwicklung und Differenz: Migration. In: Bohl, T.; Helsper, W.; Holtappels, H.G.; Schelle, C. (Hrsg.) (2010): Handbuch Schulentwicklung. Bad Heilbrunn: Klinkhardt, 42-45

Horstkemper, M. (2010): Schulentwicklung und Differenz: Gender. In: Bohl, T.; Helsper, W.; Holtappels, H.G.; Schelle, C. (Hrsg.) (2010): Handbuch Schulentwicklung. Bad Heilbrunn: Klinkhardt, 37-42 
Huber, C. (2009): Gemeinsam einsam? Empirische Befunde und praxisrelevante Ableitungen zur sozialen Integration von Schülern mit sonderpädagogischem Förderbedarf im Gemeinsamen Unterricht. In: Zeitschrift für Heilpädagogik, Jg. 60, H. 7, 242-248

Huber, C.; Wilbert, J. (2012): Soziale Ausgrenzung von Schülern mit sonderpädagogischem Förderbedarf und niedrigen Schulleistungen im gemeinsamen Unterricht. Empirische Sonderpädagogik, 4, 2012, 147-165

Huber, S.G.; Schneider, N. (2011): Qualitätsmanagement in Schulen. In: Huber, S. G. (2011): Handbuch für Steuergruppen: Grundlagen für die Arbeit in zentralen Handlungsfeldern des Schulmanagements. Köln: Link Verlag, 355-388

Hüther, G. (2011): Was wir sind und was wir sein könnten - ein neurobiologischer Mutmacher. Frankfurt: S. Fischer

Hug, R. (Hrsg.) (1994a): Integration in der Schule der 10-14jährigen. Innsbruck: StudienVerlag

Hug, R. (1994b): Integration in der Schule der 10-14jährigen: Situationsdarstellung, Erfahrungen, Perspektiven. In: Hug, R. (Hrsg.) (1994): Integration in der Schule der 10-14jährigen. Innsbruck: Österreichischer StudienVerlag, 89-112

Hurrelmann, K.; Timm, A. (2011): Kinder, Bildung, Zukunft. Drei Wege aus der Krise. Stuttgart: Klett

Husinsky, B. (1994): Sonderpädagogik in einer Integrationsklasse? In: Hug, R. (Hrsg.) (1994): Integration in der Schule der 10-14jährigen. Innsbruck: Österreichischer StudienVerlag, 13-38

Idel, T.-S.; Ullrich, H. (2013): „Die Gruppe ist doch gut, auch wenn wir uns nicht ausgesucht haben." Kooperation in Zwangsgemeinschaften. In: Keller-Schneider, M.; Albisser, S.; Wissinger, J. (Hrsg.) (2013): Professionalität und Kooperation in Schulen. Beiträge zur Diskussion über Schulqualität. Bad Heilbrunn: Klinkhardt, 57-70

Innerhofer, P.; Klicpera, C. (1991): Integration und Schulreform. Eine Untersuchung zur Integration Behinderter an den Pflichtschulen Südtirols. Heidelberg: Heidelberger Verlagsanstalt, Edition Schindele

Jäntsch, J.J.; Bosse, S.; Spörer, N. (2015): Fortbildung und Beratung zum inklusiven Unterricht. In: Landesinstitut für Schule und Medien Berlin-Brandenburg (LISUM), Universität Potsdam, Deutsches Institut für internationale Pädagogische Forschung (DIPF) (2015) (Hrsg.): Inklusives Lernen und Lebren im Land Brandenburg. Abschlussbericht zur Begleitforschung des Pilotprojekts „Inklusive Grundschule“. Potsdam; Unter: http://www.inklusion-brandenburg. de/fileadmin/daten/inklusion_im_land_brandenburg/pilotprojekt_inklusive_grundschule/wissenschaftliche_ begleitung/Abschlussbericht_PING.pdf; Stand: 14.03.2018; 155-180

Jennessen, S.; Lelgemann, R.; Ortland, B.; Schlüter, M. (Hrsg.) (2010): Leben mit Körperbehinderung. Perspektiven der Inklusion. Stuttgart: Kohlhammer

Jennessen, S.; Wagner, M. (2012): Alles so schön bunt hier!? Grundlegendes und Spezifisches zur Inklusion aus sonderpädagogischer Perspektive. In: Zeitschrift für Heilpädagogik, 08, 2012, 335-344

Jeynes, W.H. (2005a): Effects of Parental Involvement and Family Structure on the Academic Achievement of Adolescents. Marriage and Family Review, Vol 37 (3) 2005, 99-116

Jeynes, W.H. (2005b): A Meta-Analysis of the Relation of Parental Involvement to Urban Elementary School Student Academic Achievement. Unter: http://uex.sagepub.com/cgi/content/abstract/40/3/237; Stand: 09.08.2014

Jeynes, W.H. (2007): The Relationship Between Parental Involvement and Urban Secondary School Student Academic Achievement: A Meta: Analysis. Unter: http://uex.sagepub.dom/cgi/content/abstract/42/1/82, Stand: 09.08.2014

Joller-Graf, K.; Tanner, S. (2012): Wie gelingt Integrierte Sonderschulung? In: Lanfranchi, A.; Steppacher, J. (Hrsg.) (2012): Schulische Integration gelingt. Gute Praxis wahrnehmen, Neues entwickeln. Bad Heilbrunn: Verlag Julius Klinkhardt, 148-165

Kamiske, G.F.; Brauer, J.-P. (2002): ABC des Qualitätsmanagements. München: Hanser

Kane, T.J.; Taylor, E.S.; Tyler, J.H.; Wooten, A.L. (2010): Identifiying effective classroom practices using student achievement data. Massachusetts: National Bureau of economic research. Unter: http://www.nber.org/papers/w15803

Kanter, G.O. (1991): Kennzeichnungen der aktuellen Situation des Bildungswesens und Zukunftsperspektiven der Behindertenpädagogik. In: Zeitschrift für Heilpädagogik 1991, 42 (2), 92-103

Kelle, H. (2013): Theorie-Empirie-Verhältnis und methodische Standards in der qualitativen Forschung. In: Einsiedler, W.; Fölling-Albers, M.; Kelle, H.; Lohrmann, K. (2013): Standards und Forschungsstrategien in der empirischen Grundschulforschung. Eine Handreichung. Münster: Waxmann, 59-92

Keller, G.; Hafner, K. (1999): Soziales Lernen will gelernt sein. Donauwörth: Auer

Keller, G. (2010, 2. Aufl.): Disziplinmanagement in der Schulklasse: Unterrichtsstörungen vorbeugen - Unterrichtsstörungen bewältigen. Bern: Verlag Hans Huber

Keller-Schneider, M.; Albisser, S. (2013): Kooperation von Lehrpersonen und die Bedeutung von individuellen und kollektiven Ressourcen. In: Keller-Schneider, M.; Albisser, S.; Wissinger, J. (Hrsg.) (2013): Professionalität und Kooperation in Schulen. Beiträge zur Diskussion über Schulqualität. Bad Heilbrunn: Klinkhardt, 33-56

Keller-Schneider, M.; Albisser, S.; Wissinger, J. (Hrsg.) (2013): Professionalität und Kooperation in Schulen. Beiträge zur Diskussion über Schulqualität. Bad Heilbrunn: Klinkhardt 
Kiefer, W. (2010): Verfassungsrechtliche Grenzen einer Schulreform im Saarland. In: Zeitschrift für Landes- und Kommunalrecht Hessen, Rheinland-Pfalz, Saarland, 2010, Heft 4, 131-135

Kiel, E. (Hrsg.) (2012, 2. Aufl.): Unterricht sehen, analysieren, gestalten. Bad Heilbrunn: Klinkhardt

Kiel, E.; Frey, A.; Weiß, S. (2013): Trainingsbuch Klassenführung. Bad Heilbrunn: Klinkhardt

Kiper, H.; Mischke, W. (2008): Selbstreguliertes Lernen - Kooperation - Soziale Kompetenz. Fächerübergreifendes Lernen in der Schule. Stuttgart: Kohlhammer

Kirchhoff, S.; Kuhnt, S.; Lipp. P.; Schlawin, S. (2008, 4. Aufl.): Der Fragebogen. Datenbasis, Konstruktion und Auswertung. Wiesbaden: Springer VS

Klafki, W. (1963): Studien zur Bildungstheorie und Didaktik. Weinheim: Beltz Verlag

Klafki, W.; Stöcker, H. (1976): Innere Differenzierung des Unterrichts. In: Zeitschrift für Pädagogik 4, 22, 497-523

Klafki, W. (2003): Allgemeinbildung heute - Sinndimensionen einer gegenwarts - und zukunftsorientierten Bildungskonzeption. In: Berg, H.-C. (Hrsg.): Bildung und Lehrkunst in der Unterrichtsentwicklung. Schulmanagement Handbuch. München: Oldenbourg, 11-27

Klauß, T.; Terfloth, K. (Hrsg.) (2013): Besser gemeinsam lernen! Inklusive Schulentwicklung. Heidelberg: Universitätsverlag Winter

Kleber, E.W. (Hrsg.) (1977): Zur Revision sonderpädagogischer Praxis. Berlin: Marhold

Klemm, K. (2009): Sonderweg Föderschule: Hoher Einsatz, wenig Perspektiven. Eine Studie zu den Ausgaben und der Wirksamkeit von Förderschulen in Deutschland. Gütersloh: Bertelsmann Stiftung

Klemm, K. (2010): Gemeinsam lernen. Inklusion leben. Status Quo und Herausforderungen inklusiver Bildung in Deutschland. Gütersloh: Bertelsmann Stiftung

Klemm, K. (2018): Unterwegs zur inklusiven Schule. Lagebericht 2018 aus bildungsstatistischer Perspektive. Gütersloh: Bertelsmann Stiftung

Klieme, E. (2007): Bildungsstandards, Leistungsmessung und Unterrichtsqualität. In: Labudde, P. (Hrsg.): Bildungsstandards am Gymnasium. Korsett oder Katalysator. Bern: h.e.p. verlag, 75-84

Klieme, E.; Jude, N.; Rauch, D.; Ehlers, H.; Helmke, A.; Eichler, W.; Thomé, G.; Willenberg, H. (2008): Alltagspraxis, Qualität und Wirksamkeit des Deutschunterrichts. In: Klieme, E. (2008) (Hrsg.): Unterricht und Kompetenzerwerb in Deutsch und Englisch. Ergebnisse der-DESI-Studie. Weinheim u.a.: Beltz Verlag, 319-344

Klieme, E.; Artelt, C.; Hartig, J.; Jude, N.; Köller, O.; Prenzel, M.; Schneider, W.; Stanat, P. (Hrsg.) (2010): PISA 2009. Bilanz nach einem Jahrzehnt. Münster/New York/München/Berlin: Waxmann

Klingberg, L. (1995): Lernen Lehren - Unterricht. Über den Eigensinn des Didaktischen. Unter: https://publishup.unipotsdam.de/opus4-ubp/frontdoor/deliver/index/docId/445/file/ KLINGBER.pdf; Stand: 18.04.2017

Klippert, H. (2008): Besser lernen. Kompetenzvermittlung und Schüleraktivierung im Schulalltag. Stuttgart: Klett

Klippert, H. (2010): Heterogenität im Klassenzimmer. Weinheim und Basel: Beltz Verlag

Kobi, E. (1997): Was bedeutet Integration? Analyse eines Begriffs. In: Eberwein, H. (Hrsg.): Handbuch Integrationspädagogik. Kinder mit und ohne Behinderung lernen gemeinsam. Weinheim und Basel: Beltz Verlag, 71-79

Kobi, E.E. (2008): Alternative Integration als integrierte Alternative. In: Heilpädagogik Online, 02/08, 13-28

Kocaj, A.; Kuhl, P.; Kroth, A.J.; Pant, H.A.; Stanat, P. (2014): Wo lernen Kinder mit sonderpädagogischem Förderbedarf besser? Ein Vergleich schulischer Kompetenzen zwischen Regel- und Förderschulen in der Primarstufe. In: Kölner Zeitschrift für Soziologie und Sozialpsychologie 2/2014, 165-191

Köbberling, A. (1994): Integration in der Sekundarstufe I in Hamburg - Wie Schulen sich verändern. In: Hug, R. (Hrsg.): Integration in der Schule der 10-14 jährigen. Innsbruck: Österreichischer Studienverlag, 163-190

Köppel, K. (1987): Das Zentrum für Verhaltenspädagogik, Wien - ein Projekt zur integrativen Betreuung verhaltensauffälliger Schüler durch ein Beratungslehrer- und Förderklassensystem. In: Mutzeck, W.; Pallasch, W. (Hrsg.): Integration von Schülern mit Verhaltensstörungen. Praktische Modelle und Versuche. Weinheim: Deutscher Studien Verlag, 152-169

Kopmann, H.; Zeinz, H. (2015): Classroom-Management bei Heranwachsenden mit herausforderndem Verhalten im inklusiven Unterricht: Wie begegnen Lehrkräfte Kindern mit ADHS? In: Zierer, K. (Hrsg.): Jahrbuch für Allgemeine Didaktik. Klassenmanagement / Klassenführung - Perspektiven, Befunde, Kontroversen. Baltmannsweiler: Schneider Verlag Hohengehren, 25-39

Kounin, J.S. (2006, Reprint; Original der deutschen Ausgabe 1976): Techniken der Klassenführung. Münster, New York, München, Berlin: Waxmann

Kreßierer, C. (2006): Die Förderung von Schülern mit Lernschwierigkeiten im Integrationsnetzwerk Südtirol. Entwicklung, Bedingungen, Herausforderungen, Perspektiven. Unter: http://www.foerderpaed.net/kressierer/integration. pdf; Stand: 09.04.2012

Kronauer, M. (2007a): Inklusion - Exklusion: ein Klärungsversuch. Unter: http://www.die-bonn.de/doks/kronauer0701.pdf, veröffentlicht am 31.10.2007; Stand: 14.08.2014

Kronauer, M. (2007b): Neue soziale Ungleichheiten und Ungerechtigkeitserfahrungen: Herausforderungen für eine Politik des Sozialen. In: WSI Mitteilungen 7/2007, 365-372 
Kronauer, M. (2010): Inklusion - Exklusion. Eine historische und begriffliche Annäherung an die soziale Frage der Gegenwart. In: Kronauer, M. (Hrsg.): Inklusion und Weiterbildung. Reflexionen zur gesellschaftlichen Teilhabe in der Gegenwart. Bielefeld: Bertelsmann, 24-58

Kronauer, M. (2013): Soziologische Anmerkungen zu zwei Debatten über Inklusion und Exklusion. In: Burtscher, R.; Ditschek, E.J.; Ackermann, K.-E.; Kil, M.; Kronauer, M. (Hrsg.): Zugänge zu Inklusion: Erwachsenenbildung, Behindertenpädagogik und Soziologie im Dialog. Bielefeld: Bertelsmann Verlag, 17-25

Kronig, W. (2007): Die systematische Zufälligkeit des Bildungserfolgs. Theoretische Erklärungen und empirische Untersuchungen zur Lernentwicklung und zur Leistungsbewertung in unterschiedlichen Schulklassen. Berne: Haupt

Kucharz, D.; Wagener, M. (2009): Jahrgangsübergreifendes Lernen. Eine empirische Studie zu Lernen, Leistung und Interaktion von Kindern in der Schuleingangsphase. Baltmannsweiler: Schneider Verlag Hohengehren

Kucharz, D. (2010): Jahrgangsgemischtes Lernen. In: Bohl, T.; Helsper, W.; Holtappels, H.G.; Schelle, C. (Hrsg.) (2010): Handbuch Schulentwicklung. Bad Heilbrunn: Klinkhardt, 333-335

Kuckartz, U.; Dresing, T.; Rädiker, S.; Stefer, C. (2008, 2. Auf.): Qualitative Evaluation. Der Einstieg in die Praxis. Wiesbaden: Springer VS

Kuckartz, U. (2012): Qualitative Inhaltsanalyse. Methoden, Praxis, Computerunterstützung. Weinheim und Basel: Beltz Verlag

Kulik, J.A.; Kulik, C.L. (1997): Ability Grouping. In: Colangelo, N.; Davis, G. (Hrsg.): Handbook of gifted education (1997, 2. Aufl.) Boston: Allyn and Bacon, 230-242

Kullmann, H.; Lütje-Klose, B.; Textor, A. (2014): Eine allgemeine Didaktik für inklusive Lerngruppen - Fünf Leitprinzipien als Grundlage eines Bielefelder Ansatzes für inklusive Didaktik. In: Amrhein, B.; Dziak-Mahler, M. (Hrsg.) (2014): Fachdidaktik inklusive. Münster: Waxmann, 89-107

Kuper, H.; Kapelle, N. (2012): Lehrerkooperation aus organisationssoziologischer Sicht. In: Baum, E.; Idel, T.-S.; Ullrich, H. (Hrsg.) (2012a): Kollegialität und Kooperation in der Schule. Theoretische Konzepte und empirische Befunde. Wiesbaden: Springer VS, 41-51

Landesinstitut für Schule und Medien Berlin-Brandenburg (LISUM), Universität Potsdam, Deutsches Institut für internationale Pädagogische Forschung (DIPF) (2015) (Hrsg.): Inklusives Lernen und Lehren im Land Brandenburg. Abschlussbericht zur Begleitforschung des Pilotprojekts „Inklusive Grundschule“. Potsdam; Unter: http://www. inklusion-brandenburg.de/fileadmin/daten/inklusion_im_land_brandenburg/pilotprojekt_inklusive_grundschule/wissenschaftliche_begleitung/Abschlussbericht_PING.pdf; Stand: 14.03.2018

Lanfranchi, A.; Steppacher, J. (Hrsg.) (2012): Schulische Integration gelingt. Gute Praxis wahrnehmen, Neues entwickeln. Bad Heilbrunn: Klinkhardt

Lange, V. (2015): Inklusion in der Schule und der beruflichen Bildung im Ländervergleich. Die UN-Behindertenrechtskonvention und das Recht auf inklusive Bildung. In: Lange, V.; Sastges-Schank, A.: Inklusive Bildung im Saarland. Berlin: Friedrich-Ebert-Stiftung, 7-8

Lange, V.; Sastges-Schank, A. (2015): Inklusive Bildung im Saarland. Berlin: Friedrich-Ebert-Stiftung

Lange, V. (2017): Inklusive Bildung in Deutschland. Ländervergleich. Berlin: Friedrich-Ebert-Stiftung

Langfeldt, H.P. (1991): Editorial: Integration und empirische Forschung. In: Heilpädagogische Forschung 17, 1-12

Laubenstein, D.; Lindmeier, C.; Guthöhrlein, K.; Scheer, D. (2015): Auf dem Weg zur schulischen Inklusion - Empirische Befunde zum gemeinsamen Unterricht in rheinland-pfälzischen Schwerpunktschulen. Bad Heilbrunn: Klinkhardt

Lee, Ju-Hwa (2010): Inklusion. Eine kritische Auseinandersetzung mit dem Konzept von Andreas Hinz im Hinblick auf Bildung und Erziehung von Menschen mit Behinderungen. Oberhausen: Athena

Leonhardt, A. (Hrsg.) (1996): Schulische Integration Hörgeschädigter. Neuwied, Kriftel, Berlin: Luchterhand

Leonhardt, A. (Hrsg.) (2009): Hörgeschädigte Schüler in der allgemeinen Schule. Theorie und Praxis der Integration. Stuttgart: Kohlhammer

Lichtblau, M.; Blömer, D.; Jüttner, A.-K.; Koch, K.; Krüger, M.; Werning, R. (2014): Forschung zu inklusiver Bildung. Gemeinsam anders lehren und lernen. Bad Heilbrunn: Klinkhardt

Liebers, K.; Seifert, C.; Kolke, S. (2014): Gestaltung inklusiven Unterrichts in den ERINA-Oberschulen. Erste Befunde. In: Lichtblau, M.; Blömer, D.; Jüttner, A.-K.; Koch, K.; Krüger, M.; Werning, R.: Forschung zu inklusiver Bildung. Gemeinsam anders lebren und lernen. Bad Heilbrunn: Julius Klinkhardt, S. 230-243

Lienhard-Tuggener, P.; Joller-Graf, K.; Mettauer Szaday, B. (2011): Rezeptbuch Schulische Integration. Auf dem Weg zu einer inklusiven Schule. Bern: Haupt Verlag

Lindmeier, C. (2012): Heilpädagogik als Pädagogik der Teilhabe und Inklusion. Sonderpädagogische Förderung heute, $57(1), 25-44$

Lindsay, G. (2007): Educational psychology and the effectiveness of inclusive education/ mainstreaming. In: British Journal of Educational Psychology, 77, 1-24

Little, J.W. (1990): The persistence of privacy. Autonomy and initiative in teachers' professional relations. In: Teachers College Record 91, 509-536 
Lüders, C.; Reicherz, J. (1986): Wissenschaftliche Praxis ist, wenn alles funktioniert und keiner weiß warum - Bemerkungen zur Entwicklung qualitativer Sozialforschung. In: Sozialwissenschaftliche Literatur-Rundschau, 9 (1986) 12, 90-102

Lütje-Klose, B. (1997): Wege integrativer Sprach-und Kommunikationsforderung in der Schule. Konzeptionelle Entwicklungen und ihre Einschätzung durch amerikanische und deutsche ExpertInnen. St. Ingbert: Röhrig

Lütje-Klose, B.; Urban, M. (2014a): Professionelle Kooperation als wesentliche Bedingung inklusiver Schul- und Unterrichtsentwicklung. Teil 1: Grundlagen und Modelle inklusiver Kooperation. In: Vierteljabreszeitschrift für Heilpädagogik und ibre Nachbargebiete, 83. Jg., 112-123

Lütje-Klose, B.; Urban, M. (2014b): Professionelle Kooperation als wesentliche Bedingung inklusiver Schul- und Unterrichtsentwickung. Teil 2: Forschungsergebnisse zu intra- und interprofessioneller Kooperation. In: Vierteljahreszeitschrift für Heilpädagogik und ihre Nachbargebiete, 83. Jg., 283-294

Lütje-Klose, B.; Neumann, P.; Gorges, J.; Wild, E. (2018): Die Bielefelder Längsschnittstudie zum Lernen in inklusiven und exklusiven Förderarrangements (BiLieF) 1-Zentrale Befunde. In: Die Deutsche Schule. Münster Bd. 110, Ausg. 2, (2018): 109-123

Luhmann, N. (1995): Soziologische Aufklärung 6. Die Soziologie und der Mensch. Opladen: Westdeutscher Verlag

Maikowski, R.; Podlesch, W. (1988): Zur Sozialentwicklung behinderter und nichtbehinderter Kinder in der Grundschule. In: Projektgruppe Integrationsversuch (Hrsg.): Das Fläming-Modell. Gemeinsamer Unterricht für behinderte und nichtbehinderte Kinder an der Grundschule. Weinheim: Beltz Verlag

Marvin, C. (1990): Problems in school-based speech language consultations and collaboration services: Defining the terms and improving the process. In: Secord, W.A.; Wilg, E.H. (Hrsg.): Collaborative Programs in the Schools. Concepts, Models and Procedures. Hartcourt: Brace, 37-47

Marzano, R.J.; Marzano, J.S. (2003): The key to classroom management. In: Educational Leadership, September 2003, 163-167

Mattes, W. (2011, 2. Aufl.): Methoden für den Unterricht. Kompakte Übersichten für Lehrende und Lernende. Paderborn: Bildungshaus Schulbuchverlage

Mayer, W. (1994): Anfang ist das Vergessen viel wichtiger als das Lernen. Erfahrungen mit Teamteaching der Volksschule. In: Sertl, M.; Khan-Svik, G.; Wallner, B. (2004): Team Teaching. Zwischen Reparatur-Pädagogik und neuer Lehrer-Rolle. Wien, München: Jugend \&Volk, 9-19

Maykus, S.; Hensen, G.; Küstermann, B.; Riecken, A.; Schinnenburg, H.; Wiedebusch, S. (2014): Inklusive Bildung Teilhabe als Handlungs- und Organisationsprinzip. Eine Matrix zur Analyse von Implementierungsprozessen inklusiver Praxis. In: Hensen, G.; Küstermann, B.; Maykus, S.; Riecken, A.; Schinnenburg, H.; Wiedebusch, S. (Hrsg.) (2014): Inklusive Bildung. Organisations- und professionsbezogene Aspekte eines sozialen Programms. Weinheim und Basel: Beltz Verlag Juventa, 9-45

Mayring, P. (2002, 5. Aufl.): Einführung in die qualitative Sozialforschung. Eine Anleitung zu qualitativem Denken. Weinheim und Basel: Beltz Verlag

Mayring, P. (2010, 11. Aufl.): Qualitative Inhaltsanalyse. Weinheim und Basel: Beltz Verlag

McDuffie, K. (2012): Overview of Co-Teaching Research. Unter: https://education.ufl.edu/325t/files/2013/06/McDuffie_031612.pdf; Stand: 13.04.2013

Meiers, K. (1982): Schulversuch in Reutlingen: Differenzierung im Anfangsunterricht. In: Reinartz, A.; Sander, A. (Hrsg.) (1982): Schulschwache Kinder in der Grundschule. Pädagogische Maßnahmen zur Vorbeugung und Verminderung von Schulschwäche in der Primarstufe. Weinheim und Basel: Beltz Verlag, 195-208

Meijer, J.W. (2003): Special Education across Europe in 2003. Trends in provision in 18 European countries. Unter: https://www.european-agency.org/sites/default/files/special-education-across-europe-in-2003_special_education_ europe.pdf; Stand: 18.10 .2012

Mentzel, W. (1997): Unternehmenssicherung durch Personalentwicklung. Freiburg: Haufe Verlag

Merkens, H. (2010): Unterricht - Eine Einführung. Wiesbaden: Springer VS

Meuser, M.; Nagel, U. (2009): Experteninterview und der Wandel der Wissensproduktion. In: Bogner, A.; Littig, B.; Menz, W. (Hrsg.): Experteninterviews. Theorien, Methoden, Anwendungsfehler. Wiesbaden: Springer VS

Meyer, H. (2004): Was ist guter Unterricht? Cornelsen: Scriptor

Ministerium für Bildung des Saarlandes (1987): Verordnung - Schulordnung - über die gemeinsame Unterrichtung von Behinderten und Nichtbehinderten in Schulen der Regelform (Integrations-Verordnung). Saarbrücken: Ministerium für Bildung

Ministerium für Bildung des Saarlandes (2003): Verordnung - Schulordnung - über die gemeinsame Unterrichtung von Behinderten und Nichtbehinderten in Schulen der Regelform (Integrations-Verordnung). Saarbrücken: Ministerium für Bildung

Ministerium für Bildung des Saarlandes (2011a): Das Saarland macht Schule: Die Gemeinschaftsschule. Unter: https://www. saarland.de/dokumente/thema_bildung/Praesentation_GemS_Lehrkraefte_08.11.2011.pdf; Stand. 14.03.2013 
Ministerium für Bildung des Saarlandes (2011b): Erlass zur Einrichtung des Pilotprojekts zur Entwicklung eines inklusiven Förderkonzepts an Regelschulen im Saarland. Unter: https://www.saarland.de/dokumente/thema_bildung/ Erlasstext_Schulversuch_Inklusion_14_6_2011_2_.pdf; Stand: 14.03.2013

Ministerium für Bildung des Saarlandes (2011c): Arbeitsgruppe Inklusion. Eckpunktepapier zum geplanten Pilotprojekt zur Entwicklung eines inklusiven Förderkonzepts an Regelschulen im Rabmen eines Schulversuchs vom 13.04.2011, unveröff. Dokument

Ministerium für Bildung des Saarlandes (2016a): Arbeitsplatzbeschreibung von Regel- und Förderschullehrkraft. Unter: https://www.saarland.de/dokumente/thema_bildung/ Arbeitsplatzbeschreibung_FoeSL-Endfassung_Januar_2016.pdf; Stand: 13.08.2018

Ministerium für Bildung des Saarlandes (2016b): Rundschreiben zur Zusammenarbeit zwischen Förderschullehrkraft, Regelschule und dem regionalen Förderzentrum. Unter: https://www.saarland.de/dokumente/thema_bildung/Zusammenarbeit_Foerderschullehrkraft_Regelschule_Foerderzentrum_Januar_2016.pdf; Stand: 13.08.2018

Miteinander Leben Lernen e.V. (2014 Entwurf zu einem Gesetz zur Umsetzung des Menschenrechts aufinklusive Bildung gemäß Art. 24 des Übereinkommens der Vereinten Nationen vom 13. Dezember 2006 über die Rechte von Menschen mit Behinderungen in das Saarländische Landesrecht. Synopse zum Vergleich des geltenden Schulrechts mit Art. 1-11 des Gesetzentwurfs der Leben Lernen e.V. sowie Darstellung der Art. 12-14. Unter: https://www.mll-saar.de/wp-content/plugins/download-attachments/includes/download.php?id=729; Stand: 18.11.2016

Montessori-Schule Aktion Sonnenschein (o.J.): Inklusion. Unter: https://aktionsonnenschein.com/montessori-schule/ inklusion/; Stand: 21.02.2012)

Moser, S. (Hrsg.) (2004): Konstruktivistisch Forschen. Methodologie, Methoden, Beispiele. Wiesbaden: Springer VS

Moser, V. (2009): Die Geschichte der Behindertenpädagogik. Enzyklopädie. Erziehungswissenschaft Online. Weinheim und München: Juventa

Moser, V.; Schäfer, L.; Redlich, H. (2011): Kompetenzen und Beliefs von Förderschullehrkräften in inklusiven Settings. In: Lütje-Klose, B.; Langer, M.-T.; Serke, B.; Urban, M. (Hrsg.): Inklusion in Bildungsinstitutionen. Bad Heilbrunn: Klinkhardt, 144-147

Moser, V. (2012): Inklusion statt Rehabilitation? Zur Zukunft einer Disziplin. Antrittsvorlesung. Unter: https://www. reha.hu-berlin.de/de/lehrgebiete/arp/materialien/vera-moser-2012-inklusion-in-berlin; Stand: 24.12.2018

Moser, V.; Schäfer, L.; Kropp, A. (2014): Kompetenzbereiche von Lehrkräften in inklusiven Settings. In: Lichtblau, M.; Blömer, D.; Jüttner, A.-K.; Koch, K.; Krüger, M.; Werning, R. (2014): Forschung zu inklusiver Bildung. Gemeinsam anders lehren und lernen. Bad Heilbrunn: Klinkhardt; 124-143

Moser, V. (2017): Inklusion und Organisationsentwicklung. In: Moser, V.; Egger, M. (Hrsg.) (2017): Inklusion und Schulentwicklung. Konzepte, Instrumente, Befunde. Stuttgart: Kohlhammer, 15-30

Moser, V.; Egger, M. (Hrsg.) (2017): Inklusion und Schulentwicklung. Konzepte, Instrumente, Befunde. Stuttgart: Kohlhammer

Moser Opitz, E. (2014): Inklusive Didaktik im Spannungsfeld von gemeinsamem Lernen und effektiver Förderung. Ein Forschungsüberblick und eine Analyse von didaktischen Konzeptionen für inklusiven Unterricht. In: Zierer, K.; Lamers, D. (Hrsg.): Jahrbuch für Allgemeine Didaktik 2014. Baltmannsweiler: Schneider Verlag Hohengehren, 52-68

Mühl, H. (1987): Integration von Kindern und Jugendlichen mit geistiger Behinderung. Gemeinsame Erziehung mit Nichtbehinderten in Kindergarten und Schule. Berlin: Marhold

Mühl, H. (2018): Abriss zur Geschichte der Beschulung von Schulpflichtigen mit geistiger Behinderung. Unter: http://50jahre.lebenshilfe.de/50_jahre_lebenshilfe/ 1960er/downloads/60Muehl.pdf; Stand: 23.12.2018

Müller, C.; Hofmann, V.; Studer, F. (2012): Lässt sich individuelles Problemverhalten durch das Niveau an Verhaltensschwierigkeiten unter den Mitschülern vorhersagen? Ergebnisse einer Querschnittstudie und ihre Relevanz für die Fragebe einer integrativen vs. Separativen Beschulung verhaltensauffälliger Schüler. In: Empirische Sonderpädagogik, 2012, Nr. 2, 111-128

Müller, G.N.; Steinbring, H.; Wittmann, E.Ch. (2002): Jenseits von PISA: Bildungsreform als Unterrichtsreform. Ein Fünf-Punkte-Programm aus systemischer Sicht. Seelze-Velber: Kallmeyersche Verlagsbuchhandlung

Müller, H. (1987): Rahmenbedingungen für die Integration behinderter Kinder in der Grundschule. In: Lebenshilfe e.V. (1987): Bericht: Gemeinsames Leben und Lernen geistig behinderter und nichtbehinderter Kinder und Jugendlicher im Schulalter. Symposium vom 11. bis 13.12.1986, 158-164)

Müller-Stump, K.; Rossi Marty, S. (1994): Integration auf der Oberstufe. Begleitung eines Projekts. Luzern: Edition SZH Muijs, D.; Reynolds, D. (2005, 2.Auflage): Effective teaching. Evidence and practice. Thousand Oaks: Sage

Munder, R. (1988): Meinungen der beteiligten Eltern zum Integrationsversuch. Projektgruppe Integrationsversuch (Hrsg.) (1988): Das Fläming-Modell. Gemeinsamer Unterricht für behinderte und nichtbehinderte Kinder an der Grundschule. Weinheim und Basel: Beltz Verlag, 293-296

Muth, J. (1976): Einführung. In: Deutscher Bildungsrat (Hrsg.) (1976): Schulversuche zur Integration behinderter Kinder in den allgemeinen Unterricht: Materialien zur Bildungsplanung. Heft 6. Braunschweig: Westermann, 14-20 
Muth, J. (1977): Möglichkeiten und Grenzen schulischer Integration behinderter Kinder. In: Weigt, M. (Hrsg.) (1977): Schulische Integration von Behinderten. Beiträge zum Verhältnis von Sonderschulen zum Regelschulsystem. Weinheim und Basel: Beltz Verlag, 90-100

Muth, J. (1988): Die Fläming-Grundschule im Kontext der Ausbreitung integrativer Schulen. In: Projektgruppe Integrationsversuch (Hrsg.) (1988): Das Fläming-Modell. Gemeinsamer Unterricht für behinderte und nichtbehinderte Kinder an der Grundschule. Weinheim, Basel: Beltz Verlag, 17-23

Mutzeck, W.; Pallasch, W. (Hrsg.) (1987): Integration von Schülern mit Verhaltensstörungen. Praktische Modelle und Versuche. Weinheim: Deutscher Studien Verlag

Mutzeck, W. (1987a): Sehen und Verstehen von Verhaltensstörungen in der Schule. In: Mutzeck, W.; Pallasch, W. (Hrsg.): Integration von Schülern mit Verhaltensstörungen. Praktische Modelle und Versuche. Weinheim: Deutscher Studien Verlag, 4-18

Mutzeck, W. (1987b): Integrative Förderung und Prävention bei Verhaltensstörungen. In: Mutzeck, W.; Pallasch, W. (Hrsg.): Integration von Schülern mit Verhaltensstörungen. Praktische Modelle und Versuche. Weinheim: Deutscher Studien Verlag, 19-35

Nassehi, A. (1997): Inklusion oder Integration?: zeitdiagnostische Konsequenzen einer Theorie von Exklusions- und Desintegrationsphänomenen. In: Rehberg, K.-S. (Hrsg.); Deutsche Gesellschaft für Soziologie (Hrsg.): Differenz und Integration: die Zukunft moderner Gesellschaften; Verhandlungen des 28. Kongresses der Deutschen Gesellschaft für Soziologie im Oktober 1996 in Dresden; Band 2: Sektionen, Arbeitsgruppen, Foren, Fedor-Stepun-Tagung. Opladen: Westdeutscher Verlag, 619-623

Nassehi, A. (2004): Die Theorie funktionaler Differenzierung im Horizont ihrer Kritik. In: Zeitschrift für Soziologie, Jg. 33, Heft 2, April 2004, 98-118

Nollmann, G.; Strasser, H. (Hrsg.) (2004): Das individualisierte Ich in der modernen Gesellschaft. Frankfurt a.M.: Campus Verlag

OECD (1963): Resources of Scientific and Technical Personnel in the OECD Area. Statistical Report of the Third International Survey on the Demand for a Supply of Scientific and Technical Personnel. Paris: OECD-Publications

OECD (2004): Anwerbung, berufliche Entwicklung und Verbleib von qualifizierten Lehrerinnen und Lehrern - Länderbericht: Deutschland. Paris. Unter: www.tango-sophia.de/resources/.../Germany_Country_Note_Endfassung deutsch.pdf

Opalinski, S. (2014): Einstellungen von Lehrkräften in Thüringen und Finnland zur schulischen Inklusion. In: Lichtblau, M.; Blömer, D.; Jüttner, A.-K.; Koch, K.; Krüger, M.; Werning, R. (2014): Forschung zu inklusiver Bildung. Gemeinsam anders lehren und lernen. Bad Heilbrunn: Klinkhardt; 76-90

Opp, G.; Theunissen, G. (Hrsg.) (2009): Handbuch schulische Sonderpädagogik. Bad Heilbrunn: Klinkhardt

Paradies, L.; Linser, H.-J. (2001): Differenzieren im Unterricht. Berlin: Cornelsen Verlag Scriptor

Peterßen, W.H. (1974): Grundlagen und Praxis des lernzielorientierten Unterrichts. Ravensburg: Maier

Picht, G. (1964): Die deutsche Bildungskatastrophe, Analyse und Dokumentation. Freiburg im Breisgau: Walter Verlag; Unter: http://www.epb.uni-hamburg.de/erzwiss/lohmann/Lehre/Som3/BuG/picht1964.pdf(Auszug); Stand: 24.12.2018

Pfeiffer, H. (2010): Schule in erweiterter Verantwortung aus erziehungswissenschaftlicher Sicht. In: Bohl, T.; Helsper, W.; Holtappels, H.G.; Schelle, C. (Hrsg.) (2010): Handbuch Schulentwicklung. Bad Heilbrunn: Klinkhardt, 19-26

Pfrang, A.; Viehweger, A. (2015): Die Bedeutung der Lehrer-Schüler-Beziehung für schulisches Lernen. In: Blömer, D.; Lichblau, M.; Jüttner, A.-K.; Koch, K.; Krüger, M.; Werning, R. (Hrsg.) (2015): Perspektiven auf inklusive Bildung. Gemeinsam anders lebren und lernen. Jahrbuch Grundschulforschung. Wiesbaden: Springer VS, 291-296

Podlesch, W. (2003): Integrationspädagogische Lernprinzipien zum Förderschwerpunkt geistige Entwicklung. In: Eberwein, H.; Knauer, S. (Hrsg.) (2003): Behinderungen und Lernprobleme überwinden. Stuttgart: Kohlhammer, 39-53

Poscher, R.; Rux, J.; Langer, T. (2008): Von der Integration zur Inklusion. Das Recht auf Bildung aus der Behindertenrechtskonvention der Vereinten Nationen und seine innerstaatliche Umsetzung. Baden-Baden: Nomos Verlagsgesellschaft

Precht, D. (2011): Immer mehr ist immer weniger. Gesammelte Essays. München: Wilhelm Goldmann

Prell, S.; Link, P. (1974): Das Münchner Modell der schulischen Integration behinderter und nichtbehinderter Kinder. Schulversuch nach Maria Montessori. In: Weigt, M. (Hrsg.) (1977): Schulische Integration von Behinderten. Beiträge zum Verhältnis von Sonderschulen zum Regelschulsystem. Weinheim und Basel: Beltz Verlag, 302-327

Prengel, A. (3. Aufl. 2006): Pädagogik der Vielfalt. Verschiedenheit und Gleichberechtigung in Interkultureller, Feministischer und Integrativer Pädagogik. Wiesbaden: Springer VS

Preuß, E. (Hrsg.) (1985): Berliner Sonderpädagogik: Neue Alltagspraxis durch Kooperation. Berlin: Marhold

Preuss-Lausitz, U. (1977): Probleme der Integration von Sonderschülern in die Gesamtschule. In: Weigt, H. (1977): Schulische Integration Behinderter, Weinheim und Basel: Beltz Verlag, 44-54

Preuss-Lausitz, U. (1981): Fördern ohne Sonderschule. Konzepte und Erfahrungen zur integrativen Förderung in der Regelschule. Weinheim und Basel: Beltz Verlag 
Preuss-Lausitz, U.; Textor, A. (2006): Verhaltensauffällige Kinder sinnvoll integrieren - eine Alternative zur Schule für Erziehungshilfe. In: Zeitschrift für Heilpädagogik, 57, 2-8

Preuss-Lausitz, U. (2010): Schulentwicklung und Differenz: Integration. In: Bohl, T.; Helsper, W.; Holtappels, H.G.; Schelle, C. (Hrsg.) (2010): Handbuch Schulentwicklung. Bad Heilbrunn: Klinkhardt, S. 45-49

Preuss-Lausitz, U. (2011): „Wir dürfen nicht so tun, als hätten wir in Regelschulen keine Heterogenität“, 47; In: Bertelsmann Stiftung (Hrsg.) (2011): Bildung und Vielfalt, 46-50

Preuss-Lausitz, U. (2014): Wissenschaftliche Begleitungen der Wege zur inklusiven Schulentwicklung in den Bundesländern. Versuch einer Übersicht. Unter: www.tu-berlin.de/fakultaet_i/ institut_fuer_erziehungswissenschaft/menue/ institutsangehoerige/professorinnen/prof_dr_ulf_preuss-lausitz/publikationen/; Stand: 03.04.2018

Proebstel, C.H.; Soltau, A. (2012): Wieso Lehrkräfte (nicht) kooperieren - Die Bedeutung „personaler Faktoren“ in der Zusammenarbeit am Arbeitsplatz Schule. In: Baum, E.; Idel, T.-S.; Ullrich, H. (Hrsg.) (2012a): Kollegialität und Kooperation in der Schule. Theoretische Konzepte und empirische Befunde. Wiesbaden: Springer VS, 55-75

Projektgruppe Integrationsversuch (Hrsg.) (1988): Das Fläming-Modell. Gemeinsamer Unterricht für behinderte und nichtbehinderte Kinder an der Grundschule. Weinheim und Basel: Beltz Verlag

Pushor, D. (2007): Parent Engagement: Creating a Shared World. Invited Research Paper. Ontario Education Research Symposium. Unter: http://www.edu.gov.on.ca/eng/research/pushor.pdf; Stand: 09.08.2014

Reh, S. (2008): „Reflexivität der Organisation“ und Bekenntnis. Perspektiven der Lehrerkooperation. In: Helsper, W.; Busse, S.; Hummrich, M.; Kramer, R.-T. (Hrsg.): Pädagogische Professionalität in Organisationen. Neue Verhältnisbestimmungen am Beispiel der Schule. Wiesbaden: Springer VS Verlag, 163-183

Reich, K. (2002): Konstruktivistische Didaktik. Lehren und Lernen aus interaktionistischer Sicht. Neuwied u.a.: Hermann Luchterhand

Reinartz, A.; Sander, A. (Hrsg.) (1982): Schulschwache Kinder in der Grundschule. Pädagogische Maßnahmen zur Vorbeugung und Verminderung von Schulschwäche in der Primarstufe. Weinheim und Basel: Beltz Verlag

Reiser, H.; Gutberlet, M.; Klein, G.; Kreie, G.; Kron, M. (1984): Sonderschullehrer in Grundschulen. Ergebnisse eines Schulversuchs zur integrativen Betreuung bei Lern-und Verhaltensstörungen. Weinheim und Basel: Beltz Verlag

Reiser, H. (1987): Sonderschullehrer in hessischen Grundschulen - ein Ansatz zur integrativen Arbeit bei Lern- und Verhaltensproblemen. In: Mutzeck, W.; Pallasch, W. (Hrsg.) (1987): Integration von Schülern mit Verhaltensstörungen. Praktische Modelle und Versuche. Weinheim: Deutscher Studien Verlag, 131-151

Reiser, H., Deppe-Wolfinger, H., Prengel, A. (1990): Integrative Pädagogik in der Grundschule. Bilanz und Perspektiven der Integration behinderter Kinder in der Bundesrepublik 1976-1988. München: DJI

Reiser, H. (1998): Sonderpädagogik als Service-Leistung? Perspektiven der sonderpädagogischen Berufsrolle. Zur Professionalisierung der Hilfsschul- bzw. Sonderschullehrerinnen. In: Zeitschrift für Heilpädagogik 2/98, 46-54

Reiser, H. (2003): Vom Begriff Integration zum Begriff Inklusion - Was kann mit dem Begriffswechsel angestoßen werden? In: Sonderpädagogische Förderung, 48 (4), 305-312

Rheinberg, F. (2008): Bezugsnormen und die Beurteilung von Lernleistung. In: Schneider, W.; Hasselhorn, M. (Hrsg.): Handbuch der Pädagogischen Psychologie (Handbuch der Psychologie 10). Göttingen: Hogrefe, 178-186

Riedel, E. (2010): Gutachten zur Wirkung der internationalen Konvention über die Rechte von Menschen mit Behinderung und ibres Fakultativprotokolls auf das deutsche Schulsystem. Unter: http://gemeinsam-leben-nrw.de/sites/ default/files/gutachten_riedel.pdf; Stand: 19.04.2012

Rihm, T. (2008): Schule als Ort kooperativer Selbstverständigung entwickeln. In: Rihm, T. (Hrsg.) (2006): Schulentwicklung: Vom Subjektstandpunkt ausgehen. Wiesbaden: Springer VS, 393-428

Rolff, H.-G. (1998): Entwicklung von Einzelschulen: Viel Praxis, wenig Theorie und kaum Forschung - Ein Versuch Schulentwicklung zu systematisieren. In: Rolff, H.-G.; Bauer, K-O.; Klemm, K.; Pfeiffer, H. (Hrsg.) (1998): Jahrbuch der Schulentwicklung. Band 10. Daten, Beispiele, Perspektiven. Weinheim und München: Juventa, 295-326

Rolff, H.-G. (2010): Schulentwicklung als Trias von Organisations-, Unterrichts- und Personalentwicklung. In: Bohl, T.; Helsper, W.; Holtappels, H.G.; Schelle, C. (Hrsg.) (2010): Handbuch Schulentwicklung. Bad Heilbrunn: Klinkhardt, 29-36

Romey, S. (1977): Gesellschafts- und schulpolitische Bedingungen für die soziale Integration Behinderter in die Gesamtschule. In: Weigt, M. (Hrsg.): Schulische Integration Behinderter. Weinheim und Basel: Beltz Verlag, 107-132

Rossbach, H.G.; Wellenreuther, M. (2002): Empirische Forschungen zur Wirksamkeit von Methoden der Leistungsdifferenzierung in der Grundschule. In: Heinzel, F.; Prengel, A. (Hrsg.) (2002): Heterogenität, Integration und Differenzierung in der Primarstufe. Opladen: Lese \& Budrich, 44-57

Rothfritz, L.P. (2010): Die Konvention der Vereinten Nationen zum Schutz der Rechte von Menschen mit Behinderungen. Eine Analyse unter Bezugnahme auf die deutsche und europäische Rechtsebene. Frankfurt a.M., Berlin, Bern, Bruxelles, New York, Oxford, Wien: Peter Lang aus der Reihe: Dresdner Schriften zu Recht und Politik der Vereinten Nationen. Hrsg. Schorlemer, S. von; Band 10 
Rothland, M. (2013): Voraussetzungen von Lehramtsstudierenden für die Kooperation im Lehrerberuf: Berufsbezogene Einstellungen und soziale Kompetenz. In: Keller-Schneider, M.; Albisser, S.; Wissinger, J. (Hrsg.) (2013): Professionalität und Kooperation in Schulen. Beiträge zur Diskussion über Schulqualität. Bad Heilbrunn: Klinkhardt, $87-102$

Saalfrank, W.-T. (2012): Differenzierung. In: Kiel, E. (Hrsg.): Unterricht sehen, analysieren und gestalten. Bad Heilbrunn: Klinkhardt, 65-96

Saldern, M. von (1987): Sozialklima von Schulklassen. Frankfurt am Main: Peter Lang

Saldern, M. von (2011): Klassengröße. Über ein vernachlässigtes Merkmal. Norderstedt: Books on Demand GmbH

Sander, A. (1971): Die Sonderschulen im Saarland. Saarbrücken: Saarbrücker Zeitung Verlag und Druckerei

Sander, A. (1976): Sonderpädagogik in der Regelschule. Berlin: Marhold

Sander, A. (1988): Behinderte Kinder und Jugendliche in Regelschulen. Jabresbericht 1987 über schulische Integration im Saarland. St. Ingbert: Röhrig Universitätsverlag

Sander, A. (1993): Kind-Umfeld-Diagnose: Ökologischer Ansatz in der Diagnostik. In: Pädagogisches Landesinstitut Brandenburg (1993) (Hrsg.): Kinder mit Förderbedarf. Neue Wege in der sonderpädagogischen Diagnostik. Fachserien Lernort Schule. Heft 2, 23-36

Sander, A. (1994): Entwicklung der schulischen Integration behinderter Kinder und Jugendlicher im Saarland 19901993/94. In: Sander, A.; Hildeschmidt, A.; Jung-Sion, J.; Raidt-Petrick, M.; Schnitzler, P. (Hrsg.) (1994): Schulreform Integration. Entwicklungen der gemeinsamen Erziehung behinderter und nichtbehinderter Kinder und Jugendlicher im Saarland 1990-1993/94. St. Ingbert: Röhrig Universitätsverlag, 9-37

Sander, A.; Hildeschmidt, A.; Jung-Sion, J.; Raidt-Petrick, M.; Schnitzler, P. (Hrsg.) (1994): Schulreform Integration. Entwicklungen der gemeinsamen Erziehung behinderter und nichtbehinderter Kinder und Jugendlicher im Saarland 1990-1993/94. St. Ingbert: Röhrig Universitätsverlag

Sander, A. (1995): Was hat sich bewegt? Zugleich ein Versuch zu Folgerungen für die weitere Entwicklung. In: Sander, A.; Hoffmann, E.; Horstmann, M.; Raidt-Petrick, M.; Schmidt, W. (1995): Sonderpädagogische Förderzentren als Weiterentwicklung der Organisation sonderpädagogischer Arbeit für behinderte Schülerinnen und Schüler. Abschlußbericht zum Modellversuch der BLK für Bildungsplanung und Forschungsförderung. Saarbrücken: Eigenverlag, $273-290$

Sander, A.; Hoffmann, E.; Horstmann, M.; Raidt-Petrick, M.; Schmidt, W. (1995): Sonderpädagogische Förderzentren als Weiterentwicklung der Organisation sonderpädagogischer Arbeit für behinderte Schülerinnen und Schüler. Abschlußbericht zum Modellversuch der BLK für Bildungsplanung und Forschungsförderung. Saarbrücken: Eigenverlag

Sander, A. (2003): Über die Integration zur Inklusion. St. Ingbert: Röhrig Universitätsverlag

Sander, A.; Schnell, I. (2006): Länderbericht Saarland. Unter: http://bidok.unibk.ac.at/tagung/ download/laenderbericht-saarland-2006.doc; Stand: 07.11.2011

Sander, A.; Müller, F.J. (2018): Interview mit Alfred Sander. In: Müller, F.J. (Hrsg.) (2018): Blick zurück nach vorn WegbereiterInnen der Inklusion. Band 1. Originalausgabe. Gießen: Psychosozial-Verlag, 9-22.

Sandfuchs, U.; Menzel, W. (Hrsg.) (2018): Schreiben lehren. Wie Kinder Schriftsprache erlernen - ein Überblick über aktuelle Methoden. In: Grundschule, 50 (2018) 6, 6-35

Sauerwein, M.; Klieme, E. (2016): Anmerkungen zum Qualitätsbegriff in der Bildungsforschung. In: Schweizerische Zeitschrift für Bildungswissenschaften 38 (2016) 3, 459-478

Schäper, S. (2010): Inklusion als sozialethisches Projekt - Überlegungen aus fachlicher und familiärer Perspektive. In: Jennessen, S.; Lelgemann, R.; Ortland, B.; Schlüter, M. (Hrsg.) (2010): Leben mit Körperbehinderung. Perspektiven der Inklusion., 33-47

Scheer, D.; Laubenstein, D. (2018): Schulische Inklusion entwickeln. Arbeitshilfe für Schulleitungen. Stuttgart: Kohlhammer

Schenk, C. (2012, 9. Aufl.): Lesen und Schreiben lernen und lehren: Eine Didaktik des Schriftspracherwerbs. Baltmannsweiler: Schneider Verlag Hohengehren

Schinnen, M. (1988): Klassenübergreifende Förderung als Teil integrativer Unterrichtspraxis. In: Projektgruppe Integrationsversuch (Hrsg.) (1988): Das Fläming-Modell. Gemeinsamer Unterricht für behinderte und nichtbehinderte Kinder an der Grundschule. Weinheim und Basel: Beltz Verlag, 120-129

Schippen, M.; Houchins, D.; Steventon, C.; Sartor, D. (2005): A Comparison of Two Direct Instruction Reading Programs for Urban Middle School Students. In: Remedial and Special Education 26 (3), 175-182

Schley, W. (1990): Wissenschaftliche Begleitung als Team-Supervision. In: Schuck, K.D. (Hrsg.) (1990): Beiträge zur Integrativen Pädagogik. Weiterentwicklungen des Konzepts gemeinsamen Lebens und Lernens Behinderter und Nichtbehinderter. Hamburg: Feldhaus Verlag, S. 138-166

Schley, W.; Boban, I.; Hinz, A. (Hrsg.) (1992, 2. Aufl.): Integrationsklassen in Hamburger Gesamtschulen. Hamburg: Curio 
Schley, W. (2002): Teamkooperation und Teamentwicklung in der Schule. Universität Kaiserslautern: o.V.

Schmidt, M. (2016): „Ich denke wir sind hier eine gute Schule, die immer schon vorangeschritten ist..." Zur Produktivität der Unbestimmtheit von,Qualität' für Artikulationen der Wirksamkeit von Schulinspektionen. In: Hadeler, S.; Moegling, K.; Hund-Göschel, G. (Hrsg.) (2016): Was sind gute Schulen?: Teil 3: Forschungsergebnisse. Immenhausen: PROLOG-Verlag

Schnitzer, A. (2008): Kooperation von Schulen mit außerschulischen Akteuren. Abschlussbericht. Unter: https://www.dji. de/fileadmin/user_upload/kosa/Abschlussbericht_KOSA_1Neu_Januar_2010.pdf; Stand: 09.02.2019

Schön, M.; Stark, R. (2012): Zwischenbericht der wissenschaftlichen Begleitung des Inklusionsprojektes an saarländischen Schulen. Schuljahr 2011/12. unv. Dokument

Schön, M.; Stark, R. (2014): Erste Erfahrungen mit inklusiver Unterrichtspraxis an saarländischen Grundschulen. In: Schulpädagogik heute, Inklusion in Schule und Unterricht, H. 10 (2014), 5. Jahrgang, 1-16

Schuck, K.D. (Hrsg.) (1990): Beiträge zur Integrativen Pädagogik. Weiterentwicklungen des Konzepts gemeinsamen Lebens und Lernens Behinderter und Nichtbehinderter. Hamburg: Feldhaus Verlag

Schümer, G.; Weiß, M. (2008): Bildungsökonomie und Qualität der Schulbildung.Kommentar zur bildungsökonomischen Auswertung von Daten aus internationalen Schulleistungsstudien. Frankfurt: GEW-Hauptvorstand

Schumann, M. (2006): Ein wissenschaftliches Netzwerk wird 20-Zur Geschichte und Gegenwart der Integrationsforschung. Unter: https://www.inklusion-online.net/index.php/inklusion-online/article/view/182/182; Stand: 14.02.2016

Schwager, M. (2011): Beziehungsmobile - Doppelbesetzungen im gemeinsamen Unterricht. In: Ziemen, K.; Langner, A.; Köpfer, A.; Erbring, S. (Hrsg.) (2011): Inklusion - Herausforderungen, Chancen und Perspektiven. Hamburg: Verlag Dr. Kovac, 47-57

Schwartz, E. (1982): Kinderrecht auf Schulbildung. In: Reinartz, A.; Sander, A. (Hrsg.) (1982): Schulschwache Kinder in der Grundschule. Pädagogische Maßnahmen zur Vorbeugung und Verminderung von Schulschwäche in der Primarstufe. Weinheim und Basel: Beltz Verlag, 35-38

Schwarz, A.; Weishaupt, H.; Schneider, K.; Makles, A.; Tarazona, M. (2013): Mögliche kommunale Folgekosten der Umsetzung der Inklusion im Schulbereich in Nordrbein-Westfalen am Beispiel der Stadt Essen und des Kreises Borken. Gutachten im Auftrag des Städtetages Nordrhein-Westfalen, des Landkreistages Nordrhein-Westfalen und des Städteund Gemeindebundes Nordrhein-Westfalen. Unter: http://www.staedtetag-nrw.de/imperia/md/content/stnrw/ siteuebergreifend/2013/gutachten_spitzverbnrw_inklusion_130712_final.pdf; Stand: 26.02.2019

Seitz, S. (2006a): Inklusive Didaktik: Die Frage nach dem ,Kern'der Sache. Unter: http://www.inklusion-online.net/ index.php/inklusion-online/article/view/184/184/15; Stand: 24.05 .2014

Seitz, S. (2006b): Zur Bedeutung von Bildungsstandards für die inklusive Grundschule. In: Stechow, E. von; Hofmann, C. (Hrsg.) (2006): Sonderpädagogik und Pisa. Kritisch-konstruktive Beiträge. Bad Heilbrunn: Klinkhardt, 113-120

Serke, B.; Urban, M.; Lütje-Klose, B. (2014): Teamarbeit und Team-Teaching in inklusiven Grundschulen. In: Inklusion: Eine Herausforderung für die Grundschulpädagogik. Franz, E.; Trumpa, S.; Esslinger-Hinz, I. (Hrsg.); Hohengehren: Schneider Verlag, 249-260

Sertl, M. (1994): Von der Einzelverantwortlichkeit zur Teamverantwortlichkeit. In: Sertl, M.; Khan-Svik, G.; Wallner, B. (2004): Team Teaching. Zwischen Reparatur-Pädagogik und neuer Lehrer-Rolle. Wien und München: Jugend \&Volk, 40-52

SINUS-Institut (2017): Informationen zu den Sinus-Milieus ${ }^{\circledR}$ 2017. Unter: https://www.sinus-institut.de/fileadmin/user_ data/sinus-institut/Dokumente/downloadcenter/Sinus_Milieus/ 017-01-01_Informationen_zu_den_Sinus-Milieus. pdf; Stand: 10.08 .2018

SINUS-Institut (2018): Informationen zu den Sinus-Milieus ${ }^{\circ}$ 2018. Unter: https://www.sinus-institut.de/fileadmin/ user_data/sinus-institut/Bilder/Sinus-Milieus_092018/2018-09-18_Informationen_zu_den_Sinus-Milieus.pdf; Stand: 19.02 .2019

Skidmore, D. (2004): Inclusion. The dynamic of school development. Maidenhead: Open University Press

Slavin, R.E. (1995): Cooperative Learning. Boston, MA: Allyn and Bacon

Solga, H.; Dombrowski, R. (2009): Soziale Ungleichheiten in schulischer und außerschulischer Bildung. Stand der Forschung und Forschungsbedarf. Unter: http://www.boeckler.de/pdf/p_arbp_171.pdf; Stand: 08.08.2015

Soltau, A.; Mienert, M. (2013): Die Bedeutung der beruflichen Unsicherheit für die Zusammenarbeit von Lehrkräften. In: Keller-Schneider, M.; Albisser, S.; Wissinger, J. (Hrsg.) (2013): Professionalität und Kooperation in Schulen. Beiträge zur Diskussion über Schulqualität. Bad Heilbrunn: Klinkhardt, 71-85

Specht, W. (1997a): Autonomie und Innovationsklima an Schulen. Rezeption und Wirkung der Schulautonomie an Hauptschulen und allgemeinbildenden Schulen. Graz: Zentrum für Schulentwicklung, Abt. II, Forschungsberichte 26

Specht, W. (1997b): Jedes Kind ist Mittelpunkt. Ergebnisse und Gedanken aus der Evaluation der Schulversuche zur Integration behinderter Schüler in der Sekundarstufe I. In: Zeitschrift BEHINDERTE in Familie, Schule und Gesellschaft, 20, Heft 4, 9-30 
Specht, W. (Hrsg.) (1997): Fallstudien zur Integration behinderter Kinder in der Sekundarstufe I. 4 Bände. Bundesministerium für Unterricht und kulturelle Angelegenheiten. Graz: Zentrum für Schulentwicklung. Abteilung II, ZSE-Report Nummer 23. 26.

Specht, W.; Gross-Pirchegger, L.; Seel, A.; Stanzel-Tischler, E.; Wohlhart, D. (2006): Qualität in der Sonderpädagogik: Ein Forschungs- und Entwicklungsprojekt. Graz: Zentrum für Schulentwicklung, Bundesministerium für Bildung, Wissenschaft und Kultur

Speck, O.; Gottwald, P.; Havers, H.; Innerhofer, P. (Hrsg.) (1978): Schulische Integration lern- und verhaltensgestörter Kinder. München/Basel: Ernst Reinhardt

Speck, O. (2010): Schulische Inklusion aus heilpädagogischer Sicht. Rhetorik und Realität. München: Ernst Reinhardt Verlag Speck, O. (2011): Wage es nach wie vor, dich deines eigenen Verstandes zu bedienen! Ideologische Implikationen einer Schule für alle. In: Zeitschrift für Heilpädagogik 3, 2011, 84-91

Speck, O. (2014): Inklusive Missverständnisse. In: Süddeutsche Zeitung v. 21.10.2014. Unter: http://www.sueddeutsche.de/bildung/inklusions-debatte-inklusive-missverstaendnisse-1.2182484; Stand: 13.11.2014

Speck-Hamdan, A. (2015): Inklusion: der Anspruch an die Grundschule. In: Blömer, D.; Lichblau, M.; Jüttner, A.-K.; Koch, K.; Krüger, M.; Werning, R. (Hrsg.): Perspektiven auf inklusive Bildung. Gemeinsam anders lehren und lernen. Jahrbuch Grundschulforschung. Wiesbaden: Springer VS, 14-22

Spieß, E. (2004): Kooperation und Konflikt. In: Schuler, H. (Hrsg.): Enzyklopädie der Psychologie. Organisationspsychologie - Gruppe und Organisation. Göttingen: Hogrefe, 193-250

Spörer, N.; Maaz, K.; Vock, M.; Schründer-Lenzen, A.; Luka, T.; Bosse, S.; Jäntsch, C.; Vogel, J. (2015): Wie entwickeln sich Kinder im inklusiven Unterricht? Zur Anlage der wissenschaftlichen Begleitung des Projekts „Inklusive Grundschule“. In: Blömer, D.; Lichblau, M.; Jüttner, A.-K.; Koch, K.; Krüger, M.; Werning, R. (Hrsg.) (2015): Perspektiven auf inklusive Bildung. Gemeinsam anders lebren und lernen. Jahrbuch Grundschulforschung. Wiesbaden: Springer VS, 297-302

Stäbler, F.; Lambrecht, J.; Dumont, H.; Maaz, K. (2015): Lernumgebung Inklusive Grundschule - Strukturelle Gegebenheiten und Unterschiede der PING-Schulen. In: Landesinstitut für Schule und Medien Berlin-Brandenburg (LISUM), Universität Potsdam, Deutsches Institut für internationale Pädagogische Forschung (DIPF) (2015) (Hrsg.): Inklusives Lernen und Lehren im Land Brandenburg. Abschlussbericht zur Begleitforschung des Pilotprojekts „Inklusive Grundschule“. Potsdam; Unter http://www.inklusion-brandenburg.de/fileadmin/daten/inklusion_im_ land_brandenburg/pilotprojekt_inklusive_grundschule/wissenschaftliche__begleitung/Abschlussbericht_PING. pdf; Stand: 14.03.2018; S. 69-85

Ständige Konferenz der Kultusminister der Länder in der Bundesrepublik Deutschland (KMK) (Hrsg.) (1960): Gutachten zur Ordnung des Sonderschulwesens. Bonn:

Ständige Konferenz der Kultusminister der Länder in der Bundesrepublik Deutschland (KMK) (1972): Empfehlung zur Ordnung des Sonderschulwesens. Nienburg: Schulze

Ständige Konferenz der Kultusminister der Länder in der Bundesrepublik Deutschland (KMK) (1983): Bericht über die Bedingungen und Grenzen des gemeinsamen Unterrichts von lernbehinderten und nichtbehinderten Schülern in allgemeinbildenden Schulen. Bonn: Eigenverlag

Ständige Konferenz der Kultusminister der Länder in der Bundesrepublik Deutschland (KMK) (1994): Empfehlungen zur sonderpädagogischen Förderung in den Ländern der BRD. Bonn: Eigenverlag

Ständige Konferenz der Kultusminister der Länder in der Bundesrepublik Deutschland (KMK) (2005): Sonderpädagogische Förderung zwischen 1994 und 2003. Unter: http://www.kmk.org/fileadmin/pdf/PresseUndAktuelles/Dokumentation 177.pdf; Stand: 02.02.2019

Ständige Konferenz der Kultusminister der Länder in der Bundesrepublik Deutschland (KMK) (2010): Pädagogische und rechtliche Aspekte der Umsetzung des Übereinkommens der Vereinten Nationen vom 13. Dezember 2006 über die Rechte von Menschen mit Behinderungen (Behindertenrechtskonvention - VN-BRK) in der schulischen Bildung. Beschluss der Kultusministerkonferenz vom 18.11.2010. Unter: https://www.kmk.org/fileadmin/Dateien/veroeffentlichungen_beschluesse/2010/2010_11_18-Behindertenrechtkonvention.pdf, Stand: 04.11.2011

Ständige Konferenz der Kultusminister der Länder in der Bundesrepublik Deutschland (KMK) (2011): Empfehlungen zur inklusiven Bildung von Kindern und Jugendlichen mit Behinderungen in Schulen. Bitte einfügen: Unter: http://www. kmk.org/fileadmin/veroeffentlichungen_beschluesse/2011/2011_10_20-Inklusive-Bildung.pdf; Stand: 04.11.2011

Ständige Konferenz der Kultusminister der Länder in der Bundesrepublik Deutschland (KMK) (2018a): Sonderpädagogische Förderung zwischen 2007 und 2016. Unter: https://www.kmk.org/fileadmin/Dateien/pdf/Statistik/Dokumentationen/Dok_214_SoPaeFoe_2016.pdf; Stand: 02.02.2019

Ständige Konferenz der Kultusminister der Länder in der Bundesrepublik Deutschland (KMK) (2018b): Vorgaben für die Klassenbildung, Schuljahr 2018/2019, Stand: November 2018; Unter: https://www.kmk.org/fileadmin/Dateien/pdf/Statistik/Dokumentationen/Klassenbildung_2018.pdf; Stand: 03.01.2019

Statistisches Bundesamt (2018): Umfrage Lehrermangel und Lehrerüberschuss in Deutschland. Unter: https://de.statista. $\mathrm{com} /$ statistik/daten/studie/288923/umfrage/lehrermangel-und-lehrerueberschuss-in-deutschland/; Stand: 03.04.2019 
Stechow, E. von; Hofmann, C. (Hrsg.) (2006): Sonderpädagogik und Pisa. Kritisch-konstruktive Beiträge. Bad Heilbrunn: Klinkhardt

Stein, A.-D. (2008): Die Bedeutung des Inklusionsgedankens - Dimensionen und Handlungsperspektiven. In: Hinz, A.; Körner, I.; Niehoff, U. (Hrsg.) (2008): Von der Integration zur Inklusion. Grundlagen, Perspektiven, Praxis. Marburg: Lebenshilfe-Verlag, 74-90

Stein, A.-D.; Krach, S.; Niediek, I. (Hrsg.) (2010): Integration und Inklusion auf dem Weg ins Gemeinwesen. Möglichkeitsräume und Perspektiven. Bad Heilbrunn: Klinkhardt

Steiner-Löffler, U. (2008): Die Lernende Schule. Arbeitsbuch pädagogische Schulentwicklung. Weinheim, Basel: Beltz Verlag-Verlag, 208-232

Steinert, W.W. (2010): Waldhofschule Templin - Eine Schule für alle. Von der Integration auf dem Weg zur inklusiven Schule. In: Wernstedt, R.; John-Ohnesorg, M. (Hrsg.) (2010): Inklusive Bildung. Die UN-Konvention und ibre Folgen. Berlin: Friedrich-Ebert-Stiftung, 75-80

Steinke, I. (2004): Gütekriterien qualitativer Forschung. In: Flick, U.; von Kardorff, E.; Steinke, I. (Hrsg.) (2004, 3. Aufl.): Qualitative Forschung. Ein Handbuch. Weinheim: Juventa, 319-331

Stichweh, R. (1997):Inklusion/Exklusion, funktionale Differenzierung und die Theorie der Weltgesellschaft. Unter: https:// nbn-resolving.org/urn:nbn:de:0168-ssoar-138363; Stand: 06.01.2018

Stiller, E. (2008): Unterrichtsentwicklung neu denken. Überlegungen aus salutogenetischer Perspektive. In: Brägger, G.; Israel, G.; Posse, N. (Hrsg.) (2008): Bildung und Gesundheit. Argumente für gute und gesunde Schulen. Bern: h.e.pVerlag. Unter: https://www.iqesonline.net/download.cfm?id=D974921B-E0C6-B4E6-2305-4DF115342ED0; Stand: 03.12.2012

Stoellger, N. (1988): Annäherung an eine integrative Schule. In: Projektgruppe Integrationsversuch (Hrsg.) (1988): Das Fläming-Modell. Gemeinsamer Unterricht für behinderte und nichtbehinderte Kinder an der Grundschule. Weinheim und Basel: Beltz Verlag, 11-23

Tenorth, H.-E. (1994): „Alle alles zu lehren“. Möglichkeiten und Perspektiven allgemeiner Bildung. Darmstadt: Wissenschaftliche Buchgesellschaft

Tenorth, H.-E. (2006): Bildsamkeit und Behinderung - Anspruch, Wirksamkeit und Selbstdestruktion einer Idee. In: Raphael, L.; Tenorth, H.-E. (Hrsg.): Ideen als gesellschaftliche Gestaltungskraft im Europa der Neuzeit. Beiträge für eine erneuerte Geistesgeschichte. München: DeGruyter, S. 497-520.

Tent, L.; Witt, M.; Zschosche-Lieberum, C.; Bürger, W. (1991): Über die pädagogische Wirksamkeit der Schule für Lernbehinderte. In: Zeitschrift für Heilpädagogik, 42. Jg., Heft 5, 289-320

Terhart, E. (1994). Unterricht. In: Lenzen, D. (Hrsg.): Erziehungswissenschaft. Ein Grundkurs. Reinbek: Rowohlt, S. 133-158. Reinbek: Rowohlt

Terhart, E. (2001): Lehrerberuf und Lehrerbildung. Forschungsbefunde, Problemanalysen, Reformkonzepte. Weinheim und Basel: Beltz Verlag

Terhart, E. (2006): Kooperation im Lehrerberuf: Forschungsproblem und Gestaltungsaufgabe. In: Zeitschrift für Pädagogik 2, 163-166

Terhart, E. (2009): Didaktik - Eine Einführung. Stuttgart: Reclam

Terhart, E. (2010): Schulentwicklung und Lehrerprofessionalität. In: Bohl, T.; Helsper, W.; Holtappels, H.G.; Schelle, C. (Hrsg.) (2010): Handbuch Schulentwicklung. Bad Heilbrunn: Klinkhardt, 237 - 241

Textor, A. (2008): „Der lenkt uns ab und sagt Ausdrücke - aber wir hören nicht drauf." Ergebnisse zur Integration von Schülern mit dem Förderschwerpunkt emotionale und soziale Entwicklung aus einem Berliner Forschungsprojekt. In: Heilpädagogik online, 2, 85-115. Unter: https://sonderpaedagoge.quibbling.de/hpo/2008/heilpaedagogik_online_0208.pdf. Stand: 14.03.2013

Textor, A. (2009); Nötig und möglich - Gemeinsamer Unterricht mit Schülern mit dem Förderschwerpunkt emotionale und soziale Entwicklung. In: Börner, S.; Glink, A.; Jäpelt, B.; Sanders, D.; Sasse, A. (Hrsg.) (2009): Integration im vierten Jahrzehnt. Bilanz und Perspektiven. Bad Heilbrunn: Klinkhardt, 113-122

Textor, A. (2014): Die Inklusion von Schülern und Schülerinnen mit dem Förderschwerpunkt emotionale und soziale Entwicklung aus der Sicht von Mitschülern. In: Schulpädagogik heute, Inklusion in Schule und Unterricht, H. 10 (2014), 5. Jahrgang, 1-15

Textor, A. (2015): Einfübrung in die Inklusionspädagogik. Bad Heilbrunn: Klinkhardt Verlag

Thiele, A. (2009): Körperbehinderung: Erziehung und Bildung im Grundschulbereich: Allgemeine Schule, In: Theunissen, G.; Opp, G. (Hrsg.) (2009): Handbuch der schulischen Sonderpädagogik. Bad Heilbrunn: Klinkhardt, $149-153$

Trautmann, M.; Wischer, B. (2011): Heterogenität in der Schule. Eine kritische Einführung. Wiesbaden: Springer VS

Trumpa, S.; Seifried, S.; Franz, E.-K. (2014) (Hrsg.): Inklusive Bildung: Erkenntnisse und Konzepte aus Fachdidaktik und Sonderpädagogik. Weinheim und Basel: Beltz Verlag Juventa 
UNESCO (1994): Die Salamanca Erklärung und der Aktionsrahmen zur Pädagogik für besondere Bedürfnisse angenommen von der Weltkonferenz „Pädagogik für besondere Bedürfnisse: Zugang und Qualität" Salamanca, Spanien, 7.-10. Juni 1994; United Nations Standard Rules on the Equalization of Opportunities for Persons with Disabilities, A/ RES/48/96, Resolution der Vereinten Nationen, angenommen von der Generalversammlug bei ihrer 48. Sitzung am 20. Dezember 1993. Unter: https://www.unesco.de/fileadmin/medien/Dokumente/Bibliothek/salamanca-erklaerung.pdf; Stand: 04.10 .2017

United Nations (2006): Convention on the Rights of Persons with Disabilities. Unter: http://www.un.org/disabilities/ documents/ convention/crpd_english.doc; Stand: 04.10.2017

Unterleitner, I. (1990): Sozial-integrative Schule: Leistungen der nichtbehinderten Kinder und Einstellungen ihrer Eltern. In: Zeitschrift BEHINDERTE in Familie, Schule und Gesellschaft, 13, Heft 2, 9-16

Venetz, M.; Tarnutzer, R. (2012): Schulisches Integriertsein und Befinden im Unterricht. Vergleichende Analysen von Lernenden mit und ohne besonderen Förderbedarf in integrativen Schulen. In: Lanfranchi, A.; Steppacher, J. (Hrsg.) (2012): Schulische Integration gelingt. Gute Praxis wabrnehmen, Neues entwickeln. Bad Heilbrunn: Verlag Julius Klinkhardt, 103-118

Verband für Bildung und Erziehung (VBE) (2010): Verpflichtung zur Inklusion gilt seit einem Jahr. Unter: https://www. vbe.de/presse/2010/verpflichtung-zur-inklusion-gilt-seit-einem-jahr/?L=0; Stand: 20.02 .2013

Verband deutscher Hilfsschulen (1955): Denkschrift zu dem Ausbau des Heilpädagogischen Sonderschulwesens. In: Zeitschrift für Heilpädagogik, 6, 1955, 3-9

Verband Sonderpädagogik e.V. (1970): Entschließung des Bundesausschusses v. 14.11.1970 zum Verhältnis Sonderschule - Gesamtschule. In: Zeitschrift für Heilpädagogik, 12. Jg., 1970, 670f

Verband Sonderpädagogik e.V. (1975): Stellungnahme zur Empfehlung der Bildungskommission des Deutschen Bildungsrates „Zur pädagogischen Förderung behinderter und von Behinderung bedrohter Kinder und Jugendlicher“, In: Zeitschrift für Heilpädagogik 26 (1975), S. 662-665

Verband Sonderpädagogik e.V. (1997): Sonderpädagogische Förderzentren. Entwicklungen und Perspektiven. Positionen. Würzburg: Vds

Verband Sonderpädagogik e.V. (2008): Standards der sonderpädagogischen Förderung - verabschiedet auf der Hauptversammlung 2007 in Potsdam. In: Zeitschrift für Heilpädagogik 59, 42-64

Verband Sonderpädagogik e.V. Bundesverband (2010): Positionspapier zur inklusiven Bildung, Würzburg. Bundesweites Handlungskonzept inklusive Bildung auf der Grundlage des Vds-Positionspapiers", Unter: http://www.verband-sonderpaedagogik.de/con/cms/ front_content.php; Stand: 18.03.2012

Vester, F. (1999): Die Kunst vernetzt zu denken. Ideen und Werkzeuge für einen neuen Umgang mit Komplexität. München: Deutscher Taschenbuch Verlag

Vester, F. (2002, akt. und erw. Ausgabe): Die Kunst vernetzt zu denken. Ideen und Werkzeuge für einen neuen Umgangmit Komplexität. München: Deutscher Taschenbuch Verlag

Vester, F. (2006): Die ständische Kanalisierung der Bildungschancen. Bildung und soziale Ungleichheit zwischen Boudon und Bourdieu. In: Georg, W. (Hrsg.): Soziale Ungleichheit im Bildungssystem. Eine empirisch-theoretische Bestandsaufnabme. Konstanz: UVK, 13-54

Wagner, M. (2013): Sind sie der Rest? Kinder und Jugendliche mit schwerer Behinderung in einem inklusiven Schulsystem. In: Zeitschrift für Heilpädagogik 64, 12, 496-501

Wansing, G. (2005): Teilhabe an der Gesellschaft. Menschen mit Behinderung zwischen Inklusion und Exklusion. Wiesbaden: Springer VS

Wartha, S.; Schulz, A. (2013, 2. Aufl.): Rechenproblemen vorbeugen. Berlin: Cornelsen

Watkins, C.A.; Slocum, T.A. (2003): The components of direct instruction. In: Journal of direct instruction, 2003, $75-110$

Weigt, M. (Hrsg.) (1977): Schulische Integration von Behinderten. Beiträge zum Verhältnis von Sonderschulen zum Regelschulsystem. Weinheim und Basel: Beltz Verlag

Weiner, J. (2009): Die deutsche Bildungsreform. Unter: http://www.deutschlandfunk.de/die-deutsche-bildungsreform.1184.de.html?dram:article_id=185315; Stand: 04.04.2015

Weinert, F.E. (1997): Notwendige Methodenvielfalt. Unterschiedliche Lernfähigkeiten erfordern variable Unterrichtsmethoden. In: Friedrich Jahresheft 1997: Lernmethoden - Lehrmethoden - Wege zur Selbständigkeit. Velber, 50-52

Weinert, F.E. (2001): Qualifikation und Unterricht zwischen gesellschaftlichen Notwendigkeiten, pädagogischen Visionen und psychologischen Möglichkeiten. In: Melzer, W.; Sandfuchs, U. (Hrsg.) (2001): Was Schule leistet. Funktionen und Aufgaben von Schulen. Weinheim: Juventa, 65-85

Wellendorf, F. (1967): Teamarbeit in der Schule. In: Die Deutsche Schule 59 (1967), 518-528

Wember, F.B. (2001): Adaptiver Unterricht. In: Sonderpädagogik, 31. Jg, 3/2001, 161-181 
Wember, F.B. (2009): Individuelle Förderung - Kern der sonderpädagogischen Förderung und zentrales Instrument der Qualitätssicherung. In: Wember, F.B.; Prändl, S. (Hrsg.) (2009): Standards der sonderpädagogischen Förderung. München, Basel: Ernst Reinhardt Verlag, 89-108

Wember, F.B.; Prändl, S. (Hrsg.) (2009): Standards der sonderpädagogischen Förderung. München, Basel: Ernst Reinhardt Verlag

Wenning, N. (2013): Die Rede von der Heterogenität. Mode oder Symptom? In: Budde, J. (Hrsg.) (2013): Unscharfe Einsätze: (Re-) Produktion von Heterogenität im schulischen Feld. Wiesbaden: Springer Fachmedien

Werning, R.; Arndt, A.K. (Hrsg.) (2013): Inklusion: Kooperation und Unterricht entwickeln. Bad Heilbrunn: Klinkhardt

Werning, R.; Lohse, S. (2011): Gutachten Kompetenzzentren für sonderpädagogische Förderung im Bereich der Lern- und Entwicklungsstörungen in Nordrhein-Westfalen. Hannover: Leibniz-Universität

Werning, R. (2013): Inklusive Pädagogik - Eine Herausforderung für die Schulentwicklung. In: Klauß, Th.; Terfloth, K. (Hrsg.) (2013): Besser gemeinsam lernen! Inklusive Schulentwicklung. Heidelberg: Universitätsverlag Winter; 17-28

Wernstedt, R.; John-Ohnesorg, M. (Hrsg.) (2010): Inklusive Bildung. Die UN-Konvention und ibre Folgen. Berlin: Friedrich-Ebert-Stiftung

Wiechmann, J. (2004): Das Methodenrepertoirevon Lehrern - ein aktualisiertesBild. In: Wosnitza, M.; Frey, A.; Jäger, R.S. (Hrsg.): Lernprozess, Lernumgebung und Lerndiagnostik. Landau: VEP, 320-335

Wiechmann (2006, 4. Aufl.): Zwölf Unterrichtsmethoden. Vielfalt für die Praxis. Weinheim und Basel: Beltz Verlag

Wiechmann (2010, 5. Aufl.): Zwölf Unterrichtsmethoden. Vielfalt für die Praxis. Weinheim und Basel: Beltz Verlag

Wild, E.; Lütje-Klose, B. (2017): Schulische Elternarbeit als essenzielles Gestaltungsmoment inklusiver Beschulung. In: Lütje-Klose, B.; Miller, S.; Schwab, S.; Streese, B. (Hrsg.) (2017): Inklusion: Profile für die Schul-und Unterrichtsentwicklung in Deutschland, Österreich und der Schweiz. Theoretische Grundlagen - Empirische Befunde - Praxisbeispiele. Münster, New York: Waxmann, 129-139

Wildemann, A. (2010): Lesen und Schreiben erfolgreich unterrichten: Wege im sprachlichen Anfangsunterricht. München: Oldenbourg Fortbildung

Willmann, M. (2007). Die Schule für Erziehungshilfe/Schule mit dem Förderschwerpunkt Emotionale und Soziale Entwicklung: Organisationsformen, Prinzipien, Konzeptionen. In Reiser, H., Willmann, M. \& Urban, M. (Hrsg.) (2007): Sonderpädagogische Unterstützungssysteme bei Verhaltensproblemen in der Schule - Innovationen im Förderschwerpunkt Emotionale und Soziale Entwicklung. Bad Heilbrunn: Klinkhardt, 13-69

Winkel, R. (1974): Theorie und Praxis des Team-Teaching. Braunschweig: Westermann

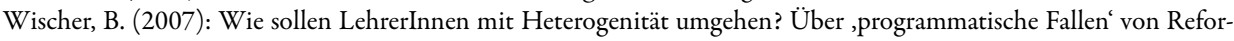
merwartungen im aktuellen Diskurs. In: Die Deutsche Schule, 99, 2007,4, 422-433

Wischer, B. (2013): Konstruktionsbedingungen von Heterogenität im Kontext organisierter Lernprozesse. Eine schulund organisationstheoretische Problemskizze. In: Budde, J. (Hrsg.) (2013): Unscharfe Einsätze: (Reproduktion von) Heterogenität im schulischen Feld. Wiesbaden: Springer, 99-126

Wocken, H.; Antor, G. (Hrsg.) (1987): Integrationsklassen in Hamburg. Erfahrungen, Untersuchungen, Anregungen. Oberbiel-Solms: Jarick

Wocken, H. (1987): Eltern und schulische Integration. In: Wocken, H.; Antor, G. (Hrsg.) (1987): Integrationsklassen in Hamburg. Erfahrungen - Untersuchungen - Anregungen. Solms-Oberbiel: Jarick-Oberbiel, 125-202

Wocken, H. (1987): Integrationsklassen in Hamburg. In: Wocken, H.; Antor, G. (Hrsg.) (1987): Integrationsklassen in Hamburg. Erfahrungen - Untersuchungen - Anregungen. Solms-Oberbiel: Jarick-Oberbiel, 65-89

Wocken, H. (1987): Schulleistungen in Integrationsklassen. In: Wocken, H.; Antor, G. (Hrsg.) (1987): Integrationsklassen in Hamburg. Erfahrungen - Untersuchungen - Anregungen. Solms-Oberbiel: Jarick-Oberbiel, 276-306

Wocken, H. (2005): Andere Länder, andere Schüler? Vergleichende Untersuchung von Förderschülern in den Bundesländern Brandenburg, Hamburg und Niedersachsen. Unter: http://bidok.uibk. ac.at/download/wocken-forschungsbericht.pdf; Stand: 27.08.2012

Wocken, H. (2009b): Von der Integration zur Inklusion. Ein Spickzettel für Inklusion. In: Gemeinsam leben. Zeitschrift für integrative Erziehung. 2009, 4, 216-219

Wocken, H. (2012): Qualitätsstufen der Behindertenpolitik und -pädagogik. Unter: https://www.ev-akademie-boll.de/ fileadmin/res/otg/501909-Wocken.pdf; Stand: 02.05.2018

Wocken, H. (2014): „Inklusive Missverständnisse?“ Einspruch gegen Falschmeldungen über Inklusion. In: magazinauswege.de, 28.11.2014; Unter: http://www.magazin-auswege.de/ data/2014/11/Wocken_Replik_Speck_Sueddeutsche-Zeitung.pdf; Stand: 13.10.2015

Wocken, H. (2018): Das Scheitern der Pseudo-Inklusion. Unter: https://bildungsklick.de/schule/meldung/das-scheitern-der-pseudo-inklusion/; Stand: 13.01.2019

Woolfolk, A. (2008): Pädagogische Psychologie. München: Pearson Studium

Wunder, R. (2000): Führung und Zusammenarbeit. Eine unternehmerische Führungslehre. Neuwied: Luchterhand 
Wyosnick, C.R. (1987): Der multidisziplinäre Ansatz. Integrationskonzept an einer amerikanischen High School. In: Mutzeck, W.; Pallasch, W. (Hrsg.) (1987): Integration von Schülern mit Verhaltensstörungen. Praktische Modelle und Versuche. Weinheim: Deutscher Studien Verlag, 188-205

Zech, R. (2009): Latente Regeln des Funktionierens der Organisation Schule. In: Bartz, A. u.a.: PraxisWissen SchulLeitung 2570.15. Basiswissen und Arbeitshilfen zu zentralen Handlungsfeldern der Schulleitung. Köln: Wolters Kluwer, 1-9

Zech, R. (2013): Organisation, Individuum, Beratung: systemtheoretische Reflexion. Göttingen: Vandenhoeck \& Ruprecht

Zick, A.; Klein, A. (2014): Fragile Mitte. Feindselige Zustände. Rechtsextreme Einstellungen in Deutschland 2014. (hrsg. von RalfMelzer für die Friedrich-Ebert-Stiftung). Berlin: Dietz

Ziemen, K.; Langner, A.; Köpfer, A.; Erbring, S. (Hrsg.) (2011): Inklusion - Herausforderungen, Chancen und Perspektiven. Hamburg: Verlag Dr. Kovac

Ziemen, K. (2011): Inklusion und „kulturhistorisches Denken“. In: Ziemen, K.; Langner, A.; Köpfer, A.; Erbring, S. (Hrsg.) (2011): Inklusion - Herausforderungen, Chancen und Perspektiven. Hamburg: Verlag Dr. Kovac, 9-19

Zinnecker, J.; Stecher, L. (2006): Gesellschaftliche Ungleichheit im Spiegel hierarchisch geordneter Bildungsgänge. Die Bedeutung ökonomischen, kulturellen und ethnischen Kapitals der Familie für den Schulbesuch der Kinder. In: Georg, W. (Hrsg.) (2006): Soziale Ungleichheit im Bildungssystem. Eine empirisch-theoretische Bestandsaufnahme. Konstanz: UVK, 291-310 


\section{Abbildungsverzeichnis}

Abb. 1: Grafische Darstellung des Aufbaus der Dissertation .......................... 17

Abb. 2: Fördersystem eines kooperativen Schulzentrums (in Anlehnung an

Deutscher Bildungsrat, 1973, S. 79) ................................ 28

Abb. 3: Übersicht über das Kategoriensystem für schulische Integration $\ldots \ldots \ldots \ldots \ldots \ldots \ldots \ldots . \quad 94$

Abb. 4: Übersicht über das Kategoriensystem schulische Integration mit Subkategorien ......... 217

Abb. 5: Phasen des Forschungsprojekts ......................................... 234

Abb. 6: Qualitätskategorien und -subkategorien im Bereich der Organisationsentwicklung ....... 313

Abb. 7: Qualitätskategorien und -subkategorien im Bereich der Personalentwicklung ........... 314

Abb. 8: Qualitätskategorien und -subkategorien im Bereich der Unterrichtsentwicklung ........ 315

Abb. 9: Wohlbefinden der SuS mit spF in den Regelschulen ......................... 328

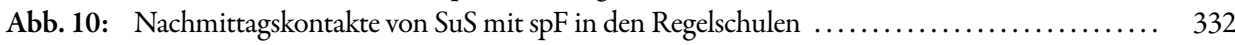

Abb. 11: SuS mit spF werden durch die Lehrkräfte der Regelschulen akzeptiert .............. 332

Abb. 12: Häufigkeit der gemeinsamen Unterrichtsvorbereitung von RSL/FSL $\ldots \ldots \ldots \ldots \ldots \ldots \ldots . \ldots \ldots 2$

Abb. 13: Häufigkeit der gemeinsamen Unterrichtsdurchführung von RSL/FSL $\ldots \ldots \ldots \ldots \ldots \ldots . . \ldots 52$

Abb. 14: Häufigkeit der gemeinsamen Unterrichtsreflexion von RSL/FSL . . . . . . . . . . . . 353

Abb. 15: Sicherheit der RSL im Umgang mit Verhaltensauffälligkeiten ..................... 371

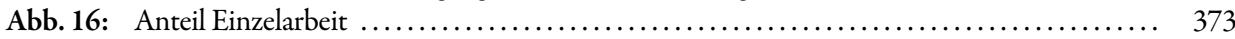

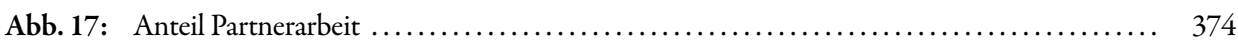

Abb. 18: Anteil Gruppenarbeit ............................................ 375

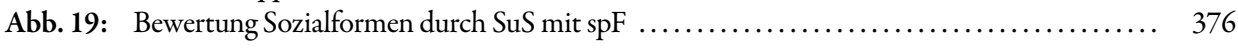

Abb. 20: Qualitätskategorien und -subkategorien im Bereich der

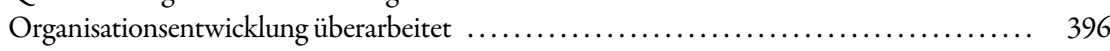

Abb. 21: Qualitätskategorien und -subkategorien im Bereich der Personalentwicklung überarbeitet ........................................................ 403

Abb. 22: Qualitätskategorien und -subkategorien im Bereich der Unterrichtsentwicklung überarbeitet ............................... 407

Abb. 23: Beispiel für Zusammenhänge Doppelbesetzung/ Co-Teaching ................. 438 


\section{Tabellenverzeichnis}

Tab. 1: Schüler mit sonderpäd. Förderung in allgemeinen Schulen (absolut)

(in Anlehnung an KMK, 2018a, S. 34)

Tab 2: Schüler mit sonderpäd. Förderung in allg. Schulen, Verteilung nach

Förderschwerpunkten in \% (in Anlehnung an KMK, 2018a, S. 34)

Tab. 3: Vergleich Schüler mit sonderpäd. Förderbedarf in allg. Schulen und in Förderschulen, Quoten und Anteile (in Anlehnung an Klemm, 2018, S. 7; Berechnungen durch Klemm auf der Grundlage von KMK. 2016 und KMK, 2018a)

Tab. 4: Förder-/Exklusions-/Inklusionsquoten und Inklusionsanteile in den verschiedenen Bundesländern (Schuljahr 2016/17) (in Anlehnung an Klemm, 2018, S. 18, auf der Basis von KMK, 2016 und KMK 2018a

Tab. 5: Spannungsverhältnis zwischen Institutionalisierung und Individualisierung . . . . . . . . . .

Tab. 6: Qualitätsmerkmale guten Unterrichts (erstellt durch Verf.)

Tab. 7: Einsatz von Unterrichtsmethoden (Anteile in Prozent) monatlich

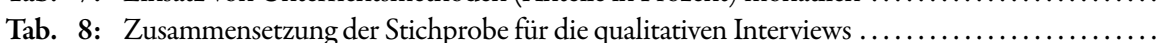

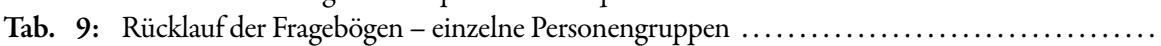

Tab. 10: Dauer der Erfahrung mit Unterrichtung von SuS mit spF SL $\ldots \ldots \ldots \ldots \ldots \ldots \ldots \ldots \ldots \ldots$

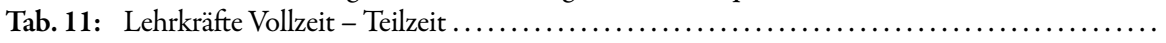

Tab. 12: Dauer der Erfahrung mit Unterrichtung von SuS mit spF RSL $\ldots \ldots \ldots \ldots \ldots \ldots \ldots \ldots \ldots$

Tab. 13: Tätigkeit der RSL und FSL in verschiedenen Klassenstufen . .......................

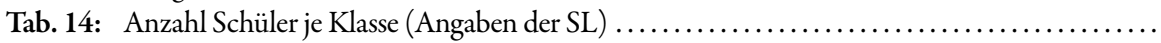

Tab. 15: SuS nach Förderbedarfen - Antworten der Schulleitungen GS ......................

Tab. 16: SuS nach Förderbedarfen - Antworten der Schulleitungen GMS . .....................

Tab. 17: Dauer der sonderpäd. Förderung Sohn/Tocher (Angaben der Eltern) . . . . . . . . . . . . .

Tab. 18: Verteilung der SuS mit spF auf die verschiedenen Klassenstufen . . . . . . . . . . . . . . . .

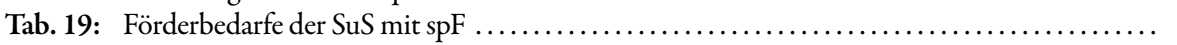

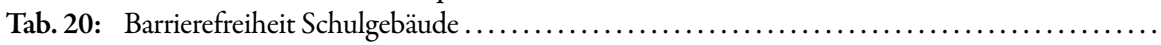

Tab. 21: Anzahl der SuS mit spF körperlich-motorische Entwicklung im Verhältnis zur Anzahl der Schulen (Angaben der SL)

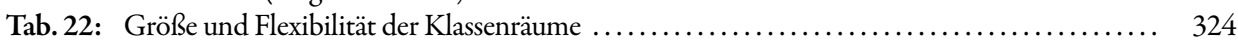

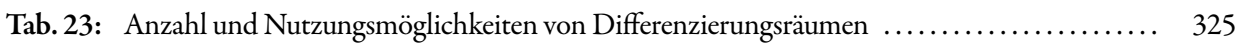

Tab. 24: Differenzierungsmaterial ............................................ 326

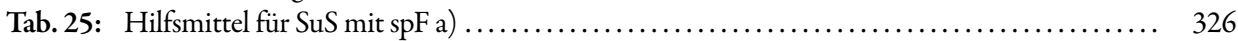

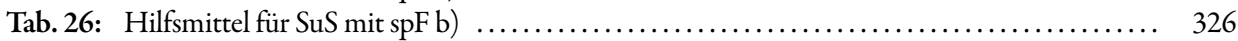

Tab. 27: Offenheit der Schulleitung für die Integration von $\mathrm{SuS}$ mit spF $\ldots \ldots \ldots \ldots \ldots \ldots \ldots \ldots \ldots \ldots \ldots \ldots \ldots$

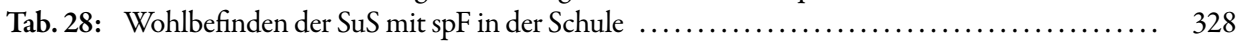

Tab. 29: Einschätzung der sozialen Beziehungen der SuS an der Schule untereinander ........... 330

Tab. 30: Verbesserung des sozialen Miteinanders der $\mathrm{SuS}$ in der Schule durch die integrative

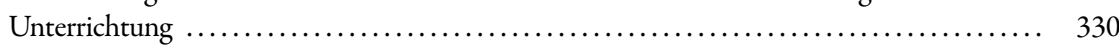

Tab. 31: Akzeptanz der SuS mit spF durch andere SuS . . . . . . . . . . . . . . 331

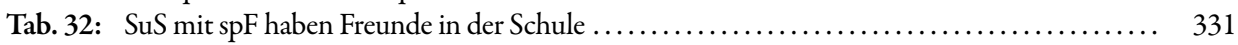

Tab. 33: Nachmittagskontakte der SuS mit spF ................................. 331

Tab. 34: SuS mit spF werden durch die Lehrkräfte der Regelschulen akzeptiert ............... 332

Tab. 35: Das Elternwahlrecht sollte erhalten bleiben .............................. 333

Tab. 36: Bewertung der Zusammenarbeit zwischen Eltern und SL (Angaben Eltern) ........... 333

Tab. 37: Bewertung der Zusammenarbeit zwischen Eltern und Klassenleitung (Angaben Eltern) ... 334

Tab. 38: Bewertung der Zusammenarbeit zwischen Eltern und FSL (Angaben Eltern) . .......... 334

Tab. 39: SL - Formen der Zusammenarbeit mit Eltern ............................. 335

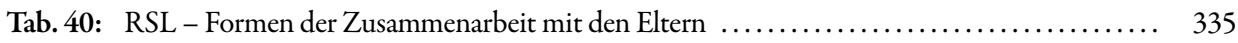

Tab. 41: FSL - Formen der Zusammenarbeit mit den Eltern ......................... 335 


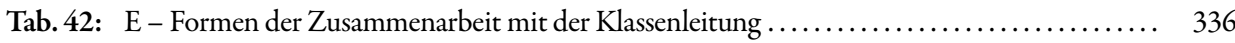

Tab. 43: E - Formen der Zusammenarbeit mit den Eltern - FSL . . . . . . . . . . . . . . . . . 336

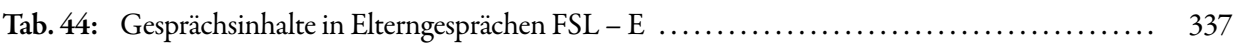

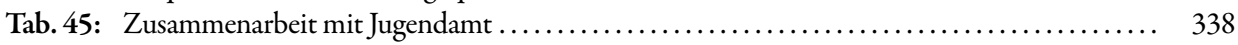

Tab. 46: Zusammenarbeit mit Schulpsychologischem Dienst ........................ 338

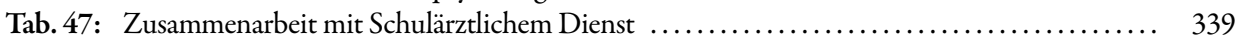

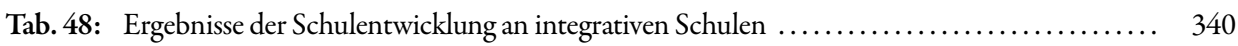

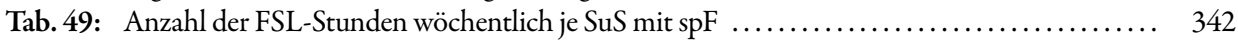

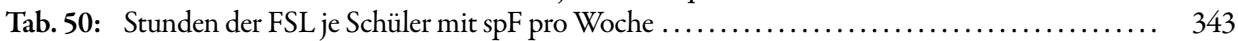

Tab. 51: Einschätzung der Anzahl der FSL-Stunden je SuS/Woche: Ich finde, das ist... .......... 344

Tab. 52: Beratungszeiten zwischen RSL und FSL, die im Stundenplan fest verankert sind ......... 345

Tab. 53: Reduktion der Unterrichtsverpflichtung von RSL in integrativen Klassen .............. 346

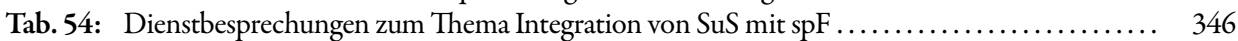

Tab. 55: Gesamtkonferenzen zum Thema Integration von SuS mit spF .................... 346

Tab. 56: Bewertung der Zusammenarbeit (bei FSL mit Blick auf RSL) . ................. 347

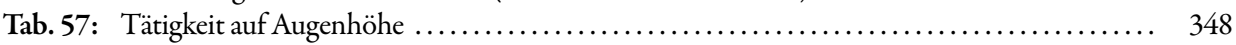

Tab. 58: Kontinuität der Tätigkeit von FSL in Regelschulen trägt zur Akzeptanz der integrativen Unterrichtung bei ....................................... 349

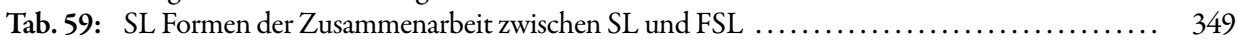

Tab. 60: RSL Formen der Zusammenarbeit mit den FSL . . . . . . . . . . . . . . . . . . . . . 349

Tab. 61: FSL Formen der Zusammenarbeit mit den RSL ........................... 350

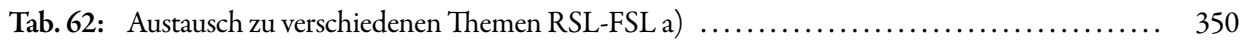

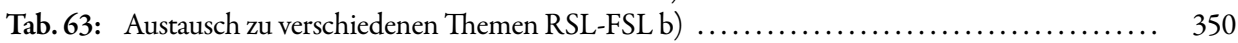

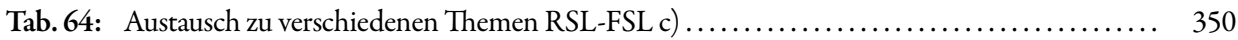

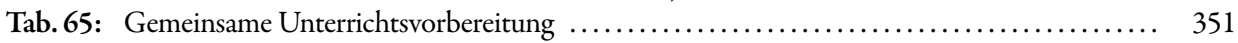

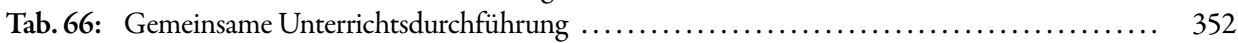

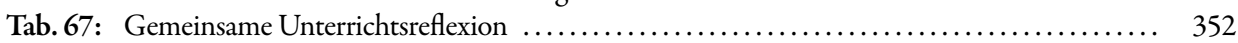

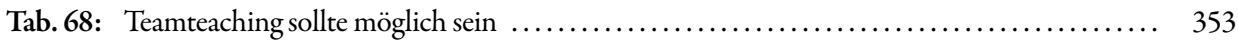

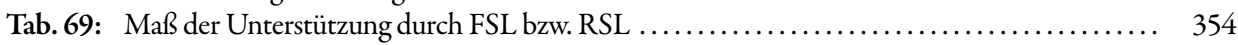

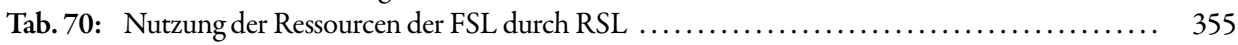

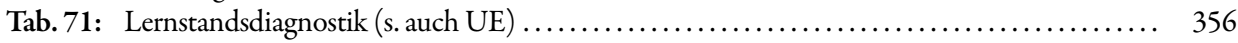

Tab. 72: Individuelle Förderung durch FSL (bei RSL gemeinsam mit FSL) $\ldots \ldots \ldots \ldots \ldots \ldots \ldots \ldots \ldots$

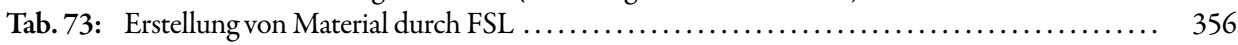

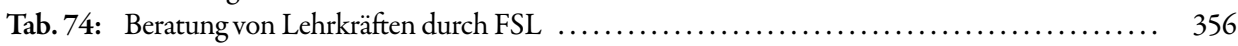

Tab. 75: Vernetzungsarbeit mit außerschulischen Diensten durch FSL

(bei RSL gemeinsam mit FSL).

Tab. 76: Schulische Integration von SuS mit Förderbedarf setzt ein hohes Engagement der

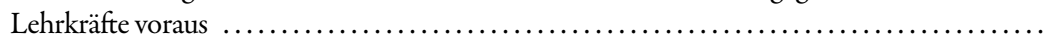

Tab. 77: SCHILF zum Thema Integration von SuS mit spF (alphabetisch sortiert) . ...............

Tab. 78: Pädagogische Tage zum Thema Integration von $\mathrm{SuS}$ mit $\mathrm{spF}$ (alphabetisch sortiert) .........

Tab. 79: Häufigkeit des Besuchs von Fortbildungen zu schulischer Integration ...................

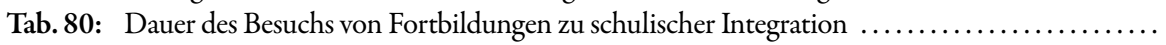

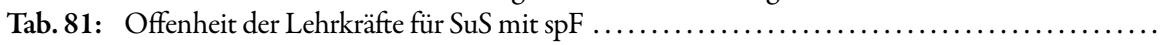

Tab. 82: Maß der Akzeptanz der bisherigen Form der Integration $\ldots \ldots \ldots \ldots \ldots \ldots \ldots \ldots \ldots \ldots$

Tab. 83: Förderung der SuS mit spF durch RSL so gut wie möglich ........................

Tab. 84: Die Haltung der Lehrkräfte in unserer Schule ist der zukünftigen inklusiven Bildung

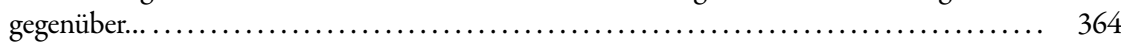

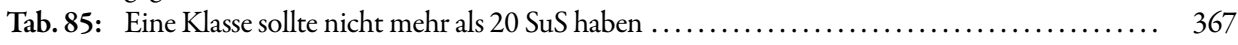

Tab. 86: Es sollte darauf geachtet werden, dass nur drei bis vier $\mathrm{SuS}$ mit Förderbedarf in einer

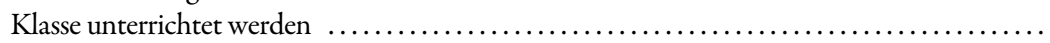

Tab. 87: Wenn möglich sollten in einer Klasse mehrere SuS mit Förderbedarf unterrichtet werden ... 
Tab. 88: In der Regelschule sollten auch SuS mit schweren Behinderungen unterrichtet werden. .... 368

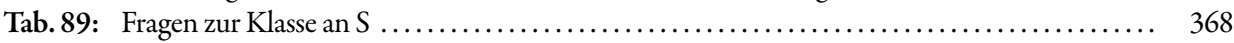

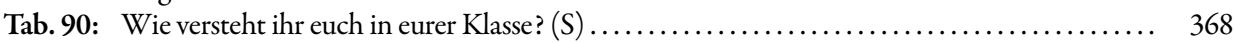

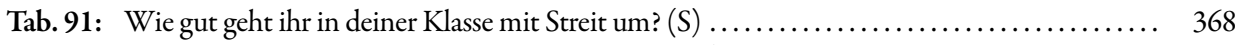

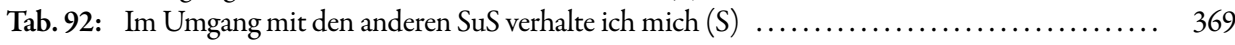

Tab. 93: Wie gut halten sich die Schüler an Regeln? .............................. 369

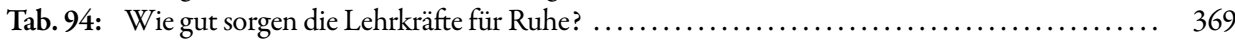

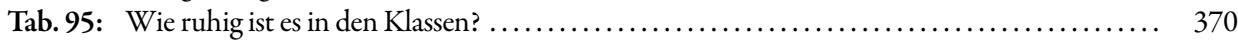

Tab. 96: Wie gut können sich die Schüler in den Klassen konzentrieren? . . . . . . . . . . . . . . 370

Tab. 97: Wie konsequent reagieren die Lehrkräfte auf Fehlverhalten? .................... 371

Tab. 98: Wie sicher fühlen sich die Lehrkräfte im Umgang mit Verhaltensauffälligkeiten von SuS? ... 371

Tab. 99: Wie hoch ist das Maß an klarer Strukturierung des Unterrichts? .................. 372 


\section{Abkürzungsverzeichnis}

\begin{tabular}{|c|c|}
\hline Abb. & Abbildung \\
\hline Abs. & Absatz \\
\hline AK & Arbeitskammer \\
\hline Art. & Artikel \\
\hline AVIVA & Ankommen und Einstimmen, Vorwissen aktivieren, Informieren, Verarbeiten, Auswerten \\
\hline BDA & Bundesvereinigung der deutschen Arbeitgeber \\
\hline BDI & Bund der deutschen Industrie \\
\hline BIJU & Bildungsverläufe und psychosoziale Entwicklung im Jugend- und jungen Erwachsenenalter \\
\hline BiLief & $\begin{array}{l}\text { Bielefelder Längsschnittstudie zum Lernen in inklusiven und exklusiven Förderarrange- } \\
\text { ments }\end{array}$ \\
\hline BGG & Bundesgesetzbuch \\
\hline BMAS & Bundesministerium für Arbeit und Soziales \\
\hline BRD & Bundesrepublik Deutschland \\
\hline BRK & Behindertenrechtskonvention \\
\hline CFT 1 & Grundintelligenztest Skala 1, Hogrefe Verlag \\
\hline DESI & Deutsch-Englisch-Schülerleistungen-International \\
\hline DIMDI & Deutsches Institut für Medizinische Dokumentation und Information \\
\hline DIN & Deutsches Institut für Normierung e.V. E \\
\hline EN & europäische Norm \\
\hline ISO & International Organization for Standardization \\
\hline ERINA & $\begin{array}{l}\text { Erprobung von Ansätzen zur inklusiven Beschulung von Schülern mit sonderpädagogi- } \\
\text { schem Förderbedarf in Modellregionen in Sachsen }\end{array}$ \\
\hline etc. & etcetera \\
\hline EU & Europäische Union \\
\hline FLEX & flexible Eingangsphase \\
\hline FSL & Förderschullehrkraft \\
\hline G & geistige Entwicklung \\
\hline GeSchwind & $\begin{array}{l}\text { Gelingensbedingungen des gemeinsamen Unterrichts an Schwerpunktschulen in Rhein- } \\
\text { land-Pfalz }\end{array}$ \\
\hline GEW & Gewerkschaft Erziehung und Wissenschaft \\
\hline GG & Grundgesetz \\
\hline ggf. & gegebenenfalls \\
\hline GGG & Gemeinnützige Gesellschaft Gesamtschule \\
\hline GMS & Gemeinschaftsschule \\
\hline GS & Grundschule \\
\hline HmbSG & Hamburger Schulgesetz \\
\hline ICF & International Classification of Diseases/Internationale Klassifikation von Krankheiten \\
\hline IGLU & Internationale Grundschul-Lese-Untersuchung \\
\hline InkVO & Inklusionsverordnung \\
\hline IQB & $\begin{array}{l}\text { Studie des Instituts zur Qualitätsentwicklung im Bildungswesen, Humboldt-Universität, } \\
\text { Berlin }\end{array}$ \\
\hline IVO & Integrationsverordnung \\
\hline KMK & Ständige Konferenz der Kultusminister/Kultusministerkonferenz \\
\hline KonnexAG & Konnexitätsausführungsgesetz \\
\hline KOSA & Kooperation von Schule mit außerschulischen Akteuren \\
\hline $\mathrm{L}$ & Lernen \\
\hline MARKUS & $\begin{array}{l}\text { Mathematik-Gesamterhebung Rheinland-Pfalz: Kompetenzen, Unterrichtsmerkmale, } \\
\text { Schulkontext }\end{array}$ \\
\hline MLL & Verein „Miteinander leben lernen“ e.V. \\
\hline
\end{tabular}


NELS National Educational Longitudinal Study

OECD Organisation for Economic Co-operation and Development/ Organisation für wirtschaftliche Zusammenarbeit und Entwicklung

PiNG Pilotprojekt inklusive Grundschule (Brandenburg)

PISA Programme for International Student Assessment

RSL Regelschullehrkraft

$S \quad$ Schüler

SchoG Schulordnungsgesetz

SchulG Schulgesetz

SE soziale und emotionale Entwicklung

SEIS Selbstevaluation in Schulen

SFZ Sonderpädagogisches Förder- und Beratungszentrum

SGB Sozialgesetzbuch

SISSI Soziale Integration, Schulzufriedenheit und Schulleistungen in Klassen mit und ohne Integration von Schülern mit dem Förderschwerpunkt emotionale und soziale Entwicklung

SL Schulleitung

spF sonderpädagogischer Förderbedarf

STAR Student-Teacher-Achievement-RatioStudie

StEG Studie zur Entwicklung von Ganztagsschulen

SuS Schülerinnen und Schüler

Tab. Tabelle

TALIS The OECD Teaching and Learning International Survey

TIMSS Trends in International Mathematics and Science Study

UN United Nations/Vereinte Nationen

UN-BRK Vereinte Nationen - Behindertenrechtskonvention

UNESCO United Nations Educational, Scientific and Cultural Organization/ Organisation der Vereinten Nationen für Bildung, Wissenschaft, Kultur und Kommunikation

VDH Verband deutscher Hilfsschule

VDS/vds Verband Sonderpädagogik (Schreibweise (groß/klein) wurde mehrfach verändert)

vs. versus

VERA Vergleichsarbeiten

ZDF Zweites Deutsches Fernsehen

ZVA Zentrale Vergleichsarbeiten 


\section{Tabellarische Übersicht des Kategoriensystems für schulische Integration}

\begin{tabular}{llc}
\hline Ziffer & Subkategorien/Indikatoren & Anz. \\
\hline & OE_1.1_Ressourcen Organisation & \\
\hline
\end{tabular}

\subsubsection{Ressourcenausstattung allgemein}

gute materielle Bedingungen (Ausstattung) (Bächthold et al., 1990); zusätzliche Investitionen für Integration notwendig (Innerhofer \& Klicpera, 1991); materielle Ausstattung (Feyerer, 1998); großzügige Rahmenbedingungen (Bless, 2003); wirtschaftliche Maßgaben (Speck, 2010)

Im Haushalt sind spezifische Mittel für die Umsetzung der schulischen Integration vorgesehen; sie können Literatur, spez. Lehr- u. Lernmittel vorsehen, sowie andere für die Integration zweckmäßige Ankäufe u. Ausgaben betreffen (Brugger-Paggi, 2003)

Die für den Ankauf spezifischer Lehrmittel für Schüler und Schülerinnen mit Behinderung vorgesehenen Finanzmittel werden ausschließlich für diesen Zweck verwendet (Brugger-Paggi, 2003)

\subsubsection{Barrierefreiheit}

behindertengerechte Ausstattung von Räumlichkeiten (Innerhofer \& Klicpera, 1991); lernanregende und behindertengerechte Gestaltung von Schulraum und Schulgelände (Heyer et al., 1997); Zugänglichkeit für alle (Boban \& Hinz, 2003); behindertengerechte Architektur (Specht et al., 2006); Barrierefreiheit (Wember \& Prändl, 2009); barrierefreie räumliche Gegebenheiten (Speck, 2010)

barrierefreie Umgestaltung ist geplant (Boban \& Hinz, 2003)

Die notwendigen architektonischen Veränderungen werden umgesetzt, bevor die betroffenen Schüler und Schülerinnen die Schule besuchen (Brugger-Paggi, 2003)

Sämtliche Räume der Schule sind auch für Schüler und Schülerinnen mit Körperbehinderung erreichbar (Brugger-Paggi, 2003)

Die Schule ist mit behinderungsspezifischen Einrichtungen ausgestattet (Treppenlift, bzw. Fahrstuhl, Rampen ....) (Brugger-Paggi, 2003)

Behindertengerechte Sanitärausstattung (nach DIN-Norm) (Brugger-Paggi, 2003; Booth \& Ainscow., 2003; Wember \& Prändl, 2009)

Zugänglichkeit für gehörlose Menschen (Boban \& Hinz, 2003)

Zugänglichkeit für blinde und sehbehinderte Menschen (Booth \& Ainscow., 2003)

Das Schulgelände ist barrierefrei (Booth \& Ainscow., 2003)

Schaffen von angemessenen Hör- und Absehbedingungen (Teppichboden im Klassenraum, Filz unter den Stühlen, Fenster im Rücken des hörgeschädigten Schülers, Vermeidung von Störschall) (Leonhardt, 2009); Klassenräume mit guter Raumakustik (Bsp.: Hörbehinderung) (Wember \& Prändl, 2009)

Bei der Ausstattung des Klassenraumes werden behinderungsspezifische Bedürfnisse berücksichtigt (Schallisolierung, Lichteinfall, angepasste Stühle und Bänke ...) (Brugger-Paggi, 2003) Ausstattung des Klassenraums mit höhenverstell- und neigbaren Tischen sowie individuell anpassbaren Stühlen (Wember \& Prändl, 2009)

Nichtelektronische Hilfen (Buchstaben- und Zahlentafeln) (Thiele, 2009)

Computerarbeitsplatz (Thiele, 2009)

Individuell angepasste Computer (Wember \& Prändl, 2009)

Punktschriftmaschine, PC mit Braillezeile (Wember \& Prändl, 2009); Sehbehinderten- oder blindenspezifische Unterrichtsmedien (visuell vereinfachte Karten/Grafiken, Modelle, taktile Grafiken) (Wember \& Prändl, 2009); Material z.B. in Großdruck, Brailleschrift, Hörkassette (Boban \& Hinz, 2003) 


\begin{tabular}{|c|c|c|}
\hline Ziffer & Subkategorien/Indikatoren & Anz. \\
\hline & Blindenarbeitstische (Wember \& Prändl, 2009) & 1 \\
\hline & Microportanlagen & 1 \\
\hline & Stehständer (Thiele, 2009) & 1 \\
\hline & Sandsack (Thiele, 2009) & 1 \\
\hline & Sitzstütze (Thiele, 2009) & 1 \\
\hline & rutschfeste Schreibtischunterlage (Thiele, 2009) & 1 \\
\hline & Möglichkeiten zur Lagerung von Kindern (Thiele, 2009) & 1 \\
\hline & Blattwendegeräte (Thiele, 2009) & 1 \\
\hline & Lernsoftware (Thiele, 2009) & 1 \\
\hline Summe & & 33 \\
\hline
\end{tabular}

\subsubsection{Räumliche Flexibilität}

Größe der Schulzimmer (Speck, 2010); geeignete Gestaltung der Klassenräume, die gleichzeitige Unterrichtung mehrerer Gruppen möglich macht (Innerhofer \& Klicpera, 1991); ausreichendes Raumangebot (PH Steiermark 2011); ausreichend große und für verschiedene unterrichtliche Arbeitsweisen strukturierbare Klassenräume (Wember \& Prändl, 2009)

klare räumliche Strukturierung des Klassenzimmers und schulischen Lernumfelds (Wember \& Prändl, 2009)

Gestaltung der Lernumgebung als Lebens-, Handlungs- und Erfahrungsraum (Wember \& Prändl, 2009)

Räume mit Rückzugsmöglichkeiten (Wember \& Prändl, 2009) 1

ausreichende Arbeitsfläche für jeden Schüler im Klassenraum (Wember \& Prändl, 2009) 1

Summe

\subsubsection{Differenzierungsräume}

unterschiedliche Räume für unterschiedliche Förderzwecke (Heese, 1977); es sind Zusatzräume vorhanden, die für die individuelle Förderung von Schülern u. Schülerinnen, für Kleingruppenarbeiten und als zusätzliche räumliche Ressource für alle Schüler und Schülerinnen verwendet werden (Brugger-Paggi, 2003); mehrere Kleinräume zum individuellen Arbeiten (Innerhofer \& Klicpera, 1991)

einsehbare Gruppenarbeitsräume (Wember \& Prändl, 2009)

Raumangebot für Sport und psychomotorische Förderung (Wember et al., 2009); Räume für Bewegungsangebote (Wember \& Prändl, 2009)

Differenzierungsräume für Wahrnehmungsförderung, therapeutische Angebote und Kleingruppenarbeit (Wember \& Prändl, 2009)

mindestens ein Therapieraum, der auch als Erholungsraum dienen kann (Innerhofer \& Klicpera, 1991)

Besondere Räumlichkeiten für Kinder mit schweren Behinderungen (Snoezelen-Raum, Pflegebetten) (Wember \& Prändl, 2009)

Werkräume für berufsvorbereitenden Unterricht (Wember \& Prändl, 2009) 1

Computer- und Medienräume (Wember \& Prändl, 2009)

Medienzentrum zur Herstellung adaptierter Medien (Schulbuchübertragung in Punktschrift 1 u.ä.) (Wember \& Prändl, 2009) 


\begin{tabular}{lll}
\hline Ziffer & Subkategorien/Indikatoren & Anz.
\end{tabular}

\subsubsection{Differenziertes Material}

Die Klassen sind mit zusätzlichen Lehr- u. Lernmitteln ausgestattet, die differenzierende Angebote u. handlungsorientiertes Arbeiten ermöglichen (Brugger-Paggi, 2003); Bereitstellung von speziellen Unterrichtsmaterialien (Holzinger et al., 2011)

Schulbehörde sollte Sammlung von Unterrichtsmaterialien und -hilfen für integrativen Unterricht anlegen (Innerhofer \& Klicpera, 1991)

Erstellen von Hilfsmitteln durch Lehrer (Boban \& Hinz, 2003; Wernstedt \& Ohnesorg, 2010) (darin Steinert: Leiter der Waldhofschule Templin)

Ausstattung mit Spielzeug und Medien, die Interaktion und Kooperation erleichtern (Mühl, 1987) 1

Medien- und Materialangebot für unterstützte Kommunikation (Wember \& Prändl, 2009) 1

In der Lehrerbibliothek werden Bücher zu integrationsspezifischen Themen geführt (Brugger- 1 Paggi, 2003)

In der Schülerbibliothek finden Schüler und Schülerinnen Bücher, Informationen, Erfahrungsberichte zu behinderungsspezifischen Themen. (Brugger-Paggi, 2003)

Summe

\section{OE_1.2_Professionelles Management}

\subsubsection{Aufgaben der Schulleitung im Zusammenhang mit schulischer Integration}

Koordination und Beratung der Beteiligten

Die Leitung und Koordination des sonderpädagogischen Bereichs ist gut gewährleistet. (???) 1

Organisation der Koordination aller Erziehungspartner (Innerhofer \& Klicpera, 1991) $\quad 1$

Hilfestellung bei der Einschulung eines behinderten Kindes (Innerhofer \& Klicpera, 1991) 1

Elternberatung (Innerhofer \& Klicpera, 1991) $\quad 1$

Beratung der Lehrkräfte (Innerhofer \& Klicpera, 1991) 1

Die Lehrpersonen und weiteren Mitarbeitenden werden in ihrer Arbeit unterstützt und geför- $\quad 1$

dert. In schwierigen Schulsituationen erhalten sie die notwendige Unterstützung. (???)

Summe

\subsubsection{Schul- und Unterrichtsentwicklung}

Die Leitung sorgt dafür, dass inhaltliche Fragen systematisch angegangen werden. (Lienhard- 1 Tuggener et al., 2011)

Ermutigung und Anregung zur Anpassung des Unterrichts an Anforderungen integrativer Be- $\quad 1$ schulung (Innerhofer \& Klicpera, 1991)

stärkere Individualisierung des Unterrichts einfordern (Innerhofer \& Klicpera, 1991) 1

Unterstützung kooperativer Unterrichtsformen (Bsp.: klassenübergreifender Unterricht) (In- 1 nerhofer \& Klicpera, 1991)

Summe

\subsubsection{Fortbildung}

Lehrerfortbildung (Innerhofer \& Klicpera, 1991); Die Weiterbildung der Mitarbeitenden im Hinblick auf die Unterstützung von Lernenden mit Beeinträchtigungen wird unterstützt und gefördert.

spezielle Fortbildungen für Schulleitungen (Innerhofer \& Klicpera, 1991)

Summe

\subsubsection{Unterstützung der Schulleitungen}




\begin{tabular}{llr}
\hline Ziffer & Subkategorien/Indikatoren & Anz. \\
\hline & OE_1.3_Schule als sozialer Raum & \\
\hline
\end{tabular}

\subsubsection{Schulklima}

Pädagogisch besonders gute Schule (Weigt, 1977, darin Heese); Schulklima, Wertschätzung, Akzeptanz (Boban \& Hinz, 2003); Schulklima der Akzeptanz und Wertschätzung (Wember \& Prändl, 2009)

Harmonisches Miteinander (Schley et al., 1992) 1

respektvoller Umgang miteinander (Boban \& Hinz, 2003); Die Mitarbeitenden pflegen einen offenen und wertschätzenden Umgang miteinander und nach außen. (Lienhard-Tuggener et al., 2011)

Wertschätzung der Schüler (Wember \& Prändl, 2009)

respektvoller Umgang mit den Schülern (Ansprache mit Namen) (Boban \& Hinz, 2003)

Die Beziehung der Lehrpersonen und der weiteren Fachpersonen zu den Schülerinnen und 1 Schülern basiert auf Wohlwollen und Verständnis. (Lienhard-Tuggener et al., 2011) respektvoller Umgang der Schüler mit allen Mitarbeitern der Schule (Boban \& Hinz, 2003) 1 Annahme des (behinderten) Kindes (Thiele, 2009) 1 Zufriedenheit der Schüler (Schley et al., 1992) 1 Offene Auseinandersetzung der Schüler (Schley et al., 1992) 1

Die Schülerinnen und Schüler fühlen sich im Unterricht und in den anderen Bereichen des 1 Schulalltags (Pausen, Betreuungszeiten, Schulweg) wohl und sicher. (Lienhard-Tuggener et al., 2011)

Rituale im Schulalltag (Begrüßungen, Verabschiedungen) (Boban \& Hinz, 2003) 1

beim Erstkontakt der Eltern erfolgt ein freundlicher Empfang durch die Schulleitung (Booth 1 \& Ainscow, 2003)

respektvoller Umgang mit den Eltern (Boban \& Hinz, 2003) 1

respektvoller Umgang der Eltern mit den schulischen Mitarbeitern (Boban \& Hinz, 2003) 1

Einstellungen und Mentalitäten im Umfeld der Schule (Speck, 2010) 1

Summe

\subsubsection{Integration als gemeinsame Aufgabe}

Unter den Beteiligten und im Kollegium besteht ein annähernder Konsens, dass eine teilweise oder gemeinsame Unterrichtung von Schülern mit Schulschwierigkeiten in Regelklassen möglich und richtig sei (Bächthold et al., 1990); Integration als Aufgabe für die gesamte Schule (Feyerer, 1998); Alle Lehrpersonen übernehmen gemeinsam Verantwortung auch für Schüler und Schülerinnen mit Behinderung (Brugger-Paggi, 2003); gesamte Schulgemeinschaft fühlt sich dafür verantwortlich die Schule inklusiv zu gestalten (Boban \& Hinz, 2003); Integration als Aufgabe aller Lehrer (Deutsches Schulamt, 2004); Bemühen um ein Arbeitsklima, in dem sich alle Lehrer in gleicher Weise für alle Schüler verantwortlich fühlen (Holzinger et al., 2011);

Aufgeschlossenheit und Vorbereitung aller Beteiligten (Mühl, 1987)

Es gibt in der Schule eine klare gemeinsame Ausrichtung in der Unterstützung von Schülerin- 1 nen und Schülern mit Beeinträchtigungen. (Lienhard-Tuggener et al., 2011)

Offenheit für Aufnahme aller Schüler (Boban \& Hinz, 2003)

Reintegration von Schülern aus Förderschulen (Boban \& Hinz, 2003)

offene Auseinandersetzung mit den Einstellungen zu Grenzen von Inklusion (Bsp.: schwer beeinträchtigte Schüler) (Boban \& Hinz, 2003) 


\begin{tabular}{|c|c|c|}
\hline Ziffer & Subkategorien/Indikatoren & Anz. \\
\hline \multirow[t]{12}{*}{1.3 .3} & Partizipation & \\
\hline & die Schule besitzt Organe für die Zusammenarbeit (Bächthold et al., 1990) & 1 \\
\hline & Partizipation der Lehrkräfte & \\
\hline & $\begin{array}{l}\text { Mitverantwortung und Entscheidungskompetenzen des Kollegiums bei der Gestaltung der } \\
\text { Schule (Bächthold et al., 1990) }\end{array}$ & 1 \\
\hline & Partizipation der Schüler & \\
\hline & Infoveranstaltungen für Schüler (Mühl, 1987) & 1 \\
\hline & Einbeziehung der Schüler in Schulentwicklungsprozesse (Boban \& Hinz, 2003) & 1 \\
\hline & Schülerforum (Boban \& Hinz, 2003) & 1 \\
\hline & Partizipation der Eltern & \\
\hline & $\begin{array}{l}\text { Eltern von Schülern und Schülerinnen mit Behinderung sind in verschiedenen Gremien auf } \\
\text { Schulebene vertreten (Elternvertreter im Klassenrat, Schulrat) (Brugger-Paggi, 2003) }\end{array}$ & 1 \\
\hline & $\begin{array}{l}\text { Informationsveranstaltungen für Eltern (Hug, 1994b); Die Schule informiert Eltern regelmäßig } \\
\text { über Themen der gesamten Institution. (Lienhard-Tuggener et al., 2011, S. 165); Eltern werden } \\
\text { rechtzeitig und regelmäßig informiert (Bless, 2003); (zum Beispiel über Schulprogramm) }\end{array}$ & 2 \\
\hline & $\begin{array}{l}\text { Die Eltern sind in die Schulentwicklung einbezogen (Bsp.: Erstellung Schulprogramm) (Boban } \\
\& \text { Hinz, 2003) }\end{array}$ & 1 \\
\hline Summe & & 9 \\
\hline
\end{tabular}

\subsubsection{Zusammenarbeit mit den Eltern}

Einbeziehung der Eltern (Bächthold et al., 1990; Hug, 1994b); enge Zusammenarbeit mit den Eltern (Innerhofer \& Klicpera, 1991); Zusammenarbeit mit den Eltern (Husinsky, 1994); funktionierende Zusammenarbeit mit den Eltern (Feyerer, 1998); gute Kommunikation (Boban \& Hinz, 2003)

Konzept für die Zusammenarbeit mit den Eltern (Innerhofer \& Klicpera, 1991)

Aufgabe der Schule, mit den Eltern über Ziele, Wege und Bewertungsmaßstäbe einer integrativen Schularbeit im Gespräch zu bleiben (Wocken, 1987); Eltern erhalten klare Informationen über die spezifischen Angebote der Schule in Bezug auf die Förderung von Schülern und Schülerinnen mit Behinderung (Hug, 1994b; Brugger-Paggi, 2003)

Gesprächsabende mit Eltern (Mühl, 1987)

regelmäßige Beratungstermine (Boban \& Hinz, 2003)

Informationsveranstaltungen für Eltern (Hug, 1994b)

Es wird ein Konsens zwischen Eltern und Schule in Bezug auf die Förderung des Sohnes/der Tochter angestrebt (Brugger-Paggi, 2003)

Gemeinsame Vereinbarungen werden schriftlich festgehalten und den Eltern zur Kenntnis gebracht (Brugger-Paggi, 2003)

Es findet eine laufende Information der Eltern über die Entwicklung Ihres Sohnes/Ihrer Tochter statt (Brugger-Paggi, 2003); Die Klassenlehrperson informiert die Eltern regelmäßig über die Inhalte des schulischen Lernens, allfällige besondere Lern- und Förderziele sowie über die Fortschritte des Kindes oder Jugendlichen. (Lienhard-Tuggener et al., 2011)

Die Eltern sind bei Standortgesprächen dabei. Sie werden angemessen in Entscheide und ihre Umsetzung einbezogen. (Lienhard-Tuggener et al., 2011)

Es gibt auch andere Formen des kontinuierlichen Informationsaustausches zwischen Lehrpersonen, Behindertenbetreuer/innen und Eltern (z.B. Mitteilungsheft, Planungsraster) (BruggerPaggi, 2003)

Information der Eltern dahingehend, wie diese das Lernen ihrer Kinder zu Hause unterstützen können (Boban \& Hinz, 2003)

Mitarbeit von Eltern im Unterricht (Innerhofer \& Klicpera, 1991)

Fortbildungen für Eltern (Innerhofer \& Klicpera, 1991) 


\begin{tabular}{|c|c|c|}
\hline Ziffer & Subkategorien/Indikatoren & Anz. \\
\hline \multirow[t]{11}{*}{1.3 .5} & Vernetzung & \\
\hline & $\begin{array}{l}\text { enge und verbindliche Zusammenarbeit mit externen Diensten (Innerhofer \& Klicpera, } \\
\text { 1991); Schule pflegt den Kontakt mit ihren externen Partnern (andere Schulen, spezia- } \\
\text { lisierte Fachstellen, Personen und Institutionen im nachschulischen Bereich, Öffentlich- } \\
\text { keit usw.) (Lienhard-Tuggener et al., 2011); Netzwerk; Vernetzung (Specht et al., 2006) }\end{array}$ & 3 \\
\hline & $\begin{array}{l}\text { Die im Kreis bestehenden Beratungseinrichtungen werden für anstehende Problemlösungen } \\
\text { hinzugezogen (Brugger-Paggi, 2003) }\end{array}$ & 1 \\
\hline & $\begin{array}{l}\text { Es besteht eine kontinuierliche Zusammenarbeit und ein Informationsaustausch mit den } \\
\text { Diensten der Sanitätsbetriebe (Brugger-Paggi, 2003) }\end{array}$ & 1 \\
\hline & die Beteiligten entwickeln Verfahren, wie diese gestaltet werden kann (Bächthold et al., 1990) & 1 \\
\hline & $\begin{array}{l}\text { Vereinbarungen werden auch mit außerschulischen Einrichtungen und Trägem geschlossen } \\
\text { mit dem Ziel der Vernetzung der schulischen mit der außerschulischen Arbeit, um so die soziale } \\
\text { Integration zu fördern (z.B. Projekte mit den Sozialdiensten, Jugenddiensten, Freizeitvereinen } \\
\text { usw....) (Brugger-Paggi, 2003) }\end{array}$ & 1 \\
\hline & $\begin{array}{l}\text { Vereinbarungen werden mit anderen schulischen Institutionen getroffen, um auf die einzelnen } \\
\text { Schüler und Schülerinnen abgestimmte individuelle Projekte durchzuführen (Brugger-Paggi, 2003) }\end{array}$ & 1 \\
\hline & $\begin{array}{l}\text { Zusammenarbeit mit lokalen Gruppierungen [Bsp.: Agenda, Stadtteilkonferenz] (Boban \& } \\
\text { Hinz, 2003) }\end{array}$ & 1 \\
\hline & Zusammenarbeit mit anderen Schulen zur Erleichterung von Schulwechseln (Boban \& Hinz, 2003) & 1 \\
\hline & Koordination von Unterstützungsmaßnahmen (Boban \& Hinz, 2003) & 1 \\
\hline & koordinierte Strategie von Schule und sozialen Diensten (Boban \& Hinz, 2003) & 1 \\
\hline Summe & & 11 \\
\hline
\end{tabular}

1.4.1 Unterschiedliche Organisationsformen integrativer Unterrichtung (äußere Differenzierung)

Fächerkanon umgestaltet in Bereiche (Hug, 1994b); Blocken von Unterrichtsstunden (Hug, 1994b; darin Köbberling zu Schulversuch Sek 1 Hamburg); flexible zeitliche Gestaltung der Unterrichtseinheiten (Holzinger et al., 2011)

Ausarbeitung von Modellplänen für die Durchführung klassenübergreifenden Un- 1 terrichts durch die Schulbehörden (Innerhofer \& Klicpera, 1991)

Es gibt schulinterne Unterstützungsangebote zusätzlich zum Klassenunterricht. Die zusätzlichen Angebote sind mit dem Unterricht und miteinander vernetzt. (Lienhard-Tuggener et al., 2011, S. 164)

Der Auftrag der zusätzlichen Unterstützungsangebote (z.B. integrative Förderung, Logopädie, Deutsch als Zweitsprache) ist klar festgelegt und benannt. (Lienhard-Tuggener et al., 2011, S. 164)

Summe

\subsubsection{Jahrgangsübergreifender Unterricht}

jahrgangsübergreifende Lerngruppen (Boban \& Hinz, 2003)

\subsubsection{Gestaltung von Übergängen}

Vorbereitung durch integrative Kindergärten (Bless, 2003)

Verhaltenstraining mit behinderten Kindern zur Vorbereitung auf die Regelschule (Mühl, 1987)

Vorbereitungen Regelschule allgemein

$$
\text { rechtzeitige Vorbereitung seitens der Schule (Berges, 1996; Feyerer, 1998) }
$$

Der Übertritt von Schülern und Schülerinnen mit Behinderung wird bereits ab Januar des vorhergehenden Schuljahres geplant und mit allen Beteiligten (Eltern, nächste Schulstufe, Schüler selbst, Dienste) abgesprochen (Brugger-Paggi, 2003) 


\begin{tabular}{|c|c|c|}
\hline Ziffer & Subkategorien/Indikatoren & Anz. \\
\hline & \multicolumn{2}{|l|}{ Vorbereitung Beteiligte Regelschule } \\
\hline & $\begin{array}{l}\text { Vorbereitung zum Umgang mit behinderten Kindern (Mühl 1987); Umgang mit Kindern mit } \\
\text { schweren Beeinträchtigungen (Bürli et al., 2009) }\end{array}$ & 1 \\
\hline & Vorbereitung der Regelschullehrer und der Eltern (Mühl, 1987) & 1 \\
\hline & $\begin{array}{l}\text { Lehrpersonen werden rechtzeitig (auf alle Fälle vor Schulbeginn) über die Anmeldung neuer } \\
\text { Schüler/innen in ihrer Klasse informiert (Brugger-Paggi, 2003) }\end{array}$ & 1 \\
\hline & $\begin{array}{l}\text { Alle Fachlehrer/innen sind über die Kinder mit besonderem Förderbedarf informiert. (Brug- } \\
\text { ger-Paggi, 2003) }\end{array}$ & 1 \\
\hline & $\begin{array}{l}\text { unterrichtende Lehrpersonen setzen sich rechtzeitig mit Grundsätzen der Integrationspädagogik } \\
\text { (innere Differenzierung, Teamkooperation, etc.) auseinander (Hug, 1994b) }\end{array}$ & 1 \\
\hline & \multicolumn{2}{|l|}{ Übergänge verzahnen und begleiten } \\
\hline & $\begin{array}{l}\text { Verzahnung von Frühförderung, Schule und Berufsberatung (Wember \& Prändl, 2009), von } \\
\text { Kindergärten, Schulen, Berufsschulen (Boban \& Hinz, 2003) }\end{array}$ & 2 \\
\hline & Begleitung des Übergangs vom Kindergarten in die Grundschule (Innerhofer \& Klicpera, 1991) & 1 \\
\hline & $\begin{array}{l}\text { Begleitung des Übergangs von der Grundschule in die weiterführende Schule (Innerhofer \& } \\
\text { Klicpera, 1991) }\end{array}$ & 1 \\
\hline & \multicolumn{2}{|l|}{ Übergänge innerhalb einer Schule } \\
\hline & $\begin{array}{l}\text { Innerhalb der Klassen wird durch zielgerichtete Information und die Weitergabe von Planungs- } \\
\text { instrumenten die erzieherische und didaktische Kontinuität auch bei Wechsel der Personen } \\
\text { gewährleistet (Brugger-Paggi, 2003) }\end{array}$ & 1 \\
\hline & $\begin{array}{l}\text { Zwischen den Klassenstufen wird durch zielgerichtete Information und die Weitergabe von } \\
\text { Planungsinstrumenten die erzieherische und didaktische Kontinuität gewährleistet (Brugger- } \\
\text { Paggi, 2003) }\end{array}$ & 1 \\
\hline \multirow[t]{2}{*}{ Summe } & & 15 \\
\hline & \multicolumn{2}{|l|}{ OE_1.5_Ergebnisse } \\
\hline \multirow[t]{4}{*}{ 1.5.1 } & Schulentwicklung (Specht et al., 2006) & \\
\hline & Pragmatische Veränderungen - keine radikale Schulreform (Feyerer, 1998) & 1 \\
\hline & $\begin{array}{l}\text { pädagogisch-didaktische Umsetzung der Integrationsidee in tragfähige pädagogische Struktu- } \\
\text { ren (Bürli et al., 2009) }\end{array}$ & 1 \\
\hline & tragfähiges Gesamtkonzept (Berges, 1996); & 1 \\
\hline
\end{tabular}

\subsubsection{Schulprogramm}

Im Schulprogramm sind Ziele und Maßnahmen festgesetzt, welche die Integration von Schülern und Schülerinnen mit Behinderung zum Ziele haben (Brugger-Paggi, 2003); Schulprogramm verdeutlicht Inklusion als Anspruch öffentlich (Boban \& Hinz, 2003); Schulprogramm, das behinderte Kinder einbezieht (Wember \& Prändl, 2009)

Im Schulprogramm sind Ziele und Maßnahmen festgesetzt, welche die individuellen Bedürfnisse aller Schüler und Schülerinnen berücksichtigen (Brugger-Paggi, 2003)

alle Beteiligten am schulischen Leben kennen das Schulprogramm (Boban \& Hinz, 2003)

die Planung einer barrierefreien Umgestaltung der Schule ist Teil des Schulprogramms (Boban \& Hinz, 2003)

In das standortspezifische Förderkonzept der Schule wird die Förderung der Schüler mit besonderen Bedürfnissen explizit mit einbezogen. (BMBWF Österreich, 2008)

Das Förderkonzept besitzt für alle Lehrkräfte Verbindlichkeitscharakter und wird laufend evaluiert. Über die Ergebnisse der Evaluation ist dem Schulforum zu berichten. (BMBWF Österreich, 2008) 


\begin{tabular}{llc}
\hline Ziffer & Subkategorien/Indikatoren & Anz. \\
\hline 1.5.3 & Dokumentation/ Außendarstellung & \\
& Integrationsprozesse werden auf vielfältige Weise dokumentiert und auch anderen zur Verfügung \\
& gestellt, z.B. durch Berichte, Fotodokumentation, Videos, CD-Rom usw. (Brugger-Paggi, 2003); & 2 \\
& Dokumentation integrativen und differenzierenden Unterrichts (Innerhofer \& Klicpera, 1991) & \\
& Erfahrungen, Informationen und Unterrichtsmaterialien werden mit anderen Klassen und & 1 \\
& Schulen ausgetauscht (Brugger-Paggi, 2003) & \\
& Es wird auf einen korrekten Gebrauch der Sprache in Bezug auf den spezifischen Bereich so- & 1 \\
& wohl in den offiziellen Dokumenten, als auch in den Gesprächen mit Eltern, Schülern und & \\
& Schülerinnen und Fachkräften geachtet (Brugger-Paggi, 2003) & \\
& Informationen über die Schule sind für alle zugänglich und verständlich (barrierefreie Inter- & 1 \\
& netseite, verschiedene Sprachen, einfache Sprache, Braille, Großdruck, Sprachausgabe) (Boban & \\
& \& Hinz, 2003) & \\
& Präsentation von Gruppenarbeiten/individuellen Arbeiten im Schulgebäude (Boban \& Hinz, & 1 \\
& 2003) & \\
& Darstellung integrativen Unterrichts in öffentlichen Medien (Innerhofer \& Klicpera, 1991) & 1 \\
Summe & & 7 \\
\hline
\end{tabular}

\subsubsection{Evaluation}

Die Schule evaluiert laufend den eigenen Prozess im Bereich der Umsetzung der schulischen Integration (Brugger-Paggi, 2003)

Für die Umsetzung spezifischer Vorhaben und Projekte im Bereich der Integration werden an einer Schule eigene Vereinbarungen getätigt (Brugger-Paggi, 2003)

Die Einhaltung der Qualitätsstandards auf Schul- und Klassenebene an den einzelnen Standorten bedarf neben der internen auch einer regelmäßigen externen Überprüfung. Diese wird durch die regionale Schulaufsicht - und sollte im Hinblick auf die Bündelung weiterer erforderlicher Fachkompetenzen - sinnvoller Weise im Zusammenwirken mit einem Expert/innenteam (z.B. Leiterin bzw. Leiter eines Sonderpädagogischen Zentrums, speziell ausgebildete Sonderpädagogin/speziell ausgebildeter Sonderpädagoge, Schulpsychologin/Schulpsychologe usw.) erfolgen (BMBWF Österreich, 2008)

Im Hinblick auf eine nachvollziehbare Dokumentation aller durchgeführten Maßnahmen und der sich daraus ergebenden Anpassungserfordernisse sowie im Sinn einer evidenzbasierten Bildungspolitik sollte vom regionalen Bildungsmanagement ein jährlicher Bericht zur Qualitätsentwicklung sonderpädagogischer Förderung in der Region unter besonderer Berücksichtigung der Bewährung der Qualitätsstandards für die Integration von Schülerinnen und Schülern mit besonderen Bedürfnissen erstellt werden. (BMBWF Österreich, 2008)

Summe

\section{PE_2.1_Ressourcen Personal}

\subsubsection{Personelle Ressourcen allgemein}

mehr personelle Ressourcen (Deutsches Schulamt 2004); ausreichende Versorgung mit Sonderpädagogen (Dumke et al., 1989)

\subsubsection{Doppelbesetzung}

Zwei-Lehrer-System (vor allem bei schwierigen Behinderungen) (Bless, 2003); I-Klasse grundsätzlich im Zwei-Pädagogen-System, dabei können durchaus zwei Regelschullehrer unterrichten, wenn diese durch Integrationsberater beraten werden können (Heyer et al., 1997); Zwei-Lehrer-Modell (Specht et al., 2006); eine Sonderschullehrerin oder ein Sonderschullehrer sollte mit einer gesamten Unterrichtsverpflichtung in der Integrationsklasse unterrichten (BMBWF Österreich, 2008); Zwei-Pädagogen-System (Thiele, 2009); Doppelbesetzung (Sonderpädagoge 1/2 Stelle, 30 Stunden Sozialpädagoge) (Köbberling, 1994); (Innerhofer \& Klicpera, 1991); (Textor, 2009) 


\begin{tabular}{|c|c|c|}
\hline Ziffer & Subkategorien/Indikatoren & Anz. \\
\hline & Auflösen der Doppelbesetzungen nur in Ausnahmefällen (Holzinger et al., 2011) & 1 \\
\hline & $\begin{array}{l}\text { Regelschullehrer und Sonderschullehrer müssen zur Verfügung stehen und zu Kooperation } \\
\text { bereit sein (Bless, 2003) }\end{array}$ & 1 \\
\hline & $\begin{array}{l}\text { Vertretungspool an Schule (ohne dass Spezialkräfte eingesetzt werden müssen) (Boban \& Hinz, } \\
\text { 2003) }\end{array}$ & 1 \\
\hline & $\begin{array}{l}\text { Drei-Pädagogen-System (1 Grundschullehrkraft, } 1 \text { Unterrichtshilfe (Erzieher), } 1 \text { Sonderschullehr- } \\
\text { kraft mit } 10 \text { (11+4-Modell) bzw. } 5 \text { (18+2-Modell) Stunden wöchentlich) (Wocken 1987) }\end{array}$ & 1 \\
\hline & Sonderpädagog/inn/en in Regelschulen (Weigt, 1977; darin Heese) & \\
\hline & $\begin{array}{l}\text { Förderschullehrer/innen werden ausschließlich in integrierten Klassen eingesetzt (außer zur } \\
\text { Beratung) (Brugger-Paggi, 2003) }\end{array}$ & 1 \\
\hline & quantitative Ausstattung mit Personal & \\
\hline & $\begin{array}{l}\text { Orientierung der Ausstattung mit Sonderpädagogen an tatsächlicher Zahl der Schüler mit son- } \\
\text { derpädagogischem Förderbedarf (nicht einfach mit Stundenpool); höchstens Betreuung von } \\
6 \text { Regelklassen für einen Sonderpädagogen (Bless 2003); Die Unterrichtszeit der Klassenlei- } \\
\text { tung in der Integrationsklasse sollte mindestens einer halben Lehrverpflichtung entsprechen } \\
\text { (BMBWF Österreich, 2008) }\end{array}$ & 3 \\
\hline & Entlastung beteiligter Lehrpersonen durch Deputatsstunden (Berges, 1996) & 1 \\
\hline & $\begin{array}{l}\text { Stundendeputat für Integrationslehrer/innen zur Teilnahme an schulinterner Arbeitsgruppe } \\
\text { (Brugger-Paggi, 2003) }\end{array}$ & 1 \\
\hline & 4 Wochenstunden Stundenentlastung für Fortbildung (Köbberling, 1994) & 1 \\
\hline \multicolumn{2}{|l|}{ Summe } & 19 \\
\hline \multirow[t]{5}{*}{2.1 .3} & Zusammensetzung der Lehrerteams & \\
\hline & Mitspracherecht bei der Zusammensetzung der Lehrerteams (PH Steiermark 2011) & 1 \\
\hline & $\begin{array}{l}\text { Das Lehrer/innenteam der Klasse soll klein gehalten werden und nach Möglichkeit acht Lehr- } \\
\text { kräfte nicht überschreiten (BMBWF Österreich, 2008); Kleines Lehrerteam (PH Steiermark } \\
\text { 2011); kleines Lehrerteam (Husinsky, 1994); möglichst kleines PädagogInnenteam (nicht } \\
\text { mehr als acht Lehrer/innen) (Hug, 1994b; Köbberling, 1994) }\end{array}$ & 4 \\
\hline & $\begin{array}{l}\text { verschiedene Fachleute (Innerhofer \& Klicpera, 1991); Multiprofessionelle Teams (Wember } \\
\text { \& Prändl, 2009) }\end{array}$ & 2 \\
\hline & einzelne Lehrkräfte übernehmen möglichst viele Fächer in der Klasse (Köbberling, 1994) & 1 \\
\hline
\end{tabular}

\subsubsection{Bündelung von Ressourcen}

Die sonderpädagogischen Ressourcen sind am Standort so zu bündeln, dass damit maximale Wirksamkeit erzielt werden kann (BMBWF Österreich, 2008)

Im Hinblick auf eine umfassende und durchgängige sonderpädagogische Förderung ist der Bündelung von Ressourcen an einzelnen Standorten in Integrationsklassen nach Möglichkeit der Vorrang vor der wohnortnahen Einzelintegration („Stützlehrer/innenklassen“) zu geben (BMBWF Österreich, 2008)

Eine bedarfsgerechte, transparente, nachvollziehbare und die Erfordernisse der einzelnen Integrationsstandorte berücksichtigende Zuteilung von Ressourcen durch die regionale Schulaufsicht ist eine wesentliche Voraussetzung, welche eine qualitätsvolle Umsetzung des integrativen Unterrichts fördert. Die „Treffsicherheit“ wird durch die Mitwirkung eines Teams von Expertinnen und Experten bei der Planung der Ressourcenzuteilung erhöht. (BMBWF Österreich, 2008)

Integrationslehrpersonen werden gemäß ihrer fachlichen Qualifikation den einzelnen Integrationsmaßnahmen zugewiesen (Brugger-Paggi, 2003)

\subsubsection{Kontinuität}




\begin{tabular}{llc}
\hline Ziffer & Subkategorien/Indikatoren & Anz. \\
\hline 2.1.6 & Feste Teamzeiten & \\
& Für die gemeinsame Planung von Unterricht zwischen Integrationslehrperson, Integrationshel- \\
& fer/in und Regellehrperson sind fixe Zeiten vorgesehen (Brugger-Paggi, 2003); Zeitliche Res- \\
& sourcen für Kooperation und Teambesprechungen (Wember \& Prändl, 2009); im Stundenplan \\
& zeitlich fixierte Teambesprechungen (Holzinger et al., 2011); Festlegung der Zeitkomponente \\
& (Preuß, 1981); im Stundenplan verankerte Planungs- und Koordinationszeiten für Teams (Bo- \\
& ban \& Hinz, 2003); eine Koordinationsstunde (Köbberling, 1994); Es bestehen angemessene \\
& Gefäße resp. Organisationsformen für den kindbezogenen, den unterrichtsbezogenen und den \\
& allgemeinen fachlichen Austausch. (Lienhard-Tuggener et al., 2011, 165); Der Informations- \\
& austausch und die Zusammenarbeit zwischen den Lehrpersonen und den sonderpädagogischen \\
& Fachpersonen sind verbindlich geregelt. (Lienhard-Tuggener et al., 2011, S. 165) \\
\hline
\end{tabular}

\subsubsection{Spezifisches Personal in Regelschulen}

Therapeutisches Personal in der Schule (Physiotherapeuten, Ergotherapeuten, Logopäden)

(Heese, 1977); (Wember \& Prändl, 2009); Physiotherapeuten und Logopäden (Innerhofer \& Klicpera, 1991)

Medizinisches und pflegerisches Personal (Wember \& Prändl, 2009) 1

Sozialpädagogen (Wember \& Prändl, 2009) 1

Schulpsychologen (Innerhofer \& Klicpera, 1991) $\quad 1$

Schulsozialarbeit (Wember \& Prändl, 2009) 1

Erzieher (Wember \& Prändl, 2009) 1

Gebärdendolmetscher (Boban \& Hinz, 2003) 1

Dolmetscher für verschiedene Sprachen (Boban \& Hinz, 2003) 1

Beratungsdienst für seh- und hörbehinderte Kinder (Innerhofer \& Klicpera, 1991) 1

psychopädagogische Berater für Lehrer (Innerhofer \& Klicpera, 1991) 1

Integrationshelfer (Wember \& Prändl, 2009; Thiele 2009); Behindertenassistenten (Innerho- 2

Summe

\section{PE_2.2_Kooperation}

\subsubsection{Kooperation von Lehrkräften allgemein}

kollegiale Kooperation (Heyer et al., 1997); Verbesserung der Kooperation (Deutsches Schulamt, 2004); Teamarbeit und Kooperation (Thiele, 2009); Kooperation im Lehrerkollegium (Speck, 2010);

direkte Initiierung des Kooperationsprozesses durch ein Mitglied einer Institution (Preuß, 1981) 1

Unterstützung (Specht et al., 2006)

\subsubsection{Teamarbeit}

Teamarbeit (Berges, 1996); Teamarbeit (Boban \& Hinz, 2003; Specht et al., 2006)

Teambildung

Teambildung (Lehrer/innen) sollte ein Jahr vor dem Wechsel einer Grundschulklasse in die Sek 1 erfolgen (Hug, 1994b)

Gemeinsame Besprechungen

Um Treffen und Besprechungen zielgerichtet und effizient durchzuführen erfolgen rechtzeitige Terminabsprachen (Brugger-Paggi, 2003)

Besprechungen werden langfristig geplant, um allen Beteiligten die Teilnahme zu ermöglichen (Brugger-Paggi, 2003)

Bei gemeinsamen Besprechungen, an denen mehrere Dienste und Personen teilnehmen, wird für eine Moderation gesorgt (Brugger-Paggi, 2003) 


\begin{tabular}{|c|c|c|}
\hline Ziffer & Subkategorien/Indikatoren & Anz. \\
\hline & Die Besprechungen sind vorbereitet und werden zielführend moderiert (Brugger-Paggi, 2003) & 1 \\
\hline & Für die Besprechungen ist genügend Zeit eingeplant (Brugger-Paggi, 2003) & 1 \\
\hline & Es wird ein gesprächsfreundliches Klima angestrebt (Brugger-Paggi, 2003) & 1 \\
\hline & $\begin{array}{l}\text { Die getroffenen Vereinbarungen werden von allen als verbindlich angesehen und gehandhabt } \\
\text { (Brugger-Paggi, 2003) }\end{array}$ & 1 \\
\hline & $\begin{array}{l}\text { Kommunikations- und Entscheidungsprozesse des Teams, die der Komplexität der Aufgabe } \\
\text { gerecht werden (Feyerer, 2007) }\end{array}$ & 1 \\
\hline \multirow[t]{12}{*}{ Summe } & & 12 \\
\hline & Kooperative Problembearbeitung & \\
\hline & $\begin{array}{l}\text { Problembearbeitung im Team aller Beteiligten (verbindliche Formen der Zusammenarbeit) } \\
\text { (Bächthold et al., 1990); Konsensfindung bei Problemen (Feyerer, 1998); Gespräche über } \\
\text { Schwierigkeiten bei der Arbeit (Boban \& Hinz, 2003); Fallbesprechungen (Hug, 1994b) }\end{array}$ & 4 \\
\hline & Konkretheitsgrad des Kooperationszieles (Preuß, 1981) & 1 \\
\hline & Transparenz der Erwartungshaltung aller am Kooperationsprozeß Beteiligten (Preuß, 1981) & 1 \\
\hline & Zusammenarbeit aller in Integration involvierten Personen & \\
\hline & $\begin{array}{l}\text { Alle in die Integrationsmaßnahme involvierten Personen werden in die spezifischen Maßnah- } \\
\text { men der Integration eingebunden (Umgang, Hilfestellungen, Besonderheiten) (Brugger-Paggi, } \\
\text { 2003) }\end{array}$ & 1 \\
\hline & Austausch der Heilpädagogen mit anderen Fachpersonen (Bless, 2003) & 1 \\
\hline & Einbindung in regionale sonderpädagogische Teams (Bürli et al., 2009) & 1 \\
\hline & Interdisziplinarität (Bürli et al., 2009) & 1 \\
\hline & Gemeinsame Konferenzen/Dienstbesprechungen & \\
\hline & $\begin{array}{l}\text { gemeinsame Konferenzen (Mühl, 1987); Dienstbesprechungen (Boban \& Hinz, 2003); Ge- } \\
\text { samtkonferenzen (Thiele, 2009) }\end{array}$ & 3 \\
\hline
\end{tabular}

\subsubsection{Unterrichtsbezogene Kooperation}

Kooperation zwischen Klassenleitung und Sonderpädagogin, intensive Zusammenarbeit der beteiligten Lehrer/innen (Feyerer, 1998); Kooperation mit der Klassenleitung (Bürli et al., 2009); Kooperation mit Stützlehrer/in (Bürli et al., 2009)

Hospitationen gegenseitige Hospitationen (bspw. auch an Förderschule) (Berges 1996); (Booth et al., 2003); kollegiale Hospitation (Wernstedt et al., 2010)

Kollegiale Beratung (Wember et al. 2009); Erfahrungsaustausch zwischen Lehrer/inne/n (Innerhofer et al. 1991)

Kooperative Unterrichtsplanung

Gemeinsame kooperative Planung des Unterrichts (auch mit Integrationshelfern etc.) (Berges, 1996; Holzinger et al., 2011; Boban \& Hinz, 2003; Husinsky, 1994; Deutsches Schulamt, 2004)

Kooperative Unterrichtsdurchführung

Gemeinsame, kooperative Durchführung des Unterrichts; kooperatives Arbeiten im Team (Berges, 1996; Holzinger et al., 2011; Husinsky, 1994; Deutsches Schulamt, 2004) TeamTeaching (Heese, 1977; Mühl, 1987; Bürli et al., 2009); Teamteaching (Husinsky, 1994) Sonderpädagogische Fachpersonen werden so weit wie möglich in den Unterricht einbezogen. (Lienhard-Tuggener et al., 2011, S. 165)

Kooperative Unterrichtsreflexion

Gemeinsame, kooperative Reflexion des Unterrichts (Berges, 1996; Holzinger et al., 2011; Boban \& Hinz, 2003; Husinsky, 1994)

Lernprozessevaluation und -reflexion als Inhalt von sonderpädagogischen Fallbesprechungen zur Erstellung und Weiterentwicklung individueller Förderpläne und zur kollegialen Abstimmung des geplanten sonderpädagogischen Prozesses (Preuß, 1985) 


\begin{tabular}{llr}
\hline Ziffer & Subkategorien/Indikatoren & Anz. \\
\hline Summe & 26 \\
\hline
\end{tabular}

\subsubsection{Rollen- und Aufgabenklärung}

Kenntnis der professionellen Kompetenzstruktur (Preuß, 1981); die Beteiligten haben eine klare Rollendefinition für die Aufgaben der Bezugsklassen- und Fördergruppenlehrer (Bächthold et al., 1990)

Die unterschiedlichen Kompetenzen aller Lehrpersonen werden optimal genutzt und für verschiedene Angebote eingesetzt (Brugger-Paggi, 2003)

umfassende Institutionskenntnisse und Handlungserfahrungen in Institutionen mit sonderpädagogischen Aufgabenstellungen (Preuß, 1985)

Spezifische Aufgaben RSL

Die Sonderschullehrerin als gleichwertige Partnerin (Feyerer, 1998)

weitere Aufgaben: Beratung von Regelschullehrern bezüglich diagnostisch auffälliger Schüler 1 (Brugger-Paggi, 2003)

spezifische Aufgaben FSL

kennt die Lernausgangslagen der Schüler/inenn mit besonderen und sonderpädagogischen 1

Förderbedürfnissen (Holzinger et al., 2011)

legt für diese die relevanten Förderziele fest (Holzinger et al., 2011) 1

schreibt für diese einen Förderplan (Holzinger et al., 2011) 1

dokumentiert die Förderarbeit (Holzinger et al., 2011) 1

informiert die Kollegen über die Förderschwerpunkte (Holzinger et al., 2011) 1

sorgt für die methodisch-didaktische Umsetzung der Förderziele (Holzinger et al., 2011) 1

Tutor für neue Integrationslehrpersonen an der Schule (Brugger-Paggi, 2003) 1

Leitung von Fortbildungsmaßnahmen bei Regelschullehrern (Brugger-Paggi, 2003) 1

in der Arbeitsgruppe für Integration auf Schulebene (Brugger-Paggi, 2003) 1

Kooperation mit Assistenten der behinderten Kinder (Bürli et al., 2009) 1

Zusammenarbeit mit externen Diensten (Wember \& Prändl, 2009) 1

bezieht die Erziehungsberechtigten in die Förderarbeit mit ein (Holzinger et al., 2011) 1

Bereitstellung spezieller Lernhilfen (Preuß, 1985) 1

Summe

\subsubsection{Spezifische Kenntnisse}

allgemeine und fachspezifische Beratungskompetenz (Preuß, 1985); sonderpädagogische Beratungskompetenz (Preuß, 1985)

Koordinations- und Kommunikationsfähigkeit mit anderen Berufsgruppen (Preuß, 1985); generelle Bereitschaft und Fähigkeit zur Zusammenarbeit statt Abgrenzungstendenz (Preuß, 1985) sonderpädagogisches Problembewusstsein (Preuß, 1985)

Relativierung der eigenen Kompetenz, d.h. Erkennen und Eingestehen eigener Grenzen im sonderpädagogischen Handeln (Preuß, 1985); Kennen und Akzeptieren berufsspezifisch anders gewichteter Kompetenz (Preuß, 1985)

Verfügen über Konfliktlösungsstrategien (Preuß, 1985) 


\begin{tabular}{|c|c|c|}
\hline Ziffer & Subkategorien/Indikatoren & Anz. \\
\hline & $\begin{array}{l}\text { sonderpädagogische Bildung für alle Lehramtsstudierenden (Specht et al., 2006; Wernstedt \& } \\
\text { Ohnesorg, 2010; Hug, 1994; Deutsches Schulamt, 2004) }\end{array}$ & 4 \\
\hline & $\begin{array}{l}\text { abgeschlossene Ausbildung der Sonderpädagogen (Bless, 2003); Qualifikationsniveau der Pädago- } \\
\text { gen (Berges, 1996); sonderpädagogische Fachkräfte (Wember \& Prändl, 2009); Ausbildung (Bürli } \\
\text { et al. 2009); Stützlehrer mit sonderpädagogischer Ausbildung (Innerhofer et al., 1991); zusätzlich } \\
\text { eingesetzte Lehrer sollten über eine sonderpädagogische Zusatzausbildung verfügen (BMBWF Ös- } \\
\text { terreich, 2008); Die Mitarbeitenden verfügen über eine staatlich anerkannte Ausbildung, die den } \\
\text { Anforderungen ihrer beruflichen Tätigkeit entspricht. (Lienhard-Tuggener et al., 2011) }\end{array}$ & 7 \\
\hline Summe & & 15 \\
\hline 2.3.2 & Vermittlung von Erfahrungswissen (Heese, 1977) & 1 \\
\hline \multirow[t]{2}{*}{ 2.3.3 } & Supervision & \\
\hline & $\begin{array}{l}\text { Supervision für Lehrer (Specht et al., 2006); kollegiale Supervision (Wember \& } \\
\text { Prändl, 2009); fachliche Begleitung und Supervision (Bürli et al., 2009; Husins- } \\
\text { ky,1994); verpflichtende Supervision (Hug, 1994b) }\end{array}$ & 5 \\
\hline
\end{tabular}

\subsubsection{Fortbildungen}

Fortbildungen für Lehrer und pädagogische Fachkräfte (Wember \& Prändl, 2009); berufsbegleitende Fortbildungen (Bürli et al., 2009); Fortbildung (Bürli et al. 2009; Specht et al., 2006); Fort- und Weiterbildung (Thiele, 2009); Fortbildungen für das gesamte Lehrerkollegium (Brugger-Paggi, 2003); Fortbildungsangebote (Boban \& Hinz, 2003; Husinsky, 1994); Weiterbildungsangebot (Hug, 1994b); abgestimmtes Fortbildungskonzept (Innerhofer \& Klicpera, 1991; Deutsches Schulamt, 2004)

Studientage (Thiele, 2009) 1

Fortbildungen für einzelne Lehrer/innen (Brugger-Paggi, 2003) 1

Fortbildung von Schulaufsichtsbeamten (Hug, 1994b) 1

Fortbildung von Schulleitungen (Hug, 1994b) 1

Beratungstreffen und gemeinsamer Besuch von Fortbildungen für Regel- und Förderschulleh- $\quad 1$ rer/innen (Bless, 2003)

innerschulische Fortbildungen (auch für Eltern und Schüler/innen) (Boban \& Hinz, 2003) 1

praxisorientierte Fortbildung (Berges, 1996) 1

Fortbildung zur Planung und Durchführung effektiver Zusammenarbeit (Boban \& Hinz, 2003) 1

Untersuchungen zum Fortbildungsbedarf (Innerhofer \& Klicpera, 1991) 1

Abstimmung von Weiterbildungsmaßnahmen aufeinander (Innerhofer \& Klicpera, 1991) 1

Fortbildungsveranstaltungen müssen hinsichtlich ihrer Effizienz überprüft werden (Innerhofer 1

\& Klicpera, 1991)

Im Haushalt sind Mittel für die spez. Fortbildung von Lehrer/inne/n vorgesehen (Brugger- 1

Paggi 2003)

\section{UE_3.1_Ressourcen}

\subsubsection{Klassengröße}

Klassengröße: kleine Klassen (Weigt, 1977; darin Heese); Lerngruppen verkleinern

(Mühl, 1987); Klassenfrequenz von 20 (Köbberling, 1994); Klassen mit Schülern mit Behinderung werden in der Regel mit 20 Schülern gebildet. Abweichungen davon sind klar begründet (Brugger-Paggi, 2003; Reduzierung der Klassenfrequenz auf 20 Schüler (Thiele, 2009); niedrige Klassenstärke (Wember \& Prändl, 2009); Größe der Lerngruppe (Speck, 2010) 


\begin{tabular}{|c|c|c|}
\hline Ziffer & Subkategorien/Indikatoren & Anz. \\
\hline & $\begin{array}{l}\text { Für die Grundschule bedeutet das, Frequenzen von höchstens } 25 \text { Schülern pro Klas- } \\
\text { se zu haben, innere Differenzierung radikal einzuführen und mit einer Curriculum- } \\
\text { Reform auch in der Grundschule zu beginnen, die von den unterschiedlichen Sozia- } \\
\text { lerfahrungen der Kinder ausgeht und nicht mehr den Anspruch erweckt, daß Kinder } \\
\text { mit gleichen Voraussetzungen in die Schule kommen. (Preuss-Lausitz, 1977, S. 50) }\end{array}$ & 1 \\
\hline & $\begin{array}{l}\text { Durch die starke Individualisierung im Lern- und Verhaltensbereich ist eine Klassen- } \\
\text { stärke von höchstens 20-22 Kindern erforderlich. (Speck et al., 1978, S. 135) }\end{array}$ & 1 \\
\hline & $\begin{array}{l}\text { An erster Stelle steht die Forderung nach kleineren Schülerzahlen. (Dumke et al., } \\
\text { 1989, S. 146) }\end{array}$ & 1 \\
\hline
\end{tabular}

Summe

\subsubsection{Klassenzusammensetzung}

ausgewogene Klassenzusammensetzung (Feyerer, 1998); Heterogenität: heterogene Klassenzusammensetzung (Streuung IQ) (Bless, 2003); Berücksichtigung der Leistungsheterogenität bei der Klassenbildung (Holzinger et al., 2011)

für die Zusammensetzung einer Klasse werden Kriterien erarbeitet, sodaß die Ansammlung von Problemkindern in einer Klasse vermieden wird (Innerhofer \& Klicpera, 1991); Zusammensetzung der Schüler - möglichst normale Zusammensetzung nach Einzugsgebiet (Husinsky, 1994); als Richtlinie für die Klassenzusammensetzung gilt, dass die Zusammensetzung der Schüler in Integrationsklassen in etwa repräsentativ für den gesamten Schülerjahrgang an der Schule sein soll (BMBWF Österreich, 2008)

Die Häufung von Problemsituationen in einer Klasse wird vermieden. (Brugger-Paggi, 2003); eine Überrepräsentation erzieherisch schwieriger, verhaltensauffälliger oder lernschwacher Schüler, für die keine zusätzlichen sonderpädagogischen Ressourcen ausgewiesen sind, ist in Integrationsklassen zu vermeiden. (BMBWF Österreich, 2008); Keine vermehrte Ansammlung von Kindern mit besonderen Erziehungsbedürfnissen im Vergleich zu den Parallelklassen (Holzinger et al., 2011) stabiles Selbstbewußtsein der behinderten Schüler (Mühl, 1987)

Die Schulleiterin bzw. der Schulleiter hat die Kriterien der Zusammensetzung der Klasse auf Antrag offen zu legen. (BMBWF Österreich, 2008); Es gibt klare Kriterien für die Bildung integrierter Klassen, die auch den Eltern bekannt sind (Brugger-Paggi, 2003)

Planung der Klasse erfolgt vor Einrichtung (Bless,2003)

\section{UE_3.2_Klassenführung}

\subsubsection{Klassenführung}

feste Regeln innerhalb der Klasse (Boban \& Hinz, 2003); Regeln und Rituale (Wember \& Prändl, 2 2009)

klare Kommunikation von Verhaltensanforderungen (Textor, 2009)

klare Vorgehensweisen als Reaktion auf störendes Verhalten (Boban \& Hinz, 2003)

\section{UE_3.3_Schulklasse als sozialer Raum}

\subsubsection{Klassenklima}

Positive Lehrer-Schüler-Beziehung (Wember \& Prändl, 2009)

Erleben guter sozialer Beziehungen zwischen allen am Unterricht Beteiligten (Bless, 2003)

unterstützendes Lernklima mit einem individualisierenden und differenzierenden Unterricht (Bächthold et al., 1990)

alle in einer Klasse tätigen Lehrer/innen fühlen sich für das Lernen aller Schüler/innen verantwortlich (Boban \& Hinz, 2003)

Lehrereinschätzung des (positiven) Klassenklimas (Bless, 2003) 


\begin{tabular}{llr}
\hline Ziffer & Subkategorien/Indikatoren & Anz. \\
\hline 3.3.2 & Soziales Lernen (PH Steiermark 2011) & 1 \\
& Gezieltes Beobachten der Klassensituation (Leonhardt, 2009) & 1 \\
& Maßnahmen zur sozialen Integration von Kindern mit Behinderungen in den Unterricht (In- & 1 \\
& nerhofer \& Klicpera, 1991) & 1 \\
& Herstellung strukturierter Kontakte (Mühl, 1987) & 1 \\
& Rollenspiele (Mühl, 1987) & 1 \\
& Training individueller Konfliktlösungsstrategien (Wember \& Prändl, 2009) \\
& Klassenrat (Boban \& Hinz, 2003) & 1 \\
\hline
\end{tabular}

\section{UE_3.4_Didaktik}

\subsubsection{Grundlegende Aspekte}

Erweiterung didaktischer Modelle um Kategorien integrativer Förderung (Berges, 1996)

Im Sinne eines integrativen Unterrichts werden methodische und fachliche Innovationen er- 1 probt (Brugger-Paggi, 2003)

größerer Freiheitsspielraum bei Unterrichtsgestaltung (für die Lehrer) (Bless, 2003) $\quad 1$

mittelfristige Themenverteilung (Berges, 1996)

Curriculare Vereinbarungen, Absprachen zur Auswahl der Lerninhalte (Wember \& Prändl, 2009)

\subsubsection{Didaktische Prinzipien}

handelndes Lernen (Berges 1996); (Thiele 2009); handelnde Auseinandersetzung mit Themen (Köbberling 1994)

Anschaulichkeit des Unterrichts (Wember \& Prändl, 2009)

ganzheitliches Lernen (Berges, 1996); ganzheitliche Lernerfahrungen (Köbberling, 1994); Umsetzung von Interdisziplinarität und Ganzheitlichkeit durch interne und externe Kooperation (Feyerer, 2007)

Nähe zwischen schulischer und außerschulischer Lebenswelt (Heyer et al., 1997);Lerninhalte zumindest teilweise zu Vorhaben bündeln und realitätsnaher ansiedeln (Mühl, 1987)

Schülerorientierung (Berges, 1996); Orientierung an der Unterstützung des Lernens der Schüler, nicht an Lehrplänen (Boban \& Hinz, 2003)

gemeinsames Lernen und Differenzierung/Individualisierung wechseln sich ab (Köbberling, 1994); Ausgewogenheit von individueller Förderung und sozialem gruppenbezogenen Lernen (Berges, 1996)

Abstimmung von Leistungslernen und sozialem Lernen (Speck, 2010)

Förderung der Gemeinsamkeit durch die Gestaltung gemeinsamer Lebens- und Lernsituationen (Wocken, 1987)

zunehmende Eigenverantwortung der Lernenden (Heyer et al., 1997); das Erfahren eines höheren Masses an Eigenständigkeit und Eigenverantwortung bei unterrichtlichen Aktivitäten (für die Schüler)(Bless, 2003); Beteiligung der Lernenden an Entscheidungen über Unterrichtsinhalte und Lernwege (Boban \& Hinz, 2003); größere Bedeutung der Aktivität der Schüler (Innerhofer \& Klicpera, 1991); lehrerunabhängiges Arbeiten (Köbberling, 1994)

Verbindung von Curricula und individuellen Förderprogrammen (Bsp.: Orientierung in der Umwelt, basale Fähigkeiten Mathe und Deutsch, Selbstversorgung) (Wember \& Prändl, 2009)

Herstellung gemeinsamer Lernsituationen (Köbberling, 1994); Ausrichten der Arbeit auf eine gemeinsame Erlebens-, Verständigungs- und Verhaltensebene (Feyerer, 2007); Organisation von Tätigkeiten mit gemeinsamen Zielen (Mühl 1987); Ermöglichung sinnvoller Teilhabe an gemeinsamen Lernsituationen (Mühl 1987); Unterricht geht von gemeinsamer Erfahrung aus, die in unterschiedlicher Weise entfaltet werden kann (Boban \& Hinz, 2003); gemeinsame Ausgangssituation und unterschiedliches Bearbeiten (Köbberling, 1994); gemeinsame Rückbindung von Ergebnissen (Köbberling, 1994) 


\begin{tabular}{llc}
\hline Ziffer & Subkategorien/Indikatoren & Anz. \\
\hline 3.4.4 & Berufs- und Lebensvorbereitung & \\
& Konzepte zur Berufsorientierung und zur Vorbereitung auf die Arbeitswelt (Wember \& Prändl, & 1 \\
2009) & Förderkonzepte hinsichtlich eigenverantwortlicher Lebens- und Wohnperspektiven (Wember & 2 \\
& \& Prändl, 2009); Hilfen und Angebote zur Verwirklichung von Lebensplanung (Feyerer, 2007); \\
& Unterrichtsplanung, die Curricula, Lebens-, Arbeits- und Berufsorientierung sowie individuelle & 1 \\
& Lern- und Entwicklungsziele berücksichtigt (= hohe Anforderung) (Wember \& Prändl, 2009) \\
& Spezifische Maßnahmen der Orientierung und der Lebensplanung für Schüler und Schülerin- & 1 \\
& nen mit Behinderung werden bereits ab der 1. Mittelschule begonnen (Brugger-Paggi, 2003) & \\
\hline
\end{tabular}

\subsubsection{Kompetenzorientierung}

Klare Beschreibung von Inhalten und Kompetenzen bezogen auf die pädagogischen, psycholo- 1 gischen und therapeutischen Anteile (Feyerer, 2007)

fachlicher Unterricht zum Thema Behinderung (Berges, 1996; Mühl, 1987)

UE_3.5_Methodik

\subsubsection{Klare Unterrichtsstruktur}

das Empfinden, dass der Unterricht verständlich und gut organisiert ist (Bless, 2003);Klare unterrichtliche Strukturen (Abläufe, Medieneinsatz, Unterrichtsmaterialien) (Wember \& Prändl, 2009); Gestaltung von Lernprozessen, die durch Rhythmisierung, Strukturierung, Differenzierung, Individualisierung, Begriffsbildung und deren Sicherung gekennzeichnet sind (Wember \& Prändl, 2009); Ruhepausen (Thiele, 2009)

\begin{tabular}{lll}
\hline 3.5.2 & Vielfältige methodische Sozial- und Arbeitsformen (Wember \& Prändl, 2009) & 1 \\
\hline 3.5.3 & $\begin{array}{l}\text { Kooperatives Lernen } \\
\text { kooperative Handlungsmöglichkeiten und Methoden (Berges, 1996); kooperatives Arbeiten } \\
\text { im Team (Specht et al., 2006); kooperative Aufgabenstellungen (Textor, 2009) }\end{array}$ & 3 \\
&
\end{tabular}

\subsubsection{Offene Unterrichtsformen}

offene Lernformen; offene, schülerzentrierte Unterrichtsformen (Berges, 1996; Specht et al., 2006); offener Unterricht (Deutsches Schulamt, 2004); offenes Lernen (Thiele, 2009); Offene Unterrichtsformen (Wember \& Prändl, 2009); Einsatz offener Unterrichtsformen (Holzinger et al., 2011) Projektunterricht (Berges, 1996); Projeke und fächerübergreifender Unterricht (Köbberling, 1994); projektorientierter Unterricht (Bürli et al., 2009); Projektunterricht

Wochenplanarbeit (Köbberling, 1994b)

\section{UE_3.6_Differenzierung und Individualisierung}

\subsubsection{Differenzierung (Heese, 1977)}

binnendifferenziert, nicht in Leistungsgruppen (BMBWF Österreich, 2008); innere Differenzierung (Specht et al., 2006), Lernzieldifferenzierung (Bless, 2003), differenzierende Förderung und Betreuung (Heyer et al., 1997); differenzieren (Mühl, 1987); innere Differenzierung mit ähnlichen Lerninhalten (Bürli et al., 2009), binnen- und zieldifferenziert (Wernstedt \& Ohnesorg, 2010; Boban \& Hinz, 2003); innere Differenzierung (Hug, 1994b); Binnendifferenzierung (Köbberling, 1994); differenzierter Unterricht (Innerhofer \& Klicpera, 1991); Differenzierung von Leistungsanforderungen (Textor, 2009)

äußere Differenzierung und innere Differenzierung wechseln sich ab (Feyerer, 1998; Thiele, 2009)

Problemschüler besuchen einen hohen Anteil des Unterrichts in einer Bezugsklasse (Regelklasse) unter Berücksichtigung der individuellen Unterstützungsbedürfnisse (Bächthold et al., 1990), möglichst viel regelklasseninternes Arbeiten (Bless, 2003); nur selten Herausnahme von Schülern für spezielle Förderung (Boban \& Hinz, 2003); Die zusätzliche Unterstützung und Förderung findet möglichst integriert in den Unterricht oder unterrichtsnah statt. (LienhardTuggener et al., 2011); Unterricht in Kleingruppen (Wember \& Prändl, 2009)

Einzelunterricht (Wember \& Prändl, 2009) 


\begin{tabular}{ccc}
\hline Ziffer & Subkategorien/Indikatoren & Anz. \\
\hline & $\begin{array}{l}\text { Es besteht ein vielfältiges Angebot an Maßnahmen zur Förderung von Schülern und Schülerin- } \\
\text { nen mit verschiedenen Begabungen. (Brugger-Paggi, 2003); Der Unterricht ist auf die Vielfalt } \\
\text { der Lernenden ausgerichtet. (Lienhard-Tuggener et al., 2011) }\end{array}$ & \\
& Einzelmethoden der Differenzierung (Innerhofer \& Klicpera, 1991) & 1 \\
& Orientierung an der Unterstützung des Lernens der Schüler, nicht an Lehrplänen (Boban \& & 1 \\
& Hinz, 2003) & 1 \\
& gesonderter Unterricht, insbesondere zur lebenspraktischen Förderung (Mühl, 1987) & 29 \\
\hline
\end{tabular}

\subsubsection{Leistungsmessung und Bewertung}

häufige Rückmeldung zu individuell erreichten Entwicklungsfortschritten (Wember \& Prändl, 1 2009)

Differenzierte Leistungsfeststellung (Holzinger et al., 2011) 1

differenzierende Bewertung (Heyer et al., 1997) 1

differenzierende Klassenarbeiten (Heyer et al., 1997) 1

Leistungsdruck abmildern (Mühl, 1987) 1

Alternative Formen der Leistungsbeurteilung (Holzinger et al., 2011) 1

Es werden vielfältige Formen der Überprüfung der Zielerreichung durchgeführt (Beobach- 2

tungen, Experimente ...) (Brugger-Paggi, 2003); vielfältige Möglichkeiten Lernfortschritte zu zeigen (Boban \& Hinz, 2003)

Multiple-Choice-Aufgaben (Thiele, 2009) u.a. für K-Kinder 1

Prüfungszeitpunkte sind flexibel (Boban \& Hinz, 2003) 1

Die Bewertungskriterien werden für Schüler und Schülerinnen und Eltern transparent formu- $\quad 1$

liert (Brugger-Paggi, 2003)

Differenzierte Bewertungskriterien werden gemeinsam von allen Mitgliedern des Klassenrates erstellt. (Brugger-Paggi, 2003); individuelle Bewertungsmaßstäbe (Deutsches Schulamt, 2004)

Die Bewertung bezieht sich auf die Zielsetzungen des Individuellen Förderplans (BruggerPaggi, 2003)

Notengebung durch Verbalbeurteilung o.ä. verändern, ersetzen, ergänzen (Mühl 1987); verbale Leistungsbeschreibung (Husinsky, 1994); Berichte (Lernentwicklungsbeschreibung) (Köbberling, 1994)

Die Bewertung wird dem Schüler/der Schülerin auch in Form einer mündlichen Rückmeldung mitgeteilt (Brugger-Paggi, 2003)

Nachteilsausgleich (Wember \& Prändl, 2009) 1

Verständigung über die Definition des Fortschrittes (Feyerer, 2007) 1

Einschätzung und Bewertung von Schülerleistungen auch durch Schüler selbst (Boban \& Hinz, 1 2003)

Schemata und Hilfen zu einer effizienten Lernzielüberprüfung (Innerhofer \& Klicpera, 1991) 1

die Aussicht, für Anstrengungen auch belohnt zu werden (für die Schüler/innen) (Bless, 2003) 1

Summe

\subsubsection{Förderdiagnostik}

professionelle Diagnostik (Specht et al., 2006) 1

Am Kind orientierte Lernortentscheidung (Wember \& Prändl, 2009) 1

Ermittlung der individuellen Lernvoraussetzungen bei Vermeidung einer ausschließlich defizitären Beschreibung (Preuß, 1985); individuelle Diagnostik der Lernausgangslage (Wember \& Prändl, 2009); Berücksichtigung individueller Lernausgangslagen (Holzinger et al., 2011); Die individuellen Kompetenzen und Bedürfnisse der Schülerinnen und Schüler sowie diejenigen des Umfelds werden sorgfältig erfasst. (Lienhard-Tuggener et al., 2011) 


\begin{tabular}{llc}
\hline Ziffer & Subkategorien/Indikatoren & Anz. \\
\hline 3.6 .4 & Förderplanung &
\end{tabular}

individuelle Entwicklungspläne (Feyerer, 2007); individuelle Lernzielerstellung unter den Aspekten des ganzheitlichen Lernens mit Zieltransparenz für Schüler und Eltern (Preuß, 1985); individualisierte Lernziele (Bürli et al., 2009); Förderpläne (Specht et al., 2006); Erstellung individueller Förderpläne gemeinsam mit Schülern, Eltern und allen Mitarbeitern (Boban \& Hinz, 2003); Ausarbeitung individueller Erziehungspläne nach Unterlagen vom Schulamt (Innerhofer \& Klicpera, 1991)

Der individuelle Förderplan wird vom Klassenrat spätestens innerhalb November erstellt und mit Eltern besprochen (Brugger-Paggi, 2003)

Der Individuelle Förderplan für Schüler mit Behinderung wird für alle Schüler und Schülerinnen mit Funktionsdiagnose vom Klassenrat erstellt, regelmäßig überprüft und wenn nötig an veränderte Situationen angepasst. (Brugger-Paggi, 2003)

Festlegung, wer für die Durchführung verantwortlich ist und wie Erreichen der Ziele kontrolliert werden kann (Innerhofer \& Klicpera, 1991)

Im FÖP sind auch organisatorische und therapeutische Maßnahmen festgehalten (BruggerPaggi, 2003)

konkrete Fördervorschläge (Bless, 2003)

kooperative Entwicklung von individuellen Förderplänen (Wember \& Prändl, 2009)

begleitende Diagnostik zur Evaluation der Erreichung der individuellen Lern- und Erziehungsziele (Wember \& Prändl, 2009)

gesicherte Kontinuität des Förderprozesses (Feyerer, 2007); kontinuierliche Lernprozeßdokumentation unter Einbeziehung lerndiagnostischer Verfahren zur Bereitstellung von speziellen Lernhilfen und zur Rückmeldung des Lernprozessverlaufs für Schüler und Lehrer bei Relativierung der vergleichenden Bewertung durch Zensuren (Preuß, 1985)

individuelle Lernprozessorientierung, flexible Lernorganisation in Form und Inhalt zur Erreichung des Lernziels unter besonderer Berücksichtigung der individuellen Lernzeit, Lernart und des individuellen Lerninhalts (Preuß, 1985)

Interventionen, die an den Stärken der Schüler/innen ansetzen (Wember \& Prändl, 2009) 1

Summe

\subsubsection{Individualisierung}

Individuelle Förderung (Weigt, 1977; darin Heese), Individualisierung (Heyer et al., 1997) (Specht et al., 2006); Berücksichtigung individueller Lernausgangslagen; Zuschneidung auf individuelle Eigenart eines (behinderten) Schülers; Ermitteln der Bedürfnisse der NutzerInnen (Feyerer, 2007); Im Sinne einer integrativen Schule geht die Schule vom individuellen Entwicklungsstand eines jeden Schülers, einer jeden Schülerin aus (Brugger-Paggi 2003); individualisieren (Mühl, 1987); Individualisierung (Husinsky, 1994); (Boban \& Hinz, 2003); Unterricht zugeschnitten auf das individuelle Lern- und Leistungsniveau (Innerhofer \& Klicpera, 1991)

Die individuellen Förderschwerpunkte der Schüler und Schülerinnen mit Behinderung werden in die gemeinsame Planung der Klasse eingebaut (Brugger-Paggi, 2003)

L Bereitstellung spezieller Lernhilfen (Preuß, 1985)

SE Handlungsstrategien zum Umgang mit Verhaltensschwierigkeiten (Boban \& Hinz, 2003)

Spra- Spezifisch sprachliche Lernprozesse (Pragmatik, Semantik, Grammatik, Morphologie, che Syntax und Phonologie/Phonetik) (Wember \& Prändl, 2009)

G Hilfen bei der Formulierung von Lernzielen für Kinder mit geistiger Behinderung (Innerhofer \& Klicpera, 1991)

gesonderter Unterricht, insbesondere zur lebenspraktischen Förderung (Mühl, 1987) 1

H Cochleaimplantate, mobile Hörsprechanlagen) (Wember \& Prändl, 2009)

Gebärdensprache (Wember \& Prändl, 2009)

Elektronische Kommunikationsmöglichkeiten (Wember \& Prändl, 2009) 


\begin{tabular}{|c|c|c|c|}
\hline Ziffer & \multicolumn{2}{|c|}{ Subkategorien/Indikatoren } & Anz. \\
\hline & & $\begin{array}{l}\text { Pädagogische Einweisung in den Umgang mit technischen Hilfsmitteln (Bsp.: Micro- } \\
\text { portanlage) (Wember \& Prändl, 2009) }\end{array}$ & 1 \\
\hline & & zugewandtes Sprechen (Leonhardt, 2009) & 1 \\
\hline & & $\begin{array}{l}\text { schriftliches Fixieren wesentlicher Unterrichtsinhalte und Kerngedanken oder auch } \\
\text { Aussagen an der Tafel, auf Folie o.ä. (Leonhardt, 2009) }\end{array}$ & 1 \\
\hline & & Schriffliche Fixierung der Hausaufgaben (Leonhardt, 2009) & 1 \\
\hline & $\mathrm{K}$ & Lagerung von körperbehinderten Kindern (Thiele, 2009) & 1 \\
\hline Summe & & & 23 \\
\hline
\end{tabular}

\section{UE_3.7_Ergebnisse}

\subsubsection{Ergebnisse allgemein}

Subjektiv wahrnehmbarer Prozess der Entwicklung der Schüler/innen (Schley et al. 1992) 1

Lerngewinne für alle Kinder (Speck 2010)

Objektivierbarer Lernerfolg (Schley et al. 1992) 1

Wissensinhalte (Schley et al. 1992) 1

Die Wirksamkeit der durchgeführten Maßnahmen wird laufend überprüft (Brugger-Paggi 2003) 1

Durch kontinuierliche Rückmeldung über die Lernfortschritte wird die Selbsteinschätzung des 1 Schülers/der Schülerin mit Behinderung gefördert (Brugger-Paggi 2003)

Die Ziele beziehen sich nicht nur auf den kognitiven sondern gleichwertig auch auf den kom- $\quad 1$

Summe munikativen, sozialen und affektiven Bereich (Brugger-Paggi 2003)

\subsubsection{Kognitive Kompetenzen}

Lern- und Entwicklungsfortschritte (lebenspraktische Fertigkeiten, Einhalten sozialer Regeln, sprachliche Entwicklung) (Wocken 1987)

Vermittlung spezifischer Schrift- und Kommunikationstechniken (z.B. Brailleschrift, Gebär- 1 densprache) (Wember \& Prändl, 2009)

Vermittlung grundlegender Kenntnisse in den Kulturtechniken (Wember \& Prändl, 2009) 1

Kommunikation/Sprache (Wember et al. 2009); Kommunikationsfähigkeit (Speck, 2010) 1

Ausschöpfen aller individuellen Entwicklungsmöglichkeiten (Wember \& Prändl, 2009) 1

Akzeptanz der eigenen Behinderung und bewusster Umgang mit ihr (Wember \& Prändl, 2009) 1

Realistische Selbsteinschätzung (Wember \& Prändl, 2009)

Geregelter Übergang ins Arbeitsleben (Wember \& Prändl, 2009) 1

Kognition (Wember \& Prändl, 2009) 1

Summe 9

\subsubsection{Emotionale Kompetenzen}

Förderung der Persönlichkeitsentwicklung $\quad 1$

Emotion (Wember \& Prändl 2009) 1

tragfähiges Selbstkonzept (Speck 2010) 1

Ich-Stärke (Speck 2010) 1

emotionale Sicherheit (Speck 2010) 1

Selbstbestimmung (Speck 2010) 1

Selbständigkeit (Schley et al. 1992) 1

\subsubsection{Soziale Kompetenzen}

Sozialverhalten (Wember \& Prändl 2009) 1

Erwerb sozialer Kompetenzen; Sozialkompetenz (Speck 2010) 1 


\section{Beispiel für Interviewleitfaden (Regelschullehrkraft Grundschule)}

\section{Interviewleitfaden für Regelschullehrkräfte an Grundschulen und weiterführenden Schulen}

1. Seit wie vielen Jahren unterrichten Sie Schüler/inne/n mit sonderpädagogischem Förderbedarf?

2. Welche Veränderungen nehmen Sie an Ihrer Schule aufgrund der integrativen Unterrichtung dieser Schüler/innen wahr?

3. In welcher Form hat sich Ihre Schule mit Anforderungen des gemeinsamen Unterrichts befasst (Bsp.: pädagogische Konferenzen, pädagogische Tage, Fortbildungen, Schulprogramm o.ä.)?

4. Wie setzen Sie die integrative Unterrichtung in Ihrer Klasse organisatorisch um (Bsp.: Einzelförderung, Förderung innerhalb der Klasse)?

5. Welche Aufgaben übernehmen Sie im Zusammenhang mit dem gemeinsamen Unterricht (Bsp.: Förderung, Teamteaching, Beratung, Vernetzung)?

6. Welche Veränderungen beobachten Sie in Ihrem eigenen Unterricht, seit Sie Schüler/innen mit sonderpädagogischem Förderbedarf in Ihrer Klasse haben?

7. Welche Qualitätsmerkmale zeichnen Ihrer Meinung nach den gemeinsamen Unterricht aus?

8. Wie schätzen Sie die Lernentwicklung der Schülerinnen und Schüler mit sonderpädagogischem Förderbedarf in Ihrer Klasse ein (Bsp.: kognitive Entwicklung, soziale Entwicklung, emotionale Entwicklung)?

9. Wie schätzen Sie Ihre Kooperation mit den Förderschullehrkräften ein?

10. Wie gestalten Sie die Zusammenarbeit mit den Eltern (der Schüler/innen mit sonderpädagogischem Förderbedarf)?

11. In welchen Bereichen sehen Sie an Ihrer Schule/für sich persönlich Fortbildungsbedarf im Zusammenhang mit integrativem Unterricht?

12. Wie schätzen Sie die Haltung der Lehrkräfte an Ihrer Schule im Hinblick auf die Entwicklung inklusiver Bildungsangebote ein?

13. Welche Tendenzen sehen Sie an Ihrer Schule hinsichtlich der Weiterentwicklung des gemeinsamen Unterrichts?

14. Welche Probleme und welche Chancen erkennen Sie mit Blick auf die Zukunft inklusiver Bildungsangebote? 


\section{Fragebogen für Regelschullehrer/innen - Grundschule}

Fragen zu Ihrer Person und Ihren Integrationsschüler/inne/n

\begin{tabular}{|c|c|c|c|c|c|}
\hline 1 & Sind Sie weiblich oder männlich? & $\square$ männlich & \multicolumn{2}{|c|}{$\square$ weiblich } & \\
\hline 2 & Wie alt sind Sie? & \multicolumn{4}{|c|}{ Ich bin___ Jahre alt. } \\
\hline 3 & $\begin{array}{l}\text { Seit wann arbeiten Sie mit Schüler/inne/n } \\
\text { mit sonderpädagogischem Förderbedarf? }\end{array}$ & $\begin{array}{l}\square \text { seit einigen } \\
\text { Monaten }\end{array}$ & \multicolumn{2}{|c|}{$\begin{array}{l}\square \text { seit etwa } \\
\text { einem Jahr }\end{array}$} & $\square$ seit \\
\hline 4 & $\begin{array}{l}\text { Seit wann arbeiten Sie in dieser } \\
\text { Grundschule? }\end{array}$ & $\begin{array}{l}\square \text { seit einigen } \\
\text { Monaten }\end{array}$ & \multicolumn{2}{|c|}{$\begin{array}{l}\square \text { seit etwa } \\
\text { einem Jahr }\end{array}$} & $\square$ seit \\
\hline 5 & Arbeiten Sie Vollzeit oder Teilzeit? & $\square$ Vollzeit & \multicolumn{2}{|c|}{$\square$ Teilzeit } & \\
\hline 6 & $\begin{array}{l}\text { In welcher Klassenstufe unterrichten Sie als } \\
\text { Klassenleitung? }\end{array}$ & $\square 1$ & \multicolumn{2}{|c|}{$\square 3$} & 4 \\
\hline 7 & $\begin{array}{l}\text { Welche Fächer unterrichten Sie als Klassen- } \\
\text { leitung? }\end{array}$ & \multicolumn{4}{|c|}{$\begin{array}{l}\square \text { Deutsch } \\
\square \text { Mathe } \\
\square \text { weitere Fächer und zwar }\end{array}$} \\
\hline 8 & Wie viele Schüler/innen sind in Ihrer Klasse? & \multicolumn{4}{|l|}{ Anzahl ___ } \\
\hline 9 & $\begin{array}{l}\text { Welchen hauptsächlichen sonderpädago- } \\
\text { gischen Förderbedarf haben die Schüler/ } \\
\text { innen, die Sie unterrichten? } \\
\text { Sie können mehrere Möglichkeiten } \\
\text { ankreuzen. }\end{array}$ & \multicolumn{2}{|c|}{$\begin{array}{l}\square \text { Lernen, } \\
\square \text { Verhalten, } \\
\square \text { Sprache, } \\
\square \text { Motorik, } \\
\square \text { Sehen, } \\
\square \text { Hören, } \\
\square \text { geistige Entwicklung, }\end{array}$} & \multicolumn{2}{|c|}{$\begin{array}{l}\text { Anzahl } \square \text { Anzahl } \square \\
\text { Anzahl } \square \\
\text { Anzahl } \square \\
\text { Anzahl } \square \\
\text { Anzahl } \square \\
\text { Anzahl }\end{array}$} \\
\hline 10 & $\begin{array}{l}\text { Benötigen diese Schüler/innen in der } \\
\text { Schule besondere Hilfsmittel? } \\
\text { Sie können mehrere Möglichkeiten ankreu- } \\
\text { zen. }\end{array}$ & \multicolumn{4}{|c|}{$\begin{array}{l}\square \text { andere Bücher } \\
\square \text { andere Arbeitshefte } \\
\square \text { handlungsorientiertes Material } \\
\square \text { eine Mikroportanlage } \\
\square \text { einen großen Bildschirm } \\
\square \text { einen barrierefreien Zugang } \\
\square \text { besondere Möbel } \\
\square \text { etwas anderes und zwar }\end{array}$} \\
\hline
\end{tabular}


Aussagen zur Schule

\begin{tabular}{|c|c|c|c|c|}
\hline Bewerten Sie folgende Aussagen zur Schule: & $\begin{array}{l}\text { stimme } \\
\text { sehr zu }\end{array}$ & $\begin{array}{l}\text { stimme } \\
\text { eher zu }\end{array}$ & $\begin{array}{l}\text { stimme } \\
\text { eher nicht } \\
\text { zu }\end{array}$ & $\begin{array}{l}\text { stimme } \\
\text { gar nicht } \\
\mathrm{zu}\end{array}$ \\
\hline $\begin{array}{l}11 \text { Die Schüler/innen mit Förderbedarf werden } \\
\text { von den anderen Schüler/inne/n akzeptiert. }\end{array}$ & $\square$ & $\square$ & $\square$ & $\square$ \\
\hline $\begin{array}{l}12 \text { Die Schüler/innen mit Förderbedarf haben } \\
\text { Freunde/Freundinnen in der Schule. }\end{array}$ & $\square$ & $\square$ & $\square$ & $\square$ \\
\hline $\begin{array}{l}13 \text { Die Schüler/innen mit Förderbedarf haben auch } \\
\text { nachmittags Kontakte zu Mitschüler/inne/n. }\end{array}$ & $\square$ & $\square$ & $\square$ & $\square$ \\
\hline $\begin{array}{l}14 \text { Die Schüler/innen mit Förderbedarf werden } \\
\text { von den Lehrer/inne/n akzeptiert. }\end{array}$ & $\square$ & $\square$ & $\square$ & $\square$ \\
\hline $\begin{array}{l}15 \text { Die Lehrer/innen sind offen für die Integration } \\
\text { von Kindern mit Förderbedarf. }\end{array}$ & $\square$ & $\square$ & $\square$ & $\square$ \\
\hline $\begin{array}{l}16 \text { Die bisherige Form der integrativen Unterrich- } \\
\text { tung von Schüler/inne/n mit sonderpädagogi- } \\
\text { schem Förderbedarf erfährt in dieser Schule ein } \\
\text { hohes Maß an Akzeptanz. }\end{array}$ & $\square$ & $\square$ & $\square$ & $\square$ \\
\hline $\begin{array}{l}17 \text { Die Kontinuität der Tätigkeit von Förderschul- } \\
\text { lehrkräften in Regelschulen trägt zur Akzeptanz } \\
\text { der integrativen Unterrichtung bei. }\end{array}$ & $\square$ & $\square$ & $\square$ & $\square$ \\
\hline $\begin{array}{l}18 \text { Regel- und Förderschullehrkräfte arbeiten auf } \\
\text { Augenhöhe miteinander. }\end{array}$ & $\square$ & $\square$ & $\square$ & $\square$ \\
\hline $\begin{array}{l}19 \text { Die Lehrer/innen dieser Schule nutzen die } \\
\text { Ressourcen, die die Förderschullehrkräfte } \\
\text { einbringen. }\end{array}$ & $\square$ & $\square$ & $\square$ & $\square$ \\
\hline $\begin{array}{l}20 \text { Die Lehrer/innen dieser Grundschule fördern } \\
\text { die Schüler/innen mit Förderbedarf so gut wie } \\
\text { möglich. }\end{array}$ & $\square$ & $\square$ & $\square$ & $\square$ \\
\hline $\begin{array}{l}21 \text { Die Schulleitung ist offen für die Integration } \\
\text { von Kindern mit Förderbedarf. }\end{array}$ & $\square$ & $\square$ & $\square$ & $\square$ \\
\hline
\end{tabular}

22 Das möchte ich zu dieser Schule noch ergänzen: 
Fragen zur Ausstattung des Klassenraums/der Schule

\begin{tabular}{|c|c|c|c|c|c|}
\hline 23 & \multicolumn{2}{|c|}{$\begin{array}{l}\text { Sind die Größen der Klassenräume angemessen } \\
\text { im Hinblick auf die Größe der Klassen? }\end{array}$} & $\square$ ja & $\square$ teilweise & $\square$ nein \\
\hline 24 & \multicolumn{2}{|c|}{$\begin{array}{l}\text { Lassen die Klassenräume eine flexible Gestal- } \\
\text { tung zu? (Bsp.: Ruheecke) }\end{array}$} & $\square$ ja & $\square$ teilweise & $\square$ nein \\
\hline 25 & \multicolumn{2}{|l|}{$\begin{array}{l}\text { Können Sie einen Differenzierungsraum } \\
\text { nutzen? }\end{array}$} & $\square$ ja & $\square$ teilweise & $\square$ nein \\
\hline 26 & \multicolumn{2}{|c|}{$\begin{array}{l}\text { Sind die Klassenräume mit Differenzierungs- } \\
\text { material ausgestattet? }\end{array}$} & $\square$ ja & $\square$ teilweise & $\square$ nein \\
\hline 27 & \multicolumn{2}{|c|}{$\begin{array}{l}\text { Ist die Schule mit Differenzierungsmaterial } \\
\text { ausgestattet? }\end{array}$} & $\square$ ja & $\square$ teilweise & $\square$ nein \\
\hline \multicolumn{6}{|c|}{ Fragen zur Schule } \\
\hline 28 & \multicolumn{2}{|l|}{ Verfügt die Schule über ein Leitbild? } & $\square$ ja & $\square$ nein & $\begin{array}{c}\square \text { weiß ich } \\
\text { nicht }\end{array}$ \\
\hline 29 & \multicolumn{2}{|c|}{$\begin{array}{l}\text { Verfügt die Schule über ein Schulprogramm, } \\
\text { in dem die Integration von Schüler/inne/n mit } \\
\text { sonderpädagogischem Förderbedarf Thema ist? }\end{array}$} & $\square$ ja & $\square$ nein & $\begin{array}{l}\square \text { weiß ich } \\
\text { nicht }\end{array}$ \\
\hline \multirow[t]{4}{*}{30} & \multirow[t]{2}{*}{$\begin{array}{l}\text { In welcher Form hat sich die Schule } \\
\text { bislang mit dem Thema der Unter- } \\
\text { richtung von Schüler/inne/n mit } \\
\text { Förderbedarf beschäftigt? }\end{array}$} & \multicolumn{4}{|c|}{$\begin{array}{l}\square \text { Dienstbesprechungen } \\
\square \text { Gesamtkonferenzen } \\
\square \text { schulinterne Fortbildungen am Nachmittag zu }\end{array}$} \\
\hline & & \multicolumn{4}{|c|}{$\square$ Pädagogische Tage zu } \\
\hline & \multirow[t]{2}{*}{$\begin{array}{l}\text { Sie können mehrere Antworten an- } \\
\text { kreuzen! }\end{array}$} & \multicolumn{4}{|c|}{$\square$ Treffen mit außerschulischen Diensten wie } \\
\hline & & $\square$ etwa & s ande & & \\
\hline
\end{tabular}

\section{Fragen zu den Klassen}

\begin{tabular}{|c|c|c|c|c|c|}
\hline 31 & $\begin{array}{l}\text { Wie schätzen Sie die sozialen Bezie- } \\
\text { hungen der Schüler/innen untereinan- } \\
\text { der ein? }\end{array}$ & $\square$ sehr gut & $\square$ gut & $\begin{array}{l}\square \text { weniger } \\
\text { gut }\end{array}$ & $\begin{array}{l}\square \text { gar nicht } \\
\text { gut }\end{array}$ \\
\hline 32 & $\begin{array}{l}\text { Wie gut halten sich die Schüler/innen } \\
\text { an die Regeln in Ihrer Klasse? }\end{array}$ & $\square$ sehr gut & $\square$ gut & $\begin{array}{l}\square \text { weniger } \\
\text { gut }\end{array}$ & $\begin{array}{l}\square \text { gar nicht } \\
\text { gut }\end{array}$ \\
\hline 33 & $\begin{array}{l}\text { Wie ruhig ist es in Ihrer Klasse norma- } \\
\text { lerweise? }\end{array}$ & $\begin{array}{l}\text { sehr } \\
\text { ruhig }\end{array}$ & $\square$ ruhig & $\begin{array}{l}\square \text { weniger } \\
\text { ruhig }\end{array}$ & $\begin{array}{l}\square \text { gar nicht } \\
\text { ruhig }\end{array}$ \\
\hline 34 & $\begin{array}{l}\text { Wie gut können sich die Schüler/in- } \\
\text { nen in Ihrer Klasse konzentrieren? }\end{array}$ & $\square$ sehr gut & $\square$ gut & $\begin{array}{l}\square \text { weniger } \\
\text { gut }\end{array}$ & $\begin{array}{l}\square \text { gar nicht } \\
\text { gut }\end{array}$ \\
\hline 35 & $\begin{array}{l}\text { Wie gut sorgen Sie selbst Ihrer Mei- } \\
\text { nung nach für Ruhe? }\end{array}$ & $\square$ sehr gut & $\square$ gut & $\begin{array}{l}\square \text { weniger } \\
\text { gut }\end{array}$ & $\begin{array}{l}\square \text { gar nicht } \\
\text { gut }\end{array}$ \\
\hline 36 & $\begin{array}{l}\text { Wie konsequent reagieren Sie auf } \\
\text { Fehlverhalten? }\end{array}$ & $\begin{array}{l}\square \text { sehr kon- } \\
\text { sequent }\end{array}$ & $\begin{array}{l}\square \text { konse- } \\
\text { quent }\end{array}$ & $\begin{array}{l}\square \text { weniger } \\
\text { konse- } \\
\text { quent }\end{array}$ & $\begin{array}{l}\square \text { gar nicht } \\
\text { konse- } \\
\text { quent }\end{array}$ \\
\hline 37 & $\begin{array}{l}\text { Wie sicher fühlen Sie sich im Umgang } \\
\text { mit verhaltensauffälligen Schüler/ } \\
\text { inne/n? }\end{array}$ & $\begin{array}{l}\square \text { sehr } \\
\text { sicher }\end{array}$ & $\square$ sicher & $\begin{array}{l}\square \text { weniger } \\
\text { sicher }\end{array}$ & $\begin{array}{l}\square \text { gar nicht } \\
\text { sicher }\end{array}$ \\
\hline
\end{tabular}


Fragen zur Zusammenarbeit mit Eltern

38 Welche Formen der Zusammenarbeit mit den Eltern der Schüler/innen mit sonderpädagogischem Förderbedarf nutzen Sie?

\begin{tabular}{lcccc} 
& $\begin{array}{c}\text { sehr } \\
\text { häufig }\end{array}$ & häufig & $\begin{array}{c}\text { eher } \\
\text { selten }\end{array}$ & gar nicht \\
\hline$\square$ Telefonate & $\square$ & $\square$ & $\square$ & $\square$ \\
\hline$\square$ E-Mail & $\square$ & $\square$ & $\square$ & $\square$ \\
\hline$\square$ Gesprächstermine & $\square$ & $\square$ & $\square$ & $\square$ \\
\hline$\square$ Mitteilungsheft & $\square$ & $\square$ & $\square$ & $\square$ \\
\hline$\square$ etwas anderes und zwar & & & \\
\hline Rückmeldung zu Lernfortschritten & & \\
$\square$ Probleme der Schüler/innen & & \\
$\square$ etwas anderes und zwar
\end{tabular}

Sie können mehrere Möglichkeiten ankreuzen.

\section{Fragen zur Zusammenarbeit mit außerschulischen Diensten}

40 Mit welchen außerschulischen Diensten arbeiten Sie zusammen?

\begin{tabular}{lcccc} 
& $\begin{array}{c}\text { sehr } \\
\text { häufig }\end{array}$ & häufig & $\begin{array}{c}\text { eher } \\
\text { selten }\end{array}$ & gar nicht \\
\hline$\square$ Jugendamt & $\square$ & $\square$ & $\square$ & $\square$ \\
\hline $\begin{array}{c}\text { Schulpsychologi- } \\
\text { scher Dienst }\end{array}$ & $\square$ & $\square$ & $\square$ & $\square$ \\
\hline $\begin{array}{l}\text { Schulärztlicher } \\
\text { Dienst }\end{array}$ & $\square$ & $\square$ & $\square$ & $\square$ \\
\hline$\square$ etwas anderem und zwar & & & \\
\hline
\end{tabular}

Fragen zum Unterricht

41 Wie hoch ist Ihrer Einschätzung nach das Maß an klarer Strukturierung des Unterrichts?

\begin{tabular}{|c|c|c|c|}
\hline $\begin{array}{r}\square \text { sehr } \\
\text { hoch }\end{array}$ & $\square$ hoch & $\begin{array}{l}\text { we- } \\
\text { niger } \\
\text { hoch }\end{array}$ & $\begin{array}{l}\square \text { gar } \\
\text { nicht } \\
\text { hoch }\end{array}$ \\
\hline $\begin{array}{l}\text { sehr } \\
\text { häufig }\end{array}$ & häufig & $\begin{array}{l}\text { eher } \\
\text { selten }\end{array}$ & aich \\
\hline
\end{tabular}

$42 \mathrm{Zu}$ welchen Anteilen setzen Sie die folgenden Formen im Unterricht um?

\begin{tabular}{lcccc} 
& & & hoch & hoch \\
& $\begin{array}{c}\text { sehr } \\
\text { häufig }\end{array}$ & häufig & $\begin{array}{c}\text { eher } \\
\text { selten }\end{array}$ & gar nicht \\
\hline Frontalunterricht & $\square$ & $\square$ & $\square$ & $\square$ \\
\hline Einzelarbeit & $\square$ & $\square$ & $\square$ & $\square$ \\
\hline Partnerarbeit & $\square$ & $\square$ & $\square$ & $\square$ \\
\hline Gruppenarbeit & $\square$ & $\square$ & $\square$ & $\square$ \\
\hline Stationenarbeit & $\square$ & $\square$ & $\square$ & $\square$ \\
\hline Lerntheke & $\square$ & $\square$ & $\square$ & $\square$ \\
\hline Wochenplanarbeit & $\square$ & $\square$ & $\square$ & $\square$ \\
\hline $\begin{array}{l}\text { Binnendifferenzie- } \\
\text { rung nach Menge der } \\
\text { Aufgaben }\end{array}$ & $\square$ & $\square$ & $\square$ & $\square$ \\
\hline $\begin{array}{l}\text { Binnendifferenzierung } \\
\text { nach Qualität der } \\
\text { Aufgaben }\end{array}$ & $\square$ & $\square$ & $\square$ & $\square$ \\
\hline
\end{tabular}




\section{Fragen zum integrativen Unterricht}

\begin{tabular}{|c|c|c|c|c|c|c|}
\hline \multirow{9}{*}{\multicolumn{2}{|c|}{$\begin{array}{l}43 \text { Welche Tätigkeiten } \\
\text { übernehmen Sie } \\
\text { gemeinsam mit der } \\
\text { Integrationslehrerin/ } \\
\text { dem Integrationsleh- } \\
\text { rer? }\end{array}$}} & & sehr häufig & \multirow{2}{*}{$\begin{array}{c}\text { häufig } \\
\square\end{array}$} & \multirow{2}{*}{$\begin{array}{c}\text { eher selten } \\
\square\end{array}$} & \multirow{2}{*}{$\begin{array}{c}\text { gar nich } \\
\square\end{array}$} \\
\hline & & individuelle Förderung & $\square$ & & & \\
\hline & & $\begin{array}{l}\text { Erstellung von Material } \\
\text { für Schüler/innen mit } \\
\text { Förderbedarf }\end{array}$ & $\square$ & $\square$ & $\square$ & $\square$ \\
\hline & & Lernstandsdiagnostik & $\square$ & $\square$ & $\square$ & $\square$ \\
\hline & & $\begin{array}{l}\text { gemeinsame Unter- } \\
\text { richtsvorbereitung }\end{array}$ & $\square$ & $\square$ & $\square$ & $\square$ \\
\hline & & $\begin{array}{l}\text { gemeinsame Unter- } \\
\text { richtsdurchführung }\end{array}$ & $\square$ & $\square$ & $\square$ & $\square$ \\
\hline & & $\begin{array}{l}\text { gemeinsame Unter- } \\
\text { richtsreflexion }\end{array}$ & $\square$ & $\square$ & $\square$ & $\square$ \\
\hline & & $\begin{array}{l}\text { Vernetzungsarbeit } \\
\text { mit außerschulischen } \\
\text { Diensten }\end{array}$ & $\square$ & $\square$ & $\square$ & $\square$ \\
\hline & & etwas anderes und zwar & & & & \\
\hline
\end{tabular}

\begin{tabular}{|c|c|c|}
\hline 44 & $\begin{array}{l}\text { Meistens lernen meine Integrationsschüler/ } \\
\text { innen mit der Integrationslehrerin/dem Integ- } \\
\text { rationslehrer: }\end{array}$ & $\begin{array}{l}\square \text { außerhalb der Klasse } \\
\square \text { innerhalb der Klasse } \\
\square \text { das ist unterschiedlich }\end{array}$ \\
\hline 45 & $\begin{array}{l}\text { Wenn die Schüler/innen mit Förderbedarf } \\
\text { außerhalb der Klasse gefördert werden, erfolgt } \\
\text { diese Förderung meistens: }\end{array}$ & $\begin{array}{l}\square \text { als Einzelförderung } \\
\square \text { als Gruppenförderung } \\
\square \text { das ist unterschiedlich }\end{array}$ \\
\hline 46 & $\begin{array}{l}\text { Ich halte die Form der individuellen Förderung } \\
\text { außerhalb der Klasse für: }\end{array}$ & $\begin{array}{cccc}\text { sehr } & \square \text { gut } & \square \text { weniger } & \square \text { gar nicht } \\
\text { gut } & & \text { gut } & \text { gut }\end{array}$ \\
\hline 47 & ...weil & \\
\hline 48 & $\begin{array}{ll}\text { Der Integrationslehrer/ } & \square \text { 1 Stunde } \\
\text { die Integrationslehrerin } & \square \text { 2Stunden } \\
\text { kommt in der Woche } & \square \text { mehr als 2 Stunder } \\
\text { je Schüler/in durch- } & \\
\text { schnittlich: } & \\
\end{array}$ & und zwar __ Stunden \\
\hline 49 & $\begin{array}{l}\square \text { zu viel } \\
\square \text { genug } \\
\square \text { zu wenig }\end{array}$ & \\
\hline
\end{tabular}


Fragen zum integrativen Unterricht

50 Wünschenswert ist $\quad \square$ die feste Zuteilung einer Integrationslehrkraft für mehrere Jahre

für die integrative $\quad \square$ eine Doppelbesetzung in Deutsch und Mathematik

Unterrichtung meiner $\square$ eine Doppelbesetzung in allen Stunden

Meinung nach:

$\square$ etwas anderes und zwar:

51 Die Integrations- $\quad \square$ mit denselben Materialien wie die anderen Schüler

schüler/innen, die $\quad \square$ mit anderen Büchern

zieldifferent unterrich- $\square$ mit anderen Arbeitsheften

tet werden, arbeiten im $\quad \square$ mit anderen Arbeitsblättern

Fach Deutsch $\quad \square$ mit anderen Klassenarbeiten

Sie können mehrere Mög-

$\square$ weiß ich nicht

lichkeiten ankreuzen.

52 Die Integrationsschüler/ $\square$ mit denselben Materialien wie die anderen Schüler

innen, die zieldifferent $\square$ mit anderen Büchern

unterrichtet werden, $\square$ mit anderen Arbeitsheften

arbeiten im Fach Mathe- $\square$ mit anderen Arbeitsblättern

matik $\square$ mit anderen Klassenarbeiten

Sie können mehrere Mög-

$\square$ weiß ich nicht

lichkeiten ankreuzen.

53 Für die Integrationsschüler/innen gibt es einen individuellen

nur für die zieldiffe-

rent unterrichteten $\square$ nein

Förderplan.

Schüler/innen

54 Zu den Lernfortschritten gebe ich den Schüler/ inne/n mit Förderbedarf

$\square$ häufig

weniger häufig

gar nicht

Rückmeldung.

55 Und zwar in Form $\square$ Hinweisen im Mitteilungsheft

von

$\square$ Beratungsgesprächen

$\square$ Entwicklungsberichten

$\square$ Beiblättern zum Zeugnis

$\square$ etwas anderem und zwar 
Das denke ich über die Förderung der Schüler/innen mit sonderpädagogischem Förderbedarf:

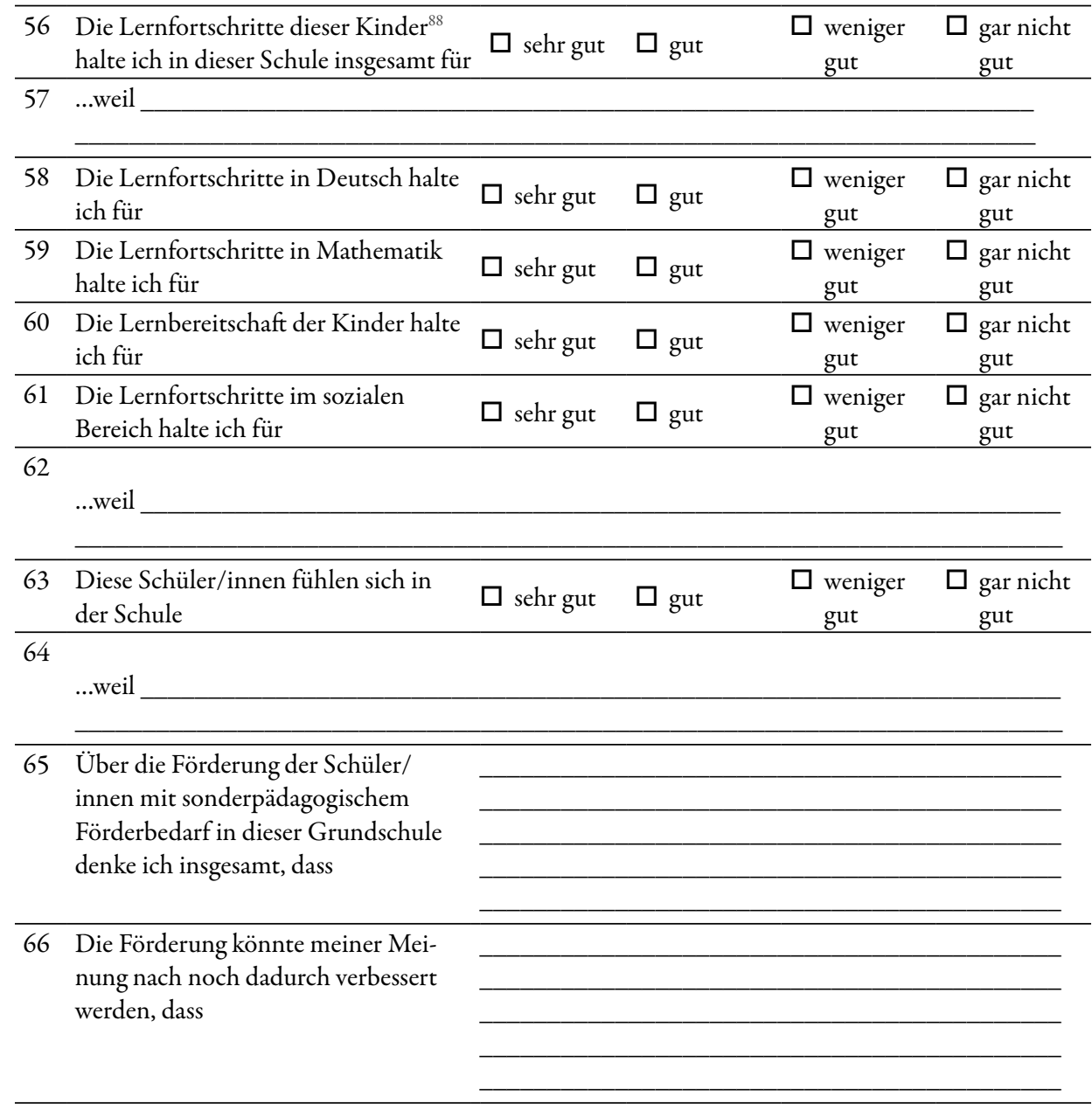

88 Bei dieser Frage geht es um eine Gesamteinschätzung hinsichtlich der Lernfortschritte von Kindern mit sonderpädagogischem Förderbedarf in Ihrer Klasse, auch wenn klar ist, dass diese von Kind zu Kind sehr unterschiedlich sein können. 


\section{Fragen zu Fortbildungen}

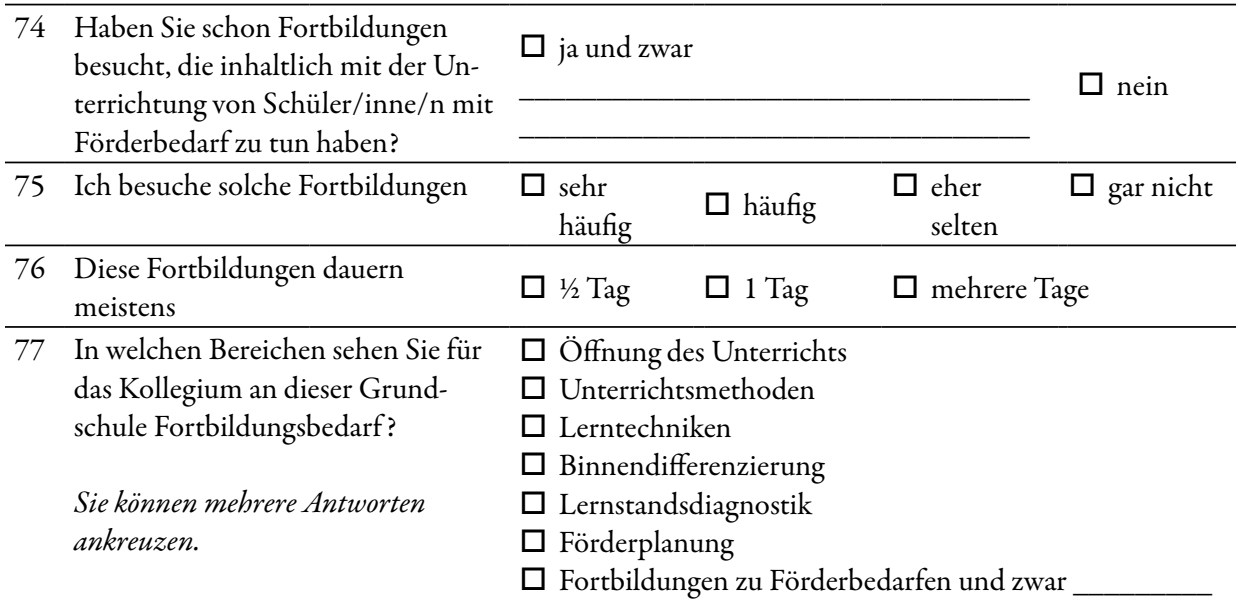

etwas anderes und zwar

78 In welchen Bereichen sehen Sie für $\square$ Öffnung des Unterrichts

sich selbst Fortbildungsbedarf? $\quad \square$ Unterrichtsmethoden

$\begin{array}{ll}\text { Sie können mehrere Antworten } & \square \text { Lerntechniken } \\ \text { Binnendifferenzierung }\end{array}$

ankreuzen. $\square$ Lernstandsdiagnostik

$\square$ Förderplanung

$\square$ Fortbildungen zu Förderbedarfen und zwar

etwas anderes und zwar 
Aussagen zur (Zukunft der) integrativen Unterrichtung

\begin{tabular}{|c|c|c|c|c|c|}
\hline \multicolumn{2}{|r|}{ Bewerten Sie die folgenden Aussagen. } & \multirow{2}{*}{$\begin{array}{c}\text { stimme } \\
\text { sehr zu } \\
\square\end{array}$} & \multirow{2}{*}{$\begin{array}{c}\text { stimme } \\
\text { eher zu }\end{array}$} & \multirow{2}{*}{$\begin{array}{c}\text { stimme eher } \\
\text { nicht zu } \\
\square\end{array}$} & \multirow{2}{*}{$\begin{array}{c}\text { stimme gar } \\
\text { nicht zu } \\
\square\end{array}$} \\
\hline 79 & $\begin{array}{l}\text { Eine Klasse sollte nicht mehr als } 20 \text { Schüler/ } \\
\text { innen haben. }\end{array}$ & & & & \\
\hline 80 & $\begin{array}{l}\text { Das Elternwahlrecht zwischen Regelschule } \\
\text { und Förderschule sollte erhalten bleiben. }\end{array}$ & $\square$ & $\square$ & $\square$ & $\square$ \\
\hline 81 & $\begin{array}{l}\text { Wenn möglich sollten in einer Klasse } \\
\text { mehrere Schüler/innen mit Förderbedarf } \\
\text { unterrichtet werden. }\end{array}$ & $\square$ & $\square$ & $\square$ & $\square$ \\
\hline 82 & $\begin{array}{l}\text { In der Regelschule sollten auch Schüler/in- } \\
\text { nen mit schweren Behinderungen unterrich- } \\
\text { tet werden. }\end{array}$ & $\square$ & $\square$ & $\square$ & $\square$ \\
\hline 83 & $\begin{array}{l}\text { Es sollte darauf geachtet werden, dass nur } \\
\text { drei bis vier Schüler/innen mit Förderbedarf } \\
\text { in einer Klasse unterrichtet werden. }\end{array}$ & $\square$ & $\square$ & $\square$ & $\square$ \\
\hline 84 & $\begin{array}{l}\text { Die Kinder mit Förderbedarf sollten immer } \\
\text { innerhalb der Klasse gefördert werden. }\end{array}$ & $\square$ & $\square$ & $\square$ & $\square$ \\
\hline
\end{tabular}

Aussagen zur (Zukunft der) integrativen Unterrichtung

\begin{tabular}{lcccc}
\hline Bewerten Sie die folgenden Aussagen. & $\begin{array}{c}\text { stimme } \\
\text { sehr zu }\end{array}$ & $\begin{array}{c}\text { stimme } \\
\text { eher zu }\end{array}$ & $\begin{array}{c}\text { stimme eher } \\
\text { nicht zu }\end{array}$ & $\begin{array}{c}\text { stimme gar } \\
\text { nicht zu }\end{array}$ \\
\hline
\end{tabular}

85 Die integrative Unterrichtung von Kindern mit Förderbedarf in der Regelschule setzt ein hohes Engagement der Lehrkräfte voraus.

86 Das soziale Miteinander der Schüler/innen in einer Schule wird durch die integrative Unterrichtung verbessert.

87 Bei Bedarf sollten Kinder mit Förderbedarf in einzelnen Stunden individuell gefördert werden.

88 Die Unterrichtsverpflichtung von Lehrkräften, die Schüler/innen mit sonderpädagogischem Förderbedarf in ihren Klassen unterrichten, sollte reduziert werden.

89 Teamteaching sollte regelmäßig möglich sein.

90 Die Haltung der Lehrkräfte in dieser Schule ist der zukünftigen inklusiven Bildung gegenüber

eher zustimmend $\quad \square$ eher ablehnend

$91 \quad$...weil 
Was ist Ihre Meinung?

92 Was wünschen Sie sich mit Blick auf die Zukunft der inklusiven Unterrichtung?

93 In welchen Bereichen halten Sie eine Weiterentwicklung Ihrer eigenen Professionalität im Hinblick auf die inklusive Bildung für besonders wichtig?

Vielen Dank für Ihre Unterstützung! 
Welche Qualität hat das, was als schulische Integration bezeichnet wurde und inzwischen als schulische Inklusion bezeichnet wird? Wie sind inklusive Schulen organisiert? Wie arbeitet das Personal in diesen Schulen zusammen? Wie wird der gemeinsame Unterricht in diesen Schulen gestaltet? Welche Qualitätsbausteine spielen im Zusammenhang mit der Weiterentwicklung inklusiver Bildung eine wichtige Rolle? Um diese Fragen zu beantworten werden im Rahmen einer theoretischen Einbettung zunächst geschichtliche Aspekte schulischer Integration sowie unterschiedliche Perspektiven der Soziologie, Pädagogik und Sonderpädagogik auf die Begriffe Exklusion und Inklusion analysiert. Es folgt ein umfassender Überblick zum Forschungsstand für die Bereiche Organisations-, Personal- und Unterrichtsentwicklung an (inklusiven) Schulen. Am Beispiel des Saarlandes wird anhand einer qualitativen und einer quantitativen Studie untersucht, wie unterschiedliche Beteiligte - Schülerinnen und Schüler, Eltern, Regel- und Förderschullehrkräfte sowie Schulleitungen - die Qualität schulischer Integration wahrnehmen und einschätzen. In Form einer Zusammenschau aller Ergebnisse werden Schlussfolgerungen und Perspektiven für das deutsche Bildungssystem aufgezeigt. Hinsichtlich der in Schulen notwendigen Transformation wird deutlich, dass zwischen der Qualitätsentwicklung allgemein und der Weiterentwicklung inklusiver Bildung ein sehr enger Zusammenhang besteht.

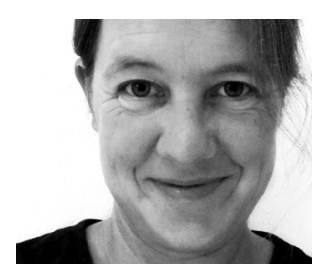

Die Autorin

Katharina Wirtz, Dr. phil., Jg. 1972, ist seit 2004 als Stellvertretende Schulleiterin bzw. Schulleiterin an verschiedenen Schulen mit dem Förderschwerpunkt Lernen tätig. Von 2012 bis 2016 war sie zudem als Lehrbeauftragte mit dem Schwerpunkt „Grundlagen inklusiver Bildung“ am Lehrstuhl „Pädagogische und Soziale Rehabilitation“ von Herrn Prof. Dr. Jennessen im Institut für Sonderpädagogik der Universität Koblenz-Landau beschäftigt.

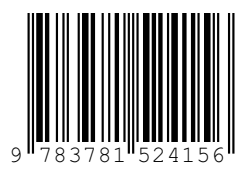

